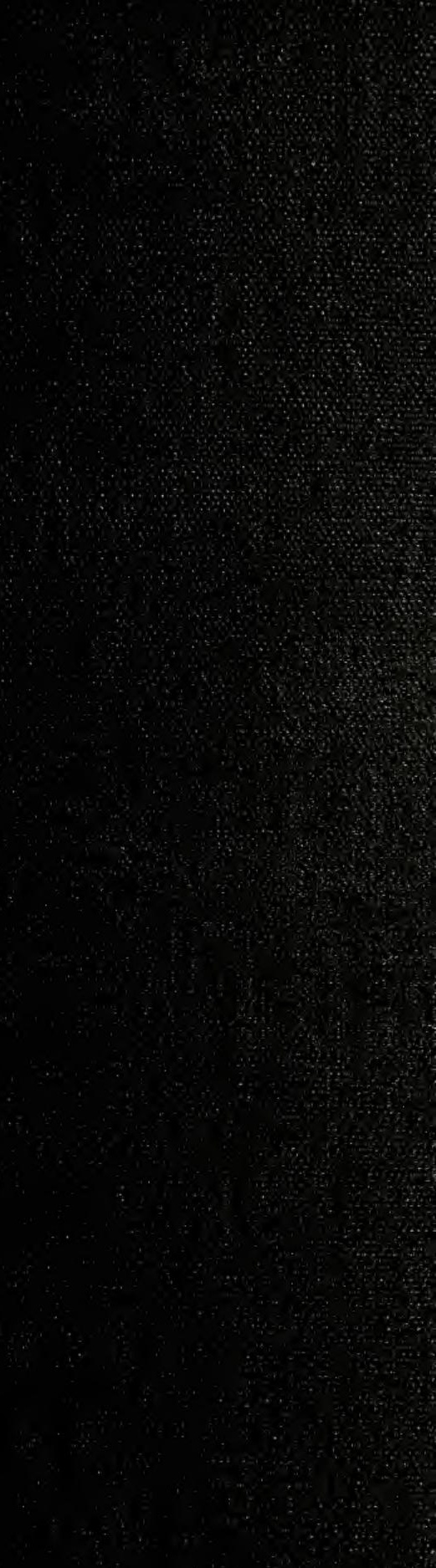




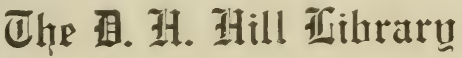

Worth Caralina State 隹niupraity

This book was presented by

MILTON M. LEONARD, D.V.M.

TO THE

VETERINARY MEDICAL LIBRARY 
THIS BOOK IS DUE ON THE DATE INDICATED BELOW AND IS SUBJECT TO AN OVERDUE FINE AS POSTED AT THE CIRCULATION DESK.

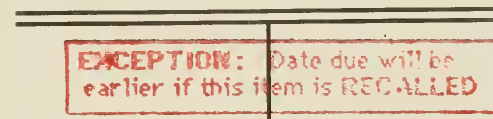







\section{SPECIAL PATHOLOGY AND THERAPEUTICS \\ OF THE \\ DISEASES OF DOMESTIC ANIMALS}

BY

DR. FRANZ HUTYRA

Professor of Infectious Diseases, AND

DR. JOSEF MAREK

Professor of Special Pathology and Therapy; both of the

Royal Veterinary College at Budapest

Authorized American Edition

From the Third Revised and Enlarged German Edition

JOHN R. MOHLER, V. M. D.

Chief of Pathological Division, U. S. Bureau of Animal Industry, AND

ADOLPH EICHHORN, D. V. S.

Senior Bacteriologist, Pathological Division, U. S. Bureau of Animal Industry, Washington, D. C.

EDITORS.

VOLUME I.

Infectious Diseases; Diseases of the Blood and of the Hematopoietic Organs; Diseases of Metabolism, of the Spleen, Urinary and Circulatory Organs.

With 198 illustrations in the text and $\mathbf{1 0}$ plates.

CHICAGO, U. S. A.

ALEXANDER EGER, PUblisher

1912. 


\section{Copyrighted at Washington, D. C.}

BY

ALEXANDER EGER

1912 


\section{TRANSLATORS OF THE FIRST VOLUME}

\section{JOHN R. MOHLER, V. MI. D.}

('hief of the Pathological Division, U. S. Bureau of Animal Industry

Washington, D. C.

ADOLPH EICHHORN, D. V. S.

Senior Bacteriologist, Pathological Division, U. S. Bureau of Animal Industry Washington, D. C.

\section{DR. PAUL FISCHER}

State Veterinarian, Columbus, Ohio

\section{DR. H. J. ACHARD}

of the Editorial Staff, American Journal of Clinical Medicine Chicago, Illinois 



\section{Authors' Preface to the Third German Edition}

Hardly two years have passed since the publication of the second edition of this work, which was accorded a very favorable reception by veterinarians. While the arrangement of the earlier editions as well as the former division of labor (Infectious diseases written by Hutyra, Organic diseases by Marek, each working independently) is maintained it again became necessary in this latest edition to add a few new chapters, to supplement the text and even to rewrite it in some portions, in order to do proper justice to the results of the most recent investigations in the field of internal veterinary medicine and of comparative pathology.

The continued and active investigations in immunology produce always new scientific facts and views which are of great importance to the practitioner. This is particularly true of the allergic reactions, of the sero-diagnostic methods, and of the development of methods for protective immumization as well as their practical valuation and application; all these subjects have been considered more especially in the chapters on tuberculosis, glanders, infectious abortion, and swine plague.

The section on protozoal diseases was carefully rewritten. Aside from their great economic importance for tropical countries, these diseases may claim a general interest, owing to the splendid results of chemotherapy, because they permit a very promising outlook for the future treatment of other diseases as well, and lead our therapentic endeavor into new paths.

Aside from a few diseases of minor importance (Malta fever, Three Days' Disease, etc.) some more important diseases were included, viz., Leukemia of chickens, Dochmiasis and Oesophagostomiasis, Enzootic Cretinism in animals, Serum diseases and Necrobacillosis; the chapters on Hemorrhagic Septicemia 
of sheep, Diphtheria and Contagious Epithelioma of fowls, Scurvy, Coecidial Dysentery of eattle, Enzootic Paralysis of the spinal cord in horses, Paralysis of the bladder and the Psychoses have been rewritten entirely. Finally essential ehanges will he found in the chapter's on Speeific P'aratuberculous Enteritis of eattle, the parasitic diseases and the skin diseases; also in the therapentic portions of most chapters.

Great eare has again been taken with the illustrations. Some less good illustrations in the earlier editions were replaced ly new ones, and the number of illustrations in the text (some of them in color's) has been inereased from 319 to 361 , that of the plates (which are mostly in colors) from 6 to 15 . TVe wish to express our acknowledgments to Messrs. Ladislaus 'T'atz and Leopold Kalmár for the colored designs, and to Messrs. Funen Raitsits and Eugen Schwamner for the photographs and drawings.

In order not to enlarge the already considerable size of the work, the text was condensed wherever possible and small type was made use of more freely. In consequenee this edition contains only five signatures more than the seeond, in spite of the many additions and alterations. We have persisted in our ilim to produce a work which should reflect the present state of veterinary medicine as faithfully as possible and should also satisfy the requirements, both of veterinary practitioners and of investigators and human practitioners who desire information on the problems of comparative pathology. The favorable reeeption aceorded to the second edition permits us to hope that the present one will be received with like favor.

The book has recently been published in the Italian language ancl a Russian edition is being prepared.

While this erlition was in press, the publishers suffered the loss of the foumder of the house, a loss which we deeply regret. We are indebted to the publishers for their courtesy in meeting our wishes in the preparation of the work. 


\section{Editors' Preface}

Hutyra \& Marek's Spezielle Pathologie und Therapie der Haustiere is generally recognized as the most comprehensive and anthoritative treatise on the theory and practice of reterinary medicine in Germany, Austria and Hungary, and has been adopted as a textbook by all the veterinary colleges in these countries, as well as in Switzerland.

The favorable reception of previous editions in foreign languages has convinced us that the work has found faror with the professions abroad, and the present edition, which has been prepared in response to a frequently expressed desire for such a translation, should prove equally valuable to the Englishspeaking veterinarian. The chapter on infectious diseases will also appeal to physicians and sanitarians who have to deal with the diseases communicable from animals to man, and it may reasonably be anticipated that the work as a whole will serve as an authoritative guide, and also as an admirable textbook and a standard of reference for students and practitioners, especially those who graduated before modern science had thrown its light upon the etiology of disease.

We wish to express sincere thanks to our eminent collaborators for their able assistance in translating portions of this work, and to Drs. Henry .J. Washburn and Jacol Traum, of Washington, D. C., for their aid in proofreading and in the preparation of the index. Acknowledgment is also due the publisher, Mr. Alexander Eger, for his uniform courtesy and assiștance in many ways.

Washington, D. C., Aug. 16, 1912.

MOHLER \& FICHHORN. 



\title{
Table of Contents
}

\author{
Infectious Diseases.
}

GROUP I.

Acute General Infectious Diseases.

PAGE

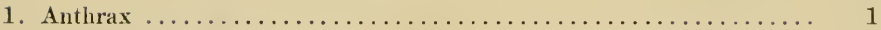

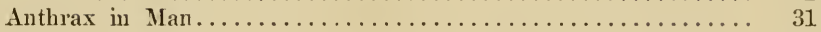

2. Malignant Edema ............................. 32

3. Blackleg .................................. 39

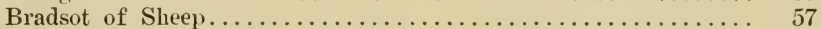

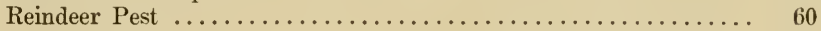

4. Swine Erysipelas ................................. 60

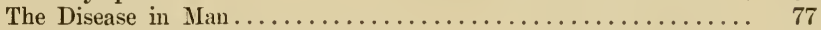

5. Hemorrhagic Septicemia ........................ 77

(a) Fowl Cholera $\ldots \ldots \ldots \ldots \ldots \ldots \ldots \ldots \ldots \ldots \ldots \ldots \ldots \ldots \ldots$

Other Diseases of Fowl of a Septicemic Cliaracter....... 97

Septicemic and Pyemic Affections of Rabbits........... 101

(b) Hemorrhagic Septicemia of Cattle................. 103

Septic Pleuro-pneumonia of Calves................ 111

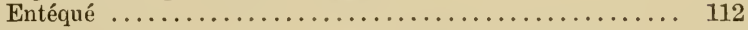

(c) Buffalo Disease .......................... 113

(d) Hemorrhagic Septicemia of Sheep................. 118

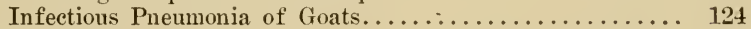

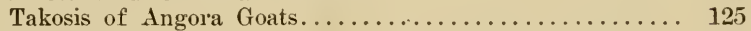

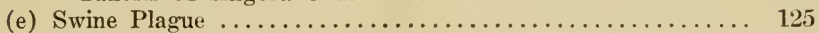

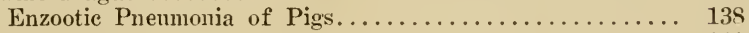

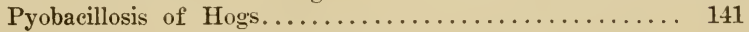

6. Septicemic Affections of Newborn Animals . . . . . . . . . . . . 145

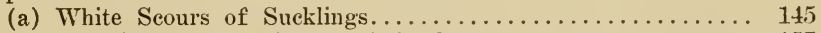

7. Influenza of Horses . . . . . . . . . . . . . . . . . . . . . . 170

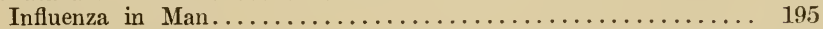

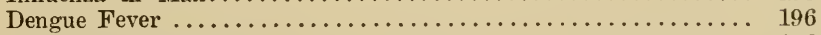

Three-days' Sickness $\ldots \ldots \ldots \ldots \ldots \ldots \ldots \ldots \ldots \ldots \ldots \ldots \ldots \ldots \ldots \ldots$

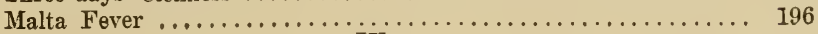


8. Distemper of Dogs.............................. 19

Distemper of Cats................................... 210

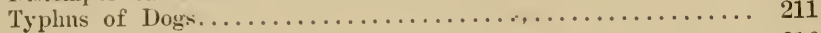

9. Purpura Hemorrhagiea ........................ 216

Purpura Hemorrhengica of ('attle.................... 232

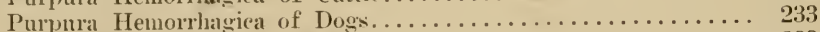

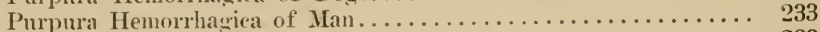

Searlatinoid of Horses. . . . . . . . . . . . . . . . . . . .

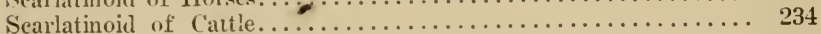

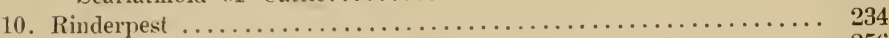

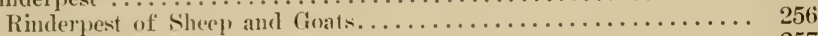

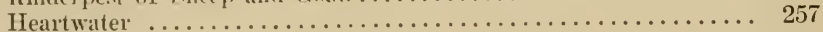

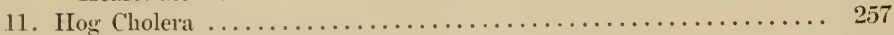

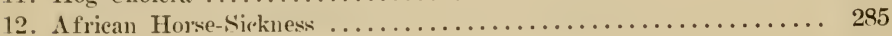

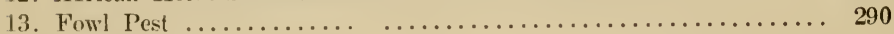

GROUP II.

Acute Exanthematous Infectious Diseases.

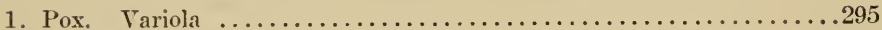

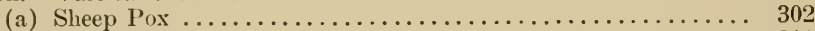

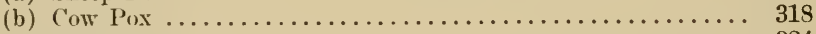

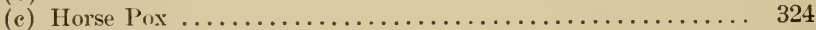

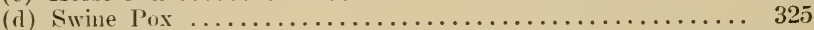

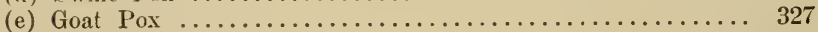

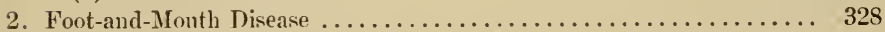

Foot-and-Month Disease in Horses, Carnivorons Animals and in

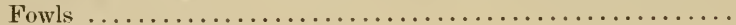

Foot-and-Mouth Disease in Man................... 355

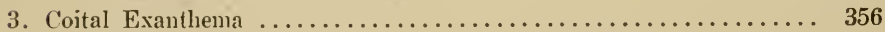

Infections sexual Disease in Rabbits and Hares............. 360

4. Infectious Pustular Stomatitis of Horses............... 360

\section{GROUP III.}

\section{Acute Infectious Diseases with Localization in Certain Organs.}

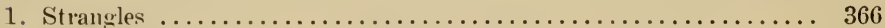

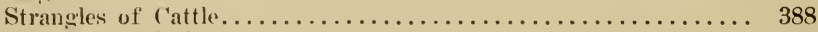

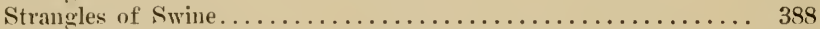

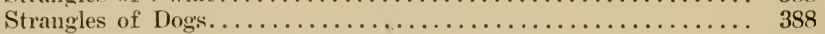

2. Contagious Pleuro-pneumonia of Cattle ............... 389

Infections Plenro-pmemumia of Goats.................. 406

Chronic Premunia of Sheep........................ 407

3. Malignant Catarrh of Cattle...................... 408

Croup of ('attle............................... 417

Catarrhal Fever of Sheep.......................... 418

4. Diphtheria of Calves............................. 419

Diphtheria of Other Mammalia..................... 423

5. Avian Diphtheria and Chicken Pox.................... 424

Diphtheritir Inflammation of the Eves in Ducks............. 436

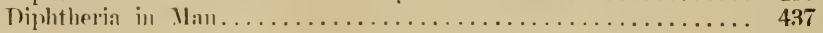


GROUP IV.

Infectious Diseases with Special Involvement of the Nervous System.

1. Tetanus

PAGE

2. Rabies

439

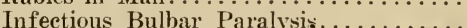

Epizootic of Cats with Servous i.

\section{GROUP V.}

\section{Chronic Infectious Diseases.}

1. Tubereulosis $\ldots \ldots \ldots \ldots \ldots \ldots \ldots \ldots \ldots \ldots \ldots \ldots \ldots \ldots \ldots \ldots . \ldots \ldots$

(a) Tuberculosis in Mammals..................... 498

Tubereulosis in Man .......................... 606

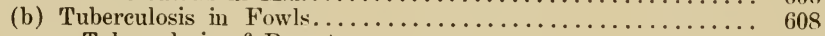

Tuberculosis of Parrots......................... 615

(e) Etiological Relation between Human and Animal Tuberenlosis titi Tubereulosis of Cold-Blooded Animals.............. 630

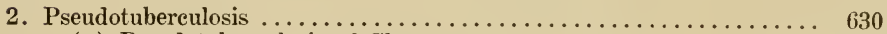

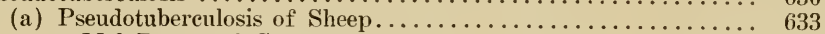

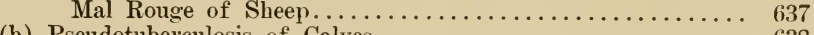

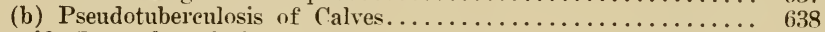

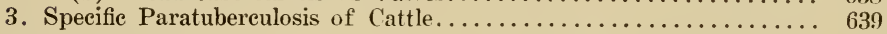

4. Actinomyeosis ................................... 642

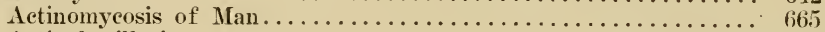

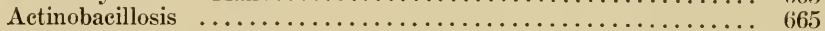

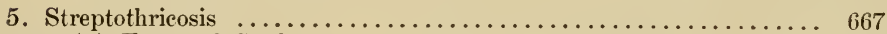

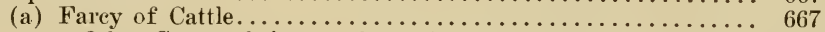

Other Streptothricoses of Ruminants and Horses.......... 669

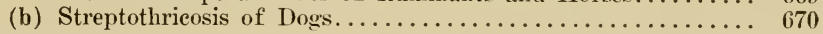

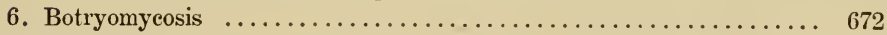

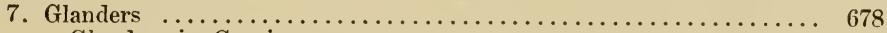

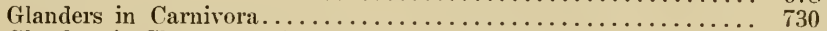

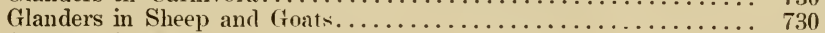

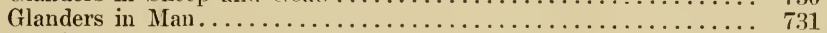

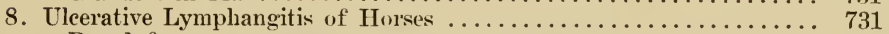

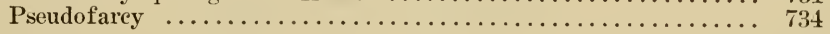

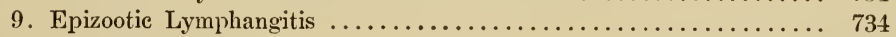

Malignant Fungus Disease of Horses. . . . . . . . . . . . . 739

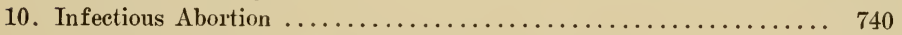

11. Infectious Vaginal Catarrh of Cattle................. 750

GROUP VI.

\section{Infectious Diseases Produced by Protozoa.}

1. Piroplasmoses $\ldots \ldots \ldots \ldots \ldots \ldots \ldots \ldots \ldots \ldots \ldots \ldots \ldots \ldots \ldots \ldots \ldots \ldots$

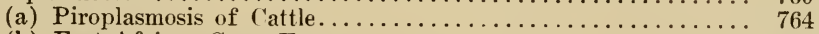

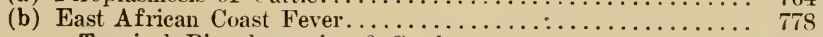

Tropical Piroplasmosis of Cattle................... 782

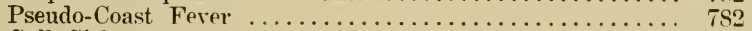

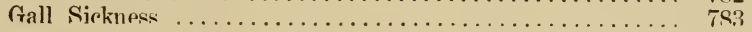


(c) Piroplasmosis of Horses...................... 784

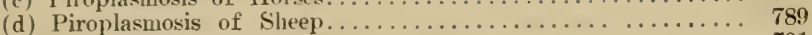

(e) Piroplasmosis of Dogs...................... 791

Piroplasma in Man....................... 796

Malaria of Man....................... 797

2. Trypanosome Diseases .......................... 797

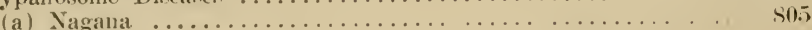

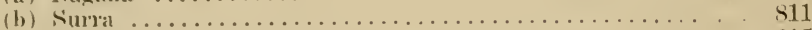

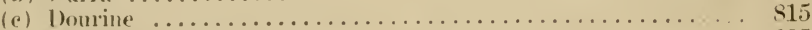

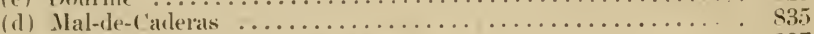

Other Trypanosome Diseases.................. 837

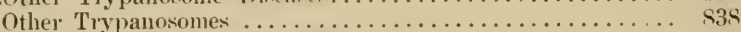

Trypanosome Affections in Man............... 839

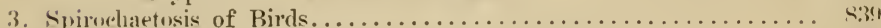

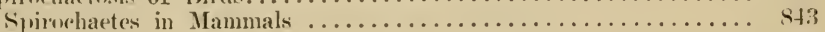

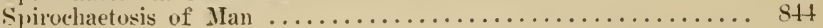

\section{Diseases of the Blood and Blood-Producing Organs.}

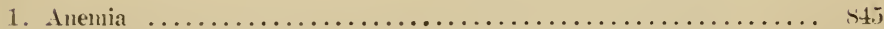

2. Infectious Anemia of Horses . . . . . . . . . . . . . . . . . 551

3. Progressive Pernicious Anemia...................... 8 8 . 3

Progressive Pernicious Anemia in Cattle................. Stis

Anemia of Sheep and Goats in Punjab................ sis

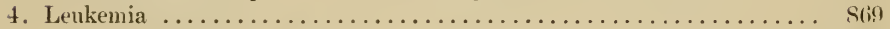

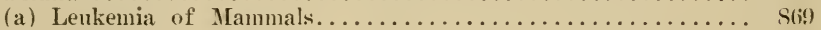

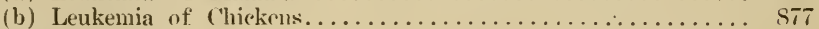

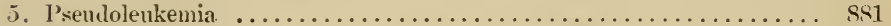

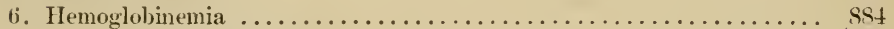

7. Paralytic Hemoglobinemia .......................... SSt

Paralytic Hemoglobinemia of Cattle................. 900

8. Hemophilia ................................. 900

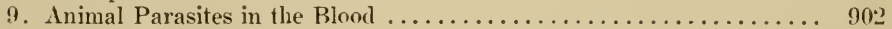

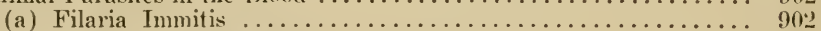

Filaria Samguinis equi.................... 905

(b) Other Animal Parasites in the Blood................ 906

\section{Diseases of the Spleen.}

Diseases of the Spleen

\section{Diseases of Metabolism.}

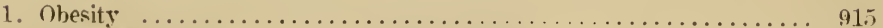

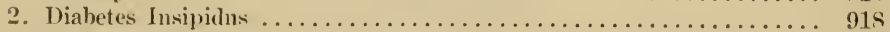

3. Diabetes Mellitus ......................... 922

4. Diseases of Metabolisu Which Partienlarly Affect the Osseons System. 930

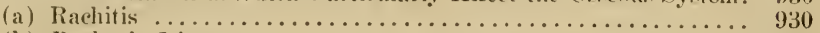

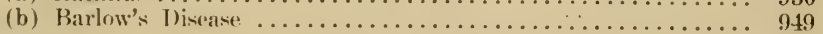

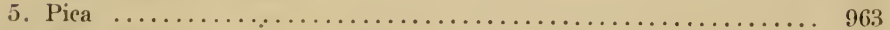

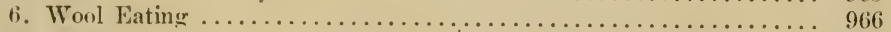

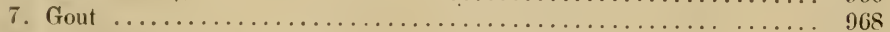

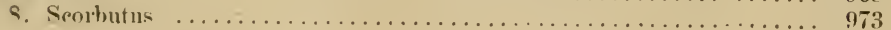




\section{Diseases of the Urinary Organs.}

\section{SECTION I.}

\section{Diseases of the Kidneys.}

1. Albuminuria

. Hematuria ............

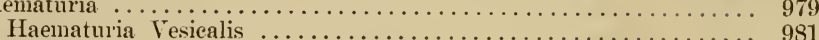

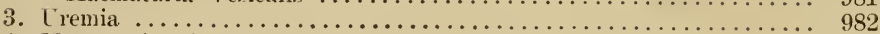

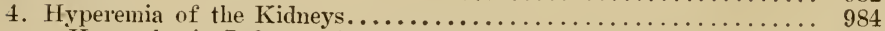

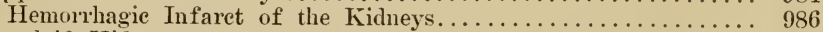

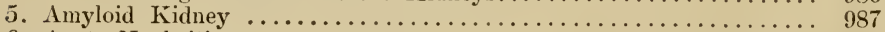

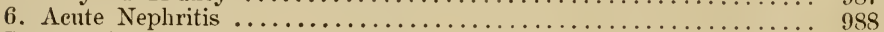

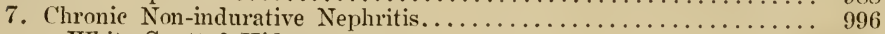

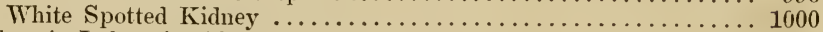

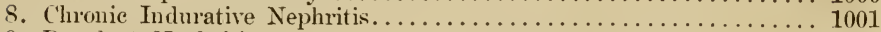

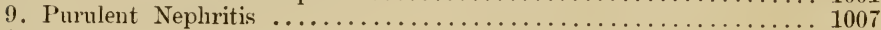

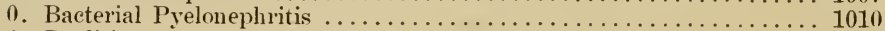

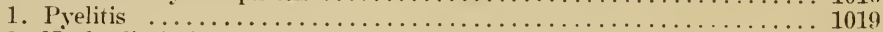

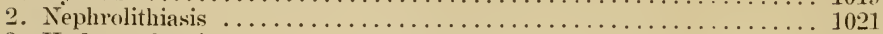

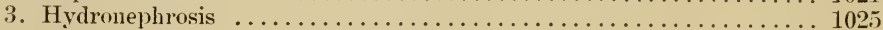

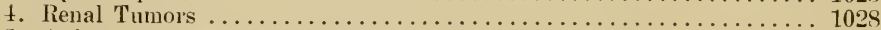

5. Animal Parasites in the Kidneys . . . . . . . . . . . . . . . . 1028

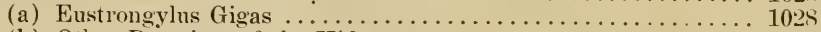

(b) Other Parasites of the Kidneys................... 1029

\section{SECTION II.}

\section{Diseases of the Bladder.}

1. Catarrh of the Bladder........................... 1031

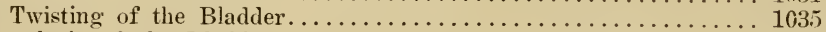

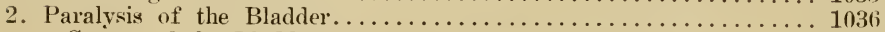

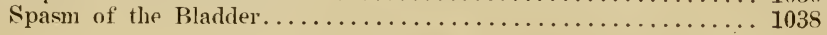

\section{Diseases of the Circulatory Organs.}

\section{SECTION I.}

\section{Diseases of the Pericardium.}

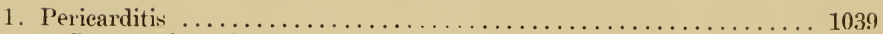

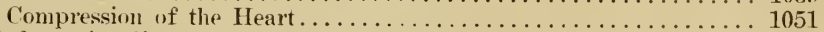

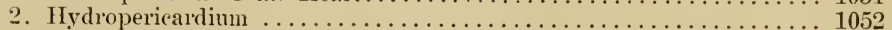

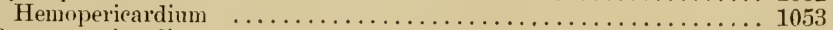

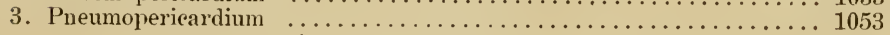

SECTION II.

Diseases of the Heart.

1. Palpitatio Cordis . ............................. 1055

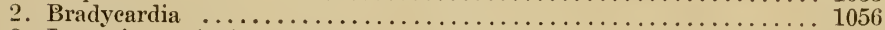

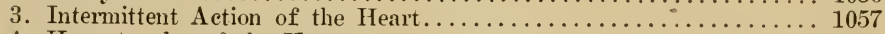

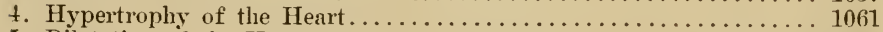

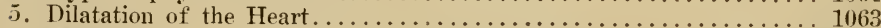

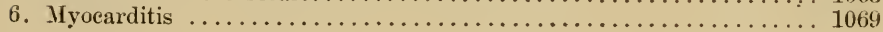


(a) Nente $\ldots \ldots \ldots \ldots \ldots \ldots$...... 1069

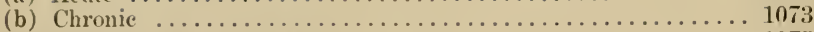

Ossification of the Heart Miks.le ................... 1075

7. Fatty Heart ................................. 1075

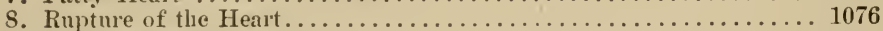

9. New-formation in the Heart . . . . . . . . . . . . . . . . . . 1077

10. Animal Parasites in the Heart....................... 1078

11. Acute Endocarditis ............................ 1078

12. Talvular Diseases ............................. 1085

(a) Insulficiency of the Aortic Valves................... 1088

(b) Stenosis of the Aortic Openin................... 1091

(c) Insufficieney of the Bienspid or Mitral Valve . . . . . . . . 1092

(d) Stenosis of the Left Amriculo-Tentricular Openin......... 1093

(e) Insufficiency of the Pulmonary Valves.................. 1093

(f) Stenosis of the Opening of the Pulmonary Artery. . . . . . . 1094

(g) Insufticiency of the Tricuspid Valve.................... 1094

(h) Stenosis of the Right Auriculo-Tentricular Opening........ 1095

(i) Congenital Heart Lesions...................... 1096

(k) Combined Valvular Lesions...................... 1096

\section{SECTION III.}

\section{Diseases of the Blood Vessels.}

1. Chronic Inflammation of the Arterial Wall ................. 1104

2. Dilatation of the Aorta............................ 1106

Aneurysms of Other Blood Vessels.................... 1109

General Diminution in the Tonus of the small Arteries... . . . . 1110

3. Obstruction of the Larger Arteries ...................... 1111

Thrombosis of the Pulmonary Artery.................... 1115

4. Obstruction of the Large Venous Trunks ................ 1116

5. Rupture of the Large Blood Vessels ................... 1117

\section{APPFNIIX.}




\title{
Explanation to the Abbreviations in the References to Literature.
}

\author{
A. L. $\quad=$ Állatorvosi Lapok (Budapest). \\ Amer. V. Rev. = American Veterinary Review (New York). \\ An. (Anim.) Ind. = Annual Report of the Bureau of Animal Industry (Washington). \\ Ann. \\ A. P. \\ = Annales de médecine vétérinaire (Bruxelles). \\ - Annales de l'Institut Pasteur (Paris). \\ Arb. d. G: A. = Arbeiten aus dem Kaiserlichen Gesuntheits-Ante (Berlin). \\ A. d'Alf. \\ $=$ Archires vétérinaires d'Alfort.
}

Arch. f. exp. Path.

u. Phmk. = Archiv für experiment. Pathologie und Pharmakologie (Leipzig).

A. f. Tk. = Archiv für wissenschaftliche und praktische Tierheilkumile (Berlin).

Arch. f. Tet.-Wiss.= Archiv für Veterinärwissenschaften - (St. Petersburg).

A. vet. $=$ Arhiva veterinara (Bucarest).

Beitr. z. path. An.= Beiträge zur pathol. Anatomie u. allgem. Pathologie (Jena).

B. kl. W. $=$ Berliner klinische Wochenschrift.

B. t. W. = Berliner tierärztliche Wochenschrift.

B. Mt. $\quad$ = Badische tierärztliche Mitteilungen (Karlsruhe).

Bull. $\quad=$ Bulletin de la Société centr. de médecine vétérinaire (Paris).

Cbl. f. B. (Bakt.) = Centralblatt für Bakteriologie, Parasitenkunde und Infektion:krankheiten. Originale (Berlin).

Cbl. f. med. Wiss. = Centralblatt für medizinische Wissenschaften (Berlin).

C. R. = Comptes rendus des séances de l'Académie des Sciences (Paris).

Comp. Path. = The Journal of comparative Pathology and Therapeutics (London).

Dict.

D. A. f. kl. M.

$=$ Dictionnaire pratique de mêdecine et de chirurgie vétérinaire (Paris).

D. m. W.

D. t. W.

D. Z. f. $\mathrm{Tm}$.

= Deutsches Archiv für klinische Medizin (Leipzig).

$=$ Dentsche medizinische Wochenschrift (Berlin).

- Deutsche tierärztliche Wochenschrift (Hanmover).

$=$ Deutsche Zeitschrift für Tiermedizin und vergleichenłe Pathologie (Leipzig).

Diss. (Inaug.-Diss.) = Inaugural-Dissertation.

Dresin. B. = Jahresbericht der tierärztlichen Hochschule in Dresilen.

Ergebn. d. Path. = Ergebnisse der allgemeinen Pathologie und pathol. Anatomie (Wiesbarlen).

F. d. M.

F. d. Vhyg.

Hi. d. p. i.

= Fortschritte der Medizin (Berlin).

= Fortschritte der Veterinärhygiene (Berlin).

- Handbuch der pathogenen Mikroorganismen; resligiert von Kolle \& WASSERManN (Jena).

H. (Hann.) Jhb. = Jahresbericht der Kön. Tierarzneischule zu Hannover.

Holl. Z. $\quad$ Tijdschrift voor Veeartsenijkunde.

Hyg. de vianıle $=\mathrm{L}$ 'hygiène de la viande et du lait (Paris).

J. du Mirli = Journal des vétérinaires du Midi.

J. of comp. Path.= The Journal of comparative Pathology and Therapeutics

J. vét. (London).

Kongr.

= Journal de médecine vétérinaire et de zootéchnie (Lyon).

Közl.

Leipz. B.
Maanedsskr.

= Internationaler tierärztlicher Kongress (Bern, Bailen-Baden, Budapest, Haag).

= Közlemények az összehasonlító élet-és kórtan köréböl (Builapest).

$\begin{array}{ll}\text { Mag. } & =\text { Magazin für die gesamte Tierheilkunde } \\ \text { M. Orv. Arch. } & =\text { Magyar Orvosi Archivum (Budapest). }\end{array}$

= Bericht des Veterinär-Institutes zu Leipzig.

= Maanedsskrift for Dyrlaeger (København).

Med. vet. $\quad$ Il medico veterinario (Torino).

Mitt. d. G.-A. = Mitteilungen des Kaiserlichen Gesundheitsamtes (Berlin).

M. (Münch.) Jhb. = Münchener Jahresbericht (Jahresbericht der königl. Zentraltierarzneischule in München). 
II, ni. IV.

M. t. IT

Mod. Zooiatro

Monh.

Neur. Cbl.

N. Ere.

Ors. $\mathrm{H}$.

O. M.

O. $\mathrm{R}$.

O. $\mathrm{Vj}$.

O. Z. f. Vk.

Pr. Mt.

Pr. Mil. Vb.

Pr. Vb.

Pr. vét.

Rec.

Rep.

Rerista

Rev. f. Tk.

Rev. gén.

Rev. vét.

Schw. A.

Schw, A. f. Tz.

S. B.

Soc. biol.

The Vet.

Tidsskr.

Tm. R.

T. R.

T. $\mathrm{Z}$

Trop. Vet.

Ung. Yb.

Vet.

T. A.

V. J. (V. Journ.) Tet. Jhb.

Vet. Rec.

V. f. T. (Vortr. f.

Tzte.) $=$ Vorträge für Tierärzte (Leipzig).

Ti. f. T'k.

Z. f. Biol.

7. f. Flhyg.

Z. f. Hyg.

Z. f. Imn.

Z. t. Infkr.

Z. f. kl. Med.

Z. f. Vet.-Wiss.

Z. $\mathrm{f}$. $\mathrm{Tm}$

Z. f. Tk.

7. f. Phys

Zool. méd.

= Il moderno Zooiatro (Torino)

= Il nuovo Ercolani (Pisa).

$=$ Orrosi Hetilap (Budapest). (Tien). Staate (Berlin). (Berlin).

$=$ Le progrès vétérinaire (Alfort).

= Rerue vétérinaire ('Toulouse). Königreich Sachsen).

$=$ The Veterinarian (London).

= Veterinarius (Budapest). für klin. Medizin (Berlin). närmedizin (Berlin).

= The Veterinary Record (London).

= Wocheuschrift für Tierheilkunde un

$=$ Zeitschrift für Biologie (München). (Bern).
= Münchener nedizinische Wochenschrift.

= Münchener tierärztliche Wochensehrift.

= Monatshefte für praktische Tierheilkunde (Stuttgart).

= Neurologisches Centralblatt (Leipzig).

= Osterreichische Monatsschrift für Tierheilkunde (Wien).

= Revue für Tierheilkunde und Tierzucht (Wien).

= Österreichisehe Vierteljahrsschrift für Veterinärkunde (Wien).

= Üsterreichisch Zeitschrift für wissenschaftliche Veterinärkunde

$=$ Mitteilungen aus der tierärztlichen Praxis in preussisehen

= Statistischer V eterinärsanitätsbericht über die preussische Armee

$=$ Veröffentlichungen aus den Jahresveterinärberichten der beamteten Tierärzte Preussens (Berlin).

$=$ Recueil de médecine vétérinaire (Alfort).

= Repertorium der Tierheilkunde (Stuttgart).

= Rerista di medecina veterinaria (Bucarest).

$=$ Rerue für Tierheılkunde und Tierzucht (Wien).

$=$ Revue générale de médecine vétérinaire (Toulouse).

$=$ Schweizer Archiv für Tierheilkunde (Zürich).

$=$ Schweizer Archiv für Tierheilkunde und Tierzucht (Bern).

= Sächsischer Bericht (Bericht über das Teterinärwesen im

$=$ Comptes rendus de la Société de biologie (Paris).

$=$ Tidsskrift for Veterenaerer (København).

$=$ Tiermedizinische Rundschau, mit besonderer Berïcksichtígung der vergleichenden Pathologie (Halle).

$=$ Tierärztliche Rundschau (Friedenau-Berliu).

= Tierarztliches Zentralblatt (Wien).

= The Journal of Tropical Veterinary Seience (Calcutta).

= Jahresbericht über das Veterinärwesen in Ungarn (Budapest).

$=$ Virchow's Archiv fur pathol. Anatomie u. Physiologie und

$=$ The Teterinary Journal (London).

= Jahresbericht über die Leistungen auf dem Gebiete der Veteri-

= Zeitschrift für Fleisch- und Milchhygiene (Berlin).

$=$ Zeitschrift für $\mathrm{H}$ ygiene und Infektionskrankheiten (Leipzig).

- Zeitschrift f. Immuntätsforschung und exper. Therapie (Jena).

$=$ Leitschrift für Infektionskrankheiten, parasitäre Krankheiten und Hrgiene der Haustiere (Berlin).

Zeitschrift für klinische Medizin (Berlin).

$=$ Zeitschrift für praktische Veterinär-Wissenschaften von PÜTz

- Zeitschrift für Tiermedizin. Neue Folge der Deutschen Zeitschrift für Tiermedizin und der Österreichischen Zeitschrift für wissenschaftliche Veterinärkunde (Jena).

$=$ Zeitschrift für Veterinärkunde (Berlin).

$=$ Zentralblatt fiir Physiologie (Leipzig u. Wien).

- Traité de zoologie mérlicale et agricole (Paris). 


\section{Infectious Diseases}

\section{Group}

\section{ACUTE GENERAL INFECTIOUS DISEASES}

\section{Anthrax. Febris carbunculosa}

(Splenic Fever; Milzbrandfieber, Milzfieber, Karbunkelkrankheit [German]; Fièvre charbonneuse, Charbon bactéridien [French]; Antrace, Febbre carbonchiosa [Italian].)

Anthrax is an acute, febrile, infectious disease of domestic animals, particularly of ruminants and horses, caused by the bacillus anthracis, and characterized anatomically by an acute swelling of the spleen and by serous hemorrhagic infiltrations of the subcutaneous and subserous connective tissue.

History. The disease was known by its present name in the oldest times $(\ddot{\alpha} v \vartheta \varrho \alpha \xi=$ =coal, on account of the black color of the blood), but it was confused with other diseases disclosing similar symptoms until the middle of the last century. Its contagiousness was first established by Eilert in 1836 by successful inoculations as well as by the feeding of anthrax blood, and by Gerlach in a similar manner a few years later (1845). In the year 1850 Davaine, and Rayer, in 1855 Pollender and soon after Brauell found in the blood of animals which died of anthrax peculiar rod-shaped bodies, which Brauell also demonstrated in the blood of living animals, attributing a diagnostic value to his findings. After Delafond (1860) had already recognized the vegetable nature of these bodies and named them bacteria, Davaine (1865) established their etiological importance, as in his experiments blood containing these bodies produced the disease even in high dilutions, while inoculations with blood without such rods gave negative results.

The fungous nature of anthrax bacilli was definitely established by Cohn. However, they were first artificially eultivated by R. Koch (1876) and by Pasteur (1877), whose fundamental investigations opened a new path for the theory of the infeetious diseases in general through the study of the biological characteristies of the anthrax virus, as well as the etiology of the disease. At the same time Pasteur's classical experiments in attenuation and protective inoculation established the foundation for the modern theory of immunity, the development of which has already 
produced highly important and valuable results, and for which the anthrax bacterium furnishes very satisfactory material.

Occurrence. As a disease of the soil prevalent in progres.sive agricultural countries, anthrax is usually confined to certain localities (so-called anthrax districts) where the moist, marshy or periodically inumlated soil is suitable for the growth of the infective agent. In such localities the disease appears enzootically almost ammally with a variable virulence, principally among animals at pasture in the summer time. On the other hand, and particularly in stable-fed animals, it is observed more rarely and mostly sporadically.

In Germany anthrax formerly existed very extensively, and the losses in Prussia alone amounter in the years from 1878 to 1883 to over one and a half million narks $(\$ 375,000)$. During the period from 1886 to 1905 the number of eases occurring quarterly rose from 0.22 to 0.62 for 10,000 cattle, in which the highest numbers occurrel mostly in the second quarter of the year. In the period of 10 years (1899-1908) the average cases were 172 horses, 4,459 cattle, 3,194 sheep, 10 goats and 100 hogs; and in the year 1908 the disease oceurred on 4,536 farms of 3,832 towuships, affecting 125 horses, 4,865 cattle, 369 sheep, 13 goats and 216 hogs, that is of each 10,000 animals, 0.29 horses, 2.36 eattle, 0.48 sheep, 0.04 goats, 0.10 hogs; in all 9.5 per cent less than in the previous year. In the last year the disease showed the largest extension in the government districts-Schleswig, Posen, Breslau and Liegnitz; in 86 per cent of the infected farms only one animal each was found to be affected.

In France the disease at one time existed to such an extent that Delafond estimated in 1842 the aunual loss at seven million francs $(\$ 1,400,000)$; in 1908 the number of infected stables fluctuated between 23 and 47 monthly, and the largest extension occurred in the northern districts.

In Great Britain the annual average during the period of 1899-1908 showed 835 outbreaks, that is 43 cases in horses, 893 in eattle, 58 in sheep and 223 in hogs. In the year 1908,80 counties were infected.

In Austria the average annual loss from anthrax during the years 1591-1900 amounted to 195 horses, 1,334 eattle, 128 sheep, 40 goats and 60 hogs; in the last years the affection existed per 10,000 animals in 1.3 horses, 1.7 cattle, 0.3 sheep, 0.06 goats, 0.05 hogs. In 1908 the number of affected townships fluctuated during the summer nonths between 6 and 27, and of farms between 6 and 123 .

In Hungary there were affected during the years $1899-1908$ an average of 273 horses, 2,542 cattle, 1,008 sheep and about 28 hogs, which involved from 821 townships in 1899 to 2,491 in 1908 ; the number of affected animals in 1899 were 2,962 , and 7,540 in 1908 . In 1908 the disease occurred in 2,491 townships, that is on 5,090 farns, with 358 cases in horses, 6,411 in eattle, 729 in sheep and 42 in hogs. The greatest number of cases occurred in the low, flat lands of southern Hungary along the Tisza and Maros rivers, as well as along the lower Danube.

In Russia the disease formerly existed to such an extent that in some periods (1864-1866) numerous persons fell victims, and in the District of Novgorod alone, during the years 1867 to $1870,56,000$ horses, eattle and sheep as well as 526 human beings suceumbed to the infection. At the present time the disease is still extensively prevalent, particularly in southern Russia, where the official reports for 1908 show 8,555 infected townships with 34,650 cases.

In Switzerland the disease appeared in 1908 in 160 townships, affecting 9 horses and 192 eattle.

Anthrax also exists in the other European countries in the north but not as frequently as in the south. Thus, in 1908, there were 3,853 cases in Italy, 466 in IIollant, 395 in Norway, 878 in Roumania, while in Denmark 143, and in Sweden 214 premises showed new outbreaks.

The disease is prevalent in all parts of Asia; in Asiatic Russia, the "Siberian pest" (there known as Jaswa) which is identical with anthrax, causes great losses annually, 95,316 cases being reported in 1899 . The disease has likewise been well known from the earliest times in Africa and America. (In Brazil it is known as "gorrotilho" of cattle and sheep.) In Australia it was not supposed to have been introduced until the year 1847, but at present it eauses an annual loss of 300,000 sheep. 
Etiology. The bacillus anthracis is 1.5 to $4.0^{\mu}$ long, and 1.0 to $1.5^{\mu}$ broad. It is a nonmotile bacterium which multiplies by fission and spore formation, and forms in the body fluids, and still more on artificial media, long chains.

Staining. The bacilli stain well, while living, with diluted aqueous fuchsin or methylene blue solution; on dried cover glass slides with the usual solutions of aniline stains, as well as by Gram's and Weigert's methods. Bacilli just stained with fuchsin disclose in their bodies one or more intensely stained granules adherent to the wall (Fig. 1). In stained dry specimens the ends of the bacilli appear sharply cut, sometimes even slightly thicker (resembling the form of I): in the chains they are separated by straight lined or double convex light spaces (bamboo-rod forms). The light parts are formed by the mucin capsule (Serafini, Johne) which surrounds the plasma of each

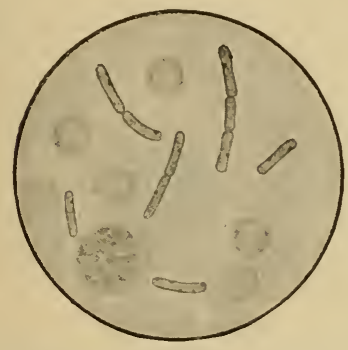

Fig. 1. Bacillus anthracis. Fresh sheep blood stained with diluted fuchsin solution.

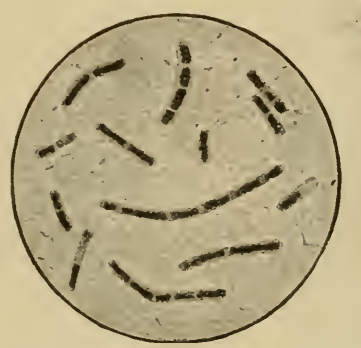

Fig. 2. Bacillus anthracis, with capsules. Spleen juice from a horse. Gram-eosin staining.

individual bacillus and holds them together in chains (Fig. 2). The capsule is well developed in fully virulent bacilli taken from the blood of animals, particularly of ruminants, as well as in those cultivated in fluid blood serum, while on other media the capsule formation is incomplete (see also page 11).

Cultivation. Anthrax bacilli grow on alkaline media in the presence of oxygen at body as well as room temperatures. The highest limit which is suitable for their growth is $43^{\circ} \mathrm{C}$., while the lowest temperature is $12-15^{\circ} \mathrm{C}$. In gelatin of not too strong concentration a white streak develops after 1 to 2 days along the stab from which horizontal radiations branch off in all directions (test-tube brush forms, Fig. 3). After 3 to 4 days the gelatin commences to liquefy under the influence of a peptonizing ferment (Fermi) along the streak from the surface downwards, while the bacterial masses later form a layer of flaky sediment on the bottom of the stocking-shaped liquefied part. The liquefied gelatin above this, however, remains entirely clear. On gelatin or agar plates the surface growth appears in the form of white points which consist of braid-like interwoven chains. The streak culture on agar and on potato forms a grayish white, reticulated, viscous 
growth of dull luster, with wavy borders, while in bouillon it forms a flaky, slimy sediment over which the liquid remains clear or only slightly cloudy. The bacilli may also multiply and form spores in greatly diluted albuminous solutions and in ordinary

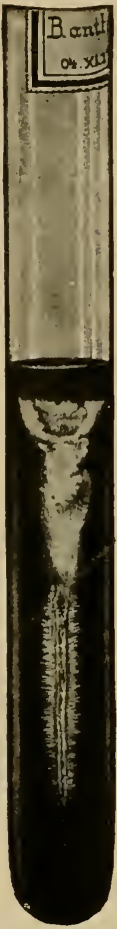

Fig. 3. Six-day old gelatin culture of the anthrax bacillus.
In the animal body anthrax bacilli multiply exclusively by direct division, and in this way short chains of 3,4 or 8 links result in the tissue fluids (Fig. 2). On artificial media very long chains develop by similar division, and the bacilli, which on these media are usually more slender, grow even to form long, homogenous threads, which subsequently become divided by cross sections into chains of bacilli (Figs. 5 and 6 ).

In the presence of a sufficient quantity of oxygen and at a temperature of $43^{\circ} \mathrm{C}$. maximum or $12 \mathrm{C}$. minimum spores develop in the bacilli (Figs. 5 and 6 ). One pole of the bacillus separates by a cross section from its body and incloses one or a half of a chromatin granule; after the disappearance of the granule the fertile pole segment separates from the wall whereupon its central part forms the body, and the peripheral part forms the covering of the spore (Preisz). Under favorable conditions the oval spore swells, its covering becomes extended and soon forms one of the poles. The young germ then makes its appearance and grows, developing into a bacillus, which later again propagates either by fission or spore formation. (Spores never develop in the tissue fluids of the living animal, in the unopened carcass, or inside of the meat of slaughtered animals.)

According to Brotzu bacilli produce spores in the intestinal tract of the dog which is only slightly susceptible to anthrax. According to Oppermann spores form in the caecum of the rabbit and according to Piana in the intestinal tract of birds.

At $42-43^{\circ} \mathrm{C}$, as well as on media containing substances which retard their growth (for instance, $0.1 \%$ carbolic acid), and by limiting the air supply, anthrax bacilli may temporarily, or forever, lose the faculty of spore formation, (so-called asporogenic mordification [Behring, Roux, Pfersilorf]). On artificial media various irregular forms develop occasionally after 2 to 3 days (club-, pear-, anil screw-forms), although the culture may retain its original virulence (Lignières \& Durrien).

Tenacity. There is a marked difference in the tenacity of the bacilli and spores. While the bacilli are less resistant, the spores are only destroyed by substances which have a very energetic action.

The bacilli are destroyed by drying in thin layers in the sunlight in from $6 \frac{1}{2}$ to 15 hours, in thick layers and in the dark in from 2 to 3 weeks, and by heating to $55.58^{\circ} \mathrm{C}$. in $10-15$ minutes. In dried blood, however, they may remain virulent for one month or longer, and may then form spores on addition of water and under proper temperature conditions (Bongert). They are only destrosed in dried blood when heated to $92^{\circ} \mathrm{C}$. for $2 \frac{1}{2}$ hours (Momont). On the other hand, they die in putrid blood, particuiarly in anærobic putrefaction inside the unopened carcass, in warm weather in 2 to 3 days. The bacilli manifest a great resistance against low temperatures, as for instance, at $10^{\circ} \mathrm{C}$. they are destroyed in 24 , and at $24^{\circ} \mathrm{C}$. in 15 days (Klepzof). In the gastric juice they die in $15-20$ minutes; in pure or putrid water in 1 to 2 days. Ichor destroys them if mixed with anthrux blood in 2-4 hours (Schipp). The ordinary disinfectants destroy the bacilli even in high dilutions (Koch).

Simple drying does not destroy the spores at all, so that in such condition, for instance, when dried on silk threads, they may remain germinative for $181 / 2$ 
hours (Székely). They also remain virulent for a long time in pure or putrid water, in putrid blool and in the ground (accorling to Sirena \& Seagliosi, they live in the ground for 3 years, in water for 17 and in ichor for 15 months). They resist the direet sunlight for 100 hours (Momont). Dry heat $\left(120-140^{\circ} \mathrm{C}\right.$.) destroys them in 3 hours, while blowing steam of $100^{\circ} \mathrm{C}$. kills them in 5 minutes (Kocli). They may still germinate after 5 minutes boiling (Geppert). On the other hand, the action of a falling temperature from 76.5 to $72^{\circ} \mathrm{C}$., inside of a manure pile is sufficient to destroy the spores (Pfeiler).

Of the disinfectants, corrosive sublimate is the most effective, since a solution of $1: 1000$ destroys the spores in 20 minutes (the addition of $0.5 \%$ hydrochloric acid or $2 \%$ earbolic acid increases the action of the solution). The following are also effective: fresh chlorine and iodine water $(2 \%)$, formaldehyde $(1 / 2 \%)$, permanganate of potash $(5 \%)$, lysol $(5 \%)$, carbolic acid $(5 \%)$, fresh chlorate of lime $(5 \%)$, chlorine gas $(5 \%)$, fluorhydrogen $(0.1 \%)$, and the vapors of formaldehyde, which will positively disinfect hair containing spores in 48 hours (Gruber).

Pickling destroys the bacilli in meat after $11 \frac{1}{2}$ months (Peuch), but it does not affect the spores (Abel). The drying and salting of hides does not destroy the attached spores which may remain virulent for 125 days on hides that have been

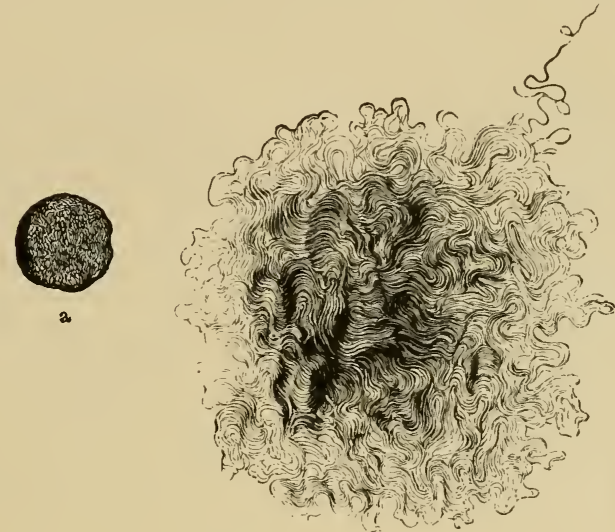

Fig. 4. (a) deep, (b) superficial colonies of the anthrax basillus on gelatin plates. Enlarged 80 . (After Flügge.)

treated with fresh milk of lime (Griglio). In sunlight hides containing the bacilli are sterilized in $6 \frac{1}{2}$ to 7 hours, while those containing spores are not sterilized evell after exposure for 13 hours (Esmarch). Quick lime solution and lime kill the spores in the tanning process in 12-17 days. Infusion of quebracho bark does not affect them in 12 days (Keszler), the lime pits (0.5-0.8\% lime contents) are not effective against the spores (Gartner \& Dammann. See also p. 23).

Pathogenicity. Rabbits, guinea pigs and mice are usually killed by subcutaneous or intravenous injections of anthrax blood or cultures, in 1 to 3 davs, while infection is less certain when the spores are fed. By the first method horses and sheep may easily be infected, while in cattle a local infection only results sometimes, even after the injection of larger quantities of virus. The infection of herbivora is also quite easily accomplished by feeding spores, even when the mucous membrane of the digestive organs is in a healthy condition. Although hogs 
may sometimes be infected by subcutaneous injections and by feeding (Perroncito, Crookshank, Garth), yet in most instances they resist artificial infection (Rátz, Tschernogoroff). Dogs occasionally become ill from intravenous injections with pure cultures (Bardach, Martel), and exceptionally from the feeding of anthrax meat. However, as a rule they manifest considerable resistance to artificial infections. Young pigeons and small birds in general may be quite easily infected artificially, while in large birls as well as in chickens infection is difficult.

The high virulence of the blood of animals dead of anthrax has been established by experiments, according to which a millionth part of a drop of blood injected subcutaneonsly kills a guinea pig in 23-24 hours (Davaine). By repeated passage of the virus through the body of susceptible animals (mice, guinea pigs) as well as through resistant animals (chickens,

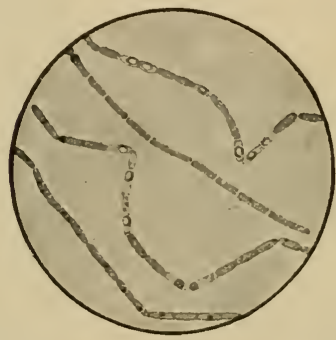

Fig. 5. Spore formation in anthrax bacilli. Eight-hours old agar eulture stained with diluted fuchsin solution.

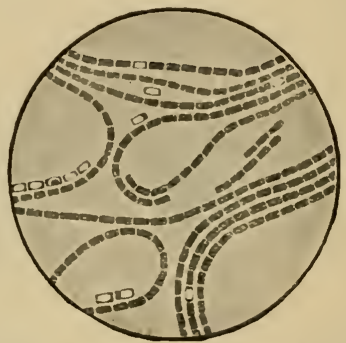

Fig. 6. Anthrax threads with spores. Cover glass preparation from an eight-hours old agar culture. Fuchsin staining.

dogs) its virulence is increased, and in this manner a virus which is fatal in 24-30 hours may be obtained (Martel). On the other hand, influences which check the development (high temperature, disinfectants as well as the continuous action of the oxygen of the air), have attenuating effects on both the bacilli and the spores. (See protective immunization.)

In the presence of certain other hacteria the multiplication of the anthrax bacillus is hindered or entirely checked. As such antagonists the following are known: Bac. pyocyaneus, prodigiosus, fluorescens, phosphorescens, Gärtner's bacillus, Friedländer's pneumococeus, streptococci and staphylococei, and the anærobic putrefactive bacteria. The antagonism manifests itself in such a way that virulent anthrax bacilli when mixed with such antagonists have little or no pathogenic action.

The natural infection in herhivorons animals occurs usually through the ingestion of food and water which contain anthrax 
spores. When taken into the digestive tract with the food or water some survive the action of the gastric juice and pass through the stomach unimpaired, germinating in the alkaline contents of the intestines where they multiply by fission. The bacilli enter the lymph spaces of the intestines and finally reach the blood through the lymph stream.

The contamination of the food with bacilli and spores usually takes place from the infected soil on which it was grown. Anthrax bacilli which in some way, especially by means of blood or intestinal contents of affected animals, are deposited in the superficial layers of the soil may there remain alive for some time, and under favorable moisture and temperatures multiply or form spores. The spores are for a long time capable of propagation, resisting dryness and moisture, cold and heat, as well as putrefaction, until favorable germinating conditions arise, whereupon the bacilli continue to multiply and produce new spores. Under unfavorable conditions, such as excessive dryness or cold, the bacilli are destroved, but the spores resist such influences. In this manner the soil of a certain locality which has once become infected may remain a source of infection for a very long time.

The elevation as well as the moisture and temperature of the soil are naturally of great importance. The infective agent thrives best in moist soil rich in organic matter, generally in swampy, marshy soil, or in localities subject to periodical inundations. In such places anthrax usually appears when by a rapid recession of the ground-water the superficial layers of the soil become exposed, and then, in the presence of proper moisture and temperature, germination of the spores and multiplication of the bacilli results. Consequently the disease is observed most frequently during the summer time, particularly in warm days following a period of rainy weather.

Forage plants are very apt to transmit the disease since the animals always ingest with their food a certain quantity of dirt which adheres to the dried and stored feed. Further, in the infected localities the water contained in pools as well as that from springs may become contaminated. As a matter of fact various outbreaks in pastures may be due to the use of certain springs, particularly if they are shallow.

The infectious material contaminates the soil in many ways. The running surface water may carry along the spores and deposit them elsewhere. Likewise, the wind may carry them to previously uninfected localities from places where the water has receded and the surface laver of the soil has become dried. Most frequently, however, the soil becomes infected by carcasses or parts of carcasses as well as by the excrements of affected animals. While the bacilli do not produce spores in the carcass, yet they pass to the outside, either through the body orifices or through decomposition of the carcass, when they form 
spores; from previously opened carcasses spore-containing material may be disseminated at once. When carcasses are not buried sufficiently deep or are left unburied the soil becomes heavily infected; even burying opened carcasses rather deeply does not offer a sufficient protection in this respect. The spores retain their germinating power for a very long time even in the deeper layers of the soil, and from here they may be conveyed to the superficial layers by the rising groundwater. They may also be brought to the surface by earthworms and deposited in their excrement (Pasteur, Bollinger). According to Karlinski the contagium may also be spread through certain snails ( $\Lambda$ rion subfuscus). Investigations of local outbreaks have frequently proved shallow burying of an anthrax carcass on a pasture or meadow to be the cause; besides this, the disease frequently occurs in pastures in the immediate vicinity of a decomposing anthrax carcass.

Wholesome food grown on uninfected soil is sometimes subsequently infected by the excrements or the blood of affected animals which have been bled. The bacilli which contaminate the food may continue to multiply and form spores if the conditions of moisture and temperature are favorable, and in this way they may cause stable infections.

The intestinal contents of affected animals is particularly dangerous as it may not only contain bacilli but also spores. Horse or cattle manure or its mixture with dirt represents an excellent medium for the propagation of the virus, and particularly for sporulation (according to Oppermann, spores which are formed on such soil are particularly resistant).

In anthrax districts the intestinal contents of healthy animals may not infrequently also contain spores which have been ingested along with the food or drinking water and passed out through the intestinal canal without molesting the host. It is therefore possible for such spore-carriers to spread the contagion into uninfected territories without becoming affected themselves.

According to Marchoux \& Salimbeni the disease is spread in Brazil by the Urubus, a species of vulture, as they consume the anthrax carcass and then pass masses of spores from the intestinal tract with their feces.

The food may also occasionally become infected by the hides of animals dead of anthrax. Hides are frequently dried in the hay-loft where the blood drops on the hay or other food stored there, or with the accumulated dust, into the mangers below. Sheep stables are particularly liable to become infected in this manner, as it is a frequent practice secretly to skin carcasses which died of anthrax and to dry the peits in the stable. It has also heen demonstrated that hides which have been thoroughly dried or even salted or treated with milk of lime (Griglio) may contain virulent spores. They may also be present in bone meal made from anthrax carcasses dne to incomplete decalcification and the consequent retention of more 
or less fat in the prepared product (Lehnert). Lastly, the soil, as well as the food and drinking water may occasionally become infected by drainage water and waste from tamneries, woolworks and horse-hair mills (Grebe, Uhlich; Gärtner \& Dammann found that while keeping the skins in the lime pit during the process of tanning the anthrax spores are not destroyed).

The introduction of a large quantity of spores is necessary for an active infection, and accordingly anthrax infections usually appear only after the ingestion of a considerable quantity of infected food or drinking water. The necessary quantity of spores varies with the individual susceptibility or resistanee which may be influenced by internal and external conditions. Animals which are affected with the so-called "latent infection" (or the above mentioned "spore carriers") not infrequently develop the disease as a result of external weakening influences through colds, physical exertion, starvation, ete. Furthermore, by repeated though slight intercurrent attacks the resistance of the animals is reduced, and under such conditions the anthrax bacilli begin to multiply, and thus produce the specific affection. (According to Oppermann healthy sheep may be infected by the feeding of 200,000 spores, while sheep which were previously starved were infected by feeding 51,000 spores). A reduction in the natural resistance is also claimed to be cansed by protective inoculation, according to Leclainche \& Vallée, and some of the losses from inoculation may be explainable in this way.

Carnivorous animals nsually become infected after ingesting meat or blood of anthrax animals. If the meat is fresh it contains only bacilli which are usually destroyed in the stomach. On the other hand, if the meat has stood for a sufficient length of time for the spores to develop on its surface and a large amount is ingested at one time the spores may pass throngh the stomach and germinate in the intestinal tract. In some instances, indeed, it may be assumed that even fresh anthrax meat may cause infection, provided the animals have eaten at one time such a large quantity as to cause a portion of it to leave the stomach undigested. In all cases there exists the possibility of the infection occurring through the mucous membrane of the mouth and pharynx, particularly in the presence of bone splinters; from the clinical appearance of the affection in hogs which is usually manifested as a pharyngitis and ulcerative stomatitis, it seems evident that the entrance of anthrax bacteria into the tissues occurs through the pharynx.

The milk of affected animals does not transmit the infection as the bacilli do not pass into the milk from the blood unless during the last hours of life. Moreover, the milk secretion ceases almost completely very early in the disease.

Anthrax very rarely develops throngh skin abrasions, and only in those instances in which the injury to the skin extends through the epithelial layer. In this way bacilli as well as spores may enter the tissue fluids, and in such eases the infection is transmitted through the blood, the excretions and 
secretions of the affected or dead animals. The disease may also be transmitted through infected food, straw, stable utensils, surgical instruments, harıess, hands and cloths of attendants, the stable floors and water. The injuries may be of the most varied origin (bites, phlebotomy, shearing of sheep, surgical wounds, spinous plants, wounds caused by sharp pointed objects, etc.), whereas the transmission by flies and insects is considered doubtful according to recent investigations (Nuttal, Mühling).

According to Dalrymple the carbuncular form of anthrax predominates in Louisiana, the infection being transmitted to healthy animals by horse flies known there as charbon flies.

Inasmuch as an artificial infection through inhalation of air containing spores is possible even when the respiratory mucous membrane is uninjured (Feser, Buchner, Enderlen) such infection may occur under natural conditions when the animals are fed very dirty infected feed. However, inhalation anthrax has never yet been positively established in domestic animals (in man a primary affection of the lungs does actually occur. See page 31 ).

Anthrax is not frequently transmitted directly from animal to animal. This kind of infection occurs only when the blood or infectious excrement of affected animals comes in direct contact with injuries on the skin or mucous membrane. Nevertheless the diseased animal is a source of danger for its neighbors as its excrement contaminates the straw, food and the ground by contact with which other animals are indirectly infected in the manner above mentioned.

Susceptibility. Of the domestic animals the lierbivorous mammals are the most susceptible, among which may be first mentioned sheep, horses and cattle, and the goat to a less extent. Considerably less susceptible are hogs, dogs, cats, and carnivorous mammals in general. Buffalo appear to become affected but rarely. Wild ruminants may be classed with the domestic ruminants as regards their susceptibility to anthrax. Domestic fowls are very resistant to artificial infection with the exception of the somewhat more susceptible pigeon (the occurrence of anthrax in fowls through natural infection has not yet been established with certainty).

The susceptibility is probably influenced to some extent by the breed, since certain breeds are more susceptible than others. Thus, according to the experiments of Chauveau, Algerian sheep manifest a remarkable resistance to artificial infection which was only overcome by large doses of the virus. Age has an influence, inasmuch as young animals are more susceptible than older ones. Fatigue, starvation and chilling increases the susceptibility; it also appears that well nourished and carefully stabled animals are more easily affected than 
those which are more used to hardship. Shedding of teeth as well as pre-existing catarrh and digestive disturbances render the animal particularly susceptible to antlirax.

Susceptible animals acquire a long lasting immunity against later infections after a recovery from the disease (see immunization).

Pathogenesis. The spores taken into the digestive tract germinate there and grow into bacilli. They then pass through the mucous glands and lymph follicles into the lymph spaces of the mucous membrane and submucosa, while spores which directly enter the connective tissue through injuries in the skin or mucous membrane germinate at the point of entrance. In the lymph spaces of the tissues the bacilli multiply by fission, although they are more or less inhibited and even partly destroyed by the hostile anthracocide substances which are present in the tissue fluids. The final result of the infection depends them on the one hand upon the resisting power of the infected organism, and on the other hand, upon the virulence of the antlurax bacilli. This again depends principally upon their capability of forming capsules. According to the investigations of Preisz, which correspond with the results obtained by Gruber \& Futaki and also by Weil, the virulence of the anthrax bacilli depends first of all upon the character of their capsules, that is, the virulence of the different strains of bacilli is more or less dependent upon the density and solubility of the capsules, inasmuch as dense capsules protect the bacilli against the destructive action of the dissolved hostile substances and the engulfing action of the phagocytes.

In immune animals even bacilli capable of producing dense capsules are quickly destroyed by the numerous anthrax destroying substances which are present in the tissue fluids, before they have time to form protecting capsules. Consequently an infection in such animals remains ineffective. On the other hand, in susceptible animals which possess a smaller quantity of anthrax destroying substances a greater or lesser number of the bacilli remain active, form capsules and become more resistant, producing also capsuled progeny. In this manner the capsule forming bacilli multiply at the point of infection, but in the meantime their destruction is also in progress, part of them being taken up and digested by the migrated leucocytes. A part of the destroyed extracellular bacilli are dissolved, and in this way the capsule substance, which has been named anthracomucin by Preisz, on account of its mucin-like action against reagents, reaches the circulation of the body. fluids. Hereby the anthracocide substances are gradually weakened. They are further affected by the capsule substance which results from the bacilli entering the blood circulation in the earliest stages there to be destroyed and dissolved. Finally, the anthrax destroyed substances of the body fluids are completely 
or almost completely neutralized, and therefore the bacilli gaining entrance into the blood circulation multiply therein very rapidly with little or no hindrance. The blood becomes heavily charged with the bacilli and in a short time the animal dies, showing manifestations of a severe disease-frequently in a few hours or even less.

At points where large numbers of bacilli accumulate as at the point of infection, the great amount of capsule substance which is present swells from the gradual absorption of fluid. These body fluids are absorbed by the capsule substance which becomes gelatinous, and consequently the fluids are prevented from re-entering the circulation, causing in these parts edematous swellings. The bacilli also accumulate in great masses in the capillaries, and because of the resulting interference with the blood circulation, principally on account of the injury to the walls of the vessels, more or less extensive hemorrhages develop. These occur very frequently in the center of the edematous swellings as well as in the organs with slow circulation (spleen, liver, brain).

The importance of the capsules as a protection against injurious action of the phagocytes and bactericidal substances in the body fluids is recognized by the majority of authors, among them being Babes, Metschnikoff, Gruber \& Futaki, Preisz and others. There are others, however, such as Bail, Fischoeder, Toyosumi and Menokawa, who are even at the present time inclined to consider the capsule formation as an abnormal swelling.

Regarding the origin of the bactericidal substances in the body fluids, Preisz accepts the opinion of Metschnikoff that they originate from leucocytes. Gruber and Futaki also attribute a certain amount of importance to the leucanthracocidin which is given up by the leucocytes on certain stimulation, but they believe that the destruction of the extracellular anthrax bacilli is due to the action of the placqanthracocidin which is secreted by the blood plates into the blood plasma under the stimulation of the anthrax substances, and which also confers upon the serum its germicidal action after the coagulation of the extravaseular blood.

Some of the symptoms of the disease (fever, severe general disturbances) as well as analogy to other diseases point to the fact that anthrax bacilli produce toxie substances similar to those of other pathogenic bacteria. When these are absorbed into the circulation their action, on the one hand, is injurious to the central nervous system, particularly the 'respiratory center, and on the other hand, at the places where great numbers of bacteria are accumulated, on the blood vessels. However, the filtrates of the cultures are not toxic, and as a matter of fact, the existence of an anthrax toxin has not been satisfactorily proved (Conradi, Levy \& Beckmann). Nevertheless, through the process of autolysis a rennet ferment, a fatty acid splitting and a peptonizing ferment, may be extracted from the bacilli (Pfersdorff), while Boidin produced from them fatty substances which, when inorulated subcutaneously, caused inflammatory edema and progressive emaciation. Lastly, the observations of Johne show a production of hemolysins wherehy in a hanging drop of blood charged with anthrax 
bacilli the red blood corpuscles dissolve in a few hours. This explains the destruction of erythrocytes, the pronounced dyspnoea in the later stages of the disease and the laked condition of anthrax blood. Recently Casagrandi, Wunschheim, Heyrovsky \& Landheimer, as well as Krogh proved the presence of hemolysins also in cultures.

Anatomical changes. The carcasses of animals dead of anthrax decompose rapidly on account of the blood remaining in a liquid condition, and therefore they become, as a rule, greatly bloated. Rigor mortis is also incomplete. Dark red blood escapes from the body openings, the visible mucous membranes are cyanotic and frequently hemorrhagic, especially on the prolapsed rectum. The autopsy shows, in addition to the general manifestations of asphyxiation, extensive serous infiltrations and blood extravasations in the connective tissue at different parts of the body. The subcutaneous and intermuscular connective tissue is infiltrated in places, having a jelly-like consistency, and being permeated with small circumscribed or large flat, dark red hemorrhages. Similar changes such as gelatinous infiltrations and hemorrhages also occur in the loose subserous connective tissue, principally in the mediastinum of the pleura, mesentery and that surrounding the kidneys; also under the mucous membrane of the pharynx, in the epiglottis and tissue surrounding the frenum of the tongue. Hemorrhages may be present in places without serous infiltrations, principally under the serous membranes, in the catarrhal mucous membranes, under the endocardium, and in the subcutaneous connective tissue. In rare cases a circumscribed swelling consisting of fixed, infiltrated cordaceous tissue may be present in any part, over which the dirty brown skin may be broken, forming a deep sinuous ulcer covered with necrotic shreds of tissue. The superficial lymph glands appear greatly swollen, particularly near areas of gelatinous infiltration. The cut surface is reddened, moist and frequently studded with small hemorrhages.

The spleen with rare exceptions shows the characteristic picture of an acute swelling. In most cases the entire organ is greatly swollen, the capsule distended, the pulp blackish red, softened and even fluid, and the swelling may be so large as to burst the spleen capsule. In rare cases the knobbed surface of the spleen shows only a few blackish red, softened areas of various sizes, and fresh hemorrhages.

The parenchymatous organs, principally the liver and kidneys, are also congested, swollen, and as a result of parenchymatous degeneration they are brittle and soft. Frequently the muscles and heart muscle have a grayish-red, flabby appearance. The mucous membrane and submucosa of the intestinal canal, especially in the duodenum and small intestine, but rarely in the stomach and large intestine, are uniformly swollen in considerable portions, intensely red, and permeated with small blackish-red hemorrhages. The swellings may be 
very marked and confined to smaller areas, particularly those corresponding to the Peyer's patches and solitary follicles, thus causing the mucous membrane to project into the lumen of the intestine in the form of spherical protuberances (carbuncles), or as elongated, thick pads. On the protuberances or pads the mucous membrane appears necrotic, which sometimes affects only the superficial layers, although its entire thickness may be involved, giving it the appearance of a pale yellow pulpy deposit. The inner surface of the abdominal wall may also show various sized ulcers with greatly swollen blackish-red borders, their bases being covered with necrotic tissue shreds. Around the nodules and ulcers the mucous membrane and subserous connective tissue usually manifests a marked gelatinous infiltration and permeation with hemorrhages.

The lungs are highly congested, and edematously infiltrated. The respiratory mucous membrane is hyperemic, catarrhal and swollen, particularly at the opening of the larynx, and contains numerous ecchymoses. The brain and meninges are also hyperemic, and between the latter sometimes extensive flat blood coagula are present (apoplexia intermeningialis). Hemorrhages may also occur exceptionally in the brain substance. The body cavities of the carcass frequently, especially in warm weather, contain a reddish fluid. The internal lymph glands show a condition similar to that seen in the external glands except that the condition is more marked, particularly in the mesenteric glands.

The blood is dark to blackish-red in color, contains only a few loose fibrin coagula, and clears up only slowly in the air. On microscopic examination the leucocytes appear greatly increased (hyperleucocytosis).

Anthrax bacilli are usually present in great numbers in the blood, spleen, lymph glands and parenchymatous organs. Occasionally, however, but few are present, especially in the rapid, apoplectic form of the disease.

The pathological ehanges described are the more distinctly developed, the more slowly the disease had progressed. Their distribution depends in the first place upon the nature of the infection as the edema and hemorrhages are most pronounced at the point of infection. However, superficial erlemas may also develop after infections through the intestines, whereas the described intensive changes in the intestinal mucous membrane develop almost exclusively in this mode of infection, although they are not invariably present in such a conspicuous form in all cases.

The manifestations of the changes in hogs differ inasmuch as in most cases the lesions are principally confined to the pharyngeal region. In addition to a marked gelatinous-hemorrhagic infiltration of the pharyngeal connective tissue and the retropharyngeal lymph glands, the tonsils are covered by strongly adherent pale-yellow, pseudomembranes, and are surrounded by the greatly swollen mucous membrane. The tissue of the tonsils under the pseudomembranes appears vellowishgray, lusterless and several millimeters in thickness. The in- 
flammatory swelling extends also to the perilaryngeal connective tissue and superior cervical lymph glands.

Intestinal anthrax occurs more rarely in hogs. When present the mucous membrane of the small intestine shows severe inflammatory changes and developing ulceration in patches or over more extended areas (Carl).

The spleen usually presents a normal appearance, but may at times be greatly swollen and softened. Zimmerman, Carl and Bongert describe as rare occurrence sharply circumscribed nodules in the moderately swollen pulp, from a hemp to a milletseed in size, in which the cut surface appears brownish-red to grayish-red in color, dry and lusterless.

In one case of Wyssmann the lesions corresponded to a serofibrinous pneumonia (inhalation anthrax?).

Anthrax bacilli are found in great numbers in the gelatinous-hemorrhagic connective tissue as well as in the swollen lymph glands. On the other hand, they are only sparingly present in the blood, and in the spleen nodules mentioned they are mostly found in a degenerated form.

Symptoms. The time of incubation of anthrax after artificial infection varies from 1 to 14 days, depending to a great extent on the place and intensity of the infection. After subcutaneous or intravenous inoculation the manifestations of the disease appear in 24 to 48 hours. Sheep usually succumb in 2 to 3 days to the feeding of large quantities of spores. However, the time of incubation under natural conditions, when the animals take up a considerably smaller quantity of spores, is undoubtedly longer. Thus in the experiments of Pasteur, $\mathrm{R}$ oux \& Chamberland sheep, which were infected with food grown on infected pastures, developed the disease only after 10 days. In horses and cattle the time of incubation seems to be hardly less than 3 days.

In horses, cattle and sheep anthrax when developing as a result of an intestinal infection usually manifests itself in the form of a intestinal infection usually manifests itself in the form of a
general, acnte infectious disease in which the individual

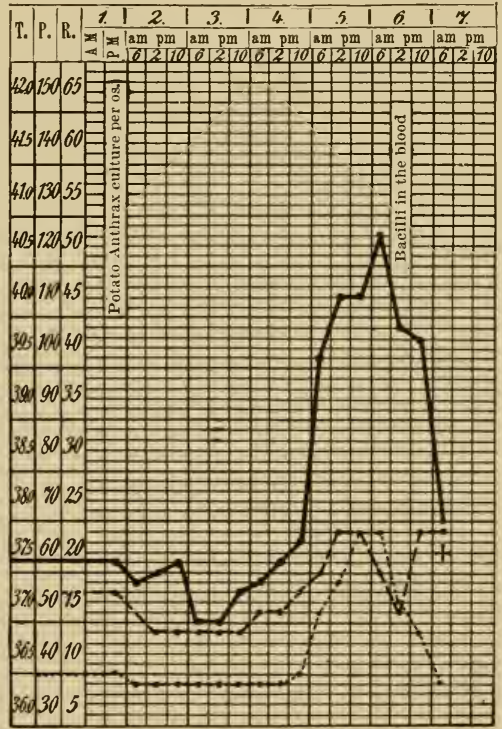

Fig. 7. Fever curve in anthrax of a horse. 
symptoms are only rarely characteristic of the specific affection.

In the peracute cases the course of the disease simulates cerebral apoplexy (anthrax acutissimus s. apoplecticus). Well nourished and apparently healthy animals, which, however, were probably feverish, suddenly go down in the stable or in the pasture and remain lying on the ground in convulsions; the respiration is rattling; bloody foam oozes from the mouth and nose, the mucous membrane of which shows a dark bluish-red discoloration, and from the anus pure blood even may escape, until finally in a few minutes, or at most within an hour, the animal dies. This form of anthrax is observed most frequently at the beginning of an outbreak, and particularly among sheep.

In the acute and subacute cases which represent by far the largest percentage of cases the disease commences with a quick rise in the temperature to $40-42{ }^{\circ} \mathrm{C}$., at which height it remains for a time, dropping quickly just before death. (Fig. 7.) Some animals, especially cattle, manifest quite a lively disposition at the onset of the disease and take their food in the usual manner.

In some cases the disease commences with restlessness and excitement which soon pass into depression. In most eases, however, the malady is preceded by symptoms of general depression, the animal refusing to eat and standing in one place with head hanging, staring look and dilated pupils. In the pasture the affected animals trail behind the others, remain for a long time in one place or lie down; when urged to move they stagger and sway. At the same time they have chills and their temperature is unevenly distributed over the surface of the hody. The pulse is accelerated and small while the heart beats are metallic in sound.

The respiration is very labored and accelerated, and the dark bluish-red cyanotic mucous membranes, which are frequently studded with petechial hemorrhages, are indicative of insufficient oxidation of the blood. A mechanical cause for the difficult respiration cannot be established except in those cases in which edema of the glottis is suspected, becanse of pharyngitis, swelling of the neck and whistling inspiration. Examination of the lungs reveals nothing abnormal except a harsh vesicular breathing.

Very frequently disturbances in the digestion are observed. Apart from affected appetite horses manifest severe colicky pains but without any indication of meteorism or bloating. Ruminants are less restless and frequently show moderate hloating (in one case, a cow observed by Schmidt, the course of the disease manifested the symptoms of an acute paralysis of the rumen with left-sided pleurisy). At first there is constipation, but diarrhea soon sets in, during which the feces, particularly in cattle, are fluid and frequently intermixed with 
blood, or occasionally large masses of blood may be passed by the affected animals. In horses it is sometimes possible to demonstrate a marked enlargement of the spleen by rectal exploration or by percussion. The urine is dark red and sometimes even bloody. (This is a particularly frequent symptom in sheep, in which animals the compression of the nasal openings causes immediate urination.)

The milk secretion usually ceases shortly after the onset of the disease; if there is a slight secretion of milk it is yellowish or bloody in appearance, slimy, and possibly bitter. Pregnant animals frequently abort. After the difficulty in respiration has reached a high degree, death finally results with signs of asphyxiation.

Besides these symptoms, which clearly indicate the presence of an acute infectious disease, in some cases, particularly in horses, more rarely in cattle and very exceptionally in sheep, acute edematous swellings develop on the surface of the bodv, which make a more accurate orientation of the nature of the affection possible. In any region of the body, but especially on the neck, breast, flanks, or lumbar region, a more or less extensive, rapidly growing swelling develops. The swellings are hot, and of a doughy or dense consistence, but the skin covering them usually shows no changes. Exceptionally on a circumscribed area of the skin proper a warm, painful and dense swelling develops surrounding which the subcutaneous tissue also appears greatly swollen as a result of the inflammatory edema. With the swelling of the neck there is usually associated a severe pharyngitis or erlema of the glottis which considerably increases the pre-existing dyspnoea. On the rectal mucous membrane carbuncular growths develop occasionally as a result of infection introduced by a rectal examination or by mannal removal of the feces. In such cases the feces are usually evacnated under severe straining, and contain dark, tar-colored blood or exfoliated tissue shreds. The rectum is prolapsed; the perineal region is edematously swollen, while the hand introduced into the rectum may detect flat or nodular swellings in the hot mucous membrane.

A primary affection of the skin only rarely occurs in animals, and consists in the appearance of circumscribed, warm, painful and hard swellings which later become gangrenous, commencing in the center, and change into nlcers (carbuncles) which have ragged borders and are covered with necrotic tissue.

According to some authors anthrax may occur in sheep in the form of an erysipelas-like dermatitis accompanied by a subcutaneous edema which starts at one extremity and soon extends to the body, whereby the patients show high fever and die inside of $1 / 2$ to 2 days. The anthrax nature of the affection has not yet been positively established.

The occurrence of the so-called gloss anthrax is also doubtful, as most cases in cattle designated under this name belong to hemorrhagie 
septicemia. The presence of gloss anthrax can only be accepted as probable when the tongue is greatly swollen, cyanotically discolored, or when the buccal mucous membrane contains hard nodules and eysts filled with bloody fluid.

In hogs anthrax usually runs its course with manifestations of febrile pharyngitis with marked swelling of the subparotidal and laryngeal regions. The swelling may extend from the neck to the head and cause considerable difficulty in deglutition and respiration. At the same time the mucous membranes are cyanotic, bluish-red spots develop on the skin, diarrhea sets in, and the difficulty in respiration and swallowing reach such a state that the animals finally die from asphyxiation. In experimental cases cysts filled with bloody fluid develop on the mucous membrane of the tongue, cheeks and lips.

In those cases where there is no swelling of the neck the symptoms observed are only debility, suppressed appetite and hiding under the straw.

In dogs and in other carnivorous animals the disease is of rare occurrence and is usually manifested by severe gastroenteritis as well as pharyngitis. Sometimes after eating anthrax blood an inflammation develops on the lips and tongue, or general infection may follow the development of a carbunculous ulcer upon the toe pads.

Course. The development of the disease and the successively appearing symptoms as well as the general picture of the affection show great variations from case to case. While sometimes only half an hour passes from the onset of the disease until death, in other cases the duration extends to two and more, exceptionally to 7 days. In slow cases the intensity of the symptoms may decrease temporarily so that the patients are somewhat brighter, and take some food, but the improvement is followed after a shorter or longer period by new attacks, and the disease may even assume an intermittent character (anthrax intermittens) in which the animal becomes greatly emaciated under periodical febrile symptoms.

The species of the animal affected, influences considerably the manifestation of the clinical picture. Anthrax in horses is usually marked by symptoms of a severe spasmodic colic in which quite frequently edematous swellings develop on the neck and chest. In cattle only symptoms of a general febrile affection are usually observed, but hemorrhages from the intestines as well as edematous swellings are not very rare. Anthrax in sheep usually runs a rapid course without localization to certain regions, acting like a pure septicemia, and the apoplectic form occurs most frequently in these animals. Anthrax in goats is less acute. Hogs usually die in 1 to 2 days with symptoms of asphyxiation due to the extension of pharyngitis. 
Diagnosis. It is hardly possible to diagnose with certainty the peracute case of anthrax on the living animal, as acute hyperemia and acute edema of the lungs as well as edema of the glottis, further congestion of the brain (sunstroke) and hemorrhages into the brain substance resulting from other causes may kill the animal under very similar manifestations. In the less acute cases anthrax is indicated principally by the appearance of the rapidly extending edematous swellings as well as by the bloody feces and urine, but even in this respect hemorrhagic septicemia very closely resembles anthrax (on post mortem the acute swelling of the spleen is always absent in the former).

Likewise anthrax may be also suspected in severe gastrointestinal inflammations, especially when associated with intestinal hemorrhages, while in horses, on account of the severe abdominal pain, colicky affections, invagination and volvulus of the intestine, may be chiefly confounded with it, and particularly so when, as sometimes happens, there are no characteristic symptoms present other than those of colic. In cattle blackleg and malignant edema are differentiated by the cold and crepitating consistence of the swelling in the latter diseases, as well as by the peculiar sour, disagreeable odor of the fluid which is contained in the swellings of anthrax edemas. In hogs, especially when suffering with pharyngitis as a result of an infection with the virus of buffalo disease, the resemblance to anthrax is so pronounced that a differentiation is only possible by additional circumstances (prior cases in buffaloes). Finally, poisonings occasionally come into consideration, in which, however, a rise in the body temperature occurs only in the later stages.

The diagnosis is certainly established by the demonstration of bacilli in the blood of the living animal which in size and form resemble anthrax bacilli, as there is no other disease known in which bacteria in any way resembling these would circulate in the blood; unfortunately, they can be demonstrated in the blood only a short time-not earlier than 16 to 18 hours -before death.

Characteristic indications on post mortem are the dark-red color of the uncoagulated blood, the acute swelling of the spleen and the lymph glands, bloody edema in the subserous and subcutaneous connective tissue and also hemorrhagic enteritis. These findings retain their importance even in case of negative bacteriological examination. However, in exceptional cases the enumerated changes and also swelling of the spleen may be absent. In such cases the bacteriological examination alone will give enlightenment. If the carcass is fresh, not older than 24 hours, then the presence of large, non-motile rods and short chains in the blood which has been taken from vessels distantly located from the intestines, such as a superficial vein, 
is certain proof of anthrax, particularly when by staining the presence of a capsule can be demonstrated. If, however, a longer time has elapsed after death, especially in summer time, other bacilli, first in importance bacilli of malignant edema, may have migrated from the intestines into the blood, and these in many instances can only be differentiated from anthrax bacilli by cultural methods and animal inoculations.

Bacteriological diagnosis. Relative to distinguishing anthrax bacilli from other bacteria more or less closely resembling them the following is to be noted:

1. Anthrax bacilli are present in the blood of fresh earcasses; they are thick, rounded at the ends or squarely cut. They usually have a distinctly recognizahle eapsule and form chains of 2 to t, rarely more, segments, in which the individual segments are separated from one another by light cross lines; they are aerobic and never produce spores inside the animal tissues and body fluids. Subcutaneous or intravenous injections kill mice, guinea pigs and rahbits, in which after death the blood usually contains the bacilli in great numbers.

2. The bacilli of malignant edema are more slender, motile, rounded at the ends, and besides, the edematous swelling, fluid of the abdominal eavity and blood of the portal vein contain curved thread-like forms of various lengths, some homogenous, others beaded. In older earcasses many of the bacilli contain in the center one oval spore each; they are strictly anærobic, liquefy gelatin, and form gases even in coagulated blood serum. Intravenous inoculations are harmless; subcutaneous inoculations into test animals produce a rapidly extending edematous inflammation with gas formation.

3. Blackleg bacilli are present only in the serous fluid of the crepitating swelling in a fresh carcass; they are comparatively short and thick, motile, never grow in form of threads, and produce in the animal body shortly after death, oval spores located either at the end or in the center of the bacillus. They may be grown anaerobically but also aerobically; liquefy gelatin slightly; and in coagulated blood serum they do not produce gas. Intravenous inoculations are harmless; subeutaneous or intramuscular inoculations kill the guinea pig and mouse, but rabbits only as an exception.

In the examination of material which is no longer fresh, various putrefactive bacilli (Bac. subtilis, Bac. termo) come into consideration, particularly those resembling saprophytes, the Bac. pseudoanthracis described by Burri \& Baas, as well as the Bac. anthracoides found by Hueppe \& Wood. They are found frequently in blood or parts of organs that have been stored, and they have some characteristies in common with the anthrax bacillus, but may be distinguished by the absence of other characteristies of the true anthrax bacillus (Hoppe). Further, they coagulate milk after a short time and do not form capsules in inactivated horse blood serum.

The anthrax bacillus may le demonstrated in most instances in sufficiently fresh material by a microscopical examination for which the various capsule stain. ing methods are especially adapted (accorring to Johne, staining with heated $2 \%$ gentian violet solution follower? by a quick discoloration with $2 \%$ acetic acid; according to Olt with $3 \%$ safranin solution also with heating; according to Raebiger staining of the air-lries smears with formol-gentian violet $(100-150: 10-15)$; further, Gram's stain with subsequent eosin treatment). The material for examination should be taken from the blood or spleen of very fresh carcasses; later 
from a peripheral vein (ear or jugular) as putrefaction sets in later in these parts than in the internal organs. As a result of putrefaction the bacilli change their form after a time; they become more sleuder; their plasma does not stain well, and later not at all; at the same time other bacteria and especially anaerobic putre. factive bacteria multiply very rapidly. The capsule of the anthrax bacillus is preserved somewhat longer so that after a time only empty capsules or the remaius of plasma envelopes can be seen (See fig. 2 on page 3 ). They finally become unrecognizable (according to Olt, in the summer time in about 48 hours). Its diagnostic importance, however, should not be overestimated as a capsule may oceasionally occur on some putrefactive bacteria resembling the anthrax bacillus (Noetzl), and on the other hand sometimes they cannot be demonstrated even on true anthrax bacilli, especially in the blood of horses and carnivorous animals.

According to Cinea \& Fenca the feces of affected animals (sheep and hogs) invariably contain anthrax spores which can be isolated through cultural methods by a previous heating to $65^{\circ} \mathrm{C}$. This is possible even in eases in which the carcass is already putrid and the examination of the blood gives negative results.

In the examination of stale blood or material from organs good results may sometimes be obtained by the mucin reaction which was first described by Weichselbaum (1592), later by Heim and recently recommended by MacFadyean. This reaction is manifested in smears stained with $1 \%$ methylene blue solution by the appearance of violet or purplish-red granules which are seen near the bacilli and originate from their capsules.

Positive results are obtained in doubtful eases by the isolation of the anthrax bacillus by the plating method. According to Bongert the bacilli remain active in dried blood for 36 to 50 days, in putrefied dried blood or tissue fluids for a shorter period. However, they retain their virulence on an average of from 8 to 20 days. In carcasses which have lain mopened the results from the examination of smear preparations are uncertain even after 24 hours, while by the plating method they may be demonstrated for 2 or 3 days after death.

Finally, the diagnosis may be proved by animal inoculations, for which the usual laboratory animals, with the exception of the rat, may be utilized (to prevent an intoxication only small quantities of the material should be used for inoculations; the scarified shaven skin is best adapted for the inoculation of putrid material).

Suspicious material forwarded for an accurate bacteriological examination is best prepared by spreading the softened pulp of the spleen from 3 to $4 \mathrm{~mm}$. tinick on a slide, across each end of the slide a strip of thick cardboard is placed, and another slide is then placed on these pieces of cardboard. They are then securely tied and forwarded in a box for examination (Kitt, Bongert). Another method consists in breaking a boiled potato in two; the blood or spleen tissue having been smeared on one of the broken surfaces, the pieces are placed together again and the whole forwarded to its destination (Olt). Further, the blood or spleen pulp may be dried on the inner surface of a test tube, or on woolen threads or cotton pads (Heim, Mazzini).

Jakobsthal \& Pfersdorff, as well as Marxer and Eberle, recommend for the above purpose quadrangular pieces of gypsum rods saturated in Loeffler's bouillon and placed in strong test tubes. Before use they are dipped in pure water, then covered with a thin layer of blood or splenic pulp, placed in the tube, which is packed in a wooden case and forwarded to the laboratory. Inasmuch as the bacilli form spores on the rods in a proper temperature the diagnosis is possible even after 14 months. Instead of gypsum rods small pieces of burned bricks are also adapted (Reinecke), or even better filterpaper rolls about the thickness of the small finger and moistened in water (Schüller, Grabert).

Prognosis. Obviously anthrax terminates not infrequently in recovery, although it is hardly possible to estimate the percentage of recoveries with any degree of certainty. In the course of various outbreaks it is a quite frequent occurrence that some of the animals which were exposed to the infection show an indisposition and fever, but recover completely in 1 to 2 days. Such passing affections are probably the result of an anthrax infection, which, however, is overcome by the energetic resistance of the animal. Toward the end of some epizooties the disease often assumes a subacute form and then 
cases of recovery are more frequent. The prognosis is most unfarorable in the peracute cases, and most favorable in the primary local skin affections as long as there are no intensive general disturbances to indicate the entrance of the bacteria into the blood circulation. The development of edematous swellings in the course of the febrile affection is almost invariably of unfarorable significance, while the appearance of bacilli in the blood is indicative of approaching death.

Treatment. In primary local affection of the skin, subcutaneous tissue or the visible mucous membranes, surgical treatment is first indicated. This consists in deep incisions of the swellings, and after thorough squeezing and scraping, the wound is repeatedly washed and cauterized. Cross incisions are advisable, while large swellings may be incised in several places. As disinfectants the following are recommended: Corrosive sublimate (1-1000), carbolic acid (3\%), creolin (5\%), etc. Good results are also obtained from subcutaneous injections at various places in the periphery of the swelling with $2-3 \%$ carbolic acid or $5 \%$ creolin solution, and covering with a carbolic dressing.

In cases of generalized disease which are not yet far advanced the treatment with immune serum (see page 28) gives remarkably good results. After the intravenous injection of $40-80 \mathrm{~g}$. of sufficiently active serum the temperature falls rapidly, frequently within as short a period as 6 hours, and this is followed by a complete recovery after 12 hours (Jaeger, Detre, Raebiger, Gál and others). In cases in which no improvement is observed after a few llours, or in which the temperature rises, it is advisable to repeat the serum injection. Serum of high potency proves effective even in small doses and in subcutaneons applications.

Sometimes favorable results may be obtained from symptomatic treatment, viz., repeated bathing witl cold water, rubbing with camphor, turpentine or pure alcohol. Internally wine, alcohol or camphor may be given; also subcutaneous injections of ether or camphor, etc. In the presence of an intestinal infection the administration of disinfectants in large doses is indicated, such as creolin or lysol (larger animals 20-50 gm., smaller animals 5-10 gm. or even larger doses); creosote (5-10 and 1-2 gm., respectively), carbolic acid (according to Hess 40-50 ce. of a $1 / 2 \% \%$ solution daily), calomel in small and frequent doses (for horses, with $1 / 2$ gin. opium) and oil of turpentine (for larger animals 200-300 $\mathrm{gm}$. mixed with sweet oil). Enemas with disinfecting fluids may also be tried, especially in cases in which the symptoms indicate a severe affection of the rectum. Intravenous injections of collargol (1 gm. dissolved in 50-100 gm. of water) are also recommended.

The sick animals should be provided with well-ventilated, shady and cool quarters. They should receive an abundant 
supply of fresh water, preferably water which has been slightly acidified with hydrochloric acid.

Bleeding, which is still practiced, proluces only temporary beneficial action in the presence of pulmonary congestion, but should be performed cautiously on account of the danger of spreading the infection.

Prevention. In order to protect healthy animals from infection care should be taken that the food, drinking water, utensils, etc., which are accessible to them, and also the ground, should not become contaminated by the excrement, blood, etc., of animals affected with anthrax. In cases, however, where this has occurred the infectious substances should be destroyed or at least propagation of the infection should be prevented.

This can be accomplished by the destruction of, or by rendering harmless all products and excretions of the affected animals and carcasses. The safest method for the destruction of the infectious material is by burning or rendering (steaming the carcasses under high atmospheric pressure should be employed to the greatest extent possible owing to the advantages afforded by this method). When this is impossible the carcasses should be buried at least 2 meters deep in ont-of-the-way, fenced-off places. They should also be covered with disinfectants, preferably powdered unslaked lime. The stalls occupied by the affected animals should be thoroughly disinfected. All food and straw that has come in contact with affected animals or their carcasses, or which has been contaminated by their excrement or blood should also be burned.

In common practice the best disinfectants are boiling water and steam, $0.1 \%$ corrosive sublimate, $5 \%$ carbolic acid, creolin or lysol solutions; also formalin vapors. However, it is necessary for these substances to remain in prolonged contact with the material to be disinfected owing to the great and variable tenacity of the anthrax spores.

According to Grossenthaler peat possesses disinfecting qualities on account of the ulnic acid it contains, and this action may be increased by the addition of $3 \%$ sulphuric acid. Hides may be disinfected with $5 \%$ ereolin or lysol, but this method is only absolutely successful when employed within two hours after the death of the animal (Lignières \& Zabala), otherwise it frequently fails. As a matter of fact at the present time there is no method known for an effective disinfection of hides without injuring them for their technical utilization (Xylander). Brekle believes that the question may be solved by subjecting the spore-containing hides in a moist condition to a temperature of $43-44^{\circ} \mathrm{C}$., by which all spores would germinate and the hides accordingly would contain only sporeless bacilli which could be destroyed with milk of lime. Horse hair is effectively disinfected by dry heat at $110^{\circ} \mathrm{C}$. followed by steam at $100^{\circ} \mathrm{C}$. (deRossi; see also page 5 ).

The officially required disinfection of railroad stock ears in Germany with $5 \%$ "Kresulfol" (cresol-sulfonic acid) was found by Schnürer to be absolutely ineffective against anthrax spores, and he recommended instead the disinfection with $1 / 2 \%$ formaldehyde solution.

When the disease appears careful search should be made for the source of the infection (origin of food, drving of hides in the hay loft, or in the stable, etc.). If suspicion is directed to the forage from certain fields or meadows this supply should not be fed, at least not while the animals are susceptible to the disease. Should the disease occur in a pasture the healthy 
animals should be removed to another higher and dryer place. When necessary they should be stabled. Not infrequently a careful investigation of the pasture will disclose dangerous places from which the animals should be kept. In other cases it is possible to suppress an outbreak by the exclusion of polluted, muddy watering places. In suspected places the dangerous character of the soil may be eliminated by draining and cultivation.

If under existing circumstances the danger of infection cannot be excluded, and the presence of a threatening infection exists, it is advisable to reduce the natural susceptibility of the animals by protective inoculations thereby protecting them from the danger of infection.

Immunization. Domestic animals may be immunized in practice by attenuated living cultures of the bacilli, by sporecontaining cultures, by imnune serum, and finally by immune serum and living cultures.

I. Immunization with attenuated cultures (Pasteur's method). In 1881 Pasteur established by conclusive experiments that anthrax bacilli, when subjected to a temperature of $42-43^{\circ} \mathrm{C}$., will continue for a time to multiply actively and no longer form spores. He further found that in such cultures the bacilli die in about a month, but that in the meantime their virulence decreases gradually, and finally he proved that the bacilli, when attenuated to a certain degree, will retain this degree of virulence if their cultivation is continued at body or room temperature. On this experimental observation is based the practical method of protective inoculation worked out by Pasteur with the co-operation of Roux and Chamberland.

The vaccine is prepared by growing the anthrax bacilli in bouillon at a temperature of $42.5^{\circ} \mathrm{C}$. The cultures which are at first fatal for rabbits, guinea pigs and mice are, after 12 days' growth at the above temperature, attenuated to such degree that they are only exceptionally fatal to rabbits. After an additional 12 days the culture kills only young guinea pigs and mice. After the virulence of the culture has been attenuated to these degrees their cultivation is continued at $35-37^{\circ} \mathrm{C}$. With such stock cultures fresh bouillon cultures are inoculated, and in this manner the vaceine is prepared in any desired cuantity.

The more attenuated culture represents Pasteur's first, the weaker vaccine, while the less attennated is the second or stronger vaccine (premier et deuxième vaccin). The protective inoculation is carried out by first injecting subcutaneously the weaker, followed in 10 to 12 days by an injection of the stronger vaccine. The immunization is based on the principle that the first inoculation reduces the natural susceptibility of the animal to such an extent, without endangering life, that it withstands 
without injury the second inoculation with the stronger vaccine. Without the first injection the second inoculation would probably prove fatal, while the two inoculations confer the necessary immunity upon the animals.

The results of the vaccination are in general satisfactory in view of the experience obtained from a large number of cases. Some of the immunized animals do not resist an artificial infection by feeding repeated large quantities of spores (in Koch's experiments 2 out of 10 sheep died after feeding them for days with virulent spores in quantities of the size of a hazelnut). These are exceptions which have no influence on the practical value of vaccination, inasmuch as under natural conditions the intensity of the infection is far below the amount given in these artificial infections. Where vaccination is practiced in large herds the losses from anthrax in the year following are in general greatly reduced compared with periods in which the animals were not vaccinated. In numerous localities where the disease raged regularly year after year it has almost entirely vanished as a result of vaccination of the animals. Not infrequently vaccination immediately checks the spread of the disease in a herd.

The immunity reaches the necessary degree in about 10 to 12 days after the second inoculation, and lasts about one year. (In the experiments of Azary vaccinated sheep resisted the artificial infection even after 8 months.) A longer duration of the artificially produced immunity is doubtful, and therefore a repetition of the vaccination is indicated annually in pronounced anthrax districts. The breed, age and sex of the animals do not appear to influence the results of the vaccination, and young animals which were only shortly weaned may also be vaccinated without danger and with promising results.

Pasteur's protective vaccination is therefore indicated in all places where the annual losses from anthrax exceed the cost of the vaccination.

The effectiveness of the vaceination has been proved by Pasteur beyond a doubt through control experiments which he earried out before a commission appointed for this purpose in 1881 at Pouilly-leFort. Of 50 sheep 25 were regularly vaceinated with his two vaceines and 14 days after the second vaceination they were inoculated with anthrax material, together with the 25 control animals. The results showed that inside of 2 days all of the 25 control animals died of anthrax, while all of the 25 vaccinated sheep remained alive.

Up to the end of the year $1899,708,980$ cattle and $4,971,494$ sheep had been vaccinated successfully in France, as a result of which the entire annual loss over a period of 12 years dropped to an average of $0.34 \%$ in cattle and to $0.94 \%$ in sheep.

In Hungary the first vaccinations were earried out in 1881 on Azary's initiative. Thuillier, Pasteur's assistant, applied them first at the Veterinary Institute at Budapest with very favorable results, while the later ones in Kapuvár were less favorable. As raccination gave very good results in practice, this protective treatment was soon taken uj very extensively. During the period of 15 years between 1886 and $1900,53,843$ horses, $1,015,700$ eattle and $2,279,221$ sheep were vaccinated. 
The official report contains the results on 39,506 horses, 718,266 cattle and $1,247,331$ sheep, and accorting to its statements the percentage of raccinated animals giren below died of anthrax:

\begin{tabular}{|c|c|c|c|}
\hline $\begin{array}{l}\text { After the first vaccination. } \ldots \ldots \ldots \ldots \ldots \ldots \\
\text { In the course of following years. } \ldots \ldots \ldots \ldots\end{array}$ & $\begin{array}{l}\text { Horses. } \\
0.10 \% \\
0.09 \%\end{array}$ & $\begin{array}{l}\text { Cattle. } \\
0.02 \% \\
0.02 \%\end{array}$ & $\begin{array}{l}\text { Sheep. } \\
0.26 \% \\
0.33 \%\end{array}$ \\
\hline Total & $0.19 \%$ & $0.04 \%$ & $0.59 \%$ \\
\hline
\end{tabular}

In Germany the results were at first less farorable (in Packish during the period 1852 to 1588 the entire loss of raceinated cattle amounted to $3.1 \%$ and of vaceinated sheep $2.8 \%$ ). Later, particularly in Württemberg and Alsace-Lorraine, the results were more satisfactory. Since 1900 in the vicinity of Magdeburg over 2,000 cattle, mostly draft oxen, have been vaccinated annually with uniformly good results, and since that time the previously frequent intestinal catarrhs (slight anthrax infections?) are no longer observed (Jungklaus).

In Austria vaccination was earried out on 9,456 fattening eattle with eomplete suceess during the period between 1894 and 1598. It was observed, however, that the vaccination of such animals during the summer months caused a severe reaction.

In Russia vaccination has also been followed by uniformly good results, with the exception of the fatal vaceination in the Crimea (1888), which was supposed to be due to an error in the vaccines, in which instance 4,564 sheep were vaccinated and 3,478 died in the course of a few days. The total loss in the district of Cherson, in which 20,000 sheep were vaccinated during the years 1885-1888, amounted to $0.87 \%$ compared with previous losses of $10 \%$ to $33 \%$ in former years (Wysokowicz).

Similar good results followed vaccinations in Holland (Wirtz), in Switzerland (Hess), as well as in America (Dalrymple), and Australia.

Direct results from the vaccination. In the vaccinated animals a slight elevation of temperature is observed as a rule in 2 to 5 days, which, however, is rarely accompanied by perceptible disturbances in the general health. Not infrequently in cattle an extensive subcutaneous edema develops in the vicinity of the point of inoculation, which, however, disappears in 1 to 3 days, especially if cold applications are made. Exceptionally, pregnant animals abort or give premature birth. In milk cows a diminished quantity of milk may be observed. Anthrax bacilli, however, do not pass into the milk as a result of the vaccination (Nekljudow).

Fatal results may sometimes follow even when the vaceination is carried out correctly, with faultless vaccine, probably as a recult of the low individual resistance of the animal. In several instances of this kind numerons losses were obserrer. Thus, excepting the case mentioned in the Crimea after the first vaccination in 1859 of 1,383 sheep in the Hungarian township of Lukáes, 77 sheep and 44 lambs died of anthrax, and in Guta in 1893 out of 1,564 vaccinated horses 72 died within 2 weeks after the first vaccination. The autopsies in this case revealed a septicemic complication in the form of a suppurative inflammation of the serous membranes, and a necrosis of the muscles at the point of inoculation, which was caused by a rotl-shaped bacterium (Preisz). In Foktö, of 680 horses 38 died in 1896 of anthrax in two weeks following vaccination. In this case the post mortem revealed a serere intoxication and anthrax bacili in association with other bacilli were found exclusively in the edematous swellings at the point of inoculation (Preisz). In the vicinity of Debreczen, of 30 horses vaccinated in 1902 with vaccine prepared for eattle, 9 became severely affected, 6 of which died of anthrax. In Austria, 316 eattle were vaccinated in the township of Storozynetz (Bukowina) in 1593, and amoug these 265 were fattening eattle. On the day following all animals manifester ferer, dyspnea, bloody, foamy discharge from the nose and rectum, and 24 died insirle of a few hours from peracute anthrax.

In cases in which the vaccine acter too severely the indication appears inside of a week following the first, or more frequently the second vaccination in which a marked edema develops from the point of inoculation followed by a general infection and the usual post mortem lesions of anthrax. In some cases the animals do not become visibly affected until after three wecks of incubation, in the meantiue manifesting a disturber appetite, finally succumbing to eachexia, which points to a chronic intoxication. After the vaccination of alreally infected herds the oceurrence of deaths may le the result of latent infection (see page 8 ) as in these bacilli carriers the resistance may possibly be reduced by the vaccination.

The attenuated hacilli contained in the vaccine retain their cultural characteristies in the body of the vaccinated animal (according to Preisz they form soft, 
rapidly liquefying capsules) as well as their reducer virulence, which eannot readily be increased even if passed through guinea pigs (Ascoli).

Technic of vaccination. The vaccine prepared by the "Institut Pasteur' or by the "Laboratoires Pasteur-Chamberland", which are established in the various countries, is marketed in suall bottles closed with rubber stoppers. The vaccine is drawn up into a sterilized syringe of about 1 ec. capacity, the plunger of which is graduated into 8 parts, and sheep, calves and foals are injected subcutaneously with $1 / 8$ of the contained vaccine, while mature eattle and horses receive $1 / 4$ (about 0.12 and 0.25 ce.). The injections are made in sheep on the inner surface of the thigh; in cattle posteriorly to the shoulder; in horses on the side of the neck. Prior to the injection the site should be clipped and carefully disinfected. Twelve days following the first vaccination the second injection is made at the corresponding place on the opposite side of the bocly.

The vaccine retains its potency for several days when kept in a dark and moderately cool place. Its effectiveness, however, is changer in time. It should therefore be fresh, and the contents of an opened tuke should be used up by all means on the same day. Good vaceine when shaken is only very slightly cloudy, rather opalescent; more pronounced cloudiness is an indication of deterioration (contamination with foreign bacteria), and such vaccine should not be used under any circumstances. The vaccine is prepared in a somewhat different way for the various species of aninals, and therefore vaccine for a specified species should be used. This is particularly applicable in the vaccination of horses (in horses, a mixture of the first and second vaceine of cattle is used for the second vaccine, and is therefore less severe than the second vaccine for eattle and sheep). The best time for vaccination is the spring, as anthrax usually occurs during the summer months, and an attempt should therefore be made to establish an immunity of the susceptible animals shortly before the period of danger.

Results obtained from the use of contaminated and ineffective vaccines prepared by the various laboratories indicate that official control is urgently desirable.

Bad results from vaceination eannot be excluded with certainty. On the one hand they may be due to the uncertainty of the degree of virulence and purity of the raccine, while on the other hand the variance in the individual resistance and susceptibility of the animals must be taken into consideration; and the veterinarian making the vaccinations is unable to judge these factors. Therefore owners of animals should be informed beforehand of the possible dangers. Errors committed during vaceination, especially neglect in the required cleanliness, may naturally endanger the results, as in such procedures other pathogenic bacteria may enter the body and procluce a passing or fatal disease. During the days immediately following vaccination the animals should be fed regularly and should not lie exposed to cold or heat, or overtaxed by hard work. As they are particularly susceptible to the infection during this time (negative phase) they should be kept from suspected pastures.

\section{Immunization with Spore-containing Vaccines. Cien-} kowsky (1884) in Charkow produced two vaccines by attenuation of living cultures at higher temperatures, the weaker of which kills the mouse and the earless marmot, but not the rabbit and sheep, while the stronger kills 30 to $50 \%$ of rabbits, and 10 to $20 \%$ of sheep. The cultivation of the attenuated cultures is then continued in bouillon at body temperatures until an abundance of spores are formed, when it is mixed with two parts of glycerin and placed in stock. Before the vaccination fresh cultures are made from the spore-containing fluid, and with these the animals are injected (Gordzialkowsky) or the spore-containing fluid itself is employed for the vaccination.

According to Rajewsky the fluid containing the bacilli and spores remains effective for a longer time in a cool, dark place. Otherwise both in the technique as well as the valuation of this form of vaccination the same rules are followed as in Pasteur's method. The dose of the vaccine consists of 0.1 cc. for sheep, and $0.2 \mathrm{cc}$. for larger animals. The animals usually stand the vaccination well, but disagreeable accidents are not totally excluded. Thus, 100 cattle were vaccinated in 1902 
in one estate and in three weeks' time $4 t$ beeame affected with anthrax, 35 dying of the disease.

Satisfactory results are olitainel in many places with spore-containing vaceine. This methou was also employed several years ago in Hungary (aceorling to Detre, with vaccines which he prepared by washing old agar eultures with a salt-glycerinwater mixture). In 1901 to 1902 the losses amounted, accorcling to Detre 's estimate, to $0.26 \%$ in horses, $0.03 \%$ in cattle and $0.12 \%$ in sheep.

Accorling to a report of the Veterinary Institute at Charkow, Russia, 5,584 horses, $19,5 \div 2$ eattle and 174,172 sheep were vaccinated in 1597 in the southwestern part of Russia with losses of $0.25,0.09$ and $0.35 \%$ respeetively. In Russia a methoul of vaceination originated ly Lange is also used. The preparation of this vaceine, however, is unknown.

Accorling to Beinarowitech the direct loss in reindeer from vaceination amounts to $1 \%$.

III. Immunization with Immune Serum. If animals susceptible to anthrax are injected with increasing loses first of attenuated and later of fully virulent anthrax cultures, they will after a certain time prodnce a serum, which, while possessing no direct bactericidal action, protects rabbits, guinea pigs and sheep against a fatal infection. If injected shortly after the infection such serum even prevents the development of the disease. Such immune serum also renders good service in practice, especially in cases in which infection threatens, or where anthrax lias already appeared in a herd. The action of the injected serum becomes effective in the course of a few hours. However, the established passive immunity is only of a short duration, lasting only from 1 to 2 weeks. It is therefore advisable in cases of continued danger of infection to render the animals actively immune. This may be accomplished by a subsequent regular vaceination with attenuated cultures by one of the ahove described methods.

Potent anthrax sermu was first prepared from sheep hy Marchoux, and also by Sclavo almost at the same time, in 1595. Later Sobernheim, Mentez, Detre, Carini and Ascoli carried on investigations along this line, but they employed large animals, especially horses, for this purpose. This method was also followed by Sclavo. The horses withstand after a certain time an intravenous injection of $500 \mathrm{ec}$, and even more of a virulent eulture; and they produce a very potent serum. Of Selavo's serum 0.5 ce. protects a rabhit and from 5 to 10 ee. protect a sheep against a fatal infection. Detre's serum has a similar effect on rabbits, while from 3 to $5 \mathrm{ce}$. will protect a guinea pig against an intraperitoneal infection, which is ordinarily fatal in from 1 to $1 \frac{1}{2}$ iays. In the experiments of Sobernheim, of whose serum 2 ce. protects rabhits against $1 / 1000$ of a loopful of a virulent culture, the serum provel a protective agent even against the feeling of antlirax spores. Sanfelice prepared a serum by immunizing dogs, and $3.5 \mathrm{ce}$. of this serum per kilo weight hail a protective effect, while 7 ce. had a curative effect on rablits even when alministerel 40 hours after the infection.

In practice the lose for large animals eonsists of 10 to 20 ec., while for small animals it is 5 re. Solernheim uses a mixed serum which is obtained from eattle, horses and sheep; Selavo obtained it from asses. Fven when exposed to the harmful influences of light and air the serum retains its potency for $21 / 2$ years (Sobernheim).

In alrealy infected herds the sprear of the outbreak may be ehecked by treating only those animals with serum in which a rise in temperature is observed; the temperature of all animals being taken twice daily (Keleti). Of course, if fever is present the serum should be used in correspondingly higher doses, and the injeetions should be repeated until a drop in the temperature is noticed.

In some cases after the administration of the serum, anaphylactic symptoms appear. These oceur in from 5 to 20 minutes, and are manifested by edematous 
swellings of the head, at the point of inoculation and other parts of the body. Nodular eruptions resembling urticaria, frothy discharge from the mouth, snorting, reddening and swelling of the nucous membranes, uneasiness, and in cattle bloating, may also be observed. Kovárzik observed these symptoms in two herds in about $20 \%$ of the inoculated cattle, anong which the two showing the strongest reactions died from asphyxiation. Inasmuch as the reaction appears well pronounced in animals that were especially exposed to the danger of infection, Kovárzik and also Zimmer, from his experience in horses, are inclined to believe that such an affection is a reaction, indicating an alrearly existing latent infection (anaphylaxis due to proteids of the bacilli which circulate in the blood of the latent infected animals, against the analagous proteid contained in the immune serum).

\section{Immunization with Immune Serum and Cultures} (Sobernheim's method). Sobernheim (1902) recommends for a lasting, combined, passive and active immunization of domestic animals a simultaneous vaccination with serum and anthrax cultures (cattle and horses are injected on one side of the body with $5.0 \mathrm{cc}$.; calves with 3.0 to $5.0 \mathrm{cc}$., and sheep with $4.0 \mathrm{cc}$. of an immune serum; and five minutes later, they are injected on the opposite side with 0.5 or $0.25 \mathrm{cc}$. of an attenuated culture, washed in salt solution, the degree of virulence of which corresponds with Pasteur's second vaccine). This method has the great advantage of requiring only a single treatment of the animals, and the results obtained from it are, with the exception of a few failures, quite satisfactory. Svarde (1906) however, claims that the superiority of this method over Pasteur's has not been establisher.

After vaccination there is usually a moderate elevation in temperature of 0.5 to $1.0^{\circ} \mathrm{C}$., slightly diminished appetite and milk secretion. A swelling often occurs at the place of inoculation. The nasal mucous membrane may be swollen, and an exanthema resembling urticaria may be observed. Occasionally the vaccination results in the death of the animal (most often olsserved in work oxen).

In Argentine and Truguay this method of vaceination was employed from the spring of 1904 to September, 1905 , on 140,000 cattle, 30,000 sheep and 2,000 horses. According to Sobernheim no fatalities resulted from the vaccination, and almost everywhere a complete eradication, or at least a marked restriction of anthrax was noted.

In Germany, according to Sobernheim's statisties, up to the year of 1906 about 4,500 cattle and sheep were successfully vaccinatel. Raebiger \& Jöhnk reported very satisfactory results, although some failures were also observed by them. According to other reports, however, the results appeared unfavorable. Thus, according to Burow, of 5,000 eattle vaceinated by this method in Pommern, $8(0.15 \%)$ died as a result of the vaccination; besides, from 4 to 7 weeks later $3(0.08 \%)$ adaitional deaths resulted from anthrax. In one instance, of 39 vaceinated eattle, 3 died; in another ease, of 137 cattle, 37 showed indications of anthrax insile of 5 days of which 8 died (Heine). Unsatisfactory results are further reported by Hummel (losses from vaccination, 139 sheep) and Lothes (of 78 eattle, 2 deaths, 17 severe affections, marked diminution in milk secretion in cows).

V. Other Methods of Vaccination. Previous to the experiments of Pasteur, Toussaint (1880) vaccinated with anthrax blood heated for 10 minutes at $55^{\circ} \mathrm{C}$, or mixed with $1 \%$ carbolic acid.

Chauveau (1883) prepared a vaccine by heating bouillon cultures which were grown at 42.5 to $47^{\circ} \mathrm{C}$., then kept at $37^{\circ} \mathrm{C}$. until sporeformation, and finally heated to $80-84^{\circ} \mathrm{C}$. Other vaccines were made 
by suhjecting the spores to a pressure of approximately 8 atmospheres, also by growing the virus in pure oxygen and under pressure of $21 / 2$ atmospheres.

According to Sobernheim, Chauveau's method of immunization is used in Chile, where 80,000 to 85,000 animals are vaceinaterl annually by that method; the results are inclicated as being favorable in cattle, but only fairly good among sheep.

According to Chamberland \& Roux, the action of a mild solution of carbolic acid (1:600) on anthrax bacilli for 24 days will attenuate them to such an extent that they represent protective vaccines. The same result is obtained by the action of bichromate of potassium ( $1: 2000-5000)$ for 10 days, and according to Gibier by cooling to $-45^{\circ} \mathrm{C}$, whereas Murillo prepares a vaccine which is also supposed to be satisfactory in practice by growing the culture alternately in diphtheria toxin and in bouillon.

Arloing, and also Hankins succeeded in immunizing test animals with bacteria-free filtrates of culture. Similar results were obtained by Ogata \& Jasuhara with a ferment from the blood serum of anthrax animals. Aujeszky also succeeded with a mixture of anthrax bacilli and spleen tissue, and also with the spleen pulp alone. According to Emmerich, Pawlowsky, Bouchard, Gilruth and others, an immunization is possible by the treatment with organisms which are antagonistic to the anthrax bacillus ( see page 6).

Finally, Bail (1904) used for the immunization the sterilized edema fluid of anthrax animals. He presumed that the aggressins (lysins in the sense of Kruse) of the anthrax baillus, which are present in the edematous fluid, favor the development of the pathogenicity of the bacillus, and if injected into an animal they would stimulate the production of antiaggressins which would protect the animal against a bacillary infection. Sheep which received 2.5-10 ec. of such edema fluid subcutaneously developed an immunity even against severe subcutaneous infection with bacilli in from 8 to 10 days. If, however, a certain quantity of the edema fluid is exceeded the body is unable to use it up, and because of the increased aggressins present a hypersensitiveness to infection develops. This method has not yet been tried in practice.

Veterinary Police. The veterinary police regulations refer in the first place to the harmless disposition and destruction of all excrements of anthrax animals, and to the cremation, rendering or deep burying of their carcasses. Diseased animals should be kept separated from healthy ones, and their stalls should be disinfected. The food and straw which have come in direct contact with affected animals should be rendered harmless; meat, milk and raw products in general (also hides) of affected animals should not be permitted to enter the trade. On the other hand, quarantine restrictions for healthy animals with the exception of severe outbreaks do not appear justified as the disease is not directly contagious.

Literature. Gerlach, Mag., 1845, XI, 113; 1846, XII, 321.-Heusinger, D. Milzbrandkrkh. d. Tiere u. ґ. Mensehen, Erlangen, 1850 (Old Lit.).-Pollender, Vierteljsehr. f. ger. Med., 1855, 102.-Brauell, V. A., 1857, XI, 131. Davaine, C. R., 1863, LVII, 320; 1864, LIX, 393.-Bollinger, Beitr. z. vergl. Path., 1872, 122; D. Z. f'. 
Tm., 1585, XI, 160.-Koch, Beitr. z. Biol. d. Pflanzen, 1876, II ; Mitt. d. G.-A., 1881, I, 49.-Pasteur, C R., 1877, LXXXIV u. LXXXV.-Pasteur, Chamberland \& Roux, C. R., 1880, XC u. XCI ; 1881, XCII.-Oemler, A. f. Tk., 1800, VI, 401.-Koch, Mitt. d. G.-A. 1581, I, 134; 1884, II, 161.-Löffler, Ibid., p. 49.-Roux \& Chamberland, A. P. 1887, I, 513; 1888, II, 405.-Kitt, Monh., 1894, V, 215 (Review on diagnosis). - Sclavo, Cbl. f. B., 1895, XVIII, 744.-Sobernheim, Z. f. Hyg., 1897, XXIV, 301; Hb. f. p. M., 1904, III; 1904, IV, 793 (Lit. on etiol. and vaceination).-Conradi, Z. f. Hyg., 1899, XXXI, 323 (Lit. on toxins).-Bongert, Cbl. f. B., 1903, XXXIV, 497.-Preisz, Cbl. f. B., 1904, XXXV, 280; 1907, XLIV, 209; 1909, XLIX, 341.Ernst, Monh., 1905, XVII, 172 (Lit. on diag. meth.).-Oppermann, A. f. Tk., 1905, XXXII, 41 (Lit.).-Gruber \& Futaki, M. m. W., 1907, 249; D. m. W., 1907, 1558.Wyssmann, Schw. A., 1907, XLIX, 287 (Lit. on anthrax in hogs).-Xylander, Arb. d. G.-A., 1907, XXV, 457.-Fischoeder, Cbl. f. B., 1909, LI, 320 (Lit. on capsule formation).-Hoppe, Diss., Leipsic, 1909 (Lit. on pseudo-anthrax bacilli).

Anthrax in Man. Anthrax in man usually occurs as a local affection of the skin and subcutaneous tissue (carbuncle, pustula maligna, carbunculus malignus) as a result of skin infections. Therefore it is chiefly persons who come in contact with diseased or dead animals who become affected. The persons who most commonly receive accidental infections are veterinarians, herders, butchers and laborers who work on hides, hair, bristles and wool. In these forms of employment there is also a possibility of infection through the inhalation of spores, and it appears that the so-called "rag disease" or "woolsorters' disease" in many cases develops in this manner. Infection takes place very rarely through the intestinal canal, as the meat of anthrax animals as a rule only contains bacilli and not spores, and they are digested by the gastric juice; furthermore the virus is usually destroyed in the cooking of the meat. Therefore this method of infeetion only occurs after the ingestion of putrefactive and improperly kept meat, or from sausage prepared from infected meat which was not fresh. It is also possible that infection is transmitted by flies.

The development of the carbuncle usually commences with a lancinating pain at the point of infection and is soon followed by the appearance of a bright red nodule, from which a blackish-red vesicle forms, containing a reddish serous fluid. After the rupture of the vesicle the breaking down of the tissue commences, whereupon in the surrounding parts new nodules and vesicles form and the affected area shows marked edematous infiltration. The subcutaneous connective tissue, and the skin appear reddened as in erysipelas. In the meanwhile general febrile manifestations develop until the infection becomes general and the patient succumbs from septicemia. The intestinal infection manifests itself in a severe (frequently hemorrhagic) inflammation of the intestines which is accompanied by severe abdominal pain, vomiting and chills, until collapse and symptoms of asphyxiation terminate the disease. Both forms may occur combined, and especially may an intestinal infection be followed by the appearance of edematous swellings in various parts of the body. Inhalation anthrax is manifested by an acute pleuritis or broncho-pneumonia.

The carbuncle developing after a local infection can be cured by early surgical treatment; and frequently in such cases, as well as in intestinal infections, the administration of immune serum is followed by good results.

Literature.. S. Korányi, -Nothnagel's Handbuch, 1909, V, Bd., 1. T. 


\section{Malignant Edema. Oedema malignum}

(Septicaemia gangracnosa, Septicémie gangréneuse [French]; Malignes Ödem [German]; Setticemia gangrenosa [Italian].)

Malignant edema is an acute, febrile, infectious wound

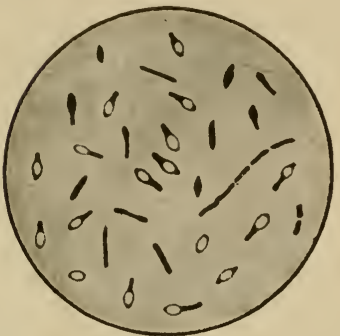

Fig. 8. Edema bacilli with flagella. Agar culture; flagella staining.

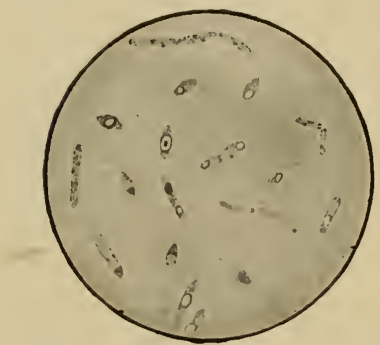

Fig. 9. Bacillus of malignant edema. Sipore formation three-day old agar culture, stained with diluted fuchsin solution.

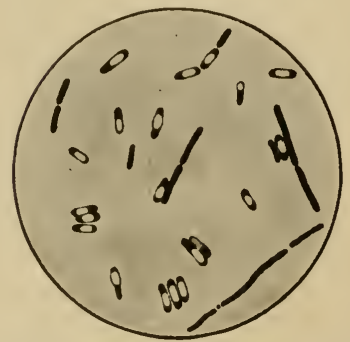

Fig. 10. Bacillus of malignant edema. Peritoneal exulate from guinea pig; dried cover glass preparation; fuchsin staining. disease of domestic animals, which is characterized principally by edematous, later crepitating swellings at the place of infection. Its cause is the anaerobic bacillus oedematis maligni.

History. The development of erepitating swellings following injuries, seatons, eastrations, ete., has been known since earliest times, and Girarıl (1880) proved that erepitating swell. ings in sheep may be produced by putrid animal substances. Chauveau (1873) showed by experiments carried out on male goats that the living organisms contained in injected putrid blood were closely associated with the development of the disease. Pasteur (1877) studied more closely the bacterium, named by him as "Vibrion septique," and also succeeded in growing it in pure culture. Later Koch \& Gaffky (1881) extensively investigated the disease produced by the inoculation of dirt, and which they termed malignant edema. Since that time Kitt, Jensen \& Sand, and Leclainche, have contributed to the bacteriology, while new observations regarding its occurrence in domestic animals have been published by Jensen \& Sand, Rátz, Fröhner, Carl and others.

Occurrence. Malignant edema occurs everywhere since its causative agent is ever present in the superficial layers of the soil. Nevertheless it is not a very frequent disease, but may occur in horses and cattle, in cows particularly after parturition. Occasionally in sheep it causes great losses, and may in reterinary hospitals among horses assume an enzootic character as a sequel of operations (L. A. Merillat). 
Etiology. The bacillus oedematis maligni, Koch, (Vibrion septique, Pasteur) is an anaerobic bacterium resembling the anthrax bacillus, but appearing somewhat more slender, rodshaped, peritrically flagellated, motile and multiplying by fission and spore formation. (Figs. 8 to 10.) Several rods unite to form longer chains in which the ends in contact with each other, as well as the free ends, appear rounded. In artificial media, as well as inside the animal body after death, the bacilli grow to long, eurved unsegmented threads. In the bacilli, oval spores develop either in the middle or ends (exceptionally even the edematous fluid taken from the living animal contains sporebearing bacilli [Jensen \& Sand]).

Staining. With aqueous aniline dyes the bacilli stain readily, and they also take the Gram stain after being carefully treated with aleohol.

Cultivation. The eultivation of this organism is only possible under oxygen-free conditions. Stab cultures in agar or gelatin show along the stab cloudy or lentil-shaped colonies which usually develop at the bottom of the medium and close to which the gelatin is later liquefied. In coagulated blood serum and also in dextrose bouillon (not in saecharose and lactose, Smith) gas having a characteristic sweetish putrid odor is formed, which later causes disruption of the media. (Fig. 11.) Kerry found in the gases hydrogen, hydrogen sulphide, carbon dioxide and methane. Milk is changed in a few days into a watery fluid with gas formation and the colleetion of a layer of fat on the surface (Smith). In bouillon cultures the bacteria produce toxins in small quantities (Roux and Chamberland).

The spores show great vitality. Dry muscle substance and sporc-containing edena fluid enclosed in glass tubes prove virulent even after years. In fluid media spores are destroyer after heating to $100^{\circ} \mathrm{C}$. for fifteen minutes (Sanfelice), while in the dry state they are killed in ten minutes at $120^{\circ} \mathrm{C}$. (Arloing). Direct sunlight kills them only slowly. Disinfectants are only effective in strong solutions and after prolonged contact. Sporeless bacilli are quickly destroyed in the presence of air and sunlight as well as by putrefaction.

Pathogenicity. After the inoculation of a pure culture in the depth of the connective tissue, a rapidly extending swelling develops at the point of injection. It becomes crepitating as

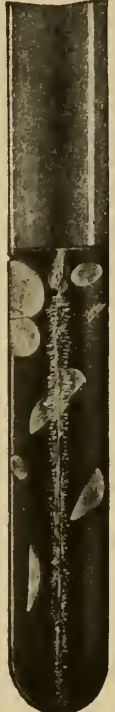

Fig. 11. Stab eulture of ellema bacillus in dextrose agar. a result of gas formation within, and the animal soon dies of a form of septicemia. Guinea pigs and mice suceumb in from 24 to 28 hours. Cattle can be infected only with great difficulty, and feeding of spore-containing material will not produce the disease even in susceptible animals. Edema bacilli injected into the blood produce the disease only when they find a suitable soil for their development somewhere in necrotic tissue (for instance, in previously ligated testicles [Chauveau]). 
Probably there are also other bacteria capable of producing a disease resembling malignant edema. Thus, Novy observed the appearance of a similar disease in guinea pigs following subcutaneous injections of milk nuclein, and he proved that an organism closely resembling the above-described bacillus was the cause. However, it was somewhat more slender and provided with strikingly long and thick flagella. It is also an anacrobic but sporeless bacillus (Bac. od. maligni II). Klein isolated from garden soil (Bac. oed. sporogenes) and Sanfelice from guinea pigs inoculated with dirt containing putrid organic substances, a similar, aithough only facultative anaerobic, bacillus (Bac. od. ærogenes). According to Fraenkel the Bac. phlegmones emphysematosie produces in man a similar disease (gangrène foudroyante). On the other hand, according to Kirsten the edema bacilli, like most of the species of bacteria, form groups of varieties which may be distinguished from each other by their morphological and biological characteristics. However, in their clinical relation the most important characteristic is the absence of pathogenicity in most of the varieties.

For natural infection of domestic animals solipeds are most susceptible, while cattle, sheep and goats are less susceptible. Hogs, dogs and cats are rarely affected.

The infection results in most instances from the entrance of the edema bacillus into the deeper parts of the subcutaneous or submucous tissue through breaks in the continuity of the skin or the mucous membrane of the mouth and esophagus. There are many opportunities for such infections as the bacilli are widely disseminated in the dirt, especially in dust of hay, in garden or field soil, in street dust, in household drainage, in putrid organic material, and in excrement of healthy animals. These substances are very prone to come in contact with wounds on the body, and yet the rare occurrence of the disease can be explained by the fact that successful infection occurs only when large masses of bacilli enter the lymph spaces of the connective tissue which contain but little oxygen. On the other hand, where the infectious material enters superficial wounds on the skin, granulating ulcerations, or even directly into the blood stream, the bacilli cannot propagate on account of the presence of oxygen. Hence the infection occurs either through contused or lacerated wounds or during parturition by means of contaminated hands or instruments. In other cases the infection may also follow vaccination, castration, shearing, bleeding, introduction of seatons, subcutaneous injections with unsterilized medicines (eserine, morphine, creolin, arecoline), unclean syringes, bites, penetration of straws into the canthus of the eye, etc. The disease has also developed in association with necrotic processes.

Lembeken observed 50 out of 600 and Gilruth 300 out of 4,000 sheep dying from malignant edema during or after shearing.

Infection may also occur through injuries or necrotic ulcerations on the mucous membrane, whereas the healthy mucous 
membrane affords an effectual protection. Feeding and inhalation have failed to successfully transmit the infection to experiment animals.

Pathogenesis. After the germination of the spores in the lymph spaces the bacilli continue to multiply by fission at the point of infection until large bacterial colonies develop. In the meantime gases are formed by the dissociation of the tissue fluids, and toxins are produced under the influence of which the blood ressels are dilated, allowing the blood plasma to transude into the connective tissue spaces through the relaxed walls of the vessels. With the penetration of the bacilli the swelling enlarges, and in case the point of infection is near the abdominal cavity, the bacilli multiply rapidly on the surface of the peritoneum where they grow into long threads. At the same time the absorbed toxins act also on the central nervous system, particularly on the respiratory and heat centers, as a result of which respiration becomes difficult and the temperature elevated.

The edema bacilli are capable of multiplying in the tissues only under special conditions. The spores will germinate only in the presence of toxins (from older cultures) in the tissues, because otherwise they would soon be taken up by the phagocytes and rendered harmless. Their propagation is also facilitated by the simultaneous entrance into the tissues of other micro-organisms (Bac. prodigiosus, staphlycocci, diplococcus pyogenes and other's). In addition propagation is aided by the presence of particles of dirt or sand which protect the spores mechanically against the phagocytes, while toxins and substances like lactic acid, keep away the cells through negative chemotaxis (Besson, Leclainche \& Vallée). At the same time the presence of other bacteria has an influence on the development of the pathological changes and gives rise to numerous eases of mixed infection.

Anatomical changes. During the formation of a swelling in any part of the body the connective tissue becomes distended and infiltrated with a yellow or reddish fluid which contains gas bubbles and liberates a peculiar disagreeable odor. The gelatinous infiltration of the connective tissue may also extend between the deeper layers of the muscles, while the muscle substance proper turns pale yellow or dark red in color, becomes brittle and tears readily. The connective tissue appears in places permeated with smaller or larger hemorrhages. The abdominal cavity contains a small quantity of reddish serous fluid; the peritoneum is markedly injected, iustreless, but is not covered with an exudate.

If the malignant edema has developed after parturition, the uterus is found insufficiently contracted, and the subserous connective tissue of the small pelvis shows edematous infiltration. The wall of the uterus is also edematous, the mucous membrane 
swollen and covered with a dirty, pasty, ill smelling mass which also covers the cotyledons that are in the process of breaking down (Carl).

The spleen as a rule appears normal in size or slightly swollen. Exceptionally, however, an acute swelling of this organ with gas formation in the pulp has been observed (Fröhner). The liver shows cloudy swelling and the intestinal mucous membrane shows indications of an acute catarrh. The lymph glands are acutely swollen, while the lungs manifest well marked active hyperemia and also edema (which, according to Kitt, are occasionally the only lesions found in sheep); the heart muscle usually shows marked parenchymatous degeneration. The blood is slightly coagulated, and the carcass decomposes rapidly.

In the fluid squeezed out from the crepitating swellings or taken from the cut surface, or frequently in the abdominal fluid, edema bacilli may be found in large numbers even in the unstained miscroscopical preparations. The bacilli, which not infrequently contain spores, occur singly, in chains or as curved threads, and are often found in association with other bacteria. Several hours after deatl the bacilli are found in the blood, particularly the blood of the lungs where they often occur in long threads similar to those seen on the peritoneum.

The presence of the bacilli of malignant edema is not in itself proof that the particular animal died from this disease. These bacilli occur frequently in association with other bacilli in the intestinal contents of herbivorous animals, and from there they may spread after the death of the animal throughout the body even to its most distant parts. This is particularly the case when the blood remains fluid, as for instance, in a suddenly appearing asphyxiation, and thus promotes their multiplication and dissemination. From the intestines the organisms first enter the peritoneum, next the blood vessels of the intestines and mesenteries, and then the portal vein from whence they may be disseminated throughout the blood.

Symptoms. After infection an edematous swelling, which is not sharply circumscribed, may develop in 12 to 24 hours in any part of the body. The swelling is at first tense, warm, very painful, later cooler, soft, doughy and less sensitive, and may even become entirely insensitive in the center, so that the animal does not manifest pain when incisions are made into the swelling (at the borders, however, it shows the characteristics mentioned). At the same time the swelling reveals crepitation on palpation. The swelling usually develops at places where considerable loose connective tissue occurs under the skin; by spreading over the surface it may occasionally involve the entire side of the body, anteriorly to the neck, posteriorly to the upper parts of the extremities, resulting in difficult and painful movements of the body. Upon incision a reddish-yellow, reddish-brown, or a colorless serous fluid is 
discharged which contains gas bubbles. In some cases the skin over the swelling acquires a dark color, and may finally become extensively necrotic.

In cases where the swelling develops in association with an evident injury the wound takes on a bright color and a peculiar lustre, and a dirty red, fetid, serous fluid oozes from its surface. Later the margins of the wound become necrotic in portions.

If the infection occurs through the genital passage during or shortly after parturition the first manifestation of the disease appears after 2 to 5 days. The vulva swells in the form of a cushion, and a dirty, brownish-red, very fetid, thick secretion is discharged from the vagina. Soon a crepitating swelling develops in the surrounding parts of the external genitals which is at first warm and painful but later cool and painless, and gradually extends over the perineal region and the lower abdomen. Besides high fever, bloating and diarrhea are observed.

Malignant edema developing in association with parturition in cattle is frequently mistaken for blackleg. Recently Alrert \& Carl established with a certain degree of probability that the eases described as parturition blackleg are actually malignant edema, and that blackleg never develops in this form.

Following castration the first symptoms appear after a few days or sometimes after several weeks; aside from high fever, an extensive edema develops under the skin of the abdomen, emanating from the castration wound, and later shows crepitation on palpation. In addition the animal manifests colicky pains, sensitiveness over the abdominal wall and bloating; in other words, there are indications of peritonitis, and finally signs of a general infection and exhaustion.

Of the other symptoms of the disease the markedly difficult respiration, the cyanotic mucous membranes, the accelerated and weak heart action, and the corresponding small, wavy pulse are conspicuous. The body temperature reaches a high degree even at the onset of the disease, remains at a uniform height, but as death approaches it drops to normal or subnormal. In later stages of the disease diarrhea is very frequently observed in which fetid and watery feces are discharged.

The symptoms follow each other rapidly; the crepitating swelling increases in size from hour to hour, and death usually occurs in twelve hours to three days after the appearance of the first symptom. In some of the cases, however, the disease terminates in recovery, especially after appropriate treatment.

Livesey observed the disease in a dog. On the left side of the head an emphysematous swelling developed and progressed to the neck and shoulders. At the same time the lips and cheeks became necrotic. Pankul demonstrated the disease in hogs; a crepitating swelling developed in this instance from a wound in the skin. 
Diagnosis. In cattle malignant edema may be mistaken for blackleg. Malignant edema is indicated by the appearance of the disease in localities where blackleg is not prevalent, further by the advanced age of the patient, the oceasional localization of the swelling in parts which are poor in muscle tissue, and by the relatively severe affection of the commective tissue compared with the slight involvement of the muscles. Parturient apoplexy is differentiated from malignant edema by the absence of the crepitating swelling, and the different bacteriological findings.

In horses and sheep the development of a crepitating swelling under febrile manifestations indicates malignant edema. The crepitation distinguishes the disease from edema, which often resembles it very closely, as well as from the inflammatory edema which is causerl frequently by a streptococcus infection following injuries; whereas in the simple subcutaneous emphysema there is in addition to the pre-existing skin or lung injury an entire absence of febrile symptoms. On post mortem care should be taken not to mistake the emphysema caused by putrefaction with the crepitating swelling which develops during life. (For differential diagnosis regarding the bacilli, see p. 20.)

Treatment. The only possible method of treating the patients consists in incising the swellings as they are forming in one or several places. This is to be followed by squeezing out the serous fluid and by thorough disinfection of the wounds; at the same time it is advisable to inject disinfecting substances subcutaneously into the tissue immediately surrounding the swelling.

While the disinfectant treatment is practically without effect upon the spores of the edema bacilli, it indirectly prevents the germination of the spores by destroying the foreign bacteria which entered the wound and also prevents the multiplication of the bacilli.

Prevention. In order to prevent the occurrence of the disease, wounds of the skin and mucons membranes should be guarded against contamination with dirt. Those wounds which have already become contaminated should be disinfected, whereas, after difficult parturitions in which the genital passages have been injured a thorough irrigation of the vagina and uterus will prevent the disease.

Immunization. Experimental animals may be immunized against virulent infections by injections of spore-containing tissue fluids which have been heated for 7 hours to $92^{\circ} \mathrm{C}$. (Leclainche \& Vallée); by bouillon eultures heated for 10 minutes to $105-110^{\circ} \mathrm{C}$., also by filtrates of the edematous fluid, or through the toxins contained therein (Roux \& Chamberland); further by hlood serum of immunized animals which have received repeated injections of virulent material (Duenschmann, Leclainche \& Morel). These inoculations protect only against malignant 
edema but not against anthrax also, as was proven by Leclainche \& Vallée, and which is contrary to the former views of Roux \& Duenschmann.

Literature. Chauveau, Ree., 1873, 263.-Pasteur \& Joubert, C. R., 1877, LXXXV, 101.-Koch, Mitt. d. G.-A., 1881, I, 49.-Affky, Ibirl., S. 83.-Chauveau \& Arloing, Acad. de Méd., 1884, XIII, 604.-Kitt, Münchn. Jhb., 1883-84 u. 1884-85; Monh., 1897, VIII, 206 (Review) ; 1900, XI, 49.-Jensen \& Sand, D. Z. f. Tm., 1887, XIII, 31.-Roux \& Chamberland, A. P., 1887, I, 561.-Leclainche \& Vallée, Ibid., 1900, XIV, 590.-Frohner, Monh., 1901 to 1903, XII-XIV; Allg. Chir., Wien, 1905 (Lit.).-Carl, A. f. Tk., 1903, XXIX, 225.-Jensen, Hb. f. p. M., 1903, II, 619 (Lit.).Livesey, Vet. Rec., 1906, 356.-Hohneker, B. t. W., 1907, 267.

\section{Blackleg. Gangraena emphysematosa.}

(Black Quarter, Quarter Ill [English]; Rauschbrand, Kalter Brand, Rauschender Milzbrand [German]; Emphysema gangraenosum, Sarkophysema haemorrhagicum; Charbon symptomatique, Charbon bactérien, Mal de cuisse, Mal de montagne [French]; Carbonchio enfisematosa o sintomatico, Acetone, Quartô nero [Italian].)

Blackleg is an acute, infectious, but not contagious, epizootic disease of cattle, and exceptionally of other ruminants and of swine, in the course of which crepitant swellings develop in the musculature of certain regions of the body, accompanied by febrile symptoms. It is caused by the anaerobe, bacillus gangraenae emphysematosae.

Historical. Until the seventies of the last century, the disease was considered as identical with anthrax or as a particular form of that disease. As early as 1782 , Chabert had differentiated the disease from anthrax under the name of charbon symptomatique, while Bollinger (1875) and Feser (1876) showed that blackleg was to be separated from anthrax from an etiological point of view. Both investigators observed the bacilli contained in the crepitant swellings, and produced the disease in ruminants through injection of the serous fluid; Bollinger also caused it by introducing subcutaneously mud from places in which blackleg was frequent. Later the etiology of the disease and properties of the bacteria were investigated thoronghly by Arloing, Cornevin \& Thomas (1879-1884), and through these stridies not only the etiology of the disease was determined upon, but they also introduced a practical method of protective vaccination. The bacteria which the French call bacterium Chauveaui were developed in cultures by Roux (1887) and later by Kitasato (1889), while still later Kitt, Leclainche \& Vallée, as well as Grassberger and Schattenfroh, eompleted our knowledge in that direction.

Occurrence. Blackleg appears mostly in mountain regions and in valleys with swampy pastures, but is also observed on low lands exposed to inundation. In such regions it appears chiefly during the hot season and causes nearly every year con- 
siderable losses among the young cattle, while older animals are attacked only exceptionally. During the winter, and when the cattle are fed in the stable, the disease appears very rarely.

In Germany, blackleg was olsserved during the years 1888 to 1905 most frequently in the P'russian provinces, Schleswig-Holstein, Rhimeland and Westphalia, and on the mountain pastures of the Rhine provinces; before the introduction of the protective vaccination, 4 to $8 \%$, at some places even 12 to $15 \%$, of the young cattle were destroyed by the disease. During the 10 years 1899 to 1908 , in about 188 districts 7 (?) horses, 1,193 cattle, 36 sheep and 2 goats were infected with the disease; the number of districts varied between 157 (1897) and 214 (1908), while the number of cases varied between 928 (1902) and 1973 (1906). More than two-thirds of the communities and cases were in Prussia, where the government district Schleswig was particularly attacked; then follow Bavaria, Hesse, and Württemberg. According to Scheibel, blackleg is often observed in Upper Hesse in sheep and goats.

In Austria the disease appears chiefly in Lower Austria and Tyrol, while the coast regions, Silesia and Dalmatia, are nearly free from it. During the years 1891 to 1900 , the average loss amounted to 552 head of eattle or $0.006 \%$ of the total stock in the involved regions. The number of infected localities varied between 142 (1892) and 227 (189s).

In Hungary, the disease appears on the mountain pastures of Siebenbürgen and in the Northeastern part of the country, and also in the former inundation clis trict of the Tisza River. Although the disease is not subject to obligatory notification, 1,460 cases were reported from 30 communities in 1907.

In Switzerland the average annual loss during the years 1897 to 1202 announted to 687 head of cattle, two-thirds of which must be charged to the cantous of Berne, Friebourg and Waadt. During the year 1908, in 272 communities, 779 animals were attacked.

In the other European countries blackleg is also in evidence, and in 1908 Belgium had 274 cases of the disease, mostly in the provinces Liége and West Flanders; Italy had 407, and Norway 67 cases, while in Sweden there were 56 infected farms. In France the northerly and southeasterly mountain districts are severely infected, while in Great Britain it is Scotland, and in Denmark, Jutland. In Holland the disease causes greater losses than anthrax.

In Africa, blackleg has been observed in Algeria and the southern part of the continent (there known as Spons-Ziekte and Swamm-Ziekte), and it is said that in many regions of the Transvaal 10 to $25 \%$, sometimes even $50 \%$, of the young cattle are destroyed annually by the disease. In America the disease has been observed in most of the northern states and seems to be very common. During the year 1906, from the Federal Bureau of Animal Industry alone, there were shipped 1,250,000 doses of protective vaccine to 40 states and territories. Most exposed to attacks are the vast regions between the Mississippi Valley in the east and tlie Rocky Mountains in the west. In South America (Chile) the disease has also been observed.

Etiology. The blackleg bacillus, bacillus gangraenae emphysematosae (Clostrydium sarcophysematos bovis Kitt, bacterium chaureaui) is on an average 2 to $6^{\mu}$ long, $0.5^{\mu}$ thick, slender and straight, rounded at the ends, and motile by means of flagella. At a certain stage of its development, the bacillus assumes a whetstone or lemon-like shape (clostrydium form), while in the interior a spore develops at the center or at the end. (Figs. 13 and 14.)

Staining. For this the common watery solutions of aniline dyes as well as Gram's method are suitable.

Culture. Cultures develop in the presence of hydrogen and illuminating gas, with the exclusion of oxygen, as well as in vacuum, particularly in Martin's pepton-bouillon (Leclainche \& Vallée); also in chicken bouillon (Arloing), and on gelatin and agar containing blood-serum, pepton, glycerin or a reducing substance (Kitasato). The bacillus thrives best at 36 
to $38^{\circ} \mathrm{C}$, poorly at $14^{\circ} \mathrm{C}$. The gelatin becomes liquefied; in the depth of the agar culture medium along the needle puncture radiating white growth develops (Fig. 15). The bouillon at first becomes uniformly turbid, small gas bubbles appearing on the surface and later a white sediment is formed, above which the solution is clear. From dextrose and lactose (not from saccharose) the bacillus develops a gas which is either odorless or resembles rancid butter (Preisz, Smith). Milk is curdled only imperfectly.

Tenacity. Fresh blackleg meat is sterilized in 2 hours at a temperature of $80^{\circ} \mathrm{C}$. or in 20 minutes at $100^{\circ} \mathrm{C}$.; fresh virus inclosed in glass tubes is made inactive in 2 minutes when kept in hot water. On the other hand, in dried meat, the spores are made inactive only by heating to $110^{\circ} \mathrm{C}$. for 6 hours, or boiling in water for 2 hours, while at room temperature the spores in dried muscular tissue are kept virulent at least 2 years (according to Mattei even 10 years); in salted meats they are kept virulent for more than 2 years, and in decaying flesh 6 months. Temperature between 85 and $100^{\circ} \mathrm{C}$. decreases the virulence of the spores through the destruction of the toxins. Extreme cold has hardly any effect In the summer, exposed to direct sunlight, the dried virus loses its activity in 24 hours, and the fresh virus in 18 hours. The spores show also considerably more resistance to chemical disinfectants than the bacilli. The nost effective are carbolic acid $(2 \%)$, salicylic acid $(1: 100)$, nitrate of silver $(1: 1000)$, and bichloride of mercury $(1: 5000)$; vapors of thynol and eucalyptus weaken fresh virus, as it is destroyed by the action of these media in 100 hours (Arloing, Cornevin \& Thomas). Virus contained in blackleg meat possesses a greater resistance than do cultures (A. Schmidt).

Pathogenicity. After subcutaneous or intramuscular injection of larger quantities of meat juice containing spores there appear in the inoculated cattle, marked feverish symptoms, and, at the place of injection, a painful, warm, edema-

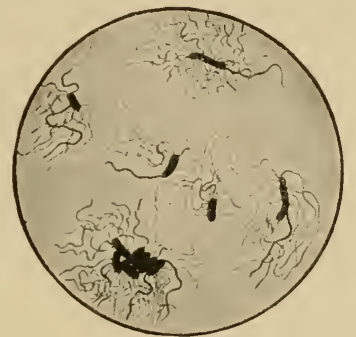

Fig. 12. Blackleg bacilli from an agar culture. Flagella staining.

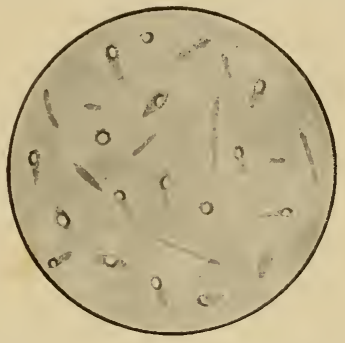

Fig. 13. Blackleg bacilli with spores. Muscle juice from a guinea pig; unstained.

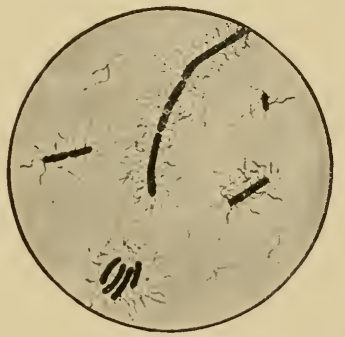

Fig. 14. Blaclleg bacilli with spores. Muscle juice from a guinea pig; dried cover glass preparation; fuchsin staining. tous, and later crepitant swelling, which, after the death of the animal produces a black color of the musculature of the involved region. These muscles together with the serous infiltrated subcutaneous and intramuscular tissue are found interspersed with gas bubbles. If an animal is inoculated subcutaneously, with only a small amount of the infectious sub- 
stance, no edema will, as a rule, appear at the place of injection itself, but at some distance a small swelling appears, which disappear's in a few days. If the inoculated amount has been very small, the injection will only cause a small indisposition and a moderate increase in temperature for a few days.

The intravenous injection of a larger amount of virus produces typical blackleg, but a smaller amount causes only 2 or 3 days of indisposition, indicated by trembling and an inconsiderable increase of temperature. A similar effect follows the intratracheal injection of the virus. In animals which have gone through the short indisposition, the disease cannot be produced by a second injection of even a larger amount. Inoculation in the tail of the animal only causes a crepitant swelling and fever, if the tail is covered up, since its temperature, which is normally $29.8^{\circ} \mathrm{C}$., is thus artificially raised to $38.6^{\circ} \mathrm{C}$.

In calves less than six months old, the subcutaneous or intravenous injection of an amount of fresh and virulent virus which in $90 \%$ of cases would be fatal to a grown animal (1. to 6 drops) only produces a slight indisposition. Larger amounts of virus, 7 to 10 drops for 1 to 14 days old and 10 to 20 drops for 3 to 10 weeks old calves, produce typical blackleg (Arloing, Cornevin \& Thomas).

Small amounts of virus inoculated intramuscularly kill guinea pigs, goats and sheep within 12 to 36 hours; mice are also very susceptible, while rabbits, rats and pigeons are infected only in exceptional cases. In horses and asses only an inconsequential swelling appears after the injection; hogs are slightly and other animals are not at all susceptible to the infection.

In artificial culture media both toxins and gases are produced, but most abundantly in Martin's bouillon. Intravenous injection of 10 to 12 ce. of an old culture kills horses in 5 minutes, evidently through the action of the toxin. The toxin is mostly confined to the bodies of the bacteria, culture filtrates being much less effective. The toxin very soon becomes inactive in the air, while heating for $10 \mathrm{~min}$ utes at $115^{\circ} \mathrm{C}$. only weakens without destroying it (Roux); its negative chemotaxis turns positive at $75^{\circ} \mathrm{C}$. (Leclainche \& Vallée).

According to Grassberger and Schattenfroh, the toxin is a product of the bacilli and is particularly in evidence in the presence of carhonate of calcium. In calves 40 ce. of the filtrate of a culture cause edematous swelling, fever, perspiration, diarrhea, prostration and, after 5 to 6 days, death. Contrary to other bacterial toxins, these show their poisonous effect on the injected animal at once, without any distinct period of incubation (Leclainche \& Valleé, Eisenberg).

In many cases natural infection results from the fact that mud from the swampy and rough soil of certain regions penetrates under the skin of the cattle, particularly through wounds on the tail and the extremities (Lüpke observed the disease after castration).

More frequently the infection results from the consumption 
of infected feed or drinking water. Artificially it is successful in this way only in exceptional cases (Bollinger, Arloing), but the fact that in blackleg regions the intestinal canal as a rule contains blackleg bacilli (Leclainche \& Vallée), while lesions on the surface of the body are observed only in exceptional cases, and furthermore, that crepitant areas are present in the internal organs, speaks in favor of the frequency of infection through the intestines. In the rare cases when such swellings originate in the region of the throat or in the wall of the esophagus, the infection is evidently brought about through the mucous membrane of the throat or the mouth.

Attacks of the disease in swine have so far only been observed in fattening establishments, where the symptoms also have indicated infection of the tonsils (Marek).

Blackleg is a disease connected with infected soil, and transmission from animal to animal, as in contagions diseases, or through intermediary objects, evidently occurs very seldom. The blackleg bacilli seem to be capable of multiplying in the soil, and through their resisting spores, to preserve their capacity of development and infectiveness even under unfavorable conditions. This, as well as the fact that bacteria from carcasses of dead animals again infect the soil, explains the stationary occurrence of blackleg in certain regions. In fact, it appears most frequently in swampy, low pastures, and in regions subjected to occasional inundations; also in pastures where carcasses of cattle infected with blackleg had previously been buried, and sometimes after feeding hay or other feed in the neighborhood of which carcasses had been skinned, or after drinking from creeks in which parts of blackleg carcasses had been thrown.

Sauer suggests the possibility that flies which have been feeding on blackleg carcasses, and in whose bodies the bacilli have increased in number, may be capable of transmitting the infection.

Susceptibility. In blackleg regions the cattle contract the disease with few exceptions only at the age of from $1 / 2$ to 4 years; sucking calves are only attacked in exceptional cases (Strebel observed the disease in a 3 days old calf), and animals over 4 years as a rule only when they are transferred at an advanced age from disease-free places to an infected place. Whether or not the breed has any influence on the susceptibility is not yet determined with certainty; it is a fact that cattle of the native breed contract the disease, but not as often as the improved breed; the same also is true of the Algerian cattle (Brémond). As to the susceptibility of the buffalo, no satisfactory data are so far available (Cronevin observed in 2 buffaloes inoculated with virulent material only intermittent changes of temperature, while, according to Makoldy these animals contract blackleg). In sheep Vogdt and Monseur. and in swine Marek, Battistini and Born have found 
isolated cases of the disease, but it is still a matter of contention whether the disease appears in horses or not.

According to a compilation by Tillmann, out of 614 animals suffering from blackleg, 7 were from 1 to 4 weeks old, 23 from 2 to 3 months, 94 from 3 to 6 months, 449 from $1 / 4$ to 4 years, and, finally, 41 were above 4 years old.

Symptoms similar to those of blackleg, and analogous anatomical changes may also be produced by other bacteria (the so-called psendoblackleg bacilli). Thus Kerry has isolated a bacillus from a guinea pig which had been inoculated with blood from a cow supposed to have dicd from blackleg. This bacillus grows at a higher temperature than the blackleg bacillus, produces no spores, and is pathogenic not only for guinea pigs, but also for rabbits (bac. edematis thermophilus). Preisz found in one case, which otherwise was similar to blackleg in every way, a considerably longer and thicker bacillus than in common blackleg.

Lignières and Bidart in their clinical reports on diseases similar to blackleg in Argentine refer to an affection which is known under the name of Mancha (=spot, owing to the dark color of the skin over

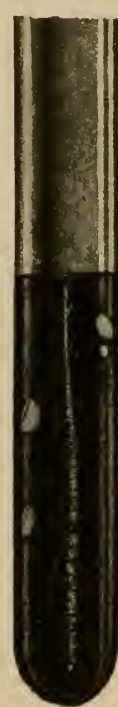

Fig. 15.

Stab culture of the Blackleg Bacillus in grapesugar agar.

the edema). Calves from 6 to 10 months old contract the disease exclusively and succumb within 24 to 28 hours. The disease is caused by a bacillus similar to the blackleg bacillus, but it is pathogenic for young rabbits, horses, and pigeons. Protective vaccination with blackleg virus is without effect.

Pathogenesis. Toxin-free spores which have penetrated into normal healthy tissues are soon taken up by phagocytes and digested; if, however, at the same time, other bacteria or grains of dust have gathered, and the tissue, particularly the musculature, is not quite sound, or if, at the involved place an extravasation of blood exists, then the spores, protected against the phagocytes, begin to germinate, and the bacilli thus created continue to multiply through fission. Under natural conditions a small disturbance in the tissue, a strain of the muscles or a liemorrhage (blow by a stick or a horn), may cause the bacteria which liave reached the blood from the intestines, and otherwise are soon destroyed, to locate at these points, to increase in number, and excite the pathological process.

Where the bacilli appear in abundance, a serous inflammation and sometimes also small hemorrhages will be observed. Under the influence of the toxins, the musculature degenerates, and, through the destruction of the nitrogenous matter, gases are formed. The toxins are also absorbed by the body fluids and cause the general symptoms accompanying the local process; they also reduce the power of resistance of the tissues against the pathogenic effect of the virus (Roger).

According to the investigations of Leclainche \& Vallée, cultures containing spores and heated from 80 to $85^{\circ} \mathrm{C}$. are inactive, as the 
spores in the tissues are digested by the phagocytes within 48 hours. These spore-containing cultures cause the disease, however, if active toxin is added or if they are injected mixed with sand or saprophytic bacteria (bac. prodigiosus, proteus vulgaris, staphylococcus albus). The spores will also show a greater pathogenic activity by the addition of a small amount of $20 \%$ lactic acid, salt solution or diluted alcohol, and also through a traumatic injury immediately before or after the infection. These saprophytic bacteria, the sand and the dead tissue protect the spores mechanically against the phagocytes and make their development possible. The same result is produced by the chemical substances as well as by the inoculation of toxins contained in unheated cultures, through negative chemotaxis.

Anatomical Changes. Characteristic of blackleg is the crepitant swelling occurring anywhere on the rump or extremities, more or less distinctly circumscribed, above which the skin appears sound, or in rare cases, partly necrotic, and therefore, stiff, parchment-like, and dark colored. The subcutaneous tissue is red or infiltrated with a yellowish, gelatinous exudate, at places intermixed with hemorrhages and gas vesicles, and in the deeper parts similarly changed connective tissue separates the single muscle fibres from each other. The musculature of the swollen part is dirty brown or dark red; at the periphery it is darker red or dull yellow with black stripes, and either intersected with bloody serous liquid or is spongy and dry (the latter with great development of gas). The dirty red, or claret colored liquid squeezed from the swellings contains gas bubbles in abundance which give off an odor suggesting rancid butter. In the tissue surrounding the swelling the lymph vessels are sometimes distended, filled with lymph and gas, while the lymphatic glands are acutely swollen, edematously infiltrated, and sometimes intermixed with hemorrhages.

Crepitant swellings appear in rare cases in the musculature of the tongue, throat, and diaphragm, from which points they may also involve the muscles of the shoulder and arm (Henninger). They may likewise be found in the loin or in the muscles between the ribs.

The internal organs show few conspicuous lesions and no constant changes. Relatively often a reddish serous liquid is found in varying amounts in the thoracic cavity. Further, there may be present on the pleura, as well as on the epicardium, a reddish gray fibrinous exudate sometimes mixed with dirty red, jelly-like substances, or the parietal pleura may be covered with soft, jelly-like, wine-colored deposits (Foth, Katona, Tillmann). In the lungs, the interlobular septa may be infiltrated, and the lobules themselves edematous. In very rare cases the heart muscle at localized points is dark red and edematous (Tillmann) or necrotic (Blome), and the epicardium is covered with strips or tufts of exudate.

In the abdominal cavity lesions are frequently found which are similar to those in the thoracic cavity. The spleen is gen- 
erally unchanged or only moderately swollen, but sometimes a well dereloped tumefied spleen is found where the capsule is distended by gas bubbles, but without hemorrhages.

The liver frequently appears mottled on section, owing to ochre-yellow foci from a pea to a walnut in size, which increase after death, and owing to the development of gas, assume a porous, friable consistency (Kasselmann, Foth, Warringholz, Tillmann). According to Warringholz, there exist in the cortex of the kidneys, light gray foci of the size of a millet grain. The nucous membrane of the intestines is catarrhal, reddened and loosened; sometimes it is dark red, swollen, intersected with hemorrhages, and covered with bloody mucus. Finally, the blood is dark red and forms a coagulum in the heart and the large vessels.

In rare cases with rapid course the characteristic external swelling is lacking, but by thorough examination dark red discolored portions of muscles may be found in hidden places, as under the shoulder blade, in the sexual organs, masseter muscles, psoas muscles or in the pillars of the diaphragm.

Ræbiger found vesicles under the endocardium varying in size from a pea to a pigeon egg, which were filled with a dark red, turbid liquid containing numerous blackleg bacilli. Scheibel sometimes found a crepitant swelling of the spleen and a gray-brown, porous, and spongy appearance of the kidneys. According to Schmitt, an intestinal infection is especially indieated by numerous small hemorrhages in the omentum and in the serosa, particularly in the neighborhood of the spleen which is swollen on account of hemorrhagic infiltration. There is also a serous infiltration of the reticulum and a bloody discoloration of its mucous membrane.

In swine a swelling of the region of the throat is mostly observed where the subcutaneous, intermuscular, and peripharyngeal tissues show a serous gelatinous fluid containing gas vesicles. The adjoining muscles appear striped with dark red streaks, the lymphatic glands are acutely swollen, and the mucous.membrane of the throat shows a catarrhal condition with small hemorrhages (Marek). In other cases the crepitant swelling has been found on one of the posterior extremities (Battistini).

In horses the oceurrence of blackleg has not been definitely determined. The literature contains reports by Ganter, Hafner, Schmidt, Velmelage, Fischer, and others, but, in these cases, a confusion with pseudo-blackleg or malignant edema is not only not excluded, but even probahle. Hasenkamp, for instance, found in a horse a serous-hemorrhagic fluid in the thoracic eavity and pericardial sac, hemorrhages in the serous membranes, and black, erepitant, softened muscles of the shoulder region, the neck, and the hind leg which were also infiltrated with a sero-sanguinolent fluid. Quadekker observed in a similar case a sweetish odor in the diseased parts which was neither pleasant nor foul.

Blackleg bacilli are generally present in large numbers in the serous liquid of the crepitant swellings as well as in the yellow foci of the liver (frequently in company with various other bacteria, as staphyiococci, mierococcus prodigiosus, proteus vulgaris, bacillus coli and others, particularly at the edges of the swelling), and a certain time after death they are also found in the transudate of the serons cavities, in the bile, in the lymphatic glands, and in the blood. In the juices 
of the diseased museles numerous clostrydian forms are seen, and if the course of the disease is not acute the bacilli will be found to contain spores at the time of death and still more 24 to 48 hours later.

Müller recommends to submit for bacteriological examination pieces of muscles covered with salt, because the decaying process is arrested by the salt and the blackleg bacilli are stimulated to a rapill formation of clostrydian forms and spores.

The gases formed in the erepitant swellings have not always the same composition; according to Roux, they consist of carbonic acid and hydrogen in equal proportions, while Tappeiner's chemical analysis shows $13.15 \%$ of carbonic acid, $76.51 \%$ of hydrogen, and $10.34 \%$ of nitrogen.

Symptoms. The period of incubation of the disease is generally 3 days, although in exceptional cases it may be more, but does not, however, exceed 5 days.

In cattle the disease starts with sudden febrile symptoms; the animals stop eating, grazing and ruminating, the temperature rising in a few hours to $41-42^{\circ} \mathrm{C}$. (Fig. 16.) In many cases, however, the disease seems to progress with a moderate temperature or without

fever (Schütt \& Warringholz, Steinbrück). In grazing animals lameness or stiff dragging of an extremity is also frequently observed.

Shortly thereafter may be seen the characteristic blackleg swelling, particularly in portions of the body with thick layers of muscle. Most frequently they appear on the upper leg, on the buttocks, in the region of the loin, on the shoulder, chest or neck (in exceptional cases also in the submaxil-

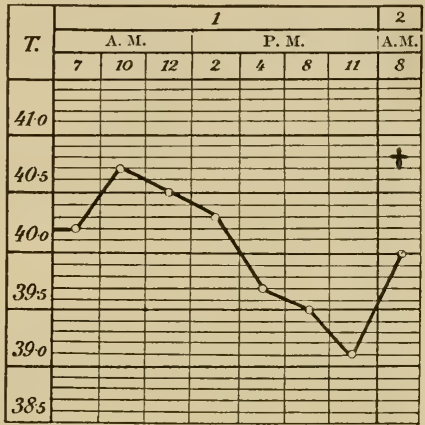

Fig. 16. Fever curve in blackleg. The animal became affected on the previous evening. lary space [Fig. 17], on the tongue or on the throat), while they never appear below the carpal or tarsal joints or on the tail. At one of the above named places a distinctly bordered or diffuse swelling of irregular shape develops rapidly, which, from the beginning, is hot and painful, later becoming cold and torpid in the center, so that the animal finally does not feel pain on touch or when an incision is made. The skin over the swelling is dry, stiff, dark colored, incidentally black and parchment-like, and in exceptional cases also necrotic. On palpating or pressing the tumor a peculiar crepitation is observed, while percussion brings forth a pronounced tympanitic sound. If the swelling is cut at the beginning of the disease, dark red blood, later a dirty red, foamy and singularly sweet snelling liquid will be discharged from the wound. The tissues surrounding the tunıor are edematously infiltrated. The lymph glands are swollen and may be felt under the skin as firm knots the size of apples. 
In the meantime, pronounced respiratory disturbances develop, while the activity of the heart is accelerated (90 to 100 contractions a minute) and becomes weak. Sometimes attacks of colic are observed. At last the animal lies on the ground stretched out motionless, the temperature is lowered to $37-35^{\circ} \mathrm{C}$. or rises once more, and then death follows immediately.

The disease generally lasts from $1 / 2$ to 2 days; in rare cases, however, it may last from 4 to 10 days.

The crepitant swelling mostly develops at one place, but in some cases tumors appear at several parts of the body, grow rapidly and coalesce to

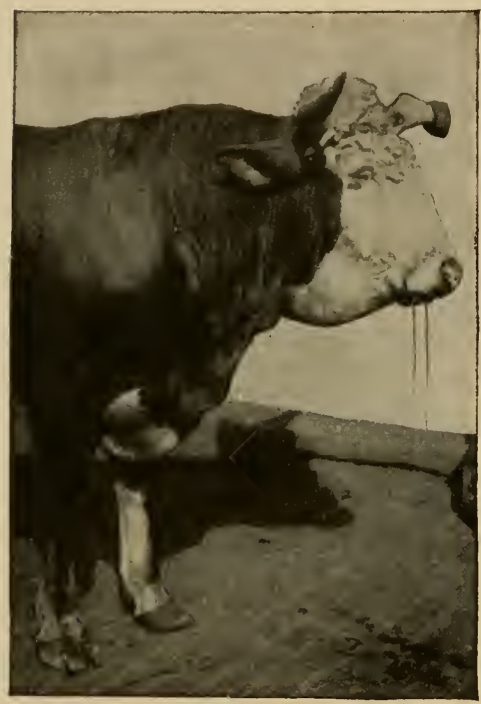

Fig. 17. Blackleg. Crepitating swelling of the forehead and submaxillary space. form a single large swelling.

If the swelling is dereloped in the deep layers of the hip or shoulder muscles, its other properties cannot be exactly determined, aside from the increase in volume of - the involved part of the body. The skin appears relatively healthy, and no crepitation is $o b$. served on touch; however, by a stronger per cussion, a hollow sound nay be observed in the periphery of the swell ing even in these cases

If the cavity of the mouth or the throat is the seat of the disease symptoms of a severe inflammation of $t$ he throat are usually observed whose specific

nature is indicated by the crepitation of the swollen parotid region. Infection of the tongue is indicated by considerable swelling, while the tip of the organ crepitates on touch and projects from the mouth. In the extremely rare cases of primary infection of the diaphragm, the cause of the fever as well as of the difficult respiration can only be determined by autopsy.

According to Arloing, Cornevin \& Thomas, and also Feser, blackleg, especially in older animals, sometimes appears in a much milder form. In such eases there may or may not appear a moderate, indistinct swelling on any part of the body, the animals show moderate fever 
for 1 to 3 days, stop eating and ruminating, and also develop symptoms of colic or tympany, after which recovery takes place. After intravenous injection of blackleg virus a similar mild infection sometimes appears.

Very severe cases of infection occur sometimes in which the animals suffer from general indisposition, tympany, and diarrhea and succumb in 8 to 12 hours, without the appearance of a distinct swelling.

The existence of a genuine parturient blackleg, according to the latest investigations, seems doubtful (see page 37 ) and could at best be ascertained by autopsy, and then only in cases where the muscles in the neighborhood of the genital organs show the characteristic necrotic changes (such a case was observed by Kosselmann).

In sheep the disease has a sudden onset, and is indicated by a stiff walk, weakness of the back, moderate tympany, champing of foam, and high fever. On the surface of the front portion of the body, particularly on the neck and lower chest, the wool is raised at one place, and here a swelling may be found the size of the palm of the hand (Scheibel).

In swine blackleg mostly appears with a severe acute inflammation of the throat and heavy phlegmonous swelling of the surrounding parts (Marek, Born). The development of the tumor which may extend backwards and to the shoulder is also accompanied with feverish symptoms, by vomiting and diarrhea, and the latter symptoms are also observed when the crepitant swelling has developed on a posterior extremity (Battistini).

Diagnosis. The rapid course and the development of a crepitant, edematous swelling, in cases where the latter appears on the surface of the body, make the diagnosis fairly easy. If, however, the swellings are in the interior of the body, the nature of the acute feverish attack can only be surmised through other factors, particularly by the fact that blackleg frequently appears in the particular region. The disease may be mistaken, especially for malignant edema, in the course of which crepitant swellings appear on the surface of the body also. The appearance of the swellings, which are restricted to certain regions and to young animals, in those parts of the body with abundant musculature is suggestive of blackleg. Besides, the swelling is here more emphysematous, the contained fluid dark red and foamy, and the muscles are black colored, while in malignant edema the swelling is more edematous, the fluid gray-red or colorless, and the muscles diffusely reddened or only red-gray (Gutzeit). The development of an edematous crepitant swelling in the neighborhood of the external genital organs at the time of birth will in itself be an indication of the presence of malignant edema.

Blackleg is differentiated from anthrax more readily, for in the latter edematous swellings are rare and, if present, always hot and painful without crepitation. In plilegmonous inflammations of the subcutaneous tissue, from pyogenic wound infection the local character of the disease is distinctly indicated 
and the course, as a rule, is slower. In subcutaneous empliysema, leaving out of consideration the etiological factors, the total absence of acute symptoms of inflammation and the normal condition of the skin are characteristic.

At the autopsy, aside from the microscopic results and the serous-empliysematous infiltration in the region of the swelling, blackleg is indicated especially by the odor of rancid butter, the black discoloration of the musculature, and the absence of a strictly acute tumefaction of the spleen.

In doubtful cases the diagnosis is verified through microscopic examination of the diseased muscles or the yellow foci in the liver and the kidneys, and through injection of such material into test animals. For this purpose Wulff recommenis drying the piece of muscle in an air current at $30-32^{\circ} \mathrm{C}$. and, after pulverization, to heat the material 5 minutes in distilled water at $65^{\circ} \mathrm{C}$. From a mixture narle of $0.1 \mathrm{gm}$. of dried muscle substance and $10 \mathrm{ec}$. water, $0.002 \mathrm{ec}$. is sufficient to kill guinea pigs by internuscular inoculation. At the autopsy of such guinea pigs numerous bacilli are found in the liver, which, however, never form chains (Foth; forming of chains characterizes the bacilli as edema bacilli). On the other hand, pigeons, rats and rabbits are never, or at least only exceptionally, killed by $0.01 \mathrm{ce}$. of the emulsion.

Owing to the extreme rarity of blackleg in other kinds of animals, the diagnosis should always rest upon an exact bacteriological examination, because, otherwise, confusion with pseudo-blackleg and malignant edema is liable to occur.

Treatment. The treatment of blackleg is hardly ever successful and only consists in a local antiseptic treatment of the swellings. For this purpose, it is advisable to incise the tumor at several places and then press it out and wash the exposed diseased tissue carefully and repeatedly with disinfecting liquids. The most suitable for this are: $3 \%$ carbolic acid, $5 \%$ lysoformic acid, $0.1 \%$ salicylic acid, $0.1 \%$ formalin solution, and tincture of iodine, of which liquids the first ones should also be subcutaneously injected at several places in the immediate surrounding tissue.

If the swelling has developed in the middle of an extremity, a bandage or other ligature may be applied above the tumor, and the latter may then be scarified (Wallraff). Tolstouchow obtained good results from applications of ice and snow, and by rubbing with oil of turpentine.

Leclainche \& Vallée recommend the treatment with immune serum, of which first 60 to $100 \mathrm{cc}$. are injected into the jugular vein, and thereafter this amount is injected every $2 \mathrm{~d}$ to 4 th hour under the skin in the region of the shoulder. In the beginning of the infection and in cases of a protracted course, the results are said to be favorable.

Prevention. As the disease seems to be limited to certain regions, and to certain places within those regions, especially damp, swampy pastures, the cattle should be kept away from such places. Through rational amelioration of the soil (draining away the water, regulating the rivers, planting trees and plowing) places which have been considered dangerous may be made harmless. In Denmark, according to Jensen, the 
disease has decreased considerably in the course of the last 30 to 50 years, owing to such improvements of the ground. When this is not practicable, and the danger of infection remains, the appearance of the disease can be prevented by means of protective vaccination; otherwise, the immediate change of pasture or stable often results in the disappearance of the disease.

Immunization. Arloing, Cornevin \& Thomas have stated that cattle raised in blackleg districts mostly contract the disease at a young age, while animals brought there from disease-free regions are liable to be infected at an older age. This is explained by the supposition that the young animals, during their stay in blackleg districts, become immunized early by repeated absorption of smaller amounts of virus. When these authors produced a mild attack of the disease by means of injection of small amounts of virus, they also found that the inoculated animals later resisted otherwise fatal infections. Based on this experience they have devised a very valuable and practicable method of protective vaccination, which has since been modified in many ways by other authors.

I. Two inoculations with attenuated virus. (Method of Arloing, Cornevin \& Thomas.) A vaccine is prepared from diseased, powdered muscles by attenuating the virus through heating to a high degree of temperature. For this purpose, the diseased part of the muscle is ground in a mortar with some water, and dried in a thin layer at $37^{\circ} \mathrm{C}$; 保en the dry mass is mixed with 2 parts of water, and the one half heated at $100-104^{\circ} \mathrm{C}$., the other at $90-94^{\circ} \mathrm{C}$. for 7 hours. The former makes the first weaker vaccine, the latter the second stronger one.

The high temperature attenuates the virulence of the virus, and the longer and stronger it has been heated the more attenuated it becomes. The spores, however, in spite of the influence of the heat, remain capable of germinating, but the toxin having been weakened through heat, the spores are not capable of exerting their pathogenic influence. Being to a certain degree protected against the phagocytes by the coagulated albumin, the spores begin to germinate and to multiply slowly, and thus little by little, the newly produced toxin immunizes the tissues. The immunity reaches the necessary degree in 8 to 10 days after the second vaccination. Until then, however, the animals have a lower resistance aganst an artificial or natural infection (negative phase).

The practical value of the protective vaccination is already definitely established. The first experiments proved that animals, vaccinated at the end of the tail, subsequently withstand the intramuscular inoculation of a very virulent culture without harm, and that the immunity obtained lasts at least 17 or 18 
months. Later vaccinations on thousands of animals performed in many sections have shown that the artificial immunity also protects against natural infection. The vaccinations have resulted in a large decrease in cases among vaccinated animals (11 to 12 times fewer cases than among non-vaccinated animals); besides in the infected localities the percentage of mortality has decreased during recent years, which evidently is owing to the fact that there have been fewer diseased carcasses and less contaminated material to infect the ground. The protective vaccination is now practiced with good results in those districts where the disease usually appears as an epizootic.

The method was modified by Preisz so that the powdered muscle substance is heated for 6 hours in live steam, and $0.05 \mathrm{gm}$. is used for the first, and $0.10 \mathrm{gm}$. for the second vaccination.

In 1890, Arloing, Cornevin \& Thomas subjecterl 13 head of cattle to protective raccination at Chaumont before a comnission; when these eattle were later inoculated with virulent material they all remained healthy, while out of 12 non-vaccinated animals 11 acquired blackleg as a result of a similar artificial inoculation, and 9 of them died.

According to Strebel, ont of 82,550 cattle vaccinated during the years 1884 to 1898 , in Switzerland, $0.75 \%$ contracted blackleg, while out of 35,400 non-vaccinated animals, kept under the same conditions, $2.6 \%$ succumbed. According to another compilation by Strebel, during the years 1885 to 1894 , out of 325,892 vaccinated cattle in various countries, $0.056 \%$ died from vaccination blackleg, and later $0.35 \%$ died from spontaneous blackleg. There were 129,705 vaccinated animals kept during the summer on the same pasture with 240,560 unvaccinated animals; out of the former $0.42 \%$ and out of the latter $1.76 \%$ were the victims of blackleg. Strebel consilers raccination in the tail as less dangerous than in the shoulder, since with the former, 23 out of 107,080 vaccinated animals $(0.021 \%)$ and with the latter 181 animals out of $26,816(0.67 \%$ or 31 times more) died as a result of the vaccination. On the other hand, Suchanka at Salzburg was satisfied with the vaccination in the shoulder. In the canton of Berne in the years 1885 to 1908 , out of 463,613 vaccinated animals $580(0.125 \%)$ died after vaccination, and $1,915(0.41 \%)$ in the following 14 months (Belavoine). In the Austrian alpine regions, during the year $1909,33,056$ head of eattle were vaccinated, out of which, during the pasture season, $114(0.345 \%)$ died, against $1.094 \%$ among the unvaccinated herd-mates.

In Hungary the protective vaccinations have had good effect both on the improved breeds and on the native cattle. First the Lyons vaceine, and later Preisz' vaccine were used. According to Hutyra's compilations, during the years 1893 to 1900 , out of 16,679 vaccinated animals $22(0.13 \%)$ were lost. In the year 1907 , out of 14,029 animals vaccinated in infected herds, 30 died after the first vaccination, and subsequently by the end of the year 36 had died; on the other hand, in previously uninfected herds, the loss amounted to only 1 and 15 respectively out of 14,148 vaccinater animals.

Technique of Vaccination. The 10 doses $(1.0 \mathrm{~g}$.) of vaccine contained in folded paper-wrappers (yellow-red powder) are ground carefully with 5.0 ce. of sterilized water in a small mortar, and the mixture is filtered through sterilized, moistened linen eloth. The filtrate is drawn into a syringe 5 cc. capacity made for the purpose, and the fluid kept agitated in order to aroid sedimentation. The piston of the syringe is divided into 10 divisions; after filling the syringe the indicator is placed at the 1st division.

Accorling to the original French method (Lyons), the place of injection is on the lower part of the tail; the first injection is malle 3 , and the second 2 handbrearlths from the end of the tail. While the animal is secured at the head and at the hind part, the tail is taken up with the left hand, the hair is shaved from the site of injection and the skin disinfected; then the trocar going with the syringe is pushed under the skin to the handle, thereby making a canal about $5 \mathrm{~cm}$. long. The trocar is then carcfully withdrawn and in its place the dull hollow needle is inserted, and one dose is injected into the canal. Then the needle is pulled out, the place of injection pressed together with the left thumb, the syringe delivered to the assistant, and the injected vaccine pressed upward by means of the right thumb; finally, the tail is bandaged with a narrow linen bandage over the place of injection. 
The bandage is removed after four hours, although it may safely be left on in eattle kept in the pasture. After 10 to 12 days the animals are again vaccinated in the same manner but with the stronger vaceine.

Many firms ship emulsions of the vaccine powder in sealed glass tubes for immediate use. Such vaccine is easily contaminated and should be used as soon as possible, but the powder vaccine keeps unchanged for months.

According to recent experiences, the vaccination, at least on the hardy range eattle, may be performed behind the shoulder with the same results and more conveniently (see, however, Strebel's statisties). Here it is also advisable to perforate the skin by means of a trocar, while the bandage after the injection of course is dispensed with. The second vaccination likewise follows 10 to 12 days later, but on the opposite side of the chest.

It is advisable to apply the vaccination at the end of winter or in the spring, if possible, before the cattle are sent to pasture. Otherwise the animals are liable to be infected before vaccination, and the summer heat may cause unpleasant complications after tail vaccination, according to the Lyons methou, because the animals do not keep the vaceinated part of the body quiet. Calves less than six months old and cattle over 4 years old raised in blackleg districts, as well as pregnant heifers in the last four weeks of pregnancy, should not be vaccinated. Sucking calves are not inmunized by the vaccination, and old animals as a rule do not contract the disease.

Immediate results of the vaccination. Vaceinations if properly performed only cause a slight local swelling at the place of injection, which rarely is accompanied by general indisposition and only by a slight rise in the temperature. Unpleasant complications are seldom observed and, as a rule, oceur only when the vaccination has not been performed with the necessary care and cleanliness. Such complications, which usually appear 3 to 6 days after the vaceination, consist in necrosis of the 3 or 4 last vertebra of the tail, bending of the tail, formation of abscesses, inflammation, ete.

Protective vaccination of sheep. Scheibel vaccinated sheep successfully with vaccine prepared from diseased muscles of sheep. The meat is dried at $30^{\circ} \mathrm{C}$. and ground; one half is heated at $100^{\circ} \mathrm{C}$. and the other half at $85^{\circ} \mathrm{C}$. for 6 hours; of this $1.0 \mathrm{gm}$. is used for every 20 sheep. The vaccination is performed, as with cattle, near the end of the tail, and in docked sheep on the inside of the leg. Such vaccinated animals prove immune against the infection with fresh diseased muscle or blackleg virus.

II. One inoculation with attenuated virus. According to experiments by Kitt, a powder prepared from diseased muscles, after having been heated for 6 hours in dry air at 85 to $90^{\circ} \mathrm{C}$., or still better, in live steam at $97^{\circ} \mathrm{C}$, gives a suitable vaccine. One injection of such vaccine into cattle immunizes them sufficiently and lastingly, without causing conspicuous symptoms of disease (the vaccine kills sheep in doses from 0.2 to $0.6 \mathrm{gm}$.; smaller doses produce only slight fever).

This method which is conducted in the same way as vaccination with Lyons vaccine, except that the operation is performed in one act, is especially to be recommended for vaccination of the more hardy native cattle, but it also gives satisfactory results in the improved breeds which have less power of resistance.

In Bavaria, during the years 1898 to 1906 , out of 62,178 vaccinated head of cattle 8 died shortly after the vaccination and 134 later, in all $0.22 \%$, while in the first 5 years, on the same pastures out of 23,324 non-vaccinated animals 398 or $1.70 \%$ died from blackleg (Kitt). In the years 1903 to 1905, out of 25,609 vaccinated animals 68 or $0.26 \%$ died later from natural infection. In Austria, according to the official reports, in the years 1891 to $1894,28,855$ head of cattle were vaccinated by one inoculation with the Kitt vaccine; ont of those 47 or $0.16 \%$ died from vaccination blackleg and 86 or $0.29 \%$ from spontaneous blackleg.

In Hungary, in the year 1892, Makoldy vaccinated 230 head of cattle from 1 to 4 years old by Kitt's method, one-half belonging to the Hungarian-Siebenbürgen breed, and the other half to the Pinzgau breed. The vaccination itself 
eansed no loss, and no case $0_{2}$ blackleg appeared among the raceinated animaıs during the following year.

In the United States this method has been much in use since 1897 , with the morlification however that Nörgaard heats the dried ground muscle intended for vaceination only to $93-94^{\circ} \mathrm{C}$. The Bureau of Animal Industry sends out $11 / 4$ millions of doses of vaceine every year, and the resuits of the vaccinations have been favorable. Thus, in the years $1907-0 \mathrm{~s}$, out of 690,528 raceinated animals 227 died within 48 hours after vaccination, 507 from 2 to 7 days after, and 2,734 later within a year (total loss $0.50 \%$ ).

In India, Holmes obtained the best results from one injection of a mixture of the first and seconi Lyons vaceine, which by means of a partienlarly constructed syringe was placed under the skin in the shape of a pill.

III. Immunization with serum and attenuated cultures. Kitt (1893-1899) obtained from sheep which had been treated repeatedly with intravenous and later with subcutaneous injections of muscle juice from blackleg tumors, a blood serum which in 5 to $10 \mathrm{cc}$. doses protected sheep against an injection of the virus made 3 to 8 days later, and 15 cc. injected into a goat cured the already developing disease. According to later investigations (1899), goats, eattle and horses also produce similar active blood sera, and the last named animal particularly seems to be suitable for the purpose.

These results were later confirmed by Arloing, and still later by Leclainche \& Vallée; besides, the latter authors have ascertained that blood serum and virus mixed together, or each for itself when injected at the same time, do not immunize, but that the successive application of blood serum and virus affords a mode of immunizing, which may be used for practical purposes.

The method which has so far peen tested only in France is as follows: For vaccination two different vaccines are used. The one is blood serum from horses, which, through repeated intravenous injections with bouillon cultures of the blackleg bacillus containing toxins, have been highly immunized; the other is a bouillon culture of the same bacillus attenuated by being heated to $70^{\circ} \mathrm{C}$. for 3 hours. First the animals are injected with 10 to 20 ce. of blood serum, according to their weight, in the region of the shoulder, and 5 to 6 days later a second injection is given subcutaneously with 0.5 to 1.0 ce. of attenuated pure culture at the same place or in the neck, ear or tail. This form of protective vaccination may also be applied without hesitation in already infected herds.

Leclainche \& Vallée vaceinated in the year 1902, 447 heal of cattle without any direct loss, but subsequently one animal died from blackleg; later statisties indicate 8 deaths among 7,987 raccinated animals $(0.1002 \%)$.

According to Leclainche \& Vallée some of the animals on blackleg pastures harbor the virus in the intestinal canal (latent infection), but they are protected against its pathogenic action by the intestinal juices and phagocytosis; if, however such animals are inoculated with live, even attenuated, virus, the body thus attacked from two sirles, ean no longer combat the infection. Through the precerling vaceination with serum, the resistance of the animal is inereased to sueh a degree through the production of passive immunity as to make it efficient even against the double danger. This conception ean hardly be recognized as correct in its present form, because after vaccination of already diseased herds with live virus direct losses from the vaceination are seldom observed. To the oceurrence of such losses other factors are contributory, particularly the relative susceptibility of the animal in comparison with the degree of virulence of the vaceine. 
IV. Protective vaccination with thread covered with spores. (Method of Thomas.) Thomas, a veterinary surgeon at Verdun, recommends a spore-containing vaccine, the preparation of which is not given (supposedly through growth in frog's bodies); with this vaccine silk threads (fils virulents, blacklegine) are impregnated which he introduces into the subcutaneous tissue of the tail. In his opinion, this method is more effective than the original Lyons method, because a blackleg culture develops around the threads left under the skin which exerts a continued immunizing action on the organism (in an experiment with sheep, he found virulent spores and bacilli between the silk threads 328 days after the vaccination).

The vaccination instrument consists in a trocar fastened to a handle of wood behind the tip of which is a cut for placing the silk threads. One dose of the vaccine consists of 7 to 8 short threads which are fastened together at the ends by means of a metal nipper. At the vaceination, after the threads have been placed in the noteh and the nipper is cut away, the needle is stuck deep into the subcutaneous tissue near the end of the tail (in calves behind the shoulder) as nearly as possible parallel with the surface of the skin, and immediately pulled out again, whereby the threads slip off from the needle and remain in the depth of the vaccination canal. The vaccination is either performed twice with a weaker and a stronger vaceine, or only once with the latter.

In America 135,000 head of eattle were vaccinated in the years 1899 and 1900 according to this method (one single vaccination). According to the reports of the results, the vaccination itself caused no losses, while later, as a result of natural infection, 0.4 to $0.7 \%$ of the vaccinated animals died.

Lately this method has also been used in Germany, and Warringholz obtained good results in 406 and Witt in 700 animals; on the other hand, in Alsace-Loraine (1901) 22 vaccinated animals out of 260 died, while in Hungary, Viasz lost 16 out of 70 vaccinated animals within 3 weeks.

According to investigations by Régn, the vaccine is very impure and contains particularly a large number of staphylococei; in connection herewith, Guillebeau frequently observed vaccination abscesses among cattle in the canton of Berne.

V. Other methods of immunization. It is also possible to immunize cattle by means of other methods, which, however, are little used in practice, and only deserve attention from a scientific point of view.

1. Protective vaccination with non-attenuated virus. Cattle may be immunized by subcutaneous injection of a small amount of the juice from the swellings into parts of the body with low temperature and dense subcutaneous tissue. The subeutaneous injection of 1 to 6 drops into the end of the tail causes neither local nor general symptoms; 10 to 15 drops will cause an increase in temperature of $1.5^{\circ} \mathrm{C}$., while 20 drops will cause a local swelling. The further away from the trunk the injection is made the milder the symptoms appear; injections in the vicinity of the root of the tail, however, often cause severe disease.

According to Arloing, the direct intravenous injection, although it requires more manual skill, is the most favorable. It consists in injecting 3 to 4 drops of the serous fluid of a swelling into a vein of the animal, care being taken that none of the liquid gets into the perivascular connective tissue. After a regularly performed vaccination. general symptoms, such as a slight elevation of temperature and loss of appetite, will appear, after the disappearance of which the animal 
will resist a subsequent artificial or natural infection. The results of these experiments are very favorable, but the operation requires great care and skill, because, if some of the vaccine should locate in the porous tissue of the neck, fatal blackleg will almost surely result (Eloire). Intratracheal injection with fluid from the swellings is subject to the same consideration as to the immunizing effect and the danger.

Poels used virulent cultures of blackleg bacilli for protective vaccination. As soon as the formation of spores has begun in the culture small cotton pads are immersed and afterwards dried; at the time of vaccination they are pushed under the skin of the tail where they cause a violent, but harmless, inflammation. During the year's 1904 to $1906,21,329$ calves from 2 to 10 months old were vaccinated in Holland according to this method with an annual total loss of from 1.0 to $1.4 \%$ (Balavoine).

2. Protective vaccination with attenuated pure culture. Kitasato found that fresh bouillon cultures more than 2 weeks old or heated to $80^{\circ} \mathrm{C}$. for half an hour did not kill guinea pigs, but immunized them effectively. Kitt had a similar experience (1894) with subcutaneous injections of 1 to $5 \mathrm{cc}$. of bouillon culture in sheep and cattle, which, 6 weeks later, resisted a virulent infection. Owing to these favorable experimental results the method was used in practice for a time. (In Austria, out of 7,434 cattle vaccinated during the years 1894 to 1895,8 , or $0.11 \%$ died as a result of the vaccination and 93 or $1.25 \%$ from spontaneous blackleg.)

Leclainche \& Vallée injected cattle subcutaneously with a 5 to 8 days old bouillon culture which had been heated at $70^{\circ} \mathrm{C}$. for 2 hours, whereafter the animals thus treated were subjected one week later to a subcutaneous injection of 2.0 cc. of á very virulent culture without any harmful effect. This single vaccination, however, has not been kept up in practice, because out of 39 animals 4 died as a result of the vaccination. Later the vaccination was performed in 2 acts, the first with a culture heated for 3 hours to $75 \mathrm{C}$. and the second with one heated to $68-70^{\circ} \mathrm{C}$. The result this time was also unsatisfactory, one animal dying after the first vaceination and six after the second.

Of the vaccine prepared by the sero-therapeutic institute at Toulouse 0.5 ce. is injected into calves less than 6 months old, and $1.0 \mathrm{cc}$. into older animals, under the skin of the ear or the end of the tail.

Detre recommends a liquid vaccine which he prepares by separating the musele fibers and albumin coagula from the spores and toxins in the Lyons vaccine, obtaining a liquid which contains the same active substances as the Lyons vaceine. The spores and toxins attenuated through higher temperature at two different degrees of exposure in a hypertonic solution of salt, are offered for sale in the market, and the vaccine is said to prove its efficiency in practical experiments.

3. Immunizing with toxins. Roux succeeded in immunizing guinea pigs against blackleg by repeated injections of 15-day old bouillon culture heated to $115^{\circ} \mathrm{C}$. or a filtrate of such culture, into the abdominal cavity, and also by subeutaneous injection of a bacteria-free filtrate of the juice of the swellings. Duenschmann obtained similar results with only the filtrate of the juice of the swellings from animals that had died from spontaneous blackleg. Finally, Schattenfroh used pure solutions of toxins from which the bacteria had been removed by means of clearing powder, but with very unfavorable results, as 23 out of 306 vaccinated animals died from the effect of the poison, and 40 to 50 more became very sick. 
4. Protective inoculation with immune serum. In 1905 Grassberger \& Schattenfroh injected about 800 head of eattle in Tyrol and Lower Austria with serum from an animal which had been immunized by means of toxin and afterwards with virulent material. The results were unsatisfactory, inasmuch as 8 animals later succumbed to blackleg when placed on infected pastures.

5. Immunization with toxins and immune serum. Grassberger \& Schattenfroh prepared a very effective antitoxic serum $(0.0025$ ce. of serum neutralized $1.0 \mathrm{cc}$. of toxin) by treating cattle with their toxin (page 43), 40.0 cc. of which killed young cattle, and used this for vaccination after having mixed it with toxin. According to their experience immunity may be attained by means of such a mixture as well as with toxin alone, the former, however, having the advantage that it does not cause edema nor interfere with the general health of the animal. The vaccination consists in one single subcutaneous injection with 5 to 10 ce. of the neutralized mixture of toxin-serum. In the Austrian mountain districts, more than 4,500 head of cattle were vaccinated in 1904 without direct loss, but later 78 died from natural blackleg while at pasture.

Veterinary Police. Owing to the enzootic appearance of blackleg in many regions, as well as to the considerable losses caused by it, the disease has recently been made subject of obligatory notification in several countries, among others Germany and Austria. The legal methods of prevention are similar to those employed for anthrax, special atteution being paid to the destruction of the carcass, and also to the indemnification of the owner and the application of vaccination.

According to the results of the investigations of Saner, hides from animais having died from blackleg may be thoroughly disinfected by being placed for 10 days in a 1:1.000 solution of bichloride of mercury, $5 \%$ creolin or carbolic acid solution, and also in freshly prepared whitewash.

Literature. Bollinger, D. Z. f. Tm., 1875, I, 297.-Feser, Z. f. pr. Vet. Wiss., 1876, IV, 13; D. Z. f. Tm., 1880, IV, 371.-Arloing, Cornevin \& Thomas, Le Charbon sympt. du. boeuf, Paris, 1887.-Roux, A. P., 1887, I, 62.-Kitt, Cbl. f. Bakt., 1887 I, 684, and 1888, III, 572; Monh. 1893, IV; 1896, VIII, and 1901, XIII (Review); Hb. d. p. M., 1903, II, 600 (Etiology), and 1904, IV, 1001 (Immunization; with literature).-Kitasato, Z. f. Hyg., 1889, VI, 105.-Leclainche \& Vallée, A..P., 1900, XIV, 202 and 590 (Lit.).-Thomas, Rép. de. pol. san., 1900, 31-Kerry, Ö. Z f. wiss. Vk., 1894, V, 228.-Marek, Monh., 1896, VII, 499; 1897, VIII, 174.-Grassberger \& Schattenfroh, Uber d. Rauschbrandgift, 1904.-Smith, Z. f. Infkrkh., 1906, I, 26.-Scheibel, D. t. W., 1907, 61.-Balovoine, Diss., Bern, 1909 (Literature and vaccination statisties).- Tillman, Diss., Bern, 1909.-Foth, Z. f. Infkrkh., 1909, VI, 201.-Wulff, Z. f. Tm., 1909, XIII, 241.

"Bradsot" of sheep (Gastromycosis ovis). This destructive disease of sheep occurs on the western coast of Norway, in Iceland, and on the Faroe Islands of Denmark, in Scotland where it is known as Braxy, also in Mecklenburg and Prussia (Peters, Dammann \& Oppermann, Hilbrand). Ivar Nielsen established the fact that the disease is caused by a specific bacterium which causes severe inflammation especially of the abomasum and duodenum.

The disease (Bradsot, Braasot or Brosot-quick plague) is chiefly observed late in the fall or winter when the sheep are driven from 
the mountain pastures to the farms and are pastured on lower lands which have been infected by unburied carcasses. The disease also occurs frequently during stable feeding in the winter, and exceptionally in summer during pasturing. Yearlings are most susceptible, while lambs and animals over 3 years old rarely become affeeted. Well nourished animals appear more predisposed.

Frequently the course of the disease is very rapid, and the animals die within a few hours. In the slower form a marked weakness is ohserved. Later the animals pass into a comatose stage, grind their teeth, respiration is difficult, and soon death results. In some cases frothy saliva drips from the mouth, and frequently tympanitis develops in association with colicky symptoms. R. Fröhner also observed swelling of the head, throat and tongue, bleeding from the mouth, nose and vulva, swelling and bluish-red discoloration of the vaginal mucous membrane.

On post mortem the most conspicuous change is a severe acute inflammation of the abomasum and duodenum, the mucous membrane

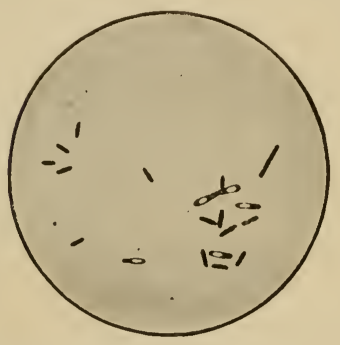

Fig. 18. Bacilli of Bradsot with spores. Kidney fluid from sheep; staining by Gram. (After Jensen.)

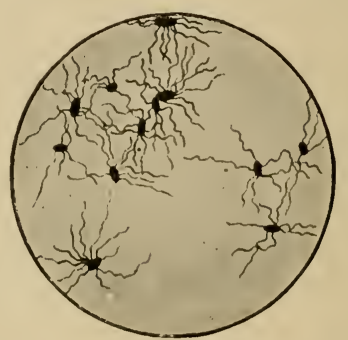

Fig. 19. Bacilli of Bradsot with flagella. (After Tokishige.)

and submucous connective tissue of which appear edematously swollen and permeated with extensive hemorrhages which may sometimes be necrotic. Similar changes are occasionally found in the small intestine, whereas in some of the cases the autopsy reveals a general infection with parenchymatous degeneration of all organs and a considerable enlargement of the spleen, which may erepitate. A sero-hemorrhagic exudate is present in the abdominal cavity, and gelatinous infiltration of the subcutaneous comnective tissue may be observed while the serous fluid may also contain gases.

Bacilli $2.6^{\mu}$ long, $1^{\mu}$ thick which stain by Gram, may be demonstrated in the affected mucous membrane and in the subjacent gelatinous connective tissue, while in a general infection they are also present in the blood. They frequently form chains, and mostly contain centrally located oval spores, and may grow into long threads. The bacillus is supplied with numerous long screw-like flagella (Fig. 18 and 19). It is an anaerobe and multiplies in the depth of the media, forming gas, and therefore resembling the bacillus of blackleg. From this, however, it may be distinguished by the formation of threads and chains. It is also pathogenic for pigeons, chickens and mice (Jensen, Tokishige). 
Subcutaneous injections of pure cultures produce only exceptionally a generalized affection in sheep when the clinical picture resembles blackleg (hemorrhagic-gelatinous infiltrations and gas formation in the subcutaneous connective tissue and muscles). In most instances only a local swelling develops which later disappear's. Animals thus affected which recover later resist natural infection. Guinea pigs, rabbits and pigeons are also susceptible. The natural mode of infection is at present not known with certainty, but it is possible that the infection occurs through slight injuries along the upper digestive tract. After the appearance of the disease a change of pasture and stable as well as a thorough disinfection of the premises is indicated.

Immunization. Nielsen used a heated powder made from kidney tissue, containing numerous bacilli and spores obtained from sheep which had died spontaneously. This method was prineipally employed in Iceland, but with uncertain results. Tokishige, on the other hand, proved that horses and goats treated intravenously with the virus yielded a potent serum. Jensen worked up the three following methods:

1. Eight-day old spore-containing bouillon-serum cultures of the bradsot bacillus are rapidly evaporated at $50^{\circ} \mathrm{C}$., finely pulverized and mixed with a small quantity of gum tragacanth. The dose (0.005-0.03 gm.) must be established by test inoculations, as the susceptibility of sheep varies in different breeds and ages. The immunity is satisfactory, and no losses were observed from the vaccinations in the Faroe Islands, while in Iceland they amounted to $1.5 \%$.

2. Blood serum of horses immunized by intravenous injections of virulent cultures $(0.02 \mathrm{gm}$. serum protect guinea pigs against fatal infection) is dried, pulverized and mixed with the vaccine described under 1. For practical purposes a mixture of $0.005 \mathrm{gm}$. each of culture and immune serum is used.

3. Threads are placed in 3-4 weeks old dextrose bonillon cultures and then dried at $40-50^{\circ} \mathrm{C}$. These threads are inserted with a suitable needle under the skin on the inside of the thigh and left there. The practical results appear uniformly satisfactory. In some cases, however the acquired immunity was insufficient.

In Iceland and the Faroe's about three quarters of a million sheep were vaccinated by these three methods previous to 1906. The losses in the first group which included 208,805 animals amounted to $1.45 \%$ as a result of the vaccination, and $0.32 \%$ later on. In the second group of 52,327 the losses were $0.03 \%$ and $1.93 \%$ respectively. In the third group of 15,934 animals 0.03 and $4.65 \%$ succumbed, while in the unvaccinated sheep it reached $4.21,5.04$ and $5.80 \%$ respectively (Jensen).

In opposition to the above conception of the etiology of the disease, Miessner believes it is questionable whether bradsot represents a uniform disease, being rather a clinical-anatomical name for any disease in sheep which causes death within a few hours, and which manifests on autopsy an extensive hemorrhagic inflammation of the abomasum in addition to an injection of the subeutis. He also concluded from his numerous bacteriological examinations of cases considered as representative of the disease, that the etiological significance of the bradsot bacillus is very questionable, principally on the ground that it has not been possible to produce the anatomical picture of the disease with bradsot bacilli. He also failed as a rule in demonstrating the mentioned micro-organism in fresh carcasses of animals dead of the disease or which were killed shortly before the fatal termination. $\mathrm{He}$, however, failed to ascertain the true causative agent. 
Literature. Krabbe, D. Z. f. Tm., 1875, I, 34.-Nielsen, Monh., 1896, VIII, 55. -Jensen, D. Z. f. Tm., 1896, XXII, 249 (Lit.); Hb. f. p. M., 1903, II, 685 (Lit.).Hamilton, The Vet. J., 1901, 286.-Peters, A. f. Tk., 1897, XXIII, 73.-Tokishige, Monh., 1901, XII, 1.-Hilbrand, Z. f. Infkrkh., 1907, III, 325 (Lit.).-R. Froehner, D. t. W., 1906, 359.-Miessner, Mitt. d. Inst. f. Landw. in Bromberg, 1909, I, 217 (Lit.).

Reindeer Pest. This devastating disease occurs periodically (the last time in 1895 and 1896) among the reindeer herds of the Laplanders in northern Sweden and probably also in Norway. In such outbreaks many thousands of animals, mostly ealves, but also yearlings and two year olds become victims of the disease. It usually rages during the summer time when the animals drift during the warm weather from the cool high pastures into the valleys. The etiology of the disease was established by Lundgreen (1897), and the virus has been more extensively examined by Bergmann (1901).

The symptoms of the disease, which is usually fatal in from 6 to 12 hours, are excitement, inappetence, cessation of rumination, increased thirst, staggering and uncertain gait, difficult and accelerated respiration, cough, and particularly the appearance of edematousemphysematous swellings at various points of the body. The temperature which is at first high, drops at the approach of death. There is bleeding from the nose, and a very repulsive odor is apparent.

The post mortem examination reveals fluid containing considerable gas in the subcutaneous connective tissue, as well as collection of gas in the internal organs. In addition to a reddish transudate in the body cavities a yellowish discoloration of the liver and a moderately acute swelling of the spleen may be observed.

From the subcutaneous serous fluid, the transudates of the body cavities and the blood the reindeer pest bacillus, which resembles the blackleg organism, may be obtained in pure culture. It is Gram-positive, motile, forms oval spores in the animal, and in artificial media at a temperature of $30-38^{\circ} \mathrm{C}$. It may be cultivated on the ordinary media aerobically as well as anaerobically, and the growth is especially luxuriant at hody temperature, when a great amount of fetid gas and acid is formed; gelatin becomes liquefied.

By subcutaneous and intramuscular inoculation of pure cultures the disease may be reproduced in reindeer. Sheep and guinea pigs as well as mice and cattle are also susceptible to artificial infections, whereas rabbits, hogs, dogs and chickens are immune. Natural infection probably results through wounds in the skin and intestinal mucous membrane.

Inoculations of sheep into the tail produce only passive elevation of temperature, and result in an increased resistance against an otherwise fatal subcutaneous infection into the rump. Animals immunized against reindeer pest are not immune to blackleg or bradsot. 241.

Literature. Lundgreen, Z. f. Tm., 1898, II, 301.-Bergmann, Ibid., 1901, V,

\section{Swine Erysipelas. Erysipelas suis.}

(Stäbchenrotlauf, Rotlaufseuche [German]; Rhusiopathia suis, Rougei du porc [French]; Red fever [English]; Antrace. eresipelatoso, Mal rosso [Italian].)

Swine erysipelas is an acute, septicemic infectious disease of young hogs caused by a very fine, rod-shaped bacterium, 
Bacillus erysipelatis suis, which is found in the blood of the affected animals.

History. The disease which until the eighties was not distinguished from anthrax was first studied by Pasteur \& Thuillier (1882). These investigators did not establish the etiology of the disease, since they accepted as its cause the eight-shaped bacterium which was first recognized by Detmers. Nevertheless their investigations resulted in the preparation of a satisfactory serum for practical immunizations. The bacillus of erysipelas was discovered by Löffler in 1885, who together with Schütz differentiated the disease from hog cholera. The differentiation had, however, also previously been made by Eggeling from his clinical observations. Later the etiological knowledge was advanced by the work of Lydtin \& Schottelius, Bang, Jensen, Preisz, Lorenz, Voges and Schütz, while Lorenz, Leclainche and Schütz worked extensively on the problem of serum immunization.

Occurrence. Swine erysipelas occurs everywhere on the European continent, and is usually prevalent in the infected territory in summer, with varied severity in an enzootic form, and not infrequently epizootically. During the last decades the disease has become more widespread as a result of the propagation of pure-bred animals. The disease usually appears during the warmer periods, increases in intensity during the course of the summer, and abates at the approach of autumn; whereas, during the winter only sporadic cases are observed.

In Germany erysipelas is very extensive. Since the inauguration of compulsory reporting, the number of infected townships increased from 8,491 in 1897 to 21,522 in 1907 ; and the number of individual infections in hogs from 33,950 to 75,619 , of which $80 \%$ died or were killed. During the last years the disease raged in the Prussian government districts, particularly in Posen, Oppeln; Bromberg, Marienwerder, Liegnitz, and Breslau.

In France the disease also occurs extensively. Optimistic figures show that the annual losses amount to at least 100,000 hogs valued at over $\$ 1,000,000$ (Nocard \& Leclainche).

In Austria the disease is more prevalent in Bohemia, Galicia; also in Moravia and Southern Austria; while in the Alpine countries the erysipelas invasious are very slight. During the period from 1891 to 1900 the number of infected districts increased from 953 to 2,368 ; that of the affected animals from 9,286 , to 14,891 ( 0.25 and $0.32 \%$ of all the hogs).

In Hungary the disease was hardly known three decades ago in the flat lands around the river Tisza, but since that time it has become quite prevalent. In the years between 1899-190s the average number affected amounted to 30,757 hogs, but fluctuated between 7,624 in 254 townships (1899) and 56,455 in 1878 townships $(1906)$.

The disease is also widely spread in Belgium (annually about 2,000 cases), in Holland 1,514 cases in 1908, in Denmark 330 new herds, in Russia 55,996 cases in 3,929 townships; further it prevails in the southern European countries, whereas in Sweden and Norway it oceurs more rarely, while in Great Britain only sporadically, and in a chronic form. The disease also occurs in the United States of North America, but in the benign form mentioned. [Urticaria but not infectious swine erysipelas occurs in the United States].

Etiology. The causative agent, Bacillus erysipelas suis (Bac. rhusiopathiae suis, Kitt) is a very slender, straight or slightly curved, non-motile organism which multiplies by fission, and is from 1 to $1.5^{\mu}$ long (1-4 to 1-5 the diameter of 
a red blood corpuscle, Fig. 20). In bacilli grown in cultures fine Gram-positive granules may be demonstrated which are considered by Fedorowitsch and by Rosenbach to be lasting forms (protospores).

Staining. They stain readily with aqueous aniline dyes as well as by Gram's method.

Cultivation. The bacilli grow well on the ordinary artificial media with the exception of potato. It is aerobic as well as anaerobic and grows in cultures sometimes as long rods or curved threads. On gelatin plates the cultures form whitish, cloudy, very fine flakes with coarse granular centers, which spread out towards the periphery, into a delicate thready meshwork. More rarely they form bright shiny, branching small colonies, whereas in stabs pinhead sized, white points develop in 2 to 3 days which later fuse into grayish-white, cloudy colonies, from which fine horizontal radiating branches run in all directions of the gelatin (test tube-brush shape, Fig. 21). On agar and on blood serum the

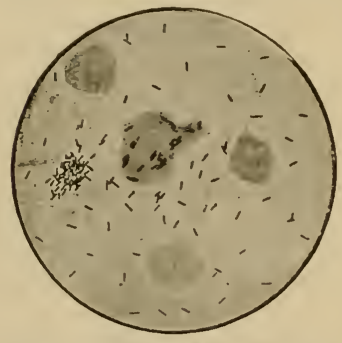

Fig. 20. Bacilli of swine erysipelas. Smear prepared from blood of a hog. Gram-eosin staining. bacillus forms very fine punctiform, dew-droplike colonies. Bouillon is made slightly cloudy, while later a fine. flaky sediment forms on the bottom of the tube.

Tenacity. The erysipelas bacilli manifest considerable resistance towarils harmful influences, which they probably owe to a waxlike eapsule (Schïtz \& Voges). Drying kills them only gradually as they remain alive when subjected to a temperature of $37^{\circ} \mathrm{C}$. for 31 , and to direct sun-

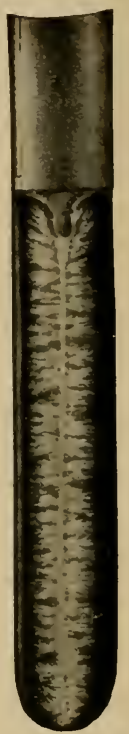

Fig. 21. Gelatin stab culture of $\mathrm{s} w$ in e erysipelas bacilli.

light for 12 days (Sirena \& Alessi). Heating to $70^{\circ} \mathrm{C}$. destroys them in about 5 minutes. Pieces of meat not orer $15 \mathrm{~cm}$. thick require $21 \% 2$ hours boiling for a complete sterilization (Stadie). Putrefaction does not destroy the bacilli in meat in 4 months (Stadie). Salting and pickling destroys them very slowly; pieces of meat and bacon may contain the virulent bacilli after lying in pickle for 170 dars, and in a mixture of salt and saltpeter for 30 days, while in smoked ham virulent bacilli were found even after 3 months (Petri). On the other hani, continued smoking for 2 weeks or shorter periods of smoking once repeated destroys the rirus of erysipelas in pieces of picklerl meat not over $21 / 2 \mathrm{~kg}$. in weight (Stadie).

Of the disinfectants the following are effective: Chlorate of lime (1\%), lot lye $(1 \%)$, soda $(5 \%)$, iron sulphate $(3 \%)$, copper sulphate $(1 / 4 \%)$, corrosive sublimate $(.1 \%)$, carbolic acid, creolin and lysol (Petri).

Pathogenicity. Pure cultures inoculated subcutaneously kill white and gray mice as well as pigeons in 2 to 4 dars. In rabhits the inoculation produces errsipelas-like reddening and swelling at the point of inoculation, which is occasionally followed in 5 to 6 days by a fatal general affection. In hogs an inunetion of virulent bouillon cultures into superficial wounds 
of the skin as well as subcutaneous injection produces a typical affection of erysipelas which terminates fatally in 6 to 9 days (Preisz). The feeding of cultures also makes hogs ill (Cornevin, Kitt), but this mode of infection is not always suceessful. Other animals camnot be infeeted. Repeated passage of the bacilli through the body of hog's increases their virulence for hogs (Schütz), and passage through pigeons results similarly for mice (Stickilorn), while the virulence is gradually diminished by continued cultivation.

Natural infection usually occurs through the intestinal canal, more rarely through injuries to the skin. 'The greater frequency of the infection per os is proven by the presence of the bacilli in the intestinal eontents of affected hogs, also by the numerous affections which occur in a herd. The infection may also occur through the uninjured intestinal nucous membrane. Injuries such as those from the echinorhynchus, however, facilitate infection.

Food and drinking water are usually the carriers of the infective agent, and become readily contaminated through feces or excrement, urine and other offal of affecterl animals. The infection may also occur through the ingestion of blood and meat of dead or slaughtered hogs.

The disease is introduced into territories which have been free from the infection, and is thus spread mostly by animals sick or dead of the disease, or by their products. The baeilli are present both in the blood and the excrement of the affected animals, of which the infectivity has been established by the experimental work of Cornevin and Kitt. The infeetion is present in the excrement even when the hogs are infected through the skin. Therefore the bacilli reach the lumen of the intestines through the blood. The urine of affected animals may also contain erysipelas bacilli. Therefore pastures in which affected animals have been kept or fields which have been fertilized with the manure of such hogs are particularly dangerous. The annual occurrence of erysipelas in herds which have been exposed to such pastures or fields is thus readily explained. On the other hand, pastures are frequently contaminated by improperly buried earcasses, especially since the carcasses of such animals are frequently dug up by dogs and hogs which scatter the infection over the pastures. If dead animals are thrown into flowing water the infection is transmitted to herds of hogs which are pastured along the banks of the stream.

Peddling of hogs and hog markets greatly aid in the dissemination of the disease, inasmuch as affected animals driven over the roads contaminate the roads and the halting places with their manure.

The infection is further spread through the meat of hogs slaughtered in emergency, particularly when healthy animals 
are given water in which contaminated meat has been washed.

The spread of the disease is also frequently brought about by wandering gypsies who carry the carcasses given to them, or which they dig up, from one town to another, at the same time scattering the viscera in the pastures. Furthermore, butchers, hog herder's and castrator's may spread the infection, particularly through utensils and containers in which meat and meat offal have been kept and which have afterwards been used in feeding or watering.

By the findings of Olt, Jensen, Bauermeister and Pitt it has been proven that true erysipelas bacilli may be present in the intestinal canal, particularly in the secretion of the tonsils as well as in the mucous plugs of the ileo-cecal valve of healthy animals. It is therefore possible that on the one hand the contagion may be spread through healthy hogs, while on the other the disease may break out in healthy herds without a direct introduction of affected animals or products originating from them. Healthy carriers of bacilli may thus become affected, if through weakening influences such as cold, heat, starvation, etc., their normal resistance is reduced, and the bacilli which were present in the intestinal canal may then set up the disease process.

In examining the intestines of 66 animals Pitt found the true erysipelas bacilli in 26 cases, and 28 times in 50 tonsil examinations. He believes that through their presence the hog becomes gradually immunized which explains why animals strictly guarded from exposure have been found to possess a lower resistance, such animals becoming severely affected if exposed to infection in spite of all precautions.

In territories once infected, erysipelas usually recurs almost annually, although with varying intensity, provided that in the meantime no measures have been undertaken for its suppression. This fact also proves that the infection may remain virulent in the soil and in stable floors for a considerable length of time. It will multiply in the summer time and infect young hogs which will then reinfect the soil with their excrement and carcasses.

The bacillus of mouse septicemia (Bac. murisepticus) which corresponds morphologically and in its cultural characteristics with the erysipelas bacillus, and which has been formerly distinguished from the latter by its very slight virulence for hogs must at the present time be considered identical with the erysipelas bacillus or as an attenuated variety of it. These conclusions were reached by Jensen, Lorenz, Löffler and Prettner, who proved that animals immunized against erysipelas are also immune against the virus of mouse septicemia and vice versa. As the virulence of the mouse septicemia bacillus may increase under natural conditions, and as it has been repeatedly found in stagnant, polluted water (Koch), in putrefactive meat (Johne), and in blood of cattle (Preisz), it is possible that the mice affected with septicemia contribute to the dissemination of erysipelas.

Rohrbach has recently (1909) been inclined to consider the bacillus of swine erysipelas and mouse septicemia as two different micro-organisms, and to group 
them with the erysipeloirls of man in a special class (Erysipelotriches: Erysipelothrix porei, E. erysipeloides and E. muriseptica). This conception, however, appears to have insuficient foundation as the norphological differences are only slight and since the variance in the rapidity of their growth in artificial media is pronouncer. (The quickest growth is oltained with the mouse bacillus; less rapid is tlie Bac. erysipeloiles, while the slowest is the erysipelas bacillus). Stiekilorn observed similar differences in the erysipelas bacilins after passage though mice and pigeons in spite of the fact that serum examination shower no differences in the straius or species; and Orerreck also found that the erysipelas bacillus and the mouse bacillins agglutinate practically uniformly $(1: 2000$ to 4000$)$ when the organism of the oue is applied to the immune serum of the other.

Schipp found a bacillus in the blood of a chicken which was identical with the erysipelas bacillus. On that particular farm many animals died in a short time and the autopsies revealed principally an enteritis and a parenchymatous degeneration of the heart muscle. The same author also isolatel a bacillus from the spleen of a cow which died with symptoms of anthrax and which on autopsy revealed only petechiae on the serous membranes and on the heart. This organism eorrespronded morphologically and culturally with the erysipelas bacillus. It was, however, pathogenic for fielı mice and was not influenced by erysipelas sera. As a secondlary finting erysipelas bacilli were also obtained from a cow which died from vaginal diphteria and septic metritis, and by Hüuser in diphtheritic mentbranes of chickens.

Susceptibility. Hogs are particularly susceptille to natural infection between the ages of three months and one year. Sucking pigs manifest a higher resistance while animals over one year old become only exceptionally affected as they have probably acquired immunity during their earlier life.

According to Lydtin the English breeds are more susceptible, particularly the Suffolk and Poland China breeds, while Yorkshires are less susceptible. The German logs are highly resistant, while the Hungarian breeds (Mangalicza) oceupy a middle position in the order of susceptibility. The disease usually rages more severely among imported hogs than in the hogs which have been raised in the infected locality. The disease has not yet been established in wild hogs.

Pathogenesis. The entrance of highly virulent erysipelas bacilli in large quantities into the intestinal canal of healthy hogs will reduce the normal resistance of the animals, and on the other hand the entrance of such bacilli will promote the pathogenic action of such bacilli as were already present in the intestinal canal, by their weakening influences upon the system. Their penetration into the depth of the intestinal mucous membrane is facilitated by injuries through intestinal parasites (strongylids, echinorhynchus gigas), whereupon they multiply in the lymph spaces of the tissues, enter the lymph glands and finally the blood circulation. The bacilli which enter the blood in this manner or through injuries in the skin will continue to multiply there, and then they accumulate in large masses in certain blood vessels, producing weakening of the vessel walls, serous transudations and small hemorrhages probably by the action of the metabolic products. The reddening of the thimner parts of the skin may be traced to this cause, whereas the necrosis of the skin which occasionally occurs is the result of the obstruction of the blood 
vessels. The accumulation of the bacilli in certain parts is probably favored by their being engulfed by leucocytes which then adihere to the intima of the vessel. In chronic cases when they become attached to the valves of the heart they produce an affection of the valvular tissue (endocarditis verrucosa s. bacillosa) or a bacillary thrombosis develops in some of the vessels of the corresponding valves.

In some cases especially those in which the bacilli are not very virulent, their multiplication occurs only in the lymph spaces of the skin whereby reddening of the skin with circumscribed edema develops (urticaria, diamond-skin disease) which symptoms disappear after the death of the bacilli.

These changes prove that the erysipelas bacilli produce toxins in the body tissues, and in cases of general infection the fever, depression, paralysis and finally death may also be traced to the action of the toxins. This is substantiated by the experience that hogs sometimes die as a result of a cutaneous infection with the symptoms of the disease, yet bacilli are only found at the point of inoculation and in the immediately surrounding tissue (Preisz). Until the present time, however, it has been impossible to demonstrate toxic substances either in artificial cultures of erysipelas bacilli or in the carcasses of animals succumbing to the disease. (According to Petri \& Maassen, erysipelas bacilli produce toxin in media containing hydrogen sulphide.)

Anatomical changes. In the acute form of hog erysipelas the post mortem on animals which have succumbed to the disease shows in most instances only slight changes in the internal organs. The mucous membrane of the stomach, particularly at the pylorus, shows an inflammatory swelling, reddening, and numerous small hemorrhages. The surface is covered with a sticky, glassy mucus. The mucous membrane of the small intestine, especially in the duodenum and ileum, appears catarrhal and congested. The solitary follicles and Peyer's patches are also swollen, the superficial layer of the mucous membrane may sometimes show desquamation and even ulceration (Schottelius). Small ulcers also occur on Bauhin's valve as well as in the large intestine.

The spleen is usually only slightly swollen and congested. The liver and kidneys show indications of cloudy swelling; the cortical substance of the latter is mostly darker and often mottled by dark red points (glomerulonephritis). The lungs are hyperemic and edematously infiltrated; sometimes they are atelectatic at their borders as a result of catarrhal inflammation. All the lymph glands are markedly swollen, rich in blood and fluids. The serous membranes may be covered with fine fibrin membranes. Small hemorrhages also occur with relative frequency on the serous and mucous membranes, under the epiand endocardium as well as in the subcutaneous connective tissue. 
Haase found, 2 to 4 weeks after the disappearance of the disease, small dark red areas in the kidneys and in the later stages, purulent nodules, sometimes accompanied by extensive inflammation of the kidney tissue.

The blood vessels, corresponding to the red spots of the skin, appear dilated, filled with blood, while the skin and subcutaneous connective tissue is edematously infiltrated and studded with small hemorrhages. The muscles either appear healthy or they are grayish red and lustreless, while the intermuscular connective tissue may be edematous. In rare cases certain parts of the body (ears, tail, nostrils, etc.) or larger areas of the skin may be necrotic (e. g., on the back).

In the chronic form the disease manifests an endocarditis (verrucosa or ulcerosa) in the majority of cases. In one or more orifices of the heart, particularly in the left arterial orifice, the convex surface of the valves is covered with a cauliflower-like fibrin coagulum which sometimes rednces the orifice to such an extent that it is hardly possible to insert the quill of a pigeon feather. After the removal of the fibrin deposit small wart-like vegetations or ulcerations appear on the thickened valve. Such carcasses also manifest the secondary changes which are usually produced by affections of the heart, such as hydrothorax, passive ltyperemia of the lungs, liver and spleen, infarcts of the kidney's, etc.

In some cases the autopsy also reveals chronic enteritis, hypertrophy of the lymph glands (without necrotic areas), small necrotic foci in the liver and chronic inflammation of the serous membranes as well as of some of the joints.

The bacilli may be demonstrated easily in acute cases in the blood in the capillary vessels of the internal organs (especially spleen, liver and kidneys), in the lymph spaces of the skin, and the subeutaneous connective tissue, in the red areas of the skin and plaques, as well as in the regional lymph glands. In the blood they are present either free in the plasma or inclosed in the leucocytes. In chronic cases the bacilli are usually only present in the affected tissues, particularly in the valves of the heart and in the fibrin coagulum as well as in the bile where they remain for a longer time even after the acute affection has subsided (Kitt). With Gram's stain they may be demonstrated even in putrefactive tissues (Opalka, Rosso). However, it is advisable to cover the parts of organs with salt in order to prevent putrefaction when shipped for laboratory examination.

Symptoms. The time of incubation of erysipelas after artificial inoculation is 3 to 5 days (Preisz, Kitt). After natural infection from the ingestion of infectious meat or of contaminated water the first indications of the disease appear as early as 24 hours, otherwise the time of incubation is usually 3 to 5 , and exceptionally probably as long as 7 days.

The clinical symptoms show a considerable variance in different cases; in general the following three readily distinguishable forms of the disease may be observed which may also differ from each other in their course.

1. Urticaria (Diamond-skin disease) represents the mildest form of erysipelas. In its course the animals show after 1 to 2 days a disturbance of their general health, and sharp 
circumscribed, roumd or more frequently quadrangular or rhomboidal spots develop in various parts of the body, especially on the chest and back, outside of the thighs and on the neck, but sometimes over the entire body, which are warm to the touch and dark red or violet in color. Later they appear elevated above the surface of the surrounding skin as much as 2 to 3 mm. The center usually becomes pale while the border retains the bright color. In other cases the raised parts remain red and even become darker, while on their surface a serous fluid exudes, which raises the epithelium in the form of small vesicles which later dry to crusts. As a rule the plaques are of dollar size, but by their confluence swellings may be formed as large as the palu of the hand.

The development of the eruptions is usually associated with fever (up to $4^{*} .8^{\circ}$ C.), dullness, loss of appetite, thirst,

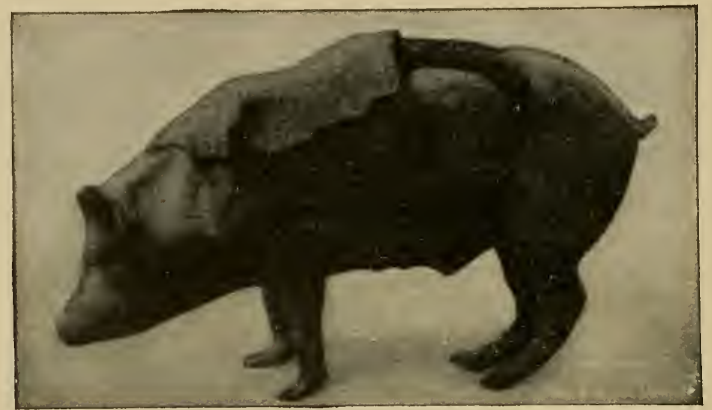

Fig. 22. Swine erysipelas. Gangrene of the skin of the back. (After Schwarzbart.)

constipation, conjunctivitis, sometimes symptoms of paralysis and nausea. After the development of the eruptions the symptoms diminish. Soon afterwards the eruptions also disappear, and after 2 to 3 days, sometimes however only after 8 to 12 days, the animal recovers.

In some of the cases the changes in the skin take on a malignant character. On the parts of the body mentioned, more frequently, however, on the ears, head and tail, the skin swells considerably in certain spots, becomes dark red and warmer; soon these places become insensitive, cooler and dry, and finally become separated from the surrounding and underlying tissues, or the animal loses one or both ears, its tail or distal digits. Sometimes the inflamed skin over the back dies off in its entire extension so that it covers the upper part of the body in the form of a shield (Fig. 22), and gradually loosens from the borders and is cast off. The resulting raw surfaces heal later should the animal remain alive, causing dis- 
figurement in the form of thick, sometimes cartilage-like cicatrices.

In some cases an erysipelatous endocarditis develops after the disappearance of the eruptions which terminates fatally, or a paralytic condition results which may remain for a longer time.

Jensen (1891), Lorenz, Lüpke and others found in urticaria the erysipelas bacillus in the lymph spaces of the affected parts; on the other hand, Markus succeeded in producing a verrucose endocarditis as well as kidney infarets in a pig by intravenous injection of 2 ce. of a bouillon culture which was derived from a dermatitis, while Schütz observed urticaria in ordinary country hogs and erysipelas in English breeds after the injection of the same culture. Jensen and Lorenz proved the presence of the erysipelas bacillus in the lymph spaces of the chorion to be a cause of dry gangrene.

2. Erysipelas septicemia. In this, the most frequent form of the disease, the animals suddenly cease to root and to run. They lie exhausted and dull upon the ground, they crawl under the straw in the stall and when caught they do not squeal or show the usual resistance. They show inappetence, nausea and vomiting. The body temperature rises even at the onset very high (up to $41^{\circ}$ to $42^{\circ}$ C.) and remains practically constant. The eyelids are frequently edematously swollen, the conjunctiva is injected, the canthus of the eye is filled with a slimy secretion. At first there is constipation, later, however, diarrhea appears, when the animals pass frequently soft, mushy, later entirely fluid stools, which are light gray or dark brown from the admixture of blood.

On the second day, rarely earlier, or only immediately before death the characteristic reddening of the skin appears on various parts of the body. On the abdomen, the inner surface of the thighs, in the axillary region, sometimes also on the neck and ears, various sized, irregular, pale red spots appear, which later take up a darker and brighter color, and confluate. The skin over the affected areas is only slightly swollen if at all, and not painful. The redness disappears when pressure is applied but returns when it is removed. In a few cases hempseed sized vesicles containing a serous fluid develop on the surface of the spots which later dry and form brownish crusts. Exceptionally gangrene of the skin is observed in some parts, especially on the ears, nose and the point of the tail.

In severe cases in addition to the described symptoms there are weakness of the hind quarters, cyanosis of the mucous membranes as well as manifestations of pulmonary edema. As the symptoms become more progressive, a sudden drop of temperature follows, and finally the animals die in 3 to 4 davs. Exceptionally death results at the end of the first day or then only after 8 to 9 days. In milder cases the intensity of the 
symptoms diminishes from the second to the third day on, whereupon the animals recover in a short time, or, on the other hand, a chronic type of the affection ensues.

In France, according to Cagny, they distinguish a mild and severe form of acute swine erysipelas; to the latter belongs the "rouget foudroyant" which terminates in a very short time in death, and the "rouget blane" in which death results so quickly that the reddish coloration of the skin cannot develop (also observed by Zschokke in Switzerland).

3. Chronic erysipelas. It occurs most frequently as chronic erysipelatous-endocarditis in animals which have recovered from the acute affection. After the disappearance of the acute manifestations the pigs are usually lively and have a good appetite for a time, but on careful observation it may be noticed by comparison that they are stunted in their development. After 6 to 12 weeks they eat less, are dull, dislike to move around, and lie down much, and almost always on the sternum and elbows. In the meantime a cough, accelerated and superficial respiration as well as a bright red discoloration of the skin, ears, neck and sometimes the rump, is observed. If the animal is urged to move about the dyspnœa and cyanosis in a short time become more marked. The heart action is increased, at times pounding, and may be easily detected over the region of the heart. The pulse is threadlike; besides this, instead of one, mostly the systolic, or both heart sounds, a blowing or harsh murmur may he heard (endocarditis verrucosa, stenosis of the orifices). The body temperature is normal or sometimes increased from one to one and a half degrees.

With such manifestations the animals may live for several weeks. However, they become emaciated, very weak, a crusty eczema frequently develops on the skin and even paralysis of the hind quarters may result. (According to Eisenmann, the duration of the disease in four cases was 34 to 112 days.)

As a particular form of chronic erysipelas Cornevin, Hess and recently Fisenmann describe an affection which is in general only manifested by chronic debility. It develops several weeks after the acute affection and consists principally in accelerated and difficult respiration, digestive disturbances, emaciation, hemorrhages and ulceration of the buccal mucous membrane, hemorrhages into the hair follicles with subsequent falling out of the bristles, eczema or also necrosis of the skin, erlema of the extremities as well as serous inflammation of the joints and tendons which may later result in deformity (most frequently the hip joint).

Course and Prognosis. The urticaria of erysipelas terminates as a rule in recovery. Exceptionally, however, manifestations of a general infection or a chronic disease may develop, when it results in death. The septicemic form terminates in the majority of cases in death, but the number of fatalities varies 
within wide ranges in different years and territories. In Hungary it fluctuated in the last ten years between $53.2 \%$ and $83.8 \%$; according to Friedberger \& Fröhner the average loss amounts to $50-85 \%$; according to Lydtin to $50-75 \%$. Chronic erysipelas always terminates fatally.

If, in the acute cases, the affection lasts over four days, it is a favorable indication; otherwise the disease takes a threatening character particularly in poorly nourished and badly developed young animals. Complete recovery is sometimes very slow and takes place only after several weeks. In most cases, however, the convalescence lasts only a few days. Nevertheless there always exists the possibility that the chronic form of the disease may develop.

Diagnosis. The septicemic form of erysipelas may be especially easily mistaken for swine plague and hog cholera, but these diseases may be distinguished from it by their usual slow course as well as by the predominating affection of the lungs and intestinal tract, or by the indications of symptoms indicating a diphtheritic inflammation of the throat. Red discolorations may occur in the course of the above diseases but they result usually from hemorrhages and therefore do not disappear on pressure. On autopsy erysipelas is distinguished from the peracute cases of hog cholera and swine plague only by the more pronounced hemorrhages, whereas from the more frequent chronic cases it can be differentiated by the necrotic pneumonia and fibrinous pleuritis or round intestinal ulcers, further, by the extensive exfoliation of the intestinal mucous membrane and caseation of the lymph glands which does not occur in erysipelas.

Anthrax, which rarely occurs in hogs, is differentiated from erysipelas by the frequent swelling in the region of the neck and the difficulty in swallowing, on post mortem by the characteristic affection of the tonsils as well as by the difference in the bacteriological findings. Wound erysipelas occurs only on the head when the reddened skin is greatly swollen and painful and the temperature higher. The cerebral hyperemia which causes death during transportation in the warm weather is characterized by sudden death of animals which were well only a short time previous, and usually occurs in older and wellfattened animals. The autopsy reveals marked lesions of asphyxiation. On the other hand, there are no lesions of gastro-intestinal inflammation, or of acute swelling of the lymph glands.

The erysipelatous nature of urticaria is indicated by the preexisting general symptoms of the infection, whereas chronic erysipelas is distinguished from other similar affections (rheumatic arthritis, catarrhal pneumonia, chronic swine plague) only by the post mortem lesions. 
In douhtful cases, especially if acute swine plague he suspected, the diagnosis of erysipelas can only be assured by microscopical demonstration of the erysipclas bacilli in the blood or in the tissue of the spleen, kidneys or lymph glands, and if necessary by subcutaneous inoculation of such material into mice or pigeons. (See also diagnosis of swine plague.)

In shipping material to laboratories for examination, squeeze preparations or thin smears from the spleen or kidneys should le made, as according to Joop, the virulence of the hacilli of such material is retained for 10 to 12 days, while they remain alive from 16 to 1 s days.

Treatment. In acute cases the treatment with immune serum gives the best results, provided the animals are treated immediately after the onset of the disease, as the injections when undertaken not later than 6 to 12 hours after the appearance of the first symptoms, exert a curative action in many cases (Leclainche; according to Schütz the serum destroys the bacilli which are present in the body or which may enter the body later). The blood serum is the same as is used for protective inoculation (see page 76), only it is necessary to use considerable amounts; 10 to 30 ce, according to the hody weight, should be injected subertaneously; it is also advisahle to repeat the treatment every 6 to 8 hours until tha appearance of a decided improvement.

In fermany 1315 hogs affected between 1597 and 1901 with erysipelas were treated with Lorenz's serun, of which 1111 that is $84 \%$ recovered, of 656 afiected animals treated with susserin 535 , that is $82 \%$, recovered. In Hessen 1451 ont of 1570 treaterl animals, that is $92.5 \%$, recovered between 1901 and 1903 (Lorenz) ; in Holland $89.3 \%$ of 5830 animals were successfully treaterl (Wirtz); in Hungary Detre's serum was employed in 1903 on 711 animals of which 677 , that is $95 \%$, recovered; and since then results have also been favoralle in different localities in which the serum treatment has heen employed. According to Nocard, in France 8483 hogs affected with erysipelas had been successfully treated up to 1901 with Leclainche's serum.

The symptomatic treatment has hardly any beneficial influence upon the disease. Nevertheless, the occasional internal administration of remedies which possess an intestinal disinfectant action may be tried, and for this purpose is recommended; calomel (2-3 gms.; only in the early stages of the disease, as later when the kidneys are affected it is harmful), creolin or lysol (5\% solution in tablespoonful doses), which may be given with mild cathartics. Besides, the nervous system may be stimulated by donches and washing with cold water.

Prevention. The fact that the erysipelas bacilli remain as saprophytes for a long time in the ground in infected localities, and that they are also frequently present in the tonsils as well as the intestinal canal of healthy animals, renders the combating of the disease very difficult. As, however, the infection is most successfully conveyed by affected hogs ancl their carcasses, healthy animals must in the first place be protected from 
this source of danger. Accordingly, healthy animals should be separated from the affected ones, and they should be kept from contaminated pastures and stables. Carcasses should be deeply buried, or still better cremated. Infected stables and premises should be properly disinfected, and the excrement of the affected animals collected and destroyed. Infected pastures should not be used as ranges for swine for a long time.

If the disease has already appeared in a herd it is advisable to place the apparently healthy animals in an unsuspected pasture, or in the absence of such, pasturing should not he permitted until the complete disappearance of the disease; in this way it is frequently possible to eradicate the outbreak. Inasmuch as the infective agent is present in all parts of the emergeney slanghtered hogs the disease may be disseminated by the marketing of such carcasses, and therefore the sale of meat of such carcasses should not be permitted until it has been sterilized. Such a procedure appears the more arlvisable, as according to Petri's and Stadie's investigations ordinary conservation of the meat and the products prepared therefrom (salting, pickling, smoking) destroys the erysipelas bacilli only after a long time. (See page 62.)

When the possibility or even probability of an infection exists, the occurrence of the disease may be successfully prevented by means of protective inoculation.

Immunization. One attack of erysipelas conveys immunity to hogs against later natural infection. This experience would also indicate that the artificially produced mild affection affords a similar immunity. This idea was practically demonstrated by Pasteur in 1882 by inoculations of artificially attenuaterl cultures, while later Lorenz as well as Leclainche established the value of protective inoculation with immune serum and cultures in practice.

I. Protective inoculation with attenuated cultures (Pasteur's method). Pasteur showed in his experiments that the passage of erysipelas virus through the body of rabbits increases its virulence for these animals. On the other hand, it reduces it for hogs, so that the inoculation with such virus in the latter animals causes only a slight febrile disease. Virus attenuated up to a certain degree retains this degree of virulence even if transferred from the rabbit blood to a suitable artificial medium, for instance, bouillon and if further cultivated at body temperature. The bouillon culture attenuated by the above method produces the vaccine which is injected subcutaneously into young pigs in form of a weaker and a stronger modification.

The effectiveness of Pasteur's protective inoculation has been positively established. Besides Pasteur, Schütz, Schottelius and others found that vaccinated hogs cannot be infected 
either by subcutaneous injections of highly virulent virus nor by feeding with organs from animals which died of erysipelas; on the other hand, the experiences made in practice proved that the immunity produced by the inoculation protects the animals against natural infection.

In Hungary the considerable losses caused by erysipelas have been reduced to 1 to $2 \%$ in formerly badly infected herds. In the great majority of cases erysipelas has not caused losses either immediately after the inoculation or later during the favorable summer weather among the vaccinated animals, whereas among the control animals which were not vaccinated, the disease raged, and in many cases the vaccination suppressed already existing outbreaks. The age of the animals influenced the results of the vaccination in such a way that the inoculation failed to produce in the sucking age a sufficient immunity or none at all; on the other hand, it has not proven dangerous in animals even up to 3 years of age. The vaccinated animals were partly of the native Mangalicza breed. However, animals of the PolandChina and Suffolk breeds were also vaccinated with the same beneficial results (Wirtz also found that vaccination of the Mangalicza breed was without danger), whereas in Yorkshire hogs sometimes dangerous affections resulted (Bleyer).

Pasteur's protective inoculation accordingly appears to be especially effective in herds of common or less highly bred animals, particularly in localities where erysipelas annually causes considerable loss, and it may be employed without danger in already affected herds of such breeds.

At the initiative of Azary, Pasteur's vaccination has been extensively used in Hungary since 1887. From 1889-1894, 1,085,686 hogs were vaceinated and after the first vaccination $0.14 \%$, after a second $0.07 \%$, and later in the year $0.54 \%$ died of erysipelas. On account of hog cholera which appeared in 1895 the results of the later inoculations cannot be determined accurately as the affections and deaths which resulted from the heretofore unknown hog cholera were often simply attributed to erysipelas. But since greater stress was placed on differential diagnosis the results appear favorable. Thus in 1907 of 28,642 hogs in already infected herds 637 died after the vaccination, later 421 additional fatalities were observed. In healthy herds out of 288,950 vaccinations 125 and 623 died.

Pasteur's vaccination is used less extensively in France and Russia probably on account of the fact that in some of the herds the losses following vaceination amounted to 6 to $10 \%$, which condition may be attributed to $a$ feebler resistance of the native breeds. In Germany this method has not attained extensive application owing to the unfavorable results obtained by Lydtin \& Schottelius, but in recent years it has been more extensively used, especially in the soutbern part of the Empire.

Technique of Vaccination. The vaccination is carried out in the same way as anthrax vaccination in sheep (see page 27 ); the quantity of the immunizing material is in this case also $0.12 \mathrm{cc}$. (1/8 of the contents of Pasteur vaccination syringe), and the second vaccination follows 12 days after the first. It is advisable to vaccinate pigs in the spring after weaning at the age of $3-5$ months, before the appearance of hot weather.

Direct results of vaccination. This is manifested in some cases by debility lasting from 1 to 2 days, impaired appetite, and sometimes a weakness of the hind quarters; on the second and third day after the vaccination the temperature rises on an average of $0.8^{\circ} \mathrm{C}$. Sometimes, however, it may rise up to $41.7^{\circ} \mathrm{C}$. (Mihály), whereupon the animals rapidly recover their normal condition. Oceasionally a swelling of considerable size may appear at the point of inoculation, and stiffness of the joints may also develop, particularly in the higher breeds, and here and there death may result from erysipelatous endocarditis. 
II. Protective inoculation with immune serum and cultures (Simultaneous method of Lorenz and of Leclainche; serovaccination). Blood serum of animals which have been highly immunized by repeated subcutaneous or intravenous injections of living cultures of the erysipelas bacillus will protect healthy animals for a short time against fatal infection. The passive immunity produced in this manner may be changed into active immunity by simultaneous or subsequent injections of virulent cultures of the bacilli.

After Emmerich and Mastbaum (1891) found that the blood serum of rabbits which had been immunized against erysipelas possessed immunizing properties, Lorenz (1893) produced an effective serum from artificially immunized hogs. This serum, concentrated to one-third of its original volume, was effective for protective inoculation of hogs. As hogs as well as sheep (Voges) produce only small quantities of serum, Leclainche (1898) and Schütz \& Voges hyperimmunized horses for that purpose, and at the present time serum for the inoculation of hogs is obtained almost exclusively from these latter animals. Potent blood serum may also be prepared from cattle (Schreiber, Kitt), and the "double serum" of Landsberger consists of a mixture of horse and cattle immune serum (Schubert).

The results of protective inoculations with immune serum and bacilli culture have generally given good satisfaction in practice. Hogs which have not yet become infected withstand the vaccination as a rule without marked reaction, and they will later resist natural infection. As a sequel of the vaccination, however, urticaria, swelling at the point of inoculation extending to the throat, stiffness of the joints, occasionally erysipelatous endocarditis, and even death after 36 hours from severe toxic action, have been observed to follow injections of the culture. The advantage of this method over Pasteur's consists in the development of the passire immunity immerliately after the serum injection, which condition in case of immediate danger of infection is of great importance-and further it is well tolerated even by breeds of hogs with low resistance. The somewhat high cost of the vaccinating material is a disadvantage.

Latent diseased processes are unfavorably influenced by the inoculation. Thus, it was repeatedly observed in Germany that chronic pneumonia of pigs (the so-called chronic swine plague of pigs) became aggravated after vaccination, and in Hungary also it was noticed that the first year after the appearance of hog cholera severe outbreaks of the latter disease developed following the vaccination of herds against erysipelas.

In Prussia 217,376 hogs were vaccinated in the years 1897-1899 by Lorenz's method, and after the first injection $0.018 \%$, after the second $0.042 \%$, later in the course of the year $0.058 \%$ died as a result of erysipelas (Joest \& Helfer). In Baden 102,448 hogs were vaccinated in the years 1899-1906 of which 21,466 animals received the serum alone; of these $116(0.09 \%)$ died inside of 3 days, later until the close of the year, $108(0.05 \%)$ suceumbed. In Hessen only 2 out of 35,258 inseulated animals died in the period from 1901 to 1903 (Lorenz). In Württemberg 
only 4 out of 59,625 hogs vaccinated in the years $1904 \cdot 1905$ died of acute erysipelas; of the original number, however, 29,249 animals received the usual two injections (Beisswanger), while in the I'rovince of Saxony in the year 1904-1905 of 175,150 animals, 13 clied of vaccination erysipelas, 6 of endocarditis, and 15 succumbed to erysipelas later (Raebiger). In Holland in the years 1599 to 1905 out of 165,884 inoculaterl hogs $136(0.05 \%)$ died as a result of the vaccinations. Reports from Balen (Fehsenmeier), Moravia (Rudovsky), C'antonzurich (Zschokke) and others are much in faror of the value of the simultaneous injeetions, and the Leclainche methou is also used extensively in Hungary with favorable results.

Preparation of the immune serum. For this purpo:e healthy horses are nsed which receive weekly injections, into the jugular vein of virulent bouillon cultures of erysipelas bacilli in increasing quantities (50 to 500 ce.). The animals react to the injection with fever, muscular trembling ancl sometimes with diarrhea. After 2 to 3 months of such treatment they produce a blood sermm which strongly agglutinates the erysipelas bacilli of which $0.5 \mathrm{cc}$. protects a pigeon against a like quantity of virulent culture which ordinarily would kill it in $2 \frac{1}{2}$ to 3 days (Leclainche); exceptionally 0.1 of such serum has such a protective action (Deutsch); it retains this action for months after heating for $1 / 2$ hour to $55^{\circ} \mathrm{C}$. In Germany only serum can be marketed, 0.015 ce. of which will protect a mouse of 15 . grams weight against a simultaneous subcutaneous (Lorenz), or against an intraperitoneal (Marx) injection of $0.1 \mathrm{cc}$. of virulent boullon culture 24 hours later.

The inmune serum used for the inoculation of hogs must possess the strength mentionel and should not be more than one year old, but the virulent bouillon cultures should he fresh and not over a week old. In Germany the immune serum is prepared in various laboratories under different nanes (Prenzlauer \& Landsberger's serum, susserin), and is placed on the market with the necessary direetions for its use.

Technique of vaccination. Leclainche's method: In a carefully sterilized syringe of 10 ce. eapacity, $1 \mathrm{ce}$. of the boullon culture is drawn up and then the syringe is filled up with the inmune serum. After the contents are well mixed hogs of not over $50 \mathrm{~kg}$. weight are injected subcutaneously with $5 \mathrm{ec}$. on the inner surface of the thigh. If the hogs are heavier, 1 ce. of adlitional blood serum is given for each $10 \mathrm{~kg}$. above $50 \mathrm{~kg}$., or in general each heavy hog is injectell subeutaneously with $9.5 \mathrm{ce}$. of serum and $0.5 \mathrm{cc}$. of culture. The second injection follows 10 to 12 days atter the first, when the syringe is filled with bouillon culture, and of this each animal receives $0.5 \mathrm{cc}$. without consideration of the animal 's weight. The injection is also made on the inner surface of the thigh.

Methoil of Lorenz. In this method the immune serum is first injected under the skin of an ear and immediately afterwards 0.25 to 1 ce. of culture is injected into the other ear; 12 to 15 days later the hog is injected with double the quantity of the culture used in the first dose. The second injection is also made unler the skin of an ear. Relative to the results of the inoculation, it is immaterial whether the blood serum and virus are injected in a mixture (Leclainche) or simultaneously, but in different parts (Lorenz, Schiitz), or if the virus is injected several lays following the serum (Lorenz).

A sufficient protection is only assured after both inoculations, as the fir:t simultaneous inoculation produces only a passing rise in the resistance as proven experimentally by I'rettner, inasmuch as the bacteria are influencer by the injecter serum to such an extent that a sufficient quantity of immume bodies is not pro iluced.

III. Passive immunization. The immune serum may also be used by itself for the immunization of already infected herds ( 5 to 10 cc.). The serum very rapidly produces a passive immunity which prevents the development of the disease in animals threatened by the infection or when the infection is in the stage of inculiation (see also page 72 ). The immunity prodnced in such a way lasts, however, only a short time. (According to Leclainche, 17 days.) Therefore, these animals should be given the combined or Pastemr's inoculation after their recovery or from 8 to 10 days after the serum treatment in order to prodnce a lasting immunity. 
Veterinary Police. Police measures aclopted for other contagions diseases may also be recommended for erysipelas, and particularly quarantine of the premises-in more extensive outbreak even the township, prohibition of marketing the animals, harmless disposition of carcasses, disinfection of infecterl localities, etc. From the quarantined premises or townships the transportation of animals to ahattoirs for immediate slaughter should be permitter. The observation periorl after the apparent disappearance of the disease should extend from $1 \mathrm{~s}$ to 15 days.

Literature. Pasteur, Rev. vét., 1883, 39.-Pasteur \& Thuillier, Bull. de l'Acad. de mérl., 1883, Nr. 45.-Eggeling, D. Z. f. Tm., 1884, X, 234.-Loeffler, Arb. d. G.-A., 1885, I, 46. - Schütz, Ibid. p. 56.-Lydtin \& Schottelius, D. Rotlanf d. Schweine, Wiesbarlen, 1885.-Bang, D. Z. f. Tm., 1892, XVIII, 27.-Jensen, Ilir., p. 278.-Kitt, Monh., 1894, V, 19.-Lorenz, D. t. W., 1897, 91; Cbl. f. Bakt., 1893, XIII, 357.-Leclainche, Soc. biol., 1897, 428; 1899, 346.-Voges \& Schïtz, A. f. Tk., 1898, XXIV, 173.-Banermeister, 1902, XXVIII, 66 (Lit.).-Preisz, Hb. d. p. M., 1903, III, 710 u. IV., 1236 (Lit. on etiol. and immunization).- Starlie, Diss., Berlin, 1904 (Lit. on biol. of eryeip. bac.).-Eisenmann, Monh., 1906, XVII, 07.Schipp, D. t. W., 1910, 98.

The Disease in Man. From the literature a considerable number of cases are known (Casper, Nevermann, Welzel, Hennig, Gleich, Zipp and others) to have occurred in men occupied in handiing hogs affected with erysipelas, or who worked with cultures of the bacilli (immunization). It was observed that after slight injuries to the skin erysipelaslike reddening of the skin and swelling of the neighboring lymph gland developed sometimes even exfoliation of the blackish-red epidermis with exudation of serum, and occasionally even swelling of the neighboring joints. The disease usually terminated within four weeks in recovery (in Röder's case it lasted for 12 weeks), which is considerably hastened by subcutaneous injections of immune serum (10 cc.).

From eating meat of hogs affected with erysipelas no attacks have been observed. Lubowsky, however, found large yuantities of hacteria in the feces of a boy which were identical with the erysipelas bacillus. The boy suffered from jaundice and intestinal catarrh, the cause of which was unknown.

Literature. Rosenbach, Z. f. Hyg., 1909, LxIlI, 343 (Lit.).-Roiler, Mitt. d. bad. Tä. 1908, II.-Gleich, B. t. W., 1909, 576.-Zipp, Tijlsskr., 1909, 98.— Lubowsky. D. m. W., 1910, 116.

\section{Hemorrhagic Septicemia, Septicaemia Hemorrhagica, Hueppe}

\section{(Septicaemia pluriformis, Kitt; Pasteurellosis, Lignières.)}

Under the collective name of hemorrhagic septicemia are included all those diseases which are produced by varieties of the bacillus bipolaris septicus (Bac. multocida s. plurisepticus, Kitt; Pasteurella, Lignières) and in which the acute cases are characterized by manifestations of a general infection and hemorrhagic inflammatory processes of the internal organs.

History. Perroncito and Semmer (1878) and later Pasteur (1880) by the study of chicken cholera, Gaffky (1881) of rabbit septicemia, 
Kitt (1883) of Bollinger's Wildsenche, and Löfller (1886) of swine plague, proved that these diseases are caused by small bacilli, all of which showed the same characteristics by taking the stain intensely at the poles and only very slightly or not at all in the middle, therefore resembling somewhat the figure 8 .

Based on a comparative study of these four diseases, Hueppe (1886) came to the conchsion that their causative agents coincided in all their principal morphological and hiological characteristics, and that the diseases greatly simulated each other in their course and anatomical changes. From these he concluded that the cansative agents of the discases mentioned represent one species of bacteria, and that the existing differences, especially with reference to their virulence, justified his conclusion that they were varieties of the same species. Inasmuch as in the acute cases there are manifestations of a general blood infection and the presence of hemorrhages in the internal organs, Hueppe inciuded the four diseases in one group and recommended the name of hemorrhagic septicemia for their designation.

The observations made since these investigations proved the correctness of this conclusion, and have at the same time shown that similar bacteria play an important part in the etiology of other diseases. Thus, this was established by Poels (1886) for the infectious pleuro-pnemmonia of calves, by Oreste and Armanni (1887) for the barbone lisease of buffalo, by Galtier (1889) for the infectious pneumo-enteritis in sheep. Therefore these diseases may also be included in the group mentioned.

In the diseases mentioned and also others of less importance the bipolar bacteria appear to he the exclusive factors in the development of the pathological processes. Lignières (1900), however, includes those diseases in which the bipolar bacilli are usually involved only indirectly, hy predisposing the body for an infection with other bacteria. Such diseases are influenza or pleuro-pneumonia of horses, and distemper of dogs and cats, to which Nocard, on the strength of his own investigations, added white scours and the catarrhal pneumonia of calves which are due to navel infections. In the meantime Lignières in supporting Trevisan's suggestion, named the bacteria of chicken cholera and of the related diseases, pasteurella, and the diseases caused by these bacteria pasteurellosis. This nomenclature has been accepted in general by the French authors.

Recent experiences with the diseases mentioned and a more accurate consideration of the etiological part of the ovoid hacteria, make the correctness of Lignières' conception very doubtful, and at least they appear to be remote. While it cannot be doubted that the bacteria under discussion may independently produce diseases, their occurrence in affected organs and tissue fluids is not a sufficient proof that the disease was originally produced by them. Since they may not infrequently be met in healthy animals, it is possible for them to invade secondarily the tissues which have been already affected from other causes. It will require further investigations to establish the significance of their presence. However, their part in distemper of dogs, in pneumonia of calves and pigs, in the pectoral form of hog cholera, and in influenza, may even now be accepted as secondary. On these grounds it appears advisable to restrict the conception of Lignicres' pasteurellosis, and therefore only those diseases will be considered here in which it has been positively estahlished that the ovoid hacteria play the primary etiological part. The results of investigations in the future will show whether a change of this viewpoint will he necessary. 
In the designation of the diseases belonging to this group the former appropiate name of hemorrhagic septicemia will be used as it appears justifiable from a pathological-anatomical standpoint. The causative agent is the species of bacteria known as bacillus bipolaris septicus, while the various organisms of the group causing this disease will be designated according to the species of animals affected, viz., Bac. avisepticus, Bac. bovisepticus, Bac. suisepticus, ete.

Morphology and Biology of the Infective Agent. The bacillus bipolaris septicus (Bac. multocida s. plurisepticus, Kitt; pasteurella, Lignières, ovoid or girdle bacterium) manifests the following characteristies without consideration of the diseases belonging to this group or from what species of animals it originates (Lignières). It is short, stains intensely at the poles but only slightly in the middle, polymorphous, non-motile, does not form spores, principally aerobic; does not stain by Gram, grows in bouillon, gelatin and agar, but does not grow on acid potatoes, nor liquefy gelatin, nor coagulate milk; it does not produce indol in pancreatic bouillon, nor redden Wirtz's agar; the bouillon cultures have a peculiar characteristic odor.

Besides these characteristics of the bipolar bacillus, the special characteristics of the bacteria belonging to this group are the following:

Form. In the tissue fluids and still more so in cultures the bacillus represents a short rod about $1^{\mu}$ long, with rounded ends (Fig. 23). It also oceurs in the form of cocei, diplococei; exceptionally also as longer rods, threads and streptobacilli. Its dimensions vary according to the origin of the disease in certain although not wide limits.

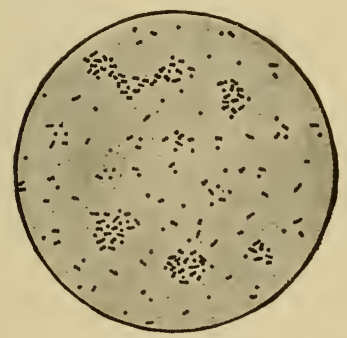

Fig. 23. Bacillus bipolaris septicus. Smear prepared from a fresh agar culture of the bacillus of fowl cholera. Fuchsin staining.

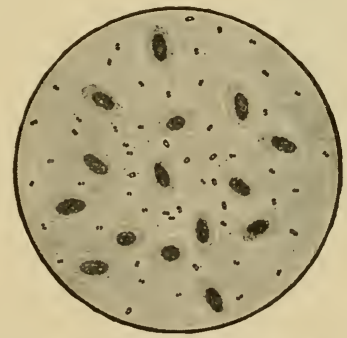

Fig. 24. Bacillus bipolaris septicus. Smear from the blood of a chicken dead from fowl cholera. Polar staining with fuchsin, and subsequent washing with a $1 \%$ acetic acid.

Staining. The bacilli stain quite readily with the aqueous aniline dyes, and in the preparations made from the tissues or from the body fluids (best in the blood of affected chickens) they appear stained only at the poles, while the middle remains unstained (Fig. 24). These results are obtained when the preparations are not stained too intensely 
or after subseruent washing in alcohol or $1 / 2$ to $1 \%$ acetic acid. On the other hand, it is difficult to obtain the polar stain in bacilli originating from artificial cultures.

Cultivation. The bacilli are easily isolated from cases of all acute septicemic character, while from chronic cases this is more difficult and sometimes impossible. The reyuirements of artificial eultivation are: temperatme of $13-28-\mathrm{C}$. and alkaline or at least neutral reaction of the medium. In racuum the bacteria grow only when the medium is inoculated with numerous organisms, and then only for a sliort time.

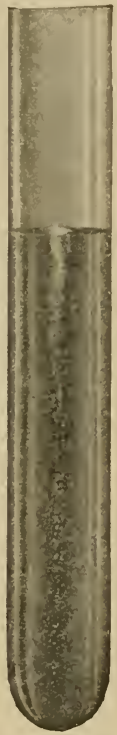

Fi:. 25. rielatin stal) eulture of the bacillus bipularis septicus.

A frequent characteristic of the cultures is their viscosity (this is also striking in the peritoneal exuclate of inoculated guinea pigs), probably due to the sticky capsule which covers the bacteria.

The eolonies formed on gelatin are no larger than a millet seed. They are at first transparent, often resembling a dew-drop, and frequently iridescent. Later they are whitish, of a hyalinecartilage-like appearance. In the depth of the medium the punctiform colonies unite along the stab and form a continuous white streak (Fig. 25). Similar colonies develop on the surface of the agar cultures, but do not extend to the wall of the test tube (Fig. 26); eultures several days old are viscid; when still older they adhere firmly to the medium On coagulated blood serum a very fine membrane forms which is at first transparent and later whitish. Bouillon with the addition of peptone or blood serum usually becomes more or less uniformly cloudy; in some of the varieties, however, the fluid clears, sometimes first forming a slimy and later a fine granular sediment; oceasionally also a delicate,

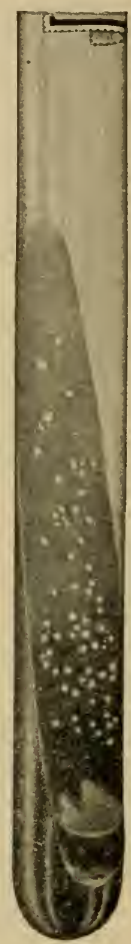

Fig. 26. Agar stab culture of the bacillus bipolaris septicus. whitish membrane develops on the sur-

face The addition of sugar or glycerin does not promote, but rather retards the growth. Dextrose bouillon is not fermented. No growth takes place on potatoes with a natural acid reaction; when artificially alkalinized a grayish-yellow deposit forms.

Animals inoenlated with a certain variety prolnce a serum which will have the highest agglutination for that particular variety (up to $1: 60,000$ ), but it will also agglutinate to a lesser legree other varieties $(1: 1000-6000)$. Sometimes, however, some of the varieties are in no way influencerl. Therefore the agglutination cannot he utilized for the identification or separation of the different varieties (Chamlerland \& Jouan). 
Pathogenicity. In contradistinction to the common characteristics described, there are marked differences in the virulence of the bacilli which depend on their origin, the nature of the disease and the species of animals affected. However, all the varieties coincide in exerting their strongest pathogenic action when inoculated with blood or exudate of animals which died of the disease (aggressin action, see page 84).

Another common characteristic of the bacteria belonging to this group is that they develop the highest virnlence towards that species of animals for which they are naturally pathogenic, while towards other species their action shows a great variance. It is, however, possible to increase the virnlence for other species throngh repeated reinoculations, but such a strain of the bacilli will even then possess the strongest pathogenic action for the species from which it originated.

The animals which are susceptible to the bipolar bacteria. although not to the same extent, are the guinea pig, rabbit and monse, while the domestic animals show a great variation in susceptibility. The guinea pig is best adapted for test inocnlations, and by using this test animal for repeated intraperitoneal reinoculations the virulence of any of the varieties may be greatly increased.

The result of artificial infection depends also on the nature and method of the inoculation. The most pronounced action is obtained by direct injection into the circulation, by which means the disease may be transmitted to any species. Somewhat less effective is the intraperitoneal and intratracheal injection of cultures, whereas the subcutaneous and intramuscular inoculation produces a severe affection only in very susceptible species of animals. The feerling of virulent material only exceptionally gives positive results, these being most frequently obtained when the cholera virus of chickens is fed.

Variability of the Virulence. The bipolar bacilli are very widely spread, and may produce disease in any species of animals, particularly in the domestic animals. As original saprophytes they occur in nature in the soil, in slow-flowing or stagnant water, on various plants, and in all kinds of dead organic material. Under normal conditions they do not manifest pathogenic characteristics. Hence they ocenr not infrequently in the digestive tract and in the air passages. Under certain conditions, which at present are still unknown, they become virulent and then they may attack the tissues of the healthy body. On the other hand, their pathogenic action is favored by all those influences which weaken the resistance of the organism, as colds, exertion, anemia, starvation, mixed infection, catarrhs, etc. Parasites in the air passages and digestive tract also favor the development of infection by loosening the epithelium of the mucous membrane, or by causing penetrating injuries to the mucons membrane, whereby the entrance of the hacteria into the lymph ressels is facilitated. 
The bacteria which through some cause become parasitic lose in some of the cases their pathogenic character after leaving the affected animal, and may change again to saprophytes. In the majority of cases, however, after having become accustomed to the organism of a certain animal, they attain an increased virulence for animals of this particular species, and therefore infect the animals more easily even without the influences of predisposing causes. This relative virulence may even increase for a time in the course of further generations, or may become constant. More frequently, however, it diminishes again after a certain time.

These characteristics of the bipolar bacteria explain the variation which is observed in the appearance and spread of the diseases belonging to this group. The common experience with these diseases is that they appear periodically in certain localities without any apparent connection to which the introduction could be traced. In such cases the outbreaks can be explained by a sudden increase in the virulence of the bacteria which are present in the soil or in the healthy animal favored by influences which reduce their resistance. Some of the infections develop through such conditions. In these the disease does not, or only exceptionally, spread from animal to animal, and it therefore remains sporadic, or at least confined to certain localities. In other cases although the disease appears spontaneously the organisms which pass through the body of the animals retain their pathogenic character for a long time, thus spreading the disease by direct or indirect infection, and disseminating the contagion to distant localities. Further, among the diseases of this group there are some in which the bacteria adapt themselves in the course of time to the animal body to such an extent that as saprophytes they are no longer capable of propagation, and therefore these diseases spread almost exclusively by being introduced from outside or by direct communication.

The acquired or inherited virulence of the different varieties of the bacteria is usually only manifested for certain species of animals, so that a certain disease spreads usually only among the animals of the same species. Exceptionally however, the infective agent may become dangerous for other species. Thus frequently the infection of buffalo disease and hemorrhagic septicemia of cattle causes in hogs symptoms resembling swine plague. On the other hand, the virus of swine plague sometimes produces a septicemic affection in sheep, while feeding it to chickens causes a disease which corresponds to fowl cholera.

Lignières succeeded in artificially producing a variety of forms of hemorrhagic septicemia in different animals by injections of cultures of the bipolar bacillus of different origin. Koske successfully infected different species of fowl per os with swine plague bacteria. On the other hand, he failed in hogs to produce a disease resembling swine plague with the fowl cholera organism.

The ovoid bacteria may retain their virulence for months in the original 
exudates or body fluids under artificial conditions, if light and air are excluded; whereas, in artificial media in the presence of air they are sometimes very rapidly attenuated and finally lose their virulence.

Pathogenesis. Bipolar bacilli which penetrate into the tissue fluids of the animal organism, produce different pathological changes, according to the degree of their virulence and the susceptibility of the animals. In case of a severe infection a peracute hemorrhagic septicemia develops. The bacilli multiply very rapidly and in a short time permeate the entire body, causing death in from 18 to 24 hours. The animals manifest high fever, weakness of the heart, debility, sometimes diarrhea. Antopsy reveals numerous hemorrhages in the tissue of the serous and mucous membranes and parenchymatous organs. The spleen is only slightly swollen, while the lymph glands show a marked acute swelling.

When the infection is somewhat less severe the acute attack lasts for several days when frequently a sero-fibrinous inflammation develops on the serous membrane, while the mucous membranes are affected with a hemorrhagic inflammation. With these an inflammation of the joints and tendons is not infrequently associated. The autopsy reveals in these cases a moderate acute swelling of the spleen and a pronounced swelling of the lymph glands in addition to blood extravasations.

An infection of a milder degree, for instance an intravenous injection of a small quantity or very weak virus, results in some cases in a chronic affection, lasting for several weeks or months. The animals become emaciated slowly but grardually, frequently in spite of their good appetite; they have an intermittent fever, and inflammatory processes develop in different joints, especially in the knee, carpal and tarsal joints. The animals also show a marked predisposition for secondary infections, especially for catarrhal pneumonia.

The bipolar bacillus may produce these varied clinical manifestations independently. In the acute cases the organism is found in great numbers in the blood and also in the exudates in pure culture. The pathological processes in some of the chronic cases may be also attributed to its action even if the bacilli have since disappeared from the body.

The local action of bipolar bacilli is manifested in the production of serous and fibrinous inflammations, later in degeneration of the exudate and tissue elements, as a result of which dry caseous masses occur, while necrotic areas develop in the affected organs. The necrosis is very probably the result of the action of toxins. Filtrates of bouillon cultures free from bacteria usually produce only a very slight or no toxic action. Killed masses of bacteria, however, possess distinct toxic properties. Inasmuch as in the body of the animal both multiplication and destruction of bacilli take place, and as dead bacilli are dissolved in the tissue fluids the toxins which are eliminated may cause general effects and local necrotic processes. There 
is also a possibility of the bacteria producing strongly acting exogenic toxic substances and aggressins in the living bocly.

According to the investigations of Klett \& Braun, the bacilli of fowl cholera and swine plague secrete even in artificial fluid cultures soluble poisons in large yuantities which ean be demonstrated. These toxins have an identical poisonous action (in pigeons and chickens lethargy and intoxication) and their endotoxins are also identical; a difference is indicated in that the Bacillus avisepticus produces greater quantities of filterable toxins and endotoxins, and these are also more constant.

According to Weil the virulence of the bacteria is considerably increased when heated to $44^{\circ} \mathrm{C}$. in the exudate of artificially infected animals, and also, aceording to Citron, in extracts of eultures. According to Bail's theory the bipolar bacilli, like the other pathogenic bacteria, produce specific substances in the animal body (aggressins) which possess the character of changing non-fatal amounts of bacteria to fatal ones, without themselves having marked toxic properties. Further, Tschistovitch believes that virulent fowl cholera bacilli contain strictly specific thermo-stable antiphagins which protect them from the digestion by leucocytes.

The bipolar bacilli manifest a particular affinity towards certain organs, and therefore pathological changes develop in the less acute cases only in certain organs. In such predisposed organs either acute or chronic inflammations may be present. The serous membranes in general, the lungs, the synovial membranes of the joints and tendons and probably the liver are the favored organs.

According to Lignières the bipolar bacilli may also give rise to the development of secondary disease processes. Through their toxins and probably indirectly through a temporary arrest of the phagocytosis, the resistance of the body may be reduced, whereby bacteria which are more or less harmless may produce disease processes, while the bipolar bacilli may in the meantime disappear from the body. Even in many of the acute cases which are associated with an inflammation of the lungs and joints other probably pathogenic organisins may be found in the affected tissues, or in the inflammatory products in addition to the bipolar bacilli. However, in the chronic cases only secondary bacteria are as a rule present.

The intravenous injection of a weak virus, for instance older cultures of the bipolar bacillus, produces as has already been mentioned, a chronie affection which results in cachexia. In these eases the bacteriological examination of the affected tissues and exudates discloses other bacteria (streptococei, colon bacteria, ete.) in spite of the fact that the infection was brought on by the bipolar bacilli. Similar results may oceasionally be obtained from the injection of filtrate free from bacteria made from cultures of the bipolar bacillus.

The very frequent occurrence of mixed infections makes it possible for other bacteria to prepare the field for the propa- 
gation of the bipolar bacilli. Through the destruction of the tissues by the other organisms the entrance and propagation of the ovoid bacteria are favored.

Their invasion is without doubt of a secondary character in those cases in which a general septic affection is caused by an ultravisible virus, as for instance in $\log$ distemper and hog cholera (see those diseases). In these affections disease processes may develop subsequently which are exclusively or at least partly the result of the action of the ovoid bacteria. In recognition of this fact, the etiological importance of these bacteria has already been considerably limited.

Immunization. Pasteur proved that eultures of the chicken cholera organism when attenuated by keeping in the air produce a suitable vaccine for the immunization of chickens against artificial infection. Similar results are obtained with cultures attenuated by higher temperature or by disinfectants. However, the results of such immunizations which are mostly limited to laboratory experiments are by no means uniform. This irregularity is probably due, on the one hand, to the variance of the virulence of the cultivated bacteria, and on the other hand, to the difference of action of the methods employed in the attenuations, accurate control of which is almost impossible.

More recently J. and M. Lignières (1902) produced a potent vaceine for all the diseases of this group from enltures which had been reinoeulated several hundred times from agar to agar at two days' intervals, as a result of which they possessed a uniform virulence. Flasks with a wide bottom are filled to a height of $1 / 2 \mathrm{~cm}$. with peptone-bouillon and inoculated with the stock eulture. The flasks are then placed in a thermostat and kept at a temperature of $42-43^{\circ} \mathrm{C}$. The cnltures which are grown for five dars at this temperature proluce the first, while those grown only for two days, the second vaceine.

With the attenuated cultures immunity may be produced not only against the same variety but also against related varieties of the bipolar bacillus. Kitt was the first to point out the possibility of a uniform procedure for the control of the diseases of this group. He successfully immunized chickens against fowl cholera with the virus of rabbit septicemia. Later Jensen obtained the same results with the virus of infections pneumonia of calves. Lignières also proved that this reciprocity obtains with regard to all strains of the varieties of bacteria belonging to this group. He succeeded in demonstrating experimentally that a vaccine prepared from a culture of a certain variety is potent in the first place against this variety, but that it also has a protective action, although of a lesser degree, against the bipolar organism of the other diseases of this group. But its protective action for the latter diseases is so slight and uncertain that it does not meet with practical requirements, and besides the preparation of the different vaccines for each of the diseases would be very troublesome. Lignières therefore prepared a so-called polyvalent vaccine 
which is potent against all the diseases of this group. The vaccine is prepared from mixed cultures of the hemorrhagic septicemia bacteria of sheep, cattle, dogs, horses, hogs and chickens and attenuated at a temperature of $42-43^{\circ} \mathrm{C}$.

The vaceine prepared from the six varieties of virus is supposed to immunize against all of the six diseases. Test animals inoculated with this vaccine will show a resistance against a virulent infection, while control animals will die or become severely affected (with the exception of very virulent intravenous infections). In practice the vaceinations are supposed to have given good results, especially against the acute septicemic forms of hemorrhagic septicenia, as for instance against chicken cholera and hemorrhagic septicemia of sheep. On the other hand, the results in the slower forms which are frequently associated with mixed infections were less favorable, although even in these cases the mortality has been supposedly reduced from $50 \%$ to $12 \%$.

The immunization consists in a subcutaneous or intraperitoneal injection of the vaceine; the second inoculation follows in from 12 to 15 days. In infected localities it is advisable to undertake the vaceination on sucking animals 8 to 10 days after their birth, or at least shortly before weaning. The immunity produced by the vaccination lasts for about a year, and therefore it is advisable in infected localities to repeat it annually.

Serum Immunization. Kitt (1897) found that animals (among others, horses and cows) which have been injected with the virus of chicken cholera produce a blood"serum which protects chickens against an artificial infection of virulent material. This fact was later confirmed in connection with swine plague by Schweinitz and Leclainche while Lignières \& Spitz (1902) showed that this principle applies to all diseases of the hemorrhagic septicemia group.

With regard to the immune sera, there exists a similar reciprocity as in the case in which the vaccines are prepared from living cultures. Kitt \& Mayr have established these facts for swine plague and fowl cholera, Grosso for hemorrhagic septicemia of eattle, fowl cholera and swine plague, while Lignières generalized the principle. Accordingly an immune serum prepared from certain varieties of the bipolar bacillus protects, in the first place, against a virulent infection caused by the respective varieties. However, it is also protective, although in a lesser degree, against the other related varieties. Blood serum of animals which have been hyperimmunized with different varieties of the bipolar bacillus has likewise a protective action against all the varieties which were used in the preparation of the serum.

Based on these experimental observations Lignières \& Spitz prepared a so-called polyvalent immune serum against all the pasteurellosæ. The serum is prepared by inoculating peptone bouillon with the above mentioned six varieties of the bipolar bacillus, and with the mixed cultures prepared in this manner horses are at first subcutaneously, later intravenously injected with increasing quantities (5 to $20 \mathrm{cc}$.). Horses highly immunized in this way produce a serum which affords protection against all of the diseases of this group, and it even possesses a curative action to a certain degree if emploved in the early stages of the disease. Similar results were also obtained by Klepzoff, while Broll 
\& Angeloff showed that by the use of swine plague serum the opsonic index is not only raised for swine plague but also for the bacteria of hemorrhagic septicemia of cattle and fowl cholera.

The polyvalence should be considered here in a different sense from that applied in the Wassermann-Ostertag's polyvalent swine plague serum (see swine plague).

The very close morphological resemblance of the bipolar bacilli from the various sources, the similarity of the cultures in their principal cultural characteristics, the great similarity of the pathological processes produced by them, the possibility of intertransmissibility to the different species of animals, likewise the common immunizing properties, indicate that the bipolar bacilli belong to one species of bacteria, while the few deviating characteristics such as the variance of virulence are only characteristics peculiar to the different strains or varieties. The bacteria which probably developed from a common strain originally have undergone modifications in the bodies of the different species of animals by adapting themselves to existing environments similar to that which occurs in other species of bacteria, and which may also be produced artificially. On the other hand, the differences in the pathological processes in the various diseases may be satisfactorily explained by the special characteristics of the different animal species. The close relationship of the causative factors of the several diseases brings the affections produced by them into closer relation. Moreover, the similarity of the etiological factors also indicates uniformity in methods to be used in the control of these diseases.

Literature. Hueppe, B. klin. W., 1886, 753.-Lignières, Bull. 1900, 329, (Lit.).-Kitt, Hb. d. p. M., 1903, II, 559, (Lit.).

\section{(a) Fowl Cholera. Cholera Gallinarium.}

(Foul typhoid; Pasteurellosis avium, Choléra des poules [French]; Hühnercholera [German]; Cholera dei polli [Italian].)

Fowl cholera is an acute, contagious and usually an epizootic affection of fowls, particularly of chickens, geese and ducks, and is manifested in a general infection associated witl a profuse diarrhea. It is caused by a bacterium of the type of the hemorrhagic septicemia organism, the bacillus avisepticus.

History. This dangerous fowl plague was deseribed by veterinarians as early as the 18th century, and in the middle of the last century some of the authors (Benjamin, 1851, Delafond \& Renault, 1851, and Hering, 1858) have recognized its contagious nature and also substantiated it experimentally. Perroncito (1878) and also Semmer described a diplococcus as the cause of the disease which they constantly found in the blood of chickens dead of the disease. Toussaint 
(1879) and later Pasteur (1850) confirmed their findings and suceeeded in growing the bacteria in artificial bouillon, thus enabling them to study their pathogenic characteristics. Pastem carried out his first fundamental experiments on the attemuation of baeterial cultures and on immunization with such attenuated cultures with the bacilli of fowl cholera. Valuable eontrihutions regarding the etiology of the disease were also furnished by Kitt and Lignières.

Occurrence. Fowl cholera occurs extensively in Europe (with the exception of Great Britain), in North America and in South Africa, and causes very heavy losses, especially among the finer breeds. The extent and value of the losses cannot be accurately estimated; as a matter of fact, in some of the localities, occasionally half or even a larger proportion of the flocks are annililated. Aside from the money value of the dead and slaughtered fowls, the total loss is considerably inereased by the temporary retrogression of fowl breeding in the affected localities as well as by the reduction of the egg production.

In the year of $1908,14,397$ ehickens, 13,877 geese, 4,795 ducks, 272 pigeons and 256 other fowls diel or were slanghtered in 776 townships in Germany on account of cholera. The greatest number of losses oceurred in the government districts, Potsdam, Bromberg, Oppeln, Allenstein, and Posen (in the last 3 years the number of eases was reduced from 74,329 to 33,597 ). The losses of the other countries are not known, but the disease exists at the present time to a great extent in Russia, Italy, Austria, Hungary, France, Bulgaria, California, etc.

\section{Etiology. The bacillus (bipolaris) avisepticus (bacillus} cholerae gallinarium s. avicida, pasteurella avium) belongs to the smallest of the bipolar bacilli with which it, lowever, corresponds in the morphological and cultural cliaracteristics (see page 79). In fowl blood the bipolar staining is particularly well marked (see fig. 24 on page 79 ) ; peptone bouillon is made uniformly cloudy with or without the formation of a slimy sediment (according to Hertel the bacilli sometimes pass through a porous Berkefeld filter).

Tenacity. The bacteria of fowl cholera remain alive in manure for at least 3 months (Gaertner), in putrefactive carcasses and in garden soil for 3 months (Kitt), in water with the exclusion of air and at a temperature of 5 to 6 degrees for 18 days (Hertel). They also show a considerable resistance to crild (according to Kitt they resist freezing for 14 days, and according to Hertel their virulence is grailually reduced at minus 13 degrees), but they are less resistant towards other harmful influences. Thus, when drierl in exulate in the air, and under the influence of sunlight they lose their virulence in 48 hours, if the light is excluted in 72 hours (Helfer), whereas organs of carcasses are sterilized by a temperature of 45 to $50^{\circ} \mathrm{C}$. in $3 / 4$ hour, and in 10 minutes at a temperature of $80-85^{\circ} \mathrm{C}$. (Kitt). The following disinfectants have a destructive action on the organisms: $1 \%$ carbolic acil, $1 / 2 \%$ sulphuric acic (Salmon), $1 \%$ chlorate of lime, ami $5 \%$ milk of lime (Jäger). The llood and loose exerements are positively disinfected by thoroughly mixing them with a $5 \%$ colper sulphate solution (Colin). Turf dust has also a disinfecting action (1Tmic acil), for when bloody intestinal contents are mixed with it the infectiveness of the bacilli is destroyed in 49 hours (Hertel).

Pathogenicity. The blood of chickens affected with cholera as well as fresh pure cultures produce the disease in fowls after 
cutaneous or subcutaneous injection even in minimal doses. The transmission may be made successfully by taking the point of a needle dipped in blood and scarifying or pricking the skin at any part of the body. In this manner as well as by subcutaneous and intramuscular inoculations most of the different species of domestic fowl (chickens, ducks, geese, turkeys, pigeons, pheasants and fancy birds) and small wild hirds (sparrows, finch, etc.) are easily infected. These bircls also become affected by the dropping of a culture into the conjunetiva, nose or the laryngeal mucous membrane, as well as on superficial wounds of the comb, wattles and feet (Hertel). Further, the disease may be produced by feeding of blood, meat, organs and intestinal contents of affected animals as well as of pure cultures. The disease produced by inoculation usually results in death in from 12 to 24 homrs, while when produced by feeding of the virus it terminates only after a longer period (according to Semmer sometimes only after 8 to 12 days).

Of other animals rabbits and mice are susceptible to the disease, whereas in guinea pigs (Pasteur), cattle, sheep and horses (Kitt) the subcutaneous inoculation produced only an abscess at the point of injection (guinea pigs, however, are easily infected by intraperitoneal inoculation). Intravenous injections of very virulent cultures are pathogenic for domestic mammals (hogs, dogs and cats), while through feeding of such material infection is successful only in rabbits. Stange \& Persdorf observed a simultaneous infection in chickens and hogs which they traced to an infection with the fowl cholera virus. On the other hand, Koske has not succeeded in infecting hogs artificially with the cholera virus. On man the eating of affected fowl has apparently no harmful effects.

Repeated passage of the hacteria through the boties of chickens, pigeons or sparrows increases their pathogenicity which is still more increased by passage through guinea pigs (intraperitoneally). In this way the virulence may be increased to such an extent that intravenous injections of such virus may kill domestic mammals in a few hours (Tignières). On the other hand, the air, and more so the combined action of air and sumlight, attenuates the virulence of the bacteria (Pasteur, see page 94).

The bacteria produce toxins in artificial cultures. Pasteur found that the subeutaneous injections of large quantities of filtrate of houillon cultures free from bacteria cause in chickens a somnolence and stupor lasting for several hours, similar to that seen in the disease. This fact was later confirmed by Salmon, Stange, Kitt, as well as by Klett \& Braun. (See page 84.) On the other hand, Calamida ohtained from a 12-day old bouillon culture a hemolysin which has the strongest action on rabbits, less on guinea pigs and still less on the red blood corpuscles of chickens.

Natural infection usually results from the ingestion of droppings or saliva of affected fowls, contaminated feed, viscera 
of slaughtered or any parts of the hody of affected animals; besides, when healthy fowl drink from the same vessels or from a pond from which infected birds have watered. The infection from cages as well as from the premises may also be disseminated in various ways to neighboring localities where an outbreak may follow.

The disease is mostly introduced into premises, which have been free from the disease, by the introduction of infected fowls, and less frequently by the sale for food purposes of birds which have been slaughtered while ill. The disease is also very frequently disseminated by poultry shows in which the veterinary supervision is very deficient. Further, it may also be disseminated by pigeons which take up the contagion in infected premises, become infected themselves and by their intestinal excrements infect the other birds of the premises (Cagny, Nocard). Finally, the disease may be spread by wild birds, particularly sparrows (Hertel demonstrated virulent bacilli in dermanyssus mites which he took from the body of sick chickens).

The possibility of the spontaneous occurrence of fowl cholera in localities in which this disease or one of this group has existed is not excluded in view of the general statements made on page 81, but this possibility has not yet been demonstrated by satisfactory observations (according to Gamaleia the intestinal canal of healthy pigeons contains fowl cholera bacteria, but Joest did not succeed in demonstrating them in the intestinal contents of ehickens. Ostertag \& Ackermann likewise failed to find them in chickens and geese).

A fluctuation of virulence in the cholera virus is observed under natural conditions as some outbreaks are limited to certain species of fowls, while other species in spite of exposure to the infection fail to contract the disease (for instance, chickens and water fowl are exclusively affected). These observations show a still closer accommodation of the virus to the organism of a certain species, as a result of which such accommodated bacteria are strongly virulent for only a certain species of fowl, whereas for another species they are less virulent or non-pathogenic. However, such differences in the virulence are rare and are not constant, and therefore the infections of various species of fowls should not be considered on this basis alone as independent affections.

Susceptibility. Purebred and water fowl are particularly susceptible to natural infection, whereas the common breeds of fowl show greater resistance. Fowl which have withstood an infection by ingestion a short time before are more susceptible to a second infection.

Pathogenesis. The bipolar bacilli penetrate the intestines, probably even through the uninjured mucous membrane, into the lymph spaces of the intestinal wall, and soon gain entrance to the blood stream where they multiply with great rapidity and cause the death of the animals. If the entrance of the bacilli takes place through a separation of the continuity of 
the skin or mucous membrane directly into the blood stream, death results in a shorter time (Tiede found bacilli in the internal organs of a mouse 15 minutes after subcutaneous inoculation). Death is caused in the first place by the action of the toxins which witlout a doubt form in the animal body in such a manner that through the negative chemotaxis of the virulent bacteria the phagocytes are unable to exert their action (Gabritschewsky, Silberbeg \& Zeliony). The chemotaxis of the attenuated bacteria is on the other hand positive, and this explains the local suppurative inflammation which usually terminates in recovery after a subcutaneous injection of such a virus.

Injections of attenuated virus into the chest muscles of chickens produce a serous inflammation of the connective tissue and degeneration of the musculature (see page 95).

The bacteria penetrate from the blood circulation into eggs, and they have been found in the yolks of eggs of affected animals (Celli \& Marchiafava, Barthelémy). The first two authors have also demonstrated the transmission of the disease to the progeny; on the other hand, Kitt found that young rabbits (4-6 weeks old) descending from artificially immunized animals resist a virulent infection.

Anatomical changes. The principal anatomical findings in acute cholera consist in an acute hemorrhagic inflammation of the intestines and lungs in association with small hemorrhages on the serous membranes and sometimes a fibrinous exudate on the same. The blood vessels of some of the loops of the intestines are injected and their peritoneal covering also contains small punctiform hemorrhages. The intestinal contents are fluid, mixed with mucus and fine bloody streaks, or of a uniform reddish color from the admixture of a considerable quantity of blood. The intestinal mucous membrane appears reddened sometimes only in spots and again in longer portions, particularly in the duodenum. It shows an inflammatory swelling and is studded with dark red hemorrhages; exceptionally small round diphtheritic ulcers are also found on the intestinal mucous membrane (frequently in water fowl).

The serous content of the pericardium is frequently cloudy and contains small fibrinous flakes; the epicardium appears as if sprinkled with blood and is frequently covered with fine fibrinous membranes, particularly in water fowl.

The lungs are congested and edematous, sometimes dark brownish red in spots and hepatized as an indication of a croupous hemorrhagic inflammation which is sometimes associated with a fibrinous pleuritis. The mucous membrane of the upper air passages shows catarrhal reddening and swelling.

In the parenchymatous organs the first stages of parenchymatous degeneration may be observed. The regional lymph glands of the spleen and cecum are almost invariably decidedly swollen and hemorrhagic. Exceptionally a fibrinous inflamma- 
tion (Kitt, Ratz) is present in all the air cells and on all serous membranes.

In the less frequent chronic cases yellowish, grey, dry caseous foci are frequently present in lungs, liver and on the intestinal nucous membrane, sometimes also under the endocardium, which may become quite extensive, especially in the lungs. Exceptionally even both lohes of the lungs may show hepatization resembling caseous pnemmonia (Jungklaus). At the same time there may be a fibrinous pleurisy and pericarditis, and in some of the joints and tendon sheaths a caseous exudate may be present (Sticker, Hensel, Willach).

In the acute form bacteria may be found in great numbers in the blood, between the blood corpuscles and only sparingly in the white blood corpuseles as well as in the exudates. The intestinal contents are also virulent. In chronic eases the caseous foci also contain virulent bipolar bacilli, while they are present in only small numbers iu the blood.

Symptoms. Ostertag \& Ackermann's experiments showed the time of incubation after a single feeding with organs of fowl affected with cholera to be 1 to 2 days in geese, and 4 to 9 days in chickens. After the recovery from a feeding infection a new infection produced death in geese (feeding) as early as 19 honrs and in chickens (subcutaneous infection) as early as 8 homrs. The time of incubation is very likely the same in natural infection.

The acute form of the disease sometimes runs a very rapid course; the animals drop to the ground while rumning, or they fall to the ground from an elevated place, such as the rod of a cage, or from a tree, and die after flapping their wings a few times.

In the large majority of cases, however, conspicuous symptoms precede death. The birds suddenly become depressed, they assume a crouching position, seek sechded places, and sit trembling. Sometimes the head is hidden under the wing or it may be turned backwards. The plumage is ruffled, the wings may droop, and if they move a staggering and wabbling gait is manifest with the wings hanging and the head drooped. The appetite is impaired from the onset, while thirst, on the other hand, is increased. A foamy slime drips from the nasal and oral openings; and from time to time the birds turn their necks convulsively, or stretch out their heads on which the bills, combs and wattles show a bluish-red discoloration. At the same time they may vomit a greyish-yellow sliny mass. At the height of the disease a profuse diarrhea develops; the droppings are at first yellowish-grey, mushy in appearance, later very fluid, dirty, greenish or reddish, mixed with coagulated albumin-like flakes, and of a repulsive odor. The plumage around the rectum becomes soiled and matted with the feces.

The affected birds show difficult respiration which is mani- 
fested by opened mouth, inspiration as well as expiration being associated with moist rales. As the weakness progresses they lie with closed eyes and in a stupor in one place. If they attempt to rise they soon collapse and finally die with manifestations of convulsions or coma.

In the chronic form of the disease progressive anemia develops after the disappearance of the acute symptoms, or from the onset of the disease, attended by emaciation; finally a persistent diarrhea occurs. Sometimes one or more joints of the wings or feet swell; later the swelling bursts, whereupon a caseous purulent mass containing bacilli is discharged from the joints. The arthritis interferes to a great extent with the movements of the already weakened birds.

The duration of the disease is from 1 to 3 days in the majority of acute cases with the exception of the peracute cases. It may, however, exceptionally extend from 7 to 12 days. In these cases the symptoms are less severe from the onset, and the exhaustion appear's later. The course of the chronic form extends over several weeks.

The prognosis is very unfavorable. Frequently entire flocks of chickens succumb to the infection, while in other cases a certain percentage of the birds escape probably owing to a slighter intensity of the infection. However, in exceptional cases recoveries may take place.

Diagnosis. Chicken cholera in its course and symptoms greatly resembles chicken pest. The latter comes into consideration particularly when it affects chickens exclusively. From this as well as from other septicemic affections of fowls, and also from acute poisonings which are frequently suspected by the owners, the disease can be differentiated with certainty by an autopsy and bacteriological examination (see also chicken pest.) Intestinal worms (for instance, the trichosoma collare) may also produce cholera-like symptoms.

Chronic cases, particularly those which are associated with arthritis, may be mistaken for tuberculosis and gout. A differentiation is here also only possible by microscopical examination of the exudates from the joints, as well as by autopsies.

On autopsy the presence of intestinal inflammation, the fibrinous exudate contained in the serous cavities, and the hemorrhages are strongly suggestive of fowl cholera, but to establish a definite diagnosis even in such cases a bacteriological examination of the blood and the inflammatory products is necessary.

The microscopical examination consists in the preparation of cover-glass smears in which usually large masses of bipolar rods can be easily recognized by the aid of the previously mentioned staining nethods (see page 79). Pigeons are best suited for test inoculations, especially since they are not susceptible to chicken pest. For this purpose the point of a knife is dipped into blood or exudate and then used for scarifying the skin, or a small quantity of the material is injected, subcutaneously or it may be introduced into the breast museles. The inoculation usually results in a short time in the death of the test animals and when the in- 
fection is proluced by an injection into the breast museles, a yellow gelatinous infiltration of the subcutaneous tissue develops at the point of inoculation around which the surrounding museles show a yellow discoloration and necrosis. Mice may also be used for diagnostic purposes. In these the bipolar bacilli may be demonstrated in large numbers in the blood.

Treatment. At the onset of the disease the treatment of the affected as well as the exposed fowls with highly potent immune serum is indicated (see page 95 ); otherwise, the affected birds are given the customary weak solutions of disinfectants, which may be added to the drinking water. These solutions, viz., iron sulphate, salicylate acid, tannin ( $1 / 2$ to $1 \%$ ), sulphuric or hydrochloric acid (2\%), may also be given in tablespoons or teaspoons (Ward and Stange give a 1 to 1500 or 2000 corrosive sublimate solution as drinking water). The same methods may be also applied as preventive measures against the disease in healthy birds which are threatened by infection. The subcutaneous injection of a $5 \%$ carbolic acid solution (Nocard, Barberio) is followed by little or no beneficial results.

Prevention. Great care should be exercised in order that the introduction of the disease by the purchase of new birds may be prevented. In addition to careful examination of the source, it is advisable to keep new birds separated for at least 1 week, and only after that period should they be allowed to mingle with the birds of the flock.

If the disease has already appeared in a flock, entirely healthy birds should be taken to an absolutely clean place without delay. Those already affected or suspected should immediately be slaughtered or taken to a separate place for treatment. The carcasses should be buried deep, or, still better, burned. After burning or burying the droppings, the walls and roosts of the chicken houses as well as the drinking vessels and containers used for feeding, should be scalded with hot water or washed with hot lye. They should also be sprinkled with lime, and the previously cleaned floors should be sprayed with a $3 \%$ creolin or lysol solution. Airing of the chicken houses for several days promotes good results from this method of prevention. The droppings from the affected as well as the healthy birds should be collected at least once daily and burned.

Immunization. Formerly attenuated cultures were used for this purpose, while recently the immunization with blood serum is more extensively employed.

I. Immunization with attenuated cultures (Pasteur's method). According to the investigations of Pasteur (1880) the bacteria of chicken cholera in bouillon cultures are gradually attenuated under the influence of the atmospheric air until after about two months standing they lose their virulence entirely. If allowed to stand for a shorter time the bacteria are attenuated 
to a certain degree, which virulence they retain if their cultivation is continued. Cultures attenuated to two different degrees produce two vaccines with which chickens may be immunized against virulent infections.

This method of immunization is of great scientific importance but has not proven successful in practice. Irrespective of the inconvenience of this method, in consideration of the relative small value of fowls, the inoculations in their practical applications sometimes cause considerable losses. Furthermore, the desired protection is not afforded which is very probably due to the uncertainty of the applied attenuation.

Pasteur's vaccine produces local necrosis of the muscles and serous inflammation of the connective tissue when injected under the skin of the breast of chickens. The corresponding part of the breast muscle separates from the living tissue towards the end of the second week, whereupon the sequestrum is reabsorbed in 3 to 4 weeks, or is cast off through a fistula which may have formed. Kitt found by repeating Pasteur's experiments with the two original vaceines that both produced such local affection in chickens and ducks, and in the majority of these birds produced an effective immunization. On the other hand, pigeons, rabbits and small birds were killed by the inoculation.

Attempts to prepare suitable vaccines from cultures through attenuation at higher temperatures (Salmon, Jess) and through the addition of chemicals (Jess) were unsuccessful.

Lignières recommends his polyvalent vaceine (see page 84 ) against chicken cholera, and in this disease the best results are supposed to have been obtained. The dose consists of $1 / 8$ ce. (subcutaneously).

II. Immunization with immune serum. Kitt (1892) established by experiments that the blood of chickens immunized against cholera has an immunizing effect upon healthy chickens, and further that the white and yolk of eggs of immune chickens ( 5 to 10 cc.) possesses a similar effect. Later Kitt \& Mayr (1897) prepared a serum by means of intravenous injections of virulent cultures in horses of which 2 to $20 \mathrm{cc}$. produced passive immunity in rabbits and $1 / 4$ to $1 / 2 \mathrm{cc}$. in mice, nevertheless it was ineffective in chickens. Still later Kitt (1902-3) produced an active immunity in rabbits by injecting them subcutaneously first with immune serum from chickens or horses (1 to $10 \mathrm{cc}$.) and following this with the injection of virulent cultures. Rabbits highly immunized in this manner produce a serum of which $1 / 2$ to 3 cc. protect rabbits and mice against a virulent infection, and in some eases chickens and pigeons were also immunized with it. Finally Kitt obtained a serum from horses by subcutaneous injection of virulent cultures, of which 2 to 5 cc. conveyed effective protection to rabbits, geese, ducks, chickens and sometimes also to pigeons against a fatal cutaneous or subcutaneous infection. The immunity develops not later than 24 hours, and is also effective against intestinal infection.

The serum inoculations naturally produce a passive immunity lasting only from 1 to 2 weeks, and therefore their 
application is indicated only in flocks already infected or in those which are threatened by infection. (Experiments to cliange inmmuity through subsequent inoculation of virulent material into an active form have thus far been unsuccessful.)

Through a procedure similar to that of Kitt \& Mayr, Schreiber succeeded in preparing a blood serum of which .5 ec. protected a pigeon against a virus which was fatal in 24 hours. The dose of this serum is for geese or ducks 1 ec., for smaller birds .5 ec. Hartel obtained a serum by treating an ass with living bonillon cultures (intravenous) of which .5 ce. protected a pigeon against a dose of virus of which $1-10,000$ part was fatal.

Jess \& Piorkowski inject fresh horse serum containing alexin under the skin of fowl before the use of the immune serum in order that the antibodies contained in the horse serum may find a sufficient quantity of alexins in the tissue fluids. The single dose is .5 to 4 ce. of immune serum, depending on the size of the bird, and 1 to $8 \mathrm{ce}$. of normal serum. The mixture is supposed to effect also a curative action when applied not later than 6 hours after the infection has taken place. Mosler found the mixture also more effective in mice than the immune serum alone. The experiments made in Prussia are, however, contradictory (Raebiger).

Klett \& Braun immunize horses with 4-day old bouillon cultures of the fowl cholera bacteria which have attained the highest degree of virulence by passage through the bodies of sparrows. The treatment commences with a dose of .25 ce. of culture subcutaneously and intravenously, which quantity of culture is gradually increased to 20 ec. and is later injected directly into the circulation. Horses thus prepared produce an immune serum of which 0.0015 to 0.005 ec. will protect gray mice for a period of about 3 weeks against the fatal action of a loopful of a 4-day old bouillon culture. The results obtained in practice met the expectations based on the laboratory experiments, and the sera sometimes appear to have a curative action in animals already affected.

In consideration of the close etiological relation of swine plague and chicken cholera Schreiber employed for the inoculation of fowl a serum (septizidin) which affords protection against the former disease, and some other authors (Hartenstein, Graffunder, Schmidt and others) also obtained with it satisfactory results. The official reports, however, of Prussia and Saxony are mostly unfavorable. Finally, Lignières' polyvalent serum may also come into consideration.

The "Höchst Farbwerke" prepare a polyvalent serum (Galloserin) with which Kováes, Sżöke \& Szzabó and others obtained quite satisfactory results in exposed as well as already affected birils. Raebiger made comparative experiments with the various vaccines on the market and found that pigeons inoculated with Höehst serum or with septizidin withstood the infection in $66 \%$, while those injected with Gans' (Frankfurter) and Piorkowski's (Berlin) sera died. The serum of KlettBraun prover effective in laboratory experiments and also once in practice. Delfino found Lignières' vaccine effective in rabbit experiments.

Technique of vaccination. Pasteur originally inoculated into the point of the wing, later the inoculation was made under the skin of the breast or into the breast muscle. According to the suggestion of Jess it is more satisfactory when made uniler the skin of the neck. An assistant secures the bird with both hanis on the rump while the olverating veterinarian raises a fold of the skin of the neck with his left hand. The dose to be inoculater is indicated specially for each preparation.

III. Immunization with Aggressins. Weil employs, according to the procedure of Bail, a pleural exudate produced by injections of 
fowl cholera bacteria into rabbits. After the addition of $.5 \%$ earbolic acid this is heated for 3 hours at $44^{\circ} \mathrm{C}$. Repeated injections of small doses (.5 to 3 cc.) or a single injection of 5 cc. protected guinea pigs and rabbits as well as a hen and a pigeon against a subsequent lethal infection. Rabbits highly immunized with sterile exudate produce serum of which $.5 \mathrm{cc}$. protected rabbits against a dose of the culture which is fatal in 20 hours. According to the aggressin theory (see page 30) the body is supposed to form an anti-aggressin as a result of the injection of aggressins which destroys the aggressiveness of the bacteria and their unlimited power of multiplication. Huntemüller, however, found that similar good results may be obtained in rabbits with washings of cholera cultures heated to $44^{\circ} \mathrm{C}$. He also learned that the filtrate of exudates was ineffective, and he therefore concurs in his belief with Wassermann and Citron, that in immunization the toxic body substances of the bacteria are the effective agents. Bisanti's experiments suggest also the possibility of a toxin immunization. This investigator sewed collodium sacs filled with virulent cultures into the abdominal cavity of rabbits and thereby protected them against a cerebral infection.

Veterinary Police. Chicken cholera has been recently in. cluded among the diseases requiring compulsory official notification in Germany, Austria and Hungary. Upon the appearance of the disease the infected premises or in case of a greater extension, localities and even entire townships are quarantined against traffic in fowls. The inland and foreign traffic in fowls as well as the premises of dealers are subject to official supervision.

Literature. Renault, Rec., 1851, 321.-Hering, Spez. Path., 1858, 355 (Lit.).Perroncito, A. f. Tk., 1879, V, 22.-Toussaint, Ree., 1879, 946.-Pasteur, C. R., 1880, XC. u. XCI.-Zürn, D. Geflügelkrankh., Weimer 1882.-Kitt. D. Z. f. Tm., 1888, XIII, 1; Hb. d. p. M., 1903, II, 543 u. 1904, IV, 969 (Lit. on etiology and immunization).-Lignières, Bull., 1900, 332, u. 1902, 444.-Klett \& Braun, D. t. W., 1904, 545.-Weil, A. f. Hyg., 1905, LII, 412; 1908, LVX, 85.-Ostertag \& Ackermann, Z. f. Infkr., 1906, I, 431.-Jungklaus, Diss. Leipzig 1906.

Other Diseases of Fowl of a Septicemic Character. Various observers have described in the course of the last decade different epizootic affection in fowl in addition to chicken pest which simulate chicken cholera more or less. In some of these their identity wit's chicken cholera was established. Others, however, were shown to be different, while the nature of some of the diseases is doubtful even at the present time. On account of their relatively slight importance and their prevention by other means than immunization for which it is essential to establish the causative agent, the same principles mentioned for chicken cholera are also applicable. Therefore these diseases will only be briefly mentioned here, emphasis being placed on their most important differences from fowl cholera.

The following diseases are identical with chicken cholera:

Lisi's chicken septicemia (Clin. vet., 1895, 176), and the hemorrhagic septicemia of ducks and chickens described by Rabieaux (Soc., biol., 1900, 141). 
Diseases simulating chicken cholera :

Infectious enteritis of chickens; observed repeatedly in England and Ireland by Klein. The causative agent (Bac. gallinarum) greatly resembles the bacillus of fowl cholera. However, it does not kill pigeons and only exeptionally rabbits. (Cbl. f. Bakt., 1889, V, 689; 1895, XVIII, 105.)

Enzootic pneumo-pericarditis of turkeys; observed by M'Fadyean and later lyy Jowett. 'The bacillus also resembles that of chicken cholera. However, it is motile and non-pathogenic for chickens as well as for water fowl. (J. of Comp. Path., 1893, VI, 334; Ibid., 1908, XXI, 324.)

Cholera of water fowl; observed in Germany by Willach. The bacillus is distinguished from that of fowl cholera by its motility, is only slightly pathogenic for hens, and non-pathogenic for pigeons. (Chl. f. Bakt., 1896, XX, 187.)

Disease of swans; observed in the Zoological Garden of Antwerp by Trétrop. The bacterium develops only slightly on potato, and eauses in swans a caseation of the lymph glands; chickens are not susceptible. (A. P., 1900, XIV, 224.)

Diseases apparently different from fowl cholera:

Cholera of ducks; observed in the Zoological Garden of Paris by Cornil \& Toupet. The bacillus stains well by Gram's method and grows well on potato; chickens, pigeons and guinea pigs are not susceptible, rabbits to only a slight extent. (C. R., 1888, CVIII, 1747.)

Epizcotic dysentery of chickens and turkeys; described by Lucet. The course of the disease is from 9 to 13 days. The bacillus thrives well on potato and kills guinea pigs by intravenous inoculation. (A. P., 1891, V, 312.)

Disease of ring-dove; observed in France by Leclainche, and is characterized by its rapid course, somnolence and diarrhea. The bacillus (Bac. choleræ columbarum) thrives on potato; chickeus are not at all susceptible, while other fowl show only a slight susceptibility. (A. P., 1894, VIII, 490.)

Disease of pigeons; observed in New Jersey by Moore. Cerebral symptoms predominate in this disease. On autopsy meningitis is found to be present, while intestinal symptoms are absent. The bacillus resembles Bac. suipestifer; it grows on potato. (Bur. Anim. Indus., Bull. No. $8,1895,71$.)

Infectious enteritis of pheasants; observed in the Zoological Garden of Nilan by Fiorentini. The affected animals are not sommolent. Autopsy does not reveal small hemorrhages. The bacillus is not motile; it forms on potato a honey-like growth and produces indol in peptone bouillon. (Chickens, guinea pigs and rabbits are not susceptible. (Soc. ital. di. se. nat., 1896, 89.)

Septicemia of ducks; observed in Italy by Lisi. The affected birds show purulent conjunctivitis and keratitis. The bacillus liquefies gelatin gradually and forms a brownish growth on potato; chickens are not susceptible to artificial infection. (Il Med. zooiatrio, 1890, 415.) 
Hemorrhagic septicemia of swans; observed in the Zoological Garden of Milan by Fiorentini in pheasants and Egyptian geese. The bacillus is motile, bouillon is made cloudy and of a yellowish discoloration, grows on potato in the form of chocolate-brown colonies; the cultures liberate a fetid odor. (Cbl. f. Bakt., 1896, XIX, 932.)

Exudative septicemia of geese; diagnosed first in Mecklenburg by Riemer, later in Stralsund by Froseh \& Birnbaum (the disease described by Bugge as infectious inflammation of the air-sacs of geese is probably identical). (Characteristic lesions; pronounced sero-fibrinous pericarditis and fibrinous peri-hepatitis. The slender rod-shaped bacillus (Bac. septicæmiæ anserum exsudativæ) which resembles Pfeiffer ${ }^{\circ}$ s influenza bacillus forms threads in cultures, is not motile, does not form spores, decolorizes by Gram, and is strongly pathogenic for geese and less for ducks. (Lit. Cbl. f. Bakt., 1909, LII, 433.)

Epizootic pneumo-enteritis of turkeys; described by Dodd, is charaeterized by swelling of the sub-orbital region, emaciation and diarrhea or hepatization and small necrotic nodules in the lungs, as well as fibrinous exudates in the pleural cavity. The causative agent resembles the ovoid cholera bacillus. (J. of Comp. Path., 1905, XVIII, 239.)

Infectious leukemia of chickens; studied in the United States by Moore; its course extends from 1 to 3 weeks with symptoms of a severe leukemia. The causative agent (Bac. sanguinarium) ; forms yellowishwhite colonies on potato. (Bur. Anim. Indus. Rpt. 1895-96, 185).

Chicken plague; observed by Mazza in northern Italy. In the course of the disease caseous foci develop in the lungs. The bacillus is motile, develops gases in bouillon containing sugar, grows on potato in the form of colorless, lustrous deposits; rabbits are slightly suseeptible. (Cbl. f. Bakt., 1899, XXVI, 181.)

Disease of canary birds; described by Rieck. A soot-like discoloration of the skin develops, while the liver contains punctiform necrotic foci. The baeillus is motile and grows on potato in the form of yellowish-gray colonies. (D. Z. f. Tm., 1889, XV, 69.)

Cholera of canary birds; studied in Budapest by F. Kern. The baeillus does not take bipolar staining, is not motile, develops fetid gases in agar containing sugar, and forms yellowish colonies on potato, the medium showing a bluish discoloration. Chickens and pigeous are not susceptible. (D. Z. f. Tm., 1896, XXII, 171; according to Nocard Zürn's canary plague is supposed to be identical with this disease.)

Infectious malady of canaries; described by Pfaff as a diffegtni disease from the above. The bacillus is somewhat larger that fithtint Kern, forms fine flakes in bouillon, does not grow on potato is hampless for chickens, and pathogenic for pigeons. Constant lesions; small ne-

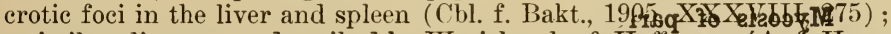

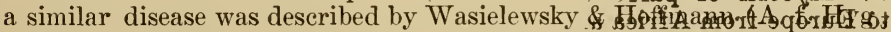

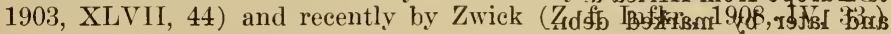

Septicemia of canary birds; descrinsgro fonr9tni 9 dj ni qol9v9b Septicemia of canary birds; descrined

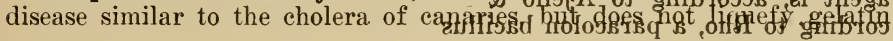


and is Gram positive; milk is coagulated and it does not ferment dextrose. There is no neerosis of the liver or spleen. (D. t. W., 1907, 501.)

Infectious necrosis of canary birds; deseribed by Miessner \& Schern as a very infectious disease which is characterized principally by necrotic foci in the spleen and liver, and is also frequently associated with diphtheria of the buceal mucous membrane. The causative agent is a short stubby, Grani negative, non-motile organism (Bac. eanariensis necrophorus). (A. f. Tk., 1908, XXXIV, 133.)

Typhoid of canary birds. As Joest had done before him, Zsupán mentions under this name a disease of canary birds caused by bacteria of the enteritis group. It runs a rapid course with symptoms of marked debility, difficult respiration and diarrhea. The constant lesions are acute gastro-intestinal catarrh, and besides, from case to case acute spleen-tumor, fibrinous inflammation of the serous membranes, cloudy swelling of the liver and kidneys. The causative agent (Bac. typhi canariensis) is very similar to Eberth's typhoid bacillus, and is pathogenic in general for small birds, as well as for rabbits, guinea pigs, rats and mice. (Dresdn. Ber. pro 1906; Közl., 1909, VIII, 149.)

Disease of guinea keets (Mykosis intestinalis phasianidum); observed in Germany by Enders; affects exclusively species of chickens. The causative agent resembles that of fowl cholera, is pathogenic for pigeons and water fowl, and grows well on potato. Autopsy shows hemorrhagic enteritis and osteomyelitis. Maggiora \& Valenti consider the disease identical with chicken pest, and the described bacillus is supposed to be a frequent inhabitant of the healthy intestines of fowl (D. t. W., 1902, 339).

Septicemia of chickens caused by coli bacteria; according to Claussen it is supposed to develop spontaneously in chickens which are exposed in long railroad transportations to thirst, hunger and cold as a result of which the normal coli organism becomes virulent. The symptoms and post mortem lesions resemble those of fowl cholera, but the blood contains bacteria of the coli bacillus type (Z. f. Infkr., 1907. III, 69).

White diarrhea of chicks; according to Rettger this disease affects chicks in the first three weeks of life, and is caused by a bacterium of the eoli-typhus group which is present in the blood, internal organs and also in the yolk (J. of Med. Research, 1909, XXI, 115).

Salmonellosis of chickens; described by Lignières \& Zabala as an infectious and mostly fatal disease which in its course resembles fowl cholera (spleen always swollen). The bacillus resembles the Bac. suipestifer, but is non-motile. Besides chickens, pigeons and rabbits are also susceptible (Bull., 1905, XXXII, 453).

Mycosis of parrots (Psittacosis); a disease of parrots imported to Europe from Africa and America, characterized by dullness, diarrhea and later by marked debility. In its course small grayish-white foci develop in the internal organs, especially in the liver. The causative agent is, according to Ajello \& Parascandolo, a specific bacillus; according to Rho, a paracolon bacillus which produces toxins in cultures. 
With this disease the cause of some forms of pneumonia in man is associated, but according to the investigations of Lichtenstein this supposition is not well founded (O. M., 1902, 385; Cbl. f. Bakt., 1899, $\mathrm{XXV}, 651)$.

Grouse disease; described by Klein as a very fatal disease of Scottish grouse. The principal symptoms are quick tiring on flying, hoarseness, cyanosis of the conjunctiva, emaciation. On autopsy necrotic foci in the liver and small blood extravasations are observed. The bacillus is motile, Gram negative, thrives on potato, quickly coagulates milk, and produces indol (Cbl. f. Bakt., 1899, VI, 36; 1890, VII, 81).

Cholera-like gastro-enteritis of fowl (Vibrionen-cholera, gastroenteritis cholerica avium). Gamaleia, who observed the disease in Odessa, described as the cause a short, curved bacillus (Vibrio Metschnikovi), which morphologically and culturally closely resembles the comma bacillus of Asiatic cholera. Affected fowl die with symptoms of somnolence and diarrhea within 2-3 days. The bacilli are present in the lumen of the inflamed intestine, and produce the symptoms by the eliminated toxins (A. P., 1888, II, 482).

Apoplectic septicemia of chickens; this very disastrous American disease runs its course in from 12 to 24 hours with paralytic symptoms and is caused, according to Nörgaard \& Mohler, by a very virulent streptococeus which in addition is pathogenic for chickens and also for rabbits, mice, ducks, pigeons and dogs. It is harmless for guinea pigs and sheep (Bur. Anim. Industry, Bull., 1902, No. 36).

Sleeping sickness of chickens; was found by Dammann \& Manegold in the vicinity of Hannover, later by Greve in Oldenburg. The most striking symptom is the more or less pronounced somnolence. Further, reddening and swelling of the conjunctiva, if the course is prolonged, also paleness of the comb and wattles, diarrhea and emaciation. In the heart blood the Gram-positive encapsulated streptococci are found in great numbers (Str, capsulatus gallinarum) thriving best on coagulated blood serum and in milk. It is also pathogenic for pigeons, rabbits, mice and lambs (D. t. W., 1905, 577; 1908, 213).

Septicemic and Pyemic Affections of Rabbits. Among rabbits mostly in laboratories, but not infrequently also in valuable rabbit breeding establishments septicemic affections occur, some of which will be described briefly.

Rabbit Septicemia. Davaine, later Koch \& Gaffky produced this disease in rabbits by subcutaneous injections of putrid fluids, unclean river water and putrid pickling fluid. After such injections the animals die in from 16 to 20 hours with rapid falling of the body temperature, diarrhea and convulsions. On autopsy the spleen and lymph glands are swollen, the lungs hyperemic, the intestinal mucous membrane inflamed, serous membranes sprinkled with petechiae. The blood contains small bacteria (Bac. cuniculisepticus s. cuniculicida), which in their form and cultural characteristies are practically identical with the fowl cholera bacterium and are very probably identical organisms (Kitt). Cultures kill fowls with manifestations of septicemia. On the other hand, rabbits infected with the virus of fowl cholera also 
die within a short time. Therefore there is a possibility that the appearance of fowl cholera may be associated with the cause of rabbit septicemia and vice versa. Selter observed the disease in a spontaneous enzo-otic form, and found swine plague serum effective in the treatment. (Koch \& Gaffky, Mitt. d. G.-A., 1881, I.-Lignières, Bull., 1900, 356 [Lit.].-Selter, Cbl. f. Bakt., 1907, XLI, 432.)

Pleuro-pneumonia of Rabbits. Tarious authors deseribe the disease under different names, thus Beck, and recently Kurita as "pleuropneumonia," Kraus as "influenza-like rabbit plague," Volk as " rabbit plague," Südmersen as "infectious rabbit pneumonia," Eberth \& Mandry as "spontaneous rabbit septicemia," all these are, however, probably identical or elosely related. In all eases there was a fibrinous inflammation of the pleura, sometimes also of the pericardium, frequently associated with a catarrhal pneumonia. The symptoms consisted in a purulent nasal discharge, difficult respiration, emaciation and rapid course. As the causative agent a fine, Gram-negative, sporeless aerobic bacillus was described which resembles the influenza bacillus of man. They are distinguished from each other by unimportant characteristics. (Lit. see Z. f. Tm., 1906, X, 429; also Cbl. f. Bakt., 1906, XLIX, 508.)

Rabbit Distemper. Described by Lucet as "septic disease of rabbits."

It manifests itself in a phlegmonous swelling of the tracheal and laryngeal regions, nasal discharge, difficult respiration and emaciation. The autopsy shows, in addition to a purulent inflammation of the subcutis at the places mentioned and regional inflammatory edema, pronounced acute swelling of the spleen, enteritis and serous exudate in the thoracic and abdominal cavities. A small bacillus (Bac. septicus cuniculi) is indicated as the causative agent. (A. P., 1892, VI, 558.)

Pyemia of rabbits; observed by Koppányi in Belgian hares usually as a severe affection. In acute cases purulent fibrinous pleuritis and pericarditis are present, while in chronic cases subcutaneous cold abscesses develop which may reach the size of a man's fist. After rupturing, which sometimes only occurs after 6 to 8 months, recovery follows not infrequently. The disease is caused by a polymorphous capsulated bacillus (Pyobacillus capsulatus euniculi). (Z. f. Tm., 1906, $\mathrm{X}, 429$.)

Streptothrichosis of rabbits is characterized by a progressive necrosis of the skin which extends to the muscles and tendons. Thrombi develop in the blood vessels and as a result emboli occur in the internal organs, especially the lungs. The streptothrix euniculi which was demonstrated by Schmorl as the causative agent of the disease, is identical with Bang's bacillus necrophorus. Basset produced an effective immune serum by treating a horse with pure cultures. (D. Z. f. Tm., 1891, XVII, 375; Bull., 1908, 345.)

Myxomatous affection of rabbits; observed by Sanarelli in Montevideo and by Splendore in Sao Paolo. At the onset a purulent blepharoconjunctivitis develops and is rapidly followed by a large swelling of the head, also in the vicinity of the rectum and the opening of the urethra and genital organs. Post mortem examination reveals tumors in the subcutis which have a gelatinous appearance. Further, there 
is hypertrophy of the lymph glands, orchitis and spleen tumor. The disease is very easily transmitted artificially and appears to be eaused by a filterable virus. (Cbl. f. Bakt., 1898, XXIII, 865; 1908, XLVIII, 300.)

\section{(b) Hemorrhagic Septicemia of Cattle. Septicaemia hemor- rhagica bovum.}

\section{(Bollinger's Wild-und Rinderseuche, Hemorrhagische Septi- kämie der Rinder [German]; Pasteurellosis bovum, Pasteurellose ou s'ipticémie hémorrhagique du boeuf [French].)}

Hemorrhagic septicemia is mostly an acute, less frequently a subacute, infectious disease. In its course the febrile symptoms are usually accompanied by manifestations of an acute gastro-enteritis, inflammatory edema of the skin or interalveolar connective tissue, and is frequently associated with necrotic pneumonia. The causative agent is Bac. bovisepticus.

History. Bollinger described in 1878 a very fatal disease, which was previously unknown, oceurring in the vicinity of Munich among the deer and wild boars. Later it was observed in cattle, and in some instances among horses and domestic hogs. The infectious character of the disease has been estahlished by Bollinger and later by Franck (1881) as well as by Friedberger (1881). The causative agent, however, was discovered by Kitt (1885) and somewhat later studied hy Hueppe (1886). Recently especially Jensen (1889) and Lignières (1898 and 1900) investigated the etiology of the disease.

Occurrence. The disease occurs everywhere either sporadically or enzootically, especially during the summer. Sometimes it originally occurs in deer and wild logs, spreading later to domestic cattle, and from the latter hogs may often become affected.

In the beginning of the eighties of the last century the disease was repeatedly observed in northern Bavaria, but it has heen since found in other parts of fermany as well as in different European countries. The losses were sometimes consillerable. In the year 1878,153 deer and 234 wild hogs died in the Reservations in the vicinity of Munich; in 1887 in the District of Bindlach more than 100, and in one district of Prussia the annual loss was more than 100 cattle. In recent times it is principally observed in Prussia (fovernment districts Posen and Bromberg) as well as in Bavaria. In 1905, 57 cases were reported from Posen and 30 from Hannover.

In Austria the disease oceurs in Bohemia, Moravia and Silesia. In Moravia 155 cattle and 29 hogs were affected in 1900 , in 44 townships, of which number 15 eattle and 15 hogs died. One hundred and one cattle and 14 hogs were slaughtered in emergency (Taufer). In Hungary the disease was first observer in 1900 in the County of Gömör, in one township where 17 eattle were affected. During the period from 1901 to 1902,12 yearlings and 2 cows died in Hanság; in 1204, 14 out of a herd of 49 yearlings in the vicinity of Kapuvár recame affected. From time to time sporadic cases occur in fattening establishments.

In France the disease was observed by Leclainche and in Switzerland by Guilleleau and Hess.

The disease also exists in North America (the broncho-pneumonia of cattle described by Nocard is a form of this disease), in Indo-China, in the Malayean Peninsula, in the Philippines and in Java. 
There is sometimes observed a septic pleuro-pneumonia in calves which occurs simultaneously with hemorrhagic septicemia in adult cattle. This disease is either closely related to or identical with hemorrhagic septicemia of cattle (See page 111).

Etiology. The cause of the disease is the bacillus (bipolaris) bovisepticus which is a variety of the bacillus of hemorrhagic septicemia. In its principal characteristics it conforms to the organisms of this group (see page 79 ).

Hueppe succeeled in growing it in spring water containing a large amount of organic substances, and also in garden soil. It grows best at body temperature, while at a temperature from $12-13^{\circ} \mathrm{C}$. it no longer propagates. A 1:5000 sublimate solution kills it in 1 minute, $3 \%$ carbolic acid in 6 hours, moist heat of $80^{\circ} \mathrm{C}$. in 10 minutes (Hueppe). The gastric juice appears also to have a bactericidal action (Taufer). Dried virulent blood loses its infectiveness in 14 days (Kitt).

The virulence of the contagion varies in the different enzootics within wide limits. Deer and wild hogs are very susceptible, cattle and domestic hogs are somewhat less predisposed. Under natural conditions the disease affects only animals of these species, but sometimes solipeds and also goats may become affected. Subcutaneous inoculations of virulent bouillon cultures produce a severe inflammatory edema which is followed by suppuration and finally by recovery. Calves, however, have died within several days after such an injection. Intravenous injection acts more energetically. By this method calves may be killed with $10 \mathrm{cc}$, and cattle with $100 \mathrm{cc}$. of the culture in 1 to 3 days, with manifestations of acute septicemia. After a less severe infection the animals gradually emaciate in spite of a good appetite; at the same time an inflammation develops in the knee and hip joints, as well as a broncho-pneumonia, whereupon the animals die from extreme exhaustion. Autopsy reveals in such cases as a rule only foreign bacteria in the inflammatory products (Lignières). Among other animals rabbits are susceptible to artificial infection; horse, guinea pig, dog and pigeon are less susceptible, while domestic fowls are not susceptible to this disease (Bollinger, Kitt, Taufer).

The natural infection results in most cases through the digestive tract; exceptionally the virus may enter the circulation through injuries in the buccal mucons membrane and the skin. The latter method of infection is possibly transmitted through flies and mosquitoes (Franck, Dammann \& Oppermann recently connected an outbreak of the disease in the Province of Hannover [1905] with swarms of insects [Simulia ornans] as they succeeded in finding in their bodies bipolar bacteria, although these were only slightly virulent for rabhits). Bollinger also believes a pulmonary infection possible, but this mode of infection has been proven so far only in rabbits. The virus may also be disseminated into remote localities through the meat and hides of affected animals.

The sporadic cases as well as the first attacks of an enzo- 
otic outbreak occur as a rule throngh infections of the soils the causative agent in some localities acquiring a strong virulence through unknown influences, or they may be due to a diminished resistance of the animals. As local enzooties frequently develop first among deer and black game the moist soil of the forests appears to be especially favorable for the propagation of the bacteria and for the change in their virulence mentioned above. The warm spring weather is particularly favorable for the development of the bacteria. During this period the disease occurs most frequently among cattle.

The virus which originates from the soil retains its increased virulence for a certain time in the body of the animals, and this may even be increased further. The blood is highly infectious in the latter stages of the disease, and during this time the blood as well as other body fluids and excrements may also infect other animals, and in this way the enzootic may continue to spread in a contagious form. (Bollinger observed an infection in a steer which died from pneumonia within 54 hours after the ingestion of 1 gram of feces from a calf affected with an acute form of the disease.) After a certain time these enzootics which do not as a rule extend over large territories, usually subside very probably as the result of an attenuation of the virus which takes place in the meantime (Carrougeau observed a gradual attenuation of the virus from repeated reinoculations of cattle).

The cornstalk disease or corn fodder disease which occurs among cattle in America, especially in the middle and upper Mississippi Valley, is also supposed to be caused by an ovoid bacterium, and to be identical with hemorrhagic septicemia of eattle. This disease usually occurs in the fall and early winter after the animals are turned into the cornstalk fields. It is characterized by a very rapid course. The autopsy reveals numerous hemorrhages in the internal organs, especially in the serous membranes. Recently, however, Moore showed that the disease was not of an infectious nature, but was due to toxic substanees (according to Mayo, potassium nitraté) which are sometimes present in large quantities in the stalks, and which are responsible for the intoxication. As a matter of fact the disease may be actually prevented by cutting the stalks early and only feeding them after they have been carefully cured and removed from the field.

Anatomical changes. The autopsy reveals a marked hyperemia of the internal organs as well as fresh hemorrhages in the serous and mucous membranes, and in some of the organs, especially the lungs and muscles. The spleen has either a normal appearance or contains circumscribed bloody areas; the liver and kidneys show clondy swelling. The other anatomical changes vary in the different cases.

In the edematous or exanthematous form the subcutaneous connective tissue of the head, the throat region and the neck, show a gelatinous infiltration, which may in some places be studded with hemorrhages. The serous infiltration may extend 
to the deeper layers of the muscles. The tongue appears more or less enlarged, dark, of a dirty brownish-red color, and of a very firm consistency. On incising this organ a large quantity of a light yellow serous fluid escapes, while in parts of the infiltrated tissue, especially under the mucous membrane, flat, diffused, fresh hemorrhages are present. The frenum of the tongue and the surrounding parts as well as the submucous

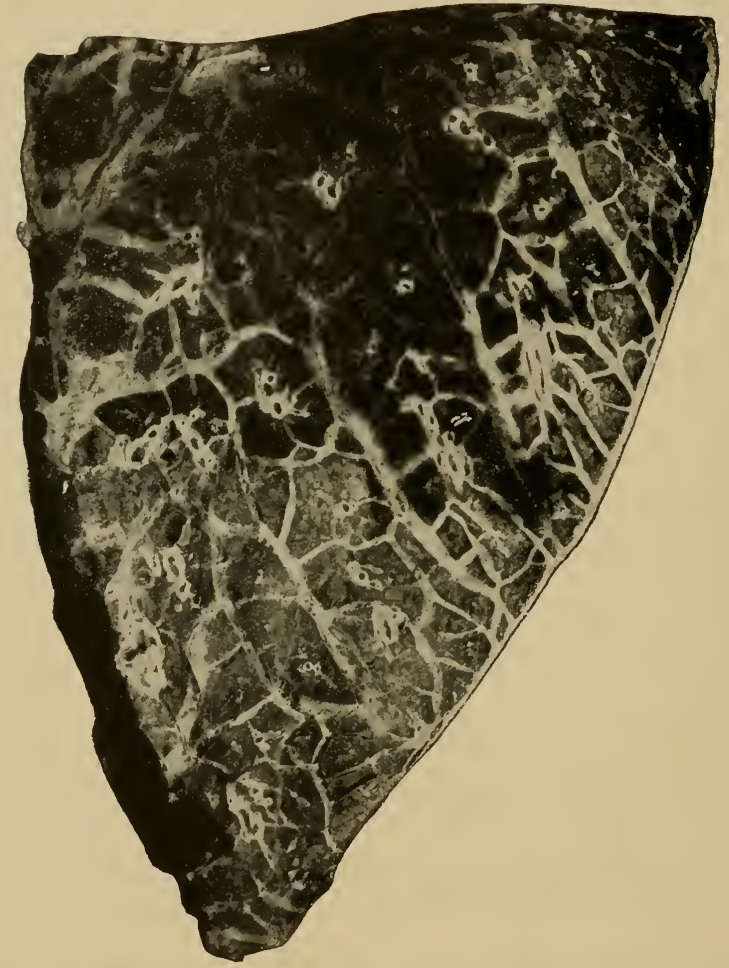

Fig. 27. Pneumonia in hemorrhagic septicemia of cattle. Above, brownish-red hepatization with hemorrhages; below, grayish-red hepatization with commeneing necrosis. Inter-lobular connective tissue edematously infiltrated, lymph spaces dilated in parts.

connective tissue of the pharynx and the larynx show gelatinous infiltration and appear as flabby masses. The mucons membrane of the upper air passages shows a catarrhal swelling and is sometimes covered with croupous membranes. The retropharyngeal lympl glands and those of the neck show pronounced acute swelling. 
The abdominal cavity sometimes contains several liters of a yellowish or reddish serous fluid. The small intestine manifests lesions of a pronounced acute hemorrhagic inflammation, which while also present in the large intestine are less marked there. The content of the intestinal canal is thin, fluid, yellowish-gray, or from an admixture of blood, dirtyreddish in color. The spleen is normal, or at most in parts, somewhat swollen. The blood is also normal in color.

In the pectoral form the thoracic cavity contains serous or sero-fibrinous exudate which is sometimes mixed with blood. The pleura is inflamed and studded with small hemorrhages, especially the visceral layer which appears to be covered with fibrinous membranes. In one or both lungs smaller or larger connecting parts are hepatized and friable. The cut surfaces are uniformly dark, brownish or redish in color, finely gran ular, and contain, in less acute cases, small, dry, caseons areas. Sometimes the interlobular connective tissue septa appear thickened and yellowish-white through serous infiltration, so that the cut surface of the lung has a mottled marbled appearance (the appearance in such cases is very similar to the lungs in contagions pleuro-pneumonia [Fig. 27]). The parts which are not hepatized show marked hyperemia and edema. The pericardium may also contain an exudate mixed with fibrinous flakes. The connective tissue of the mediastinum is gelatinously infiltrated and studded with hemorrhages. The peribronchial lymph glands are acutely swollen; usually there is also an acute enteritis present, while the spleen preserves a normal appearance even in severe cases.

Nocard, who diagnosed the disease in cattle imported from America to Paris, (broncho-pneumonia of American cattle) emphasizes the fact that the serous fluid in the lungs differs from that of contagious pleuropneumonia. It is not so markedly yellow, and has not the appearance of lymph, also the thickened lymph spaces are filled with a whitish, tenaceous, fibrinous coagulum. The individual lobules appear firmer in the center and more markedly hepatized than in the periphery, which is probably on account of the fact that the inflammatory process extends from the bronchi and not from the interlohular connective tissue.

According to the anatomical findings in the different cases, Bollinger distinguished a pectoral and an exanthematous form of the disease, the former oceurring principally in wild animals, while the latter is supposed to be more frequent in cattle. Later a so-called intestinal form was also described, which, however, does not oceur independently, but only in association with one of the previously mentioned forms (the designation "exanthematous form" is not appropriate as there is no exanthema present but only an inflammatory edema).

Symptoms. The time of incubation of the disease in inoculation and feeding experiments proved to be from 6 to 24 hours. In natural infections it is probably somewhat longer, but it hardly ever extends over 2 days. 
As the first symptom a rapid rise in the body temperature to over $40^{\circ} \mathrm{C}$. is observed.' This is accompanied by an accelerated pulse, dullness, rough coat and muscular trembling. At the same time the surface of the body feels alternately hot and cold, while the muzzle is cold and dry. There is also cessation of appetite, rumination and milk secretion. The peristalsis of the rumen and intestines is retarded; constipation at this stage may also be noticed. Later, symptoms of colic appear with much straining when the animals pass instead of the usual dry, dark brown feces, a mushy and finally a thin, fetid fluid which is not infrequently mixed with fibrin and mucous flakes as well as with blood. In the meantime blood frequently exudes from the nose and sometimes the urine may also contain blood. In addition to these manifestations one or the other form of the disease finally develops during the further course of the malady.

According to Carrougeau as well as Reynolds, Wilson and Brimhall, the temperature may be either normal or subnormal, and Mezey observed in a fallow deer a temperature of only $37^{\circ} \mathrm{C}$. with symptoms of a marked apathy, inappetence and bloody excrements.

Guerrieri observed the disease in Sardinia (Male della ferula) in a peracute form when the animals, while ruminating or feeding, suddenly ran away like mad, staggered, collapsed, and died with escape of blood from the nose.

In the edematous (exanthematous) form the head and neck swell, especially in the region of the throat and dewlap, as a result of the rapidly increasing inflammatory edema of the subcutaneous connective tissue, causing a deformity of these parts. There is also swelling of the legs and in rare cases the swelling may commence at one of the extremities and spread from there to the neck and body (Rátz). In such cases inflammatory enlargement of the different joints may be observed. Over the swollen parts of the body the skin is very tense, warm and sensitive. On the eyes an acute conjunctivitis develops, frequently with a yellowish coloration of this membrane. There is profuse lachrymation, the tears running down the face. The buccal mucous membrane is bright red, warm, dry and swollen. Deglutition is difficult or impossible, so that the saliva accumulates in the mouth, and dribbles from the corners of the mouth in long, tenacious strings. The tongue swells to such an extent that it entirely fills the buccal cavity, and a part of it may even protrude between the teeth. It is bluish-red, or dirty reddishbrown and shows on its lower surface impressions of the teeth, while at both sides of the frenum the mucous membrane of the floor of the mouth is bunched up in thick, flabby, gelatinous rolls.

Respiration is very difficult and frequently stertorous. All the mucous membranes are cyanotic, often studded with hemorrhages, and finally the animals die of asphyxiation, or the 
asthenia, which is caused by the marked enteritis, produces death.

Taufer observed the following additional symptoms during an outbreak of the disease: A very extensive infiltration of the masticatory and cheek muscles as well as of the back of the nose, the lips and the nasal mucous membrane (hippopotamus head), cloudiness of the cornea (in two cases also panophthalmia). In one case he observed necrosis of the cheeks and lips, and lastly in 24 cattle a skin eruption over the entire body. These skin lesions were developed most extensively above the carpal and tarsal joints, and resulted in circumscribed necrosis. Carrougeau has also observed skin eruptions in the form of nodules which were closely set in rows, from which the thickened epidermis separated in thick shreds.

According to Robertson and Spreull the disease of cattle known in South Africa as "lamziekte" is supposed to belong to hemorrhagic septicemia. However, a confusion with "heart water" (see this disease) is not excluded.

The pectoral form is characterized by symptoms of an acute pleuro-pneumonia. The animal stands immovable with back arched, has a dry and painful cough, and a colorless or reddish, foamy serous discharge from the nose. On one or both sides of the thorax there is dullness over different areas with bronchial breathing and vesicular rales. There may be a total absence of respiratory sounds. Friction sounds may be heard over the hepatized areas. Respiration is greatly accelerated and labored. Rumination ceases. Peristalsis of the rumen and intestines is frequently suppressed. Constipation is followed by bloody diarrhea, whereupon the greatly weakened animal rapidly succumbs.

According to Carrougeau the disease may take a chronic course. The emaciated, feverless animals cough frequently; their appetite is capricious, and there is profuse lachrymation. At the same time a persistent diarrhea is present in which feces mixed with blood are passed. Over the lungs rales can be heard, and percussion sounds are sometimes dull. The disease may terminate in recovery, but in such cases the cough persists for a long time. In other cases the animals die from exhaustion as a result of the long continued sickness and emaciation. The autopsy of such animals reveals caseated areas in the lungs, chronic catarrh of the stomach and intestines, enlargement of the lymph glands and indications of cachexia (in some cases only the latter lesions are present).

The belief of Simader that the "endemic pharyngeal paralysis (Wasenmeisterkrankheit)" is a form of hemorrhagic septicemia is not substantiated as aside from the different post mortem findings and its continuous afebrile course (Schmid) there are no proofs, and especially since an identical etiological factor has not been established.

Course and prognosis. The duration of the disease varies, with the exception of the chronic cases, between 6 hours and 8 days. The edematous form usually runs the more rapid course, mostly from 12 to 36 hours, while the pectoral form, 
which occurs very frequently in some enzootics, rarely terminates in less than 3 days.

The prognosis is very unfarorable. According to past observations 85 to $95 \%$ of the affected animals die. Recovery may exceptionally be observed in the pectoral form, but even in these cases it is not complete, as in the patients a chronic lung affection usually follows.

Diagnosis. The disease resembles first of all those cases of anthrax in which edematous swelling and signs of enteritis are present. Hemorrlagic septicemia is indicated by the development of edematous swelling of the throat, marked swelling of the tongue and the fact that hogs may be simultaneously affected. On the other hand, in anthrax the edema which is less frequent is more apt to develop on the neck, dewlap and in front of the chest; hogs are only exceptionally, while sheep are frequently affected. On autopsy the absence of acute swelling of the spleen excludes anthrax with a high degree of certainty, while bacteriological examination of the blood from carcasses recently dead of the disease makes differentiation easy on account of the great difference in the appearance of the causative agents. If the carcass is no longer fresh cutaneous or subcutaneous inoculations of the blood or inflammatory products are advisable, as the blood of such test animals (mice, rabbits and pigeons) contains the respective bacteria in great numbers.

From rinderpest hemorrhagic septicemia may be differentiated by the fact that the latter may occur without being introduced from outside into a locality, and that in its course the mucous membranes, especially those of the air passages, do not show an intense croupous inflammation and ulceration. Blackleg and malignant edema are characterized by crepitating, edematous swellings; moreover, these diseases cannot be transmitted to test animals by cutaneous or intravenous injections.

The pectoral form of hemorrhagic septicemia can easily be mistaken for pleuro-pneumonia. In the majority of cases hemorrhagic septicemia may be recognized by the appearance of inflammatory changes in the lungs, as they occur in this disease in larger extension or in numerous centers of the same stage of development. Occasionally, however, the lesions may simulate acute or subacute pleuro-pneumonia to such an extent as to render diagnosis from the microscopic findings almost impossible. In such cases a microscopical examination will establish the correct disease, as in hemorrhagic septicemia the serous fluid of the lungs contains bipolar bacilli, while in pleuropneumonia the findings are negative. Test inoculations may also be made to differentiate the disease, as the virus of septicemia is pathogenic for practically all test animals, while that of pleuro-pneumonia is harmless (in calves infected subcutaneously with the latter an edematous swelling develops at the point of inoculation in 1 to 2 weeks). The presence of 
small hemorrhages in the tissue of the inflamed pleura, and the sudden appearance and rapid course of the disease are suggestive of hemorrhagic septicemia.

Sometimes bacteriological examination may be difficult as occasionally the blood as well as the exudates of animals affected with hemorrhagic septicemia contain few bacteria and the virus may be greatly attenuated. In doubtful or negative cases, it is therefore advisable to inoculate several test animals, especially rabbits.

Lastly, the disease is differentiated from simple asphyxiation by the absence of inflammatory changes; from spontaneous (mycotic) or toxic enteritis by the absence of hemorrhages in other organs which will also differentiate septicemia from inflammatory changes in the lungs and inflammatory edemas of the subcutaneous connective tissne.

Treatment and prevention. The treatment of affected animals has so far been unsuccessful and can at best consist in the scarification of the swellings, washing of the wounds and disinfection of the intestinal canal.

For the suppression of the disease it is advisable to remove cattle and hogs from the infected premises to dry, and if possible, to elevated places. When the disease has appeared in a stable, it is well to withliold the suspected feed and to disinfect the premises.

The method of immunization which has been recommended by Blin \& Carrougeau against buffalo disease (see page 118) might perhaps be applied to hemorrhagic septicemia in cattle.

Veterinary police. Before requiring the inclusion of hemorrhagic septicemia among the diseases for compulsory reporting, it is necessary to take into consideration the fact that it is a disease of the soil. Far reaching traffic restrictions do not appear justified, while hides may be rendered harmless by simple drying.

Ostertag found in his investigations that dried hides from affected cattle are always free from the infectious agent as soon as their moist and pliable condition is lost and they become hard. Submerging pieces of skin in milk of lime or in fresh tanning fluids destroys the organisms in 24 hours. In meat the infective agent is destroyed by advancer putrefaction, by heating to $70^{\circ} \mathrm{C}$. (immerliately), with less certainty by salting, pickling or injections of brine (sometimes only after 45 days).

Literature. Bollinger, Ǔb. eine neue Wild- und Rinderseuche usw. München 1878.-Franck, D. Z. f. Tn., 1881, VII, 293.--Friedberger, Mch. Jhb. 1880-81, 21.Kitt, Sitzgsber. d. Ges. f. Morph. München 1855; Meh. Jhb. 1855-86 u. 1856-87.Nocard, Bull., 1891, 424.-Jensen, Monh., 1894, II, 188.-Lignières, Bull., 1897, 761; 1900, 537 (Lit.).-Taufer, Fortschr. d. V.-Hyg., 1904, II, 188 (Lit.).Ostertag, Z. f. Infkr., 1908, IV, 1.-Simader, W. f. Tk., 1908, 397 (Lit.).

Septic pleuro-pneumonia of calves, The disease was first described by Poels in Holland (1886) and since that time by other authors (Jensen, Liénaux, Bongartz, Galtier and others). It evidently represents a form of hemorrhagic septicemia of eattle, and the close 
relation of the two discases appears especially indicated by the fact that in certain herds adult cattle and calves are frequently affected at the same time.

The causative agent (Bac. vitulisepticus) is identical with the organism of septicemia, and natural infection appears to occur principally through the digestive tract. All excretions of the infected animals may transmit the infection.

The autopsy reveals a large amount of sero-fibrinous exudate in the pleural cavity, punctiform hemorrhages in the serous covering of the lungs, reddish-hrown or grayish-red hepatizations with serous infiltration of the interalveolar connective tissue in the lungs, and sometimes dilatation of the lymph vessels contained therein (marbled appearance). The finding is completed by the presence of small hemorrhages in the serous and mucous membranes, acute catarrh of the air passages and the gastro-intestinal canal, acute swelling of the lymph glands, and cloudy swelling of the parenchymatous organs.

The symptoms consist in high fever, weakness, inappetence, indications of acute pleuro-pneumonia such as painful cough, difficulty in respiration, sensitiveness over the thorax, dullness over the lower parts of the lungs with friction sounds, bronchial breathing, or a total absence of respiratory sounds. Difficulty in respiration increases, and in most cases death results in 1 to 2 weeks after diarrhea sets in, or after a temporary improvement a chronic form of the disease may develop.

For prevention immune sera (calf-pneumonia serum, septizidin) prepared from horses and cattle by injecting them with cultures of the bipolar bacillus were used with varied results. According to the investigations of Schirop promising results can he expected only from the use of polyvalent sera (see page 85). However, he succeeded in immunizing rabbits and guinea pigs with aggressins.

On the relation of the disease, especially in its chronic form, to other pneumonias of calves see the chapter on enzootic volume II.

Literature. Poels, Fortschr. d. Med., 1886, IV, 388.-Jensen, Monh., 1890, II, 1.-Liénaux, Ann. vét., 1s92, 465.-Bongartz, B. t. W., 1892, 529.-Sehirop, Zbl. f. Bakt., 1908, XLVII, 307 (Lit.).

Hidebound. Enteque. The disease is designated in Argentine by this name, and occurs there especially along the shores of the Atlantic Ocean in certain marshy localities, where the outbreaks cause considerable annual losses. It was described by Monfallet, Even, and particularly Lignières who gave a careful and full description of the disease. In acute cases occurring in animals from 12 to 24 months old, a persistent painless diarrhea and gradual emaciation are observed in spite of a good appetite, until the animals finally die of exhaustion in from 3 to 4 months. In the cachectic form of the disease, which occurs mostly in animals which recover from the acute affection, the course may extend over several years. In these cases the progressive emaciation and anemia which develop in the meantime and a marked exhaustion are the most conspicuous symptoms. Normal appetite is suppressed, but the animals manifest a desire to eat bones. Along the neck in the jugular groove the ealcified carotid may be felt in some animals, while in others inflammation of the joints appears, resulting in deformities.

On autopsy in acute cases besides the general anemia and emacia. tion, sometimes a pleuritis, broncho-pneumonia or a fibrinous pneu- 
monia may be observed. In the chronic cases the lungs sometimes show islands or cords consisting of spongy osseous tissue which crepitate on pressure and which have developed by ossification of the vessel walls. In the large blood vessels of the body, and in exceptional cases in the small arteries, especially those of the heart, a pronounced arterio-sclerosis exists; the endocardium may show a chronic endocarditis, while the different joints may show indications of chronic inflammation (similar changes were found by Moussu in France in chronic forms of diarrhea and by Brusaferro in a yearling steer slaughtered in Turin).

The disease was also observed in horses and sheep but the lungs failed to show the processes of ossification.

According to the investigations of Lignières, the causative agent of the disease is a very small variety of the Bac. bipolaris septicus. Intravenous injections of large quantities of fresh cultures produce septicemia which is fatal in 1 to 3 days; following injections of older cultures in cattle and sheep an articular and peri-articular inflammation develops which results in the formation of new connective and cartilaginous tissue.

Natural infection occurs in marshy localities from the water and food. The results are sometimes an enteritis, or a chronic inflammation of the joints and an affection of the vessel walls.

Lignières obtained satisfactory results in the treatment of the acute form of the disease by intrajugular injections of diluted blood serum of healthy animals (400 cc. serum and 500 ce. sterile water), or artificial serum ( 1000 cc. boiled water, 9 grams sodium chloride and 4 grams sodium sulphate). In cases where recovery was prolonged, the injections were repeated.

Literature. Even, Revista vet., 1896, 301.-Lignières, Bull., 1898, 761.

(c) Buffalo Disease. Septicaemia hemorrhagica bubalorum.

(Barbone disease, Pasteurellosis bubalorum, Pasteurellose des buffles [French]; Büffelseuche [German]; Barbone bufalino [Italian].)

This affection is an acute, febrile, infectious disease of buffalo, and is characterized by febrile manifestations, difficult respiration and especially by an edematous swelling of the throat region. It is caused by the bacillus bubalisepticus.

History. The disease was first studied in Italy by Oreste \& Armanni (1886), and included in the hemorrhagic septicemia group. They also undertook immunization experiments. In Hungary the occurrence of the disease was first estahlished by Sequens (1889), while Rátz undertook a more thorough investigation of the pathogenicity of the infective agent.

Occurrence. Buffalo disease occurs frequently in localities where large numbers of buffalo are kept during the summer, and at times causes considerable loss in some herds. It usually occurs as a disease of the soil in marshy pastures and in dry stubble fields. Exceptionally, however, cases may occur in animals fed in stables. The economic importance of the disease 
is increased by the fact that it may be transmitted to hogs, among which it may also cause considerable loss.

In Italy the infection is especially widespread in the districts of Salerno, Rome and Terra di Livorno. In Hungary the disease occurs almost exclusively in the southeastern counties (in 190s, 98 buffaloes were affected in 56 townships, and 95 of them died; considerably less than in former years). In Russia, Egypt, East and West Indies, and Indo-China the disease frequently oceurs in enzootic form.

The dangerous character of the disease is readily recognized by the following data: In Italy, in a herd of 1,400 buffaloes, 200 died in the year 1882 within ten days; in another herd of 1,300 buffaloes, 530 sucemmed. In one township of the infected districts of Hungary, 52 of 237 buffaloes died within one week; in another, of 663,89 died within a short time. The losses in the Netherland-West Indies (in Bantam and Batavia alone) amounted, between 1588 and 1591, to 11,000 buffaloes.

Etiology. The established cause of the disease, the bacillus (bipolaris) bubalisepticus, appears in all its principal characteristics as a variety of the bacillus bipolaris septicus (see page 79).

Tenacity. Blood dried in the air loses its infectiveness in 24 hours. On the other hand, when kept in elosed glass tubes at room temperature its virulence is lost in 40 days. Carbolic acid $(2 \%)$, sulphuric acid $(5 \%)$, and alcohol $(87 \%)$ render equal quantities of blood harmless (Oreste \& Arnanni, Sanfelice).

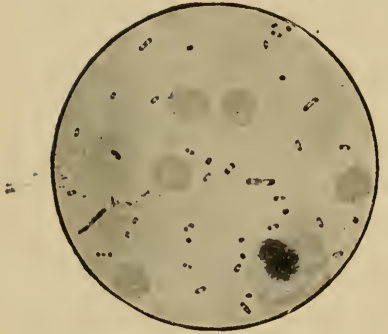

Fig. 28. Bacillus bubaliscpticus. Snear preparation from rabbit blood. Polar staining.

Pathogenicity. According to the investigations of Rátz, rabbits are most susceptible to artificial infections; somewhat less susceptible are the guinea pig, the mouse and the pigeon; to a still lesser degree, chickens, while the dog and duck are not at all susceptible. Of the large animals the horse succumbs to subcutaneous infection in an average of 20 hours, cattle in from 20 to 48 , and hogs in from 20 to 40 hours. The inoculations result in manifestations of a general infection with edematous swelling at the point of infection. Buffalo calves die from a cutaneous or subcutaneous infection inside of $24-36$ lours with similar symptoms, while infection is not successful when virulent cultures are fed, but buffalo calves treated in the latter manner will develop an immunity against a subsequent virulent infection. Sheep inoculated with virulent buffalo blood die with symptoms of an acute pharyngitis (Reischig).

The bacteria are present in large numbers in the blood, in the serous exudates and the feces of the infected animals. They may also pass into the saliva, bile, milk and the urine.

Natural infection may occur through wounds of the skin and mucous membranes. Such infections are favored in pastures which contain thorny plants and pools of water in which the animals lie for hours, thus constantly reinfecting the water.

In the great majority of the cases, however, natural in- 
fection probably results from the ingestion of infected food and water through the uninjured mucous membrane of the digestive tract. This mode of infection is indicated by the constant swelling in the region of the throat and also by the relatively frequent acute inflammation of the abomasum. On the other hand, infection through the air passages is less probable, as intratracheal injections of virulent material fail, in most cases to produce the disease. A direct transmission from animal to animal or a spreading over extensive territories is not observed in the buffalo disease.

In premises where the disease has appeared according to the experience of Hungarian veterinarians hogs also frequently become affected with symptoms of an acute pharyngitis and general infection; in many cases this has been observed after the ingestion of meat from buffaloes which had died from Buffalo disease. In exceptional cases it may also occur that hogs are first affected and the disease in buffaloes is observed only later (Sequens). The disease does not spread to hogs kept on other premises.

Exceptionally the infection may be observed simultaneously in buffalo and cattle probably as a result of an infection with the same virus (Betegh, Sequens, Sanfelice).

Anatomical Changes. A serous infiltration of the subeutaneous tissues in the region of the throat and trachea is almost a constant lesion and more or less characteristic of the disease. This may extend posteriorly reaching to the sternum. In the region of the swelling the perilaryngeal and peritracheal loose connective tissue as well as that of the lig. aryepiglottica and of the frenum is saturated with a clear watery, slightly yellow serous fluid. In rare cases the edema extends on one side to the cheeks and on the other side also to the mediastinum in which case the thoracic cavity sometimes contains yellowish clear transudate (Gál). Occasionally the serous fluid shows red coloration, and the infiltrated connective tissue is studded with small hemorrhages.

The other changes usually indicate in general a septic infection. The mucous membrane of the air passages, especially of the larynx and of the bronchi, shows a catarrhal swelling, redness and occasionally punctiform or linear hemorrhages. The lungs show a pronounced acute edema. The blood vessels of the serous membranes are highly injected, the peritoneum is sometimes covered with a fibrinous membrane.

The mucous membrane of the abomasum appears highly reddened and swollen, either over its entire surface or over certain places, while in the intestines the catarrhal manifestations are less pronounced. The spleen is congested, but only very slightly swollen or not at all. The liver and kidneys usually show no changes. The lymph glands of the mesentery as well as in other parts, manifest an acute swelling. 
Piot observed an affection of the lungs in Egypt which resembled pleural pneumonia, the lesions resembling those of hemorrhagic septicemia of cattle (see page 107). Sohns has also observed a pectoral form of the disease.

Symptoms. The time of incubation after artificial infection is usually only 12 hours, and under natural conditions it very probably extends only from one to two days.

The disease commences with a rapid rise of the body temperature to $41-42^{\circ} \mathrm{C}$. The affected animal stands indifferent with extencled neck and closed eyes. From the corners of the mouth foamy saliva drips in long strings. In the meantime the swelling of the throat region develops, becomes hot, painful, first of a doughy, later of a denser consistency. It does not crepitate on touch. During the development of the swelling the animals manifest a short and painful cough; soon the swelling extends over the cheeks, also over the parotid region and backwards along the neck to the breast, and even over the region of the shoulder. The conjunctivae of the eyes as well as the mucous membrane of the nose and of the mouth is bright red in color, and at the same time hot and dry. There is lachrymation from the eyes and a yellowish mucous secretion is discharged from the nose. On opening the mouth the tongue appears usually normal, only the surrounding of the frenum of the tongue shows a serous infiltration and swelling. In some cases, however, the tongue may swell considerably so that it is dark brownish-red, and its tip protrudes from the mouth to the length of several centimeters.

With the increase of the swelling, respiration becomes more difficult, accelerated and later rattling. The action of the accessory respiratory muscles is greatly increased, the nasal openings are widely distended. The long inspiration is followed by a short, jerky expiration accompanied by painful grunting.

The appetite is suppressed from the onset of the disease or may be capricious. Rumination ceases. There is first constipation while towards the termination of the disease diarrhea may set in when the feces are thin, fetid and mixed with dirty red mucus. The animals look from time to time towards the abdomen, otherwise they hardly move from the place and cannot be urged to walk. In case there is a water pool near by the animals like to lie down in it. The vulva and the surrounding parts of the rectum are often swollen during the later course of the disease. Pregnant cows frequently abort.

With the rapid progress of the symptoms the animals, shortly before death, grunt painfully, and the rattling respiration may be heard at a distance from 30 to 40 steps, they grind their teeth or bellow loudly. Finally the animal suddenly rises from the ground, takes several staggering steps, suddenly col- 
lapses, whereupon death ensues in a few minutes under manifestations of aspliyxiation and convulsive movements.

There are occasionally deviations from the described symptoms. Thus the swelling of the throat region may be absent, and the autopsy may show only very slight changes in the mucous membrane of pharynx and larynx, in spite of the fact that the animal died with symptoms of asphyxiation (Biró). In less acute affections symptoms of a malignant catarrhal fever develop sometimes (Makoldy).

In localities where rinderpest prevails, as for instance in Eastern Asia, buffalo disease has been frequently mistaken for the former, especially in cases of severe catarrhal symptoms of the nasal and intestinal mucous membrane as well as in the eases of somewhat more chronic forms. However, the noneontagious character of the buffalo disease and the great susceptibility of rabbits for its virus offer suffieient differential signs.

In eattle which in rare eases become affected simultaneously with buffaloes similar clinical manifestations are observed, especially high fever, extension of the head, protrusion of the swollen tongue, rattling respiration, cyanosis of the mucous membranes, further an edematous swelling of the region of the throat and cheeks.

In hogs, which sometimes also become affected on premises where buffaloes are sick, the disease runs a course of severe pharyngitis with marked swelling of the region of the throat, which sometimes extends also over the neck, when the debilitated animal suffers from difficult and rapid respiration.

Course and Prognosis. In rare cases the disease lasts only six to eight hours, but as a rule death results on the second day. Exceptionally the course may extend up to eight days, and such protracted cases sometimes terminate in recovery, especially if no swelling of the throat develops in the meantime.

According to Italian authors the percentage of mortality amounts to from 50 to 60 per cent. According to the observations made in Hungary it varies from 87 to 98 per cent and averages 94.2 per cent.

Treatment and Prevention. The treatment of affected animals promises little success. The scarification of the swell. ings in the region of the throat followed by washing of the wounds with a disinfectant, for instance three per cent carbolic acid solution or turpentine, appears most appropriate. Repeated injections of the first solution into various parts of the swelling without previous scarification may also be beneficial. Beside cold applications to the region of the throat, cold showers and laxatives (salines in large doses) as well as internal administration of disinfectants (creolin, lysol) are indicated.

The most effective method against the spreading of the disease is the removal of the healthy animals from the infected pastures, or at least the abandonment of low-lying, marshy 
pastures. In many cases the disease ceases immediately after the animals are stabled. Further preventative measures are the disinfection of the infected stables and premises as well as the amelioration of the dangerous pastures by drainage and drying of the swampy areas.

Immunization. According to Oreste \& Armanni (1887) the blood of pigeons which have been inoculated and have died in from three to four days becomes attenuated and adapted for immunization. Three injections of 0.1 ce. of such blood is supposed to produce an active immunity in buffalo. Such immunizations were carried out by the authors in their practice with good results.

Oreste and Marcone (1889) prepared a vaccine by growing the bacteria in the presence of air at a temperature of $30^{\circ}$ to $32^{\circ} \mathrm{C}$. which supposedly protected sheep and buffaloes against a virulent infection.

Blin and Carrougeau (1902) produced an immune serum by careful immunization of horses with old, then with fresh cultures (inside of 5 months 150 ce. of cultures were injected subcutaneously) of which the protective action has been increased by the addition of killed cultures. Simultaneous injections of the serum and $1 / \mathrm{s}^{-1 / 2}$ cc. of virulent culture produced a lasting immunity, but still more effective was the subcutaneous injection of a serum-culture mixture (20 ce. serum plus 2 cc. culture). The serum must have such a strength that 20 ce. of the same will protect a 20 months old calf against an infection with 2 ce. of culture of which 1 ce. ordinarily kills the animal in from 24 to 30 hours. The results are supposed to be satisfactory in buffalo as well as in cattle.

Veterinary Police. A similar procedure as in hemorrhagic septicemia of cattle is advisable. Besides buffalo, hogs should be also protected against infection.

Literature. Oreste \& Armanni, Rev. Vet. Jhb., 1887, VII, 45.-Oreste \& Marcone, Clin. vet., 1891.- Sequens, Vet., 1894, 504.-Rátz, D. Z. f. Tm., 1896, XXII, 331.-Lignières, Bull., 1900, 541 (Lit.).-Blin \& Carrougeau, Rec., 1902, 571.-Yersin, A. P., 1904, XVIII, 440.

\section{(d) Hemorrhagic Septicemia of Sheep. Septicaemia haemorrhagica ovum.}

(Catarrhal Fever of Sheep; Septicemia pluriformis ovum, Pasteurellosis Ovum, Lombriz [Spanish]; Schafrotz [German].)

Hemorrhagic septicemia of sheep is characterized in acute cases by septicemic manifestations, in subacute and chronic cases by a discharge from the eyes and nose as well as by a pleural pneumonia. The cause is the bacillus ovisepticus.

History. Galtier (1889-1890) observed in the Alps of France a disease of sheep which he associated with swine plague, describing an ovoid bacterium as its cause. Later Liénaux (1895) described an organism as the cause of infections pleuro-pneumonia of sheep which entirely corresponded with the bipolar bacillus. Conte (1897) as well 
as Besnoit and Cuillé (1898) obtained similar results during their studies of diseases of sheep in France.

Lignières (1898) studied this disease more thoroughly when he established that the disease which is known in Argentine under the name of Lombriz on account of the usual presence of intestinal parasites, represents in its character a disease of the hemorrhagic septicemia group. He as well as Besnoit and Cuillé interestingly demonstrated its relation to verminous bronchitis and verminous gastritis of sheep.

Similar results were recently obtained by Miessner \& Schern (1910) when they established the etiological relation of the bipolar bacilli in a disease of sheep which was considered by Spinola (1863) as catarrhal fever and by Friedberger (1883) as glanders of sheep, according to their clinical and anatomical manifestations. The first mentioned authors succeeded in demonstrating virulent bipolar bacilli in the lymph glands and sometimes in the blood of animals dead from this disease. The presence of this bacterium was sometimes only demonstrable by inoculations. Pure cultures of this organism produced artificially the acute and subacute form of the disease. Accordingly the so-called glanders of sheep (septicemia pluriformis ovis) is identical with Lignières' Pasteurellosis. On the other hand it is distinguished from the South African eatarrhal fever of sheep (see that disease; infection experiments with filtered material were not made). This new conception will be given consideration in the following description, especially concerning the chronic cases.

Occurrence. The disease caused considerable loss, especially among lambs, while older animals are rarely affected, and then usually in a chronic form. Most frequently it is observed in low marshy localities, more rarely in higher sections, and especially during the weaning of lambs.

In Argentine the disease occurs almost annually in an enzootic form, frequently associated with verminous bronchitis and gastritis, which has also been found to be the case in France (Lignières, Besnoit \& Cuillé). In Germany the disease appears to be quite extensive as Miessner \& Schern established its presence, in 1907, in eight herds of sheep where it usually caused great loss among the lambs (sometimes $50 \%$ of the increase of lambs). In Hungary it was observed by Keleti in 1896 when it appeared in association with an outbreak of swine plague. In this instance, out of 300 sheep of Oxford and Cotswold breeds 23 died within seven days. The disease has also been observed in Belgium, England and Turkey, and the fatal disease known in Algeria as "El $\mathrm{K}$ 'och"' is probably identical with this disease (Caze).

Etiology. The bacillus (bipolaris) ovisepticus represents a variety of the bacillus bipolaris septicus with which it is identical in its principal characteristics (see page 79). It is difficult to isolate the organism from the affected organs, and sometimes its growth suddenly ceases.

Pathogenicity. Several loopfuls of virulent culture injected into the circulation of a young sheep, and 5 cc. in an old animal, cause death inside of one to three days under manifestations of hemorrhagic septicemia. Subcutaneous injections of a culture produce a serous inflammation at the point of injection, further acute catarrh of the conjunctiva as well as of the nasal mucous membrane. The animals then become emaciated 
and finally die with symptoms of enteritis, diarrhea and anemia. The same results may also be obtained by repeated injections of one-third to one-half ec. of a fresh virulent culture as well as by intravenous injections of 5 to $10 \mathrm{cc}$. of an older culture (Lignières). The infection may also be produced with ground lymph glands and should the animals remain alive for a longer time certain parts of the lungs become hepatized, and as in the acute cases the lymph glands are markedly swollen (Miessner \& Schern). By feeding 50 to 60 cc. of culture Lignières produced the disease in sheep, although the attack was only slight. On the other hand similar experiments by Miessner \& Schern with 150 cc. of culture gave negative results. Inhalation of 50 cc. of culture killed a lamb in three days.

In animals which died from the acute form of the disease the bipolar bacilli may be found in all the organs, while in the chronic cases they are frequently absent and other bacteria may be demonstrated in the affected tissues. Mice, guinea pigs and rabbits can be easily infected while birds are more resistant. In large animals the subcutaneous injection of the virus produced a serous inflammation at the point of infection. Intravenous injections (50 to $100 \mathrm{cc}$.) cause the deatl of the animals from septicemia within a short time (Lignières). The chronic form of the disease could not be produced artificially.

Natural infection occurs very likely through the digestive tract as a result of the ingestion of infected food or drinking water on infected pastures or in contaminated stables. Young sheep are particularly susceptible immediately after weaning, and the disease usually runs a rapid course in these animals, while in older animals it is more likely to take on a chronic character.

Infectious excrements and excretions of affected animals naturally add substantially to the dissemination of the disease. In herds which are kept in a small pasture or in stables the disease usually occurs in a more severe form. Affected animals may spread the disease to healthy herds.

External influences which reduce the natural resistance of the animals, such as continued cold and exposure to rain, etc., increase the susceptibility, and therefore the disease usually occurs after damp weather and in general in marshy localities.

Furthermore, according to Lignières' view, thread worms (strongylides) favor the infection through injuries of the mucous membrane of the stomach and intestines thereby rendering possible the entrance of the bipolar bacilli, which are present in the feces or in the mucous secretions of the air passages, into the lymph spaces of the mucous membrane (according to Besnoit \& Cuille the dystomas in the bile ducts exert a similar action).

Lignières' view on the close connection of the bacterial infection with the lung and intestinal worms must be considered as too far fetched. Admitting that 
these parasites may independently cause a severe infection and on the other hand that they may be found in apjarently healthy animals, their relation to the etiology of this disease is diminisher by the fact that not infrequently hemorrhagic repticemia of sheep is present even when such parasites are not found in the animal. (Miessner \& Schern do not even mention the presence of parasites in their anatomical findiugs. Lambs only a few months old may become affected with the disease, and the disease may be produced by inoculation of pure cultures.)

Pathogenesis. After a severe infection the bipolar bacilli multiply very rapidly in the body and produce death in a short time by hemorrhagic septicemia. If on the other hand the infection was less severe or the resistance of the animal relatively higher, the action of the bacilli would be manifested only in a chronic intoxication which would result in progressive emaciation and anemia. In these more frequent forms the debility of the animals favors the entrance of other bacteria (especially streptococei) from the intestinal canal and the air passages into the tissues and body fluids. As a result of this secondary infection changes develop in the internal organs and the bipolar bacilli in the meantime may have entirely disappeared from the blood. The relatively frequent broncho-pneumonia as well as the less frequent arthritis and tendonitis are as a rule the results of such secondary infections, while the pleuro-pneumonia may be produced independently by the bipolar bacilli.

Anatomical Changes. In the peracute cases the autopsy reveals the typical findings of a hemorrhagic septicemia (see page 83 ).

In the acute form the subcutaneous connective tissue of the anterior portion of the body sometimes shows a gelatinous hemorrhagic infiltration. The mucous membranes of the head, the air passages and of the intestines also those of the abomasum are usually inflamed, reddened and swollen, the lymph glands are enlarged, their cut surface is moist and in parts shows diffuse reddening. Frequently there are small hemorrhages in the serous membranes, in the kidneys and in the lymph glands. In some cases the lungs contain even in this stage dark brownish-red broncho-pneumonic foci, besides serons infiltration of the intralobular connective tissue. The spleen has usually a normal appearance.

In the subacute form lesions of broncho-pneumonia in the anterior and inferior portions of the lung are almost invariably present. In such cases the pleura, sometimes also the pericardium, is frequently covered with fibrinous psendo-membranes, and the serous cavities contain smaller or larger quantities of a vellow clear or cloudy fluid. The mucous membrane of the bronchi is usually inflamed, reddened and swollen, while that of the intestines is affected less frequently; in some cases the nasal mucous membrane also shows yellow mucous or fibrinous deposits. The lymph glands of the thoracic cavity are moderately swollen.

In the chronic cases larger areas of the lungs usually show 
a grayish-red hepatization, the pleural covering of these parts of the lungs manifesting indications of a former pleuritic condition (thickening and adhesions). The hepatized lung tissue contains numerous necrotic foci which may sometimes be enclosed in a connective tissue capsule. Some of these smaller lesions appear in the form of onion-like layers, from the center of which a tenacious, pus-like mass may be squeezed out, while the larger ones consist of a smeary, grayish-yellow mass which is intermixed with granules. In some of the chronic cases there may only be present manifestations of severe anemia and cachexia without any affection of the internal organs.

Bacteriological examination may readily reveal the presence of the bipolar bacilli, particularly in the lungs, the exudates of the serous membranes, the lymph glands, and in the nasal discharge. On the other hand in chronic eases the bacteriological examination is quite frequently negative, or the presence of other bacteria only can be demonstrated (streptococei, coli bacilli, bacilli of pseudotuberculosis, etc.), although Miessner \& Sehern do not mention secondary organisms in either subacute or ehronic cases. In some of the cases the presence of the bipolar basteria ean be proved only by test inoculations (especially with material from lymph glands) or not at all; thus Lignières succeeded in demonstrating the organism in only 77 out of 258 acute and chronic cases.

Symptoms. In the acute form, which usually runs its course in from two to five days, sometimes even more rapidly, symptoms of severe febrile infections are present. Marked dullness, rising temperature to $41^{\circ} \mathrm{C}$. and higher, inappetence, increased thirst, colicky pains, difficult respiration, albuminuria, muscular trembling, etc., are the indications of this form of infection.

In the subacute cases, which extend from one to three weeks, the symptoms consist of febrile changes in the temperature, disturbed appetite and weakness; at first serous later purulent discharge from the eyes and nose. In addition there may be present either an acute pneumonia or pleural pneumonia, or an enteritis. In the presence of enteritis the excrements are of a greenish-yellow, later of a dark color, and fetid. Sometimes there may be present granulations and also ulcerations on the mucous membrane of the lips and in the surrounding parts and possibly caries of the teeth (Miessner \& Schern). In such cases the lips, the cheeks and the tongue swell, they show a dark red discoloration (exceptionally an ulcerative keratitis has been observed) (Friedberger, Tiede). The animals are very weak and die with symptoms of cachexia. Only in rare cases do the symptoms subsicle after a while. However, the recovery is not complete, the animals often developing later symptoms of a chronic affection of the lungs with cachexia.

The chronic form, which usually develops in older sheep, sometimes from an acute form, is characterized by the appearance of a severe chronic affection of the lungs. The animals cough frequently, they have panting respiration, there is mucopurulent discharge from the nose and eyes and gradual emacia- 
tion. In some cases there develops a swelling of the carpal and tarsal joints, or a suppurative inflammation of the coronary band and of the sensitive lamina.

In some outbreaks thread worms may be found in the abomasum and the air passages, or in both places, or tape worms in the small intestines, while in the bile ducts dystomas may be present in varying numbers.

Diagnosis. The acute cases may easily be mistaken for anthrax, from which the disease differs clinically only by the absence of the hematuria. On autopsy, however, the absence of extensive gelatinous infiltrations and hemorrhages as well as the absence of an acute swelling of the spleen makes a probable differentiation possible. In the subacute and chronic cases the presence of inflammatory changes in the lungs and serous membranes is indicative of hemorrhagic septicemia, especially in cases in which the lungs contain no lung parasites. "Bradsot", usually affects only older sheep during the fall and winter months, and is characterized principally by rapid putrefaction as well as by the severe inflammation of the abomasum and duodenum. In piroplasmosis there is a pronounced acute swelling of the spleen in addition to the characteristic condition of the blood. In cases in which the lungs and intestinal canal contain intestinal parasites in great numbers, especial care should be taken in the diagnosis inasmuch as the demonstration of a few bipolar bacilli does not of itself prove the presence of hemorrhagic septicemia.

Treatment and Prevention. As there is no method of successful treatment it is advisable to kill the chronically affected animals. The remaining animals in the band may be immunized and a further spread of the disease avoided. For this purpose the affected flock should if possible be taken to a large pasture and from this pasture the animals should be changed to others from time to time. If on account of unfavorable weather stabling cannot be avoided, the stables should be cleaned and disinfected at frequent intervals in addition to the isolation of the affected animals. As animal endoparasites favor the bacterial infection, the animals should not be pastured on damp ground, in order to prevent the disease.

Immunization. Lignières obtained good results with his polyvalent vaccine (see page 85 ) of which he injected one-quarter ce. subcutaneously to sucking lambs. Animals inoculated by this method later resisted an intravenous injection of virulent culture, while the control animals died either from an acute or a chronic form of the disease. The polyvalent immune serum (see page 124) is also supposed to have proved effective against the disease.

Miessner \& Schern obtained satisfactory results in protecting, and sometimes also in curing, animals from the disease with the serum of horses which had been hyperimmunized with cultures of the bacillus ovisepticus. However, for practical purposes they preferred a simul- 
taneous vaccination with immune sera and bacterial extracts. But as the simultaneous inoculation produces an immunity which lasts only about 6 weeks it is advisable to vaccinate again after 4 to 6 weeks with bacterial extract. The simultaneous method has proven satisfactory in large flocks of lambs by checking the outbreak of the disease, and in most cases affected animals recovered after this form of vaccination.

The bacterial extracts are prepared by washing 24-hour agar cultures with salt solution. They are killed with formalin and shaken for 7 days in a ball-mill run by electricity. Then the formalin is abstracted from the fluid, it is centrifuged and the clear fluid is utilized for the vaccination. The dose for the vaccination in practice varies between 2 and $5 \mathrm{cc}$. The bacterial extract is injected subcutaneously. The immune serum is used in doses varying from 5 to 18 cc. The subsequent vaccination was made in one case with 4 ce. of extract. If serum alone is used for the inoculation $(10$ to $20 \mathrm{cc}$.) it is advisable to repeat the injection after 4 weeks.

The serum inoculation alone was used in five herds with good results. On the other hand in the sixth herd 68 out of 171 lambs died in spite of the inoculations. In five herds over 500 lambs were inoculated with serum and bacterial extract, 364 of which received two inoculations, and all of which showed uniformly favorable results.

Literature. Friedberger, Münch. Jahrb. 1882-1883 (Lit.).-Andum, Unters. über den Schafrotz. Dorpat 1888.-Galtier, J. vét., 1889, 58 u. 1890, 481.-Liénaux, 1896, 625.-Conte, Rev. vét., 1897, 516.-Besnoit \& Cuillé, ibid., 1898, 465.-Lignières, Bull., 1898, 797; 1900, 529 (Lit.).-Miessner \& Schern, A. f. Tk., 1909, XLIV, 44 (Lit.).

Infectious pneumonia of goats. Nicolle \& Refik-Bey (1896) studied in Anatolia a pneumonia which caused periodically great losses in the goat herds, and especially among the kids. The disease is probably identical with the affection observed by Hutcheon \& Stecle in South Africa, which had been introduced there in 1880 from Angora, causing a loss of more than 12,000 goats.

The elinical manifestations are those of acute pneumonia, such as fever, cough, mucous nasal discharge, dullness on percussion, bronehial breathing and rales over one or both sides of the thorax. Towards the termination of the disease, which on the average lasts for 10 days, paralysis of the extremities usually appears. Manifestations of enteritis are as a rule absent. Three-fourths to four-fifths of the affected animals succumb, while those which remain alive retain a persistent cough for a long time.

The autopsy reveals a lobular pneumonia of the lower anterior parts of one or both lungs. The cut surface of the hepatized areas is dry, granulated, its color violet red or reddish-gray. The adjacent pleura is covered with a moist fibrinous membrane. In exceptional cases the affected part of the lung becomes necrotic and may be found, lying as a soft caseous mass, in a cavity formed by the thickened pleura and the hepatized lung tissue. The bronchi frequently contain strongylides in great numbers.

Bacteria which morphologically and in their cultural characteristies are identical with the bipolar bacilli may be demonstrated in the lung tissue and in the nasal discharge. Intrapulmonary or intraperitoneal injections of virulent cultures, especially when mixed with lactic acid, kill goats in a few days. When injected intravenously they succumb in a few hours. Subcutaneous injection results in a protracted infection towards the end of which paralysis sets in. The intratracheal infection is negative. Mice, guinea pigs, rabbits and pigeons are also susceptible to the virus. In the abdominal eavity of 
guinea pigs the virulence of the virus is increased, while passage through a pigeon increases its virulence still more.

Goats which after a subcutaneous injection remain alive acquire, to a certain degree, immunity against subsequent virulent infection. (See also Infectious Pleuro-pneumonia of Goats.)

Literature. Hutcheon, Vet. J., 1581, XIII, 171, u. 399.-Steele, ibid., 1889, XXIV, 153.-Nicolle \& Refik-Bey, A. P., 1896, X, 321.

Takosis of Angora Goats. Under this name Mohler and Washburn (1902) described a very malignant chronic infectious disease of angora goats which occurred in various localities of the United States. The principal symptoms are at first moderately high, later a subnormal temperature, pronounced weakness, gradually progressive anemia and emaciation, later diarrhea and indications of pneumonia. The disease results in death in from one to eight weeks. The autopsy reveals, besides a pronounced general anemia, small hemorrhages in the serous membranes, eatarrhal pneumonic foci in the lungs, accumulation of transudate in the pericardium, finally chronic catarrh and, in parts, superficial necrosis of the intestinal mucous membrane.

In the blood as well as in the transudates a small Gram positive coccus (nicrococeus caprinus) was demonstrated. Grown on artificial media the cultures showed great resemblance to those of the staphylococcus pyogenus albus. They are pathogenic for goats, chickens, rabbits, guinea pigs and white mice. Intra-abdominal injection of cultures produced in angora goats a fatal infection similar to the natural disease.

The disease which has been previously observed by Pegler (1885) causes in the infected herds losses amounting from 30 to 85 per cent, which can be reduced only by an early isolation of the affected animals. (An. Ind., 1902, XIX, 354.)

\section{(e) Swine Plague. Septicaemia suum.}

\section{(Pasteurellosis suum; Septicémie,Pneunonie contagieuse du porc, [French]; Schweineseuche, [German]).}

Swine plague is usually a sporadic, less frequently an enzootic infectious disease of hogs which with the exception of the peracute septicemic cases is characterized principally by a multiple necrotic pleuro-pneumonia. It is caused by the bacillus suisepticus.

History. Swine plague in its septicemic form was first differentiated from swine erysipelas by Löffler (1885) and recognized as an independent disease. Shortly afterwards Schütz (1886) established the etiological identity of the pectoral form with the septicemic form. Later the disease was identified by various authors (Billings, Silberschmidt, Voges) with hog cholera. In America Salmon (1886) and Smith (1890) separated swine plague sharply from hog cholera, while in Europe Schütz, Jensen, Bang and especially Preisz established its independent nature. (See Hog Cholera.) It was supposed until very recently that swine plague very frequently occurred in association with hog cholera as an independent disease. However, with the 
establishment of the etiology of the latter disease (see that disease) it has been proven that in hogs affected with the filterable virus of hog cholera the bipolar bacillus usually produces only secondary processes, so that these cases should properly be considered as hog cholera.

The over-estimation of the importance of the ovoid bipolar bacilli being present in the affected lung tissue caused many to connect with this disease the chronic eatarrhal pneumonia of pigs as the so-called chronic swine plague, but there are no satisfactory proofs for such a conception. On our part we separate this disease from the true swine plague and consider it closely related to other similar pneumonic processes of young animals, and principally as the result of a mixed infection. Likewise the diseased processes caused by the bacillus pyogenes suis will be discussed here under a separate heading (see pyobacillosis), while the name swine plague will be retained for the disease caused by the bacillus suisepticus. This is the disease which is originally and exclusively, or at least principally, caused by this organism and which in its anatomical changes corresponds with the pathogenic nature of this bacterium, and which in its classical form was first described by Löffler and Schütz.

Occurrence. Pure swine plague occurs mostly in a sporadic form in otherwise healthy herds. Exceptionally it may also attain an enzootic form, but even in such cases it remains confined to the directly affected herds without spreading in the usual way of epizootics from place to place. In Hungary the disease occurred as early as in the eighties in the last century as a necrotic pneumonia among fattened hogs, but always in a sporadic form.

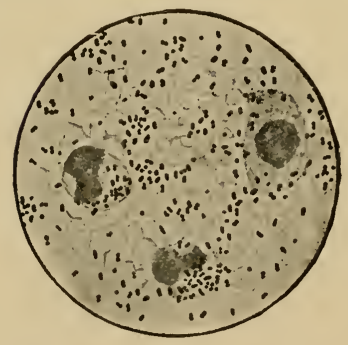

Fig. 29. Bacillus suisepticus. Smear preparation from the fluid of the lung of a hog. Fuchsin staining; and subsequent washing with $1 \%$ acetic acid.

In the conception given alove for pure swine plague the disease is only of slight economic importance. The losses which are, even at the present time, attributed to swine plague are actually the result of either hog cholera or of the catarrhal pneumonia of pigs. The occurrence of acute and destructive epizooties of pure swine plague has, at least in central Europe, never been positively established.

Borzoni (1908) reported a fatal disease in Sardinia (Angina, su fogale, su mali, de sa 'ula) which occurred especially after long floods, protracted rains, or in poorly fed animals, which cansed a loss up to 90 per cent, the blood of the affected animals showing a "coceo bacillus;" but in this disease inflammatory and necrotic changes are absent in the organs of the thoracic cavity. Jowett (1908) reports an outbreak of pure swine plague in Capland in which on one farm out of 100 hogs 40 died of pleuro-pneumonia. The lungs in these cases containerl only bipolar bacilli. The diagnosis of this outbreak was, however, based on the autopsy finding of only five carcasses. Theiler observed in South Africa in the course of years only one case of swine plague in which hog cholera could be excluded.

Etiology. The bacillus (bipolaris) suisepticus is characterized by the peculiarities of the bacilli of hemorrhagic septicemia (see page 79), but it is usually somewhat larger, can be quite readily isolated from the animal body (especially from the 
lungs and lymph glands), and also grows somewhat better anaerobically than do the other varieties of this organism.

Tenacity. In distilled water the bacilli retain their virulence up to 14 days; heating for $1 / 4$ of an hour to $58^{\circ} \mathrm{C}$. kills them in 20 minutes, direct sunlight in 6 to 8 minutes, diffused daylight in about one hour (Joest). Exudate dried on silk threads loses its virulence in daylight after 48 , in darkness after 72 hours. In sterilized milk the virus retains its virulence for 14 days, in sour milk not more than a few days. In hog and cow manure, at a depth from $20 \mathrm{~cm}$. to $1 \frac{1}{2} \mathrm{~m}$., they remain alive for about 14 days (Beck \& Koske). Disinfectants have an effective action on the organism even in mild solutions. Thus their development is checked by carbolic acid 1:2000, sulphuric acid 1:5500, potassium hydrate 1:900; while corrosive sublimate $1: 15000$ as well as $1 / 2 \%$ creolin solution destroys the bacteria in one minute, $1 \%$ copper sulphate solution in three, $1 \%$ solution of lime water and chlorate of lime in $20,1: 2000$ formalin in 40 , lastly iron sulphate solution in 40 minutes. (Erdös \& Koppányi.)

Pathogenicity. The virulence of various strains of the bacillus varies within very wide limits and changes sometimes very rapidly even in cultures of the same strain. Aside from hogs, animals susceptible to artificial infection are mice, rabbits, guinea pigs and domestic fowl. All animals of these species succumb to a subcutaneous infection of 0.1 to $0.5 \mathrm{cc}$. of bouillon culture inside of 24 hours, dying of septicemia. Rabbits are especially susceptible, and they are killed in 36 hours by 0.1 cc. of a bouillon culture diluted to a trillionth part (Preisz). Some strains are so much less virulent, that they kill guinea pigs injected intraperitoneally and rabbits intravenously only with difficulty, and are altogether harmless for chickens, which may, however, develop a chronic affection of the joints (Lignières). Carnivorous animals succumb to intravenous infections. In large animals a subcutaneous injection results in a local inflammatory process (calves, however, may die), while intravenous injections result occasionally in death. Feeding of cultures is not as effective, but rabbits may easily be affected by this method. After repeated inoculation horses develop a diseased condition with indications of a chronic intoxication and interstitial hepatitis.

Intrapulmonary injections cause multiple necrotic pneumonia in hogs (Schütz), and sometimes this may also be produced by inhalation of a virulent culture (Schütz, Olt, Pütz, Beck \& Koske). Intertracheal injections of the culture have a similar action (Lignières, Karlinski), and intravenous injection produces a double pneumonia, pleurisy and pericarditis (Smith). After subcutaneous injection in the thigh an inflammatory growth develops occasionally at the point of injection, with which then a lymphangioitis becomes associated, whereupon the animal dies in 4 to 5 days or may linger for several weeks (in the latter cases not infrequently inflammatory manifestations of the different joints develop in addition to the cachectic manifestations. Autopsy frequently reveals in acute cases a necrotic pleuro-pneumonia with hemorrhages (Preisz). In the majority of cases, however, the local inflammatory processes subside, and the animal recovers, or the inoculation does 
not produce any perceptible symptoms. After intraperitoneal infection an acute croupous diphtheritic enteritis develops. Vigorous hogs cannot be infected fatally by feeding (Gnüchtel succeeded in doing so by weakening the pig by continued cooling).

According to Ostertag it is possible to produce the so called ehronic swine plague, or the iudulent pneumonia of entirely chronic eatarrhal nature, which charac. terizes this disease, by inhalation of pathologic lung substance as well as with pure cultures of the ovoid bacterium. However, the limited number of experiments do not appear sufficiently conclusive proof for Ostertag's contention, inasmuch as other authors always produced by inhalation an acute form of necrotic pneumonia if any disease at all.

Toxins. Novy isolated from cultures of the bacillus suisepticus a basic substance which he named "Susotoxin," and which in a watery solution in quantities of 0.125 to 0.25 ce. subcutaneously injected into rabbits produced death in 36 hours. Schweinitz isolated from the cultures a toxin and an albumose which he named "Suplagotoxine" and "Suplagoalbumin." Filtrates of fresh bouillon culture, if free from bacteria, are not toxic. On the other hand, killed masses of bacteria produce a toxic action on small test animals. (Guinea pigs are destroyed by 8 to $10 \mathrm{mg}$. of killed bacteria [Voges]). Klett \& Braun. could by their method demonstrate toxins in large quantities in the filtrates of eultures which produced the characteristic symptoms of cholera in pigeons and chickens, viz., somnolence and intoxication. At the same time the toxicity of the cultures did not run parallel with their virulence (see page 137). According to Beck \& Koske fresh filtrates of cultures produce a temporary rise in temperature while killed cultures as well as filtrates of old cultures are very toxic. McFadyean succeeded in extracting from the bacterial cells a filterable poison which was found to be very toxic for rabbits, guinea pigs and mice.

Natural infection. The experience that pure swine plague occurs usually in a sporadic form or only in a limited enzootic extension indicates that the transmission from animal to animal has only an insignificant part. On the other hand, the fact that bacteria which correspond with the true swine plague organism in all their characteristics, are present in the soil and accordingly also in the food and drinking water as well as in the air passages and intestinal canal of healthy hogs proves that the development of the disease is associated with these facultative pathogenic bacteria. It may be accepted as highly probable that, in a similar way as in the other diseases of the hemorrhagic septicemia group, the usually harmless habitants of the hog produce a pathogenic action under certain conditions, which are unknown at the present time. It is possible that the bacteria attain a higher virulence before entering the animal body so that they are capable of attacking the normal tissues of the animal. However, it probably happens more frequently that the normal resistance of the organism is diminished by various weakening influences, so that now even the less virulent bacteria may exert a pathogenic action. Lig- 
nières, Uhlenhuth and Gnüchtel proved the predisposing influence of colds by experiment, while Salmon and Rátz established the fact that the infection is facilitated by the presence of parasites in the intestinal canal and in the air passages (Ascaris lumbricoides, Echinorhynchus gigas, Strongylus paradoxus). In the presence of such parasites the bacteria may more readily penetrate the affected or injured mucous membrane. There may be also other influences, such as poor condition, exhaustion during transportation, etc., especially in young animals increasing the susceptibility to the pathogenic action of these ubiquitous organisms, which is usually not marked. The most important part, however, in the infection with the swine plague organisms may be attributed to the virus of hog cholera and the primary infection caused by it, and as a matter of fact in hog cholera outbreaks there are very frequently found changes of organs which are analogous to the swine plague infection (see hog cholera).

Ovoid bacteria which closely correspond morphologically and in their cultural characteristies with the swine plague organisms were first found in healthy animals by Smith then by Moore, Bang, Jensen, Karlinski, Kitt, Klein and Haushalter. Sometimes they were only slightly virulent, or not at all. In Jensen's experiments, however, various strains killed mice promptly and Bauermeister found that hacteria which he grew from acinous glands were very pathogenic for test animals, while, on the other hand, Karlinski as well as Beck \& Koske succeeded in markedly increasing the virulence of the originally slightly virulent strains.

While the first appearance of swine plague in healthy herds may be associated with soil infection, and also frequently with weakening influences acting upon the animals, the occasional direct or indirect infection from the first affected animals, like in the other diseases of the hemorrhagic septicemia group, cannot be left out of consideration. Affected hogs pass with their excretions, and especially with the mucus which they cough up, great numbers of very virulent bacilli which then enter the bodies of healthy hogs and may by their great numbers and high virulence produce inflammatory processes in them. In this manner an infected animal which has been purchased and added to a healthy herd may produce the disease among the other animals. As a matter of fact, however, healthy hogs resist such an infection in most instances. The fact that local outbreaks usually die out rapidly, even without any particular measures of eradication may be attributerl to a rapid diminution of the virulence of the infective agent ontside of the animal body.

As the ovoid bacteria occur also in animals affected with other diseases, or may subsequently enter such affected bodies, they may exert a specific pathogenic action in already affected organs. In this manner a preexisting catarrhal pneumonia may 
by secondary infection pass into a fibrinous or necrotic pneumonia (such cases, which are observed especially after long transportation, have been taken repeatedly as proof for the identity of the primary chronic and the superimposed acute processes).

In the pneumonic lungs of animals affected with swine plague other bacteria than the ovoid bacilli may often be found in varying numbers in the acute cases, but more so in the chronic cases. Of these streptocoeei, staphylococei, colon baeilli, bae. viscosus (Preisz), bae. pyogenis suis, bac. neerophorous and others are frequently present. They come into consideration only as secondary invaders which produce local changes in the already affeeted tissues (small suppurative centers, ete.). The presence of these organisms may be explained by the fact that they are also frequent inhabitants of the air passages, and may oceasionally multiply under abnormal conditions. Clearly different are conditions in the enzootic pneumonia of pigs, this disease being in all probability the result of a mixed infection, and the ovoid bacteria play here probably an unimportant role. This is indieated by the fact that in about one-third of the cases the demonstration of this organism from the pneumonie tissue is not possible.

The susceptibility of hogs for this disease is principally affected by their condition of health or by the reduction of their natural resistance as a result of weakening influences. Very young animals rarely become affected, otherwise age does not seem to have any influence.

Those diseases of hogs which sometimes develop in association with hemorrhagie septicemia of other animals are identical in their nature with true swine plague. Thus, simultaneous affection in hogs may espeeially be ohserved during outbreaks of hemorrhagic septicemia in cattle as well as in outbreaks of buffalo disease (see the latter). Oceasionally also hogs may be affeeted while influenza of horses is prevalent (Sehindelka), or chieken cholera (Gorges). On the other hand, in outhreaks of swine plague other species of animals may become affected. Thus Galtier ohserved affections in sheep which were kept in the same stable with affeeted hogs, or which were pastured in the same fieid where earcasses of hogs were buried, while Keleti saw lambs die during outhreaks of swine plague. Both authors demonstrated in the organs of the affected sheep hipolar baeilli (see page 119). Lastly Goldstein as well as Chamberland \& Jouan observed the death of all the chickens in a flock with manifestations of cholera-like symptoms after they had eaten from a carcass of a hog which died from swine plagne. Gorges also has ohserved geese dying after ingestion of food contaminated by affected hogs.

Anatomical Changes. In peracute cases the autopsy reveals lesions of typical hemorrhagic septicemia, viz., numerous small hemorrhages in the skin or subcutaneous fat, in the serous and mucous membranes, in the kidneys and under the endocardium, larger hemorrhages in the perirenal connective tissue, in the mucous membrane of the pelvis of the kidney and the bladder, 
as well as in the meninges. There is also acute hemorrhagic swelling of the lymph glands and gelatinous serous infiltration of the subcutaneous connective tissue, especially in the region of the throat and neck.

In the more frequent acute cases lesions in the thoracic organs are constantly present. The lungs contain either small or larger hepatized areas, of which the cut surface, according to the duration of the disease, is either blackish-red or dark brownish-red to light gray, and studded with millet seed to hemp seed sized pale vellow granules (croupous hemorrhagic or multiple necrotic pneumonia; Fig. 30). Some of the hepatized areas are surrounded by a pale yellow necrotic zone. In older cases, however, larger areas, sometimes even entire lobes of the lungs, are changed into a mass of cheesy detritus. The interlobular connective tissue is broadened and shows a serous or bloody infiltration as a result of which the cut surface has a mottled appearance. The pleura over the affected areas of the lungs is covered with loose or more adhesive fibrinous deposits under which the pleura

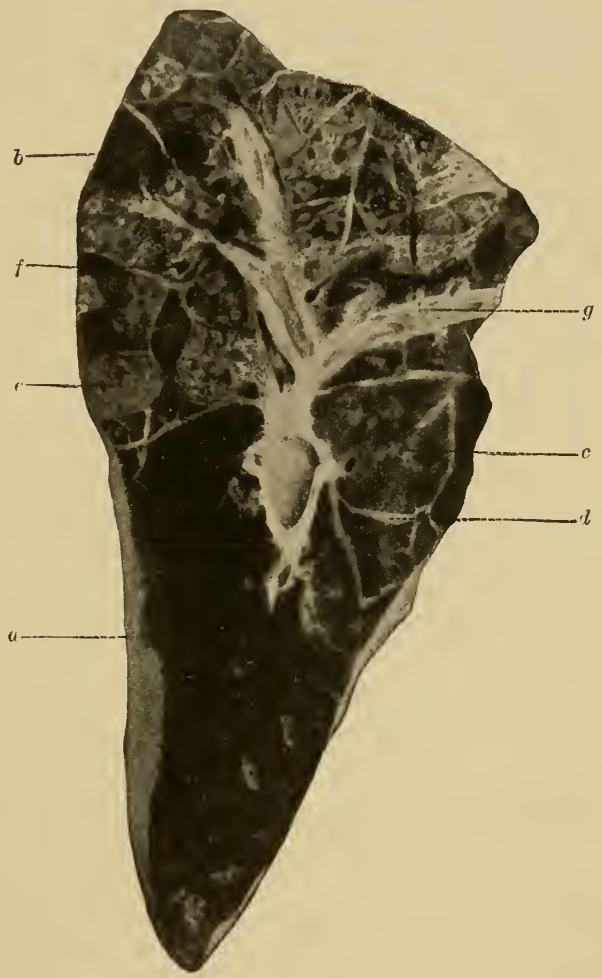

Fig. 30. Mulliple necrotic pncumonia in swine plague. (a) Congested lung tissue still containing air; (b) brownish-red hepatization; (c) grayish-red hepatization; $(d)$ dilated septum; (e) necrosis of a lobule; $(f)$ necrotic focus (center dark red, border pale yellow) ; $(g)$ bronchus. contains small

hemorrhages or shows bloody infiltration. In the thoracic cavity sero-fibrinous exudate may be present in varying quantities (exceptionally pleurisy may ocenr withont pneumonia). A similar inflammatory process exists frequently on the 
pericardium. The peribronchial lymph glands are acutely swollen, and not infrequently studded with small hemorrhages.

The mucous membrane of the stomach and intestines shows a catarrhal swelling and frequently also numerous hemorrhagic spots. In some cases the surface is covered with fine croupous pseudo-membranes, or it may appear as though sprinkled with bran as a result of the necrosis of the epithelium. These changes are especially marked in the posterior part of the small intestines or in the large intestine. The solitary follicles and the Pever's patches are swollen or contain shallow ulcers. The spleen usually shows no changes or only a slight swelling. Sometimes it contains several pale-red firm areas. The kidneys are hyperemic, and some wedge-shaped portions are sometimes grayish in color.

In the chronic cases the lungs of the greatly emaciated carcass show extensive necrotic foci without any signs of acute inflammation. Exceptionally there may be sequestra inclosed in thickwalled cavities or cavities which communicate with the bronchi. In other cases fairly large hepatized areas of the lungs are studded with numerous small yellow or gray necrotic foci.

Caseous foci may also be found in the peribronchial and mesenteric glands, in the tonsils, in some joints, in bones and in the subcutaneous connective tissue. Possibly also so-called caseous inflammation of the intestines, in which the mucous membrane of the large intestines is broken down forming a dry mushy mass, belongs to the anatomical lesions of swine plague (Schütz, Peters).

In the peracute cases the bipolar bacteria are present usually in great numbers in the blood and in all organs. In subacute cases they may be found in the affected parts of the lungs, the bronchial secretion and in the exudate of the serous membranes, sometimes also in the blood and likewise in great numbers. On the other hand, in the chronic cases they may be demonstrated only in the necrotic regions, mostly in association with other bacteria and even in such cases they may be detected only with great difficulty.

According to the histological examinations of Marek the hemorrhages develop either as a result of hacterial emboli or as a result of a toxic affection of the walls of the blood vessels (the latter especially in the kidneys). The infarcts of the lungs and kidneys may also result from thrombosis of blood vessels. The pneumonia is rarely catarrhal but more frequently croupous, developing in its later course a coagulation necrosis. Coagulation necrosis - may also be observed in small foci in the liver and in the adrenals. The nephritis, which is present in all acute cases, leads to parenchymatous degeneration of the epithelial cells, serous infiltration of the interstitial tissue, hemorrhages and necrotic foci.

Symptoms. After artificial infections by subcutaneous or intratracheal injections or by inhalation of virulent material, the time of incuhation is usually only a few hours or one to 
two days. Under natural conditions also it is probably very short, particularly if some predisposing factor exists.

The peracute cases of the disease manifest the typical symptoms of hemorrhagic septicemia. As the fever rises to $40^{\circ} \mathrm{C}$ and over, the animals become weak, lose their appetite and lie motionless on the ground. In the pasture they separate from the herd, and in the stable they usually crawl under the straw. If urged to move they have a staggering gait, may move in a circle or execute other forms of involuntary movements. Red spots develop on different parts of the body, especially under the ears, on the neck and on the rumps which do not disappear on pressure, and hemorrhages may occur from the nose, the intestines and urinary organs. In some animals symptoms of a severe acute pharyngitis may be observed. The patients usually die in 12 to 24 hours after the appearance of the first symptoms.

In the acute course the clinical picture corresponds in the majority of cases with that of acute pneumonia. The fevered animals have a short, dry and spasmodic cough, assume frequently squatting position like dogs, while slimy material is expelled from their mouths. The respiration is accelerated and labored, especially during expiration, the animal frequently standing with legs spread apart and with mouth open. The patient manifests pain on palpation of the thorax. The nose discharges a sticky, slimy secretion. Later the paroxysms of coughing become more frequent and more severe; the respiration is very labored, rattling, the temperature remaining constantly around $41^{\circ} \mathrm{C}$. The animals become visibly emaciated and finally they can hardly rise from the ground.

The mucous membranes are cyanotic, a purulent conjunctivitis may sometimes be observed. The heart action is accelerated, later throbbing. There is at first constipation, later diarrhea sometimes with bloody excrement. Towards the termination of the disease red spots may also be observed on the skin.

The duration of the acute form usually extends from 1 to 2 weeks. Complete recovery is very rare, although occasionally the condition temporarily improves, but usually it passes into the chronic form of the disease.

The chronic form usually follows the acute. After the subsidence of the acute symptoms indications of a lung affection with periodical coughing and difficulty in respiration will persist for a long time. In such cases the animals show disturbed appetite, progressive emaciation, and occasionally chronic inflammation develops in the joints. Towards the termination of these cases also there is fetid diarrhea, and the animals die in from 3 to 6 weeks from complete exhaustion. Not infrequently, however, the affection in the lungs remains stationary and the animals may even be fattened in spite of the encapsulated foci in these organs. 
In the swine plague observed by Borzoni in Sardinia (see page 126) one form of the disease is manifested by symptoms of a general febrile affection, greenish fetid diarrhea and red spots on various parts of the body, as well as by a very pronounced acute edematous swelling of the throat region. The other form commences with a marked bloody serous infiltration of the subeutis on the extremities, with which an inflammatory swelling of the neck becomes associated in the course of a few days. The autopsy shows bloody serous infiltration of the subcutaneous and intramuscular connective tissue and of the tongue, and in addition to this only an acute swelling of the lymph glands and a slight intestinal catarrh.

Diagnosis. The peracute septicemic form of the disease may be mistaken for acute hog cholera, from which it can be distinguished only by the demonstration of the bipolar bacilli in the blood.

The recognition of the lung affection in process of development is quite easy but the diagnosis of its exact nature is much more difficult. Verminous bronchitis is very similar in its manifestations. This, however, has a much slower course and attacks usually only young animals.

The greatest difficulty is met with in differentiating swine plague from hog cholera. The sporadic appearance of the disease or its confinement to individual premises, with symptoms pointing to an affection of the lungs, are indicative of swine plague. Howerer, if there exists a possibility of hog cholera or the very frequent mixed infection its differentiation in the living animal is impossible.

On autopsy pure swine plague may be accepted with a certain probability when in addition to the described changes in the lungs (acute croupous-hemorrhagic or necrotic pneumonia), the hemorrhages and acute swelling of the lymph glands, there are no diphtheritic or caseous inflammatory areas present. The differentiation from hog cholera even in such cases is absolutely established only by filtration experiments, in which positive results are indicative of the presence of hog cholera. On the other hand a negative finding does not establish the absence of hog cholera, inasmuch as the virus of the latter disease may already have disappeared from the body either partly or entirely. In such cases the sporadic character of the disease in herds which otherwise continue to remain healthy speaks for pure swine plague. The epizootic character should therefore be given a weighty consideration in the establishment of a diagnosis.

Less difficult appears the differentiation from catarrhal pneumonia of pigs. This affection occurs only in very young animals; the pneumonia has a purely catarrhal form, with or without fibrinous exudate. This applies also to those cases in which the affection proves clinically and anatomically to have commenced with a chronic catarrhal form of pneumonia, and only later developed into an acute form. In such cases, the 
upper and posterior parts of the lungs usually show in addition to catarrhal pneumonia a croupous-catarrhal inflammation.

The demonstration of bipolar ovoid bacilli even though they are highly virulent has only a very restricted and subordinate diagnostic value. Their presence is of itself by no means sufficient to establish the diagnosis of swine plague, as these bacilli may be present quite accidentally or may have taken only a secondary part in the development of the pathological processes. Only if they are present in great numbers and exclusively, or almost exclusively, in the inflammatory products, may they be accepted as the direct causative agents of the disease and even in such cases the possibility must be considered that the disease was caused primarily by the much more vigorous hog cholera virus.

Swine erysipelas usually runs a more rapid course and manifestations of pneumonia are absent. On autopsy this disease may be distinguished, aside from the different bacteriological findings, by the absence of post mortem lesions. In chronic cases tuberculosis comes into consideration and the positive differentiation is only possible by a bacteriological examination. The pyobacillosis of pigs or its complications are characterized by purulent catarrh as well as yellow or greenish suppurative areas in the lungs and in other organs, by which it may be differentiated from swine plague.

Treatment and Prevention. Medicinal treatment has up to date proved without effect.

Becker, Spitzer and Szöllös reported favorable results with the proprietary remedy called suptol and prepared by Burow (according to Andrejew it is a preparation of swine plague bacilli). On the other hand Tátray employed this remedy in 17 herds on several thousand animals with varying results, which agrees with the findings of the Prussian Official Veterinarians. Andrejew and Gellmann deny its protective and curative action.

Immediate separation of the affected animals, effective disinfection of stable and feeding trough, destruction of the affected organs and secretions and of the manure appears to be advisable; even though the danger of infection is slight, nevertheless it should be considered. From a standpoint of veterinary police it is advisable to separate swine plague from hog cholera, and to treat it in the same manner as hemorrhagic septicemia of cattle.

Immunization. Since the etiology of hog cholera has been studied more closely the former attempts of elaborating effective methods of immunization against this disease, as well as against swine plague which is frequently associated with it, from the cultures of the bacillus suisepticus, have proven of very doubtful practical value. Considering that an immuniza- 
tion against pure swine plague does not appear necessary, because of its rather harmless character, vaccines prepared from this organism could at best be considered as a probable protection against the secondary infections of hog cholera, and also perhaps to a certain degree as protective against the pneumonia of pigs depending on a mixed infection with the swine plague organism. Nevertheless the numerous experiments for the establishment of a method of vaccination which would protect against the infection with the bacillus suisepticus have a practical importance inasmuch as some of them, especially the inoculation with immune sera against the so-called chronic swine plagne of pigs (see page 142), are extensively employed in Germany.

1. Active Immunization. In rabbits and guinea pigs this may be attained with cultures killed at $58^{\circ} \mathrm{C}$. (Smith \& Moore), as well as with the toxic substances isolated from the cultures (Suplagotoxin and Saplagoalbumin (see page 128), with which Schweinitz succeeded in protecting hogs against artificial infection.

More recently Veil immunized hogs with an exudate (aggressin) obtained from infected rablits and hogs of which 10 ce. injected in two parts or as one dose into the blood afforded a protection agamst a lethal artificial infection. In order to protect the animals against natural infection, which is especially dangerous at the commencement of the treatment, the simultaneous use of antiaggressive immune serum is recommended. Similar favorable results were obtained by Citron in rabbits and guinea pigs with exudate as well as with extracts from cultures, and animals immunized by his method withstood a hundred thousand times larger dose than that necessary for fatal infection. Titze employed shake-extracts of tiltrates made from cultures, and recently Miessner \& Schern used pleural exudate and heart blood from rabhits containing aggressins and sterilized at $44^{\circ} \mathrm{C}$. with which they produced an active immunity in guinea pigs against virulent infection with swine plague bacteria.

Beck \& Koske produced an active immunity in pigs by injecting them intraperitoneally first with 0.5 ce. of fresh bouillon culture heated to $55^{\circ} \mathrm{C}$. for 20 minutes, and seven days later with $1 \mathrm{cc}$. of virulent culture into the muscles of the thigh. Broll, however, found that the animals occasionally became fatally affected with peritonitis and enteritis as a result of the first infection, due to the poisonous toxic action of the killed bacilli, and further that the vaccination in infected herds favored the development of new cases before immunity had been established.

According to Broll's experiments the administration of living fowl cholera bacteria, or of such killed by heating for 20 hours at $52^{\circ}$ to $55^{\circ} \mathrm{C}$. does not produce a sufficient resistance against swine plague infection, whereas swine plague bacteria killed in a similar manner produced in pigs a high grade of protection. The best results against artificial infection were obtained with a 48-hour shake-extract prepared by the method of Wassermann \& Citron. The extract is not filtered, and is sterilized by the addition of mustard oil. The vaccination of new horn pigs is recommended with such extract and immune serum (the serum is supposed to prevent an infection during the negative phase), 
and in ease the protection is not sufficient the animals may be revaccinated shortly before weaning with the bacterial extract.

2. Serum Immunization. Blood serum from animals treated with virulent cultures of the bacillus suisepticus protects test animals for a short time against a subsequent virulent infection. As a result of these experimental findings serum of horses hyperimmunized by subcutaneous and intravenous injection of increasing doses of virus has been used in numerous instances for the immunization of hogs (see also page 141).

Schweinitz had already in 1890 immunized horses, mules and cattle with either living or dead cultures, as well as with filtrates, and also with toxic substances isolated from cultures. He proved that the serum of such immunized animals afforded a protective action against an infection with the bacillus suisepticus or against the action of its toxins. The serum was usually employed together with the hog cholera serum against the mixed infection, which occurs in America, but with doubtful results.

Later Schreiber obtained an immune serum from horses treated with cultures, which was placed on the German market under the name "Septizidin." According to his views his product possesses both a protective and also a curative action, and is often used against the septic pneumonia of ealves.

Polyvalent immune serum. According to Wassermann \& Ostertag blood serum of artificially immunized animals usually protects only against the variety of the bacillus suisepticus which had heen used for the immunization. On the other hand its action against the other varieties of this organism is ineffective in spite of the fact that morphologically and in their eultural characteristies they are identical. According to their conception the protoplasm of the bacterial cell consists of different components which, in the different varieties of the same bacterium, are to some extent not identical. Besides a eommon domimant receptor which is to be considered as the carrier of the special characteristies of the species of bacteria, the different varieties may contain individually different side-receptors. Inasmuch as during the process of immunization the various components produce corresponding immune bodies (amboceptors) by binding the receptors adapted to them (Ehrlich's theory), all these immune bodies bind the dominant receptor; but the side-receptors of the variety which have been used for the immunization, thereby afford a protection only against the latter, and are ineffective against varieties containing other sidereceptors. The above mentioned authors accordingly treated horses with as many varieties as possible of the bacillus suisepticus and found that the serum proved effective a'gainst all the different varieties. This polyvalent or multipartial serum with a titre of not less than 0.01 ee. for mice against a dose ten times the fatal dose of a living culture has been used especially against the pneumonia of pigs.

Immunization with chicken cholera serum. Klett \& Braun employ serum of horses which have been treated for a long period with a mxiture of toxins from killed cultures and their filtrates, and which afterward have been inoculated with fully virulent chicken cholera bacteria. These authors contend that the bacilli of fowl cholera produce the same toxin, only in larger quantities, as the bacilli of swine plague. Serum from horses treated in the above manner protects gray mice, in quantities of $0.0015-0.005 \mathrm{gm}$, , against a fatal infection of a loopful of virulent 
swine plague eulture, while if the serum treatment has been given 24 hours previously, it also has projective action against the toxins of chicken cholera and swine plague bacteria. The passive imniunity lasts from 3 to 4 weeks, while active immunity could not be produced even in mice by serum and culture injections.

Literature. Löffler, Arb. d. G.-A., 1886, I, 46.-Schütz, A. f. Tk., I886, XII, 210.- Salmon \& Smith, Bur. of Anim. Ind., 1886-1894. Smith, Spec. rep. of the cause of swine-plague, Washington, 1891.-Preisz, Z. f. Tm., 1898, II, 1, u. 1907, XI, 161.-Lignières, Bull., 1900, 389 (Lit.).-Beck \& Koske, Arb. d. G.-A., 1905, XXII, 181.-Joest, Schweineseuche u. Schweinepest, Jena 1906 (Complete Lit.).-Ostertag \& Staılie, Z. f. Infkr., 1907, 1I, 112, u. 425.-Hutyra, ibid., pp. 281, and III, 235.-Citron, Z. f. Hyg., 1906, XLI, 238.-Uhlenhuth, Xylander, Hübener \& Bohtz, Arb. d. G.-A., 1908, XXVII, I (Lit.).-Broll, Z. f. Infkrkh, 1908, V, 81 (Lit. on immunization).-Gnüchtel, Diss. Leipzig 1909 (Lit.).

Enzootic Pneumonia of Pigs (so-called chronic swine plague of pigs). According to the view of Ostertag, which is also accepted by a great number of other investigators, the classical form of swine plague described above has changed since the middle of the nineties of the last century to a milder form, so that at the present time it occurs mostly as a chronic pneumonia affecting only very young pigs. Among these young animals, however, it occurs widely distributed. According to this conception the disease is generally known as "chronic swine plague of pigs," but in some localities it is also designated as pig disease, pig cough and cement disease (as it occurs frequently in new stables with cement floors).

The disease causes considerable loss, especially among the improved breeas, and occurs principally in Northern Germany (Eastern and Western Prussia, Silesia, Brandenburg, Pommerania, Schleswig-Holstein, Hessen-Nassau, etc.). Thus for instance in the abbatoir of Hamburg about 50,000 hogs annually show the anatomical lesions of the disease.

The symptoms of the disease develop sometimes early in the first days of life, more commonly, however, between the second and fourth week after birth. The animals lose their sprightliness and appetite, sometimes manifesting also a perverted appetite (eating of manure, filth, etc.). A catarrh of the nose (frequent sneezing and blowing, especially during eating), as well as of the deeper air passages, frequently develops. The young animals have a short, sharp spasmodic cough which is especially brought on by rising and eating. The cough sometimes is associated with rattling and choking. At the same time the respiration is more or less accelerated and labored, the animals in the more severe affections lying on the sternum or on the side, or they stand with arched back and legs spread apart.

Of the other symptoms conjunctivitis, muco-purulent nasal discharge, and frequently a peculiar skin eruption may be observed. This is manifested in the form of red spots on the head, on the ears and on the sides of the body, which show a slight exudation and soon become covered with scabs and scales. In other cases vesicles form which after bursting also become covered with scabs, so that finally the dry and exfoliated skin appears covered to a great extent with blackish-brown crust.

With the advance of these symptoms the affected animals are retarded in their development, the appetite being changeable or continually absent. Subacute inflammations appear in the different joints, especially in the knee and elbow joints, as a result of which the animals walk with evident pain and stiffness, and after the appearance of diarrhea and bloating the completely exhausted patients usually die in the course of the second or third month of their lives. 
In the cases terminating in recovery the animals become gradually brighter, the cough becomes less frequent, the scabs fall off, the skin regains its normal color, the appetite also improves, and the food is utilized in the normal manner. Such animals are considered as recovered; according to Foth, however, this is not the case as the cough never disappears entirely. On the other hand, animals which are still sickly may, under new conditions, become again affected with the acute manifestation, especially if exposed to cold in the presence of digestive disturbances or after infections with virulent pathogenic germs. In such relapses the animals usually die from pleural pneumonia or pericarditis (Hinrichsen).

In pigs about 4 weeks old the course of the disease may be so unfavorable that 50 to $80 \%$ of the affected animals succumb. On the other hand, the mortality in older animals hardly ever exceeds 1 to $2 \%$. The average loss is about $10 \%$, while an additional $10 \%$ remain runts. The majority, however, become only slightly affected (Grips, Glage \& Nieberle).

The anatomical changes consist principally in a chronic bronchopneumonia, frequently associated with a sero-fibrinous inflammation of the serous membranes. The autopsy reveals in the lower anterior part of the lungs, either in a large portion or only at the borders, wedge-shaped sharply circumscribed hepatized areas which have a peculiar semi-solid consistency, or the organ may be similar to the normal consistence of the pancreas (Joest). On the cut surface the affected lung is grayish-red, smooth, moist or somewhat dry, and studded with yellowish spots. At the same time the plenra over the corresponding areas is lusterless and rough or covered with fibrinous membranes which cause adhesion of the anterior lobes, and also with the costal pleura. Between the pleural layers a reddish-yellow fluid may be present in small quantities which may also contain fibrin-coagulums. Similar inflammatory changes are not infrequently found also on the pericardium. The recovered cases often show only adhesions of the serous membranes, some atelectic areas on the borders of the lungs and hardening of the thoracic lymph glands. The bronchi are usually filled with a muco-purulent secretion.

In some of the cases the affected areas of the lungs contain greenish purulent foci, and such may also be found in other organs (see pyobacillosis, page 141).

According to the histological examinations of Lüpke and Joest the changes in the lungs are, at the incipient stages of the disease, those of a typical catarrhal pneumonia, while later the productive granulating character becomes more and more prominent in which cell forms predominate such as are found in inflanmatory granulation tissue.

The bacteriological examination always reveals various kinds of bacteria in the affected parts of the lungs. The bacillus suisepticus is present in about two-thirds of all cases. Frequently, however, they are few in number and of a very slight virulence, so that it is possible to kill test animals only with large quantities of suspected material. Simultaneous with this bacillus, but oceasionally without it, streptococei and staphylococci, coli bacilli, bacillus pyocyaneus, further in about $60 \%$ of the cases the bac. pyogenes suis may be found.

The varying bacteriological findings and especially the frequent absence of the bacillus suisepticus in the pneumonic tissues make its exclusive primary etiological importance very doubtful. It appears more probable that the pneumonia 
of pigs commences with catarrhal processes of the air passages which develop in a broncho-pneumonia when the bacteria which are normally present in the respiratory passages, among them also the bac. suisepticus, will subsequently multiply and aggravate the inflammatory processes; that the bac. suisepticus is not the true causative agent of the diseave is further substantiated by the fact that this organism, at least in the artificial infections, always produces acute inflammatory and later necrotic processes, and consequently the anatomical lesions of the pneumonia of pigs do not correspond with the pathogenic characteristics of this bacillus (see also chapter, Enzootic Inflammations of the Lungs of Young Animals, in Fol. 1I.). For the correctness of the conception that the former acute swine plague has changed in Germany to a chronic eatarrhal pneumonia, there are no convincing proofs. It is only established that in certain localities this disease occurs quite extensively; it is, however, probable that the disease existed there in former times. The occurrence of the disease is in the first place influenced by local conditions and especially by exposure of the delicate young animals to cold moist stables, and possibly also by attempts to improve the breed by intensive breeding, by the confinement of too many animals in elose quarters, the feeding of ereamery products, ete.

Uhlenhuth and his co-workers have proven experimentally the correctness of the conception that the catarrhal affection of the lungs in question is independent of swine plague, and that the lisease may develop without the cooperation of the bac. suisepticus in animals in which the vitality has been reduced. They placed pigs in enameled troughs or in sheds in which the floor was covered with cinders which were frequently moistened. These animals became affected with a typical eatarrhal pneumonia and the lung tissue contained only streptococei or staphylococci.

The infection from animal to animal may also play an important part in the spread of the disease. The animals weakened by the debilitating influences above mentioned will be renclered more susceptible to infections, if they ingest, with the expectoration and excrements of affected animals, various kinds of facultative pathogenic bacteria in large numbers. Such organisms originating from affectel organs possess usually a high virulence compared with the organisms of the same species present in the air passages of healthy animals. An infection of adult animals through affected young hogs has not yet been established.

The recent findings of Frosch \& Broll regarding the nature of the enzootic pneumonia of pigs cannot yet be accepted as conclusive. They succeeded in producing hog cholera in pigs with filtered and unfiltered material of affected pigs from 4 to 6 weeks old, although it is known from experience that animals of that age very rarely becone affected with hog cholera.

The medicinal treatment promises no results. On the other hand, improvement of the hygienic conditions and good nutrition have a favorable influence on the course of the disease.

A proprietary remedy which is marketed in Germany under the name of "Wassermann's Vaceine for the Cure of Swine Plague", (aggressin preparation?) gave Mucha, Raebiger, Willenberg and Dien satisfactory results.

According to Ostertag in the prevention of the disease, which should extend also to the protection of the healthy animals in the herd where the disease has already appeared, great caution should be exercised in the purchase of animals in limiting to some extent the inhreeding, keeping of the pigs in warm and dry stables with access if possible to outside range. For parturition the animals should he placed in separate stables (cow or sheep stables), the pigs should not he weaned too soon, those pigs stunted in growth should be removed, and lastly the stables and stable utensils should be regularly disinfected with a $2 \%$ hot soda solution.

Evers considers frequent disinfection as a very difficult proceslure in practice, and in the winter time not without danger; on the other hand, he lays especial stress on the proper building of isolation stables as well as the destruction of all runts.

Immunization. In Germany the polyvalent swine splague serum of Wassermann \& Ostertag is used quite extensively (sce page 137). The 
results cannot be estimated satisfactorily, but it appears that it gives especially good results in the places where proper care is taken in general for the welfare of the pigs.

According to Ostertag's report in 218 herds which were exclusively affected with swine plague in $1902,7,944$ hogs under 3 months and 2,235 older animals were inoculated. Of these, 633 young pigs $(7.9 \%)$ and 11 (.5\%) old hogs died, 47 $(.6 \%)$ young and $42(1.9 \%)$ old hogs had to be slaughtered in emergeney. 376 $(4.7 \%)$ young and $28(1.2 \%)$ old hogs became stunted in growth, while $6,8 \times 8$ $(87.7 \%)$ young and $2,757(96.4 \%)$ old hogs remained healthy. In some of the herds in which before the vaccination 50 to $75 \%$ or almost all pigs died, the disease almost entirely ceased after the vaccination, and in numerous formerly infected herds breeding became again satisfactory and remunerative after the vaccination. According to Wassermann 17,769 pigs were vaccinated in the years of 1903 and 1904, in infected herds of which in the animals up to 3 months of age $90.7 \%$ remained healthy, while of 1,490 older hogs $94.3 \%$ also remainer heaithy.

It is advisable to vaccinate the pigs on the first day of their lives anil to repeat it shortly before weaning, at which stage the animals are most susceptible to the infection, and are also especially exposed. The injection may be repeaterl when one inoculation fails to produce a sufficient protection and the lisease las newly appeared. The inoculation of the affected animals is unsatisfactory. The dose of the serum is from 3 to $\bar{j} \mathrm{ce}$.

Raebiger as well as Joest anil Ripke also reported favorable results from the polyvalent serum (of 2,227 pigs $19.5 \%$ remaineil healthy). The reports of the Prussian district veterinarians (1902-1906) indicate partly favorable and partly less favorable results.

Immunization with extracts of swine plague bacteria and immune serum (see page 137) gave Pfeil and Diem favorable results, while Majewski and Klipstein failed to obtain such. The preparation known as Euman appears to be prepared from cultures of the bac. pyogenes suis.

Literature. Ostertag, Z. f. Flhyg., 1905, XV, 266; B. t. W., 1905, 205 и. 234; Z. f. Infkr., 1907, II, 113, u. 425.-Grips, Glage \& Nieberle, F. d. Vhyg., 1904, II, 5 (Lit.).-Hutyra, Z. f. Infkr., 1907, II, 281 u. III, 235.-Evers, Sehweinepest. Jena 1906 (Lit.).-- Uhlenhuth, Xylander, Hübener \& Bohtz, Arb. d. G.-A., 1908, XVIII, I (Lit.).

Pyobacillosis of Hogs. (Pyemic cachexia). Grips claimed in 1903 that the cause of swine plague is not the ovoid septicemia bacterium described by Löffler \& Schütz, but an organism which he found in the encapsulated caseous foci of the lungs and named bacillus pyogenes suis. Then Grips, Glage \& Nieberle attempted also to prove that the enzootic pneumonia of pigs which occurs extensively among the pigs of Northern and Northwestern Germany, and which is characterized by catarrhal suppuration and severe inflammation, by nervous symptoms, disturbances of nutrition and skin eruptions (see page 138), should be considered as the true swine plague, and that it is caused by the above mentioned organisin, whereas Löffler's ovoid bacteria only occasionally cause acute complications in the normal course of the disease.

This view stimulated O1t, Casper, Ostertag, Pütz and Preisz to undertake bacteriological work in this line, and they proved the incorrectness of the above conecption. They have proven that the bac. pyogenes suis produces suppurative processes in the lung tissue only secondarily, but on the other hand they showed that the new bacillus may also produce similar processes in other organs. Its biological characteristics were studied more extensively by Berger and Holth.

In most of the cases the disease represents the enzootic pneumonia of pigs, known also as chronie swine plague $e_{0}$ in which there is a more 
profuse muco-purulent nasal discharge, a complication of inflammations of the joints and abscesses in various parts of the body as a result of associated infection with the bac. pyogenes.

In such cases the autopsy reveals a pronounced purulent bronchitis, and numerous small greenish suppurative foci which are in the lung tissue enclosed in connective tissue capsules. Sometimes nodules of the size of a hen 's egg may be present which consist of a purulent mass, at times dry at the periphery, softened towards the center, or which may be entirely purulent. The contents of the nodules may easily be removed from the connective tissue capsule.

The intestines, especially the large intestines, frequently manifest a catarrhal purulent inflammation; in the submucous tissue there may frequently be found nodules in size from a hazel to a walnut, with dense fibrous walls and greenish caseous contents. The nodules cause the intestinal wall and the mucosa to bulge, and over them the serous membrane frecuently shows inflammatory changes.

Oceasionally generalization of the pathological process may oceur in which case the various tissues may become affected more or less frequently in the order given; muscles, joints, tendon sheaths, subcutaneous fat, liver, spleen, lymph glands, bones, more rarely the

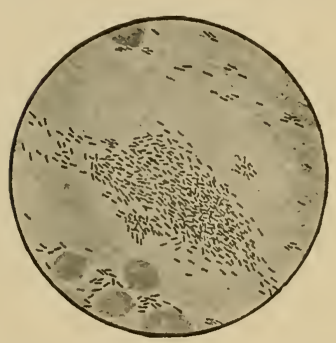

Fig. 31. Bacillus pyogenes suis GRIPS. Bronchial mueus from a hog. Carbol-fuchsin staining; and subserguent washing with $1 \%$ acetic acid. kidneys may contain greenish-yellow suppurative foci. The exudates are usually thin, mucous, of whitish-yellow or reddishgray color, frefquently with a greenish hue.

The bac. pyogenes suis which is recognized by Grips, Glage \& Nieherle as at least the principal cause of these suppurative processes, is present in the bronchial secretion (see Fig. 31), in the exudate of the serous cavities and in the suppurative foci. It is usually associated with other bacteria and among these also with Löffler's bipolar bacillus, more rarely it may be present in great quantities in pure cultures (especially in greenish pus). It is also found in great numbers in the intestinal contents, while in older suppurative foci the bacilli are present only in small numbers, or may be entirely absent.

According to the investigations of Glage, Berger and Holth the bacillus is identical with the organism deseribed by Poels (1897) as the eause of arthritis in calves and known as the polyarthritis bacillus as well as with the bac. pyogenes bovis which was found by Künnemann in abcesses of cattle (see page 144). Dunkel considers it identical with the bac. pseudotuberculosis ovis (see that disease).

In hogs Puitz found the organism in inflamed tissues and in the secretion of the bronchi in $36 \%$ of the cases; in necrotic foci of the lungs in $50 \%$; in softened purulent norlules in $100 \%$ of the cases. Preisz demonstrated the organism in 16 out of 77 eases of acute swine plague pneumonia. Olt on the other hand proved that the hacillus is present in great numbers in the pale gray mushy plugs in the tonsils of healthy hogs.

The bacillus represents delicate non-motile and sporeless rods, which in the exudate resemble the erysipelas bacillus (Fig. 31), but in cultures appear somewhat shorter and sometimes elub shaped. It stains with the basic anilin dyes, and especially well with carboi fuchsin which stains it uniformly and intensely, after a sufficiently long action of Lugol's solution; it may also be stained by Gram. 
The cultivation, although somewhat difficult, succeeds aerobically as well as anaerobically, but only at temperatures of between 24 and $40^{\circ} \mathrm{C}$., (the best growth being obtained at $37^{\circ} \mathrm{C}$.), and only in the presence of proteid. As nutritive media coagulated horse, cattle or hog blood serum is most suitable on the surface of which fine bluish points, and corresponding with these through liquefaction of the medium, small cup-shape depressions develop in from 2 to 7 days. Stab cultures in coagulated serum show. a grayish thread consisting of little round colonies or a continuous growth, which, after liquefaction of the serum, soon sink down and form a flaky gray mass. On serum agar small transparent drop-like colonies develop in the stab which confluate, forming a lustrous transparent thin layer. On serum agar plates the colonies form small thorn apple-like and later round colonies. Luxuriant growth is obtained in milk which commences to coagulate from the bottom up in 48 hours, and finally turns to a uniform whey from which a water-clear serum separates later.

The resistance of the bac. pyogenes is very slight. In cultures it does not remain active longer than from 2 to 3 months. A temperature of $57^{\circ} \mathrm{C}$., drying, formalin vapors or sulphuric acid kill it rapidly. (Pütz, Berger).

According to the investigations of Grips, Glage \& Nieberle the pathogenic characteristics of the organism develop in small test animals only after the injection of large doses of cultures, in which case they produce suppurative processes. In sucking pigs the feeding of purc cultures produces a chronic gastro-intestinal eatarrh, which, especially during weaning time, results in fatal diarrhea. In addition atelectasis, exudative pleurisy and peritonitis as well as nervous attacks may develop not infrequently. The animals which recover from the disease remain runts. Intratracheal injection produces suppurative bronchitis. Subcutaneous injection causes a spontaneously perforating abscess at the point of inoculation. In older weaned pigs the action of the inoculation is manifested in similar changes, only that in this ease also skin lesions in the form of pox-like eruptions and scabs are frequently observed. Intrapulmonary injections result in catarrhal pneumonia, exudative pleuritis or abscess formations surrounded by a hepatized area. Metastatic lesions may develop in the joints. Intrapleural injections cause a specific pleuritis with catarrhal pneumonia in the adjoining lung tissue, as well as chronic pericarditis. Intraperitoneal injections produce a specific peritonitis, while intravenous injections result in a fatal pyemia. Healthy pigs which are placed in contact with experimentally infected animals become stunted in growth as a result of chronic gastritis.

The later experiments of Pütz, Berger \& Holth gave practically the same results, as they have proved that the pyobacillus prcduces suppurative inflammation in the animal tissues, which is characterized by its chronic and slow character. A marked prolification similar to the condition occurring in actinomycosis, precedes the breaking down of the tissues (Holth). The authors mentioned above failed in producing catarrhal pneumonia in hogs by inhalations or tracheal injections of eultures.

According to these experiments the diseased condition occurs in hogs, especially in young pigs, principally associated with suppurative processes. They are caused by specific bacilli and may be artificially produced in healthy animals. The nature of the anatomical changes 
consists in a specific chronic granular suppurative inflammation which sometimes is confined to the digestive tract, at other times affecting also the mucous membranes of the air passages and especially the bronchi, in which case the lungs develop a catarrhal suppurative or a purely suppurative inflammation. With the affection of the mucous membrane an exudative inflammation of the serous membranes becomes associated and a generalization of the suppurative processes may also take place.

The changes, however, do not correspond with those pathological processes which have customarily and undoubtedly with justice been considered as swine plague lesions. Acute catarrhal croupous or necrotic pneumonia, such as is observed in acute outbreaks of pure swine plague, as well as in a more frequent mixed infection with hog cholera, has in no case been produced in the above experiments. These facts have been pointed out by Olt, Casper and Ostertag (the changes which were indicated as such were always atelectatic). The bacillus pyogenes suis exerts in all cases purely pyogenic properties.

According to Olt the disease caused by the pyo-bacillus and designated by him as "pyemic cachexia of hogs" (the name pyobacillosis was suggested by Lüpge) occurs in the majority of cases as an independent affection, but also in association with, or as a result of other infective diseases. Contrary to this Ostertag maintains, without disputing the independent occurrence of pyobacillosis, that most cases of the affections of the lungs described by Grips are chronic affections of swine plague eaused by ovoid bacteria. He claims that in the course of the disease the Grips bacillus as well as other bacteria may localize especially in the bronchial mucous membrane, and this is the inore possible as it is known that this organism occurs as a saprophyte in the upper air passages of healthy hogs. The primary etiological part. the ovoid bacterium, in pneumonia of pigs has been authoritatively established in accordance with the description on page 139. Considering, however, the fact that the pyo-bacillus occurs in pure cultures only in metastatic abscesses, while in the pneumonic lungs it is always present in association with other pathogenic bacteria, it probably takes only a secondary part in the lung affections of hogs. In such eases it is the eause of severe changes, especially of extensive suppurations.

The pyo-bacillus exerts a similar action in the suppurative processes of ruminants. According to the investigations of Poels, Künnemann, Roux, Holth and Olt it occurs in inflammation of different organs in cattle (Poels found the organism in 38 out of 56 cases). However, it is mostly present in association with staphylococei, streptococci, colon bacilli, necrophorous bacilli and other bacteria. It occurs especially in cases of peritonitis caused by foreign bodies, in bacillary pyelonephritis, metritis, to which inflammation of the valves of the heart may be added; further in arthritis in calves, various broncho-pneumonias in young as well as in old animals; finally in mastitis, especially in those cases which are associated with septic and catarrhal metritis (according to Holth the presence of the bacillus pyogenes is especially indicated by a thin milk-like secretion of the ndder with dense yellow irregular flakes; further by a purulent fetid secretion). As a causative agent of mastitis it has been established in goats (Dammann \& Freese, Olt) and in sheep (Olt). Olt also found the organism in broncho-pneumonia of lambs and sheep in which especially the terminating bronchi were in parts almost entirely obstructed with masses of bacilli. 
Literature. Grips, Diss., Giessen 1902.-Künnemann, A. f. Tk., 1903, XXIX, 128.-Grips, Glage \& Nieberle, F. d. Vhyg., 1904, II, 5 (Lit.).-Olt, D. t. W., 1904, 325; 1908, 617 (Lit.).- Ostertag, 1bid., 1903, 185.-Pütz, Diss., Giessen 1904.-Koske, Arb. d. G.-A., 1906, XXIV, 1s1.-Berger, Z. f. Infkr., 1907, III, 101.-Holth, Ibid., p. 155 (Lit.).-Dunkel, Z. f. Bakt., 1909, LIII, 61.

6. Septicemic Affections of New Born Animals. The acute septicemic affections of the newborn may clinically be collected into two groups. In the first group all those affections are considered in which manifestations of an acute gastro-intestinal catarrh are present, and its most conspicuous symptom is diarrhea, while the affections of the second group are characterized by suppurative inflammatory processes in various organs and about the body, especially in the joints and extremities. In spite of the variance of the symptoms the more recent investigations indicate with great probability that the same bacteria produce the diseases belonging to both these groups. It is also probable that several species are concerned in the etiology of the diseases and that the differences in the course of the disease depend especially on the mode of infection.

While the two forms of disease are apparently very closely related, or possibly even identical in their etiological relation, we consider that the question has not been completely solved, especially as in some of the enzootics frequently only one or the other form comes under observation. Accordingly from a practical standpoint it is deemed advisable in discussing the diseases to separate the forms from each other.

\section{(a) White Scours of Sucklings: Dysenteria neonatorum}

\section{(Diarrhea neonatorum, Diarrhée des nouveaunés [French]; Ruhr der Säuglinge [German].)}

White scours of newly born is an acute, contagious, infectious disease affecting the animals in the first days of their lives, and is observed usually as a stable affection characterized by profuse diarrhea and by rapid exhaustion.

History. The cause of this dangerous and fatal disease has been thought until recently to be either due to dietetic errors, especially as a result of feeding milk of improper constitution (Tolnay 1799), and also caused by improper feeding of the mother animal, or else as a result of climatic conditions. Based on this supposition the affection has been associated with the gastro-intestinal catarrh of adults which results from similar causes. However, Obich (1865) and Roloff (1875) proved that the disease is transmissible from stable to stable, and further investigations proved beyond a doubt the infectious nature of the disease. Frank (1876) concurred in these findings, and at the present time this view is generally accepted. The recent investigations of Poels (1899) and Joest (1902), and especially those of Jensen (1893 and 1905) have cleared the etiology of white scours in most points. 
Occurrence. The disease which with the development of intensive agriculture always attains greater extension, occurs especially in the spring and in the fall, while in winter and summer it is more rarely observed. Calves become affecter most frequently. Sometimes, however, the disease also occurs among foals, lambs and pigs (very rarely also among dogs

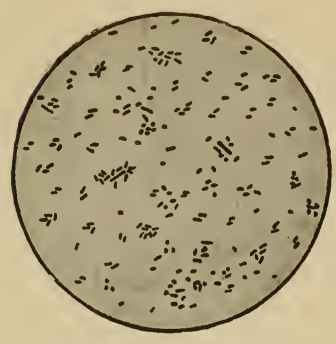

Fig. 32. Bacillus coli communis Agar culture; fuchsin staining and cats). It causes considerable loss due to its contagious nature as well as by its destructive course in the affected herds. Occasionally all the young of an entire year succumb to this malady, which moreover frequently reappears in an infected stable during the consecutive periods of parturition.

Etiology. Up to the present time white scours has been studied bacteriologically very extensively, and according to the uniform results of these studies the disease was in most cases caused by the bacillus coli communis, or by one of its several virulent varieties (Jensen, Poels, Joest, Bongert, Titze \& Weichel).

The bacillus coli communis is a small relatively thick rod-shaped or slightly oval bacterium (Fig. 32). It is motile (one, two or more flagella), does not form spores, and stains uniformly with aqueous anilin dyes; does not stain by Gram's method.

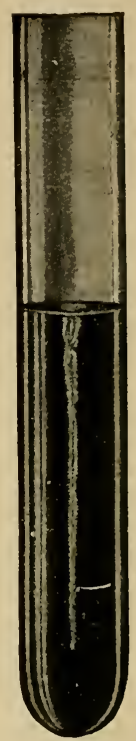

Fig. 33. Gelatin stab culture of the Bacillus coli communis.

Cultivation. The bacillus is aerobic. In the depth of gelatin it produces round or whetstone-shaped, brownish colonies which spread more on the surface of the media. They are first transparent, later white, veined like grape leaves, and irregular. Along the stab the colonies are white and small, the medium does not liquefy (Fig. 33). On agar it forms gray transparent, later white, colonies. The bouillon becomes uniformly eloudy. On potatoes a grayish, later yellowishbrown, thick deposit forms. Milk is acidified and coagulated. The bacillus causes the formation of indol and hydrogen sulphide as a result of which the cultures have a disagreeable odor. In media containing sugar the organism causes an alcoholic fermentation, but colon bacilli of different origin show in this respect marked differences, which are even more pronounced relative to their virulence.

The colon bacillus is almost a constant inhabitant of the intestinal canal of most animals and of man. In guinea pigs and chickens, however, it is usually absent. 
Pathogenicity. Colon bacilli originating from the bodies of calves which die from white scours exert a severe pathogenic action on newly born calves. If a one-day-old calf is given 5 to 6 cc. or even only $1 / 4$ to $1 / 2$ cc. of the bouillon culture mixed with milk, fever and diarrhea set in the following day and the animal dies in from $11 / 2$ to 3 days. The autopsy reveals a hemorrhagic inflammation of the abomasum, and to a slight extent also of the intestines. The contents of the intestines are fetid and the mesenteric lymph glands show hemorrhagic swelling. The injection of $5 \mathrm{cc}$. of bouillon culture into the rectum of a new born calf has similar results, and sometimes the subcutaneous injection of such a quantity of culture is fatal, while 1 cc. of culture has no ill effects upon the animal (Jensen). A fatal infection per os may be much more easily produced in calves in which the organs have not yet performed their functions than in those in which the stomach and intestines have already been set in activity by the ingestion of food (Joest). Injection of culture into the umbilical vessels, in the amniotic sac or into a vein produces in some cases a fatal septicemia (Poels, Joest). Intraperitoneal inoculation of rabbits and guinea pigs causes a fibrinous peritonitis (Jensen, Willerding), and sometimes subcutaneous injections of large quantities of culture are also fatal to these animals as well as to mice (Mazzanti \& Vigezzi, Joest).

The colon bacilli which are found in ealves affected with diarrhea correspond in all their morphological characteristies with the colon bacilli isolated from the excrements of healthy calves. They differ, however, from them by their severe pathogenic action on calves. According to Jensen the pathogenic colon bacilli are facultative parasites which enter the digestive tract soon after birth, either with the first milk or with other material swallowed by the animals (for instance with mucus contaminated with straw). Under normal condition the bacteria multiply in the intestinal canal only to a moderate extent, and are harmless, as they are not capable of attacking the mucous membrane of the healthy intestines. If, however, the resistance of the tissues is weakened from some cause (cold, poor nutrition, etc.), the bacteria penetrate into the intestinal wall and later also into the distant organs as well as into the circulation and produce in this manner an affection of the animal.

The great susceptibility of calves which have not yet taken any food may be explained by the fact that the colon bacilli which enter the stomach before the ingestion of the colostral milk pass without interference into the small intestines as the stomach has not yet produced any gastric juice. Owing to the absence of the intestinal juices the meconium which is present in the intestines affords a favorable medium for the multiplication of bacteria. Their penetration into the mucous membrane is probably also facilitated by the fact that the intestinal epithelium does not at that period possess a mucous covering, and is therefore easily penetrated (Joest).

Colon bacilli which have attained a higher virulence in the animal body retain it for that respective species of animals. Leaving the 
animal body they may again be taken up by the digestive tract of other newborn animals and produce the disease. Repeated transmission increases the relative virulenee of the bacteria which then retain the severe pathogenic action until they again lose their virulence through the influenee of certain conditions.

The lowered natural resistance of the animal is considered by Jensen to be of great importance, as he succeeded in producing the disease by feeding material which has a weakening influence only on the intestinal wall. Thus the disease developed after two administrations of $5 \mathrm{gm}$. doses of ereolin, and after repeated doses of pyoktannin (in all $0.75 \mathrm{gm}$.) or trichloride of iodine $(0.65 \mathrm{gm}$. given inside of 30 hours). This is also substantiated by the fact that the discase will appear when the newborm ealves are fed boiled milk instead of colostral milk.

It is not yet positively established whether the causative agent of the disease represents a "pathogenic variety" of the colon bacillus (Jensen, Titze \& Weichel) or is a "virulent colou bacillus" (Poels) or again whether it is "a variety of the bacillus coli communis which is pathogenic only for calves" (Joest found the isolated bacillus identical with the organism isolated by the other two authors). Of great importance is the fact that among the colon bacilli very widely distributed in nature there are such, which may as facultative parasites sometimes attain a high virulence for ealves and when possessing such properties may form a dangerous virus.

In some cases other bacteria have been demonstrated to be the cause of white scours, as the bacillus aerogenes, paracolon bacilli (identical with Gaertner's enteritidis bacillus?) as well as varieties of the proteus. These are found as a rule only when the disease develops somewhat later in life (Jensen). Further the bacillus enteritidis Gaertner and the bacillus pyocyaneus (Poels) enter into the etiology of this disease. According to the causative agent the disease may show marked clinical differences (Jensen; see page 151).

Tit: \& Weichel found among 210 strains from white scours from different parts of Prussia 160 colon, 4 paracolon and 16 pseudocolon strains, further 24 strains of the bacillus enteritidis of Gaertner and two strains each of bacillus paratyphosus B, bacillus lactis aerogenes and of the bacillus proteus mirabilis. (Paracolon bacilli or Caertner's bacilli and bacillus paratyphosus B can be only distinguished from each other by agglutination tests; pseudocolon bacilli resemble colon bacilli, but they coagulate milk). In cultures of colon bacilli the presence of toxins could not be demonstrated, but they were found in cultures of Gaertner's bacillus that were at least ten days old, and also in cultures of paratyphosus B bacilli and paracolon bacilli. The toxic action was somewhat diminished by heating the filtrate containing the toxins for a half hour at $60^{\circ} \mathrm{C}$, and at $80^{\circ}$ the toxicity was entirely destroyed. The administration of potent toxins per os caused diarrhea in test animals. Schmitt repeatedly found also, in ealves affected with scours, bacilli of the paratyphosus B group, which finding deserves especial consideration, as this bacterial group plays an important part in meat poisoning. (Titze \& Weichel could never demonstrate such bacilli in healthy animals).

Recently Krautstrunk isolated a diplococeus from the blood and organs of ealves affected with scours which resembles the pneumococeus of man. The cultures of this organism even in small quantities (few loopfuls of agar culture) administered to newborn calves, either in milk or by intravenous injections within a few days produced a fatal typical white scours which was sometimes accompanied by swelling of the joints. The diplococcus which is Gram positive and has been obtained in pure cultures, in 9 out of 73 cases, and in 16 cases was found in associa- 
tion with colon Lacilli, appears in smear preparations to be surrounded by a broad capsule. It grows only in alkaline media, especially well on blood agar, and also in serum bouillon.

The affection of the newborn of other species of domestic animals is probably similar in its etiological relation. Thus according to Hecker's investigations diarrhea in lambs is produced by the same bacteria as in calves, while Baldrey demonstrated in white scours of a foal colon bacilli as the cause.

Natural Infection. Sometimes the disease will appear in localities which have previously been free from it, without apparent introduction from the outside. In sucl cases it may be assumed that the ordinary colon bacilli which have been present in the stable, have for some reason attained virulent properties in the intestines of the newly born animals (for instance as a result of dietetic errors, feeding of boiled milk immediately after birth), and that this virulence is being retained for later born animals.

In affected stables the virus may be present in the vagina of the mother, on the floor of the stable or in the litter, from which the external genitals and the udder of the mother become contaminated. The infection results usually during the sucking of the contaminated udder, but also has taken place during parturition, and later through contact with litter, from licking of the stable walls, etc. Exceptionally the infection may also take place throngh the umbilicus (Poels, Jensen). The affected animals may then infect their surroundings with their excrements and urine, thus increasing the possibilities for infection of animals born later. Newborn animals fed artificially with boiled milk may become infected by the contaminated hands of the attendants as well as by the use of dirty feeding vessels. In many instances the unfavorable experiences, gained in places where animals are raised artificially (numerous affections with diarrhea) may be attributed to a large extent to such infections, but in such cases consideration should also be given to the fact that by heating the milk substances are destroyed which are valuable for the digestion (ferments), which, if undisturbed, counteract to some extent the development of diarrhea by stimulating the function of the digestive glands, thereby preventing the excessive multiplication of the normal intestinal bacteria.

The contagion may retain its virulence in infected stables for a long time, which may explain that in some premises where nothing is done for the prevention of the disease it reappears from year to year in the spring and in the fall. On the other hand, the infective agent may be introduced into premises, as yet free from disease, by infected sucklings as well as by adult animals. The transmission of the disease by breeding animals is especially suggested by the fact, that, where cows in advanced pregnancy are taken from an infected stable and placed on premises free from the disease, the calf born there is frequently 
affected with diarrhea (Dicekeshoff). Besides affected animals may spread the contagion among sucklings of other species of animals, especially lambs may become affected when an infected calf is placed among them (Kotelmann).

The possibility of an intrauterine infection was proven by Kitt when he injected 3 cc. of a washed agar culture of colon bacilli intravenously into a cow in advanced pregnancy. This immediately cansed a profuse diarrhea in the calf which was born eight days later.

Newborn animals are more susceptible or predisposed to the disease immediately after birth, while later the susceptibility diminishes rapidly. As a matter of fact the animals become affected in the majority of cases within 24 to 48 hours, much more rarely from 3 to 8 days after birth, whereas older animals usually do not contract the disease. Conditions which predispose the body, such as poor nutrition, congenital weakness, etc., favor the development of the disease, particularly as the animals have not a great resistance in the first days of life. Besides, the withholding of colostral milk from the calf and the immediate feeding of boiled milk after birth results almost always in the appearance of the disease.

An abnormal composition of the mother milk, high fat contents or the opposite too high dilution, as well as indirectly the feeding of the mothers with oil meal cake or swill (Kovácsy) may have an influence on the development of the disease, since such conditions favor an abnormal multiplication of colon bacilli in the intestines of sucklings.

Pathogenesis. Bacteria which accumulate in great numbers in the intestinal tract of sucklings enter the tissues of the intestinal mucous membrane, the lymph spaces and the lymph follicles. The penetration of these organisms in the first days of life is facilitated by the absence of the "mucous laver" (Disse) from the intestinal epithelium which at a later age prevents to some extent the penetration of the bacteria. From the intestinal walls the bacteria enter the mesenteric lymph glands and cause acute swelling. Later they may enter the blood circulation and flood the entire body.

According to Jensen white scours is not a simple inflammatory condition of the digestive tract, but an inflammatory process complicated with a septicemic condition, which is caused by the entrance of the bacteria into the blood circulation. It is very probable that the manifestations of the disease are to some extent caused by toxic products of the bacteria. Although filtrates free of bacteria from virulent colon cultures have proven harmless (Jensen), the negative results do not exclude the development of toxins in the animal body. Bacilli of the paratyphus group B produce severely acting toxims even in artificial culture (see page 148). 
Anatomical Changes. The carcasses are greatly emaciated, anemic, the parts around the anus are soiled and the mucous membrane protrudes. The mucous membrane of the stomach appears highly reddened, in ruminants, only in the abomasum, and in this especially around the pylorus, it is edematous, swollen, covered with a great quantity of glary mucus. The ridges of the folds are sprinkled with punctiform and linear hemorrhages and erosions. The loosened superficial layer can be easily scraped off with a knife. The small intestines contain a very fetid, yellowish or dirty gray, and sometimes reddish fluid contents, which may be nixed with mucous flakes, and a great quantity of gas. The intestinal mucous membrane is highly reddened, covered with sticky mucus and may be studded with hemorrhages. Its solitary follicles and the Peyer's patches are swollen, the submucosa and the muscular coats are edematous. Similar changes, although not to the same extent, are present in the mucous membrane of the large intestines. The mesenteric lymph glands are always intensely swollen and frequently contain small hemorrhages.

The parenchymatous organs are conspicuously pale, the spleen is not enlarged. The lungs show acute edema, and in exceptional cases catarrhal pneumonic foci. The serous membranes, especially the pericardium and the endocardium, show hemorrhages. The umbilical vessels are either filled with normal blood coagula or are empty.

The lesions described are not in all cases distinct, but may be only slight even in cases where the pathogenic bacteria are present in all organs and even in the muscles (Titze and Weichel).

According to Jensen the following forms of white scours in calves may be distinguished, depending on the canse of the disease:

1. Colibacillosis. In one form of this affection which usually occurs shortly after birtl and runs a rapid course, the lesions consist in a pronounced enteric condition, the mesenteric lymph glands are red and swollen, the spleen is in most cases enlarged, and large numbers of hacteria are present in the blood and in the organs. The other form appears from 3 to 5 days after birth and causes death of the animal in 3 to 5 days. The intestines are distended by gas and are pale, the mesenteric lymph glands are swollen, but mostly pale; swelling of the spleen is rare, the blood and the organs contain no bacteria, or only sparingly so. bacillosis.

2. Aerogenes bacillosis; resembles the first form of the coli-

3. Paracolon bacillosis or pseudocolon hacillosis. This may run a course of hemorrhagic enteritis; frequently, however, the enteric changes are not very marked while the swelling of the mesenteric lymph glands, the enlargement of the spleen and degeneration of the internal organs is very pronounced. In rare cases the serous membranes may contain fibrinous exudate, the blood and the internal organs always contain numerous bacteria.

4. Pyocyaneus bacillosis (Poels). It is manifested as a severe diarrhea and the autopsy reveals a red spotted intestinal mucous mem- 
brane, degeneration of the liver, but no swelling of the spleen. In the intestinal contents the pyocyaneus is found in almost pure cultures; the blood on the other hand is sterile.

5. Proteus bacillosis. This usually occurs when the calf is several days or about a week old and runs a slow course. Feces are fetid, never mixed with blood. On the autopsy the intestinal tract is greatly distended, pale, the mucous membrane becomes macerated shortly after death. The spleen is normal, the mesenterie lymph glands are slightly swollen but not hyperemic. Proteus organisins are present in great numbers in the intestinal contents, but are not found in the blood or in the organs.

In the cases which are caused by the diplococcus found by Krautstrunk the autopsy reveals changes similar to those found in colon bacillosis, the intestinal glands being always markedly swollen and reddened.

Symptoms. In animals born healthy the first symptom usually appears in from 1 to 3 days, exceptionally in a few hours after birth; in rare cases in from 4 to 8 days. The newborn cease to suck and stand in one place with a staring look. They are depressed and lie down a great deal of the time.

On the first day, or not later than on the second day, the diarrhea appears; the expelled very fluid and fetid feces is yellowish-brown in foals and pigs, while in calves and sheep it is first yellowish, later grayish-white, frequently foamy, and contains lumps of clotted milk as well as blood streaks (white and red diarrhea). The parts around the anus are soiled and the hair or the wool is matted. The consistency is at first slightly mucous, later watery; the odor is intensely sweetish, later penetrating, putrid (Hess). The feces are passed at first under severe straining, later it is painless and towards the end of the disease involuntary passing of the feces is observed. Colts suffer severe colicky pains before and after the passing of the feces, they show restlessness, paw with the front feet, look towards the abdomen or they draw the feet under the body. Similar, although not so pronounced, restlessness is observed in calves. At the same time there is bloating, or on the contrary the abdomen may be contracted, the flanks are sunken and the pressing of the abdominal walls causes pains. After the passages the animals stand in one place with feet drawn together, arched back and lowered head, or they lie for a long time motionless on the ground.

The weakness increases during the course of the disease, the eyes sink into their sockets, the cornea loses its lustre, saliva flows from the mouth, the dry tongue is covered with a viscir mueus, the anus is continually open, the hair is rongl, the skin is sticky from the perspiration, the body disseminates a disagreeable sweetish odor, and finally the greatly emaciated animals enter into a comatose state and die.

At the onset of the disease the body temperature rises from 1 to $2{ }^{\circ} \mathrm{C}$; later, however, with the appearance of 
the weakness, it returns to normal, and before death it may even become subnormal. The respiration is superficial and later accelerated. The heart action becomes more frequent and weaker, towards the termination of the disease, and towards the end it is hardly perceptible.

In the more protracted cases some animals develop later inflammations of the joints as well as manifestations of a pyosepticemia, which makes the close relationship of the two diseases apparent.

Course and Prognosis. The course of the disease is in most cases unfavorable. The sooner the first symptoms appear after birth and the sooner they become aggravated, the more unfavorable is the prognosis. Bloody diarrhea or a rapid drop in temperature are forerunners of death. These may appear on the second day of the disease; mostly, however, between two and three days, exceptionally even after ten days.

In some outbreaks practically all the affected animals die and even in more favorable cases the losses are rarely less than $80 \%$. The high mortality is partly the result of catarrhal pneumonia which frequently develops as a complication in cases of less severe primary infections. (See Vol. II, Enzootic Pneumonia of Young Animals.)

Diagnosis. The disease is differentiated from the simple acute gastro-enteritis caused by dietetic errors by its appearance shortly after birth, by the predominating diarrhea and by the enzootic character of the disease. Acute gastro-enteritis usually affects older animals and is characterized by less severe symptoms. In calves in this disease the intestinal contents are thick to mushy in consistence, later almost constantly of a clay-yellow color, and have an intensely sourish or somewhat putrid odor (Hess).

The pyosepticemia of the newborn resulting from umbilical infection is characterized by an early affection of the umbilicus and by the early appearance of metastatic inflammatory processes, especially in the joints.

For distinguishing the different forms of the disease in accordance with the etiological factors the descriptions given on page 151 offer sufficient points of differentiation.

Treatment. At the onset of the disease suitable immune sera (see page 156) may have beneficial effect and should be employed intravenously, and if necessary the injection should be repeated ( 5 to $20 \mathrm{cc}$.), but even in such cases suitable dietetic treatment should be carried out. This is commenced with a mildly acting purgative in order to remove the fermenting intestinal contents. Especially to be recommended is castor oil (colts and calves 40 to $50 \mathrm{gm}$., lambs 10 to $15 \mathrm{gm}$.), or calomel (colts 0.2 to $0.3 \mathrm{gm}$.). After the appearance of the purgative action it is advisable to administer various slimy gruels (barley 
gruel, lime water, decoctions of linseed, barley and oats, further of althaea or senega roots). They may be administered with or without opium ( 0.5 to 1 ce. or 0.05 to $0.10 \mathrm{gm}$.) to which some chalk or magnesia ( 3 to $5 \mathrm{gm}$.) may be added. Hertwig's mixture has a good reputation and consists of Pulv. Rad. Rhei 4, Magn. Carb. 1, Opii puri 0.3 in $100 \mathrm{gm}$. cammomile tea or in $50 \mathrm{gm}$. of diluted alcohol, given twice daily to small animals in tablespoonful doses. Of the intestinal disinfectants salicylic acid ( 0.2 to 0.5 gmı.) may be given together with tannin; further tannoform (colts 0.2 to $0.5 \mathrm{gm}$., calves 1 to $2 \mathrm{gm}$.), tannalbin (3 to $5 \mathrm{gm}$.), napthalin (1 to $2 \mathrm{gm}$.), salol (5 to $8 \mathrm{gm}$.), etc., may be used with beneficial results.

Creolin and lysol are suitable per os (1 to $2 \mathrm{gm}$.) as well as in a $1 \%$ solution of enemas. Boracic acid (3 to $4 \%$ ) may also be utilized for this purpose. In the presence of severe trismus starch flour enemas containing tincture of opium may be given.

Evers recommends the intravenous injection of collargol as an abortive remedy ( $0.05 \mathrm{gm}$. collargol in $5 \mathrm{gm}$. of $1 / 2 \%$ carbolic solution). This method of treatment was also found satisfactory by Stampel and Trost. Fumagalli obtained good results from it in well developed cases (four injections daily of 0.15 to $2 \mathrm{gm}$.). Möbius was successful with phosphoric acid ( $5 \%$ solutions in teaspoonful doses). Schmidt recommends the administration of $1 \mathrm{gm}$. tannargentan every 3 hours, to be given in a tablespoonful of milk, while Eber obtained satisfactory results in mild cases from tannothymol (4 to $5 \mathrm{gm}$. per dose).

In addition to the medicinal treatment attempts should be made to preserve the strength of the animals. For this purpose eggs should be given either raw with a small quantity of alum, or in warm wine soup. The weakness is treated advantageously with wine (port wine), further with black coffee or with subcutaneous injections of caffein. Infusions of a slightly alkaline physiological salt solution $(0.8$ of $1 \%$ salt and $0.25 \%$ sodium carbonate) into the subcutis of the neck or into the rectum ( 2 liters, repeated if necessary in from 2 to 5 hours) have often given surprisingly good results (Eber). Further rubbing with alcohol and warm blanketing of the abdomen is indicated.

As the infective agent is contained in the excrements of the animal the immediate isolation of the infected animals and the destruction of the feces should be carried out. Besides this a thorough disinfection of the stables, to be repeated at frequent intervals, is indicated.

Prevention. In order to prevent the infection of the newborn the greatest cleanliness should be exercised during births (disinfection of the stables at intervals of 3 or 4 weeks, fresh straw under the parturient animals, clean hands of the stable attendants, etc.). If the disease is already in existence it is advisable to remove the highly pregnant breeding animals 
a few weeks before birth into suitable clean stalls (Roloff, Franck), and immediately before birth to place them in a box stall especially used for that purpose. Sometimes the disease does not recur in infected stables if the stable floor and the cribs have been thoroughly disinfected and if the newborn calves are not placed in common pens, but are kept tied with the mothers for two weeks.

Further it is advisable to disinfect the litter and the vagina of the mother animal before as well as after birth, and to care for the newborn with the utmost cleanliness. In order that the calves may pass the meconium they should, as soon as possible, suck the collostral milk from the previously washed udder, and if they are kept in special pens they should be allowed to feed from the mothers at regular intervals. Further they should be protected against taking cold and therefore kept in a moderately warm, clean place, free from draughts.

In places where the calves are brought up artificially, sterilized milk should be given only after the animal has partaken of the collostral milk, and not earlier than the second day, and in such cases a painstaking vigilance should be maintained as to cleanliness, especially of the vessels and the hands of the attendants. The milk should be heated shortly before feeding and given to the calves cooled to $40^{\circ} \mathrm{C}$. in clean sucking pails. In various places keeping the calves in single stalls for the first two weeks after birth has given splendid results.

Behring recommended the feeding of raw formalin milk (1 to $10,000)$. The raw milk is supposed to have the preference over the sterilized milk as the activity of the glands of the stomach and intestinal mucous membrane are stimulated by the presence of ferments. The addition of formalin prevents the spoiling of the milk, and exerts also to some extent a slight inhibiting action on the intestinal bacteria (according to the investigations of Kolles the addition of formalin to milk in 1 to 40,000 or 1 to 25,000 , inhibits only the development of the lactic acid bacilli but not the peptonizing bacteria). Unfavorable results, however, were observed from this method of treatment (Raebiger), and it will probably not meet with favor as the formaldehyde retards or inhibits the digestion of the casein by the pepsin and the trypsin, and besides the milk containing formaldehyde no longer acts on the rennet (Löwenstein).

Poels Method. The external genitals of the mother as well as the tail and the udder are washed with a $3 \%$ creolin solution. The vagina is irrigated with a corrosive sublimate solution of 1 to 5,000 , or with a liberal quantity of luke warm followed by cold water. The ealf is eaught in a clean sheet, the umbilical cord is ligated as elose as possible to the abdominal wall, severed and the stump is painted with a $5 \%$ solution of potassium permanganate. Then the mouth is cleaned and a tightly woven muzzle applied. The calf should immediately suck out the collostral milk; however, it is recommended to first milk from each teat a few strokes into the straw, as the first few strokes may be infected. The milk may be also drawn into clean vessels and fed to the ealves (immediately after birth, and again after $1 / 2$ to 1 hour, $1 / 2$ to 1 liter each time). The quantity of milk required by newborn ealves is on the first day $3 / 4$ to 1 liter collostral milk, on the second day $1 \frac{1}{2}$ liter on the third day $21 / 2$ liters, and later up to the sixth day it increases $1 / 2$ liter per day. Later the milk should be given fresh and in small rations. The muzzle is removed only during sucking or feeding. By this method, which of course can be also applied to foals, 
the disease has been eradicated in many small farms where formerly all calves died. Raebiger recommends washing with pure water and Arying with a clean eloth for cleansing the udder. Guillebeau advises to follow the drying by the application of fat to the uidder by means of a cloth (for the treatment of the umbilicus see also under pyosepticemia.)

Evers obtained very good results from placing the calves in a transportable box in which they are retained for 4 to 5 days, and meanwhile are fed with milk freshly obtained from their mothers. Inasmuch as the box is thoroughly eleaned and disinfected before its use, infection of the newborn may be prevented, and should it occur the dissemination of the infection is limited.

Immunization. Jensen treated a horse partly with dead and partly with living cultures of the colon bacillus and prepared a serum which had a decided bactericidal action in experiments on guinea pigs. It protected the animals against an otherwise fatal artificial infection, but proved only effective against the particular strain of the colon bacillus with which the horse had been treated, while against other strains it afforded only slight protection or none whatever. As the application of the monovalent serum proved unsatisfactory in practice he produced a polyvalent serum by using colon strains of different origins with which far better results were obtained. White scours also being caused sometimes by paracolon bacilli it was necessary to produce also a paracolon serum which in suitable cases exerted a protective action.

In the preparation of polyvalent colon serums earefully selected strains of the colon bacillus are separately cultivated in bouillon at $37^{\circ} \mathrm{C}$., then the cultures are mixed in measured quantities and the mixture is injected intravenously into horses in quantities of 0.25 to 0.50 ec., and after each 12 to 14 days in increased doses up to 10 and $20 \mathrm{cc}$. If after a certain time the sermm of the horse produces precipitation in a bouillon culture of a certain colon strain in the dilution of 1 to 500 to 2000 it is probable that the protective serum may be utilized with satisfactory results against infections with that particular strain.

Bongert and Raebiger recommend goats and sheep for the serum production. At the present time, however, only large animals are used for the production of serum.

The preparation of the paracolon serum is more difficult, as the paracolon bacilli possess higher virulence, so that an injection of even 0.1 to $0.25 \mathrm{ec}$. of a bouillon culture may cause a serious infection.

As a colon serum is effective only against colon bacillosis (the action is principally bacteriolytic), favorable results can be expected only in those cases in which the disease is caused by colon bacilli. The results obtained in practice are not always uniform in this respect; thus Jensen succeeded in reducing the number of cases and the mortality to $0 \%$ in numerous premises where the disease had prevailed in an enzootic and malignant form, eausing sometimes a loss of $100 \%$. These results were obtained without the inauguration of any other protective measures, and all the control animals died. In other premises the mortality was only reduced from 70 to $20 \%$, or the serum treatment had no apparent effect on the disease.

The results in practice have since revealed the favorable action of the serum treatment (Raebiger, Jansson, Heurgren, Schupp, Piethrisen, ete.). The colon serum is injected subcutaneously into the newborn ealves as soon as possible after birth in $20 \mathrm{cc}$. doses. If the animals do not show an improvement the paracolon serum may then be used.

Raebiger observed only one death among 62 injected calves; in ardition he also reports 278 inoculations in previously infected premises in which none of the newborn calves have become affected since this protective measure has been adopted. 
Active Immunization in Advanced Pregnancy. Based on the experimental observations that protective bodies formed in the maternal organism are transmitted by way of the foetal circulation to the foetus, and that these protective bodies will convey a specific protection against a later infection to the offspring, Sande aimed to immunize calves before their birth. He repeatedly injected pregnant cows subcutaneously with sterile extracts obtained from white scours bacilli (shake-extract from colon bacilli cultures?), and the calves of cows treated in this manner are said to have withstood the natural infection in infected premises.

Sande reports the inoculation of 215 cows with good results in that $91.6 \%$ of the calves were protected from white scours. Six per cent becanie affected with the disease and died, and $2.3 \%$ had diarrhea which, however, disappeared soon after the administration of the serum treatment. Favorable results are also reported by Fehrmann in 22 cows (only 2 calves of cows which had not been vaceinated became affected), by Grajewski in 8 and by Kronacher in 16 cows (only 3 control calves lecame affected). Schmitt found, however, that the vaccines for mother animals which are marketed bear no relation to the bacteria of the colon and paratyphoid group nor to the ovoid bipolar bacilli, and elaims that this form of vaccination has not proven the efficiency of the treatment.

The vaccination of the cows is carried out by injecting under the skin of the neck $10 \mathrm{ec}$. of extract 6 weeks before parturition, and ten days later $20 \mathrm{cc}$. The animals stand the inoculation without any disturbance in their health. However, cows should not be vaccinated in the last month of pregnancy as they may abort or become fatally affected. It is dangerous also to employ fully virulent cultures.

Literature. Obich, W. f. Tk., 1865, 101.-Roloff, Trzt., 1876, 83.-Franck, D. Z. f. Tm., 1877, 376.-Hess, Lantw. Jahrb. d. Schweiz, 1897.-Poels, B. t. W., 1901, 290.- Willerding, A. f. Tk., 1899, XXV, 93 (Lit.).-Joest, Z. f. Tm. 1903, VII, 377.-Jensen, Monh. 1892, IV, 97; Hb. f. p. M. 1903, III, 761; Z. f. Tm. 1905, IX, 321 (Lit. on Etiology and Vaceination).-Bugge Kälberrukr u. ihre Behdlg., 195.-Schmitt, D. t. W., 1908, 673 ; Z. f. Infkrkh., 1909, V, 435; 1910, VII, 71.-Sande, B. t. W., 1909, 261.-Titre \& Weichel, Arb. d. G.-A., 1910, XXXIII, 516 (Lit.).-Krautstrunk, Z. f. Infkrkh., 1910, VII, 256.

Other Colonbacilloses in Cattle. Thomassen (1897) described a new septicemia in calves which causes in Holland every spring great losses. The animals become affected at the age of from 4 to 5 weeks and manifest fever of $40-41^{\circ} \mathrm{C}$., great debility, depressed appetite, in some calves a severe dry cough, normal action of the bowels and, only in very exceptional cases, diarrhea. The urine contains great quantities of albumen and a small amount of blood pigment as well as casts. In some severe forms epileptic convulsions, opisthotonus and trismus may be observed. The disease usnally terminates in death in 5 to 6 days. The autopsy reveals small hemorrhages in the endocardium, on the peritoneum, the mucous membrane of the abomasum and bladder. Acute hemorrhagic swelling of the mediastinal glands, marked acute swelling of the spleen and acute nephritis.

Bacilli resembling the bac. coli communis, but more so the bacillus typhi hominis were isolated from the blood, from the fluid of the abdominal cavity, from the liver, kidneys and urine. Bouillon cultures of these organisms when inoculated subcutaneously into calves in quantities of 1 to 2 cc., or when fed in larger quantities, caused an attack of the original disease fatal in 3 to 7 days. The cultures were also pathogenic for rabhits, guinea pigs and mice. Good results were obtained in treatment of some of the eases from a mixture of carbolic acid (1 part), alcohol (30 parts), milk of lime (300 parts), and oil menthae (3 parts). (A. P., 1907, XLVI, 542.)

Mrohler and Buckley (1902) ohserved in America among cows 
a similar and probably etiologically identical disease. In the acute eases the elinieal manifestations as well as the anatomieal changes were very similar, while in 1 cow which died only after 26 days, neerotic foci were found in the liver substance in addition to hemorrhages of the serous membranes. As the cause of the disease an organism of the paracolon type was demonstrated which possessed pathogenie properties also for pigeons, sheep and dogs. (Bureau Anim. Industry, 1902, XIX, 297 [Lit.].)

\section{(b) Pyo-Septicemia of Sucklings. Pyo-septicaemia neonatorum}

\section{(Pyemic and septic joint disease of sucklings, Joint evil, Navel ill; Omphalophlebitis septica, Pasteurellosis neonatorum)}

Pyo-septicemia of sucklings is an acute contagious, infectious disease of newborn animals occurring in the first days of life and not later than in the first four weeks. It is characterized by purulent inflammation of the joints and general pyemic manifestations. It develops as a result of umbilical infection, exceptionally as a result of an intrauterine infection of the foetus.

History. In former times all affections of newhorn animals were combined under the term navel ill (joint-evil) in the course of which lameness and disturbance in walking was present. Fuerstenberg (1864) recognized the fatty degeneration of musele in young animals as an independent disease, and Roloff (1864) inticated that rachitis may also manifest symptoms resembling those of the pyo-septicemia. Later the enzootical acute gastritis and white scours of sucklings was separated from this group of infection, which left only the cases in which the suppurating processes occurring on different parts of the body are characteristic. Bollinger (1873 and 1875) proved that one part of these cases, especially the so-ealled "navel ills" and "joint evils," are a pyemia resulting from septic umbilical infection, more rarely a septicemia, and that the infection of the umbilieus oceurs immediately or very soon after birth. This conception may well be made to agree with the possibility of a uterine infection in spite of Bollinger's exelusive view point, and recently Tátray, Pfeiffer and especially Sohnle confirmed the possibility of this method of infection.

The etiology of the disease has recently been more extensively studied in ealves hy Gmelin, Hess, Lesage \& Delmer, in colts by Casper, Sohnle and Ostertag.

Occurrence. The disease occurs principally in sucking colts and sucking calves, less frequently in sucklings of other species. In some of the breeding districts it attains not infrequently an enzootic character. The best opportunity for the spreading of the disease is present in the studs or dairies where all of the newhorn animals nay become affected, and as a result the breeding industry is endangered to a great extent.

ITp to the year 1889 the disease caused an annual loss of at least $15 \%$ in the stud of Mezöhegyes (Hungary): since that time disinfection has been carried out 
systematically, and recently the disease has not occurred. In Salzburg out of 336 colts 172 became affected in the year 1884, of which $152(92.4 \%)$ died. In the year 1885,98 , in 1886,120 succumbed to the disease.

Etiology. The etiology of pyo-septicemia has up to the present time been only elucidated to the extent that the investigations showed as the causative factor of the disease on one side a variety of the bacillus (bipolaris) septicus (Pasteurella; see page 79$)$, on the other a virulent colon bacillus.

The bacillus bipolaris septicus isolated from the body of affected animals is pathogenic for rabhits, guinea pigs, mice, and in larger quantities also for pigeons. Injections of 3 to 5 ec. of fresh virulent bouillon culture into the umbilicus of a 4 to 5 -day old ealf causes an inflammation of the umbilicus and softening and thrombi of the umbilical vessels, resulting in a serofibrinous inflammation of the joints (Gmelin). Intravenous injections of 3 cc. of culture into a 1-day old calf causes a slight rise in temperature; on the following day the animal is debilitated and at the same time the temperature drops below normal $\left(34.6^{\circ}\right)$. The animal passes white, foamy, later bloody, fluid, fetid feces with much straining, and succumbs after 30 hours to complete exhaustion. The autopsy reveals manifestations of hemorrhagic septicemia, and also extensive inflammation of the large intestines. The blood and the exudate of the serous eavities contain the bipolar bacillus in pure cultures. On the other hand, the mesenteric glands and the liver contain shortly after death foreign bacteria, principally colon and paracolon bacilli in great quantities. Animals recovering from the disease react after 4 weeks to an intravenous injection of 10 ce. of culture only with a rise in temperature which lasts for one day (Nocard).

Bacteria of the bacillus coli communis type (see page 146) were isolated by Zschokke and Wilhelmi from the joints of calves dead from the disease either in pure culture or mixed with some other bacteria. Wilhelmi produced in 8 cases in young calves, by intravenous injections of pure cultures, a sero-fibrinous inflammation of the joints, acute endocarditis and diarrhea, whereupon the animals died within a short time or after longer ailing. In one case Zschokke produced only a passing arthritis. Poels and Joest also produced fatal infection in ealves by injecting colon bacilli into the umbilical vessels. The disease, however, corresponded with the white diarrhea of calves, and inflammation of the joints was absent.

The results of the investigations accordingly appear to indicate that the disease following the umbilical infection develops as a result of infection with various micro-organisms. The organisms which are implicated are on the one hand the bipolar bacillus of hemorrhagic septicemia, and on the other hand a strong virulent variety of the colon bacillus. According to Lesage \& Delmer the colon bacillus prepares the field for the pathogenic action of the bipolar bacillus, as the injection of both bacteria under the skin of a calf produces the disease, while the bipolar bacillus alone causes only a local inflammation (in the first case the colon bacillus remains at the point of inoculation, while the bipolar bacillus passes into the blood). 
Relative to affections of sucklings of other species of animals there is a possibility that the diseases which clinically and pathologicanatomically are practically identical in various species of animals, bear a very close relationship etiologically. As a matter of fact, however, the investigators of pyo-septicemia of foals have not proven such relationship. Casper and Ostertag obtained from all organs of affected colts, sometimes only from the heart blood or from the joints and bone marrow, the streptococcus pyogenes, while Sohnle obtained from the bloed and the joints of colts, as well as from the uterus of mares a highly virulent variety of the staphylococeus pyogenes aureus. Whether these bacteria are the original causative factors of the disease or whether they play only a secondary part will have to be established by further investigation.

The natural infection results mostly through the torn or not yet closed umbilical vessels immediately after birth, exceptionally also during birth. The stump of the loose, juicy umbilical cord which is no longer nourished, as well as the blood present on the torn end of the cord, present a suitable medium for the propagation of the micro-organisms, until drying of the stump has taken place. Bacteria which reach the surface of the stump find favorable development in the thrombi inside of the vessels. Then they penetrate along the thrombus, pass the navel ring and finally spread even in the abdominal portion of the umbilical vessels.

The extra uterine infection which causes the disease in the majority of cases occurs through the soiling of the umbilical stump with the contaminated straw immediately after birth, as well as by coming in contact with the stable floor containing pathogenic bacteria or else with the infected hands of the attendants. The sucklings which have first become infected in the course of the disease contaminate the straw and the stable floor with their excrements, especially however with the purulent exudate of the umbilicus. In this way the infection accumulates in the stable, and as a result of this the animals which are born later are more exposed to the infection. In this way the disease which was at first sporadic may later occur with greater frequency, so that after a certain time almost all the newborn animals become victims of the disease.

The cases in which the animals are affected at the time of birth, or in which autopsy reveals extensive and progressive changes which could hardly have developed since the time of birth, can be accepted as due to intra-uterine infection. This is transmitter by the placental circulation in such a way that the bacteria which are present in the blood of the mother enter through the placenta into the blood circulation of the foetus, where they then multiply. For the intra-uterine infection to occur it is of course necessary that the mother suffer from an infectious disease as a result of which pathogenic bacteria circulate periodically in the blood. This form of infection has been ohserved during the course of outbreaks of influenza, when 
the foals of affected mares are infected at the time of birth (Tátray, Sohnle).

The possibility that the infection may also occur from the external genital organs cannot be excluded. The contagion which may enter the mucous membrane of the uterus might localize there and later enter the vascular system of the developing placenta, and in this manner also the body of the foetus. In this way it may be explained that some animals give birth for several consecutive years, to affected offspring, a condition not infrequently observed, especially in thoroughbred mares. In case of an intrauterine infection the infective agent remains lodged only in the finer blood vessels of the internal organs and causes at these places alone inflammatory processes, while the umbilical vessels remain intact (Peiffer found only $37 \%$ of affected foals with umbilical vessels diseased).

The infection probably does not occur through the digestive and air passages; at least up to the present time the disease could not be produced artificially in this way in calves (Nocard).

The greater or lesser frequency with which the disease occurs can in various localities be referred to existing local con. ditions. The fact that the disease is only rarely observed among common bred stock which is kept out of doors most of the time, as well as in stables with only few animals can be readily explained by the fact that under such conditions the animals are not confined in close quarters and therefore there is less chance for infection.

Since the infective agents, the bipolar bacterium, as well as the colon bacillus, can propogate in the soil, the disease may appear without introduction from outside, as a result of direct infection from the soil; nevertheless it is most frequently the result of an introduction from adjoining farms, while if the disease has already been introduced in certain premises it is propagated by the secretions and excrements from the infected animals.

Formerly breeders attributed great importance to heredity in the appearance of pyosepticemia. However except for the cases of intrauterine infection, in which the infection of the foetus is directly associated with the infection of the mother, the disease occurs only when the conditions for the infection of the sucklings are favorable.

Pathogenesis. The bacilli which enter the umbilical veins commence to multiply in the coagulated blood of these vessels, on the one hand cause the thrombus to break down, and on the other hand produce an inflammation of the intima and probably also of the external laver of the walls of the vessels. The breaking down of the thrombus progresses gradually inward until it reaches the end, when the infection may come in contact with the liquid blood contained in the same vessel, or in the portal vein, coming in direct contact with the circulating blood. 
Small particles of the softened thrombus may be carried off by the circulating blood, and with these the bacteria may enter the free blood circulation. They are taken either into the liver' or with the blood of the rena cava into the general circulation.

The micro-organisms which enter the umbilical vein from without do not necessarily remain confined in the immediate surroundings of the mubilical ring. While the circulation ceases after birth in this blood ressel, the passing blood stream of the portal vein or of the ductus Arantii, as well as the respiratory movements, produce a suction on the fluid which is present in the umbilical vein, by which the blood serum separates from the blood coagulum, is diverted toward the portal vessel, carrying with it the micro-organisms which may be contained therein. They may remain attached to any part of the intima of the umbilical rein, where they produce a local inflammation as a result of which deposits of blood coagulum occur, or a softening of the thrombus which is already present. The bacilli may even penetrate the portal vein without stopping, whence the blood conveys them into the heart. In the first case that section of the umbilical vein which is nearest to the umbilical ring remains healthy; in the other case the entire umbilical vein will be intact although the infection has passed through it.

In ruminants the infective agent enters with the blood either through the ductus Arantii or through the ramification of the portal and hepatic reins into the vena cara, and thence into the general circulation. In foals all septic material enters through the latter channels. In these animals, however, breaking down of the blood coagulum is also frequently observed in the umbilical arteries (Gmelin), as a result of which the infective agent may pass into the posterior part of the abdominal aorta, and with the blood of these vessels into the peripheral arterial ramifications of the posterior extremities.

Certain organs appear to have a special predisposition for attacks by the micro-organisms circulating in the blood. ()f these the lungs and the liver should be mentioned first, and in addition metastatic inflammations develop frequently on the synovial membranes of certain joints.

In acute cases of the disease the flooding of the blood with pathogenic bacteria, as well as the inflammatory processes which develop at different parts of the body, cause the death of the animal. In some cases, however, the acute affection is suddenly checked, and the disease process appears to turn towards recovery. After several weeks, or in one to two months, however, manifestations of the chronic affection, especially of the lungs, make their appearance. These manifestations may be associated with the pre-existing infection. According to Nocard hacteria of hemorrhagic septicemia may disappear after a time from the blood; the toxins, however, have in the meantime (xerted a paralytic action on the cells by which the resistance of 
the animal has been reduced to such an extent that other bacteria, especially the Preisz-Nocard bacillus of pseudo-tuberculosis (see that disease), may attack the tissues of the body and produce an inflammation of the lung tissue.

In these eases which have become chronic, eatarrhal pneumonia leading to caseation and suppuration can usually be discovered. Bacilli of pseudo-tuberculosis may be demonstrated in the affected tissues in association with other bacteria (pus eocei, bacillus necrophorus, streptothrix, colon and paracolon bacilli). Their oceasional pathogenic action has been proven by Nocard by the following experiment: ' A calf three weeks old was injected intra-peritoneally with $100 \mathrm{ce}$. of culture of the bipolar bacillus, which had previously been heated for one hour to $60^{\circ} \mathrm{C}$., and after six hours it was made to inhale a bouillon culture of the bacillus of pseudo-tuberculosis for five minutes. The autopsy which was performed eight days later, revealed numerous catarrhal-pneumonic areas in the lungs. In a second ealf, which was not given the injection of the killed culture of the bipolar bacilli, the inhalation of the pseudo-tubercle bacilli caused no changes in the lungs.

According to Moussu broncho-pneumonia may develop in sucking calves also, from the aspiration of the amniotic fluid into the air passages during difficult births. The infection occurs in these cases while the head is lodged in the vagina, and with the commencing respiratory movements the aspiration of the fluid takes place.

Anatomical Changes. In some of the acute cases the umbilicus is swollen and dense. The borders of the umbilical opening of the ring which has not closed are infiltrated; sometimes they are ulcerated, and a purulent ichorous secretion may be pressed out of the same. An incision frequently reveals an abscess lying in the abdominal wall.

The umbilical vein in colts, also one or both umbilical arteries (Gmelin), are greatly thickened and of a firm or fluctuating consistence in their entire length, or only in parts. In different places the lumen is filled with blood coagulum, which in various parts or along its entire length appears of a dirty red color, and sometimes liquefied to a thick fetid mass. The surface of the intima is covered with fibrinous shreds or with puriform softened masses under which there may be ulcerations. The other layers of the wall of the vessel, as well as the surrounding connective tissue, are sometimes only infiltrated with the serous fluid, in other cases again with fibrinous purulent exudate. In case the inflammation has extended to the adjacent peritoneum the abdominal organs lying close to the affected areas usually show adhesions by fibrinous psendo-membranes (intestines, omentum, liver, in colts also the bladder). All these changes may be present without an affection of the umbilicus. In the branches of the portal vein, inside of the liver tissue, similar thrombi may occur, while on the other hand, the thrombus of the vein may occasionally extend into the ductus Arantii, and may even penetrate with its blunt end into the lumen of the vena cava. The thrombi of the umbilical arteries may reach 
the aorta. The internal organs present indications of an acute blood infection in the lungs, sometimes showing areas of broncho-pneumonia.

Metastatic abscesses occur with relative frequency in the lungs, where they are always associated with a bronchial catarrh, and sometimes also with a fibrinous or purulent pleurisy. They may also occur in the liver in the peribronchial, mediastinal and mesenteric lympl glands, in the brain, in the kidneys, etc. Exudative inflammations of the peritoneum, pericardium, and of the meninges of the brain, are also frequently associated with the disease. Not infrequently ophthalmitis, with an accumulation of fibrinous and purulent exudate in the anterior chamber of the eye, is present, sometimes also a liquefaction of the crystalline lens.

Purulent inflammation is found in the majority of cases in the joints of the extremities, most frequently in the hock and knee joints. The more or less swollen joints contain a yellow or reddish semi-liquid, pus-like synovia. The synovial sack appears greatly swollen, bright red and in places ulcerated; the surface of the cartilage is uneven, its tissues softened, sometimes covered with a fibrinous or purulent exudate, which occasionally may be traced even into the spongy substance of the bone and into the bone marrow. The pariarticular connective tissue is saturated with serous fluid or with a sero-fibrinous or sero-purulent exudate and in these cases abscesses may also be found, as well as fistulous tracts communicating with the cavity of the joint. The surrounding tissue is always edematously infiltrated and the muscles frequently show a fatty degeneration. The tendon-sheaths may contain a sero-fibrinous or purulent exudate, the tissue of the tendons in such cases showing serous infiltration and loosening. Purely metastatic abscesses may be present in the subcutaneous as well as in the intra-muscular connective tissue, also in the muscles proper.

In the peracute cases the changes are less conspicuous, and usually point to general septic infection. The lesions consist of a cloudy swelling of the parenchymatous organs, acute catarrh of the mucous membranes, hyperemia of the spleen, small hemorrhages in the tissue of the serous and mucous membranes, sometimes in the small intestines an ulceration of the Peyer's patches, marked acute swelling of the lymph glands, etc.

In chronic cases (in calves) when the disease has not progressed to an advanced stage, the lungs show signs of bronchopneumonia, and there exist frequently fibrinous or sero-fibrinous pleurisy and pericarditis. In later stages the broncho-pneumonic areas show small yellow caseous foci, and still later the lungs contain large firm nodules, which inclose small cavities containing a white or greenish caseous substance. The mediastinal and peribronchial lymph glands are enlarged and sometimes partially caseated. 
The carcasses of animals dead from the disease are always greatly emaciated, and show manifestations of a general anemia and cachexia.

Symptoms. The symptoms usually appear inside of 24 to 48 hours after birth; exceptionally the disease may also occur somewhat later, and in rare cases the animals may be born affected with it.

In some of the cases symptoms of a local affection of the umbilicus precede the general affection. The umbilicus swells, becomes sensitive and feels warmer than normal to the touch. The stump dries more slowly, while from the opening of the umbilical ring purulent or ichorous secretion may be pressed out. In the median line between the swollen umbilicus and the xyphoid cartilage, on the imner surface of the abdominal wall, a dense cord of the thickness of a finger may be felt. The affected animal moves but little, its gait is laborious and stiff, the desire for sucking is diminished, the body temperature is somewhat elevated.

The symptoms of inflammation of the umbilicus may subside after a few days, especially when appropriately treated, and the patient may even completely recover. In the majority of cases, however, the local inflammatory processes increase in severity, and symptoms of a general septic infection develop sometimes rapidly, in other cases gradually. In rare cases the disease may develop without any preliminary inflammation of the umbilicus, with symptoms of a general infection.

The general affection is indicated by symptoms of a general febrile condition. The animals lose their desire to suck, they will not look for the udder of their mother on their own accord, and if led to it they suck only a little or not at all. At the same time they are depressed and dull, and lie continually on the ground. When stood up on their feet they remain with drooping head in one place, or soon collapse. The body temperature is elevated and unevenly distributed over the body surface. The pulse and respiration are accelerated. This condition is soon followed by symptoms indicating a metastatic inflammation in some of the internal organs.

Inflammations of the joints make their appearance most frequently and earliest. One or more joints, preferably at the extremities (hock and knee joints), swell simultaneously, or one after the other, they become warm, painful, and tense, while the surrounding connective tissue is edematous. In some cases the swelling soon disappears; more frequently, however, a fluctuation may after a certain time be noticed in different parts of the swelling. This is followed by a breaking at one or more points, and from the resulting opening a tenacious purulent secretion is evacuated, first in great quantities, later more sparingly. By introducing a probe into the opening, a rough cartilaginous or bony surface may be felt at different places, while 
with the secretion small tissue shreds or cartilaginous and bone particles may be discharged. The motility of the affected joints is interfered with by the pain and by the tense and swollen condition. The animal does not support the body with the affected extremity, and protects it from touching the floor. If several joints are affected at the same time, the animal no longer moves around, but lies constantly on the ground.

The affection of the lungs is indicated by a painful cough and rapidly increasing difficulty in respiration, which is later associated with a profuse nasal discharge, as an indication of a developed purulent bronchitis. Percussion reveals only in circumscribed areas a dull or tympanitic sound; by auscultation, however, the most varied catarrhal sounds, such as whistling, roaring, moist rales, and in parts also bronchial breathing, may be distinguished. Sometimes friction sounds may also be heard over some parts of the lungs and the heart, indicating an acute exudative inflammation of the corresponding serous membranes. In such cases the patients lie much on the chest with extremities drawn under the body, or with legs extended.

In the presence of an inflammation of the brain and its membranes, the patients are much depressed and apathetic. Sometimes chronic spasms may be noticed in some of the muscles or groups of muscles, or the animal may struggle considerably. With relative frequency, symptoms of meningitis may be present as the result of the extension of the inflammation to the medulla; still later paralysis may appear in the extremities, or in the territory of some of the facial nerves.

Symptoms showing disturbances of the digestive organs are hardly ever absent, and in some cases they may even be present at the onset of the disease. Severe colicky symptoms may appear when the animals lie in a cramped condition on the ground with the head turned toward the abdomen; touching of the abdomen causes considerable pain. The constipation which is at first present is followed by diarrhea, the animals passing first light, later dark colored, and very fetid, fluid feces. The feces soil the parts around the anus, and later the excrements and urine are passed involuntarily.

In the meantime the animals become greatly emaciated, they lose their strength, the eyes are sunken deep into the orbits, the action of the heart becomes accelerated and weak, the body temperature drops below normal, the extremities, the face and the ears become cold, the body is covered with perspiration, and finally the patients die, with symptoms of convulsions or without any struggle. Death may occur before the progress of the disease through the stages described, from asphyxiation or paralysis of the brain.

In cases of pure septicemia only high fever and severe diarrhea, sometimes also hematuria are observed; in such cases the patients die within a few days as they rapidly lose strength. 
In these instances the umbilicus manifests no inflammatory changes.

Animals which recover from the acute inflammation may later become affected with a chronic form of the disease. This form may develop exceptionally even at the onset, with latent symptoms. The animals fail to take proper nourishment, and therefore they do not develop well; they gradually become emaciated, and toward the termination a rise in the body temperature and diarrhea appear. Some of the joints become swollen, painful, and hinder the patient in its movements

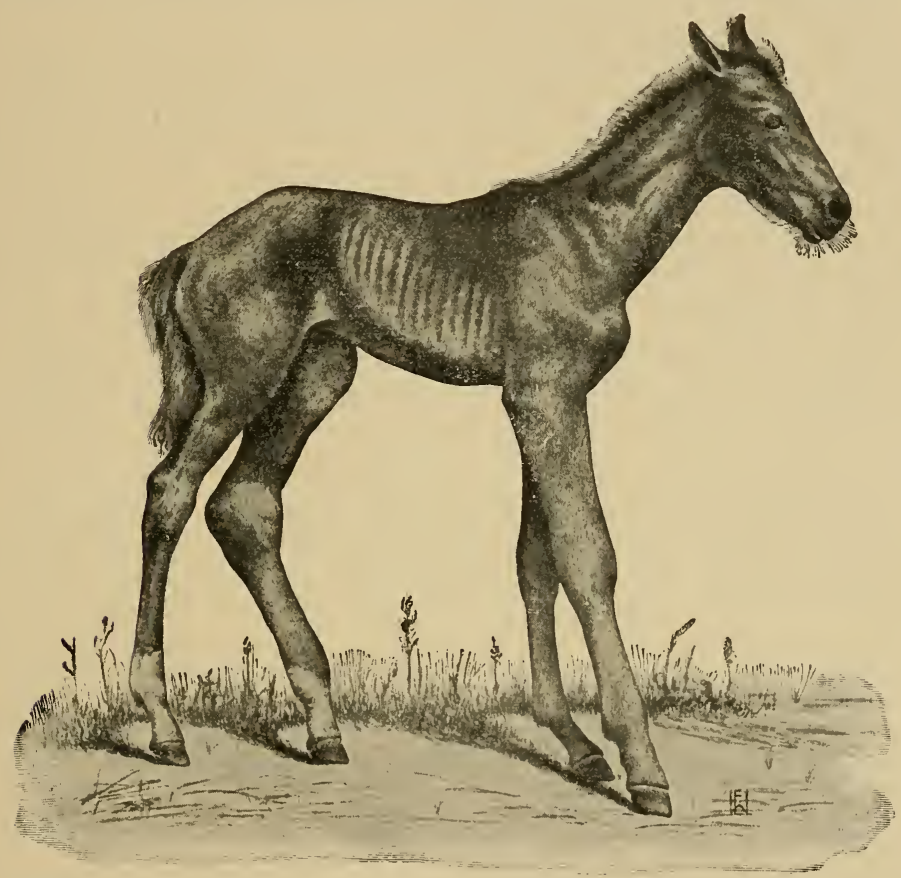

Fig. 34. Chronic pyo-septicemia of a colt. Swelling of the right carpal and left tarsal joints.

(Fig. 34). In the meantime, symptoms of a lung affection are manifested. The animals cough, there is a muco-purulent discharge from the nose, over the lungs numerous rales may be heard. Finally the animals die after several weeks, from exhanstion.

Course. The very acute septicemic cases result in death in from two to three days. In some less acute cases the disease may remain confined to the umbilicus until the termination of 
the disease, and the opening of the umbilical ring may permanently close after the breaking or opening of the abscess. Such a favorable termination is principally observed in cases where the disease appear's somewhat later after birth; that is, when the thrombi of the umbilical vessels are already more solid, the fluid parts have been absorbed, and the penetration of the microorganisms lias thereby been rendered more difficult. The inflammation of the joints may also improve after a time, but the recovery is only exceptionally complete, as usually chronic changes, such as shortening of the ligaments of the joints, deformities of the cartilaginous surface, ankylosis, ete., will remain as permanent injuries.

Severe general symptoms, such as a persisting high fever, severe diarrhea, grave and multiple arthritis, metastasis in the internal organs, etc., have a very unfavorable prognosis. In such cases the animals usually die in from two to three weeks (colts on an average in 26 days) (Gmelin).

The chronic form of the disease usually leads to complete exhaustion in from two to three months; exceptionally, however, recovery takes place, when only a thickening of the pleura is left as a result of the affection.

The percentage of mortalities varies with the different outbreaks; as a rule, however, it amounts to over $50 \%$, and only in calves appears somewhat more favorable (according to Hess it is 40 , and according to Strebel $35 \%$ ).

Diagnosis. The inflammation of the umbilicus can be recognized easily, and with certainty. An affection of the joints, or of internal organs, in association with the umbilical affection, beaves no doubt as to the nature of the disease. In cases where the urachus remains open, there may be also intense inflammation and swelling of the umbilicus. However, in such instances the dripping of the urine is sufficient to reveal the nature of such an abnormality (Baillet \& Sérès observed as a result of a urachus infection, pyelo-nephritis, with septic symptoms). Mistakes in diagnosis may only occur in cases when the local affection of the umbilicus is absent, or present only to such a very slight degree that it may pass unnoticed. The very young age of the patient, and the purulent consistency of the exudates from the joints, differentiate the disease from rachitis, in which moreover the synovial capsule is not distended but the cartilaginous diaphyses of the bones are thickened. From traumatic inflammations of the joints it is differentiated by the absence of any traces of traumatism, and by the presence of symptoms of a general disease. Internal inflammation of the eye occurs in such an early age, from almost no other cause than from a pyemic umbilical infection.

White scours of sucklings cannot be separated from the acute septicemic form of the disease. From the other forms it is distinguished by the absence of lesions in the lungs and in the joints. (According to the introductory remarks of this 
chapter there exists a possibility that both diseases have an identical etiological basis.)

Treatment. The inflammation of the umbilicus should be treated according to the rules of surgery, by disinfection of the wounds, and if necessary by opening of the swellings. The commencing inflammiation of the joints may be treated in colts by inunctions with gray mercury ointment. However, if the joint contains a great quantity of exudate, this should be removed by puncturing or incising the joint, and by subsequent disinfection of the joint cavity. This course is particularly indicated when the inflammation exists only at one place, ancl the internal organs are not affected. If the contents of the joint prove to be serous or sero-fibrinous, recovery not infrequently takes place in from one to two weeks after a single puncturing. In the presence of a general infection the treatment can only consist in the systematic internal administration of stimulants; further in controlling the gastro-intestinal catarrh which may be present. At the same time appropriate untrition should be provided for the patient, such as milk, eggs, wine-soup, or nutritive enemas. The affected animals should be kept in a moderately warm, clean, and airy place. Gott recommends for colts intravenous injections of collargol $(80 \mathrm{~g}$. of a $1 / 2 \%$ solution, for three days successively), while Bernliardt recommends ichthargan (according to Zürn it should not be used in stronger solutions than $1 / 10$ to $1 / 5 \%$, on account of the destructive action on the blood corpuscles).

Prevention. The infection of sucklings may be successfully controlled by scrupulous cleanliness in the stables. For this purpose disinfection of the stables at intervals of one to two months is recommended; but even with such precautions it is advisable to place the highly pregnant animals shortly before giving birth, in a stable or in a parturition box stall kept only for such purposes. Such places should be thoroughly disinfected before the pregnant animal is installed in them, and at the same time provision should be made that the newborn animal may be received on fresh straw or on a clean sheet. Animals affected with suppurating wounds should be kept away from the places used for parturition, as well as from the stalls of the sucking animals. Operations (opening of abscesses) should not be undertaken in these quarters. Satisfactory ventilation and dry stable air also deserve especial consideration. These measures should of course be carried out with increased vigilance when the disease has already appeared, that is, when the stable or the parturition stand are proved already infected. Cleaning of the external genital organs of the mother animals, as well as irrigation of the vagina with a disinfecting fluid, for instance with $2 \%$ lysol or creolin solution, aid also in the prevention of suckling infection.

In places where the veterinarian is present, disinfecting 
treatment of the umbilical stmmp is very desirable. This can also be executed by a competent attendant.

Technique of the Treatment of the Umbilicus. In this method the unbilical cord is washed with disinfecting fluid, ( $3 \%$ carbolie acid, 1 to 1000 corrosive sublimate, $5 \%$ creolin solution.) It is then ligated about three to five em. from the navel with a previously disinfected string. The ligation should le carried out after the pulsation ceases. The cord is then severed with scissors below the ligature, and the eut surface is moistened either with the same fluid or with a more concentrated disinfectant, for instance, concentrated carbolic acid, (Gmelin.) On the following days sponging with one of the above-nentioned fluirls, or with Burow's solution containing camphor should be repeated until the complete drying of the stump, while the cut surface may be sprinkled with disinfectant and drying powders. The parchment-like dried stump may be ent off after 4 to 5 days, and the navel wound should i.e treated daily with $1 \%$ corrosive sublimate gelatin until cicatrization is complete. Nocard washes the stump of calves first with Lugol's solution, (25 parts iodine, 4 parts iorlicle of potassium, 1000 parts water,) then with iorline-alcohol, ( 2 parts iorline, 1000 methyl-alcohol,) and after the evaporation of the alcohol he seals the stump, anil the navel with a thick layer of a $1 \%$ iodine collodion. Reindl recommends for washing the unbilicus tincture of iodine containing carbolic acid; for further treatment, linseed oil containing $5 \%$ earbolic acid. Wilhelmi eleans tnt navel and the umbilical stump with a $1 \%$ lysol of ereolin solution, whereupon he opens the sheath of the umbilical cord, paints it outside and in with a liniment con sisting of iorline, tannin, and glycerine, and then knots the lower end with two fingers. In places where the owner is poor, rejeated painting of the navel region with wood tar dissolved in oil and turpentine, gives good service.

The disinfection of the unbilicus, if earried out with sufficient thoroughness and cleanliness, gives very good results, and in this manner the disease has been checked anong colts and calves, (Gmelin, Nocard, and others.) In some cases, however, an aggravation of the condition has been observed to follow, probably because the persons in charge of the treatment have directly infested the umbilical cord with their contaminated hanis and instruments.

Immunization. As in some of the cases, the causative agent proved to be the colon bacillus, and owing to the close relation between pyosepticemia, and white scours of the newly-borns, the immune serum of animals treated with colon bacilli may be also recommended for this disease (see page 156). Considering that the bipolar bacilli have also an etiological part in this disease, the use of the serum which serves against this bacterium appears to have at least an experimental foundation.

Literature. Fürstenberg, V. A., 1864, XXIX, 152.-Roloff, Ibid., 1866, XXXVIİ, 434.-Bollinger, Ibit., 1873, LVIII, 329; D. Z. f. Tm., 1875, I, 50.Pfeiffer, A. f. Tk., 1891, XVII, 81.-Gmelin, Monh., 1891, II, 196 (Lit.) ; 1897, VIII, 259.-Hess, Lanitw, Jahrb. d. Schweiz, 1897.-Casper, D. t. IV., 1897, 159.-Sohule, Monh., 1901, XII, 337.-Ostertag, Ibid., S. 385.-Krautstrunk, Z. f. Infkr., 1910, VII, 256.

\section{Influenza of Horses. Influenza equorum.}

(Equine influenza, Pink eye, Typhoid fever, Stable pneumonia, Influenza catarthalis s. erysipelatosa et pectoralis,

Plenro-pnemmonia contagiosa equorum; Grippe,

Fière typhoide, Pneumonie infectiense, Pasteurellose du cheval [French]; Feblre

tifosa [Italian]; Pferdestaupe, Rotlaufseuche, Brustsenche

$$
\text { [German].) }
$$

Influenza is an acute febrile, contagious infectious disease of horses, which occurs frequently in stables in an enzootic form. It is characterized either by acute catarrh of the mucous 
membranes or by severe inflammation of the lungs and pleura. Accordingly two forms of the disease may be distinguished, namely: the catarrhal influenza, and the pectoral influenza.

History. The term "influenza," taken from the Italian nomenclature of human medicine (in Italian identical with "contagio"=infection), has beell used since the beginning of the last century for all acute febrile affections of horses which spread rapidly and have a pronounced miasmatic, or miasmatic-contagious eharacter. Even in the middle of the century various diseases have been collected under this term, and among others the petechial fever has also been considered in this group. Later Falke (1862) applied the term "Influenza" only to the febrile catarrhal affections of the mueous membranes, while to the eases in which the inflammation extended to the internal organs, especially to the respiratory apparatus, he applied the name of "Typhus." At the same time he considered the two forms to be different in their etiological nature.

Similar to this was the eonception of Dieckerhoff, only that he designated the first form as "horse distemper," and the latter as "contagious pleuro-pneumonia." At the same time he separated from these, as independent affections, the "Skalma" under which he collected those cases in which a moderate fever, a diffuse infectious bronehitis, with a subacute course, without an affection of the conjunctiva, or edematous infiltration of the subeutaneous conneetive tissues, were the characteristic indications. Further, he also considered "infectious laryngitis," "infectious pharyngitis," and "endemic cough" as independent diseases. Other authors have not recognized these four different forms of disease, otherwise, however, the majority of German authors, as well as many French writers, have accepted the classification of Falke and Dieckerhoff.

As a matter of fact, even at the present time there are no experimental proofs which would convineingly establish either the identity, or the difference of the two diseases. Purely practical experiences, however, speak rather for their elose relationship. It has frequently been observed in outbreaks in large stables, that while some of the animals become affected with eatarrhal manifestations, often with serous inflammation of the tendons, in others again, severe symptoms of pleuro-pneumonia develop, the characteristic affection of the eyes being frequently present in both forms of the disease. As a result of these observations the relation of these forms appears evident, particularly as the etiological studies of their nature, by which the possible difference of the two forms of the disease could be established, have not yet been completed. In spite of the marked differences of these two forms, in which, however, transitory forms are frequently observed, it appears advisable to follow the view of Siedamgrotzsky, and treat the disease as two different manifestations of the same afteetion; namely, catarrhal influenza and pectoral influenza.

Influenza has been known from earliest times under various names (Febris nervosa, F. maligna, Typhus, ete.), and its infectious nature has been recognized since the second half of last century. Schütz (1887) undertook extensive bactericlogical experiments to establish the nature of the disease, and indicated a diplococeus as the causative factor of the disease. Hell, Foth and more recently Lignières, however, proved that this bacterium is really a streptococeus, and is at least closely related to the streptococeus of strangles. On the other hand, 
Babes, Starcovici \& Calinescu (1889), as well as Galtier \& Violet (1890), demonstrated in the tissue of affected lungs bipolar staining bacteria, besides the diplococcus of Schütz. They suggested the etiological relation of this organism; Lignières (1897 to 1900) established its identity with the causative organism of hemorrhagic septicemia, and expressed his view that influenza is caused by these bacteria, while the diplococeus of Schütz plays an insignificant part in the etiology of the pathological processes. Exception may also be taken to this view, the same as against those of Schütz, and it may especially be mentioned that with neither of these two bacteria may a typical pneumonia be produced, as well as that the bipolar bacilli probably exert their pathogenic action only secondarily in the affected animal; therefore the question of the true cause of influenza, or of its two forms, must be considered at the present time as unsolved.

Occurrence. The disease occurs everywhere either sporadically, or in a pronounced enzootic form, and in the latter case it spreads rapidly among the horses of large territories. With the greatly varying character of the disease it sometimes causes only slight losses, while at other times a considerable percentage of the affected animals succumb to the disease, principally as a result of pleuro-pneumonia which develops in its course. Owing to the latter condition the disease is of great economic importance, but the owners also sustain considerable loss in the milder outbreaks as a result of the incapacity of the horses for work.

Large outbreaks occurred in Europe during the last century in the years of $1813-1815,1825-1827,1836,1846,1851,1853,1863,1870-1873$ (in connection with the German French war), and finally in the years of $1881-1883$, at which time the disease spread over almost the entire continent. In 1872-1873, the disease occurred extensively in America; starting in the eastern parts it extended all over the United States. It was introduced in South Africa by the English troops in 1599.

In 1908 the reports from the German Empire show in Prussia 622 deaths from the disease; in Bavaria and Saxony 55 and 210 eases resp., were affected with the pleuro-pneumonic form, while 12 and 48 cases respectively were affected with the catarrhal form. In Württemberg 88 eases with 12 deaths resulted from the disease. Howerer, these numbers are not considered as complete.

Among the horses of the Prussian army, in the years between 1899 and 1908 , $861,4,325,1,066,594,2,223,1,367,487,1,811,8,058$, and 1,010 horses, were affected with the catarrhal form, while the pleuro-pneumonic form oceurred in 2,301 , $1,608,1,078,1,937,1,263,1,675,3,577,7,581,3,502$, and 2,750 horses, respectively.

Etiology. The true primary cause of the disease has, up to the present time, not been estahlished. Bacteriological examinations prove only the fact that in the pectoral form (contagious pleuro-pneumonia) two bacteria are principally present, the streptococcus, pyogenes equi, and the bacillus equisepticus.

I. The streptococcus pyogenus equi, which has heen described by Schütz as the cause of contagious pleuro-pneumonia, under the name of diplococeus pneumoniae, is in its principal character identical with the streptococcus of strangles, or at least is closely related to the same. In the affected tissues it is present in the form of mono and diplococei; in the exudates in somewhat longer chains. It stains readily with aqueous 
aniline dyes as well as by Gram's method. On artificial media, it grows in the same way as the streptococcus of strangles (see that disease).

Pathogenicity. Cultures kill mice in two to three days, while rabbits are less susceptible, guinea pigs show a considerable resistance against the infection. In the experiments of Schütz 4 cc. of a bouillon culture injected in various parts of the lungs of a horse produced a gangrenous pneumonia and a sero-fibrinous pleurisy which extended from the point of inoculation. On autopsy great numbers of cocci identical with those inoculated were found in the gangrenous areas and in the tissues surrounding them, as well as in the pleural exudate. They were present in smaller numbers in the spleen and in the kidneys. The inhalation of a sprayed culture produced in the horse a circumscribed, chronic inflammation in one lobe of the lungs.

According to Lignières the streptococeus in question is identical with the diplococcus of contagious pneumonia of Schiitz, with the streptococeus of Delamotte \& Chantemesse, as well as with the streptococeus of Violet \& Galtier, and is elosely related to the streptococeus of strangles.

II. The bacillus (bipolaris) equisepticus (Pasteurella equi), which is considered by Lignières and even before him by Babes, as the true cause of the disease, represents a variety of the bacillus bipolaris septicus of the hemorrhagic septicemia. In regard to its staining and culture characteristics it is identical with the other varieties of this bacillus (see page 79).

Pathogenicity. Cultures obtained from the affected body of a horse produced a severe pathogenic action on rabbits and guinea pigs. The former succumbed to an intravenous infection in 18 to 24 hours, while the latter died in the same length of time from an intraperitoneal infection. Carnivorous animals and sheep also die of septicemia following an intravenous injection. Subcutaneous injection of 0.25 cc. of culture produces in the horse fever and a local inflammatory edema which later changes to suppuration. Sometimes however such an infection causes the death of the animal inside of four days. The abdominal exudate of guinea pigs which die as the result of an intraperitoneal infection acts much more severely. Onequarter to one-fifth cc. of such exudate, when injected subcutaneously into a horse may cause the death of the animal in 24 to 48 hours, while 1 to 2 cc. injected intravenously kills the animal in a few hours. In such cases the horse manifests high fever, great debility, accelerated and small pulse, throbbing heart action, muscular trembling, diarrhea, injection of the conjunctiva, and lachrymation. Less severe infection produces an edematous swelling of the extremities, and in some of the joints a sero-fibrinous inflammation. Subcutaneous injections of culture are sometimes followed by sero-fibrinous pleurisy, 
in which the exudate contains streptococci in great numbers in addition to the bipolar bacilli.

The bipolar bacilli may sometimes be demonstrated in the blood at the beginning of the febrile stage, they are very frequently found in the liepatized tissue of the affected lungs, and also in the pleural exudate. In the exudate they are usually present in association with streptococei, and also with other bacteria. They may also be found during the lung affectior. in the nasal discharge.

In repeating the investigations of Lignières, Hutyra found the bipolar bacilli only very exceptionally in the blood and in the nasal lischarge of affected horses; on the other hand they were present in great numbers in the affected lungs, however, usually associated with streptococei and also with other bacteria (colon bacillus, bacillus pyocyaneuts, and others). In the small hemorrhagie, or reddish brown, hepatized areas the bipolar bacilli are usually present in great numbers, while they loige in the gray hepatized or gangrenous areas, and in the pleural exudate, only sparingly, or their presence cannot at all be demonstrated. The bipolar bacillus, as well as the streptococens, apparently exert their pathogenic action through toxins. Filtrate of a bouillon culture of the bipolar bacillus, several days old, injected intraperitoneally into a horse in a dose of $500 \mathrm{~g}$., produced symptoms of severe intoxica. tion (rapid small pulse, diarrhea, profuse sweating). The filtrate of streptococens cultures is less toxic, nevertheless $350 \mathrm{cc}$. of the same injected intravenously nay also produce symptoms of severe poisoning which last for several hours.

The presence of pyogenic streptococci in the nasal discharge, and in the thoracic organs has also been established by other authors (Hell, Foth, Sven Wall, Ostertag, Tartakowsky and others). Pfeiler observed after an intravenous injection of bouillon cultures ( 35 to 120 ce.) persistent fever, nasal discharge, yellowish-red discoloration of the conjunctivae, as well as serous or fibrinous inflammation of the pleura, and in some cases also pneumonia. Tabusso found that cultures of the bacillus equisepticus exert a severe toxic action, and that the intrapleural injection may produce lobar pneumonia. Dreyer is of the opinion that the lisease may be transmitted with the fresh blood of affected horses. However, Ostertag proved in his varied experiments, which he carried out for many years, that the typical disease can be produced with neither of the two deseribed bacteria, nor with the diplobacillus, which he found in the nasal discharge and, more rarely, in the blood of affected horses. He further showed that the disease cannot be transmitted to healthy horses by means of the nasal discharge, exhaled air, pleural exudate, urine and blood of affected animals.

The experiments of Hempel \& Pfeiler also speak against the streptococei and bipolar bacilli as being the primary causes of the disease, as they failed to demonstrate complement fixing substances for these respective bacteria in the blood of horses affected with the disease.

The view of Lorenz, according to which the cause of the disease is a pleomorphous micro-organism, usually present on the surface of the skin of the pastern, is a priori extremely improbable and has never been substantiated by others. The claim of Baruchello \& Pricolo that the causative agent of the disease is a blood spirochete, and the more recent assertion of Baruchello \& Mori that it is a piro. plasma, are contradicted by the fact that the numerous blood examinations of other authors failed to demonstrate the presence of these organisms, and can be only explained by assuming that these Italian anthors studied a disease of horses which differs from the influenza occurring in middle Europe.

The etiological action of the bacteria found up to the present in influenza cannot as yet be established aceurately. For none of these organisms has sufficient proof been advanced that they can produce the disease primarily, and no one has ever suceeeded in producing croupous pneumonia in its typical course artificially. It is therefore possible that the primary cause of the disease, or the infective agent which transmits the disease from animal to animal, is a micro-organism which is not yet known, and that the streptococei, as well as the ovoid bacteria, which are normal habitants of the healthy air passages, exert their pathogenic action only after the specific virus has already affected 
the animal, and they then subsequently produce the inflammatory changes attributed to them. The resistance of the horse may be weakened also by other influences such as colds or overexertion, in which condition the action of the bacteria mentioned may be exerted and lead to the development of the inflammatory processes in the lungs. The pleuro-pneumonia which develops in such cases would anatomically and bacteriologically manifest the same character, inasmuch as it is caused by these same bacteria, but it would differ from influenza by the absence of all contagious properties. This conception, which is based only on theoretical considerations, tends to explain the peculiarities in the development of the disease, which sometimes appears as an enzo-otic, at other times only in a sporadic form, while the clinical and anatomical manifestations, especially the croupous pneumonia, are practically the same.

Poels' observation, which refers to a stallion, deserves special mention. This animal for months infected, in the act of coitus, the mares which he covered, and its semen, fresh as well as filtererl, when injecterl intravenously in healthy animals, prorlucel influenza after an incubation of from 5 to 6 days. The blood of horses infected in this manner also proved virulent, even after being filtered through a Berkefeld filter.

The natural infection is evidently transmitted usually by secretions of the lungs, and by the feces of affected animals, while infectiousness of the exhaled air is very improbable.

The secretions and excrements are especially infectious during the development and at the height of the disease. The convalescing animals may also infect, and even apparently recovered animals may do likewise, in cases when the lungs still contain gangrenous areas after the disappearance of the acute symptoms. The development of the disease among horses, by the introduction of an apparently healthy animal, can be explained by such a mode of infection. The animal introduced had probably been affected with the disease but a short time before. This may also explain the continued existence of the disease in some stables, when it is continuously transmitted to other animals from one which has not completely recovererl from the disease. Such apparently recovered animals, whose lungs contain necrotic lesions, which are not encapsulated, may carry the infection for months (Siedamgrotzky). Usually, however, the disease is introduced by animals which are in the incubation period or in the first stages of the disease, and in this respect animals bought in horse exchanges are especially dangerous, while among army horses the disease frequently breaks out after the introduction of remounts.

According to some English authors (Pottie, J. Clark, Rieks), as well as the observations of Jensen, stallions which recover from influenza may transmit the disease to mares in the act of coitus months later and even after one to two years. Grimme reports a case in which apparently 14 out of 22 mares were said to have been infected by a stallion, which, besides a slight reddening of the conjunctivae, manifested no other indications of the disease. (See also the observation of Poels, above.)

Indirect infection is transmitted by the most varied objects which may be contaminated. Such are the secretions and ex- 
crements of affected horses, contaminated food, straw, manure, stable utensils, cribs, pails; people who are employed in handling sick animals, their clothing, etc. The virus may retain its virulence for a long time in poorly ventilated, musty and damp stables, while in pure, dry air and under the influence of sunlight it loses its virulence within a short time. These conditions may explain the fact that outbreaks usually remain confined to individual stables or townships, or to limited localities, and that a wide extension of the disease, such as is the case, for instance, in foot-and-mouth disease, is rarely observed.

The possibility of the infection heing transmitted by flies, as intermerliate carriers of the virus (Giesecke), is not at all probable in consideration of the mode of appearance and extension of the disease, and especially in view of the frequent localization of the affection in individual stables.

The infective agent, as a rule, enters the animal body with the feed or drinking water, and very probably through the intestines or through the lymphatic pharyngeal ring (Walther); however the possibility of infection through the air passages should not at the present time be disregarded.

Pecus reports on observations of outbreaks in which the infection could only have occurred through the digestive tract; in several stables only those horses became affecterl which drank from a common trough, whereas the horses which were watered with individual pails escaped the disease. One particular horse drank from a pail the contents of which was contaminated by the discharge of an animal affected with influenza, and this horse developed the disease in six days.

Of the predisposing causes of the disease colds should come first in consideration. Experience also points to this fact, that the beginning, and the greatest extension of the disease, is frequently associated with northern or eastern winds, and the frequent occurrence of the disease in the cold, damp periods of the year can be traced to this. Catarrhal affection of the mucous membranes is another predisposing cause for the disease, and owing to this fact the mortality among horses which are kept in badly ventilated stables is usually greater than under more favorable hygienic conditions.

The age of the animals has an influence on their susceptibility, inasmuch as horses over one year of age are most readily affected with the disease, while it occurs less frequently among very old animals. The disease occurs with greater frequency and in a more severe form among horses of high breeding, as well as among the heavier breeds. All solipeds are susceptible to the disease.

One attack of the disease diminishes the susceptibility to infection for only a short time. We have observed horses attached to the medical (army) service which passed almost every year through a slight attack of influenzal pneumonia.

Pathogenesis. As the primary cause of the disease is at present unknown, only suppositions are admissible relative to its method of action. The infective agent produces a general blood infection which is manifested in general febrile and 
catarrhal symptoms. The disease which develops in this manner may, after the disappearance of the infective agent from the body, terminate in recovery within a short time. Most cases of the catarrhal form of influenza follow this course, while an unusually severe infection may result in a very severe septic manifestation, and canse the death of the animal within a few days. The autopsy findings in such cases are similar to those of hemorrhagic septicemia, with hemorrhages in the serous and mucous membranes, hyperemia, and small hemorrhages in the parenchymatous organs, especially in the lungs and kidneys, but as in such cases the bacteriological examination not infrequently shows the presence of the bipolar bacilli in the organs as well as in the blood, there exists a possibility that they have taken part in the fatal termination of the disease. These organisms enter the blood in an early stage of the disease, multiply in the same very rapidly, and are frequently the direct cause of death, or at least they hasten death.

The primary infection, that is, the general febrile condition, may weaken the body to such an extent that the facultative pathogenic bacteria, especially the ovoid bacteria and streptococci, can exert their specific infective action. As a result of their invasion inflammatory processes develop, especially in the lungs, in the form of pneumonia or pleuro-pneumonia, while the primary infective agent has possibly in the meantime disappeared from the body.

The bipolar bacillus, as well as streptococci, may produce such inflammation; usually, however, in the cases where pleuropneumonia caused the early death of the animal, under manifestations of a violent septicemic character, the pleural exudate and the affected lung tissue contain the ovoid bacteria exclusively, while the streptococci appear to multiply more rapidly only later in exudates which have already developed as the result of the disease. These latter are present in predominating numbers or sometimes even exclusively in the hepatized and necrosed lung tissue, while in the plemral exudate they are usually found exclusively (the pleurisy apparently being caused only by the streptococci which pass from the lungs into the pleura). Streptococci may also enter the blood, and in this way they may reach the parenchymatous organs when they may be found on autopsy in the spleen, the kidneys, and in the lymph gland .

Other bacteria may in some cases cause a secondary infection; thus, in one of our cases, hesides the bipolar bacillus, the diplococeus lanceolatus (recently also demonstrated by Meyer), in another case the bacillus pyocyaneus, were present in great numbers. Besides these the colon bacillus may often be found in the inflamed lung tissue. The eventual necrosis of the inflamed lung tissue may be due to the bipolar bacillus or to the streptococcus. In several cases the gangrenous lesions have contained the bacillus necrophorus, which indieates that in this process, at least under suitable conditions, this 
bacillus probably has an active part. Bourget \& Prevost finally found a virulent form of proteus in the pleural exudate of several horses.

Anatomical Changes. In the catarrhal form of influenza an acute hyperaemia of the mucous membranes develops in the digestive tract or in the air passages, or possibly in all the mucous membranes. This condition is associated with an edematous infiltration of the submucous connective tissue, as a result of which the mucous membrane, especially in the larynx and in the pyloric portion of the stomach, and in the small intestines, forms thick gelatinous folds. In the small intestine the solitary follicles, and the Peyer's patches are swollen, and moreover the reddened mucous membrane contains in places superficial erosions or even deep ulcers (Rips). The mesenteric lymph glands are greatly swollen, their tissue is reddishgray and moist.

Very frequently the subcutanous connective tissue is infiltrated with a clear, or somewhat cloudy, readily coagulating serous fluid. These infiltrations may occur at different parts of the brody, especially in the parts surromaling some of the tendons of the extremities. A similar fluid may be contained in the tendinous sheaths in considerable quantities. The yellow, gelatinous tissue is in such cases studded with small hemorrhages, and similar changes are sometimes present in the suband retroperitoneal connective tissue, especially in the immediate vicinity of the kidneys. The body cavities may also contain various quantities of a pure or slightly cloudy, reddish exudate.

The other internal organs are not much changed. A moderate, acute swelling of the spleen may be present, also hyperaemia or acute edema of the lungs, cloudy swelling of the heart muscles, of the liver, and of the kidneys. Hyperemia of the meninges, and even a slight acute hydrocephalus internus may be found.

In the pectoral form of influenza, necrotic pneumonia, in association with sero-fibrinous pleurisy, represents the most important and common anatomical change. The pneumonia sometimes occurs in the form of lobular broncho-pneumonia, or the inflammatory process extends in the form of a lobular pneumonia, affecting at the same time, large contiguous areas of one or both lungs. In the first case the lung contains only a few dense hepatized catarrhal or hemorrhagic areas; the tissue in immediate surroundings of these areas is serously infiltrated; while in the second case, the lower sections of the lungs are uniformly hepatized throughout large areas. The affected lung tissue breaks readily, the cut surface is granular, and depending on the stage of formation, it is either gravishbrown, grayish-red, or yellowish-gray. In the parts still containing air, besides the hyperemia or acute edema, the connective tissue walls are gelatinously infiltrated. 
In the hepatized tissue numerous necrotic areas may frequently be disseminated, which vary in size from that of a poppy seed to a hazelnut. They are of a dirty, grayish-brown or greenish color, and of a mushy consistency. In some cases a circumscribed portion of the affected lung is changed into a necrotic mass which may attain the size of a fist or a child's head, and may sometimes be surrounded by a layer of pus.

The mucons membrane of the bronchi and trachea is bright red, swollen, not infrequently sprinkled with hemorrhages, and profusely covered with sero-purulent secretion.

Inflammation of the superficial layers of the lung is always associated with pleurisy. The pleura loses its luster as a result of a desquamation of the epithelial layer, is dull, rough, and covered by loose, moist, smeary pseudo-membrane. This peels off readily, and is of icteric yellow color, and somewhat resembles pudding. Between the two layers of the pleura there is a yellowish fluid which contains. flakes of the fibrinous membrane. This exudate is cloudy, and is sometimes present in considerable quantities, exerting more or less pressure on that portion of the lung which is not hepatized. In less acute cases there is a thick, wide connective tissue layer under the loose false membrane. If the pleurisy develops in connection with superficial necrotic fields, or as a result of the rupture of such an area, the exudate is always of a purulent or ichorous character. In case the gangrenous area which opened into the pleural cavity communicates with the bronchi, pneumothorax or pyo-pneumothorax will develop.

In other respects the autopsy reveals conditions similar to those present in the catarrhal form of influenza, only the changes are usually somewhat more pronounced. On the mucous membranes not infrequently superficial ulcerations may be found besides the catarrhal reddening and swelling. The parenchymatous degeneration of the heart muscles, the liver and in the kidneys, frequently passes into a fatty degeneration. Small hemorrhages or even extensive suffusions are often present in the tissue of the serous membranes as well as in the endocardium. The lymph glands, especially those of the thoracic cavity are greatly swollen. Suppurative areas may exceptionally be found in various organs, and purulent inflammation of the joints.

Symptoms. The time of incubation in influenza extends in the majority of cases from 3 to 7 days; less frequently, especially after the animal has contracted a cold, the first symptoms may appear after 24 to 48 hours, while in other cases the disease may remain latent for 8 to 9 days. In some cases the animals are affected for a time only with a slight bronchial and stomach catarrh, while the manifestations of the pectoral form of influenza gradually develop later. In these cases the time of incubation appears to be considerably longer, although 
the preexisting catarrhal manifestations were really indications of infection with influenza.

According to Siedamgrotzky the time of incubation in influenza is from 4 to 5 days, while Dieckerhoff claims that the first manifestations appear in from 12 to 24 hours. On the other hand, even 14 days may in exceptional cases elapse before the onset of the disease. In infections which are transmitted by coitus the disease usually develops in from 6 to 9 days (Clark, Jensen, Grimme).

The disease commences almost invariably with marked depression, dullness of the sensibility, and capricious appetite. The animals stand with eyes half closed, ears drooped, supporting the head to the crib or the strap of the halter, sometimes standing in the stall, apathetic and in abnormal positions. They take the food offered them with hesitation, masticate it slowly, and with frequent interruptions. They frequently do not swallow the bolus, but it lies in the mouth on the outside of the molars apparently forgotten. If urged to move they walk lazily and with some difficulty; frequently the gait is staggering.

The body temperature is first elevated, and reaches on the first day of the affection 40 to $40.5^{\circ} \mathrm{C}$. At this point the temperature remains for two or three days with only slight variations, whereupon in the catarrhal form, after one to two days more it drops rapidly to normal (Fig. 35 on page 181).

In the case of pure or predominating pneumonia the temperature continues to remain high, with slight variations, and returns to normal only after the regeneration of the changes in the lungs (Fig. 36 on page 183). In severe cases the fever persists continuously or remittently until death. In slowly progressing cases however the fever is interrupted periodically by afebrile intervals (Fig. 37 on page 186). During the period of high fever the heat over the surface of the body is unevenly distributed, the coat is rough; muscular trembling may be also observed in some parts of the body. The skin sometimes manifests nodular eruptions or urticara. The heart's action is from the beginning more or less excited, in severe cases it is greatly accelerated and weak, as a result of the developing parenchymatous degeneration of the heart muscle. The pulse is usually from 50 to 60 per minute; in severe cases, however, the number may increase to from 80 to 100 , with throbbing heart and small, wiry pulse.

\section{Special Symptoms of the Catarrhal Form of Influenza.} In this form of the disease catarrhal manifestations of the mucous membranes develop in association with the ahove described febrile symptoms.

In the eyes the conjunctivae appear reddened (mahogany or brick red), frequently with a yellowish tint, the submucous connective tissue containing a serous infiltration. There is always photophobia as well as lachrymation present. The eyelids swell, and the opening of the eye is thereby narrowed. 
If it is forced open, tears escape profusely, or in the later stages a secretion which is mixed with mucus or pus. The swelling may extend to the sclerotic conjunctiva, as a result of which the cornea is surrounded by a yellowish-red, somewhat elevated ring. The cornea proper is often cloudy, and the epithelium is desquamated from the anterior portions. In some cases an iritis may also develop, with a contraction of the pupil, and the deposit of a yellow fibrinous exudate in the anterior chamber of the eye.

The catarrh of air passages is first manifested by a rough and dry, later by a moist but always strong and sometimes painful cough, which comes on spasmodically. From the reddened mucous membrane of the nose first a small quantity of a serous, later more profuse muco-purulent discharge escapes, which during spells of coughing is also expelled through the

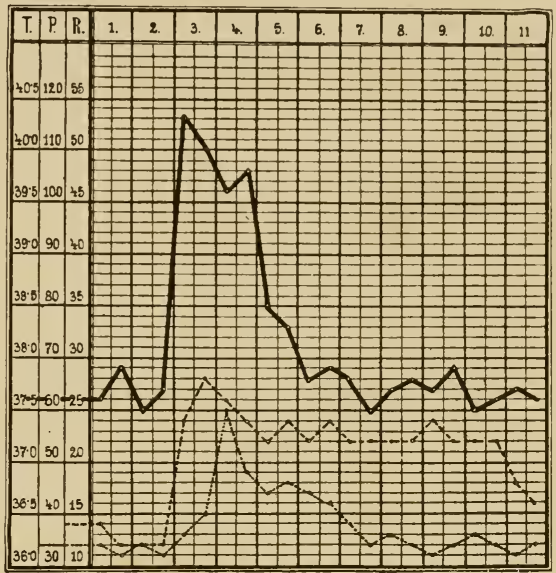

Fig. 35. Fever curve in catarrhal influenza. mouth and nose. If the nasal catarrh is severe the submaxillary lymph glands are usually swollen and sensitive. Respiration is more or less accelerated and superficial. Percussion reveals normal conditions over the thorax, whereas on ascultation, raw, vesicular breatling, dry and moist rales of varying intensity and numbers, may be heard. Sometimes an increased sensitiveness of the thorax may also be present. The appetite is at first depressed, while the animals willingly take water, although only in small quantities. The mucous membrane of the mouth is warm and dry, the tongue is also dry and coated. Not infrequently difficulty in swallowing may be observed as an indication of the coexisting pharyngitis.

Gastric catarrh is indicated by the distribution or entire disappearance of the appetite, also by frequent yawn and a desire to lick cold objects. At first there is constipation, during which the feces are dry, forming small balls, covered with mucous shreds. The abdomen is sometimes moderately bloated. Later the feces become softer, or even fluid, and towards the third or fourth day frequently fetid. Sometimes they are of a dirty reddish color, and expelled with straining. Peristalsis is at first depressed, later lively, and in some instances may. 
be uninterrupted. Not infrequently mild colicky symptoms may be observed. The persistent diarrhea and the absence of appetite weakens some of the patients considerably.

During the entire existence of the febrile condition urine is passed in only small quantities. It is of a high specific gravity, and dark yellow in color, containing a small quantity of albumen, and sometimes bile pigments. The chemical reaction in the later stages, and in the presence of continuous bad appetite turns acid. If allowed to stand a great amount of sediment precipitates, which may contain epithelial cells from the bladder and the pelvis of the kidneys, exceptionally also casts may be noted. In the presence of catarrh of the bladder and urethra the animals urinate frequently and strain during each passage. A transient polyuria may develop after the subsidence of the other symptoms. In females the mucous membrane of the vagina may show catarrhal inflammation, and in such cases a muco-purulent discharge escapes.

In some cases edematous swellings develop in the subcutis, especially on the chest and lower abdomen, or on the lower extremity of the sheath. Occasionally urticaria-like eruptions may occur on the rump; these present the form of round, beetlike elevations. Such subcutaneous and cutaneous edema may be observed in some outbreaks on practically every patient. In other instances it may be observed only in isolated cases. In some outbreaks there may be found an inflammation of the flexor tendons and tendinous sheaths of the extremities, with an accumulation of serous exudate in the latter. As a result of the pain which is associated with this form of affection the patients rest the legs intermittently and keep them flexed as much as possible.

The symptoms described may reach various degrees in the different cases. In mild forms they may disappear in one to two days, whereas severe symptoms develop only in very few instances.

Kramell reports a peculiar form of influenza in 39 out of 72 army horses. The fever, lasting from one to six days, varied between 38.6 and $41^{\circ} \mathrm{C}$., at the same time the frequency of pulse dropped to 30 and 28 beats. In about one third of the cases a disturbance in the gait of the animals was observed, which was evinced by stiffness of gait, resembling a rooster's step, or lameness in the different extremities. Respiration was not affected, the conjunctivae were usually normal, and the appetite was capricious.

Special Symptoms of the Pectoral Form of Influenza. This form is also inaugurated by febrile and catarrhal symptoms, as is the case in the catarrhal form, only they are more intense in character. In the meantime symptoms arise which point to an affection of the respiratory organs, and this develops in a short while into characteristic acute pneumonia or pleuropneumonia. The affection of the lungs represents either lohar 
croupous pneumonia, or lobular broncho-pneumonia, but the definite differentiation of these forms is only in rare cases possible because the latter also has a very acute development and a rapid extension.

In some cases the pneumonia develops in a manner similar to genuine croupous pneumonia (see Fig. 36). Accordingly the symptoms consist of difficult, costal-abdominal breathing; first a tympanic, later a dull sound on percussion over the lower parts of one or both sides of the thorax, which later changes into a tympanic and finally into a normal sound. On ascultation rales may be heard, later bronchial breathing or the absence of any respiratory sound. This may be again followed

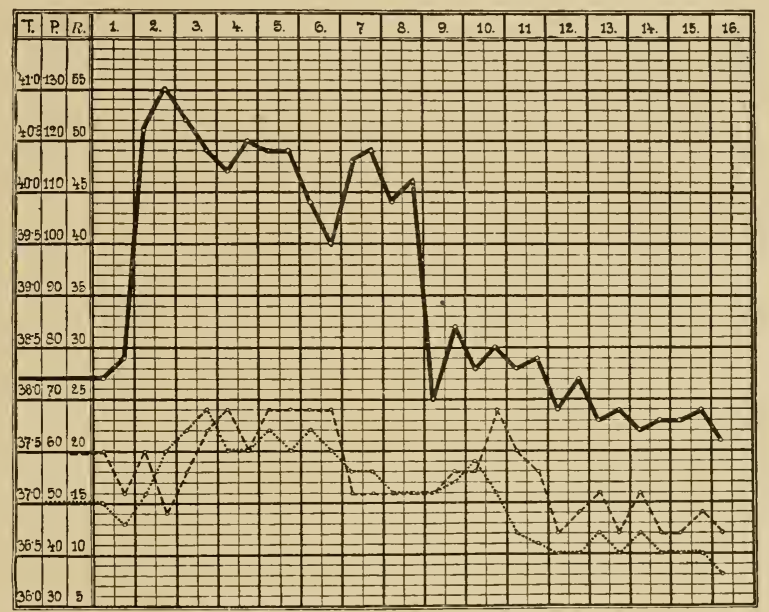

Fig. 36. Fecer curve in pectoral influenza. Type of the croupous pneumonia; recovery.

by moist rales. In numerous cases there is a saffron, or rustcolored nasal discharge (for detailed information see Vol. 2, Chapter IV). On the other hand, if the affection of the lung's develops as a lobular pneumonia the symptoms of hepatization (dullness, bronchial breathing), may be entirely absent, or they may be established only over small irregular areas, so that the existence of pneumonia is suggested only by the marked respiratory difficulties, the high fever and possibly the rustcolored nasal discharge.

The pleurisy which frequently develops in association with the pneumonia is manifested by a sensitiveness of the thorax, and also by superficial abdominal breathing, later by double 
breathing, friction sounds, and if a pleuritic exudate forms also l,y dulhess below a horizontal line (see Vol. 2, chapter on Pleuritis.)

In the Prussian army, in the years of 1899 to 1908 , out of 9462 eases in which $319 \mathrm{~s}$ were affecterl with pleuro-pneumonia, in 1817 eases pneumonia on the right, and in $17 \times 0$ pneumonia on the left side, in 1336 double pneumonia, and in 100 cases pleurisy only was present. In the elinies of Budapest, in the years of 1904 and 1906 , in 169 eases the pneumonia was unilateral, in 195 eases it was bilateral, while in 106 cases pleuro-pneumonia was established.

In association with the pneumonia gangrene of the lungs develops subsequently with relative frequency, and to a certain degree this is characteristic of the lung affections in influenza. In such cases the symptoms consist of a disagreeable odor of the exhaled air, cavity formations in the hepatized lung tissue, high fever, weak lieart action, etc., which usually develop in the course of the second week of the affection. A nasal discharge is observed in almost every case. The secretion consists first of only yellowish drops, later it becomes more profuse and purulent, or rust-colored, and tenacious, in exceptional cases it may also be bloody. After the appearance of gangrene the discharge becomes of a greenish-brown color, and has an intensely disagreeable odor.

The urine invariably contains albumen, sometimes also blood. During the incipient stage of the pleuro-pneumonia the quantity of chlorine salts is diminished, but it increases again at the convalescing period as a result of the absorption of the exudate. The appetite is entirely absent, the chemical reaction of the urine is acid. Microscopically the sediment shows epithelial cells from the urinary passages, sometimes hyaline or granular casts, and even red blood corpuscles.

The number of lymphocytes in the blood increases from the begimning of the disease, and by the fifth to seventh day may exceed the normal number by $40 \%$. After recovery has taken place, normal relation is not established until a long time after the disappearance of the clinical symptoms (Sturhan).

The patients become emaciated in the course of the disease as a result of the fever and inappetence. They usually do not lie down, or in the presence of a painless pure pneumonia they lie only on the affected side, and in the unfavorably terminating cases they succumb as a result of septicemia or aspliyxiation.

Complications occur quite frequently in the course of the disease, and may lead the otherwise mild affection to a fatal termination. Of relatively small importance are the inflammations of the tendons and tendon sheaths of the extremities, especially of the flexor tendons, also inflammations of the sensitive lamina of the hoof; this applies also to the edematous swellings of the skin, which may appear as long as the heart's action is sufficiently strong, as these processes soon disappear after the improvement of the inflammation of the respiratory organs (Fröhner observed in the later stages of contagious 
pleuro-pneumonia the development over night of a weeping exzema around the ankles of the hind legs and over the hoofs which later extended also to the front legs, causing suppuration and scab formations). The paralysis of certain nerves, which develops in the course of the first or second week of the disease is likewise usually only temporary; the nerves most frequently affected are the $\mathrm{N}$. recurrens vagi, N. facialis, $N$. trigeminus or the N. ischiadicus. In some cases, however, the paralysis subsides only after a longer period.

A more dangerous and quite frequent complication is the parenchymatous or fatty degeneration of the heart muscle, and the paralysis of the heart which develops as a result of it, or owing to a pericarditis which develops at the same time. The parenchymatous degeneration of the kidneys which is almost constantly present may later pass into an acute nephritis, manifested by a diminution of the quantity of urine, marked albuminuria, and the presence of numerous other constituents.

The manifestations of intestinal catarrh may increase in severity and a profuse diarrhea develop. If this does not persist for a long time recovery may follow. Severe intestinal symptoms however which indicate deep-seated inflammatory changes of the intestinal mucous membranes, always aggravate the course of the disease, inasmuch as it causes in the weakened patient an almost complete exhaustion; and besides the diarrhea is not infrequently a symptom of developing septicemia.

Septicemia, exceptionally pyemia, usually terminates the course of the disease, especially when the grangrenous processes in the lungs have reached great extent. Persistent high fever, chills, very weak and frequent pulse, as well as diarrhea, indicate the development of the disease in this direction.

As further complications may be mentioned pronounced icterus, hemorrhages in the lungs (into cavities and thence into the communicating bronchi), or between the layers of the pleura, marked nervous irritation, sometimes associated with spasms and trismus (Wohlmuth; Wilden observed in a case the development of general tetanus 8 days after the onset of the disease, which lasted for four days and suddenly disappeared on the fifth day). Inflammation of the cerebral and spinal meninges, paralysis of the sphincter muscles of the rectum and bladder, as well as of the tail (Friedberger), occasionally develop during the course of the disease. Further, inflammation of the eye, sometimes with hemorrhages into the interior of the eyeball, serous and sero-fibrinous inflammations of the joints, verrucose and ulcerative endocarditis, necrosis of the skin over the edematous swellings, phlegmonous inflammation of the subcutaneous connective tissue, breaking through of a cavity in the lung under the skin of the thorax, falling of the hair from mane and tail, may also result during the disease. Pregnant mares may abort in the course of the affection. 
In the Prussian army in the years of 18,99 to 1908 , among 9,925 affected animals tenclonitis and tendo-raginitis ( 724 cases), roaring $(330)$, internal opthalmia (189), purpura hemorrhagica (53), skin eruptions (62), inflammation of joints (32), ete, occurred as the most frequent complications, or sequelae of contagious pleuro-
pneumionia.

Course. The catarrhal form of influenza lasts in the great majority of cases about one week, sometimes, however, only two to three days, in which cases the fever rapidly subsides,

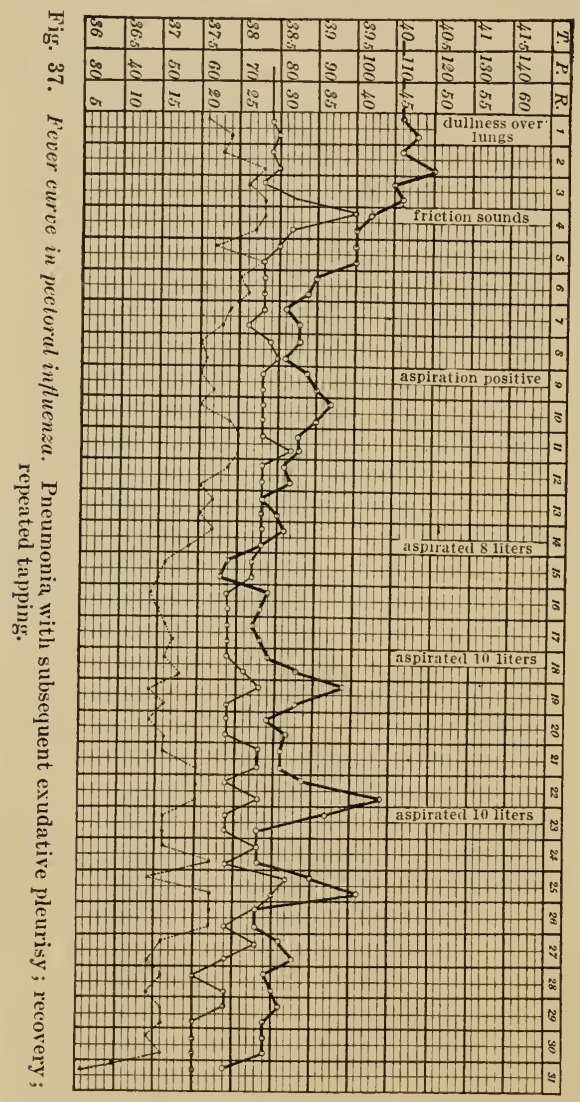
and the animals soon recover. In other cases the course may extend to two weeks, and then it requires considerable time until the animals regain their health. Fatal terminatious are exceptional, and only occur when severe complications become associated with mild forms of the affection (pneumonia, hyperemia or acute inflammation of the brain, enteritis, degeneration of the heart muscle, etc.). Much more varied is the course of the pectoral form of influenza. In the typical cases in which the different stages of lobar pneumonia follow each other with regularity, and the resolution is also undisturbed, the pleurisy being present in a mild form or not demonstrable at all, the fever may suhside at the end of the first or at the beginning of the second week. Here, however, complications and sequelae which develop frequently in association with the disease, more often cause a deviation of its regular course. Especially in cases of pleurisy in which a great quantity of exudate develops, the affection may extend for weeks (Fig. 37), and even for montlis, the patients manifesting temporary im- 
provement and repeated relapses, until finally the animal dies from exhaustion, or remains broken-winded for the rest of its life. Death may result however even earlier at the height of the pneumonia, usually following paralysis of the heart. Convalescence in this form of influenza takes a considerably longer time, and as a rule extends over a period of more than two weeks.

The termination of the disease is naturally also influenced by the existing hygienic conditions, and especially the continued working of animals already ill will usually render the course of the disease, which otherwise might have been only mild, an unfavorable one.

After the termination of the acute disease, chronic processes sometimes develop as sequelaœa After a severe pneumonia, and especially when the tissues in different parts have become gangrenous, chronic granulating inflammatory processes, with shrinkage of the lung tissue, may develop. This condition may also result in the formation of bronchiectasis, while after an exudative pleuritis, adhesions of the pleural layers may follow, as a result of which the animal may suffer from dyspne. until its death. A frequent result of pleurisy is the paralysis of the N. recurrens vagi, which causes roaring in the animal. The acute catarrh of the intestines may develop into a chronic form, which results in the gradual emaciation of the animal. Other after affects that may occur are paralysis of the hind parts, shortening and even necrosis of some of the tendons, chronic inflammation of the joints, exostoses, hemoglobinemia, and as a result of the inflammation of the eve, amaurosis. Finally, horses recovering from influenza may later become affected with purpura hemorrhagica, and the internal inflammation of the eye may also develop only two to three weeks after the passing of the disease (Hell).

The character of the disease varies greatly in the different outbreaks. While in some periods the course of the disease is very mild, so that the great majority of the animals are only slightly affected with the eatarrhal symptoms, and while also the infrequent pneumonia almost invariably terminates in recovery, there are outbreaks in which the disease is of a remarkably malignant character. This form is characterized especially by frequent hemorrhages, as well as by pneumonias which frequently assume gangrenous forms. The character of the disease may also change in one and the same outbreak; thus in some instances it may occur that at the beginning of the outbreak only cases of the catarrhal form of influenza are observed, while later the pleuro-pneumonic form becomes more frequent, and towards the termination of the epidemic the affections return again to the milder form.

Diagnosis. The catarrhal form of influenza may be easily mistaken for catarrhal manifestations of the mucous mem- 
brane from other causes, while pectoral influenza may easily be confounded with sporadic croupous pneumonia. Although the edematous infiltrations of the subcutis, as well as the tendonitis and the tendo-vaginitis, also the icteric discoloration and chemotic infiltrations developing in association with the conjunctivitis, are quite characteristic of influenza, these symptoms are not infrequently absent, especially in the early cases of an outbreak. In such cases the infectious character of the disease, and its rapid spread among horses, reveals the nature of the affection. In practice it is advisable therefore to suspend diagnosis in the first cases, until the appearance of new cases, or dependable data disclose the infectious nature of the disease, or until characteristic symptoms develop, or on the other hand, until the infectiousness of the affection may be excluded. In consideration of these diagnostic difficulties every suddenly appearing catarrhal affection, and particularly every croupous pneumonia, should be considered as if it were an attack of influenza, that is, as an infectious disease. Accordingly an immediate isolation of the affected animal is indicated in all such cases. Influenza is distinguished from the non-infections catarrhs, principally by the fact that in the former the catarrhal manifestations are preceded by high fever of sudden development, while in the latter the catarrhal symptoms are rather latent and develop only with slight rises in temperature.

If influenza has been established in a stable, or if the disease is present in nearby localities, all febrile affections should be considered as suspicious of the disease. Further, as the febrile rise of the temperature represents the first symptoms of influenza, it is advisable in order to separate immediately the newly affected animals, to take the temperatures of the horses in the infected or endangered stables systematically twice a day. A rise in the temperature of two degrees or over is sufficient to indicate the probable presence of the disease.

Infectious anemia may possibly be mistaken for the eatarrhal form of influenza, especially when it is associated with severe intestinal disturbances. The condition of the blood affords a means of differentiation since in anemia it coagulates incompletely, at the same time the serum frequently appears opalescent and of a more reddish color (Vallée). The appearance of pneumonia, or pleuro-pneumonia, or the development of similar symptoms in other horses of the stable would be indications of influenza; on the other hand, rapid emaciation and cachexia, without organic affection, as well as the presence at autopsy of a creatly enlarged spleen, would suggest the presence of infectious anemia. Strangles may also be considered in the beginning of the affection, from the standpoint of a differential diagnosis; in this, however, the swelling and suppuration of the lymph glands reveals the nature of the affection. 
Prognosis. The prognosis of the catarrhal form of influenza is usually favorable, as deaths seldom occur except as a result of complications, the appearance of which are commonly associated with unfavorable hygienic conditions. Under favorable conditions the loss hardly ever exceeds 0.5 to $1 \%$; it may however reach 4\% and even more. Accordingly in the prognosis all existing circumstances, as well as the benign or the malignant character of the disease should be taken into consideration.

The prognosis of the pectoral form of influenza is much more unfavorable. In this form the losses are never less than 4 to $5 \%$, and frequently considerably higher (according to Dieckerhoff the average is $16 \%$ ). Besides it should be also considered that in addition to the losses resulting from death some of the animals do not completely recover, and that their protracted convalescence may greatly diminish the value of the animals through the lessening of their ability to work.

The life of the affected animal is imperiled in the first place by danger of paralysis of the heart. Indications of a weak heart always render the prognosis unfarorable, and in the presence of a soft empty pulse of over 80 beats, as well as when a venous pulse can be detected, the chances of recovery are very slight.

The termination of the disease depends also on the extent of the pneumonia and pleurisy. A unilateral pneumonia with only slight pleuritis usually terminates favorably; on the other hand a bilateral affection, and especially the formation of great quantity of pleural exudate is always grave. In such cases, even at the best, the course of the disease is very prolonged, and chronic changes of the respiratory organs usually remain as a sequel to the disease.

Moderate fever, and at least a fair appetite, are favorable prognostic signs. The more regularly the different stages of the disease follow each other, the more favorable is the prognosis, while a rapid change for the worse in the respiration, with very high fever, without a distinctly demonstrable dullness (pneumonia centralis!), usually results in death after a few days. The dangers from cases of a hemorrhagic character are in most instances great (bloody nasal discharge, similar pleural exudate!).

According to Fröhner, prognostic conclusions can be marle in every case of contagious pleuro-pneumonia, from the consistence of the exudate which may be obtained by aspiration. If the exudate contains no bacteria the prognosis is always favorable; on the other hand, in the presence of bacteria (streptococci) the disease as a rule has a fatal termination.

The course of the fever is of great importance in the prognosis, especially in the pectoral form of influenza. If the temperature, after rising rapidly, remains for several days approximately at the same height, and if it falls towards the end of the first week just as rapidly, or even gradually, the prospect of a favorable termination is excellent. If, on the 
other hand, the fever curve shows a remittent character, and if the febrile condition lasts over a week, and also if a new rise in temperature takes place after the appearance of the apyrexia, an unfavorable prognosis is indicated. In the latter instance a rise in the temperature lasting only for one day (perturbatio critica), has no special importance; continuous fever is, however, always grave, as it either points to an extension of the pleurisy, or to the development of some other complication.

Unfarorable terminations are also indicated by the presence of severe inflammations of the tendons, which interfere with the standing of the animal; also persistent diarrhea, hemorrhages in the lungs or other organs, the appearance of pulmonary gangrene, or an affection of the brain, etc. A rapid reaccumulation of the pleural exudate after aspiration is also an unfavorable sign, although in exceptional cases, even after repeated aspirations recovery may finally take place, which, however, is usually incomplete. In all of these cases the dictetic conditions, the care and nursing of the patients, as well as the general strength of the animals should be given relative consideration. The more favorable the conaitions are in this respect the milder a course of the disease may be expected.

In the Prussian army, during the period of 1.5 years (1S94 to 1908), out of 26,678 horses affected with the catarrhal form of influenza, 40 died (or $0.15 \%$ ), in most instances as a result of subsequent pleuro-pneumonia; while in the same period 38,671 horses were affected with the pectoral form of influenza, of which 1,527 succumbel $(3.94 \%)$. Among the horses of private stables in which the hygienic conditions are usually more unfavorable, the losses are correspondingly higher.

Treatment. This consists in the first place, in a suitable regulation of the dietetic conditions. It is of particular importance that the animal should immediately be taken from work and placed in a clean, quiet and moderately cool stable. If the weather is favorable it should be allowed to remain outdoors, although protected against wind or rain. The diet slould consist of easily digestible, palatable, and if possible, of fresh green food, and the animal should be given fresh lrinking water in sufficient quantity (when the disease appeared among army horses the bivouacking of all the animals in the open air has repeatedly proved beneficial). As long as the course of the disease is normal, and there are no alarming symptoms, the expectant method of treatment may be followed. In such cases medicinal treatment may be omitted, with perhaps the exception of alcohol rubs which are always favorable, and the regulation of the bowels. In the presence of an extensive bronchial catarrh, inhalations of turpentine or tar vapors are advantageous (Lugano recommends also intratracheal injections of 10 to $12 \mathrm{cc}$. of a $4 \%$ formalin solution).

The fever does not require special treatment, as it usually 
subsides, without interference, after several days. Cold water in the form of donches, once or twice a day, or as rectal enemas, gives favorable results, especially during warm summer weather. Its beneficial action, however, is due rather to the stimulation of the nervous system and the heart's action, than to its antipyretic action, although continued application of cold water douches does reduce the temperature from one to two degrees. Direct antipyretic treatment appears to be indicated, when the body temperature remains abnormally high, even in the later stages (over 40.5 to $41{ }^{\circ} \mathrm{C}$.). In such instances the internal administration of antipyrin and of anti-febrin (15 to $30 \mathrm{~g}$.) is advisec.

If pneumonia or pleuro-pneumonia develops in association with catarrh of the air passages, or if the affection develops in this form from the beginning the application of Priessnitz bandages, as well as rubbing with camphor and spirits of turpentine, act favorably. During the summer weather, cold douches once or twice daily may be applied advantageously. The heart's action requires special watching, and in case it is accelerated, while still strong, the administration of alcohol in drinking water ( 25 to $50 \mathrm{~g}$. alcohol, $1 / 2$ to 1 liter of wine) will give beneficial results. In case the heart's action is greatly accelerated, and the pulse is weak, heart stimulants are indicated, such as digitalis leaves (2 to $5 \mathrm{~g}$.), tincture of strophanthus (10 to $25 \mathrm{~g}$. ), caffeine (1 to $2 \mathrm{~g}$.), camphor (2 to $5 \mathrm{~g}$. subcutaneously every 3 to 4 hours), or harium chloride ( 2 g.). Strophantine may be given subcutaneously $(0.003 \mathrm{~g}$. in $10 \mathrm{~g}$. of water, injected at 2 places).

Fröhner recommends instead of the natural eamphor, the cheaper preparation made artificially from oil of turpentine, for the treatment of contagious pleuro-pneumonia. The horses should receive daily 100 to $200 \mathrm{~g}$. of oleum camphoratum (syntheticum) forte, or as a single dose 50 to $100 \mathrm{~g}$. injected on both sides of the thorax.

Recently Eherlein \& Toepper obtained very favorable results in the treatment of influenza by oxygen inhalations. The animals were given the oxygen inhalations twice daily for 10 to 15 minutes. According to observations in the German army, however, the effect of this treatment consists only in a general improvement for 15 to 30 minutes, as well as a diminution of the rate of respiration and pulse, while the treatment has no influence on the pneumonic process, and even if inaugurated at the onset of the disease does not prevent an unfavorable course (Füchsel). Hermann observed rapid ahsorption of the exudate and recovery in a severe case from two subcutaneous injections of a $25 \%$ iodipin solution (50 g. for each injection) ; such an effect, however, was not observed in the Budapest elinic. The German army veterinarians had variable results from the intravenous injections of tallianin $(10 \mathrm{~g}$. doses). (Eichhorn found this remedy effective in the beginning of the disease.)

If the exhaled air indicates by its fetid odor that decomposition of the secretion in the air passages, or gangrene of 
of the lung tissue is progressing, the inhalation of finely atomized disinfecting fluids is advisable. For this purpose a solution of corrosive sublimate is recommended (1 to 2000-4000). If this treatment is applied sufficiently early the fetid odor frequently disappears; extensive gangrene of the lungs however is not checked by this method of treatment, likewise has it no influence on the development of a general infection as the result of absorption of septic substances.

Masson \& Vazeux recommend for the treatment of pulmonary gangrene, daily intratracheal injections of ereosote (20 ec. of a solution of $1 \mathrm{~g}$. of creosote in each $40 \mathrm{~g}$. of alcohol and water), while Gotti recommends formalin (10-20 ec. of a $4 \%$ solution).

The pleurisy should be treated in the beginning with cold applications or cold douches; however after the subsidence of the symptonis of irritation these should be changed to warm, moist compresses. The accumulation of fluid pleural exudate should be relieved by an early aspiration of the thoracic cavity. Repeated aspirations result sometimes, even in severe cases, in recovery, but in such cases the chances are not very promising, as the pleurisy frequently develops in conjunction with gangrenous processes of the lungs. The absorption of the exudate may also be promoted with diuretics, such as potassium acetate (100 to $180 \mathrm{~g}$.), oil of turpentine (10 to $15 \mathrm{g.}$.), juniper berries ( 15 to 25 cc.), etc., as well as by rubbing with irritating liniments (6 to $10 \%$ mustard, or chloroform liniment 10 to 40 ), followed by the application of moist, warm compresses.

The aspiration of the fluid should be stopped as soon as the patient manifests uneasiness, accelerated respiration, or coughing spells. The subsequent irrigation of the pleural eavity with a physiological salt solution, or a mild disinfecting solution does not appear to act farorably. Strictly aseptic technique is naturally the most essential requirement for satisfactory results. Boncheriou, after aspiration, recommends a subcutaneous, while Almy, an intravenous injection, of 2 to 3 liters of a physiological salt solution. Fairise advises an intrapleural injection of 1.5 to 4 liters of a 1 to $1.5 \%$ gelatin solution in order to prevent a possible hemorrhage, as well as the rapid re-accumulation of the exudate, the injections to be made immerliately after the aspiration of the fluid.

In the presence of symptoms of a gastro-intestinal catarrh laxative salts should be given daily to the patients, or Karlsbad salts (40 to $60 \mathrm{~g}$.), with bitter remedies (rheum, gentiana, oakbark, etc.). In consideration of the infectious character of the catarrh, especially in the presence of fetid excrements, intestinal disinfectants are indicated, such as resorcin (10 to $15 \mathrm{~g}$.), creolin or lysol (10 to $20 \mathrm{~g}$.), creosote (5 to $10 \mathrm{~g}$.), naphthalin (10 to $15 \mathrm{~g}$.), etc. If diarrhea exists astringents (oak-bark, bismuth sub-nitrate, etc.), with opiates, as well as enemas containing powdered starch are beneficial. If, however, the diarrhea is profuse, which usually is an indication of a general septic infection, the results from such treatment are not favorable. 
The conjunctivitis may be treated with washes of mild collyriums, such as boracic acid or ereolin solution, later astringent washes may be applied ( 0.5 to $1 \%$ tamin, or sulphate of zine solution, collyrium adstringens luteum). In the presence of a chemotic swelling of the conjunctiva, and keratitis, moist warm applications are advantageous. If the iris is also affected a $1 \%$ atropine solution may be dropped into the eye.

Swellings of the skin are benefited by rubbing with alcohol containing oil of turpentine. Acetate of lead $(5 \%)$ or Burow's solution, with the addition of camphor, may also be applied to this form of affection.

In the convalescing stage the animals require good nourishment and rest, although moderate exercise in favorable weather is rather profitable than injurious. Animals should be used for work only when all symptoms of the disease have disappeared, and after they regain their former strength.

The symptomatic treatment is similar to that followed in the pneumonia or pleurisy and enteritis resulting from other causes; see accordingly their respective chapters in the second volume.

Serum Treatment. Lignières recommends his polyvalent Pasteurella serum for the treatment of influenza (see page 86). In acute cases of the eatarrhal form of influenza, in the presence of a fever $\left(40.5\right.$ to $\left.41^{\circ}\right)$, he injects 40 to $60 \mathrm{cc}$. of the serum intravenously, whereupon the fever is reduced, the patient becomes brighter, and recovers inside of a few days. In subacute cases $\left(39\right.$ to $\left.39.5^{\circ}\right)$, the temperature usually rises somewhat after the injection, but drops after 10 to 12 hours, to normal. He also observed improvement in the presence of pneumonia, when the serum was administered within the first 24 to 36 hours, while later treatment promises little result, because of the associated streptococcus infection. Believing that the inflammatory processes may also be caused or aggravated by streptococci, Joly recommends the subeutaneous injection of anti-streptococcus serum(25 to 30 ee. daily).

Prevention. Healthy horses should be separated from affected animals, as well as from attendants and utensils that come in contact with diseased, animals or with their secretions. It is advisable to isolate a newly acquired horse for two weeks for observation, and it should be placed among the other horses only after that period. This applies particularly to regions in which the disease is prevalent.

If the disease has already appeared the affected animals should first of all be separated, and their stalls, as well as the neighboring stalls, should be thoroughly disinfected. As in the first place the immediate neighboring animals are under suspicion of becoming affected, they, as well as all animals showing an elevated temperature or catarrhal symptoms, should be segregated in different isolated places. At the same time the healthy animals which have no fever should be kept out of doors, as far as place and weather conditions permit, or in places which are free from the infection. They should be divided in groups, and only animals which are entirely free 
from fever should be used for work. The infected stable should be thoroughly disinfected, and scrupulous cleanliness, as well as free ventilation of the stable, should be carried out.

As the development of the disease is favored by influences which have a weakening effect on the animals, the protection of the horses from such influences, and especially from taking colds, should be given due consideration.

Animals which have passed through the disease may, in the majority of cases, and usually in the catarrhal form of influenza, be considered as recovered and free from the infection two weeks after cessation of the fever, and the disappearance of all the symptoms. Convalescent patients from the pectoral form of influenza if the changes in the lungs have not entirely disappeared, and especially if in the meanwhile relapses have occurred, are for a much longer time capable of infecting other animals.

Immunization. Shortly after the discovery of Schütz' diplococeus, Hell undertook the inoculation of over 1000 animals, with bouillon cultures of this bacterium. Healthy animals were injected 3 or 4 times with $40 \mathrm{ec}$. of the culture into the trachea, and some of the animals were given in addition subcutaneous injections of 5 to 10 ce. The injection was followed by a fever lasting from one to two days, whether the animals had had the disease or not. The subcutaneous inoculation was also followed by abscess formation. Further observation showed that animals immunized against the Schütz diplococeus did not withstand the influenza infection, and therefore this method of immunization was later entirely abandoned.

Immunizations with blood serum from horses which had shortly before recovered from the pectoral form of influenza have not given uniform results. Although Hell, at first, reported favorable results, the continued experiments among the horses of the German army showed that the serum treatment of affected animals was entirely unsuccessful and moreover produced such contradictory and even unfavorable results, that this method has also been gradually abandoned. According to the tabulation of Christiani, in the years of 1892 to 1898 the results of 17 inoculation tests showed that in 9 eases they were favorable, and in 8 they were unfavorable. In opposition to the advocates of immunization (Eichhorn, Toepper, Garrey, Giancola and especially Friis, Jensen \& Nielsen), others (Pilz, Neusse, Wittich, Zschokke, Troester, Mieckley) found it ineffective. The contradictory results may be explained to some extent by the fact that the effectiveness of the serum varies in accordance with the intensity of the disease, and also with the time which has elapsed since the recovery of the animal.

At best serum inoculation affords the animals a passive immunity which lasts but a few weeks. Such results do not meet the requirements of practice, even when the serious difficulties encountered during the execution of the inoculations are not taken into consideration. It would therefore be indicated only when it appears desirable to protect the animals, even for a short time, against a threatening infection, such as immediately before or during cavalry maneuvers.

Vaccination Technique. For the serum inoculations blood is taken under strictly aseptic precautions from a horse which has recovered from the pectoral form of influenza not more than six weeks previously. The quantity of blood drawn 
is usually between three and four liters, collected in sterilized high test tubes, and stored in a dark, cool place. The serum which separates on the following day from the blood clot, may be utilized for the inoculations immediately, or several days later. Until its use it should be kept in a cool place.

The serum is injected subeutaneonsly in doses of 40 to 100 ce., and the procedure is repeated four to six times during the following days (Krueger recom. mends the intravenous, Della Noce the intratracheal injection). To the serum several drops of chloroform may be added (Friis, Jensen, Nielsen), or $0.8 \%$ of oxalic acid, in the proportion of 1 to 10 (Prussian army). The inoculation causes in most instances only a transitory indisposition, but in one Prussian regiment, among 195 horses, extensive swellings and abscess formations were also observed (evidently the serum was contaminated). Recently various serum preparations have been recommended, for the purpose of immunization, which however have not proved satisfactory in practice; thus the results which were obtained by Bues with Dentschmann's yeast serum (and with polyvalent log serum), gave absolutely negative results in contagious pleuro-pneumonia. Thomann experienced similar unsatisfactory results with the vaccines prepared by Lorenz from eultures of his pleomorphic bacterium. The same probably also applies to the serum of Willerding, prepared from the cultures of a diploccocus which he isolated from the secretions of the nose and eye, as Lorenz found it equivalent to his own in his laboratory experiments.

Immunization with Killed Cultures. Vallée \& Carré used a vaccine prepared from killed cultures of six varieties of "Pasteurella" for the immunization of horses. The administration of this vaceine to the horses of an infected stable was followed in several animals by severe symptoms of depression and dullness, some became affected with the typical form of contagious pleuro-pneumonia, and three of them died as a result of the infection (latent infection?).

Veterinary Police. In the German Empire compulsory notification of influenza in horses was inaugurated in the year of 1908. The official measures require the separation of the affected and suspected animals, quarantine and disinfection of the infected stables. The observation period extends over five weeks.

Literature. Falke, Die Influenza des Pferdes usw., Jena 1862.-Dieckerhoff, Die Pferdestaupe, Berlin, 1882.- Sierlamgrotıky, Sachs. Jhb., 1:91-1893.-Schütz, A. f. Tk., 1887, XIII, 27.-Hell, Z. f. Vk., 1890, iI, 97; 1892, IV, 452; 1906, XVIII, 159.-Jensen, Monh., 1891, II, 196.-Foth, Ibid., 1891, III, 192.--Leclainche, Rev. vét., 1892, 78 (Ijit.).-Lignières, Bull., 1897, 355 u. 437 ; 1900, 524 (Lit.) ; Rev. gén., 1907, IX, 599.-Jensen \& Nielsen, Maanedsskr., 1897, VIII, 401.-Sturhan, Z. f. Vk., 1905, XVI1, 248.- Dreyer, Z. f. Vk., 1908, XX, 307.-Ostertag, Z. f. Infkr., 1909, V, 180.-Pfeiler \& Hempel, Ibid., 1909, VI, 28.

Influenza in Man. In human medicine influenza represents an acute, contagious, infectious disease, which at times spreads with extraordinary rapidity. The symptoms consist in pain in the joints and muscles, occasional muscular tremors in different parts of the body, sudden and intense fever with severe headache. There is also marked debility, and a progressive acute catarrh of the air passages, conjunctivitis, and frequently gastro-intestinal catarrh. The disease usually terminates in from two to six days in recovery, however it may extend to two weeks. In older, and exceptionally among younger persons, severe disease processes may develop in association with eatarrh of the mucous membrane, such as pharyngitis, catarrhal and eroupous pneumonia, occasionally also pleurisy and pericarditis, as well as inflammation of the middle ear. In the latter cases the course is usually protracted and not rarely fatal.

The disease, which has no association with influenza of horses, is caused by the very small influenza bacillus, which has been discovered by Pfeiffer (Beck, Ergebn. d. allg. Ätiol., 1896, I, 742). 
Dengue Fever. This disease of man oceurs in the tropics, especially in America, East Indies and on the coast of the Mediterranean. It sometimes is very widely distributed, and is eharacterized by suddenly appearing chills, pains in the joints and muscles (dengue-dandy, so ealled from the peculiar stiff gait as the result of pain in the knee joints), further in a polymorphous, exanthema of the skin. According to some observers (DeBrun, C'ubillas, Martialis), the disease also oceurs among domestic animals. Piot-Bey observed in 1895, in Egypt, enzootic affection of cattle which he identified with dengue fever. The animals suddenly became affected with a striking museular weakness, which was so marked that they staggered and trembled in walking, or becoming exhausted they lay down on the chest. The body temperature was moderately elevated ( 39.5 to 39.6 C.), respiration and pulse accelerated ( 25 to 30 , and 60 to 90 per min.). At the same time there was complete inappetence and constipation. Usually after three to four days diarrhea appeared, which was soon followed in all cases by complete recovery. (Fièvre dengue sur l'espèce bovine, Extrait dı Bull. de l'Inst. Egyptien, Le Caire, with literature).

Three Days' Sickness, Stiff Sickness, Ephemeral Fever. In South Africa there oceurs a disease among cattle which Sehweinfurth observed in 1867 , but to which the attention of the experts has only in recent years been directed, when it spread extensively in Rhodesia, in the Transvaal, Natal and in the Cape Colonies. It was first demonstrated in November, 1906, by Edmonds, and later described by Bevan, Theiler and Freer (it is possibly identical with dengue fever).

The symptoms in the majority of cases develop and disappear within three days, or the disease may terminate in death. Manifestations are, stiffness of the museles of one or all extremities, extending to the neck, or even over the entire body. As a result of this condition the animals are lame, and later they are hardly able to move and seem obliged to lie helplessly on the ground. Development of this condition is preceded by diminishing appetite and rumination, and also by an elevation of the body temperature, while later on there appears a conjunctivitis, with swelling of the parts surrounding the eyes, frefuent respiration, very weak pulse, distinct veinous pulse and constipation. The termination is usually favorable, as not more than $3 \%$ of the affected animals succumb, and part of these as a result of inhalation pneumonia.

The cause of the disease is unknown at the present time, but Robertson and Theiler showed that it may be transmitted with the blood of affected animals, in which ease the symptoms develop after an incubation of from 2 to 3 days. It is possible that the infection is communicated by inseets (mosquitoes?). Animals which have recovered from the disease aequire an immunity which only lasts about six weeks (Robertson), reinfections being therefore not infrefuently observed (Freer).

Literature. Bevan, J. of comp. Path., 1907, XX, 104.-Theiler, Rep. of the Transvaal Gov. Bact., 1907.-Freer, V. J., 1910, 19.

Malta Fever. (Mediterranean Fever.) Malta fever is a febrile disease of man, which most frequently occurs on the Island of Malta, then in the countries around the Mediterranean Sea, and probably also in other tropieal countries. The disease is very protracted, remittent in its course, and associated with anemia, headaches, rheumatic difficulties, constipation, and swelling of the joints. A fatal termination 
occurs only in about $3 \%$ of the eases. Bruce (1887) established as its eause a very small, motile, Gram-negative, oval coccus (micrococcus melitensis). The organism can be cultivated on the ordinary media, and shows considerable resistance against drying. In affected persons the coccus is usually present in the blood, from which it is frequently eliminated with the urine.

Natural infection occurs in most cases through the ingestion of raw goat milk. Zammit (1905) proved that about $10 \%$ of the goats at Malta eliminate the causative agent of Malta fever with their milk, and that many of these animals harbor the organism in their blood. He further found that the serum of about $50 \%$ of all goats gives a positive agglutination reaction. As it has been proved that the infection may be readily transmitted through ingestion of infected milk, the drinking of raw goat milk was prohibited in the army, whereupon the morbidity immediately dropped to one tenth of its former rate.

Goats which pass the microccocus melitensis with the milk, and also with the urine, usually show no, or only slightly marked symptoms, but if they are killed, hyperemia and acute swelling of the spleen, together with swelling of the lymph glands, especially of the mesenteric and inguinal will frequently be found. Exceptionally nephritis and lobular pneumonia are present. The causative factor may be isolated by culture procedures from the lesions in the organs, as well as from the blood and from the udder (in chronic cases only from the latter).

Goats are easily infected per os, and it appears that the natural infection occurs by this method, especially by the ingestion of feed contaminated by urine of affected animals. The virus then becomes localized in the internal organs and in the udder, where it produces inflammatory changes, from which the bacteria pass into the milk. Intravenous or subeutaneous injections of cultures produce, sometimes, a severe affection, with symptoms of septicemia. It is also possible to produce a mild form of the disease in monkeys.

The infectious properties of the goat milk may in some cases br established by the presence of the virus; it may also be considered infectious if the blood serum of the suspected animals agglutinates cultures of the organisms in dilutions of at least 1 to 30 . The agglutination test may be also made with milk (Zammit), or with milk serum (Pulvirenti), and W.right's opsonic test also gives good results.

Literature. Babes, Hb. d. p. M., 1903, IIT, 438, Report of the English Commission, London, 1905-1907, Vol. I-VII.-Mohler \& Hart, Bureau Anim. Industry, $1908,279$.

\section{Distemper of Dogs. Febris catarrhalis et nervosa canum}

(Dog ill, Pasteurellosis canum, Maladie des chiens, Maladie du jeune âge [French]; Staupe der Hunde LGerman]; Cimurro, Moccio canino [Italian].

Distemper is an acute, contagious infectious disease of young carnivorous animals, and is characterized by febrile manifestations, and an acute catarrh of the mucous membranes, which is frequently followed by catarrhal pneumonia, and in some cases by the development of nervous symptoms. The disease is caused by a filtrable virus. 
History. The frequency of the disease among dogs, and its importance, is well illustrated by the fact that it is known in almost all languages as the "dog disease." In Europe it is supposed to have occurred since the second half of the eighteenth century, having been introduced from Asia (Spinola) or from Peru (Heusinger). The infectious nature of the disease was proved in the middle of the last century, by successful experiments of transmission, with the nasal discharge (Renner \& Karle, Weisz, Laosson, Krajewsky), with saliva (Venuta), and with the contents of the skin pustules (Trasbot), from affected animals to young dogs. Its etiology, however, was first cleared up by the investigations of Carré (1905), which established that the disease is caused by a filtrable virus.

Occurrence. Distemper is a remarkably frequent disease of young carnivora, especially of young dogs, so much so that the great majority of the latter become affected before reaching the age of one year. In kennels young animals usually become affected in great numbers, but not infrequently the disease occurs in an epizootic form affecting entire townships or even large territories. It is more prevalent during warm weather, but is also quite frequent during the winter.

Etiology. According to the investigations of Carré which have since been confirmed by Lignières, distemper is caused by a filtrable virus which in the beginning of the disease is contained in the nasal discharge.

Subcutaneous injections of two drops of the serous discharge from the nose kills young animals within 6 or 7 days with symptoms of coma and a fall of the temperature at the termination of the disease. The autopsy shows only an accumulation of clear, yellow serous fluid in the pericardium. Injection of a filtered nasal discharge, or of filtered pericardial fluid produces a rapid rise in temperature after 3 or 4 days, catarrh of the conjunctivae and the nasal mucous membrane, cough, and after 8 to 10 days a pustular exanthema on the parts which are thinly covered with hair. The nasal discharge becomes more profuse and purulent, whereupon death results in from 2 to 3 weeks. The autopsy reveals catarrhal pnenmonia, the heart muscle appears to be sprinkled with small hemorrhages and yellow spots, while the pericardium, sometimes also the pleural and the abdominal cavity, contains a serous fluid in varying quantities. The exudate is only exceptionally virulent, unlike that of acute cases. The blood is only virulent at the beginning of the fever, while later, even in an unfiltered condition, it will not produce the disease; this applies also to the nasal discharge which has become purulent, and to material from the hepatized lungs. Healthy dogs may be artificially infected by cohabitation.

In view of the results of the investigations, the different bacteria which have been found by various investigators in the tissues of dogs affected with distemper must be considered as secondary participants 
in producing the disease processes. The body which is attacked by the filtrable virus prepares the field of action for the other organisms which produce inflammatory local changes. Thus Jensen found streptococci, staphylococei and a slender bacillus, Mathis isolated a diplococcus from pustules on the skin, the cultures of which resembled the ordinary staphylococcus, Schantyr obtained a motile bacillus resembling the organism of abdominal typhus, Perez incriminated the cocco-bacillus ozoenae foetidus, while Galli-Vallerio found a bacillus resembling the colon bacillus, which, however, stains by Gram. Lignières, Ceramicola and Wunschheim attributed the disease to an ovoid bacterium, and Piorkowski obtained a slender, delicate capsulated bacillus, etc.

All of these organisms appear to be normal inhabitants of the dog organism. In different cases one or the other, or several of these organisms commence to multiply more rapidly in the affected dog, and then exert their pathogenic action. This applies especially to the bacillus bipolaris septicus, which was considered by Lignières as the true cause of the disease. Cultures of this organism (they can only exceptionally be isolated) produce severe septic symptoms in dogs after intravenous injections, and frequently also secondary inflammations in various organs. Whether there exists an independent pasteurellosis of dogs, as claimed by Lignières, in addition to the distemper caused by the filtrable virus, is not established at the present time.

Contrary to the deseribed etiological findings, Kregenow succeeded in producing the disease with filtrable virus in only two instances out of 10 inoculations, ani even the two positive cases he attributes to natural infection. Accordingly he is of the opinion that the virus is not filtrable, and must be one of the numerous visible organisms. However, the experiments, as indieated by his notes, were not carried out as suggested by Carré, with the serons nasal discharge, but with a purulent discharge and material obtained from pnemonic lungs. It is possible, therefore, that the filtrable virus contained therein was retained during filtration.

Natural infection oceurs through direct or indirect contact with affected animals. The virus is present in the catarrhal secretion of the mucous membranes and is evidently in most instances taken into the digestive canal with the food and water. The infection is probably favored by influences which reduce the resistance of the body, such as colds, insufficient or improper food, such as bread, kitchen refuse, etc. The disturbances of digestion cansed by these, together with loss of blood, exhaustion, weak constitution, etc., favor the development of the disease.

Young animals are most susceptible. They may exceptionally become affected when only 2 to 3 weeks old (Friedberger \& Fröhner), but usually dogs several months old up to one year suffer most frequently from the disease (this is probably due to the fact that the animals at that age move around more freely and are more exposed to the infection). The susceptibility diminishes with advancing age, so that animals over three years old are but rarely affected. The great resistance of older animals is in all probability due to the fact that the great majority have been affected with the disease at a younger age and have thereby become immune to it.

Pampered and weakly dogs, as well as animals recently 
imported, especially those of the finer breeds, are more susceptible and usually become more severely affected than do dogs which are kept in the country and are hardened.

Pathogenesis. The action of the specific virus produces a folsrile condition, and in direct association with it catarrh of the mucous membranes, as well as inflammation of the serous membranes, especially of the pericardium. If the animal lives through the acute attack, its weakened resistance favors the development of secondary processes by other micro-organisms. These are usually facultative pathogenic bacteria, which are mostly, or probably invariably, frequent habitants of the healthy dog. Of the secondary inflammations the most frequent are preumonia, purulent inflammations of the conjunctivae, the mucous membranes of the air passages and of the intestinal canal, pustules on the skin, and also inflammatory changes in varions parts of the nervons system as a result of metastasis. The later course of the disease depends on these secondary diseases, while the filtrable virus usually disappears before, or not later than at the beginning of their development.

Anatomical Changes. In the rare peracute cases a large quantity of serous fluid is present in the pericardium, and possibly there are small hemorrhages in the heart muscles.

In the great majority of cases the respiratory organs, especially show severe clianges. The mucous membranes of the air passages are bright red, swollen, and profusely covered with sero-purulent secretion. If the smallest bronchi are affected simultaneously numerous small purulent plugs mav be squeezed out of the cut surface of the lungs (bronchitis capillaris). In most cases there is also a catarrhal pneumonia. Sometimes small broncho-pneumonic areas may be disseminated throughout the lungs, or larger lung portions, especially near the anterior and lower borders are found dense, hepatized, and according to the duration of the inflammation either dark brown-red or grayish-red. The cut surface is smooth or finely granulated, and sometimes studded with small purulent foci. The borders of the unaffected lobes of the lungs show bluish-red, depresserl, club-shaped atelectatic areas. The pleura covering the affected parts of the lumgs is either smooth and lustrons, or dull and covered with fine fibrinons membranes.

The mucosa of the stomach and intestines show frequently manifestations of acute catarrh; the solitary follicles, as well as Peyer's patches are swollen. Exceptionally there may also be superficial erosions and catarrhal ulcerations present.

The lymph glands of the thoracic cavity, and of the mesentery show inflammatory swellings; the pericardium contains sometimes a great quantity of a clear serous fluid. The heart muscles may be interspersed with small hemorrhages and yellow fatty degenerated areas. The liver and the cortical portions of the kidneys show parenchymatous or fatty de- 
generation. The spleen is only slightly, or not at all enlarged. The eyes are usually affected with a more or less intense catarrh of the conjunctivae sometimes an ulcerative keratitis, and occasionally by a panophthalmitis.

The central nervous system may in some cases show changes corresponding to a myelitis disseminata (Mazulewitsch, Bohl, Dexler).

According to Dexler's investigations the ehanges of the central nervous system show a pronounced disseminated myelitis and poliomyelitis, in which the inflam matory foci are not confined to the gray anterior lobes alone, but are always present in the entire nervous system witlout any regular arrangement. Virulent streptococci are frequently found in the affected areas.

Confirming similar reports of Standfuss, Lentz describes small roundish or oval formations which he calls distemper corpuseles and which are supposed to develop from the action of the distemper virus on the plastin substance of the nerve cells which is destroyed, and causes the chromatin substance to roll together in peculiar granules. These formations which have been found in dogs dying from a severe nervous form of distemper are distinguished from the somewhat similar Negri's rabies bodies, in that they occur outside of the nerve cells, or in the greatly degenerated cells, and possess no iuner structure.

Symptoms. The time of incubation after an artificial infection is usually from 3 to 4 , and at least 2 days (Carré); although it is probably somewhat longer under natural conditions (according to Krajewszki it may extend up to $21 / 2$ weeks).

The peracute form of the disease is manifested in a suddenly appearing inappetence, depression, groaning, fatig'te, as well as high fever. After 2 or 3 days the temperature rapidly drops below normal (to $35-33^{\circ} \mathrm{C}$ ), whereupon death soon appears under comatous symptoms (Carré).

The acute form also is inaugurated by high fever (Fig. 35), the temperature rising rapidly to $40^{\circ} \mathrm{C}$. or over, and remains at this lieight for 1 to 2 weeks or it falls slightly in 1 to 2 days, and then there exists only moderate fever, of a continuing or remittent character in the further progress of the disease. In severe cases the temperature rises again owing to the development of pneumonia, but towards the termination of the disease it falls gradually or rapidly below normal (to $32^{\circ}$ ).

The beginning of the disease is usually indlcated by a change in the disposition of the animal. The animals, ordinarily bright and playful, become capricious and less lively; they obey the call only with hesitation and unwillingly, preferring to find secluded places where they remain lying for long periods. Meanwhile they tremble over the entire body, or at times appear suddenly startled. The hair is roughened, the appetite diminished and capricious. After 1 or 2 days the more characteristic symptoms of the disease develop.

In most cases an acute catarrh of the air passages appears. The animals feel an itching in the nose because of which they frequently sneeze and puff, wiping the nose with their paws or on the forelegs. There is at first a serous or mucous, later a purulent secretion from the nose, which soils the surrounding 
parts and dries around the nasal openings in brown crusts. The secretions may also contain streaks of blood, or become ichorous, and irritate the skin of the upper lip and of the nasal wings, which then become raw. If the swelling of the nasal mucous membrane is pronounced the respiration becomes

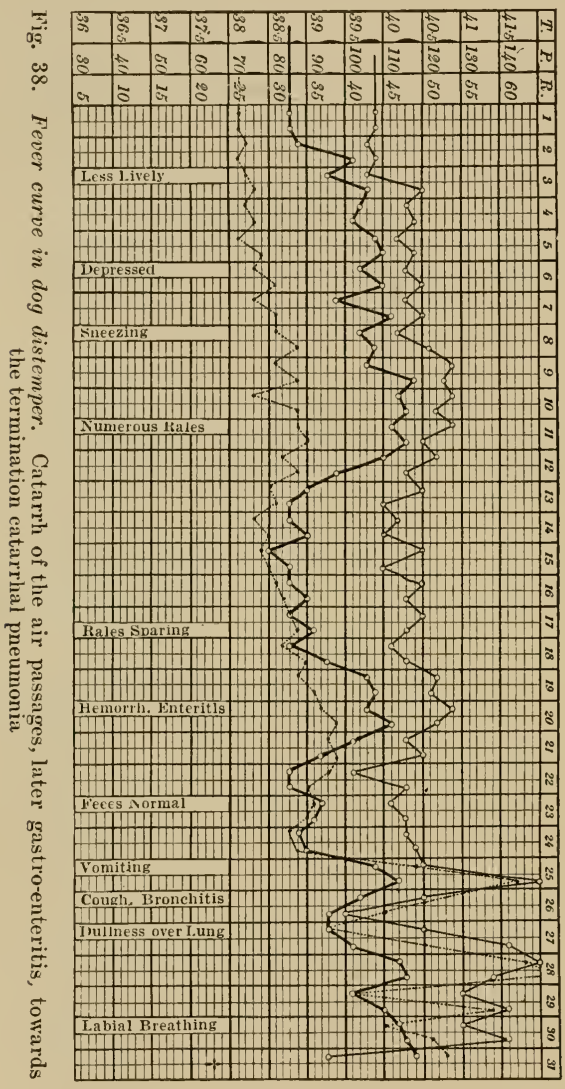
sniffling (this is an especially frequent manifestation in breeds of dogs with short heads).

The eatarrh of the larynx and the bronchi is indicated by a congh which at first is short and dry, later more prolonged and moist. It sometimes appears spasmodically, and in such cases it tortures the animals greatly, and also causes vomiting. Respiration is accelerated and labored. Anscultation of the thorax reveals rough vesicular breathing, and dry or moist rales, as well as other catarrhal sounds. If in the further course of the disease capillary bronchitis or catarrhal pneumonia develops, the number of respirations increases to $60-80$ and even more per min. ute. The accessory respiratory muscles are utilized to a great extent, and at each expiration the cheeks become distended. In such cases percussion shows dullness, or a tympanitic sound in places, while on auscultation, extensive eatarrhal murmurs are heard, in other places bronchial breathing or no respiratory sounds at all are observed. The cough now becomes very weak and feeble.

The affection of the eyes mostly develops simultaneously with the catarrh of the respiratory organs. It commences with 
conjunctivitis, to which are added photophobia, pain and swell. ing of the eyelicls. The conjunctiva is bright red, has a moist luster, and at times is raised above the cornea. The lachrymal secretion is increased from the onset, but later the tears are mixed with a mucous and purulent secretion, which, to some extent accumulates in the inner canthus, sticking the eyelids together, especially at night. Ulcerative keratitis develops with relative frequency in association with the conjunctivitis, small depressions appearing in different parts of the cornea, which may later heal entirely. Sometimes, however, these lesions extend rapidly in size, so much so that even on the first day loss of substance may result which reaches the extent of a lentil, and penetrates to Descemet's membrane, or in some instances the entire cornea may be destroyed. The border's of the depressions, as well as the entire cornea may at the same time be clear, or pus may be present only at the base of the depressions. Descemet's membrane thus exposed is then forced out as a result of the internal pressure from the eye, and in this manner a staphyloma may develop, the rupture of which may result in the prolapse of the iris, followed by the well-known severe results. In other cases a parenchymatous keratitis develops, associated with a uniform cloudiness and a marked ciliary injection of the cornea, which retains its character until the termination of the disease, is not followed by ulceration, and gradually disappears or results in a permanent white cloudiness. The pupil usually becomes constricted, while severe iritis with accumulation of a plastic or a purulent exudate in the anterior chamber occurs only rarely except in cases in which there is a perforation of the cornea.

The involvement of the digestive organs is shown early by diminished appetite. At the onset of the disease an increased thirst, as well as vomiting is observed, which is an indication of acute gastritis. The patients vomit mostly slimy, yellowish material mixed with bile. At the same time the buccal mucous membrane is dry and warm, the tongue is coated, and the region of the stomach sensitive. As a result of the intestinal catarrh which is usually present together with the gastritis, there is at first constipation; which soon changes to diarrhea. The excrements which are often passed under severe straining are in a fluid state and may contain mucous flakes or blood. The feces are very fetid. The abdomen is at first somewhat bloated, later however it becomes drawn up and painful.

In some cases the urine contains albumen, and occasionally bile pigments as well as hyaline and cellular casts.

Nervous symptoms are hardly ever absent even in the cases which are not very severe, and sometimes they may reach such a degree that they practically control the entire disease. Besides the dullness and the change in the disposition of the animals already mentioned, the patients sometimes manifest considerable excitement which, however, usually lasts only a 
few hours. In many cases muscular tremors or clonic spasms develop, and are either confined to certain groups of muscles, or extend over the entire body. Local spasms are usually observed in the face, especially on the lips, nasal wings, cheeks, and in the muscles of mastication (chattering teeth and foaming lips). In other cases spasms occur in the muscles of the neck or extremities so that the affected portion of the body, in the former case the head and the neck, trembles sometimes constantly or only at certain periorls, or agam it may be moved regularly in a certain direction.

The spasms of the entire body muscles which frequently develop from the above condition are manifested either by contimous trembling or by periodical epileptiform fits. In the latter case the animal becomes restless, then suddenly collapses unconscious, while chronic spasms occur over the entire body, and the rapid movements of the jaws churn the saliva to a foam. The expression of the face is distorted, and the head is forcibly extended forward or turned sidewise. During the attack feces and urine may be passed involuntarily. These attacks exhaust the animals greatly, and sometimes occur several times within an hour, whereupon a deep coma develops which soon is followed by death. As the reflex irritability is greatly increased the local, as well as the general spasms may be brought on by slight external irritations, such as loud calling, irritation of the skin, sudden exposure to light, etc. Occasionally involuntary movements may be also observed.

The convulsions may gradually become less frequent and finally disappear; in some cases, however, they terminate in paralysis, which may appear in certain parts of the body, while others are still irritable. The paralysis is most frequent in the hind parts, and is associated with movements of incoordination, and not infrequently by paralysis of the sphincter muscles of both bladder and rectum. Paralysis of a facial nerve results in a distortion of one-half of the face; paralysis of an oculomotor nerve causes an abnormal position of the eye, strabismus and inequality of pupils. Simultaneously with the paralysis contractions may develop in some of the extremities.

According to Dexler the virus of distemper may produce three great groups of nervous disturbanees: 1, loeal clonie-tonie eontimued spasms of different parts of the hody, and chorea-like movements; 2 , flaccid paralysis of the extremities and sphineters; 3, clonic-tonic cpileptiform fits occurring during attacks of uneonseiousness. All these symptoms are brought on by isolated or general inflammatory ehanges which ean be demonstrated histologically (see p. 201), and since the motor disturbanees differ from those of chorea of man, Dexler appears to be justified in his eoneeption that the convulsions of distemper do not represent a neurosis, and accordingly should not be designated as chorea, but that the affeetion should be considered as an independent condition, and for the present should be named "distemper-Tic," or rhythmical post-infectious eonvulsions. 
In about one-lialf of all cases a peculiar pustular exanthema (distemper exanthema) is observed on the skin. Small red spots may appear on the inner surface of the thigh, on the contiguous abdominal wall, or more rarely on the parts surrounding the nose and the eyes, in the external ear, on the conjunctiva, and exceptionally on the entire surface of the body. At these places small nodules soon develop, which change to lentil-sized purulent vesicles. They dry to brown crusts, or burst, leaving reddened moist places which later become also covered with scabs. If the eruptions become extensive the animals at this stage diffuse an unpleasant sweetısh odor. Finally the scabs drop off, and in their place reddish spots covered with epithelium are left, which gradually become paler, and finally disappear entirely. In rare cases the pustular exanthema may also develop on the border's of the lips, whence it may even extend to the buccal mucous membranes, and there produce a severe ulcerative condition which may even lead to a general septic infection. (Semmer, Arnous, Hürlimann; see also typhus of dogs, p. 213.)

Trasbot considered the pustules of the skin as true pox eruptions, and this conception appeared to be strengthened by suecessful experiments of transmission. However, their rapid development, as well as the lack of the characteristic structure of the true pox vesicles, further the fact that the artificial production of such vesicles does not influence the susceptibility of the togs for distemper infections, contradicts this supposition. Moreover, Carré succeeded in producing the exanthema even with the virus of foot-and-mouth disease.

The action of the heart is more or less disturbed according to the severity of the different cases. In severe cases the heart beat is greatly accelerated, irregular, throbbing, and the pulse wiry, owing to the parenchymatous degeneration of the heart's muscles.

Lange ohserved in ahout $50 \%$ of animals affected with distemper, a painless external otitis, with a thin fluid secretion and moist eczema of the external ear passage. Sabrazès \& Muratet found in the blood of dogs affected with distemper a neutrophilic polynucleareytosis and hypoeosinophilia, as well as an iodophilia.

The animals usually become greatly emaciated in the course of the disease. At the approach of death they lie in a deep stupor, trembling on the ground, until they finally die of the convulsions.

Course. The disease shows a most varied course. Frequently, especially in benign cases, only a certain group of the symptoms described are present, and even these may be developed in different cases with varying intensity. Thus in some cases catarrhal symptoms are present exclusively, while in others only nervous manifestations are seen. Sometimes the disease is manifested by pustular exanthemata of the skin only. Taking the etiological identity of these cases into consid- 
eration, a catarrhal, a nerrous and an exanthematous form of distemper may be distinguished. Such sharp characteristic forms of the disease, however, are only rarely observed, as in most instances the symptoms of two or even of all three forms are present simultaneously, although in such a way that the symptom complex of one of the forms usually predominates. Most frequently the catarrhal or nervous form occurs separately, while the skin exanthema usually occurs conjointly with one or the other. Besides the character of the disease may not infrequently change during its course, as it is often noted that nervous manifestations associate with the catarrhal symptoms, and later these may even predominate.

Just as there are great variations in the clinical appearance of the disease, so its duration varies greatly from case to case; very mild cases may thus terminate in recovery inside of a week, whereas severe cases may extend over several months. The average duration, however, is from three to four weeks. The recovery is not infrequently incomplete, or it may become complete only after a long time. The symptoms of paralysis are most prone to persist, or may improve only after several weeks. Incurable degenerations may be present in some of the nerves of the brain, leading sometimes to blindness, deafness, loss of the sense of smell, etc.

Occasionally after the acute stage of the disease has passed a certain predisposition of the mucous membranes for catarrhal affections becomes noticeable which leads for months afterwards to repeated catarrhs. The catarrhal pneumonia may develop into a chronic inflammatory condition leading to caseation of the exudate and an increase of the connective tissue, which considerably interfere with the later development of the young animals.

Permanent changes sometimes develop in the eyes, such as white cicatrices at the sites of the cornea ulcerations, synechiae after a perforation of the iris, and occlusion of the pupil or atrophy of the eyeball after a severe iritis.

Diagnosis. Distemper may be readily distinguished from other catarrhal affections whenever nervous or pustular exanthema are present in addition to catarrhal symptoms. This also applies to older animals, in which the diagnosis is otherwise more difficult. In the presence of purely catarrhal srmptoms, the high fever which occurs in the beginning of the disease, the pustular exanthema, the early age of the animals, and frequently also the possibility of tracing the infection, may reveal the nature of the disease. Gastro-intestinal catarrhs originating from other sources, such as for instance from dietetic errors or from poisoning, are differentiated from distemper by the absence of the catarrh of the air passáges and conjunctivae. The change in the disposition of the animals, 
especially the occasionally noticeable excitement and also the convulsive mastication with the formation of foam at the mouth, may create a suspicion of rabies. However, this condition exists in distemper only for a very short time, and is soon followed by a period of depression, then by muscular spasms, while the paralysis of the muscles of the pharynx and of the muscles of mastication which are characteristic of rabies, are absent. The severe convulsive attacks are distinguished principally from those of epilepsy by the fact that in distemper they occur at frequent intervals, are preceded by iocal muscular spasms, and that these spasms are present even between the attacks.

Prognosis. The average mortality in the disease amounts to about $50 \%$. The cases in which the purely exanthematous and catarrhal symptoms are present are the most favorable with about $70 \%$ recoveries. Least unfavorable are the cases in which the nervous manifestations predominate, as in these the recoveries sometimes do not reach 10 to $15 \%$. The more rapidly the symptoms increase in severity and the higher the degree they attain, the more unfavorable becomes the prognosis. The convulsions which affect the entire body, as well as the appearance of catarrhal pneumonia, and a severe persistent diarrhea are unfavorable prognostic indications. Continuous high fever indicates a severe infection as a result of which grave changes in the myocardium may be expected. A still more unfavorable sign is the rapid fall in the temperature below normal, as it usually indicates the approach of death.

Pampered, very young or improperly nourished dogs usually become more severely affected than older and hardened animals of ordinary breeds. The existing hygienic conditions should also be given consideration in the prognosis.

According to statistics of Wirth, which include 2855 cases, an average of $358 \%$ suceumb to the disease. In these cases the mortality was the lowest among fox terriers, and highest in hounds. Nervous symptoms were observed in $15.1 \%$ of the cases, females appearing to be more predisposed to this form of affection; in some outbreaks the exanthema was entirely absent.

In localities in which the disease occurs only periodically as a result of introduction from the outside, it rages with peculiarly destructive force, and it has been observed, for instance in Greenland, that sometimes such outbreaks destroy about one-third of the entire number of dogs (Hjortlund).

Treatment. First of all the hygienic condition, and especially the diet, should be suitably regulated. The affected animals should be placed in a clean, uniformly warm place, they should be protected from draughts, and if possible should be furnished concentrated nourishment. For this purpose milk and good strong meat broth, with the yolk of an egg beaten into it, is most suitable. Several daily feedings with chopped, salted meat cooked in steam, is very beneficial (Gerstner). In the presence of gastro-intestinal catarrh, gruel may be given to advantage. 
As an abortive treatment subcutaneons injections of trichloride of iodine ( 3 times daily, $3-5 \mathrm{~g}$ of a solution of 1 to 1000-500), as recommended by De Bruin, are indicated. This method of treatment when applied in the early stages of the disease is very beneficial, as the animals after a few days become much brighter, their temperature returns to normal, and the catarrhal manifestations diminish. In some of the animals, however, in spite of the greatest care, a necrosis of the skin at the point of injection develops. Bass recommends for the early treatment, Ichthargan (Ichthargan, gummi arab. aa. 5.0 , aqu. $100.0,3$ times daily in teaspoonful to tablespoonful doses), while Liebert \& Schmidt recommend tannargentan (1 g. 2 or 3 times daily).

For the depression and weakness nervous stimulants are recommended, such as alcohol (wine, cognac) in proper dilutions, and in small but frequently repeated doses. In severe cases coffee or caffeine, liquor anmonii anisatus in any desired infusion, also subcutaneous injections of $30 \mathrm{cc}$. of a physiological salt solution (Parent) are indicated. To the salt solution it is adrisable to add 10-20 cg. caffeine (Cuny).

The fever does not require any special treatment, unless very severe (over $40^{\circ}$ ), when the administration of antipyretics (phenacetin, antipyrin, in doses of 0.25-0.50 g.) is recommended.

The catarrh of the respiratory organs is treated to the best advantage with inhalations of warm vapors, containing expectorants ( 1 to $3 \%$ solution of carbonate or bicarbonate of soda). If the secretion is very profuse inhalations of disinfectant fluds (creolin 2-3\%, creosote $0.5 \%$, turpentine, aqua-picis, septoform) may be given three or four times daily with Siegel's or Bulling's apparatus. In painful dry cough antispasmodics such as Morpheum salts (morphii muriat 0.10, aqua. amygd. amar. 12.0, 10 to 15 drops every 2-3 hours), or codein (cod. phosph. 0.30 , aqu. $150.0,1$ to 2 teaspoonfuls every $2-3$ hours) give beneficial results. In the presence of profuse secretions, however, suppression of the cough is rather harmful. In such cases ipecacuanha infusions and senega decoctions, given either with apomorphine (1 to $2 \mathrm{mg}$. per dose) or with ammonium chlorate (0.1-0.5 g.) may be used to better advantage. If pnenmonia has appeared inhalations of oxygen may he tried (Kantorowicz).

The gastro-intestinal catarrh should be treated first by regulating the diet as already described, and in the first davs of the affection a cathartic such as castor oil or calomel $\left(0.0^{2} 2\right.$ $0.05 \mathrm{~g}$. 2-3 times daily), as well as salol, should be given. If vomiting is present this may be controlled by the administration of ice, water containing soda, salicylate of bismuth $(0.5 \mathrm{~g}$.$) ,$ in emergency with opium $(0.05$ to $0.10 \mathrm{~g}$.). For the stimulation of the digestion hydrochloric acid ( 0.2 to $0.5 \mathrm{~g}$.) in the drinking water, or pepsin (0.1 to 0.5 g.) alone or with the hydrochloric acid, is advisable. Besides the various bitter tinctures (Tinct. 
rhei aquosa and vinosa, Tinct. gentianae, Tinct. chinae composita 5-10 g. per dose) are often used. If diarrhea has appeared it is advisable to administer opiates ( 0.1 to 0.2 opium or 5 times that amount of the opium tincture every $3-4$ hours) ; this may be given together with tannin (0.1 to $0.5 \mathrm{~g}$.), bismuth subnitrate $(0.2$ to $0.5 \mathrm{~g}$.), tannoform, tannalbin, tannopin ( 0.5 to $1 \mathrm{~g}$. of each), or with red wine (in teaspoonful doses).

Raleigh obtained recoveries in $60 \%$ of his cases by treating the affected animals first with 0.25 to $0.40 \mathrm{~g}$. of calomel, and followed four times daily with $0.60 \mathrm{~g}$. sodium hyposulphite, while Liebert \& Schmilt obtained splendid results from the administration of tannargentan (2-3 times daily $1 \mathrm{~g}$. doses).

In the presence of nervous symptoms anodynes and antispasmodics are recommended. However a beneficial action from their use on the neuritic or myelitic affection, can hardly be hoped for. Of these may especially be mentioned bromide of potassium and bromide of sodium (1:250 4 or 5 times daily in tablespoonful doses), chloral hydrate (0.5 to 2 g.), sulfonal ( 1 to 2 g.), or morphium in larger doses $(0.05$ to 0.1 g.) subcutaneously. Paralysis may be treated with massage, alcohol rubs, or by the application of electricity, as well as by subcutaneous injections of veratrin ( 1 to $5 \mathrm{mg}$.) or strychnia (0.5 mg.).

The conjunctivitis, while the symptoms of irritation last, should be treated witl warm applications 2-3 times daily, and washing with a $1 \%$ creolin and boracic acid solution, while astringents are indicated only after the subsidence of the acute symptoms. Of such astringents may be mentioned 0.5 to $1 \%$ solutions of sulphate of zinc, alum, or tannin, as well as the "collyrium adstringens luteum" (diluted 1-3 to 1-2). If the catarrh has become chronic, or if there exists a continuous purulent secretion, the painting of the eye with a $1 \%$ silver nitrate solution may be tried. It should be applied with very great care, and the superfluous portion should be neutralized with a $1 \%$ salt solution. For the keratitis, as well as for the internal inflammations of the eye, warm applications and the dropping of a $1 \%$ atropin solution into the eye are recommended. For clearing away the clondiness in the cornea, the blowing of powdered calomel or rubbing of a iodide mercury salve (1-20), with subsequent massage of the eye, is often beneficial; this, however, should be only undertaken after the disappearance of the acute srmptoms. For threatening blindness as a result of paralysis of the optic nerve, injections of strychnia into the temporal region might be favorable.

The exenthema of the skin does not require a special treatment, but the sprinkling of the moist surfaces with a drying powder, or the application of a salve, may assist in the healing of these lesions.

Prevention. Since the affected animals easily infect others, they should be kept from healthy young dogs and cats, and the 
place where they are kept should be disinfected. Young animals should be kept from contact with sick ones, and by this the advantage will at least be gained that the animals may not become affected until an older age, when, according to observations the chances for recovery are much better.

Immunization. The various sera for distemper are not very promising, as they are prepared with the aid of bacteria, which at best are of importance only in the secondary organic lesions.

Iignières uses for this purpose partly attenuated cultures of bipolar bacilli ohtaned from affected dogs, partly his polyvalent vaceine and his polyvalent serum (see pp. 85 and 86). Physalix uses cultures of the same organism grown in glycerin bouillon, while Piorkowski prefers a serum prepared with the aid of his "distemper l,acillus." But with none of these preparations was it possible to obtain satisfactory results in practice. Richter proved that the vaccines of Physalix and Piorkowski are valueless for the treatment of the disease, and Boden also found that Piorkowski's serum at the best only produced an improvement in the first stages of the purely eatarrhal form of distemper.

Bissange \& Naudin recommend the antistreptococcus sera of the Pasteur Institute, and of Dassonville \& Wissoeq (twice daily 2-4 doses of 4 ec. each); and at the same time the administration of Pury's Swiss yeast in warm milk.

The yeast serum (antistreptococcus serum), obtained by Deutschmann by treatment with increasing amounts of yeast, is according to Lamche absolutely useless as a prophylactic agent, and as a curative it has only a moderately beneficial action in those cases which show no complicating intestinal affections. Blanck and Wolff, however, report favorable results from this method of treatment.

The distemper-antigourmin (Furonculine), which has been highly recommended by Diffiné and is prepared from equal parts of yeast and starch, proved unreliable in the experiments of Meltzer, Hochstein, Meckelburg and Lamche both as a curative and proplyylactic.

Literature. Bollinger, A. f. Tk., 1878, IV, 214.-Krajewski, Rev. f. Tk., 1881, 177 (Lit.) ; D. Z. f. Tm., 1887, XIII, 324.-Laosson, Diss. Dorpat, 1882 (Lit.).-Dexler, Arb. d. Wiener Inst. f. Nervenanat., 1892; D. t. W., 1909, 313 (Lit.).-Jensen, Maanedsskr., 1895 and 1896. -Jess, Zbl. f. Bakt., 1899, XXV, 541.Lignières, Bull., 1900, 469 (Lit.); 1906, 622.-Physalix, Bull., 1901, 131.-Carré, Bull., 1905, 335; Rev. gén., 1906, VII, 649; C. R., 1906, 962.-Piorkowski, B. t. W., 1905, 830; 1906, 377.-Wirth, T. Zbl., 1908, 200.-Richter, Die Hundestaupe, Diss. Bern, 1908 (Lit.).-Kregenow, Zbl. f. Bakt., 1909, L, 326 (Lit.).-Lamehe, Diss. Zürich, 1909 (Lit. on yeast treatment).

Distemper of Cats. Although young cats are also susceptible to the virus of distemper (Krajewski, Laosson), the disease occurs in these animals much less frequently than in dogs. The symptoms and the anatomical changes correspond with those of dogs affected with distemper, but according to Friedberger \& Frohner nervous symptoms are less common and the exenthema of distemper is usually absent. In the treatment the above-described principles should be followed. (Literature same as in dog distemper.)

A new disease of cats was described by Gaertner, in which the symptoms consisted of depression, conjunctivitis, muco-purulent discharge from the nose, and marked difficulty in respiration. The disease "usually terminated in death in from three to six days; exceptionally, however, it lasted for several weeks. The autopsy revealed a multiple necrotic pneumonia, principally of the posterior lobes of the lungs, and as the causative agent a bipolar ovoid bacillus (Bact. pneumoniae felis) was established (Cbl. f. Bakt., 1909, LI, 232). 
An ovoid bacterium (Bac. felisepticus) was also found by Boucek in young cats, which died in an enzootic. These animals suffered from disturbed appetite, vomiting and convulsions, while the autopsy revealed a mild intestinal catarrh, moderate swelling of the spleen and of the mesentery lymph glands, as well as punctiform hemorrhages in the pericardium and in the heart muscles (Cbl. f. Bakt., 1909, LIII, 279).

\section{Typhus of Dogs. Typhus canum.}

\section{(Hundetyphus, Stuttgarter Hundeseuche; Gastro-enteritis haemorrhagica.)}

Typhus of dogs is an acute infectious disease which periodically occurs in an epizootic form, in the course of which severe symptoms of a gastro-intestinal inflammation are observed frequently complicated by ulcerative stomatitis and severe nervous symptoms. The etiology of the disease has not been determined up to the present time.

History. Hofer described the disease under the name of "typhus" in 1850; however, it did not create a general interest until it appeared in Germany in 1898, and in the following years also in other countries, as a severe plague. In this outbreak the disease was first described clinically by Klett, who observed it in the fall of the year in and around Stuttgart (hence the name "Stuttgart dog disease"). Other outbreaks of the affection, and also deviations from the usual manifestations were described by Scheibel of Frankfort, Albrecht of Munich, Zschokke of Switzerland, Trevisan \& Gambarotte of Italy, Bîmes \& Sérès and others of France, Héhrant of Belgium, Némo of Edinburgh, Mattel \& Tremmel of Vienna and vicinity. A similar disease was observed in 1903 at Budapest, when several cases eame under observation.

Occurrence. According to the above mentioned writers the disease existed in the years of 1898-1900 in Middle Europe in the form of a wide sprear, epizootic extension; since that time however it has again disappeared. In the period mentioned the disease caused great losses especially among older dogs of the affected localities.

Etiology. The epizootic occurrence of the disease in various localities which are not far apart, appears to indicate its infectious nature. On the other hand various investigators have observed almost uniformly that direct transmission has no, or only an insignificant, importance, in its appearance and spread. Frequently dogs became affected which had not previously come in contact with affected animals, while on the other hand among dogs kept in kemnels, not infrequently only isolated cases were observed. Test inoculations have not given uniform results up to the present time.

In the outhreaks which have occurred usually the older dogs and not infrequently those over 10 years old became 
affected. Some of the animals contracted the disease in spite of the fact that they had passed through an attack of distemper. The condition, sex and breed of the animals appeared to have no noticeable influence on their susceptibility.

Attempts at artificial transmission have so far given variable results. Pirl observed after subcutaneous injection of the bile of a severely affected animal (5.0 or $2.0 \mathrm{~cm}$.) into two healthy dogs, that an inflammatory edema developed at the point of inoculation, followed by fever, salivation, vomiting, bloody diarrhea and convulsions. One of the dogs died after three days, the other recovered after six days. The subeutaneous injection of blood, fluid from the lungs, or an emulsion prepared from the walls of the stomach or intestines, produced only a local suppuration (Albrecht, Scheibel), while the feeding of organs and of the contents of the stomach of animals dead from the disease proved harmless. Scheibel observed in only one instance the development of a gastro-intestinal inflammation, after he had previously neutralized the gastric juice in the animal which was fed with the infected material. Scheibel \& Zschokke found a bacterium in the blood resembling the organism of fowl cholera or swine plague, while Leclainche \& Vallée demonstrated in the pathologic secretions and tissues the presence of a "Pasteurella." Based on these findings Bimes \& Sérès identified the disease with dog distemper (?).

In the winter of 1903 to 1904 several cases were observed at the Budapest clinic which in their manifestations, except for the absence of the ulcerations in the mouth, were similar to the cases observed in Germany. From the contents of the severely inflamed and hemorrhagic intestines, also from the intestinal mucous membrane and the mesenteric lymph glands, a colon bacillus and a virulent type of proteus has been isolated in every instance. An intravenous injection of $1 \mathrm{~g}$. bouillon culture of these bacilli per $1 \mathrm{~kg}$. of body weight, produced in dogs a very intense hemorrhagic gastro-enteritis, which produced death inside of six hours, while the subcutaneous injection of the colon bacillus caused extensive but painless suppuration, and necrosis of the skin. It was impossible to produce the disease by feeding the bouillon or meat cultures, not even after a previous neutralization of the hydrochloric acid of the stomach (Hutyra).

Anatomical Changes. The autopsy establishes in all cases severe inflammatory changes in the digestive tract. The mucous membrane in the entire stomach and in the duodenum, is greatly swollen, corrugated, and of a blackish-red color. Its surface shows numerous hemorrhages varying in size from that of a lentil to a pea, and is covered profusely with mucous or with a chocolate brown, semi-liquid mass which has an alkaline reaction (exceptionally fresh blood may be also present in the stomach). Similar, but less plainly marked inflammatory changes, are present in the intestinal canal, especially in the small intestines and in the cxeum. The peritoneum over the stomach and the intestines is markedly injected, the mesenteric lymph glands, and to a greater or lesser extent the spleen also show acute swelling. The liver and the kidneys are hyperemic, the latter also contain small hemorrhages; the bladder is 
usually distended, its mucous membrane is usually smooth and pale red. The lungs show only hyperamia and acute edema. The muscle of the heart often tear's easily, and on the cut surface it is sprinkled with butter-yellow streaks. The brain and its meninges are hyperemic. The buccal cavity frequently contains inflammatory as well as necrotic changes (see symptoms).

Symptoms. The disease almost invariably commences with sudden vomiting, which usually attacks the animal while eating or drinking, and which may re-appear very frequently. The vomited material consists of remains of the ingested food or water stained yellow by the bile, but may later become bloody. From this stage on, the animals refuse to eat, or they accept food unwillingly; on the other hand they drink water very frequently and feverishly. There is first constipation, but later diarrhea appears.

The animals are greatly depressed from the beginning of the disease, and are unwilling to move about, and in doing so they manifest a staggering gait. They show indifference to their surroundings, which condition later develops into great dullness and sleepiness. The animals become greatly emaciaterl as a result of the frequent vomiting, and persistent inappetence, and the disease develops in its characteristic form in from 3 to 5 days.

The conjunctivae show a brownish-red discoloration, are greatly injected, the eyeballs are sunken in their sockets, the pupils are dilated. The nasal mucous membrane is of a similar color and dry.

The mouth emits a very unpleasant, penetrating odlor, which sometimes may be noticeable at a great distance. The buccal mucous membrane is dry, dark brownish-red, and covered with a tenacious coating which is at first gray, later thick and chocolate brown; this coating is especially noticeable on the tongue. Later the mucous membrane loses its luster in spots, these areas soon changing into erosions, and becoming covered with pale yellow or dirty brown, tinder-like scabs; sometimes they are surrounded by bright red inflammatory tissue. These lesions are principally observed on the inner surface of the cheeks opposite the rows of teeth, on the gums, in the corners of the month, on the inner surface of the lips, especially in the region of the corner teeth, and on the borders of the lower surface of the tongue. Later the scabs drop off and in their place dark red ulcerations appear, which are covered with tissue shreds or with a dirty brown debris. In severe cases the ulcers may confluate forming large ulcerative areas, while the tongue may change into a dry, chapped, frequently entirely insensitive, shrunken body.

The abdomen is very sensitive, especially over the region of the stomach, and it is either bloated or drawn up; loud peristaltic sounds may be heard when close to the animal. The 
movement of the bowels is at first retarded, sometimes the animals after severe straining, pass balls of feces covered with mucous or blood, which may also contain blood; in other cases again severe diarrhea may appear, the feces being fluid, bloody and very fetid. The mucous membrane of the rectum is occasionally greatly inflamed, very painful, and exceptionally also ulcerated. The body temperature is usually normal, only towards the end of the disease it sometimes drops below normal. The heart's action becomes accelerated in the later stages of the disease, being weak and beating arhythmically. The respiration is quiet, and deep, exceptionally however in the presence of pneumonia it becomes accelerated. The urine which frequently can be passed only on pressing the greatly distenderl bladder, contains in severe cases a great amount of albumen, and more or less bile pigment. In some cases chronic spasms of the muscles of the head, or over the entire body, may be present.

From the described clinical appearance of the disease, which was observed by Klett at the time of the outbreak at Stuttgart, various deviations are observed in the different outbreaks, especially in the extension and intensity of the ulcerative and necrotic processes on the buccal mucous membranes, which may even be entirely absent in fatal cases (Albrecht). In other cases there occurs considerable swelling of cheeks and lips, without ulcerations, a livid discoloration of the buccal mucous membrane, profuse salivation, and an acute swelling of the cervical lymph glands (in Scheibel's eases the disease was always introduced by high fever). In some of the outbreaks nervous symp. toms predominated over the clinical manifestations (Bîmes \& Sérès).

Among other symptoms may also be mentioned rigidity and tenderness of the muscles, similar to those occurring in rheumatism; total paralysis of the hind quarters, hematuria (Mattel); in some of the otherwise typical cases the body temperature may be high at the beginning of the disease, and may be associated with severe chills (Mattel, Tremmel). There may be hemorrhages into the anterior chamber of the eye (Richter) with which keratitis may become associated (Pirl). Some patients may become hard of hearing, and later deaf (according to Richter probably as a result of the extension of the inflammatory process from the pharynx to the internal and middle ear).

Course and Prognosis. The average duration of the disease is usually from 8 to 10 days; in most severe cases the animals usually die in from 4 to 6 days, exceptionally lowever death ensues towards the end of the second day. The rate of mortality fluctuates between wide limits in different epizootics. In the beginning of an outbreak sometimes almost every animal dies, while later the course of the disease becomes less and less severe. A very rapid development of the disease, complete inappetitence, profuse diarrhea and great debility indicate infavorable terminations, whereas a slow course and mild symptoms warrant hopes for recovery. Young animals are more apt to withstand the disease than older ones. 
In the favorable cases the animals sometimes recover entirely within a short time, even the ulcers in the mouth heal with remarkable rapidity; as a rule it requires from 2 to 3 weeks for the animals to regain their former appetite and disposition. The recovery is usually complete; permanent sequelae develop only in exceptional cases (paralysis of a posterior extremity, deafness).

Diagnosis. The sudden vomiting, the inappetence and great depression, the ulcerations on the mucous membrane of the buccal cavity, the striking injection of the sclerotic conjunetiva, in connection with the infectious character of the disease, usually afford sufficient grounds for a diagnosis. Distemper is distinguished from this disease by the almost invariably present catarrh of the conjunctivae and air passages, as well as by the fact that in distemper usually young animals become affecterl. In cases of gastro-enteritis resulting from dietetic errors or poisonings (meat poisoning), the ulcerative condition in the mouth is absent, besides the more uniform development of the clinical manifestations, the appearance of the disease in numerous localities, as well as the exclusive affection of $\operatorname{logs}$, are indications against poisoning. Ulcerative stomatitis and scorbutus develop more slowly, furthermore the violent vomiting and great depression seen at the beginning of this disease are absent.

Treatment. For the violent vomiting washing of the stomach appears to be indicated; besides this Klett recommends subcutaneous injections of morphine, swallowing of ice, and the placing of ice bags over the region of the stomach. Mattel advises the administration of mild Russian or bitter tea, Wohlmuth prefers a tablespoonful of black coffee every hour, and tincture of valerian, 15 drops 3 times daily, later warm or Priesznitz bandages applied to the abrlomen may diminish the pains. In obstipation or diarrhea, enemas ( $4 \%$ boracic acid, $1 \%$ alum solution) may be used. Bass administers internally ichthargan (ichthargan, gummi arab. aa 15.0, aqua 50.0, teaspoonful doses every 2 hours), and ichthargan ointment externally to the inner surface of the thighs. It is advisable to wash the buccal cavity carefully several times daily with fresh water or a mild disinfectant (potassium hypermang. in a $1 \%$ solution). The animals should be kept in a quiet place, and if they evince great weakness or lose considerable blood, subcutaneous infusions of luke warm salt solution $(0.8 \%)$ are indicated (Klett).

After the vomiting subsides it is advisable to give the animals water acidified with hydrochloric or citric acid, while in the period of convalescence the animals should be given highly nutritious food (gruels, milk, eggs, and later meat). 
Literature. Hofer, Repert., 1852, XII, 201.-Hürlimann, Schw. A., 1896, 120.-Klett, D. t. W., 1899, 43.--Seheibel, B. t. W., 1899, 73.-Albrecht, D. t. W., 1899, 189.-Zschokke, Schw. A., 1900, 241.-Mattel, O. M., 1900, 491.-Tremmel, T. Zbl., 1900, 454.-Wohlnuth, T. Zbl., 1905, 113.

\section{Purpura Hemorrhagica. Morbus maculosus equorum}

(Petechial fever, Acute haemorrhagic-anasarcous toxemia, Typhus equorum, Anasarque essentielle ou idiopathique,

[French]; Blutfleckenkrankheit der Pferde, [German]; Febbre petechiale, Morbo maculoso, [Italian].)

Purpura hemorrhagica represents an acute, non-infectious disease or process, probably caused by a septic bacterial intoxication, as a sequel to a specific infection. It is principally characterized by extensive edematous infiltrations of the scbcutaneous connective tissue, and by hemorrhages in the sweilings as well as in the mucous membranes, and in the internal organs.

History. In olden times the disease was grouped among the putrid fevers (Febris putrida) or with the typhus affections (horse typhus), and it was thought to be essentially a primary decomposition of the blood. Later it was considered by some to be a form of anthrax (Roell), by others an affection related to scarlet fever of man (Percivall, Zschokke). Hering (1841-1850) recognized its non-infectious nature and at the same time separated the disease as an independent affection (petechial fever) from other similar ailments. Recently the idea became generally accepted that the disease is to be taken as an intoxication caused by bacterial toxins (Dieckerhoff, Lignières and others).

The names given at the present time to the disease (petechial fever, Hering, purpura hemorrhagica) are based upon important symptoms, particularly the hemorrhages into the mucous membranes. The designation of horse typhus which was used in former times does not appear to be appropriate, as the disease differs decidedly from typhus affections of man, and especially from the abdominal typhus.

Occurrence. The disease usually occurs sporadically, but many cases may appear within a short time, especially in stables where influenza and strangles have existed. The disease is of importance on one hand because of its frequently fatal termination, and on the other hand by the very slow course and the loss sustained by the continued disability of the patient for work.

According to Javorsky in 1892-1901, 544 cases occurred in Moscow; the mortality among the animals received in the eity hospital was as high as 0.3 to $0.8 \%$, the average being $0.42 \%$.

Etiology. Purpura hemorrhagica with rare exceptions develops as a secondary affection, as a sequel to diseases in which suppuration or necrosis of tissue have occurred in any part of the borly. Such diseases are especially strangles, pneu- 
monia, pharyngitis, influenza, in rare cases catarrh of the upper air passages or of the intestines, and pyemia of the sinuses of the head, caries of bone, exanthema of the skin, abscesses from any cause, and gangrenous areas in the subcutaneous connective tissue, or in the internal organs. As all of these diseases are associated with the presence of micro-organisms, and as the purpura hemorrhagica sometimes occurs in the form of an enzootic, it may be accepted that micro-organisms are either directly or indirectly incriminated in the development of the disease. The indirect influence of the microbes, and the appearance of the disease, is substantiated by the fact that the affection is not transmitted from infected to healthy animals, and cannot be transmitted artificially either by inoculation of secretions from the affected animals, or with blood (Haubner, Hering), nor by direct transfusions of blood (Arloing).

The characteristic hemorrhages and serous exudations in the disease indicate a severe affection of the vessel walls which in all probability may be explained by the fact that chemical substances which circulate in the blood reduce the normal elasticity and resistance of the vessel walls. While this may be the result of a change in the blood, through which the nutrition of the walls of the vessel becomes diminished, and the watery consistency of the blood in itself facilitates the transudation of the blood plasma, a direct toxic action appears much more probable in consideration of the quick, sometimes very abrupt hemorrhages and serous infiltrations. Since, according to observations, pathogenic bacteria produce toxic substances, poison of similar origin may be suspected as the etiological factor of this disease.

According to Dieckerhoff's conception these toxins form in necrotic or suppurative lesions which develop in any part of the body through the action of the micro-organisms during the course of the primary disease. This supposition is very probable, as the diseases preceding petechial fever as a rule are those in which the abscesses or gangrenous areas which develop in the course of the disease communicate either originally or later with the outside world, whereby micro-organisms may readily gain entrance into the body. These organisms may then multiply in the exudates or the necrotic tissues, and produce chemical poisons which are later absorbed by the circulation. In rare cases petechial fever apparently develops independently of other diseases, or in connection with fresh injuries (castration), but even in such cases local necrotic processes cannot be excluded with certainty. Accordingly purpura hemorrhagica should be considered as an infections disease in which, however, the incriminated virus (probably the pyogenic streptococcus which because of its ubiquity easily gains entrance into the necrotic tissue), exerts its pathogenic action indirectly with its specific toxic products. 
According to the bacteriological examinations of Lignières (18951898), the internal organs and the blood of animals dead from purpura hemorrhagica contain, as a rule, besides other bacteria, streptococci, principally pyogenic, and more rarely streptococci of distemper. The bacillus equisepticus is also met with relative frequency (among 26 cases this organism was found in 9 instances), and this seems to indicate that both diseases are closely related. This may be also substantiated by observations in Argentine where influenza oceurs mostly in a very acute form without inflammation of the thoracic organs and purpura hemorrhagica very frequently develops as a sequel (similar observations were made by Theiler in Transvaal). Accordingly both bacteria play a part in the etiology of purpura hemorrhagica, the severe symptoms, however, are caused by the streptococei or their toxins.

Lignières failed to produce the disease even in a single instance in its characteristic form, in spite of the fact that he injected horses intravenously with quantities up to $8000 \mathrm{~g}$. of streptococci culture, and subsequently exposed the animals to cold rains. He considers the importance of the streptococci proven, however, from the cbservations made by Frasey, who has seen a disease corresponding clinically with purpura hemorrhagica develop in two horses, which had been injected with highly virulent cultures of streptococci for the preparation of antistreptococcic serum. The fact that purpura hemorrhagica develops quite trequently as a sequel to strangles and influenza, appears to indicate the indirect association of streptococci with the disease (Bruin observed the development of purpura hemorrhagica in its severe form in two cases after the accidental injury of a vein in puncturing an abscess in strangles)

Cadéac also consider: purpura hemorrhagica as an intoxication, which, according to his view develops as the result of vasodilatatory toxins of various bacteria, especially of different pyogenic cocci, in animals which have been weakened by another disease. Mouilleron \& Rossignol, as well as Cormier, also attribute the greatest importance to the stieptococci, especially on the ground of the favorable results which they have obtained in the treatment of the disase with antistrepto coceus serum, although they do not deny the possible action of other micro-organisms. Coquot considers the disease as a Pasteurellosis.

Contrary to other observers Javorsky succeeded in only 7 cases out of 148 , in demonstrating the occurrence of purpura hemorrhagica as a sequel to othei affections (twice strangles, 5 times pleuro-pneumonia). Wohlmuth also believes that the disease may develop independently, and therefore he distinguishes an illiopathic and symptomatic form, in which the vascular system, especially the intima of the capullaries, is always the point of attack for the toxins which produce the disease.

Recently Baruchello \& Nori, and later also Perrucci expressed the improbable view that purpura hemorrhagica represents a piroplasmosis, as they found protozoa resembling piroplasma in the red blood corpuscles of four affected horses. However, the severe initial symptoms (high fever), which were observed ir these cases indicate that these affections were not true cases of purpura hemorrhagica.

The mode as well as the determining factors of natural infections are not yet sufficiently clear. It has been observed that the disease occurs principally in badly ventilated stables, with dirty floors, but it also occurs in well groomed horses kept under favorable conditions. The disease is most frequent in the spring and in the beginning of summer.

The age of the animals appears to have no influence except that colts under two years of age do not become affected (Dieckerhoff); otherwise there appears to be no factor which affects 
the susceptibility of the animal, nor does the care the horses receive or the work they do exert any influence.

Anatomical Changes. The characteristic lesions of the disease are the hemorrhages in the skin and in the mucous membrane, as well as in the subcutaneous and submucous connective tissue, in association with extensive, very marked, edematous swellings. Inflammation and tissue necrosis may develop as secondary processes with swelling of the affected parts of the body.

Upon the cut surface of the edematous swelling the subcutaneous connective tissue forms a yellow, gelatinous layer of several centimeters thickness, which shows dark red hemorrhages and in some cases contains cavities filled with purulent ichorous fluid, or with necrotic tissue. The gelatinous infiltration extends also between the muscles while the muscle substance itself appears pale grayish-brown or clay color, and friable, as a result of parenchymatous and fatty degenerations of the muscle fibers. The affected muscies feel greasy to the touch, and are sprinkled with small hemorrhages. In different places small areas of the muscles are necrotic and changed to a clay-like detritus, which sometimes appears dirty brown-red from admixture of blood. Hemorrhages may also occur in the tendons and tendon sheaths, under the periosteum, or in the joints; in the latter the cartilaginous tissue may even become necrotic (Prevost).

Of the mucous membranes those of the nose, pharynx and larynx become affected in the first place, then those of the stomach and the small intestines. The mucous membrane is affected less frequently in the deeper air passages and in the other parts of the digestive tract, as well as in the gental organs. In addition to catarrhal injection and swelling, the mucous membrane, as well as the submucous connective tissue of the intestinal canal, also in the muscular layer and under the serosa, small punctiform or streak-like or even larger hemorrhages may be present. The loose connective tissue surrounding them is always more or less gelatinously infiltrated. Over the larger extravazations the mucous membrane is often necrotic, or after the pale yellow necrotic parts have been thrown off, irregularly formed ulcerations develop which are covered with dirty tissue shreds. In the intestines they may extend to the peritoneum, or the intestinal wall may be perforated and lead to purulent or ichorous peritonitis, while in the nose the ulcerative process may cause a destruction of the nasal septum.

Hemorrhages are as a rule present also in some internal organs, especially in the lungs and the spleen, and also under the capsule of liver and kidneys. The lungs may contain hemor. rhagic areas the size of a fist, the cut surface may show spots like those of a tiger's skin, which are the result of blood aspirated into the bronchi. The spleen is sometimes consider- 
ably enlarged due to hemorrhages in its tissue and under its capsule, and sometimes a rupture of the spleen and a fatal internal hemorrhage may be observed. Far less often are instances of fatal bleeding into the retroperitoneal or into the perirenal connective tissue, into the digestive tract, or into the air passages; finally hemorrhages may also be found in some cases in the inside of the eyes, in the bone marrow, in the heart muscles, and under the pericardium.

The immediate cause of death usually is septicemia, pyemia or gangrenous pneumonia, less frequently asphyxiation may be observed. Autopsy may reveal the mode of origin of the disease by disclosing the presence of older organic changes.

Symptoms. The disease usually commences with the appearance of small punctiform or linear hemorrhages in the nasal mucous membrane. In some instances the animals do not manifest any indications of disturbed health while in other cases the appetite becomes somewhat diminished in the earlier stages, and the patient manifests slight dullness and depression.

Later the hemorrhages in the mucous membranes become more numerous, and those lying close together confluate, so that extensive blood extravasations result. At the same time there is a yellowish mucous discharge from the mucous membrane, which dries on the surface first to yellow, later to dirty brown crusts. In severe cases the mucous membrane covering the hemorrhages becomes necrotic, and ulcerations develop. In this condition the discharge which at first is scanty becomes more profuse, discolored, mixed with shreds of necrotic tissue, and not infrequently also with blood The discharge emits a fetid odor. In the meantime swelling of the eyelids is frequently observed and if they are forcibly spread apart a reddish fluid or even pure blood flows ont of the slit; in such cases the bullbar as well as the tarsal conjunctiva, shows shallow hemorrhages (exceptionally necrosis of the conjunctiva and hemorrhages into the inside of the eyes [Roell] may be observed, also atrophy of the optic nerve [Schindelka]).

In all of the 148 cases olserved hy Jarorsky hemorrhages were present on the nasal mucous membrane On the other hand they were found in only 58 cases in the mouth and in only 38 eases on thy conjunctiva.

Immediately after the appearance of the hemorrhages in the nasal mucous membrane, swellung of the skin and the subcutaneous connective tissue make their appearance. In some cases numerous urticaria-like vesicles develop over the entire body, which disappear after a certain time, or confluate forming dollar-sized flat roundish swellings (Fignre 39; Kleinpanl observed simultaneous shedling of the hair). In most instances, however, diffuse swelling developed, especially on the extremities, on the chest and abdomen, especially on the sheath and udder, and sometimes on the front part of the head (Figure 
40). The swellings often grow rapidly assuming great dimensions, so that the outline of the affected part of the body which is much deformed, becomes lost. In severe cases the circumference of the extremities becomes enormous, so much so that sometimes the legs from the coronary band to the ellow or to the knee swell to 2 or 3 times their normal size, and they appear like posts on which some transverse furrows are the only indications of the joints. On the head the swelling first affects the alae of the nose and the lips, soon however the lower part of the face becomes affected, and the fore part of the head

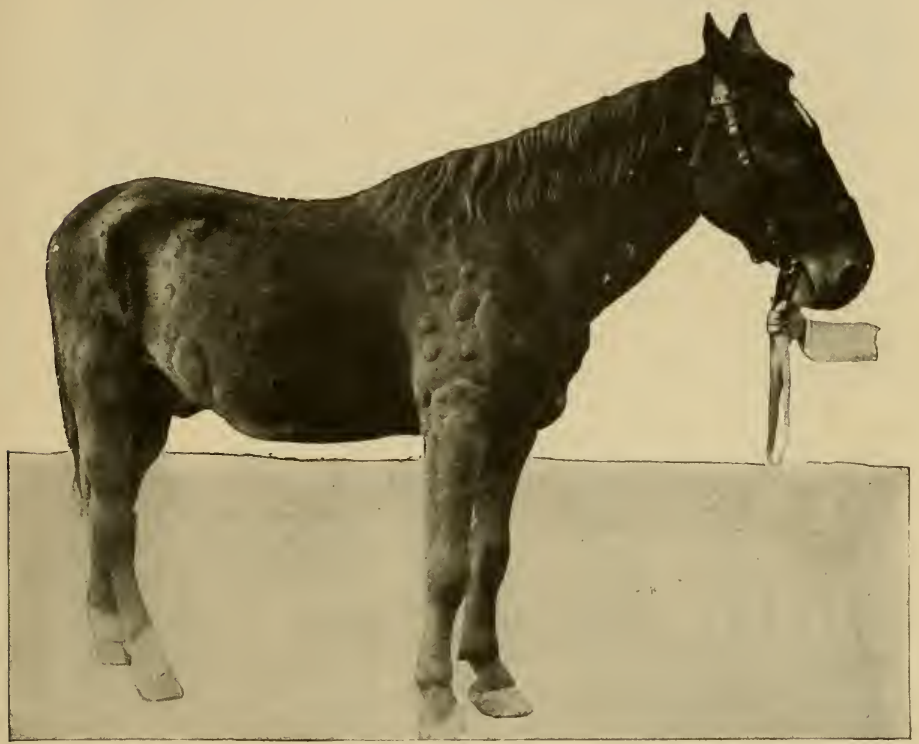

Fig. 39. Purpuru hemorrhagicu. Eruptions resembling urticaria at the beginning of the disease.

may become so broad that the transverse diameter greatly exceeds that of the upper portion of the head, the nose piece of the halter making a deep impression, and the head in such cases resembles somewhat the head of a rhinoceros (Figure 40). The swellings are characterized by being always sharply defined from the healthy surrounding parts. They are firm, almost of the hardness of a board, so that the surface may be pitted only with difficulty, the resulting depressions disappearing but slowly.

The skin over the swelling becomes stretched, and from its surface a yellowish tenacious serous fluid oozes out which later dries, forming brown crusts. In the flexor side of the 
joints the skin shows cracks which expose a moist reddened wound. In places where the stretching of the skin is the greatest, therefore, especially over the bony protuberances, as well as in the places where it is exposed to outside pressure, excep-

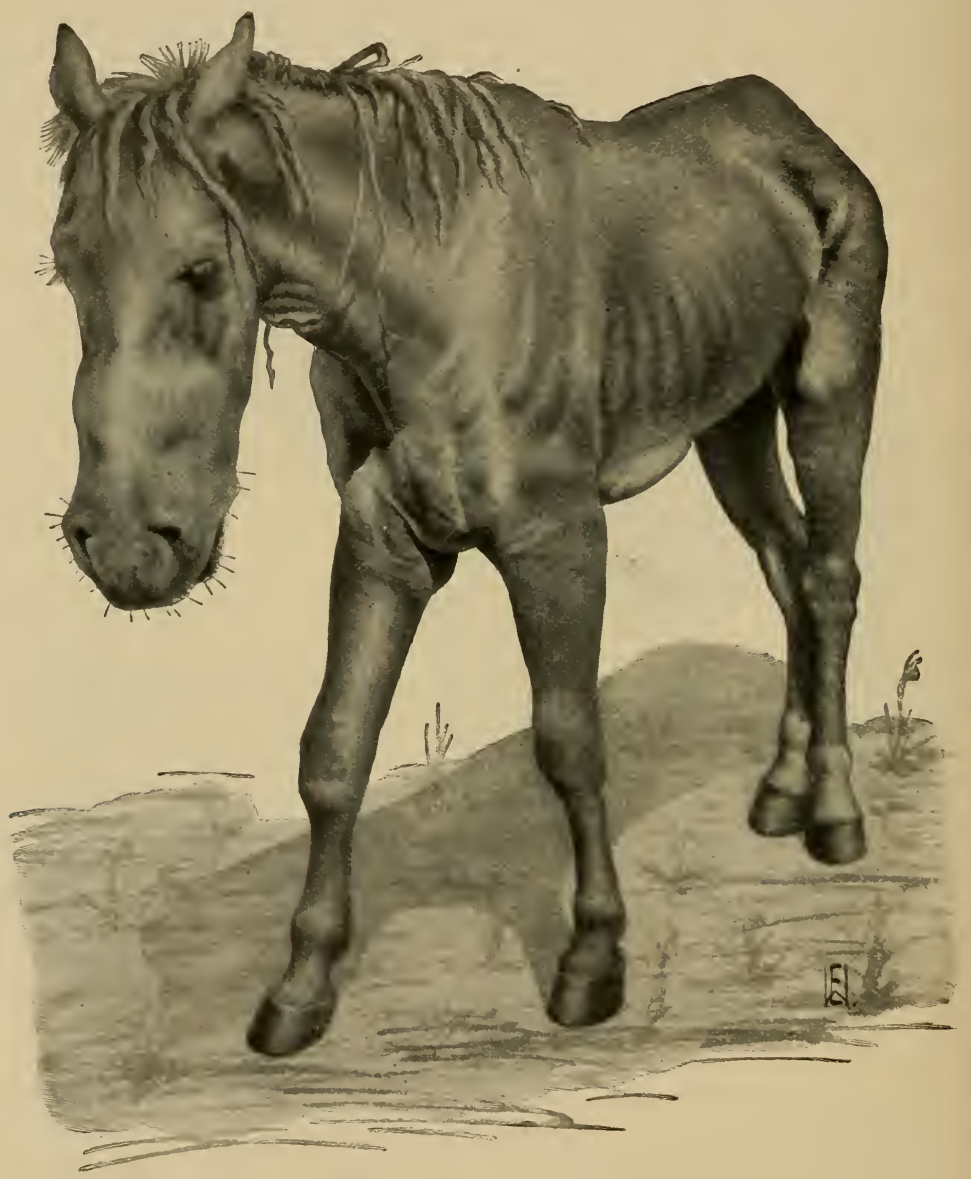

Fig. 40. Purpura hemorrhagica. Swelling of the forehead and of the lower chest.

tionally also in other parts of the body, for instance on the upper lip (Figure 41), the skin becomes insensitive in circumscribed areas, cold and dry, and takes on a darker color. Soon dry, round portions of skin as large as a dollar or even larger, dark brown and parchment-like are separated and fall off. The 
gangrenous process may extend deeper, affecting the muscles, while on the back of the nose and below the carpal and tarsal joints it may extend to the bone, leading to ugly ulcerations, which continually discharge a purulent, ichorous, fetid secretion containing necrotic tissue shreds.

According to Javorsky's statisties swellings on the abdomen are observed in $47.3 \%$, on the posterior extremities in $74.3 \%$, and on all 4 extremities in $45.5 \%$ of the eases.

The swelling impairs the physiological functions and the mobility of the affected part of the body. With the increase of the swelling of the nasal mucous membrane the respiration becomes snuffling, and is especially difficult during the act of inspiration. The labored respiration is still more severe when the swelling extends to the folds of the mucous membrane in front of the larynx, or to the mucous membrane of the larynx itself. In such cases the animals show all the indications of an inspiratory dyspnœea (anxious look, whistling $\mathrm{or}^{*}$ rattling respiration, spreading of the legs, forced extension of the

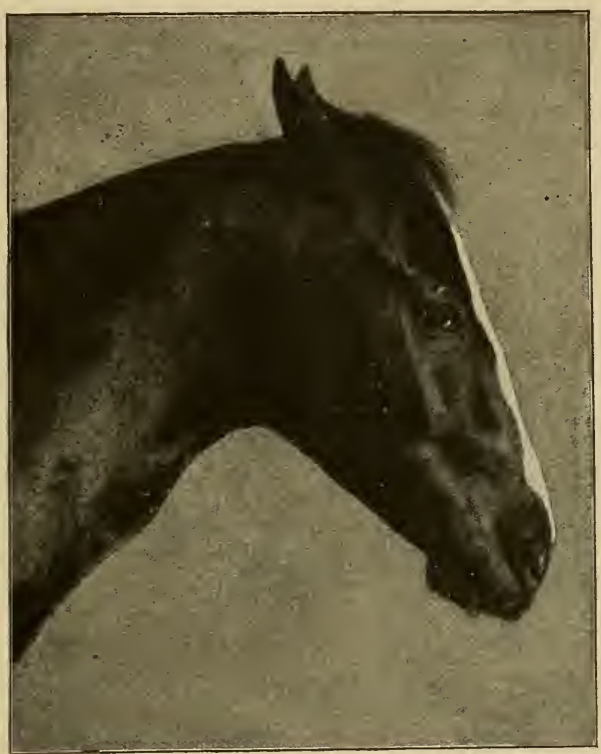

Fig. 41. Purpura hemorrhagica. Commencing necrosis of the skin on the swollen upper lips. thorax, with the intercostal spaces sunken in) and with further progress of the stenosis asphyxiation may result.

The great swelling of the forehead renders the motion of the jaws and with it mastication more difficult, while the infiltration of the pharyngeal mucous membrane and of its underlying connective tissue interferes with deglutition, so that in severe cases the animals are mable to take nourishment, although their appetite is not impaired, or they succeed only with great effort in swallowing small quantities of food or a little water.

The swelling of the extremities interferes more or less with the gait of the animals. At first and even later in mild cases, 
the disturbance is manifested only by stiffness and chumsiness in gait and inability to lie down, or if they succeed in this, in their inability to rise. In the presence of large swellings they remain motionless in one place, and it is impossible to make them move (the inability to move may be partly due to involvement of the muscles). The swelling of the sheath hinders the animal from protruling the penis, so that the urine trickles ont of the narrow opening of the sheath, and its borders as well as the surrounding parts of the skin become irritated by it.

The nutrition of the animals, except in the very mild cases, is always insufficient, through the difficulty in mastication and deglutition which was already mentioned. Althongh the appetite remains unimpaired for a considerable length of time, and in mild cases even during the entire course of the disease and the thirst is even increased; in severe cases disturbances in the appetite are noticeable which are dne to the involvement of the gastric mucous membrane. Srmptoms of colic sometimes follow the severe changes, and these indicate hemorrhages and inflammatory edematous infiltrations of the gastric and intestinal wall. The colic may occur either in severe attacks or less severely but more continuonsly. In some cases they are followed by manifestations of a rapidly progressing anemia and by collapse, which is an indication of an internal hleeding into the abdominal cavity, while in other cases paralysis or perforation of the intestine lead to sudden death. Feces are passed in moderate quantities, a bloody consistency pointing towards intestinal bleeding, while frequent movements of the bowels and a covering of the feces with a muco-purulent material points to inflammation of the rectum. In the presence of a severe inflammation of the intestines profuse diarrhea appears. In severe cases the urine contains albumen and also blood.

In mild cases the body temperature remains through the entire conrse of the disease at normal height, or is only very slightly elevated. In the presence of necrosis and ulceration of the skin, and of the mucous membrane, the fever may rise high from absorption of septic substances. The character of the fever in such cases indicates a septic form. The temperature may also change in those cases in which the affection developed immediately after a febrile affection (Fig. 42), or if complications arise during the course of the disease.

In the beginning the heart's action is somewhat accelerated hut sufficiently strong. Later it becomes excited, and because of the difficult respiration the changes in blood pressure due to hemorrhage and to pyemia or septicemia, the heart's action becomes very rapid and throbhing, while the pulse steadily diminishes in volume.

A microscopical examination of the blood shows nothing characteristic, with the exception of an occasional hyperleucocytosis (according to Smith the quantity of albuminous sub- 
stances and fats in the blood is increased, that of fibrin, water and iron is diminished).

Modification of the Course.

The clinical picture described may s li o w marked modifications. The disease may become checked in any stage of its development, and pass on to recovery. On the other hand in some cases in the early stages the origin a l disease, to which purpura hemorrhagica forms a sequel, may yet persist; but in a still $\mathrm{gr}$ e a t e r $\mathrm{number}$ of cases the disease itself may be changed by the appearance of complications arising during its development.

One of the most frequent complications is pneumonia, which usually is lobular in character, and d evelops in connection with h e morrhages into the lung tissue; and may

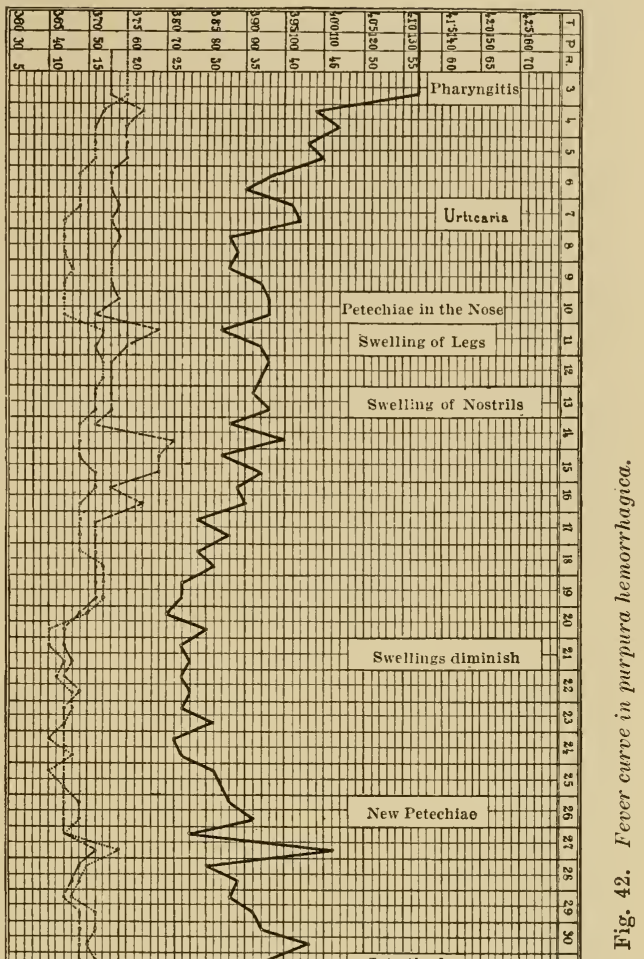
be, less frequently, the result of the aspiration of necrotic parts of mucous membrane, assuming then often a gangrenous form. 
Rapid and labored respiration, especially in cases when the upper air passages are not markedly constricted, in association with a rise in temperature, are indicative of pneumonia, and renders a careful examination of the thoracic organs necessary.

$A$ serere and not infrequently nlcerative inflammation of the stomach and intestinal mucous membrane also develops with relative frequencr, and causes a putrid diarrhea, which greatly weakens the animal.

In the cases with unfarorable termination the clinical symptoms are almost invariably terminated by manifestations of pyemia or septicemia. They usually develop either from the above cited causes, or from the sores which develop on the body of the animals when they persistently lie down.

According to Payrou petechial fever as it is observed as a sequel to distemper, may be manifested either as a toxemic (lymphatic) form, brought on by the absorption of toxins into the blood, or in a septicemic form caused by a streptococcus infection of the blood. The former develops in the second week after the beginning of strangles, when, in the presence of a moderate fever, the extremities slowly swe!l from lelow upwards, and the nasal mucous membrane manifests only small petechiae. The course is frequently mill. The second form develops with high fever, and extensive petechiae, running a peracute course, causing swellings everywhere, especially on the head and on the conjunctivae, which have a great tendency to necrosis. In these cases the disease usually terminates fatally in from 8 to 10 days. As a result of the great frequency of intermediate forms the separation of these two types might often be very difficult, and it is doubtful whether such a classification is justified.

Course. The course of purpura hemorrhagica varies from case to case. From the very mild forms which manifest only petechiae of the nasal mucous membrane, and a slight simultaneous swelling of the skin and the symptoms of which may disappear inside of two or three days, to the cases of extreme distortion of the body and resulting in death from asphyxiation or sepsis, all the possible degrees may be observed. In the successive development of the symptoms the affection of the nasal mucous membrane usually appears first, and it is hardly ever absent. This is followed in one or two days by a swelling of the skin, and only later by the affection of the pharynx, whereas the involvement of the intestinal canal is usually observed only in advanced stages of the disease. However, there are rare cases where the sequence of symptoms differs; thus the edema of the skin may proceed to development of petechiae in the nose, or severe colicky symptoms may develop in the beginning of the disease.

The evolution and course of the disease depends especially on the localization and the more or less rapid development of the hemorrhages and of the edematous swellings. Apparently severe cases, in which however the changes are exchusively confined to the nasal mucous membrane, the skin and the subcutaneous connective tissue may terminate in complete recovery even when the skin becomes necrotic over a considerable area; on the other hand affections of the internal organs, as well as hemorrhages and inflammations, exert an unfavorable influence 
on the course of the disease, and such cases usually terminate fatally.

In the favorable cases the swellings after reaching a certain degree retrogress either rapidly or gradually. Frequently, however, the improvement is only temporary, as the condition later becomes aggravated again, and with such variations the disease may run for several weeks until finally the animal recovers. The average duration of the mild cases may be placed at about two weeks, although it may occasionally extend over a period of 6 to 8 weeks. In cases where large areas of the skin become necrotic it may require several months until a definite recovery takes place, and even in such cases the recovery is not complete, as in place of the extensive wounds contracted scars remain which interfere with the function of the extremities, and thereby reduce the value of the horse for work.

In fatal cases the duration of the disease also varies. There are cases in which the swellings form with such rapidity and become so extensive that death ensues in from 3 to 5 days (Dernbach saw a horse in which death occurred after 7 hours as a result of a hemorrhage in the neighborhood of the medulla oblongata). Complications (gangrenous pneumonia, enteritis, pyemia, septicemia) usually develop only after the swellings have progressed extensively and have produced functional disturbances as well as necrotic processes; up to that time, however, usually 1 or 2 weeks have elapsed. If any of these complications have appeared death always ensues after several days.

Diagnosis. Recognition of the disease is difficult as a rule only when it is associated from the beginning with fever, or when edematous swellings and hemorrhages appear in the course of a preceding febrile affection. Edematous infiltrations may develop from local causes (phlegmons, lymphangioitis) as well as in general infectious diseases (anthrax, malignant edema) ; they may, however, be distinguished from the swellings of purpura hemorrhagica by the diffused borders and by the localization of the inflammatory processes. Besides this the swellings in purpura hemorrhagica are usually sharply circumscribed and occur in most instances symmetrically on both sides of the body; they also appear after or simultaneously with the development of petechiae on the nasal mucous membrane. In the early stages of the disease urticaria may also come under consideration, as it is sometimes manifested by extensive and sharply eircumscribed swellings of the skin; however, in this affection the petechiae are absent on the nasal mucous membrane, and the eruptions disappear entirely after a short time. Acute glanders may likewise resemble purpura hemorrhagica to some extent, especially in cases in which the swelling of the nasal mucous membrane and its surrounding tissue, as well as of the extremities and abdomen develops rapidly; however, in 
such eases there are no petechiae on the nasal mucous membrane, but nodules and ulceration, and at the same time there is also a swelling of the submaxillary lymph glands. In addition to this, the rapid development of the swellings on other parts of the body is always accompanied by fever and by symptoms of a general infection (it must be remembered that purpura hemorrhagica may develop in the presence of glanders).

Prognosis. This is very uncertain in consideration of the varied course of the disease, and therefore should be made with especial care. Although with proper treatment on an average $40-50 \%$ (according to Javorsky $60-65 \%$ ) of the affected animals recover, the mortality fluctuates between wide limits. The slow development of the hemorrhages and swellings, and their presence in small numbers, are indications of a favorable comrse, whereas their rapid increase and extension point toward an unfavorable prognosis. There are cases, however, which show a very severe affection from the onset, and in which after a certain time sudden improvement is noticeable, the animals finally recovering. This is especially the case when by proper treatment the development of threatening complications is prevented. A recovery may be expected as long as the patients are without fever or have only a slight fever, and take nourishment at least in moderate quantities. High fever renders the situation usually grave, as it points to septic infection or to inflammation of internal organs. Other unfavorable indications are pronounced cyanosis or yellow discoloration of the mucous membranes, the presence of albumen or blood in large quantities in the urine, continuous lying down, frequent and weak heart action, further the excessively rapid disappearance of the swelling. The latter apparently favorable symptom indicates in most instances a rapid breaking down of the tissues, or that great quantities of blood or blood plasma have, under the influence of the high fever, transuded into the internal organs. The development of after affections should also be taken into consideration in forming a prognosis, as they are of a decided influence in determining whether treatment extending over a long period of time is justified, in view of the inability of the animals to work and the expense of such prolonged treatment.

At the elinic at Budlapest between 1887-1908, out of 296 affected horses $167(56.4 \%)$ recovered; the numbers of maximum and minimum recoveries in these years was 20 and $95 \%$. In the Prussian army in the years of 1895-1903, 149 horses became affected, of which $110(79 \%)$ recovered (the maximum and minimum recoveries in these years were 56.2 and $85 \%$ ).

Treatment. Symptomatic treatment has a great influence on the further development of symptoms and on the termination of the disease, especially as it is not infrequently possible to avert the danger of asphyxiation, and to prevent the appearance of complications.

Above all it is necessary to assure suitable feeding of the 
affected animals, and to provide a sufficient supply of fresh water. Hydrochloric or sulphuric acid (8-10 g. to a pail) may be added to the water and in the presence of fever wine or alcohol (11/2-1 liter to a pail). If the animals show difficulty in mastication and swallowing they should be given green food and bran-or flour-gruels. If this condition becomes aggravated the animals may be fed artificially per rectum, and in this manner they may retain their strength for a considerable time. Pure fresh air is likewise an important requirement for a favorable course, and it is advisable to keep the animals out of doors in the summer time and in bad weather in roomy box stalls. The halter should be removed as it may have a harmful effect by its pressure on the swollen cheeks and nose.

Wounds, ulcers and abscesses require antiseptic surgical treatment. In the presence of a considerable swelling of the nasal mucous membrane the nasal cavities should be carefully syringed 2 to 3 times daily with a non-irritating disinfecting solution (3-4\% boracic acid, 2-3\% creolin or lysol solution, $1 / 2-1 \%$ alum solution). The swellings of the skin are in many cases favorably influenced by rubbing with spirits of turpentine 2 to 3 times daily, since under this treatment gangrene of the skin occurs more rarely. Various other fluids may be used for the same purpose, especially Burow's solution with camphor, which is highly recommended by Dieckerhoff (Camphorae 50.0, Plumbi acet. 200.0, Alum. 100, which is used in the proportion of 1 tablespoonful to 1 liter of water), Goulard's extract, subacetate of lead or alum solution $(5 \%)$, spirits of camphor, etc. Counterirritants should positively be avoided as the stimulation of an inflammation enhances the development of necrosis of the susceptible skin.

If in spite of this treatment gangrenous abscesses develop over the swellings of the skin, such defect as well as tears should be treated after the removal of the necrotic tissue shreds, by washing with one of the above mentioned solutions, or still hetter they may be dusted with a drying powder. For this purpose charcoal powder containing corrosive sublimate (charcoal mixed with a $1 \%$ corrosive sublimate solution and then dried), further Dermatol, Alumnol, Iodoform, Thioform, Tannoform, etc., may be used to good advantage. A 5-7\% creolin liniment (smeared on wool and placed on the wound) may also act bene. ficially whereas tinctures (tincture of myrrh, tincture of aloes) are less effective. This treatment should be continued until the wound has filled up and healed over, but in the meantime protuberant granulations which may develop should be controlled by cauterization (nitrate of silver or with fuming nitric acid). Sores of decubitus should be treated in like manner. T'ho conjunctivitis may be controlled by blowing sodium borate into the eye, while in gangrene of the conjunctiva washing with a $2 \%$ creolin solution is advised.

If the patient is threatened with asphyxiation as the result 
of marked constriction of the upper air passages, the performance of tracheotomy is advisable. The stenosis of the nose may be relieved by elevating the median alae of the nostrils passing threads through them and tying them together over the hack of the nose, or according to Johne's suggestion, by the introduction of tin tubes into the nostrils. These metlods, however, are not to be recommended as the threads and even more the tin tubes may produce necrosis of the skin and of the mucous membrane. The wounds caused by tracheotoniy should be cleaned 2 or 3 times daily, and in order to prevent a harmful pressure between the outer plate of the tube and the skin a ring of cotton should be inserted. The operation may even be undertaken before asphyxiation threatens, especially in cases when a rapid development of the swellings indicates the approach of a dangerous nasal stenosis.

In consideration of the difficulty in swallowing which is present in most instances only such remedies should be used in the treatment of gastro-enteritis as may be administered in the drinking water or in soft food. Calomel is to be mentioned as a remedy, as it not only has a disinfecting action, but also facilitates the absorption of the transudated serous fluid as a result of its uniting with the blood albumen (Jendrássik) (3-4.0 g. daily, divided in several doses), then boracic acid, tannin, sugar of lead, also ichthyol (sodium or ammonium, sulfo-ichtliyolicum, 20-30 g. pro die), cinchona bark, etc., may be given. In cases where the rectum is severely affected enemas of astringents and disinfecting fluids (for instance 2-4\% lukewarm boracic acid) are indicated.

If improvement becomes apparent the convalescing animals should be exercised out of door's for a short time 2 or 3 times a day, as soon as the fever disappears, and the loss of the body fluids should be restored through profuse, rich nourishing.

Various remedies were recommended for the purpose of an abortive treatment, but none of these can be considered as specific.

Thus Azary recommended the fluid extract of hydrastis canadensis (5-8.0 g. subeutaneously). Payrou advised adrenalin (15-25 drops in $1 / 4$ l. of water) early in the disease, because of its vasoconstrictor action; while recently iodine and silver preparations are used very extensively.

Dieckerhoff employed intratracheal injections of Lugol's solution (iodi puri 1.0 , potass. iodati 5.0 , arquae dest. $100-200.0 ; 30-40 \mathrm{~g}$. for one dose ; at the same time $10-20 \mathrm{~g}$. of iodide of potassium may be added to the drinking water). This treatment has been given extensive trial in practice, but without any remarkable results. The literature shows that out of 96 cases treated in this manner only 61 horses $(63.5 \%)$ recovered (in the Prussian army inside of three years out of 41 horses 31 recoveries, $75 \%$ ) and some of the anthors (Zschokke, Cadéac, Lemke, Johne) have even observed bad effects from this treatment (gangrene of the mucous membrane of the trachea, symptoms of poisoning).

Jodvasogen, which has been introduced into veterinary practice hy .Jess, has also been tried (intratracheal injections of 15-20 g. of a $6-10 \%$ solution, or $50-80 \mathrm{~g}$. per os daily). The results, however, have 
been doubtful, as alongside of the favorable results reported by Straube, Thomas, Clausen, Richter \& Feuerhack, there are those of Zerler \& Giesenschlag as well as Gutzeit, which are unfavorable. The value of the iodipin treatment as recommended by Franz, Thomas and Tantos ( $10 \mathrm{~g}$. of a $25 \%$ solution of iodipin injected daily subeutaneously, and on every second day $50 \mathrm{~g}$. of a $10 \%$ iodipin solution per os), cannot be judged at the present time.

Of the silver preparations Dieckerhoff recommended Crédé 's colloid silver or Collargol (50 g. of a freshly prepared $1 \%$ solution intravenously 2-4 times a day at the beginning of the disease); the results from this method of treatment varied. While Thomassen, Zimmermann, Roder, Dresdow, Mollerau and others obtained satisfactory results, the experiences with this method in the Prussian army were not so eneouraging; in the years 1899-1902, 51 animals were treated, with Collargol, of which $40(78.5 \%)$ recovered, while of 35 animals which were treated by other methods or were not given any treatment $26(74.3 \%)$ reeovered. At the Budapest clinic in the years of 1898-1902 25 animals received the silver treatment, and $14(56 \%)$ recovered, while 16 other cases were treated otherwise, of which $8(50 \%)$ recovered. It was observed that even when this remedy was employed at the earliest stages of the disease it did not influence its normal course (Fettiek). Payrou declared this remedy to be worthless, and Brohle ohserved a fatal termination in three eases as a result of embolism of the pulmonary arteries following an intravenous injection of $0.4: 40.0 \mathrm{~g}$. Collargol.

Conditions are similar in the ease of Ichthargan $(50 \mathrm{~g}$. of a $1 \%$ solution intravenously), as recommended by Lange and Jost. The results obtained with this preparation in the Prussian army in the years of 1904-1905 were not uniform. Zürn calls attention to the hemolytic and strongly irritating action of this remedy, and therefore recommends only 0.1 to $0.2 \%$ solutions for intravenous injections. (Lövy saw after an injection of $0.5 \mathrm{~g}$. of a $1 \%$ solution, the development of severe toxic symptoms in a horse of advanced age).

Bertelotti employed spirits of turpentine $(5.0 \mathrm{~g}$. intravenously), Howe oil of turpentine (per os), Pelz, Thomas and Fenerhack tallianin (10-30 cc. intravenously), Gorjaew corrosive sublimate ( 10 ce. of a $1 \%$ solution intravenously), Beck lastate of silver or Actol (40 ce. of a $1 \%$ solution 3 times daily intravenously), apparently with good results.

Serum Treatment. Stimulated by the good results obtained in human medicine with Marmorek's anti-streptoeoecus serum in streptococcus infections, various authors used the serum for the treatment of purpura hemorrhagica in horses, accorling to the prevailing idea that the disease is also caused by a streptococeus infection. This appeared justified in view of Lignières' assertion that the streptococei associated with purpura hemorrhagica was identical with the pyogenic streptococei of man, and in view of his good results with Marmorek's serum in mice. The treatment consists of subcutaneous injections of $10-40 \mathrm{~g}$. serum, 3 to 6 times daily, or repeated even every hour. (Sommermeyer injects first $10 \mathrm{~g}$. of immune serum subcutaneously and later $20 \mathrm{~g}$. normal serum intravenously).

The reports published so far on the use of Marmorek's serum are favorable (Lignières, Mouilleron \& Rossignol, Pench, Pecus, Maier who used in one case 190 and in another $440 \mathrm{~g}$. serum inside of two weeks!), as well as about the Jess-Piorkowski serum prepared in a similar way (Sommermeyer, Bolz). Lignières' results show only two deaths in 
15 patients, the tabulation of Mouilleron and Rossignol only 11 deaths with 62 patients, while of 209 cases treated by other methods 126 suecumbed to the disease. The Pasteur Institute of Paris has recently prepared a polyvalent serum with the aid of streptococci of man and of the horse, and with this serum Payrou obtained favorable results in army horses (of 45 cases only 7 died, 15.5\%). However, as in the etiology of purpura hemorrhagiea other bacteria appear to have also a part and Caliot obtained equally good results with normal blood serum (100-125 g.), the value of the rather expensive serum treatment cannot be definitely judged at the present time.

Literature. Percivall, The Vet., 1844.- Hering, Spez. Path., 1858, 371 (Lit.).Dieckerhoff, Spez. Path., 1892, 417; B. t. W., 1898, 541.-Mouilleron \& Rossignol, Bull., 1898, 168.-Lange, Z. f. Vk., 1903, XV, 117.-Jost, B. t. W., 1904.-Lignières, Bull., 1895, 587; 1898, 722; 1903, 363.-Javorsky, A. f. Tk., 1905, XXXI, 601.Payrou, Bull., 1905, 491.-Mollerau, Ree., 1905, 24.-Drouin, Rev. gén., 1906, VII, 289.-Barthel, Z. f.' Vk., 1906, XVIII, 430 (Lit.).-Franz, D. t. W., 1906, 38.

Purpura Hemorrhagica of Cattle. Recent literature contains several reports (Schleg, Eichhorn, Frank, Röder, Plate, Ehrhardt, Koppitz, Reinhardt and others), which render the occurrence of purpura hemorrhagica among cattle, especially younger animals, and perhaps also in buffalo (De Does) probable. In the respective cases there was a diminished appetite and cessation of rumination, as well as great depression and weakness. At first small, later more extensive hemorrhages occurred in the skin, and in the subcutaneous connective tissue, in the conjunctivae, in the mucous membranes of nose and lips, and in the udder; while in some cases edematous swellings, although usually only in a moderate development, were observed on the extremities, and on the abdomen. From the surface of these swellings a bloody serous fluid oozed out. At the same time a bloody discharge from the nose and the vagina was occasionally observed, and the mash-like excrements were also mixed with blood. In exceptional cases there appeared a necrosis over the hemorrhages in the buccal mucous membrane, and in the conjunctivae, as well as in the skin. In some of the cases the temperature remained normal, in others, however, the disease was inaugurated with high fever. In the cases where the animals were not slaughtered during the disease, recovery usually followed in from six to ten days, but in some instances not for three weeks, while some of the animals died from intestinal hemorrhage or septicemia. Autopsy usually revealed more or less extensive hemorrhages in the muscles, in the serous and mucous membranes, in the subserous and submucous connective tissues, as well as in the internal organs. In some eases there was also yellowish-gray or grayish-brown discoloration of the liver. The spleen usually appeared normal.

In the majority of eases the healthy animals became affected without any apparent cause; exceptionally the disease developed in connection with a local inflammatory process, especially mastitis (Röbert), puerperal inflammation of the uterus (Augstein), or rheumatism of the joints (Zehl). The disease was also olserved as a stable infection, although a transmission from animal to animal could not be established in these cases.

Before establishing a diagnosis of purpura hemorrhagica it should be differentiated from other diseases in which hemorrhages oceur, especially the various kinds of septicemias (anthrax, malignant edema, hemorrhagic septicemia, etc.). The disease is mainly distinguished from these affections by the absence of fever in the early stages. 
In the treatment the same principles apply as given in purpura hemorrhagica of the horse. In the cases where in one stable several animals became affected consecutively, the disease was checked by changing the feed and the drinking water (Minder) as well as by disinfecting the stable (Eichhorn).

Literature. See Reinhardt, Monh., 1909, XXI, 221.-Zehl, B. t. W., 1909, 715.

Purpura Hemorrhagica of Dogs. Lellmann observed in a 6-year old dog symptoms which indicated an affection of purpura hemorrhagica. The emaciated animal, which had no fever, showed weakness and a weak heart action, also swelling of the hind parts, ascites, epistaxis, hematuria, and bloody excrement. The autopsy revealed hemorrhages in the spleen, liver, kidneys, in the serous membranes, in the skin, in the subcutaneous connective tissue, and in the muscles. In another case there was anemia (pale mucous membrane, small pulse, anemic heart murmurs) bloody vomiting and diarrhea, also numerous hemorrhages in the skin which comprised the principal manifestations. The autopsy findings in this case resembled those of the first. Lellmann considers both eases identical with purpura hemorrhagica of the horse. (B. t. W., 1897, 509; 1902, 266.)

Raitsits observed in a dog bluish-red, later confluating spots on the abdomen and on the inside of the thighs, petechiae on the conjunctivae, swellings of the gums, hazel-nut sized abscesses on the phrenum of the tongue, albuminuria and hematuria. The antopsy revealed hemorrhages under the skin, also in the conjunctivae. and a hemorrhagic inflammation of the rectum. (A. L., 1906, 421.)

Purpura Hemorrhagica of Man. This disease (morbus maculosus Werlhofii, purpura hemorrhagica), is cansed according to Kolb, by a small Gram-positive bacillus, the bacillus haemorrhagicus, while according to Letzerich it is caused by the bacillus purpurae haemorrhagicae. The hemorrhages which appear in different parts of the body in association with the disease cause it to somewhat resemble purpura hemorrhagica of animals; however, the possible relations of the two diseases have not yet been established. It should be mentioned that in a case reported by Bock a stable attendant became affected with Werlhof's disease several days after the death of a horse with purpura hemorrhagica.

Literature. Kolb, Arb. d. G.-A., 1891 VII, 60.-Bock, Z. f. Vk., 1902, $\mathrm{XIV}, 117$.

Scarlatinoid of Horses. Under this name Champetier described a disease of young horses which resembled scarlet fever of man. In a mild form of the affection there is a fever reaching $103-104^{\circ} \mathrm{F}$., inappetence, frequent pulse and cough, injection and petechiae of the mucous membranes, further a peculiar pustular exanthema of the skin, and edematous swellings of the extremities. In the more severe form the enumerated symptoms are more intense and bronchial catarrh, eatarrhal pneumonia, and nephritis may also develop. (Les maladies du jeune cheval. Paris, 1892.)

The latter of these forms has also been observed by Marek in an 8-year old mare. Simultaneously with a marked swelling of the nasal mucous membrane diffuse edematous swellings appeared on the abdomen and on the hind extremities; nodules developed on the skin of the rump, the head and neck, and later round ulcerations appeared 
in their places. The antopsy on the animal, which died sixteen days after the appearance of the disease, revealed a partial necrosis of the nasal mucous membrane, fresh ulcerations on the tracheal mucous membrane, and a hemorrhagic nephritis. There was also present a striking inflammation of the lymph vessels, and a pronounced acute congestion of the lymph glands of the mesentery. (Monh., 1896, VII, 346.)

Scarlatinoid of Cattle. Hetzel observed in a $11 / 2$-year old steer an affection resembling the clinical appearance of the preceding disease of the horse. The symptoms consisted in a high fever with sudden onset, great depression, catarrh of the pharynx and larynx. Two to four days later petechiae appeared on the nasal mucous membrane, and various kinds of erythematous spots on the skin. At these places nodules teveloped in the later course some of which disappeared, while others developed into pea-sized vesicles containing clear serous fluid. Later the contents of these vesicles became purulent, and finally they turned into thick scabs, the animals manifesting severe itching. Purulent conjunctivitis and bronchitis had also developed, and diarrhea exhausted the animals to a great degree. The fever subsided after a duration of six to twelve days, the catarrhal symptoms became milder, and after a desquamation of the scabs complete recovery took place. (A. L., 1903, 33.)

\section{Rinderpest. Pestis Bovina}

(Cattle plague, Typhus bovum contagiosus, Peste bovine; Typhus contagieux [French]; Orientalische Rinderpest

[German]; Peste bovilla [Italian].)

The oriental rinderpest represents an acute febrile, contagious, infectious disease of cattle, caused by an ultra-microscopical micro-organism, and which aside from its typical course is characterized by a croupous diphtheritic inflammation of the mucous membrane. Exceptionally the disease passes from cattle to other ruminants.

History. The attention of experts has been directed to rinderpest from earliest times, because of its destructive character, and the most varied theories have been formulated regarding its nature. At times the affection was considered identical with human pox (Ramazzini, Vicq D'Azyr, F. Müller), and again with typhus (Veith, Spinola) and dyser1tery of man (Lorinser). Its infectious nature was recognized early, and was established by successful inoculations (Dodson, 1744; Camper, 1770 ), and veterinary police measures were inaugurated against the spread of the disease (in Prussia in the year of 1711, in France in 1714). Since the beginming of the last century the belief of a spontaneous origin of the disease has been more and more abandoned, but the causative agent remained unknown up to the most recent times in spite of the numerous investigations conducted along this line (Semmer, Gamaleia, Nencki and others), until Nicolle \& Adil-Bey (1902) established its filterability through porcelain filters. Deliberate experiments conducted by Semmer \& Raupch, more recently by Nencki and his co-workers in Russia, Koch, Danysz \& Bordet, Edington, Koller \& 
Turner in South Africa, Nicolle \& Adil-Bey in Turkey, led to the elaboration of several methods of immunization adapted for practical requirements.

Occurrence. Rinderpest causes enormous losses in the infected herds of cattle, and may even result in the almost complete extinction of animals in large territories. Sometimes it may also cause considerable losses among flocks of sheep and goats. At the present time it prevails in Europe only in the southern part of the Balkan peninsula, but in Asia it is continually present, and recently it has become prevalent in Africa.

Rinderpest was probably known in the early ages, and not only in its original home, Asia, but also in Europe. With the great migrations of people it was introduced from the Black Sea over the entire continent westward, where it has raged almost continuously since that time until the '70's of the last century. Extensive outbreaks usually appeared associated with great wars, inasmuch as the cattle which were driven after the troops disseminated the disease over distant territories. Such invasions occurred in Germany during the period of Charles the Great in connection with his wars; in France and Italy at the same time; in the thirteenth century after the intrusion of the Mongolians; at the time of the thirty-years' war, etc.

In the first two decades of the eighteenth century the disease existed throughout Europe with such vehemence that for instance in the years 1711-1714, one and a half million of cattle fell victims to it (Paulet). Later Holland lost almost its entire stock of eattle as a result of the disease. Through the great Napoleonic wars the disease attained again a great extension especially in Germany and in France (the first French veterinary schools were established as a result of the great losses caused by rinderpest at that time).

Since the middle of last century it has generally been accepted that the disease spreads only by infection through which knowledge the only rational way of control and eradication of the disease has been established. The complete eradication of the disease, which in some countries caused severe losses, appeared as a very urgent necessity, and consequently the European governments adopted measures by which they succeeded in pressing it back to the far East. The plague attained considerable extension in middle Europe for the last time during the Franco-Prussian war, but in the course of the following decade it was completely suppressed, so that since 1881 it is contined to Russia and Turkey and more recently has apparently disappeared even from European Russia.

The losses in Germany during the eighteenth century are estimated at 30 million cattle. The disease was distributed most extensively in the beginning of the last century during the Napoleonic wars, in the ' 30 's through the Polish Revolution, and later throngh the Franco-Prussian war. During the latter, 13,000 eattle and 4000 sheep died in Alsace-Lorraine, while in Germany 8151 cattle succumbed or were killed (the disease was also brought to Switzerland by the eastern army which entered that country). In 1877 the disease was spread from Poland to Posen and from Austria to Wiesbarlen and caused a loss of 1591 cattle, and in the following year 2349 cattle died in Prussia from the disease. It appeared for the last time in 1881 in the province Schlesien, and since its eradication from this territory the German Empire has remained free from the plague (Dieckerhoff).

In France the disease raged to an alarming extent during the great Revolution and the Napoleonic wars, especially after the unsuccessful Russian expedition. 
It appeared for the last time after the Franco-Prussian war, and caused in the course of two years, in 43 districts, a loss of 56,533 cattle valued at $15,000,000$ franes.

Great Britain was free of the disease from the middle of the eighteenth century for a period of 120 years. In 1865 the disease was brought to the London stock market, and at the same time to different provincial cities by a cattle boat from Finland, and towards the end of that year 85 counties were infected. During the $1 \frac{1}{2}$ years of its prevalence 500,000 eattle died, valued at $100,000,000$ franes. The last outbreak occurred in 1877 as a result of its introduction from Hamburg, and caused a loss of 1193 cattle.

In the middle of the year 1866 the disease spread from England to Holland, where it extended rapidly and in barely a year's time had affected 156,594 cattle, of which 75,110 died and 36,919 were slaughtered, while 41,56.5 recovered.

The disease was introduced into Italy toward the end of the eighteenth century by Austrian troops, and spread southwards with such rapidity that inside of three years over $3,000,000$ cattle became its victims. Later in the years 1862-1868, the disease was widely distributed, having been introdnced from Dalmatia, in which time the kingdom of Naples alone sustained a loss of 50,000 cattle. Rinderpest occurred in Italy for the last time in 1878 in the province of Naples.

In Austria, Galicia and Bukowina sustained great losses in the middle of the last century. In both of these crown possessions about 54,000 cattle succumbed in the years of $1848-1864$, and about 35,000 head in the years 1868-157\%. In the first perioul Moraria suffered a loss of 12,000, while southern Austria lost 4,500 head. The plague invaded Dalmatia for the last time during the Bosnian War, in the year of 187s, and towards the end of the following year Slavonia, Styria and the Krain experienced a visitation. During this outbreak, in a period of three months 1785 cattle died of the disease. Since the year 1880 Austria has been free of the disease.

Hungary suffered most severely after the invasion of the Mongolians, and during the time of the Turkish wars, but the disease appeared repeatedly in that country in later years. During the time of the Russian-Turkish war, in the years 1827-1828, the disease was introduced from Moldall and from Roumania, and prevailed continuously until 1842 , the losses in the first two years amounting to 30,000 head of cattle. After the conclusion of the wars of $1848-1849$ the disease was almost entirely eradicated from the western part of Europe, but was brought to Hungary in 1849 by the Russian army, where it raged continuously until 1961 . (The loss in this period amounted to over 350,000 cattle.) After the war between Austria and Prussia the plague again increased, and lasted up to 1874 . From the middle of the year of 1861 until the end of 1873 the total loss was 165,732 cattle, valued at over $8,000,000$ florins; while the execution of the veterinary police measures required an expenditure of over half a million of florins (Roll). The ereation of the Rinderpest law in 1874 finally resulted in a complete eradication of the disease in the year of 1881 .

In Russia during some of the outbreaks more than a million cattle, in some localities even 3 to $5 \%$ of the total stock, were annihilated by the disease (Jewtachiew). In more recent years it was possible, with the aid of the measures of eradication adopted in 1879 , to restrict the disease within narrow limits. In 1892 it again commenced to spread westward, but gradually diminished since 1894, and since 1897 it recurs only east of the Cancasian Mountains, where it prevailed in 1907 in six government districts affecting 812 townships. In November, 1908, three infected transports were brought from Petropawlowsk (Siberia) to the abattoir of St. Petersburg, but the localization of the disease appears to have been successful. Rinderpest occurs continuously in Turkey, and appears especially in the vicinity of Constantinople, as well as on the coast of the Marmara Sea. Detailed statistics of its occurrence are not available.

The entire territory of Asia, including the East Indian groups of islands, is infected; and in Asiatic Russia alone the losses in 1906 amounted to 25,093 cattle; (according to Yersin the disease rages in Indo-China also among the buffaloes). In 1892 the plague was carrier from Corea to Japan, but in two years its successful eradication was accomplished with energetic measures.

Previous to 1864 rinderpest was known to exist in Africa only in Egypt, but during the Italian invasion of 1890 it spread southward along the Nile, and inside of five years reached the South African States as well as the West African Colonies of Germany. Transvaal lost 980,000 cattle in 1897, while in the Cape Colony 1,300,000 animals died of the disease in the years of 1897-1898. After that time the plague diminished somewhac, but gained again in severity in the rears of 1901 and 1904, until during recent years it has been considerably limited by the persistent employment of protective vaccination. According to Littlewood the disease was again introduced into Egypt from Minor Asia in June, 1903, appearing in the Province Behera, where it spread with great severity, and according to 
official reports, 147,285 animals, out of a total number of about $3,000,000$ cattle and buffaloes, had fallen victims to the disease up to the end of 1906 . Ninety-three thousand cases of these occurred in northern Egypt. Arloing estimates the losses at 5,000,000 cattle, and the valuation at $150,000,000$ franes.

Etiology. Nocard, as well as Tartakowsky, proved that the pure tissue fluids of affected animals contain no micro-organisms visible witl the microseope, and that no organisms could be cultivated with the ordinary methods of procedure. Nicolle \& Adil-Bey demonstrated that brain emulsion, intestinal contents, cerebro-spinal fluid or peritoneal exudate, when diluted with water pass through the Berkefeld or Chamberland filter; that such filtrates are infectious, and that therefore the contagion belongs to the group of ultramicroscopical microorganisms.

Pathogenicity. The contagion is present during the entire disease in all tissue fluids (the nasal secretion of inoculated animals is infective twenty-four hours after inoculation according to Raupacli). Blood taken at the height of the disease and injected subcutaneously into a full grown animal in as small a quantity as $0.001 \mathrm{~g}$. will produce the disease with the same intensity as if for instance 41. of blood had been injected subcutaneously (Nicolle \& Adil-Bey). Besides the tissue fluids the virus is present in the different secretions (saliva, nasal discharge, urine, feces, bile, tears, vaginal discharge, perspiration), while the infectiousness of the exhaled air is doubtful. Infection is readily accomplished by subcutaneous injections or by feeding infectious material, while by intravenous injections infection results only exceptionally (Kolle). Repeated passage of the virus through the bodies of cattle does not modify its virulence (Nicolle \& Adil-Bey); on the other hand when passed through the bodies of sheep and goats it becomes attenuated (Galambos, Koch).

Besides cattle and buffaloes, sheep (Galambos) and goats (Woronzow \& Eckert) as well as camels (Tartakowsky) may be infected by subcutaneous injections, while infection is less certainly transmitted by feeding infectious material.

The question whether hogs are also susceptible to the virus of rinderpest has not yet been positively established. While Carré \& Fraimbault succeeded in infecting these animals with subeutaneous injections of virulent blood, and Penning obtained positive results on wild hogs, and later reinfected calves from them, Theiler failed in all attempts to produce the disease in hogs in a similar manner.

Tenacity. Blood or other infectious fluids, sealed in a glass tube, remain virulent for several months (Semmer); the same applies to spleen tissue when kept in a dark place. Distilled water mixed with blood loses its virulence within 4 to 5 lays (Nencki), and if mixed with glycerin inside of 8 days (Edington); blood mixed with an equal quantity of bile becomes avirulent in two hours (Lingard). According to older observations (Haubner, Dieckerhoff) the virus remains virulent on hay kept in the stable, or in the hay loft for 3 to 4 months (?), but when exposed to the sunlight it is destroyed in two days. When dried on wool out of doors it becomes avirulent in three days (Woronzow \& Eckert). Hides salted for two 
to three days, then kept for 4 or 5 days in a shady place, also bone marrow after the bone has been dried for 30 days, may still contain infectious virus. By heating to $58-60^{\circ} \mathrm{C}$. it is destroyed immediately, whle a temperature of $20-25^{\circ} \mathrm{C}$. destroys its virulence only after it has been subjected for a longer period. Blood becomes arirulent when subjected to a temperature of $37^{\circ} \mathrm{C}$. for 2 to 3 days (Theiler).

According to older data the virus is destroyed very slowly by putrefaction. Thus meat which has been buried for three months is supposed to have been still infective (Ticq D'Azyr). Arloing, however, found that the virus retains its virulence in meat only for 4 days. The infectiveness of manure which has been contaminated with the exerements of affected animals contains the virus for one month, according to Bouley, and according to Krajewski it may sometimes be infectious even after five months. More recent investigations in this direction provel, however, that the virus is destroyed by putrefaction in a few days (Kolle).

Besides glycerin carbolic acid (2\%), sublimate (1-1000), milk of lime (1\%) are effective as disinfectants; on the other hand the virulence of the blood is not modified by a $1 / 2 \%$ earbolic aeid solution (Kolle).

Natural infection results either br direct contact with affected animals or is transmitted through the raw products of such animals, such as parts of carcasses, as well as by persons contaminated by such carcasses or by infected secretions and excretions. Food, drinking water, stable utensils, clothes, etc., may also transmit the infection. In most instances, however, the infection is disseminated by affected animals in the ordinary traffic with cattle, which mode of transmission is the more important as some animals may be only very slightly affected and yet retain the virus in the body for 14 days, in chronic cases probably even somewhat longer (Stockman; the maximum time in which the animal may be infectious for others is supposed to be 30 days).

The infection usually occurs through the digestive tract, probably through the lymph spaces, even of healthy mucous membranes. The possibility of infection through the air passages has not been positively established, as it is questionable whether the contagion is disseminated through the air. Out of doors the spread of the disease may be prevented by digging a trench around the infected herd which the animals are unable to pass, and in such cases cattle on the other side of the trench remain healthy (Raupach). Likewise it is possible to prevent its spread by isolating affected cattle for a distance of 10 meters, when it will be found that healthy animals will not contract the disease (Piot-Bey). In the stable healthy cattle may be protected from the neighboring infected animals by simple board partitions (Nencki).

The susceptibility is very great in cattle, especially in the young animals, although there appears to be considerable difference in the various breeds. According to some authors (Jessen, Haupt) cattle exposed to the infection become affected as a rule without consideration of the breed. Some Russian authors, however, claim that the long horned ranch cattle are less susceptible to the infection than the breeds of Western Europe. Cattle of the gray breeds show a greater resistance to artificial infection, and the course of the disease in them is usually milder.

According to Semmer only 5 to $10 \%$ of ranch eattle succumb to an artificial infection with virulent virus; against 90 to $98 \%$ of other breeds. Nicolle \& Adil-Bey 
also observed that the time of incubation after virulent inoculations is 24 hours shorter in the higher bred animals, and death as a rule follows half a day earlier than in the black Anatolian cattle, and that these latter may be much more readily immunized. In South Africa (Stockman) and in India (Rogers) the eattle of the mountains appear to be much more susceptible than the animals in the lowlands.

Buffaloes are much less susceptible. During the existence of an outbreak they become affected only exceptionally, and even young animals may withstand artificial infection (Nicolle \& Adil-Bey; although the disease exists among buffaloes on the East Indian islands in an epizootic form, Van Ecke, Blin \& Carrougeau).

Of other animals camels are susceptible (according to Tartakowsky the disease is always mild in these animals); also sheep, goats and ruminants living wild, especially deer, zebu, gazelle, etc., and these animals bear an important part in the distribution of the disease. Solipeds and carnivora are not susceptible, and the reliability of the observations relative to the occurrence of the affection in hogs (Pereari, Driessa, Pluning) has not been proven beyond a doubt (see p. 237). Man is not susceptible to rinderpest.

One attack of the disease usually abolishes the susceptibility of the animal; and while repeated attacks were observed in exceptional cases the infection was usually of a mild form. (In the Vaccine Institute of Karlowka it was not possible to reproduce the disease in a steer which had recovered from the affection produced by inoculation six years previously.) Calves from cows which hecame ill during advanced pregnancy are also resistant against the infection (Gerlach, Semmer, Rogers), or they become affected only with mild symptoms (Yersin).

Pathogenesis. The first symptoms are apparently produced by the virus which enters the blood and propagates with great rapidity. The virus appears to have a predilection for exerting inflammatory changes on the mucous membranes, producing catarrhal symptoms in the very beginning of the disease. The croupous-diphtheric inflammation which then develops with great rapidity is evidently the result of secondary infection by other micro-organisms from the digestive tract on the weakened mucous membrane. These bacteria cannot be designated exactly at present (colon and necrophorus bacilli ?) in the absence of accurate bacteriological and histological examinations. The tissue changes consist principally in that the epithelial layer of the hyperemic and swollen mucous membrane changes together with the exudate which comes from the blood ressels into an easily detachable and friable pseudo-membrane. Sometimes the tissue of the mucous membrane proper becomes necrotic to a certain depth, forming thicker membranes that are more adherent. The mucous glands, especially those of the intestinal mucous membrane swell as a result of cellular infiltration, and later become necrotic. The inflammatory process is sometimes 
also observed in the eutis of the skin, which is a direct result of the softening and sloughing of the epithelial layer.

The continuous high fever and great debility, the weakness of the heart, the hemorrhages of the serous and mucous membranes, as well as the pronounced difficulties in respiration, indicate also a severe action of the filterable virus on the central nervous system.

Anatomical Changes. Carcasses dead of rinderpest are greatly emaciated. The skin surrounding the sunken eyes, the nostrils and the lips are profusely covered with tenacious mueus and saliva, the anal region is soiled with dried feces, the mucous membrane of the rectum is greatly swollen and cyanotic. In some of the cases the skin shows the changes which can be noticed already during life (see p. 246), while in the subcutaneous connective tissue the flabby and easily torn veins are dilated and filled with blood. The abdominal and thoracic eavities contain in some cases a yellowish-red or dirty-brown fluid, which is odlorless or may have a sickening, sweetish odor.

The most important changes appear in the mucous membranes, and especially on that of the digestive tract.

In the mouth, especially on the mucous membrane of the lips, lying opposite the gums, under the tongue and on the phrenum, more rarely on the cheeks and on the hard palate, on the soft palate and on the borders of the tongue, there are yellowishgray nodules and granular pulpy deposits. There may be also areas the epithelial layer of which has exfoliated, or even deeper ulcers, while in other spots the mucous membrane usually shows reddish maculae, or appears as if it had been sprinkled with bran as a result of a necrosis of the epithelial layer (sometimes pin-head sized ulcerations are present on the tongue, gums and in the pharynx). The mucous membrane of the pharynx is hyperemic, and contains small hemorrhages, while in the cesophagus it is usually normal; exceptionally a necrosis mav be observed in the superficial layers of the osophagus which are of hemp-seed to lentil sized dimensions.

The omasum contains very dry food; the abomasum, which usually contains only a small quantity of a tenacious mucus, shows the most varied shadings from a brick-red to a darkhrown coloration in its mucous membrane. The reddening is most intensive in the region of the pylorus, and especially on the tops of the folds. At the same time the mucous membrane is swollen, and the submucosa thickened as a result of an edematous infiltration. In a later stage plate-like deposits may be observed, especially on the folds of the mucous membrane, which are hemp-seed to lentil in size, dirty-gray or brownish in color, and flat or rounded in form. These deposits are only loosely attached with their soft borders, while in the center they adhere more strongly to the hase. If they are picked off highly reddened depressions remain in their places. 
The intestinal canal contains dirty-gray or from the admixture of blood, darkish-brown, very liquid feces which have a sweetish stale odor and are often mixed with membranous shreds. The mucous membrane is much reddened, the subjacent connective tissue gelatinously swollen. The nucous membrane of the duodenum and of the jejumum shows plate-like deposits similar to those in the abomasum, and occasionally they are also found in the ileum. The solitary follicles are swollen to the size of a hemp seed or even larger, and form small nodules from which a purulent or yellow caseous mass may be squeezed. (If the necrotic epithelial cells are not thrown off, they together with the swollen glands cause the mucous membrane to resemble the skin of a boiled eel.) The Peyer's patches are at first uniformly swollen, and rise with a flat prominence over the surrounding tissue; later small dirty-gray deposits appear around them, which subsequently confluate to a dry, caseous and strongly adherent or a mush-like, soft deposit, several millimeters in extent. In rare cases the epithelial layer forms with the coagulated exudate a membranous cylinder several millimeters thick on the inner surface of the intestinal tube; in other cases the necrotic tissue of the mucous membrane changes into a friable, dry, caseous mass, when after its removal the smooth muscular layer is exposed.

The large intestines show similar changes, but in these the lesions are less pronounced than in the small intestines. Frequently they show only indications of a simple acute catarrh, or the mucous membrane appears as if sprinkled with bran because of the superficial necrosis of the epithelial layer. Sometimes the mucous membrane of the large and small intestines presents broad, bright-red transverse stripes or extravasations of blood so that it greatly resembles the skin of a zebra (Verney).

In the bright-red air passages, punctiform or linear hemorrhages and catarrhal swelling of the mucous membrane, as well as edematous infiltration of the submucous connective tissue, especially in the nose and in the larynx, are almost constant lesions. There are frequently croupous, pseudo-membranous deposits in the nose, and especially on the nostrils, also in the larynx and in the trachea, under which ulcerative erosions are found. The mucous membrane of the bronchi usually manifests only a ring-form deposit, and is covered with a purulent secretion, which may in places entirely fill the small bronchi and pop out on the eut surface on slight pressure in the form of white plugs.

Similar changes may frequently be observed on the mucous membrane of the female genital organs, and especially in the anterior part of the vagina, while further back the lesions are usually less marked.

The liver shows parenchymatous or fatty degeneration. The gall bladder is distended and contains thin, green or yellow, 
or even dirty-gray bile. The mucous membrane is in some instances at first covered with vesicles and ulcers, later with greenish-yellow patches of necrotic tissue. The spleen is usually unchanged, sometimes, however, it may here and there be acutely swollen. The kidneys manifest cloudy swelling or fatty degeneration, the mucons membrane of the hylus of the kidneys and of the urinary bladder shows catarrhal swelling and small hemorrhages.

The lungs show an acute hyperemia, and sometimes contain scattered areas of catarrhal pnemmonia. Frequently an interstitial emphysema is present, and in some cases air bubbles are found under the pleura, in the mediastinum, in the surrounding tissue of the trachea, as well as in the subcutaneons and subperitoneal connective tissue. The heart is flabby, the muscles are friable, of a dull, grayish-brown color, sometimes showing butter-yellow streaks. Under the pericardium as well as beneath the endocardium, hemorrhages are frequent. The blood is dark red and only partially coagulated.

The lymph glands, especially in the mesentery, show an acute swelling, their tissue is reddened and moist, but contains no hemorrhages.

In the brain and in the cerebral meninges only a hyperemia and moderate edema of the white brain substance may be found.

The described anatomical changes are present in animals which die at the height of the disease, or which were killed not earlier than on the third or fourth day of the affection. Pronounced lesions on the mucous membrane of the digestive tract, and especially in the abomasum, are present in almost every instance, while the mucous membrane of the air passages and genital organs may only occasionally manifest an acute catarrh. However the affection of the intestinal tract is not always uniform, for instance, the very decided affection of Peyer's patches which was observed in every case by Gerlach is according to Bristowe, Murchison and Dieckerhoff in some cases entirely absent. Again Koch observed in South Africa, and Nicolle in Turkey, that the diphtheritic changes in the buceal eavity and in the intestines were only slightly pronounced in those countries in the rinderpest of cattle. In the gray range cattle the anatomical changes are usually less prominent than in cattle of Western European breeds.

Symptoms. The time of incubation in cattle extends from 3 to 9 days.

Raupach inoculated 865 eattle, of which $70(8.1 \%)$ developed the disease on the fourth, $227(26.25 \%)$ on the fifth, $303(35 \%)$ on the sixth, $208(24.15 \%)$ on the serenth, and $57(6.6 \%)$ on the eighth day. Sanderson and Semmer have observed in exceptional cases a febrile rise in temperature 36 to 48 hours after inoculation. Of 390 animals inoculated by Tode 55 became affected on the fourth to tenth, 21 on the elerenth to twentieth, 20 on the twenty-first to twenty-sixth day. Of the more recent authors Koch observed the development of ferer as the first indication of the disease in from 3 to 9 , Theiler from 3 to 6 , Nicolle \& Adil-Bey from 4 to 6 days.

The establishment of the accurate time of ineubation was of special importance during the time when cattle imported from Roumania and Russia to AustriaHungary and Prussia, were subjected to a quarantine on the borilers. For this reason the international reterinary congresses repeatedly discussed this question (in 
1563 in Hamburg, in the years 1865 and 1867 in Vienna). Nine days was established as the maximal duration, and after the international hygienic conference in 1872 had also substantiated this finding, the duration of the quarantine was reduced from 21 to 10 days.

The first symptom of the disease which usually precedes the other manifestations by one or two days is the rise in the temperature which in the Western European cattle reaches on the first or not later than on the second day $40-42.2^{\circ} \mathrm{C}$. (Gerlach). In range cattle however it usually does not reach that height (Medvedski. According to Dieckerhoff the rise in the temperature commences in exceptional cases only 10 to 20 hours after the appearance of the other symptoms).

At the same time the animals manifest a marked depression and dullness. They stand in the stable, or apart from the other animals in the barnyard or pasture, with head drooped, sometimes supporting it on the crib, the ears drooped, and the back arched. Exceptionally the affected animals first show great excitement, constantly moving about, attempting to break loose when tied, shaking their heads, tearing up the ground with their horns and feet, and sometines even assuming a fighting attitude. This excitement however lasts only a short time, not longer than a few hours, and is soon relieved by the above-mentioned depression.

The appetite is depressed, the thirst on the other hand being sometimes increased (according to Dieckerhoff many animals manifest frequent yawning, and the swallowing of pieces of mortar) ; rumination is delayed or sometimes ceases, the feces which are passed at long intervals are dry, darkcolored, and do not form curled cakes. The urine is diminished in quantity and of a darker color. The milk secretion is considerably diminished from the beginning of the disease.

In the meantime the animals tremble, shaking severely over the entire body at short intervals. They grind their teeth, and certain groups of muscles, especially on the neck and near the flank, show fibrillar twitching. The temperature is unevenly distributed over the body surface, the horns and the ears feel warm to the touch, the muzzle is dry, the skin at the flexor surfaces of the extremities and in the surrounding parts, is covered with perspiration, the hair especially over the vertebral column is lusterless and erect.

Respiration and pulse become somewhat accelerated, and the frequency of the pulse is affected by even slight external influences. Many of the affected animals cough frequently, even in the first stages of the disease, without. it being possible to detect any changes in the thoracic organs.

From the second day of the manifest affection inflammatory changes of the mucous membrane appear in the foreground, which render the symptoms of the disease characteristic.

The conjunctivae are bright red, the lids are swollen, cushion-like, while tears run profusely from the narrow opening of the eye. Later the secretion becomes mucous and soon 
purulent. On forcibly separating the lids a great quantity of discharge rums out, and dries below the inner canthus of the eye into dry crusts. On the surface of the inflamed conjunctivae fine pseudo-membranes form, and sometimes the cornea becomes cloudy (according to Krajewski especially in dry warm weather).

From the nasal opening there is first a glassy transparent, tenacious, later a more purulent and at last a dirty gray or brown, fetid, ichorous or bloody discharge running down the

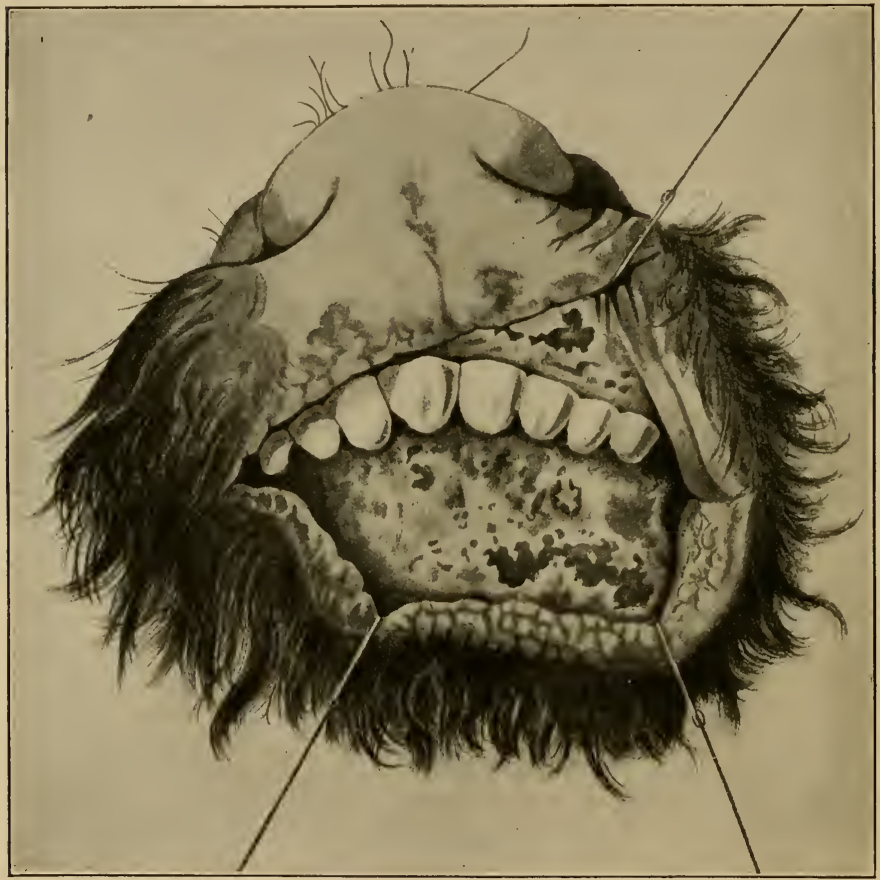

Fig. 43. Stomatitis in rinderpest. (After the reports of the English Commission of the year 1866.)

muzzle. The nasal mucous membrane as far as it may be examined, is at first spotted, later uniformly reddened, and sprinkled with dark red, punctiform hemorrhages. The itching produced by the inflammation of the mucous membrane causes the animals to blow frequently, and to continuonsly move the head up and down, or from right to left, and sometimes to even shake it violently. As a result of the developing difficulty in respiration the animals sometimes shake their whole bodies, 
thus causing a rattling of the chain with which they are fastened by the head or neck, by the sound of which affected animals may frequently be recognized in an infected stable.

In the buccal cavity the mucous membrane is at first reddened in spots, later assuning a mniform, livid or bright red color, and not infrequently becomes covered with dark red ecchymoses; these changes appear first on the gums, and on the imner surface of the lips, later in the corners of the mouth, on the borders of the tongue, in the vicinity of the phrenum of the tongue, on the upper lips, and on the cheeks. Soon hemp-seed to lentil-sized gray spots appear on the surface, which confluate forming larger, comnecting, gray or gravishyellow, patch-like deposits; after their loosening, wiping off or throwing off, the bright red, bleeding, deeper laver of the mucous membrane becomes visible (so called erosion ulcerations; see Fig. 43).

As a result of the affection of the intestinal tract, there is in the first two days obstipation, later profuse diarrhea. The feces which at first were firm and sometimes mixed with blood, become softer and later very thin; they are passed under great strain, in which the dark red, or livid, greatly swollen rectal mucous membrane protrudes. The parts surrounding the anus are soiled by the watery, later dirty gray and then dark brown, fetid, sometimes bloody feces, which contain mucous shreds, gray detritus, or even large pieces of pseudomenibranes. Towards the end of the disease, about the fifth day, involuntary passages of the bowels take place at short intervals and in small quantities, from the flabby and open rectum of the animals which lie on the ground at this stage.

Even in the earliest stages a mucous or muco-purulent discharge, which is sometimes mixed with blood flows from the vagina of cows and heifers, the vulva showing a swelling and higher temperature. The mucous membrane of the vagina and the vulva is reddened, and on the surface small gray, or yellow, pseudo-menbranes form, which later are thrown off leaving dark red, bleeding, superficial erosions in their places.

The animals urinate frequently. The urine flows almost continually in drops at short intervals, and is sometimes yellowish-red to coffee brown in color (Eggebrecht).

As an indication of an affection of the respiratory organs a frequent painful cough is observed as the first symptom, which however disappears after the disease has completely developed (Dieckerhoff). The respiration is accelerated, 40-60 per minute, later it is labored to a ligh degree, and the grunting of the animal is andible for a great distance. Percussion of the thorax reveals nothing abnormal, or only a londer percussion sound. Auscultation reveals intensified vesicular breathing, later dry and moist rales. In some cases a subcutaneous emphysema develops in association with the interstitial lung emphysema, which results from the very difficult respiration. 
In such cases the emphysema is first indicated on both sides of the vertebrae, then on the neck, shoulders and thorax.

The heart's action is at first sufficiently strong, but gradually weakens. The constantly increased frequency in pulse is at first 50-60, later $80-100$ and even more per minute. The pulse is weak, small, and toward the end of the disease wiry.

The leucocytes increase in the blood with the onset of the fever up to the fifth day of the affection. From this time on their number diminishes to 4,000 per cubic millimeter in the unfavorable cases. If the animal remains alive their number increases towards the sixteenth or seventeenth day of the disease to $28,000-30,000$, which is again followed by a rapid diminution, whereupon by the twentieth day the normal condition is re-established.

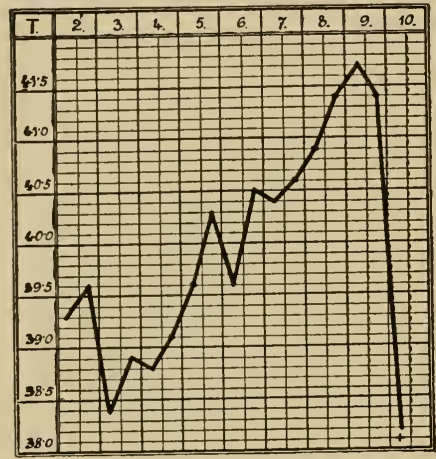

Fig. 44. Fever curve in rinderpest, following a subcutaneous injection of a drop of virulent blood; three year old cow. (After Nicolle and Adil-Bey.)

The continuous fever usually reaches its height on the fifth or sixth day, the daily differences varying by one degree, and rapidly drops with the onset of the diarrhea in the severe cases to below normal; whereas if there is an indication of improvement the fever subsides gradually (Fig. 44). The height of the fever does not always correspond with the severity of the other symptoms.

In some cases, particularly in range cattle, the skin becomes affected in a peculiar manner. Two or three days after the appearance of the disease, sometimes even earlier, small protuberances and vesicles develop on the neck, before and behind the shoulders, along the vertebral column, on the shanks, on the udder or on the scrotum, and in the flank. Over these areas the hairs stick together, later thick scabs cover the wrinkled skin. In case the animal improves the scabs are thrown off, the epithelium desquamates, at the same time the hair falls out, and sometimes the animals may even lose the hair from the tips of their tails.

Russian authors and also Zlamál, laid great stress on the affection of the skin which occurs rquite frequently in range eattle, and accordingly they distinguished an exanthematous form of rinderpest, from one in which no exanthema developed. In confirmation of older conceptions, they consider the appearance of the exanthema a favorable prognostic indication. The skin affection however which develops in some of the outbreaks quite frequently, in others but rarely, may exceptionally occur also in Western European cattle and in spite of this favorable symptom the disease may terminate unfavorably. (The exanthema was frequently observed in Hungary in the outbreak of 1852 , 
whereas in the years 1861-1863 it was not observed in a single instance [Zlamál].)

With the diminishing milk secretion the udder becomes flabby, wrinkled and cold; the milk which is secreted in small quantity is watery and of a pronounced yellow color. Pregnant animals usually abort at the height of the disease.

During the development of the described symptoms the animals have no appetite and do not ruminate, become rapidly emaciated and steadily lose in strength. The skin loses its elasticity, becomes stiff and dry, the hair dull and lusterless. The advanced stage of the disease appears characteristic in the presence of a dull look from the deeply sunken eyes, purulent secretions from the eyes, dirty nasal discharge, foamy saliva in the corners of the month, grinding of the teeth, accelerated and grunting respiration acconpanied by shaking of the body, muscular trembling, and the peculiar repulsive odor of the excrements. Soon the animals are not capable of standing up, they lie constantly on the ground with head extended or turned on the shoulder, and finally they succumb, completely exhausted, in convulsions or without struggle.

Course. The described symptoms do not all develop in every case of rinderpest. Furthermore according to Lorinser the entire symptom complex is never present in one animal or even in the herd, but from case to case the changes in the respiratory organs, or those of the digestive tract predominate, and in other instances symptoms of the genital organs; in most instances all the organs mentioned may be affected, at the same time but in varying intensity.

In the great majority of the severe cases the disease lasts on an average of from 4-7 days. The affection of the mucous membrane can usually be detected on the second day after the appearance of the febrile symptoms. The clinical manifestations develop in their full intensity on the third or fourth day; in exceptional cases the condition becomes very rapidly aggravated, when death may result on the second or third day, while in other cases the course may extend from 14-16 days. The convalescence lasts from 2-3 weeks, and the symptoms, especially those of the intestinal inflammation diminish very gradually.

In the milder cases, such as are usually observed among range cattle, the symptoms do not develop to such a degree, and usually commence to recede about the fourth or fifth day. According to the observations of Russian authors and Eggebrecht, the disease among these breeds of cattle may be manifested only by a febrile condition lasting for a few days, and by a mild catarrh of the mucous membranes of the respiratory organs, as well as of the digestive tract. As a result of these the disease may sometimes even pass unnoticed in the larger herds. This mild form of rinderpest is observed in very 
exceptional cases also among cattle of the Western European breeds (Dieckerhoff; according to Stockman the disease sometimes may run a very chronic course in the presence of mild symptoms).

In Turkey, Russia and Africa animals frequently become affected which have previously been infected with the parasites of infectious hemoglobinuria, or coast fever. In such animals rinderpest runs the usual course, but at the same time the piroplasma which were present prior to the infection with rinderpest only in unimportant numbers in the blood increase very rapidly (Nicolle \& Adil-Bey, Kowalewsky, Theiler, Koch). Latent cases of trypanosomiasis are likewise influenced hy rinderpest (Holmes, Woolley).

Rinderpest as a plague does not spread very: rapidly in large herds. After placing an affected animal in a stall, usually after 1-2 weeks the animals in close proximity first become affected, whereupon however the disease attacks the other animals with a greater rapidity in a similar way. According to Haupt it requires from 5-6 weeks for the disease to affect all the animals in a herd of 15-20 head.

Diagnosis. The sudden appearance and the rapid development of the symptoms, among which the characteristic affection of several mucous membranes is of great importance, aid in the recognition of the disease. In establishing a diagnosis careful data on the history, first on the possibility of an introduction of the infection as well as the mode of spreading in the affected herd, must be carefully considered, because of the far-reaching consequences which may follow the presence of the disease. The diagnosis is formed with difficulty, in the beginning of an outbreak, especially if the disease affects the first animals in an unusually mild form, but a sudden rise in the temperature is sufficient to justify a suspicion of the presence of rinderpest.

From the standpoint of differential diagnosis the following diseases are principally to be considered:

1. Coccidiosis (red scour). The resemblance consists in the fact that in this disease there is also a febrile diarrhea and rapid loss of strength; further autopsy may reveal necrosis of the epithelium and even of the deeper layers of the gastric and intestinal mucous membrane. It is distinguished from rinderpest in that the digestive disturbances precede the febrile rise in temperature, further the excrements retain their original color for a longer period, but may become mixed with blood even at the beginning of the disease. The affection remains exclusively confined to the gastro-intestinal canal, and in older animals the affection of the rectum is especially marked; whereas the mucous membranes of the head, and of the eyes, remain normal. Further the disease is not contagious, and cannot be transmitted, even by inoculation. Similar differences 
exist between the acute gastro-intestinal catarrh, the mycotic and toxic enteritis, on one side, and rinderpest on the other.

Tschudinow observed an affection in eattle after feeding swill heated to a temperature of $53-54^{\circ}$, which consisted in fever of $40-42^{\circ}$, difficult respiration, reil spots on the buceal mucous membrane, eatarrh of the conjunctivae, seanty hair, mucous discharge from the nose, and a bloody mucous diarrhea. The autopsy revealed in one case hyperemia, and hemorrhages in the mucous membrane of the rumen and of the intestinal canal.

2. Malignant catarrhal fever of eattle, with which rinderpest has been frequently confouncled. This disease resembles rinderpest inasmuch as it also runs a severe course, and as all mucous membranes may become affected. However the most severe changes are found in the mucous membranes of the eyes, nose and sinuses of the head, while the digestive tract and the genital organs are usually only slightly affected. The head feels warm to the touch, the horns sometimes fall off, the depression of the animals is pronounced from the onset of the disease. Besides cloudiness of the cornea, a fibrinous iritis may also develop, and it should be taken into consideration that the disease is not contagious and usually occurs only sporadically.

3. Foot-and-mouth disease can only be mistaken for rinderpest when the ulcerations in the mouth extend to the deeper parts, and when the ulcerations later become covered with tissue detritus and coagulated exudate; further when high fever and indications of enteritis can be associated with the disease. In foot-and-mouth disease however the ulcerations develop at the site of the vesicles; the nose and the eves are not affected, while frequently inflammatory changes are present in the interdigital spaces; furthermore foot-and-mouth disease spreads more rapidly through the affected herds than rinderpest.

4. Piroplasmosis may cause suspicion of rinderpest, especially on autopsy, in cases where the mucous membrane of the abomasum and of the small intestines contain ulcerations, and even patch-like deposits. Normal appearance of the mucons membrane of the mouth, air passages and genitals, the presence of hemoglobin in the urine, acute swelling of the spleen, yellowish discoloration of the subperitoneal fat, as well as an enzootic character of the outbreak, afford sufficient grounds for excluding rinderpest. However the establishment of the piroplasmosis, or the presence of piroplasma in the blood does not exclude the simultaneous presence of rinderpest, as mixed infections of this kind occur quite frequently (see pp. 248 and 255 ; according to Arloing \& Ball the swollen lymph glands are free from hemorrhages only when piroplasmosis exists in association with rinderpest). $\dot{A}$ demonstration of trypanosomes in the blood also has no importance from the standpoint of a differential diagnosis.

Prognosis. The termination of the disease depends in the first place on the character of the outbreak, and on the breed 
of the affected animals. The mortality fluctuates within wide limits, and is estimated among cattle of the colored breeds at $75 \%$, in range cattle at less than $50 \%$.

In England during the outbreak in 1966 the affected animals died almost without exception, whereas at the same time in Holland only two-thirds of the diseased animals succumbed, and in France in $15: 1$ the mortality amounted in places to only 20\%. In Hungary the losses in 1852 did not exceed $10 \%$, on the other hand in the years 1861-1863 they reached $75 \%$. According to Krajewski the disease sometimes runs a mild course, especially after a rapid spread through large herds (losses of 15 to $20 \%$ or even less), but occasionally, even among range cattle, $85 \%$ may die. In India the mortality among cattle of the lowlands is about $20 \%$, while in mountain cattle it reaches $90 \%$.

Experience has shown that if the disease returns after a long period of absence, a greater number of animals fall victims, and on the other hand the mortality immediately after the outbreak is greater than in its later course. Under favorable hygienic conditions, pure air and dry weather, the losses are lighter than under adverse conditions.

Treatment. Medicinal treatment of the affected animals gives no result, as was proven by the completely negative results obtained in experiments in Fngland and Holland on the occasion of the last outbreak. A favorable influence can be hoped for only from serum treatment, in which the patient should be given $40-80$ ce. of immune serum subcutaneously or intravenously at the beginning of the febrile stage, or not later than the appearance of the first manifestations (see p. 252; Fedetzky found the curative inoculation valueless).

The opportunities for therapeutic treatment are remote, as all of the European countries which are free from the disease, with the exception of Turkey, require the killing of all affected and suspected animals.

Immunization. The possibility of an effective immunization against rinderpest is indicated by the numerous instances in which recovery from the disease has afforded the animals an immunity lasting for a long time, usually as long as they live. The intensity of the immunity thus obtained develops in a degree relative to the severity of the affection. However, as affected animals infect others during the course of the disease, and as they may disseminate the infection, immunization except with pure serum inoculations, is only indicated in barly affected localities, in order that the otherwise very great losses may be reduced.

As this purpose was not attained with older methods of immunization, owing to the considerable losses from the immunization, it has been abandoned not only in the Central and Western European countries but also recently in Russia. The occurrence of the disease with great severity in the last decarle, especially in Soutl and North Africa, has again placed the question in the foreground and the experiments conducted 
there have resulted in more effective methods by which more satisfactory results are being obtained in affected localities.

I. Immunization with Secretions of Affected Animals. Stimulated by the favorable results of smallpox vaccination, immunization against rinderpest was undertaken in the middie of the eighteenth century (1744) in England (Dodson), in France and in Germany, as well as in Holland, and later was employed to a great extent in Russia.

The procedure consisted in the introduction of the nasal discharges or lachrymal secretions of affected cattle into healthy animals, under the skin of the tail or other parts of the body. After the inoculation the disease developed in a very mild form, especially in the gray range cattle, and the mortality reached only a small percentage. Jessen experimented later with a supposedly attenuated virus, which he carried through several generations of eattle. After such inoculations in the Vaccine Institute of Karlowka, in the years 1857-1873, the entire loss among 2629 eattle amounted to only $5.97 \%$.

This method of immunization, however, did not appeal to Western Europe, inasmuch as the breeds of cattle in those countries became severely affected from the inoculation and the losses were considerable, and further than this, the inoculated animals endangered the lives of those which had not been treated. After the international conference in Vienna in 1872 had resolved that the immunization against rinderpest should not be considered as a valuable method, it was abandoned even in Russia, and in 1874 the last serum institute was given up.

II. Immunization with the Bile of Affected Animais. The blood and the bile of animals dead from rinderpest has been used for a long time for immunization purposes by the Boers in the Transvaal and in the Orange Free States. Koch later demonstrated (1897) that the bile of such animals in quantities of 100 cc. injected into healthy animals afforded them a very valuable immunity against the infection. The immunity can be established by the tenth day following the immunization, and is of such intensity that the immunized animals withstand an injection of $40 \mathrm{cc}$. of virulent blood without ill effects even four weeks after the inoculation, and they are also able to resist natural infection. The immunity probably results from the virus contained in the bile which gives the animals a mild local affection, due to the retention of the virus at the point of inoculation through the action of certain ingredients of the bile (Kolle).

The immunization employed in practice showed that the immunity following the injection of bile sometimes appears on the sixth day, and lasts from four to six months (Kolle). In other cases, however, it lasts only from 3-4 weeks (Kohlstock), and the inoculation oceasionally causes not only a local swelling at the place of inoculation, but it may also cause a severe general affection, and exceptionally the death of the animal, especially if the bile contained some blood (Theiler). Further, as the bile inoculation in already infected animals during the time of incubation is entirely without effect, the application of this method could only come into consideration if rinderpest suddenly broke out in certain localities and a more effective method of immunization could not be employed.

Potent bile can be obtained only after the seventh day of the dis- 
ease; it should be of a green color, odorless, and on shaking should form a white foam. An animal dead from the disease produces a quantity sufficient for not more than 20-25 cattle.

In South Africa in the years from 1896 to 1898 over 2,000,000 eattle were immunized with bile with apparently satisfactory results, and according to Ailani it has proved beneficial in Erythräa. Rogers also found this method effective in India, howerer only in cattle of the iowlands, since mountain cattle, which are much less frequently exposed to the infection, nearly all succumbed to the disease, when inoculated with virulent blood 10 days to $4 \frac{1}{2}$ months after they had been immunized with bile. (ln some localities of South Africa the immunizations were repeated every three months, and in spite of this deaths from the disease were occasionally observed).

Edington mixes bile (2 parts) with glycerine (1 part) and injects this mixture subcutaneously, 20 to 25 ce. to adlult cattle and 15 ee. to calves. According to Turner immunity cannot be established by this method, as the glycerin kills the virus contained in the bile; Rogers likewise asserts the ineffectiveness of the addition of glycerin. Filtering the bile before the injection through a porcelain filter (Turner \& Kolle) also reduces its immunizing action, and at best only has the advantage that by this procedure bile which is no longer fresh may be utilized for immunization (Rogers). Lingard on the other hand found that bile from healthy animals if subcutaneously inoculated four times at several days intervals in 50 ce. doses, also gave an effective immunity against subcutaneous virulent infections.

Lingard uses instead of fluid bile a precipitate which he obtains by the addition of Aimen's reagent ( $5 \mathrm{~g}$. tannin, 10.0 ec. acetic acid, and 240 ce. of $45 \%$ alcohol). The precipitate which is washed in a $13 / 4$ soda solution, neutralizes the virus in vitro, and inoculations by this method are supposed to have given good results in India even in the mountain cattle.

Active immunization with bile and with virulent blood. In order to produce a more lasting immunity, Kohlstock, and later also Henning \& Edington injected subcutaneously virulent blood ( 0.2 ce.) about two weeks following the bile inoculation. The animals thus injected became sick, however with only a few exceptions they recovered and later proved that they possessed a lasting immunity. In German South Africa this method has found extensive use, and with its aid the disease was successfully eradicated in a few months at the time of the severe outbreak of 1897 (Rassau). It has likewise proven satisfactory in Tientsin (Asia) where of the immunized animals $66 \%$ showed no disease whatsoever, while $24 \%$ became only slightly affected, and $10 \%$ died as a result of the inoculation (Haedicke); Edington on the other hand observed at Kimberley great losses from such combined inoculations.

III. Immunization With Blood Serum. Semmer found in 1893 that blood serum from cattle which recovered from rinderpest possessed immunizing properties, and these finding's have since been confirmed. However such serum exerts its protective action only when relatively large quantities are used (100-200 cc.), still its action may be increased by injecting the animals with large quantities of virulent hlood.

The passive immunity produced by the sermm inoculation only lasts from 10-21 days, and the duration camnot be increased to over four months even by the use of greater quantities of serum (100-200 cc.). Accordingly it may he suitably applied whenever, after the slaughtering of the affected animals, the remaining cattle, if treated with serum, will not be likely to become exposed later to the infection; further it may be issed where infection is threatened by outbreaks in neighboring 
localities when the serum will be found to afford protection for at least a short time. One advantage of this method lies in the fact that it is also effective during the period of incubation, and that the inoculated animals do not become affected and therefore do not spread the disease.

Tokishige (1897) in Japan, and Pitchford \& Theiler, as well as Bordet \& Danysz, during the invasion of the disease in South Africa, aimed to increase the potency of the blood serum of recovered (socalled salted) eattle, by injections of virulent blood. Kolle \& Turner injected at first 100 cc. subcutaneously in recovered animals after the disappearance of the fever, then at intervals of several days increasing quantities of virulent blood (the last time 5 liters in one injection). After 3-4 pronounced reactions the animals produced a serum of which 20 ce. protected an animal weighing $300 \mathrm{~kg}$. against a pathogenic action of 1 cc. of virulent blood (for use in practice the blood serum must have at least such a potency). Animals which have not recovered from rinderpest must be treated first with bile, or with blood serum and blood, and only later with virulent blood.

Nicolle \& Adil-Bey observed in Constantinople that animals which recovered from rinderpest could not again be made sick by a subcutaneous or intraperitoneal injection of a large quantity of virulent blood (up to 10 liters), even when inoculated immediately after the recovery. Accordingly recovered animals were injected subcutaneously at first with 4 liters of blood, and later the quantity was increased. Another method of these authors consists in the injection into the abdominal eavity of an affected animal which already has diarrhea, of 6 liters of a physiological salt solution containing peptone. Three to six hours after the injection the animal is bled to death, and a healthy animal is then injected subcutaneously with 2-3 liters of the fluid collected from the abdominal cavity. The blood taken after 10, 15 or 20 days from this animal produces a potent serum; later the animal again receives the abdominal fluid subcutaneously, and the method is continued in this manner. Two weeks following the injection of 4 liters of blood the animals produce a similarly potent serum, no maiter whether this quantity has been injected immediately after the first opportunity or only in gradually increasing quantities. Sheep are also adapted to the production of immune serum, the horse and the goat however are not. By adding to the immune serum $1 / 4$ to $1 / 2 \%$ of carholic acid and keeping it in a dark place it remains potent for four years (Theiler).

The dose of the serum is 50-100 cc., according to the size of the animal. In India the very susceptible mountain cattle are injected with 18 times larger doses than are given to the cattle of the lowlands, and the quantity also depends on the severity of the disease before the inoculations have been undertaken (Walker).

According to the statisties of Turner, in 189733 herds on several farms, with a total of 3318 eattle, of which 455 animals had already been affected, were subjected to the sermm treatment. Of these inoculated animals 455 died, while $2857(86.1 \%)$ remained healthy. In the English Egyptian Sondan Head lost 2\% ont of 7386 inoculated animals. The serum inoculation has been applied in China to about 11,000 cattle, with very satisfactory results (Keylock).

As it is sometimes difficnlt to obtain virulent blood, Rüdiger injects cattle intraperitoneally with 5 liters of a $1 / 2 \%$ potassium citrate solution. The animals are slaughtered an hour later. The fluid thus injected proves very virulent and when mixed with equal quantities of virmlent blood prodices a very effective and a correspondingly cheaper material for the preliminary treatment of serum cattle. 
Dschunkowsky \& Kupziz produced a dry preparation from the liquid serum by evaporation, at $30.32^{\circ}$, and by adding at the same time $1-5 \%$ of sodium hydrate; this has, however, not yet leen tested in practice.

IV. Immunization With Immune Serum and Virulent Blood. (Simultaneous Method.) Kolle \& Turner produced an immunity in cattle which lasted for at least five months, by injecting the animals subcutaneously on one side of the rump, with one cc. of virulent blood, and on the other side, according to the size and age of the animal, 8 to 25 cc. of immune serum. This immune serum is the same as that which is used for ordinary serum inoculations, while the blood originates from animals either affected with the natural disease or from such as were artificially infected, and should be taken on the fifth or sixtl day of the disease (sheep infected with blood are also suitable for this purpose). Some of the animals inoculated by this method react with a fever lasting for several days, while about $10 \%$ of the vaccinated cattle, especially common range cattle, do not manifest any reaction whatsoever. The blood of the reacting animals is infectious during the febrile stage. In exceptional cases severe symptoms of rinderpest develop in the inoculated animals, and some (about 1\%) may even die with the disease.

The simultaneous method has the advantage over the other methods that it causes a relatively small loss, and with very rare exceptions it produces a lasting immunity. However while only a small proportion of the inoculated animals become affected with severe symptoms, they may transmit the disease to healthy animals, and therefore its application is only indicated in already infected territories, and in these localities only under assurances that the blood does not contain other poisonous substances in addition to the virus of rinderpest, and it should especially be free of protozoa (see p. 255).

This method has been employed for several years in Africa, in Asiatic Russia (Nencki, Siebert \& Wyznikiewicz), in Turkey (Nicolle \& Adil-Bey), and in India (Rogers), and the first reports from all these places indicated favorable results. In India, however, in the less resistant mountain eattle the immunity obtained was not sufficient, although the dose of the serum was increased 6 to 9 times. Rogers therefore injects another $10 \mathrm{cc}$. of virulent blood subcutaneously 10 days after the simultaneous inoculation. The simultaneous method may be also adopted for the immunization of buffaloes as well as goats ( 3 to 5 ec. of blood serum, and 1 ec. of blood).

According to Turner, 10,407 cattle were inoculated by this method in South Africa (Rhodesia, Cape Colonies and Pretoria), and the loss from inoculation amounted to 136 animals $(1.31 \%)$; Head inoculated 7,386 animals in the Egyptian Soudan, with a loss of only $2 \%$, Fedetzky and Gorain observed a loss of $1-2 \%$, Stockman on the other hand observed in one herd deaths amounting to $12 \%$. Very good results are reported by Eggebrecht in Eastern Asia.

For a time another method of immunization was used in South Africa, which has since been introduced into practice by Borlet \& Danysz, as well as by Pitchford \& Theiler, and which consisted in injecting subeutaneously into the animals 100-200 ce. of defibrinated blood from animals which had recovered from rinderpest. 
This injection was followed by rubbing infectious saliva or blood into the nose, and after five days $100 \mathrm{cc}$. of blood was again injected subcutaneously. Conti immunized animals with 20 ce. of serum from animals which had recovered from the disease, to which 3 ec. of blood from an animal which had been affected for 47 hours with rinderpest was added. The author reports favorable results from this method of immunization.

Complications. Several years ago Koch (1904) and later Theiler and Woolley, pointed out the danger's in the application of the simultaneous inoculation. In localities where piroplasmosis or trypanosomiasis is prevalent there is the danger that parasites of the mentioned diseases may be transmitted with the blood from cattle affected with rinderpest, causing diseases from which the animals die; thus in Egypt in 1903 of animals which had been inocnlated with serum obtained in Constantinople (25 cc.), and virulent blood (1 cc.), 80-90\% died within a short time, and the losses appeared at least partially to be caused by a piroplasmosis which resembled the East African coast fever. The existence of this disease in Egypt has been established by Koch (he therefore recommends large serum doses of 100 ce. to be used repeatedly instead of the simultaneous inoculation). Theiler also observed a mortality of $6.8 \%$ among 452 eattle in South Africa which were inoculated by the simultaneous method. In cattle coming from localities free of Texas fever (124 head), for which a possible piroplasma infection is much more dangerous, the loss amounted to $27.4 \%$. As it is impossible in practice to exclude the blood from animals which have recovered from Texas fever, but still contain in their blood piroplasms, it was deemed advisable in the Transvaal to alostain from the simultaneous inoculation, and serum has been used exclusively, by which means an eradication of rinderpest was accomplished in 1903. Woolley reports a case in which all of the 56 cows imported from the United States to the Philippines died after the simultaneous inoculation, probably from piroplasmosis, while according to Nesom's observations, in the Philippines, the otherwise effective simultaneous inoculation causes a loss of $4 \%$ as a result of trypanosomiasis, which develops after the injection. Spirillae, or the virus of heart water, may also be the cause of possible bad results from this method.

Veterinary Police. The safest prophylactic measure consists in a quarantine against countries in which rinderpest prevails. Since the practical application of this principle Western and Central Europe have been freed of the disease, although the plague has appeared repeatedly in an epizootic form in Russia and on the Balkan Peninsula. The introduction of the disease may also be prevented to some extent by establishing a carefully executed registration of cattle at the endangered borders of the countries, which would considerably facilitate the establishment of the origin of the disease, if it has been introduced.

If the disease should make its appearance in any locality the immediate slaughter of all affected, as well as all exposed animals, together with the thorough disinfection constitutes the surest method for its eradication. If however such a procedure cannot be executed it is advisable to segregate the infected herds into small groups, keeping them completely isolated under observation. (According to Piot-Bey better results were ob- 
tained in Egypt with this method than from serum inoculations.) At the same time the other ruminants, especially sheep and goats, should also be considered as carriers of the virus. In the meantime the losses in infected herds may be diminished by the application of one of the above-described modes of immimization and in neighboring herds the animals should all be given serum inoculations thereby producing an immune zone around the infected herds.

Literature. Dieckerhoff, History of Rinderpest and Its Literature, Berlin, 1890.- Semmer, B. t. W., 1893, 590.-Koch, Zbl. f. Bakt., 1897, XXI, 526; Verötf. d. G.-A., 1904, V, 681.-Kohlstock, Ibid., 1897, XXII, 787.-Kolle, Z. f. Hyg., 1898, XXYII, 45; Ergebn. d. allg. Path., 1899, VI, 470 (Lit.).-Kolle \& Turner, Z. f. Hyg., 1S98, XXIX, 309.-Theiler, D. t. W., 1898, 205 ; Monh., 1901, XIII, 145.Rogers, Z. f. Hyg., 1900, XXXV, 59.-Nicolle \& Adil-Bey, A. P., 1899, XIII, 329; 1902, XVI, 56.-Blin \& Carrougeau, Bull., 1902, 107.-Yersin, A. P., 1904, XVIII, 417.-Hädicke, B. t. W., 1904, 823.-Arloing, Journ. de méd. vét., 1905, LVI, 38.5.-Holmes, J. of comp. Path., 1905, XVIII, 207.-Stockman, Ibid., p. 207.Head, Ibid., 1906, XIX, 12.-Rassau, Z. f. Infkr., 1906, I, 382.-Woolley, Rev. gén., 1907, X, 68.-Kowalewsky, J. vét., 1908, 146.-Rüdiger. D. t. W., 1909, 400.Eggebrecht, Z. f. Infkr., 1910, VII, 54.

Rinderpest in sheep and goats. In 1861 Galambos established with certainty a disease in sheep identical with rinderpest, and he succeeded also in transmitting it artificially to sheep (6 animals inoculated became affected on the 10th day). Since that time the susceptibility of sheep as well as of goats has been confirmed repeatedly, and according to Roll only about $55 \%$ of these animals resist artificial infection.

Extensive outbreaks among sheep were observed in 1862 in Poland, where $64-74 \%$ of the flocks became affected (Seifmann); in 1863 in Sicily, where 20,000 sheep and goats succumbed to the disease (Chicoli); further in 1870-1871 it affected the sheep in France and Alsace-Lorraine, in the latter provinces eausing a loss of 4,000 animals (Zundel).

The clinical manifestations consist in the milder forms, in fever, catarrh of the conjunctivae and the nasal mucous membranes, accelerated respiration, dry cough, and passing of very dry feces. In severe cases there is great debility and apathy, a yellowish-white discharge from the eyes and nose, moist cough and frequent passage of thin, greenishbrown excrements mixed with mucus.

In favorable cases the patients improve very rapidly after an illness lasting 4-5 days, while in severe cases death ensues in the same length of time. The mortality in sheep and goats is generally smaller than in cattle, rarely exceeding $25-30 \%$, Tamilini however observed a loss of $75 \%$.

The anatomical changes resemble those in cattle, but extensive pseudo-membranes and ulcerations on the mucous membranes do not usually occur. The ordinary changes consist in a hemorrhagic inflammation of the mucous membrane of abomasum and intestines, with reddish-yellow, loosely adhering deposits. Similar changes are also present in the mucous membrane of the air passages and in the bladder. There is also anemia of the lungs, and an interstitial emphysema (according to Röll a catarrhal pneumonia is noted with relative frequency).

Literature. Galambos, Gyógyászat, 1862, Nr. 4,-Mareseh, Ö. Vjschr., 1863, XIX, 34.-Röll, Rinderpestähnl. Krankh. d. Schafe u. Ziegen. Wien., 1864. 
Heartwater. According to Theiler a disease of cattle occurs in several localities of Transvaal, which is known there as Veldt-sickness. The most striking symptom of the disease is the filling of the pericardium with a colorless or yellowish fluid (hence the name heartwater). There is also a pronounced indication of acute gastro-enteritis present. The micro-organism is, according to Counsbury, transmitted from the affected to the healthy animals, by a tick (Amblyomma hebraeum); sheep and goats are also very susceptible.

The same disease occurs also among sheep and goats, and may readily be produced by the subcutaneous or intravenous injection of blood, but not of the pericardial fluid. The transmission may be made from sheep to cattle, and vice versa. The symptoms develop 5-15 days after the injection, and consist of high fever, dullness, exceptionally also in nervous symptoms, such as continuous moving of the tongue and the jaws, turning in a circle, later a stiffness of the neck (hence the name "brainwater"). The autopsy reveals a great quantity of fluid in the pericardium and pleural eavity, also petechiae on the pericardium, edema of the lungs and moderate acute swelling of the spleen.

The disease, which has been known in South Africa for about 40 years oceurs only in eertain localities, principally during the summer time, and in pastures located upon high lands. The Kaffir goat and the native fat-tailed sheep are not susceptible, while the Persian sheep are only slightly susceptible to the disease.

Hyperimmunized cattle, sheep and goats produce an effective serum for their respective species, a practical immunization however has not yet been established.

The supposition of Edington that the disease is identical with "horse-sickness" is considered by Theiler as incorrect, as horse-sickness ean be transmitted artificially only to horses (see page 286).

According to Edington's view the disease is identical with the African horsesickness (see that disease). Spreull on the other hand contends that the diseases of cattle occurring in Sonth Africa uniler the names Lamziekte, Gall-sickness, Velitsickness, Blood-lung and Inapunga are identical with Heartwater, and he considers their causative agent to be a motile bipolar bacillus. Clinically he distinguishes an edematous form, with swellings on the neck and hearl, a thoracic form with congestion and hepatization of the lungs, and a paralytic form which is associated with a stiffness of the gait.

Literature. Theiler, The Vet. J., 1904, IX, 300; The transv, agric. J., 1904, II, 163; Bull. Pasteur, 1905, III, 617 ; J. of comp. Path., 1905, XVIII, 155.-Edington, J. of comp. Path., 1904, XVII, 141.-Sprenll, V. J., 1908, 358.

\section{Hog Cholera. Pestis suum.}

(Swine fever, Typhoid fever; Schweinepest [German]; Cholera suum, Peste du porc [French]; Peste porcina [Italian].)

Hog cholera is usually an acute, sub-acute or chronic, more rarely a peracute, contagious, infectious disease of hogs, caused by a filterable virus. In the course of the disease, with the exception of the peracute cases, inflammatory and necrotic processes develop, produced either by the bacillus suipestifer when the lesions occur in the intestinal canal and in the abdominal lymph glands, or by the bacillus suisepticus when the lesions are manifester in the lungs. 
History. The disease has heen recognized in their studies of American diseases of hogs by Salmon \& Smith (1885) as an independent affection (hog cholera) as the eause of which they designated a short motile rod. Based on these findings they sharply separated the disease from swine septicemia (swine plague), considering this as identical with swine plague of Germany, which was recognized just about that time. At the same time they proved that the two diseases are frequently present in one and the same animal, and that the severity of the American outbreaks is brought on by such mixed infections. The same standpoint was accepted in America by Moore and de Schweinitz, in Europe by Raceuglia and Affanasieff. The freyuent simultaneous occurrence of the two forms of diseases, that is the frequency of the mixed infections, eaused Billings in America, and Silberschmidt and Voges in Europe, to take a stand against the identity of these two diseases and their supposed causative agent (the common name of "pneumonoentérite infectieuse" used in France and England corresponds with this conception), while Bang, Schütz, Jensen and Preisz (1898) confirmed the correctness of the original stand of Salmon and Smith.

Through the experimental investigations of the last decade the etiology of the disease appears to have been definitely established. In 1904 the American investigators de Schweinitz \& Dorset made the discovery that a disease occurring in the State of Iowa which clinically resembled hog cholera was transmissible from affected animals to healthy hogs by filtered bacteria-free blood. Soon afterward Dorset, Bolton and MicBryde proved that the disease was identical with hog cholera, and therefore that this disease is also caused by a filtrable virus, whereas the formerly supposed causative agent, the bacillus suipestifer, produces the characteristic changes in the intestines only secondarily in the already affected animal. Similar results were also obtained in Michigan almost at the same time by Boxmeyer (1904), while Hutyra (1906), then the Board's Laboratory of England, Ostertag (1907), as well as Uhlenhuth, Xylander, Hübener \& Bohtz (1907), later Carré, Leclainche \& Vallée, also Wassermann, confirmed the correctness of the new conception of the disease in Europe and Theiler in South Africa. At the same time the views concerning the part of the bacillus suisepticus in the production of the secondary affection of the organs were changed, whereby the disease formerly considered swine plague, is now considered in most cases to be a complication of hog cholera.

The aceurate determination of its etiology directed the combating of the disease into new lines, and the attempts at immunization by Dorset, McBryde and Niles, as well as those by Uhlenhuth and his co-workers, also those by Hutyra, have given valuable practical results.

Occurrence. Hog cholera is at present very widely spread in America, and in almost every European country, causing annually great losses among hogs. The disease, which in itself is not very malignant, becomes very fatal as a result of the frequent, secondary inflammatory processes in the intestines and in the lungs, which, especially in young animals, exist in an epizootic form. It causes the greatest loss in places where hogs are kept in large herds, whereas in localities with smaller droves the disease is combated with better success.

Hog cholera is supposed to have first appeared in North America in the State of Ohio in 1833, and from there it spread over the entire 
territory of the United States. It was probably carried to Europe in the 60 's of the last century. Its oceurrence was first established in England in 1862 (swine fever, typhoid fever), where it has since appeared from year to year, eausing in 1896 the loss of as high as $30 \%$ of all hogs in the country. From England it was supposed to have been spread to Sweden in 1887, with an importation of boars, and from there in the same year to Denmark, to the Island of Amagar, and from there it later extended to other localities in the country. At the same time it appeared in France in the vicinity of Marseilles (it is supposed as a result of an introdnction from Algeria), spreading to all parts of this country, as well as Spain and Italy.

Roloff reported in 1866 in Germany the occurrence of an infectious "caseous intestinal inflammation" in young hogs of English breed in the Province of Saxony, which according to the accurate description of the changes in the eaecum and colon was possibly identical with hog cholera. Hog cholera was observed in 1893 as a severe fatal affection in Posen, and shortly afterwards in Prussian-Silesia, from which country it has been disseminated to the neighboring territories of Austria. Extending in an eastern and southeastern direction it reached Hungary in 1895, where it first made its appearance in the fattening establishments of Köbanya and Györ, causing great losses. At the occurrence of the outbreak at Köhanya of the 180,000 animals 8,368 died, or had to be slaughtered in May; 10,288 in June, and at the end of that year with the exception of Siebenbürgen, all counties were infected. In the following year the disease existed throughout the entire country.

In Germany during the period of 1S98-1906 the number of townships infected with hog cholera and swine plague inereased from 1,817 to 11,513 , and the number of the affected animals from 11,813 to 104,728 . Subsequently up to the year of 1908 the number of townships infecter diminished to 8,782 , and the number of cases to 75,658 . The total loss amounted in 1908 to 60,101 hogs, and the disease was most widely spreal in the government districts of Breslau, Liegnitz, Posen, Bromberg, Potsiam, Frankfurt, and Oppeln. At the end of 1907 there were infected 1,339 townships, ani $86.6 \%$ of all outbreaks were confined to Prussia.

Hog cholera is widely spread in France, and all parts of the country are infecterl with the disease. In 1908 from 13 to 20 districts were said to be infected during different months; however the reports are not accurate.

For a time the disease has greatly diminished in Great Britain, as a result of the measures of eradication which were inaugurated in 1893 . While in 1894 5,862 outbreaks were established in 73 counties, in 1905 only 817 outbreaks occurred in 58 counties. In 1894 to 1896 the number of hogs, which were excluded from traffic on account of the disease or on suspicion, amounted to $56,296,69,931$ and 79,586 , while in the year $190 . \overline{5}$ only 3,876 aninals were affecterl by this measure. In 1906 the disease spread again more extensively, and in $190 \mathrm{~s}$ there occurred 2,067 outbreaks in 68 counties, and 14,096 affected or suspecter hogs were slaughtered.

The disease diminished in Austria as a result of regulations passed in 1895 , and consequently from 1896 to 1895 a gradual decrease in the number of ontbreaks resulted; later however it again spread to a considerable extent. In 1895, 2,767 outbreaks with 28,142 affected animals were recorled; in 1898,654 outbreaks with 4,610 affected animals; and in $1900,1,100$ localities with 35,984 affections. In 1908 the weekly number of outbreaks fluctuated between 104 and 208. The disease was most extensive in Galicia, Bohemia, Moravia and Lower Austria, while Dalmatia was free from it.

In Hungary in the first year (1895) after the appearance of the affection 1,478 and in the following year 3,569 townships, with 413,562 and 868,777 animals, were affected. From this time on the disease gradnally diminished, but even in 19082,200 townships were noted, with 263,945 cases, of which $148,110(56.8 \%)$ died (in the first 2 years the mortality amounted to $81.4 \%$ and $73.6 \%$ ). The disease is most widely spread in the section south of the Tisza and Danube rivers. Of other countries the disease was discovered in 1908 in Bulgaria, affecting 
73 townships, in Jtaly 14,499, in Roumania 1,829, in Russia 27,223, in Servia 45 cases, while in Dennark and in Holland the disease appeared less frequently, in Sweden and Norway (in 1903468 cases, of which 450 occurred in Dép. Akershus) only sporadically.

In the Inited States the disease (hog cholera and swine plague) eansed in the year of $1 \$ 73$ losses amomnting to 20 million, in 188213 million, in 1854 and 188525 and 30 million dollars respectively, while in 1888 the loss was estimated to have affecterl $45,000,000$ hogs of a value of 200 million dollars, and in 1903 Salmon estimated the loss at 50 million dollars.

Etiology. According to the results of investigations by Dorset and his co-workers which, as already mentioned, have since been substantiated by other investigators, hog cholera is caused hy an ultra-microscopic virus, which passes the ordinary porcelain filter. Attempts at cultivation, or even increasing the amount of virus have up to the present been fruitless.

Pathogenicity. Subcutaneous injections of 1-2 cc. of filtered, defibrinated blood, blood serum or organ juices, produce in young hogs, after an average incubation period of 8 to 10 days, sometimes however in four days or only after 20 days, and without any local reaction, an acute febrile affection witl inflam-

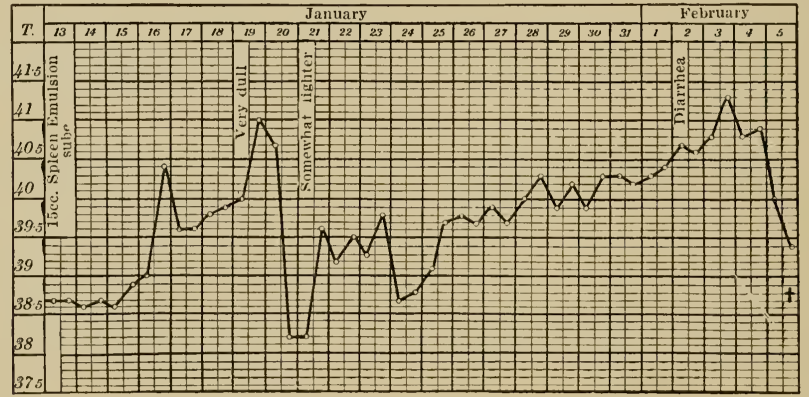

Fig. 45. Hog Cholera. Artificial infection with filtered material from a hog affected with cholera. The first rise up to the fonrth day of sickness is eaused by the primary infection, the second by the secondary infection.

matory swelling of the conjunctivae, eczematous eruption of the skin, followed by diarrhea (Fig. 45; the quantity of blood does not affect the outcome of the disease). In some of the cases the animals die in 3 to 4 days with an acute affection, whereupon the autopsy reveals exclusively hyperemia and acute swelling of the internal organs, as well as hemorrhages on the serous and mucous membranes (sometimes also a great quantity of serous transudate in the pericardium). If the animals remain alive for a longer time ulcerative and necrotic processes may be found in the intestines, in other cases again pneumonic changes will be olserved which are similar to those found in the acute pure swine plague. If the animals remain alive still longer the affection of the intestines and the lungs develops in a more chronic form, whereupon the animals die greatly emaciated, 
or recover completely. Such recovered animals are subsequently immune against artificial, as well as against natural infection.

Animals artificially infected in the above manner may readily transmit the disease to healthy hogs by cohabitation, and their blood even in a filtered condition, and in small quantities, is also infectious. By serial re-inoculations the virus may be propagated from generation to generation.

The artificial affection is, although with less certainty, also successful, by the administration of blood and blood serum in gelatine capsules per os, or by feeding the same with food, when unfiltered material acts more strongly than filtered. The virus is as a rule also present in the bile and the urine, while the intestinal contents frequently prove noninfectious (Uhlenhuth). By feeding internal organs of diseased hogs the disease may readily be produced artificially. Other species of animals aside from the $\log$ are not susceptible to the virus.

Tenacity. Fluid material containing the virus retains its virulence at room temperature for 10 to 14 weeks. Heating for one hour at 60 to $70^{\circ} \mathrm{C}$. destroys the virus, while in the dried condition subjected to 72 to $76^{\circ}$ it requires an hour to destroy its virulence. Blood frozen below $-15^{\circ}$ and thawed out after 24 hours, proved fully virulent, likewise blood which had been dried for three days. The addition of a 1:1000 corrosive sublimate solution in the proportion of 1 to 2 , or of a $5 \%$ glyeerine-carbolic acid solution in the proportion of $2: 5$ does not destroy the virus inside of 8 days; on the other hand the virus in serum is rendered ineffective by the aldition of . $2 \%$ formalin in 15 days, by $2.5 \%$ antiformin in 2 hours. The virus in old putrefied organs loses its virulence in eight days (Uhlenhuth).

Besides the filtrable virus, two species of bacteria, the bacillus suipestifer and the bacillus suisepticus, play an important part in the etiology of hog cholera. The secondary affec-

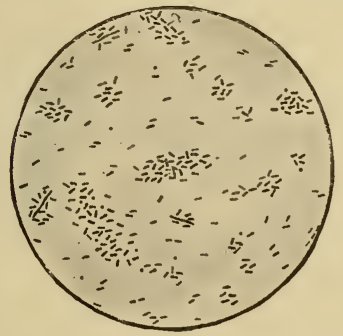

Fig. 46. Bacillus suipestifer. Agar culture, fuchsin staining.

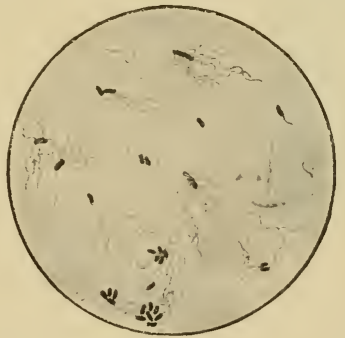

Fig. 47. Brcillus suipestifer, with flagella. Agar culture.

tions of the intestines and of the lympl glands or of the lungs are usually produced by the pathogenic action of these bacteria.

1. The bacillus suipestifer (Bac. cholerae suis) resembles the colon bacillus and belongs to the paratyphus B-group. It is motile, possessing peritrically arranged (3-9) flagellae, and multiplies exclusively by fission (Fig. 46 and 47). In tissues 
it occurs singly or in pairs, in cultures howerer it also forms longer chains or even slightly curved filaments.

Staining. With aypueous aniline dyes the organism usually stains uniformly, sometimes, however when stained with diluted methylene blue solution, it may stain more intensively on the poles. It does not stain by Gram's methoc.

Cultivation. The bacillus grows aerobically as well as anaerobically on all artificial media, in room temperature as well as body temperature; in the latter however considerably faster (the temperature limits are according to Frosch +8 and $+42^{\circ} \mathrm{C}$.). On gelatin plates bluish, trans-

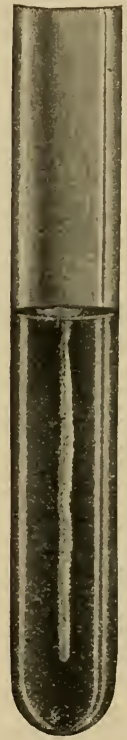

Fig. 48. Four day old gelatin stab culture of the bacillus suipestifer. parent, uniformly rounded colonies develop, while in stab cultures a continuous grayish-white streak forms along the stab, surrounding which the nutritive medium is sometimes turbid. Gelatin is not liquefied (Fig. 48). On agar bluish, transparent, flat, round colonies develop which do not closely adhere (Fig. 49) ; the growth on coagulated blood serum is similar. On Löffler's malachite-green plates, and on endo-plates, colorless colonies form, while on Drigalski's plates the colonies are bluish. Peptone bouillon becomes uniformly cloudy, later a loose sediment settles on the bottom of the tube; indol does not form in the cultures. On potatoes a colorless, moist, lustrous, or a brownish-yellow deposit forms (the variance is probably due to the different kinds of potatoes). Milk becomes grayish, in 3 to 4 weeks saponified, and still later it is changed to a stiff, alkaline jelly. Litmus milk is somewhat reddened, but after 2 to 4 days it becomes decidedly bluish. Dextrose is fermented by the bacillus, lactose on the other hand is not.

Tenacity. The cholera bacillus withstanis simple drying for four months, but in the influence of sunlight and by alternate drying ancl moistening it is quickly killed. In ordinary drinking water, also in soil infected with feces of affected animals, it retains its virulence for 65 to 95 days, while a temperature of $50^{\circ}$ destroys it in 66 hours, and one of $55^{\circ}$ in one hour (Preisz).

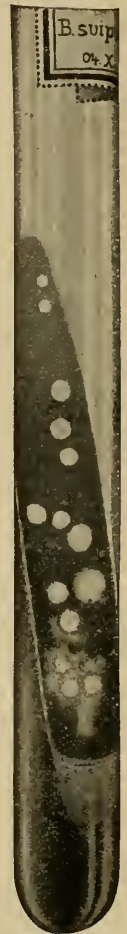

Fig. 49. Twoday old agar culture of the bacillus suipestifer. Of the disinfectants the growth of the bacillus in inhibited by 1:4000 sulphuric acill, 1:900 potassium hydrate; it is destroyed in one minute by a $1 \%$ carbolic acid, solution, $1: 10,000$ corrosive sublimate, $1 \%$ creolin, in 10 minutes by $1 \%$ milk of lime, in three minutes by a $1 \%$ copper sulphate solution, in 11 , nimutes by $0.1 \%$ formalin solution, and in three hours by a $2 \%$ iron sulphate solution (Koppányi \& Erdös). The bacillus suipestifer is considerably more resistant than the bacillus suisepticus.

Pathogenicity. Mice are susceptible to artificial infections, and after an inoculation with $0.1 \mathrm{mg}$. of bouillon culture they die of septicemia in from 3 to 5 days. The guinea pig and rabbit 
are less susceptible (after a successful subcutaneous infection they die in 6 to 7 days; showing enlarged spleen and necrotic foci in the liver). Pigeons are only very slightly susceptible (muscle necrosis develops after an intra-muscular infection), while chickens are not susceptible. If the culture is injected into the cæcum of a rabbit, small white dots at first develop on the mucous membrane (swollen lymph follicles); later ulcers appear in sizes up to a lentil.

By feeding large quantities of cultures or injecting cultures into the intestines of hogs, either acute or more chronic inflammatory and necrotic processes develop, in some cases according to the intensity of the infection (dysenteric necrosis and ulcerations on the intestinal mucous membrane, swelling and caseation of the lymph glands, etc.). After previous starvation or neutralization of the hydrochloric acid of the stomach, the disease results in a much more acute and severe form, and the animal may die on the third day with symptoms of septicemia (Salmon \& Smith). The subcutaneous injection of the culture results in an inflammation of the neighboring lymph vessels and lymph glands, leading to necrosis, and the animals die in from 2 to 3 weeks, or even later. In some of the cases ulcerations develop in the meanwhile on the intestinal wall, and metastatic, caseous areas appear in various internal organs. Intravenous injections may result in a hemorrhagic septicemia producing death in 1 to 3 days. The infection cannot be produced by inhalation.

Contrary to the infection produced by virulent blood, hogs which are infected with pure cultures of the bacillus suipestifer do not transmit the disease to healthy hogs, and if they recover they continue to be susceptible to natural infection.

2. The bacillus suisepticus is in every particular identical with the organism of pure swine plague (see p. 125).

Natural infection results apparently from the digestive tract and appears to be transmitted especially by the strongly virulent urine of affected animals, while the excrements are frequently only slightly or not at all virulent, and therefore play a less important part in the transmission of the disease. In piggeries of localities which were previously free of the disease it always results from an introduction from the outside, while in already infected premises and stables the affection may repeatedly appear without a new introduction, as the virus retains its virulence for a long time outside of the animal body, in fluids as well as in a dry material. This is proved by the fact that the disease which was formerly unknown in Europe spread in the course of the last decade along the road of swine traffic, and then remained permanently in the infected countries, causing severe losses from year to year.

The very extensive traffic in hogs which exists everywhere and which is difficult to control, is generally the most effective disscminator of the disease, and in this the hog markets, the 
stock dealers, castrators and wandering grpsies (the latter by taking with them the hog carcasses and throwing the viscera about in different pastures) contribute materially to the spread of the contagion.

In herds of hogs which have been healthy the disease is in most cases introduced by sick animals, and not alone by those in which acute symptoms may be detected, but also through the so-called runts in whose bodies the filterable virus may also be concealed. Infection may also occur during the pasturing of the animals, in the piggeries, during driving on highways or transportation in railroad cars, which have been contaminated by the excrements of affected animals days or weeks previously. The contagion may be spread from one locality to the other by flowing water, into which carcasses of logs have been thrown, and carcasses which have been left in pastures or which have not been buried satisfactorily may also occasionally be the cause of an outbreak of the disease.

Although hogs of all breeds and of any age may become affected with hog cholera, the higher bred stock and young hogs are much more susceptible to the disease. Sucking pigs become affected only very exceptionally, and as a rule the influence of age is especially marked in localities in which the disease has existed for a long time. Thus during the first years of the existence of the disease in Hungary the older hogs became affected quite frequently, while since that time the disease is confined more and more to younger animals. The greater resistance of the older animals in infected localities is in all probability due to the fact that they passed through a mild form of the disease while young.

Pathogenesis. According to the most recent experimental investigations the disease is without a doubt caused by a filterable hog cholera virus, which enters the body fluids and multiplies there. It causes directly a febrile condition, with acute catarrh of the mucous membrane, and in case of a severe infection death results with manifestations of hemorrhagic septicemia. In the great majority of cases however secondary inflammatory changes of different organs may become associated with the general blood infection. Such secondary changes occur especially in the pharynx, stomach and intestines, in the corresponding lymph glands and in the lungs, being caused without a doubt by the bacillus suipestifer or the bacillus suisepticus. The etiological part of these organisms came into the proper light only after the virulence of the filtered blood of affected animals had been established.

Contrary to the former conception by which the bacillus suipestifer was considered as the original and true cause of the disease, there now exists hardly any doubt that it participates only in a secondary manner, yet though it is only granted an inferior part in the transmission of the disease it is very active, 
and has a great share in the development of the organic affections which derelop in association with the primary affection. Ascording to Dorset, MeBryde and Bolton the following factors proved that this organism is not the primary cause of hog cholera: 1. The artificial transmission of the disease can be much more readily affected by blood from an affected animal, than by subcutaneous injection of pure cultures of the bacillus suipestifer. 2. Hogs which were affected either by natural infection or by subcutaneous injections with virulent blood transmit the disease very readily to healthy animals, whereas animals which were infected with cultures of bacilli do not infect healthy hogs. 3. Blood from hogs which have been infected with blood, proves virulent by subcutaneous injection, while the blood from hogs infected with cultures of the bacilli is harmless for healthy animals. 4. Recovery from the natural or the artificially produced disease confers a lasting immunity, whereas hogs inoculated with the bacillus suipestifer continue to be susceptible to the natural infection.

Besides these considerations it should also be noted that the bacillus suipestifer eannot be demonstrated bacteriologically in all cases of true hog cholera. Thus Uhlenhuth found the organism only in 76 out of 178 affected hogs, while in the other cases in seven so called varieties of this organism (some of which do not ferment dextrose) three times varieties resembling the paratyphus A-Group, once the bacillus enteritidis Gärtner, 50 times the hacillus pyocyaneus, 36 times streptococei, 27 times staphylococci, and 110 times colon bacilli, were isolated from the organs; while in 14 cases the organs proved sterile. In some of the outbreaks the bacillus appears to occur only rarely, as Boxmeyer in Michigan and Theiler in South Africa failed to demonstrate the bacillus in typically affected hogs. These findings make it probable that in different localities various bacteria, especially of the paratyphus $\mathrm{B}$, or of the hog cholera group, may produce the usual changes in the intestines found in hog cholera.

According to views accepted at the present time the bacillus suipestifer forms a group in common with the bacillus paratyphi B and Bac. botulinus, which are pathogenic for man, also including the mice typhus and the psitticosis bacillus, as well as the Bac. Sanarelli. This group is designated as the hog cholera, or the paratyphus B-Group, and the various organisms of this group cannot be distinguished from each other, either by their morphologic and eulture characteristies, or by sero-diagnostic methods (agglutination). The only distinguishing signs consist, excepting when their origin may he demonstrated, in certain, not constant, and therefore not characteristic pathogenic properties. The very elose relation which almost borders on identity, would explain in hogs, for instance, why it is that various representatives of this group may produce very similar pathological changes, and attain pathogenic properties for man (this theoretical possibility has not yet been proven for the true suipestifer bacillus). Bang and Th. Smith, also Uhlenhuth and his co-workers cultivated strains of the bacillus suipestifer from hog cholera pigs, which showed certain deviations from the common type, and therefore are considered as varieties (some strains produce no gas in dextrose, others coagulate milk, and Smith \& Moore even deseribed a non-motile variety). 
Against the idea that the bacillus suipestifer is the primary virus of hog cholera are the experimental ohservations of Uhlenhuth and his co-workers, who found that virus which was treated for one hour with $2.5 \%$ antiformin still proved virulent, while cultures of the bacillus suipestifer lost their pathogenic action even for the very susceptible rabbit, after being subjected to such a solution from 30 to 40 minutes, the organism being readily dissolved by the antiformin. A similar difference is also shown to exist by the fact that the hog cholera virus is destroyed by shaking with a $10 \%$ uric acid only in four days (Marxer), whereas the bacillus suipestifer is killed in 23 hours (Stilling); the olsservation that the bacillus suipestifer agglutinates the serum from hogs affected with aeute cholera in dilutions of only $1: 5-20$ (Uhlenhuth), whereas in animals artificially infected with this bacillus the agglutination takes place in higher dilutions (1:1000 and over) should also be noted. Moreover in virulent hog eholera seruin there are no opsonins or bacteriotropins, which have an influence on the bacillus suipestifer (Broll).

Recently (1907) Lourens took a stand relative to the importance of the bacillus suipestifer as the primary cause of hog cholera, when he proved that this bacillus passes through the porcelain filter in a granular form, and that it may later nultiply in the filtrate. Filtrates containing bacilli, however, produce inflammatory changes at the place of injection, and since the disease always develops in one to two days, Lourens' findings are in no way sufficient to weaken the importance of the results obtained with fresh blood and filtrates of organs, or to shake general belief in the correctness of recent discoveries. Moreover McBryde found recently that the bacillus suipestifer may pass through some of the Berkefeld filters, but not Chamberland $B$ and $F$, which however allow the hog cholera virus to pass.

The same applies to the theory of Schreiber, accorling to which the blood of hogs affected with hog cholera supports, through the agressins which it contains, the pathogenic action of the bacillus suipestifer, or mobilizes the same, the bacillus being a frequent habitant of the intestines of hogs. This theory stands in contradiction to the fact that even small quantities of blood, and especially from hogs which have not yet develojed intestinal changes, produce the disease, and further that artificial infection may also result from feeling such blooi, and that antiforminblood mixtures prove effective, in spite of the fact that the endotoxins of the bacillus suipestifer are destroyed by this product.

The view that the bacillus suipestifer plays only a secondary part in the etiology of hog cholera is further substantiated by the discovery that bacilli which morphologieally, eulturally, and biologically correspond to the bacillus suipestifer not infrequently occur in the intestines of entirely healthy hogs; thus Garbert succeeded in demonstrating such in seven out of 23 , Uhlenhuth in $8.4 \%$ of 600 healthy hogs. Similar results are also reported hy Morgan from England, Gardenghi from Italy, and Velzen from Holland. Furthermore Titze \& Weichel found organisms in the intestinal contents of calves affected with white scour, which absolutely corresponded to hog cholera bacilli, and also in one healthy ealf.

The facts mentioned here, as well as the experimental observation that hogs which were inoculated with the filtrable virus develop intestinal changes caused by the bacillus suipestifer, lead to the belief that the bacillus suipestifer being a frequent normal liabitant of hog intestines, penetrates into the tissues injured by the filtrable cholera virus, and exerts in the same its pathogenic properties. 
A similar view would probably also apply to the part of the bacillus suisepticus. Ovoid bacteria which correspond in all their characteristies to these organisms occur as saprophytes in healthy hogs (see p. 129). Animals which have been injected subcutaneously with filtered cholera blood frequently develop acute, fibrinous, catarrhal or necrotic pneumonia, in which the ovoid bacteria are present in great numbers.

These findings can be explained satisfactorily only by considering that the bacillus suisepticus, like the bacillus suipestifer, finds favorable conditions for its propagation in the body affected by the cholera virus, and thereby exerts its pathogenic action in the production of a multiple necrotic pneumonia. From case to ease one or the other of these bacilli, and frequently both at the same time or following each other, may become conspicuous in the pathological changes.

Therefore in association with the primary cholera infection there may be not only the characteristic anatomical changes of hog cholera present, but also those of swine plague; thus not only the anatomical hog cholera, but also the anatomical swine plague as it occurs in outbreaks of cholera, either in association with the former, or alone, is primarily brought on by the filterable cholera virus. In all of these cases only one disease comes into consideration, and that is hog cholera, which however manifests itself in various clinical and anatomical pictures, depending on the secondary infection, namely either as a pure septicemia, as hog cholera or as pure swine plague, or the changes may indicate a mixed infection with the last two forms. Accordingly a septicemic, a pectoral and a mixed form of hog cholera may be distinguished. It should however be distinctly understood that the intestinal and the pectoral changes in the organs are only secondary complications, and that strictly they should not be considered as belonging to the manifestations of hog cholera.

Contrary to the above-aescrused views relative to the secondary action of the bacillus suisepticus, Ostertag contends that pure swine plague may occur as an independent acute disease even under favorable hygienic conditions, infecting old, as well as young animals, and causing losses amounting to $75 \%$. He further claims that chronic swine plague is directly connected with the acute, destructive, classical swine plagne. He bases this view mainly on the observation that the acute form may pass into chronic, and as such may again introduce the acute fatal form, and that with the material of both forms, either of the forms may be alternately produced (see also p. 128).

On the other hand Uhleuhuth and his co-workers perfectly agree with the view of Hutyra. They found that pigs infected with filtrated blood frequently develop a pleuro-pneumonia, and that the treatment of pigs with immune serum protects them also against a secondary pneumonia. They therefore emphasize the suggestion that changes in the lungs belong to the accompanying hog cholera symptoms, and therefore are to be considered as symptoms of the disease. Therefore the so called pure cases of swine plagne in outhreaks of cholera shonld be considered as cholera infections, and the previously considered mixed 
forms, that is the cases which were heretofore considered as results of two entirely different infectious discases, are etiologieally due to one primary cause, namely to the infection of the body by the cholera virus.

At the present time it eamnot even be imagined in what way the filterable virus exerts its pathogenie action; on the other hand the pathogenicity of the bacillus suipestifer and the bacillus suisepticus is quite well understood. Regarding the first one it must be aceepted that as a rule it penetrates into the follicles of the already catarrhal intestinal mueous membrane, where it produces a cellular infiltration of the follicles, with which a neerosis of the tissues soon becomes associated. In this manner nodules first develop, then small, deep round uleerations covered with caseous material, which gradually become larger. At the same time usually, but in some instances quite independently, a necrosis of the epithelium and of the superficial layers of the mucous membrane results from the immigration of the bacilli into the lymph spaces of the mucous membrane, whereupon diphtheritie or croupous inflammation results. Such deposits also develop very frequently on the mucons membrane of the stomaeh, and of the pharynx.

From the intestinal wall the bacilli pass through the lymph vessels into the mesenteric lymph glands, where they produce a cellular infiltrition, and consequently a tissue necrosis, as a result of which dry easeous areas develop in the enlarged glands. In chronie cases the process may remain confined to the intestinal wall and to the mesenterie lymph glands, and may by disturbing the digestion finally result in complete exhaustion of the animals.

In other eases the bacilli may be disseminated along the lymph vessels, to distant lymph glands and into the blool cirenlation. The infeetion of the blood may sometimes oceur in the early stages, and contribute to the aggravation of the already existing septic condition. Besides baeillary emboli may cause the development of metastatic inflammatory lesions, which will later easeate begimning at the center (such caseated areas are most frequently found in the lungs, kidneys, spleen and the liver).

The necrosis (caseation) which is quite characteristic of hog cholera, is probably to some extent the result of an ohstruction of the blood vessels by masses of bacilli, and the eonsequently developing nutritive disturbances. In the first place however it is probably due to the toxic action of the bacilli.

Tp to the present time experiments for the demonstration of toxins in cultures of the bacillus suipestifer have not given uniform results. While Preisz found that neither inactivated cultures nor bacteria-free filtrates of eultures were toxic for test animals, Voges and Karlinski elaimed that the killed bacteria contain an intra-cellular toxin (according to Voges $10 \mathrm{mg}$. of killed bacteria are fatal to guinea. pigs). De Schweinitz profluced a ptomaine from cultures by precipitation with alcohol (Sucholotoxin) and also a poisonous albumose (Sucholoallmmin). He further found two kinls of enzymes in aqueous extracts of agar cultures, as well as in milk in which the bacilli were grown for three weeks, of which the one converts starch into dextrose (Diastase), while the other liquefies gelatin anil digests fibrin and albumen (trypsin-enzym). Prettner also foum in the cultures lacteriolytic enzymes, which slowly dissolve gelatin. According to the investigations of Prettner and Emmerich the bacillus proluces nitrous acid in material containing nitrates, as well as in the intestinal canal, which may cause a nitrite poisoning in the body of the animal (cyanosis, necrosis of the intestinal epithelia, paralysis or gangrene of the sympathicus, serous transulations in the intestinal lumen, formation of methemoglolin in the bloo(l). F. Schmilt aimed to obtain specific sulsstances from cholera hacilli, by the use of Brieger's metlod (suspension of cultures grown on agar in water and shaking) as well as through the method of Conraci (autolysis), 
and further with the aid of MeFadyean's apparatus for breaking up bacteria, but he failed in immunizing test animals with the substances obtained by any of the three methods. The toxicity of killed bacilli (endotoxins) is shown by the experiments of Uhlenhuth and his co-workers, who sncceded in producing a typical anatomical picture of hog cholera by intravenous injections of cultures heated to $60^{\circ} \mathrm{C}$.

According to Bang's investigations, the necrophorus bacillus is also a participant in the production of the necrotic processes, and as a matter of fact it is very frequently found in large clumps at the base of the ulcers as well as in the nodules along the border between the necrotic and living tissue.

The bacillus suisepticus exerts its pathogenic action in a similar way in eases of pure swine plague (see p. 127). In the peracute cases it is found quite frequently in the blood more often than the baeillus suipestifer. The lesions of hemorrhagic septicemia which are found at the autopsy of such cases are evidently largely due to its action. Often, however, the frequent pneumonia and pleurisy is directly associated with the activity of this organism.

The fact that the bacillus suipestifer and the bacillus suisepticus are very frequently actively concerned in hog cholera besides the filtrable virus, explains why clinical as well as anatomical findings should vary to such a great extent from case to case. In the majority of the dead or emergency slaughtered animals in infected herds examination discloses an ulcerative inflammation of the intestines and caseation of the lymph glands, also necrotic inflammation of the lungs, fibrinous deposits on the serous membranes, as well as hemorrhages, while careful bacteriological examinations prove the presence of both species of the bacteria mentioned. In the course of some outbreaks, as well as after the artificial infection of herds (by introducing affected animals into healthy herds, the placing of a healthy herd on infected premises), septicemic affections usually predominate. The first animals dead from the disease, which probably had the least resistance, show acute catarrh of the mucous membranes exclusively, and more or less numerous hemorrhages, somewhat later pleuro-pneumonia may also be present, while on the mucous membranes of the intestines small follicular ulcerations or superficial desquamation of the mucous membrane may be seen here and there. Later the characteristic intestinal changes of cholera develop, in constantly greater extension and intensity; first a superficial desquamation of the epithelium, then a follicular ulceration, or a diffuse, diphtheritic inflammation of the mucous membrane. At the same time the pleuro-pneumonia occur's with greater frequency, so much so that toward the end of the outbreak it is present in the great majority of cases. In the animals which still remain debilitated after the acute outbreak has passed off changes of a chronic character are present either in the intestines or in the lungs.

These successive anatomical findings may be explained by the fact that the most susceptible and the least resistant animals are killed by the specific cholera virus at the beginning of the outbreak, whereas the more resistant withstand the primary infection for a time and are only exhausted by the organic affections which develop later. 
Anatomical Changes. According to the presence and the nature of the seconclary infection, the following forms of the disease may be distinguished:

1. Pure Hog Cholera (septicemic form). In this the autopsy findings correspond to those of an acnte hemorrhagic septicenia, namely hemorrhages in the serous membranes and in the mucous membranes of the pliarynx, stomach and intestines, in the acutely swollen lymph glands and probably also in the kidneys. Further there is also a moderately acute swelling of the
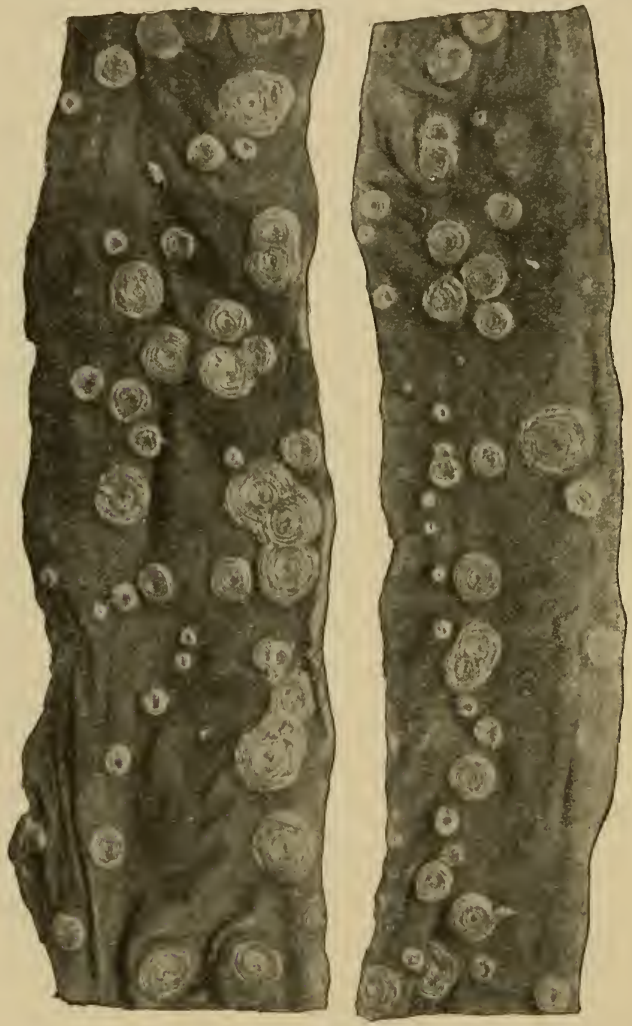

Fig. 50. Intestine of hog with follicular ulceration of hog cholera. spleen. In the meantime in some cases the peritoneum is covered with a fibrinous pseudomembrane, and the intestinal walls are hemorrhagic. Even in such eases fine, croupous pseudo-membranes may frequently be found on the swollen and hyperemic mucous membranes of the stomach and intestines, sometimes also a diffuse, superficial necrosis (bile stained, mushy deposits), orhere and there yellowish scales.

\section{Intestinal}

Form. In this most frequent form of the disease, the intestinal tracts, especially the cecum and the large intestines, slow intensive changes. In the less acute eases a hard round nodule may he felt on palpation of the wall of the large intestines, or this is thickened either in part or uniformly 
in its entire length. At the same time the flexures of the intestines may stick together, and this condition may also be seen in the convolutions of the small intestines, or there may even be adhesions. In acute cases there are lentil to dollar-sized, flat, round, yellow, greenish-yellow, brown or blackish-gray, dry scabs on the mucous membrane of the colon and cæcum, especially around the Bauhin's fold (ileo-caecal valve), and sometimes also in the adjoining small intestines, under which the submucosa or the muscularis is infiltrated or thickened. Between these the follicles are swollen to hemp-seed size hard nodules, from which a caseous mass may be squeezed. In some cases the affection may be confined exclusively to an ulceration of the follicles, in which deep ulcerations, with raised, thickened margins, cover the mucons membrane of the intestines. These ulcerations increase in numbers posteriorly, and are of the size of lentils, round and covered with dry tissue debris (Fig. 50).

In more chronic cases the imner surface of the intestines usually contains thick, hard scabs, in small numbers, sometimes flat, at other times hemispherical, colored similarly to the abovedescribed lesions, so-called buttons or boutons, which protrude over the neighboring mucous membrane (Fig. 51). The cut surface of these buttons appears sometimes in layers, they adhere firmly to the submucosa or the muscularis, and are surrounded by a thickened ring of the mucons membrane. Around these nodules the intestinal wall is frequently thickened and stiff.

In some cases the mucous membrane may be necrotic over large sections of the large intestines; in less advanced cases the necrotic epithelium forms a mushy deposit on the surface of the mucous membrane, while later it may change in its entire thickness to a dirty yellow, or greenish-yellow, dry, mushy detritus. At the same time the outer layers of the walls of the large intestines are greatly thickened, so much so that the affected section represents a stiff tube with a constricted lumen.

The mucous membrane of the stomach, especially in acnte cases, shows a pronounced hemorrhagic inflammation, and is frequently covered with croupous psendo-membranes.

In the acute cases the lymph glands are swollen, hyperemic, and even hemorrhagic. In the later stage they contain small, grayish points, disseminated in reddish tissue; in still more severe cases they change to hard growths up to the size of a hen's egg, which on the cut surface prove to consist of a grayish-red, dry, homogenous necrotic tissne, or of a dry or soft caseous mass. These changes are most frequently and most prominently present in the mesenteric glands, less frequently in the other lymph glands of the abdominal cavity, are sometimes also to be found in the glands of the thoracic cavity, and in rare cases even in the body lymph glands.

In some cases tumor-like lesions which resemble those of the lymph glands may be found in other internal organs, which 
may reach the size of a walnut, and contain caseous masses. Such are most often found in the liver, the kidneys, lungs, spleen, udder, bone marrow, etc. The lungs may in exceptional cases also contain circumscribed pneumonic areas, with a yellowishgray cut surface, without necrosis or pleurisy (Preisz).

In the pharynx, at the base and on the borders of the tongne, sometimes also in other parts of the buccal cavity, and in the larynx hemorrhagic inflammations of the mucons membrane, with croupons psendo-membranes and ulcerations, together with a superficial or deep necrosis may very frequently be found.

Among other changes which may occur are necrosis of the mucous membrane of the gall bladder, the vagina, and the bladder, localized or extensive necrosis of the skin, cloudy swelling of the parenchymatous organs, etc.

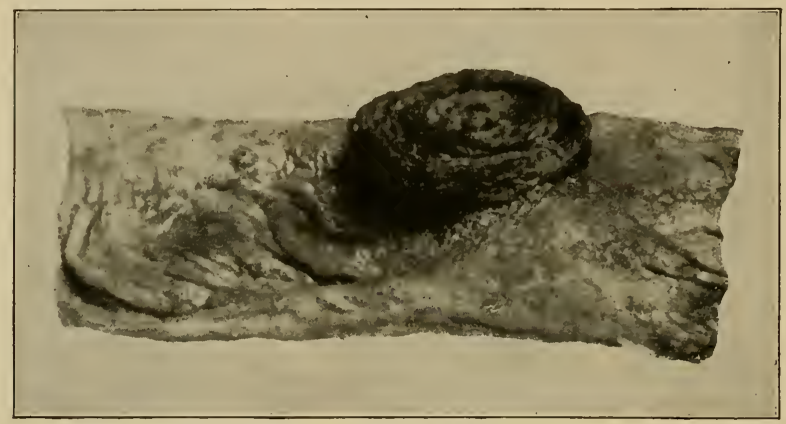

Fig. 51. So called button on the mucous memibrane of the large intestine in hog cholera.

The intestines of animals slaughtered during convalescence show at the places of the thrown-off necrotic nodules, ulcerations with a granulating base, or small white scars may serve to indicate the pre-existing ulcerations (Gerosat and Bilitz observed in exceptional cases cicatrized constrictions of the intestines). The lymph glands of other organs may at times also contain caseons foci.

The bacillns suipestifer may be demonstrated only in acute cases in the blood, and even then only in small numbers, while in the spleen it may be present in greater numbers. The same organism may also be found in the affected lymph glands, in the necrotic nodules of the intestinal mucons membrane and in the caseons metastatic lesions of the internal organs.

The necrotic and ulcerative processes on the intestinal mueous membranes develop quite slowly. Thus in an animal 12 days after the infection there are, lesides the bean-sized mesenterie lymph glands, only hemp-seed size yellow deposits of exudate on the intestinal mucons membrane; after 14 days they attain the size of a lentil, and after 18 days the exudate or nlcerations may be the size of a dime. At this stage the mesenteric lymph glands reach the size of an almond; 
21 days after the infection large sections of the intestinal mucous membrane may be covered with a deposit or exudate, and the intestinal wall may be 3 to $4 \mathrm{~mm}$. in thickness. The mucous membrane of the eecum however may show necrotic areas as large as a quarter in size.

According to Marek's histological examinations the nature of the changes in the intestinal mucous membrane are those of a croupous, or crompous-diphtheritic character. The epithelial layer and the propria mucosae changes throngh a coagulation necrosis, into a homogenous layer, which contains fibrinous threarls and obstructed blood vessels. This layer is surromnded by a wall of polynuclear leucoeytes. The bacillus suipestifer and the Bac. necrophorus are found in great numbers on the border line between the necrotic and healthy tissue. The patho-

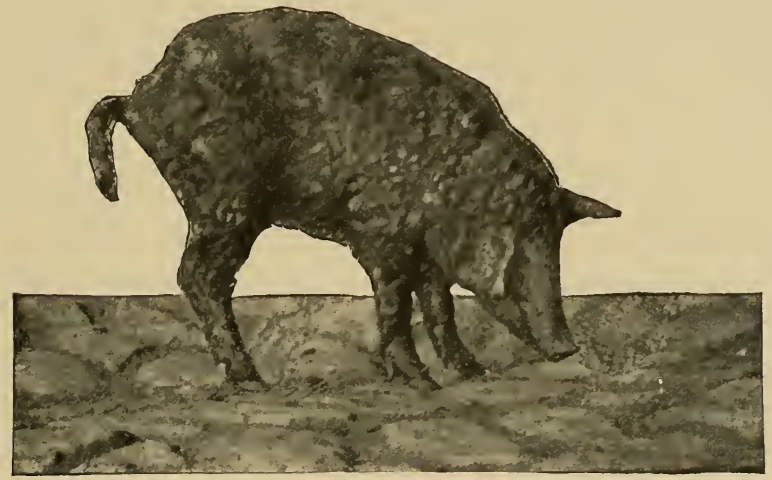

Fig. 52. Hog Cholera. Pig affected for two weeks.

logical process frequently commences in the follicles, occasionally however the necrotic layers may contain healthy follicles. If larger bacterial colonies develop in other organs they may cause coagulation necrosis in such places, which later results in the formation of sequesters.

3. Pectoral Form. This form is characterized by an acute, usually croupous, more rarely catarrhal pneumonia, which in the later stages passes into multiple necrosis. Frequently there is also a fibrinous or sero-fibrinous pleurisy and in some instances also a similar pericarditis. Besides this, the mucous membrane of the gastro-intestinal tract reveals a diffuse, acute inflammation, while in acute cases the other organs indicate a general septic infection. Otherwise the autopsy findings are identical with those of swine plagne (see p. 130), and the lisease can only be distinguished from this by the fact that the filtered blood or material from the organs is virulent (see diagnosis).

4. Mixed Form. The great majority of eases which are found in infected herds belong to this class. The lesions of the intestinal and pectoral forms, with the corresponding changes of the organs, are present simultaneously from case to case but in varying intensity; therefore the autopsy findings vary greatly, even in the animals of one and the same herd. In 
acute cases the mucous membranes of the gastro-intestinal tracts, as well as of the lungs, are simultaneously affected in the above-described manner, while in chronic cases one or the other form is often exclusively present.

Symptoms. The time of incubation of hog cholera in artificial infections, carried out as closely to the natural mode of infections as possible (cohabiting healthy animals with affected hogs, or in infected premises, Preisz, Hutyra), is at least 4 days, usually however 13 to 18 days. After a subcutaneous injection of virulent blood the first symptoms also appear at the earliest on the fifth day, more frequently however in 8 to 10 days, and in some cases only after from 2 to 3 weeks (Uhlenhuth).

1. Pure Hog Cholera (septicemic form). Excepting the very rare peracute cases which develop almost exclusively at the beginning of an outbreak and terminate in from 2 to 3 days with manifestations of an acute hemorrhagic septicemia, the clinical development of the acute cases is usually somewhat slow. One of the first indications of the presence of the disease is impairment of the appetite. The animals take their food less eagerly, they separate, apparently quickly satisfied, from the other animals of the herd, stand at one side, alone, or crawl into some secluded corner of the stable or yard. Later they appear only hesitatingly or not at all for their feed. If urged to leave their place they show great reluctance to move, and do so slowly and feebly. They soon stop, and remain for long periods in one place with back arched, and with head depressed.

One of the first symptoms and one almost invariably present is an acute conjunctivitis, with the production of a mucous or muco-purulent secretion. Vomiting is frequently observed on the first or second day, the vomited material containing mucus and being stained yellow by bile. There is at first constipation, but diarrhea sets in after a short time. The feces are thin and sometimes bloody. In this stage the patients become more or less weakener, and if the disease does not turn toward improvement, or if it does not become chronic, death ensues in from 4 to 7 days, with septic manifestations.

2. Intestinal Form. This develops like the first form, but is less acute. The more intense affection is indicated by a distinctly yellowish or greenish coloration, and by the very fetid odor of the feces. At the same time inflammatory or diphtheritic changes appear on the buccal mucons membrane. The borders, the dorsum and the base of the tongue, the cheeks and the pharynx hecome covered with a dirty gray or yellow, loose deposit of exudate, the tonsils are greatly swollen, or ulcerations covered by a mushy mass develop upon them. These changes may be observed by examining the buccal cavity. There is also 
pharyngitis, associated with difficulty in swallowing, frequently also by difficult, rattling respiration, and death may result from asphyxiation.

In the less severe form these symptoms continue to exist, and in some cases several small or larger growths may after a time be felt in the abdominal cavity by palpating the relaxed abdominal wall (lymph glands and adherent intestinal loops).

In severe cases the animals, having lost their appetite, become more and more emaciated, the diarrhea alternates with constipation (this is sometimes also observed in extensive ulcerations of the intestines), they become very weak and anemic, move only with difficulty and with a staggering gait, or they stand in one place with back arched, and with croup sunken (Fig. 52). Towards the end they continuously lie on the ground, and die completely exhausted, at the earliest on the 11th day after the infection, mostly however in from 2 to 3 weeks.

In other cases the acnte symptoms of an intestinal affection diminish in 1 to 3 weeks, the appetite gradually improves, the diarrhea ceases, the animal moves about more lively, and finally a complete recovery takes place. Sometimes the animals continue to be sickly, in spite of the fact that the acute symptoms become milder. The appetite remains irregular, normal movement of the bowels alternates with diarrhea, the animals do not show a normal development, gradually a severe anemia and cachexia sets in, which finally leads after some months to complete exhaustion.

3. Pectoral Form. With the initial symptoms of pure hog cholera manifestations of an acute pneumonia or pleuro-pnenmonia become associated after several days and from this stage on the clinical development is identical with the acute or chronic swine plague (see p. 132).

4. Mixed Form. In this form symptoms of varying intensity develop in connection with the symptoms of hog cholera infection, which indicate gastro-intestinal trouble, as well as an affection of the thoracic organs. Besides inappetence and diarrhea there is a cough, and increasing difficulty in respiration, and in unfavorable cases death results as a rule directly from the affection of the thoracic organs. In the later course the symptom-complex of both forms may exist for a long time, and they are not infrequently observed simultaneously even in the chronic cases; while occasionally the manifestations of the lung affection are less pronounced in the early stages, they later become milder, and the dominating symptoms then consist of a changeable or depraved appetite and diarrhea.

The cases belonging to this form manifest with relative frequency changes in the skin. Aside from the extensive necrosis which is sometimes present, resulting from the action of the hog cholera bacillus, and being principally observed on the ears, 
red spots frequently appear on the finer parts of the skin, which develop as a result of a simple dilation of the vessels. Later small vesicles form in these places, and soon the superficial epithelial layer separates in fine lamellae. The abdomen sometimes shows numerous punctiform hemorrhages, which remain noticeable for a long time even after recovery. Occasionally urticaria may develop, when vesicles will appear on the flat, raised lesions (Schindelka). In some cases the bristles may fall out on different spots or over the entire body, animals with dark pigmented skin may become white, and if the animals remain alive white bristles grow on the bald places, which however may later be replaced by black.

Diagnosis. The clinical symptoms do not afford sufficient grounds for the establishment of a positive diagnosis. Although they cause a well-founded suspicion of hog cholera, especially in already affected herds or if the disease is prevalent in the locality, the determination however must be made dependent on the autopsy findings. As in accordance with the present state of our knowledge the bacillus suipestifer or other bacteria of the hog cholera group under natural conditions produce only in the body weakened by the filtrable cholera virus follicular lesions on the intestinal mucous membrane, and the ulcerations and buttons resulting from these with simultaneous caseation of the regional lymph glands, these changes must be considered as characteristic of hog cholera. Therefore where they are present the diagnosis of hog cholera may be established without consideration of accessory circumstances.

The fact that it is sometimes possible to produce the anatomical hog eholera artificially, with the bacillus suipestifer alone, does not indicate that this occurs under natural conditions, especially as positive results have only been obtained in feeding experiments with very large quantities of culture. Glasser is nevertheless of the opinion that an organism which he isolated from two apparently spontaneously affected pigs, and which biologically differed from the bacillus suipestifer resembling more the human typhoid bacillus (Bac. typhi suis), may independently produce a contagious epizootie infection (typhus of hogs). However the occurrence of such a spontaneous enzootic, or even epizootic, has not been confirmed from any other source. According to a preliminary report of Stedeferler the bacillus suipestifer also may produce spontaneous affections independently, without any preexisting accessory influence of an ultravisible virus.

Contrary to the formation of intestinal lesions, the presence of a necrotic pneumonia, even in a typical form, has not the same pathognomic significance, as this form may occur independently withont any participation of the filterable hog cholera virus, and in such case it must be considered as pure swine plague. The decision whether in a single case one is dealing with pure swine plague, or hog cholera with swine plague symptoms, is in practice sometimes very difficult. If besides the inflammatory infection of the lungs there are at the same time scabs, round ulcers with discolored covering, or even buttonlike scabs on the mucous membrane of the intestinal canal, with or without swelling of the lymph glands, a cholera infection 
must always be considered. On the other hand, it there is present a pure hemorrhagic septicemia, or only an acute croupous eatarrhal, hemorrhagic, or a necrotic pnemmonia, with or without a fibrinous pleurisy, or if the case shows chronic remnants of a pre-existing' acute pneumonia(large necrotic areas, seqnesters) then the anatomical changes, together with the microscopical and cultural findings, do not clear the true nature of the affection. In such cases filtration tests are final, and if a positive result is obtained with these the presence of a cholera infection is thereby established. Still, a negative result of such a test is not conclusive; as the cholera virus which has been present in the body fluids may already have disappeared from the body to a great extent or entirely. In such cases the diagnosis must depend upon whether the animal comes from a herd infected with hog cholera, and whether in any of the other animals there were any lesions pointing to an infection of hog cholera, or if a case represents a sporadic affection from a herd which has proved healthy. It is essential therefore, in such cases, to consider the epidemiologic factors, and to attach to them their appropriate value, establishing the diagnosis.

Of course this presents far greater difficulty in reaching a diagnosis, when compared with the former conditions, in which the bacteriological demonstration of bipolar ovoid bacteria or cholera bacilli in the organs showing the inflammatory changes were alone sufficient for the determination of the disease. By such findings the disease was designated as hog cholera or swine plague. At present however the demonstration of these bacteria, whether in the blood or in the organs, is not of conclusive importance for the establishment of the diagnosis of hog cholera or swine plague. It now becomes necessary to consider the gross anatomical changes of the organs, in doubtful cases also the epidemiologic accessory circumstances, and the nature of the affection of other animals in the same herd.

In bacteriological examinations the simple microscopic examination does not always give positive results, but with the aid of culture and agglutination tests it is in most instances possille to demonstrate the bacteria which participate in causing the pathological processes. Animal inoculations with material from lesions as a rule is only adopted for the demonstration of the septicemia bacillus, as small animals are killed by these organisms in from 24 to 36 hours, and their blood or the exudate of the alodominal cavity contains in such instances only this bacillus, even thongh the inoculated material also contained the cholera bacillus.

Swine erysipelas frequently greatly resembles a septicemic form of hog cholera, especially when there are only few and small hemorrhages present in the internal organs, such cases usually occurring in the begimning of outbreaks. The microscopical finding of erysipelas bacilli in the blood or in the spleen of course establishes the diagnosis of erysipelas. It is advisable however to postpone the final decision in negative findings and to depend on the autopsies of other animals which may die or which are slanghtered in emergency. If hog cholera 
exists it is certain that in one of the other animals there will be found intestinal or puhmonary lesions pointing to the disease. Chronic cases of hog cholera may be mistaken for tuberculosis; a clistinction is only possible by a bacteriological examination, unless the intestines contain the characteristic ulcerations of hog cholera.

Neither the allergic eutaneous, the ophthalmo-reaction, nor the complement fixation method ean he utilized for the diagnosis of hog cholera (Uhlenhuth and others; the authors also failed to obtain satisfactory results with the second of these methods).

Course and Prognosis. The septicemic and the intestinal form of hog cholera is considered among the less fatal diseases, as it leads to recovery with relative frequency. In herds infected with the mixed infection those animals usually recover in which symptoms of a lung affection are absent or occur only in a mild form.

On the other hand the pectoral and the mixed form has a much more malignant cliaracter, but even in these cases the mortality fluctuates within wide limits. While in localities in which the disease lias appeared for the first time, the mortality, especially among the young hogs, may reach $80-90 \%$, in later rears it will be much lower. In herds which are kept under favorable hygienic conditions very frequently only $10-15 \%$ of the animals may die, and by taking proper precautions it is possible to reduce even this number.

Herds in which cholera has already caused a loss of about $15 \%$ and over usually resist later infections permanently, therefore a repeated appearance of the disease in the same herd is observed only very exceptionally.

The case reported by Tóth is probably isolated, in which in a herd of 550 hogs $18 \%$ died first, several months afterwards $32 \%$, and after another two months $12 \%$.

In giving a prognosis the character of the disease should be considered, that is the more the septicemic and the pectoral manifestations predominate, the less favorable is the prognosis. Hemorrhages from the body cavities are almost certain forerunners of approaching death; likewise the severe pneumonias terminate in most instances unfarorably. On the other hand in cases where only the intestinal affection appears to be present, the recovery of the animal is more or less certain, according to its intensity.

Treatment. At the beginning of the disease, when no complications, especially of the thoracic organs, have become associated with the affection, therefore in the first 4 or 5 days of the febrile condition, treatment with the specific immune serum frequently gives good results. Quite often a rapid recovery takes place after a single injection of serum, while in other 
cases 1 to 2 repetitions of the injection bring about recovery. (See further under immunization.)

Medicinal treatment has not given any decided results. In consideration of the supposition that the infection results from the intestinal canal, the administration of disinfectants was tried, but without much benefit. Of such remedies milk of lime, naphthol, iron sulphate, carbolic acid, calomel (2 g.), creolin ( $2 \%$ solution), arsenous acid $(0.2$ g.), and formalin milk $\left(1 / 4^{-1 / 2} \%\right)$ have been tried. According to observations such a treatment could be only considered for the purpose of protecting if possible healthy animals from the infection. (The carbolic acid enemas recommended by Trevisan, $800-1500$ g. of a $0.5 \%$ solution twice daily, has also been found effective in several cases by Kolisch).

Some authors recommended the intravenous or subcutaneous injection of antiseptics; thus Marenghi advised corrosive sublimate (1 part corrosive sublimate, 3 parts salt, 1000 parts water; 0.5 to $2 \mathrm{~g}$. of this injected into the auricular vein); Hajnal suggests lysol (subcutaneous injection of $5 \mathrm{~g}$. of a $20 \%$ alcoliolic solution) and also antipyrin; Révész chinosol (3 ce. of a $2 \%$ solution subcutaneously). The effectiveness of these treatments however has not yet been confirmed by other observers.

It is essential to place the patients in a clean airy place, and they should be given easily digestible feed; if the elinical manifestations indicate a severe infection the early slaughter of the animal should be recommended.

Prevention. Herds which are free from the disease should be protected as much as possible from infection. Animals just purchased should be kept isolated for at least three weeks. In large herds infection of the animals is not infrequently avoisc. $l$ by preventing the contact of the herd with strange hogs, especially with those belonging to the farm hands, as well as by keeping them from strange pastures and highways. Cleanliness of the piggeries and their surroundings, as well as periodical disinfection aid considerably in maintaining the health of the hogs.

If the disease has already appeared, the affected animals should be slanghtererl or left in the infected locality, while the healthy hogs should if possible be divided into small groups, and driven to uninfected places. Should new cases develop in these parts the procedure should be repeated, and the infected pasture should always be avoided. If the disease has appeared in the pulblic pasture, and if it is not possible to remove the remaining healthy animals to a clean pasture, the spread of the disease may be prevented by subjecting the animals to a continuous stabling, for at least two weeks. Of course the premises should be placed under veterinary supervision, and where the disease appears the contagion should be destroyed rapidly by early slaughter of the affected animals and thorough disinfection of the stables. 
Immunization. Practical results can be only hoped for from a method of immunization which will afford effective protection against primary infection with the filterable cholera virus. After the demonstration of the latest facts regarding the etiology of the disease by establishing the virulence of the filtered tissue fluids of affected animals, experiments were undertaken to work out a suitable method of immunization, and they have already led to valuable results.

Purely practical observations showed years ago that animals recovering from hog cholera were usually immune for their entire lives. This fact has been utilized by Hungarian hog feeders, as they prefer to feed animals from herds in which the disease has already caused some losses. As a matter of fact they pay considerably higher prices for such animals.

The idea of using serum from hogs which recovered from cholera for immunizing purposes, was first utilized by Preisz (1897). He obtained serum from a hog which hat been convalescent for three weeks after a serere attack of cholera, and in which the autopsy revealed marked lesions of cholera in the intestines anil mesenteric lymph glands. The serum was injected into 30 pigs from 3 to 4 months old, each animal being injected with 10 ce. of such serum. These 30 animals were later placed in a stable with 30 healthy pigs, and with several affected hogs. In the course of one month, 18 of the inoculated animals became sick, and 9 of them died, while all of the control animals suceumber (the last one after 4 months).

As a result of these findings immunization with blood serum from slaughtered or recovered animals has been used extensively in Hungary. The results however failed to come up to expectations, which probably was due to the fact that the material was not always taken from recovered animals which had passed through a severe form of the affection.

After the presence of the causative factor of the disease had been recognized in the filterable virus, the attempt was immediately made to hyperimmunize hog's artificially, according to the method of inmmization used against rinderpest, and thereby prepare a suitable serum for passive immunization, and by the simultaneous use of virulent blood to produce, if possible, an active immunity. These expectations have been fulfilled, insofar as satisfactory methods of immunization have been developed which are practical for both purposes.

1. Immunization with Immune Serum. (Passive Immunization.) According to the experiments of Dorset, McBryde \& Niles, Uhlenhuth, Xylander, Hübener \& Bolıtz, Ostertag and Stadie, as well as Hutyra \& Tetzl, hogs which acquired an immunity from a natural or artificial infection of hog cholera, when injected by a single large dose, or by several small doses of virulent blood, produce a serum which protects susceptible hogs for at least three weeks against artificial, as well as against natural infection. This immunity is also effective during the first six days of the incubation period (Hutyra \& TVetzl). The results in practice proved satisfactory, as with the aid of this method it is possible to considerably reduce the losses in herds which have been affected only for a short time, and in many cases to check the outbreak abruptly. Besides this, in animals which are in the stage of incubation, or which have only been affected for a few days, the serum possesses a curative action. It was found further that in infected herds the animals which 
were protected by the serum inoculation will not become affected by the disease later on, or only exceptionally, in spite of the existing dangers of infection. This leacls to the belief that the animals which were given a passive immunity by the immune serum, have acquired an active immunity as a result of a natural infection which has taken place in the meantime.

On the basis of this experience it is advisable to treat all herds which are threatened by the infection with the immune serum, and especially those in which the disease has already appeared, and to retain the drove in the same place until the infection is eradicated. At the same time the apparently affected animals should be separated, and those which are affected severely should be slaughtered. In case the disease appears again in the herd it is advisable to repeat the injection with the immune serum.

Dorset, McBryde \& Niles (1905-1907) treated immune hogs by two methods. In the "quick method" they injected one dose of 900 to 1500 ec. of virulent blood (10 ec. per pound of body weight) subcutaneously, in the "slow method" gradually increasing doses of 500 to 900 ce. ( 1 to 5 ce. per pound of body weight) of such blood at intervals of 3 to 4 weeks or even longer periods. About three weeks after the single injections, or after the last injection, as well as later at various intervals, they have drawn blood from the injected animals. The serum from such blood when obtained by defibrinating, or by allowing it to stand immunized animals weighing from 25 to 50 pounds when injected in $20 \mathrm{cc}$. doses, against a simultaneous infection of 1 to $2 \mathrm{~g}$. of virulent blood, and also against subsequent natural exposures.

Later the forced immunization was accomplisherl by injecting the hogs once or twice intravenously with $5 \mathrm{ce}$. of virulent blood per pound of body weight, or with 10 ec. intraperitoneally by a single injection.

The immune serum is used in the United States mostly for simultaneous inoculations (see below), and is only rarely employed alone. Experiments regarding the curative action of the serum showed that if the serum treatment is unilertaken not later than four days after infection, the onthreak of the disease may be prevented. According to the reports of the Bureau of Animal Industry the results from the practical application of the serum are very satisfactory.

Uhlenhuth and his co-workers (1907) produced a similarly potent serum from hogs, to which they had given several subentaneous injections of 25 to 100 ec. of virulent material, mostly blood. The serum in doses of 10 to 50 cc. protected healthy pigs from a subsequent severe, natural infection, while animals which were not inoculated, or which were given large doses of horse serum, died from hog cholera. Doses of 10 to $20 \mathrm{cc}$. also protected against the pathogenic effects of 0.5 ce. of virulent blood, when injected subcutaneously at the same time. Potent serum can be prepared only from hogs, not from horses or asses.

Practical experiments with immune sermm have heen made in various herds, most of which were severely affected, 330 out of 743 hogs being immunized while 413 were not given the treatment. The loss among the inocnlated hogs was 61 $(18.4 \%)$, among those which were not inoculated $212(51.3 \%)$. The dose of the serum was 20 to 50 ee.

Ostertag's laboratory experiments with serum of successively highly immunized hogs have also given good results. With this serum 
pigs may be protected against an infection of cholera, and Stadie \& Raebiger have obtained mostly good results in practice with his serum, testing it upon $2 t$ herds.

In Germany a serum prepared by Gans is also in use, and Spitzer, Stock and Pfeil obtained beneficial results from its application.

Hutyra \& Wetzl treat hogs weighing from $60-100 \mathrm{~kg}$. which have recovered from the disease, either with a single injection of $1000-1200$ cc. or 2-3 injections of 500-600 cc. of fresh defibrinated blood from slaughtered hogs affected with hog cholera. Ten days after the last subeutaneous injection, and at intervals of 2 to 3 weeks afterwards, they are bled from the tail, and finally they are bled to death. The blood is used for immunizing purposes, either defibrinated, or it is used after centrifugation as pure serum. Finding that the preliminary tests gave favorable results, serum immunizations have been employed regularly in Hungary since 1909, and on the whole with satisfactory results.

In the preliminary tests 285 hogs of five herds were injected once or twice with 10 ec. of filtered serum, or $15 \mathrm{cc}$. of defibrinater blood, while 240 animals were not injecterl. After placing the animals in badly infected stables, the losses among the inoculated animals in the various herds amounter to 6.1 to $35.4 \%$, among the hogs which were not inoculated 40 to $66 \%$, the difference in favor of the inoculated animals amounting to 20 to $60 \%$.

Up to October 15, 1909, reports from 106 farms, with a total of 10,661 apparently healthy hogs, gave the results of the serum treatment as follows: in 42 herils with 3,125 inoculations the disease was immerliately cheekell; in 22 herds with 3,754 inoculations the loss amounter to $1.9 \%$, in 7 herds with 730 inoculations the loss was $8.2 \%$, in 14 herds with 1611 inoculations $15.7 \%$ snceumbed, in the remaining 21 herds the losses amounted to 23.7 to $57.6 \%$. In 46 herds in which 4,189 animals were inoculated, 5,008 hogs not being given the treatment, $8.8 \%$ of the inoculated animals dier, and $30.9 \%$ of those which were not inoculated. The inocnlations were absolutely without results in only eight herds, with a total of 599 inoculated, and 659 non-inoculated animals. The losses in these cases amounted to 63.8 and $57.9 \%$, respectively.

The treatment of animals which were already manifestly diseaserl $(2,681$ cases) was in only a few cases successful and then the animals improved rery rapidly and recovered.

The recurrence of the disease in herds which har been treated previonsly with the serum, and from which the dlisease harl heen eradicated, has up to May, 1910 , been observed in only five instances. (The dose of serum was 10 ec. per aninal weighing from 20 to $40 \mathrm{~kg}$.)

Favorable results with the serum are also reported by Szurán, Szabó, Tokayer and Márton, but Szurán and Márton failed to obtain good results in one herd each where the majority of the animals were already affecter. In one instance the ontbreak was abruptly ehecked by the immunization, but 55 days later the disease recurred with great severity.

\section{Immunization with Immune Serum and Virulent Blood.} (Simultaneons Method.) In the United States this method is mostly used according to the procedure of Dorset, McBryde and Niles, the animals being injected subcutaneously with $20 \mathrm{cc}$. of immune serum, and 1-2 cc. of virulent blood. This treatment has also by our numerous observations been proven to confer an active immunity upon the animals which lasts at least six months, and probably even a longer time. Nevertheless the practical application of this method does not, at least for the present, appear to be altogether without danger, as some of the inoculated animals apparently become affected, and scatter 
the virus. Such accidents cannot be excluded at the present time, as the virulence of the blood fluctuates considerably in the affected animals, and there is no satisfactory method by which the virulence of each can be established rapidly; there is likewise no quick method for the standardization of the serum.

In 1905-1906 168 hogs were treated in the United States by the simultaneous method (2.5-20 ce. serum, ani 0.25-5.0 ce. of virulent bloorl, each separately, but simultaneously injected). Of these $35(21 \%)$ became slightly affected, while $15(9 \%)$ succumbed. On the other hand 54 hogs became affected after the injection of the same quantities of virulent blood, and $50(92.5 \%)$ of these succumbed to the disease. When the hogs which had been treated by the method described were exposed on infected premises, they remained healthy with very few exceptions, while all control animals became severely ill, and most of them died. Some experiments on suckling pigs showed that immunization may also probably be used with goorl results at a very early age (Dorset, McBryle \& Niles). Up to November, 1909, about 2,000 hogs were treated with satisfactory results, the losses among the inoculated animals in infected herils amounting to $5-15 \%$, in herds free from the disease $0 \%$, whereas among the animals which were not inoculated the losses amounted to $75-89$ and $35 \%$ (Melvin).

3. Other Methods of Immunization. The preparation of a potent serum from horses and cattle has not been successful (Uhlenhuth and Hutyra), and except for the method described above there is at the present time no procedure which has been tried in practice sufficiently.

Marxer prepared a raccine by shaking virulent hog blood at $37^{\circ} \mathrm{C}$. for four days in $10 \%$ uric acil, or for three days with $25 \%$ galactose, this vaccine proving effective in immunizing pigs in laboratory experiments. Inoculated animals lid not become infected, and failed to infect healthy pigs. For its practical applieation it is recommender to inject the animals at intervals of several months, from 3 to 4 times, with $5 \mathrm{ce}$. of the vaceine. Uhlenhuth and his associates found on the other hand that pigs treated by this method do not acquire an immitunity, and that it is not possible to establish an immunity with virus attenuated or killed by either physical or chemical means.

King employed for a vaccine the serum of horses which had shortly before been injected intravenously with $85-200 \mathrm{ce}$. of virulent hog serum. On the assumption that the virus is grailually attenuated in the body of the horse, he used the blood of a horse which was drawn six hours after the intravenous injection as a vaccine. He treated several hundred hogs by this method in practice, the results however were varied. While apparently favorable results were obtained in several herds, the disease appeared shortly after inoculation in 11 other herds which had hitherto been free from the disease.

Immunization Against the Bacillus Suipestifer. Before the establishment of the etiology of hog cholera, the immunization experinents were directed against the bacillus suipestifer, as the supposed causative factor of the disease. The practical results however proved entirely unsatisfactory in spite of the apparent goor results in laboratory tests on small animals. At present the terlious experimental investigations are only of generally scientific interest, as it appears very improbable that the treatment of hogs with a serum which, although successful against the secondary infection of the bacillus suipestifer, will ever prove of any advantage in its practical application.

Recently Stedefeder reports on several experiments in which he succeeded in immunizing pigs against the suipestifer disease, which is said to occur independently (see p. 276 ). The serum was obtained by a preparatory treatment of rabbits, horses and hogs.

Veterinary Police. The observations made in recent years have proved repeatedly that hog cholera may be limited to single premises or farms, and the losses be reduced in a great measure by the immediate application of suitable methods of 
prevention and eradication. Accordingly the veterinary police measures (compulsory reporting, isolation of affected animals, farm or town quarantine, isolation and if possible slaughtering of the affected animals, destruction of the carcasses, closing up of the hog markets, and prohibition of the peddling of animals, etc.), when applied at the right time and if energetically executed will lead to favorable results.

As hog cholera always develops as the result of the introduction of the virus, and as it is of a decidedly contagious nature, there remains no doubt that it may be eradicated from large territories by obligatory slanghter of the affected and suspected animals. This procedure, when vigorously executed, has brought good results in several places in which the disease has not become prevalent to a great extent, and where hogs were kept in small groups, and the traffic with these animals was not very extensive (for instance in Sweden and Norway). Such a method of eradication would also bring about the desired results in countries in which hog breeding is well developed, where the traffic in hogs is very extensive, and the disease has already spread considerably. Eradication based on this principle would of course necessitate a corresponding indemnity to the owners, and would also have to be aided by immunization. At the present time the most powerful obstacles in the way of such a procedure are the financial difficulties.

The erarlication has been in progress in Great Britain since 1893, and the results are indicated, at least up to 1905 , by a marked reduction in the number of cases (see p. 259).

In Austria a law of 1899 requires the obligatory slaughter of affected, exposed and suspected animals, for which an indemnity reimburses the owners. Until the end of 1901102,769 hogs were slaughtered, and the entire cost of the eradication amounted to $2,625,470$ crowns. The results of the erarlication dill not come up to expectations, as repeated introductions from foreign countries have greatly hampered it, although in recent years a diminution of the disease has been observed in the Alpine countries.

Veterinary police measures may be aided greatly by immunization, inasmuch as outbreaks may be cheeked and the spreading of the disease to surrounding herds may be prevented by serum inoculations.

In the United States a procedure is follower to a great extent; in which the newly infected herds are treated with serum, and the droves of the immediate vieinity which are not yet infected are inoculaterl by the simultaneous method. At the same time further extension is prevented by quarantine of the infected heris, slaughter of the affected animals, and the disinfection of the premises (Melvin).

Literature. Ip to the year 1905 Joest, Hog Cholera and Swine Plagne, Jena, 1906.-De Schweinitz \& Dorset, Bureau of Animal Industry, XX, Rep., 1903. 157.-Dorset, Bolton \& McBryde, Ibiu., XXI, Rep., 1904, 138; Ibid. Bull. Nr., 102, 1908.-Clintock, Boxmeyer \& Siffer, Journ. of Diseases, 1905, II, 351.-Hottinger, Schw. A., 1905, XVII, 255.-Hutyra, B. t. W., 1906, 607.-Ostertag, Ibiu., p. 623.Ostertag \& Stadie, Z. f. Infkr., 1907, II, 113 u. 425.-Theiler, F. d. Yhyg., 1906, IV, 121.-Glässer, D. t. W., 1907, 617.-Lourens, Zbl. f. Bakt., 1907, XLIV, 420.Thlenhuth, Xylander, Hïliener \& Bohtz, Arb. ג. G.-A., 1908, XXVII. 1 (Lit.): 1909, XXX, 217 (Lit.).-Marxer, B. t. W., 1908, 401.-Hutyra \& Wetzl., Z. $\mathbf{c}$ 
Infkıkh., 1909, VI, 1; B. t. W., 1909, 863.-Stailie, B. t. W., 1909, 113.-Stedefeder, D. t. W., $1909,546$.

\section{African Horse-Sickness. Pestis equorum.}

(S̈̈dafrikanische Pferdesterbe, [German]; Peste du cheval, [French]; Paardenziekte, Perrezielte, [Holland].)

African horse-sickness is an acute or subacute, infectious disease of solipeds, caused by an ultravisible virus. It exists in an epizootic form in Africa, and is characterized by extensive edematons swellings, and hemorrhages of the internal organs.

History. The disease, which has been known in South Africa for over a century, has in recent times been confused either with anthrax (Lambert, Sander), or with piroplasmosis (Rickmann, Edington). Both views were abandoned since McFadyean (1900), and later Nocard (1901), proved that the virus passes through the porcelain filter. The disease has been studied especially by Theiler, from its etiological and clinical standpoints, while the question of immunization has also been investigated by Edington, Koeh and Rickmann.

Occurrence. The disease occurs annually in South Africa during the rainy, warmer months, January to March, especially in low, moist localities. In years with great precipitation the disease rages among horses and mules to such an extent that in the affected localities $35 \%$ of the animals succumb, and some of the localities are devastated to such a degree that not a single horse remains alive unless some of the animals have previously withstood the disease in some other place (has been "salted").

In the Cape Colony 64,850 horses and mules perisherl from horse-sickness in 1854 and 1855. Since that time the annual losses are estimated at 14,000 animals. In German Southwest Africa the losses amounted in some years to $66 \%$, in Rhodesia to as much as $90 \%$ of the entire number of animals.

According to Memmo and Brumpt the disease occurs also in Abyssinia, and according to Friedrichsen in East Africa.

Etiology. The causative factor of the disease belongs to the ultra-microscopical micro-organisms, as the filtrate of the blood diluted with physiological salt solution (Berkefeld or Chamberland $\mathrm{B}$ and $\mathrm{F}$ filters) proves infections. Blood taken from affected horses in any stage of the disease, and injected subcutaneously, intratracheally, intravenously or into the lungs (0.01-1.0 ce., or only 0.0005 ce.) or administered per os (100-150.0 cc.), produces the disease in the horse. The exudates and the bronchial secretions also contain the virus, but the infectiousness of the excrement is considered to be doubtful. Blood from recovered animals has no infectious action.

Horses are highly susceptible to artificial infections, mules and asses to a lesser extent. According to Edington it is also possible to infect cattle fatally, and to produce febrile reactions in goats and sheep. Theiler \& Stockman found, however, that 
this is only possible in young Angora goats, their blood becoming infectious to lorses and mules, but not for other goats. On the other hand they succeeded by intravenous injections of large quantities of unfiltered horse blood, to cause severe illness in dogs, and to infect other dogs as well as horses with their blood; McFadyean however failed to observe any manifestations of disease in three dogs after subcutaneous injections of small quantities of virus. Man is not susceptible, as has been established by Rickmann and Kaestner through subcutaneous injections on their own bodies.

Edington and Morton Coutts consider horse sickness and heart water (see p. 257) as identical, or at least as closely related diseases. They succeeded in producing in horses a disease resembling horse sickness, with the virus of goats affected with heart water. The virus of this disease however possessed only a slight virulence for horses. This view is disputed by Theiler and Stockman, as their experiments have shown that goats treated with the virus of horse sickness remain susceptible for heart water, and likewise horses inoculated with heart water blood may later become affected with horse sickness.

Whether there exists a close relation between horse sickness and infectious anemia (see that disease), in the sense that the anemia represents a mild form of horse sickness, is not conclusively established, and requires closer investigation.

Natural infection occurs in the periods of the year mentioned, usually out of doors and at night; keeping the horses in the same places in day time from the time when the dew dries until sunset, as well as during rainy weather, is not dangerous for them. The first frost usually terminates the disease, so that from the eighth day following it no more cases occur (in dry weather the disease occurs only very exceptionally). Although the disease may be produced by feeding great quantities of virus, the observation that in the dangerons pastures horses are not protected by muzzles, indicates that the infection is transmitted by some insect or fly. According to Pitchford anopheles mosquitoes and stegomya which have been within 48 hours upon the body of an affected animal serve to transmit the disease to healthy horses; Reinecke succeeded by a test in Germany in producing a fatal disease in one horse by subcutaneons injections with an extract from ticks which he had collected in the African horse-sickness district one year before. This may explain why smoke near the horses (Theiler), or keeping them in a stable protected by a net (Pitchford), prevents the disease.

Anatomical Changes. The autopsy reveals in locations varying from case to case, a gelatinous infiltration of the subcutaneous and intermuscular connective tissue, especially around the eyes and the region of the throat. There is acute swelling of the superficial lymph glands, severe catarrhal swelling of the mucous membrane of the stomach, the anterior portion of the small intestines and occasionally also of the large 
intestines. The intestines may also show hemorrhages and ulcerations, and in about two-thirds of the cases a well marked, acute edema of the lungs with serous infiltration of the interlobular connective tissue; sometimes gelatinous pseudo-membranes will be-found on the pleura, and hemorrhages under the peri- and endocardium, as well as in the heart muscle. The spleen is normal or only slightly enlarged, the liver hyperemic, the kidneys show hyperemia, or an acute inflammation with small hemorrhages.

Symptoms. Horse-sickness usually develops after a period of incubation averaging 6 to 7 days in two forms (Theiler).

In the acute cases (Dunkop-Paardenziekte, Dun-Perreziekte, pulmonal form) the body temperature rises within a few days up to $40-42^{\circ}$. The patients show corresponding signs of feeling ill, although the appetite remains unimpaired for a long time. At the height of the disease, towards the end of the first week, the animals are strikingly feeble, the pulse is frequent and small, the heart throbbing, the respiration accelerated and labored. In a small number of cases improvement and subsequent recovery take place in the course of the second week, the fever diminishing. In most cases however at this time, 1 to 2 days before death, manifestations of edema of the lungs develop, as a result of which the animals show very labored respiration, at the same time passing great quantities of a yellowish-white foam from mouth and nose, which is also expelled by spasmodic coughing. Swelling of the superficial lymph glands may also be observed, and frequent manifestations of metritis (Friedrichsen). In rare cases the course takes a sudden unfavorable turn, after slight prodromal febrile manifestations lasting 4 to 6 days. The condition of the animal becoming worse from hour to hour, the temperature drops and death ensues inside of from 4 to 6 hours with symptoms of dyspnea and heart weakness (Theiler's peracute septicemic form).

In the subacute form (Dikkop-Paardenziekte, Dikkopziekte) the disease develops in a similar manner, although considerably more slowly. At the height of the fever, or possibly somewhat later, the parts surrounding the orbits become edematously swollen, and at the same time an exophthalmia develops; then in some cases edematous swellings appear on the forehead, on the head, on the chest and abdomen, on the back and on the extremities, and sometimes even the tongue is swollen to a great extent (blue tongue). In the meantime marked muscular weakness is noticeable in the animals, in the majority of cases however recovery finally takes place.

Frei demonstrated by extensive physical-chemical blood and serum examinations the following differences from normal blood: the volume of red blood corpuseles and the viscosity is increased at the height of the disease, later diminished; specific gravity, inside friction, and 
conductivity of the serum is always below the normal average. Similar changes are also present in immune horses.

The course of the aeute and peracute horse-sickness is usually unfavorable, inasmuch as horses, espeeially those brought from non-infected territories, frequently suecumb almost without exception, while the subacute form runs a much milder course.

The disease is distinguished from anthrax by its slower course, and especially by the absence of the pronounced, acute enlargement of the spleen; from piroplasmosis, by the absence of icterus, by the edematous swellings, the severe inflammation of the left half of the stomach and by its oceurring only at certain periods of the year. Microscopical examination of the blood in the diseases mentioned gives positive results, however the presence of piroplasmas does not exclude horse sickness.

Prevention. The treatment attempted up to the present time has generally proved ineffective. In order to prevent the disease, horses are in the summer driven to high pastures, which are known to be non-infections, and in suspected places they are allowed to pasture only in day time. By persistent breeding of immune lorses and mules it will probably be possible to produce resistant breeds in the infected localities (Rickmann).

Immunization. Recovery from the disease reduces the susceptibility of the animals, but does not entirely eliminate it, as even comparatively immune ("salted," "gezouten") horses may become affected by a virulent infection (Theiler). Nevertheless this acquired immunity protects the animals from natural infection, in about two-thirds of the cases, and when they do become affected the course of the disease is much milder.

Immunity may be produced artificially in various ways, but only in mules without great losses. In these animals immunization has been applied in practice in South Africa for several years, but it must be repeated annually, as the immunity gradually vanishes.

Koch's Method of Immunization. Koch obtained a serum from "salted horses" which were injected subcutaneously and intravenously with increased doses (up to $2000 \mathrm{ce}$.) of virulent blood at intervals of from 3 to 4 weeks, which produced passive immunity on being injected into healthy horses without exerting a hemolytic action, as was the case with sera prepared by other methods. Further experiments showed that by repeated injections of virulent blood and then of serum horses may be actively immunized. The quantity of virus and serum must be proportioned in such a way that the animals become affected by the disease without dying of it.

It was found most effective to make an inoculation of 0.01 ce. of virus and $100 \mathrm{cc}$. of serum for the first injection, the dose of the virus to be increased to 0.05 and $0.2 \mathrm{ce}$, at the same time redncing the serum to $50 \mathrm{cc}$. and finally continuing the inoculations with the pure virus $(0.5,1.0,2.0$ cc.). The serum is 
always injected four days after the virus, while each subsequent inoculation is made 12 days after the last. In $1 \frac{1}{2}$ to 2 months the horses may be immunized to such an extent that they withstand $5 \mathrm{cc}$. of virulent blood without any reaction.

The blood necessary for the immunization is taken from affected animals shortly before death, it is defibrinated, filtered, mixed with an equal quantity of glycerin and water, and 5:1000 carbolic acid, and allowed to stand from 2 to 3 weeks. Afier repeated filtration it is stored in small bottles. Immediately before inoculation the required quantity of virus is diluted with 5 ec. of physiological salt solution.

For the preparation of the immune serum older (salted) horses are injected in the jugular with fresh defibrinated virulent blood at two-weekly intervals (the first time 2,000 ce., later somewhat smaller doses). The blood, which is subsequently drawn, produces the sermm, and is preserved by Ehrlich's method (to 90 parts serum 10 parts of a mixture of 5.5 parts earbolic acid, 20 parts glycerin and 74.5 parts of water is added).

Theiler's Method of Immunization. According to Theiler the safest immunization consists in the injection of immune serum (300 ec.) into the jugular, and virulent blood (1-2 ec.) under the skin; after the appearance of the febrile reaction a second serum injection (50-100 ec.) appears indicated.

In the Transvaal and Rhodesia 8,766 mules were treated by this method, with a loss of $3.7 \%$ from the inoculation. Subsequently only $1.3 \%$ fell victim to the disease from natural infection in affected territories. As it has since been found that serum prepared with a certain virus is sufficiently effective, only against this virus, Theiler recommends the preparation of a polyvalent immune serum.

Other Methods of Immunization. Rickmann employs material originating from mules which are first injected subeutaneously with 0.1 ce. of virulent blood, three days later with 100-200 ce. of serum, and after 12 to 14 days again with 1 ec. of virus (ineubation method).

A second method consists in the simultaneous but separate subcutaneous injection of 0.2 ce. of virns, and $200 \mathrm{ce}$. of serum, and 21 days later the subserquent subeutaneous inoculation of 25 ce. of virus (simultaneous method).

According to Reinecke of 280 mules treated by the first method, and of 160 injected by the second method $8 \%$ died, and the percentage being somewhat higher in the simultaneous than in the incubation method. Practically all of the inoculated animals remained well, while the losses among the mules which were not inoculated, and kept at the same place, amounted to $3.3 \%$ as a result of natural infection.

Leipziger gives for the first dose subcutaneous injections of 1 ce. of virus, and $300 \mathrm{ce}$. of highly potent serum; for the second dose $20 \mathrm{ec}$. of virus. The losses from the inoculations amounted to $3.5 \%$. For the immunization of horses he recommends first the subcutaneous simultaneous method, with 0.1 ec. of virus and $400 \mathrm{cc}$. of serum; three weeks later an injection of $0.3 \mathrm{cc}$. of virus and 100-200 ec. of serum; then the animals should be injected daily, first subeutaneously, later intravenously, with diminishing quantities (down to 0.01 ce.) and later again with increasing quantities of virus, until a reaction takes place.

Literature. Theiler, Schw. A., 1893, 145; D. t. W., 1901, 209; Bull. P., 1905, III, 617 ; Rep. of the Gov. Vet. Bact., 1905-1908.-Edington, The Vet., 1895, XLI, 595; J. of comp. Path., 1900, XIII, 223 u. 281.-Rickmann, B. t. W., 1895, 289; A. f. Tk., 1907, XXXIII, 372; B. t. W., 1908, 883.-Sander, A. f. Tk., 1896, XXI, 249.-MacFadyean, J. of comp. Path., 1900, XIII, 1; 1901, XIV, 103.-Nocard, Bull., 1901, 37.-Koch, A. f. Tk., 1905, XXXI, 330.-Reinecke, Diss. Bern, 1909 (Lit.) ; Z. f. Vk., 1910, 76.-Leipziger, Diss. Bern, 1909 (Lit.).-Frei, Z. f. Infkr., 1909 , VI, 363.

Vol. 1-19 


\section{Fowl Pest. Pestis avium.}

(Pestis gallinarum, Typhus exsudativus gallinarum Rrvolta \&

Delprato, Peritonitis epizootica Scheuerlen \& Buhl, Kyanolophiaea Lode \& Gruber; Peste aviaire, [French]; Geflïgelpesi, [German].)

Fowl pest is an acute, contagious, infections disease of fowls, especially of geese, which in its course greatly resembles chicken cholera; it is however caused by an ultra-microscopical virus.

History. The disease was first described in 1878 by Perroncito, and was shortly afterwards recognized as a disease differing from fowl cholera by Rivolta \& Delprato (1880). Later on Italian authors treated the disease repeatedly as a dangerous plague, which caused great losses especially in Northern Italy. Centanni \& Savonuzzi (1901) proved that the virus passes the porcelain filter, and similar results were also obtained by Maggiore \& Valenti, as well as by Lode \& Gruber, Ostertag \& Wolffhügel, Maue and others. Recently Kleine, Möller, Rosenthal and Schiffmann established the occurrence of the disease in geese, Prowazek, Kraus \& Doerr, as well as Marchoux contributed to its etiology, and Freese to its pathological anatomy.

Occurrence. The disease raged, especially since the year 1894, independently of chicken cholera, as a very fatal plague in Northern Italy, from which region it was recently introduced into Tyrol and Germany, and later into Belgium and France.

In Southern Germany it appears to have occurred since 1898 (Schenerlen \& Buhl), but was spread more extensively by the Brunswick fowl exposition in 1891, at first in Württemberg, later in the Northern states, especially in Prussia. In 1908 the disease was officially recognized in Germany in 51 townships, affecting 1,826 chickens, 159 geese and 3 ducks (considerably less than in previons years). In Hungary it has leeen known since 1903.

Marcone observed the disease in Capodimonte in a pheasantry, Eggebrecht in Tsingtau as a severe plague of chickens.

Etiology. The infective agent is contained in the blood, in the nervous system, in the nasal secretion and in the droppings, as well as in the exudate of the serous cavities and in the bile of the affected animals. By the inoculation of minimal quantities of these substances (for instance $0.000,001$ ce. of blood), the disease may be positively transmitted to chickens. Similarly effective, although in a lesser degree, are the filtrates of the above mentioned substances through porcelain.

According to Landsteiner the virus appears to arlhere to the blood corpuseles as serum alone proved only slightly or not at all virulent. Rosenthal, Kleine and Schiffmann found in the brain, intra- and extra-cellular, round or oval, sometimes also ring-shaped bodies, the true nature of which (protozoa or products of degeneration?) has not been established at the present time. Prowazek could not confirm these findings, and believes that the carriers of the virus are 1 to $1.5 \mu$ sized, sometimes dumb-bell shaped, forms in the brain, which frequently lie elosely against the red blood corpuseles; further fine single and double granules in the filtrates. Marchoux reports the successful eultivation of the virus by spreading blood on peptone-agar containing sugar; $0.2 \mathrm{cc}$. of culture of the 10th generation killed a chicken in two days. 
Tenacity. Blood placed in glass tubes, the ends of which were closed by melting, and kept in a dark place, retained its virulence for three nonths, while the filtrate was virulent for only one week. Heating to $55^{\circ}$ destroys the virus within a half hour, at $60^{\circ}$ in 5 minutes, at $65^{\circ}$ immediately (Centanni). According to Maue the virus remains active in dried spinal cord, or liver substance, for 200

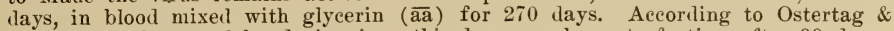
Bugge it is destroyed by drying in a thin layer, or by putrefaction after 39 days; on being subjected to a teniperature of $70^{\circ}$ it is killed instantly. A $1 \%$ corrosive sublimate solution destroys it in 30 , a $1 \%$ sulphuric acid and a $3 \%$ chlorate of lime solution in 10 minutes (Lode). A hot $2 \%$ soda solution, as well as a hot $5 \%$ creolin solution are also effective (Ostertag \& Bugge).

Pathogenicity. Chickens and turkeys, pheasants, sparrows, black-birds, sparrow-hawks, owls and parrots are susceptible to the virus, while water fowls and pigeons as a rule resist the natural, and frequently also the artificial infection; mammalia are not at all susceptible.

Old geese can be infected only by subdural inoculations, those only half a year old however by subcutaneous or intramuscular inoculations, as well as per os. In these birds the disease manifests itself principally in nervous symptoms (see symptoms). Lode \& Gruber and Centanni succeeded in infecting pigeons only by repeated injections of large quantities of virus, Maggiore \& Valenti only after previous starvation with blood injections; Freese however produced the disease in them by letting them live with ehickens. Suceessful infections in ducks are reported by Leclainche, and spontaneous infection of a parrot by Stazzi.

The natural infection is probably transmitted mostly by the droppings and the nasal secretion of affected birls, and also by the blood of killed animals and by the organs of those dead from the disease, which are ingested with the food by healthy chickens (by feeding such material the disease has been produced artificially). Oceasionally the virus may enter the borly through injuries of the skin; at least it was found that the disease may be produced artificially very easily and positively in this manner (it is sufficient to dip the needle of a syringe into infected blood, and slightly scarify the skin with it; Kleine \& Möllers snceeded in producing the disease by dropping the virus into the conjunctival sac).

Parasites living on affected fowls (Dermanyssus avium) cannot transmit the disease, and it cannot be even produced by subeutaneous injections of such macerated parasites (Centanni).

Anatomical Changes. In very acute cases the autopsy sometimes reveals negative findings, or frequently only punctiform hemorrhages on the inner surface of the breast bone, on the visceral layer of the pericardium, on the peritoneum, in the fat tissue of the gizzard, and on its peritoneal attachment, as well as in the mucous membrane of the air passages. There may also be hyperemia of the spleen and of the kidneys. In less acute cases there is an edematous swelling of the subcutaneous connective tissue on the neck and breast, also a great amount of a pale yellow, slightly cloudy fluid in the pericardium, which rapidly coagulates in the air. The layers of the pericardium sometimes appear to be adherent by fine fibrinous threads. Italian authors mention as a frequent finding a fibrinous exudate on the pleura and peritoneum (therefore the name of "Typhus exudativus"').

The comb and the wattles are dark, bluish-red, the conjunctivae show catarrhal swelling. There is a considerable 
quantity of mucus in the mouth and in the nose, which is sometimes bloody. The lungs are congested and sometimes contain atelectatic areas.

The intestinal tract contains on the smooth micous membrane grayish-white, sometimes greenish excrement; at the passage between the glandular and muscular stomach, as well as between and on the conical protuberances of the glandular stomach, there are superficial or deep hemorrhages, sometimes only very small (Freese). The mucous membrane at the beginning of the small intestines shows sometimes only catarrhal reddening, in other cases it is inflamed more markedly. The liver and spleen show no striking changes. The kidneys are either grayish-brown in color and cloudy or dark brown and moist on the cut surface. The serous membrane of the egg yolk is markedly congested, revealing sometimes hemorrhages which may also be present in the yolk itself (Freese).

Symptoms. The time of incubation in the natural infection is, as a rule, 3 to 5 days, exceptionally 2, or on the contrary it may be even 7 days (Freese), while after artificial infection deatl ensues not infrequently inside of 36 to 48 hours.

The disease commences with depression and diminished appetite, which condition soon changes to a conspicuous dullness and sleepiness. The chickens creep into dark corners, and sit

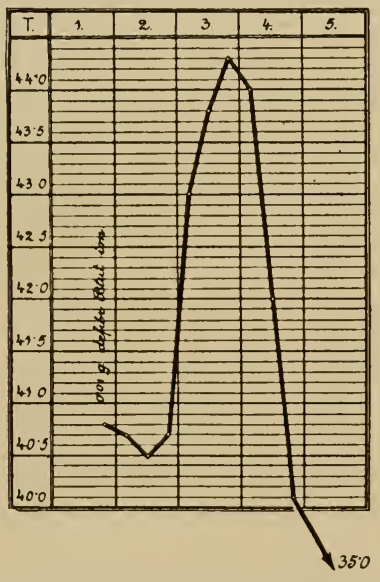

Fig. 53. Fever curve in chicken pest Following inoculation of virulent blood with drooping liead and wings as when brooding, or crouch motionless in one place. Even at the onset of the disease they do not resist being caught, and some of the birds give a peculiar loud cry when picked up. When driven about they move with a staggering, irregular walk, later it is hardly possible for them to move at all. Setting them on the perch they find their equilibrium only after long balancing, or not at all.

In the meantime the comb and the wattles become dark red, and finally are blackishred in color (hence the name Kyanolopliiaea), while towards the termination of the disease grayish scales sometimes develop on the skin. A similar discoloration is also observed around the eyes, while the eyelids are usually closed, tears accumulating under them profusely, the conjunctivae being injected and swollen.

On pressure of the bill a grayish or reddish, tenacious mucus oozes from the opening, and a similar secretion is also 
observed in the throat, while on the buccal mucous membrane small hemorrhages may sometimes be found. Some patients periodically shake their heads, at the same time giving a peculiar sound, and expelling saliva from nose and mouth. In rare cases profuse diarrhea is observed, the droppings being dirty gray or greenish, fluid and exceptionally red colored.

Towards the end of the disease the affected birds hold their heads turned toward the back, their respiration is very labored, and finally paralysis appears, which progresses from the extremities to the head.

In artificial infection the body temperature commences to rise after 12 hours, and later may exceed $44^{\circ} \mathrm{C}$. At the approach of death however it drops rapidly, even to $30^{\circ}$ (Fig. 53).

Maggiora \& Valenti describe a diphtheroid form of the disease, in which a muco-fibrinous exudate forms on the surface of the swollen, bright red mucous membrane of the mouth, throat and nose.

The same authors observed in several affected chickens peculiar nervous symptoms in addition to irregularity in walk, and symptoms of paralysis, which were manifested by moving the head in a circle; Centanni has seen disturbances in the equilibrium, lasting for weeks in pigeons which were inoculated with blood, especially turning the head, moving in a circle, and a desire to turn the body around its long or transverse axis. The examination revealed as the cause of these disturbances an exudative inflammation of the membranous, semi-circular canals of the organs of hearing (semicirculitis specifiea). Calamida has also observed the turning of the head hackwards, and besides, shortly before death, attacks of delirium which somewhat resemble rabies, the birds jumping very high during these attacks.

In artificially infected geese the disease runs a slower course (an average of 7 days), and as a rule under manifestations of striking nervous symptoms, which are indicated by tonic-clonic spasms, involuntary movements, fluttering of the wings, spasmodic extension of the neck, increased reflex irritability, etc. At the approach of death the symptoms pass into paralysis. The blood of these birds, as well as of artificially infected pigeons, is infectious only at the beginning of the affection, while later the virus disappears entirely. The brain and spinal cord, on the other hand, remain highly infective even after death (Kleine). This may be explained by the fact that the virus emigrates from the blood into the nervous cellular elements, and becomes anchored there as a result of its specific affinity for the cell plasma. This would correspond with the histological findings (proliferation of round cells, the above-mentioned bodies) as well as with the findings of Landsteiner, according to which the virus is, in contrast to bacteria, quickly destroyed by a $1 \%$ saponin solution.

Kleine observed atrophic areas in the eye ground of a chicken, and various kinds of chorio-retinitic areas in geese.

Course. The course of the disease is 2 to 4 days as a rule with the exception of the very rapidly terminating cases, and only exceptionally it extends to 7 or 8 days. The termination appears to be fatal in the great majority of cases.

Diagnosis. The disease greatly resembles chicken cholera in its course, and is only distinguished from it in that in this 
disease, at least in the somewhat protracted cases, a bloody diarrhea develops quite frequently. The cyanosis of the comb and of the wattles is an indication of pest, but does not exclude cholera, as it is also frequently present in this disease. In the autopsy decided sub-epicardial hemorrhages, fibrinous deposits on the heart, severe hemorrhagic inflammation of the intestines, and pnemmonic areas are indications of cholera, and speak against the presence of pest. The differentiation is only certain by bacteriological examination. In pest no bacteria can be demonstrated in the blood of fresh carcasses, either by a microscopical examination or by cultivation, although the fresh blood, as well as the filtrate through porcelain is virulent, but only for chickens; whereas the virus of cholera which can be shown microscopically and also by cultivation, is also patl:ogenic for other species of fowls, especially pigeons, and likewise for rodents.

Treatment and Prevention. The treatment of affected animals has thus far proven ineffective.

In the prevention of the disease the same measures are indicated as were given for fowl cholera (see p. 94), only that in chicken pest the chickens should be especially protected against the infection. Germany, Austria and Hungary have recently adopted official measures against the disease, which in their nature are similar to those established against cholera.

According to Maggiora \& Valenti serum of recovered geese which were subsequently treated with virulent blood, is supposed to protect chickens effectively against the disease, and is also said to possess a curative action. According to Ostertag \& Bugge the blood of chickens which were treated in a similar manner only renders the virus ineffective when given in large doses (10 ce.). According to Kraus \& Schiffmann, dried $\left(\right.$ at $22^{\circ}$ ) spinal cords from geese which were inoculated intramuscularly, protected young geese against a similar infection; on the other hand geese may be actively immunized with virulent spinal cords from chickens against a fatal subdural infection.

Literature. For literature see Freese, D. t. W., 1908, 173.-Also Maue, Arb. d. G.-A., 1904, XXI, 537.-Rosenthal, Cbl. f. Bakt., 1905, XL, 204.-Landsteiner, Cbl. f. Bakt. Ref., 1906, XXXVIII, 540.-Kraus \& Schiffmann, Ibid., 1907, XLIII, 825.-Schiffmann, Ibid., 1907, XLV, 393.-Prowazek, M. m. W., 1908, 165, and 1016.-Kraus \& Doerr, Cbl. f. Bakt., 1908, XLVI, 709.-Marchoux, C. R., 1908, CXLVII, 357. 
Group II.

\section{ACUTE EXANTHEMATOUS INFECTIOUS DISEASES}

\section{Pox. Variola.}

\section{(Pockenkrankheit [German]; Tariole [French]; Vajuolo [Italian].)}

As pox are designated acute febrile, infectious diseases, which usually take a typical course and are characterized by a peculiar vesico-pustular exanthema which develops on the skin, either over the entire surface of the body or only over certain parts. In its first form the disease occurs in man, in sheep, and, although more rarely, in goats and hogs; in cattle and horses it is almost invariably observed only as a benign local affection.

Accordingly only sheep pox, and to some extent also pox of goats and swine, are of any economic importance, inasmuch as sheep pox usually occurs in an epizootic form and eauses notable losses.

Etiology. All that is known at the present time regarding the virus of variola in the various species of animals and in man, is that in filtration of the contents of the vesicles under high pressure it passes through moderately dense porcelain filters, and therefore probably belongs to the ultra-microscopical micro-organisms.

The very numerous bacteriological investigations which were made principally with the infectious material from human and from cow pox, have up to the present time failed to give positive results. Formerly the causative agent of the disease was believed to be a bacterium (Kleber, Simon, Hallier, Cohn, Tonssaint, Klebs, Voigt, Garré, MIarrotta and others), in recent times it has been considered as a protozoon. Pfeiffer \& Rieck observed in the lymph of the vesieles, spherical bodies containing a nucleus, and of a greenish lustrous color, with a movement somewhat resembling that of amoebae. They considered these hodies as parasites belonging to the species of protozoa, or to the class of monocystidea, naming them sporidium vaccinale. Funck on the other hand considers them as spores of these parasites, while Guarnieri attributes the cansative role to certain contents of epithelial cells (Cytorhyctes vaccinae et variolae), which he believed to have cultivated on the cornea of rabbits; the same view is also accepted by Siegel. Other authors however consider them as parts of cells, or as remains of polynuclear leucocytes which have penetrated into the epithelial cells (Salmon, London, Schrumpf, Mïhlens \& Hartmann). Calmette \& Guérin are inclined to regard as the causative factor of pox, extremely 
fine granules which they observe in the lymph with highly magnifying lenses, and Bose describes extremely small granules in the vaccinia as well as in the ovina, which gradually become larger, and after the appearance of a nucleus in their bodies divide by schizogony, the nucleus breaking up into fine granules (merozoites).

The findings of Prowazek are similar (1905). According to these, the vaccinia contains as characteristic constituents in the smallest epithelial fragments elongated motile bodies. These stain with hematoxylin, and consist mostly of two corpuscles (lymph corpuscles) slightly varying in size, and contain in their structureless substance one to two enclosures, $1-11 / 2^{\mu}$. in size, exceptionally punctiform, mostly however rod or elub-shaped, so called initial bodies. They are also found in the cornea of rabbits after inoculation with vaccine. In sueh cases they are present within and without the Guarnieri bodies, and their development may be explained by the fact that the nucleus of the epithelial cell reacts with the production of vaccine bodies to the invasion of the initial body, which should be considered as the carrier of the pox virus. According to these findings, which were confirmed by Volpino, the pox virus would belong to Prowazek's so called chlamydozoa.

The spirochetes which were described by Bonhoff as the causative factors of pox (sp. vaccinae) were considered by Süpfle, ('arini, Mühlens \& Hartmann as artifacts.

The bacteriological examination gave positive results only insofar as it showed that the pustules of pox always contain pyogenic baeteria; thus Garré found in human pox the streptococcus pyogenes, Guttman the staphylococcus pyogenes aureus, Grigorjew the same'and the staph. pyog. eitreus. These, and probably also other bacteria (for instance the bacillus necrophorus) however play undoubtedly only a subordinate or secondary role, as compared to that of the true, as yet unknown cause of pox.

The contagion of pox is contained in the lymph of the nodules and vesicles, and also in the scabs which develop in place of the latter, while the infectionsness of the blood, and of the exhaled air, and also of the skin exhalations of affected animals during the fever period is doubtful. The secretions and excretions contain the infectious material only in those instances in which they become mixed with the virulent contents of the vesicles.

Pathogenicity. After inoculating infectious material into the skin between the cells of the epithelial layer, characteristic pox developed at the point of inoculation in susceptible individuals, sometimes however the inoculation results in a general eruption of pox. This may sometimes also be produced by intravenous or intra-tracheal injections of pox lymph.

Pathogenesis. The epithelial layers of the skin and of the mucous membrane are the most favorable places for the propagation of the pox virus. If this is inoculated or rubbed into these parts it enters directly between the epithelial cells and if the infection occurs by inhalation the virus reaches the same 
localization by transmission with the blood stream. In these parts the virus propagates, and causes the development of the characteristic exanthema of pox. The epithelial cells in the stratum mucosum swell, changing to pale flakes without nucleus, and then dissolve completely in the serous fluid. Some of these cells resist this process, and are under the pressure of the accumulating exudate drawn out to fine threads which then form a loose network in the inside of the vesicle. At this stage the contents of the vesicle are enclosed, on the inside by the papillary body, which may be still covered by a thin layer of epithelium, or may be entirely denuded, and at the same time is infiltrated with small round cells. From the outside it is closed by the corneal layer. Later a navel-like depression (pit) forms on the top of the vesicle, the development of which is explained in varions ways. According to Rindfleisch the depression in the covering of the resicle is caused by the horny layer of the epidermis of the hair follicle, or the excretory duct of the sireat gland around which the pox is arranged in a concentric manner. According to the view of Virchow and Weigert the crater-like depression is formed because the central necrotic part of the pox, which does not take part in the expansion, acts as a retinaculum of the covering of the vesicle, while the progressive accumulation of pus extends the periphery of the pox. As a result of the migration of leucocytes the contents of the vessels become purulent, and at the same time the network in the inside undergoes dissolution. Finally a scab forms, owing to the drving of the contents under which the corium becomes covered by fresh epithelium; but if the inflammation extended to the deeper parts, a scarred depression remains at the place of the destroyed papilia.

This peculiar pathological process develops to maturity as the result of the pathogenic action of the pox virus itself, while the subsequent suppuration of the contents of the vesicles is produced by pyogenic bacteria, which probably penetrate from the glandular duct and the hair follicles of the skin, into the inside of the vesicles. According to their nature and virulence the inflammatory process may be more or less intensely influenced by them. Especially the occasional hemorrhages and the deeper destruction of the corium, as well as the subsequent pyemia or septicemia should be considered only as accidental infections, and therefore the mild or severe character of different outbreaks also depends on such secondary infection. At places which are protected from this the virus of pox propagates without foreign contamination, and in such cases, as for instance in the hard nodules which sometimes occur under the skin of sheep, the contents consist of virulent pox lymph which is free of pyogenic bacteria.

Whether the eruptions of pox remain local or become generalized depends, aside from the susceptibility of the respective animal species, upon the virulence of the virus and 
on the method of infection, as well as to some extent upon the condition of the epithelial layer. The skin inoculation, even if effected with strong virus, produces as a rule only a local eruption, as in such cases the virus propagates only at the point of inoculation. A portion of the virus may later be absorbed into the circulation, and he disseminated by the blood to other parts of the skin and mucous membranes, but in these places it is no longer capable of exerting a specific action, as the hody has already been immunized dnring the development of the local eruption (Chanveau). If however inhaled virus enter's the lumgs, or if it is injected directly into the blood circulation, it is convered by the blood stream, to the papillae of the skin, as well as to the tissue of the mucous membranes, where it produces a generalized eruption of pox. The infection is farored by injury of the epithelium, or other pathological conditions (Ċalmette \& Guérin), but the exanthema may develop in the absence of any lesions.

Of course deviations from the rule may occur. If in skin inoculations very virulent or massive virus enters the tissue, or if the local exanthema produced by the inoculation develops slowly, a generalized affection may result, even after cutaneous or subcutaneous inoculation. On the other hand if only a very small quantity, or very weak virus enters directly into the blood circulation, the pox eruptions develop only in a few places or not at all.

As the virus of variola does not propagate in the blood, the blood of affected animals is not, or only exceptionally, infections. It is infectious only when the virus circulates in the blood immediately before the development of generalized pox eruptions. In this manner the fact may be also explained that the excrements and secretions are non-infectious; the milk, however, is sometimes an exception, namely, in those instances when pox develops in the milk ducts, the contents of which then become mixed with the milk.

Immunity. Recovery from pox produces in man and in animals, an immunity which lasts for several years, whether the infection was produced naturally or artificially. The immunity derelops in a generalized pox eruption in the same manner as after a local eruption from inoculation (according to Chaureau a re-infection can not be produced after the fifth day of the development of the pox), and further a pox exanthema is not absolutely necessary for the production of immunity, as calves may be immmized by intravenous injections of vaccine (Strausz, Chambon \& Ménard), rabbits by an inoculation into the lungs or into the trachea (Calmette \& Guérin), and sheep by intravenous injections of ovine (Semmer \& Raupach), although in such cases the infection does not produce manifestations of variola.

Regarding the possibility of reciprocal immunization in the 
different forms of pox, experiments prove that horses and cattle may be immunized with variola against vaccinia, man with vaccinia against the variola; but a similar reciprocal action of vaccinia and ovina is very questionable.

The mode of development of the immunity is not yet sufficiently known. According to Béclère, Chambon \& Ménard, as well as Martins, the blood of animals which were inoculated with vaceinia or with variola, and in a still greater degree that of persons who have passed through an affection of pox, contains substances which prevent the pox lymph from exerting its action. At the same time they have an immunizing action, and the favorable results obtained in recent times with the blood serum of highly immunized animals, especially sheep, make this conception very probable (see p. 317), however the nature of these substances is not even remotely known. As the pox eruptions develop only several days after the penetration of the virus into the hlood circulation, potent blood serum may exert its immunizing action even during the time of incubation. The immunization may be suceessfully accomplished also with lymph heated to $60^{\circ}$ (Süpfle) and with filtrates through porcelain (Green).

The inoculation of virulent lymph into the skin results in a slowly developing immunity, and in all probability is due to the influence of the substances absorbed from the point of inoculation, as in such instances the virus itself does not pass into the blood circulation (Süpfle). As a rule only a local eruption develops after such an infection; if however the inoculated part of the skin is removed 24 hours after the inoculation, a generalized pox eruption develops (Chauveau). In the first instance an inmunity results already during the development of vesicles, and the virus which later penetrates into the blood circulation can no longer attack the tissues, while in the second case the resistance of the tissue has not been increased to a degree in which it conld oppose the pathogenic action of the virus by the removal of the source from which the immunizing substances develop. In infections resulting from inhalation the virus enter's directly into the blood circulation, and thereby meets everywhere unresisting tissues, and in such cases a more or less generalized pox eruption invariably develops.

Offspring from animals which recovered from a general or local variola during pregnancy are immune, but this immunity becomes gradually weaker (in lambs according to Duclert from the 3d-4th month on), and disappears entirely in the second generation. The pox immunity is only exceptionally inherited, and even in such cases it affords only a partial and passing protection (Süpfle).

The etiological relation of human and animal pox to each other is not sufficiently established. The uniform anatomical structure, and the similar development of the characteristic vessels indicates the etiological identity of the different forms of pox. However observations relative to the reciprocal infec- 
tiousness or susceptibility do not seem to corroborate this conception.

Although the disease occur's in man and in sheep in the form of a severe general disease, and with an extensive pox eruption, in cattle and goats eruptions are almost invariably confined to certain parts of the bodies, their development being associated with only very mild general symptoms. The disease in man and sheep may very readily become epidemic, while among other animals the infection is usually confined within restricted limits.

Very close relations exist undoubtedly between human and cow pox. The vaccine of cow pox may be very readily inoculated into man, but the inoculation produces only an eruption confined to the point of inoculation, and never a general infection analogous to variola. On the other hand human pox are less surely transmissible to eattle. In some experimental cases characteristic pox developed in eattle at the point of inoculation (Numan, Ceely, Fischer, Éternod \& Haccius, Simpson, Freyer, Meder), in other cases only hard nodules developed after the inoculation, which healed without changing into vesicles or scabs (Chanveau) or again the inoculation remains entirely negative (Ducamp \& Pourquier obtained a positive result in only one case, Juhel \& Resnoy).

These experimental observations appear to indicate that the virus of human pox (Variola), and that of cow pox (Vaccinia) are two different modifications of one and the same pox virus. Fischer and Étermod \& Haccius aimed to prove this by reinoculating man with pox lymph of calves which had been repeatedly inoculated with variola lymph, whereupon in several thousand persons only a local pox eruption developed. From the above findings these author's, as well as Roloff and Bollinger conclude that variola may be changed into vaccinia by passage through the body of cattle, and that therefore the vaccinia represents an attenuated modification of variola. That such a transformation does however not easily and constantly result is proven by the experiments of Chauveau, who after inoculations of lymph from cows which were inoculated with variola virus, repeatedly observed in man the development of pox as a severe general affection.

The very close relation between variola humana and variola vaccinia is proven by the fact that vaccinia produces a lasting immunity against variola. Inasmuch as the local affection which develops after vaccination in man represents a very mild form of human pox, vaccinia must be considered as an attenuated variola virus. If it is further considered that the transmission of the contagion from man to man, as it has usually been practiced in former times, produces in most cases only a local affection, and further that pox in cows originally results as a rule, and probably invariably, from transmissions from 
man affected with pox, the conclusion is justified that the contagion of cow pox represents a modification of the human pox virus which has become attenuated in the human body. Such virus loses its dangerous properties for man permanently in the body. of cattle.

Jenner shared the belief of the people in the vicinity of Gloucester, that vaccinia invariably results from an infection from horses affected with pox, and that it is therefore identical with horse pox. His view was later shared by Lafosse and subsequently by Bouley, and under the influence of their authority and that of Chauveau, this opinion has been generally accepted in France. In recent times, however, after the findings of Warlemont \& Hugues, that the horse is only very slightly susceptible for the vaccinia, Dieckerhoff and Grawitz asserted that the epizootic disease observed by the French authors did not represent the pox affection, but either a stomatitis or a contagious pustular dermatitis, which diseases may be transmitted to other species of animals, and also to man; and they opposed, in consequence, the prevailing view.

Sheep pox usually spreads only among sheep, and the disease, although very fatal among these animals, does not even pass naturally to the closely-related goats, neither is it dangerous for man. On the other hand human pox can only exceptionally be transmitted to sheep (Manson, Simond), and even artificially a severe pox affection can only be transmitted to these animals by intravenous injections of variola virus (Küchenmeister, Tappe). It is not possible to immunize cattle with pox lymph from sheep against cow pox, and vice versa (Peuch, Voigt), therefore sheep pox must be considered as a specific affection of sheep.

Goats are likewise only susceptible to the contagion of goat pox, as sheep do not become infected in severely affected goat herds, and it is even difficult to infect them artificially. On the other hand the transmission of sheep pox to goats is also difficult (Bremond, Bonvicini, Nocard), while an infection with human pox is not possible (Voigt); finally cattle are not susceptible to goat pox (Bonvicini). Accordingly goat pox may likewise be considered as a specific affection of goats.

Pox of other species of animals, if such occurs at all, results from an infection from man or from the disease affecting one of the above mentioned species of animals, and therefore cannot be recognized as independent forms of the disease.

According to the present state of knowledge, therefore, human pox, sheep pox and goat pox represent independent forms of the disease, occurring and spreading independently of each other, whereas the pox of other species of animals always have some connection with one of the above mentioned forms of the disease. This view however does not at all exclude the 
mutual relationship of these forms of the disease. On the contrary, the nniformity of the development of the specific pathological processes points to the probability that the pox disease was originally a specific, independent affection of one of the susceptible species of animals or of man, and was here and there transmitted to other species of animals. However as the virus, in the course of time, passed through many generations of various species of animals it adapted itself in point of virulence, and probably also in its other properties, to the organisms of these respective species of animals. As the result of this the pathogenicity of such an adapted virus has been partly or entirely lost against other species of animals.

Literature. Jenner, Inqu. into the causes u. effects of the Variolae-Vaccinae. London 1798.-Chauveau, Rec., 1866, 305 u. 625; Bull., 1893, 51.-Bouley, Dict. de méd. vét., 1871, IX, 451.-Roloff, Pr. Mt., 1870-71.-Bollinger, Volkmanus Samml. klin. Vortr., 1877, Nr. 116 (Lit.).-Dieckerhoff \& Grawitz, V. A., 1885, CII, 148.- Prowazek, Arb. d. G.-A., 1905, XXIII, 535.-Mühlens \& Hartmann, Zbl. f. Bakt., 1906, XLI, 41 (Lit. on the so called vaceine-virus).-Voigt, Z. f. Infkrkh.. 1909, VI, 101.-Süple, A. f. Hyg., 1908, LXVIII, 237.

\section{(a) Sheep Pox. Variola ovina.}

\section{(Scabrot, Schafpocken [German]; Clavelée, Picotte [French]; Vajuolo pecorino [Italian].)}

History. Sheep pox was probably introduced from Central Asia, since human pox which is closely related to pox of animals is known to have existed in India a long time before the birth of Christ. Sheep pox was extensively studied for the first time by Joubert and Rabelais towards the end of the sixteenth century; its infectionsness however was established by Bourgelat in 1763 . As a result of the customary immunizations of sheep at that time, and through the use of Spanish sheep for the improvement of existing breeds, the disease attained a large distribution in Europe.

The etiology and pathogenesis of the disease were investigated especially hy Chauveau (1868) in more recent times by Borrel (1902), whose work has ereated great interest in the matter of immunization, which had been neglected for a time.

Occurrence. Pox among sheep has in recent times been entirely eradicated in the northern and central countries of Europe by proper measures, while in France and in the countries lying sonth and east the disease occurs even now in an epizootic form. In the affected territories extensive outhreaks appear especially during the warm weather, but even in these localities the disease does not attain the extension of former times. Its economic importance results from the great losses which oceur occasionally, and also from the considerable loss of wool which follows the disease.

Severe outbreaks are mentioned as having occurred in the beginning of the last century, thus Salmuth estimated in 1804 that the 
annual losses of sheep from pox amounted to more than one-eighth of the total number of animals, further Heintl, in 1823, gives the annual loss in Austria as 400,000 animals, and in France it is claimed that in 1819 more than one million sheep fell victims to the disease.

Germany was entirely free in the years 1859-1899, the disease however appeared in 1900 in a township of the Governmental District Lïneberg (220 cases with two deaths). In the following year it was discovered in four townships of the government districts Königsberg and Gumbinnen, affecting 1005 sheep, of which 38 succumbed. The country was free from the disease for one and a half years, hut it reappeared in the above districts, as well as in Marienweriler in 1903 , and on this occasion 1157 sheep became affected, of which 465 diecl. It is supposed that at this time the disease had been brought in by smugglers or butchers from Russia. Two years later it was brought from Russia into Eastern Prussia, by agricultural laborers, where 20 townships with 30 farms, and isolated farms in Saxony and Anhalt, were infected. The total eases amounted to 3,220 sheep, 704 of which succumbed. Since that time several outbreaks have been observed annually in Prussia, and in the year 19084 townships with 402 cases, and 154 deaths, were recorded.

The disease formerly raged in a severe form in France, especially in the Southern provinces, but also oceurred in other parts of the country, especially after the importation of sheep from foreign countries was restricted while large numbers were imported from Algeria.

Austria was free from the disease in 1594 , but since that time this has again become widely spread, especially in the years 1898-1900 in Tyrol, and still more in Dalmatia; in the latter year 21,575 sheep in 72 townships were affected, and of these 10,007 died or were slaughtered. After that time the disease diminisherl greatly, and no outbreaks have occurred since 1903.

The disease was always more or less confined in Hungary to the Southeastern part, and in recent times it occurs almost exclusively in the counties which are adjacent to Roumania and Servia. From 1899 to 1908 an average of 59 townships were infected, with 15,275 eases, of which 2,175 died. Since 1904 the rlisease attained again a greater extension, and occurred in 1908 in 113 townships of 27 counties with 19,131 cases, the total deaths being 4,443 .

In the same year 466 townships were affected in Bulgaria; 40,744 in Roumania; in European Russia 23,034 animals became affected. The disease also frequently oceurs in Spain, while the other European countries have keen free for many years. It is widely spreal in Asia, and is also known in Africa (Egypt, Algeria), while there are no statisties relative to its occurrence in America and Australia.

Etiology. Regarding the nature of the virus, it is at present only known that in diluted pox lymph and under great pressure it passes the porous Chamberland filters $\left(\mathrm{F}^{4}\right.$ and $\left.\mathrm{F}^{6}\right)$. The filtrate is not even under highest power found to contain formed bodies, and attempts at cultivation fail to give positive results (Borrel).

Tenacity. In glass tuhes, closed on both ends by melting, and kept in dark and moderately cool places, the pox lymph retains its virulence even for two years. At a somewhat higher temperature, for instance at $35^{\circ}$ it is active for months. Its virulence is attenuated by higher temperatures, and at $48^{\circ}$ it is destroyer in a short time, while when subjected to a cold of $-12-15^{\circ}$ it does not become attenuated for two months (Nocard). Glycerin attenuates it proportionately to its quantity, and a mixture of equal parts of lymph and glycerin becomes avirulent in 12 days when kept at a temperature of $25^{\circ}$ (Duclert). Of the disinfectants a $3 \%$ boracic acid, or $2 \%$ sodium salicylate have hardly any influence on the lymph (Galtier) and likewise $2 \%$ sulphate of zine and $10 \%$ chlorate of lime solutions are ineffective (Peuch). Putrefaction, diluted hydrochloric acid, 11/1\% carbolic acid, $21 / 2 \%$. sulphuric acid, $10 \%$ wine vinegar, spirits of turpentine (Grünwald) and also a mild iodine solution $(1: 10000)$ (Peuch) exert a quick destructive action on the lymph. The virus dies in the fleece of sheep affected with pox, at the latest in two months (Duclert \& Conte).

Experience gained in practice also points to the considerable resistance of the pox virus. When kept in a moderately warm place, protected from draughts, 
as for instance in sheep stables, it may retain its virulence for six months, and even out of doors it nay remain infectious for a long period of time. In Siebenbürgen it was noted in one case that lambs became affecter with pox in great numbers, in a stubble pasture in which 62 days previously sheep affected with pox had been pastured on young wheat (Dely).

Pathogenicity. A cutaneous inoculation of a small drop of pox lymph usually produces in susceptible sheep only a local pox ermption. In some cases, however, especially in lambs and by using very virulent lymph, a generalized pox eruption results. In the latter case, after an incubation period of $2-21 / 2$ days, a dark red nodule develops at the point of inoculation, which from the fourth to fifth day on changes into a vesicle. When the nodule begins to soften the body temperature rises to $39.5-40.6^{\circ} \mathrm{C}$., later to $41.5-42.6^{\circ}$, indicating the entrance of the virus into the blood circulation. This is followed by the formation of nodules, which change into pox vesicles on the mucous membrane of the lips, on the nostrils, the eyelids, later also in the axillary region, on the thighs, around the vulva and rectum. At the same time the general febrile symptoms reach a high degree until at the beginning of the third week, or somewhat later, death ensues with manifestations of septicemia. After subcutaneous infection the temperature rises with the beginning of the local inflammation; this is shown by a firm swelling which may reach the size of a fist, containing numerous nodnles, later forming confluating pox vesicles on its surface, while the general pox eruption develops in from 5-6 dars after the infection in the same manner as though resulting from a cutaneous inoculation. Intra-peritoneal injections of lymph are followed by the formation of nodules on the peritonemm, with bloating and sensitiveness of the abdomen, while injections into the trachea lead to a specific inflammation of the mucons membrane of the air passages, with marked difficulty in respiration as an immediate result, whereupon, in from 6-8 days a generalized pox eruption develops (Bose). Injections of lymph into the circulation may produce only febrile manifestations without eruptions, but sheep are immunized after such a short affection to the same extent as after recovery from a typical infection (Semmer \& Raupach). An intra-cerebral inoculation results in 6-8 dars in fever, the animals dying within one week afterwards, while injections of lymph into the milk cistern produces a pox eruption on the mucous membrane of the milk ducts, the milk in such cases containing the infective agent for two weeks (Nocard).

Outside of the contents of the vesicles the virus is not present in the pure secretions or in the blood, or in the latter only exceptionally immediately before or at the beginning of the pox eruption (Bose; Nocard \& Roux failed to obtain any positive results from the inoculation of blood).

Natural Infection. The virus of sheep pox probably enters the lungs with the inhaled air, and from there passes through the alveoli directly into the blood, which disseminates the virus 
into the superficial vessels of the skin and mucous membranes. The disease may be invariably produced by injecting or atomizing pox lymph into the trachea of susceptible sheep, while the feeding of lymph, when the possibility of iphalation is excluded, is not effective (Nocard).

The pox virus contained in the vesicles and in the scabs becomes pulverized and disseminated in the air, as a result of which a certain area surrounding the infected animals becomes infectious (according to Gilbert in calm weather for $20-25$ paces, in windy weather even further). If the food is contaminated by scabs the virus is inhaled by sheep with the dust disturbed during feeding.

The disease usually develops in flocks of sheep when they come in contact with affected animals or when sick or not entirely recovered sheep are placed among healthy animals. In this respect sheep which become ill from immunization, especially young lambs, are also dangerous. Susceptible sheep associating with such lambs may also become affected with a generalized, severe form of pox, as the virus used for the immunization is not attenuated in any way. The frequent occurrence of the disease among lambs in the spring of the year is often associated with the usual immunization carried out at that season in many localities. The infection may also be transmitted by contaminated utensils or attendants, by skins, wool, feed, manure, straw, pasture, highways, transportation wagons, further by shepherds and their clothes, dogs, cats, fowl, etc.

The infectionsness of a diseased animal is especially great when the eruption becomes matured on the hody surface, and to a still higher degree during the stage of scab formation and desquamation. The contagion may remain virulent in the scabs under the wool from 6-8 weeks, and therefore the infection may be communicated by animals which have already passed through the disease.

The susceptibility of the various breeds of sheep is not uniform. The most susceptible are the Merino sheep, somewhat less the more hardy, coarse-wooled sheep. In the first the disease develops as a rule in a more severe form than in the latter. In northern countries it is less severe and less frequent than in the south, which is probably due to the higher temperature which favors the development of pox. According to Nocard the sheep of Bretagne are not susceptible to pox, and the same opinion is also held by Chanvean concerning the Algerian sheep. Waldtenfel \& Nocard however found that pox exists continuously among the sheep of Algeria, although in a very mild form (if affected Algerian sheep come in contact with French sheep, the latter become severely affected).

Young sheep are more susceptible to pox than older animals, and in affected herds the lambs as a rule become severely ill without exception.

Affected ewes may transmit the disease to their offspring, 
which in such cases are born with pox eruptions; in other cases the fetuses in utero remain healthy in spite of the infection of the mother, such lambs having developed an immunity against the disease.

Rickert vaceinated about 700 breeding ewes in the last week of pregnancy. Repeated inoculations of the lambs born from these animals were ineffective, while 36 lambs, from ewes which were not vaceinater, dereloped pox nine days after the inoculation. Three years later the inoculation gave positive results in the first, while the latter failed to contract the disease. Anacker likewise failed to produce the disease in 60 , Peuch in three lambs from affected sheep.

Other species of animals as well as man, are not susceptible to sheep pox, or only in a very slight degree. Goats occasionally become affected as a result of contact with sheep affected with pox, and according to Borrel \& Konew the sheep virus gains in virulence for goats by passage through their bodies, while it becomes attenuated for sheep (see p. 327). Supposed infections of horses by affected sheep are reported by Berger \& Pecus.

Anatomical Changes. The autopsy of slieep dead from pox reveals, as a rule, besides the characteristic changes of the skin, hemorrhagic inflammation of the mucous membrane of the air passages and of the gastro-intestinal canal. On the mucous membrane of the pharynx and trachea, less frequently in the abomasum, pox vesicles are occasionally present, or ulcerations which have developed from them. The lungs, especially near the plemrae, contain small, gray transheent nodules, which may show a central caseation; these nodules are located within preumonic areas (Morel \& Vallée). In the other internal organs clianges indicating pyemia or septicemia may be found (acute swelling of the lymph glands, petecliae on the serous membranes, parenchrmatous and fatty degeneration of the parenchymatous organs, subpleural infarets in the lungs, initial acute interstitial nephritis, etc.).

Under the skin hard nodules the size of hazelnuts and showing a homogenons cut surface, may occur; they consist of thick masses of polynuclear lencocytes, lodged between shrerls of subentaneons connective tissue, many of these lencocytes containing broken down nuclei.

Symptoms. The pox exanthema is preceded by an incubation period of six to eight days, which is somewhat longer in cold weather. After this has passed the disease commences with febrile and catarrhal symptoms, which consist in dullness, loss of appetite, and elevation of temperature up to $41-42^{\circ}$, accelerated pulse and respiration; sometimes trembling and chills may be also observed. Pressure on the vertebral column, especially in the lumbar region, causes many of the affected animals to show signs of pain. The eyelids are swollen, the conjunctivae reddened, and from the narrowed slit of the eyes tears mixed with mucus are discharged. From the nose there 
is also a muco-purulent discharge, and sometimes a snuffling respiration is observed. The tongue is coated, and the exhaled air frequently disseminates a disagreeable odor. The nucous membranes of the cheeks and of the pharynx show either nniform or spotted reddening. The animals are constipated, the urine is diminished in quantity and has a high specific gravity. Auscultation of the lungs reveals intense vesicular breathing.

$\mathrm{T}$ h i s condition lasts from one to two days, whereupon, r. o u n d red spots appear mpon the skin (roseola variolosa), which in some places may be surrounded by a moderate serous infiltration, manifested by an urticaria - like elevation of the affected parts of the skin. Such spots are found in the vicinity of the eyes, on the cheeks, lips, and nostrils, on the vulva, or on the borders of the prepuce, on the udder, or on the scrotum, on the surface of the tail and on the inside of the thigh, therefore principally on those parts of the borly which are free from, or only slightly covered by wool. Less frequently they occur also on the chest and on the lower abdomen.

On the following day hemp-seed size, round reddish nodules may be observed in the middle of the red spots, which lose

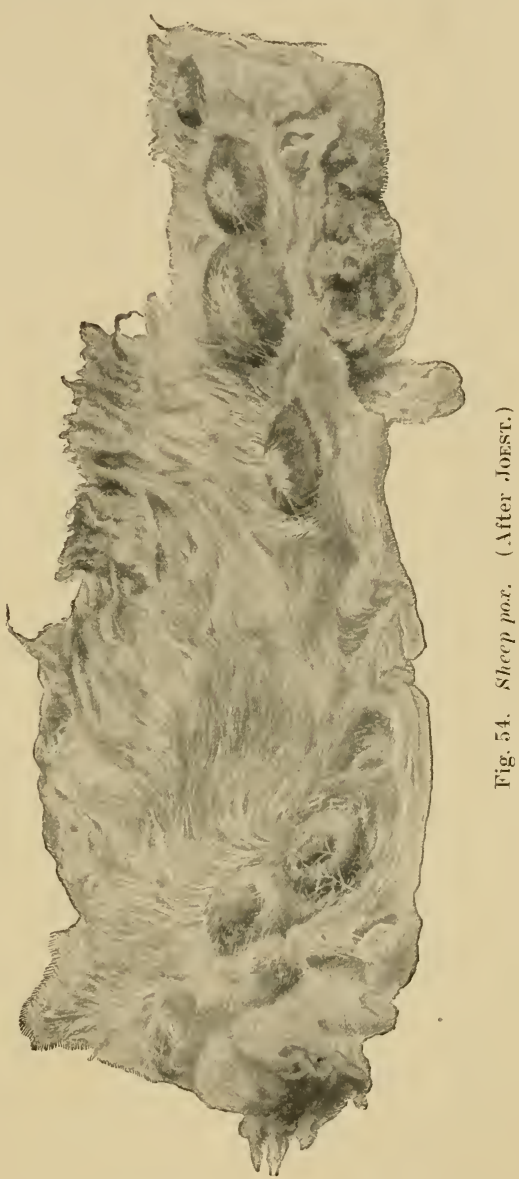
their color on pressure.

They continue to increase in size on the following days to such an extent that the base may reach the size of a ten cent piece (stadium papulosum). From the surface of the nodules a tenacious fluid oozes out; soon the superficial layer's become raised, and a clear yellowish serous fluid accumulates under them, 
whereby a fine, bluish-white vesicle develops with a transparent wall. This continues to enlarge on the following day, and at the same time a slight depression (navel) develops on the top (stad. vesiculosum; Fig. 54). The development of the vesicles occur's in the same succession as is the case in the appearance of the red spots and the nodnles, and it may take several days for the development of all the vesicles. The vesicles attain a size ranging from that of a lentil to that of a bean; while the larger vesicles are more flat and in their center the navel is never absent, the smaller ones are less elevated, resembling more blunt ended pins and on them the navel may frequently be absent. The closer the vesicles are located to each other the smaller their size, and vice versa. In pricking the vesicles their serous contents ooze out slowly, on account of their compartment-like construction.

Immediately surrounding the vesicles the skin is as a rule hyperemic, and therefore forms red zones around them; the skin and the subcutaneous comnective tissue of the affected parts are also edematous, as a result of which the respective regions of the body, especially the head, and on this particularly the eyelids and the region of the nose are swollen, so that the corresponding openings and slits become constricted. The development of the exanthema up to this degree usually takes from 5 to 6 days.

In the completely developed ripe vesicles the clear yellowish or pale red lymph-like contents changes after 2 to 3 days, or 6 to 7 days from the onset of the disease, into a cloudy fluid owing to the admixture of pus cells, finally becoming entirely white, at the same time the vesicle may increase somewhat in size and the navel-like depression may also disappear (stad. pustulosum s. suppurationis), while the edematous infiltration of the skin in their immediate surroundings becomes as a rule still more intense. This stage usually lasts three days.

This completes the development of the vesicles; a depression again appears on their tops, the wall becomes withered and wrinkled, and after the horny layer has taken on a brownish color the vesicle dries with its contents to a $1 / 2-1 \mathrm{~mm}$. thick hrown scab (stad. crustosum s. exsiccationis). N'ew epithelia form under this from the borders and from the base of the vesicle, whereupon the scab drops off in from 4 to 6 days (stad. decrustationis). In its place a red spot or a red sear remains for a time, which gradually turns pale and at the same time becomes somewhat depressed.

The development of the eruption is associated more or less with severe general symptoms. The sudden high fever at the onset of the disease diminishes from the second to third day on, and in the pustular, as well as in the vesicular stage only a morlerate fever is present. With the appearance of suppuration it again increases, and again drops rapidly after 2 to 3 days, so that the drying of the vesicles, as well as the falling off of the scabs occurs under normal temperature. 
With the appearance of the eruption the general condition improves, and during the development of the vesicles the patients usually are quite bright. They partake of some food, and also ruminate periodically. Profuse secretion continues to be discharged from the eyes and nose, the respiration may be snuffling owing to the swelling of the skin of the head, and especially of the nostrils. From the corners of the month a tenacious, foamy saliva hangs in long strings; the extremities are usually edematously swollen. The animal disseminates a peculiar, sweetish, repulsive odor, which becomes more pronounced during the period of suppuration. The general condition later becomes worse, but as soon as the suppurative process is concluded, and the drying commences and with the disappearance of the fever the catarrh of the mucous membranes also diminishes whereupon the patients usually regain their normal health in a relatively short time, on an average in 3 to 4 days.

The pox develop in a similar manner on the mucous membrane, and simultaneously the eruptions on the skin. Their appearance in the mouth is usually associated with profuse salivation, and when they are present in the pharynx there is difficulty in swallowing, while their appearance on the tongue sometimes causes a marked swelling of this organ.

Pox with an Atypical Course. Deviations from the regular course are very frequent. It is probable that the catarrh of the air passages and of the eyes which is sometimes observed in some sheep of an infected herd without other symptoms, and withont their developing a severe affection, is the result of a pox infection, which however, owing to the resistance of the animals, was not sufficiently severe to produce an eruption of pox (variola sine exanthemate).

Frequently it is also observed that the ermptions do not develop into the vesicular stage, but the red and frequently elongated nodules remain stationary for a long time, peel off after a few days and disappear (so called abortive or stone pox). These abortive lesions occur sometimes also between well developed vesicles, especially on the parts of the body which are exposed to a greater pressure (variolae compressae, flattened pox), while in other cases the affection is exclusively manifested in an eruption of such nodules. The disease frequently occurs in this mild form among Algerian sheep, in which case small, hard nodules develop only on the head, around the eyes, nasal openings and lips; and in the outbreaks in Germany, in 1905, the manifestations were similar, although the course was quite severe (see p. 303).

After Ostertag had as the first recognized the disease to he pox, its symptoms were described more accurately by $R$. Fröhner, Kleinpaul, Ėber, Roessler, Noack, Klebba, Haak, and the autopsy findings by 
Joest. The difference from the usual course is not shown so much in the general symptoms (fever, acute catarrh of the mucous membranes, dullness, ete.), as these greatly resembled those of the typical form, but in this atypical form hard nodules as large as lentils or

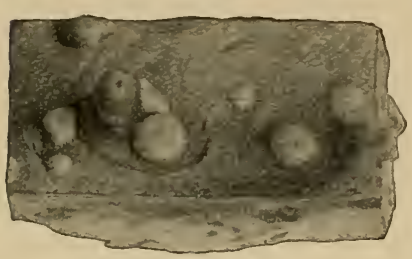

Fig. 55. Atypical sheep pox. (After JoEst.) hazelnuts usually developed on the skin (Fig. 55). They were not surrounded by a red zone, and showed no indications whatsoever of vesicular or pustular formation. Although the exanthema often extended over the entire body, it appeared that the lesions occurred preferably in the vicinity of the nose, lips, vulva, and on the udder. The affected parts of the body frequently showed a marked inflammatory swelling. Later the papules dried, and dropped off entirely. Not infrequently they turned into suppuration or gangrene, whereupon quite deep ulcerations developed, which were covered by thick scabs and erusts. After the scabs dropped off, cicatrices without pigment or hair remained. Typical pox vesicles develop but rarely and only later in an outbreak. Here and there purulent keratitis, sometimes with subsequent panophthalmitis and blindness resulted, and lameness resulted frequently from an inflammation of the interdigital space, also erysipelas-like swellings of the skin of the thighs; further a colliquative diarrhea was observed in severe cases. In some cases goats became affected with similar symptoms, and the transmission from sheep to goats was also successful by artificial means (Foth). Losses in affected flocks vary among the different animals from $25-50 \%$, or even a larger proportion. (According to Lungershausen the disease showed a similar character in the outbreaks of 1862-3.)

The number of papules varies greatly from case to case, sometimes they occur only sparingly, while in other cases the parts of the body not covered by wool, as well as the other parts, are covered with great numbers. As a rule they are thickest and most numerous on the face.

If the eruptions appear very closely together they may confluate into large purulent vesicles (variola confluens), and if this occurs in many places the neighboring skin and subcutaneous connective tissue becomes greatly edematous, so that the head, for instance, becomes greatly deformed, and the face appears as though covered by a mask. In such cases the papillary layer under the suppuration forms a continuous wounded surface, from which a general pyemia or septicemia may readily develop. In such cases numerous papules may develop on the mucous membrane in the mouth, pharynx and nose, the development of these being associated with difficulty in swallowing and respiration, purulent nasal discharge, and profuse salivation. Vesicles may occasionally develop on the conjunctivae, and also on the corneae. In such cases the disease usually terminates in death, and if the animals should recover after the falling off 
of the thick scabs, an extended ulcerative surface remains, which heals only after a long time leaving irregular shaped cicatrizations.

Most unfavorable is the course in those cases in which, during the development of the eruptions extensive hemorrhages appear (variola hemorrhagica s. nigra). Although disseminated small hemorrhages may occur in the mild cases, the hemor'rhagic character of the affections is at other times pronounced. Between papules of normal appearance, dark red eruptions are observed, which do not disappear on pressure, hemorrhages also occurring on the smooth skin and in the subcutis. In other cases the exanthema develops at first normally, although accompanied by severe general disturbances. Suddenly, however, the serous contents of the vesicles become hemorrhagic and then the eruptions appear dark red, almost blackish. At the saine time a bloody nasal discharge, hematuria and bloorly diarrhea, indicate similar hemorrhages in the internal organs.

The severity of the disease is also manifested in the behavior of the affected animals. They stand in one place, swinging their bodies with drooping heads, and at times grinding the teeth, or lie motionless on the ground. The respiration is very labored, and is usually done through the mouth. The body disseminates an unpleasant repulsive odor. The affected parts of the skin turn into ulcers, which become covered with a dirty ichorous mass, while other parts of the skin may become gangrenous, forming dirty brown masses. Gangrene may also extend to the muscles, and occasionally the ears, lips, eyelids, etc., may also become gangrenous, these parts later dropping off (variola gangraenosa, gangrenous pox, rot pox; such necrosis is sometimes observed in the confluent but non-hemorrhagic papules). Death may result in any stage, even before the development of the vesicles.

The so called black pox does not represent a special form of the disease, but is primarily ideutical with the ordinary sheep pox, while the hemorrhages are very likely originally associated with an especially severe accidental septic infection.

Most frequent are those complications which occur in the animals as a result of scratching and rubbing on account of itching. Thus a severe dermatitis may sometimes develop at the places of the ruptured vesicles, which retards the subsequent healing, and as a result of this marked edematous swelling may develop, even in mild cases, especially on the head. After healing scarred depressions result from such wounds at the place of the vesicles. If the patients rub their noses much, which they frequently do, the inflammation may also extend to the nasal septum, and to the periosteum of the nasal bones, and for this reason these bones sometimes become necrotic.

Chemosis of the conjunctivae, which in severe cases of conjunctivitis develops not infrequently, may result in an ulcerative 
breaking down, or necrosis of the cornea, with which occasionally an acute inflammation of the internal parts of the eyes becomes associated, leading finally to atrophy of the bulbus. Vesicles which develop on the sclerotic portion of the conjunctiva may extend to the cornea, leaving a permanent cloudiness behind.

The catarrh of the air passages may sometimes extend to the finest bronchi, when it causes intense difficulty in respiration, and a catarrhal pneumonia may develop as a result.

The pox eruptions are nsually associated with gastrointestinal catarrh; in severe eases it becomes more intense, and the resulting complete inappetence combined with the profuse diarrhea exhausts the strength of the affected animals.

The eruptions on the lower extremities, on the coronary band, and in the interdigital space, lead not infrequently to purulent inflammation of the sensitive lamina, and of the neighboring joints, as in these places septic infection readily occurs, whereupon the horny capsules drop off, and general pyemia develops from pus absorption.

Of other complications there should be mentioned, suppuration of the lymph glands, metastatic abscesses in various internal organs and in the subcutaneons connective tissue, diphtheritic inflammation of the mucous membranes, pneumonia and pleurisy, necrosis of the tendons, etc.

Course. The course of pox is always acute, and except for some of the outbreaks (see p. 309), is in the majority of cases typical unless the development is hindered by outside conditions. The development of the vesicle occurs with few exceptions always in the same order, and the other manifestations also follow a typical course. Accordingly the average duration of the disease, from the begimning of the exanthema until the scabs fall off and to the new formation of the epithelium, is from 3 to 4 weeks. The course may however be influenced by external conditions, as it is known that the disease runs a slower course in dry winter weather than in summer, and cold rainy weather has an unfavorable effect on its course. Further, the disease develops more slowly in anemic, poorly nourished animals, and if they survive at all the period of desquamation especially requires a longer time.

In severe cases, with very extensive pox eruptions, death may ensue eren in the early stages of the disease, possibly from hemorrhage. Usually however unfavorable changes take place during the suppuration period, although in exceptional cases animals may die even if they are on the road to recovery, as a result of exhaustion or some complication.

The mortality varies greatly in different outbreaks. While the loss sometimes amounts only to from 2 to $5 \%$, in other cases, especially when the coalescing and hemorrhagic forms are more frequent, more than half of the affected animals die. 
If an outbreak has appeared in a large flock the cases first appear at intervals of 10 to 14 days, later however in more rapid succession, until finally, with the exception of about 2 to $3 \%$, all animals have become affected. In some cases only a small part of the herd becomes affected at first, and only 3 to 4 weeks later when the animals that were stricken first already show indications of recovery does the disease break out suddenly in a great number of sheep, and finally after a further interval all of the animals of the flock which have been spared become affected.

Diagnosis. The character of the exanthema and the typical course of the disease as a rule make a positive diagnosis possible. If the danger of contagion exists the first prodromal symptoms are sufficient to arouse a suspicion of the disease, otherwise however the diagnosis should be suspended until typical vesicles have developed from the nodules. The presence of vesicles and pustules is not absolutely necessary in the clinical develorment of the disease, as was shown by recent German and Algerian observations, and therefore in such an atypical course a papular exanthema is sufficient to establish at least a suspicion of the disease, especially when such lesions develop in association with fever and eatarrh of the mucous membranes. The diagnosis may be confirmed later by inoculations of the contents of the nodules into healthy animals.

The disease may be mistaken for scabies and pustular eczema, especially in the stage of pustule and scab formation. In these diseases lowever there are no severe prodromal symptoms preceding the development of the lesions, besides this, eczema is not infectious, and in its course no larger vesicles develop, while scabies, although infectious, spreads very slowly, and scabies mites may be readily demonstrated with the microscope in the scabs and crusts.

Prognosis. In cases in which the pox eruptions are few and the indiridual papules develop in the regular order the prognosis is favorable. On the other hand in those cases in which the exanthema extends over a larger portion of the body, and in which the resicles show a tendency toward coalescing, it is doubtful. Hemorrhages are always unfavorable indications, irrespective of the stage in which they appear.

Very old or very young animals show, under otherwise like conditions, feeble resistance and sucking lambs especially become severely ill with otherwise mild pox on account of the usual association of gastro-intestinal catarrl. In flocks where the animals are confined in narrow quarters the disease runs a more unfavorable course than in animals kept out of doors. In the latter again great heat or damp chilly weather has a bad effect. Sheep which come from localities in which pox is prevalent become more readily affected tlan those which come from localities where the disease has not existed for a long period. 
In the prognosis, the possibility of complications and after effects (lameness, chronic ailment) should be given due consicleration.

Treatment. In the treatment of sheep pox, regulation of hygienic conditions is the essential factor to be considered. The animals should be given proper food and pure, fresh, uniformly warm air, wherefore in favorable weather it is best to keep the sheep out of doors. The early slaughter of the severely affected animals appears indicated, because through this procedure the danger of a subsequent septic infection of the animals with a milder attack is diminished.

During the stage of incubation in the beginning of the affection, immune serum of high potency (see p. 317) may give good service.

Pox Immunization (Ovinatio). As recovery from pox usually confers upon the animals an immunity for their entire lives, the production of the disease in a mild form appears advantageous in all cases in which it is feared that the sheep will become severely affected from natural infection (emergeney immunization). This danger exists especially when the disease has already attacked some sheep in a flock, and if its further spread is expected. The situation is similar when the disease has appeared in nearby localities, and the local conditions appear to be favorable for the transmission of the disease to the liealthy herds. Under such conditions it should be aimed to assure as much as possible a mild course for the disease, and at the same time to reduce its duration. The vaccination of the herds exposed to the infection answers for both purposes. The disease which develops subsequently usually takes a more favorable course than in the natural infection, provided that the vaccination has been carried out properly and with good vaccine. If the entire flocks have been vaccinated the disease usually disappears within a few weeks. At the same time the spread of the infection to non-vaccinated herds should be prevented by quarantining all infected herds.

Vaccination of lambs at times when the disease does not exist (protective or preventive vaccination) is not indicated.

This procedure is at the present time used extensively in countries in which sheep pox is prevalent, and is in many places carried out annually by the shepherds. It appears to have a very old origin, but has attained a wider extension in Europe since Bourgelat in 1765 and shortly afterwards Venel and Teisier, Hurtrel d'Arboval and others subjected the method to scientific examination, and recommended it as a suitable protective method. In the first half of the last eentury farmers and shepherds applied the vaccination very generally, and. the necessary vaccine was prepared for this purpose in specially established institutes.

As a matter of fact the vaccination produces a lasting immunity against subsequent natural infection. The vaccination is followed with 
few exceptions, by only a local and very mild pox eruption, and the time for vaccination may be chosen according to convenience. The unfavorable results which are observed in flocks suffering from natural infection and the severe form which develops in unfavorable weather, together with prolonged duration of the disease, may thereby be eliminated. The vaccination of the animals therefore serves entirely the purpose for which it is intended.

The vaccine is the original, not attenuated virus of pox, which continues to propagate in this highly virulent condition in the inoculated animal. As a result of this, flocks which have not been vaccinated are threatened to a great extent by the vaccinated animals, and particularly so as the resulting infection usually appears generalized, and frequently causes a malignant eruption of pox. If the vaccination were carried out from year to year on all sheep of a country all subsequent infection of the native animals could be excluded; since however vaccination is practiced only in certain localities, and there mostly in single flocks, every vaccinated herd represents annually reproduced artificial infection, from which the artificially propagated virus may sometimes become disseminated and produce severe outbreaks of pox.

Considered from a general viewpoint the vaccination of lambs is therefore positively harmful, especially as the location of the flocks is but rarely changed, and they may be easily protected against the infection by safer measures.

Observations on this point made in Germany afford convincing proof. Siuce 1875 the vaccination in Prussia has been depenilent upon official permits. This resulted immediately in a conspicuous reduction of pox, and consequently the Imperial law on animal diseases in 1818 prohibited vaceination except in emergeney cases. The results eame up to expectations, inasmuch as Germany has been almost entirely free from the disease since that time. Pox has appeared from time to time, owing to importations from foreign countries, but in all instances the disease has been controlled and limited to the primarily affected flocks. In Austria a law was passed in 1880 by which the vaceination was permitted only with official sanction, and this also resulted in a striking reduction of the disease. On the other hand in France, Hungary, and in the eastern and southern European countries where vaccination is still in vogue, consiclerable loss from pox results from year to year among the sheep.

Technique of the Vaccination. The elear, yellowish, or yellowish-red, serous contents of the ripe vesicle which is obtained from young vigorous sheep, in which the eruption develops only in moderate extension and in association with only slight febrile symptoms is used as vaccine. The vaccine should never be taken from a purulent vesicle, and is still more dangerous if it originates from animals in which the papules have coalesced, or in which the presence of hemorrhages indicate a severe character of eruption. At the beginning of an outbreak it is advisable to vaccinate only a few sheep from the first affected animals, and then from these the lymph should be taken for the remaining part of the flock. In such cases the lymph may be taken ten to twelve days after vaccination, otherwise in from 5 to 7 days after the appearance of the eruptions. If however lymph from the animals vaccinated the previous year is available it is best to use such for the vaccination.

The contents of the ripe vesicle are collected in capillary tubes. The tubes are previously heatel, and one end of the tube is stuck into the vesicle. In an emergeney however the lymph may be collected from an open vesicle in glass bottles. It is less advisable to utilize the scabs which develop at the place of the vesicles for the vaccination. Vaccinations may also be carried out directly from animal to animal by first inserting the vaccination instrument in the vesicle, and then immediately into the skin of the animal to be vaccinaterl. Serous fluid obtained by squeezing incised nodules may be also utilized for vaceine.

The most favorable place for vaccination is the under surface of the tail which is free from wool, $5-10 \mathrm{~cm}$. from the rectum, or the inner surface of the ear $3.4 \mathrm{~cm}$. below its point. Less suitable is the inside of the thigh, the scrotum and the abdominal region. As instruments, the vaceinating lancet or still better 
the vaccinating neclle is used, which after the opening has been filled with lymph, is stuck into the stretcbed skin in an oblique direction, in orler that the vaceine may euter the deep layers of the epithelium, and not under the skin; however the skin may be also superficially scarified, and the vaccine rubbel into the scratched area.

In susceptible animals a typical pox exanthema develops if the vaccination has heen carried out properly, usually a single vesicle develops at each place of pricking, others develop exceptionally in the surrounding parts, whereas a generalized eruption of pox results only very exceptionally.

The vaccinated animals slould le kept isolated during the entire duration of the local affection from sheep suffering from natural infection, as well as from those which have not leen vaceinated. After a period of about a week the raccinaterl animals should be examined individually, and in those which have failed to take the vaccination the operation should be repeated. If the vaccination is negative this tine, although good lymph has been used, the respective animals should be considered as immune. Vaccination with contaminated material may of course result in rarious wound infections (necrosis of the skin, purulent inflammation of the connective tissue, pyemia, tetanus).

The vaccination of lambs is carried out in a similar manner, but usually with lymph which was taken the previous year from vaccinated lambs, and kept in air tight bottles in a cool room.

Production and Preservation of Pox Lymph. In the first half of the last century special institutions were established for the production of pox lymph in France, and also in other countries, where the lymph originating from natural benign pox was propagated in more or less constant virulence by continnous transmission from sheep to sheep, and was increased in accordance with the demand. Vaccination with such lymph usually resulted in very mild, localized pox, but it was repeatedly noted that the artificially kept vaccine was capable, without any apparent canse, of producing a generalized eruption, although in the meantime it had passed through 65, 98, and even 140 generations of sheep (Roll).

Recently Soulié produced a vaceine in large quantities in the Pasteur Institute of Algeria, ly injecting single drops of lymph in various places into the clipped skin of the rump of healthy sheep. In 10 to 12 days he collects the contents of the ripe vesicles and stores them in a diluted state (see below). Every sheep treated in this manner produces $500 \mathrm{cc}$. of diluted lymph, which is sufficient for the vaccination of 10,000 animals. This vaccine has been used exclusively in Algeria for several years, and the results are uniformly satisfactory; the lymph proves effective in $80 \%$ of the cases, and the losses amount to only $0.1-0.5 \%$. The action of the lymph however is not constant, as occasionally, especially in resistant breeds, the vaceination may be effective in only $20 \%$, and even in only $8 \%$ of the cases (Martel).

Borrel strengthens the virus by injecting pox lymph and then salt solution mixed with bread crumbs into the pleural carity of sheep. As early as in 4 to 5 days a consilerable quantity of a virulent fluid may he obtained from the thorax. Another method of Borrel consists in injecting subcutaneously in the abdominal region a mixture of $10 \mathrm{cc}$. of pox lymph and $500 \mathrm{cc}$. of salt solution, of which $300-400$ ce. is injected into the animal. The fluid, which should be rubhed over as large a surface as possible, is usually absorher by the third day, and from the fourth day on a marked serous infiltration develops in the respective parts. On the eighth day the animal is destroyed, and the fluid which has collected under the skin, together with the infiltrated tissue, is ground in salt solution; in this manner about two liters of a liquid are obtained, of which $1 / 20,000$ of a ce. is effective.

Various methoils are adopted for the preservation of the raceine. The simiplest methou consists in placing it in capillary tubes, or in fine glass tubes, the center of which has been blown into spindle shape. The ends of such tubes, after filling, are closed by melting, or sealed with sealing wax. For the same purpose two plates of glass of 4 centimeters square may be used; the surfaces facing each other are painted with the vaccine and the borders are closed either with mastix or wax. The lymph may be also preserver in elean bottles, which are closed air tight. (According to Peuch old lymph which on eutaneous vaccination is ineffective may produce typical pox if injected subeutaneously.) Soulié stores the lymph with 2-5 parts of a $3 \%$ boracic acid, or $2 \%$ salicylate of sodium, and inmediately lefore vaccination he dilutes it with 3 parts of its quantity, of boiled sterile water. Aceorling to Bose sheep pox virus remains unattenuated for at least two years in the intestinal canal of leeches. which have sucked themselves full on opened, ripe pox postules.

Other Methods of Vaccination. In order to prevent a generalized eruption of pox the attennation of the virus was undertaken, but with unsatisfactory results. 
Peuch diluted for this purpose the lymph obtained from the vesicles with 60-100-160 times its quantity of water, Nocard \& Mollereau with water containing oxygen, Semmer \& Raupach by heating to $55^{\circ}$. After the inoculation of such vaceines no eruptions resulted, nevertheless the respective sheep proverl immune. Duclert kept fluid lymph, Conte dried lymph, at a temperature of $25^{\circ}$, and both observerl a gradual attenuation of the virus. The vaccinations undertaken with such attenuated virus, however in numerous cases failed to give positive results.

Supposing that the ovina becomes attenuated in virulence for sheep by passage through goats Konew employs for the vaccination of sheep, edema fluid obtained from goats (Kaprino) which have received serial subcutaneous vaccination with ovina. Up to $1907,91,735$ sheep were vaccinated with such lymph in Russia with uniformly satisfactory results, and Bridré also obtained the same goorl results in an outbreak. Voigt also convinced himself that sheep eaprinized with goat ovina became immune, only he found that a necrosis of the point of vaccination developed from the subcutaneous, as well as from the cutaneous injections.

Following the initiative of Sacco, sheep were vaccinater for a time with cow pox lymph, but without results. At the same time it was learned that vaceination with cow pox lymph took only in few sheep (Pessina), and it was observed that after such vaccinations a generalized pox eruption with considerable loss occurred (Fürstenberg, Gips).

Serum Immunization and Serum Therapy. Duclert (1896) found that blood serum of sheep which have passed through an attack of pox, and more so from those which were repeatedly treated with virulent lymph, has a protective action for lambs against an energetic infection of virulent lympl. Borrell (1902) inangurated this procedure in practice.

Sheep which have recovered from pox are injected subcutaneously every month with $200-300$ ec. of lymph prepared by his methort (see p. 316). After 5-6 of such injections the sheep produce a potent blood serum. If such serum is mixed with lymph obtained from pox vesicles, and diluted with 53 parts of bouillon, a cutaneous inoculation with such a mixture produces results accorling to the quantity of the serum, smaller and smaller vesicles are developer as the amount of serum is increased while a mixture of 1 cc. of dihted lymph with $1 / 2$ ce. of blood serum proved entirely ineffective. The cutaneous inoculation of $1 \mathrm{ce}$. of diluted lymph produces a blister with a diameter of 6-7 $\mathrm{cm}$, and subsequently a general pox eruption. If on the other hand such sheep receive two days after the inoculation, from 2-10 ce. of blood serum, the size of the vesicles is diminished in accordance with the serum used, and if $15-20$ ce. of blood serum is injected, no eruption whatever develops.

Immune serum has been used in practice in France with good results, it is claimed, although mostly under very unfavorable conditions. According to Martel's view the serum inoculation checks the disease in infected herds, and also prevents the eruption of pox in already febrile (40 degrees) sheep. If the eruptions are already in progress usually only a few small vesicles develop, and even if they do become larger, recovery takes place within a short time. In some sheep only small nodules which do not suppurate, develop under the skin. The serum immunization is supposed to confer also an immunity on the otherwise very susceptible lambs, which lasts for at least 40 days (Borrel).

The dose of the blood serum is for the immunization proper 5 ce., for therapentical purposes at least $10 \mathrm{cc}$. In alrearly infected aninals the serum affords only a protective or curative action in the incubative stage or, at best, in the prodromal stage of the disease.

The value of serum immunization cannot yet be definitely establisher. In the fall of 1902 , and in the spring of 1903, six herls were given the serum treatment, in which previous to the treatment $10.3 \%$ of the animals died from pox. Of 581 sheep and 352 lambs which were treated, 49 sheep and 24 lambs, that is in all $7.8 \%$ lied, the loss fluctuating in the individual herds between 0.0 and $27.7 \%$. In a herd consisting of 500 sheep and 150 lambs, in which pox hat developel two weeks previously, the disease ceased immediately after the animals had been injected subcutaneously with 5 ec. of serum. Conte treated 689 sheep 
and 63 lambs in 40 infected herils in the Department Herault, in the years 1903-1909; of these 10 became infected subsequently, two of which died, whereas in 137 animals which were not given the treatment the mortality amounted to $38.7 \%$.

Bose found that the serum, as well as the defibrinated blood of lambs severely affected with pox (hyper-infected) possessed immunizing properties, inasmuch as 20 ce. injected into healthy lambs protected them against virulent infeetion. Lambs, and also donkeys, which have been treated repeatedly with such serum and pox lymph, produce a still more potent serum, which may confer total immunity, or only jrevent the generalized pox eruption.

Simultaneous Immunization (Séro-clavelisation). For producing a lasting immunity Borrel recommends the simultaneous inoculation with his immune serum (5-15 ee. injected subeutaneously in the rump) and virnlent lymph ( $0.05 \mathrm{cc}$. in the ear). In Arles over 10,000 sheep were treated by this method, and none of the animals became affected with pox, and from subsequent complications only $0.2 \%$ died. Good results were also obtained by this method by Bridré in Tunis, and by Conte in France.

Poenaru vaccinated 262 sheep with threads saturated in a mixture of immune serum and lymph, the thread was introduced under the skin, whereupon in 164 animals only a nodule developed at the point of inoculation, in 94 a small postule, aud in only one instance had several postules appeared.

Veterinary Police. Outside of the measures for compulsory reporting of the outbreaks, the quarantine of the premises or townships, and the harmless disposition of the carcasses, a compulsory immunization of the healthy animals in an affected herd appears indicated. In case the disease is disseminated more extensively, or if infection threatens, vaccination may also be applied to the flocks that are in danger. The vaccination may be omitted if the sheep which are not affected are slaughtered within a short time. Vaccinated sheep should be subject to the same police measures as animals affected with the natural disease, and be quarantined for the duration of the affection. The raising of the quarantine from infected premises should be preceded by a period of observation of at least 45 days. The pelts of sheep affected with pox may be considered non-infectious after complete drying, the meat however, if its condition permits its passing for food, is to be considered non-infectious even in a fresh state.

Literature. Chauveau, J. vét., 1868, 548.-Gerlach, Ger. Tk., 1872, 65 (Lit.).Nocard, Rec. 1888, 272; Bull., 1898, 43 and $331 ; 1899,263 ; 1900$, 21.-Peuch, C. R., 1888, CVII, 425.-Soulié, Rev. vét., 1896, 421.-Borrel, Soe. biol., 1902, 59; A. P., 1903, XVI, 123.-Bose, Cbl. f. Bakt., 1903, XXXIV, 413; Rev. gén., 1904, IV, 273.-Poenaru, Arhiva vét., 1904, 339.-Joest, Z. f. Infkr., 1906, I, 221 (Lit. on the last pox invasion in Germany).- Borrel \& Konew, Cbl. f. Bakt., 1907.Foth, Diss. Leipzig, 1907.-Conte, Rev. vét., 1910, 65.

\section{(b) Cow Pox. Variola vaccina.}

\section{(Kuhpocken [German]; I'accinia [French]; Vajulo vaccino [Italian].)}

Occurrence. Cow pox, the relation of which with human pox has already been recognized by Jenner (1796), occurs usually only sporadically, or as an enzootic confined to individual herds. In most cases it may he attributed to an infection of true pox, or vaccination-pox of man. 
In the affected herds a great number of the animals may become infected within a short time. Thus Bartos observed among 210 cows 90 infecter inside of two weeks. In this instance the children of the owner had leen raccinaterl 6 days previous to the first ease. The disease was observed in an epizootic outbreak by Fréger in the Arrondissement de Bayeux, where the milkers disseminater the disease from stable to stable.

According to Leese pox oceurs in Pendschab among eamels with such frequency that most of the animals become affected in the first or seconil year of their lives. As a result of this, older camels rarely become affecterl, but if so, usually with generalized pox and pyemie manifestations.

Etiology. According to the investigations of Negri (1905), which have since been confirmed by Remlinger \& Osman Nouri, Carini, Nicolle \& Arlil-Bey and others, the cow pox virus passes through not too dense porcelain filters, therefore it apparently belongs to the ultra-visible micro-organisms.

Negri filtered vaccine fluid collected from young cows through Berkefeld filter under 3 atmospheric pressures, and produced with the filtrate, the characteristic vaccinia keratitis in rabbits, and true pox postules on the udders of cows. The fact that filtrate from lymph which has stood for some time is frequently more infective than similar substance from fresh material, is explained by Carini thus that the virus is contained in cells, and is freed only after long maceration. (According to Nicolle \& Adil-Bey this may be hastened by pancreatic digestion). Up to the present time only ahortive pox could be produced with filtrates.

Pröscher is said to have succeeded in cultivating the ultra-visible pox virus on artificial media. On solid merlia preparesl especially for this purpose, smeary deposits develop by changes of the nutritive substrata, which do not contain microseopically visible micro-organisms, but which yet produce pox postules in ealves up to the third or fourth passage.

Pathogenicity. Inoculation of cow pox lymph into the skin of cattle results in a typical pox eruption, which is always confined to the point of inoculation, or to the parts immediately surrounding it. The ermption is associated with very slight general disturbances. Young cattle are especially susceptible to infection, which is favored by a previous injury of the epithelium of the skin (shaving, etc.). Man, buffaloes, camels and horses are susceptible to a similar extent; hogs, sheep and dogs, to a lesser degree, and in these animals cutaneous inoculation results only in a local exanthema. The rabbit is very susceptible, and according to Calmette \& Guérin, the guinea pig likewise.

Subcutaneous inoculation of the virus produces in cattle an edematons infiltration, in horses in addition to this an eruption of pox, while an intravenous infection causes only fever without exanthema in these animals. Chanveau and Arloing observed the development of a vesicular exanthema in young horses following an intravenous infection.

The injection of lymph into the milk ducts of cows results in 2 to 3 days in moderate fever, swelling and sensitiveness of the udder, wherempon on the ninth day the secretion of the udder becomes purulent and soon bloody. The secretion retains 
its virulence even after 14 days. The symptoms are caused by the development of vesicles on the walls of the milk ducts (Liénaux \& Hébrant).

The tenacity of the virus is apparently similar to that of the virus of sheep pox. It resists drying for several weeks, but is destroyed at a temperature of $57.5^{\circ}$ in five minutes (Power). On the other hand it is not destroyed by - 180 degrees, and its virulence is not greatly influenced by the action of glycerin, as when mixel with it and kept in a dark place the virus retains its virulence from $s$ to 10 months.

Natural Infection. In healthy herds the infection usually occurs through vaccinated persons. This has been proven by the positive results of inoculation experiments and also by the observations that the disease attacks quite frequently large numbers of cows shortly after the vaccination of children. For instance the disease is usually observed among cows in the spring, at which time the vaccination of children is customary, when the dissemination of the virus is given a very favorable opportunity.

Infection usually takes place luring milking, when the virus is easily transmitted from the hands of the milkers to the superficially injured skin of the udder. The disease is also frequently transmitted from animal to animal by the milkers during milking, but contaminated straw, food and the stable floor may play a part as intermediate carriers of the virus, and this infrequent node of infection has been observed in young stock, steer's and oxen. In all probability the infection is not transmitted through the air.

True human pox may also in all probability be transmitted to cattle, although the results of artificial inoculation do not prove it (see p. 300). As a matter of fact in the eighteenth century and at the begimning of the last century, at which periods human pox still occurred frequently in an epidemic form, the disease was also observed with great frequency among cows.

Symptoms. The disease usually commences after an incubation period of 47 days, with moderate febrile symptoms (rise in temperature of 1/2-1 degree, diminished appetite, irregular rumination, weakness, etc.). In many cases however these prodromal symptoms are so mild that they pass unnoticed. During milking it is noted that the udder is sensitive, the milk is thinner, of a lower specific gravity and coagulates more quickly. On the slightly warm and swollen teats, as well as on adjoining parts of the udder, hard nodules in size ranging from that of a lentil to a pea appear on the second to third dav, which change inside of 1 to 2 days into bean-sized vesicles, filled with a clear, transparent lymph. The vesicles are reddish or bluish, or vellowish-white, of a mother-of-pearl or metallic luster, sometimes lusterless, depending on the thickness of the skin, and on its color. On the body of the udder the vesicles are uniformly 
round, on the teats they are oval in the direction of the long axis of the teat, and they are surrounded by a red zone of 1 to $2 \mathrm{~mm}$. width. Toward the eighth to eleventh day they usually become ripe, and then they show in their center a welldefined depression ("Delle" navel), which however may sometimes be absent. Later the contents of the vesicles turn purulent, and dry to scabs (Fig. 56). After the scabs drop off the underlying skin is at first pale red and swollen, later however it shows shallow, white, cicatricial depressions in the affected parts.

According to the severity of the case one, two or numerous, but only exceptionally more than 15 to 20 , vesicles develop. They do not appear at the same time, but mostly at intervals of a few days, thus between the appearance of the first and the last

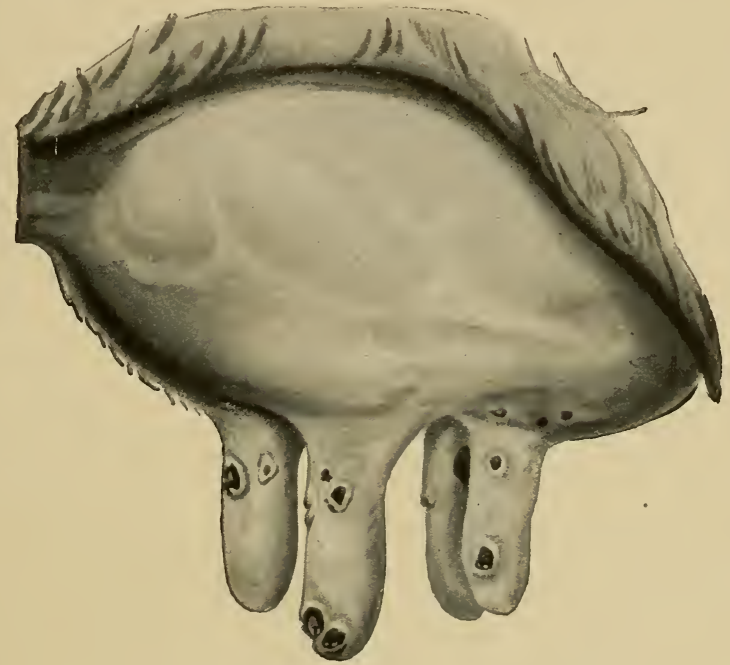

Fig. 56. Cow pox on the udder. (After Freger.)

vesicle 4 to 6 and sometimes even 14 days may elapse, as may be seen by the varying stages of development of the vesicles. Frequently the later vesicles are smaller than the early ones.

Outside influences, especially traumatic, have frequently an unfavorable effect on the otherwise mild course of the disease. During milking the vesicles may easily rupture before they obtain their full ripeness. On such an exposed, readily bleeding wound the scab formation cannot take place on account of the repeated handling, and in the meantime the regeneration of the epithelium does not take place in the usual way, but on the contrary the inflammation of the surrounding connective tissue is aggravated from the frequent irritation of the wound.

Vol. 1-21 
Occasionally infection of such wounds may lead to the development of severe ulcerations, which considerably retard the healing process. Frequently 30 to 40 days may be required in such cases for recovery. Exceptionally a parenchymatous mastitis may be associated with the eruption of pox. As the milk is mixed with the secretion of the wounds it of course becomes contaminated.

In rare cases eruptions resembling those which occur on the udder of cows may develop on the scrotum of male animals.

A generalized pox eruption occurs only very rarely (Dupuis observed it in two cases, Strebel in one instance). In such cases the eruptions, which may reach the size of hazelnuts, appear, associated with febrile symptoms, on the udder, also on the inner surface of the thigh, on the croup, rump, chest, neck and in the muzzle. (In Strebel's case paralysis of the stomach and intestines was observed in the later course of the (lisease, and after a temporary improvement of 8 to 10 days, a fatal peritonitis developed.)

Diagnosis. The diagnosis of cow pox is based on the presence of roundish or oval vesicles containing a depression in the center. In some cases the spreading of the eruption to other animals and a similar exanthema on the hands of the attendants handling the affected animals, may reveal the nature of the affection. The injuries and irritations which are usually caused by the milking, may sometimes interfere with the establishment of a diagnosis.

It is possible to mistake the exanthema for that of footand-mouth disease, but in this the vesicles do not develop from nodules, they are larger and less uniform in appearance, and similar vesicles are present in the mouth and in the interdigital spaces of the affected animals in the stable. The benign, coital vesicular-exanthema is readily recognized by the simultaneous affection of the genital organs. Pustules may sometimes also develop on the udder in rinderpest, but these findings are unimportant as compared with the other general and local manifestations of the disease.

The nature of the eruptions, which have been described by German authors as "wind and stone pox," is as yet doubtful, nevertheless the possibility does not appear to be excluded that they represent abortive forms of true cow pox. According to description they form hard nodules which develop on the lower part of the udder and on the teats, and change into purulent vesicles. In doubtful cases the inoculation of a calf would readily reveal the nature of the affection in a short time.

Ehrhardt described under the name of false cow pox (pointed pox, varicellae) a stable disease of cows, in the course of which desquamation of epithelium from the teats occurs, causing severe inflammation. In one case the milker also became affected, his hand was greatly swollen, and numerous vesicles up to the size of a pea appeared on his skin, eausing considerable pain. 
In human medicine the term "varicellen" (sheep pox, water pox, wind pox), designates an acute exanthema of children, in which in the course of only a few hours small vesicles develop on the skin, and sometimes also on the mucous membrane, which however soon dry into scabs. This condition is associated with mild general disturbances. Recovery from this disease does not produce immunity against the true pox, and therefore the affections are considered etiologically different.

Treatment. Besides keeping the affected udder clean and dry, it is advisable to milk the animals daily with great care, or to draw the milk with the aid of milk catheters. If ulcerations have appeared at the place of the vesicles they may be treated with neutral fats, with zinc or lead ointment. If milking has been discontinued, uneventful recovery takes place within 10 to 15 days.

Immunization. Fréger vaccinated all cows of an infected herd with calf lymph used for man. The vaccination was carried out on the perineum by superficially scarifying the skin. Nodules developed upon the already affected cows, while on the healthy animals typical pox postules appeared, and these animals did not contract the infection. The same procedure was also employed with good results by Krause in a large herd, in which the affection existed for $11 / 2$ years; newly introduced animals which previous to the vaceination had usually become affected within 3 to 4 weeks, failed to develop the disease after the inauguration of this method.

Literature. Jenner, Inqu. of the causes and effects of Variolae-Vaccinae. London. 1798.-Bollinger, Volkmann's Samml. Klin. Vortr., 1877, Nr. 110 (Lit.).Ehrhardt, Schw. A., 1896, 81.-Strebel, Schw. A., 1898, XL, 113.-Negri, Esper. sulla filtr. del virus vace. Pavia, 1905.--Pröscher, Cbl. f. Bakt., 1906, XL, 337.Carini, Cbl. f. Bakt., 1906, XLII, 325.-Nicolle \& Adil-Bey, C. R., 1906, CXLII, 1196.-Fréger, J. vét., 1906, 385.-Leese, Trop. Vet., 1909, I, 1.

Pox Vaccination of Man. The discovery of the English physician Edward Jenner (1796) established the fact that the inoculation of cow pox lymph in man produces at the point of inoculation a mild pox eruption, the recovery from this producing in the vaccinated person a lasting immunity against true pox. This method eaused the abandoning of the older procedure, in which the lymph of man affected with pox was used for pox vaccination, and as a result of which the vaccinated persons not infrequently became severely affected with generalized pox. Jenner used at first exclusively original animal lymph, but as it was later observed that this virus could be transmitted from man to man, in which ease its character does not change, the vaccination with such humanized vaccine, that is, with the contents of vesicles from man vaccinated with original cow pox lymph, has latterly attained continuously increasing use.

In the meantime various disadvantages have been observed in this procedure. In order to practice this vaccination, a vaccinated person in the proper stage of the disease must be available, which is often very difficult. This condition cannot be remedied by conserving the lymph obtained from vaccinated persons, as in this manner only small quantities of lymph may be collected. A still greater disadvantage lies in 
the proven fact that occasionally diseases of persons producing the lymph (syphilis, tuberculosis) are transmitted by the vaccination to the persons being vaccinated.

On account of this danger, bovine lymph is used exclusively in recent times for vaccination. The vaceinating lymph is eultivated artificially in state or private establishments erected for the purpose. The lymph is constantly re-inoculated from calf to ealf, and then preserved hy a special method. For the original vaceine either original cow pox lymph, or lymph of a calf vaccinated with humanized vaccine is used. By employing calf lymph the danger of transmitting other diseases may be excluded with certainty, by using young calves exclusively for cultivation, and by placing the collected lymph on the market only after the health of the vaccinated animals has been proven by an autopsy.

At the present time the great practical value of pox vaccination is recognized to such an extent that in most countries compulsory vaccination is established by law, in spite of the fact that here and there opposition is made against it.

For the cultivation of the vaceine, weaned, healthy calves, preferably male animals, are commonly used. These should previously be tested by tuberculin for the presence of tuberculosis. The animal is secured lying on the side upon a suitable table, the abilomen from the navel to the pubic-symphysis, or one-half of the thorax, is shaved and thoroughly cleaned. Then the lymph from a ripe vesicle of another calf, or of a vaccinated person, is inoculated into the skin of the calf. The procelure is carried out by puncturing the skin in 100-200 points into the deeper epithelial layers, or in such a manner that upon the surface of the skin superficial incisions $1-2 \mathrm{~cm}$. long are made, and the lymph is then rubbed iuto the wounds. In from 4 to 5 days bluish nodules and resicles on a bright rediened base develop at the point of vaccination, from which the lymph is pressed out with suitable clamp forceps, and preserved either as pure lymph, or together with epithelium, that is scraped off, mixed with equal parts of glycerin.

The lymph is preserved in glass tubes, or painted in thin layers on ivory plates $5 \mathrm{~cm}$. long and $7 \mathrm{~mm}$. wide, the scraped fluid is enclosed between two small glass plates and sealed with paraffin. As in the lymph prepared in this manner bacteria are destroyed within a few weeks without affecting the potency of the lymph, only vaceine which is from 2 to 3 months oll is used for human vaccination.

Literature. S. Huguenin, Ergebn. d. allg. Path., 1897, IV, 246.

\section{(c) Horse Pox. Variola equina.}

\section{(Sore heels; Pferdepocken [German]; Tariole équine [French]; Vajuolo equino [Italian].)}

Occurrence. Horse pox which was extensively studied by Jemner, supposedly existed more frequently 3 or 4 decades ago, and at that time periodically attained an epizootic extension among hor'ses. The descriptions however do not reveal a sufficiently clear conception regarding the true nature of the affection observed, as English and French authors (among them Bouley and Lafosse, also Nocard \& Leclainche) understood under the name of "horse pex" that disease which since the studies of Eggeling and Ellenberger (1878) is otherwise known as contagious pustular stomatitis, and considered as an independent affection. (see p. 360). Jenner's horse pox is at the present time so rare that some authors, among them Dieckerhoff, doubt its occurrence entirely. 
Etiology. Jenner searched for the source of cow pox in horse pox, and did so in that form of the disease which is manifested as a vesicular eruption in the posterior part of the pastern. Later, as las been mentioned, the disease was identified with pustular stomatitis or dermatitis, and therefore the infection experiments supposedly earried out with horse pox virus must be regarded as indecisive.

Loy transmitted horse pox snecessfully to man in seven eases, and Hertwig, also Pingaud observed the natural transmission of horse pox to man. Recently Cameron (1908) reported a ease in which on the arm of a driver who treaterl a horse affected with pustular stomatitis, a pox eruption developed. Chanveau, on the other hand, prodnced general pox eruptions in older horses, by injections of vaceine into the lymph vessels, and in young animals with an injection into the blood circulation (large vesicles developed especially on the nostrils, lips and on the rump; they first appeared eight days after the infection). Similar results were obtained in transmission experiments by Warlemont and Pfeiffer.

Symptoms. At the present time the vesico-pustular exanthema, in the flexor region of the pastern of young animals, is considered as horse pox, in which it is supposed that the disease is transmitted to the skin of the pastern principally during shoeing by the hands of the blacksmiths and drivers (Friedberger \& Fröhner). The sensitiveness of the pastern, and the functional disturbance of the extremities produced by this, sometimes also a febrile elevation of the body temperature, indicate the development of the affection. The skin on the posterior surface of the pastern is swollen and reddened, soon vesicles develop to the size of a lentil, which burst shortly after, whereupon scabs develop on the reddened, moist epidermis, under which new epithelium forms. In rare cases the eruption develops also on other parts of the body, as on the head, especially around the opening of the month and nostrils, exceptionally also on the mucous membrane (Berger observed the appearance of pox in 15 horses on the conjunctiva).

Diagnosis. A disease which is manifested with the above symptoms could be considered as pox and differentiated from the common exanthema of the pastern only when typical pox vesicles develop on the affected part of the skin, or when the inoculation of the contents of the vesicles produces a typical eruption of pox on the skin of cattle. From the standpoint of differential diagnosis the contagions pustular dermatitis, coital exanthema, and the contagious pustular stomatitis come into consideration.

Literature. Jenner, s. p. 319.-Bouley, Dict. de méd. vét., 1871, IX, 451.Chauveau, Rec., 1866, 305 u. 625.-Hertwig, Chirurgie, 1874, 182 (Lit.).-Dieckerhoff, Spez. Path., 1892, I, 999.- Noeard \& Leclainche, Maladies mierob. des animaux, 1903, I, 598.-Cameron, Brit. med. J., 1908, I, 1292.

\section{(d) Swine Pox. Variola suilla.}

Occurrence. Swine pox represents a very rare disease. In some years it appears to occur in certain parts of Southern Hungary with considerable frequency (in 1907 it existed in 
seven counties of that territory), and recently it was also reported from Roumania (Poenaru).

Etiology. The susceptibility of hogs for the pox virus was established experimentally a long time ago. Chauveau succeeded in several cases in transmitting cow pox to hogs, Gerlach swine pox to goats, Koch the same to calves. Szántó placed two pigs affected in the pustular stage of the disease among 7 to 8 weeks old, healthy pigs, wherempon six developed typical pox after an incubation of four days, and the disease was further transmitted to healthy pigs by cutaneous inoculation. An inoculation of lambs always resulted in a local, flat swelling, as well as an acute conjunctivitis and nasal catarrh. Poenaru also succeeded in producing the disease in pigs with blood and with contents of pustules, in one instance in the generalized form with a fatal termination.

Originally the disease develops in pigs through infection from eattle, from man and probably also with material originating from goats. In the infected piggeries the disease may easily spread to a great extent, and may also be disseminated to other herds.

Szántó observer 64 out of 74 pigs affecter with the disease, of which 3 sucumberl, while Lövy recorded a loss of $18 \%$ in a herd of 155 pigs. Laquerrière observed swine pox as a severe plague with usually a fatal termination as early as in 1864 in Algeria. Old hogs became severely affected in this outbreak.

Symptoms. According to the descriptions (Spinola, Szántó) the disease as a rule affects young pigs, and is usually manifested in an exanthema affecting a large part of the body. Several davs after infection the animals show symptoms of a general disturbance, such as fever $\left(41.5-41.8^{\circ}\right.$ C.), weakness, loss of appetite, sluggish movement, stiff gait, straightened tail, chills, bristles standing up and greasy, on account of the marked evaporation from the skin. In the early stages catarrhal symptoms, especially conjunctivitis, are often observed. Soon small red spots appear in places only slightly or not at all covered by bristles, as on the snout, eyelids and inner surface of the thighs, the abdomen, less frequently on the neck and on the back. These grow rapidly to sizes like a ten cent piece, in the center of which hard nodules develop. After 2 to 3 days pea-sized vesicles develop, which at first contain pure lymph, later pus. They dry to dark brown scahs, and fall off after several days. The number of vesicles varies greatly, and the intensity of the prodromal symptoms, also the course of the disease is not uniform. In severe cases the vesicles coalesce, their contents become bloody, eruptions appearing also on the mucous membrane of the mouth, pharynx, in the trachea and in the bronchi. In the meantime diarrhea sets in, whereupon the disease terminates in death from exhaustion, broncho-pneumonia, or from a general infection. (Lövy also found on the 
mucous membrane of the stomach, flat ulcers surrounded by a red zone, we have also observed such lesions with pale yellow elevated borders, and a dark, yellowish-red, granular base.)

Diagnosis. Before establishing a diagnosis, the various forms of eczema, granular eruptions, and erysipelas-urticaria should first of all be excluded. If necessary it conld also be established by artificial inoculation of the contents of vesicles upon healthy animals.

Immunization. In emergency this could be undertaken in a similar way, and probably with the same results as in vaccination of sheep for the protection of the animals which are not yet affected.

Literature. Spinola, Krankheiten d. Sehweine, 1892, 204.-Koch, Ö. M., 1887, 57.-Szántó, Á. L., 1906, 541.-Poenaru, Arh. vet., 1907, 67.-Lövy, Á. L., $1908,103$.

\section{(e) Goat Pox. Variola caprina.}

Occurrence. Goat pox has been observed principally in Norway, where it occurs with relative frequency in certain localities (Boeck, Hansen); it was also established in Italy, Spain, France, Germany and Algeria, where the disease runs sometimes a very unfavorable course.

Etiology. The virus of goat pox may be transmitted to goats with the contents of the pox eruptions, and also with the saliva of the affected animals if the vesicles have appeared in the mouth. Cutaneous inoculation results in the development of a local pox eruption at the point of inoculation, associated with very mild, general symptoms. In some cases the transmission to sheep was also snceessful, however it only produced an incomplete pox exanthema at the point of inoculation (Bonvicini, Marcone). Man is not entirely without susceptibility, as occasionally persons working around affected goats develop an exanthema with small vesicles on the hands and arms (Hansen, Marcone).

Goats are sometimes infected by sheep affected with pox, whereupon they become affected either with mild or severe symptoms. A subeutaneous inoculation of sheep pox virus, when the transmission is successful, results in fever after the third day. On the seventh to eighth day a papular eruption appears, and if the animals die nodules are found in the lungs and in the liver. There is also a swelling of the intestinal follicles, and of the mesentery lymph glands (Voigt). Subsequent inoculation of the fluid from the lesions at the place of injection into goats is supposed, according to Konew, to increase the virulence of the virus for goats, while for sheep it becomes attenuated (see p. 306). Voigt, on the other hand, found that it later possesses a lesser virulence, even for goats.

Natural infection results from direct contact, and in this manner the disease spreads rapidly in goat herds, so that as 
a rule almost every animal becomes affected (sheep kept together with goats usually escape the infection, or become only very slightly ill, Garbuti \& Reali). The disease shows no tendency to spread over large territories, but is mostly confined to single flocks.

Symptoms. The clinical appearance closely resembles that of sheep pox. In association with moderate, febrile and catarrhal symptoms, various sized partly coalescent vesicles, containing a depression on top, develop on various parts of the body, especially however on the udder, on the inner surface of the extremities, on the face, in the parts surrounding the lips and eyes. They form scabs in the usual manner, and heal with radiating scars. In some cases abscesses develop in the tissue of the udder, when the milk may become bloody. In some animals, especially in kids, pox also develop in the mouth and on the mucous membrane of the.upper air passages, their appearance being manifested by cough, accelerated respiration, and purulent nasal discharge.

The course of the disease is usually favorable; although the skin may become gangrenous at the places where the resicles coalesced, the recovery is however only retarded therehy, not prevented. Cases in which the disease develops with severe svmptoms from the onset, and in which a general infection causes death, are very rare (such severe outbreaks were observed in Algeria).

Diagnosis. The disease can only be confused with footand-mouth disease, as the pox vesicles in goats may sometimes attain a size up to that of a hazelnut (Conte); the characteristic appearance of the pox exanthema, and the absence of lesions on the feet, together with the non-transmissibility of the disease to other cloven-footed animals, will prevent errors in diagnosis.

Treatment and Prevention. The indications which hold for the treatment and prevention of sheep pox apply also to this disease.

Literature. Hertwig, Mag., 1840, 339.-Boeck, D. Z. f. Tm., 1879, IX, 298.Hansen, Ref. Rep., 1890, 135.-Bonvicini, Il nuovo Ercolani, 1898, 216.-Mareone, La Rif. vet., 1900, 387.-Garbuti \& Reali, Clin. vet., 1905, 234.-Voigt, A. f. Tk., 1909, XXXV, 204.

\section{Foot-and-Mouth Disease. Aphthae epizooticae.}

(Maul-und Klauenseuche [German]; Fièvre aphtheuse, Cocotte [French]; Febbre aftosa [Italian].)

Foot-and-mouth disease is an acute, febrile, contagious, infectious disease of cloven-footed animals, in the course of which a vesicular exanthema develops on the mucous membranes, and the skin, especially in the month and interdigital spaces. The causative factor is an ultra-microscopical microorganism. 
History. The cause of the disease was attributed previous to the middle of last century, to various atmospheric influences, as well as to spoiled food, although Sagar recognized its contagious nature during his observations in Norway in 1764. In the second half of the last century, especially since Bollinger reached his definite conclusions, it has been generally recognized that the disease develops exclusively as the result of a specific infection, but its causative agent is even at the present time not clearly established. While various authors deseribed certain bacteria, or protozoa, as the cause of the disease (Siegel, Schottelius, Kurth, Behla and other's), Löffler \& Froseh (1897-1900) and Heeker (1899) showed that the virus passes the ordinary porcelain filter, and the first authors, with Nocard, and recently also Poels, eontributed valuable information relative to the eradication of the disease with serum inoculations.

Occurrence. From time to time foot-and-mouth disease spreads rapidly over extensive territories, as a severe disease, affecting principally cattle, in a lesser degree hogs and sheep. Its spread was especially rapid in former times, when, spreading from the east westward it swept unchecked during a period of 2 to 4 years, passing over the entire European continent, whereupon a period of freedom from the disease followed its disappearance which lasted for several years. In recent times the disease usually spreads more slowly, since its dissemination is more or less controlled by veterinary police measures. In the course of the various historical outbreaks, recurrences were repeatedly observed, but uniformity between the appearance of outbreaks, and the periods of freedom from the disease is not very regular. Formerly almost all the susceptible animals of large territories became affected within a short time by the rapidly spreading disease, and therefore the disease disappeared of itself, when there were no longer any susceptible animals left, while in recent times the growing young stock continually affords suitable material for the perpetuation of the virus, so that infective herds remain, even after the apparent disappearance of the disease, from which in a short time new outbreaks may develop.

The disease spreads with particular rapidity during warm weather, while in the winter it does not occur so extensively. With the appearance of warm weather, however, it frequently gains a wide prevalence, as a result of the greater traffic in cattle.

The economic importance of foot-and-mouth disease lies in the severe losses, which the owners sustain from the long period of inactivity of the affected animals, from the loss in body weight, and from the diminished milk production, also by the considerable limitation of the traffic in cattle, and through the loss of calves and young stock, sometimes also of mature cattle. These losses caused the inauguration of measures for the eradication of the disease by the government, and the efforts thus marle have in recent times brought gratifying results in many places. 
The last great epizootic in Europe occurred in 1887, its starting point being Russia. The disease invaded all the countries of the continent, and raging with varying intensity has not been eradicated even up to the present time.

In Germany the disease commenced to spread rapidly in 18s8, and in 1892 it reached its height (number of animals in the newly infected premises: 1,504,299 eattle, $2,193,187$ sheep, 17,782 goats and 438,262 hogs); from that time on it diminished, but in 1899 it again reached a wide extension (1,885,774 eattle, 1,505,830 sheep, 59,535 goats and 814,862 hogs); since then the disease has rapilly retrogressed, so that in November, 1905, the entire Empire was free from the infection. However at the end of this month it appeared in isolated heris in western Prussia, from which it spread to the abattoir of Berlin, and thenee to the Province of Saxony. At the end of the year 1906, 55 townships were infected. In the following three years it again reached a great extension, first by an introduction from France to southern Germany, later oceurring also in the other states, and in the year 1908 , 324 townships, 18,773 eattle, 19,450 sheep, 179 goats, and 16,081 hogs were affected. In May, 1909, the disease was entirely suppressed, and only one outbreak was afterwari established in December at Mittlefranken.

In France the disease raged severely in 1893 . Soon it spread rapilly, and reached its height in the years 1899 and 1900 , and from 1901 it gradually retrogressed, again gaining a great extension in 1906. In 1907 it retrogresserl, and at the end of 1908 only two premises in two townships were affected.

Great Britain was free from the disease between 1895 and 1899 . Since 1900 however, several townships have been infected annually, in spite of the fact that the importation of living cloven-footed animals from the continent has been prohibited since that year. In each instance, the outbreaks were localized by slaughtering the affected herils. In the years between 1903-1908 the entire territory of the Kingdom was free from the disease, with the exception of an outbreak in the vicinity of Ipswich in January, 1906, and another in Edinburgh in 1908.

Holland was very severely infected in the years of 1897-1899 ( 868,206 and 122,567 eases of infections). Since that time however the disease has rapidly diminished. In 1907 it gained again a greater extension (17,816 outhreaks with 341,287 eases in 11 provinces). Since the second half of 1908 only isolated cases have oceurred.

In Austria the infection was very widely spread in 1891 (247,946 eattle, 32,423 sheep, 5,013 goats, and 18,741 hogs were affected). It diminished rapidly until 1894 ( 7,462 eattle, 617 sheep, 45 goats, 1,379 hogs). From that time on a rapid extension up to 1896 took place (305,934 eattle, 11,422 sheep, 1,032 goats, and 18,816 hogs), whereupon an improvement was noted, and the Empire was considered entirely free in 1901. Since that time it spread again, and in the years from 1904 to 1906 was widely spread, especially in Poland and Bohemia. In the subsequent two years it raged with varying, but mostly with mild intensity, and toward the end of the year only 12 prenises in seven townships were infected.

In Hungary the disease which had been introduced in 1889 from the southeast, reached its height in the subsequent year (561,950 eattle, 337,566 sheep and goats, and 370,425 hogs); later it showed a dimimition up to 1894 , but in 1896 it again attained wide extension (572,809 eattle, 178,612 sheep and goats, 82,931 hogs). In 1901 the entire Kingdom was free for six months; in the fall of this year the disease reappeared as the result of an introduction from Roumania, and since that time has again attained wide extension. In 1903 , in 2,516 townships, 434,221 cattle, 30,533 sheep and goats, and 10,951 hogs, were affected. From that time on the eondition improved, in 1907 however, in the northwestern part of Hungary, on the Polish border a badly infected locality was discovered which railiated all through the country, and in 1908, in 108 townships, 16,988 cattle, 491 sheep and goats, and 48 hogs were still affected.

Roumania was severely affected in 1907 ( 677,465 cases of infection). Since that time the disease has diminisher (in 1908 11,038 eases). In Russia in 1907, 596,977 eases occurred in 6,320 townships; in 1908, in 3,274 townships, 292,302 cases. The disease also occurred in 1908 in Belgium, in 11 townships, with 147 cases; in Italy, in 47 provinees, with 138,887 eases; in Switzerland, in 96 townships, with 13,555 cases (in 1909 the disease attained in the latter country a great extension), while in Bulgaria 61 townships were affected; Denniark, Norway, Swerlen and Servia were free from the infection.

Since the outbreak of 1870 in the United States, only a few limited outbreaks have occurred. In the fall of 1902 the disease appeared in the States of Massachusetts, Vermont, New Hampshire and Rhode Island, but was eradicated in a few months by energetic measures (see veterinary police). In the fall of 1908 the 
disease reappeared in the States of New York, Pennsylvania, Michigan and Marylancl. (See 1. 353.)

Argentina was severely affected in 1900.

In Asia and Africa the lisease prevails from time to time over large areas, while Australia is free from the infection.

At certain times, and in certain localities the disease shows a deviation from the normal conrse, by exhibiting a very severe character, and in such instances many animals are destroyed by it. Thus according to Rychener 2,000 cattle died in 1839, in the Swiss Kantons Bern and Freiburg, while in 1872 in the District of Nièvre in France $20.3 \%$ of calves and $22.2 \%$ of hogs suceumbed to the disease inside of two months. During the summer of 1892 nore than 3,000 animals died in Bavaria; in the course of $1596,1,500$ in Württemberg, two years later 1,300 . In 1901 the disease appeared in Spain with such a malignant character, that in Barcelona $50-70 \%$ of the young stock snecumberl, and these heavy losses caused a suspicion that the disease in question was the oriental rinderpest. In Hungary the disease ragerl in a severe form in 1893 in certain parts of the counties of Gömör and Pest, in 1599 in Siebenbürgen (in the counties of Csik, Maros-Torda and Udvarhely of 7,498 eattle, 711 died, amounting to $9.4 \%$ ).

Etiology. Löffler \& Frosch, also Hecker, and later Nocard \& Roux proved that the virus of foot-and-mouth disease in dilution will pass the porous (Chamberland, Berkefeld) porcelain filters, while it is partially retained by the denser Kitasato filter. It has not yet been possible to demonstrate the organisms either by methods of staining or cultivation. The virus is therefore an extremely minute, ultra-microscopic micro-organism.

Recently Ferni elaimed that the cansative agent is a protozoon $0.5 \mu$ in size (Cytoryctes Jenneri), which he succeeded in demonstrating in the lymph of the eysts, and also in the internal organs of 472 affected cattle, arranged like the pavement of a street, or radiating like the spokes of a wheel. A confirmation of this finding has not yet been marle.

The virus is contained in the purest state in the contents of the vesicles which derelop in the course of the disease, and greatly diluted also in the blood though only during the beginning of the fever. The saliva, the tear's, the nasal discharge, the milk, and the other secretions become infectious only after being. contaminated with the contents of the ruptured vesicles. In the later course of the disease the virulence of the serous fluid of the vesicles diminishes, and after ten days the saliva of infected cattle is no longer infections for other animals (Schütz).

Tenacity. Virus dried at room temperature and in daylight, loses its virulence insirle of 24 hours, diluterl in proportion of 1:10 and kept in glass tubes closed at both ends by melting, it remains infective for 3 to 4 months. A temperature of 37.5 degrees destroys it in 12 to 24 hours, one of 50 degrees in 15 minutes, and 70 degrees in 10 minutes. Heating to 100 degrees destroys its virulence immediately (Löffler \& Frosch). The action of a temperature of $-8-9$ degrees destroys it in several hours (Perroncito). Of the disinfectants, milk of lime, a $1 \%$ carbolic acid, $2 \%$ formalin, $3 \%$ soda, and $1 \%$ hydrochloric acid destroy its virnlence in one hour (Löfler \& Frosch). Infectious milk which has stood for 3 to 4 days, becoming soured and coagulated, is no longer infections (Ebertz). In fresh cow and hog nanure the virus is destroyed at a depth of more than $20 \mathrm{~cm}$. within a short time (Hecker).

Pathogenicity. Intravenous injection of fresh pure lymph will sometimes, even in a quantity of $1 / 5000$ cc., produce the disease in susceptible cattle. Depending upon the quantity and the virulence of the virus, typical vesicles develop in from 
1 to 3 days after the injection, in the montl, in cows also on the udder, and 1 to 2 days later also on the feet, these eruptions being associated with febrile manifestations. Infection may also result from rubling the lymph upon the superficially scarified mucous membrane of the mouth, by dropping the virus on the conjunctiva, the rectum or the vaginal mucous membrane, and further by intra-muscular, intra-peritoneal injections, likewise by inoculation of the milk ducts. Placing woolen threads dipped in lymph, between the teeth, and sometimes also feeding gelatin capsules filled with virulent lymph, may give positive results, while a subcutaneous infection is uncertain. Should virulent lympl be injected into the milk system of a fresh milking cow, local inflammation of the milk ducts develops, and the milk proves virulent for a period of eight dars (Nocard). The most effective is the lymph of infected hogs, then that of cattle, while the least efficient is that from sheep. The artificial infection of goats and sheep is in general quite difficult. Young dogs and cats are very susceptible to artificial infection (the vesicles develop in these animals between the digits), exceptionally guinea pig's and rabbits may also become affected (Hecker), while mice and fowl, as well as older, carnivorous animals, are resistant to inoculation. Young pigs die after several dars from paralysis of the heart after an inoculation of $1 / 10$ to $1 / 50$ ce. of fresh lymph. The autopsy in such cases reveals a great amount of serous fluid in the pericardium, which contains the virus in pure culture (Löffler, Nocard).

Natural infection often occurs directly, healthy animals coming in contact with affected cases, in the stable, pasture, or yards, by means of which they receive the virus upon their mucous membranes. Infection with inhaled air has not yet been proven, and could only occur within very short distance, in damp weather, or in the stable.

Indirect transmission has a far greater importance in the spread of this disease. The saliva, which flows from the months of affected animals, and also the contents of the ndder or the resicles which develop on the feet, contaminate the food, the cribs, the drinking water, the straw, the stable floor, the pasture, the highways, the railroad cars, etc., as well as the hancls and the clothes of the attendants. From such contaminations, or from the floor, the virus reaches the mucous membrane of any body opening, or with the food or drinking water it enters the month and stomach of the healthy animal, from which points it enters the blood eirculation. The virus is accordingly disseminated in the first place by affected animals during driving, railroad transportation, stabling, by common drinking places, common pastures, and yards. In the second place food, manure, and raw animal products, especially raw hides, wool and milk may be considered as carrier's of the infection. Butchers, dealers, peddlers, castrators, and other persons, first of all farm hands, 
and also animals which are not susceptible, coming from infected premises, may contribute to the transmission of the infection. Stock markets play an especially important part, through trading, further also changes in farm hands.

The obscure ways by which the disease sometimes spreads are especially well illustrated in the periodical appearance of the affection in England. Although the importation of rloven-footel animals from the continent into that country has been entirely prohibited since 1892 , and from other parts of the world, permission was granter only after the lapse of the period of inculation, yet since 1900 the disease has appeared in several different places (for the outbreak in 1908 it is assumed that it was introduced with hay from Hollanil). The last outbreak in the United States originated from several ealves which were inoculated with pox lymph imported from Japan, and Mohler \& Rosenau proved by experiments that this tymph contained the virus of foot-and-mouth disease even after one year.

Whether the disease can be disseminated by bloor sucking insects, especially by the tabanus bovinus, as suggested by Roch Marra after making several experiments, must at the present time be questioned.

In recent times where isolated outbreaks occur more frequently in territories which have heen free from the infection for a time, and in which careful observations luve been made, the possibility enter's more and more into the foreground that animals which pass through the disease may harbor the virus in their bodies for a long time, and expel it periorlically or continually (so called virus carriers). This may explain why it is that animals occasionally become affected when placed in herds in which the animals have apparently entirely recovered from the disease, or that the disease breaks ont on the premises where such animals are introduced ( $\mathrm{Pr}$. Vb., Bartolucci, Löffler, Lourens). Although an introduction by other means cannot be excluded with certainty in such cases, and the presence of the rirus in recovered animals has not yet been established experimentally, nevertheless the possibility camnot be ignored that virus carriers have heen recognized beyond a loubt for other diseases (typhoid fever, Asiatic cholera, hog erysipelas, tetanus). Therefore in ascertaining the cause of new outbreaks this contingency should be given due consideration.

According to Barsolucei eattle are infectious two months, and accorling to Löffler even six (?) months after the disappearance of the vesicles, and in accordance with the Pr. Vb. for 1907 a bull was suprosed to transmit the disease to other cattle after a period of $2 \frac{1}{2}$ years (?).

Although successful infection may occur through the uninjured mucous membranes, nevertheless it is favored by loss of substance and by excoriations in the mouth, on the skin of the udder, and in the interdigital space (in feeding dry food, also while pasturing on stubble fields, the disease sprearls with particular rapidity).

The infection of sucking animals, or those which are fed with milk, occurs almost invariably through the milk of affected animals, or through the skim milk from creameries, and is usually acquired through the uninjured mucous membrane of the 
digestive tract, which at that age is sensitive, owing to its covering of loose epithelium.

Susceptibility. Cattle are most susceptible to natural infection, then follow hogs, sheep and goats. Differences are observed in this regard in the various outbreaks, inasmuch as the last named species of animals may become affected in the same degree as cattle, or at least in considerable numbers. Buffaloes may also become affected, oceasionally however they remain healthy among affecter cattle, while in other cases the cattle may become affected through buffalo. Wild cloven-footed animals, including boars (Borzoni) are also susceptible to the disease, and sometimes it exists among these animals in an epizootic form. Reindeer and camels are also susceptible, and very exceptionally horses, dogs and cats may becone affected.

Among the animals of a certain species a variation in susceptibility may be observed in the various breeds, as under the same conditions a greater number of animals of common breerls will resist the infection, than of the finer breeds. While under conditions favorable to the infection, animals of the western European breeds become affected in the proportion of $95-100 \%$, among the common breeds only $40-50 \%$ contract the disease, and even after an energetic, artificial inoculation, not infrequently $20-30 \%$ of the animals remain healthy (Kovaesy, Born). Accoriling to Hartenstein hogs of the Mangalieza breed are more resistant than the Polish or the German breeds. In Köbánya hogs from the lower Danube region became more sererely affected than the native hogs.

Recovery from the disease reduces the susceptibility for a certain time. The immunity thus acquired lasts in the majority of cases longer than a year (according to Mazzini from 3 to 5 , and according to Schwenck even up to 7 years). Exceptionally the same animal may become re-infected by the disease within a short time. Strebel observed re-infection by the disease in cattle after 6 to 10 weeks, Makoldy after 12 days, while Kovacs observed a re-occurrence of the disease in a herd of hogs 10 days after the recovery of the animals. Warnesson reports a case in which a cow became affected three times within a year. Calves from cows which recovered from the risease at an advanced stage of pregnancy are sometimes immune towards the natural, as well as the artificial infection, while the feeding of milk from immune cows does not reduce the susceptibility of the calf (Fröhner, Löffler \& Frosch).

Pathogenesis. Observations that the disease develops in a typical form after intravenous inoculations of the virus, that the development of vesicles is preceded by fever, and that the hlood is infectious at the onset of the disease, indicate that the virus, at least in some of the cases, reaches the tissues predisposed for the development of the exanthema, by transmission through the blood stream. As such should be considered the deeper epithelial layers of the mucous membranes, and the finer portions of the skin. The frequent affection of the mouth, udder, and feet may possibly be associated with traumatic irritation, or with superficial defects of these parts of the body., 
The virus which penetrates between the epithelial cells of these parts of the body in all probability commences to multiply immediately, later however it passes into the blood stream, by which it is distributed to distant parts of the body. The affection of feet and udder, which later becomes associated with the affection of the mouth, may be of such metastatic origin.

The virus may be transmitted from the mother to the offspring, in which ease the young may be born showing manifestations of foot-and-mouth disease (Möbius).

According to Kitt the development of the vesicle commences with hyperemia and serous infiltration of the papillae of the skin or mucous membranes. The serous exudate loosens the epithelial cells from the mucous layer between the papillae, then it raises the more resistant, superficial layer of cells, while the contents of the developed resicles in the meantime becomes cloudy, as a result of migration of white blood corpuseles. The epithelial defect which develops after the bursting of the vesicles hecomes covered by epithelial cells which form either from the remaining part of the stratum mucosum, or by a multiplication from the periphery, and complete healing takes place in this manner, except that the papillae become somewhat smaller than they were originally.

Besides the unknown virus, the fluid of the vesicles may contain various other bacteria; such as Kurth's streptococens involutus, the staphylococeus pyog. aureus, the micrococeus tetragenus, sarcinae, etc. (Sanfelice). These pus-producing organisms may modify the later course of the disease, and the occasional complications which develop (deep ulcerations and suppurations, purulent inflammations of the feet, mastitis, pyemia, ete.) may be attributed to their action.

Anatomical Changes. Autopsies on animals which are slaughtered during the development of the disease, or which suddenly die, reveal as a rule, in addition to the characteristic exanthema, only an acute catarrhal swelling of the mucous membrane of the mouth, of the pharynx, and of the air passages, or there may also be present small hemorrhages on some of the serous membranes, especially on the visceral layer of the pericardium. In some eases however vesicles, or ulcerations may be observed in the wall of the pharynx, on the mucons membrane of the bronchi and esophagus, in the stomach (in ruminants in the first three stomachs, Uhirich), and in the intestinal canal. The ulcerations may sometimes be covered by a fine fibrinous deposit.

Animals which die as a result of complications, reveal severe suppurating processes, especially on the lower parts of the extremities, which are frequently associated with caries of the neighboring bones. There may also be found purulent or ichorous inflammation of the joints, or metastatic abscesses in varions parts of the body, and acute degeneration of the parenchymatous organs. In some cases a gangrenous pneumonia is the immediate cause of death, which results from inhalation of saliva or food particles.

If the animal dies suddenly in the stage of commencing recovery, from the malignant type of foot-and-mouth disease, 
the macous membrane of the abomasum, and of the intestines, is found to be dark red, covered by numerous hemorrhages, while in the rumen, especially in the vicinity of the folds, also in the leaves of the third stomach, and in the intestinal tract, and sometimes in the pharynx, erosions and ulcerations, sometimes of the size of a silver dollar, may be observed. The parenchymatous organs show fatty degeneration, which change is sometimes so pronounced in the muscles of the heart that its cut surface appears mottled, because of the rellow spotting (Johne, Nocard; according to Trattñer there is also hyaline degeneration in the affected parts). The spleen is moderately swollen, dark red and softened. Hemorrhages may also be found on the mucous membrane of the respiratory organs, and in the serous membranes. Finally in some cases the interlobular and subpleural connective tissue of the lungs may show an edematous infiltration, and the contents of the ventricles of the brain may be increased and cloudy (Willach).

Symptoms. After an intravenous injection of the virus, the time of incubation in cattle varies from six hours to six days (Löffler \& Frosch) ; after inoculation into the buceal mucous membrane it is from 48 to 60 homrs (Schütz); after natural infection the time is between 2 and 7 days, exceptionally even 11 days (Siedamgrotzky, Born). Data regarding a longer period of incubation (according to Schrader, Lies and Möbius 14, according to Isepponi even 21 days) are doubtful.

Without considering the manner and method of the infection as well as the place of the eruption of the vesicles, the disease commences with fever, which is quite evirlent in young, strong animals, while in older animals it may sometimes be so moderate that it is not observed at all. The body temperature in the first 1 or 2 days may reach $40-41^{\circ}$, but as soon as the vesicles develop, possibly in from 6 to 24 hours, it rapidly drops, and the later course of the disease runs without fever, with the exception of those cases in which inflammatory complications resulting from a subsequent septic infection become associated with the original disease. The rise in temperature is usually accompanied by a somewhat accelerated pulse, and diminished appetite.

The other symptoms vary in accordance with the localization of the affection.

The affection of the mouth is most frequently observed in cattle, while in sheep, goats and in hogs it occurs with considerable less frequency. At the onset of the disease a diminished appetite and retarded rumination is observed. The animals chew their food very slowly and carefully, on account of the sensitiveness of the buceal mucous membrane, and also swallow the bolus slowly. In the periods between feeding times they keep the moutli closed, and only open it now and then with a 
characteristic smacking sound. Later they entirely cease to eat. The animals stand immovable with a staring, expressionless look, saliva dropping in long, sticky threarls from the corners of the mouth. They like to play with their mouths in the water offered them, and some patients evince an increased thirst.

The mucous membranes on the inner surface of the lips, and on the gums is warm, dry and reddened; the opening of the mouth, which is followed by a flow of great quantities of accumulated saliva, reveals a similar change in the mucous membrane of the other parts of the mouth. On the second to

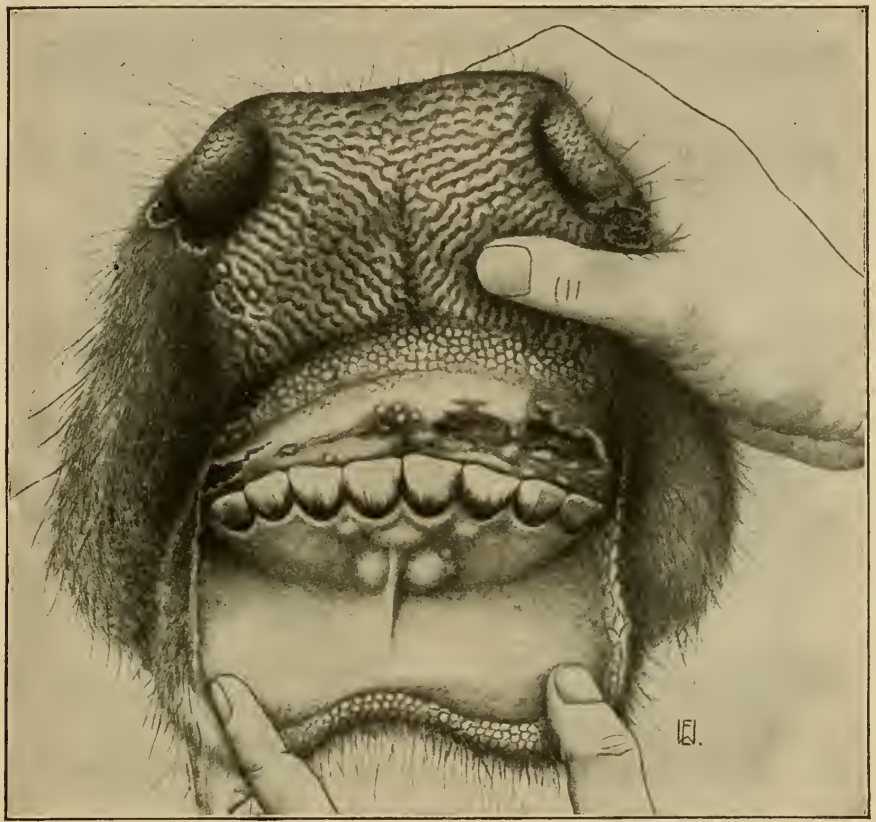

Fig. 57. Foot-and-mouth disease. Vesicles and ulcerations on the gum, the latter also on the nostrils.

third day of the affection smaller and larger vesicles develop on the inner surface of the lips, and on the gums lying opposite, or on the toothless part of the upper jaw, on the top and borders of the tongue, in the vicinity of the frenum of the tongue, as well as on the inner surface of the cheeks (Fig. 57). These vesicles are in small animals hardly ever larger than the size of a pea, while in larger ruminants they may attain the size of a nut, and on the dorsum of the tongue may even reach the size of an egg. The vesicles on the tongue (Fig. 58) possess a thick 
wall, in other parts however the walls are thimner. These contain first a clear, watery, colorless or yellowish fluid, which later beeomes cloudy and grayish-white. Ifter 1 to 3 days the vesicles burst, the contents eseape, the wall is thrown off, and in their places highly reddened, moist, painful, flat erosions with widle borders remain. After 1 to 2 days these become covered with fresh epithelium, whereupon for a certain time yellowish-

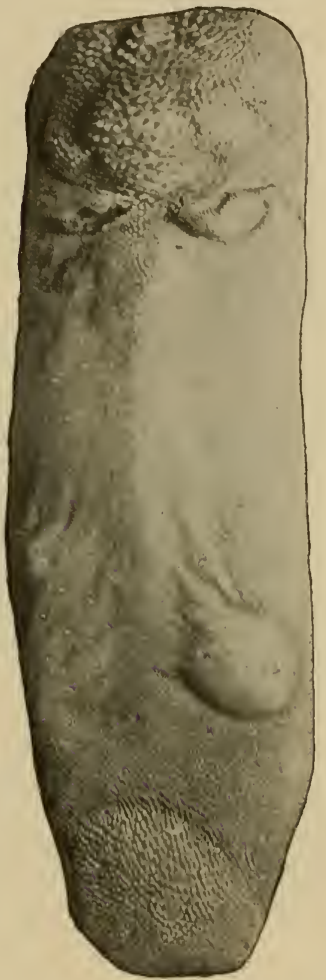

Fig. 58. Foot-and-mouth discase. Subepithelial vesicles on the point of the tongue, on the body of the tongue, and on the thick portion.

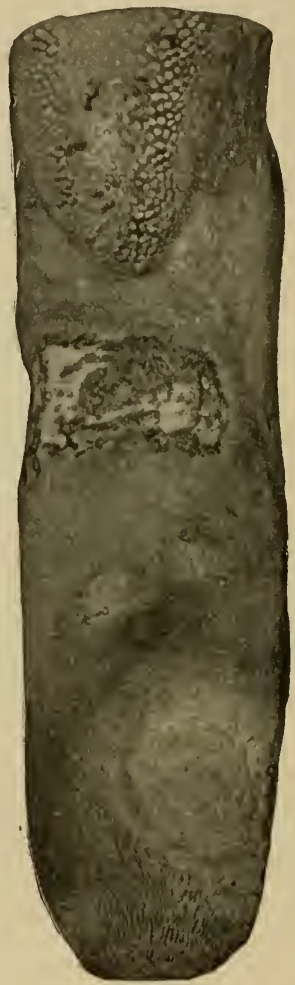

Fig. 59. Foot-rud-mouth diseuse. Erosions on the dorsum of the tongue in stages of early liealing.

brown spots remain, which finally also disappear (Fig. 59). As soon as the erosions become eovered by epithelium the animals, which in the meanwhile frequently have become greatly emaciated, commence to eat.

In animals which were in poor condition previous to the affection, sometimes a lemon-colored deposit of 3 to $4 \mathrm{~mm}$. thickness develops at 
the seat of the vesicles, and healing occurs only after the shedding of the same (Born). Russian veterinarians (Ravitsch, Korsak, Michailow, Schadrin) have also ohserved in some outbreaks the appearance of such pseudo-membranous deposits but only in strong animals, and in these eases vesicles were not present, or only in an abortive development.

Simultaneously with the affection of the mucous membrane, similar vesicles develop, in cattle on the muzzle ancl at the base of the horns (rarely), in hogs on the snout (frequently), which may reach the size of a nut, and correspond with the described vesicles (on the snont sonietimes only a single large vesicle develops). Vesicles may also be observed in rare cases on the nasal mucous membrane, and on the conjunctiva. The spreading of the affection to the pharyngeal mucous membrane is inclicated by symptoms of a pharyngitis (difficulty in swallowing, cough, regurgitation). In cattle the cornea is sometimes uniformly cloudy, and small vesicles may develop on its surface. The ulcerations which subsequently develop heal withont leaving a trace, or white cicatrices may remain, which however later disappear partly. In some instances vesicles may also develop on the epiglottis, on the mucous membrane of the trachea and bronchi, in which eases difficulty in respiration, or manifestations of edema of the lungs may be noticed (Leyendecker).

Foot Affection. Sheep are quite frequently and hogs are in most instances affected only on the feet, whereas in cattle affection of the feet may oceur sinultaneously with the formation of lesions in the mouth, but it usually develops subsequent to their appearance.

The first symptom, which may he recognized even from a distance, is always the lameness or the stiff gait, which is eaused by the pain in the lower extremities, and is especially manifested during walking on liard ground. If more than one foot is affected the pain may be so severe that the animals lie lown continually, and it is only with difficulty that they can be made to rise.

A careful examination of the affecter feet reveals the skin of the coronary hand, the heel of the foot, and the interdigital space at first warm, swollen, painful, and in case it is not pigmented it appears also highly reddened. After 1 to 2 days, small vesicles develop in these places, especially in the interdigital space, more rarely also at the base of the dew-claws, which soon reach the size of a hazelnut, and are filled first with a clear, and later with a cloudy fluid. After their hursting, which at these places results earlier than in the month, owing to the continuous friction, the lymph-like fluid, together with the dirt from the ground, dries to hrown crusts. If they are removed, the underlying surface shows sore places, which are desquamated of epithelium, highly reddened, moist, and very sensitive. The formation of new epithelium under the scab progresses 
very slowly, and the sensitiveness and swelling of the feet disappears completely only after from 1 to "2 weeks.

If in eattle several vesicles develop adjacent to each other, along the coronary band, it may frequently be observed that the coronary seam separates from the horny border, and this may even beeome loosened from the sensitive lamina. In the comrse of the healing process this crack remains between the newly formed horn and the old wall, and it is only after several weeks that the coronary band finally becomes covered with new horn. (Dirt and sand may penetrate the horny erack, produeing bruises and inflammation of the sensitive lamina, as a result of which the animals remain lame for a long time.) In heary, fattened hogs the loss of the hoofs is often observed.

In sheep the inflammatory process quite frequently affeets the biflex gland which is located in the interdigital space, whereupon from the excretory duet a great quantity of muco-purulent material may be squeezed out (hoof worm).

The vesicular exanthema occurs rel'y frequently on the udder in cattle, simultaneously with the infection in the mouth or hoof. As a rule resicles develop on the teats, sometimes reaching the size of a nut. In such eases the whole ndder swells, and appears reddened in the immediate surroundings of the vesicles. The affected parts are very painful. The vesicles are usually broken on the first day during milking, while in cases in which they are protected from meehanical interference they may remain intact for 5 to 6 days, until finally their thin wall bursts of its own aecord, and the resulting loss of substance soon yields under the formed scab. The sensitiveness and swelling, as well as the redlening of the affected parts of the skin, may however remain for several days.

With the exanthema a catarrh of the milk ducts may beeome associated. In such eases the milk, the quantity of which is usually considerably diminished even in healthy ndders, has a more or less colostrum-like consistence, and is therefore of a vellowish-white eolor and acid reaction, coagulates easily, and ean only with diffienlty be worked into butter and elseese. In exceptional cases it is watery, and contains white mucous flakes, which collect in the milk diets, and therefore may render the milking diffieult. In the meantime the milk may have a raneicl, bitter taste.

The milk production is always diminished in the course of the disease, but the deerease varies from ease to ease. Relative to this, Siedamgrotzky ohserved in a dairy farm of 43 cows, that during the disease in two cows the milk entirely ceased, in the others the shrinkage was the greatest on the sixth lay, at whieh time the loss in old milk cows amounted to $75 \%$, in fresh to $55 \%$, and in those which were about in the midlle of their milking perion it reachei $43 \%$. After the disappearance of the disease the quantity of milk rose only to $3 / 4$ of the former volume. The entire quantity of milk dropjerl at the height of the disease from 745 to 364 liters, and after the disappearance of the disease came back to 522 liters. In a second dairy farm 32 cows gave instead of 300 , only 30 liters of milk, and the quantity remained at the same height for $s$ days. In a third place the quantity of milk dropped on the fifth day from 510 to 260 liters, it increased from 
the eighth day on, but even after six weeks it amounted to only 350 liters. According to Weber cows in the period of the highest milk production give during the course of the disease 5-6 liters less milk daily. Born observed on a farm that the entire quantity of milk eight days after the outbreak of the disease diminisher from 750 to 280 liters; after the next eight days it rose to 400 liters, but failed to reach its former height at any later period. The reduction amounted on a monthly average, to 3.6 liters daily per cow.

The milk of affected cows contains more salts (Lavena, Herberger) and albumen (Kalantar), but less fat and sugar (Herberger;- according to Kreis the proportion of fat is increased), and in the fat the volatile fatty acids are greatly diminisherl (Tögler); under the nicroseoje colostral borlies, mucous epithelial and pus cells, and sometimes also red corpuscles may be found.

Complications occur very frequently in the course of the disease, and may greatly modify the otherwise mild and typical course.

The development of resicles on other parts of the body than those mentioned is of relatively slight importance. The exanthema may sometimes be observed on any part of the body which is not, or only slightly, covered by hair, especially on the vulva, and on the mucous membrane of the entrance to the ragina, also on the inner surface of the thigh, and on the skin of the chest, or abdomen (Harns).

More frequent and of greater importance are those complications which develop as the result of an extension of the inflammatory processes to the surrounding parts, or as a result of an infection of the erosions, or those which develop as the result of continuous mechanical interference. In this way in the further course of the disease, catarrh of the larynx, trachea, and bronchi may develop, besides pharyngitis, and further, owing to the difficulty in swallowing, saliva or food may enter the air passages, and lead to a suppurative or gangrenous pneumonia.

The superficial erosions on the mucous membrane may sometimes change to deeper ulcerations, which are covered with purulent matter, or a thick deposit, as a result of which the surrounding part is greatly swollen, through inflammatory edema. The suppuration increases, spreading not only sideways, but also in depth. The ulcers finally heal by cicatrization.

Similar severe suppurating processes occur much more frequently on the feet, and these are much more exposed to a subsequent infection. The inflammation is greatly increased by walking on hard, stony, rough roads, or on stubble. Of still niore unfavorable influence is the uncleanliness to which the affected extremities are exposed in the stables, on dirty floors, which are sometimes soaked with ichor or other filth, as well as the mud to which they are exposed in rainy weather. As the result of the mechanical irritation, in association with the local inflammatory process, great swelling of the coronary cushion and of the heels develops, as a result of which the hoofs are spread apart. The swellings may extend upward to the hock joint. The animals suffer very severe pain, and therefore they favor the affected feet in every possible way, or they lie 
down continually. If they are urged to walk they move very slowly, displaying much lameness, while small animals hop on three, or sometimes even on two feet, or they move about on their knees.

The progressive inflammation leads finally to the formation of abscesses (panaritium), which are mostly observed on the coronary band above the affected interdigital space, more rarely on the heels of the feet. The abscesses break outwardly provided they have not been opened, or the suppurating process continnes to burrow into the deeper parts. This results in caries or necrosis of the coronary or pedal bone, or the pus breaks into the eoronary or perlal joint, sometimes also into the sheaths of the tendons, while in other cases the inflammatory process extends along the sensitive lamina, resulting in the formation of fistulas, partial loosening of the horny wall and sometimes in the loss of the hoofs.

According to lytdin in eattle with transheent hoofs, red spots may be seen in the sensitive lamina of the hoof, and the loss of the hoofs, as well as the changes of the horny wall, and the horny sole, in eattle and hogs, when severely affected with foot-and-month disease, is probahly associated with an affection of the matrix of the hoof.

The affection of the udder may lead to deep, penetrating abscess formation in the affected quarter, due to the irritation during milking, and to possible infection, as a result of which slowly healing defects develop on the skin. Sometimes a considerable quantity of the milk remains in the udiler, and this commences to decompose, through an infection originating in the milk ducts, whereupon a parenchymatous inflammation of the ndder develops, which finally leads to atrophy of the affected quarter, and sometimes also to obliterating strictures of the canals of the teats.

In severe eases septicemia or pyemia, with their well-known symptoms and fatal termination may result, and if death shonkl not immediately follow the patient beeomes greatly emaciated, and finally sucembs to complete exhaustion. Such a termination may be noticed in affected animals, which although the loral process is not very malignant, yet have remained continuonsly lying, hecause of the pain in their feet, until decubitus gangrene lias set in.

In young animals acute gastro-intestinal catarrh is a very frequent complication, often resulting in death. In such eases the feeble and depressed animals do not suck, or only indifferently. The feces are fluid and fetil, the abdomen is sensitive to pressure, the pulse is accelerated and wiry. The young patients frequently die in from 2 to 3 days, sometimes even on the first day after the appearance of the gastro-intestinal infection.

The gastro-intestinal catarrh is without a doubt produced by the pathogenic action of the virus, which is swallowed with the saliva or 
milk, and acts directly upon the mueous membrane of the digestive apparatus. In sucking animals the changed ehemieal consistence of the milk of the affected mother comes also into consideration. On the other hand the virus may also enter the gastro-intestinal wall by way of the blood cireulation, and this mode of development must be suspected in those cases in which the manifestations of the gastrointestinal catarrh develop in the early stages of the disease in animals which are not fed on milk, and in which vesicles and erosions develop in the mueous membrane of the stomach.

Of the other complications abortion, internal inflammation of the eyes, inflammation of the frontal sinuses, which sometimes leads to the dropping off of the horns, may be mentioned. Finally the disease may run a course with febrile symptoms only, and without a vesicular exanthema.

Malignant Foot-and-Mouth Disease. Under this designation all those cases are usually combined which appear oceasionally, and usually only restricted to certain territories, and in which the discase, without apparent complieations, results in the death of the cattle.

In the cases belonging to this group the disease usually develops in a typical form; but in the stage of the commencing reeovery, on the fifth to sixth day, a sudlen change to the worse takes place, without any apparent canse. The affecterl animals which are already free from fever and commence to eat, sucldenly become very feeble and apathetic; they cease eating and ruminating, tremble, stagger, grind their teeth, respiration is diffieult, and they suddenly drop to the ground, when death soon follows. In some animals, constipation, delayed or completely suppressed peristalsis, sensibility of the abdomen, and hemorrhages from the intestines and vagina may be observed (Faber), while other's suddenly die under apoplectic eonditions without any apparent prodromal symptoms. Those cases should also be includerl in this group, in which in the course of the foot-andmouth disease a paresis or complete paralysis of the hind quarters develops, and the symptoms of paralysis extend anteriorly, the clinical appearance then resembling somewhat parturient apoplexy; in such instances the animals usually die from paralysis of the pharynx (Wester).

The cause of this malignant type of foot-and-mouth disease has not been established with certainty; however the manifestations on the live animals, as well as the severe changes found at antopsy, inclicate an intoxication. The supposition appears very probable that the unusually strong virus attacks also the mueous membrane of the stomach and intestines, whereby poisonous substances are absorbed into the organism from the uleerations developed in these places. Whether these products originate from the virus of foot-and-mouth disease proper, or from other bacteria present in the digestive tract has not yet been determined. Noeard having shown that typical foot-and-mouth disease may he produeed by inoculation into cattle of degenerated 
parts of the heart muscle, the presence of the virus in the heart muscles suggests at least the symptoms of the heart weakness. The malignant character of the affection is not influenced by outside conditions, especially by dietetic or hygienic conditions, and Imminger observed in Bavaria that most of the deaths during the disease occurred in clean, well ventilated stables.

Course. In the great majority of cases foot-and-mouth disease usually runs a typical and favorable course. The development and healing of the vesicles require on an average 5 to 6 days. In spite of this however the disease extends even in the mild cases, from 2 to 3 weeks, as the different parts of the body do not become affected simultaneously, but usually successively. The cases in which the affection is localized in the mucous membrane of the mouth usually recover inside of a week. Recovery is protracted somewhat longer in the hoof affections, on account of the delayed healing of the ulcerations. In those cases however in which the affection of the mucous membrane of the mouth is associated with lesions in the hoofs, a still longer time is required for recovery. After healing of the local changes the animals usually regain their former condition very rapidly.

In cases rumning an unfavorable course with the exception of young animals affected with gastro-intestinal catarrh, death ensues as a rule from pyemia or septicemia, as a result of absorption of septic substances from the ulcers, abscesses or suppurating wounds, while in other cases a gangrenous pneumonia is the cause of death. In the malignant form of foot-andmouth disease toxin poisoning, or paralysis of the heart may be the immediate cause of deatl.

In a certain percentage of cases various permanent affections remain, which more or less reduce the value of the animals. Such affections are, in the first place, chronic disturbances of the hoofs, which develop from extensive inflammation of the feet, and especially from the loss of hoofs. Such conditions result in an irregular development of the new hoofs, as well as in chronic arthritis and tendonitis. In exceptional cases a proliferation of granulation tissue is observed, which originates from the ulcerations on the coronary band, heels, and the interdigital space. In such instances the hoofs are forced apart, and the lesions consist in sore, ulcerative masses (Schenkl). Of further importance is the diminished milk production which usually lasts until the following parturition, but may also remain constant, especially when the severe affection of the udder has resulted in atrophy of the glandular substance.

Horvath observed in the course of an acute affection a marked difficulty in respiration, and that the animal failed to shed the hair during the summer. The number of respirations during rest amounted to $60-80$ per minute, but after short exercise as well as in warm weather, it rapidly increased to $120-160$, while the pulse numbered 40-60 per minute. In the meantime the appetite and the milk production were diminished; pregnant animals gave birth to poorly developed calves, 
weighing oniy from $10-15 \mathrm{~kg}$. In 16 townships of the affected district in Hungary a year previously 9,087 cattle were affected with foot-and-nouth disease, of which 300-400 manifested the above disturbances in respiration. A similar affection sequel to foot-and-mouth disease was observed by Laszlo, also in Hungary, in connection with the very severe outbreak in which out of 2,500 eattle of a township, $86^{\circ}$ (about $20 \%$ of the animals heing over 5 years of age) died suddenly from paralysis of the heart, usually while in the stage of recovery. In the other animals the symptoms greatly diminished during the cold winter weather; complete recovery however was not observed in any of the eases.

Diagnosis. While in typical cases the disease may be easily recognized, or at least suspected, in the stage of the vesicular development, especially when several animals are affected at the same time, the correct recognition of sporadic cases, especially during the beginning or towards the end of the outbreak, frequently causes considerable difficulty.

In the peracute and fatal course the suspicion of hemorrhagic septicemia, anthrax, and the oriental rinderpest may be awakened, but these may be excluded by the autopsy findings.

With the infectious mouth disease there may be mistaken: Traumatic injuries in the mouth, which occur most frequently on the inner surface of the lips; in these cases however the losses of substance are sharply defined, frequently straight, and penetrate to considerable depth. The vesicular stomatitis (incorrectly also named as aphthous stomatitis) in cattle, in which the mucous membrane, especially on the gums, or on the toothless portion of the upper jaw, discloses small vesicles and later thin pseudo-membranous deposits form in their place; the development of this exanthema is however not associated with fever, nor is the affection infectious. The ulcerative stomatitis of hogs, in which however the gums become affected only in the vicinity of the teeth, and no vesicles develop (similar is the mercurial stomatitis). In aphthous stomatitis of calves and lambs, the vesicles usually appear only on the skin of the lips, while in the different parts of the mouth white, or yellowish membranous deposits develop on the mucous membrane without a previous vesicular formation. In cattle the actinomycotic ulcerations on the back of the tongue may also come into consideration. These however are always located immediately in front of the transverse groove on the dorsum of the tongue, and also extend to the papillæ fungiformes, whereas in footand-mouth disease these remain intact even in the places of vesicles or erosions (Leutsch). The stomatitis which is sometimes observed after feeding musty, moldy food, may be distinguished from foot-and-mouth disease by the absence of vesicles, and by the diffuse inflammation of the mucous membrane.

Concerning affections of the mucous nembrame of the mouth, which were recently described under various names, for instance pseudo-mouth disease, and which may come into consideration from the standpoint of differential diagnosis, see Chap. "Aphthous Stomatitis", in Vol. 2.

The affections which may be mistaken for hoof disease, are to be distinguished in general, in that the affections on the lower 
extremities are not preceded by felorile symptoms, but that, if they are present, they follow upon the appearance of the lesions. Further there is no formation of vesicles, and the affection is confined to the feet, while the mucous membrane of the mouth is not simultaneously affected (this fact in itself of course does not exchule the infectious foot-and-mouth disease).

The diseases which should be considered in this regard are: The interdigital panaritium of cattle, which occurs especially in filthy stables, also in pastures, sometimes in enzootic extension; in this affection the skin of the interdigital space swells to a thick roll, which extends from the interdigital space and over which the skin sometimes may even become gangrenous, resulting in a discolored ulceration, and in a separation of the interdigital band. - A necrotic (malignant) inflammation of the hoofs in cattle, which occurs in cows shortly after parturition or towards the termination of pregnancy, exceptionally also in bulls, and which is probably produced by the bacillus necrophorus (Bang, Hess); in this affection rapid softening necrosis of the skin in the interdigital space takes place, which also penetrates into the deeper parts, but fever develops only in the later stages of the affection.-The inflammation of the interdigital space in sheep (Moderhinke), from which many animals may suffer at the same time, in continnously moist weather. The affection consists in a serous, and later purulent inflammation of the skin, which may easily lead to a separation of the coronary band, and the horny wall; the suppuration usually occurs at the heels, while the anterior part of the interdigital space is less affected. The so called hoof worm (inflammation of the biflex gland) only oceurs sporadically, provided it does not develop as the result of foot-and-mouth disease, and the inflammation is confined to the vicinity of the swollen gland, which is filled with smeary material.-The so called malignant (FrenchSpanish) disease of the hoof commences with an inflammation of the hoof proper, which feels warm to the tonch, while an affection of the interdigital space becomes apparent only in the later stages, frequently after suppuration of the sensitive lamina has already developed. Furthermore this affection spreads more slowly in the herd than is the case in foot-andmouth disease.

To the latter probably belongs also the disease of the hoofs known in America as foot-rot of sheep, in England as contagious foot-rot, in France as piétin contagieux, in which the inflammation commences on the coronary band, whereupon small ulcers with purulent discharge of a peculiar odor develop on the heels. The process extends to the deeper parts, forming fistulas, and resulting in the separation of the horns of the hoofs, and sometimes also in necrosis of the ligaments, tendons and bones (Mohler and Washburn).

Exanthema on the udder might be mistaken for pox, but this affection remains as a rule exclusively confined to the 
udder, the vesicles are only the size of a pea, the surrounding skin is elevated, and in a certain stage of their development navel-like depressions form on the vesicles.

Indications of recovery from the disease within a short time are profuse salivation, greater moisture on the surface of the mucous membranes, yellowish, straight cicatrices, or flat, yellow spots on the gums and on the toothless portions of the upper jaw, small, red points and depressions on the gums and on the border of the muzzle. According to Koninski these changes may be noted even 3 to 4 months after the disappearance of the acute symptoms.

Prognosis. Foot-and-mouth disease is in its nature a mild affection, death occurring only very rarely under normal conditions, except in very young animals, in the malignant form of the disease. The mortality hardly amounts to 0.2 to $0.5 \%$, and even these figures are principally due to unfavorable hygienic conditions (filthy stables, using the animals for work, or driving the affected animals on the road, negligence in the treatment, etc.). These conditions must therefore be taken into consideration in giving a prognosis.

Of the different species, cattle are most mildly affected under similar conditions; in hogs also the affection usually runs a mild course, except in fattened hogs, in which, owing to the heavy weight of their bodies, the loss of hoofs is frequently observed, and therefore complete recovery usually takes a longer time. In sheep the course of the disease is less favorable on account of the relatively frequent after-effects to the hoofs.

In very young animals, especially in the sucking age, the prognosis is unfavorable on account of the associated gastrointestinal catarrh.

The deviation of the course, according to sex and age of cattle, is illustrated by Bouley's reports, which also correspond with the observations marle by others. In the District of Nevers in 1872, in the course of five weeks of 7469 steers 9 died $(0.12 \%)$, of 6625 cows 59 suceumbed $(0.89 \%)$, of 3.947 calves $781(20.30 \%)$. According to Mari the foot-and-mouth disease in Russia assumes sometimes such a malignant character that 70 to $95 \%$ of the affected animals die. In this form the patients suffer aside from the characteristic but severe symptoms of the disease, from a pronouncer eatarrh of eyes and nose, from diarrhea, and a severe depression of the nervous system.

The prognosis of malignant foot-and-mouth disease is unfavorable, as the mortality in this form usually amounts to from 50 to $70 \%$. As death occurs mostly after rather light symptoms in the stage of commencing improvement, it appears advisable, in making a prognosis in instances when sudden deaths had been observed in the vicinity, to take these facts into consideration.

Further, in making a prognosis in severe affections of the hoofs, the possibility of sequelae, and in the affection of the udder, a lasting shrinkage in the milk production should be given due regard. 
Treatment. Rest and cleanliness are the two principal requirements under which mild forms of the disease terminate in recovery within a short time, and without leaving subsequent affections.

In the mouth affection care should first of all be taken that the affected mucous membrane be protected from mechanical injury, and that the animals are kept in good condition, in spite of the existing pain which interferes with the partaking of food. For this purpose the diet should be regulated and the animals should be given, instead of coarse and rough food, fresh, green food, tine hay, or silage. Severely affected patients should be supplied with flour and bran gruels, while young animals should be given boiled milk.

For the cleaning of the bnceal cavity the animals should frequently be given pure fresh water, besides the mouth should be syringed out 2 to 3 times a day with pure water with the aid of an irrigator, or with a hard rubber syringe. Medicines are of subordinate importance, and only serve to prevent the lecomposition of the accumulated saliva, and to hasten the lealing of the erosions. For this purpose mild disinfectants and astringents may be used for the irrigation, such as salt and vinegar water, alum, copper sulphate, iron sulphate, potassium chlorate, boracic acid, creolin, carbolic acid, lysol (in 2 to $3 \%$ solutions), pyoctanin (1:1000), formalin $(0.5 \%)$, ete. These remedies may be used in camomile-sage or flax-seed decoctions.

In order that a possible eruption on the base of the horns should not cause a severe inflammation of the horn matrix, and subsecpuent loss of the horns, it appears indicated, especially in cows, to tie them with chains on the neck instead of on the head.

The hoof affection is in greater need of treatment, as the affected feet are exposed to irritating influences, although even this affection usually heals without interference, if the ground is sufficiently clean and dry, and the affected animals keep quiet. If the animal is affected only with the mouth lesions, it is advisable to wash the healthy feet at least once daily with a disinfecting fluid, or still better, to paint them repeatedly with tar. If the hoofs are already affected, care should be taken that the floor under the animals is kept dry; therefore the bedding should be changed frequently. For bedding hygroscopic and antiputrefactive material is recommended (turf, gypsum, etc.).

The treatment proper consists in washing the affected extremities with one of the disinfectants mentioned, or an astringent solution, which in this instance may be used in a somewhat concentrated form. Painting with wood tar, creolin, or lysol liniments, in milking cows with thick milk of lime, or sprinkling with drying powders, will be found beneficial (powdered copper sulphate mixed with oak bark, tannoform, wood charcoal mixed 
with corrosive sublimate, see p. 229, powdered oxide of zinc, etc.). Bor'n recommends placing oakum balls sprinkled with alum between the hoofs, Pilger painting the ulcerations on the hoofs with a $10 \%$ bacillol ointnent, in severe cases the application of bacillol cataplasms.

Complications which result from improper treatment, or from unfavorable external influences should be treated in accordance with surgical rules.

In affections of the udder, first of all regular milking, if possible with milk catheters, is necessary. In addition to this washing with luke warm water, or with a $2 \%$ boracic acid solution, is sufficient. If the inflammation is more intense, and the sensitiveness is greatly increased, it is advisable to apply warm applications to the sores, after previously softening and loosening the scabs, also lead or zinc ointment, lanolin, boracic acid vaseline (20\%), or dermatol vaseline; tincture of opium may be added to the salves, or a $5 \%$ cocaine salve may be used from the beginning. Rubbing with glycerin gives likewise good results. Parenchymatous inflammation of the udder is best treated with inunctions of green soap, containing tincture of iodine $(1: 10)$, or camphor ointment $(1: 4)$, repeated twice daily for 10 minutes at a time, and in order to prevent the formation of strictures the udders should be milked ont every 2 to 3 hours. During the intervals iodoform bougies may be introduced into the milk ducts (Born).

In maligmant forms of the disease it is advisable to watch the activity of the heart in the affected animals carefully, especially from the fifth to the ninth day, and in case its function is disturbed, the threatening heart depression should be treated by stimulation. In case of severe symptoms the early slaughter of the animals is indicated.

In 1901, Baccelli warmly recommended intravenous injections of corrosive sublimate (0.05:0.1 g. in physiological salt solution). According to later experiences this treatment is useless, sometimes even dangerous. It has no favorable influence on the local lesions, nor does it influence the course of the disease favorably, and further poisoning was oceasionally observerl in the treated animals, which sometines failed to appear until 3 to 4 weeks later (Sacchini, Lanzillotti-Buonsanti, Löffler). The treatment with intravenous injections of collargol (1\% solution), which has been reconmended by Winkler, was in the comparative experiments of Barabás found to have no favorable influence on the course of the disease, and to be incapable of preventing it.

Perroncito recommends his Hämoaphthin for the treatment of affected cattle which he prepares as follows: blood which is taken from recovered cattle, that have been repeatedly treated with filtered lymph, is defibrinated, filtered through hydrophylic cotton, and mixed with 3 to $4 \%$ sulphuric ether, and $0.75 \%$ formol. After the injection of this preparation ( 0.5 to $1 \mathrm{~g}$. per $\mathrm{kg}$. borly weight), which keeps for a long time, the recovery is supposed to be almost instantaneous (?).

Prevention. The highly contagious character of foot-andmouth disease, and the fact that the infection is not only transmitted by living animals, but also by the most varied objects, render its control at the time of its prevalence very difficult. Nevertheless it has recently been observed that outbreaks which have not yet spread very extensively may be eradicated by 
suitable measures, and herds, especially those on large farms, may be protected from the infection. The principles for prevention are, in general, the same as those applied to other infections diseases, and expressed briefly are, that healthy animals should be protected in every possible way from coming in contact with the unhealthy ones, or with those which have recovered within a short time (see also page 334 ), and also from coming in contact with contaminated utensils.

A quarantine of at least two weeks duration for newly introduced animals appears to give good results in this respect. It is furthermore advisable, during threatening periods, especially in already infected herds, to prevent the calves from sucking their mothers, but they should be given milk that has been heated to 70 degrees.

Emergency and protective vaccination. Emergency vaceination with virulent material, and immunization proper, possess a marked difference in their value and in their practical importance.

I. Emergency vaccination. This method, in which the disease is produced in healthy animals by the inoculation of virulent material, is employed to shorten the disease in already infected herds, when more energetic measures of eradication are not adopted.

The disease usually affects all of the susceptible animals in an infected herd owing to its remarkable contagiousness, but inasmuch as its transmission from animal to animal occurs only successively, a long time usually elapses before the last animal recovers and the entire herd may be considered free from infection. In order to diminish the loss due to inability of the animals to work by a more rapid passing of the infection through the herd, and to reach as soon as possible the removal of the quarantine period, artificial infection of all animals in the herd after the appearance of the first cases, appears indicated. The advisability of this procedure is also supported by the experience that the inoculated disease usually runs a milder and frequently also a more rapid course than the natural infection. In order however to prevent severe losses, the inoculation should be undertaken only in outbreaks in which the disease is mild in character, and in which deaths in adult animals have not been observed in the vicinity. (In the spring of 1904 in a county in Hungary, all of 35 inoculated cattle, and 39 sucking calves died within 11 days.)

Technique of Inoculations. The simplest procedure consists in rubbing or superficially searifying the nucous membrane of the mouth lightly with a clean coarse piece of linen, and then applying the saliva of affected cattle; or the animals are given coarse food contaninated with such saliva. The saliva should be taken only from animals which have vesicles in their mouths, as the saliva is rendered infectious by their contents alone, whereas it is no longer infectious when the erosions are healing. Likewise very simple and certain is the method in which a piece of linen is placed in the mouth of the affected animal, which is allowed to chew at it for several minutes, then the well saturated cloth is introduced 
into the mouth of the next 6 to 8 cattle, and they are also allowed to chew upon it. Less certain is the procedure in which threads impregnated with saliva of affected animals are inserted into the subcutaneous connective tissue of the ear, or of the tail; the feeding of affected milk is less commendable, as this may sometimes produce a severe gastro-intestinal eatarrh.

Twenty-four to 48 hours after inoculation the temperature of the animals commences to rise, and 2 to 3 days later vesicles appear on the mucous membrane of the mouth, exceptionally also between the hoofs, which continue to develop in the usual form until recovery takes place, mostly in the course of the second week. The inoculated animals should be subjected to the same treatment that they would have received had they contracted the disease by natural infection.

Other animals than eattle are usually not subjected to the inoculation as it is usually less certain in them, e. g. in hogs (in these it may be undertaken on the snout with the aid of an inoculation lancet).

II. Immunization. Although animals may easily be immunized by the deliberate production of the disease, and the immunity thus produced as a rule lasts for a long time, yet such a procedure fails to meet the requirement that the immunity be produced without a pronounced affection of the animals, especially without the development of the vesicular exanthema. Attempts to work out a method, which would be sufficiently effective, and at the same time without danger, have so far produced only partially satisfactory results, in establishing the possibility and the nature and method of passive immunization, while the problem of general immunization is not solved at the present time.

1. Active immunization. For this purpose either pure lymph or a mixture of lymph and immune serum have been used, however, without practical results, and in some instances even with unfavorable effect.

Nosotti in Northern Italy attempted to immunize cattle, by injecting them subcutaneously with pure lymph taken from closed vesicles, diluted with aqueous humor or blood serum, the results however were not satisfactory, as a considerable proportion of the inoculated animals became affected in the usual way.

According to Löffler and Frosch, virulent lymph which has been subjected for 12 hours to a temperature of $37^{\circ}$, may produce an immunity if injected into the blood in quantities of $0.01-0.1 \mathrm{ce}$. As however this method is effective only in 30 to $50 \%$ of the eases the procedure has not been inaugurated in practice.

Löfler and Frosch later found that a mixture of virulent lymph and blood serum from artificially hyper-immunized animals (1/50-1/40 ec. lymph plus 10-20 ce. blood serum), if allowed to stand for a short time, and then injected subcutaneously into cattle and hogs, produces only a passing febrile affection, without the development of a vesicular exanthema. The animals however become immune so that in 3 or 4 weeks they stand an inoculation with $1 / 50$ ec. of virulent virus without danger.

The mixture of lymph and blood serum was placed on the market at the end of 1898 under the name of "Seraphthin," but it did not prove satisfactory in practice. In different localities a large portion of the inoculated animals became affected with typical foot-and-mouth disease (Flatten, Schrader, Schmidt, Leonhardt, Lothes and others) while the disease produced by the inoculation was sometimes malignant, and even fatal (Schimlelka, Geist). Equally unfavorable results were obtained from application of Hecker's vaceine.

In Löffler's later procedure (1905) the eattle to be immunized received subcutaneous injections, first of $0.5 \mathrm{cc}$. of highly valent eattle serum, and $0.03 \mathrm{cc}$. of fresh virulent lymph. After 24 to 26 days they receive, also subcutaneously, 0.0033 ce. of lymph, 12 to 14 days later $0.01 \mathrm{ce}$, and after another. 12 to 14 days $0.04 \mathrm{ce}$. This method has not been tried in practice because of its impracticability.

The most recent experiments of Löfler show that in the inoculation of a lymph serum mixture, the quantity of serum must be in a certain proportion to the lymph if it is desired to produce a uniform immunity, and that the lymph 
should te attenuated by the serum only to the extent that it should prevent its disease producing action, but should not entirely render it ineffective, that is, it should still be able to produce a reaction in the body for the establishment of an immunity. The difficulty of measuring the quantity of the lymph and serum accurately can of course hardly be overcome, and therefore, at least at present, there is very little possibility that immunization based on this principle will prove practical.

Ory recommended cow pox lymph as a vaceine against foot-and-mouth disease, inasmuch as he supposed that in the close relation of the two diseases there exists a reciprocal immunizing relation. He first inoculated horses with ealf lymph, and used the lymph from the vesicles for the vaccination of cattle. These are claimed to have proven immune against foot-and-mouth disease after passing through a mild inoculation disease. Confirmation tests by Anker and Starcovici, Calinescu and Sitaru however proved that it is not possible to produce immunity in this manner, and Seibert could not obtain practical results from the use of original pox lymph.

The Roumanian authors just mentioned later modified the procedure, in using as a vaccine a mixture consisting of two parts of cow pox lymph, two parts salt solution, and one part foot-and-mouth disease lymph. The practical value of this method is supposed to be found in that the animals only become mildly affected and in the meanwhile may be used for work (the danger of spreading the disease is of course present in the same degree in this procedure as in the emergency inoculations).

2. Passive immunization. Blood serum from animals which have passed through foot-and-mouth disease, in quantities from 500 to $1,000 \mathrm{cc}$., increases the resistance of the susceptible animals; by the repeated intravenous injections of virulent lymph this resistance may be increased to such an extent that the serum, even in small doses, will produce a passive immunity. In this way cattle may be immunized for a period of several weeks, and by serum inoculations repeated at intervals, the immunity may be prolonged even for several months.

A single serum inoculation would then be indicated only in those cases in which it is desired to protect the animals for a period of about three weeks; thus, for instance before they are taken to stock sliows or to markets. On the other hand inoculations repeated two or three times, at intervals of 10 days, may serve beneficially in the eradication of the disease, inasmuch as by such a treatment of animals threatened with infection, its spread to surrounding herds may be prevented, and thereby the results from other measures of eradication and prevention may be assured.

Löfler's method of preparing the immune serum is as follows: For the inmunization of large animals a virus propagated in the body of pigs weighing 15 to $20 \mathrm{~kg}$. is used. The pigs are inoculated with $1 / 25 \mathrm{ce}$. of lymph, whereupon they become ill in from 2 to 3 days, and the lymph is collected from the vesicles. They then receive subentaneously 10 ec. of inmme horse serum, which results in their recovery. The lymin, which is filtered through a porcelain filter, is inoculated intravenously to eattle in inereasing quantities, and after such treatment for $21 / 2$ to 3 months they produce a sufficiently potent serum. The serum is suitable for practieal application when 100 ec. protects attle of $200-252 \mathrm{~kg}$. weight against an intravenous injection of 0.1 ce. of virulent lymph, 24 hours later. Twenty ec. of serum neutralizes the action of $0.05 \mathrm{ec}$. of lymph; as in the natural infection usually only small quantities of virus produce the disease, it is sufficient, except in unusually severe eases, to nse 20 ec. of serum for immunization purposes. In alrearly affecterl animals the ontbreak of the disease is prevented only by a considerably greater quantity $(100-200$ ce. $)$, and even then not with certainty. 
The possibility of passive immunization of eattle with highly potent serum has also been established experimentally by Nocard, Roux, Carré \& Vallée, and Nocard recommended it also for the treatment of affected animals, as he found that 20 to 40 ce. of the serum reduced the convalescent period, and that in severe outbreaks with a mortality of 10 to $20 \%$, it prevented fatal complications.

The utilization of immune serum as an aid to other measures of eradication and prevention has been warmly recommended by Löffler, also by Leclainche and Lourens.

Veterinary Police. Owing to the severe losses which agriculture sustains from the foot-and-mouth disease its compulsory notification has been inaugurated everywhere, and various special measures have been adopted for its eradication. The special regulations aim to prevent the further spread of the disease, especially by the immediate isolation or destruction of the affected animals, and consist especially in the application of strict farm and township quarantine. Strict measures are also justified in the supervision of cattle markets (prohibition of such markets in the infected towns and their vicinity), the transportation and driving of cattle, as well as the control of trading by dealers. The utilization of the meat of affected animals for consumption should depend on the decision of the veterinarian, the skins should be disinfected before they are worked up, milk from the affected dairies or cow stables should be permitted on the market only after boiling or after being subjected to at least $85^{\circ}$ of heat. Artificial infection of animals which are directly threatened by the disease should be made dependent on official approval. The lifting of the farm or town quarantine appears permissible at the earliest two weeks after the recovery of all affected animals, or after the last death.

Proper disinfection is of great importance in the control of the disease. It is advisable to undertake this immediately after the appearance of the first cases, likewise it should be repeated every week, until after the suljsilling of the disease. After thorough cleaning of all parts of the stables the disinfection could be made with a $2 \%$ hot soda or lye solution; besides it is arivisable to paint the woolen parts with tar, and the walls with fresh milk of lime, and if possible the feet of the animals should also be cleansed (with lysol soap, etc).

It is advisable to cover the manure of cows and hogs with horse manure. It should be left in the manure pit for eight days, and should only then be taken to the field where it is to be plowed under with horse teams. The virus is lestroyert by the heat which develops inside of the manure pile, as according to Hecker's investigations the temperature in the inner part of the manure pile, at a depth of $30-40 \mathrm{~cm}$. rises to $70 \mathrm{deg}$, in a greater depth to $48 \mathrm{deg}$., and remains for sereral days at this height.

If the disease appears in regions which were previously free from the infection, immediate slaughter of the infected herds is indicated as the most effective and also as the most practical measure. This may be greatly supplemented by the serum treatment of neighboring herds (see above).

With the immediate slaughter of the infected herds the erarlication of several local outbreaks was suecessfully accomplished in recent times in England, and the spread of the infection in the United States in 1902-3 was also prevented, when in

Vol. $1-23$ 
a period of seren months in 244 heris 4712 cattle, 360 hogs and 229 sheep and goats were slaughtered representing a value of $\$ 128,908$. In a similar way Australia has remained free from the infection after the slaughter in Victoria of two herds in 1865 . In recent times Holland and Germany also adopted the slaughter of the infected herds in sporadic outbreaks.

Literature. Hertwig, Mag., 1842, VIJI, 3s.9-Haubner, Teterinary Police, 1869, 359 (Lit).--Johne, S. B., 1881, 62; D. Z. f. Tu1., 1854, 186.-Siedangrotzky, S. B., 1892, 71.-Hess, Klauenkrankh. d. Riniles, 189.- Sehütz, A. f. Tk., 1894, XX, 1.-Kitt, Monh., 1894, V, 319 (Revue).-Möbius, S. B., 1S95, 75.-Löftler \& Frosch, Cbl. f. Bakt., 1597, XXII, 257.-Hecker, B. t. W., 1895, 61; 1899, 6 u. 130.Imminger, B. t. W., 1898, 517.-Löffler, D. t. W., 1899, 317; D. m. W., 1903, 685 ; Kongr. Haag, 1909.-Eluertz, A. f. Tk., 1900; XXVI, 199.-Nocard, Rev. gén., 1903, I, 369; Acad. đe méd., 1901.-Arb. z. Erforschung d. M. u. Kls., Denkschr. d. kais. Ges.-Amtes 1901.-Mohler \& Washburn, Bureau Animal Industry Bull. Nr. 63, 1905.-Casper, B. t. WV., 1907, 399 (Lit. on immunization).-Ory, Sem. vét., 1907.-Terni, D. t. W., 190s, 747 .

Foot-and-Mouth Disease in Horses, Carnivorous Animals and in Fowls. Besides ruminants and hogs, carnivorous animals and the horse are also susceptible to the disease, althongh in a considerably lesser degree, so that in these animals the disease is observed only very rarely.

The disease has been noted in various places among horses simultaneously with its occurrence among ruminants and hogs. In most instances it could be traced to direct contact of the horses with affected cattle, to the drinking of water contaminated with infectious saliva, or to pasturing in fields in which affected ruminants were previously kept. (The transmissibility to horses was proven experimentally by Brauer and Woestendieck; Albrecht, and also ourselves, failed in such inoculation experiments.) From the affected horses the disease is usually not transmitted to healthy horses.

In horses the disease occurs only as a mouth affection; vesicles develop with febrile symptoms, on the sometimes greatly swollen tongne and on the lips, sometimes also on the nasal mucous membrane, the conjunctiva, and on the immediately surrounding skin. The erosions which develop in place of the ruptured vesicles are for a time covered with yellow, flat deposits, the horses may sometimes also show slight indications of gastro-intestinal catarrh. Usually however recovery takes place within a short time (although Perazzi reports two cases of death).

By its clinical manifestations alone the disease can hardly be distinguished from the contagions pustular stomatitis, except that in the latter the development of the pustules takes place from nodules. Usually the establishment of a diagnosis is only possible with the aid of the history, and by determining the method and nature of the infection, also from the fact that pustular stomatitis spreads very rapidly among horses.

Literature. Bräuer, S. B., 1876, 84.-Woestendieck, Pr. M., 1883, 9.-Albrecht, W. f. Tk., 1896, 37.-Perazzi, Clin. vet., 1907, 633.

The disease is very rarely observed in carnivorous animals ; sporadically it has been established in dogs (Adam, Lukas, Schäffer) and cats (Uhlich, Esser), which were kept on infected premises, or used for guarding an affected herd of cattle. Vesicles develop on the mucous membrane of the mouth, the erosions showing a tendeney to form deep, penetrating ulcers. The development of the disease is associated with febrile manifestations, sometimes also with vomiting and diarrhea. If the feet are involved a vesicular exanthema develops on the balls, between the toes, and on the soles, with which swelling and lameness are associated. 
Literature. Sichäffer, A. f. Tk., 1894, XX, 331.-Hecker, B. t. W., 1898, 61.Borzoni, N. Erc., 1907, 292.

In fowls cases of the disease which were mentioned already by Spinola are exceedingly rare. The location of the exanthema is either on the head (around the nostrils, comb, conjunctiva, mucous membrane of the bills), or the feet (claws, in water fowl on the webs). Sometimes however the vesicles may also develop on other parts of the body. Wildner observed the disease in two townships in an enzootic form, where it affected chickens and pigeons severely. The buccal mucous membrane sealed off in shreds, the feet swelled up to the tarsus and the skin between the claws was covered with numerous small vesicles; in some birds not only the claws dropped off, but also the last joints of the toes. On the feet of the animals which remained alive great deformities developed.

125.

Literature. Spinola, Spez. Path., 1858, II, 883.-Schindelka, Monh., 1892, IV,

Foot-and-Mouth Disease in Man. As man is also susceptible to the virus of foot-and-month disease, it may frequently be transmitted to him if it occurs in an epizootic form. The infection is mostly transmitted by raw, or insufficiently heated milk from affected cows, or by whey (Dieckerhoff), cheese and butter (Schneider, Frick, Fröhlich) prepared from such milk. In rare instances persons engaged in work around the infected animals may contract the disease by direct contact with the affected parts of the body, as in milking, slaughtering, or during the treatment of the patients. Bertarelli succeeded in one case in transmitting the disease back to a calf.

Bussenius \& Siegel report 16 epizootics in the years of $1878-1896$, in the course of which entire families, sometimes all inhabitants of certain houses, and even townships became affected. During different outbreaks the cases terminated fatally in 36,23 , and 16 instances respectively.

The disease develops in man usually in a mild form, except in children, in which an associated gastro-intestinal catarrh may lead to death. During some of the outbreaks however, adult persons also became severely affected.

After the ingestion of raw milk the symptoms are as follows: at first mild fever, sonetimes with vomiting, soon a feeling of warmth and dryness appear in the mouth, the mucous membrane of which becomes reddened, especially on the lips, gums and on the cheeks; later vesicles as large as peas develop at these places, sometimes also on the borders of the tongues, and even further back. After they burst the lost epithelium is soon replaced. Similar vesicles also develop oceasionally on the conjunctiva and on the skin of nose and face. The skin exanthema appears most frequently on the hands, on the points of the fingers, on the base of the nails, and on the volar surface of the finger tips, more rarely also on the toes or on other parts of the body. After the vesicles burst a thin scab forms, under which the new formation of epithelium progresses rapidly.

Besides these local changes headaches and pains in the extremities, dizziness, stomach cramps, vomiting, diarrhea and great dullness may be observed from case to case.

Literature. Korányi, Nothnagels Handbuch, 1900, V. Bd. I. T. 


\section{Coital Exanthema. Exanthema vesiculosum coitale.}

\section{(Vesicular exanthema; Bläschenausschlag [German]; Exan- thème coital [French].)}

Coital exanthema is a disease of cattle and horses, more rarely of sheep and hogs, characterized by vesicular exanthema of the external genital organs. It is usually transmitted from animal to animal during the act of coitus, and usually terminates in complete recovery. The cause of the disease is at present unknown.

Occurrence. The disease occurs more frequently among cattle than in horses. It is observed annually, especially in spring and summer, in Germany, Austria and Hungary, while in Denmark and on the Scandinavian peninsula it also appears sometimes. However it is probable that it is known also in other countries (in France it is considered identical with pox). Considerable losses are sustained in extensive outbreaks of the disease, because of the diminished milk secretion in cows, the inability of draught animals to work, and from the prevention of impregnation, and consequently the deferring of the lactation period in cows.

In Germany in 1908, 206 horses and 4680 cattle were affected in 1194 townships, the disease oceurring principally in Württemberg, Prussia, Bavaria and Baden. In Austria during the period $1891-1900$, on an average 26 stallions, 168 mares, 84 bulls, and 510 cows were affected; the number of the affected townships in 1908 reached 51, and in these 159 premises were infected. In Hungary between 1897 and 1908 the affected townships increased from 43 to 278 , the number of affected horses from 81 to 2780 , of eattle from 134 to 1662 . The disease oceurs most frequently on the right banks of the Danube, and in the territory lying between the Danube and Theiss rivers. In other countries the disease is not recorded officially.

Etiology. The virus, which is unknown at present, is contained in the serous or purulent contents of the vesicles, and in the secretion of the ulcers, and is easily transmitted with these fluids from the affected to healthy animals.

The infection occurs mostly during copulation, in which male animals may infect females and vice versa; in large herds however the disease is transmitted mainly by the males. Stallions or bulls may transmit it without themselves being affected, after covering an affected mare or cow, and transmitting the infectious secretion with the penis to the genitals of a healthy female. This method of infection is however only observed when male animals repeat the copulation inside of a few hours. The spread of the disease is probably also facilitated by the fact that affected females show in the early stages of the disease symptoms resembling the period of oestrum, and consequently are taken to the male.

In some cases the disease may also occur in advanced stages of pregnancy, or in animals which have not copulated, among which it sometimes may spread extensively (Fenner observed the disease in the course of one year among 746 heifers), and 
exceptionally $1 / 2$ to 1 year old colts and calves and even steers may become affected. In these cases the infection develops by contact with the infected animals, being transmitted by straw contaminated with infectious secretions, stable floors or manure, further by sponges and by the hands of the attendants. Affected mares may transmit the disease to the lips of their colts during sucking.

Cattle are most susceptible to the disease, horses considerably less, and to a still slighter degree sheep, goats and hogs. Recovery from the disease reduces the susceptibility for a time; the duration of the immunity however is unknown, and exceptionally the same animal may become repeatedly infected at short intervals (Fenner, Kalb).

The relation of the affection to other diseases, which are also associated with a vesicular exanthema, has not yet been established. The supposition towards its identity with foot-and-mouth disease (Zlamal, Hertwig) or with dourine (Spinola), has been discarded, but French authors (Trasbot, Peuch, Nocard \& Leclainche) consider it, even in recent times, as a form of pox. The majority of authors consider the affection as a disease by itself, which view corresponds with its independent occurrence, and its almost invariable localization upon the genital organs.

It has likewise not been definitely established whether the disease is caused by the same virus in the various species of animals, as inoculation experiments have failed to give uniform results. According to observations made it usually spreads among the animals of the same species, and does not pass from horses to eattle, or vice versa. Nevertheless the almost complete resemblance of the clinical appearance in the various species indicates an identity, or at least a very close relationship of the affections, and the veterinary police treat the trouble among horses and eattle in this sense.

Exceptionally the transmission of the virus to man has also been observed, when a pustular exanthema appears on the hands and on the face, and the affected person infected other members of the family.

Symptoms. The time of incubation is as a rule from 3 to 6 days, in exceptional cases the symptoms may appear even in from 24 to 48 hours after the infection, while in some instances the period of incubation may extend to 10 or 11 days (Friedberger \& Frölmer, Dieckerhoff).

The characteristic signs of the disease develop on the external genital organs. In female animals the reddened, and exceptionally also the hemorrhagic nucous membrane of the vagina, shows in from 2 to 3 days, lentil to pea-sized vesicles, which are filled with a clear, yellowish fluid. The vesicles appear on a reddened and swollen base, especially on the upper commissure, and in the vicinity of the clitoris, as well as on the inner surface of the vulva, in male animals on the glans penis in the vicinity of the urethral opening, in rare cases also on the immediately surrounding skin. Small nodules may also appear, later chang- 
ing into vesicles, which at first contain a serous, later a purulent fluid. After a short existence the thin walls of the vesicles burst, and in their place erosions remain, which show desquamation of epithelium, or deep, penetrating ulcerations may follow, with redlened bases and sharp borders, producing a yellowish, sticky, lymphatic or a more purulent secretion, which dries in the air to yellow or brownish scabs. The ulcers later become covered by gramulation tissue, on which a new layer of epithelium soon forms. In the place of the deeper ulcerations white scars remain, which later take on a yellowish-red color, and are almost unrecognizable.

In male animals the glans penis swells, in severe cases also the sheath or the scrotum, and the prepuce. Vesicles may also develop on the skin of these parts. The swelling sometimes reaches such a degree that a true phymosis, or a paraphymosis develops. At the same time contimuous erections of the penis are frequent, and a desire for urination exists, as well as a yellowish, mucous or purulent discharge from the urethra.

In female animals the lips of the vulva are especially swollen, but the edematous infiltration extends also to the surrounding parts, and even to the legs. In mares frequent contractions of the clitoris are observed, while the vagina discharges a muco-purulent material. A similar discharge occurs also from the genitals of cows and smaller animals.

The affected animals manifest great itching, as a result of which the females rub their hind parts against solid objects, they wag their tails, arch their backs, strike at the abdomen, and stand frequently in a position for urinating.

The lymph glands of the groin and of the vicinity of the rectum are usually swollen, elastic, dense, and more or less sensitive.

The nervous condition is hardly affected with the exception of the restlessness produced by the itching; sometimes slight digestive disturbances, diminished appetite, delayed rumination, a reduction of milk secretion, etc., are observed. In the presence of very numerous pustules these symptoms may reach a high degree, and in such cases symptoms of restlessness and straining are especially severe. Pregnant animals exceptionally abort.

The described symptoms may become more aggravated as a result of external, harmful influences. The original slight inflammatory process is sometimes considerably increased by the continuous severe rubbing, when the open surfaces of the wounds may subsequently become infected by dirty material. In such cases the ulcers have a malignant appearance, they are covered by yellow crusts, and penetrate into the deeper parts, as a result of which crater-like depressions develop at the base of the ulcers. The edematous infiltration in the vicinity of the genital organs may reach a high degree, and may extend also to the lower abdomen, and to the inner surface of the thighs. 
The neighboring lymph glands may suppurate, learling to abscess formation in the vicinity of the anus and in the groins; exceptionally such may also occur in the tissue of the udder. At the same time the gait is stiff, sensibility is greatly depressed, the appetite impaired, the body temperature increased, and the fever may be remittent. Finally pyemia or septicemia with their usual severe results may follow the local affection through the development of a general infection.

Course. In the great majority of eases the disease runs a course of 2, more rarely of 3 to 4 weeks, and terminates in complete recovery. The severe cases may always be traced to a subsequent septic infection, or to trammatic influences; in such cases the infection may be so intense, and the changes in the tissue so severe, that convalescence may extend to several months; sometimes entire recovery fails to follow or the disease may terminate in death. Althongh even larger wounds may heal without scar formation, in some cases a chronic vaginal catar'rh follows, which may result in sterility of itself, or through an extension to the uterus. In bulls the deep penetrating abscess formation may result in a deformity of the penis (Kampmann), or a weakness of the penis remains, which prevents the animals from the act of coitus.

Diagnosis. The thin walled resicles on the genital organs assure the diagnosis, and distinguish the disease from the other inflammatory local affections. If ulcer's have already developed from the vesicles, it can be distinguished from traumatic inflammation only by its infectious nature, and by the rapid recovery. In infectious vaginitis of cattle, with which this disease has been frequently confounded, hemp-seed size, grayish-red nodules on the mucons membrane are characteristic, which remain for a long time, and only exceptionally change to small ulcers. In horses dourine comes into consideration; in this however there is also no vesicle formation, and it develops with less acute symptoms and is later associated with nervons manifestations.

Treatment. Mild cases recover without any treatment. Keeping the genital organs clean and washing with mild disinfectants and astringents hasten the recovery. For this purpose alum, sulphate of zine $(1 / 2 \%)$, boracie acid $(3 \%)$, iron and copper sulphate $(1 \%)$, acetate of lead (1-2\%), carbolic acid, creolin, lysol, or chinosol in 2-3\% solutions, may be used, with which the affected parts should be washed two or three times a day. In the presence of a severe vaginal catarrh, irrigations of the vagina with the same solutions may he undertaken. If the nlcerations are unclean, with deep penetrating destruction of tissues, they may properly be cauterized with nitrate of silver, or copper sulphate. J. \& H. Streit recommend the blowing into the vagina of a powder consisting of four parts of sulphate of 
zine and one part of boracic acid ( 1 teaspoonful per eow); Gallia advises the introduction of bacillol capsules into the vagina.

Veterinary Police. The most important measures for the prevention of the spread of the disease consist in the exclusion of the affected animals from breeding luring their disease. The infectiousness of the animals may be considered extinct when all inflammatory manifestations of the genital organs and the discharge have disappeared, and the mucous membrane has regained its former smoothness and pale red color. During a general prevalence of the disease, the prohibition of breeding is indicated until the disappearance of the disease in the affected localities.

Literature. Hertwig, Mag., 1842, VIII, 269; 1947, XIII, 376.-Spinola, Spez. Path., 1858, IV, 14\$6.-Hamm, D. Z. f. Tm., 1901, XVII, 147.-Kampmann, Ö. Revue, 1887, 81.-Fenner, Monh., 1891, II, 196.-Ehrhardt, Schw., A., 1896, XXXVIII, 79.-Streit, B. t. W., 1901, 496.-Nocard \& Leclainche, Mal. microb., 1903, I, 598.

Infectious Sexual Disease in Rabbits and Hares. Friedberger \& Fröhner observed an infectious disease of the external genital organs (vulva, preputium) in a rabbit breeding establishment, which was characterized by swelling of these parts, and by a muco-purulent discharge. Treatment continued for weeks, with a 1 to $2 \%$ copper sulphate solution, resulted finally in recovery. (Spez. Path. 1908, II, 496.)

A similar disease in rabbits was noted by Strangeways, only that in this case ulcerations developed on the genitals, and also on the lips and nostrils. The autopsy revealed abscesses of the internal organs (J. of comp. Path., 1906, XIX, 9).

\section{Infectious Pustular Stomatitis of Horses. Stomatitis pusto- losa contagiosa equorum.}

Infectious pustular stomatitis is a benign, acute febrile infectious disease of horses, which aside from its pronounced contagious nature, is principally characterized by a pustular exanthema of the mucous membrane of the mouth. The causative factor is at present unknown.

History. The disease was first described under the present name by Eggeling \& Ellenberger (1880), as an independent affection. Formerly it was identified with foot-and-mouth disease of the eloven-footed animals (Roll), or with coital exanthema (Hertwig), and again by some authors (Gauthier, Silvestri, Flemming) was considered identical with horse pox. Since the publications of the first mentioned authors, whose observations were later confirmed by Friedherger, the independence of the disease is generally recognized except in France and England, where according to Bouley's conception it is even at the present considered as horse pox (see pp. 301 and 324).

Occurrence. The disease is observed especially in younger horses, and mostly as a stable infection. Its economic importance lies in the fact that the affected horses are incapacitated for work during its duration, and are poorly nourished. 
Among the horses of the Prussian Arny, from 1904-1906, 48, 113 and 3 cases were observed, and in 1908, 16 cases were found.

Etiology. The virus is apparently contained in the nodules and pustules on the mucous membrane and skin, and becomes mixed with their contents, also with the secretions of the mucous membrane, as well as with the saliva of the infected animals. Horses may be infected by rubbing the infectious saliva into the mucous membrane of the mouth or nose, or by superficial scarification of the skin; sometimes transmission is also successful to cattle, sheep, hogs and chickens (Eggeling \& Ellenberger, Friedberger). Man is also susceptible to a certain degree, as febrile dermatitis with pustular formation with salivation and difficulty in swallowing, has been observed on the hands and on the face of persons who were employed around infected animals (Greswell, Ellenberger, Schultz). Under natural conditions the disease occurs only among horses.

The natural infection is as a rule transmitted by the saliva and the nasal discharge from affected animals, which reaches the mucous membrane of healthy animals with the drinking water, food, straw, or from the liands of the attendants. The fact that the disease does not spread over large territories, but disappears of itself in a relatively short time, indicates that the virulence of the virus is attenuated during its passage from animal to animal, or that the virus is soon destroyed in the onter world.

Young horses are much more susceptible than older animals, but variations in the susceptibility are also noted in the individual animals. In large stables most horses usually become successively affected, some however remain healthy. Horses which recover from the disease resist a new infection for a long time, the extent of which is not known.

Symptoms. After a period of incubation which in artificia? inoculation is 3 to 5 , and in natural infections 8 days, the disease commences with a moderate fever, which is manifested by a rise in temperature of from 1 to $11 / 2$ degrees, as well as a moderate acceleration of the pulse and debility. The animals take their feed more slowly, masticate cautiously, and enjoy playing with the lips in the water offered them. At intervals they keep the mouth closed, when from its corners pure saliva drips out in long threads.

The mucous membrane of the mouth at first has a higher temperature; it is painful and reddened in spots; soon the red spots coalesce, and at the same time hemp-seed to lentil, or even pea-size hard nodules develop on the inner surface of the lips, on the gums lying opposite, and also on the intervening folds, further in the vicinity of the frenum of the tongue, in the vicinity of the commissures of the mouth, and on the inner surface of the cheeks. Fresh nodules continue to develop for 2 to 3 days, until they appear in great numbers upon these places and 
coalesce in part. In mild cases however they only occur in small numbers. With the reduction of the fever the nodules change into vesicles owing to dissolution of the cellular elements and accumulation of pus cells. Those which develop from very small

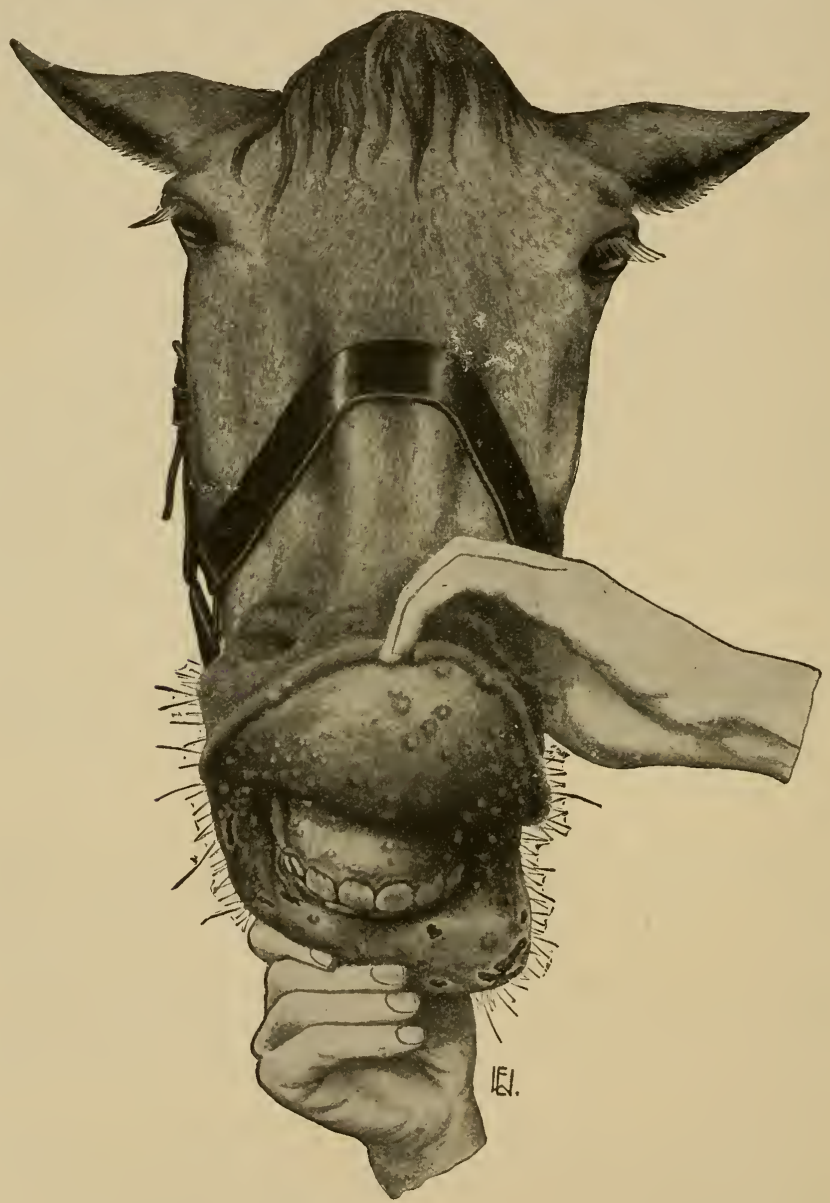

Fig. 60. Nodules, pustules and ulcerations in the infectious pustular stomatitis.

nodules contain clear serum, and after they burst very superficial erosions remain which become covered with fresh epithelium in from 1 to 2 days. The large nodules change into pustules after the conical point turns gray and later white. 
At their places somewhat deep, round ulcers develop later, which extend to the submucosa, and hardly ever exceed the size of a lentil (Fig. 60).

The center of the ulcers is formed by easily-bleeding granulation tissue, while the periphery appears covered for a time with a grayish-white deposit. After granulation tissue fills the ulcers they heal with fine scar tissue, which later gradually becomes pale. Only when the suppuration penetrates deeper does a thicker scab form, which becomes distinct from the other healthy tissue of the mucous membrane by its white color and moderate depression.

In some cases the process also extends to the nasal mucous membrane, when nodules, and from these, pustules develop on the inner surface of the nostrils, more rarely on the nasal septum, but always in the vicinity of the border of the skin. The highly reddened, and swollen mucous membrane secretes a mucopurulent discharge, which dries on the borders of the nostrils to brown crusts. The inflammation sometimes extends to the eyes by transmission through the lachrymal duct, the conjunetivae then showing catarrhal swelling, while from the slit of the lids lachrymation is profuse. The secretions sometimes take on a purulent consistency, and sometimes small pustules may make their appearance on the eyelids. (Mieckley observed keratitis, and iritis, associated with an affection of the mucous membrane.) The exanthema has exceptionally been observed also on the inner surface of the vulva.

Nodules and pustules develop on the mucous membranes, and sometimes on the skin, which result in ulcerations. Such are especially observed on the skin of the lips, the nostrils and the cheeks; in exceptional cases also on distant parts of the body, as on the chest, on the upper arms, on the thighs, in the vicinity of the rectum, and on the genitals, occasionally also around the pastern, or over the entire skin (Rehnitz). On the affected parts nodules up to the size of a pea develop in a similar way as on the mucous membrane, only that dry secretions, together with sticky tufts of hair, cover the ulcers in the form of brown scabs the healing prog'ressing subsequently under these scabs.

Dieckerhoff observed repeatedly that the exanthema in some horses of large stables developed exclusively on the skin, while the mouth remained free from the infection, although the origin and the nature of the infection could not be doubted, as typical cases occurred at the same time in other horses of the same stable. On the other hand Friedberger observed a case in which the nasal mucous membrane and the bordering skin were alone affected.

An inflammation of the mouth similar to pustular stomatitis has been observed by Bochberg, which appeared in five horses of a large stable. In this instance pustules with depressions in the centers developed also in the neighboring skin.

In regard to an epizootic stomatitis observed by Theiler in South Africa, see under Chap: Stomatitis vesiculosa Vol. II. 
The lymph glands in the immediate vicinity of the affected part of the body, that is the retro-pharyngeal glands, become more or less acutely swollen. The swelling diminishes with the formation of the ulcers, and soon disappears entirely. Exceptionally however it may lead to abscess formation (Mariott, Gröner). The lymph vessels leading to the swollen lymph glands are thickened to distinctly perceptible cords in those cases in which the exanthema develops on the skin. They likewise disappear after the glands have regained their normal condition. If the inflammation of the mouth extends posteriorly the clinical manifestations include symptoms of a mild pharyngeal and laryngeal catarrli. Exceptionally the hair falls out in the course of the disease, and the animals become greatly emaciated (Gröner).

Course. The disease lasts as a rule from 10 to 14 days, but in somewhat more severe cases it may extend to 3 or 4 weeks. The pustules develop in from 3 to 6 days after the appearance of the first symptoms. The suppuration requires another 4 to 6 days, and the healing of the ulcers requires about the same length of time. A longer course is observed in cases in which the pustules develop in slower succession, or the suppuration becomes malignant, through a subsequent septic infection, in which cases the healing of the deeper destruction of tissue requires a longer time.

Very exceptionally the disease may also terminate in death. Pöschl observed in a stud the deaths of three colts with manifestations of severe pharyngitis and septicemia, in which the autopsy revealed marked swelling and suppuration of the follicles of the mucous membrane of the pharynx and larynx, hemorrhages in the serous membrane, the spleen and the mucous membrane of the stomach, also an acute swelling of the mesenteric lymph glands. In one case (Bayr. Mil. V.-B.), the affection became complicated later with a diphtheritic inflammation of the buccal mucous membrane, later diphtheritic ulcerations appeared also on the skin and on the nasal mucous membrane, and the animal died from general septicemia. Goldschmidt observed the death of five affected horses from similar causes.

Diagnosis. Vesicular or follicular stomatitis somewhat resembles pustular stomatitis, but in the former no nodules precede the development of vesicles, and the vesicles contain only a clear serous fluid. Inflammations resulting from injuries or corrosions are distinguished from this affection by the history of the case, and by the deeper penetrating destruction of tissues in such conditions.

A mistake is more readily possible when, besides the mucous membrane of the mouth, that of the nose and the skin is also affected, and still more when the affection is exclusively localized to these parts. In such cases the following diseases enter into 
consideration: contagious acne; in this however the pustules are always larger, they usually develop only in the region of the withers, and in its immediate surrounding, while they never occur on the mucous membranes;-the coital exanthema, in which however the vesicles are confined to the mucous membrane of the genital organs and the neighboring parts of the skin, and usually develop after copulation;--pox, which usually occurs only on the skin, and around the flexor region of the pastern;-glanders, a suspicion of which may sometimes be aroused by the affection of the mucous membrane and the neighboring parts of the skin, also by the swelling of the lymph glands. In this disease however there are no changes in the buccal cavity, the ulcerations in the nose develop from nodules, and the ulcers of the skin show no tendency towards healing; in doubtful cases the rapid spread of the disease in stables, and the rapid and complete recovery excludes glanders.

Treatment. In the mild form of the disease a purely dietetic treatment is usually sufficient. Less oats should be given to the horses, and instead they should be given green feed, soft, fine hay, or flour and bran gruels, also pure, fresh water frequently. It is advisable to flush the mouth 3 to 4 times daily with any mild disinfecting fluid (1 to $2 \%$ solutions of iron or copper sulphate, or potassium chlorate, 2 to $3 \%$ creolin or lysol). The ulcers on the skin shonld be treated in a similar way or with dusting powder (dermatol, tannoform, etc.). In conjunctivitis, sulphate of zinc or tannin $(1 / 2 \%)$ gives good results.

Prevention. Not infrequently the spread of the disease in the threatened stables may be checked by immediate isolation of the horse first affected. For this purpose it is sufficient to separate the affected animal together with its neighbor by an empty stall from the other animals, only they must be attended by special persons, and separate utensils, special pails and sponges must be used in their care.

If several horses are already affected in a stable, and if a spread of the disease is expected by the existing conditions, it is advisable to undertake emergency inoculation of the healthy animals in order to shorten the duration of the disease. The inoculation is readily accomplished by rubbing saliva from the affected horses on the inner surface of the lips, which have been previously rubbed with a coarse linen cloth.

After the disappearance of the disease the stalls of the affected horses and all stable utensils should be washed with a hot solution of lye.

Literature. Hertwig, Mag., 1841, VIII, 305.-Bouley, Diet. de méd. vét., 1871, IX, 451.-Eggeling \& Ellenberger, A. f. Tk., 1878, IV, 334.-Friedberger, D. Z. f. Tm., 1879, V, 265; Münchn, Jhb., 1882-83, 66; 1886-87, 50; 1989-90, 141.-Dieckerhoff, Spez. Path., 1892, I, 391.-Schultz, Münch. m. W., 1894, 201.-Gröner, Vet. Jhb., 1895, 97.-Pöschl, A. L., 1904, 681.-Rehnitz, S. B., 1906, 176. 


\section{ACUTE INFECTIOUS DISEASES WITH LOCALIZATION IN CERTAIN ORGANS}

\section{Strangles. Adenitis equorum,}

(Coryza contagiosa equorum; distemper; Druse der Pferde, [German]; Gourme [French]; Adenite equia [Italian].)

Strangles is an acute, contagious, infectious disease of horses, in the course of which catarrhal symptoms of the upper air passages develop in association with suppurative inflammations in the adjoining lymph glands, and sometimes in a metastatic form in more distant lymph glands. The streptococeus equi is considered at the present time as the cause of the disease.

History. The disease, which is very prevalent among horses, and especially among colts, has long been considered as a specific affection of the young, which was supposed to be essential to the normal development of the animal, while similar affections of older animals were considered as an entirely different disease (Solleysel, Vitet, Lafosse). It was also supposed (Spinola), that it might sometimes lead to glanders (Traeger [1836] was the first to take a stand against this view).

Bowinghausen recognized the contagious nature of the disease towards the end of the eighteenth century, while Viborg demonstrated the infectiousness of the nasal discharge of the infected horses by inoculation experiments at the beginning of the last century. Schütz in 1888 , and independently of him, Jensen \& Sand, as well as Poels, established the etiological importance of the streptococci which are present in the nasal discharge and in the pus of the glands.

Occurrence. Strangles occurs almost annually in studs and sale depots, when it usually affects practically all the young foals in a varying degree. In later ages it is rarely observed, and almost exelusively in horses which have not passed through the disease while young. It occurs almost everywhere (Ireland and Argentine are supposed to be free of the infection), and although its course is usually favorable, yet it may cause considerable loss to the horse owner through frequent disturbances in the development of the colts, and also by occasional deaths.

In the years 1899-1908, 1753 horses were affected with strangles in the Prussian Army, of which 43 died, and $49 \%$ of the affections occurred in the last 
quarter of the year. In the French Amy in the years 1900 and 1901, 18,165 eases with 283 deaths oceurred anong about 100,000 horses.

Etiology. Streptococci (Streptococcus equi Schütz) are invariably present in the lymph glands and in the nasal discharge of affected horses, formed by cocci arranged in the manner of a string of pearls; these are not always uniform in size and are frequently split transversely. The rounded chains consist of 50 to 60 , and even more segments, but much shorter chains, and also diplococei and single cocei may occur. They stain well with aqueous aniline dyes, as well as by Gram's method (Fig. $61)$.

Cultivation. The streptococeus erfui may he cultivated aerobically and anaerobically, at room as well as body temperature. On gelatin very fine white points develop along the inoculation streak, on agar the colonies have hardly the size of a poppy seed; they are grayish-white, not transparent, not coalescing, and closely adhering to the medium. On coagulated blood serum glassy transparent, yellowish-gray droplets develop, which later may coalesce forming a tenacions layer. In the water of condensation a fine precipitation results, which consists of very long chains. In bouillon the organisms grow in fine flakes, which accumulate on the bottom of the tube and over which the fluid remains clear; the growth is favored by the addition of blood serum. No growth results on potatoes. Milk is coagulated; dextrose is

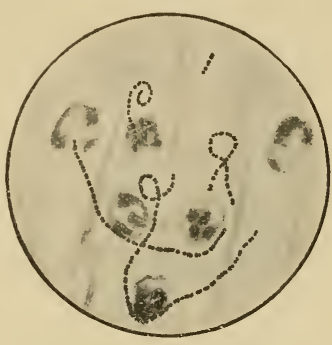

Fig. 61. Streptococcus equi. Pus from gland ; fuchsin staining. not fermented.

The relation of streptococei from different derivations to earh other has not yet been sufficiently establisherl. While Schütz considers his streptococens as an independent species, Foth helieved it identical with Schütz's diplococeus of influenza, and also with the streptococcus pyogenus of man. Lignières considers the identity of streptococeus of strangles anil that of influenza proven beyond a doubt, while he believes that Rosenbach's pyogenic streptococcus, which among other diseases plays a part in purpura hemorrhagiea of horses, is an independent species. This conception is supported also by the experience that blood sermm from animals immunized with 1 of the 2 above mentioned streptococei immunizes only against the respective varieties (Lignières), and the serum from a horse treated with the streptococcus of strangles agglutinates this streptococeus in the proportion of $1: 100$, while other pyogenic streptococei from animals are agglutinated only at $1: 25$, and the human, on the other hand, have only a very slight or no agglutinating powers (Piorkowski). Similar results were also observed by Angeliei. He found that the serum of a horse hyper-immunized with strangles strentococei had an agglutinating value of 1:10000-20000, whereas streptococei cultivater from wounds and intestinal contents agglutinated in much lower dilutions. Marmorek consilers the two forms of streptococei as not identical, hased on his observation that horse streptococei thrive in filtrates of cultures of the streptococcus of man. Besides this Lulwig found some differences in inoculation of eultures on Löffler's serum, which were manifester by the fact that the streptococeus of strangles produced larger colonies than the streptococcus of man, or the streptococeus of mastoitidis. The identity of the streptococei of different origin is still an unsettled question, the solution of which is surrounded by great difficulties inasmuch as in animal experiments one and the same streptococcus may produce various disease processes (erysipelas, suppuration).

Baruchello found streptococci 92 times in 97 samples of feces from 87 horses, 
which he considers as harmless habitants of the intestinal tract, but which may become harmful under certain conditions, alone or in connection with colon baeilli.

Tenacity. Streptococei belong to the more resistant bacteria. They resist drying, especially in pus or blood, for several weeks. A temperature of $70-75$ degrees destroys them in 1 hour, while frozen bouillon eultures are still active after two days. They are destroyed inside of 15 minutes by sulphuric acid $1: 150$, sodium hydrate $1: 55$, corrosive sublimate $1: 1500$, iron sulphate $1: 125$, earbolic acid or lysol 1:200, and ereolin 1:s0 (Lingelsheim).

Pathogenicity. Subcutaneous inoculations of a pure culture, or pus from a gland produces in mice a purulent inflammation of the subcutaneous connective tissue and lymph glands, also metastatic abscesses in the internal organs. Rabbits are less susceptible, and guinea pigs to a still lesser degree. Intraperitoneal inoculations, however, are fatal to rabbits (Ludwig), while cutaneous inoculations into the ears produces erysipelas (Jensen \& Sand). A subcutaneous injection in horses causes a purulent inflammation at the point of inoculation, with partial necrosis of the tissues; an injection of pure culture into the nasal cavity, especially if the nucous membranes have been previously rubbed, develops an acute nasal catarrh, and suppuration of the submaxillary lymph glands; therefore the typical clinical manifestation of strangles (Schütz). Rubbing pus containing streptococei upon the scarified skin produces a febrile vesicular dermatitis, which heals within a short time. The infection of cattle is sometimes successful (Jolly \& Leclainche).

Natural Infection. The results of the experiments just alluded to indicate that the causative factor of strangles, when reaching the nasal cavities, produces there a purulent or mucopurulent inflammation of the mucons membrane; but there exists also a possibility that the virus may primarily attack the tissnes from other organs, especially from the plarynx and the intestines. Scliütz based the belief on his microscopical examinations of liver sections from mice, that streptococci penetrate the walls of the blood capillaries, and that therefore the infection may take place throngh the uninjured mucous membranes. As streptococci may easily colonize in the numerous recesses of the nasal cavities, where they may continue to multiply, the nasal secretion of infected animals may transmit the disease to a healthy horse when it reaches its nasal cavities.

Under natural conditions the infection occurs usually through the nasal secretion or pus from affected animals entering, directly or by transmission with contaminated substances (food, drinking water), the upper air passages of a healthy horse (nasal cavities, buccal cavities), where it adheres to the mucous membrane. It is also very possible that infection takes place through the uninjured mucous membrane, where the bacteria very likely penetrate the exeretory ducts of the mucous glands. The disease which usually attacks all the colts in a stable, conld hardly be explained otherwise; on the other hand the infection is favored by conditions of the mucous membranes in which 
there is a desquamation of epithelium, or by deep penetrating injuries.

The disease occurs usually when affected or not entirely recovered animals are introduced into the stable. The outbreak of the disease in the studs of Mezohegyes is usually associated with the introduction of colts purchased from different parts, and from similar causes the disease frequently develops in colts at remount depots. According to the investigations of Jolly \& Leclainche scabs from an exanthema of the skin of colts affected with strangles may also disseminate the infection.

The air evidently plays an important part in the transmission of the infection, inasmuch as in the warm and moist stable air the virus expelled during coughing and blowing may float for a considerable time attached to the droplets, and later enter the upper air passages of healthy animals. This form of transmission through the air is less dominant ont of doors, first on account of the smaller quantity of moisture, and second on account of its more rapid movement, which results in the rapid dilution of the expelled virus.

Through contamination with nasal discharge and pus from the glands the most varied objects may become carriers of the infection, such as cribs, drinking utensils, the food, the drinking water, straw, walls, floor of the stables, the grass in the pasture, further the hands and clothes of the attendants. The fresher the secretion the greater its virulence; drying however does not wholly destroy its infectiousness. The disease usually occurs annually almost at the same time of the year in stables of colts, if they have not in the meantime been cleaned and disinfected. It usually appears in the spring, and it is not possible in all instances to prove a fresh introduction from the outside. In such cases it must be assumed that the virus remained in the stable after the disappearance of the disease, and was there dormant for months until it again attacked the susceptible individuals of the new generation of colts, and thereby cansed a new outbreak of the disease.

The infection probably enters from the digestive tract, and especially through the intestinal mucous membranes in those cases in which it affects primarily or exchsively, the lymph glands of the mesentery. However up to the present time it has not been possible to produce this form of the disease by feeding infected food.

In copulation an infected stallion may transmit the disease to mares, and in such cases there appear symptoms of a vaginal catarrh, while the lymph glands in the vicinity of the external genital organs and of the rectum, as well as in exceptional cases also the udder, become affected (van Leenwen, Prenss. V.-B., Bierstedt). Affected colts mav infect their mothers during sucking and cause an inflammation of the udder and the neighboring lymph vessels (Bermbach; in one case necrosis of one half of the udder and later a fatal septicemia resulted).

Vol. 1-24 
There is another possibility which has been emphasized by Schütz, that among other streptococci which are very widely distributed in nature the streptococci of strangles may live outside of the animal bodies as saprophytes, and under favorable conditions may attack the colts, especially when the animals are weakened by outside influences. In this manner those cases can be explained in which the disease appears in localities after years of absence, without any demonstrable introduction.

Solipeds are exclusively susceptible to the disease, and they become infected at the age of $1 / 2$ to 5 years, mostly howerel while they are colts. In rare cases the disease may occur at the age of 1 to 2 months and also in animals over five years of age. The greater susceptibility of young animals is associated with the lesser resistance of their mucous membranes. The greater resistance of older animals is however probably the result of their having already passed through an attack of the disease. According to common experience it usually attacks animals only once during their lives, and the immunity resulting from one attack may be proven experimentally (Toggia). In exceptional cases the acquired immunity does not last through the entire life, as some horses may become affected repeatedly, but in such cases the attacks are usually separated by intervals of several years, while cases in which an animal becomes repeatedly infected in one year (Holterback) are exceptionally rare.

According to Viborg and Hertwig out of 100 horses affected with strangles 70 were less than 5 years old, 20 more than 5 years, and 10 over 15 years. Accorrling to Humbert's observations eovering four years in the vicinity of Snippes (France), of all horses over two years of age 2195 were affected once, 543 twice, 121 three times, and 1641 animals did not contract the disease.

Outside influences which reduce the natural resistance of the animals, especially colds, favor the infection, by causing a catarrh of the mucons membrane of the air passages, as a result of which the epithelia become loosened, and thereby the bacteria colonize more readily in the profusely accumulated secretion, from which place they may penetrate the tissue of the mucous membrane.

Usually weakened and poorly nourished colts are particularly susceptible to the disease. In this regard, stabling in poorly ventilated excessively warm stables, insufficient feeding, sudden changes of weather, fatigue during transportation, also pre-existing illness have a modifying effect on the individual susceptibility. The cases in older horses usually have a direct connection with such factors. On the other hand the resistance is increased by work, acclimation to the changeable outside influences and liardening. Other accessory conditions, such as temperament, teething, etc., have no influence on the susceptibility.

Peterson observed an instance in which all the colts of a stud became affected with strangles after standing for half an hour in a pond, and later being exposed on 
a high elevation to cold winds, and being further provided with cold water to drink. According to Cagny the disease occurs in certain localities of France less frequently and runs a milder course since the colts are driven home from the pastures in the month of September.

Pathogenesis. The streptococci which reach the surface of the nasal mucous membrane, colonize in the numerous side cavities of the upper air passages, and after multiplying there they penetrate the mucous glands, from which they pass into the lymph spaces of the tissue of the mucous membranes, along which they gain entrance to the submaxillary lymph glands. In case of an infection from the mucous membrane of the pharynx, intestines, or vagina, they are held up in the neighboring lymph glands.

Under the local influence of the penetrating bacteria hyperemia and serous infiltration of the tissues develop, also in some places necrosis of the superficial epithelial layer of the mucous membrane. Serous fluid oozes from the surface, and at the same time the secretion of the mucous glands is increased. In the lympl glands a migration of the leucocytes commences under the positive chemotactic action of the streptococci or their toxins, and probably also because of an increase of lymphocytes. The glandular tissue proper however becomes partly dissolved, probably under the influence of a peptonizing ferment. In this manner large suppurative areas develop in the lymph glands, the tissues surrounding them showing usually a marked swelling, due to inflammatory edema.

Superficial abscesses nsually open through the outer skin, in which case the body rids itself of the infective substance. From the first affected lymph glands, however, the bacteria may pass along the lymph vessels to the neighboring, as well as distant lymph glands in the body, in which they cause similar inflammatory processes. Besides this the streptococci may enter the blood stream by their power to penetrate the walls of the vessels, and may thus be carried to all parts of the body, producing metastatic abscesses in various organs aside from the lymph glands, or they may cause a general septicemia.

The acute inflammatory character of the local disease process, and the associating febrile manifestations indicate that the streptococei exert their pathogenic action through toxins. As a matter of fact the experiments conducted with Rosenbach's streptococeus gave positive results (Marmorek, Baginsky \& Sommerfeld, Marmier and others), and the pathogenic action of the toxin of this streptococeus manifested itself in local acute inflammation, fever, and diarrhea. In less severe intoxications, emaciation and sometimes paralysis resulted. Some strains produce toxins which possess hemolytic properties (Besredka, Neisser \& Wechsberg and others).

In exceptional cases foals may be born with the affection (Nocard, Choisy). The ability of the streptococei to penetrate the walls of the vessels explains the occurrence of intrauterine infection. In such cases pharyngitis and suppuration of the 
submaxillary lymph glands were repeatedly observed in pregnant mares ('T'relut).

Anatomical Changes. In addition to inflammatory changes in the superficial lymph glands, frequently the size of a fist, autopsy exceptionally reveals abscesses the size of a child's head in the mediastinum and in the vicinity of the bronchi. They are filled with white, creamy pus containing streptococci, and are frequently divided into several sections by connective tissue walls. By their presence the trachea or the neighboring bronchus may be pushed to one side and may also become compressed. At the same time there may be present a purulent inflammation of the pleura and pericardium. In the lungs there is sometimes only hyperemia and acute edema, at other times a catarrhal pneumonia, or in its tissue various sized abscesses may be imbedded, which are sometimes very numerous. The mucous membrane of the bronchi and trachea, also of the pharynx is always hyperemic, loosened and sprinkled with ecchymoses, and covered with considerable quantities of mucous or purulent secretions. There is frequently a gelatinous infiltration of aryteno-epiglottic ligaments.

The lymph glands of the mesentery frequently change to large suppurating masses, when the peritoneum shows marked injection, and the abdominal cavity contains larger or smaller quantities of purulent exudate. Animals which die after long illness are sometimes found to be affected with one or more large abscesses with thick, purulent or caseous contents and adhesions of the neighboring loops of intestines by dense connective tissue. Abscesses may also be present in the kirlneys, liver, spleen (this is sometimes greatly enlarged and contains numerous suppurative areas, Lesage; fatal bleeding may take place into the cavity of a large abscess, Berton), pancreas and in the psoas muscle, further in the brain and in the spinal cord, in which they are usually associated with purulent inflammation of the meninges. They may also be present in the muscles of the neck, and in the axillary region, in the inguinal region, in the tissue surrounding the anus, in the different joints, testicles, the udder, thymus gland, heart muscle, etc. In exceptional cases the mucous membrane of the stomach may be separated from the muscular coat by a flat, diffused layer of pus several millimeters in thickness.

The autopsy may sometimes reveal degenerative changes in the parenchymatous organs indicating pyemic or septicemic infection.

Symptoms. After a period of incubation of 4 to 8 days, which possibly may last only 1 to 2 days, especially after colds, exhaustion, etc., the disease commences with symptoms of an acute fehrile affection, such as debility, diminished appetite, and increased body temperature. Almost at the same time, or not later than after 1 to 2 days, symptoms of acute 
nasal catarrh appear. The mucous membrane of the nose is first highly reddened, warm, and dry, soon the secretion becomes profuse, consisting at first of a clear or only slightly turbid fluid, later it is tenacions, after 3 to 4 days it is muco-purulent, and finally purulent (rhinitis blenorrhoica). At this stage the discharge is profuse, and flows in great quantities from the nasal openings over the upper lips. In colts the discharge is usually crumbly, less sticky, and the patients expel it in great balls during conghing or blowing. The animals cough much, and frequently manifest more or less pronounced symptoms of an initial pharyngitis; in this stage an acute catarrh of the buccal mucous membrane and the conjunctiva may also be observed.

In rare cases the mucous glands of the nasal mucous membrane are swollen to hemp-seed sized hard nodules, or small vesicles develop on the membrane, which later suppurate (on the basis of such experi-

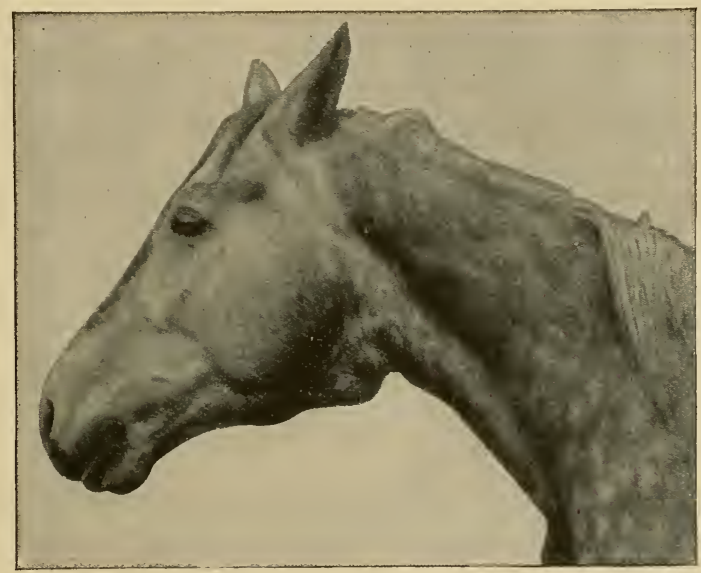

Fig. 62. Strangles. Commeneing swelling of the submaxillary lymph glands.

ences Trasbot incorrectly considered strangles as a disease identical with, or at least related to pox). Still less frequently the mucous membrane may reveal urticaria-like, flat plaques, which are the result of serous infiltrations of the submucous connective tissue. According to Rabe the streptococcus may also cause an extensive, ulcerating inflammation of the nasal mucous membrane, which can only be distinguished from ulcers of glanders by their vivid, reddened horders, and similarly reddened base, also by the numerous streptococei which are found in the discharge. Peters observed in a colt a necrosis of the bony parts of the nose as the result of an infection with strangles.

In the great majority of cases an acute swelling of the submaxillary lymph glands may be noted with the first appearance of the catarrhal symptoms (Fig. 62). The lobular strue- 
ture is still markedly perceptible at this time, but the individual lobules are larger, firmer, and painful on pressure. They soon coalesce, forming a uniformly hard growth, which rapidly increases in size, fills up the intermaxillary space, later also the throat and parotid regions, and may extend also to the lower border of the cheeks. It is not sharply circumscribed, but is firm, taut, warm and painful, and the animals hold their heads stiffly extended. After the swelling reaches a certain size it remains apparently unchanged for several days; finally however a distinct fluctuation appears in one or more places, while in other parts it remains hard and tense. The hair falls out, over the affected places, and from these hairless surfaces a yellowish sticky fluid oozes out, the skin proper becomes thin, and at certain points a dark brown discoloration, and finally a conical prominence appears, which soon bursts, whereupon from the resulting opening white or slightly yellowish, creamy pus is evacuated in profuse quantities. If the abscess is opened the pus squirts out. If several fluctuating places form on the surface of the swelling ruptures may occur at several places, usually following each other in rapid succession. Adjoining abscesses may coalesce by the breaking down of the separating walls, and evacuate their pus from a single opening; or after the bursting of an abscess the contents of the adjacent abscess may break into the first evacuated cavity through the sudden relaxation of pressure.

After the evacuation of the pus the inflammatory edema of the surrounding parts diminishes rapidly, and the swelling subsides in 1 to 2 days. From the opening however pus and later a lymph-like fluid oozes out for several days, until finally the cavity fills up with granulation tissue, the borders of the opening close, the swelling disappears entirely, and recovery takes place.

The microscopical examination of the nasal discharge discloses loosened pavement and cylindrical epithelial cells, fatty degenerated cells, and leucocytes. The discharge from the abscesses contains pus cells, sometimes intermixed with granular, broken-down tissue elements. Cover glass preparations stained with aqueous aniline dyes or after Gram's method, show long, eurved chains of cocei in the pus (see fig. 61, p. 367), while in the nasal discharge the chains are shorter, and other forms of bacteria are present. According to Bermbach and Baruchello the nasal discharge and in the early stages also the pus of the glands contain pyogenic staphylococci, which aid the streptococei in the execution of their pathogenie action. The pus of the pharyngeal or mesenteric abscesses may also contain other bacteria, (Cuillé found in them a Gram-positive, thread-forming anaerobic bacillus), which evidently gained entrance by subsequent migration.

The respiration is accelerated even in the milder cases, but it is of a normal type if there is no severe pharyngitis and laryngitis present. iVhen the inflammatory process extends to the larynx, a moist, painful and spasmodic cough is observed, which, together with the sensitiveness of the larynx persists for 1 to 2 weeks after the abscesses break. If there is a pronounced swelling of the aryteno-epiglottic ligaments the respiration becomes rattling, whistling and very labored. 
At the onset of the disease the heart's action is only slightly accelerated; with the advance of the suppurating process however the number of heart beats may double, but the pulse remains even then full and tense.

The body temperature reaches a thigh degree even on the first day $\left(40^{\circ}-41^{\circ}\right)$. On the following days it drops slightly, but on the appearance of suppuration a new rise is observed, and then the fever remains at a comparatively uniform height, until the termination of the suppurating process, or until the bursting of the abscesses. From this stage on the temperature diminishes rapidly, and becomes normal after 1 to 2 days.

The nutrition is disturbed in all cases, not only on account of the difficult mastication caused by the swelling of the submaxillary tissue, but also becanse of the difficult deglutition brought on by the pharyngitis. The appetite is diminished from the onset of the disease, and the patients take, at best, only small quantities of hay or green feed.

Defecation is at first retarded, and later an intestinal catarrh sets in not infrequently with the well-known symptoms.

The urine is passed in diminished amount, its specific gravity is increased, and in the later stages it often contains considerable quantities of albumen. With the ripening of the abscesses the quantity of indican is considerably increased, but drops to normal after the evacuation of the pus; during convalescence polyuria lasting for several days is occasionally observed.

During the beginning or the termination of the disease, the skin shows not infrequently an extensive urticaria, lasting only for a short time, or small vesicles may develop rapidly, which are filled with clear serous fluid. Thin scabs form later at these places, and finally fine white scars remain (according to Woronzow such a skin eruption may precede the characteristic symptoms of strangles). Places of predilection for the eruptions are the cheeks, the parts around the nose and mouth, the neck, the sides of the cliest, and the flexor sides of the joints.

Atypical Forms. In every outbreak there occur cases in which the manifestations deviate considerably from the described symptoms, and accordingly the course of the disease appears varied.

In this category belong undoubtedly those catarrhal affections of the upper air passages and the pharynx, which are observed during the disease in a smaller or greater number of animals of the same stable, withont the development of the characteristic suppurative inflammation of the lymph glands, or without their having any participation in the disease process. These catarrhs also develop apparently as. a result of the specific streptococeus infection, but inasmuch as the bacteria do not penetrate to the lymph glands, these remain intact or show only an insignificant swelling. The catarrh of the affected 
mucous membranes is manifested by the ordinary symptoms; the nasal catarrh is sometimes very mild, but the mucous membrane may secrete a profuse purulent discharge, while suppurative inflammation of the lymph glands does not take place.

The inflammation of the lymph glands does not in all cases lead to suppuration and abscess formation. Especially in older horses the swelling of the lymph glands may come to a stop at a certain time, whereupon it gradually retrogresses, and finally disappears entirely, only a slight thickening remaining in some cases.

More important are those complications which result from the extension of the inflammatory process to the immediate surroundings, or to metastatic processes.

The inflammatory process extends most frequently to the region of the pharynx, which then, together with the subparotid glands, and the laryngeal region, swells to a high degree. The swelling is usually caused by an inflammatory edema of the subcutaneous connective tissue, which results from severe inflammation of the submaxillary lymph glands.

Unfavorable significance attaches to cases in which pharyngitis becomes associated with purulent inflammation of the retropharyngeal lymph glands. In such instances the swelling always attains a very high degree, and is either uniformly distributed on both sides, or more marked on one side. At the same time symptoms of severe pharyngitis are present. Excluding the regurgitation cansed by the serous infiltration of the pharyngeal muscles, swallowing is rendered difficult or even impossible, by a marked extension of the posterior wall of the pharynx. Besides this, the larynx may be forced downwards and forwards by the swelling and thereby cause inspiratory dyspnea and stenosis. Finally the suppurating abscesses in the pharyngeal wall may burst in any direction. This occurs most easily into the pharyngeal cavity, especially when the mucous membrane is necrotic in places. In such cases a great quantity of pus is suddenly evacuated by the mouth and nose, and then it is always possible that the suddenly eliminated pus may be aspirated through the laryngeal opening into the deeper air passages. In other cases the suppurative process penetrates to the outside, and finally reaches the lower parotid region, or the ricinity of the larynx under the skin; after the perforation of this part the pus is evacuated outside. Exceptionally the perforation may take place both to the outside and inside, which leads to the formation of a pharyngeal fistula, the healing of which usually requires a long time. 'The condition becomes still more aggravated in case the inflammation extends to the soft palate, or downwards and forwards to the muscles of the hyoid bone (musc. omohyoideus and musc. hyoideus), in which cases swallowing is impossible and the danger of an inlialation pneumonia becomes greater. The catarrl of the 
nasal cavities may also extend to Highmore's cavity, and lead to necrosis of the bony walls, and to cerebral meningitis (Nagy). This may also develop by the suppurating process progressing along the nerve trunks into the cranial cavity (Augustin). The abscess may also burst into the vertebral canal, whereupon paralysis of the fore parts develops suddenly; with this symptoms of general spinal meningitis become rapidly associated (Fröhner).

An affection of the subparotid lymph glands results also in marked swelling of one or both of the lower regions, but the abscesses burst either into the laryngeal cavity or the pus sinks downwards along the jugular groove until it finally finds its way out in the middle of the neck or immediately anterior to the entrance into the thorax. The inflammatory process may further extend to one or both guttural pouches, which in such cases become filled with pus, and the swelling thus produced may reach the size of a child's head (in such cases a marked dyspnea is always present). In rare cases the subparotid abscesses burst into the guttural pouches, from which the pus is discharged by the nose through the existing openings of communication; a portion however may remain in the guttural pouch and change into a caseous, or later a mortar-like mass.

If the abscess develops in the deeper layers of the laryngeal region, directly under the floor of the buccal cavity, painful, hard, later fluctuating swellings develop in the vicinity of the frenum of the tongue, by which the veins and lymph vessels of this region are compressed, and a serous infiltration of the tongue results. This organ is then considerably enlarged, and the tip protrudes between the teeth, while saliva flows profusely from the mouth. The abscess usually opens into the buceal carity, whereupon the swelling of the surrounding parts and the tongue diminishes promptly, and the abscess cavity rapidly fills with granulation tissue.

The affection of the submaxillary lymph glands is sometimes associated with an inflammation of the superficial lymph vessels of the head. In such cases the lymph vessels leading from the eyes, cheeks, nose and lips swell to painful cords, the thickness of a pencil, while the surrounding connective tissue appears diffusely infiltrated. Later small nodules appear along the lymph vessels, which develop into abscesses. At the same time the swelling of the surrounding tissues reaches a very high degree, and the nostrils, cheeks and lips become greatly thickened (formerly known as "benign farcy"). There may also be associated a diffuse purulent inflammation of the subcutaneous connective tissue of the head and neck. In such cases the affected parts of the body are greatly deformed, at the same time high fever is present, and the respiration because of the swelling around the nasal openings is very difficult. If the inflammatory process reaches such a high degree it almost invariably results in fatal septicemia or pyemia. 
The spread of the inflammation to the lymph glands of the neck results in inflammatory swellings of one or both sides of the neck, which later open to the outside. More dangerous are those abscesses which develop from the retrotracheal connective tissue. In these cases bursting not infrequently takes place both to the inside and outside, and the resulting fistulae usually heal very slowly or not at all. Abscesses developing in this part may also canse severe dyspnea and difficulty in swallowing by pressing on trachea and esophagus. If inferior cervical glands in front of the chest are involved, a marked swelling results, which may gradually extend to the lower two thirds of the neck. The Hascia of the neck affords usually a sufficient guard against the pus breaking into the thoracic cavity; but sometimes the swelling exerts such a pressure on the trachea and the large blood vessels that the animals finally die from asphyxiation. In rare cases the inflammation may extend also to the organs of the thoracic eavity, and in such instances there exists danger of general fatal infection.

The inflammation of the axillary, inguinal, popliteal and precrural lymph glands is not dangerous in itself on account of the superficial location of these glands. Their involvement is indicated by the inflammatory swelling of the affected parts and by the disturbance of locomotion, which however disappears rapidly after the abscesses burst. Suppuration in the perianal glands, which also usually burst to the outside, may cause an obstruction of the feces, sometimes also symptoms of inflammation of the rectum; there may be added an involvement of the pelvic lymph glands with extensive abscess formation. An affection of the mammary lymph glands is usually associated with severe inflammatory swelling of one or both halves of the udder.

The inflammation of certain joints, which sometimes develops in association with the disease, causes painful, hot swellings and marked lameness. The knee, pastern and coronary joints become affected relatively most frequently, and the inflammation occurs in one alone or in several joints at the same time. In some cases a phlegmonous inflammation of the subcutaneous tissue develops, which later results in necrosis of the skin and inflammation of the adjacent tendon sheaths, especially in the pastern and the fetlock joints.

The mediastinal and the mesenteric lymph glands are among the internal lymph glands most frequently affected. An involvement of the mediastinal lymph glands can he established in the living animals only with very great difficulty. Under febrile symptoms severe disturbances in respiration develop gradually withont it being possible to estahlish an affection of the thoracic organs by percussion or auscultation. The contrast between the striking disturbance in respiration and the negative results of the physical examination points to an affection of the diaphragm if there is a suspicion of infection. This 
may infrequently lead to rapid death from purulent pleurisy, through an extension of the inflammation to the pleura or the bursting of an abscess between the layers of the pleura. In the meantime catarrhal or purulent pneumonia may develop with invariably fatal results. In affections of the peribronchial glands Chaussée observed in one case a venous pulse on the neck and murmurs of stenosis.

The affection of the mesenteric lymph glands which sometimes develops primarily, more frequently however in the later course of typical strangles, and after the healing of the submaxillary lesions, is very difficult to recognize in the living animal. Usually only disturbances of digestion lasting for several weeks are observed, indicated by constipation alternating with diarrhea, moderate colicky symptoms, and persistent fever, as well as rapid emaciation, until finally the animals die from exhaustion, or a purulent peritonitis sets in suddenly which then rapidly results in death. In rare ases abscesses in the mesentery may open into the intestines, whereupon recovery takes place, while the development of abscesses in the gastric region may result in a rupture of the stomach (Bolz).

The primary affection of the mesenteric lymph glands is, according to the observation of Szöllös, inaugurated by a sudden illness, with symptoms of acute gastro-intestinal catarrh, which either leads to death in a short time, or disappears almost entirely in a period varying from several days to 1 to 2 weeks. The symptoms reappear after a certain time, and then in a more severe form. In these cases frequent stretching of the hind legs, arching of the back and grunting are observed, while in the region posterior to the xiphoid cartilage palpation causes pain. Defecation and urination are associated with symptoms of restlessness. After this condition has continued for a time, or in some cases suddenly, an aggravation is observed, which leads to death from purulent peritonitis in from 6 to 48 hours.

Abscesses which develop in the pelvic eavity, and which may sometimes be found by manual examination through the rectum, exceptionally penetrate towards the perineum, or under the skin of the tail, where they finally break on the outside (Altmann). Sometimes they compress certain nerves (for instance the $\mathrm{N}$. obturatorius), or an inflammation results in certain nerves with subsequent atroply of the corresponding groups of muscles (Lahat); in both of the latter cases the locomotion of one or both hind extremities is greatly interfered with.

If metastatic abscesses in the internal organs (brain, lungs, liver, spleen, kidneys, thyroid glands, etc.) are in the course of development, high fever, rapid weakening of the heart's action, great debility and depression of the sensibility as well as rapid emaciation are observed, which usually resuit in death in the course of a few days. Extension of a brain abscess to the meninges is sometimes indicated by sudden staggering, whereupon the animals drop to the ground and die. (According to Kofler an inflammation of the uveal tract of the eyes is also a frequent complication of strangles.) 
Course and Prognosis. The instances in which the symptoms represent typical cases, the inflammatory process being confined to the submaxillary lymph glands and the nasal mucous membrane, are favorable indications of the disease, while the deriations from this type render the prognosis more unfavorable. In the typical cases the disease lasts two to four weeks, and terminates in complete recovery. Localization of the inflammatory swellings in the submaxillary space, rapid progress towards abscess formation, and correspondingly high fever indicate a farorable course, while in less pronounced inflammatory symptoms the disease may persist for a longer time. The extension of the processes to the mucous membrane of the pharynx, or to the superficial lymph glands of other parts always lengthens the course, but even in these cases recovery takes place in most instances. This is likewise the case in affections of the retropharyngeal lymph glands, but in these instances there is always danger of an inhalation pneumonia, and therefore the prognosis should be cautious. Symptoms indicating an affection of the intrathoracic or intraabdominal lymph glands, and metastasis in the internal organs, have always an unfavorable significance.

Sometimes the course is very prolonged, inasmuch as after the healing of one abscess others appear in other parts of the body, and this may repeat itself (wandering strangles). Finally however healing may take place, or the animals may die from exhaustion or from the involvement of internal organs leading to chronic pyemia.

The course depends considerably on the age and strength of the patients. While in horses over one year old the disease almost always runs a favorable course, very young or poorly developed and badly nourished animals, or foals which have already been weakened by other diseases, do not stand the disease as well.

Accordingly the mortality varies greatly. While ordinarily it amounts to only $1-3 \%$, it may be considerably higher under unfavorable hygienic conditions.

The course also depends on the character of the outbreak, inasmuch as in some years, or in some localities, the suppurating process, with or without participation of the submaxillary lymph glands, attacks the abdominal and thoracic lymph glands with remarkable frequency.

Szöllös observed in 1906130 cases in a small territory with severe manifestations, of which 37 terminated fatally. At this time the disease frequently affected oliler horses. Prussian official veterinarians likewise report severe outbreaks, es. pecially Bernt in 1904, which occurred in the District of Gumbinnen.

In the Hungarian Government studs, 1711 colts were affected between the years $1886-9$, almost all uniler the age of one year, of which $49(2.8 \%)$ died. In the same periorl the disease had heen observed in the government stallion depots, among 118 stallions, and in only one instance was the course so severe that it became necessary to restroy the affected animal. Anong the horses of the Prussian cavalry, in the years $1900-1903$, out of 3954 affected remount and service horses S2 $(2.1 \%)$ died (the loss varied in the different years between 0.0 and $3.4 \%$ ). In 
the French army, in the period between 1885-1897, 90,3\$1 horses were affected with strangles, of which 992 (1.1\%) succumbed. In the Italian army colt depots the number of cases in the years from 1903-1907 varied between 645 and 1329, among $5000-6000$ colts, the number of deaths varying between 16 and 30 .

After the subsidence of the acute affection, various sequels occasionally develop; thus the inflammation of the mesenteric glands may come to a stop in case no acute peritonitis or pyemia result. But the mutrition of the animals usually remains more or less affected through suppuration in the glands, through new formation of comective tissue and the resulting thickening of the mesentery and sometimes also through a constriction of the intestinal lumen. Consequently a severe cachexia gradually develops (tabes mesaraica). The inflammation of the guttural ponches, and of Highmore's cavities, which is at first acute, may sometimes change in its further course and become chronic, while contracted tendons, which may result from the disease on the extremities, produce lasting lameness. In some animals symptoms of hydrocephalus develop gradually as a result of encapsulated abscesses in the brain (Kofler found in 12 out of 40 horses which were slaughtered on account of hydrocephalus, softened areas in the brain, with streptococei in the contents of the abscesses). Further purpura hemorrhagica may develop in horses recovered from strangles, while roaring is a frequent sequel and is due to paralysis of the recurrent laryngeal nerves (according to Wiart out of 100 colts affected with strangles 25 later became roarers, while according to Nocard, in Argentine, where strangles does not occur, roaring is supposed to be unknown).

Diagnosis. In those cases in which the infection of strangles is indicated only by acute nasal catarrh or pharyngitis, withont suppuration of the lymph glands, the specific nature can only be established with more or less probability, by the condition of other horses in the same stable in which attacks with characteristic symptoms of strangles might be present. A demonstration of streptococei in the nasal discharge would have to be considered with caution as streptococci may be present in the discharge without true strangles.

In affections of the lymph glands in the pharyngeal region strangles may be confounded with pharyngitis resulting from other causes, or with inflammation of the parotid gland. If the disease process is exclusively localized in the region of the throat, only the further course of the disease will reveal the true nature of the affection, while previous to that the diagnosis can be based only on the mode of infection, or upon the fact that in strangles the symptoms of pharyngitis usually reach a very high intensity. In cases of inflammation of the parotid gland the inflammatory swelling is less diffuse, at least at the beginning, but is more or less sharply circumscribed following the borders of the glands or its lobules.

In the cases in which the affection is exclusively confined 
to the lymph glands of the body cavities the diagnosis can be established correctly only with sufficient information regarding the development and the presence of the infection, as the symptoms proper usually only indicate disturbances of the functions in the neighboring organs. Nevertheless a repeated examination of the urine for the presence of inclican may be beneficial in such cases, as its considerable increase points with a degree of certainty to a suppurative process.

The differentiation of strangles from glander's and the so called suspected strangles will be discussed fully in the diagnosis of glander's.

Treatment. Not much can be expected from abortive treatment; yet as in some cases the symptoms diminish, and the swelling of the lymph glands retrogresses after antiphlogistic treatment, it is advisable to attempt such treatment if it ean he done at the commencement of the disease (cold applications, inmetions with gray mercurial ointment). If however there is no improvement perceptible after a few days, it appears practical to discontinue this treatment, and allow the suppurative process a free course.

If expectant treatment has been decided upon, suitable regulation of the care and nursing, as well as of the diet, should first be carried out. The animals should be placed in clean, and uniformly warm stables, or in warm weather they may during the day be kept out of doors in shady places free of dust. It is advisable to feed the animals, in addition to good hay and some oats a few beets, or during the summer fresh grass, clover or alfalfa; if swallowing is difficult, bran slop is advisable. Sucking foals should be taken to their mother at regular intervals, and during sucking they should be supported; if however there is great difficulty in swallowing, artificial feeding through the rectum must be considered (see Tol. II).

Great care should also be exercised in maintaining the greatest possible cleanliness, for which purpose the straw should be removed frequently, the cribs washed during the day, and the purulent excretion should be frequently removed from the nasal openings and the eyes, with clean sponges or cotton swalos, dipped in luke warm water containing boracic acid. Periodical rulsbing of the body with alcohol has a beneficial effect on the general condition of the patient.

Swelling of the glands may be left without interference, or if the progress of suppuration is too slow it may be stimulated with warm or moist warm poultices. Counter irritants, which are frequently applied in such cases (cantharides ointment, mustard dough, etc.) have a doubtful value, and may easily cause serere inflammatory swellings, especially if they are applied in the region of the throat. If fluctuation is perceptible at any part of the swelling which is indicated by the softening, the abscess should be opened without delay. Althongh the pus ultimately works its way to the outside of its own accord, the 
destruction of tissue in these cases becomes very extensive; and there is increased danger of breaking on the inside, when healing will require longer time.

The abscess eavities should always be opened with a long incision. If the opening is too small it should be enlarged after the partial evacuation of the pus, and subsequently the cavity should be thoronghly cleansed with a disinfectant (boracic acid, earbolic acid, creolin, Burow's solution), whereupon the wound should be treated in accordance with the requirements of surgery. After evacuation of the pus the condition of the patient improves immediately; the temperature drops to normal inside of 12 to 24 hours, the quantity of indican in the urine is diminished, the swelling of the abseess reduces rapidly, rendering the movements of the head and mastication easier.

If the swelling persists after the opening of the abscess or should it even inerease and at the same time the borly temperature continue to remain high, it is an indication of the presence or development of other abscesses in the vicinity of the one which has heen opened. In such cases the separating wall may suceessfully be broken by the finger introrluced into the open abscess cavity, whereby a passage into the open cavity is provided for the pus; in other cases the abscesses must be opened individually.

If the pharyngeal region is affected, the necessity for feeding the animals with easily swallowed food (gruels, sloppy food) becomes still more imperative. At the same time the existing pharyngitis requires special treatment, for which the administration of potassium chlorate $(8-10 \mathrm{~g}$. to a pail of water), or the application of Priesznitz's poultices is appropriate. The opening of the superficial abscesses eauses no difficulties; if however abscess formations are present in the deeper parts, between the parotid glands and the lower jaw, or in the retropharyngeal tissue, their opening requires special care. These abscesses should be opened as early as possible on account of the threatening danger from asphyxiation. On account of the nerves and blood vessels lying in these parts, which may be displaced from their normal position, it is advisable only to split the skin with a knife at the most prominent place of the swelling, and then to spread apart the nerves and ressels by boring movements with the index finger, and if possible by this means to pierce the wall of the alscess cavity. In this way not only subparotid, but also retropharyngeal abscesses may be opened. Sometimes this procedure may not immediately lead to results, but in the course of 1 to 2 days the pus penetrates without aid in the direction of the eanal which has been bored as this constitutes the place of least resistance, and therefore ultimately it breaks through at this point.

For the local treatment of the mucous membrane Kagel introduces a thick. walled rubber tube $60 \mathrm{~cm}$. long in cases of pharyngitis through the nose into the pharyngeal cavity, and injects a disinfecting fluid $(0.5 \%$ salt solution, 1:1000 sublimate, or $0.3 \%$ of a potassium permanganate solution). Frick employs for this 
purpose a tulje perforater in several places at the end, with the aid of which the afiected nasal and pharyngeal mucous membrane is irrigated, while Payrou injects the pharyux with a thin urinary eatheter 40-42 cm. in length. He injects 10-15 g. of a fluid consisting of Guaiacol, Acid. carbol. cryst. $\overline{a a} 15.0$, Menthol 40.0, Ol. oliv. 100.0. Bringard uses a stiff sound $40 \mathrm{~cm}$. in length, to the eud of which is tied a wall of eotton dipped in encalyptus vaseline (1:15), with which the soft palate and the pharyngeal walls are swabbel. This treatment is supposed to shorten the duration of the disease considerably (in 40 horses treated in this manner the time was reduced from 1112 days to 470 days). None of these methorls are without danger in lively animals, ani especially if difficulty in swallowing exists.

Pöschl recommenls to open the submaxillary swelling before the appearance of suppuration. According to his observations recovery is more rapid after such a procedure and takes place without abscess formation, and without nuch destruction of tissues. Szöllös also obtained favorable results in many cases ly this treatment, but nevertheless abscesses not infrequently developed in distaut lymph glanis, especially in oliler horses.

If the difficulty of respiration has reached a high degree because of much swelling in the throat region, and the threatening danger of asphyxiation eannot be eliminated by artificial evacuation of pus, tracheotomy should be performed, in which case the tube should be allowed to remain in the wound until the conclusion of the suppurating process, or until the complete disappearance of the threatening symptoms.

The affection of the guttural pouches also demands surgical treatment in order to remove the pus which has accumulated therein; further abscesses formed in the superficial lymph glands or in other parts, as well as the lymphangioitis and phlegmons which become sometimes associated, also require surgical interference.

Internal treatment is only indicated in the presence of digestive disturbances. If constipation exists it is advisable to mix tablespoonful doses of artificial Carlsbad salt with the food. Beside the administration of antimony preparations, and of mucilaginous substances which are still given extensively, salicylic acid and sodium salicylate ( $8-10 \mathrm{~g}$. per day) may be given advantageously (Hardon).

Inlialations of steam vapors may be used as a remedy for the catarrh of the nose, throat and the upper air passages; it being advisable to add to the boiling water salt, carbolic acid, or oil of turpentine. In such cases intralaryngeal or intratracheal injections of mild solutions of astringents (subnitrate of bismuth $5 \%$, alum, iron sulphate or tannin 1-2\%, Lugol's solution $1: 5: 100$ ), or according to Levis' suggestion antipyrin (2-4.0 g.) may also be userl.

The fever requires special treatment only when it exceeds 41 degrees, and then the usual antipyreties, in doses adapterl to the age of the patients, are indicated. In the presence of a weak heart cardiac stimulants should be administered (for instance caffein 5-6 g.).

Metastases in the internal organs are excluded from direct treatment; in such cases and also when symptoms of severe general infection are present only stimulating treatment comes into consideration. 
Dieckerhoff recommends in protracted cases, as well as in phlegmonous inflammations and septic fever, intravenous injections of Argentum colloideale or Kollargol (40 ce. of a $1 \%$ solution repeated for several days) but Baumgart, Kegel and Werner failed to obtain favorable results from this treatment. Moretti employs corrosive sublimate (for 5 days $30-60$ ec. of a $1: 1000$ solution intravenously), Franz administers Iodipin ( $50 \mathrm{~g}$. of a $25 \%$ solution subcutaneously), with supposedly favorable results. Lindner however found Iodipin without effect. Bass recommends Ichthargan (50 ec. of a $1 \%$ solution intravenously, or 1.5 to $3 \mathrm{~g}$. in a $5 \%$ solution intratracheally), Kettner however observed in two cases no beneficial results from this treatment. The yeast treatment recommended by Ludwig and Petersen (100 g. of a mixture of brewer-yeast cells and flour of starch, which is marketed as Antigourmine and Furonculine, dusted 2 to 3 times daily on the food; the healthy horses receive as a prophylactic, thin, sour-dough soup), failed to give results according to Hausmann, Zerler, Schultz and Mitrowitsch, also in the Prussian remount depots, Feuerhack (only Diercks and Deich express approval of this treatment).

In recent times the treatment with antistreptococeus serum has been extensively used, but its value cannot yet be exactly established (see p. 386).

Prevention. This consists in keeping healthy horses, and especially colts, from affected animals, as well as from stables and stands occupied by the latter. In this way it is frequently possible to protect the animals from infection through their early years and if they should later contract the disease it will run a milder course. The colts born upon certain premises should be kept as much as possible separate from strange colts. If the disease appear's in spite of it, it may be checked by transporting the healthy animals as early as possible to localities free of the disease; in such instances the animals should be guarded especially against cold, which would reduce their resistance. After the extinction of the disease thorough disinfection of the stables and the stable utensils is very desirable, as otherwise it reappears annually in the infected establishments. Repeated disinfections will prevent the reappearance of the disease still more efficiently.

Immunization and Serum Treatment. As one attack of the disease as a rule affords an immunity which lasts for several years, the possibility of an artificially produced immunity is evident. Even in former times experiments were made in this direction, always however for the purpose of artificially producing a mild affection in the animals, thereby protecting them from the natural, usually more severe form which may occur during an unfavorable time of the year.

Viborg and Toggia in the beginning of the last century rubbed the nasal discharge of affected animals into the noses of healthy horses, while Tatray injected subcutaneously in the neck near the thorax the lymph-like fluid oozing from the broken abscesses. The affection produced in this manner is usually mild, and as the peripharyngeal lymph glands are spared, no dangerous symptoms appear. Jolly \& Leclainche injected scabs taken from sick animals and emulsified in water subcutaneously into healthy horses and thus always produced a mild attack of the disease.

Vol. 1-25 
1. Active Immunization. At first experiments were made with living cultures of the strangles streptococcus, but inasmuch as this procedure proved dangerous, dead cultures have recently been used for this purpose. As a matter of fact however there is at present no immunization method at our command which has been tried out sufficiently in practice.

After the discovery of the strangles streptococeus, Jensen \& Sanil observed that intravenous injections of pure cultures do not prorluce strangles, but only an inflammation of the vein, after the healing of which the horses resist an intranasal infection. This method howerer could not be utilized in practice on account of the severity of the local inflammation.

Kitt treated two colts which had not been affected with strangles, in the course of two months with repeated intravenous injections of 5-10 ce. of strangles streptococcus serum bouillon cultures killed at 53-55 degrees, and found later that these animals resisted an artificial infection through the nasal cavities and in. testinal canal.

Gabritschewsky used a bouillon culture concentrated to $1 / 10$ of its volume, and killed with $0.5 \%$ carbolic acid, of which he injected subcutaneously six colts with 60 ce., in 1, 2 , or 3 injections. The animals resisted an infection from rubbing a virulent culture on the nasal nucous membrane, and displayed also a higher resistance against subcutaneous injections of $1.5-2$ ec. of virulent eulture than did the control animals. Cultures of virulent streptococei also killed by $0.5 \%$ carbolic acid but not concentrated, are according to Umeno used in Japan against strangles of horses which is prevalent there.

Baruchello used as vaccine a mixture of dense streptococci culture, and pleural exudate (aggressin) of artificially infected horses or donkeys $(1: 8)$ which had been previously sterilized with toluol $(3 \%)$, and preserved with ether $(2 \%)$. The results in practice are claimed to have been satisfactory.

Wiedenmann immunized rabbits by treating them with eultures which had been shaken for $3 \frac{1}{2}$ days with $25 \%$ uric acid at $37^{\circ}$, and then concentrated by evaporation. Baldrey recommends vaccine prepared from 24 hour agar or bouillon cultures, according to Wright's method, by washing them off with salt solution, and heating to $60^{\circ}$. These however proved unreliable even in experiments on rabbits.

Concerning "strangles extract"' (shake-extracts of cultures of strangles streptococci?) of Jess \& Piorkowski, Otto obtained very satisfactory results from their use in his experience with 694 horses. All healthy animals which had been vaccinated by these methods showed no affection with strangles for a period of eight months.

2. Passive Immunization. Blood serum of horses artificially immunized against streptococcus infections in man, is recently being used more extensively, and this method appears to be giving satisfactory results when utilized against strangles in horses. According to numerous laboratory experiments it is possible to actively immunize animals with artificially attenuated streptococci, as well as with toxins, and such animals later hyperimmunized with fully virulent material, produce a blood serum which confers a passive immunity upon other animals, but as a rule only against that strain of streptococci with which the serum-producing animals have been treated. Therefore Marmorek injected his horses with various strains of streptococci of man, and the polyvalent serums obtained thereby have also given better results in human medicine.

Marmorek's polyvalent anti-streptococcus serum has also been used by French veterinarians (Jacoulet, Drouin and others) against strangles of horses, since this is a disease also produced by streptococcus infection. However the results were not promising. As these more or less unsatisfactory results 
indicate that the streptococcus of strangles, in spite of its morphological similarity, is different from the pyogenic streptococci of man, the immunization of horses has lately been undertaken with the specific streptococci of horses. The treatment with serum obtained from horses treated by the various strains of pyogenic and strangles streptococei consequently gave better results.

According to Rohr's observations, the serum injection of horses affected with strangles diminishes the quantity of the profuse purulent nasal discharge, the fever is reduced, and not infrequently the treatment results in the absorption of the abscesses already in the stage of development (the serum was subcutaneously injected in doses of $10 \mathrm{cc}$. three times on the first day, and once on the second and third day). The results of Jacoulet's experience with this treatment on 30 horses were also favorable; since the fever in this mode of treatment was of short duration, complications and relapses were more rare, and the recovery of the patient was hastened. (The daily dose amounted to from 20 to $30 \mathrm{cc}$; 80 to $100 \mathrm{cc}$. brought the development of the disease process to a standstill).

Dassonville \& Vissocq produced a serum by treating horses with virulent streptococci cultures, of which 20 to 30 ec. was supposed to protect healthy horses against natural contact infection. By following the serum injection with a subcutaneous injection of 3 to 5 ce. of virulent cultures, it should be possible to convert the passive into an active immunity. French veterinarians (Desoubry, Breton, Argoud and others) obtained favorable results with this method of immunization, and partly also with the serum alone.

Jess \& Piorkowski produced a strangles serum from horses by treating them with cultures made from growing various strains of strangles streptococei directly from the abscesses on strongly alkaline media, of which 0.0005 ce. is said to protect mice against double the fatal dose of virulent culture, and to agglutinate these cultures in dilutions of at least $1: 100$. The single dose in practice is $10 \mathrm{cc}$. or even more, which if necessary may be repeated 2 to 3 times.

The reports regarding the protective and curative action of this serum vary. While Aronson in his laboratory experiments, Angerstein, Reimers, Feuerbach and also Prussian district veterinarians in practice found it without effect, according to Stramnitzer it is supposed to be beneficial at the beginning of pure strangles, and also to improve the general condition in advanced cases in 50 ce. doses. A farorable influence on the course of the disease was also observed by Brandt, Pflanz, Franz and Otto (in 124 horses), in Hungary by Demeny \& Szende; after the serum inoculations the fever dropped and the inflammatory swelling disappeared rapidly, mostly however after the opening of the abscesses. Likewise favorable are the reports of Cederberg on the serum treatment of horses affected with strangles in the Danish army; the preventive inoculations however failed in several cases, and the experiments of Wucherer and Wagenheuser gave similar results.

Ruppel's strangles serum (Gurmin) is derived from horses which have been treated with a mixture of strains from strangles, together with a streptococeus culture made highly virulent by passages through animals. It is supposed to protect mice in doses of 0.005 to $0.00025 \mathrm{cc}$. against 10 to 100 times the fatal dose of virulent culture. In 36 out of 44 horses affected with strangles it is supposed to have given a pronounced curative result, in daily doses of 20 to $50 \mathrm{cc}$., while in healthy horses it afforded a protective action. 
Recently a strangles serum, which is prepared in Rome according to Vicchi and Gatti, was used quite extensively in Italy with satisfactory results.

Finally Delvos uses a serum from horses which have recently passed through an attack of strangles for protection of healthy and treatment of affected animals. Of this serum previously heated to $70^{\circ}$, and mixed with $1 / 2 \%$ carbolic acid, the dose amounts to $20 \mathrm{cc}$., the curative dose from 30 to $40 \mathrm{cc}$. (if necessary to be repeated). The results are said to have been satisfactory in the treatment of 94 horses.

Literature. Haubner, Mag., 1843, 227.-Bouley, Rec., 1849, 89; 1855, 537.Hering, Spez. Path., 1858, 83, (Lit).-Schütz, A. f. Tk., 1888, XIV, 172.-Sand \& Jensen, D. Z. f. Tm. 1888, XIII, 437.-Poels, F. d. M., 1888, VI, 4--Noeard, Bull., 1888, 22 ; 1890, 187.-Foth, Z. f. Vk., 1891, III, 192.-Lignières, Bull., 1895, 369 ; 1896, 173.-Marmorek, A. P., 1902, 172.-Jess, B. t. W., 1902, 171; 1905, 242.-Piorkowski, Ibid., 1902, p. 1124.-Jolly \& Leclainche, Rev. vét., 1893, 289 (Lit.).-Schnürer, Z. f. Tm., 1903, VII, 286 (Literature on Immunity).-Lingelsheim, Hb. d. p. M., 1903, III, 303; 1904, IV, 1185 (Literature on Streptococeus and streptococeus immunity).--Stramnitzer, A. f. Tk., 1904, XXX, 519.-Dassonville \& Vissoeq, Bull., 1905, 176.-Feuerbach, Z. f. Vk., 1905, XVI, 12.-Nitrovitseh, B. t. W., 1905, 77.-Ludwig, Monh., 1906, XVII, 289. (Literature on Immunization).-Gabritschewsky, Cbl. f. Bakt., 1906, XLI, 719.-Baruchello, Rev. gén., 1908, XI, 497.-Otto, B. t. W., 1909, 921.

Strangles in Cattle. Nagy observed in two mature cattle marked and painful swellings of the submaxillary lymph glands, which developed with symptoms of high fever, and purulent nasal catarrh, and led in a few days to abscess formation. After opening the abscesses and evacuating the creamlike pus, recovery soon resulted (the pus was not examined bacteriologically; according to Kuennemann the bacillus pyogenes bovis is most frequently found in the pus from cattle, and is considered by Glage identical with the bac. pyog. suis, see p. 144; streptococci and staphylococci occur in pus of cattle only very rarely). Peschke also observed multiple abscess formation in the glands on the heads of calves. (Peschke, A. f. Tk., 1896, XXII, 346.-Nagy, Vet., 1897, 580.)

Strangles in Hogs. Starcovici observed in Roumania (1898) an affection in young hogs in the course of which the submaxillary and subparotid lymph glands developed an inflammatory swelling and later suppurated. The animals also showed debility, capricious appetite and fever. During the disease, which lasted several weeks, they became greatly emaciated, as the condition prevented them from taking nourishment. Finally however, with a few exceptions, all recovered. In the pus from the abscess bipolar bacilli and streptococci were found, which were pathogenic for rabbits, guinea pigs and mice. The treatment consisted in opening and subsequent washing of the abscesses. (Revista, 1902,11, H.)

Dog Strangles. Fröhner describes under this name a rare affection of dogs in the course of which the lips, cheeks and the region of the pharynx become greatly swollen without a febrile rise in the temperature. On the skin of these places lentil-sized pustules develop, filled with bloody pus. Later the submaxillary and the parotid lymph glands swell to the size of a pigeon egg, and form abscesses (purulent lymphangioitis and lymphadenitis). Following surgical treatment recovery takes place in most instances; sometimes however metastatic suppurations may develop in the internal organs, when the animals succumb to pyemia. The purulent inflammation appears to commence in the hair follicles 
of the lips, with which an inflammation of the subcutaneous connective tissue, lymph vessels and the lymph glands becomes associated. Treatment consists in opening the suppurative formations, while in high fever and in the later course the administration of quinine and camphor is indicated (Monh., 1894, V, 301).

\section{Contagious Pleuro-pneumonia. Pleuropneumonia contagiosa bovum.}

\section{(Lung plague; Lungenseuche der Rinder [German]; Peripne»- monie contagieuse [French]; Polmonera, Pleuro- polmonite essudativa [Italian].)}

Contagious pleuro-pneumonia is as a rule an acute or subacute, but sometimes chronic contagious disease of cattle, which is characterized by an exudative inflammation of the interlobular lymph vessels, and of the alveolar tissue of the lungs, with a simultaneous, sero-fibrinous pleurisy. It is caused by a filterable virus.

History. Since the disease was described in detail by Bourgelat (1765), and Haller (1773), it was according to the changes in medical views considered at one time as a severe typhus affection, and again as a paralysis of the lungs, and the possibility of its spontaneous development was generally accepted. Chaberts (1794) insisted upon its contagiousness, which was since recognized by the majority of authors. This view has been substantiated by the investigations of commissions appointed in the middle of the last century in France, Germany, and in other parts, for the study of the disease. In the meantime Willems (1850-1852) succeeded in proving that cattle may be effectively immunized with fluid from the lungs of affected animals. The causative factor was discovered by Nocard \& Roux in 1898, and by the use of pure cultures a new method of immunization has been worked out.

Occurrence. Contagious pleuro-pneumonia was extensively prevalent in Europe prior to the last but one decade of the last century, and caused great losses among the cattle herds by its severity. Through the energetic measures which were inaugurated at that time the disease was successfully eradicated in a great part of the continent and also in Great Britain, so that it exists at present only in Russia and Spain; it also prevails in Africa, Australia and Asia.

Contagious pleuro-pneumonia was known as early as the end of the 17 th century in Switzerland, as well as in the neighboring mountainous regions of Germany and France. At the beginning of the last century it caused great losses among cattle herds in Belgium and Holland. With the expansion of animal traffic about the middle of last century it rapidly extended over large territories, and was spread especially by breeding stock from Switzerland and Holland, which was at that time preferred for breeding and crossing purposes, to the east and also to distant parts of the world. In this manner it was earried to Austria, in 1841 with Dutch cattle to England (in this country however, it had, according to Barker, been observed already in 1736), and in 1854 it was imported to South Africa supposedly with a Friesland bull. Further the disease was in 1843 spread from England, also with breeding stock, to Sweden and North America, and in 1858 to Australia. 
In Belgium in the period between 1882 and $1887,5,152$ affected and 451 suspected cattle were slaughtered representing a value of $2,165,938$ franes. In 1890-1896 the disease was decreasing (from 893 to 3 eases), since 1897 the country has been free from it.

In Germany the formerly wide-spread disease has in later times confined itself to the Prussian provinces of Brandenberg, Posen, and Saxony, also to the States of Saxony-Weimar and Anhalt. While in 1596, as many as 1608 cases were still diagnosed, the number diminished since that time to such an extent that at the beginning of 1904 the disease was considered to be eradicated. After $11 / 2$ years one case was discovered in Saxony and after this the entire Empire remained free from the affection for $1 \frac{1}{2}$ years. Since July, 1907, however new outbreaks oceurred first in Prussia, then also in Saxony and Saxony-Koburg-Gotha, and in the year 1908,448 cases (with 28 deaths) were recorded in 24 townships.

In France 4762 animals became affected during the period from 1893 to 1903 , the disease being most virulent in the northern part of the country, as well as in the Department of the Seine. Since 1890 the number of cases diminished with some fluctuations, in 1905 only 14 cases were recorded, in 1906 and since that time no outbreaks were recorded officially.

Contagious pleuro-pmeumonia had raged in former times in Great Britain with such severity that in 1860 for instance, 187,000 animals, with a value of 19 million pounds sterling, fell victim to the disease. From 1870 to $1890,63,333$ affecterl eattle were slaughtered, and 7,110 died; since 1891 the disease has rapidly decreased; in 1898 it was diagnosed only in one single case, and since that time the entire Kingdom has been free from the affection.

In Holland in the period between 1833 to $1850,64,989$, and between $1851-1869$, 37,706 eattle died; from 1881 to $1887,22,183$ affected and 12,783 suspected cattle were slaughtered, by which measure the disease was entirely suppressed.

Formerly the disease raged in Austria, most extensively in Moravia, Bohemia, Southern Austria, and Silesia. In 1590, 15.57 cases were recorded, and up to 1592 the number increased to 2,524. As a result of the eradication measures inaugurated in that year, it has rapidly decreased, so that in 1897 only one case was observed in Silesia. Since that time the entire empire has remained free from the disease.

In Russia the disease has become widely spread during the last decade; in 1899 five government districts were officially recorded with $7,72 \mathrm{~s}$ cases; in 1906, 19 government districts and 389 townships with 2964 cases; in $190 \mathrm{~S}$, 1 s government districts and 965 townships with 4,971 eases.

In Hungary contagious pleuro-pneumonia was introduced, in all probability, in the middle of last century from the west and spread extensively later to the northwestern part of the country, (in 18932,352 cases occurred in 351 townships of 17 counties). As a result of the measures inaugurated in 1892 the infecterl territory was more and more limited; in 1901 only 1 case was recorded, and since 1902 the entire territory has been free.

Spain appears to be badly infected, as even in recent times the disease was introduced from that country into France, while in Italy no eases have been recorded since 1889.

The northern countries, Denmark, Norway and Sweden, have been free from the affection for a long time, and in the countries of the Balkans it does not appear to oceur.

In Asia the disease appears especially wide-spread in the Russian territories, (in 1908 14,115 cases in 857 townships), as well as in India; it occurs likewise frequently in Central and South Africa (in Capeland alone 9,062 cases were recoriled in the period of 1897-1898, and in Egypt five cases were officially established in 1908), in Australia (Loin estimates the loss in 1899 at 16 million franes), and also in South America. The United States of North America, where in 1856, 10,000 cattle were affected in the State of Illinois alone, has been free from the disease since 1892 .

Etiology. Contagious pleuro-pneumonia is caused by an extraordinarily small, polymorphic micro-organism, which passes through the Berkefeld filter, and also through the Chamberland F-candle. In cultures under the microscope it is perceptible at a magnification of about 1500 and with strong illumination, in the form of extremely small, refracting dots, fine vibrios, and very short spirillae, as well as of branching and asteroid bodies. 
Lipschütz describes the bodies of contagious pleuro-pneumonia as roundish protoplasmic elumps almost $1 / 4 \mu$ in size which occur single or in pairs, or, though rarely, in numerous ehains containing 3-4 segments, lying close to each other. Plump forms with angular borders may be also found (degenerated elements?).

According to Bordet spirochrtes resembling the organism of syphilis develop on blood-potato agar, and also in transfers to alkalime peptone bouillon containing rabbit serum, they are however much shorter and finer spirillae. Borrel observed in preparations obtained according to Löffler's mordant method, a pronounced pleomorphic appearance of the virus. According to his and his co-workers' findings, pure cultures consist of cocci, streptococei, and morula-shaped bodies, further short, spiral threads, which show fork-shaped branchings, and asteroid figures as well as mycelioid moulds, and are always surrounded by fine, mucin coverings. On account of its last-named properties he called it asterococcus mykoides.

Staining. In bouillon cultures the bodies may be stained with aqueous, and still better with aniline dyes containing carbolic acid; also with Löffler's flagella stain, and according to Giemsa's method, while they cannot be stained by Gram's method.

Cultivation. Nocard \& Roux cultivated the virus by placing a mixture of bouillon and a small quantity of lymph from the lungs in collodion sacks and sewing these into the abdominal cavity of rabbits. By such a procedure the bacteria are protected from the phagocytes, and the bouillon is rendered more suitable for the propagation of the virus by osmosis between it and the peritoneal fluid. After 15 to 20 days the fluid became cloudy and opalescent, and the above mentioned small granules were, on microscopical examination, found present in great numbers.

Later the cultivation succeeded by a similar procedure in the abdominal cavity of cattle (not in guinea pigs) also in Martin's bouillon, to which had been added blood serum from eattle or rabbits $(6$ to $8 \%$ ). Outside of the animal body they may be cultivated on agar prepared with such bouillon, the surface of which has been moistened with a few drops of serum (Dujardin-Beaumetz) as well as in Löffler's bouillon containing $25 \%$ cattle blood serum (Schmidt). In bouillon the culture is noticeable after 2 to 3 days by a very delicate turbidity of the fluid, while on agar the colonies form very fine transparent, later whitish points adherent to the surface of the medium. The optimum temperature is $37^{\circ}$ to $38^{\circ}$; below $30^{\circ}$ the growth ceases.

Tenacity. Cultures kept in the thermostat and transferred every two weeks, retain their unattenuated rirulence not longer than 6 to 8 days, while in glass tubes closed by melting at the ends, and kept at a temperature not exceeding $12^{\circ}$, they retain their virulence for almost a year (Nocard, Roux \& Beaumetz).

The fluid from the lungs remiains virulent for eight days if kept in a cool place; after a month the virulence has become attenuated, in the air and in sunlight, however, the attenuation occurs much earlier. A temperature of $58^{\circ}$ destroys the virus, while frozen pieces of lungs kept at a temperature of $-5^{\circ}$ to $-6^{\circ}$ contain virulent lymph even after three months (Laquerrière). The virulence is not influenced by concentrated glycerin, nor by $0.5 \%$ earbolic acid (Schütz \& Steffen, Nocard).

Pathogenicity. After the subcutaneous inoculation of a few drops to $10 \mathrm{cc}$. of pure culture into the subcutaneous connective tissue of the rump or neck of cattle, the temperature commences to rise after 6 to 27 days. Soon an edematous swelling develops starting at the point of inoculation, and finally the animal dies, when the autopsy reveals a pronounced serous infiltration of the subcutaneous and intramuscular connective tissue; or the swelling disappears after 1 to 2 weeks and the animal recovers. 
Inoculation of a culture into the pleural cavity produces, after a similar period of incubation, sero-fibrinous pleurisy and peritonitis, serous infiltration of the lung tissue and acute swelling of the mediastinal lymph glands. Inhalation of a virulent culture may result in fever and symptoms of bronchial catarrh, which however disappear after 1 to 2 weeks, while animals which are slaughtered in the meantime show serous infiltration of the interstitial connective tissue of the lungs. The feeding of cultures is without effect, likewise intravenous or intratracheal injections, provided that none of the culture enters the subcutaneous comnective tissue. Intracerebral injections of a few drops of pure culture results in 6 to 14 days in fever, symptoms of cerebral irritation, later apathy and drowsiness, and invariably in inflammation of the joints (experiments of Nocard, Roux \& Dujardin-Beaumetz).

The fluid of the lungs and the pleural exudate of affected cattle possesses a similar pathogenic action, according to the older investigations of Willems. The reactive inflammation however is much less pronounced when the lymph is not injected into the rump or the neck, but in the connective tissue at the point of the tail, which is more tense and has a lower temperature (such inoculations in sucking calves frequently result in serous or fibrinous inflammations of the joints, and Dschunkowski observed similar manifestations from injections into the rump of reindeer). Injections of lung juice into the milk cistern of a cow produces a severe mammitis, which results in recovery after four weeks, but the pus-like, tenacious milk contains the virus even after two months, and its virulence has in the meantime been increased (Nocard).

Cattle which have recovered from an inoculation disease later resist subcutaneous or intrapleural inoculations without reaction, while by feeding and also by intravenous or intraocular inoculations no immunity can be produced.

Besides cattle and closely related species (buffalo, reindeer, camel, yak, bison) other animals and man are not susceptible to either artificial or natural infections.

Dujardin-Beaumetz obtainer cultures by cultivating the virus on merlia prepared from mutton and from sheep serum, or from goat meat and goat serum, which produced a typical action in eattle, and similarly also in sheep and goats. The lesions produced in cattle, however, were no longer virulent for the last named species of animals.

The channels for the natural infection are not yet clearly known. In addition to being found in the affected lung tissue, the pleural exudate and the lymph glands of the thoracic cavity, the virus is also present in the bronchial secretions and in the nasal discharge, probably also in the moist exhaled air, and is possibly introduced into the bodies of healthy animals with the inhaled air. Up to the present however it has not been possible to produce artificially, by inhalation of infectious exudate and secretions or in other ways a disease which completely corre- 
sponds to contagious pleuro-pneumonia. The possibility of infection by means of inhaled air is proven to some extent by the successful experiments of Nocard \& Roux, as well as by an older experiment of Chauveau, in which a cow that inhaled air from an affected animal through a sack passed over the heads of both animals, became affected with contagious pleuro-pneumonia.

As was shown by experience, the disease spreads by direct contact of healthy with affected animals, while the transmission of the virus by intermediate hosts has not yet been positively established. The contagion appears to remain virulent in infected stables for a long time, and infects animals which are subsequently placed in such stables. On the occasion of outbreaks careful investigation usually reveals the fact that at some time previously one or more affected animals had been introduced into the affected herd. At the same time the disease spreads preferablyt among cattle which are stabled closely for a long time, but sometimes a rapid spread may also be observed during the pasturing season.

Affected animals may transmit the disease in all stages to healthy individuals, the transmission being however easiest and most effective during the acute symptoms. The virus may remain effective in the affected lungs for years, and may even retain its virulence in the sequestrated and later encapsulated, necrotic parts of the lungs. If an entirely closed connective tissue capsule forms around such a sequestrum and other parts of the lungs remain healthy, the infectiousness of the animal is suppressed for a time; however as soon as a communication opens between the cavity and a bronchus, some parts of the sequestrum and with it micro-organisms enter the air passages, whereupon the animal may again infect other's. The disappearance of the acute symptoms and improvement or apparent recovery does not therefore mean that the animal is no longer infectious.

According to Walley the virus may remain active in the encapsulated cavities for 15 months; according to other authors the infectiousness may last even considerably longer. Minette traced outbreaks in herds to cows purchased two and three years previously.

The susceptibility of cattle to the disease varies greatly in accordance with the breed and method of living, and also with the individnals. The more hardened range cattle resist natural infection far more than the more highly bred stable cattle. The less frequent occurrence of the disease among range cattle may also be explainer by the fact that such animals live more out of doors, and are therefore less exposed to an intensive infection than milk cows or fattening cattle kept standing closely in stables. Sometimes in herds of one and the same breed some animals may withstand the disease in spite of favorable conditions for infection. 
A commission (Commission Dumas), appointed in 1850 for the study of the disease under the presidency of Bouley, found that out of 100 cattle 32 resisted infection. According to Lutz on three premises in the County Bars (Hungary) out of 246 Hungarian fattened steers four $(1.63 \%)$ became affected, out of 174 fattened steers of higher breeds $22(17.32 \%)$, of 32 cows of the same breed $5(15.63 \%)$, of 15 similarly bred young stock $9(60 \%)$ were attacked by the disease, although all animals were kept under the same conditions and were kept together in the stables.

Pathogenesis. The virus which in all probability enters the lungs with the inhaled air, appears to propagate first in the lymph spaces of the interlobular connective tissue, where it causes an inflammatory process, as a result of which the spaces become filled with serous exudate and therefore distended. With this a serous inflammation of the surrounding connective tissue soon becomes associated, and the inflammation spreads to the neighboring lobules, which from their periphery towards the center become filled with a serous, cellular, later coagulating exudate, containing also a few red blood corpuscles. The inflammation gradually involves an increasingly larger part of the lungs, producing inflammatory infiltration of the alveoli, while the exudate contained in the previously affected parts of the alveoli undergoes fatty degeneration. The virus also reaches the pleural tissue, as well as the connective tissue of the mediastinum, and there produces serous infiltration, while acute swelling occurs in the neighboring lymph glands.

As the inflammation extends to the lympl spaces of the walls of the vessels, it causes coagulation of the blood in parts of these vessels, by which a smaller or larger part of the lungs is excluded from the blood circulation, and therefore becomes necrotic (ischemic necrosis). This affected part of the lungs separates later from the still sufficiently nourished surroundings, and a connective tissue capsule forms around it, in the cavity of which the necrotic piece of lung lies free as a socalled sequestrum.

The sequestration represents essentially a healing process, and as a matter of fact when only a small part of the lung becomes necrotic, it is occasionally later absorbed, and actual recovery may take place. Mostly however the sequestrum and the virus contained therein remain unchanged for a long time, until the capsule breaks, for instance by a severe coughing spell, whereupon the virus penetrates the surrounding lung tissue, causing acute inflammatory changes. Sometimes the primary inflammatory process may come to a standstill in the tissue which is not necrotic, whereupon the enclosed lobules of the lungs become atrophied through shrinking of the interlobular connective tissue. If the inflammation was confined only to a small area, the process mav entirely heal in this manner by the formation of cicatricial tissue.

The circulating blood does not appear to be favorable for the propagation of the virus, as blood from affected animals is usually not infectious, and the injection of virulent lymph into the blood circulation is also ineffective. Exceptionally however the blood may contain the virus and disseminate it 
through the body; this is substantiated by the experience that in sucking calves inflammations of the joints develop sometimes by way of metastasis, and that in some cases changes characteristic of contagious pleuro-pneumonia are found in the lungs of fetuses of diseased pregnant animals.

The virus evidently exerts its pathogenic action through toxins. This conception is supported by the character of the inflammatory processes and the fever, also by the fact that rabbits, in the abdominal eavity of which the virus propagates for a time inside of collodion sacks, die months afterwards from cachexia, although the virus proper has apparently not passed into the circulation of the body. According to Arloing the exudate contains a toxic substance, which may be precipitated by alcohol, does not lose its activity when heated to $80^{\circ}$, but produces a very severe intoxication if inoculated even in very small quantities into the blood of cattle and goats.

\section{A n a t o mica 1} Changes. In acute cases a smaller or larger part of the lungs is found hepatized, free from air and not elastic; on incision a ele a $r^{\circ}$ serous, yellow fluid oozes from the cut $\mathrm{s} u \mathrm{r}$ a ce in great quantities and later coagulates to a gelatinous mass. The

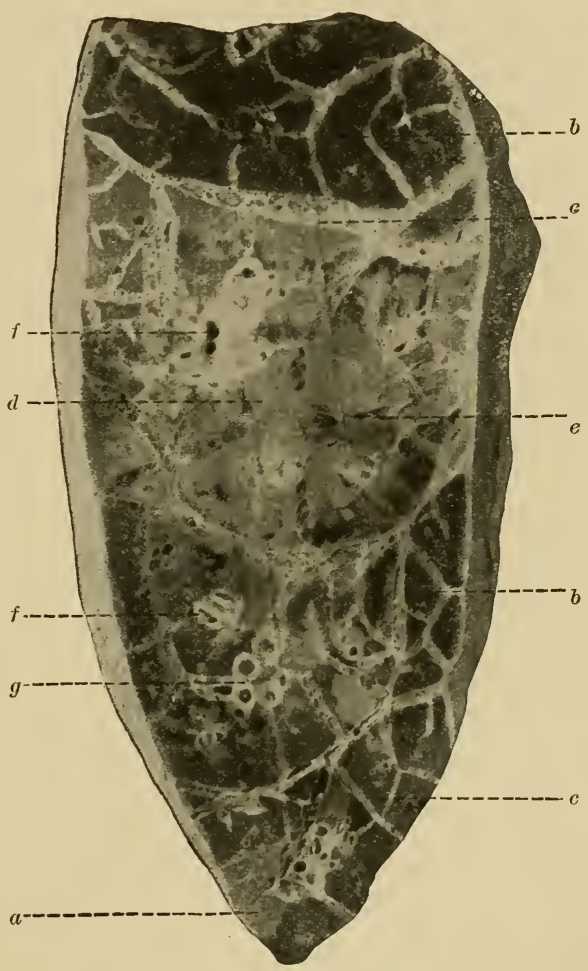

Fig. 63. Cut surface of the lung in contagious pleuropreumonia: (a) normal lung tissue; (b) red hepatization; (c) gray hepatization; (d) incipient necrosis ; (e) interalveolar septum with dilated lymph spaces: (f) blood vessels with thrombus; (g) bronchi. (After a preparation of the pathologic anatomical collection.) cut surface discloses a pale yellow network, formed by an increase of the interlobular connective tissue, which separates the hepatized lung into various sized islands, and according to the stage of hepatization 
they appear gray, pale red, or dark brownish-red in color (variegated, marble-like cut surface; Fig. 63). In the broader beams of the network the dilated lymph spaces are found as round, oval or fissured depressions, filled with yellow lymph or more fibrinous exudate, while the immediately adjoining connective tissue appear's uniformly gelatinously infiltrated. The enclosed airless foci are pale red and firm especially at the border of the hepatized lung portion, sometimes on the periphery, while the center is still dark red and elastic. The walls of the bronchi in the affected parts of the lungs also show serous infiltration and are dilated, while their lumen contains much fibrinous exudate. The peribronchial and mediastinal lymph glands are swollen and filled with fluid.

The pleura over the affected parts of the lungs is sometimes lusterless, injected and covered with a very fine, veil-like fibrinous membrane. Usually however friable deposits $1-2 \mathrm{~cm}$. thick, pale yellow, are found on its surface, under which the pleura appears thickened, and the underlying connective tissue is frequently serously infiltrated. In the majority of cases the pleural cavity contains at the same time more or less (sometimes up to 15 to 20 liters) clear yellow, or turbid, yellowishgray exudate mixed with fibrin-flakes, and in rare cases, the mediastinal connective tissue is also saturated by a similar exudate.

In more chronic cases the connective tissue streaks on the cut surface of the lungs consist of white, firm connective tissue, while the lobules lying between them are uniformly hepatized and necrotic, sometimes even calcified. In such cases so called sequesters are found frequently in one or both lungs, that is one or rarely several necrotic lung portions which are from nut to fist size or even larger. Their surface is knobbed, the outside layers mushy and softened, while the inside is still firm, and the cut surface is diffusely mottled. The necrotic portions are enclosed in thick comnective tissue capsules, to which they are sometimes still partly adherent, and their inner surface is covered with a gray, smeary mass. The capsule may be closed or may communicate with the neighboring bronchus (Arloing found a sequester enclosed in a connective tissue capsule as early as the fiftieth day). Exceptionally even in the lung tissue proper, dull yellow areas may be found, which are only partly separated from the surrounding parts and show evidences of acute inflammation, while in other cases evidences of fresh pneumonia may be observed in the vicinity of an old, encapsulated sequester. In the chronic cases the pleura is usually much thickened, but the surface may still be covered with loose pseudo-membranes, or the pleural membranes may adhere.

In some cases the autopsy may further reveal serous or fibrinous pericarditis, similar inflammation on the peritoneum, on the diaphragm, and in the portal portion of the liver; in young calves sero-fibrinous inflammation may exist in different 
joints and their surroundings, further gelatinous infiltration of the subcutaneous connective tissue in the dewlap, on the chest, and sometimes also on other parts of the body.

Symptoms. The period of incubation, after subcutaneous injection of the virus, in this disease is 6 to 27 days, after inhalation 12 to 16 days (Nocard \& Roux), and it is probably not much longer under natural conditions. The reports of a very long time of incubation may probably be traced to the fact that the disease may be obscured for a time even after the initial rise of temperature, and symptoms of lung affection are only later perceptible.

As the first symptom of the affection a rise in temperature of $1 / 2$ to 1 degree is observed, which may become even higher during the following days.

The accurate observation of the body temperature in suspected eases is of special importance. According to Lydtin, Fleming and Brown animals in which the temperature is over $39.5^{\circ}$, and according to Dèle those in which it goes to over $40^{\circ}$, may be suspected of the disease even if they fail to manifest other abnormalities.

Simultaneously with the rise in temperature the animals manifest more or less pronounced symptoms of general illness, depending on the more acute or more chronic character of the disease (debility, capricious appetite, delayed rumination, diminished milk secretion, etc.; according to Berndt, an edema of the region of the throat appears at the beginning of the affection, which however soon disappears again). Almost at the same time there is a short dry painful cough, which appears especially in the morning after the animals rise at the opening of the stable door, or on leaving the stable, and while drinking. This may also be brought on by percussion of the thoracic wall. Later the cough becomes more frequent, although without resonance, moist and painful. In some animals there occurs a moderate muco-purulent nasal discharge.

This still indefinite clinical picture changes only slightly for a time, gradually however symptoms of difficult respiration become more emphatic. About 2 to 4 weeks after the appearance of the first symptoms the respiration becomes accelerated (30 to 40 per minute) and labored; the stronger extension of the thoracic wall is followed mostly by double contraction of the abdominal muscles, with a simultaneous trembling of the rump. During inspiration the intercostal spaces sink in, the nostrils are opened wide, while the expiration is carried out with grunting (later the respiration may become superficial and very much accelerated). At the same time the animals turn their elbows outward, they utilize the accessory muscles of respiration, and also show the want of air by an anxious expression. On pressure on the vertebral column this is bent laterally.

Percussion reveals, in accordance with the extension of the pneumonia, on one or both sides, behind the shoulder blade 
and up to a certain height, a partly or entirely dull sound, which is limited above by a horizontal or slightly convex line. During percussion, as well as on pressure of the intercostal spaces the animals manifest serere pain, and attempt to avoid the pressure. Auscultation at first shows a weakened, resicular breathing and some crepitation in the anterior and lower portions (Trinchera); later in the region of the partially or entirely dull area no vesicular breathing or bronchial breathing may be heard, but this is usually perceptible around the borders of the affected area, while near the spinal column and, if the affection is confined to only one lung, over that entire side the vesicular breathing is increased, and frequently associated with moist rales. In severe cases expiration is accompanied by grunting, which covers up the respiratory sounds. The heart sounds are either clear and strong, or can be heard only slightly on both sides (in cases of serous pleural exudate). Friction sounds may sometimes be heard in the early stages of the disease, over areas in which the dullness has not yet completely developed.

With the progression of the lung affection the skin loses its elasticity, the hair its smoothness and luster. The appetite becomes worse and worse, the patients rest frequently in the pasture, and separate themselves from the healthy animals. Rumination is also delayed and sometimes entirely absent. Later the patients fail to take any food, they pull away from the crib, or remain apathetically in one place in the pasture. At tho same time rumination ceases, and instead they occasionally eructate. The animals enjoy taking water, especially if it is pure and fresh. Peristalsis is diminished, the flanks are sunken in, constipation alternates with diarrhea, so that the animals pass either dry, solid and dark brown, or fluid and fetid feces, usually with straining.

U'ine is passed at long intervals and very sparingly. It is dark yellow in color and acid in reaction, the specific gravity is as high as 1.022-1.036, the amount of phosphoric acid is considerably increased, up to $0.30 \%$, while chlorin can only be demonstrated in certain localities (Bugarszky).

The milk production, as already mentioned, diminishes even in the earlier stages of the disease, and in the later course good milking cows give hardly 1 to 2 deciliter of milk which contains a considerably reduced quantity of fat and sugar, while the casein and albumen are increased.

The body temperature rises in the further course of the disease up to $41^{\circ}$ to $42^{\circ}$, and the fever curve corresponds to a "febris continua." The body heat is distributed unevenly over the surface of the body, the ears and horns feel sometimes warm, at other times cold, the extremities are cold. The muzzle is dry and warm, later its surface is chapped and covered with dried nasal secretion. The heart beat is correspondingly increased during the height of the fever (80-100-120 beats per minute), in the later course it is throbbing, the pulse is weak. 
In fatal cases the animals become greatly emaciated while the described symptoms become aggravated, they lie with extended neck and extremities on the ground, they breathe with groans and with open mouths, and finally die of exhaustion. Toward the termination of the disease a subcutaneous edematous infiltration develops on the lower chest, lower abdomen, and on the neck, and in other cases a serous inflammation of the joints may follow. Cows usually abort in the course of the disease.

Course. The disease commences as a rule in a latent form, and 2 to 4 and even more weeks may pass before it is distinctly perceptible. From this on the process progresses rapidly in about one half of the cases, and death ensues inside of 2 to 3 weeks more. Exceptionally the disease may have an acute onset with severe symptoms, the pneumonia develops with a sharp rise in temperature in from 2 to 3 days, and death ensues even in the course of the first week. In other cases the affection develops in the described manner, but before reaching an intense degree the animal suddenly dies (paralysis of the heart due to an inflammation of the pericardium and of the mediastinal connective tissue?).

- With the exception of these peracute cases the disease may come to a standstill at any stage, whereupon the symptoms gradually subside, the temperature drops, the cough becomes stronger, the difficulty of respiration subsides, the appetite, rumination and milk secretion returns, the skin becomes elastic, and the animal is more lively in general and improves in nutrition. The shorter the duration of the disease and the milder the symptoms, the more complete is the improvement, and some animals may appear entirely recovered after a certain time. This condition may be permanent, especially in cases in which the symptoms were less intense from the beginning, and no pronounced dullness of the lungs could be found. Usually however the recovery is only apparent, as after a shorter or longer time the disease breaks out anew, and takes on a severe form, or it is possible even later to ascertain a dnllness in the lungs, with the other manifestations of a constricted respiratory area in the improved animals, which meanwhile may even increase in body weight. If in severe cases improvement occurs at all it usually requires months, until at least the digestive functions are again performed normally. Notwithstanding this the respiration may even later remain more or less difficult, a slight cough may be noted periodically, and the animals are easily fatigued. Such patients become again emaciated after a while, and finally die from exhaustion, or the disease again assumes an acute form and leads to rapid death.

The outcome of the disease can hardly be prognosticated during its development. At present the prognosis is of no importance, as the diagnosis of the disease, or its suspicion requires the immediate slaughter of the animal. 
As an epizootic contagious pleuro-pneumonia usually runs a slow course. After the first introduction the suspicion of a general outbreak, because of its occurrence in several animals, is aroused only after about 4 to 6 weeks. In stables the immediate neighbor's of the affected animal become first affected, and the number of cases increases only from week to week, so that in large herds it requires several months before all susceptible animals are affected. If the dead or emergency slaughtered animals are replaced by new ones, as for instance in fattening establishments, the disease may exist with varying intensity for years, and it may also be propagated for years among animals in a township when kept upon public pastures.

Diagnosis. In the living animals a positive diagnosis can only rarely be made with sufficient assurance. Physical examination shows only that the animal is affected with pneumonia, or pleuro-pneumonia, the nature of which however can be established accurately only by reliable historical data. If there is a suspicion of infection every disease of the lungs which shows a latent development and which gradually becomes more intensive is a suspicious indication of the disease, especially when several cattle become affected successively under similar manifestations. Under such conditions the diagnosis of contagious pleuro-pneumonia may be established with great probability, especially when the disease becomes more advanced, and the symptoms become more pronounced. Absolute certainty however is even in such cases revealed only by the autopsy.

From the standpoint of differential diagnosis, the subacute pectoral form of hemorrhagic septicemia comes into consideration in the first place. Although in most cases it is distinguished from pleuro-pneumonia by the sudden onset, the acute course, the severe intestinal inflammation which is commonly present, as well as the simultaneous affection of several animals, isolated cases of the disease which run a slower course may be easily mistaken for the acute stage of pleuro-pneumonia. The diagnosis is sometimes difficult even on postmortem examination; although in hemorrhagic septicemia a larger part of the lung is usually uniformly affected and the cut surface shows no marbled appearance, yet the changes in the lungs may entirely simulate those of acrite pleuro-pneumonia. Numerous hemorrhages in the pleural tissue and in the mucous membrane of the air passages are indications of hemorrhagic septicemia, nevertheless even in such cases the diagnosis can be established with certainty only by bacteriological examination. In this respect the difference is that in pleuro-pneumonia the exudate of the lung's reveals no bacteria under the microscope, further the inoculation of the fluid from the lungs has no effect on test animals, and in calves the subeutaneous injection is followed after one week at the earliest by an edematous swelling, while in hemorrhagic septicemia the exudate of the lungs contains small, polar stained bacilli; the inoculation of the fluid from the 
lungs kills guinea pigs, rabbits, and as a rule also calves in 1 to 2 days. Nevertheless the diagnosis may prove difficult even by this method of examination, as in some subacute cases of hemorrhagic septicemia the lung tissue may contain only a few ovoid bacteria, which are only slightly virulent for rabbits.

The characteristics given above hold also for the differentiation of septic pleuro-pneumonia of calves caused by bipolar bacilli, from pleuropneumonia.

Tuberculosis may be mistaken both for the early stage and for the chronic form of pleuro-pnemmonia. The severe cough, the absence of extensive dullness, bronchial breathing and sensitiveness of the thorax, further the normal temperature or only moderate and sometimes remittent fever and the very slow evolution suggest the probability of tuberculosis. The differentiation may sometimes be difficult, as tuberculosis may take on an acute character, and in pleuro-pneumonia the course may become chronic after the disappearance of the acute symptoms. Moreover the establishment of tuberculosis, for instance by microscopical examination of the discharges, does not exclude pleuro-pneumonia, as both affections may exist simultaneously in one and the same animal.

Traumatic pericarditis has in common with pleuro-pneumonia the sensitiveness of the thorax, the dullness on percussion and the difficulty in breathing. The sensitiveness however is mostly confined to the region of the heart, the dullness is bordered by a concave line running downwards and forwards, the heart's action is quite easily disturbed, and besides a venous pulse is sometimes perceptible in the neck, while on the lower chest an edematous swelling is present. If however the lung is simultaneously affected, the differentiation of the affection from pleuro-pneumonia is impossible.

A genuine croupous pneumonia in which the fully developed clinical picture entirely corresponds with the svimptoms of pleuro-pneumonia, could only be suspected when the possibility of contagious pleuro-pneumonia appears to be excluded, and when the disease takes a typical course and disappears completely within a short time after a rapid dissolution of the lung infiltration. On autopsy the slight affection of the pleura, and the entirely uniform color of the hepatized portion of the lung are indications of a genuine pneumonia (the cut surface is sometimes marbled even in these cases but not variegated).

Finally in infected territories chronic bronchial catarrh, catarrhal pneumonia, verminous bronchitis, pneumomycosis, (nasal discharge!), the echinococcus disease (good nutritive condition, no sensitiveness of the pleura), as well as other affections of the lungs, may give rise to suspicion of pleuro-pneumonia.

According to Nocard the diagnosis of pleuro-pneumonia may be marle with certainty in a short time by the examination of small portions of the lungs or of

Vol. $1-26$ 
the exudate in the following way: The exudate from the lung is diluted with Martin's bouillon 60-80 times and filtered through a Chamberland's F-candle or through a Berkefeld filter. The filtrate is collected in a sterilized flask, on the bottom of which eattle blood serum has been placed in an amount corresponding to $6-8 \%$ cf exudate. Then the flask containing the filtrate is placed in a thermostat, and should the fluid become opalescent in 3 to 4 days without the microscopical examination demonstrating therein distinguishable bacteria, the diagnosis of pleuropneumonia may be considered as established.

Immunization. Immunization against pleuro-pneumonia has been employed to a considerable extent since the stand taken by Wrillems in 1850 , as a procedure by which healthy animals may be protected against natural infection. Since that time it has been employed widely, especially in Holland, Belgium, Great Britain, France, and Germany, in recent times also in Africa and Australia, while in England, Austria and Hungary it has not met with favor.

I. Immunization with Fluid from the Lungs. This method of immunization, the principle of which consists in the injection of the living virus into the stretched subcutaneous connective tissue of the point of the tail, which has a low temperature, affords, as proved convincingly by Willems and later also by other observers, the animals an immunity lasting over one year. This can easily be proved by injections of virulent lymph into the loose connective tissue of the dewlap or rump. While it produces an extensive inflammatory edema in non-immunized cattle which sometimes results in the death of the animal, in cattle which have been immmized a swelling not exceeding the size of a nut results at the point of inoculation, and an injection of virulent lymph directly into the tissue of the lung does not in any way affect their health. Practical experience coincides with. these results, it having been observed that immunized animals become much more rarely affected throngh natural infection than cattle which have not been immunized.

In Willems' experiments 108 immunized cattle remained healthy after being exposed to the natural infection, while out of 50 animals which were not immunized, 17 became affected under the same conditions. Thiernesse \& Degive injected 96 immunized cattle with virulent lymph into the loose connective tissue of the rump, whereupon they became affected only mildly or not at all, while of 33 animals which were not inmunized and subjected to the same infection, all contracted the disease. Schütz \& Steffen inoculated 31 animals partly with warm, partly with cooled lymph from the lungs; 12 were later placed among infected animals, but remained healthy, while of four control animals three died. The remaining 19 head received later virulent lymph injected either into the connective tissue of the dewlap or into the lungs, whereupon they contracted the disease only in a mild form or not at all, while all of the six control animals became severely affected and three of them died.

Under certain conditions the immunization may prove an effective method of controlling the disease, especially when it is very widely spread in a country, and the general obligatory slaughter of the suspected animals cannot be carried out for some reason, and where the danger of subsequent exposure must continue to threaten the animals (in case of infection involving large herds, public herds, valuable large dairy herds, industrial fattening establishments). 
As a matter of fact the procedure was mostly disapproved of in the past, and played only a subordinate part in the attempts to eradicate the disease. This may be explained principally by the fact that in immunizing large herds those animals are of course also inoculated which, although apparently healthy nevertlieless are already infected. In these animals the already existing inflammatory process continues to develop in the usual manner in spite of the inoculation and thus these animals, which are believed to be no longer susceptible, continue to spread the disease among those which have not been immunized. A further disadvantage exists also in the frequent loss of the tail as well as in the occasional fatal results of the method.

Immunization was introducer in the Spoelinger District of Holland in 1874. as this territory had been so badly infected that the slaughter of animals could not be carried out, and as a result of this procedure up to 1884 the annual loss was rerluced from 1,208 to 135 eattle. Aceording to Degive $2.7 \%$ out of 6,706 immunizer animals acquired the disease between 1850 and 1883 in the various countries, while out of 2,453 animals, which were not immunized, $26.9 \%$ became later affected with pleuro-pneumonia. In the German Empire, according to official reports, out of 3,868 immunized animals $151(3.9 \%)$ became affected in the period of 1891-1900 in previously immmized herds, while out of 546 not immunized animals $73(13.4 \%)$ became ill. In herds which were immunized only after the outbreak of the disease, out of 3,287 immunized animals $394(11.9 \%)$ became subsequently affected with pleuro-pneumonia.

Results of the Immunization. After the inoculation of fresh, pure lymph, a hard, painful and warm swelling in size like a nut to an egg develops in about $90 \%$ of the animals, at the point of inoculation; the animals keep the tail quiet and slightly bent upward, and at the same time showing marked febrile symptoms. In favorable eases the swelling disappears together with the febrile symptoms inside of 1 to 2 weeks; not infrequently however the swelling increases in size and suppuration or even necrosis of the tip of the tail sets in. Occasionally the inflammation may extend even to the root of the tail and to the part around the anus, as well as to the connective tissue of the pelvis, whereupon the animal dies from peritonitis which may be associated with it, or from pyemia or septicemia. The cause of these dangerous complications may almost invariably be attributed to the fact that the vaccine originally contained foreign pathogenic bacteria, or that such organisms entered it during its preparation and preservation, or during the inoculation. The number of deaths and losses of tails varies accordingly from case to case. On an average 1 to $3 \%$ of the immunized animals die, and 5 to $15 \%$ lose their tails.

According to Haubner the deaths amount on an average to $1.2 \%$ (at the highest $10 \%$ ), the losses of tails to $5-10 \%$. In Holland, in the Spoelinger District, out of 147,971 immunized animals 1,285 (0.94\%) died in the years 1878-1887, while in Hasselt, the home of Willems, the mortality among 184,275 immunized animals did not exceed $1 \%$. In Germany out of 17,520 immunized animals $141(0.8 \%)$ died as a result of the inoculation between 1891-1901. In some cases, however, the losses were considerably greater. Thus Hildebrandt observed among 365 inoculated animals $69(18.9 \%)$ deaths, while on another occasion $49 \%$ of the animals remaining alive lost their tails. Similar unfarorable results are reported by Omler.

Technique of Inoculation. In the oldest method recommended by Willems, the material which is used for the immunization consists of fluids from the lungs of an animal affected with the acute form of pleuro-pnenmonia, which had been slaughtered immediately before the immunization. The hepatized part of the lung is incised by a knife sterilized in a flame, whereupon the lymph accumulates in the depth of 
the incision. The inoculation is carried out by dipping a grooved racciuating needle into the lymph, and sticking the same about $10 \mathrm{~cm}$. from the point of the tail into the subentis, or the lymph may be allowed to coagulate in a glass vessel and 1-2 drops of the serum are subcutaneously injected into the same place by meaus of a Pravaz syringe.

In procuring the virus special care should be taken that the lungs are only affected with characteristic acute changes of pleuro-pnemmonia, and that the lymph is very elear and light yellow. Lungs in which necrotie centers are already present must not be used for this purpose, as they frequently contain pyogenic or gangrene producing bacteria. Some filter the collected lymph through pure linen and inoculate the filtrate. If pure serous exuclate is present between the layers of the pleura, this may also be utilized for the inoculation.

The described procedure has repeatedly been modified; thus Martin introduces uncler the skin of the end of the tail, immediately under the last caudal vertebra a hair cord three mm. broad and $\$-10 \mathrm{~cm}$. long saturated with lymph. This is allowerl to remain in place for 8 to 12 days and is in the meantime repeatedly moistened with lymph. A similar method is practiced by the Boers in South Africa, in which they saturate raw, woolen threads with lymph and introduce them under the skin at the point of the tail. Defays, Bouley, Thiernesse and Degive injected 2 ce. of virus directly into a superficial vein, for instance into the jugular.

Others again, among them Willems, utilize the serous fluid of the swelling at the point of inoculation as vaccine, whereas in Australia, in accordance with Pasteur's suggestion, the fluid of the lung is first inoculated subcutaneously into the dewlap, or behind the shoulder of a calf two or three months old, and from the resulting swelling after the death of the animal the lymph, which sometimes amounts to several liters, is collected in glass tubes, the ends of which are closed by melting. The lymph thus obtained is supposed to remain unchanged and effective for six to eight weeks if kept in a cool place (according to Schmidt adult cattle are still better adapted for lymph production than ealves).

Laquerrière allows the lung to freeze at $5^{\circ}$ to $6^{\circ}$ below zero, and then places small pieces of it in sterilized glass vessels. The lymph which oozes out has the same effectiveness after one year as when taken out from the fresh lung. Sichütz \& Steffen grind the fresh lung juice in a mortar with glycerin and then place it in a cool place. This substance remains effective for at least eight days, as coagulation is prevented by the glycerin. Nocard incises the hepatized lungs, which have been washer in hot water, deeply, and covers the incision with a plate. The collected lymph is drawn off with a sterilized pipette, mixed with water containing carbolic acid and glycerin (1 part lymph, 1/2 part of a 1:2\% solution carbolic acid, $1 / 2$ part pure neutral glycerin). This is filtered through paper and kept in glass tubes in cool dark places. Such lymph remains unchanged for months. The natives of Senegambia for a long time employed the method of taking the point of a knife or dagger, dipping it first into the affected lungs, and then sticking it uncter the skin of the back of the neck of the animal which was to be inoculated.

Besides the methods described Theiler recommends the administration of virulent material (fluid of the lung or culture) per os, (6 oz. diluted with water), as has been practiced for a long time in South Africa. The immunity is supposed to last for 18 months.

II. Immunization with Pure Cultures. In France immunization with pure cultures has been carried out since 1899 according to the suggestion of Nocard \& Roux, and the results have in general been more favorable than with the old method. An eight-day old culture of the virus in Martin's bouillon serves as vaccine, of which cattle receive $1 / 8$ to $1 / 2$ cc. subcutaneously, inoculated into the tip of the tail. The immunity thus produced lasts for at least two years, as immunized animals, which have distinctly reacted to the inoculation, withstand after two years a subcutaneous injection of $0.5 \mathrm{cc}$. of pure culture without any reaction (Constant \& Mesnard).

In the beginning of $1901,2,359$ cattle were immunized by this method in Southern France, on 597 premises; of these $3(0.12 \%)$ died, 18 head $(0.7 \%)$ lost their tails. All of these losses oceurred in one township, where a veterinarian had not carried out the inoculation in accordance with the requirements; otherwise no 
losses were observed (Nocard). During 1902, out of 1,955 immunized animals nine died, while 257 lost their tails (the inoeulations were made in this instance during unfarorable weather). In 1903 and 1904 attenuated eultures were used for the immunization, and on this oceasion out of 5,554 animals only one died, and only 88 eattle lost their tails.

According to Yohowsky's report immunization with pure eultures also gave satisfactory results in Russia, only he emphasizes the fact that eattle which had not reacted to the inoculation should be inoculated the seeond time after 20 to 25 days. Very farorable results are likewise reported from Russia and Siberia by Dorofeew, Mukarewsky and Stahl.

C'attle may also be immunized effectively by intraocular injections of pure enitures (Nocard \& Roux).

III. Serum Inoculation. Although the serum of healthy cattle and those which have recovered from the disease, possesses no immunizing properties, animals which become affected as a result of subcutaneous injections of a small quantity of pure culture and have again recovered, and which have subsequently received during several months subcutaneous injections of up to over 6 liters of culture, produce a potent sermm, although it has no agglutinating or bactericidal properties. A subcutaneous injection of 40 cc. of such serum produces an immunity lasting from 8 to 10 days, while $40-60$ cc. checks the further development of an inoculation-swelling produced by a virulent injection. After the appearance of the fever repeated injections of $100-200$ cc. of serum are required to exert a curative action; at still later stages it is not possible, even by rery large injections, to check the process in the lungs (Nocard).

Veterinary Police. As the treatment of affected animals does not promise results, the complete eradication of the disease by reterinary police measures is of special importance. As the virus remains virulent in the body of affected animals for a long time, even in cases showing improvement, it can only be destroyed by the slaughter of all cattle which are supposed to harbor it in their bodies. In this regard, besides the affected and exposed animals even those should be considered which are suspected of having been affected, as suspicious symptoms of the disease appear only relatively long after the infection in a perceptible manner.

This attitude has been assumed by the legislative bodies of those countries which earnestly desired the complete eradication of contagious pleuro-pnemmonia, and by an energetic practical execution of this principle, and by satisfactory compensation of the owners, the desired end has been achieved. However the killing of all the suspected animals in localities where the disease is widely spread, especially in large dairy herds, fattening establishments, and public cattle herds, is hardly possible of execution without great danger to the stock industry. Therefore in such herds the isolation or quarantining of the suspected animals for a period of at least six months should be considered, and further, in order to reduce the individual susceptibility the application of occasional immunization, particularly since by 
the utilization of pure cultures for immunization, the danger has been greatly lessened as compared with former times. Further a thorough disinfection of the infected stables, as well as the most thorough airing possible of the evacuated buildings, aid greatly in obtaining the desired result.

The law of 1850 concerning the infectious diseases of the German Empire ordered only the slaughter of the affected animals; in 1895, however, this measure was extended also to suspected animals, and the authorities were further authorized to destroy cattle which were suspected of liaving been infected. In the period of 1890-1903 11,975 cattle were slaughtered by authoritative order, and the governmentinclemnity to the owners amounted to $2,987,795$ marks.

In Great Britain the measure of 1888 did not have satisfactory results in spite of the fact that over 30,000 eattle were slaughtered, as the slaughter of the affected and suspected animals and the compensation of the owners had to be carried by the local funds; therefore by the Act of July 4, 1890, the government was entrusted with the execution of the eradication. Since that time the situation has improved rapidly, so that in 1898 the entire country was free from the disease (the cost of eradication amounted to over $1 / 4$ million pounds sterling).

In accordance with the contagious pleuro-pneumonia law of August 17, 1892, the eradication was carried out in Austria until the suppression of the disease in 1897 , during which time 21,013 eattle were slaughtered, at a valuation of $3,555,886$ crowns; of this amount the State paid an indemnity of $2,711,651$ crowns.

In Hungary, according to the law of 1893, the slaughter of the affected and suspected animals was ordered, eventually also of those which were suspected of being affected, and the time of observation for the latter was extended to six months. The owners were assured of an indemnity by the State, which in accorlance with the law of 1897, included for healthy animals the full value, and for affected cattle $90 \%$ of the appraised value. Until the eradication of the disease in 1901, 56,238 cattle were slaughtered in accordance with this law, with a total indemnity amounting to $3,651,905$ crowns. In a similar way, by obligatory slaughter of the affected and suspected animals, with indemnity from the State, the disease has been eradicated in Holland (1884-1888), in Denmark (1849-1856), and in North America (1887-1892).

Literature. Willems, Rec., 1852, 401; 1887, 11.-Magendie \& Bouley, Ibid., 1854, 161.-Sussdorf, D. Z. f. Tm., 1879, V, 353.-Thiernesse \& Degive, Ann., 1852, 620.-Schütz \& Steffen, A. f. Tk., 1889, XV, 217 ; 1890, XVI, 29; 1891, XVII, 290.Mac Fadyean, J. of comp. Path., 1892, V.-Nocard, Bull., 1892, 158, 203 u. 317.Roeckl, Ber. über d. Kongr in Bern, 1894, 11.-Thomassen, Ibid., 1894, 51.-Nocard \& Roux, A. P., 1898, XII, 240; 1901, 416.-Nocard, Roux \& Dujardin-Beaumetz, Bull., 1899, 430.-Schmidt, B. t. W., 1898, 159; D. t. W., 1899, 265.-Techn. Dep. f. d. Vet.-Wesen, A. f. Tk., 1899, XXV, 312.-Theiler, Schw. A., 1899, XLI, 57. -Constant \& Mesnard, Rec., 1903, 436; 1904, 552.-Dujarlin-Beaumetz, A. P., 1906, XX, 449.-Bordet, A. P., 1910, XXIV, 161.-Borrel, Dujardin-Beaumetz, Jeantet \& Jouan, Ibid., 168.

Infectious Pleuro-pneumonia of Goats. In the years of 1894 and 1895 a severe pneumonia developed in various localities of Germany, especially in Saxony in the vicinity of Dresden (Pusch) and in Thuringia (Storch, Holzendorff), among the bucks imported to this locality from the Swiss Simmental shortly after their arrival, which later spread also to the native goats. At the same time the disease was observed by Leclainche in the Pyrenees, two years later by Mazzini in Italy, and the first mentioned author established the fact that the disease is identical with the affection of goats in Algeria known there as "boufrida," and which occurs there in some years in an epizootic form causing great losses.

The disease appears to affect goats exclusively; the transmission to other animals, even to sheep, has not yet been observed. According to recorded observations the disease is prevalent only in mountainous regions, where it usually runs a mild course. If however the affected animals are brought to other localities the disease attains, under the influence of the transportation and the changed climatic conditions, a severe form, and causes severe losses even among the native goats. 
Symptoms. The disease commences after a period of incubation of 8 to 10 days (Pusch) with fever and catarrh of the air passages (cough, nasal discharge, increased vesicular breathing). After 3 to 5 days the respiration becomes very laborious and on physical examination of the thorax exudative pleurisy or hepatization in a portion of the lung may be established, which as a rule is confined to one side. Subsequently the condition becomes aggravated sometimes rapidly, other times only gradually, and as a rule about one half of the affected animals die. In some animals a herpes-like eruption is observed on the lips, sometimes also on the udder, which as a rule heals without interference.

The course is at times so rapid that the hepatization of the lungs develops in a few hours and death ensues in from 12 to 46 hours; mostly however the disease lasts, even in severe cases, from 3 to 4 weeks, while in other animals it runs a more chronic course, and the animals, which in the meantime become greatly emaciated, finally recover.

The autopsy sometimes reveals only hepatization of a small or larger area of the lung in which there is at the same time an infiltration of the interstitial connective tissue. The cut surface therefore resembles somewhat that of the lungs in contagious pleuro-pneumonia. In other eases, which are more frequent, there is also a sero-fibrinous plemrisy with profuse exudate in the thorax. At the same time the peribronchial lymph glands, and the spleen show acute swelling, while the other organs show no special changes.

Bacteriological examinations have failed to reveal, up to the present time the presence of micro-organisms, either in the affected lungs or in the pleural exudate. Inoculation of material from the lesions into goats, and also into other animals was entirely negative. This condition differentiates the disease from contagious pleuro-pneumonia of goats (see p. 124), and also excludes a relationship with that disease. Nevertheless the infectiousness of the disease cannot be denied, especially in consideration of the experiences in Germany, and therefore quarantine measures, isolation, and early slaughter of the affected animals, disinfection of the stables, etc., are fully justified on its appearance.

Literature. Pusch, D. t. W., 1894, 403.-Storeh, B. t. W., 1896, 567.-Holzen. dorff, A. f. Tk., 1896, XXII, 346.-Leclainche, Rev. vét., 1897, 1. (Lit.).-Mazzini, Giorn., 1898, 350.-Castelet, Progr. vét., 1906, 324.

Chronic Pneumonia of Sheep. According to Robertson (1904), a disease occurs in South Africa among sheep, known there as Jagziekte (hunting-disease), or Hartslagziekte, which is usually observed during the winter months in the higher altitudes in the Cape Colonies, and is manifested by difficulty in respiration, with flank breathing. The autopsy reveals a chronic catarrhal pneumonia, without tendency to degeneration, caseation or suppuration; the sharply circumscribed, hepatized areas have the appearance of bacon, and are greasy to the touch; in older lesions the connective tissue is increased to such an extent, and is so tough, that it cuts like cartilage. In the later stages the adjoining pleura also appears thickened and adheres to the costal plcura. The affected lung tissue contains half-moon shaped, centrally well-stained, and also cylindrical bodies (supposed to be protozoa?). Attempts to transmit the disease artificially to healthy sheep failed to give positive results. (J. of comp. Path., 1904, XVII, 221.) 


\section{Malignant Catarrhal Fever of Cattle. Coryza gangraenosa bovum.}

(Rhinitis gangraenosa, Bösartiges Katarrhal-fieber der Rinder

[German]; Coryza gangréneux, mal de tête de con-

tagion $[$ French]; Febbre catarrale infettiva

[Italian].)

Malignant catarrhal ferer is an acute, non-contagious, infections disease of cattle and buffalo, which is characterized especially by an inflammation of the mucous membrane of the head, with a tendency to formation of membranes and ulcerations, with a simultaneous affection of the eyes and severe nervous symptoms.

History. The disease has been observed and described repeatedly since the end of the eighteenth century; its nature however was, even np to the present time, not sufficiently established. Anker (1832) describes it as typhus of eattle, he as well as Spinola were of the opinion that the benign nasal catarrh may change to the malignant type under certain conditions. Haubner and Röll, later also Lucet, laid particular stress on the diphtheritic affection of the mucous membranes, the disease however was not considered as a specific infection of cattle until Bugnion (1877) classified it as such, his view being at present generally accepted. Transmission experiments were carried out by Brusasco (1887) and Lucet (1892), with negative results, while Leclainche (1898) added further contributions to the etiology of the disease.

Occurrence. The disease is observed either sporadically or as a stable infection. In some localities, and especially in stables of poor cattle owners it is more frequent than under more favorable hygienic conditions.

In Europe the disease occurs everywhere, most frequently, however, in northern parts, on the Scandinavian peninsula (in 1904, 599 cases occurred in Norway), and in Denmark. Severe outbreaks have repeatedly been reported from Russia, but it cannot be asserted with certainty that it was not mistaken for rinderpest or a mixed infection. In Hungary most cases were observed in the mountainous region of Northern Hungary and Siebenbürgen. The disease is also known in South Africa (in Transvaal as "Snottziekte," Theiler).

The disease has been observed among buffaloes in the Netherland-Indies, and on the island of Java (Pascola, Paszotta).

Etiology. The etiology of the disease is not yet entirely clear. This can only be accepted with great probability, that the supposed infective micro-organism produces through its toxic products a general intoxication of the organism, in the course of which the resistance of the tissues, and especially of the mucous membranes is diminished, so that under the influence of other bacteria which subsequently reach these parts, inflammatory processes develop on the mucous membrane with the formation of fibrinous membranes, and later necrosis of the tissue.

Various authors (Ow, Semmer, Franck, Lucet and others) found different bacteria in the deposits of the mucous membrane; their part 
in the etiology of the disease has however not been definitely established. Nocard found in the pseudo-membranes of the larynx and the nasal cavity, bacilli resembling the bacteria of hemorrhagic septicemia.

The etiology has been more closely studied by Leclainche, and the results of his investigations may be summed up as follows: A virulent variety of the colon bacillus may be demonstrated in the intestinal canal, in the lymph glands of the mesentery, sometimes also on the nasal mucous membrane, in the horn core and in the sub-lingual glands. This organism produces very poisonous toxins in bouillon culture, especially for young cattle. Two ce. of a 15-day old bouillon culture injected intravenously or $10 \mathrm{cc}$. injected subcutaneously into a calf about nine months old, produces after a few minutes severe symptoms of poisoning (restlessness, trembling, rising temperature, colie, serous discharge from the nose, and lachrymation), which after 24 to 48 hours gradually disappears. Intravenous injection of 10 to $40 \mathrm{ce}$. of a one-day old culture produces diarrhea, nasal discharge, and salivation, whereupon the animal dies in from 5 to 6 hours, and on autopsy all mucous membranes are found markedly reddened. Feeding of one liter of the culture canses only a passing diarrhea.

Leclainche considers these symptoms and changes as identical with those which are observed in the early stages of the disease, and believes that the bacteria which multiply rapidly in the intestinal canal migrate into the neighboring lymph glands and produce toxins, which after absorption produce a general intoxication and catarrhal hyperemia of the mucous membranes, whereupon streptococei and other pyogenic bacteria subsequently canse suppuration and necrosis in these tissues. Inasmuch however as Leclainche did not succeed in producing the disease in its typical form, and as the observed symptoms of intoxication may be bronght on in a very similar manner by the inoculation of eultures of other pathogenic bacteria, and as the virulent coli bacteria may also multiply subsequently in the intestinal canal and later migrate into the lymph glands, the conclusions drawn from the above investigation must for the present be accepted with reservations.

The nature and method of the natural infection is not yet known accurately. The experience that the disease occurs mostly, although not exclusively, in poorly constructed, low and dark, badly ventilated stables, with unclean floors, appears to indicate that the virus propagates on the contaminated floors of such stables, and probably also in stable filth. From such places it reaches the digestive tract of the animal with the food or drinking water. In rare instances animals in pasture may also become affected, these cases however, are frequently associated with bad, polluted drinking water.

The disease appears to be non-contagious. Experiments of transmitting the disease by inoculation with the nasal secretions of affected animals were without results (Brusasco, Esser, Lotzer). Furthermore, animals which stand next to the affected cattle do not become affected in spite of the very close contact. If the disease spreads in a stable, or if it appear's at the same time in various places in a township or locality, this cannot be attributed to its contagiousness, but rather to the simultaneous influences of a common local cause. Instances are recorded in which the disease appears annually in certain 
stables, which also indicates the influence of a local cause, and likewise proves that this may continue to exist for a long time in one place.

Frentzling observed in the course of a year and a half in one stable 32 cuses of the disease, while Dieckerhoff \& Junkers saw in a herd of 15 cattle, eight fatal affections within 17 days. According to Isepponi the disease occurs enzootically amoug the cattle and goats in the mountains of the Swiss Canton Graubinden, especially in the spring, for instance in the period of 1889-1892, of a total number of 45,000 eattle, 407 became affected, while in the Canton Zürich, out of 94,738 cattle only three fell victims to the disease in 1900 . In the fall of 1893 Kramarew observed the disease in an epizootic form among pastured animals in four townships in Southern Russia. Of 1,244 cattle 322 became affected, 139 (40\%) of which died (the mortality raried in the different townships between $34-67 \%$ ). The disease was supposed to have resulted from polluted river water.

Unfavorable outside influences, such as sudden change in the outside temperature or moist cold weather and colds caused by such, furtlier fatigue during work, etc., appear to be not infrequently accessory causes in the appearance of the disease. If it could be proved that the disease is caused by a variety of the colon bacillus its development could be explained in that the otherwise harmless habitant of the intestinal canal sometimes multiplies rapidly under the above mentioned weakening influences and at the same time attains an increased virulence as a result of which it produces an intoxication of the organism.

The disease occurs frequently in certain localities, especially in swampy places, while in other's it is unknown in spite of otherwise similar local conditions. It is usually observed in spring and fall, more rarely in summer, and very exceptionally in winter. (Melirdorf has known a farm on which the disease existed uninterruptedly for several years as a stable infection.) Young animals are affected mostly; according to Ow only up to the third year, while calves under one year show but very slight susceptibility; steers appear to be more susceptible than cows. A recovery from the disease does not protect the animals from a new attack, as sometimes recovered animals become reinfected after several weeks or months, and then usually with more severe symptoms than the first time (Nagy).

Similar affections were observed in other species of animals than cattle. Thus Hable has seen a similar disease in two horses, Schlossleitner in two hogs. In these cases the symptoms were fever, severe nasal eatarrh, conjunctivitis and keratitis, while the horses also showed pharyngitis. According to Isepponi's observations the disease may occur frequently in goats.

Anatomical Changes. The most constant findings at autopsy are the inflammatory changes on the mucous membrane of the upper air passages. The mucous membranes of the nose, and those of Highmore's and the frontal sinuses, which contain various quantities of a thick, fetid pus, are markedly reddened or cyanotic, swollen, and sprinkled with small hemorrhages. Its surface is covered with purulent secretion, and 
in places with loose, croupous, easily detachable, flat deposits, under which the epithelium is desquamated, sometimes also ulcerated. The ulcers may also extend into the deeper layers, so that their base is formed by the periosteum; indeed, the necrosis and the gangrene may even involve the bones especially the ethmoid, or the turbinates. The inflammation sometimes involves the cores of the horns, also the meat platelettes of the horns, as a result of which they appear reddened and juicy, provided that the horns have not dropped off during life, or are only loosely attached to the skin. The hemorrhagic or sometimes also croupous inflammation may occur in the larynx and in the trachea and in rare cases in the bronchi. Exceptionally a catarrhal pneumonia, and sero-fibrinous pleuritis may be present.

The buccal mucous membrane is bright red in color and shows catarrhal swelling; the gums, the inner surface of the lips, as well as the palate may contain round, flat deposits, with underlying erosions. Similar changes are frequently present in the greatly swollen mucous membrane of the pharynx, which shows a gelatinous infiltration of its deeper layers.

The eyes manifest the changes which are recognizable in the living animal (see p. 412). The cerebral meninges are hyperemic, and saturated with a serous fluid; the ventricles contain a profuse yellowish, slightly turbid or reddish exudate.

Among other changes there is frequently an acute catarrh of the mucous membrane of the stomach and intestinal canal, with small superficial hemorrhages, in rare cases covered with fibrinous pseudo-membranes, and necrosis of the superficial layers, especially on the Peyer's patches. The liquid contents of the intestinal canal are frequently mixed with blood, sometimes also with fibrinous shreds.

The spleen shows a moderately acute swelling, the liver and the kidneys cloudy swelling; the mucous membrane of the urethra shows an inflammatory reddening in its entire length, and is sometimes sprinkled with punctiform hemorrhages and covered with fine pseudo-membranes. The lungs are hyperemic, and show acute edema, sometimes also interstitial emphysema and infarcts. The lymph glands, especially in the mesentery are acutely swollen; further fresh hemorrhages may be seen in some cases in the subcutaneous and subserous connective tissues, as well as in the muscles.

Symptoms. Older authors (Bugnion, Franck) estimated the time of incubation of the disease as from 3 to 4 weeks; it extends however very probably only to several days (according to Lucet it is not more than 12 hours).

The disease commences usually with rapidly progressing febrile symptoms. Within a few hours the temperature reaches $40^{\circ}-42^{\circ}$; the heat on the body surface is unevenly distributed, and the skin feels hot, especially at the base of the horns, and over the cranium. The muzzle is warm and dry, the hair rough- 
ened, the skin tight and dry. The animals manifest at the same time great debility, and dullness of the sensorium, they stand with staring look and drooped head or support the head on the crib, or they lie down much of the time with extended neck. If urged to rise they stand up only with difficulty, stagger on their legs, and arch their backs. Some patients grind their teeth from time to time, bellow, stand in the manger, and even attack objects standing before them. In the meantime trembling is observed in some of the groups of muscles, especially on the neck, shoulders and abdomen, which, under the influence of outside irritation, for instance sunlight, may extend to all muscles and even increase to epileptic convulsions (Anker). The animals show great thirst, while appetite and rumination are suppressed. The milk secretion diminishes and constipation is present. Exceptionally the disease may commence without fever or conspicuons dullness, but the manifestations are indicated in digestive disturbances and salivation (Balog).

On the first, and not later than on the second day of the disease inflammation of the conjunctiva, as well as of the nasal and buccal mucous membranes develops.

The eyelids swell and are continuously kept closed on account of photophobia, at the same time tear's run profusely orer the cheek from the inner canthus of the eye. After 18 to 24 hours the cornea commences to show a smoky cloudiness, which starts at the periphery, and the entire surface gradually becomes opaque. In the meantime in some of the cases a serous exudate is eliminated from the inflamed areas and the ciliary body, which fills the anterior chamber of the eye, either partly or entirely giving the still transparent cornea a yellowish hue. Exceptionally the cornea remains transparent in spite of the internal inflammation of the eye (Bugnion, Dieckerhoff, Isepponi). At the same time a purulent secretion accumulates under the eyelids, the vessels of the sclera are extended, and a fine wreath of vessels may appear on the border of the cornea.

A nasal discharge becomes noticeable; the secretion is at first mucoid, yellowish, but soon becomes purulent, fetid, and mixed with fibrinous shreds, occasionally also with blood, and dries on the nostrils into brown crusts so that it chaps the skin. The nasal mucous membrane is highly reddened and covered with dirty gray, loose pseudo-membranes which are easily detachable leaving an ulcerated surface.

The inflammatory process always extends from the nose to the neighboring Highmore's and frontal sinuses, which is evident by the conspicuous dullness, as well as by the increased heat and swelling of the corresponding parts. Sometimes it extends from the wall of the frontal simuses to the bony wall of the matrix of the horns and in consequence the horns loosen from their cores, and drop off either spontaneously, or as the result of a blow.

Respiration becomes snuffled as a result of the swelling of 
the nasal cavities sometimes also of the larynx, later rattling and snoring are observed. It is always accelerated and labored; in some cases the difficulty in respiration increases to symptoms of asphyxiation, which may finally cause the death of the animal. Increased vesicular breathing may be heard over the chest walls, sometimes together with moist rales. The heart's action is excited from the beginning, the pulse is at first full and hard, later small and soft.

The mucous membrane of the mouth appears reddened, hot and dry (especially striking on the dorsum of the tongue); soon gray or yellowish pseudo-membranes develop in some parts of its surface, especially on the gum, on the inner surface of the lips, and on the hard palate, which later are thrown off, leaving superficial granulating ulcers. Exceptionally vesicles lying in close proximity appear on the skin near the corners of the mouth, and on the muzzle (Wahl), which soon leave ulcers in their places. Still more readily the inflammation extends to the pharynx, as a result of which difficulty in swallowing appears or swallowing becomes entirely impossible. At the same time the earlier salivation becomes still more profuse, and the saliva itself shows a dirty discoloration, mixed with membranous shreds, and is fetid, as is also the exhaled air (Pascolo and Paszotta in a buffalo observed trismus). In rare cases croupous membranes and ulcerations develop also in the rectum and in the vagina. The feces which are at first dry become softer, soon fluid, at the same time may be mixed with blood and fibrinous shreds, and are passed under great straining.

The urine lias an acid reaction (Franck), is cloudy, contains considerable albumen, and sometimes also blood; under the microscope renal epithelia and cylinders are found.

The body temperature, which usually reaches its height on the second day, drops later $1^{\circ}-2^{\circ}$, fluctuates then between $39^{\circ}-40^{\circ}$, and at the approach of death it drops below- normal (Lucet).

In some cases a papular or vesicular exanthema may develop on the skin of the entire body or only in some places, such as the neck, back, udder and interdigital space, resulting in the formation of brown scabs, which on dropping off remove also the hair, while on the udder the thin skin bursts in places, and subsequently a parenchymatous mastitis may develop. Later profuse scaling of the epidermis is observed on the hairless parts. Finally a diffuse inflammation of the skin of the interdigital space may result in exceptional cases. (According to Franck in this form the horns, as well as the hoofs are most frequently thrown off.)

During the disease the animals become greatly emaciated, they lie continually on the ground, and finally die from exhanstion, usually with convulsions.

The enumerated symptoms are observed in the majority of cases 
in quite a uniform development in the respiratory and digestive apparatus; sometimes however the respiratory organs are only slightly affected in spite of the severe affection of the digestive tract (intestinal form).

According to Isepponi the disease occasionally shows an atypical course, inasmuch as in the presence of a high fever, but almost normal respiration and heart action, the patients suddenly pass thin, fetid, sometimes also bloody feces, whereupon death ensues in 2 to 3 days. The cornea in such eases is not clouded.

Lucet also described a form of the disease in which a catarrhal croupous pleuro-pnemmonia becomes associated with the inflammation of the upper air passages after a short time. According to his observations, in this always very rapid type of the disease the affection of the eyes is only slightly pronounced, and especially on the cornea hardly any changes are noticeable. He considers the croupous inflammation of the pharynx, larynx, bronchi, and of the lungs as different forms of a special disease, the diphtheria of cattle (?).

Lichtenheld reports on a malignant catarrh of eattle in German East Africa, which with otherwise similar symptoms is differentiated from the catarrhal fever occurring in Europe, by its epizootic form, with losses up to $75 \%$, further by the marked predominance of the affection of the intestines, and the usually hemorrhagic character of the mucous membranes.

Course and Prognosis. The disease runs with few exceptions an acute course. The clinical picture is usually fully developed in 3 to 4 days, and death follows in 4 to 12 days. In some of the cases the symptoms diminish after the disease has rapidly developed, and the animals commence to eat, although they still have fever and continue to emaciate; a little later the condition again becomes aggravated, and the animals finally die in 2 to 3 weeks.

The prognosis is quite unfavorable, as the mortality varies between $50-90 \%$. Severe nervous symptoms and a great extension of the inflammatory process should be considered as unfavorable indications. The body temperature deserves special attention, as from the third day on a new rise (indication of an associating pneumonia or septic infection), or as a rapid drop have a very unfavorable significance.

In the case of a favorable termination the local changes are not so marked, and the necrotic processes are either absent, or they do not penetrate deeply. After a certain time lachrymation ceases, the nasal discharge becomes again mucous, and loses its fetid odor, the respiration is no longer rattling, the appetite and rumination return, and the animals become brighter, while the temperature gradually returns to normal.

Recovery, which is usually preceded by a convalescence lasting for several weeks, is rarely complete, as the animal frequently remains blind in one or both eyes, or a chronic catarrh of the nose and its sinuses, and sometimes lasting digestive disturbances remain.

Diagnosis. Besides the rapid development of the clinical 
symptoms, the inflammation of the eyes (cornea), and the nasal mucons membrane, the marked depression of the sensorium are characteristic of the affection. From these signs the disease is easily recognized when it occurs sporadically and no intensive affections of other organs are associated. If however several cases follow each other rapidly, or if the intestinal canal and the genital organs are severely affected, and the course is very rapid, a differentiation from other similar diseases requires great care, and very accurate observation.

The greatest similarity exists with rinderpest, especially when at the same time there exists a severe inflammation of the intestinal and raginal mucons membranes. However in malignant catarrh the symptoms of the affections of the eye (diffuse keratitis, which may exceptionally be absent), and sinuses of the head, as well as the severe nervous symptoms, are in the foreground of the clinical picture, and besides the disease is not contagious, which condition may in emergency be established by subentaneous inoculations of blood (in rinderpest in 4 to 5 days after such inoculation fever and a severe catarrh of the mucous membranes develops).

The fibrinons rhinitis (croup of cattle), which sometimes also occurs in an infectious character and manifests quite similar symptoms, usually attacks cows shortly after parturition, and at the same time nervous symptoms as well as indications of an eye affection are absent (Grunth). By the affection of the nose and its sinuses, as well as by the great dullness, the disease is distinguished from foot-and-month disease, and from the various forms of intestinal inflammation (scours, mycotic and toxic enteritis). The croupons character of the mucous membrane distinguishes it from the non-infectious nasal catarrh, while in infectious keratitis the process is confined exclusively to the eyes. Finally tuberculous meningitis may enter into consideration on account of the great apathy, in which however inflammatory changes of the mucous membranes are absent, and instead paralysis of certain nerves of the head is observed.

Treatment. At present the treatment is purely symptomatic. To alleviate the acute inflammation of the mucous membranes of the head energetic cold applications, or cold irrigation of the head are indicated first of all. The accumulation of discharges should be prevented by repeatedly washing the conjunctiva, as well as the nasal and buccal mucous membranes with mild astringents and disinfectants (boracic acid, alum, copper sulphate, creolin, lysol, in 1-2\% solutions), further by inhalation of water vapors, and if possible by manual removal of the necrotic tissue. In attacks of asphyxiation tracheotomy must be performed, and it is advisable to retain the tube in the opening of the trachea for a time, even after the respiratory difficulties diminish.

If a great quantity of exudate accumulates in the frontal 
sinuses or in the cavities of the bony extensions of the horns, this may be removed artificially by trephining, or if this does not produce results, if necessary one or both horns may be sawed off, whereupon the cavities should be washed 2 to 3 times daily as long as they contain the discharge. The exudate accumulating in the submaxillary sinuses usually discharges througli the natural channels from the nose.

If the apathy is very pronounced alcoholic rubs, and the addition of coffee or alcohol to the drinking water are indicated. Very high fever is subdued by antipyretics.

The constipation which exists at first, and later the diarrhea, are treated in the usual manner (see vol. II, Acute Intestinal Catarrls). Suitable feeding during the disease, as well as during convalescence must be especially provided for.

The derivative treatment, which is still greatly in favor with French veterinarians, and which consists in the application of counter irritants to the frontal part of the head and the neck, is hardly ever used elsewhere. Eggeling and Esser obtained good results in some cases from intratracheal injections of Lugol's solution (15 to $20 \mathrm{~g} .1$ to 2 timnes daily), while Lotzer observed rapid recovery from the administration of large doses of iron sulphate. Temnebring, Meissner, Peter, Werner and others recommend the colloid-silver preparations (Collargol, Protargol; 40 to $50 \mathrm{~g}$. twice daily intravenously, of a $1 / 2$ to $1 \%$ solution, for 2 to 3 days); in severe cases however even this treatment fails, or produces at best only a temporary fall in temperature (Schlossleitner, Hohmann, Burgies). Périgaud obtained good results from subcutaneous injections of a $0.75 \%$ salt solution ( 7 recovered out of 11 cases), and this procedure is also recommended by Moussu, as well as recently by Schlotte; of the luke-warm solution 4 to 6 liters must be injected daily subcutaneously until improvement is noticed, and later 1 liter for several days. Isepponi recommends profuse bleeding at the onset of the disease; if no improvement is noticed after 24 hours the animal should be slaughtered as incurable.

As the convalescence lasts for weeks, even in the favorable course, and not infrequently blindness results, it is advisable from an economic standpoint at the appearance of severe symptoms, to slanghter animals of little value rather than to pursue the unpromising treatment. (Ingestion of the meat is not harmful for man according to investigations of Esser.)

Prevention. According to our present knowledge of the etiology of the disease, cleanliness and satisfactory ventilation of the stables, as well as feeding good, unspoiled food, should receive first consideration.

Literature.-Anker, Schw. A., 1832, VI, 81.-Bugnion, D. Z. f. Tm., 1877, III, 63 (Lit.).--Semmer, Ibid., 1885, XI, 77.--Franck, Ibil., 137.-Brusasco, II, med. vet., 1886, 145.-Lucet, Rec., 1892, 481.-Kramarew, A. f. Vm., 1893, I, 53.-Pascola, B. t. W., 1893, 562.-Paszotta, Tä. bl. f. Niederl. Indien, 1894, VIII, 16.-Theiler, Schw. A., 1895, XXXVII, 1.-Keleti, Vet., 1895, 167.-Nagy, Ibid., 231.-Mehrdorf \& Peschke, A. f. Tk., 1897, XXIII, 194.-Leclainche, Rev. vét., 1898, 69.-Péricand, Bull., 1902, 388.-Isepponi, Schw. A., 1904, XLVI, 1.-Lichtenheld, Z. f. Infkr., 1910, VII, 290. 
Croup of Cattle. Grunth descrihes under this name a disease which in addition to disturbances of the general condition is associated with croupous inflammation of the nasal mucous membrane, in whieh the local affections do not result from loeal irritation, neither do they belong to the clinical manifestations of rinderpest, malignant catarrh, or to croupous inflammation of the intestines. Bang however considers the affection as closely related to the malignant eatarrh of cattle.

The disease appears to prevail ehiefly in Denmark, and especially in Copenhagen and vicinity, where it was first ohserved by Sand, later by Hansen, Kaufmann, Olsen, Bang and Jensen. Grunth made a careful study of it. The fibrinous rhinitis observed hy Berndt in Germany may also belong here.

Etiology. According to Grunth the disease is produced by an unknown infectious substance, which weakens the resistance of the body, thereby rendering the mueous membrane susceptible to seeondary infections. Accordingly all complications, and probably also the croupous inflammation of the nasal mucous membrane should be considered as secondary manifestations.

Cows which have recently calved are espeeially susceptible, but in rare eases the disease may appear shortly before or several months after parturition, if the animals have meanwhile been otherwise weakened. Other cattle than cows are only exceptionally affected by the disease in infected herds.

Symptoms. As a rule the animals become affected suddenly by high fever intermittent in character. The pulse and respiration are accelerated, appetite and rumination are disturbed, the milk secretion eeases.

By sufficiently illuminating the nose the nasal mueous membrane appears more or less reddened, and sometimes eovered with petechiae. On the nasal septum or on the nostrils, more rarely in the other parts of the nasal cavity, white or yellowish, loosely adherent croupous membranes of various extension may be ohserved attached to the mucous membrane. Only exceptionally do they attain the thickness of several millimeters, sometimes however they may even be diphtheritic in nature, in which ease even perforations of the nasal septum may result. There is always a nasal discharge, first serous, later beeoming mueo-purulent and containing croupous membranes, sometimes also streaks of blood. Here and there snuffling or wheezing respiration may be olserved with head extended and the mouth wide open.

Complications occur very frequently (according to Grunth in $70 \%$ of the cases). Tery often a broncho-pneumonia sets in (Grunth observed it in $42 \%$ of the eases) which sometimes may also give rise to an interstitial lung emphysema, in which the air may pass under the skin, or into the subperitoneal connective tissue. Simultaneously with the broncho-pneumonia a croupous inflammation of the larynx, traehea and the bronchi frequently develops.

The genital passages are also affected in some cases (according to Grunth in $27 \%$ ). These affections take the form of a croupous, diphtheritic or hemorrhagic inflammation of the mucous membranes of vagina and uterus, and in some cases a parametritis and peritonitis, or an affection of the mucous membrane of the urinary organs is associated with it.

Likewise a profuse, fetfd diarrhea is frequently observed, in which the feces may be mixed with coagulated blood or croupous membranes. In conjunctivitis, which is by no means rare, the eyelids swell, and there is usually serous lachrymation, which may become purulent. An accumu-

Vol. 1-27 
lation of blood in the anterior chamber of the eye, as well as keratitis has also been observed. Here and there fibrinous deposits may form on the buceal mucous membrane.

The course is acute, and its duration averages nine days. It fluctuates however in the different cases between one and thirty days. The convalescent stage is always protracted.

The prognosis appears to be unfavorable, as according to Grunth $45 \%$ of the patients die from the disease, or must be slaughtered. In the recovered animals a permanent shrinkage in the secretion of milk may result.

In eroup without complications Grunth observed $33.3 \%$ deaths, while in the cases complicated with broncho-pneumonia the mortality was $42.8 \%$, in those which are associated with diarrhea $48 \%$, in complications with metritis $52.9 \%$, and in the cases showing a simultaneous affection with broncho-pneumonia and metritis $78.6 \%$.

Differentiation from malignant eatarrh should be based on the facts that in the primary nasal croup there usually exists no keratitis, the affection runs a more favorable course when compared with eatarrhal fever, it principally affects cows, and produces no cerebral disturbances. If the pseudo-membranes developing in the nose are obscured, the disease may be confused with broncho-pneumonia, metritis, or eroupous enteritis.

The treatment is purely symptomatic. The same remedies which come into consideration in other inflammations of the nasal mueous membrane or in broncho-pneumonia, enteritis, metritis, etc., are adapted. At the same time it is advisable to separate the affected animals from those which are healthy.

Literature. Grunth, Z. f. Tm., 1905, IX, 232.

Catarrhal Fever of Sheep. (Malarial catarrhal fever, Geel dikkop, Bekziekte, Ouil bek, Blaw tong.)

Under this name a disease of sheep and goats is known in South Africa, which occurs there under similar local and periodical conditions as horse sickness (see p. 285) and which in some years causes great losses, especially among sheep herds. It was first described by Hutcheon and Spreull, then by Paine, and recently by Theiler.

The symptoms which develop from artificial infection, after an incubation period averaging four days, consist in diminished appetite, dullness and fever (up to $42.5^{\circ}$ ), severe hemorrhagic stomatitis, with shedding of great shreds of epithelium; further a marked edematous swelling of the forehead and the laryngeal region, and a cyanotic discoloration of the tongue. Later the swollen parts become hard and wrinkled, sometimes ulcerative keratitis and panophthalmitis, as well as diarrhea and icterus set in, whereupon in about $40 \%$ of the cases death follows. In some animals an inflammation of the corium of the hoofs develops in the meanwhile. In favorable eases the duration of the disease until recovery is about three weeks. Quinine and calomel, also searifications of the tongue are supposed to be beneficial in action.

The autopsy reveals, aside from the local changes on the head, only a general blood infection, and in protracted cases anemia. The spleen is, even in acute cases, only moderately swollen.

Although the disease is not directly contagious, Spreull succeeded in transmitting it with filtered blood to sheep but not to goats, and the natural infection appears to be transmitted by insects. Sheep recovered from the disease are immune against later infections, their blood however proves virulent sometimes even after 50 days. 
Simultaneous inoculationś with serum of highly immunized sheep (4 ce.), and with virulent blood ( 2 cc.), gave very favorable results in a herd of 295 merino sheep. A mixture of serum and virus $(2: 1)$ is also suitable for immunization. Theiler observed that in serial inoculations of sheep the virulenee diminishes gradually and finally from the tenth operation, and from then on, a harmless vaccine is obtained.

Literature. Spreull, J. of comp. Path., 1905, XVIII, 321.-Paine, Ibid., 1906, XIX, 5.-Theiler, Sehw. A., 1895, XXXVII, 1; Bull. P., 1905, III, 617 ; Z. f. Tm., $1907, \mathrm{XI}, 301$.

\section{Calf Diphtheria. Diphtheria vitulorum.}

\section{(Angina diphtheritica vitulorum.)}

Calf diphtheria is an acute infectious disease of sucking calves, in the course of which croupous diphtheritic pseudomembranes form on the mucous membrane of the mouth and the pharynx, and which, as a result of a general septic infection usually results in death. The causative factor of the disease is the bacillus necrophorus, which was closely described by Bang.

History. The disease was first deseribed in 1877 by Dammann, who observed it in enzootic extension in two consecutive years on an estate near the East Sea. He established its contagious and transmissible nature and considered it identical with diphtheria of man. Later Blazekovic and Vollers observed similar affeetions, while Löffler (1884) proved by histological examinations that the affection differed from human diphtheria, and was probably eaused by long rods, which were present at the border of the necrotic tissue. Later Bang (1890) established the identity of the Löffler's bacillus with his bacillus necrophorus.

Occurrence. According to the observations made up to the present time the disease occurs but rarely; in some localities however it occurs as a stable disease among the sucking calves and causes by its malignant course material loss. Thus in Dammann's case all calves born in the winter and spring became affected, and with a few exceptions they died of the affection.

Etiology. The bacillus necrophorus, Bang, which is considered to be the cause of the disease, appears as a thin slender rod, which in the tissues as well as in artificial cultures, grows into long threads, the plasma of which is either homogeneous or at uniform distances contains dark, fine

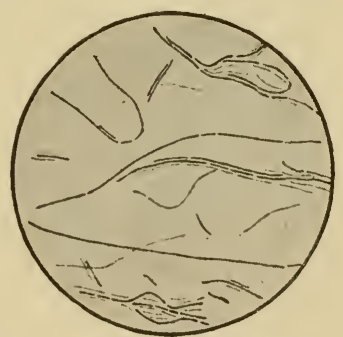

Fig. 64. Bacillus necrophorus Bang. Agar eulture; staining with methylene blue. granules (Fig. 64). The bacillus shows no motility.

Staining. The bacillus stains only slightly and uniformly with aqueous solutions of aniline dyes, stains well with carbol-fuchsin, but not with Gram. 
Cultivation. The bacillus develops only at body temperature, and only anaerobically. Artificial cultivation suceeds on blood serum, agar containing blood serum, bouillon, and in milk. On serum agar, colonies 2 to $3 \mathrm{~mm}$. in diameter develop with striped surface and consisting of felt-like braided threads. At the same time indol and gas are produced, which have an unpleasant eheesy odor (Jensen, Ernst). Bouillon is uniformly clouded in a few days, liquid blood serum is coagulated. No growth results on potatoes.

Pathogenicity. Inoculation of pus eontaining bacilli or of a pure culture into or under the skin of a rabbit canses progressive necrosis of the skin (see p. 102). Inoculation into mice produces also skin necrosis, while guinea pigs, dogs, cats and chickens are not susceptible to the infection. A subcutaneous injection in cattle, hogs and pigeons causes only local necrosis of the skin, in lorses cold abscesses. (According to L. Roux the experimental necrosis develops especially after adding other bacteria to the pure culture, whereas alone it frequently causes only cold abscess.) The introduction of pseudo-membranes of affected calves into the nose and into the mouth of lambs only several days old, produces a diphtheritic stomatitis, while if sewed in under the eyelids a severe conjunctivitis with keratitis results. Feeding experiments always give negative results.

The bacillus necrophorus, as has been proven by Bang and later hy Jensen, plays an important part in various diseases of animals, in which, during their course necrotie and gangrenous processes develop as complications. Thus the neerotic and croupous diphtheritic processes of the mucous membranes in general, especially however of the mucous membranes of the pharynx and the intestines (among others in hog cholera), in sheep, on the lips and on the borders of the nostrils, or on the external genital organs (see Vol. II), further in gangrene of the hoofs and the teats, in necrotic dermatitis, ete., are frequently produced by this bacillus. In addition to these the occasional necrotic areas in the liver, kidneys, lungs, etc., may he attributed to this cause.

In the great majority of cases the bacillus necrophorus attacks only tissue that is already affected. Successful infection experiments, as well as metastatic neerotic areas, proved however that under specially favorable conditions it may attack even healthy tissues. Its characteristic location at the border of the necrotic and the living tissue, proves that it exerts its pathogenic action, the necrosis of the tissue elements, by the elimination of toxic products.

The bacillus necrophorus is described in literature under various names and is identical with the: Bacillus necrophorus, Flügge; Bacillus diphtheriae vitulorum, Loeffler; Bacillus necroseos, Salomonsen; Bacillus filiformis, Schütz; Bazillus des Kälbernoma, Ritter; Streptothrix cuniculi, Schmorl; Actinomyces euniculi, Gasperini ; Actinomyces necrophorus, Neukirch, and Streptothrix necrophora, Ernst (L. Roux).

Natural infection may probably be explained by stating that the bacillus necrophorus, which is very extensively distributed in nature, is among others frequently contained in the feces of herbivorous animals, and owing to its anaerobic character 
remains alive for a long time on the dirty floors of damp stables, in musty food and straw, and from there reaches the buccal cavity of young animals. Healthy animals may become infected by contact with affected ones, by licking them, thereby introducing diseased products (shreds of pseudo-membranes hanging from the mouth, nasal secretions) into their mouths. The easy transmissibility of the disease has been fully established by Dammann, since he watched a healthy calf which was placed in a stable between two affected calves, become affected in five days.

The disease attacks young sucking calves exclusively, which sometimes become affected as early as on the third day after their birth. The great susceptibility of the young can probably be explained by the loose and delicate consistency of the epithelia of the mucous membrane. The susceptibility of the newly born may also be increased by any other physical weaknesses, the residue of the milk remaining in the mouth of such animals being especially adapted for the localization and propagation of the virus. Injuries of the buceal mucous membrane, such as the eruption of the milk teeth, may constitute the principal entrance for the infective agent.

Anatomical Changes. The autopsy reveals on the mucous membrane of the mouth and pharynx, various sized and coalescing croupous membranes, which extend higher than the elevation of the surrounding tissue. They are several centimeters thick, sharply circumscribed, dirty gray or brown in color, ragged on the surface, and firmly adherent to the underlying tissues. Ulcerations also occur with thickened borders and ragged bases; between these lesions the mucous membrane is greatly swollen, reddened, the submucosa shows serous infiltration. Preferred places for the formation of these psendomembranes are, the inner surface of the cheeks, the hard palate, the borders of the tongue, the parts surrounding the frenum of the tongue, the gums, the soft palate and the pharynx. In some cases the mucous membrane over a considerable area and in its entire thickness changes to a yellowish-gray, brittle, fetid mushy mass. The muscles of the tongue may also be necrotic under the affected parts of the mucous membrane to a greater or lesser depth, the destructive process on the hard palate may even extend to the bone producing caries, and changing it to a soft mushy mass. Similar changes occur also on the lower parts of the nasal cavity, on the larynx and in the trachea, where they may affect the cartilage.

The lungs usually contain either dry or purulent, softened, necrotic areas, which are surrounded by zones of catarrhal pneumonia, and sometimes also a purulent pleurisy may be present as a result of perforation of such superficial, necrotic areas. The small intestines reveal acute catarrh, whereas posterior to the cecum sometimes pea-sized dirty yellow, dry deposits may be 
found. The spleen is not enlarged and the lymph glands show a moderately acute swelling.

Symptoms. The disease commences on about the fifth day after the infection with marked weakness and diminished appetite, as well as a slight rise in temperature. On the second to third day salivation appears, and at the same time on one or both cheeks a hard painful swelling is noted. With a finger introduced into the buccal cavity, rough deposits may be felt on the mucous membrane in the corresponding places. On inspection of the mouth however, on these as well as on other places, especially on the hard palate and on the swollen tongue, yellowish deposits are found, which in part show a ragged surface.

In the meantime there is a yellowish discharge from the nose, and the nostrils fill with dirty yellow, brittle masses, on account of which the respiration appears snuffling. At the same time the animals become greatly emaciated, and are able to take, only from time to time, and with difficulty, small quantities of water. In some cases cough, and a very labored, rattling respiration, indicate an affection of the larynx and the lungs, while diarrhea points to an involvement of the intestines. (Dammann observed in one case a necrosis of the skin and of the subcutis in the interdigital space in both forelegs.) Exceptionally swelling and a suppuration of all glands of the head have also been observed.

Eggeling found repeatedly that the disease commenced in calves with high fever, difficulty in respiration, and inability to swallow. At the same time cows which had recently calved showed a diphtheritic vaginal and uterine involvement.

Course. The disease terminates in the majority of cases in death after 4 to 5 days, in other cases again in about three weeks, pneumonia being usually the immediate cause. In the rare cases with favorable termination the convalescence lasts several weeks, as the defects on the mucous membrane after the shedding of the necrotic deposits heal reluctantly, and the strength of the affected animals is regained very slowly.

Diagnosis. The thick deposits on the lesions, consisting principally of necrotic tissue of the mucous membrane, distinguish the affection from foot-and-mouth disease, in which the erosions are at the worst only covered with fine, loose, readily removable pseudo-membranes. In aphthous stomatitis and in thrush only superficial, readily removable deposits form on the only very slightly swollen mucous membrane.

Treatment. After removing the deposits, which are not very firmly adherent, it is advisable to paint the ulcerating surfaces, as well as the firmly adhering psendo-membranes 
1 to 2 times daily with a paste consisting of water and salicylic acid (Dammann), Lugol solution (Dieckerhoff), tincture of iodine, $10 \%$ citric acid (Poels), or with a $5 \%$ solution of permanganate of potash. Besides it is advisable to wash the mouth repeatedly with lukewarm water. Salicylic acid (1-2 g.) or potassium chlorate ( 2 g.) may be administered internally. At the same time the attempt should be made to keep the animals in good strength by supplying them with nutritious food (milk, eggs).

Careful ventilation and disinfection of the stables is indicated for the prevention of the spread of the disease.

Literature. Dammann, D. Z f. Tm., 1877, III, 1.-Blazekovic, Rec., 1878, 64. -Vollers, W. f. Tk., 1879, 432.-Loeffler, Mitt. d. G.-A., 1884, III, 401.-Bang, Maanedsskr., 1590, II; 1892, IV.-Jensen, Hb. d. p. M., 1903, II, 693.-Eggeling, Pr. Mt., 1903, II, 17.-L. Roux, Cbl. f. Bakt., 1905, XXXIX, 531.

Pharyngeal Diphtheria of Other Mammals. As the bacillus necrophorus has been demonstrated in the diphtheritic processes of various species of animals, it appears probable that a diphtheria analogous to the affection in calves may also occur in other animals as an independent affection. In the cases however which have been observed the bacillus necrophorus as a rule attacked the buccal mucous membranes only after the animals had become sick from other causes.

Mayr observed a diphtheritic inflammation of the pharynx in three head of cattle, while Strebel observed it in five head. The symptoms consisted in salivation, dyspnea, difficulty in swallowing, nasal discharge, sensitiveness and swelling of the throat region. The autopsy revealed diphtheritic deposits, and ulcerations on the palate, in the pharynx and larynx, as well as along the esophagus. In some cases ulcers were found on the muzzle and in the interdigital space, with necrotic deposits as large as a quarter on the mucous membrane which was without papillae, in the pillars of the rumen, membranous inflammation of the small intestines, etc. In these cases the disease had probably developed as a complication to a septicemic infection, which is apparently also the case in the affection of horses (Cobbett, Pr. Mil.-Vb.) occurring as a diphtheritic process in the mouth, in which case it is probably a complication of an influenza infection.

Roche-Lubine observed a disease among lambs which was manifested in difficult respiration, salivation, laryngeal and bronchial eatarrh. The autopsy revealed extensive pseudo-membranes on the mucous membrane, of the upper air passages. Diem reports a similar affection which occurred as a local enzootic, while Hasenkamp observed an outbreak of panaritium of sheep caused by the bacillus necrophorus, in the course of which diphtheritic processes developed on the mucous membrane of the pharynx and mouth, in some of the animals.

In hogs the so called angina represents a frequent symptom of hog cholera, in which the flat deposits on the mucous membrane of the pharynx and larynx contain the bacillus necrophorus, together with the bacillus suipestifer. This is likewise the case in the inflammations of the pharynx, which are frequently observed as a symptom of swine plague, especially on premises where hemorrhagic septicemia exists among cattle.

According to Johne and Kitt the pharyngeal diphtheria may also occur among hogs as an independent disease. In such cases the crypts 
of the greatly swollen tonsils are filled with yellowish, brittle masses, and the mucous membrane of the surrounding part is covered with numerous yellowish deposits which may reach the size of a pea, or the diffuse, swollen and reddened, buccal and pharyngeal mucous membrane appears to be covered with caseous deposits.

A severe plaryngitis, with the formation of pseudo-membranes, has also been obscrved in dogs and cats (Gray, Symes, Bell), and Brandt reports a case in which a s'cotch collie became affected with a diphtheritic inflammation of the throat, and from which he isolated bacteria which microseopically, culturally, and biologically appeared identical with diphtheria bacilli (several days later a girl who attended the dog became affected with diphtheria, and $31 / 2$ weeks later still another girl). During the course of canine distemper, and still more frequently in typhus of dogs, necrotic processes ocenr on the buccal mucous membrane, which are also caused by the haeillus necrophorus (Jensen), while in one ease showing also symptoms of albuminuria and hemorrhages, Ball found only streptococei.

Literature. Wayr, Bayr. W., 1902, 185.-Strehel, Schw. A., 1899, 173.Cobbett, Cbl. f. Bakt., 1900, XXVIII, 631.-Diem, W. f. Tk., 1900, 339.-Johne, S. Jhb., 1893, 61.-Kitt, Mneh. Jhb., 1893-94, 81.-Ball, J. vét., 1906, 449.-Gray, J. of comp. Path. 1896, IX., 46.-Symes, Brit. med. Journ., 1896, I, 1385.-Bell, Amer. Rev. 1901, XXV, 115.-- Brandt, J. of the Americ. med. Assoc., 1908, L, 15.-Hasenkamp, D. t. W., 1909, 237.

\section{Avian diphtheria and chicken pox. Diphtheria et epithelioma, contagiosum avium.}

Until very recently avian diphtheria was considered to be a contagious, epizootic disease of domesticated fowls, characterized by croupous and diphtheritic pseudo-membranes on the mucous membranes of the head, while chicken pox was held to be a contagious, epizootic disease in which hyperplastic, epithelial nodules of the skin occurred especially on the comb and wattles, but in addition to which croupous-diphtheritic deposits frequently developed on the mucous membranes of the head. The two diseases were considered as independent; more recent research, however, indicates that they are produced by the same organism, and they will here be treated accordingly.

History. In avian diphtheria as well as in chicken pox investigators were inclined, since the experiments of Rivolta and Silvestrini (1873 and 1878), to consider protozoa as the etiological factors (in diphtheria the flagellate, Cercomonas gallinae; in pox gregarines, hence the former name Gregarinosis). Later bacteriological experiments, particularly those of Loeffler (1884) on pigeon diphtheria, seemed to indieate that the diphtheria was due to a rod-like bacterium which he called Bac. diphtheriae columharum. Furthermore other. observers, as Krajewski, Moore and Galli-Valerio found the same organism in the diphtheritic membranes of sick chickens, while subsequently Guérin demonstrated its identity with the oroid bacillus described by Haushalter and Loir \& Ducloux. Streit insisted on the etiological importance of his roup baeillus for the disease called roup in America, and Müller was of the opinion that the virus of chicken diphtheria was a corynebacillus, 
an organism similar to the human diphtheria organism. But these and other similar findings influenced but slightly the view that bird diphtheria is a bacillary infectious disease, and were explained by the assumption that various bacteria participate in different cases in the diphtheritic diseased processes, a view which indicated a degree of uncertainty as to any positive knowledge in this regard.

The etiology of chicken pox was elucidated acceptably when Marx and Sticker (1902) brought proof that the pock virus will pass through a porcelain filter and therefore belongs to the filterable infectious substances. This has since been confirmed by Juliusberg, Borrel, Loewenthal, Burnet and others.

Experiments following this discovery have led to results which completely shattered the existing view that the etiological factor of the two diseases differed. It is true that Roell (1867) suspected that the changes in the oral mucosa and skin were due to the same causative agent, and this view is also expressed in the work of Rivolta \& Delprato (1880) as well as Pfeiffer (1889) and Kinsley (1907). Moreover from a practical point of view, considering the difficulty in differential diagnosis, this did not seem unlikely, but experimental support was not obtained until Carnwath (1908) succeeded in producing a diphtheritic disease of the mucous membranes from pure pox material, and with diphtheritic material produced chicken pox. These experimental results, doubted by Bordet \& Fally and also by Jowett, were confirmed by Schmid, Uhlenhuth \& Manteufel, and also by Rátz, and further experiments directed towards the solution of some contested details such as virulence of the blood in pure diphtheria, role of the bacteria present in the pseudo-membranes, ete., will no doubt soon establish the etiological identity of the two forms of disease definitely.

Prevalence. Both forms of disease occur particularly in fowls (chickens, turkeys, pheasants, peacocks), also in pigeons, and much less commonly in water fowls. In the southern regions (Italy, Tunis) the diphtheritic form rages with greater virnlence and sometimes presents a septicemic character, but in northern regions the losses, especially in pedigreed fowl farms, are heavy on account of the frequent severe invasion of the disease and high mortality.

Etiology. The experiments of Marx \& Sticker have shown that the fluid obtained by maceration of epithelial nodules in physiological salt solution is infectious after passing through a Berkefeld filter, since after the inoculation of the filtrate into the skin of chickens the characteristic epithelioma developed, which could be inoculated from one animal to another.

Borrel, Lipschütz, as well as Prowazek, are inclined to consider as etiological agents, very minute, hardly $0.25 \mu$ sized, spherical or biscuitform organisms, found in large quantities in virus-emulsions, and which can be demonstrated by Giemsa's as well as by Loeffler's flagellum stain. By penetrating into cells they may cause the development of the frequently mentioned "inclusions" in the epithelial cells, as products of reaction. Prowazek named them, as well as similar formations in vaceinia, rabies, etc., Chlamydozoa, since they caused vacuoloid reaction products in the infected cells.

Tenacity. The pox virus is rather resistant to external influences. Enclosed in epithelial masses it is not destroyed by complete drying or exposure to diffuse daylight for several weeks; heating to $60^{\circ} \mathrm{C}$. for three hours, cooling to minus $12^{\circ} \mathrm{C}$. for 
five weeks, and one per cent carbolic acid solution for half an hour also failed to destroy it. Portions of the nodules preserved in glycerin for several weeks still produced the typical disease (Marx and Sticker). The virus further resists the effect of $10 \%$ atoxyl and $1 \%$ saponin solutions, as well as a $10 \%$ solution of sodium taurocholate (Lipschütz). When the tumor masses are finely pulverized it is killed at $60^{\circ} \mathrm{C}$. in 8 minutes (Burnet), by $1 \%$ potassium hydrate solution, $1 \%$ acetic acid or $1: 1,000$ corrosive sublimate in 5 minutes (Sanfelice).

Culture. Bordet reported successful cultivations from diphtheria material on his blood-glycerin-potato-agar, minute colonies developing on the surface, composed of granules staining by Giemsa's method and having a dimension of barely $0.2 \mu$. With such cultures he succeeded in producing diphtheritic membranes on the oral mucosa of chickens, but not epithelioma on the skin. Similar attempts at culture by Lipschütz and by Uhlenhuth \& Manteufel with pox material gave no positive results.

Pathogenicity. When finely pulverized epithelial nodes are rubbed into the skin of the comb, wattles or eyelids of chickens there occur after 5 or 6 days swelling and pallor of the affected parts of the skin, and on the following day a nodule as large as a millet seed appears on its surface. After three or four more days hemorrhages suddenly appear over the affected region; later the blood dries forming thick scabs, beneath which the papillae enlarge considerably and become covered with a turbid, gray exudate. Beneath this bloody scab healing takes place after a shorter or longer period of time; but when the diseased portions are exposed to traumatic influences (rubbing, scratching) there develop extensive papillary proliferations which delay healing. The result from inoculation of the filtrate is similar, only the incubation period is from 8 to 10 days on account of the dilution or attenuation of the infectious substance (Marx \& Sticker). Juliusberg, who demonstrated the filterability of the infectious virus of pigeon pox, observed an incubation period of 14 days after inoculation of the filtrate into pigeons.

When pox material is rubbed into the scarified oral mucosa or injected into the submucosa, there either arises a local reaction in the form of a light yellow membrane, or there develops a cliaracteristic croupous-diphtheritic inflammation, which may extend from the oral mucosa to the nasal cavities and their sinuses (Loewenthal, Schmid, Uhlenhuth \& Manteufel). Conjunctival inoculation produces a diphtheritic conjunctival inflammation, which eventually causes a panophthalmia.

The results are similar when diphtheria material is employed for inoculation on the skin and mucous membrane only here negative results are more common than after inoculation with pox material (Schmid, Uhlenhuth \& Manteufel):

Loewenthal and Burnet produced pox on the skin by intravenous injection of pox material. Uhlenhuth \& Manteufel used intravenous injection's of pox and diphtheria material and produced severe diphtheritic symptoms on the mucosa of the mouth and on the conjunctivae. 
Both pox and diphtheria are easily transmitted from chickens to chickens and from pigeons to chickens, whereas the transfer of pox material from chickens to pigeons is accomplished with difficulty, and transmission of diphtheria material has thus iar been unsuccessful. The infective virus transferred from the pigeon to the chicken appears to undergo an attenuation even in the first generation; but also in the pigeon the virus becomes attenuated by successive passages even to aviruleney (Juliusberg).

Aside from its presence in the epithelial nodules and mucous membranes the virus is also found in the blood, at least in the beginning of the disease, for it was repeatedly found possible to produce the characteristic changes on skin and mucous membrane, especially with liver substance (Loewenthal, Burnet, Schmid, Uhlenhuth \& Manteufel).

The above mentioned experimental results permit the conclusions, that epithelial nodes and diphtheritic pseudo-membranes contain the same virus which produces pox on the skin and diphtheritic processes on mucous membranes, and therefore chicken pox and avian diphtheria form an etiological entity. This view is supported by an experimental result obtained by Uhlenhuth \& Manteufel, according to which recovery from the affection of the mucous membrane produced with diphtheria material gives immunity against a subsequent artificial skin infection with virulent pox virus.

In consideration of the present viewpoint the question arises what significance attaches to the more or less pathogenic bacteria found by different authors in the diphtheritic pseudo-membranes and considered by them as causes of the disease on account of the partly successful experiments of transmission. The following come under consideration:

1. The bacillus diphtheriae columbarum obtained in pure culture by Loeffler from a case of pigeon diphtheria. This bacillus, which takes a bipolar stain, is similar to the organisn cansing rabbit septicemia, but slightly longer and more slender; its pure cultures, after subcutaneous inoculation, produce in pigeons a necrotic inflammation of the skin, while after injection into the oral mucosa of chickens circumseribed pseudo-membranes form. Loir \& Ducloux no doubt found the same bacillus in diphtheritic chickens in Tunis, only this organism was characterized by a marked virulence, while its cultures after subentaneous inoculation soon produced a rapidly fatal septicemia, or heavy deposits in the throat and in the larynx in addition to general septic symptoms.

2. 'The "Roupbacillus," an organism in many ways similar to the bacillus pyocyaneus, described by Streit as the cansative factor of American " roup," a disease clinically identical with chicken diphtheria. He found it in abundance in the disease products of sick birds and made with his pure cultures experiments of transmission to chickens and pigeons, which were in part snceessful.

3. The "Chickendiphtheriabacillus" of R. Müller, which he describes as related to the human diphtheria organism or Loeffler's bacillus, althongh a smaller corynebacillus. He obtained pure cultures which mpon inoculation in mucous menbranes or injection into the submucosa in pigeons produced a diphtheritic inflammation.

4. A colon-like bacillus, which Hausser was enabled to constantly demonstrate in spontaneous diphtheria in the deposits, and with whose pure cultures he succeederl several times in prodncing a diphtheritic inflammation in chickens and pigeons.

Finally there may be found in the diphtheritic deposits in some cases the bacillus pyocyaneus, streptococci, chicken cholera bacteria, necrosis bacilli and sometimes swine erysipelas bacilli. 
These variable results of the several observers preclude any specific role of the bacteria found; on the other hand, however, in view of the positive inoculation results obtained with pure cultures the possibility is by no means cxcluded that under certain conditions, especially when the requirements for their propagation in the body of the fowl are for any reason favorable, local diphtheritic inflammatory processes may be caused or already existing processes be aggravated. When it is considered that the disease of the mucous membrane may also be produced by filtrates of disease products and by intravenous injection of such filtrates the assumption or supposition can hardly be rejected that avian diphtheria, as well as chicken pox, is primarily produced by the same filterable virus, whereupon facultative pathogenic bacteria subsequently settle on the mucous membranes and on their own part participate more or less in the development of the clinical picture of the disease.

According to Bollinger and Csokor the epithelioma contagiosum of man is identical with the epithelioma of birds, for material from the former, when inoculated into the comb of birds, gave rise to a disease identical with chicken pox. The close relationship is inclicated further by the fact that the virus of the disease is also filterable (Juliusberg, Casagrandi, Serra, and others).

Natural infection probably occurs in most cases by the intermediation of exfoliated epithelial material or of exudate discharged by the diseased animals while sneezing or coughing, or otherwise. These, or the intestinal contents or portions of internal organs of diseased or dead animals, may come in contact with superficial abrasions on the skin or mucous membranes. It is possible that infection mav occur also through the unimpaired mucosa, which is favored, no doubt, by a preceding injury, and the frequent disease of the eyes may be associated with the habit chickens have of rubbing the vicinity of the orbit with their feet (according to Mégnin older pigeons, which frequently show small caseated nodules in the esophagus, may infect their young while feeding them).

Healthy fowls are usually infected by sick animals, contaminated drinking water, food, etc., serving as media of infection. The disease is spread mostly through transportation of fowls to markets and poultry exhibits; but free-flying birds (pigeons, sparrows, etc.) may carry the infection for long distances to healthy poultry farms.

Pigeons are most susceptible to the disease, chickens less so, and water fowls to a considerable degree less. The susceptibility to infection is in general greater in young than in older animals.

An attack of the disease, no matter whether skin changes or diphtheria of the mucous membranes was manifested, confers immunity against subsequent infection, for chickens which have recovered from the artificially produced disease, can not be reinfected after two weeks. The immunity so acquired is, however, sometimes, only of short duration (Loewenthal).

Anatomical changes. These may be confined to the skin, or to the mucosa of the mouth and nose, but in the animals 
which have died from the disease the vast majority of cases will show the skin as well as the mucous membranes seriously affected.

In addition to the epithelial nodes of the skin one finds croupous-diphtheritic deposits on the mucosa of the mouth and throat, the nose and accessory cavities, sometimes also in the larynx, trachea and bronchi as well as in the intestinal mucosa. The membranes in the mouth are dark gray or brown, at other points yellowish-gray, usually croupous, sometimes softened and cheesy, while the subjacent mucosa is denuded of epithelium, finely granular and occasionally appears necrotic to a certain depth. In individual cases a croupous-caseous exudate is found in the bronchi and in the cella infraorbitalis in such quantities as to fill the canals and cavities involved completely.

The intestinal mucosa also shows similar inflammatory changes and small extravasations of blood. Occasionally the epithelium is removed for a considerable extent, and here and there shallow ulcers may form, while on other areas, especially in the caeca firm dry nodes composed of proliferated epithelium and detritus may adhere to the inner surface of the intestine.

In the more acute cases, in addition to the above changes on the mucous membrane which may be very indefinite, a large splenic tumor may be found, fine yellowish points or spots in the liver, acute edema of the lungs, hemorrhages and occasionally fibrinous membranes on the serous coats. and finally a serous exudate in some joints.

The microseopic examination of the epithelioma of the skin shows, according to Polowinkin, the epithelial layers thickened and the epithelial papillae elongated inward; in the papillary layer the vessels are dilated and, like those of the subeutis, surrounded by migrating leucocytes; in addition there are small hemorrhages into the tissue of the papillary layer. Cell-inclusions are found in the swollen epithelium, similar to Guarnieri's pox bodies (Loewenthal, Burnet; s. p. 293). In a general way the characteristic features of the efflorescences are composed of a more or less pronounced hyperplasia and proliferation of the cell elements of the skin, which sooner or later succumb to degeneration. This is associated with the extension of the process to the external skin and there follows a condition which, according to Reischauer, is similar to true pox; the difference lies mainly in the fact that in bird pox the more ehronic character of the disease causes the nodular and tumor formation to predominate.

Microseopic examination of the diseased mucous membrane shows a fibrinous network in the superficial layers, in the folds of which lie irregularly formed flakes and granular detritus, while in the mucous tissue are seen cellular infiltrations and occasionally neerosis of the tissue elements. The exudate may also show various bacteria and occasionally protozoon-like organisms, but their significance has thus far not been ascertained. Rivolta found a flagellated, spherical or ovoid protozoon, the Cercomonas gallinarum, in the membranes of the oral mucosa, while Pfeiffer found in membranous inflammation of the trachea and the intestines, flagellates in large numbers with three, more rarely with two or four flagellae, which he believed to be Trichomonads, and both observers were inclined to associate their findings etiologically with the pathological conditions. Babes \& Puscariu consider the flagellates as harmless and only accidentally present in the air passages and in the intestinal eanal, because they nerer succeeded in producing a disease of the mucous membranes with them.

Symptoms. The clinical picture of the disease depends upon whether there is an affection of the skin or the mucous membranes or if both these tissues are involved, and whether 
the disease runs an acute or chronic course. Thus there are several clinical forms of disease to be differentiated.

In one form, which has always been designated as chicken pox, morbid changes develop in the skin, and in the majority of cases the skin of the head is first affected. On comb, ears

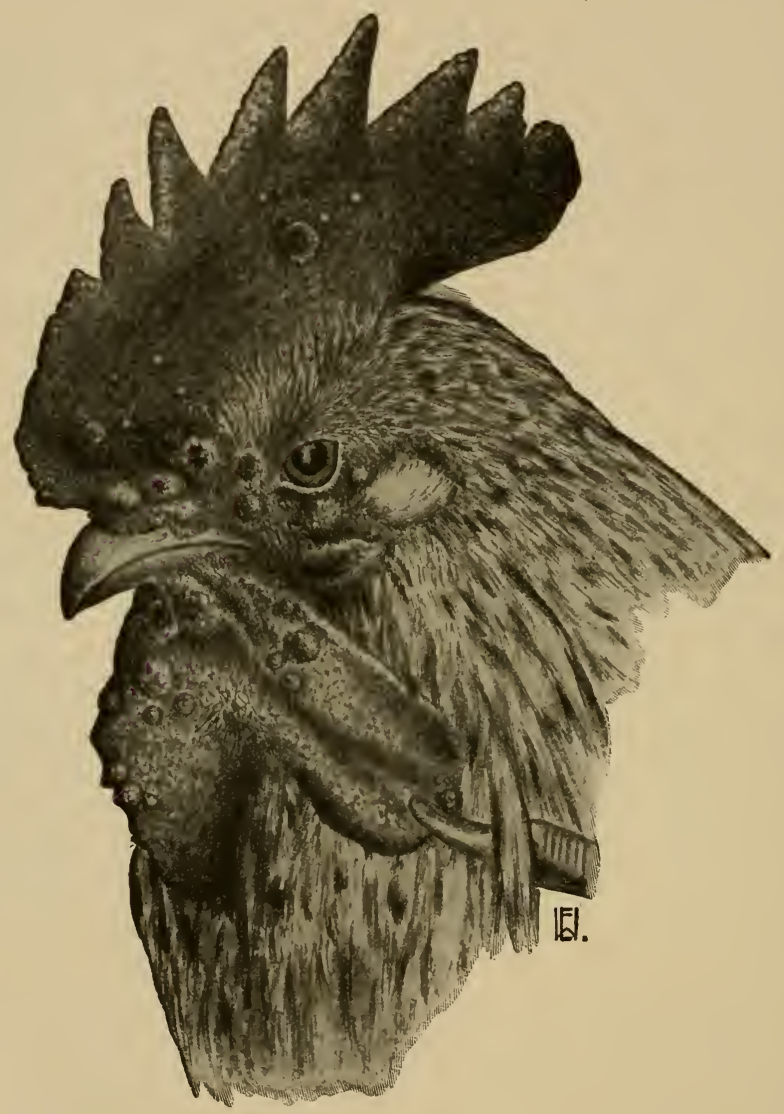

Fig. 65. Chicken pox. Epithelial proliferations on the comb, on the wattles, and in the vicinity of the corners of the month.

and wattles, in the vicinity of the natural openings and especially on the parts of the body which are slightly covered or totally devoid of feathers there arises at first a fine, branlike, gray deposit, which is soon followed by little nodules, at first reddish-gray in color and with a mother-of-pearl luster, 
but which later assume a more grayish-yellow color, and are composed of horny or fatty degenerated epithelial cells. By gradual enlargement these develop to pea-sized, yellow or darkbrown nodes whose surfaces are warty, dry and firm, the interior contents being composed of a yellow, fatty paste (Fig. 65). Sometimes they are so abundant that they touch and even coalesce with the result that the highly reddened and easily bleeding skin forms thick cohesive scabs. The comb and wattles are in consequence much thickened and deformed. The margins of the nasal orifices and still more those of the eyelids also become thickened so that eventually the eyes are completely closed. Should the inflammatory process spread to the conjunctival tissue much caseous and purulent secretion accumulates in the conjunctival sac which gradually effects a pressure atrophy of the eyes, or a corneal inflammation develops, which may become complicated with a purulent panophthalmitis.

Nodules and nodes similar to those on the head develop in some cases on other portions of the body, especially in the vicinity of the cloaca, on the under surface of the wings, on the neck, on the rump, particularly on unfeathered areas. Here they sometimes attain the size of a hazelnut and may even develop into horny or callous growths. Such large growths which have an irregular surface, may, by the exercise of considerable force, be removed or broken off from the skin, which will expose the interior cavity filled with a thick cheesy mass, while vertical sections show wavy layers running more or less parallel with the skin surface.

As long as the disease is confined to the skin the general condition usually remains undisturbed; only when it is widely distributed over the surface of the skin marked emaciation is likely to occur.

In warmer countries a virulent diffuse form of the disease occurs whereby the skin shows no tumor formation but a flat papular thickening and the affected areas become in a few days covered with dry crusts. Febrile symptoms may also occur, not followed by nodules but by reddish-violet pustules, which turn later white and finally yellow, and which eventually dry up and form brownịsh crusts, or death follows after 4 to 5 days of the disease (Reischaner). A malignant course was also observed by Klee on a chicken farm where a diffuse inflammation developed over the body associated with seborrhoea and warty growths. All hens so diseased died within 4 to 5 days.

The second form which has hitherto been considered exclusively as avian diphtheria, begins, also without any marked disturbance of the general condition, with a local affection of one of the mucous membranes of the head, usually the mouth. In some cases there develop on the otherwise smooth surface which is at times only slightly reddened, small spherical or oval yellowish-white spots, which gradually spread and finally form extensive membranes. In other cases the mucous membrane assumes a dark red color, soon followed by a gray deposit 
on its surface, which gradually becomes thicker and forms dense deposits, at first gray or yellowish, later brownish-gray and dark brown on the parts exposed to the air; when dry, the surface becomes rough and fissured. The membranes are usually adherent to the subjacent tissue. When they are removed they leave red, uneven, slightly depressed and bleeding surfaces, or the mucosa is covered with cheesy easily removable masses of exudate, and beneath these the surface is only reddened and finely granulated, but not eroded (usual appearance in pigeons).

The adjoining tissue which is not covered with membranes, is edematous, but here also oftimes exudative masses occur in the later stages; these gradually become conflnent and finally completely cover the affected cavity. Such abundant deposits may occur within the first or second day, and after their removal new ones form; sometimes, however, the ulcerative process extends. deeper leading to extensive destruction. If the animal survives the membranes are in the course of time loosened spontaneously, and the loss of substance is replaced.

Such croupous-diphtheritic membranes develop most commonly on the oral mucosa, especially on the gums near the longitudinal cleft, on the borders and the under surface of tongme, the corners of the month and cheeks, as well as on the pharyngeal wall and in the vicinity of the larynx. The inflammatory process not infrequently extends to the larynx and from here to the trachea and bronchi; on the other hand it may extend from the pharynx to the mucosa of the esophagus and crop. As the inflammation progresses respiration and deglutition become more and more difficult; the bird keeps its neck constantly extended and the beak always open or opens it every few moments snapping for air, whereby the in-rush of air is accompanied by whistling or rattling noises. In the meantime the desire for food, at first unimpaired, grows less and less, and as deglutition is more difficult or impossible, the bird ceases to eat altogether.

The affection of the nasal cavity is at first manifested by a serous nasal discharge, which soon becomes muco-purulent and later dirty-gray in color. It is discharged in considerable quantity on pressure orer the dorsum of the nose; otherwise it dries in the nasal orifices, closing these in part or entirely and occasionally raising the upper nasal wall. In the meantime the birds breathe heavily, show a desire to sneeze and shake their heads thus hurling out masses of pus and single flecks of thickened pus and bits of membranous shreds. From the nasal carity the inflammation extends to the lachrymal canal, which as a result becomes occluded; it also progresses quite frequently on one or both sides to the cella infraorbitalis which now becomes filled with exudate and bulges under the internal angle of the eye in the form of a growth, which on pressure discharges a mncopurulent fluid from the nasal orifice on the affected side. The 
tumor is hot, painful and in the later stages shows on palpation a harder nucleus (Fig. 66).

The constantly increasing swelling forces the orbit upwards and backwards while the corresponding wing of the palate is arched against the oral cavity and later is entirely destroyed, so that the cavity appears to be surrounded only by mucous membrane or by a dense pseudo-membrane. This produces a marked disfigurement of the head while the movements of the head or beak are greatly interfered with by the tumor. On incision the g'rowth discharges a yellowish-white, cheesy or caseated mass, or dry and brittle limps may be removed from its cavity.

When the eyes are involved the first indications are acute catarrhal symptoms of the conjunctiva with edematous, swollen, painful lids beneath which there accumulates much muco-purulent exudate filled with air bubbles,

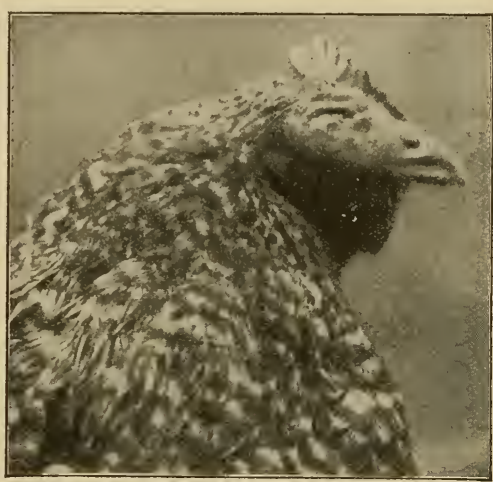

Fig. 66. Swelling of the cella infraorbitalis in chicken pox. sealing the eyes over night. If the eye slit is not opened at regular intervals the exudate dries, forming thick, yellowish-white, caseated masses, which cause a protrusion of the lids and force the eye backwards, forming on the surface of the cornea clubshaped formations whose dry brownish-yellow ends project between the compressed lids. These masses are easily removed from the conjunctival sac, but form again rapidly. In other cases diphtheritic membranes develop on the conjunctiva; the inflammation may also extend to the conjunctival sclera and from here to the cornea, which becomes opaque or ulcerated, and finally a general inflammation of the internal eye results.

Involvement of the intestinal tract occurs only in the later stages of the disease and is manifested by profuse diarrhea with frequent discharge of creamy or fluid feces, sometimes mixed with pus or blood, and causing a rapid weakening of the animal.

The general condition of the patients is at first not disturbed, but later the condition changes markedly. Unable to eat and gasping for air they sit around tired and depressed, with neck drawn in, wings hanging down, feathers ruffled, and do not resist attempts to catch them. The body temperature is raised only in the late stages, while towards the end it falls

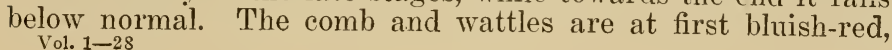


later pale and cold to the touch. 'The discharge emanating from the mouth and nasal orifices soils the breast and emits an umpleasant odor. The animals emaciate considerably, until they finally die from exhaustion due to the associated diarrhea.

Those cases in which the disease affects both the skin and mucous membranes of the head, although not necessarily at the same time, may be designated as mixed form. In the majority of cases the skin of the head is first affected, and the inflammatory process usually spreads from the corners of the month, later, to the oral mucosa and from here to the neighboring cavities; the reverse order is much less commonly observed. No matter in what direction the process progresses the two forms coalesce in such a variety of clinical pictures that in the lethal cases one usually finds the signs of pox and of diphtheria simultaneously, although developed to different degrees. Howerer, cases in which the mucous membranes alone are affected are not rare.

The acute form of the disease as it has been almost ezclusively observed in southern countries (Algeria, Cuba), hegins with marked symptoms of a general infection (marked lassitude, loss of appetite, difficulty of hreathing, etc.), followed on the second or third day by small yellowish-brown psendomembranes on the inflamed oral mucosa, which in a very short period of time develop to extensive deposits. Althongh the process mostly affects the nasal cavities and conjunctivae, deghutition and respiration are markedly impaired, and intestinal inflammation occurs causing rapid exhaustion, and ending fatally within a few days.

Course and prognosis. Cases in which the disease is confined to the skin run a favorable comrse in the majority of instances. The skin disease ceases to progress after a certain time when the nodules have become dry and have been shed, and spontaneous cure follows after 3 to 5 weeks. In this manner even large norles may fall off spontaneously; in other cases there occur fresh nodinles on other parts of the body, and in these instances the disease runs over several monthis.

Less favorable is the course of the diphtheritic disease of the mucous membranes. In a goodly number of cases spontaneous arrest and cure follows in these instances, but as the process is easily carried to the larynx and bronchi and often involves the intestinal mucosa the mortality is much higher on account of exhaustion or dyspnea. The disease usually lasts 2 to 3 weeks, sometimes 1 to 2 months, and with transitory improvement the time may he even more prolonged. According to Friedberger the mortality is $50-70 \%$, and is much higher in young birds, especially in those of highly bred varieties. 
The acute form so common in southern countries usually ends fatally in 4 to 8 days.

Diagnosis. The earlier differential symptoms between avian diphtheria and chicken pox have lost their significance since the discovery of an identical etiology for both diseases.

The contagious eatarrh of the nose (coryza contagiosa) is confined exclusively to the nasal cavity, runs a course without any development of pseudo-membranes and is therefore not associated with as severe symptoms as diphtheria.-. "Pips" which might be mistaken for the beginning of diphtheria, is confined to the tongue, the discoloration occurring by simple drying of the epithelium, acute inflammatory symptoms being absent.-In mycosis aspergillina the deposits on the palate and nose are dryer and sometimes of a greenish color; the fungi are easily demonstrated with the aid of the microscope.

Treatment. So long as the disease is confined to the skin proper treatment will usually effect a cure, and the affection of the mucous membranes may sometimes be arrested in its incipiency and a cure effected. The nodules and nodes on the skin are softened with ointments, oil or soda solution and removed; the mucous membrane deposits are caught with forceps and carefully stripped off or removed with a piece of cotton on the end of a stick or other object. The exposed surface is painted once or twice a day with $1 \%$ silver nitrate solution (if in the mouth apply an after treatment of saline solution), $1 \%$ corrosive sublimate solution (after treatment with soda solution), with lactic acid, lemon juice, $2 \%$ creolin or lysol solution, tincture of iodine (with Tinct. gallarum $\overline{\mathrm{aa}}$ ), or the skin is painted with concentrated glycerin.

The secretion which accumulates in the eyes is washed ont with warm water and the eye is subsequently treated with $2 \%$ boric acid, tannin or creolin. Tumors below the eyes are to be opened early and after thorough evacuation of the cavities these should be thoroughly cleansed.

For the difficult respiration inhalations of turpentine and tar vapors may be tried while against intestinal catarrh tannin or sulphate of iron ( $1 \%$ solution in drinking water), red wine (spoonful doses) or other astringents (in geese, glycerin) may be employed, but in most cases treatment is here of little avail.

Local treatment must be continued until the cleansed skin or mucous membrane shows no new nodules or deposits. In those instances where proper treatment is not available or impractical, or where the birds are of little value, it will prove more practicable to suppress the disease by killing the diseased animals or even destroying the entire number of fowls on the farm, and instituting thorough disinfection of the coops.

Prevention. The general prophylactic measures, such as isolation of the diseased and removal of the dead birds, cleans- 
ing and disinfection of the coops and runs, careful examination and quarantine of the newly bought birds, etc., are identical with those employed in fowl cholera (see p. 95). As the disease has a latent development it is proper, in a threatened epizootic to examine the mouths of the healthy birds from time to time, and if the slightest changes in the skin or mucosa are observed, the affected bird is to be at once isolated and treated.

Preventive inoculation. Manteufel succeeded in immunizing chickens by injecting into the circulation or under the skin a lymph that had been prepared from the serapings of epithelioma or mucous membranes mixed with saline solution. Chickens treated in this manner were immune to subsequent infection for $11 / 2$ to 2 years, even though no loeal signs of reaction followed injection. Chickens that had been inoculated with mixtures of virus and bile resisted subsequent inoculations into the comb. Serum of highly immunized chickens on the other hand possessed no preventive or curative value.

The earlier attempts at immunization, directed as they were exclusively against bacillary infection, offer very little in view of our present knowledge, and ean be considered effective only against the secondary infections; however brief mention of them will be made here.

Loir \& Ducloux immunized healthy ehickens by injecting subcutaneously, first 1.0 ec. of fresh culture of their diphtheria bacillus which harl been kept at a temperature of $55^{\circ} \mathrm{C}$. for half an hour; ten days later they injected 1.0 ec. of a two months old culture. One of twenty ehickens so treated, and four out of five not treated, succumber to a later virulent infection.

Guérin found this measure noneffective in pigeons, but obtained more farorable results by injecting intraperitoneally 0.5 ee. of a culture that had been heated to $55^{\circ} \mathrm{C}$. for an hour. He repeated this in 12 days by employing a culture that hal been subjected to $50^{\circ} \mathrm{C}$. only, for the same length of time. After an interval of 12 to 15 days the pigeons sustained a subcutaneous injection of $0.5 \mathrm{ce}$. of virulent eulture without any bail effects. The same procedure was later suceessfully employed on young ehickens. From January 1st to Angust 31, 1905, the Pasteur Institute in Lille sent out 24,602 vaccine doses to 74 parties. Of these, 39 parties whose observations encompassed 10,426 animals, declared themselves as satisfied with the results, while eight rendered an unfavorable report (Eloire).

The Behring-Roux antitoxic serum employed in human therapy against diphtheria of children and recommended by Lang and by Eloire also against bird diphtheria has been proved ineffective by Harrison as well as by Gratia \& Liénaux.

Literature. Bollinger, V. A., 1873, LVIII, 39.-Löfflér, Mitt. d. G.-A., 1884, II, 421.-Loir \& Ducloux, A. P., 1894, VIII, 599.-Marx \& Sticker, D. m. W., 1902, 893 ; 1903, 79.-Streit, Z. f. Hyg., 1904, XLVI, 407.-Müller, Cbl. f. Bakt., 1906, XLI, 621 (Lit.).-Burnet, A.-P., 1906, XX, 742 (Lit.).-Bordet, Ann. rét., 1907, 494.-Carnwath, Arb. d. G.-A., 1907, XXVII, 388.-Bordet \& Fally, Ann. vét., 1907, 494.Hausser, Cbl. f. Bakt., 1909, XLVIII, 535 (Lit.). Schmidt, Ibid., 1909, LII, $200 .-$ Thlenhuth \& Manteufel, Arb. d. (r.-A., 1910, XXXIII, 288 (Lit.).-Manteufel, Ibid., S., 305 .

Diphtheritic inflammation of the eyes in ducks. Kampmann, Hirschbruch \& Lange in Posen observed on a chicken farm during the spring and fall of 1902 a peculiar disease in 100 ont of 240 ducks, of which 25 died, while 15 were killed and 60 recovered, while other fowls remained healthy. The disease began with severe itching in the eyes followed in a few days by a conjunctival inflammation with the production of a yellow, thick exudate and diphtheritie pseudo-membranes, to which was added an ulcerative inflammation of the cornea and a 
erusty eczema of the skin due to contamination with the conjunctival secretion. The ducks became much emaciated and many died within a few weeks, yet autopsy failed to reveal any marked changes in the internal organs. Bacilli of the type of pseudo-diphtheria hacilli were found in the conjunctival exudate. (Centralbl. f. Bakt., 1903, 214.)

Diphtheria in man. This so justly feared disease of children, produced by the bacillus diphtheriae hominis discovered by Klebs and more carefully studied by Loeffler, and belonging to the group of corynebacteria, consists of an acute diphtheritic inflammation of the mucous membrane of the pharynx and upper air passages, beginning usually with fever and associated with severe constitutional symptoms. The Klebs-Loeffler diphtheria bacillus displays its pathogenic action through the medium of toxins which on the one hand produce a local inflammatory and necrotic process as well as general constitutional symptoms, and is also responsible for later nerve paralysis which sometimes follow diphtheria. Horses treated with the toxic filtrates of the cultures furnish an effective antitoxic serum.

The relationship between diphtheria of man and that of animals and particularly that of birds has not been fully explained.

There remains no doubt whatever that true diphtheria of man and the diphtheria of animals are two separate diseases, occurring and spreading entirely independently of one another. The bacillus of human diphtheria, however, possesses a pathogenic action for animals, among them for birds, and as some species, such as young guinea pigs, eats and dogs, after artificial inoculation, develop even croupousdiphtheritic processes of the mucous membranes, it cannot be denied that this bacillus under natural conditions may produce similar disease in animals and thus cause the latter to spread the contagion. This view is supported by Ferré, and by Wheeler and Brandt, who from cases of avian diphtheria and from the mouth of diphtheritic pharyngeal disease of sick dogs respectively, grew cultures of bacilli whose pathogenic action was similar to that of the Klebs-Loeffler bacillus. Further support comes from Cobbett, who found a bacillus in the nasal secretion of a horse suffering from laryngitis, which corresponded not only morphologically, but in its pathogenic action with the bacillus of human diphtheria and against which the antitoxic diphtheria serum proved effective. Gallez, Jr., Gratia \& Liénaux also found a bacillus similar to the Klebs-Loeffler bacillus but less virulent, in the contagious nasal catarrh of birds.

On the other hand the possibility cannot be excluded that oceasionally the diphtheria of animals may produce diphtheritic processes in man. This may be presupposed particularly for those cases where in the products of the diphtheritic mucous membrane inflammation in man it is impossible to demonstrate the Klebs-Loeffler bacillus, and which in all probability results from some other etiological factor. Close relationship between avian diphtheria and that of man has repeatedly been affirmed, based on experiments or observations which appeared to indicate the likelihood of transmission of the disease from man to birds. Loir \& Ducloux have succeeded in cultivating the same hacillus from the pseudo-membranes of a child suffering from diphtheria which they considered to be the virus of avian diphtheria, basing their opinion on the results of their experiments. Finally it is worthy of note that the so called psendo-diphtheria bacillus which several authors, among them Roux \& Yersin, Fränkel, et al., believe to be an avirulent 
variety of the true diphtheria bacillus, has repeatedly been found in birds (Gratia \& Liénaux).

At present the view that the Klebs-Loeffler bacillus may oceasionally produce membranous pharyngeal inflammation in animals is justified; such cases, however, are to be differentiated from true diphtheria of man and from the true diphtheria of animals, as cases of pseudo-diphtheria.

Literature. Klebs, Verh. ג. Kongr. f. inn. Med., Wiesbarlen, 1883.-Löfller, Mitt. d. G.-A., 1854, II.-Galli-Valerio, Cbl. f. Bakt., 1897, XXII, 500.-Gallez, Ann., 1895, 309.-Gratia \& Liéneaux, Ánn., 1898, 401.-Beck, Hb. d. p. M., 1903, II, 754 (Lit.). 
Group IV.

\section{INFEUTIOUS DISEASES WITH SPECIAL INVOLVEMENT OF THE NERVOUS SYSTEM.}

\section{Tetanus. Lockjaw.}

\section{(Tétanos [French]; Starrkrampf [German]; Tetano [Italian].)}

Tetanus is an acute infectious disease resulting from an intoxication of the nervous system, characterized by persisting spasmodic contractions of the entire body musculature or of single groups of muscles, without impairment of consciousness. The disease is caused by Nicolaier's bacillus tetani and its poisonous products of metabolism.

History. The opinions concerning the nature of this disease were until quite recently based on purely theoretical grounds. The association between muscle spasm and the increased irritability of the nervous system was recognized during the 18 th century, but the real cause of the nervous affection remained a mystery. The most generally prevailing view was that tetanus, which was so often associated with injuries, arose reflexly as the result of severe traction or contusion of peripheral nerves and that the irritation thus originated was transmitted to the spinal cord (tetanus traumaticus), but the efforts to produce the disease experimentally in this manner all failed (Arloing \& Trippier [1869]). Since in other cases no preceding traumatism could be demonstrated ( T. idiopathicus) the cause was sought in other circumstances, particularly in colds ( $T$. rheumaticus) and even in psychic excitement (T. psychicus), although several authors (Travers, Rose, Spencer Wells) were already inclined to consider the disease an intoxication.

Etiological research was directed into a more correct channel when Carle \& Rattone (1884) succeeded in producing the disease artificially by inoculating the contents of an acne pustule of a person who had died from tetanus into rabbits. Almost at the same time Nicolaier (1884) obtained positive results by inoculation of garden earth into rabbits, guinea pigs, and mice, and at the same time recognized the cause of the disease in the form of a spore-bearing bacillus. Later observations (Rosenbach, Baeumer, Bonome) have confirmed the infectious nature of the disease for man, while Nocard (1887) established the identical origin of tetanus of horses.

Kitasato (1889) was the first to grow the anaerobic bacillus in pure culture; Brieger (1887) demonstrated the presence of toxic sub- 
stances in mixed eultures, and Faber, (1890) in pure cultures. Finally Behring \& Kitasato (1892) worked out an effective method of immunization against the disease, while Vaillard and Vincent, Rouget and Roux, and Tiberti and Tarozzi furnished important contributions on the pathogenicity of the disease.

Occurrence. Tetanus, a rare disease in the more northern countries, occurs much more frequently in tropical regions and sometimes attains an enzootic character among both man and beast. In the temperate zone, as in Europe, the disease is considered as sporadic and here cases occur in some regions in rapid succession during the spring and fall. Considerable loss occurs usually in horses, sometimes in sheep, while other species of animals as a rule are much less commonly affected. More recently, since greater attention has been given to the cleanliness of the wounds, a decrease in the number of cases has been observed.

In the Prussian army during the years 1899 to 1908 , in an average of 87,000 horses, 42 to 64 (average of 52 ) were attacked annually with tetanus, while in the French army during 1899 and 1900, 98 and 94 cases respectively, were reported.-In Hungary the disease appears to be more common in the southern regions, and in certain communities it causes considerable losses, especially after eastrations.-The disease appears to occur more frequently in the centers and the outskirts of large eities, and during certain years or seasons.

It is much more common and widely distributed in warmer countries, and occurs frequently in horses, especially after castration, in San Domingo, in the Antilles, Algeria and Egypt.

Etiology. The bacillus tetani Nicolaier, is a straight, slender rod (Fig. 67) possessing feeble motility, and propagates

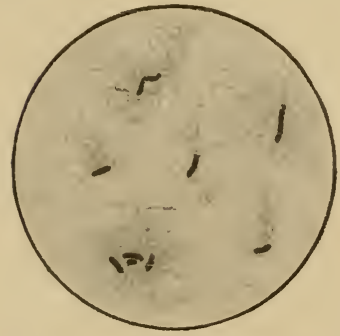

Fig. 67. Bucillus tetani with flagella. Two-day old agar culture.

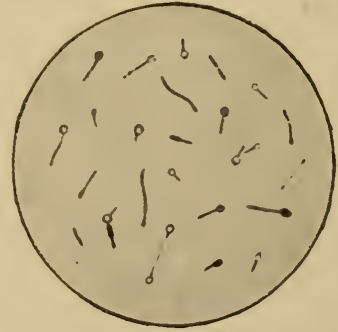

Fig. 68. Bacillus tctani with spores in various stages of derelopment. Fourday-old agar culture. Carbol-fuchsin staining.

by fission and also by spore formation. The spore is spherical, with a diameter about twice as long as the thickness of the bacillus, and is situated at one pole of the rod, thus giving 
the spore-bearing bacillus a pin-like or drum-stick-like appearance (Fig. 68). In cultures it may form longer slightly curved threads.

Staining. The tetanus bacilli stain readily and uniformly with the usual aqueous anilin dye stains as well as by Gram's method.

Cultivation. The tetanus bacilli are anaerobic, but develop also in the presence of oxygen when associated with aerobic bacteria which energetically consume oxygen. Under anaerobic conditions they grow readily, especially on media containing grape-sugar, at body temperature, less readily at room temperature. On gelatin and agar the colonies form grayish-white spots composed of clumps of fine threads, while a white streak develops at the point of inoculation, with radiations in all directions (pine tree shapes Fig. 69) Gelatin is liquefied slowly, bouillon is clouded uniformly, milk is not coagulated; on potatoes there is sometimes formed a colorless, transparent deposit. The interior of an extirpated eye is also well adapted for its cultivation (Mackie). Gas $\left(\mathrm{CH}_{4}, \mathrm{CO}_{2}\right)$ is produced on sugarcontaining media and the eultures disseminate a peculiar burnt odor. Spore formation begins in the bacilli, under favorable conditions, after 24 to 30 hours.

Tenacity. Since most of them are sporebearing, the bacilli are almost as resistant as the spores. Direct sunlight destroys them in 12 days, diffuse light in 1 to 2 months (Vaillard \& Vincent). In decomposing material they remain alive for a long time and may increase for a time accorling to experiments by Bombicci. When kept in a dark place they are still virulent after 11 years (Henrijean), and in pus and animal tissues they retain their vitality for months ( $\mathrm{Kitt}$ ). Cultures retain their vitality for two years (Kitasato). The spores are killed by live steam at $115^{\circ} \mathrm{C}$. in 5 minutes, by $5 \%$ carbolic acid in 15 minutes, by $1: 1000$ corrosive sublimate and $1 / 2 \%$ hydrochloric acid solution in half an hour (Kitasato), by a $1 \%$ nitrate of silver solution in 1 minute, by $5 \%$ creolin solution in 5 hours (Tizzoni \& Cattani). The addition of iodine trichloride in the proportion of $0.125-0.25 \%$ to culture decreases the virulence proportionally to the quantity of the drug (Behring \& Kitasato). The digestive juices have a very slight destructive action (Sormani).

Pathogenicity. Fresh cultures are so slightly virulent that guinea pigs which are very susceptible to tetanus can stand a subcutaneous injection of 0.3 or 0.5 ce. without harm, but cultures ten days old or older will in very minute quantities produce tetanus in susceptible animals. Filtrates of such cultures free from bacilli and spores act similarly and only after a certain period of incubation, showing that living cultures exert their pathogenic properties by means of toxic products (in aerobic mixed cultures there develop similarly acting toxins as in pure anaerobic cultures). Spores freed of their toxins by prolonged washing or heating for 3 hours at $80^{\circ} \mathrm{C}$. prove ineffective upon subcutaneous injection even when given in relatively large quantities, while these same spores when injected subcutaneously or brought under the skin in small paper sacs 
will produce the disease when fine sand, coal dust, foreign bacteria (Bac. prodigiosus), or negatively chemotactic fluids (as lactic acid) are added.

In animals which are infected subcutaneously or intramuscularly tetanus always begins with local symptoms. After such an infection into the thigh the muscle spasm appears first in the posterior extremity of the same, then on the opposite side and later spreads to the anterior extremities, until it becomes general. After intravenous injection of toxins there is generalized tetanus from the beginning, while after intracerebral inoculation there follow excitement, intermittent spasms and disturbances of motility (tetanus cerebralis) (Roux \& Borrel); after injection into the spinal cord severe attacks of pain occur with subsequent tetanic symptoms (tetanus dolorosus) (Meyer \& Ransom, Tiberti).

The horse possesses the greatest susceptibility for the virulent living cultures as well as for the toxins, and is followed by the guinea pig, goat, sheep, mouse, rabbit; cattle are very slightly susceptible, dogs and cats still less so, while chickens are infected with difficulty with very large doses. Man is very susceptible to both infection and toxins.

The toxic products of the tetanus hacillus were first studied by Brieger who grew mixed eultures on beef broth and ohtained four sulsstances (tetanin, hydrotetanin, tetanotoxin and spasmotoxin) which caused muscular spasms after subeutaneous injection into experiment animals. As these substances acted at once and in comparatively large doses they could not be considered as true toxins. Later, after pure cultures of tetanus bacilli were grown Kitasato, Behring \& Knorr, Tizzoni \& Cattani, Roux \& Vaillard studied the toxic culture filtrates carefully without, thus far, succeeding in establishing the nature of the toxic substances. It is probable that they represent specific secretions of the bacilli and do not develop as the result of decomposition of albumin-containing nutritive media as they are formed also on albuminfree asparagin (Buchner). The toxin is soluble in water, insoluble in alcohol, ehloroform and ether; otherwise in certain characteristics it resembles very much diastases (is not very stable, is precipitated by alcohol, dializes, ete.).

The tetanus toxin is an extremely powerful poison. $1.0 \mathrm{cc}$. of the filtrate of a highly virulent culture kills a horse $500 \mathrm{~kg}$. in weight; 0.001 ce. kills a guinea pig of 300 gram, while other species of animals require proportionally larger doses of toxin. If for 1 gram body weight of horse the lethal dose is 1 , guinea pigs require 2 , goats 4 , mice 13 , rahbits 2,000 , chickens 200,000 doses per gram of body weight (Knorr).

The toxic value of toxins is usually designated by the smallest amount of virus necessary to kill an animal of a certain weight. If therefore $0.0000001 \mathrm{ce}$. toxin kills a 15 gram monse, 1.0 cc. will kill 10 millions of mice of 15 gram body weight each, eqquivalent to 150 millions grams weight of mice (Behring).

The action of the toxin follows only upon subeutaneous, intramuscular, intraperitoneal, intravenous or intracerebral injection, while large doses of toxin when given by mouth or intestine are entirely 
without effect as the toxin is not absorbed by the mucous membrane (Ransom) nor is it decomposed by bile and pancreatic juice (Carrière, Vincent).

The powder obtained from filtrates by precipitation with ammonium sulphate is also toxic, and is so in correspondingly smaller doses (horses, for instance, are killed by 0.006 gram).

Tenacity of the toxin. The toxicity of the filtrates suffers hardly any impairment by dilution with blood, blood serum or salt solutions, but is diminished upon addition of mineral acids and alkalis. The toxin is precipitated by small amounts of alcohol, and decomposed by large quantities of alcohol. Direct sunlight destroys its effect in 15 to 18 hours, a temperature of $80^{\circ} \mathrm{C}$. in 3 hours, mineral acids and alkalis in a concentration of $1 \%$ in one hour (Kitasato, Fermi \& Pernossi). In powdered form it is much more resistant; at room temperature in a dark place it remains effective for years, while it is gradually attenuated by diffused sunlight; it can stand constant heating to $120^{\circ} \mathrm{C}$. and even $135^{\circ} \mathrm{C}$. for 15 to 20 minutes, and is destroyed only by 15 minutes' heating to $159^{\circ} \mathrm{C}$. (Morax \& Marie).

Natural infection. This occurs as a rule through contamination of wounds on the skin and mucons membranes with bacilli and spores contained in the contaminating medium. The most common carrier of the contagion is earth, less frequently pus or tissue particles emanating from sick animals or cadavers. The bacilli and spores are abundantly distributed in the superficial humus layers, especially in such places as contain much manure, especially horse manure; they may also be found in large numbers in the slime of swampy areas. Nicolaier produced the disease in experiment animals by inoculation with garden earth and since then tetanus bacilli have been demonstrated in many regions in garden and farm earth, in street lust, in manure, in various food substances, especially in hay. The experiments have also shown that the bacilli occur in the excrements of healthy horses (Sanchez \& Veillon, Nocard), also in those of cattle, hogs and smaller experiment animals (Sormani Strauss, Molinari), for by the inoculation of such material it is frequently possible to produce the disease artificially. (Joseph found tetanus bacilli in the intestinal contents of eight cattle examined for this purpose, and believes that they vegetate constantly in older animals.)

On account of these results which, by the way, also furnish proof that the tetanus bacilli may be present in body cavities and canals, with external openings, without endangering the health of the animal, Verneuil believed that the contagion of tetanus developed originally in the intestinal tract of horses and that tetanus in man always occurred as the result of coming in contact with horses and with straw or earth that had been contaminated with their excrements. It is more likely, however, that the spores gain entrance into the digestive apparatus of horses through dusty food, remain here a time and pass on unchanged into the open (in feces of man, as well as in all carnivora, tetanus spores are very rarely demonstrated).

Avenues of infection are offered by the various injuries of the skin or mucous membranes, mostly those, however, which are associated with destruction of tissue or accumulation of pathological secretions and which readily come in contact with the earth on account of their location, or those in which foreign bodies have penetrated deeply into the tissues. Especially 
dangerous in this regard, are injuries from nails, nail punctures of the hoof or foot, about the coronary band, and particularly injuries about the lower portions of extremities; fractures of bones, wounds of castration, and harness galls; in horses tail docking; furthermore injuries of the mucosa of the mouth (through carious or sharp teeth) or genital passages (from aid in difficult labors), in the newborn the contamination of the navel wound, in foals the operation of umbilical hernia, injuries of the eyes, etc. In any case the most varied injuries and accidents may give rise to the disease provided that dead tissue, extravasated blood or wound secretions facilitate the multiplication of the contagion; aside from this the penetration of the anaerobic bacillus into the deeper portions of the tissue or into hidden cavities or pockets of wounds favors the development of the disease.

The infectious material gains entrance into the wound with earth, necrotic tissue or wound secretion containing bacilli, or the infection is facilitated by wood shavings, bone splinters, the harness of a horse suffering with tetanus, or by hair ropes, manure applied to a wound, further by implements, surgical instruments, the hands of operators or those assisting them in operations. In this way, for instance, tetanus is conveyed after castration through the medium of the operator's soiled hands or by clamps which had been used before, while in other cases the virus reaches the castration wound from the horse lying down and contaminating it. The cases which point to the first mode of infection are those that occur among animals which have been castrated by the same operator within a sliort period of time, even though in different places, when cases of disease occur in rapid succession. Those pointing to the second mode of infection are the ones where year in and year out animals from the same farm become diseased. Since the antiseptic treatment of wounds, tetanus occurs much less frequently after such operations which were formerly considered dangerous (castration with ligation of the spermatic cord, especially in rams and calves).

It is therefore possible to designate the site of infection in most of the cases, but there are cases where even the most careful examination fails to reveal an injury of the body surface or of the mucous membranes. Such observations, which were formerly considered rheumatic tetanus, make it probable that the disease may occur exceptionally without any preceding injury. This might be assumed in parturition tetanus in the sense that the infectious virus is propagated in the exmdate which collects in the womb and the toxins thus produced pass from here to the lymphatic circulation. In this way could those cases be explained where the occurrence of tetanus may be associated with the retention and decomposition of the placenta. In many cases of horse tetanus it is impossible to exclude the possibility that the contagion multiplies in the follicles of the tonsils or between the folds of the intestinal mucosa to such 
a degree that colonies are formed which produce sufficient toxins to cause intoxication (Teyssandier observed several cases of tetanus following colic). Infection occurs more readily by way of inflamed mucous membranes (Thalmann) and this might explain the occurrence of the disease in dogs after eating spoiled meat (Alessandrini). The tetanus bacilli after passing through the intestinal mucosa may also find a favorable medium for development in exudates or necrotic areas of an internal organ, for example in abscess of the liver (Keleti). Recent experiments have also shown that tetanus spores may probably pass in healthy animals from the intestinal canal to the lymphatic circulation and remain latent in the internal organs, until a time when circumstances favoring their development arise.

Susceptibility. Among the domestic animals solipeds are most commonly affected by tetanus; the disease occurs much less frequently in cattle, sheep and goats, while hogs and dogs are only occasionally attacked (in dogs the disease occurs usually after extensive and contaminated destruction of tissue, more rarely after docking the tail). Cows are attacked in the majority of instances after labor, only exceptionally following a traumatic gastritis (Friedrich), calves after castration, while in sheep castration, pox inoculation, ear marking, etc., are the leading factors in the occurrence of infection. Among goats the disease was observed almost exclusively in rams which were castrated at an advanced age.

Young animals are more susceptible than older ones, and the disease may become enzootic among lambs particularly, sometimes also among foals, as the result of infection of the umbilieus (tetanus neonatorum).

Pathogenesis. Healthy animal tissue is not a good medium for the propagation of the tetanus bacteria. Toxin-free spores, when not inoculated subcutaneously and in excessive numbers (in guinea pigs 2,500 spores according to Vaillard, Vincent \& Ronget), are ineffective, as they are engulfed by phagocytes and immobilized. On the other hand toxic spores produce the disease even in very small quantity, as the phagocytes are kept away from them by the negative chemotaxis of the toxins, and these germinate under the protection of the toxins and enable the bacilli to multiply. In the same manner spores are protected by the simultaneous injection of negatively chemotactic lactic acid or trimethylamin (according to Hektoen this is not the result of chemotactic but antiopsonic actions). This explains the effectiveness of the toxin-containing older cultures as compared with the harmless, toxin-free, young cultures.

When the spores enter necrotic tissue or extravasated blood they multiply rapidly and produce toxins. By injection of spores into such dead tissues (badly contused muscles, blood extravasations) the disease may positively be produced, and 
experience also proves that tetamus, under natural conditions, appears by preference associated with such wounds as are accompanied by destruction of tissues, hemorrhage or accumulation of a purulent secretion.

Spores occurring free in nature do not contain toxins, although an infection occurs with spore-containing earth in healthy tissue; this finds its explanation in the fact that, together with the tetanus spores, other bacteria gain entrance into the tissues, finding here a favorable medium for their development, and consequently causing disease of tissue which then furnishes a suitable medium for the growth of the tetanus organism. Spore-containing earth loses its power of infection when heated to $85^{\circ} \mathrm{C}$.; such a temperature is not sufficient to kill the spores, but it does destroy other bacteria present in the earth. Earth that has been freed of its infective character by heating may again produce tetanus if simultaneously with its subcutaneous injection there are introduced cultures of bacteria grown from the earth before heating it. That is why tetanus develops sometimes after comparatively slight injuries, for instance after the introduction of a wooden splinter or following a superficial suppurating wound covered by a scab.

Influences which reduce the resistance of the tissues and perhaps paralyze the activity of phagocytes favor infection, and in this sense colds or overheating (liard work in summer heat) may be of importance.

The apparently spontaneous cases which do not appear immediately associated with any injuries are at least partly explained by the latency of the tetanus spores in the animal hody, in that spores which have penetrated into the body some time previously are kept alive enclosed in cells, and later germinate under the influence of a favoring factor (disease, colds, overheating, muscular exertion) and cause a multiplication of the bacilli.

Taillard \& Rouget found viable and virulent spores $3 \frac{1}{2}$ months after injection of spores into the bodies of guinea pigs, and Tarozzi also demonstrated that in animals infected subcutaneously with spore-bearing cultures of tetanus the spores frequently gain admission to the blood, migrating to organs far removed from the original site of infection, where they may remain latent for $3 \frac{1}{2}$ months or longer. When favorable conditions arise, particularly necrosis of sporecontaining tissue, the latent spores are revived to regetation and may cause tetanus. Soprana, Canfora, also Reinhardt \& Assim have arrived at similar conclusions in their studies on the latency of tetanus spores. As the intestinal evacuations of herbivora frequently contain spores there is always the possibility that the may occasionally gain entrance to organic tissue, where they multiply and give impetus to the formation of antitoxins. This would explain Roemer's findings in eattle over two years of age in which the blood serum frequently showed antitoxin in demonstrable quantities (1/2400 to $1 / 50$ units per ec.).

The influence of an increase of the body temperature on the development of tetanus was demonstrated by Vincent who raised the body temperature of guinea pigs in the thermostat or incubator to $40.5^{\circ} \mathrm{C}$., subsequently injected spores that had been heated to $85^{\circ} \mathrm{C}$. and caused fatal tetanus in 2 to 3 days. The bacilli were found disseminated through the entire body.

Germination and multiplication of bacilli usually occur exclusively at the site of infection and therefore bacilli are rarely 
found in the blood and more remote organs (spleen, brain) and then only in minute quantities (Hoblbeck, Dor, Creite, Nicolaier, Haegler, etc., probably they are enclosed in leucocytes and carried there). This negative finding harmonizes with the fact that the bacteria exert their pathogenic function indirectly, through the toxic products absorbed from the lymphatic circulation. On the other hand, the fact that the symptoms of poisoning, different from true poisons, such as strychnine, occur after a comparatively long period of incubation (in guinea pigs at least 12 , in mice 6 hours) points to the fact that the toxin is carried very slowly to the central nervous system.

Tetanus toxin exerts a pathogenic effect on the motor nerve cells, primarily those of the spinal cord and medulla oblongata, giving rise to an increased irritability, indirectly however causing contractions of the corresponding muscle groups. If the source of the toxins is on the surface of the body some of them are at once bound by the axis cylinder (nerve endings) and conducted along the nerves to the central motor cells, whose irritation causes local tetanic muscle spasms; the other portion of the toxins enters the blood, is later, however, also bound by constantly intercepting nerve fibers and conducted to the motor nerve cells, so that muscle spasms occur in constantly widening areas. If on the other hand, the toxins immediately enter the blood, as would occur in intravenous or intraperitoneal injection, or after an intestinal infection (so called T. splanchnicus), they are almost simultaneously bound by all motor cells and in such cases the muscle spasms occur later, but in almost all the muscles simultaneously (Morax \& Marie, Behring). The fact that trismus, in both man and horse, is one of the first symptoms of the clinical picture of tetanus would indicate that the tetanus toxin is more readily bound by the trigeminus neuron than by the other motor neurons. The increased reflex irritation also points to an increased irritation of the sensitive neurons.

Bruschettini was the first to discover that the tetanus toxin was conducted from the point of infection to the nerve centers mainly through the nerve channels, and his discovery was confirmed by Meyer \& Ransom, Stintzing, v. Behring, Tiberti, etc. According to Meyer \& Ransom the toxin is taken up by the nerve endings containing no axis cylinders, according to Pochhammer by the medullary sheaths; Zupnik, however, thinks that the muscles are directly attacked by the toxin. In the central nervous system the toxin combines electively mostly with the motor nerve cells of the spinal cord and medulla oblongata.

The slow progress of the toxin along the nerve channels explains the relatively long incubation, as the toxin has to be carried to the central nervous system along these paths; when the toxin is brought in direct contact with the nerve centers the incubation is considerably shortened.

The peculiar action of the tetanus toxin on the nervous system is probably explained by Ehrlich's side chain theory. According to 
this each toxin molecule is composed of one non-toxic (haptophore) and one toxic (toxophore) atom group. The protoplasm of the motor nerve cells is composed of a vital nucleus and numerous side chains (receptors), of which many possess a special affinity for the haptophore atom groups of the toxin molecule. When such molecules of the tetanus toxin reach the nerve cells they are anchored by the aid of haptophore groups to the corresponding receptors of the cell protoplasm, whereupon the toxophore group attack the vital nucleus of the cell. The opposed affinity between tetanus toxin and nerve substance is demonstrated by the experimental observation, where it is shown that a mixture of toxin and brain substance is non-toxic for guinea pigs (Wassermann \& Takaki).

Ehrlich also demonstrated a hemolytic substance (tetanolysin) in culture filtrates, which was neutralized by antitetanic serum.

Recovery from one infection does not immunize the animal against a subsequent one. Repeated occurrence of the disease may result from renewed infection, or may follow when tetanus spores were retained somewhere, as for instance in a scar, in an abscess or blood coagulum and germinate at a considerably later date. (Repeated infection in horses was observed by Johne after 4, Hill after 5, Hell after 6, Mongnean after 10 months.)

Anatomical changes. Autopsy reveals no changes characteristic of the disease. In the central nervous system, especially in the spinal cord and its coverings, hyperemia is often seen and punctate hemorrhages are found in the gray matter of the brain, but such findings are not uncommon in other diseases. This is also true of the microscopic examination of the motor nerve cells which show slight degenerative vacuoles, disappearance of Nissl's bodies, etc. Marinesco found marked changes also in the nerve fibrils; in many cases, however, the appearance was entirely normal and the positive findings may be associated with the infections accompanving wounds. Small hemorrhages and serous infiltration are frequently found in the peripheral nerves, especially in those leading from the infected area. Fatty degeneration of the heart muscle is an almost constant occurrence. The intramuscular connective tissue of the trunk and the extremities is edematously infiltrated and contains small hemorrhages, with muscle bundles torn and colored rellow or grayish due to parenchymatous or hyaline degeneration. (Eichhorn found the muscles in a cow to look like fish meat.)

In animals in which rigor mortis sets in early the only lesions are those pointing to death by suffocation (dark color of the poorly coagulated blood, slight hemorrinages on the serous and mucous membranes, hyperemia and acute edema of the lungs) and in ardlition occasional subsequent changes (pneumonia, bone fractures, signs of general septic infection).

The sife of infection should if possible be disclosed in every case at autopsy. and this requires at times a very careful examination of all the parts of the body 
and the different organs; when external injuries camot be found great care should be exercised in the insuection of the canals and cavities lined with mucous membranes, especially the nasal eavity, the pharynx, the gums, and the uterus. In a ease reported by Merkel the infection was occasioned by a wheat kernel that had entered the air passages, while Hengst traced the infection in a cow back to an injury of the paunch by a wire.

The infectious nature of suspected material may be ascertained with certainty by subcutaneous or intramuscular inoculation of pus or necrotic pieces of tissue into guinea pigs or mice. This is not so easy by microscopic examination; however the spore-bearing tetanus bacilli are found in the inflammatory products and the diseased tissues associated with other organisms. (See Fig. 73 on page 457.)

Symptoms. The period of incubation after subcutaneous, intramuscular or intraperitoneal injection of a medium-sized amount of virus into smaller animals is about 2 to 5 days and in larger animals longer; its duration depends on the strength and quantity of the toxin, less so on the susceptibility of the animal. Under natural conditions the first symptoms have been noted as early as 24 hours after infection (mostly in very young animals); as a rule the incubation lasts longer, and in the majority of cases, especially in horses, it is 1 to 2 weeks.

From Carliot's compilation of 38 horses suffering from tetanus the disease occurred at the end of the first day in one case, on second to fifth day in six cases, fifth to tenth day in 13 eases, tenth to twentieth day in 15 cases, twentieth to thirtieth day in three cases. According to Hoffmann the period of incubation in parturition tetanus in cows is 2 to 14 days, in rams and boars after castration 8 to 14 days, in lambs after pox vaccination 13 to 18 days.-Dieudonné reports on 58 cases of tetanus following docking of the tail, where the incubation lasted $S$ to 42 days. - In a case of Roeder's a calf was attackel by the disease immediately after birth and died on the fifth day, while De Bruin noted in a colt that had stepped on a rusty nail on the day of birth the first symptoms of tetanus two days after the accident. Rietseh performed artificial inoculation and observed a period of incubation of 15 days in mules; Kitt observed it in sheep to be 6 to 7 days, and in dogs 2 days.

Excluding the rapidly developing cases where the disease is completely developed within a few hours, the beginning of the disease causes no change whatever sometimes for 1 to 2 days, with the exception of a disinclination of the animals to move and a sluggish action when they are prodded. The legs bend less than normally and the extremities are raised stiffly, while feeding is prolonged, the animals appearing to chew much more carefully and apparently having some difficulty in swallowing food; at this stage the peculiar projection of the head and the rigid station and immobility of the ears may be noted. These symptoms indicate beginning spasm of individual muscle groups, which, in the majority of cases, appears first in the head or neck, less frequently in the hindquarter's and from here spreads forwards or backwards. In some cases the spasm begins in the muscles nearest to the site of infection, as is the case in artificial subcutaneous inoculation. In rare cases the spasm may be confined to a certain region of the body, for instance the hindquarters ( $\mathrm{T}$. parietalis); usually it is universal ( $\mathrm{T}$. universalis), in which case the infected animals have a characteristic appearance. 
In an advanced stage of the disease in the horse the animal will remain with stiffened, widely separated feet, and with head and neck extended in one position (Fig. 70). The ears stand erect approaching one another; the eyes, whose pupils become dilated, are drawn deep into the orbits and are immobile on account of the spasm of the eye muscles, while the anterior surface is largely covered by the prolapsed membrane nictitans (particularly noticeable when the head is raised). The nasal wings are drawn mpwards and as a result the nostrils have a trumpet like appearance. The mouth is held closed, with the commissures usually drawn outwards and slightly upwards; it is difficult to overcome the spasm of the thick masseter muscles so as to separate the upper and lower incisors $1-2 \mathrm{~cm}$. (lockjaw, trismus). The spasm of the constrictors of the

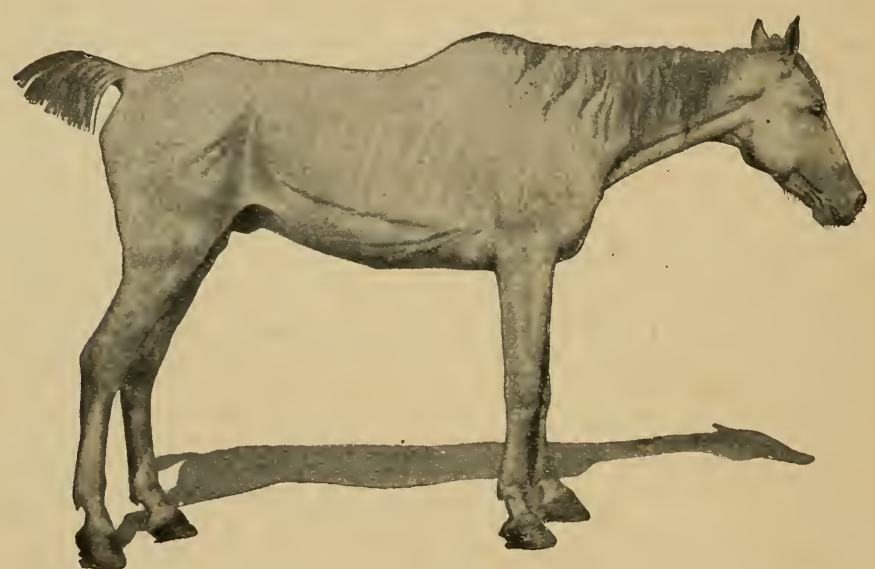

Fig. 70. Tetanus. Generalized muscular spasms in a horse.

pharynx prevents swallowing of the saliva which rums from the mouth in long tough threads.

As the facial portion of the head is raised by the contraction of the neck and upper throat muscles, the animal stretches the head forwards and upwards, while the neck is extended in a straight line or is slightly curved upwards, so that its upper margin forms a concave, its lower a convex line (deer neck). The abdominal muscles are also contracted and the abdomen is in consequence more or less retracted. The tail is held rigidly and slightly raised or usually pulled a little to one side.

The spinal column usually forms a straight line (orthotonus); exceptionally, owing to unusually violent spasms of the long back muscles there is formed a concave arch (opistho- 
tonus); or in case of excess musculature on one side a lateral curvature occur's (pleurosthotonus).

The muscular spasm of the extremities results in a straddled, sawbuck-like position, the hind feet being but very slightly arched at the hocks. Bending in these or other joints is impossible or can be accomplished only by applying considerable force; hence it is also impossible to lift the feet from the ground, and when this is done the disturbance of equilibrium is apt to cause falling. The patients avoid all possible movements; when forced they manage to move with great difficulty, lifting the feet but slightly from the ground. Turning is especially difficult, as the entire body acts like a stiff object; just as difficult, and frequently impossible, is the motion of backing.

The musculature is tense, almost board-like to palpation; some muscles project, appear of sharp contour and occasionally show fibrillary tremors. In the course of the disease the spasmodic contraction of the muscles remains for some time unchanged-in the same condition, for hours and even days; in some cases again certain variations occur. In such cases the muscles are at times less tense, movements easier and the general condition appears to be temporarily improved; this apparent improvement is soon followed by renewed severe spasmodic attacks which are easily originated by external irritation.

Reflex irritability is increased from the beginning, and the effect of light, noise and touch or movement increases the spasm, sometimes to such a degree that the animals bend the head unusually far back or even fall to the ground, trembling violently while on the ground; or sudden contraction of the dorsal muscles may cause fracture of one or more vertebrae (Zünclel). Such paroxysms are usually accompanied by profuse sweating, which is also observed in other cases of severe infection with the disease.

Conscionsness is retained to the end; the expression of the eye indicates fear, while the facial expression appears of a characteristic rigidity on account of the constant contraction of the musculature. Even slight excitement will cause the animals to shrink and to become restless.

The increased irritation of the nerves leading to the genital apparatus causes a moderate but constant erection of the penis in males.

The spasm of all the voluntary muscies of the body naturally exerts a disturbing influence on the other functions and thus irregularities in the respiratory and circulatory systems as well as in digestion are observed. In addition changes occur in the internal organs, which markedly aggravate the condition of the patient and incidentally are the direct cause of death.

Respiration is superficial and increased on account of the spasm of the abdominal and intercostal muscles, so that the number of respirations may be ten times that of normal. The 
gaseous exchanges in the lungs are not sufficient in spite of the increased respiration and soon there develop symptoms of impaired oxidation of the blood, whenever the spasm is continuous, such as cyanosis of the mucous membranes, hyperemia and later acute edema of the lungs with rales over the trachea and thorax. Breathing is frequently stertorous or groaning in nature, and sometimes one may observe on inspiration a whistling noise due to the spasm of the larrngeal muscles. Aspiration of mucus or saliva or forcible administration of drugs may easily cause a pneumonia, almost always of gangrenous character and with lethal result.

The heart action does not vary from the normal in the beginning of the disease, and in light cases it may remain unchanged throughont; the pulse, however, is small, the arterial walls tense. In severe cases the heart beat becomes very frequent and finally throbby, especially towards the fatal end of the disease.

Internal temperature is increased in severe cases only and then only on the approach of death, or when an inflammation of an internal organ has become associated (pneumonia) or when general septic infection occurs. Aside from such cases the temperature rises in uncomplicated cases only one or two days or even only a few hours before death, increasing rapidly, and reaching $42-43^{\circ} \mathrm{C}$. immediately before deatl (Fig. 71 ).

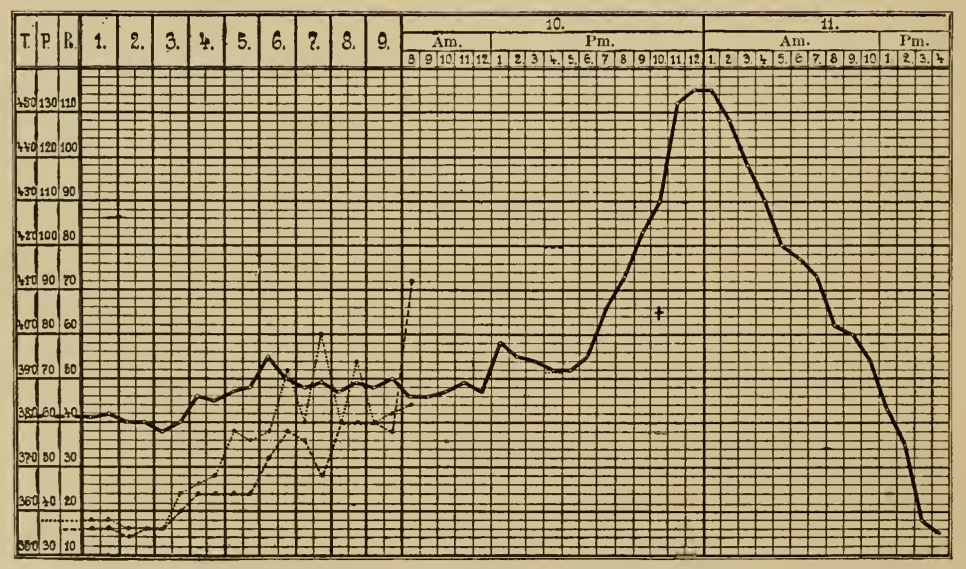

Fig. 71. Fever curve in tetanus with post-mortem rise of temperature.

The increase of temperature continues after death, and in two hours attains $45^{\circ} \mathrm{C}$. The eause of this increase in body temperature, first observed in the human cadaver by Wunderlich, is found in the amount of heat which is produced by the powerful muscle contraction which inereases after death to scme extent and then relaxes, for similar post-mortem signs have been observed after poisoning hy strychnine as well as after electrical irritation of the spinal cord (Leyden). 
Ingestion of food is lindered by the trismus, in spite of a good appetite. Patients repeatedly reach for food, chew the morsels with a lot of noise and finally swallow them with difficulty, or the food remains in the mouth and is later expelled by cough. The food particles which accumulate in the mouth are mixed with saliva and undergo fermentation or decomposition, decolorizing the saliva and imparting to the exhaled air a fetid odor. Liquid food is more easily taken, although sometimes even the drinking of water becomes impossible, in spite of every effort, regurgitation being often observed. The tongue is usually coated with a thick, dirty-grayish deposit, the margins showing the imprint of the teeth and frequently superficial injuries. Peristalsis is suppressed, defecation delayed on account of the tension of the abdominal walls, and only a few fecal balls are evacuated at a time.

Micturition is also delayed and the urine is more concentrated, with increased specific gravity as a result of the prolonged retention in the bladder as well as reabsorption of the aqueous constituents. In completely suppressed ingestion of food the reaction is acid and the urine contains debris and oxalate crystals in large amounts (Friedberger).

According to Tabusso's experiments the blood contains heterolysins, also auto- iso- and heteroagglutinins, while the freezing point is slightly diminished (an average of 0.535 against 0.558 for normal horse serum), whereas in other instances where dyspnea and asphyxia are present the molecular concentration is increased.

In fatal cases the patients, which have hitherto been constantly standing, suddenly break down, fall to the ground and die in a few hours under progressive increase of the muscular contractions. The immediate cause of death is sometimes exhaustion, or suffocation, at times also a hypostatic or traumatic pneumonia.

In localized tetanus the muscle spasm is confined to certain parts of the body, and usually to those in which the infection occurred. When the spasm affects the muscles of mastication, the course of the disease is usually unfavorable on account of impaired nutrition; in other cases the disease occasions as a rule merely a transitory disturbance of function. When the partial tetanic spasm remains local to the end, it is usually accompanied by light symptoms and the contraction of the affected muscle groups does not develop to as high a degree as in generalized tetanus. In most cases the localized tetanus is the incipient stage of general infection.

The clinical picture of the disease varies but slightly according to the species of animals affected, and deviations in the complications are usually the only changes observed.

In cattle bloating and distention of the left flank develop on account of the suppression of the movements of the paunch and of rumination due to spasms of the abdominal muscles. The patients show a more pronounced apathy or dullness and their 
reflex irritability is less marked than in the horse. Dreymamn and Shadow each observed a case with marked upward curve of the vertebral column (emprosthotonus); Balog observed marked orthotonus. In cows a suppurative inflammation of the uterus occurs frequently, which serves as the basic point of the disease.

In sheep and in goats opistlıotonus usually occurs; it is pronounced when the animals are standing, but more so after they have fallen to the ground. Moderate bloating is also noted frequently.

The lockjaw of lambs (tetanus agnorum) which occurs as a rule from a navel infection and is not infrequently observer as a stable enzootic, begins with lassitude and sluggish indisposition. Later movement becomes stiff and finally impossible; the animals hold the head to one side or backwards, curve the back upwards, suckle with difficulty or are unable to do so, and

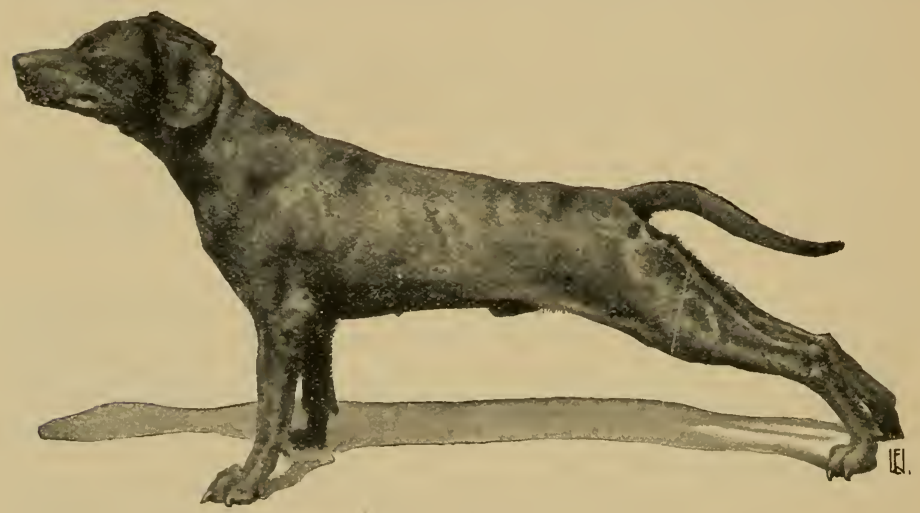

Fig. 72. Tetanus. Generalized muscular spasms in a dog.

finally die with symptoms of suffocation. The disease is often associated with an acute intestinal inflammation with profuse diarrhea.

In hogs, which usually are affected with generalized tetanus, trismus attains a marked degree (Jakab observed the disease in this form among 16 pigs of one drove); upward or downward curvature of the vertebral column also occurs frequently.

Tetanus is not common in dogs-is usually localized ani manifests itself especially in young $\log$ s by transitory trismus. In severe cases one is struck by the characteristic facial distortion, the forehead being wrinkled in a sagittal direction, hy the rigid erect position and approximation of the ears, the external strabismus of the eyes and the grinding of the teeth as well as the rigidly spread extremities; the vertebral column is straight or curved downwards (Fig. 72). 
The disease occurs very rarely in fowls. Dreymann observed it in a turkey gobbler four days after a dog bite. The symptoms were spasm of the entire musculature, stiffness of the head and neck, trismus, close approximation of the wings to the body, ruffled feathers and cyanosis of the comb and wattles.

Course. The most varied transitional stages may be observed in the course of tetanus in the rapidly progressing cases which terminate fatally in 2 or 3 days as well as in those that progress slowly and attain only a limited degree of intensity. Death usually follows in 3 to 10 days after the appearance of the first symptoms, very rarely earlier and even more rarely later. The symptoms in the fatal cases, aside from slight remissions, are progressive in intensity until death supervenes. There are cases in which the patient's condition may become improved for a time to such an extent that recovery is hoped 'for; suddenly a remission occurs, followed in a few days by death, while no cause for the change is discernible. In favorable cases the symptoms, especially the spasmodic contraction of the musculature, abate towards the end of the second week, and finally cease altogether. Convalescence will be long, however, a certain stiffness of movement being noticeable in the animals even 4 to 6 weeks later, but this also gradually disappears.

The course of the disease is influenced by nutrition in that those cases where ingestion of food and water is difficult or impossible on account of the trismus, exhaustion occurs earlier, and this is accelerated by the exertion occasioned by the constant muscular tension. Further, the superficial respiration occasions danger of suffocation, which is enhanced by the easily induced hypostatic hyperemia of the lungs. Mild cases may be shaped unfavorably by occasional complications, and in this respect pneumonia is especially dangerous. It is produced almost exclusively by aspiration of fluid or particles of food and usually runs an unfavorable course." (Schindelka has observed a marked decrease in the tetanic symptoms after an intercurrent influenza in four horses.)

Diagnosis. A characteristic picture of the disease is presented by the continuous tonic muscle spasms with retained consciousness and normal temperature. Strychnine poisoning simulates it very closely; here, however, the spasms develop much more rapidly and death results much more quickly.

Tetanus may be mistaken for other diseases only in the initial stages of the disease, as long as the muscle contractions are confined to one part of the body and while they are not very pronounced. Cerebrospinal meningitis comes into consideration on account of the rigidity of the neck, but here symptoms are also present pointing to an inflammation of the brain (dullness, paralysis in the area of certain cerebral nerves), trismus is usually absent, reflex irritability is increased, but 
external irritants do not produce a rigidity of muscles in remote parts of the body, while on the other liand muscle paralyses oceur in the later course of the disease.

Clonic spasms are characteristic of epilepsy and eclampsia. In epilepsy these are separated by intervals of freedom from spasms. Tonic spasms are exceptionally observed in single muscle groups and then they are only transitory, while the patients also lose consciousness during the attacks.-In rahies trismus is absent, the ears are not stiff and here paralyses occur later.

Acute muscular rheumatism, when rery serere, occasions a similar spasmodic condition at the parts of the body affected as occurs in tetanus; but here the muscles are painful and appear swollen rather than tense, the reflex irritability is not increased and external irritation causes no extension of the condition.

In articular inflammations of the extremities as well as in rickets the stiff gait also reminds one of tetanus, but the changes in the shape of the joints protect against error, while the cause of an apparent trismus resulting from inflammation of the maxillary joint is differentiated by the tenderness of the involved articulation and the laxity of the masseters.

Finally tetanus must be differentiated from tetany wheh is produced by traction of certain nerves or adhesion to a cicatrix. As soon as the nerve is freed these symptoms all disappear (Gumning cured the disease in one case by freeing the spermatic cord stump which had grown into the scrotum). Tetanic svmptoms are sometimes observed in painful intestinal diseases (Hoffmann observed this repeatedly during four weeks in a horse infested with ascaris). Recovery followed expulsion of the worms.

Pus from wounds may occasionally reveal spore-hearing tetanus hacilli on microscopic examination, which are mixed with other bacteria (see Fig. 73). Such material is also suitable for diagnostic inoculations of mice and guinea pigs.

Prognosis. The course of tetanus is unfarorable in the majority of cases. The mortality varies in horses between 55 and $90 \%$, in cattle it is a little lower, in sheep higher and in young animals, especially in lambs, most unfavorable (95 to $100 \%$ ). Giinther, however, saw four out of five colts 8 to 14 days old recover. The earlier the symptoms appear after infection, the more rapidly the muscle spasm develops with pro portionate intensity, the more unfavorable is the prognosis. and a marked locking of the jaw is of bad significance. In a longer incubation and more gradual development of the disease, the prospects are better, and should the animal survive the second week it may most probably be considered saved if the respiration and nutrition are satisfactory and no complications set in.

From a prognostic point of view the internal tenperature 
is also of importance, as every increase in temperature during the course of the disease is an unfavorable sign. Such an increase indicates in cases without complications that the end is near; in other cases it is due to some inflammatory process, usually pneumonia.

The cases of localized tetanus are, as already stated, always more favorable, and the prognosis of tetanus in dogs is usually better.

Among 247 horses with tetanus obserred at the Bulapest elinic in 1886-1906, 202 died, i. e., $81.5 \%$. - In the stables of the Prussian army tetanus was diagnosed from 1881 to 1908 in 1,139 horses, 84s, i. e., $74.4 \%$ died, while in the French army from 1904 to 1905 among 172 eases, 115 or 66.8\% dieil-According to Dieudonné the mortality from tetanus after docking of the tail (58 cases) is $75 \%$.

Treatment. The first essential part in the treatment of tetanus consists in a thorough disinfection of the seat of infection. If it is possible, by removing the pus and necrotic tissue, as well as by a subsequent disinfection of the wound, to render the tetanus bacilli and spores harmless, the source of the poison is thereby eliminated, and if the already absorbed toxin has not yet produced severe changes in the nervous system, the recovery of the animal may be hoped for, especially since a portion of the toxin is probably eliminated from the body with the secretions (perspiration, urine, saliva). According to these indications careful search should be made at the supposed place of infection by means of an appropriate incision, to de-

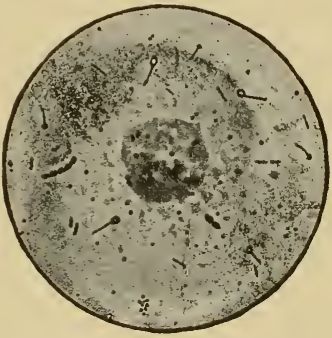

Fig. 73. Pus from a castration wound of a hog affecter with tetanus. Besides spore-containing tetanus bacilli, streptococci, staphylococei, diplococeus lanceolatus, ete., are present.

termine whether it contains any purulent or ichorous secretion. If so it should be washed, and if a foreign body is present it should be removed, the suspicious tissue should be cut from the walls of the cavity with the aid of scissors, or curetted with a spoon, and the cavity should be thoronghly washed with a concentrated disinfectant. For this purpose a $1 \%$ nitrate of silver, or corrosive sublimate solution, $8 \%$ chloride of zinc solution, or $5 \%$ iodide of glycerin (Van), are satisfactory; cauterization may also be applied. The disinfection acts also by destroying the less resistant foreign bacteria, and thereby rendering the conditions for the propagation of the tetanus bacilli unfavorable. By the application of a Bier's ligature the absorption of the toxins may also be made more difficult. If the disease appears after docking the tail it is advisable to amputate another portion, and to apply the thermocantery to the surface of the wound.

In practice the local treatment of horses is sometimes rery difficult, as the patients become much excited, anil it is hardly possible to lift the leg from the 
ground. If however the place of infection is known with sufficient certainty, it appears justified to anesthetize the animals for the purpose of local treatment.

Suitable quarters and proper food are of great importance. The patients should be put in a quiet place, if possible, with soft ground, and on account of the increased reflex irritability, they should be protected from loud noises and from direct sunlight (complete darkness is not necessary), and also from unnecessary liandling and examinations. It is only in severe cases advisable to place the horses in slings, usually when the animals are exhausted to such an extent that they can stand up only with difficulty, for once the animals have fallen to the ground the condition becomes very rapidly aggravated. If the animal is found lying on the ground it is advisable to allow it to lie for awhile on soft bedding; then it should be raised carefully, and placed in slings if this is possible without unduly exciting the patient.

As long as the patients are not unable to eat they should be given soft, easily digestible food; later, when mastication becomes difficult on account of approaching trismus, flour and bran gruels, to which eggs or red wine have been added, may be given, while if trismus is fully developed nutritive enemas (milk and egg's) may be employed. At the same time the animals should have fresh water within reach, so that they may, at their desire, rinse their mouth and quench their thirst. In order to prevent the decomposition of the saliva and of the remnants of food it is advisable to rinse the mouth from time to time with pure water or water containing a little vinegar. The retained feces should be removed by manual emptying of the rectum, or by enemas of soap water; the retention of urine is relieved by pressure on the bladder, or if necessary by the introduction of the catheter.

The internal treatment proper is of minor importance. The administration of drugs per os is dangerous even if trismus is only slightly developed, and for this reason usually only such remedies are given as the patients can take with their drinking water (salines for the prevention of constipation). In cases where the infection appears to have occurred through the intestines oil of turpentine (Ol. thereb., Ol. oliv. $\bar{a}$ a $100.0 \mathrm{~g}$. per diem) is now administered per os or per rectum, as advocated by Hartenstein and Nocard. Inhalations of chloroform, or chloroform and ether appear to act beneficially, by diminish.. ing the trismus somewhat, so that the animals can more easily partake of food. Such inhalations should be given immediately before feeding, but only up to the commencement of anesthesia. Matozzi recommends for reducing the trismus an ointment of cyanide of potassium (2:8), Schleg a mixture of gray mercury ointment and camphor liniment, to be rubbed into the cheeks. Chloral hydrate or ether (3-50 g. daily per rectum), morphine (0.2-0.5 g. subcutaneously), eyanogen (0.3-0.5 g. subeutaneously or injected into the trachea), extract of belladonna (2-4 g. in 
drinking water), salts of atropin (0.02-0.08 g. subcutaneously) etc., may be used on account of their anti-spasmodic action, relatively small doses being required for small animals. The last mentioned remedies are preferred by some authors to the opiates which cause digestive disturbances or symptoms of irritation (Barrier, Friedberger, Pethe).

The value of the subcutaneous injection of carbolic acid as recently recommended by Maccagni \& Bianchini, Croce, also by Schmid, Guerrieri, Cangini and others (20-40 cc. of a $2 \%$ solution, according to Croce $10 \mathrm{cc}$. of a $10 \%$ glycerin solution, and in addition carbolized water into the rectum), cannot yet be determined. Pilocarpin (0.20-0.50 g. subcutaneously) was recommended to promote the circulation, and to increase the secretions through which it possibly would promote the elimination of the toxins, but the writers, as well as Friedberger, failed to obtain beneficial results from its application. Profuse bleeding (10 liters at one time possibly to be repeated in one to two rlays and followed by subentaneous infusions of several liters of artificial serum) as recommended by Crinon and also by Dumas, is supposed to serve the same purpose, but its action also appears to be problematical, since together with this the administration of bromide of potassium, and antitoxic serum is recommended, while Habicht advises also injections of iodipin $(25-30 \mathrm{~g}$. alternating with the bleeding, and as after treatment). Chigos and Diffiné recommend daily intravenous injections of 250 cc. oxygenated water, Dabert peroxide of hydrogen (10 cc. daily subcutaneonsly, or 1-2 liters per os), Mayr the iodipin (100 ce. subcutaneously), with repeated rectal infusions of a $3 \%$ septoform solution. French veterinarians (Chapard, Desoubry, Cagny, Girard \& Malle) praise the tallianin (ozonized turpentine), which is supposed to have given good results even in severe cases (20-30 cc. daily intravenously).

The antitoxin serum has given only indifferent results in animals affected with tetanus, but may be beneficial if employed at the beginning of the affection, and in relatively high doses (see p. 462).

After the disappearance of the muscular spasms and during the long convalescent period care should be taken that the animals are daily exercised quite slowly for $1 / 4$ to $1 / 2$ an hour, and they should be used for work only after several weeks.

Immunization and Serum Therapy. Behring \& Kitasato (1890) successfully immunized rabbits by inoculating them first with 0.3 cc. of filtrate of a virulent culture, and then repeatedly injecting them subcutaneously with 3 cc. of a $1 \%$ iodine trichloride solution. Rabbits treated in such a manner later withstood $10 \mathrm{cc}$. of a culture of which 0.5 cc. killed other rabbits. They also withstood 20 times the fatal dose of toxin, while 0.2 cc. of their blood serum injected intraperitoneally into rabbits protected them against a virulent infection given 24 
hours later. If 0.033 ce. (300 times the fatal dose) of a culture, 0.0001 ce. of which killed mice inside of two days, is injected into mice with 5 times the quantity of immune serum $(0.2 \mathrm{cc}$. the animals will remain unaffected. The blood sertm of rabbits which were not inmmized had no protective action. Similar results were obtained by Tizzoni \& Cattani in mice and rats (1891) with blood of artificially immunized pigeons and dogs (1-2 drops of dog serum rendered ineffective $0.5 \mathrm{cc}$. of toxic filtrate of cultures).

These experiments formed the basis of the serum immunization and serum therapy, for which the serum of horses is now exclusively used, since Schütz found that horses and sheep may be also successfully immunized against tetanus, and produce a potent immune serum.

Preparation of Immune Serum. According to Behring's method, a virulent bouillon culture is obtained, $0.75 \mathrm{ce}$. of which is fatal for rabbits in 3 to 4 days, and of this bouillon 80 ce. is mixed with $0.25 \% ; 60$ ce. with $0.175 \% ; 40$ ec. with $0.125 \%$ trichloride of iodine, while $20 \mathrm{ec}$. is used without any mixing. Passing from the most attenuated to the least attenuated cultures the horses to be inmunized are treated once in eight days with subentaneous injections of $10-20$ ec. and finally with the pure eulture, 0.5 ce. being injected at first and thereafter each five days the dose being doubled for each injection.

According to the procedure of Ronx \& Vaillard immunization is carried out with filtrates of 4 to 5 weeks old cultures, of which $1 / 400$ ec. kills a mouse. At first a filtrate attenuated with Lugol's solution is injected subeutaneously in increasing doses and later the pure toxin, first injected subcutaneously, then intravenously or intraperitoneally.

Standardization of Immune Serum. According to Behring's procedure the potency of the serum is determined in such a manner that the smallest quantity of serum is ascertained by inoculations on mice, which renders a certain quantity of tetanus toxin of a known strength, ineffective (toxin and $\mathrm{s} / \mathrm{cm}$ are mixed and injected subcutaneously). As a test toxin a dry tetanus normal toxin (TeTN) is used, of which $1 \mathrm{~g}$. kills $150,000,000 \mathrm{~g}$. of mouse weight, that is $10,000,000$ mice of $15 \mathrm{~g}$. body weight each. As normal serum $\left(\mathrm{TeAN}^{1}\right)$ is designated that serum of which $0.1 \mathrm{cc}$. renders ineffective $0.03 \mathrm{~g}$. of normal toxin; serum, of which 0.01 ce. exerts such an action, possesses 10 times the strength (TeAN ${ }^{10}$ ) etc. One ce. of normal serum contains one antitoxin unit (A.-E.). In Germany only a serum can be marketed which possesses not less than at least ten times the strength $\left(\mathrm{TeAN}^{10}\right)$, of which 1 ec. contains at least $10 \mathrm{~A} \cdot \mathrm{E}$., or $0.01 \mathrm{ec}$. neutralizes $0.03 \mathrm{~g}$. of normal toxin.

Serum prepared by the Pasteur Institute of Paris renders ineffective 1 ec. of a solution diluted in proportion of $1: 100,000$, which is 100 times the fatal toxin quantity (=0.001 g. dried toxin).

Both sera when dried in the vacumm are also used in the powdered form, $10 \mathrm{~g}$. of the German preparation containing 100 antitoxin units, while of the Parisian preparation $1 \mathrm{~g}$. corresponds to $10 \mathrm{ce}$. of the liquid serum. There is also an antitoxin prepared by Tizzoni and Catanni on the market, which is obtained from dog and horse serum.

The blood serum of highly immunized horses when injected subcutaneously in sufficient quantities, protects animals against the pathogenic action of a simultaneous tetanus infection or an infection occurring shortly afterwards. The immunity is produced immediately, but lasts only a short time, about 3 to 4 weeks in horses, and then diminishes gradually, finally disappearing entirely. A simultaneous injection of immune serum and toxin causes local tetanic symptoms, which however last only a few days whereupon the animals recover. Similar results 
are obtained when the serum is injected after the toxin, but before the appearance of the tetanic muscular spasms, while if the injections of serum are made still later the fatal termination can be averted only by large doses of serum, especially by its administration into the blood circulation, or directly into the brain (Roux \& Borrel). After the lapse of a certain time after toxin injection serum treatment is entirely without effect.

The protective and curative action of tetanus antitoxin depends, according to the present conception, upon the fact that the antitoxin molecules contained therein bind the toxin molecules circulating in the tissue fluids and render them harmless. The repeated action of the toxin on the nerve cells in the serumproducing animals results in the production of excessive numbers of receptors, which are thrown off into the circulation, and are therefore present in the antitoxin molecules (Ehrlich's Theory, see p. 463). The serum therefore protects the nerve elements from the pathogenic action of the toxin molecules which have not ret reached them; but it is not capable of separating toxin molecules, which have already become anchored to the plasma of the nerve cells, and thereby restore the former condition of the cells. Accordingly the serum affords protection against infection immediately preceding or following its administration, but if the disease has already become manifest it exerts a curative action at best only when the intoxication has not greatly reduced the functional ability of the nerve cells.

I. Immunization with Immune Serum. Such an immunization is advisable and indicated in all of those cases in which tetanus is feared within a short time. Such a possibility exists especially after contusions which have become contaminated with dirt or manure, also whenever tetanus is frequently observed in certain localities to follow upon certain injuries (castration, docking of the tail operation for umbilical hernia, skin injuries, etc., and when during the operation careful asepsis cannot be carried out. Where the necessary requirements for a satisfactory surgical technique are present, such prophylactic serum injections are of course superfluous.

For immunization with Behring's serum large animals are injected subeutaneously with 20 A.-U., small animals with 4-5 A.-U., while with the Pasteur serum 20 or $6-10$ ce. respectively are used; the injections may be repeated after 10 to 12 days. The surgical treatment of the wound does not hecome superfluous by the use of this serum since the injected serum does not prevent the development of the tetanus bacilli (Roux \& Vaillard). Besides the spores are retained in the wounds for a long time, and if they germinate, after the disappearance of the artificial passive immunity, they produce toxins and may cause disease in the animal which has again become susceptible.

Inmunization is employed to a great extent in France, in accordance with Nocard's recommendations. In the period of $1895-97,2,395$ horses, asses and mules, 44 cattle, 82 sheep and lambs, and 206 hogs were immunized. More than 2,300 
of these animals were immunized immediately after an operation and none of them became ill. Of about 400 animals which were treated 1 to 5 days after an accidental injury only one horse became affected with tetanus, and this one recovered. The 63 veterinarians who carried out the immunization observed tetanus during this same period in 259 animals which had not been given the treatment. Labat in France (703 eases) and Lang in New Calerlonia likewise obtainer very goorl results with immunization in localities where the soil was bally infected. Dieudonné failed to observe a single case of tetanus in colts which had been treated with serum after operations for umbilical hernia, whereas $33 \%$ of the animals hail succumbel previously under similar conditions. Gool results are also reported by Nandrin in tetanus of newly born foals; he found however that $5 \mathrm{~g}$. of serum hall a toxic action and therefore considers $3 \mathrm{~g}$. as a suitable dose.

According to the recent experiences of Dieudonné (in 1,009 cases of castration and abdominal operations), Labat (in about 2,000 clinical operations), and (Chapellier (in about 500 castrations), a single serum injection suffices to accomplish effective results. (Merillat observed the development of fatal tetanus in a horse in sjite of the sermm injection one week after it had been operated on for a nail puncture of the hoof.)

The liquid serum keeps at least one year in a dark, cool place, while dry antitoxin keeps much longer. This should be dissolved before using in boiled water. Accorling to Calmette dry antitoxin exerts its protective or curative action (in rabbits) even when it is dusted upon the infected wounds.

II. Serum Therapy. In animals that are already affected the serum treatment has thus far not been successful. Animals in which the subjective symptoms cannot be ntilized for the diagnosis of the first stages of the disease, come for treatment as a rule in an advanced stage of the disease, and mostly with symptoms of general intoxication. In such cases the toxin has already flooder the borly, and consequently the nervous system is affected to such an extent that even the prevention of further intoxication is no longer able to save the animal. At best the beneficial results can only be expected from serum treatment when it is undertaken on the first day of the disease.

For curative purposes horses should receive subeutaneous injections as near as possible to the place of infection, with at least 100 units of Behring's serum, 50 ec. of Pasteur's serum (or 9,000 American units. Trs.). If possible the injections should he repeated the following day.

Nocard could not check the progress of the disease in horses even with intravenous injections of immune serum; not even in those cases when the injection had been undertaken 24 hours before the appearance of the first symptoms.

In the perior of 1897-1903 there were treated with antitoxin in the Prussian army 91 horses, of which $58(63.7 \%)$, died, while in the French army the mortality since the administration of inmune serum was reduced from $72.7 \%$ in 1897 , to $57.3 \%$ in 1901 . In the elinic at Budapest of 7 horses treated with serum 4 died. A farorable result was obtained only when the progress of the disease was slow and the recovery of the animal was probable even without such treatment. Knorr obtained relatively good results in some horses; however he injected 1,000 A.-U., repeating this dose after 1 to 3 days (the treatment of one animal cost $\$ 50.00 !)$.

Later reports of Dieudonné ( 31 recoveries in 79 cases), Ryder, Hammond, Labat, Rickmann and others, speak more favorably of the value of the serum treatment; all however express the necessity of using large doses repeatedly. Haubold obtained very favorable results in sheep which became affected after shearing, castration, or docking; two doses of 5 ce. of the Pasteur serum were sufficient to produce recovery even in severely affected animals. Dourille obtained recovery in four jogs after the injection of 3-5 ee. of serum.

Roux \& Borrel recomment the intracerebral injection of immune serum. Out of 4.5 guinea pigs which receiver four ec. of serum in each hemisphere after the appearance of tetanic symptoms, 35 recovered while of 17 guinea pigs which had 
been treated under similar conditions subeutaneously only two remained alive. Villar treated two horses successfully in this manner (3-8 ce. of serum were injected into the brain substance through an opening in the frontal bone). Sendrail and Cullié on the other hand saw the fatal termination in two cases hastened, while with the injection of serum into the vertebral canal they obtained a more satisfactory result (30 ce. injected through the lumbo-sacral articulation on two consecutive days).

Küster and Sawanura claim a special astion from endoneural serum injections. This method is recommended as prophylactic, especially in those cases in which museular tissue has become injured, therapeutically in tetanus ascendens, while not in tetanus descenilens; nevertheless subcutaneous and intravenous serum in. jections should also be used.

Hartenstein and Trélut obtain good results from the injection of blood serum from a horse affected with tetanus; the authors however observed a pronounced aggravation after transfusion of the blood of an immunized horse, while Pilz found the blood serum of horses that had recovered from tetanus to be withont any effect.

Treatment with Brain Emulsion. After Wassermann found that a mixture of brain substance of rabbits and toxin in certain proportions is not poisonous, and in consideration of Ehrlich's theory that the antitoxins are nothing more than receptors thrown off from the ganglion cells, Fiebiger undertook curative experiments at the Vienna clinic, under the direction of Schindelka with the injection of brain substance. In the course of 15 months 20 horses affected with tetanus, but excluding the very severe eases, each received subentaneous injections of one lamb's brain finely emulsified in physiological salt solution. Of these 20 horses eight (40\%) died. In most cases extensive and slowly healing abscesses developed at the point of injection. Schuemacher also reports favorable results from this treatment.

Literature. Friedberger, D. Z. f. Tm., 1879, VT, 33 (Lit.) ; 1884, X, 27 (Lit.).-Carle \& Rattone, Giorn. di Torino, 1884.-Nicolaier, D. m. W., 1884, 842; V. A., 1892, CXXVIII, 2.-Nocard, Rec., 1887, 617.-Kitasato, Z. f. Hyg., 18s9, VII, 225; 1S92, XI, 256.-v. Behring, D. in. W., 1903, 617.-v. Behring \& Kitasato, D. m. IV., 1890, 1113.-Kitt, Monh., 1890, I, 214 (Review).-Tizzoni \& Cattani, Cbl. f. Bakt., 1891, IX, 189.-Vaillard \& Vincent, A. P., 1891, 623.-v. Behring, Das Tetanusheilserum, Leipzig, 1892.-Vaillard, A. P., 1892, 224.-Schütz, Z. f. Hyg., 1892, XII, 58.-Roux \& Vaillard, A. P., 1893, 65.-Dreymann, Monh., 1894, V. 19.-Dieudonné, Rec., 1895, 279; 1896, 290; 1909, 433.-Roux \& Borrel, A. P., 1898, 225.-Fiebiger, Z. f. Tm., 1902, VI, 161.-Lingelsheim, Hb. d. p. M., 1903, II, 567; 1904, IV, 983 (Lit.).-Vincent, A. P., 1904, XVITI, 450; Bull. de l'Acad., 1907, LVIIT, 233.- - Hoffmann, Z. f. Vk., 1905, XVII, 118.-Tiberti, Cbl. f. Bakt., 1905, XXXVIII, 307 (Lit. on Tetanus Toxin).-Tarozzi, ibid., 1906, XL, 305.Mayr, B. t. W., 1907, 931.-Römer, Z. f. Immf., 1909, I, 363.

\section{Rabies. Lyssa.}

(Canine madness, hydrophobia; Wuthrankheit, Tollwut, [German]; Rage [French]; Rabbia [Italian].)

Rabies is an acute, contagious, and almost without exception, fatal infectious disease, the clinical picture of which is characterized by disturbed consciousness, increased nerve irritability and by subsequent symptoms of paralysis. The disease is usually transmitted by the bite of an affected animal to other animals as well as man. The infectious virus is a filterable microorganism.

Historical. The disease and its contagious nature, as well as the danger connected with the bites of mad dogs, have been well known since the time of Aristotle, but up to the most recent times, there has been a certain belief in its spontaneous development. In fact, it was supposed that hot feed, lack of drinking water, unsatisfied sexual desire, and violent nervous excitement, might cause the disease. By 
means of inoenlation experiments the infectiousness of the saliva was determined in dogs by Zinke (1804), Grïner and Salm (1813), in herbivorous animals by Berendt (1822), in human beings by Magendie, while Galtier, in 1879, found the injection of the virus into rabbits to be a convenient and harmless method of studying the disease, and in 1850-81 made immunity tests with intravenous injections of virulent saliva or brain emulsion.

The very extensive investigations of Pasteur and his collaborators, Roux, Chamberland, and Thuillier (1881-1889), proved on the one hand that the purest and most concentrated virus is contained in the central nervous system; they also established a method for the attenuation of its virulence, and solved the important question of protective vaccination against the disease. Finally, Remlinger and Riffat-Bey (1903) have determined the filterability of the virus, while Negri (1903), through the demonstration of specific eell inclusions in the nervous system of the infected animals, has facilitated the postmortem diagnosis considerably.

Occurrence. Rabies occurs in all parts of the world among dogs, cats and carnivorons wild animals (especially wolves and foxes). Other animals and human beings are seldom affected, and then almost exclusively after having been bitten hy the above named carnivorous animals (about $80 \%$ of the observed eases have heen found in (logs). While in earlier times the disease appearer often epizootically extending over large areas, in the course of the last ten vears it has decreased or ceased to appear in several countries as a result of the measures taken against it.

In Germany, the number of cases of rabies, during the years 189.5 to 1898 , increased from 480 to 1,202 , but in the year 1902 they were reduced to 612 . During the years 1903 and 1904 there was again an increase to 1,043 , but since then the disease has been diminishing. In the year 1908, 683 animals, of which 585 wero dogs and 2 cats, were found infected in 644 communities. Of these cases $87.6 \%$ were observed in Prussia where the eastern provinces, East Prussia, Posen, and Silesia were prineipally infected. Cases were also found in large numbers in Saxony and Bavaria, while in the remainder of the territory of the kingdom only isolated cases were observed.

In France, the disease is found all over the country. Since 1899, nearly 2,000 cases of rabies liave been observed every year (in the year $1907,1,892$ eases, and in 1908, 1,482 eases in dogs). In Paris, however, the number of eases has decreased from 807 in 1900 to 43 in 1907.

In Great Britain, since 1895 when there were 672 eases in 29 counties, the disease has decreased so rapidly, thanks to the energetic measures taken to eradicate it, that only 13 eases in 4 counties were observed in 1902 , and since 1903 the whole territory of the United Kingdon has been free from the disease.

In Austria, the disease showed a steady inerease during the years 18.91 to 18.99 (from 815 to 1,551 eases and in $1900,1,187$ cases). It was most common in Bohemia and Galicia, less prevalent in Moravia and Lower Anstria. In many instances the infection was introduced into Galicia and Buckovina from Russia and Roumania, in the border districts of which countries an exceedingly large number of dogs are kept without supervision, and where rabies is not only regularly found in dogs, but also in wolves. During the 10 years from 1891 to $1900,4,974$ human beings were bitten by mad dogs, $123(2.4 \%)$ of whom developed hydrophobia and died. In the year 1908 the weekly number of eases at these places amounted to from 14 to 56, while the mountain and coast districts were free from the disease.

In Hungary, temporary improvement followed inmediately after the veterinary law of 1888 became effective, but since 1893 when there were 883 cases of disease, rabies has inereased considerably, and in 1908, 2,460 cases of rabies, 1,759 being in dogs, were observed in 1,471 communities. The majority of the cases was observed at the southern frontier. 
Plate $I$. Hutyra and Marek, Special Pathology and Therapatics of Domestic Animals. Volume 1.

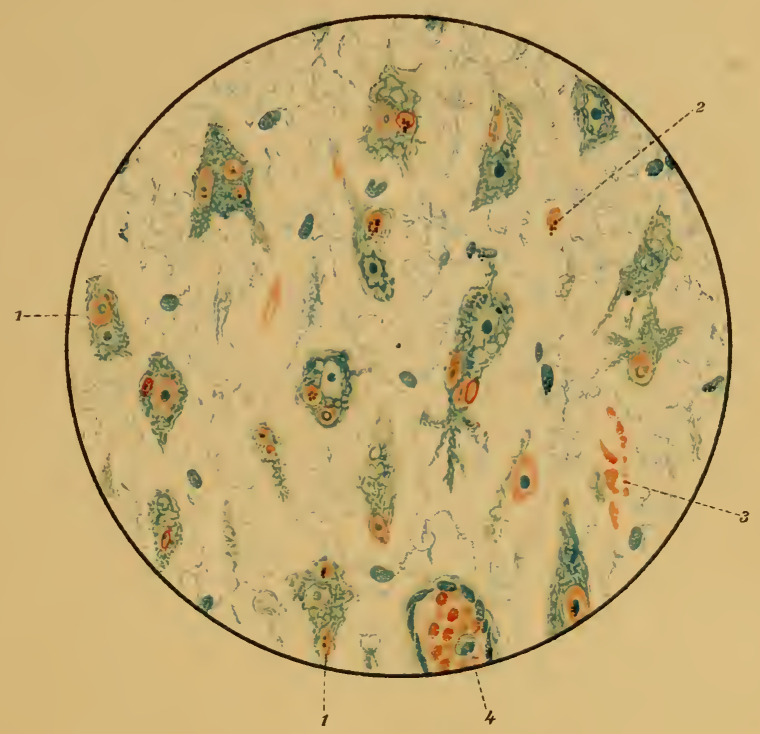

Negri Bodies.

(Ammonshorn of a dog dead of rabies) 1. Negri bodies in gangtion cells. 2.A free Negri body. 3. Fragments of red blood corpuscles. 4. Artery. Stained after Lentz. 

Russia is heavily infected as is indicated by the frequent introrinction of the disease into Germany and Austria (in 1908 there were 7,253 cases in 4,060 eommunities).

The disease is also frequently found in Servia, Bulgaria and in Rommania ( 273 cases in 1908 ; in 1906,142 mad logs were brought to the reterinary institute of Bucharest), while it is observed more rarely in Turkey.

In Belginm there were 123 eases in $190 \mathrm{~S}$, in Italy 525, in Switzerlani 2 . In Holland 41 cases of rabies were observer in 1907, and in Spain the disease also seems to appear frequently, but Demmark, Sweden and Norway have for years been free from the disease.

In North America rabies is very common (Frothingham) and has been increasing in prevalence dming the last ten years (Hart). In the Uniter States 230 human beings were the victims of rabies in 73 cities from 1890 to 1900 , while in the District of Columbia 91 cases of rabies in animals were reported from 1893 to 1900.

In Africa, the disease is also known, and in Asia it appears very frequently, while Australia has thus far been free from it.

Etiology. Remlinger and Riffat-Bey prodnced typical rabies in 6 out of 19 rabbits with a filtrate of highly diluted virulent hrain emulsion which passed through a fairly porons Berkefeld filter. According to the results of these experiments, which were later confirmed by Di Testea, Schrüider, Bertarelli \& Volpino and others, the virus of rabies must belong to the filterable microorganisms. The attempts at cultivation which have thus far been unsuccessful, justify the supposition that it multiplies only in the tissues and fluids of the body. The purest and most concentrated virus is secured in the central nervous system of the diseased animals; it is also found in the salivary glands and their secretions, and sometimes in other glånds of similar structure (lachrymal glands, pancreas, mammary glands), while in the aqueous humor (Gibier, Pasteur, Alhanesi, Courmont \& Nicolas), in the cerebro-spinal fluid (Pasteur), and in the blood (Marie) the virus is found only in exceptional cases, and in the flesh not at all. (Konrádi found it in the blood of guinea pigs, but only in small quantities.)

Negri Bodies. In 1903 Negri found in dogs and rabbits inoculated with either street or fixed virus, and also in animals which had died or had been killed after natural infection small, round, oval or three cornered inclusions in the large ganglion cells of the hippocampus major, in the Purkinje cells of the cerebellum, in the ganglion cells of the cerebral cortex, pons, and medulla ohlongata, and sometimes also in the nerve cells of the spinal ganglia and spinal cord. These structures now known as Negri bodies, are 1 to $27 \mu$ long, 1.5 to $5 \mu$ broad and appear red in color when stained with methylene blueeosin (the Mann method of staining). These cell inclosures (see Plate I) contain in their interior very small, strongly refractile, and sharply outlined gramules. Negri believes that these hodies, which have been found exchnsively in rabid animals and persons are the parasites (protozoa) of rahies, because among other reasons they appear in the nerve cells only 14 to 23 days after artificial infection, and resist both putrefaction and the effect of glycerin.

These findings have later been confirmed by Volpino, Beck, Bose, Ernst, Bohne, Lentz, and others, and the structure of the bodies has also been subjected to a closer study. According to Volpino's investigations, the large bodies consist of a hyaline ground substance, in which

Vol. 1-30 
sometimes very small, marginal, and, at other times, larger, central formations (small and large inner formations) seem to be imbedded, which contain very fine ring, rod or dumb-bell-shaped inclosures. The rod-shaped bodies contain a fine granule sometimes at one or both ends, while the ring-shaped hodies show one or more similar chromophile granules around a light central space.

This complicated structure at once excludes the eharacterization of Negri bodies as products of degeneration; besides, their specificity is demonstrated by the fact that they are only found in rabid animals. There is, however, a doubt as to their parasitic nature and their importance as the exciting agent of rabies. The filterability of the virus and the fact that in the initial stage of the disease and in animals infected with fixed virus. Negri bodies are generally lacking seem to speak against it, but still the possibility exists that the true specific virus consists in small formations, barely visible or completely invisible, which develop only in the nervous system of the infected animals, and aided by the cell reaction, first form small and simple corpuscles and later large, complicated hodies (Di V'estea, Volpino).

Negri has recently confirmed his conception of the bolies namerl for him as true ralies parasites by microscopic discoveries according to which the formations at a certain stage of evolution contain a nucleus in a homogeneous original substance, which breaks up into very diminutive chromatin granules, whereupon the surrounding plasma is divided. In this way small corpuseles are formed which eousist of a chromatin granule and its eapsule, and later grow up to new indepenitent borlies. Quite consistent with this conception are discoveries by Babes, Koch, and Rissling, who in sections of the cornu ammonis, but mainly in the degenerater ganglion cells stained according to Cajal, Giemsa or Heidenhain, have found cocci-like formations in large number, scarcely $1 \mu$. in size, surrounded by a light capsuie. They are of the opinion that these formations are the active causative agents of rabies, while the Negri bodies which inclose them are reaction products of the ganglion cells produced by their irritation.

Prowazek consider's the capsulated small formations as protozoa and designates them as chlamydozoae, the same as the causal factors of vaccinia, chicken pest, ete. In faror of their protozoan character and against their bacterial nature is the fact that rabies virus is injured by gall and glycoside (Frantzius, Kraus, Eisler, Lesieur), which substances are known to be poisonous to protozoa and cells, but are not poisonous for bacteria.

Finally, the specificity of the Negri body is also shown by the fact that its abundance is in direct proportion to the virulence of the same parts of the nervous system. According to Fermi the order of sequence is cornu ammonis, cortex cerebri, medulla oblongata and spinalis; then, cerebellum, thalamus opticus, and nucleus caudatus.

Very small boties from 0.5-2.0 $\mu$. in size, were also found by Bertarelli, Febré and Bonnard in the brains of logs inoculated with fixed virus, and by Lentz and Manqueliau in rabbits treated in the same way. Their absence or the fact that they could not be found may have some connection with the knowledge that this form of the disease has such a brief course that no larger formations are developed. Schiffmann found by frequent passages that first the complex, and then the single, smaller boties disappear from the cornu ammonis. Lentz describes as "rabies corpuscles of passage" the Negri-like formations which are larger" in size and also contain larger, compact, inner corpuseles, and besides are frequently located extracellularly. They come from nuclei or ganglion cells under simultaneous disintegration of the protoplasm.

Tenacity. The medulla oblongata of rabid animals retains its virulence for months at ordinary room temperature when protected from drying and putrefaction (Pasteur, Frothingham); on the other hand the spinal cord of rabbits becomes non-virulent in 14 or 15 days when exposed in a dry, dust-free atmosphere (Pasteur) or in 4 or 5 days if spread in a thin layer (Galtier). Fluid saliva infects even after 24 hours (Gibier); dried saliva not later than 14 hours (Celli). The virus is destroyed in one half hour at $52^{\circ}-58^{\circ} \mathrm{C}$. (Högyes), and in two minutes at 
$80^{\circ}$ (Bertarelli). The virus is scarcely influenced by severe cold $\left(-16^{\circ}-35^{\circ} \mathrm{C}\right.$.). Putrefaction destroys the virus only after long exposure; for instance Galtier found that the medulla of a dog that had been buried for 44 days was still virulent, Rátz reported virulence persisting after exposure to putrefaction for 14 to 24 days, while Klimmer found the cord of a rabbit to remain virulent which had been undergoing putrefaction for 15 days. Of the chemical agents, glycerin acts rather as a preservative, for according to Kempner the medulla retained its virulence in glveerin for at least $\&$ to 10 days; according to Roilet \& Galavielle for 10 months. On the other hand the virus is destroyed in a few minutes when exposed to the action of each of the following: Mercuric chloride 1:1000 (Celli), dilnted chlorine or bromine water ( $10-20$ drops of the official ehlorine or bromine water to $10 \mathrm{ce}$. of water), $1 \%$ solution of potassium permanganate, and $2 \%$ snlphuric acid (Bókay \& Szilágyi), 5\% hydrochloric acid, 1\% creolin solution and also to lemon juice (Blasi \& Traveli). The natural gastric juice destroys the virus of rabies in $4 \frac{1}{2}$ to 5 hours (Babes \& Talaseseu), and bile kills it in several minntes. In a vacuum from which the sunlight has been excluder the dried and pulverized brain substance will remain virulent at least 9 months (Vansteenberghe).

The Negri bodies are destroyed by weak alkaline solutions; they are however very resistant to the action of mineral acirls (Negri) and are only imperceptibly altered by drying, warmth, putrefaction, glycerin and water (Bertarelli \& Daddi).

Pathogenicity. By the inoculation of virulent saliva or oblongata emulsion the disease may be transmitted to dogs, cats, guinea pigs, rabbits, mice, rats and also to other mammals. The best results are ohtained by the injection of the virus under the dura mater of brain or cord, into the brain substance, into the anterior chamber of the eye, or into the muscles. Equally good results may be obtained by injection into the peripheral nerves. Rubbing the virus into the scarified skin of dogs and rabbits is successful in only $50 \%$ of the cases. Subcutaneous inoculation in dogs rarely gives positive results. In rabbits $75 \%$ of subentaneous injections are successful only when the muscles or the nerves have been injured at the same time. Intravenous injection sometimes produces the disease in dogs and rabbits, but not in ruminants or horses.

Konrádi has infected rabbits through the scarified skin, Galtier through the shaved skin, Högyes, Galtier, and Remlinger through the uninjured nasal mucous membrane, and Galtier and Conte through the conjunctiva, while an infection of the mucons membrane of the vagina only succeeded in exceptional cases. Several experiments have been conducted without results by feeding saliva, meat, and spinal cord of rabid animals to horses, dogs and sheep (Delafond, Rénault, Hertwig, Nocard). Nocard in the course of 2 months, had a fox eat the cerebral and spinal tissue of 6 rahid foxes and 12 mad dogs, the unusual feed not having the slightest harmful effect.

Birds may be infected artificially, but they are not very receptive, and the virulence of the virus seems to decrease in their bodies (Gibier, Löte). Cold-blooded animals are also killed by the rabies virus, and frogs succumb with paralytic symptoms.

In rabbits which have been infected successfully, the disease develops in its paralytic form, as a rule the hind parts and exceptionally the front part of the body becoming paralyzed first. The paralysis soon becomes general, spasms in some groups of muscles are observed, and at last the greatly emaciated animal dies in the course of 2 to 3 days. Indications of excitement (unrest, desire to bite) are seldom observed. but these symptoms have been often observed in gninea pigs. In dogs the nsual symptoms of rabies develop after inocnlation and if . 
this was intracranial, the disease usually commences with mania and a desire to bite.

The virulence of street virus varies within a rather wide range, and this is also indicated by the varying periods of incubation after subdural inoculation in rabbits. According to d'Amato, the virus from cats, foxes and wolves has a more violent effect than that from dogs.

The natural infection is brought about in the majority of cases by man or animal being bitten by a rabid animal, whereby the virulent saliva is inoculated through the wound into the subcutaneous tissues, muscles or nerves; in rare cases the contagion has been introduced by licking of wounds and skin abrasions. The saliva is most virulent immediately after the onset of the disease and in its further course but it is also infectious in a lesser degree 2 to 5 days before the appearance of the symptoms (Roux \& Nocard, Nicholas), and in cases described by Pampouki and Konrádi even 8 days before the appearance of the first symptoms. On the other hand during the long period of incubation preceding these days, the saliva is not infections but it may be virulent for 5 days after the animal has recovered (Remlinger). Finally, there exists a possilility of transmitting the disease by means of milk from diseased animals (Perroncito \& Carità, Nocard).

The danger from a bite depends on the one hand on the virulence of the saliva, on the other hand upon the size, that is the depth of the wound, and further on the number of nerves and lymphatic vessels in the wounded part of the body. Large wounds offer large surfaces for absorption, and from small but deep wounds, the virus once entered, can hardly he removed, while from small, superficial wounds it is easily removed by means of bleeding. Therefore, the serious wounds inflicted by animals of prey, especially wolves and large rabid dogs, anil the deep wounds inflicted by the sharp, pointed teeth of cats have an equally had reputation, while the bites of the herbivorous animals are less dangerous.

Rabies is particularly frequent after infectious bites in parts of the body where nerves and lymph vessels are plentiful and which are near to the brain or spinal cord. Wounds on the lips, cheeks, and nose are considered as especially dangerous, and in horses and cattle the infection comes mostly from these parts of the body. The density and thickness of the layer of hair or wool covering the point bitten is important as it might withhold the saliva. Similarly human beings are often protected against infection on account of the thickness and density of their clothing. Thick wool especially renders such protection, and this is in accord with the experience, that bites of rabid animals are much more dangerous for clipped sheep than for those that have a dense covering of wool.

The contagion, he it ever so virulent and concentrated, cannot penetrate through the uninjured skin, while the possi- 
bility of infection of the uninjured mucous membrane cannot be disputed in consideration of the recorded positive results of experiments.

After the bite of rabid animals the disease follows only in a portion of the cases, hardly more than half. As already mentioned there is a possibility that the contagion will not reach the wound or may be carried a way by bleeding; besides, the virus may be removed or destroyed by means of immediate clisinfection. Probably, in many cases, the rabies virus is destroyed without causing the disease after having entered the body. In favor of this possibility is the experience that bites from various animals are not of equal danger in the various parts of the body, and also that artificially infected susceptible animals do not always contract the disease, while a few cases ultimately recover. The animal organism is undoubtedly capable under certain circumstances of rendering the contagion ineffective, when the virus is present in small amount or is attenuated, and this power is decidedly increased by measures of immunization. Thus only can it be explained why barely $30-40 \%$ of dogs bitten by rabid animals eventually develop the disease. On the other hand it is possible that latent virus in the brain and spinal cord is stimulated to rapid increase by external influences, such as physical exertion, cold, etc., whereupon the disease which until this time had been latent, may develop within a short time (Marie, Remlinger).

Accorling to a compilation of Frielberger \& Fröhner, animals bitten by rabid dogs afterwards contract the disease as follows: According to Hertwig, $5 \%$; accorting to Haubner $40 \%$; accorring to the calculations of the Lyon school $20 \%$; according to those of the Alfort school 33\%, according to Röll, in Austria, in the years $1877-1887,40 \%$ of bitten horses, $50 \%$ of eattle and sheep, $36 \%$ of hogs, anil $20 \%$ of goats contracter the disease. As a general average $30 \%$ may be arlopted, although for cattle anıl sheep $50.60 \%$ will be reacher.

Regarding susceptibility to rabies there exists no considerable difference among the mammals. While the disease has been observed very frequently in certain species of animals, this may be explained satisfactorily by their mode of living, their manner of commingling, and by a temperament favorable to mutual infection. Therefore, the disease is observed most frequently in vagrant, vicious dogs, then in cats and wild carnivorous animals, and it is transferred to animals of this class by means of bites, while herbivorous animals infect each other only exceptionally.

The breed or the sex of the animal as well as climatic factors are without influence on the susceptibility, and age is a factor insofar as in young animals the outbreak of the disease will follow after a shorter period of incubation. According to Remlinger 1 to 2 months old puppies are very susceptible to rabies and the virus in their bodies is very virulent since it produces typical rabies by subdural and intraocular injection in 9 to 13 days. The more frequent appearance of the 
disease during summer time is sufficiently explained by the greater intercourse between the animals, and the same fact explains the frequent appearance of the disease in densely populated districts. In various individnals, however, great differences are noticed, as some animals, even dogs, resist every infection.

The relatively rare oceurrence of raloies in Constantinople was formerly explained by the supposition that the breed of dogs there had greater resistance against the contagion, that the disease there generally caused only paralytic symptoms, and that the rabies virus was attenuated. Remlinger found on the contrary that the disease follows its regular course, and that the infectious agent is very virulent. According to his opinion, the rarity of cases of rabies may be explained by the peculiar way of living and the distribution of the street dogs there. They are not running about the streets, but live in groups in certain streets or parts of streets, strictly watching their places of living, and when a strange dog appears it is at once chased away. When a dog develops rabies, it also remains in the street where it is born and has so far lived, while the healthy dogs by instinct avoid their diseased comrade, and, in case it shonld try to escape, it will be surrounded, barked at and compelled to withdraw to its sulking place. Only inexperienced young dogs come near it; therefore, in Constantinople, only young animals have been found with rabies, and human beings have been bitten by these dogs almost exclusively.

Pathogenesis. If the rabies virus becomes introduced in some part of the body through bites or artificial infection, it penetrates, as suggested already by the theoretical views of Duboue in 1879, as a rule along the nerve tracts of the involved part of the body towards the central nervous system usually without entering the circulation, and from there it is distributed only gradually. The researches of Vestea \& Zagari show that after the inoculation of virus into a nerve of the hip of a rabbit the paralysis will first appear in the hind leg, and from there proceed forwards, while after the inoculation into a nerve of the foreleg or under the meninges, a reverse process will be ohserved. If the nerve connecting an extremity with the spinal cord is severed, the subsequent injection of rabies virus into the respective limb will not produce the disease (Nicolas). If, before the inoculation into a peripheral nerve, the spinal cord is cut through in its entire breadth, only the portion connected with the infected nerves up to the place of division becomes virulent, but not the part on the other side. The results of the inoculation experiments have been confirmed by the histological researches of Schaffer, who always found intensive changes in the portions of the spinal cord which were connected with the nerves coming from the place of the bite. If the disease occasionally develops after injection into the blood stream or into the peritoneal cavity, the explanation is that simultaneously a nerve was also injured.

The virus which has reached the central nervous system, also moves in centrifugal direction along the nerve tracts. Thus, when the disease has a rapid course, the virus will be contained only in the nerves connected with the place of infection and in the cerebral and spinal tissue, but when the course is slow, the virus after a certain time will disappear from the nerves 
of the infected extremity, and reappear in the nerves of the other side of the body (Roux, Högyes) undoubtedly after having passed the involved segment of the spinal cord. In the same way, proceeding in centrifugal direction, it reaches the acinous glands where the conditions for propagation are favorable; thus the tissues of the salivary glands first become virulent, and from there the virus invades the secretion of these glands, the saliva.

In the central nervous sristem, the virus exerts its pathogenic effect on the walls of the ressels, causing small-celled inflammatory foci in the nerve tissues, and on the other hand it attacks the nerve cells. The irritation of the nerve cells causes nervous excitement, such as disturbances in consciousness, increased reflex irritability, and, after involvement of the medulla oblongata, high temperature, polyuria, and diabetes. After a time however, the degeneration of the nerve cells leads to consecutive paralysis affecting finally the respiratory muscles which forms the immediate cause of death. The stage of irritation preceding the degeneration may be of a very short duration, and may be so little pronounced, especially in rabbits, that the disease will show symptoms of paralysis apparently from the start.

The virus of rabies probably exerts its pathogenic action through some poisonous chemical products, the character of which is yet unknown.

Anrep has produced out of the cerebral substances of rabid animals a very poisonous, alkaloidal principle, $0.0003-0.0004 \mathrm{~g}$. of which, injected subeutaneously, eaused lowering of the temperature, paralysis of the extremities, salivation, and death from suffocation. Udránszky has obtained a solution from the medulla oblongata, which, injected under the meninges of rabbits, killed the animals with symptoms of street rabies. Babes observed only the development of marasmus after injecting large quantities of brain emulsion of rabid animals, which had lost its infectiousness while, according to Galtier, this substance heated to 100 to $105^{\circ} \mathrm{C}$. produced serious toxemia in sheep, goats, and dogs (somnolence, weakness, paralysis). According to Aujeszky, the disease in rabbits injected intracranially lasts only a few hours, while in the experience of Remlinger rabbits injected with virus-free hrain filtrate, succumb sometimes in a short time with paralytical symptoms. (From such animals the disease cannot he transmitted.)

Very rarely does the virus pass from the female to the offspring; leaving a few positive experiments out of consideration (Kolesnikow, Perroncito \& Carità, Loir, Dammann \& Hasenkamp) the medulla oblongata of the offspring did not appear virulent (Pasteur, Celli, Zagari, Galtier, Rátz; Konrádi found it virulent but the virus therein was weakened).

Anatomical Changes. The post mortem examination of an animal infected with rabies does not show any characteristic changes. Besides the hanging lower jaw, in carnivorous animals and especially in dogs, the stomach is contracted and empty, 
or instead of normal food products, it contains indigestible substances such as straw, hay, pieces of wool or bones, sand, stones, hair, feathers, pieces of leather, etc., the mucous membrane being injected and swollen, and the summits of the rugae studded with hemorrhages (Jolme) and shallow erosions. Foreign bodies are occasionally found in the esophagus (Franke) or in the intestinal canal in which case the mucous membrane of the latter is likewise inflamed. In other species of animals, the stomach is as a rule empty or contains little food. The bladder is nearly empty, the urine as a rule containing sugar, and the blood being varnish-colored and fluid.

Tortley Axe found foreign bodies in $90 \%$ of 200 rabil dogs on which he performed post-mortem examinations during twenty years, while Galtier in the course of thirteen years found foreign bodies in only 657 cases out of 1,434 rabid animals autopsied ( 1,304 of which were dogs).

\section{The post-mortem examination may also show an acute} catarrh of the mucous membranes of the respiratory and digestive organs, hyperemia of the salivary glands and serous infiltration of their connective tissues, hyperemia of liver, spleen, and kidneys and finally inflanmmation or edema of the cerebral and spinal meninges as well of as the gray matter of the brain.

The ehanges in the eentral nervous system were the subject of investigation some time ago by Cantani, Zagari, Bahes, Csokor, Dexler, and others, and recently Schaffer studied the subject thoroughly in connection with six eases of hydrophohia in man. In the segment of the spinal cord where the nerves coming from the place of infection enter, in the perivascular lymph spaces, as well as in the walls of the ressels of the gray matter of the anterior cornn, in the vicinity of the central canal, and also along the connective tissue trabeculae of the white substance, Schaffer found cell infiltration, with either capillary or larger hemorrhages. These changes became less and less marked anteriorly and posteriorly; in the nerres passing from the point of infection toward the cord there was also an infiltration of small cells. In the nerve cells he found fibrillation, hraline and vacuolar degeneration, granular disintegration, and pigmentation atrophy of the cellular bodies; the white substance contained numerous amyloid granules. In the medulla oblongata, inmediately below the floor of the fourth ventricle, and especially around the origin of the $12 \mathrm{th}, 10 \mathrm{th}$, and $7 \mathrm{th}$ pairs of nerves, there were noted pronouncer? hyperemia, perivascular cell infiltration, and small hemorrhages, while the nerve cells showed signs of degeneration. In the brain no changes of importance were found, as a rule, excepting hyperemia, slight cellular infiltration, and rarely capillary hemorrhages.

Csokor and Dexler ascribe great diagnostic importance to the small scattered foci of inflammation in the brain, and to the small cellular lencocytic infiltration of the perivascular lymph spaces as constant lesions of rabies. Trolldenier, however, conchuled after the examination of 52 cases, that these lesions are absent in the brain and still more in the medulla oblongata (in the latter in $60 \%$ of the cases), and that, on the other hand, they may be found in the nervous system of dogs suffering from distemper and eancer.

Tan Gehuchten and Nélis found in the cerebro-spinal ganglia anit those of the sympathetic nerve, but especially in the plexus noilosus ragi as well as in the upper cervical ganglia of the svmpathicus, a cellular infiltration of the interstices, which later causes the sensitive nerve cells themselves to degenerate and atrophy, and in their place, inside of the inspisated enclothelial capsule, there appear enilothelial and small round cells (Fig. 74). In many cases the ganglion consists exclusively of dense connective tissue infiltrated with small cells, only once in a while atrophied nerve cells being observed. These changes are most marked in the ganglia of rabid logs, and less so in those of rabbits and of human beings.

In the salivary glands Elsenberg and Kossjökow have demonstrated cellular infiltration of the interacinous connective tissue, and partly also of the acini, as well as granular fatty degeneration of the glandular cells. 
Aceording to Kolesnikoff and Babes, there exists in the immediate neighborhood of the motor center of the merlulla oblongata and the motor cells of the spinal cord, an aceumulation of small round cells, which also invaile the plasma of the nerve cells and cause degeneration and atrophy. Babes consiclers these foci as characteristic of rabies (rabic tubercles) as they were present in 389 cases of rabies and absent in 35 other cases, but, according to Bohl, such changes are sometimes also found in cases of distemper.

Symptoms. The period of incubation of rabies after a natural infection is generally sereral weeks, but may extend to some montlis. Its duration is influenced by the virulence of the virus, the distance of the place of infection from the central nervous system, and the age of the animal. Generally the first symptoms appear 2 to 8 weeks after the bite; sometimes, however, they occur as early as the end of

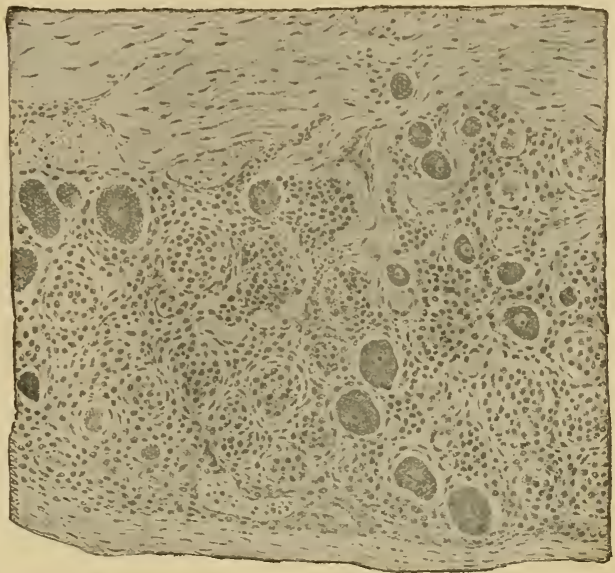

Fig. 74. Rabies. Cervical ganglion of a dog affecterl with rabies. The nerve cells are partly atrophied, and in their place clumps of round cells appear. (After Van Gehuchten \& Nélis). the second week or again only after several months and as late as 1 to 2 years. In dogs and swine the period of incubation is generally brief (frequently only 10 to 15 days); in horses and cattle it is longer (mostly 1 to 3 months). The disease develops earlier in young animals than in old ones.

Concerning the duration of the period of incubation, Bouley, Haubner, and Zündel have gathered numerous statistical data. According to a compilation by Röll, the periods of incubation were as follows: Dogs: according to Bouley (144 cases) in $43 \%$ less than 30 days, in $40 \%$ between 30 and 60 , in $14 \%$ between 60 and 90 days, and in $3 \%$ between 4 and 12 months. According to Haubner (out of 200 $\operatorname{dogs})$ in $83 \%$ less than 2 months, in $16 \% 9$ to 12 weeks, in $1 \% 13$ to 16 weeks, and in only a few 7, 8, and 24 months. Horses: Accorling to Bouley (out of 23 horses) in 1 more than 1 month, in 11 from $21 \%$ to 3 months, in 3 more than 100 days; according to Zündel (out of 121 eases) in $28 \%$ less than 1, in $46 \%$ less than 2, in $9 \%$ less than 3 months, and in the rest more than 3 months. Cattle: According to Zündel (out of 579 animals), in $5 \%$ less than 15 days, in $23 \% 15$ to 30 , in $39 \% 30$ to 45 , in $13 \%$, 45 to 60 days, in $17 \% 3$ to 6 months, and in 1 ease each 42 and 95 weeks; accorling to Hauber (out of 234 cases), in $82 \%$ less than 3 months, and in $18 \% 4$ to 9 months. Sheep and Goats: According to Zündel (out of 188 sheep), in $6 \%$ less than 15 days, in $41 \% 15$ to 21 , in $10 \%$ 21 to 30 , in $26 \% 30$ to 45 , in $2 \% 45$ to 60 days, and in $7 \%$ still longer. Out of 20 goats, in $15 \%$ less than 20 , in $60 \% 20$ to 60 days, and in $25 \%$ longer. Swine: According to Zündel (out of 123 hogs), in $3 \%$ less thán 8 (?), in $8 \%$ 8 to 15 , in $51 \% 15$ to 30 , in $27 \% 30$ to 60 days, and in $11 \%$ still longer. According to the observations of the official Prussian veterinarian, the average period of 
inculation is: in horses $461 / 2$, in eattle 70 , in dogs 22 , in swine 26 , in goats 14 , and in sheep $261 / 2$ days. Finally, schüder found ont of 395 carefully observed cases of rabies $21(5.3 \%)$ where the period of incubation was 3 to 7 months.

Unusually long periods of incubation have been observed: in horses, by Saikowitsch 17 months, by Virág 18, Cosswinter 20, Bahr 21, Swain 25 months; in eattle, by Szabó 323 days (bite in the snout), Mieckeley 327 days, Leipert 19 montlıs and 21 days, and by Kalt 23 months. The reports relating to periods of more than 2 years, however, must be accepted with reservation, as a new infection may have occurred in the neantime.

After artificial infection, dogs develop the disease 13 to 19 days after subcutaneous injection, 10 to 15 days after subdural injection; rabbits show symptoms 12 to 21 days after subdural injection, but in exceptional eases later, occasionally only after 43 days. After a series of inoculations, the period of incubation following the subdural injection may be shortened to 5 days (see protective vaccination).

The clinical picture of rabies is on the whole the same in the various species of animals, and the only variations observed are owing to the fact that the symptoms are more or less subject to modifications in accordance with the individual temperament and the physical structure of the animal.

Rabies in Dogs. At the initial stage of the disease (stadium prodromorum or melancholicum), the animals show a peculiar change in behavior, which, particularly in house dogs and with the so-called "dumb rabies," is so inconspicuous that it is completely overlooked, and the owners associate with them as usual without suspecting anything. Most dogs become capricious; they are at times irritable and gloomy, and aroid all noise, hiding in dark places under or behind furniture, in a dark corner of the room, or in the stable. They obey the call of their master very unwillingly, but then jump about and fawn on him even more markedly than usual. In other cases they show a remarkable uneasiness, scratch with the fore feet, frequently change their place of rest, walking uneasily around in the yard or in the room, suddenly stopping, becoming attentive, and without any canse barking and biting at the air (so called "fly-catching"). If resting on the ground, they frequently become startled and attempt to catch imaginary flies, growling at the same time.

The reflex excitability is in this stage already decidedly increased. This is shown by the fact that the animals when approached in a friendly way, and still more when teased, become excited and snap at the caressing hand and any other objects or their companions. This excitability is particularly conspicuous toward strange persons and dogs, even when they are still friendly and familiar with their habitual attendants. The reflex excitability is still more noticeable in that the patients become startled or jump up frightened even on slight external causes, such as a strong light, sudden noises, touching of the body, etc. During such paroxysms there are also observed sudden but very transitory respiratory disturbances, spasm of the diaphragm and dilatation of the pupils. Many rlogs scratch and gnaw or bite at the place of infection or the cicatrix formed there. 
At the same time, a particular change of appetite or taste is observed, as the animals scarcely tonch their favorite food or let it drop again after having it in the mouth. Later they reject all food, although they will chew all objects within their reach, also their own excrements, and swallow them, if possible. It may then be noticed that the patients swallow with greater difficulty stretching the neck forward in swallowing, allowing the food to fall out of the mouth, and showing a desire to vomit or actually do vomit. At the same time, the patients repeatedly seek water, but in spite of all exertions they can swallow small amounts of it only with difficulty.

Sometimes there is also trouble with the voiding of excrement and urine, the animals frequently attempting it but with little or no results. Besides, they continually lick their own genitals or those of other dogs and otherwise show an increased sexual desire.

By this time salivation exists, which symptom subsequently becomes more and more marked.

In the second stage (stadium excitationis or acmes) which develops in $1 / 2$ to 3 days, the described symptoms attain a higher degree and the unrest and excitement, probably under the influence of hallucinations, increase to a violent rage. It is now particularly observed that the dogs frequently lick the earth furiously, chew any objects they find, tear them to pieces, and swallow them eagerly. They want to get away from their usual quarters; if shut up in a room, they show this desire by standing near and scratching at the door ; if tied, they try to break their chains; if in a cage, they try to bite the walls and hars. Once escaped, they will run aimlessly around, and sometimes cover long distances without resting. They scarcely ever return to their homes, but if they do happen to come back, they are usually exhausted, covered with wounds of bites, and with hair disheveled. For a while they crawl about dejectedly fearing punishment and fawning upon their master, or exhausted hide away and rest. If the rabid animals on their journey encounter other dogs, they will attack them without cause or noise, and, if possible, bite them in the head (healthy dogs running around in strange places are mostly scared and run away from other dogs). If the attacked dog rums away in fright, the diseased animal will continue its journey, but if it resists, the rabid dog will fight furiously, and it will be observed that, while the attacked dog howls, snarls, and barks, the diseased animal will hardly emit a sound. The desire to bite and the aggressive behavior, are shown toward other species, particularly small animals though less conspicuously, and in a herd of sheep or swine a rabid dog may in a brief time wound many animals. Human beings are less in danger, as rabid dogs mostly avoid them and as a rule only attack them when irritated or excited by threats.

In dogs shut up in eages rabies generally appears in 
paroxysms. The dog, his eyes bloodshot, jumps sidewise against the bars and grabs them between his teeth with such violence that one or more teeth are broken; or he takes the straw on the floor, the excrement or any other object, and shakes it furiously around, always without barking or growling. If approached with a stick or a bar and teased, the dog will make a mad attack, hold the stick or bar between its teeth, and shake it. The nature of the ohject held out is alssolutely immaterial, as the dog will bite red lot iron or burning coal withont hesitation. Some animals even bite their own bodies and tear the flesh to the bone.

Such attacks of rage are interrupted by shorter or longer periods of depression. After an animal lias been wandering aimlessly around for a time or, if shut up, has been raging, it finally falls down exhausted and remains unconscious on the floor breathing with difficulty. Soon it will rise, remain standing at one place with staring eyes, dilated pupils, and a peculiarly changed expression of the face indicating cumning and fright (Fig. 75 and 76 ), until, owing to some outside influence, such as the sight of other animals or some object, but sometimes without any visible cause. the attack breaks out anew.

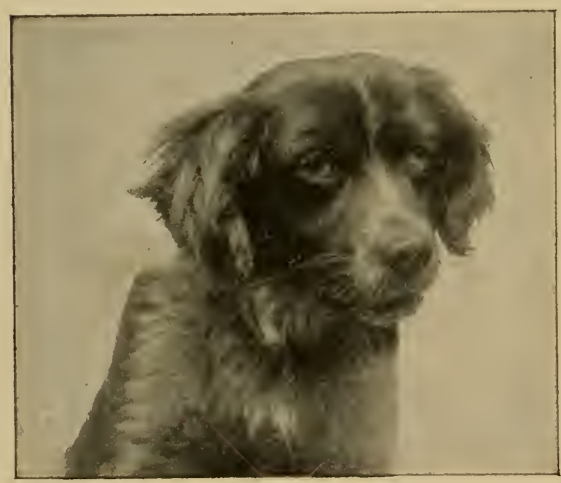

Fig. 75. Rubies. Characteristic peculiar watchful and distmbed expression of the face, with an outparalysis of the masticatory muscles. ward position of the eyes; at the same time

In this stage, as a lule, symptoms of paralysis of some of the nerves appear. Most conspicuons is the peculiar clange in voice owing to a paralysis of the laryngal II e rves or muscles, which causes the bark to become hoarse and double and accompanied by loud, long drawn howls. This hoarse barking, which is so characteristic that the trained ear may suspect a case of rabies from it alone, is emitted frequently by many dogs, by others only when they are excited. Swallowing becomes very difficult owing to the progressive inflammation, and later to the degeneration of the 11 th and 12th pair of nerves. The regular food is wholly rejected, and the animal is at the best able to swallow only small amounts of water with difficulty. These exertions, sometimes even taking an object in the mouth, may produce violent 
attacks of suffocation and even a raljies paroxysm by reflex action from the mucous membrane of the mouth and throat. Therefore, animals thus affected do not touch food or water at all. In rare cases, the mere offer of water is sufficient to cause an attack (hence the term hydrophobia), which is only the result of the difficulty in swallowing, and, on the whole, is only observed in a portion of the cases.

At this stage, salivation increases, partly because of the more abunclant secretion of saliva, and partly owing to the existing difficulties in swallowing. Difficulty in defecation and urination is also frequently observed.

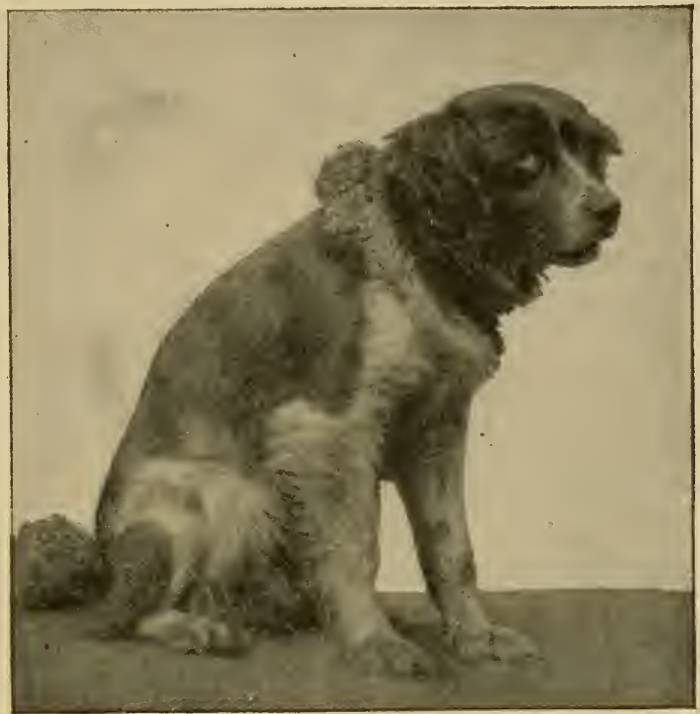

Fig. 76. Rabics. The same dog as in Fig. 75.

As an extraordinary symptom, Nicolas ohserved spasmodic movements at this stage (pushing forward and moving in circles) in 3 dogs, and also in a goat that had been infected intraocularly.

The stage of irritation generally lasts 3 to 4 days and is then followed by the paralytic stage (stadium paralyseos or depressionis). The attacks of rage become less violent and also less frequent, but in the meantime symptoms of insensibility and dullness may appear. As a result the symptoms of an already' existing paralysis become more conspicuous, and other parts of the borly may also become paralyzed.

As the changes in the medulla and cranial nerves already 
mentioned also affect the other nerves, there develops besides total aphony and inability to swallow, a partial and shortly a total paralysis of the muscles of the lower jaw, tongue, and eyes. The mouth is kept open most, if not all the time, the dry and livid tongue hangs out of the mouth paralyzed, and the saliva flows in long threads from the lips. Owing to the irregular position of the eyes the animals squint, the cornea is dull and lusterless and the pupils are dilated. These symptoms give the face a peculiar cunning and troubled expression (Fig. 75 and 76 ).

Owing to the process of degeneration in the spinal cord, paralysis of the muscles of the tail and the extremities appears. The hind limbs are generally first attacked, which causes the animal to stagger and tumble, at first when running and later when walking, and to drag the hind legs along the ground. The paralysis then progresses to the tail, which hangs down between the legs. The paralysis of the hind limbs finally reaches a stage which makes it impossible for the animals to move and they remain recumbent on the ground; if urged to rise they will make only a few fruitless attempts with the fore legs.

Before the disease has reached this stage, the animals are already quite emaciated; the exhaustion now increases rapidly, and the patients succumb within a brief time, usually in convulsions.

Owing to the danger connected with intimate association with the animals little is known of those symptoms which can only be determined by a close examination. As to the temperature, Hertwig and Monin have found that it rises 1 to 3 degrees during the maniacal stage, but that during the paralytic stage it falls down to several degrees below normal. The pulse is rapid and tense from the beginning (Blaine). In the blood the polynuclear lencocytes are multiplied, and the hyperlencocytosis increases toward the end of the disease (Courmont and Lesieur). The urine usually contains sugar (Nocard; Rabieaux \& Nicolas found sugar in the urine in 33 out of 44 examined cases).

In artificially infected dogs the temperature is increased during the initial stage for $1 / 2$ to 3 days, but afterwards it is low; in rabbits infected with street rabies on the contrary the temperature commences to fall at the onset of the disease and continues thus until the death of the animal (Löte). In human beings, the symptoms of the stage of irritation are accompanied by a moderate rise in temperature, with morning and evening remissions, but before death the temperature becomes very high and may continue to be high for some time after death Moravesik, Schaffer).

The described clinical picture of rabies in $\log s$ is in some cases subject to considerable modification. Above all, variations occur in the duration of the various stages and in their mutual relations, the several stages being briefer than here stated, or longer at the expense of the others. This is especially true of the second stage, that of irritation, which is often very 
short and indicated by few conspicuous symptoms of excitement; then not infrequently the melancholic stage is followed almost without interruption by the paralytic symptoms, particularly paralysis of the muscles of the jaw and throat, to which is then added the paralysis of the hind parts of the body. This form of the disease, during the course of which most patients are weak from the beginning and neither bark nor bite, and which is generally fatal in 3 to 4 days, is usually called dumb rabies in contrast to the typical violent rabies.

The cause of the differences in the course of the disease is not exactly known, but it seems in some way to be connected with the species and earlier life of the animals. Experience teaches that symptoms of violent excitement are particularly observed in biting dogs of a lively temperament living in the open, while gentle house dogs, in which aggressiveness and the desire to bite appear more rarely and less conspicuonsly, are attacked more frequently by dumb rabies (according to Hjortland, in Greenland the disease nearly always assumes the form of dumb rabies).

Rabies in cats shows the same symptoms as in dogs, but, these animals being of a less conficling nature, generally seek dark corners in the beginning of the disease and do not leave them any more or they show by continuous mewing a more aggressive hehavior and a desire to escape. Then they attack both persons and animals, including dogs, which come in their way, jumping at their faces or biting their legs from behind, inflicting severe wounds with their teeth and claws. Ainslie observed in one case that the cat jumped at ohjects held before it, did not lap water, but was attacked with violent spasms when sprinkled with water. Later the voice becomes hoarse with a peculiar howling tone owing to paralysis of the vocal cords, and 2 to 4 days after the appearance of the first svmptoms, paralysis of the hind parts appears.

In artificially infected eats, Dammann and Hasenkamp first observed great unrest, fright and a desire to take hold of strange objects; later the movements became staggering, and finally total paralysis of the hind extremities appeared (but not of the masseter muscles or the constrictor muscles of the anns or bladder). In the beginning very little water and no food was consumed; the animals bit furiously at a stick held before them mewing and spitting at the same time, and when sprinkled with water they fell into violent convulsions.

In horses, the increased sensitiveness of the scar formed at the point of infection (mostly the lips, nostrils or front extremities) often gives the first indication of the beginning disease. The animals gnaw at the site of the bite on the body or rub it against objects, which behavior in otherwise quiet horses, particularly if they have been bitten several weeks previously by a $\mathrm{dog}$, arouses a reasonable suspicion of an approaching attack of rabies. The increased reflex excitability is also indicated early 
hy fright and unrest, which appear on the slightest provocation. They change their position every moment, paw with their feet, gnaw at the manger and other objects near them, and show staring, restless eyes with dilated pupils; at times, they draw the lips convulsively up and backward so that the teeth are visible, while foamy saliva runs from the corners of the mouth.

With increasing restlessness some horses also show an aggressive behavior toward other horses or human beings, and still more toward dogs, which they attempt to bite and kick. They will furiously attack a stick held before them, and sometimes in biting the manger will show strengtli enongh to break several teeth and even the neck of the inferior maxillary bone. Many patients do not spare their own bodies, but tear off large pieces of the skin; as a rule, however, such-rage is mot; observer. More frequently attacks of spasms lasting 1 to 2 minutes are observed in the masseter and respiratory muscles. Further the appearance of increased sexual desire causes male animals to have erections and to eject semen involuntarily, while mares behave as when in heat; in connection herewith is also observer a frequent resire to urinate. (Göhre once found a temperature of $39.3^{\circ}$ C.)

Soon the symptoms of paralysis become marked, first in the region of the nerves originating from the medulla oblongata. On this account swallowing is difficult and liquids are returned from the mouth through the nostrils. Owing to the change of taste, the animals eat nothing or at best only indigestible substances, sometimes even their own excrements. They will smell of the water offered them, but will not drink it, and with raised hear draw the lips upward as if attempting to yawn. When neighing, a hoarseness of the voice is observed.

Later the symptoms of excitement hecome less frequent and less severe, while indications of severe paralysis develop, as a result of which the patients fall to the ground and finally suecumb on the 3rd or 4th day of the disease, in most cases with violent convulsions.

Darmagnae ohserved aseending paresis in one case 4 months after a bite in one of the hind legs. First there was paresis of the same extremity, then paraparesis with hyperesthesia of the hind quarters, and finally, paralysis of the muscles of deglutition; the animal died after 3 days of sickness.

Cattle also show unrest and excitement as well as an aggressive tendency, which behavior, so uncommon except in cattle raised in the open, at once attracts the attention of the attendant. In such cases the animals remain standing at one place, at times drawing the upper lip backward, tearing up the earth with feet and horns, looking around with the head high, the eyes wild or staring, and finally furiously attacking the other animals of the 
herd and even human beings, but particularly dogs which happen to be near. Animals tied in the stable attempt to get loose from the chains, jump up suddenly from their resting place, shake the head, rise on the hind legs, bite at and climb into the manger and run the head against the wall sometimes with enough force to break the horns. Such outbreaks of rage are interupted by nearly normal pauses and are then repeated at first every 20 to 30 minutes, but later at increasingly longer intervals.

The other symptoms are similar to those in other animals: the increased reflex excitability, licking or biting the place of the bite or its scar, hoarseness, sometimes rapid bellowing which the animals may emit for hours without interruption, salivation and foaming, gritting of the teeth, difficulty in swallowing, loss of appetite, bloating (owing to the paralysis of the intestinal muscles ?), cessation of rumination, constipation with following diarrhea and tenesmus, drying up of the milk, convulsions in some groups of muscles (through spasmodic convulsions of the muscles of the neck the head is sometimes turned to one side), increased sexual desile, tenderness of the wrethra on pressure throngh the rectum (Bézaguet), sugar in the urine (Nocard, Nicolas, and Rabieanx), rapid emaciation, exhanstion, and extended paralysis, until finally the animal succumbs on the 3 rd to 6 th day of its illness. Similar symptoms have also been observed in buffaloes (Esser).

In other cases the symptoms of irritation are only slightly marked or entirely absent, but there appear instead early weakness of the hind extremities and marked downward curving of the spine, accompanied by moderate bloating, difficulty in swallowing, and constipation. In walking or in raising the head the animals fall down readily, and then kick with their feet, they hreathe laboriously and discharge foamy saliva from the mouth: standing up they emit a painful bellowing, move stiffly, smell at the hay and drinking water but consume nothing or let the water inn out again through the mouth or the nose, while they make constant movements of mastication. The next day they lie groaning on their chest or with the head resting on the shoulder, while spasmodic convulsions appear in the diaphragm and in other groups of muscles, and death follows mostly at the end of the first week with symptoms of bulbar paralysis and rapid lowering of the temperature.

Nessl ohserved 9 cases of rabies in cattle which were conspicuous by the fact that the initial symptom was vomiting while the typical paralytic symptoms were absent. Heks saw in cows a tendency to press against ohjects and to move around in a circle.

Intermittent rabies was observed by Galtier in two dogs and by Lignières in one. Following the first attack there was a remarkable improvement, but after 36,27 and 28 days respectively, a considerable change for the worse occurred with fatal results.

Vol. 1-31 
In sheep and goats rabies appears with quite similar symptoms, except that the stage of irritation is often absent or of very brief duration. If such a stage develops, the unrest (stamping with one front foot, running to and fro, climbing the wall of the stable or the fence, etc.) and aggressive behavior toward their herd mates or toward dogs are the more remarkable in sheep as these animals otherwise are shy and easily scared. In goats the symptoms of irritation are always more violent; they become very uneasy and pugnacious, run against the walls, other objects, animals, and persons. Female animals butt and bite their own kids, while in bucks a remarkably increased sexual desire is observed. In goats sugar has been found in the urine (Poreher).

The disease usually lasts from 3 to 5 days and ends with symptoms of paralysis.

In swine the affection begins nearly always with very decided symptoms of irritation. The animals run around in all directions, grunt hoarsely, root the litter and the earth, gnaw and rub the site of the bite, then hide under litter and remain quiet for a while or continue to grunt while lying down. If they are aroused by a noise or when touched, they jump up and run aimlessly around, attacking other swine, their own young, other animals, and even human beings. While rooting, they swallow various substances and saliva runs in abundance from the mouth. They seek water, but can only drink with difficulty. Young pigs sometimes fall down while running around, kneel on their front legs, lay the neck on the ground, and, in this position, push their bodies in a straight line 25 or 30 feet forward or move around in circles in this same position (Papp).

The other symptoms are, on the whole, similar to those in dogs. After the appearance of paralysis, the animal succumbs on the 2nd to 4 th day of the disease, but sometimes on the first day.

According to observations of veterinarians (Zürn, Gibier) the disease begins in fowl also with conspicuons fright and unrest, when the animals run around with ruffled feathers, frightened look, and hoarse cries, and attack the healthy birds, other animals, and also human beings with their heaks and claws, until they finally succumb after 2 to 3 days with symptoms of paralysis.

After intracranial injection, Galtier, Pasteur and Löte observed only dullness and paresis of the feet and neck, when the animals would awkwardly pick up grains of corn and with staggering gait would now and then tumble down. In the course of the disease improvement was often observed and the infected birds not infrequently recovered.

Course and Prognosis. The disease generally has a course of 4 to 7 days; in some cases it last only 3 days, but in exceptional cases may last 11 to 13 days (Nicolas).

The duration of the various stages and their mutual relation, leaving out of consideration the fact that they cannot be 
defined exactly, appear unlike in different cases. Increased excitability is also present in the first stage, and most of the symptoms differ only in intensity from those of the second stage. In the latter stage the desire to bite and the aggressive conduct, when these symptoms are present, are the most characteristic. Just as indistinct is the transition from the second to the third stage since, on the one hand, partial paralysis may be apparent in the stage of irritation and, on the other hand, symptoms of irritation, and particularly cases of suffocation are not uncommonly observed in the stage of paralysis. The more rapid the course of the disease, the more rapidly the symptoms change and intermingle, wherefore it is nearly impossible to make an exact distinction between the various stages in the course of the affection. In cases of dumb rabies striking symptoms of irritation are absent.

The disease is, with very few exceptions, fatal. The possibility of recovery has been proved in cases where artificially infected dogs have recovered from the typical disease (Pasteur, Roux, Högyes, Remlinger, and other's). Consequently we must not conclude that the bite is not infectious from the fact that the biting dog has remained alive, but this opinion shonld only be reached if, during the two weeks following the bite, no suspicious symptoms are observed in the animal.

Pasteur observed recovery from rabies in dogs; later Högyes noticed that out of 159 dogs, which had contracted rabies in different ways, 13 or $8.1 \%$ recovered. Of these 6 were not treated at all, while the remaining 7 were given the antirabies vaccination treatment. Remlinger found the saliva of a cured dog virulent 20 days after the begimming of the disease and 5 days after recovery.

Cases where dogs were still alive while persons bitten by them had already died from rabies, have been reported by Talko and Johne.

Diagnosis. The establishment of the diagnosis is not connected with any particular difficulties in cases where the various stages are distinctly separated and there has been an opportunity to observe the whole course of the disease or at least the greater part of it. An exact diagnosis being extremely important and necessary, particularly in cases where human beings or other animals have been bitten by suspected animals, it is advisable not to kill the suspected animals, but to keep them under strict observation and await their natural death. The symptoms of irritation, the aggressive conduct and subsequent paralysis, particularly the appearance of bulbar paralysis form a very characteristic picture of the disease, and the diagnosis is made easier by the existing suspicion of infection. Statements to the effect that no bite has been received must be taken with reservation, as light wounds are inflicted not infrequently without the knowledge of the owner. In making the diagnosis the former behavior and temperament of the animal must be taken into consideration. 
If no reliable history of the case is available or if it has only been possible to have the animal under observation for a short time, the diagnosis may present considerable difficulty. From a practical point of view every animal in which a sudden change of conduct is observed should be considered as suspicious and only declared unaffected when for 3 to 4 days no symptoms appear to confirm the suspicion.

Differential Diagnosis. Diseases more or less similar to rabies are observed, especially in $\log s$, and they may cause confusion, particularly if symptoms of nervous excitement are present. Thus, a violent irritability, sometimes connected with aggressive conduct and a desire to bite, may appear as a result of acute inflammatory processes of the meninges and the cerebral substance. Here belongs the acute cerebral hyperemia as well as acute meningitis and encephalitis without regard to whether these diseases occur from unknown causes or outside influences, or are caused by animal parasites or tumors existing in the brain. In such instances the further course of the disease will decide, as cases of paralysis in other diseases are not developed in the same sequence and uniformity as in rabies. Eclampsia and epilepsy are characterized by clonic spasms all over the body while in epilepsy there is also the clear conciousness between the attacks. Nervous distemper is characterized by the simultaneous symptoms in the eyes and respiratory organs, as well as by distemper exanthema.

Repeated or continuous pains in some parts of the body may irritate the animal to a degree which will cause attacks of frenzy or delirium that are sometimes confounded with rabies. To these belong the acute inflammation of the intestines caused by intestinal worms, particularly tenia echinococcus, foreign bodies, poisoning, perforation of the intestines, as well as many cases of colic with violent symptoms in horses; further, dog diseases caused by pentastomum tenioides, eustrongylus gigas, and spiroptera sanguinolenta, and finally dermanyssus mites in the external auditory canal of cattle, scabies mites in the ears of dogs, as well as muscular rheumatism. In the course of these diseases no paralysis appears, but the clinical aspects of the disease may, owing to the exhaustion and comatose condition on approaching death, he very similar to rabies sometimes to a degree that makes an autopsy necessary to determine the real nature of the disease.

After lodgement of foreign bodies in the pharynx or between the teeth of $\log s$ and cats, rabies may be suspected, because the animals show restlessness, and, owing to difficulty in swallowing and to stomatitis, saliva is abundant and the voice is hoarse from the perilaryngeal edema. On account of difficulty in moving the lower jaw the mouth is kept continuously open, which condition is very similar to the paralysis of the masseter muscles. In such cases, as well as in the apparent paralysis 
resulting from dislocation of the inferior maxilla, the mouth is continuously open, while in rabies the jaw moves slightly up and. down but only in limited degree. Finally a trigeminal or facial paralysis sometimes develops independently or in the course of distemper; however, in such cases the total absence of other symptoms of paralysis, especially in the hind limbs, is against rabies.

In cats, dogs, and cattle, perhaps also in other animals, rabies is easily confounded with paralysis bulbaris infectiosa, which disease is to be considered especially when the animals rub and gnaw at the place of the bite (see p. 496).

Finally, owing to confinement of hitherto free animals, to the lack of opportunity for copulation, nymphomania and satyriasis, removal of the young from their dams, as well as in many acute infectious diseases, attacks of frenzy may occur, but they may be readily distinguished from rabies by proper observation of the accompanying conditions, particularly the absence of paralytic symptoms.

As to the post-mortem diagnosis, the absence of normal food and the presence of foreign bodies in the stomach form the only finding that is in any way characteristic, but even this does not afford any real proof, because, in exceptional cases, foreign hodies are also found in the stomachs of dogs not suffering from rabies, especially in young dogs and in those with digestive troubles (in the latter the bodies are usually found with normal food). On the other hand, the presence of food substances in the stomach does not by any means exclude the possibility of rabies. The presence of sugar in the urine strongly supports the diagnosis of rabies, but even this does not constitute a full proof, because sugar is also found in the urine in other diseases. The difficulty of diagnosis is enhanced by the fact that many rabid animals are killed before the complete development of the symptoms, so that even partially characteristic changes have no time to develop.

The existence of rabies is established definitely by animal experiments and also by microscopical examination of the brain, at least if the results are positive.

Complement fixation with nerve substance as antigen, has not yet succeeded in showing the presence of the complement fixing amboceptors in the serum of immunized or rabid animals (Centanni, Heller \& Tomarkin, Friedberger).

Microscopical Diagnosis. In this connection the detection of Negri bodies has a very high diagnostic value, while the examination of the nerve ganglia gives less certain results.

1. Detection of Negri Bodies. The numerous experiments following the discovery of these formations (see p. 465) have shown uniformly that they are only present in animals or human beings suffering from rabies, and that, through their detection, the diagnosis of rabies may be definitely established. On the other hand, they may not be present in the initial stage of the disease, and rabid animals are often killed shortly after the affection develops; therefore the negative result 
of the mieroscopical examination does not exclude a diagnosis of rabies. In the case of animals which have died from rabies or have been killed at an advanced stage of the disease, the microscopical examination of the brain (cornu ammonis, medulla oblongata, cerehellum) with positive results right after the autopsy, makes an exact diagnosis possible, and in such cases one may also conclude from a negative result that rabies has not been present. In the latter ease, if human beings have been bitten by the animal, the final diagnosis should be made subject to the results of inoculations into animals.

Negri examined nicroseopically 75 brains of animals suspecter of rabies; in 50 cases the results were positive, and the liagnosis was always confirmed by the biological method, while in 5 out of 72 cases with negative results the inoculation experiments resulted positively. Accorling to Luzzani, up to the year 1905,457 brains of animals had heen examined by Italian students for Negri bodies; out of these 297 were proved positive throngh inoculation, and in only 9 of this number were there no Negri bodies found, while Krajuschkin in 4 ont of 34 , Nicolas in 7 ont of 61 , Nélis in 2 out of 61 , and Bohne in 10 out of 109 cases of estal)]ished rabies did not succeed in detecting Negri bodies (according to Ball, the number of such non-confirmed findings amounts to about $3.5 \%$ ). At the Berlin institute for infectious diseases, Lentz on applying the method of Mann found Negri bodies 370 times or in $86.4 \%$, and, on applying his own method, in 52 ont of 5.5 or in $94.5 \%$. Rátz, at Budapest, saw the histological findings confirner by animal experiments in $25 \mathrm{~s}$ cases. Hart, at Vienna, found hoth methods to agree in 498 cases while in 22 cases the histological examination gave negative, the inoculation of animals positive results.

All students agree that Negri bodies appear exclusively in cases of rabies, and in none of the many cases examined were Negri bodies found without the inoculation experiments confirming the presence of rabies.

Technique. For the examination the cornu ammonis is most suitable and secondly the cerebellum; but in cats the cerebellum is more satisfactorily examined, because, according to Luzzani, in the cornu ammonis of normal cats formations sometimes appear which in stained preparations are very like Negri bodies in their initial stage. Besides, Standfuss calls attention to the fact that, particularly with nervous distemper in dogs, ganglion cells abont to degenerate may contain red colored granules; further, that the karyosome, which niay he donble has a similar structure and also may be found ontside of the miclens. "Distempercorpuseles" have also heen oliserved by Lentz, partly in the tissues, partly in highly decomposed ganglion cells; according to his opinion, they originate from the conglomeration of the chromatin substance, and are different from Negri boclies in that they contain no inner granules.

[At the present time the most suitable method of quickly demonstrating Negri borlies is by Frothingham's impression preparations, stainerl according to Tan Gieson. A transverse section is cut from the Ammon's horn and placed upon a block of wood near the edge, thus permitting a slide to be pressed evenly upon it. By lifting the slide with a quick motion, an impression of the cut surface of the organ remains on the glass, and, as the histological structure is preserved, an excellent substitute for a microscopic section is obtained. At least four such impressions should be made on one slide, increasing the pressure for each preparation until the tissue becomes quite flat. Before the preparations have dried in the air; fix in methyl alcohol for one half ninute, or longer; drain off the alcoliol and immediately cover the slide with Van Gieson's stain heating gently for one-half to one minute; then wash in water, dry with filter paper and examine withont a cover glass. The Negri bodies stain a pinkish to purplish red, their inner structure blue. The nerve cells are blue and the red blood corpuscles colorless or yellowish. Van Gieson's stain consists of $10 \mathrm{cc}$. distilled water, two drops of a saturated alcoholic solution of rose aniline violet, and one drop of saturated aqueons solntion of methylene b]ue; as modified by Frothingham: 20 ce. of tap water, three drops saturated alcoholic solution of basic fuchsin, and one drop saturated aqueous solution of methylene blue. This stain remains effective for abont twenty-four hours. Trs.]

Very pretty pictures, particularly suitable for an exact stuily of the structure, are given in cover glass preparations and in sections by the staining methocls of Mann, Bohne, and Lentz.

2. Detection of Changes in the Nerve Ganglia. The changes in the ganglia of the peripheral nerves described by van Gehuchten \& 
Nélis, especially in Gl. nodosum, in Gl. cervicale supremum, and in Gl. Gasseri (see p. 472), which are only conspicuous when the animals have died from street rabies, merely justify a diagnosis of suspected rabies, because, according to investigations by Bohl, McCarthy \& Ravenel, Frothingham, and others, they are also present in other infectious diseases. Besides, according to Manquélian and Vallee, the ganglia of old dogs show a similar microscopic picture; Raymond, however, found that both in nervous distemper and in healthy old dogs the small cellular infiltration was considerably less pronounced, while the nerve cells showed normal nuclei and no migratory round cells were contained in the perivascular spaces. Therefore, this method may still render good service, particularly in cases where suspicion of rabies already exists, and when the detection of Negri bodies is not successful.

The examination always requires the preparation of regularly stainer sections, but may be finished in 12 to 24 hours by means of the aceton-paraffin imbedding method.

Diagnostic Inoculations. If material from the animal is successful in producing rabies artificially in another animal, there ean be no doubt that the former was infected with rabies. For inoculation purposes the medulla oblongata of the suspected animal is most suitable, from the center of which a small piece is excised with sterilized instruments, carefully rubbed into an emulsion with boiled water and then filtered through clean, fine, linen eloth. For test animals rabbits and dogs are most suitable, but guinea pigs (Konrádi), mice and rats (Fermi) may also be used.

The subdural injection (Pasteur) is performed in rabbits by trephining one of the parietal bones of the animal with a small trepan (diameter 5-6 mm.), ancl then 1-2 drops of the previously prepared emulsion is injected between the dura and the surface of the brain by means of a hypodermic needle which is bent in a right angle. The opening is then covered with the skin, and the edges of the wound are united by means of sutnres.

For the intracerebral injection (Leclainche), a small opening is made in the cranium by means of a fine drill, and $1 / \mathrm{s}^{-1 / 4} \mathrm{ce}$. of the above named emulsion is injected about $1 \mathrm{~cm}$. deep into the brain with a straight neetle.

The intraocular injection (Gibier, Nocard, Johne) recommenis itself owing to its simplicity. The cornea which previously has been anesthetized by the application of cocaine (10-20 drops of a $2-5 \%$ solution), or acoin ( $0.1 \%$ solntion), is perforated with the straight needle, and 1-2 drops of the emulsion is injected into the anterior ocular chamber.

In the intramuscular injection which also has stood the test (HeIman, Klimmer), $1.0 \mathrm{ce}$. of the emulsion is injected into the museles of the legs or shoulders of a rabbit or guinea pig. This method is particularly to be preferred in eases where the brain substance is not fresh, or the purity of the injection material is questionable for some reason or other.

For the same purpose Lebell recommends the injection under the meninges of the spinal cord; Galli-Valerio mentions the injection into the cranial cavity through the foramen occipitale magnum, or rubbing the infecter material into the mucous membrane of the nose by means of a piece of cotton fastened to a wire (according to Konrádi, the perior of incubation in this case is sometimes very long). Dawson and Oshida recommend an injection in the cranial eavity through the foramen opticum (inserted beside the inner angle of the eye, along the wall of the ocular eavity); finally Szpilmann recommends the injection under the conjunctiva (Högyes, however, found this proceeding mostly ineffective). The subcutaneous and the intravenous injections are very unreliable, and therefore they are not to be recommended. It is also advisable to inject at least 2 animals by the above mentioned methods, because sometimes the injection of the same virulent material does not affect every animal.

The methorls just deseribed are easy to adopt in general veterinary practice, owing to their simplicity; if, however, the laboratory of an institution has been secured for this purpose, the test material must be sent there in a condition fit for 
inoculation. For this purpose, the medulla oblongata, the cerebellum, and the cornu ammonis, that is the whole nucleus of the brain, or the whole brain, are to be preserved in $50 \%$ glycerin, or the whole head is separated from the trunk and sent unopened.

Injections with infectious material by the four first methods named result, as a rule, in producing rahies within 2 to 3 weeks, but sometimes the period of incubation is longer. Aujesky observed a period of incuhation of 43 days after subdural injection, Gal one of 51 day's after intraoeular injection, and Klimmer one of 235 days after intramuscular injection. This relatively long period reduces the practical value of the method, as it does not permit the use of protective vaccination on bitten human beings who depend on the results of the diagnostic inoculation. Besides while the positive result of the inoculation surely indicates the presence of rahies, a negative result does not in the same way preclude its presence; in addition the rabbits may die from septicemia when the technique or the material has not been quite clean. Unclean or putrid material, after a previous dilution with physiological solution of sodium ehloride $(1: 250)$, may be elcansed by filtration through a Berkefeld filter, and with such filtrates a positive result may be obtained with tissue kept for 63 days (Mazzei).

In rabbits inoculated with positive results, the disease is, as a rule, attended with symptoms of paralysis (see p. 467), and in dogs it takes the usual course. According to Beck, rabhits are also killed within 1 to 2 weeks hy subdural injections of cerehral substance of dogs which have died from nervous distemper, in which cases, however, the intestines and bladder are also paralyzed, and the disease cannot be transmitted further from one rahbit to another. In paralysis bulbaris infectiosa (see p. 495), the inoculated rabhits violently scratch and rub the place of injection, and as a rule hecome sick after 2 to 4 days, or at the latest, after 8 days.

Treatment. Rabies which is already developed cannot be arrested or changed in its course.

More successful is the treatment of wounds from bites for the purpose of preventing the future derelopment of rabies, by destroying the infections saliva already introduced. One of the most effective methods consists in having the wound bleed profusely, because the flowing blood washes the virus ont of the tissues; at the same time it is advisable to wash the wound thoroughly with a rlisinfectant solution, such as strong vinegar, absolute alcohol, permanganate of potash $(1 \%)$, carholic acis $(3 \%)$, and particularly $1 \%$ solution of bichloride of mercury. In cases where the wound comes too late under medical treatment, the elimination of the contagion may be attempted by means of a red hot iron or of caustics. The caustics mostly used are muriatic acid, sulphuric acid, nitric acid, iron chloride, lunar caustic, ammonia, etc., in the most concentrated solutions. This treatment is possibly aided by subcutaneons injections in the region of the bite of $1: 10,000$ solution of bichloricle of mercury, 1:1000 of silver nitrate, $1 \%$ solution of carbolic acid, $1: 1000$ of collargol. In ardition the application of a Bier's ligature might also prove helpful (Fermi thus saved all test animals, even when the ligature was applied fom hours after the infec- 
tion). Experience teaches that these procedures, when applied within the first quarter of an hour after the bite, will often succeed in postponing an attack of rabies, wlile later the result becomes more and more uncertain, according to the length of time passed after the bite.

A favorable result of the treatment, however, no matter how soon it has been applied, can never be predicted with certainty and, therefore, the bitten animals, in spite of the treatment, must be considered as suspicious, they should be placed under careful observation and also be subjected to a protective vaccination (see p. 491). Dogs and cats should be destroyed as soon as possible upon reasonable suspicion of infection.

The treatment with radium rays studied by Tizzoni \& Bongiovanni seems, accorling to inrestigations by Calabrese, Danysz, and Rhens, to be of little value from tests on rabbits, and it can hartly be applied to domestic animals.

Protective Inoculation. The disease which develops in dogs injected with rabies virus may in rare cases come to a stop, and finally a complete cure may he effected. Such recovered cases cannot be infected later with rabies even with large doses of virus, the disease having immunized the organism against the virulent infection. This fact was first, and almost at the same time ascertained by Pasteur and Azary, and, on account of the favorable results of the experiments, Pasteur later introduced an efficient methor of anti-rabies vaccination.

First of all, he ascertained the important fact (1884) that the virus, through artificial transmission from dog to monkey and then further to other monkeys, is grarlually so attenuated that it does not any longer cause rabies in dogs, but makes them immune against a later artificial infection with unattenuated virus. On the other hand, the virulence may he increased by repeated passage through the bodies of rabbits and guinea pigs. The increased virulence manifests itself by the fact that material originating from later generations injected subdurally in rabbits will cause rabies after a considerably shorter perioil of incubation.

The rabies virus, in the shape in which it is obtained from animals infected in a natural way (street rabies), is inconstant since animals infected with it contract rabies after periods of incubation which vary in their duration. Transmitterl through bodies of rabbits and guinea pigs, the effect on the animals is not only increased, but also becomes so constant that the spinal cord from the 25th rabhit regularly causes symptoms of rabies on the 8th day, that from the 50th on the 7th, and that from the 90th on the 6th day. Virus that has been thus strengthened also keeps this quality later on, wherefore Pasteur designates it as "fixed virus of rahies." (Later, Högyes has reduced the period of incubation to 5 days by using young rabbits for the series of injections.) 
With the increase in virulence for rabbits, a similar inerease does not seem to follow for other animals and for human beings. Thus Helman injected $1-10 \mathrm{~g}$. of fixed virus into 34 dogs and 8 monkeys 4 days in succession without rabies appearing in a single animal; further, Nitzsch inoculated himself under the skin with an emulsion from a 4-5 mm. piece of rabbit cord without experiencing any bad results whatever, and also found the fixed virus without effect on dogs and monkeys.

After securing a rabies virus of known and constant virulence, Pasteur set to work to obtain its attenuation. Having harl to discontinue the passage through monkeys, owing to its high cost, he chose the following procedure:

The medulla and spinal cord of a rabbit, which has contracted rabies after a subdural injection with fixed virus, are suspended free by means of threads in a bottle closed by a plug of cotton and the bottom of which is covered with pieces of caustic potash. At a constant temperature of $23^{\circ} \mathrm{C}$., the spinal cord will dry, and also gradually lose its virulent properties, so that, for instance, spinal cord dried for 5 days will produce the disease on the 8 th day, cord dried for 9 days on the 15th day, and the same dried for 14 days will be absolutely without effect. If spinal cord from a rabbit dead of rabies is treated in this mammer daily for 14 days, there will be, after 2 weeks, material on hand of 14 different degrees of virulence.

Pasteur then immunized dogs by injecting at first the most attenuated virus, and then every second hour, a virus gradually less attenuated until he reached spinal cord which had been dried only one day. Dogs which had been subjected to 2-3 series of such inoculations were later bitten by a rabid dog, and were also injected subdurally with fixed virus or street virus, without suffering harm.

Through further experiments, Pasteur proved that this method of immunizing likewise prevented the appearance of the disease in dogs previously bitten by rabid animals. The immunity obtained by protective vaccination lasts about one year, but is still effective after two years insofar as rabies develops after a considerably longer period following inoculation (Pasteur). In exceptional cases the immunity may be effective up to 5 years (Högyes); it may be further prolonged by vaccinations repeated at intervals.

Finally, in July, 1885, Pasteur successfully applied the method, which he had proved to be effective in dogs, to a boy who had been bitten. Since then, the anti-rabies protective vaccination has always been practiced on human beings in Pasteur Institutes established for this purpose, either according to Pasteur's or to a somewhat modified method.

I. Protective Vaccination with Dried Substance of Spinal Cord. (Pasteur's method.) The method which is nearly always followed in human beings consists in taking $2 \mathrm{mg}$. of the dried substance of the spinal cord of rabbits dead of rabies after inoculation with fixed virns, 
triturating it with sterilized bouillon into an emulsion and then injecting it subcutaneously in the abdominal region. The first injection is made with the spinal cord dried for 14 days, and then the substance dried one day less is injected at first every half a day, and later every day, until finally the cord dried for only 3 or even 2 days is injected. The inoculation with cord dried 5,3 , and 2 days is repeated once or twice, especially in the case of wounds from bites on the head. (Pflanz in practice applied this method with favorable results on a dog.)

II. Protective Vaccination with Attenuated Virus. (Method of Högyes.) Högyes prepared from virulent spinal cords of rabbits by trituration with sterilized physiological solution of sodium chloride, emulsions of different concentrations $(1 / 5000,1 / 2000,1 / 500,1 / 200$, $1 / 100,1 / 10$ ) and injected them subeutaneously every second hour from the weakest to the strongest. Dogs thus treated later resisted bites from rabid animals as well as the subdural injection of fixed virus or street virus, and moreover this method is effective after the appearance of the disease. (This also proves that not the strength, but the amount of the vaccine is of most importance, and that with the Pasteur protective vaccination, not an attennation, but rather a reduction of the amount of virus is important.) Since 1895, this method has been exclusively applied at the Budapest Pasteur Institute and also at other places to human beings.

Högyes in this way produced immunity in 36 dogs, and in most cases ihe immunity was absolute, as it also protected the animals against a later subdural infection. He experimented further with 16 other dogs which were infected by bites of rabid dogs; 8 of them were not subjecter to treatment, while 8 were inoculated according to the above namerl methou with attenuated virus. Of the former group 5 contracted rabies, while all the animals of the latter group remained alive.

Kurz \& Aujeszky applied the method at a stock farm, where inmediately before 3 animals har died from rabies, by injecting 44 colts, 6 of which had wounds of bites on their bodies. The method was as follows: On the first day about $15 \mathrm{ce}$. of the emulsion 1:2000 were injected, and 2 hours later a similar amount of the emulsion 1:1000; on the second day, about 8-10 ec. of the emulsion 1:500, and 2 hours later a similar amount of emulsion 1:300; on the third day 8-10 ce. of the emulsion 1:100. After an interval of 5 days the three-days' eycle was repeated in the same manner, and after a further interval of 3 days the colts received $3-5 \mathrm{cc}$. according to their body weight of the emulsion $1: 10$. The raccination had no injurious results for the colts, and none of them later contracted rabies. Aujeszky further vaccinated a pack of hounds in the following way: In the forenoon of the first day $5 \mathrm{cc}$. of emulsion $1: 5000$; in the afternoon, 3 ce. of emulsion 1:3000; in the forenoon of the second day $4 \mathrm{cc}$. of the latter emulsion, in the afternoon $2.5 \mathrm{cc}$. of emulsion $1: 1000$; on the third day, in the forenoon 2 ce. enulsion 1:500, in the afternoon $1 \mathrm{cc}$. emnlsion $1: 200$. The hounds suffered no ill effects from the vaccination.

\section{Protective Vaccination by Intravenous Injection of Cerebral}

Substance (Galtier's Nethod). Galtier immunized sheep and goats as early as 1885 by injecting saliva or, in another series of experiments, medulla oblongata from rabid dogs into the jngular vein; the animals resisted both a subsequent and a previous virulent subeutaneous injection, while the control animals (sheep and rabbits) contracted rabies after a similar infection. Nocard \& Roux later ascertained that sheep immunized in this way also resisted the intraocular infection, provided that the protective vaceination was done within 24 hours after the virulent infection; they found further that the immunity lasted at least 9 months. The method, applied at the latest 3 to 4 days after the bite, 
is said to be effective only in ruminants. while it has not proved reliable in horses (Nocard, Conte) and dogs (Protopopoff, Helman). Wysokowitz has also tried the method on 70 human beings.

The inoculation is conducted as follows: Pieces of spinal cord from dogs having died of street rabies are ground up with sterilized water, and 5.7 ce. of the thin emulsion, filtered through linen cloth, are slowly injected twice in succession into eattle and horses, and $2-3$ ce. into sheep or goats, with an interval of from 6 hours to one day; the enulsion is injected into the jugular rein or one of the ear reins.

Recently Nicolas also found the method efficacious in larger ruminants but without effect in goats, while Forgeot and Rarnaud had unfavorable results in cattle (out of 8 vaccinater animals 5 died afterwards from rabies). According to Remlinger and Mustapha-Effendi, sheep sometimes die after the vaceination from rabies or an intoxication, while Delannoy states that it is only suitable for large ruminants.

Bahes found that dogs might be immunized against a subsequent rirulent infection by subcutaneous injection of cerebral substance of healthy sheep, and also, that an emulsion of rirulent cord was made harmless by the addition of nornial cerebral substance. Aujeszky, Calabrese, and others, however, did not obtain any immunizing effect from the normal cerebral substance. In agreement herewith, Friedberger \& Eisler showed that an emulsion of fixed virus $(1: 100)$ is neutralized by 0.005 ce. of serum from a horse which had often been treated with such virus, while, on the contrary, normal cerebral substance was not capable of absorbing the proved antirabic substance from the immune serum. Recently, Fermi expressed the opinion that normal nerve substance possesses immunizing properties, and he succeeded in immunizing rodents per os; Kraus and Fukuhara, however, could not confirm the results of these experiments, as they proved that normal nerve substances are not able to produce antirabic substances in animals treated with them.

Immunizing experiments with liver tissue and bile (Marx) have not produced good results, but Protopopoff succeeded in immunizing dogs hoth against subsequent and previous infection by means of subcutaneous or intravenous injection of virulent cerebral substance which had been kept in glycerin for a long time (30 to 60 days).

Protopopoff and Helmann immunized dogs, sheep and goats, and Marx immunized rabbits and dogs by intraperitoneal injections of relatively large amounts of virulent cerebral tissue. Marx believes, howerer, that only fixed rirus immunizes in this manner. The immunity is obtained after 12 to 14 days and lasts from 6 to 11 months.

IV. Serum Immunizaion. Babes \& Lepp found that hlood serum from dogs which had been made immune against rabies br repeated vaccination with rabies virus protects dogs against the subdural infection, when subcutaneously injected with doses of 5-10 ec. Later, Tizzoni and Centanni prepared an effective serum hy successive immunization of sheep, $1.0 \mathrm{cc}$. of which protects $25 \mathrm{~kg}$. of body-weight of rahbits against the effect of street rabies virus, and in vitro makes $800-1600 \mathrm{~g}$. of virulent cerebral substance harmless. According to their opinion, the dried serum keeps unchanged for a long time, and is effeetive both at the beginning and at a later stage of incubation, immediately before the appearanee of the first symptoms. Recently, however, Kraus \& Fukuhara found hy numerous experiments that serum ohtained from sueh sheep, dogs and horses may destroy the rabies virus by direct contact. but that neither a preventive nor a curative application has any protective action of any kind.

In order to obtain an active immunity, Remlinger repeatedly injected sheep with a mixture of fixed virus and immune serum with good results, and Schürer succeeded in protecting dogs against intramuscular and natural infection hy a single subcutaneous injection of a similar mixture, while Marie obtained effective immunity against the subdural infection of dogs by subcutaneous injection, at first, of a 20 ce. mixture of virus and serum, and. 2 weeks later, of $12-20$ ce. of fixed virus. Rem- 
linger also applied this method to 4 horses suspected of infection, injecting subcutaneously 700 ce. of a mixture of serum and fixed virus each in a dilution of $1: 1000$. The horses remained healthy.

Veterinary Police. According to the veterinary police ordinances, which are nearly ever'ywhere alike, all animals suffering from rabies must be destroyed, and all those suspected of having the disease or of being infected must he placed under veterinary observation; dogs and cats, however, with the exception of those which have bitten human beings, are to be killed on the first suspicion of infection. The time of observation must be at least 3 months, and even then an outbreak of the disease cannot with certainty be excluded. Meat or milk from rabid or suspicious animals must not be used for consumption, but such products from animals only suspected of being infected may be put on the market without hesitation as long as these animals appear completely healthy.

The most important and effective prophylactic measure for eradicating rahies in large sections of country is the general obligatory dog tax. The latter has caused a reduction of cases where it has been adopted and in some countries the complete eradication of the disease by reducing the number of dogs, particularly vagrant and ownerless $\operatorname{logs}$, and thereby reducing the opportunity for infection. On the other hand, the obligatory muzzling of dogs was found effective only in connection with the dog tax and with other measures of prevention (killing of bitten dogs and cats and the longest possible quarantine), and it can moreover only be applied in larger towns and cities. A reduction in the number of cases of rabies can only be accomplished by carrying out the same preventive measures in large districts.

Bavaria offers an instructive illustration of the favorable results obtained in the reduction of rabies. From 1863 to 1876,14 to 18 human beings were victims of the disease annually, while the number of rabid dogs amounted to about 500 every year. Under the influence of the obligatory dog tax introduced in 1876, the number of rabid dogs was reduced to 61 in 1893 , and from 1883 to 1898 no human beings died from rabies. In the years 1898 to 1900 fatal cases of rabies and suspicious instances were again observed, but only through being introduced from outside, particularly from the East (Kitt). Like favorable conditions prevail in Saxony where the number of rabid dogs was reduced to 22 from 1879 to 1893. In the Grand Duchy of Baden, the introduction of the dog tax in connection with obligatory muzzling, in the year 1875, resulted in a rapid decrease and finally in the complete eradication of rabies.

In Hungary, the favorable results of the dog tax were apparent during the first years after the enactment of the veterinary law of 1888 , as the number of rabid dogs was reduced from 1,286 to 609 during the years 1890 to 1892; later, however, the disease again increased owing to the faulty enforcement of the tax law and to the fact that it had not been made obligatory in all communities. Szakall showed from statistical data that rabies was more rare in communities with a general obligatory dog tax, but appeared more frequently where exemptions from the dog tax had been granted for certain kinds of dogs.

That muzzling alone is not sufficient to eradicate the disease completely is shown in the City of Berlin. During the years 1853-1875 obligatory muzzling was in force, but in spite of it rabies sometimes assumed an enzootic character (1865-1867, 1871-1873). After the promulgation of the Prussian law of 1875, the disease decreased rapidly, and since 1883 only isolated cases of rabies have been observed. 
That obligatory muzzling, at least when supported by other strict measures, also gives good results has been shown by the experience in London where the fatal cases in human beings immediately ceased every time after its introcluction, while such fatalities always occurred again when obligatory muzzling was abandoned. In the same way, the cases of rabies of logs in England raried according to the enforcement or the repeal of the muzzling ortinance. (From 1890 to 1893 obligatory muzzling causer a reluction of eases of ralies from 312 to 129,79 , and 38 ; from 1893 to $1 \times 96$ the absence of obligatory muzzling increased the cases of rabies to $93,248,672$; since 1896 obligatory muzzling again reduced the cases of rabies from 438 , to $151,17,9$ [Salnon].)

The efticieney of suitable measures against rabies has been proved not only in Denmark, Sweilen, and Norway, but also in Germany, where it has succeeded in restricting the disease to such a degree that it is only observed to any extent in the eastern horder provinces of Prussia, while in the interior of the country only very cireumserilied foci exist. Finally in England the disease rapidly decreased and in 1903 was completely eradicated as a result of obligatory muzling, and after the importation of dogs from the continent (1597) and Ireland (1899) had lieen prohibited or maile depenilent ou a 6 montlis quarantine. Rabies has not heen observed in Englami since its eradication in 1903.

Literature. Galtier, C. R., 1879; J. vét., 1904, 274 and 330.-Pasteur, 1881, XCII, 1259 ; 1882, XCVIII, 457; 1885, CI, 705; 1899, CVIII, 1228.-Roux, A. P., 1888, II, 18; 1859, III, 69.-Nocard \& Roux, A. P., 1888, II, 341; 1890, IV, 163.Högyes, A. P., 1888, II, 133; 1889, III, 449.-Schaffer, A. P., 1889, III, 644.Kitt, Monh., 1590, I, 314 (Review).-Johne, Z. f. Tm., 1898, II, 349.-Van Gehuchten \& Nélis, Aun. vét., 1900, XLIX, 243.-Vallée, Rev. vét., 1900, 763.-Negri, Z. f. Hyg., 1903, XLIII, 507; 1904, XLIV, 519; 1909, LXIII, 421.-Remlinger \& Riffat-Bey, Soc. biol., 1903, July II.-Sehüder, Z. f. Hyg., 1903, XLII, 362; A. f. Tk., 1904, XXX, 610.-Remlinger, A. P., 1904, XVIII, 150; 1909, XXIII, 430.Schnïrer, Z. f. Hyg., 1905, LI, 46 (Lit. on immunity).-Tizzoni \& Bongioranni, Cb1. f. B., 1905, XXXIX, 187; 1906, XL, 745.-Bohne, Z. f. Hyg., 1906, LII, 87 (Lit.).-Nitsch, Cbl. f. Bakt., 1906, XLII, 647.--Panisset, Rev. gén., 1906, VIII, 113 (New lit.).-Lentz, Cbl. f. Bakt., 1907, XLIV, 374; 190s, LX̃11, 63.-Frosch, Hb. d. p. M., 1, Ergzbd., 1907, 626.-Nicholas, J. vét., 1908, 198.-Marie, A. P., 1908, XXII, 271.-Standfuss, A. f. Tk., 1908, XXXIV, 109 (Lit. on Negri bodies).Fermi, Cbl. f. Bakt., 1907-1910, XLIII-LIII.-Rátz, Kongr. Haag, 1909 (Lit.).Koch \& Rissling, Z. f. Hyg., 1910, LXV, 85.

Rabies in Human Beings. In countries where rabies occurs frequently among the animals, it is also often observed in human beings. In about $90 \%$ of the cases, they are infected by the bites of dogs, while in the other cases (ahout 7 to $8 \%$ ) cats, wolves and other animals transmit the infection. Of the persons bitten by rabid animals, about 16 to $20 \%$ die of rabies (according to Babes 60 to $90 \%$ die after hites of wolves) mostly after bites in the face or on the hands. The period of incubation is at least 13 days, msually from 20 to 60 days, and only in exceptional cases is it longer than 90 days (after bites from wolves the disease usually appears in the third week).

The symptoms of rahies in human beings are at first dejection, itching and trembling in the bitten part, also fever; soon respiratory troubles, uneasiness, difficulty in swallowing, aversion to liquids, abundant salivation, in general increased reflex excitability, and in consequence attacks of delirium, appear; lastly paralysis of the muscles of the face, eyes, and tongue, as well as the extremities and the trunk is observed. In exceptional cases, the disease develops from the beginning in the paralytic form.

The results of the anti-rabies protective vaccination are as a rule favorable since, after regularly conducted vaccination, less than $1 \%$ of persons who were undoubtedly bitten by rabid animals die subsequently of rabies. On the whole, results are the more satisfactory the earlier after the hite the vaccination begins, and, everything else being equal, they are most favorable after bites on the legs and body, somewhat less favorable following bites on the hands, and the least so after bites on 
the head and face. The inortality figures computed according to these points of infection on 36,891 vaccinated persons, amount to $0.32,0.56$ and $1.78 \%$ respectively.

During the years 1886 to $1908,31,759$ persons were subjected to protective vaccination at the Pasteur Institnte in Paris, and after the completel treatment 129 persons, or $0.41 \%$, died. The annual percentage of mortality during the above named period has been lowered from 0.94 to $0.19 \%$. In Berlin 1,416 bitten persons were subjected to protective vaccination during the years 1 s9s to 1902 , and of these 12 or $0.84 \%$ dier afterwards of rabies, while among non-raceinated persons the percentage of mortality amounted to $14.8 \%$ (Doebert). At the Pasteur Institute in Budapest 45,067 bitten persons were vaceinater during the years 1890 to 1909. Of these 181 or $0.4 \%$ died of rabies afterwards (last year only $0.19 \%$ ), while during the years 1890 to 1903 , ont of 1,861 bitten but non-vaccinated persons 266 or $14.29 \%$ later contracted the disease. During the years 1890 to 1595 , Pasteur's method was used, while subsequently that of Högyes was employerl.

According to a compilation by Bernstein in 1905, 104,3.57 persons were subjected to treatment in 40 Pasteur Institutes in all parts of the world, outside of Australia. Of these 560 persons or $0.54 \%$ died more than 14 davs after the conclusion of the treatment while $0.19 \%$ died within 14 davs, making the total of deaths $0.73 \%$. According to the location of the bite, whether it was inflicted on the head or on the hands, the percentage of mortality was 1.99 and $0.36 \%$ respectively.

Literature. S. Högyes, Lyssa, Nothnagel's Handbuch, 1897, V, 1, T.Bernstein, Vjschr, f. ger. Med. u. öff. Sanitätsw., 1905, XXXI, 1.

Infecticus Bulbar Paralysis (Pseudo Rabies). After the inoculation of rabbits with the medulla from a cow and a dog which died with suspicious symptoms of rahies Aujeszky observed a disease in the experiment animals which resulted in their death within 48 to 50 hours. The most conspicuous symptom was a very severe itching at the point of inoculation.

According to his and to Schmiedhoffer's investigations the disease may he produced by subdural, intraocular, intramuscular, or subeutaneous inoculations of brain substance from animals dead of the disease, or with parts of the tissues from the point of inoculation. The disease may also be produced in guinea pigs, rats and mice, very easily in earnivorous animals, cattle and sheep, while the ass is less susceptible and horses can be infected only with difficulty. Fowls are resistant to the infection. Artificial infection occurs also by the feeding of organs containing the virus to eats and dogs, as well as rodents. The virus is most concentrated in the tissue fluids at the point of inoculation, then in the blood and in the central nervous system. It is not present in the bile, saliva, or urine $(0.001 \mathrm{cc}$. of blood is sufficient to produce an infection). Dense porcelain filters retain the virus, while the filtrate through coarser filters, which however do not let bacteria pass, is infective in large quantities. Attempts at cultivation have so far been fruitless.

Tirulent brain substance remains infective for months in $50 \%$ glyeerin, while by slow drying it is rendered avirulent in from 3 to 6 days. Defibrinated blood kept in an ice chest, retains its virulence for from 1 to 3 weeks. Heating to $55^{\circ}-60^{\circ} \mathrm{C}$. destroys it in from 30 to 35 minutes, $80^{\circ}$ in three ninutes, and $100^{\circ}$ immediately. Of chemical agents $1 / 2 \%$ hydrochlorie acid destroys it in 3 minutes, $1 \%$ corrosive sublimate immediately, $5 \%$ carbolic acid in 2 minutes, $3 \%$ lysol in 10 minutes, $2 \%$ formalin in 20 minutes, and absolute alcohol in 30 minutes (Schmiedhoffer).

After a subcutaneous injection a local reddening develops at the point of inoculation, which later results in a necrosis. The animals seratch and rub the affected part severely: in the ineantime they become very weak, and usually die within a few hours. The experimental dis- 
ease appears similar to the spontancous cases in animals. After the infection per os a marked inflammatory swelling and sensitiveness of the lips and the buccal mucons membrane were observed.

The disease, which up to the present time has been observed only in Hungary, occurs not very infrecpuently in eats and dogs, as Marek diagnosed it in the elinic at Buclapest from 1902 to 1908 in 118 cats and 29 dogs. Some of the cats had previously been fed spoiled meat; in the majority of cases, however, the history relative to this point was negative. Some owners lost all their cats from the disease, without consideration of sex and age. Szabo reports 16 cases occurring among a pack of hounds. The disease has been repeatedly observed among eattle and also in rats.

Symptoms. After an incubation period of 20 hours to 10 days (following artificial infection), a peculiar ehange in the hehavior of the animal is observed. Dogs and cats become apathetic, change their resting place often and sit erouched up; cats meow and yawn, sometimes painfully. There is profuse salivation; inappetence is present from the onset of the disease with frequent vomiting, and constant symptoms of paralysis. In about half of the cases the patients manifest severe itching on any part of the head, which they scratch with the fore paws, or rub against some object, sometimes so severely that inside of several hours extensive abrasions or even deeper injuries result. In other eases this symptom is absent, but the groaning or painful meowing indicates that the animals are in pain. With rare exceptions the examination of the nervous system shows paralysis of the pharynx and pruritis, also an uneven dilation of the pupils associated with at first increased reflex irritability, later diminished pupillary reflex, muscular sensibility, and superficial and deep reflexes. In most eases periodical twitching may he observed in the flexor muscles of the head and neck, sometimes also the muscles of the lip. The respiration is markedly labored, the temperature is normal or only slightly elevated and the patients succumb almost exclusively inside of 24 to 36 hours. In cattle a persistent rubbing of the muzzle or of other parts of the face is ohserved, which results in more or less extensive bloody areas, dennded of hair and surrounded by edematous swellings. At the same time the animals bellow londly, and kick spasmodically with their hind legs. In the meanwhile a pronounced swelling and weakness of the hind quarters develop, while the appetite is for a time normal. Finally the disease terminates in death after 12 to 36 hours.

The autopsy reveals inflammatory lesions which occur at those parts of the bodies of the animals which were painful during life; perhaps there are also hyperemia or small hemorrhagic extravasations in the cerebral meninges. Recovery has so far been observed only in one cat. This animal, which on the first day had no appetite whatever, ate more frequently on the following day, at the same time manifesting spasmodic contractions of the muscles. The spasms became less frequent, salivation ceased after the fourth day, and on the eighth day the eat had recovered.

The disease is distinguished from rabies on the one hand by the absence of furious attacks, aggressive behavior, and of paralysis of the jaw; on the other hand by the fact that the blood as well as the brain tissue is infectious, while the saliva is not, and finally that the disease after a period of incubation of not more than several days, runs a very rapid course. Especially characteristic of infectious bulhar paralysis is 
its sudden appearance, accompanied by the very acute and usuaily fatal course, and the severe itching of a certain part of the body; if this latter symptom is absent the disease may be readily mistaken for acute poisoning in dogs and cats.

The nature and mode of natural infection are not yet sufficiently known. The observation that rats sometimes die in great numbers with the disease simultaneously with numerous cases in dogs, eats or cattle (Balas, Hutyra), and that the conspicuons itching occurs mostly on a portion of the face, renders the supposition very probable that domestic animals are infected by diseased rats (possibly also mice), although the possibility that the infection of the various species of animals may occur simultaneously through a common source cannot be excluded.

Literature. Aujeszky, Cbl. f. Bakt., 1902, XXXII, 353.-Marek, Jahresber. d. Hochschule Budapest, 1902-03, 1908-09.-Hutyra, B. t. W., 1910, 149.-Sehmiedhoffer, Közl., 1910.

Epizootic of Cats with Nervous Manifestations. Mori observed an epizootic of eats in Siena which commences with sudden severe excitement, when the animal becomes frightened, and with widely opened eyes rums up and down, turns in a circle, and finally collapses as if paralyzed, passing urine involuntarily. On the following days depression and coma alternate, the attacks continually becoming more pronounced and of longer duration; further there is a conjunctivitis with paleness of the other mucous membranes, yawning, difficult respiration, accumulation of feces in the colon, and relaxation of the anus. The autopsy revealed in one case a marked swelling, softening and hyperemia of the spleen, fatty degeneration of the liver, as well as dark, fluid blood.

This disease, the symptoms of which decidedly resemble those of acute lead poisoning (Chyzer), is supposed to be caused by a facultative anaerobic, Gram negative bacillus 0.8-2.0 $\mu$ long, having peritrichally arranged flagellae. The bacillus is pathogenic for guinea pigs, rabbits, pigeons and white mice. Feeding one ce. of the culture to a cat resulted in an elevation of the temperature, followed later by hypothermia and diarrhea (Cbl. f. Bakt.; 1905, XXXVIII, 40.) 
Group $Y$.

\section{CHRONIC INFECTIOUS DISEASES.}

\section{Tuberculosis.}

\section{(Tuberculose [German and French]; Tubercolosi [Italian].)}

Tubereulosis is a chronic contagious infections disease of man and domestic animals. It is caused by Koch's Bacillus tuhereulosis or one of its varieties. Anatomically it is characterized, in its incipient stage at least, by small non-vascular nodules known as tubercles which have a tendeney to undergo cheesy degeneration.

\section{(a.) Tuberculosis of Mammals.}

(Pearl disease, Grapes; Perlsucht, Skrofulose, Lungenschwindsucht, Stiersucht, Monatreiterei, Drïsenkrankheit, Franzosenkrankheit [German]; Cachexia boum tuberculosa, Phthisis pulmonum [Latin]; Pommelière. [French]; Malattia glandolare [Italiain]).

History. Tuberculosis, and particularly that form known as pulmonary consumption, has from the remotest time been known as the most prevalent disease of man. A large pereentage of all deaths is due to tuberenlosis. The study of its nature had, therefore, until the beginning of the last century, been confined almost exchusively to man. Intil reeently, however, the results of these investigations amounted to little more than theories or hypotheses. The peculiar charaeter of the tubercle was necessarily recognized by pathologists in the early history of the study of the disease. Morton, as early as 1689, regarded the tubercle as the anatomical foundation of pulmonary consumption. At a later date special stress was laid upon the importanee of eheesy degeneration as characteristie of tubereulous lesions. While Bayle, in 1819, restricted the definition of the tubercle to non-caseating, gray and translncent nodules, he as well as Baillie (1794) and Laënnec (1819) considered pulmonary consumption and scrofula as identical morhid processes. Laënnec held the view that the tubercle owed its origin to a speeifie tuberele-produeing substanee, soft and homogenous in consistency, which was secreted from the blood, and that the inoenlation of this primary tuberele substance eould produce the local development of tuberculous tissue. As he, however, like later on Magendie and Rokitansky, attached undue importance to the cheesy character of the tubercle, he included many other morhid processes which consisted of simple degenerations. 
Virchow, in 1847, had a more restricted conception of the nature of tuberculosis. This was based upon a histological foundation and recognized as tuberculous such lesions only as presented small, well marked non-vascular nodules or groups of nodules, or cheesy masses that were evidently the result of the degeneration of such nodules. According to this view, however, he excluded several forms of diseases which are undoubtedly tuberculous in character, viz., tuberculosis of the lymphatic glands (scrofula), tuberculous caseous pneumonia and tubereulosis of animals.

Of the various forms of tuberculosis in animals, so-called pearl disease in cattle in particular long ago attracted general attention, and the regulations concerning meat inspection in various countries concerned themselves with this question in detail. In the beginning of the eighteenth century pearl disease was generally supposed to be identical with syphilis of man, and supposed to be the result of unnatural intercourse of syphilitic persons with animals (Franzosenkrankheit). As a result of this view all cattle affected with pearl disease were excluded from use as food. At the end of the same century, however, this view of the nature of pearl disease was found to be incorrect. But the appreciation of the true nature of the disease was the result of a very gradual development of our knowledge. During the first half of the last century some author's (Gurlt, Hering, Fuchs) regarded pulmonary tuberculosis of cattle and pulmonary consumption of man as identical, and others (Gerlach, Spinola, Leysering) placed pearl disease of the peritoneum of cattle in the same group. On the other hand, Virchow dispnted the occurrence of tuberculosis in animals altogether. According to his view which was subsequently shared by Röll, the morbid growths of the lungs and serous membranes were in reality neoplasms, sarcomata, which might be considered identical with lympho-sarcomata of man.

The question assumed a new aspect when in the year 1865 Villemin proved by means of experimental infection that tuberculosis was transmissible from man to animals, and from animal to animal respectively. Morgagni had already 200 years ago believed in the infectiousness of pulmonary consumption and Klencke produced in 1843 tuberculosis in rabbits by the intravenous injection of tuberculous material. But in spite of this the spontaneous development of the disease according to the humoral pathological theory continued to be asserted. Villemin obtained positive results in his experiments with tuberculous material from man as well as with material from cattle affected with pearl disease, and thus furnished proof of the infectiousness of tuberculosis as well as the identity of the disease in man and in animals. In recent years Villemin's experiments have been abundantly verified by numerous repetitions, and at the same time the various modes of infection have been elucidated. These latter experiments have shown not only that tuberculosis is transmissible by means of subcutaneous or intraperitoneal infection (Klebs, Bollinger, Kitt), intraocular inoculation (Cohnheim \& Salomonssen, Baumgarten), as well as through inhalation (Tappeiner, Weichselbaum) of tuberculous material, but furthermore they have shown that other foreign substances could not produce similar pathological processes (Martin, Ponfick, Langerhans). Semmer observed that the injection of milk and blood from a cow with advanced pulmonary pleural tuberculosis into the jugular vein of a pig produced a pronounced general tuberculosis. Chauveau (1868) as well as Gerlach (1869) succeeded in producing the disease by feeding tuberculous material from cattle. Klebs produced pearl disease of the peritoneum by means of intraperi- 
toneal injection of tuberenlous sputum. Bollinger obtained the same result by the injection of tuberculous lung tissue.

As the result of a statistical study of the various feeding experiments with the transmission of tuberculosis, Cohnheim declared in 1879 that the correct diagnosis of tubereulosis could no longer be made from a purely anatomical point of view and that the etiological viewpoint alone was justifiable. He maintained further that the cellular structure of the nodules was as little eharacteristic of a purely tuberculous process as the tendency to cheesy degeneration which had been emphasized by Laënnec. He held that either process could be considered as tuberculous only when their infectious nature had been demonstrated. Further, since other tubercles or cheesy products are not infectious, thongh similar in their anatomical structure, the infectiousness of the true tubercle must he due to a specific virus.

Nothing now was lacking in the chain of evidence but the recognition of this specific virus in order that the infectious nature of tuberculosis might be recognized as a scientifically demonstrated fact. This was accomplished by Robert Koch in 1882 when he proved by incontrovertible evidence that tuberculosis in man as well as tuberculosis in animals was dependent upon the pathogenic action of a morphologically and culturally characteristic bacterium. The theory of the infectiousness of tuberculosis was herehy placed upon a firm foundation and further research was theneeforth directed to the establishment of the various conditions under which infection took place and the means by which the discovery of efficient methods of prevention was possible.

In recent years the study of the etiology of tuberculosis received a new stimulus when Koch, in an address before the Tuberculosis Congress of London in 1901, declared that bovine and human tuberculosis were different diseases, that the human disease was not transmissible to cattle and that it was not necessary to observe special precautions to protect man against pearl disease or bovine tuberculosis. This opinion was diametrically opposed to former views expressed by him or held by the medical profession in general. The various commissions which were thereupon appointed by the German government as well as the English Royal Commission for the study of this question did not express an unqualified endorsement of the views expressed by Koch. Nevertheless they produced valuable information regarding the morphological, cultural and pathogenic characteristics of tubercle bacilli of different origin. Simultaneously the question of the exact mode of infection was again forced into the foreground of investigation. At this time two theories, sharply opposed one to the other, received general recognition, viz., the view of Flügge who held that infection occurred chiefly through inhalation of the virus into the air passages, and that of Calmette who held that infection by way of the alimentary canal was of most frequent occurrence. In recent years, however, the tendency is to accept a combination of these two views on the modes of infection, while the importance of either mode is recognized.

These as well as the extraordinarily numerous and painstaking investigations of other details of the tuberculosis question, the numerous associations, congresses and conferences which were organized in rapid succession, and the active social and economic interest which developed with reference to this question, give cause to hope for a gradual repression of this devastating disease. As far as tuberculosis of man is concerned the various public sanatoria and dispensaries have produced happy results in the way of disseminating popular knowledge concerning the disease. The results of a similar campaign for the suppression 
of the disease among animals, though less far reaching in their effects, have also been gratifying.

The results which were for a time expected from the curative value of tuberculin (Koch, 1890) were realized to a slight extent only. On the other hand tuberculin has proven itself a valuable diagnostic agent and has been used with particular success among domestic animals. The diagnostic properties of tuberculin and the recognization of the conditions which produce infection constitute the basis of Bang's system for the extermination of tuberculosis. By means of this system the eradication of the disease can be accomplished without serious economic loss even in badly infected herds. On the other hand, the advantages expected from protective inoculation of cattle by means of human tubercle bacilli (Behring, 1902) have by no means been realized in the same degree, and the problem of employing this agent in the fight against tuberculosis remains for the future to solve.

Among the more recent discoveries which deserve special mention are Pirquet's local "allergic" tubereulin reactions and Much's non-acidfast form of tubercle bacillus.

Occurrence. The prevalence of tuberculosis, especially among cattle and swine, stands in intimate relation to the development of intensive agriculture. Among cattle its spread is favored to a great extent by our modern soiling system, forced maturity of young animals and increased traffic in live stock. Thus tuberculosis is practically unknown on the extensive prairies of North America, in Central Africa and on many islands (Iceland, Sicily). It is almost unknown among the cattle of the steppes of Eastern Europe, while it is particularly prevalent in the central and western countries of Europe, especially in the neighborhood of large cities where it is increasing in prevalence. Large dairy establishments near cities suffer comparatively greater losses than the smaller herds of farmers in mountainous regions. Young animals are less frequently affected than older stock. Among swine the disease has gained a foothold through the practice of feeding the products of creameries and skimmed milk to young swine. Among other domestic animals, with the exception of fowls, the disease is much less prevalent, although no species is entirely free from its ravages.

The importance of tuberculosis to the cattle industry lies in the fact that the average age of usefulness of the young animals has decreased in direct proportion to the increased prevalence of tuberculosis, and to the fact that the losses from the elimination of indifferent and poor milk producers is continually increasing. The raising of young animals is attended with greater difficulties and expense; the volume of milk produced by infected herds is decreased, and the emaciation of affected animals rednces their food value. Moreover the large number of carcasses of diseased animals which are condemned for food purposes causes annually a considerable economic loss to the national wealth.

Our knowledge of the prevalence of the disease has been gained on the one hand from statistics furnished by public 
abattoirs and on the other hand from results furnished by the tuberculin test. The value of slaughter house statistics where only the more or less conspicuous lesions are noted, depends to a great extent upon the experience and care of inspectors in charge. The tuberculin test furnishes evidence of the existence of the disease in its most incipient stage, hence the percentage of cases disclosed by the latter is much larger than that indicated by the former.

In Germany investigations of the Imperial Board of Hualth for the year 1888.1889 show that from 2 to $8 \%$ of all cattle were tuberculous. Since then, however, meat inspection statisties and the results of extensive tuberculin tests have shown the disease to be far more prevalent. Thus according to Siedamgrotzky's compilations in $1,110,252$ animals slaughtered in the abattoirs of Saxony, Prussia and Bavaria in the period from 1895 to $1897,13.1 \%$ were found tubereulous. During the same period the abattoirs in the Empire of Germany furnished 353,162 tuberculous animals. Of these 9,705 were condemned and 18,322 were sold under declaration. The resulting annual loss is estimated at six and one-half million marks, or a little over one and a half million dollars. During the same period, of $1,107,552$ slaughtered swine, $1.22 \%$ were found tuberculous. This resulted in the condemnation of the carcasses of 2,600 head of swine while 39,000 head were sold under declaration. The actual loss from tuberculosis in swine (carcasses) was one and three-quarter million marks or over four hundred thousand dollars.

The following table which is taken from the official report on sanitary police control of ahattoirs of the German Empire shows the percentage of animals condemned on account of tuberculosis:

$\begin{array}{rrrrrrrr} & \text { Cattle } & \text { Calves } & \text { Swine } & \text { Sheep } & \text { Goats } & \text { Dogs } & \text { Horses } \\ 1904 & 17.89 \% & 0.26 \% & 2.46 \% & 0.20 \% & 0.69 \% & 0.86 \% & 0.15 \% \\ 1905 & 19.16 \% & 0.30 \% & 2.87 \% & 0.22 \% & 0.76 \% & ? & 0.16 \% \\ 1906 & 20.66 \% & 0.35 \% & 2.81 \% & 0.17 \% & 0.72 \% & ? & 0.17 \%\end{array}$

Abattoir reports of the varions states of the German Empire show that tuberculosis is on the increase among cattle as well as among other species of animals. A few localities only show a decrease in the prevalence of tuberculosis among swine and calves (Ostertag). Thus the kingdoms of Bavaria, Prussia and Saxony furnish the following tables of information:

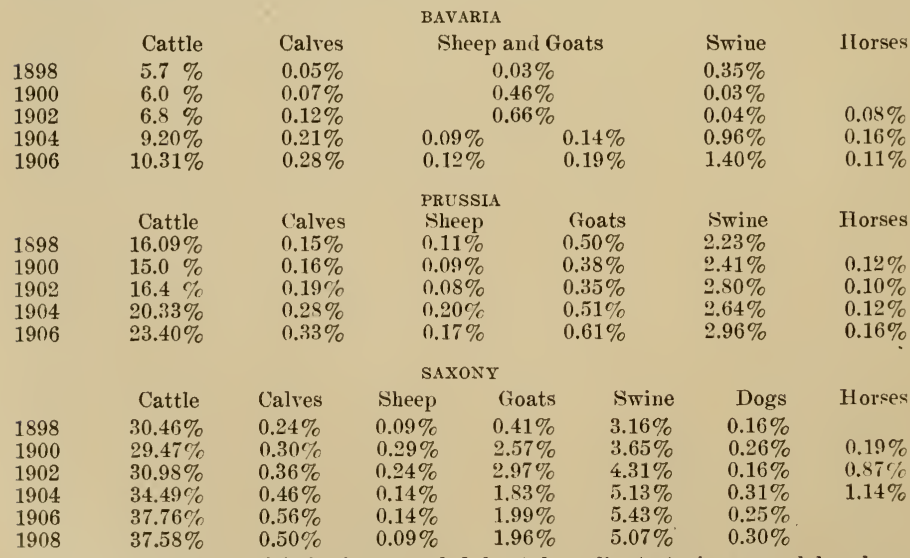

The percentage of infection revealed by tuberculin tests in general has been very high. Thus in the kingdom of Saxony for the years 1891 to 1897 it varied hetween 57 and $84 \%$. Up to the middle of the year $1904,68 \%$ of 3,083 cattle that were tested reacted. Thus the assumption of Klimmer that $2 / 3$ of all cattle 
harbor germs of tubereulosis has a basis of fact. On the other hand Ostertag, basing his estimate upon personal experience in Northern Germany and in the region surrounding Stuttgart, estimates that $25 \%$ of all cattle are affected with tubereulosis.

In France the disease is very prevalent. In several regions (Bretagne, Nivernais, Hautes Vosges, etc.) estimates of veterinarians place the percentage of tuberculous cattle at 30 to $40 \%$ or more. In some instances herds that were apparently free from disease showed by the tubereulin test that they contained from 50 to $80 \%$ of reactors. Over ten per cent of all cattle in the French Republic are claimed to be tuberculous (Nocard \& Leclainche). In the abattoir La Vilette in Paris the percentage of tuberculous calves was from 1899 to 1903 reduced from 0.31 to $0.15 \%$ (Césari). Autopsies held on logs in Alfort in 1900 to 1904 revealed $4.6 \%, 3 \%$, $7.2 \%$ and $9.1 \%$ respectively, to be tuberculous (Petit).

In Great Britain Walley found as early as 1879 tuberculosis to be a rery prevalent disease among Alderney and Shorthorn eattle. From 1897 to $1899,4,105$ or $26.6 \%$ of 15,392 cattle that were tested with tubereulin reacted.

In Austria tuberculosis occurs in all of the Crown Lands. Some abattoir reports, however, show very low percentages of infection. Thus in 1900 lower Austria is credited with $1.4 \%$, upper Austria with $2.9 \%$ and the other provinces with $1 \%$ of infected animals. In Vienna 1.3 to $1.8 \%$; in Moravia, in the years 1896 and $1897,39.5 \%$ of 2,314 tested cattle gave positive reactions and $4.4 \%$ doubtful reactions. During the following years tests that were made in Salzburg revealed from 41 to $44 \%$ of reactors; in Silesia 25 to $40 \%$; in lower Austria $53.4 \%$; in Gallicia among Polish eattle $14.2 \%$; among milk cows $60.3 \%$ of reactors.

The slaughter house reports of Budapest in Hungary, give the following exact statistics:

\section{Cattle :}

1899

1901

1903

1905

1907

1909

$\begin{array}{cc}\text { Longhorned } & \text { Improved } \\ \text { Breeds } & \text { Breeds } \\ 12.2 \% & 13.8 \% \\ 14.1 \% & 13.9 \% \\ 15.0 \% & 16.9 \% \\ 16.6 \% & 21.6 \% \\ 19.8 \% & 25.2 \% \\ 16.8 \% & 21.8 \%\end{array}$

\section{Calves Buffalo Swine}

$0.006 \%$
$0.01 \%$
$0.01 \%$
$0.03 \%$
$0.04 \%$
$0.03 \%$

$2.6 \%$
$1.42 \%$
$1.41 \%$
$1.53 \%$
Horses

$$
0.07 \%
$$

$0.26 \%$

$0.07 \%$

$0.11 \%$

$0.89 \%$

$0.58 \%$
.....

.....

$\ldots \ldots$

$\ldots \ldots$

$\ldots \ldots$

..... results :

Tubereulin tests conducted on eattle of the colored breeds revealed the following

No. of Herds Cattle under $21 / 2$ years Tested Reacted

$\begin{array}{lll}1902 & 34 & 1723 \\ 1903 & 34 & 1145 \\ 1904 & 56 & 2847 \\ 1905 & 85 & 3152 \\ 1906 & 93 & 3859 \\ 1907 & 88 & 2548\end{array}$

\section{Cattle over $21 / 2$ years Tested Reacted}

1871

2.588

3639

4404

5999

4422

$$
\begin{aligned}
& 250=13.3 \% \quad \text { culosis } \\
& 323=13.3 \% \quad 3 \\
& 966=26.8 \% \quad 8 \\
& 1033=23.4 \% \quad 22 \\
& 1345=16.5 \% \quad 12 \\
& 760=17.1 \% \quad 20
\end{aligned}
$$

Herds free culosis

According to investigations by Ujhelyi covering 20 estates, 1,517 or $75.36 \%$ of 2,013 head of eattle tested, reacted; of 1,495 calves and yearlings tested, 526 or $35.18 \%$ reacted. On the other hand of 117 herds belonging to peasants of the county of Moson 53 were found free from tuberculosis. Among the remaining herds of this district, of 501 eattle tested $25.7 \%$ reacted and of 177 ealves and yearlings that were tested only one reacted. The infected herds of these peasants were all located near the larger states from which discarded cattle were occasionally bought.

In Belgium in 1906 among 2,905 herds that were investigated and of 19,004 cattle that were tested, $48.8 \%$ reacted. During the year $1900,10,269$ tuberculous cattle were officially condemned and slaughtered. In the Netherlands the large abattoirs revealed from 2 to $13 \%$ of all eattle as tuberculous. In Switzerland statisties show that the percentage is much lower although in several cantons (Bern, Geneva, Zürich, Winterthur) the percentage in slaughtered cattle is reported to be as high as $19 \%$. Tuberculin tests show that from 40 to $50 \%$ of all cattle are tuberculous (Zschokke).

Denmark was free from tuberculosis at the beginning of the 19 th century, but hecame infected by the importation of Swiss, Holstein and English (Shorthorn) cattle. As a result, the first tubereulin tests made in this ecuntry (1893-1894) shower that from $38.5 \%$ to $40 \%$ of the cattle were tuberculoss. The ahattoir 
statistics of Copenhagen for 1895 showed that $29.7 \%$ of all slaughtered eattle and orer $10 \%$ of all swine were tuberculous. In the period beginning April, 1893, and ending with the year 1908, 19,717 herds were tested with tuberculin. Of these 11,553 were first tests. A total of 503,879 cattle were tested of which 108,378 or $21.5 \%$ reacted to the tuberculin tost. During this period the number of reactors was reduced from $40 \%$ to $8.5 \%$ of 10,344 lierds tested for the first time in the year 1903, 2,664 herds (consisting of 33,946 animals) were found free from tuberculosis. Most of these herds contained less than 25 animals each; 153 herds were composed of from 26 to 55 animals (Bang).

In Norway, in 1895 to 1909 , of 25,832 herds that were tested, 4,217 or $16.3 \%$ were tuberculous. Of 219,925 animals tested 12,523 or $5.5 \%$ were infected (Malm).

In Sweden, from 1897 to 1908 , of 12,720 herds which were tested for the first time, 5,527 heris were free from reactors. Of 306,372 animals tested $30.7 \%$ reacted (Regnér).

In Finland, from 1594 to 1900 , of 75,447 eattle that were tested $13.7 \%$ reacted (Hójer).

Of the other European countries tuberculosis is very prevalent in Northern and Central Italy, in Spain and in Portugal. According to Nogueira the disease exists to the extent of $3.8 \%$ among bulls which are reared in the open to supply the market for bull fights. The disease is prevalent to an equal extent in Russia, Roumania, Servia and the Herzegovina. On the other hand the cattle of the steppes of Russia are practically free from the disease. Of 30,000 Servian cattle killerl annually in Budapest up to the year 1906 , only .20 to $.23 \%$ were found tuberculous.

Outside of Europe tuberculosis occurs wherever European breeis of cattle have been imported. Thus the disease is very prevalent in North America where, according to Salmon, among suspected herds that were tested, $25 \%$ were found infected, while among unsuspected herds $9 \%$ were found infected. Reports of the Bureau of Animal Industry show that of 400,008 cattle tested $9.25 \%$ reacted. According to Melvin the disease causes an annual loss of $\$ 23,000,000$ in the United States. Tuberculosis is also quite prevalent in South America. In Buenos Ayres 3.7\% of all cattle and $10.8 \%$ of all swine were found tuberculous. In the State of San Paoblo $20 \%$ of milk cows are tuberculous. Finally the disease is also prevalent in Africa (Egypt and Southern Africa) as well as in Australia. In Japan where tuberenlosis has always been prevalent among human beings, it has been known in cattle only for the past 30 years and since then has been gaining ground rapiclly. The disease was introduced by the importation of American cattle (Kanda).

Etiology. The Bacillus Tuberculosis Koch is a slender, slightly bent bacterium, 1.5-4.0 $\mu_{\text {. }}$ long and $0.3-0.5 \mu$. in diameter, which seems to be covered with a thin waxy substance. It is non-motile and non-sporulating. In tissues it occurs singly; in morbid secretions it is frequently found in groups, in parallel hundles or in pairs placed at an acute angle (see Fig. 77). In rare cases it is composed of a chain of two or three links. In fresh cultures it grows in wavy lines, while older cultures produce long threads which may show a tendency to branch and form club-shaped swellings. Stained bacilli from morbid secretions show colorless spaces which give them the appearance of coccus chains.

Besides the typical acid-fast form of the tubercle bacillus there has recently been discovered by Much a non-acid-fast granular form which consists of small granules arranged in straight rows. This probably represents a young culture variety of the species from which the rod shaped acid-fast bacterium develops (see Fig. 78 and Fig. 79). The granular form which is found especially in pearl disease of cattle and in cold abscesses of man, is just as virulent as the acid-fast rod-like species.

$\mathrm{Br}$ means of special staining methods the interior of the tubercle bacillus reveals a granule (rarely two or four granules), 

Plate 11 .
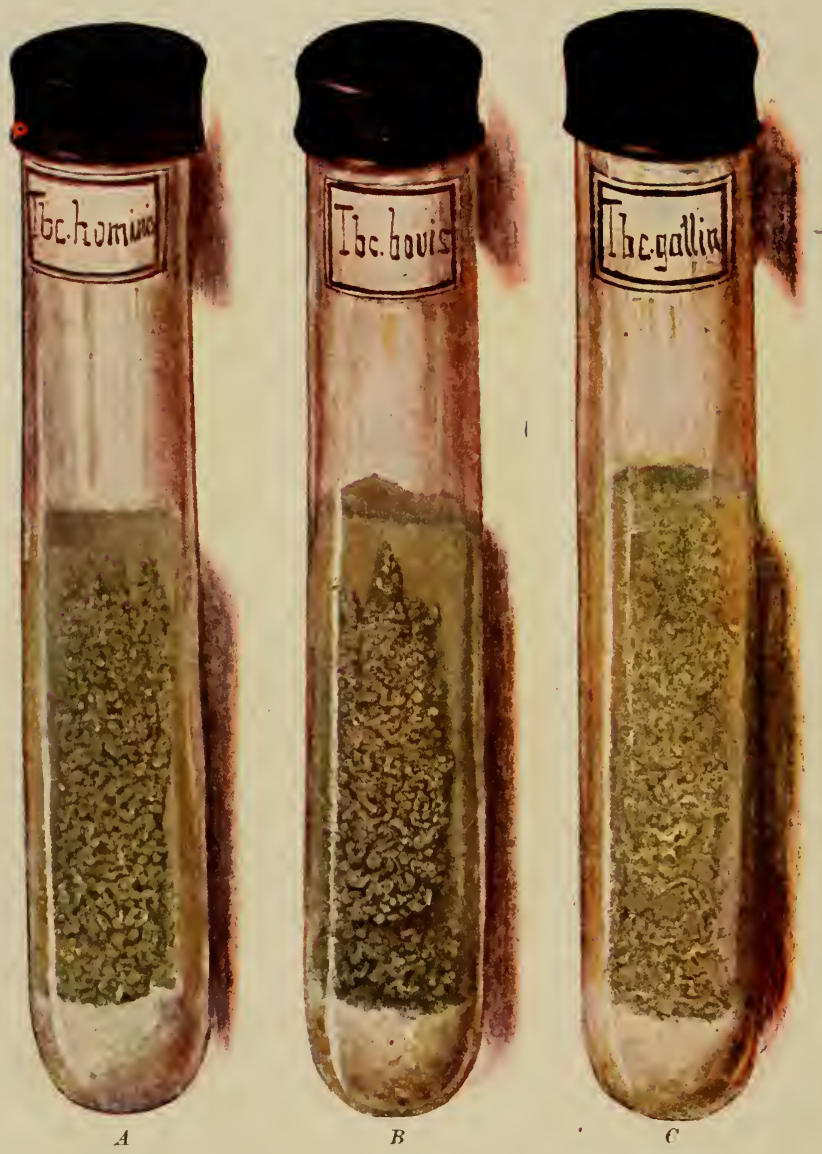

Cultures of the Bacillus tuberculosis on glycerin-potito.

A. Human type, 4 weeks old; $B$. Bovine type, \& weeks old; $C$. Arian trpe, 2 weeks olit. 
which Spengler refer's to as "splinters" and regards as true spores. Most investigators, however, look upon these bodies as chromatin granules such as are found also in other bacteria whether they are sporulating or non-sporulating species.

Staining. The typical tubercle bacillus is stained most readily and characteristically with a warm carbol-fuchsin solution or with any aniline water gentian-violet. After once absorbing the stain the latter becomes so well fixed that it is impossible to remove it with alcohol or mineral acid (5\% sulphuric acid$30 \%$ nitric acid). This characteristic (acid-fast or alcohol-fast) which is met also in the bacillus of leprosy and in several saprophytic bacteria, is due to the presence of fatty acids in the body of the bacillus (Deycke). No other bacteria except those mentioned possess this characteristic.

Much's granular form of the tubercle bacillus may be stained by Gram's method of subjecting it to the action of anilinegentian violet for 24 to 48 hours at body temperature. (See Diagnosis.)

Culture. The tubercle bacilli will grow only in the presence of oxygen and at a temperature varying from 29 to $42^{\circ}$ C., its growth being most prolific at blood temperature. The best culture media are those containing egg albumen, although the bacillus will grow also on common laboratory media which do not contain this substance. The chemical reaction of the medium must be neutral or slightly acid. The presence of glycerin, grape sugar or phosphoric acid favors its growth which is usually very slow. Cultures are never visible before the end of a perion of at least one or two weeks.

On blood serum and on 3 to $5 \%$ glycerin agar the cultures appear as follows: In the course of two weeks after inoculation of material small gray dry lusterless granules or scales make their appearance. These are gradually surrounded by a halo (Fig. 80 ). In the course of time they form an irregular. crumpled, dry granular continuous mass which is easily removed from the surface of the medium. On glycerin bouillon after surface inoculation there is formed a fine pellicle which ultimately develops

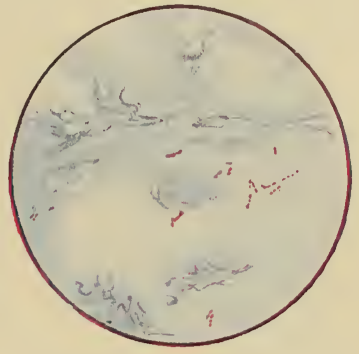

Fig. 77. Bac. Tuberculosis, acidfast form. Bronchial secretion from cattle. Stained by ZiehlNelssen's method.

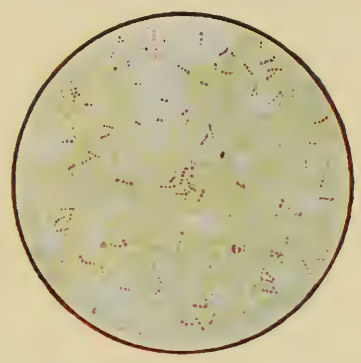

Fig. 78. Bac. Tuberculosis, granular form, Bronchial secretion from cattle. Stained by Much's $n$ ethod.

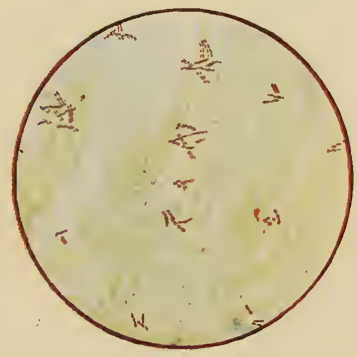

Fig. 79. Bac. Tuberculosis, Stained by Hermann's method. (The same material as in Fig. 77-78). 
into a thick membrane covering the entire surface of the fluid. In the course of time this membrane increases in thickness and becomes crumbly. Very slight jarring of the container will cause it to break into fragments causing portions to sink to the bottom of the flask where they develop into globular mulberry-like white colonies. The bouillon remains elear throughout its mass. Upon glycerin-potato medium, one of the most suitable for its growth, there develop dry crumbling masses which unite to form a rough irregular covering. (See Plate II.) Acidreacting brain-agar and brain-serum are also excellent media for its growth.

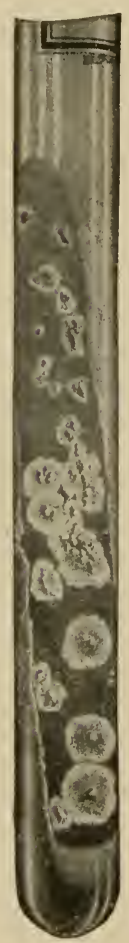

Fig. 80. Glycerin a gar culture of the tuherele bacillus, five weeks old.

Arloing succeeded in producing so-called homogeneous cultures by repeated shaking, in which the unusually long bacilli are less acid fast than usual and motile. The original potato cultures had a shiny fatty appearance.

Morphological and Cultural Differences Observed in Tubercle Bacilli of Different Origin. These were pointed out by Theobald Smith in 1898 . Later other investigators (Kossel, Weber \& Heuss) confirmed the results reported by Smith. According to these investigators two types or varieties of tubercle bacilli are recognized (human type and bovine type). These types, however, do not include the avian form of tubercle bacillus nor the varieties which occur in cold hlooded animals. Intermediate between the human and the bovine type are observed many transition forms some of which it is impossible to classify with either group. The cultural characteristics, however, are clearly manifest only when the various types are grown on the same medium and under similar conditions, particularly upon beef broth of amphoteric reaction with $2 \%$ glycerin.

The bacilli of the human type, when found in morbid secretions or tissues or in pure culture, are usually more slender and show the irregular staining to better advantage than the bacilli of the bovine type. Further, on some varieties of potato the cultures of the human type assume a dark red color in the course of three weeks (Arpad). They also show more rapid growth in first cultures than the bacilli of bovine tuhereulosis. On the other hand the latter are frequently characterized by their fine verrucose surface on potato culture.

The bacilli of the human type grow lnxuriantly on glycerin houillon, forming a wrinkled membrane of even thickness which spreads over the entire surface of the liquid, while the hovine type forms a fine, delicate and frequently fenestrated pellicle upon which small nodular protuherances develop in the course of time (Kossel, Weber \& Ileuss). Then again, the mulberry shaped colonies which develop after inoculation of small numbers of bacilli are characteristic of the bovine type.

On bile medium or on potato impregnated with $5 \%$ glycerin bile, tuhercle bacilli will develop only if obtained from the same speeies which furnished the bile. Thus the human type will grow only on bile medium produeed from human bile and the hovine type on hovine bile, the avian type on bile obtained from fowls (Calmette \& Guérin). 
Another difference observed between the two types under discussion is that in cultures of the bovine type the acidity decreases until the medium at times becomes alkaline, while media containing a growth of the human type, though at first reduced in acidity, becomes more and more acid with the age of the culture (Smith). According to Rothhaar, Fibiger \& Jensen this characteristic is inconstant.

These two types of bacilli, however, are not confined strictly to definite species of animals, both types occurring in different species. However, in the great majority of cases tuberculosis of man, dogs and cats is caused by the human type and tuberculosis of cattle and other ruminants and of swine is caused almost exclusively by the bovine type.

Tenacity. The tubercle bacillus is extremely resistant to external influences. This is probably due to the waxy capsule by which it is protected. When exposeil to the direct rays of the sun they are destroyed in a few hours. In diffuse light they will retain their virulence from 3 to 10 days (Flügge). In artificial light they are destroyed in 48 hours (Galtier). Simple drying, especially when imbedded in nitrogenous tissue or fatty medium has little effect on their vitality. Thus in dry sputum protected from light they may remain virulent 126 days (Schill \& Fischer), in dry and pulverized tissue from cattle lung 102 days, in large tissue masses 150 days (Cadéac \& Malet). Stone found that sputum was infectious after three years. Decomposition affected their vitality very little. Thus decomposing sputum harbors virulent bacilli for six weeks (Schill \& Fischer) and decomposed cattle lung 167 days (Cadéac \& Malet). In water, cultures remain virulent 70 days, in tissue 120 days.

The bacilli will remain virulent in raw milk for 10 days (Gasperini) at the end of which period they probably succumb to the influence of lactic acid (Bollinger). On the other hand they have been known to remain virulent 33 days in Emmentaler cheese and 104 days in Chedilar cheese (Harrison). In sweet eream butter they remain virulent 4 weeks. They are destroyed in the course of ten days in sour cream butter containing 4 to $6 \%$ conmon salt. Sometimes 5 days suffice to destroy them under these conditions (Petterson). On the other hand they have been known to remain virulent 45 days in such butter (Schroeder \& Cotton). Treatment with $\mathrm{H}_{2} \mathrm{O}_{2}$ ("Budderisicrung") destroys the tuberele bacilli with certainty (Bergman \& Hultman). Kefir fermentation does not affect their virulence.

The influence of high temperatures varies according to the medium in which they are found. Simple boiling or contact with live steam will destroy dry or moist sputum in 5 or 15 minutes, respectively. On the other hand dry sputum can be safely sterilized by the action of dry heat at $100^{\circ} \mathrm{C}$. continued for one hour (Schill \& Fischer). In milk, bacilli which were originally present as well as bacilli which have been added in cultures, are destroyed in 25 minutes when heated to $65^{\circ} \mathrm{C}$., providing the milk was heated in a closed vessel (Forster). On the other hand heating the milk in open vessels, which does not result in an equal distribution of temperature throughout the mass, will not destroy all tubercle bacilli in the course of six minutes, even if the temperature reaches $80^{\circ} \mathrm{C}$. All bacilli found in the film which forms on the surface of the milk are more or less protected from the action of the heat (Galtier, Smith, Bang). According to Rabinowitsch \& Beck cream can be sterilized by heating for 2 minutes at $85^{\circ} \mathrm{C}$. (Herr). According to investigations of Barthel \& Stenström bacilli will withstand an exposure of $80^{\circ}$ or $85^{\circ} \mathrm{C}$. when the milk is coagulated while they are destroyed in non-coagulated milk at the same temperature. Thus pasteurizing at a temperature of $80^{\circ}$ continued for one minute will suffice to destroy the tubercle bacilli providing no coagulation occurs. Freezing temperature of $1^{\circ}$ to $\mathrm{S}^{\circ} \mathrm{C}$. will not destroy the bacilli in 120 days (Cadéac \& Malet).

With respect to chemical agencies, bacilli occurring in albuminous substances are most effectively destroyed by such chemicals as will not coagulate albumen. Thus carbolic acid $5 \%$, acetic acid $31.8 \%$, or corrosive sublimate $1: 1000-500$ will not destroy them (Schill \& Fischer). Cultures are destroyed in solutions of $0.1 \%$ tuberculin in 48 hours, $0.2 \%$ methylene blue, $0.1 \%$ pyoktannin in 72 hours (Jacob, Bongert \& Rosenberg); $0.2 \%$ thymol, $4 \%$ boracic acid and $4 \%$ ereolin or lysol are also effective. Brine and smoke have only a slightly destructive action, virulent tubercle bacilli having been found in tuberculous organs after six weeks immersion in brine (Klepzow). Glycerin acts as a preservative of tuberculous organs (Galtier). Antiformin in solution of $15 \%$ does not affect tubercle bacilli or other acid-fast bacilli while non-acid-fast bacilli are destroyed by this agent. On the other hand $20 \%$ solution of cholin will dissolve the tubercle bacilli at a temperature of $37^{\circ}$ to $56^{\circ}$. Neurine is even more effective (Deycke \& Much). 
Gastric juice at $30^{\circ}$. C. does not affect the bacilli in 3 to 4 hours. In the course of 6 or 8 hours, however, their virulence becomes reducer and in 18 to 24 hours they are destroyed (Zagari, Strauss \& Wurtz). Bile and pancreatic juice do not affect them (Gallichi).

Pathogenicity. Then susceptible animals are inoculated subcutaneously with tuberculous secretions, tissue or virulent cultures, there develops at the point of inoculation a tumor which may break in the course of a few days, discharge a caseopurulent mass and develop into a sinuous ulcer. In some cases the ulcer will heal and the process terminate; in other cases the disease progresses. After a time the lymphatic glands which are supplied by the lymph vessels of the affected region, swell and become the seat of the formation of tuberculous foci, which, in the course of time, undergo cheesy degeneration. Following this the internal organs become affected almost simultaneously, sometimes one after another along the lymphatic circulation and at other times apparently independently through the medium of the blood. Cutaneons inoculations (rubbing on the scarified skin) usually produces only small rapidly healing nodules (Chauveau). Sometimes, however, the neighboring lymph glands become involved and a general tuberculosis may follow (Courmont, Babes, Osman Nouri).

After intraperitoneal inoculation to which animals in general are very susceptible, the peritoneum (especially the mesentery) the abdominal lymph glands, the spleen and the liver are attacked first. Adjacent organs may become involved later. The most effective methor of inocnlation consists in the injection of virus into the jugular vein. This is followed by the development of innumerable tubercles in the lungs with acute swelling of the lymphatic glands of the thoracic cavity. Animals usually die in the course of a few weeks with symptoms of high fever and rapid emaciation. Sometimes other organs are similarly affected. If inoculation is made in the anterior chamber of the eye, typical tuberculosis develops in the iris in the course of two or three weeks. This local affection is then followed by a general tuberculosis (Cohnheim, Baumbarten).

The feeding or ingestion of pure cultures or of material containing tubercle bacilli (sputum, milk tissue) to guinea pigs or calves is followed either by tuberculosis of the peripharyngeal, cervical and mediastinal lymph glands and bronchial tubes, or by an infection of the mesenteric lymph glands and the abdominal organs. The pharyngeal and intestinal mucous membrane may remain practically healthy but on the other hand, especially if the infection was severe, the tonsils may ulcerate or the lymph follicles of the intestines may undergo tuberculous vlceration. Old animals are only rarely affected by these means.

Inhalation of dry or pulverized tuberculous tissue or secretions as well as culture sprays may be followed by tuberculosis of the lungs and of the bronchial glands. The more finely the infectious material is pulverized or divided the greater are the 
chances of infection. Inhalation through the mouth is more effective than inhalation through the nostrils. The inhalation of heavily infected air usually results in miliary tuberculosis of the lungs, while slightly infected air may produce only isolated tuberculous foci which develop into cheesy pneumonia processes or cavities (Cornet \& Mayer).

Infection also follows the injection of tuberculons material into the vagina or its direct application to the mucous membrane (Cornil \& Dobroklonsky). Occasionally the first symptom of infection is a swelling of the inguinal glands without local clianges. Finally direct application of virulent cultures to the teats of the udder is followed by a progressive inflammation of the milk ducts (Nocard, Nattan-Larrier).

Variations in the Pathogenic Effect of Tubercle Bacilli of Different Origin. The results of experimental infection vary considerably according to the origin of the tubercle bacilli or of the material containing them, as well as according to the age and the species of the experimental animals. With rare exceptions the bacilli of the bovine type possess a higher degree of virulence for mammals, including the ape, than the bacilli of the human type. It seems immaterial, also, whether the particular type of bacillus in question originates from one species of animal or from another. The differences in the pathogenie action are observed even in the guinea pig which is unusually susceptible. These animals suecumb much more rapidly to infection with the bovine type than to infection with the human type (Smith, Gratia). The difference in the results of these two types is much more marked, however, in rabbits and in cattle.

The difference between the two types in their effect on rabbits was recognized by Villemin and later by Orth, Baumgarten, Vagedes, Smith, Dorset, Kossel, Heuss, Rabinowitsch and others. According to Weber this may be summed up as follows: The baeilli of the hovine type when injected intravenously in $0.001 \mathrm{~g}$. doses will kill rabbits in three weeks, while the bacilli of the human type similarly administered produce at first no conspicuous morbid symptoms, but in the course of several months symptoms of chronic tuberculosis develop which affects chiefly the articulations, the kidneys, the lungs and the testes. The bacilli of the bovine type injected subentaneously into the abdominal wall in $0.01 \mathrm{~g}$. doses, (not under the fascia into the museles) produce a lung tuberculosis which terminates in death in a short time. The bacilli of the human type will not prodnce these results.

The effect of the two types of tubercle bacilli on cattle has, in recent times, been the object of almost innumerable experiments. These experiments as far as they have been limited to the use of pure cultures have given comparatively uniform results, viz., that the bacilli of the human type possess either a slight, or no pathogenic action whatever for eattle and that the bovine type, on the other hand, possess very marked pathogenic properties with regard to cattle. Thus the subcutaneous injection of bovine bacilli, injection into the circulation or into the peritoneal cavity usually produces severe progressive tuberculosis. Young animals occasionally become affected in the same manner by the ingestion of infected material. On the other hand inoculation of cultures of the human type produce a local affection only or remain withont any pathogenic effect. This is particularly true if the dose of pure culture 
is not excessive (not more than $4 \mathrm{cg}$.). The ingestion of such material will produce local changes only in the mesenteric glands.

Kossel, Weber \& Heuss, who experimented with 38 different strains of the human type and with 27 strains of the bovine type, included in their experiments a large number of eattle. Subentaneous inoculation of $.05 \mathrm{~g}$. of culture produced at first an inflammatory swelling at the point of inoculation which developed into abscesses that broke and discharged their contents. This was follower by enlargement of the prescapular glands. When the bacillus of the human type was used this swelling attained the size of a goose egg but was consiterably larger in cases where the bacillus of the bovine type was usel, sometimes as large as a man's hear. When the bacillus of the human type was used the process stopped at this point and was followed by retrogressive ehanges. After seven months all bacilli were destroyed. On the other hand the bacilli of the bovine type continued their work of destruction and protuced a progressive tubereulosis which, in $30 \%$ of all cases encled in death in the course of 8 or 10 weeks. Feeding experiments demonstrated that one feeding of bacilli of the bovine type sufficed to infect ealves. Of eight animals some of which were given one treatment, others repeater treatments, four died in from 79 to 115 days. Post-mortem examination showed serere lesions in the intestines and the regional mesenteric glands. On the other hand all ealves fed with glycerin bouillon cultures of the human type remained alive. The bacilli which had been introduced into the intestinal canal were lodged partly in the mesenteric glands where they gave rive to the formation of small yellow calcified foci which had not eaused any reaction in the otherwise normal tissue. In the course of inhalation experiments it was shown that bacilli of the bovine type introduced in this manner were exceeringly virulent. One $\mathrm{mg}$. of bacilli was sufficient to kill a calf four months old within 48 days. Following a period of ineubation of 12 to 14 days the animals were attacked with a high fever and difficult respiration. Four died in the course of 26 to 62 days with caseous pneumonia. Four others were affected in the course of 110 to 174 days with pulmonary tuber-eulosis and tuberculosis of the adjacent lymph glanils and serous membranes. On the other hand the bacilli of the human type in these experiments produced no tuberculous disease.

The difference in virulence is observer also where injections are made into the milk ducts of eattle and goats. Thus tubercle bacilli of human origin produced only a slight redilening lasting a few days, and attended with moderate sensitiveness and swelling of the affected quarter. On the other hand the hovine bacilli produced a pronounced tuberculosis of the udder and mammary glands and, in aldition, probably as a result of an intoxication, rapicl emaciation ending in death (Nocard, Meyer, Calmette \& Guérin, Zwick).

The differences in results referred to above apply only to characteristic pure cultures. Since both types oceur in man, inoculation experiments with crude material, such as sputum, tuberculous tissue, etc., give varying results. However, these results correspond with the fact that the human type is most frequently present in man, while the bovine type is almost exclusively present in cattle. (For further information on this subject refer to the chapter on the Relationship between Human Tubereulosis and Bovine Tubereulosis.)

Results of experimental inoculations of other animals with morbid secretions or tissue show, in general, that swine are readily infected with either of the two types of bacilli, that sheep and goats are most readily infected with material containing the bovine type and that dogs may he infected with large doses of either the human or the hovine type of bacillus (Titze \& Weidanz). Other mammals possess a considerable power of resistance to infection. Again, all avian species with the exception of house birds (parrots), possess considerable resisting power against infection with the bacilli of the mammalian types.

Infectious Secretions and Products. In the bodies of tuberculous animals the bacilli are, with the exception of miliary tuberculosis, found usually only in the affected organs, and in 
fact only in the diseased portions of these organs. They are further found in the contents of body cavities and canals the walls of which contain tuberculous ulcers or which may receive pathologie proclucts from other organs (e. g., lumg secretions in the intestinal canal). In so far as these canals connect with the outer air, the secretions and excreta discharged through their opening's may contain more or less tubercle bacilli which are thus disseminated (open tuberculosis, in contrast to enclosed tuberculosis in which the bacillary foci do not communicate with the outer air).

Even in slightly adranced cases of tuberculosis of the lung's the bronchial secretions as well as the nasal secretions may contain bacilli in varying numbers either at intervals or constantly. The organisms may also be present in the saliva as a result of an admixture of bronchial secretions with the secretions of the buccal cavity.

Since animals usually swallow the secretions from the lungs which are coughed up from time to time, these become incorporated with the contents of the intestinal canal and are found unchanged in the feces. In addition to this source tubercle bacilli which are discharged from intestinal ulcers may be the cause of infection of the feces, consequently the excretions from animals with advanced tuberculosis are one of the most important factors in the dissemination of the disease.

The fact that tubercle bacilli may pass through the intestinal eanal without having their virulence impaired in the least, has been experimentally demonstrated by Cadéac \& Bourney. These investigators inoculated rabbits with the feces of a cow that had been infected by feeding tuberculous organs, and in this way produced tuberculosis in nearly every case. Even before this Zagari demonstrated that the feces of dogs that were fed with human tuberculous sputum contained virulent bacilli. The experiments by Ferranini demonstrating that gastric juice containing $2 \%$ of free hydrochloric acid does not reduce the virulence of tubercle bacilli in the least, in the course of 1 to 2 hours, are in accordance with this fact.

Schroeder \& Cotton emphasize the danger that exists in the feces of apparently perfectly healthy eattle but which are in fact tuberculous. They found that in a number of cases the feces of such animals contained tubercle bacilli constantly for periods of months and years. The enormous number of bacteria present in feces is indicated by the fact that $0.001 \mathrm{~g}$. of such material will produce a fatal tuberculosis in guinea pigs, the average ruantity of feces voided by a cow daily being $30 \mathrm{lbs}$. It is evident, of course, that this infected mass of manure may easily contaminate the milk during milking.

In tuberculosis of the kidneys, especially of the pelvis and in disease of other portions of the urinary apparatus, the urine contains tubercle bacilli (Kallina); similarly tuberculosis of the uterus, epididymis or prostate gland may cause the presence of bacilli in the urine. In tuberculosis of the uterus, the vaginal secretion, and in tuberculosis of the epididymis the spermatie fluid may contain tubercle bacilli. 
In tuberculosis of the udder the milk is always infected, sometimes to such an extent that $0.00001 \mathrm{~g}$. of milk when injected intraperitoneally into a guinea pig will produce a fatal tuberculosis (Ostertag). Further, the milk from infected cows which already show clinical symptoms of disease, may contain tubercle bacilli even though the udder is apparently perfectly healthy; since small tuberculous foci in the glandular tissue could not of course be recognized by clinical examination. Recent experiments have shown that cows in apparently perfect health which reacted to the tuberculin test may discharge tubercle bacilli with the milk. In such cases, of course, in the face of all negative clinical evidence, the mammary tissue may contain minute metastatic tuberculous foci; furthermore the possibility cannot be refuted that tubercle bacilli may occasionally gain entrance into the circulation from tuberculous foci, be carried into the mammary glands and in this way pass into the milk.

The infectiousness of milk from a tuberculous cow has frequently been demonstrated since the time of Gerlach (1869). Aside from the product of evidently diseased udders, in which ease the milk almost invariably contained tubercle bacilli in large numbers, Hirschberger succeeded in producing tuberculosis in guinea pigs with milk from clinically diseased cows with apparently healthy udders, by means of intraperitoneal injection in 11 cases out of 20 , Ernst in $26.8 \%$ out of 33 cases, Nocard 3 times in 54 cases.

As far as the use of milk is concerned coming from apparently healthy cows which have reacted to the tuberculin test, the results of experiments vary.

Ostertag was the first to conduct exact experiments to shed light upon this subject. The milk from 49 reacting cows was collected in separate containers with observance of every possible precaution, and injected intraperitoneally into guinea pigs. The results show that not a single sample of milk contained tubercie bacilli. On the other hand 14 inoculations of guinea pigs made from a mixture of the above 49 samples produced tuberculons disease in one guinea pig. Feeding experiments with this milk produced no positive infections. In a second experiment the milk of 18 cows was used. In this case neither intraperitoneal injections into guinea pigs, nor the feeding of the milk for several months to guinea pigs, swine and calves produced tuberculosis. Similar results were obtained by Müller and Ascher who used the milk of 9 and 7 reacting cows, respectively, in experiments with guinea pigs. From this Ostertag concluded that milk from merely reacting rows contained no tuherele bacilli.

Bacteriological examination of milk of 5 head by MacWeeny and of 50 head of cattle by Stenström some of which were clinically diseased, others affected with latent tubereulosis but with healthy udders gave similar negative results. Hessler obtained the same results with 61 merely reacting cows. Schroeder \& Cotton also are of the opinion that tubercle bacilli are excreted with the milk only where the udder itself is diseased. Of 11 cows, 9 of which had generalized tuberculosis and 2 advanced pulmonary tubereulosis, the milk proved virulent by inoculation in $1.78 \%$ and by feeding in $0.68 \%$ of cases. These results might of course have been due to subsequent infection of the milk.

On the other hand Gehrman \& Evans found the milk virulent in 9 out of 41 reacting cows in spite of the fact that a post-mortem failed to reveal tuberculous lesions either in the udder or in other internal organs. In 2 cows there were only slight lesions in a few lymph glands. Ravenel demonstrated the infectiousness of milk of 5 reacting cows none of which showed tuberculous lesions at post-mortem examination. Mohler, of Washington, examined the milk of 56 reacting cows only 9 of which showed symptoms of tuberculosis after months of observation, and in all eases the udder was perfectly normal. In 8 of the eows the milk proved to be 
virulent in feerling as well as in inoculation experiments; in 3 cows, by inoculation only; in one cow, ly feerling only (guinea pigs used in this experiment receiverl 80 to $120 \mathrm{cc}$. of whole milk daily for 2 to 3 months). Similar results were obtained by Moussu. The milk of tuberculous cows with clinically and anatomically healthy udders gave positive results in 7 out of 57 guinea pigs that were treated. Calves were also infected by feeding. Martel \& Guérin examined 20 udders of slaughtered animals; in all cases the parenchyma as well as the mammary glands were free from observable lesions. The secretions of 4 of these udders proved infectious for guinea pigs. Finally De Jong found virulent tubercle bacilli in the milk of 3 out of 10 cows with slight closed tuberculosis.

One reason which led Ostertag to conclude that a transmission of tubercle bacilli into the circulation did not take place under normal conditions was that the inoculation of a guinea pig with milk of a cow which had received a large dose of bacillary material into the circulation 11 days previously, produced negative results. The experiments of Prettner who made repeated injections of pure cultures of tubercle bacilli into the circulation of 2 buffalo cows, and those of Coquot \& Césari who injected an emulsion of hacilli into the mammary artery gave similar negative results. But Griffith demonstrater the infectiousness of milk in the course of the first week after the subeutaneous injection of bovine tubercle bacilli; Titze produced the same result with human tubercle bacilli. In one case they appeared 24 hours after the injection of the culture, in another they could be demonstrated daily after the third week for 144 days and in some cases as late as 16 months after the injection. As a rule they are excreted from one quarter of the udder only, probably as the result of a focal infection without local symptoms. Snith found that bovine as well as human bacilli injected intravenously into a cow, appeared in the milk after a few days. The human hacilli appeared also in the feces and the urine. The udder was not visibly affected in either case.

Then we take into consideration that even animals with a mild infection may at any time suffer an attack of the udder through metastasis, and that neither the beginning of the attack, nor the stage of the disease can be recognized by a clinical examination, the claim that milk from merely reacting cows may be dangerous is by no means groundless.

In milk of normal appearance tubercle bacilli when present are evenly distributed. If the milk is coagulated, watery or flaky, the bacilli are usually found mostly in the solid parts. If milk is allowed to stand the bacilli have a tendency to collect in the cream. Similarly the cream of separator milk and the walls of the centrifuge contain the bacilli in the greatest numbers.

The milk of a single cow with an infected udder may infect a large quantity of milk from healthy cows if added to it. For this reason the milk from creameries or milk stations is so generally infected. Thus Petri in Berlin found $14 \%$ of 64 samples infected; Beck of the same city, $30 \%$ of 56 samples; Delépine \& Niveau, of Manchester, $11.7 \%$ of 401 samples and $18.5 \%$ of 108 samples; Preisz, of Budapest, $13.8 \%$ of 58 samples; Eher, in Leipsic, $10.5 \%$ of 21 samples. Rabinowitsch found that the milk from creameries which demanded the tuberculin test for all dairies was free from tubercle bacilli, while on the other hand where only clinically infected animals had been removed the milk was found infected.

Of course, milk from healthy animals may become infected with bacilli in the process of milking. There is always abundant opportunity for this in infected stables, especially where the floors and the bedding have been soiled with the excretions and secretions of animals with open tuberculosis. Where stables are kept in a generally unsanitary condition and where careful cleansing of the udder before milking is disregarded, where the first few streams of milk are not discarded, additional opportunities for infection are offered. Dirty milk utensils and soiled hands of the milkers naturally play an important role in bringing about an infection of the milk of otherwise healthy animals.

Vol. 1-33 
In examinations made for the East Prussian Heri Book Association up to July 1, 1907, by Müller \& Hassler, tubercle bacilli were demonstrated in 156 of 2,949 milkings from herds consisting of 30 to 200 animals each. The sources of infection were afterwards found to be, the udder in 113 instances, the uterus in 17, open lung tuberculosis in 20, pulmonary and intestinal tuberculosis in one instance. In 5 cases open tuberculosis could not be demonstrated.

Until very recently the general impression prevailed that the bacilli were rarely found in the blood and then only for a short time after the discharge of a tuberculous focus, thus especially in the beginning of acute miliary tuberculosis. 'This view was based upon the experimental observations of Nocard and MacFadyean according to which all comparatively large masses of bacilli injected intravenously disappeared after a few hours. In the meantime Neumann \& Wittgenstein as well as Bongert, showed that tubercle bacilli injected into the blood could remain there for one month. More recent investigations have shown that in the course of tuberculosis and even in tlie very first stages of the disease, tubercle bacilli are by no means infrequently present in the blood and can be demonstrated by the microscopical examination of small quantities of blood. In such cases providing they are not too abundant, an eruption of miliary tubercles does not necessarily follow. Evidently the animal organism has the power to overcome an infection by a limited number of bacilli. Miliary tuberculosis seems to develop only after an invasion of germs in large masses.

After Liebermeister called attention to the comparatively frequent occurrence of tubercle bacilli in the circulating blood of consumptives, having demonstrated their presence by guinea pig inoculations in $30 \%$ of the second stage cases and in $60 \%$ of the third stage cases examined. Forsyth, Lippmann, Schnitter añd Rosenberger demonstrated their presence with the microscope. Booth showerl nimeroseopically the presence of tubercle bacilli in the blood of two apparently healthy cows suffering with pulmonary tuberculosis. On the other hand Schroeder E Cotton in an examination of 42 cattle could not demonstrate bacilli in the bloor either with a microscope or by means of guinea pig inoculations. Ravenel and McFarland arrived at similar negative results in the examination of blood of tuberculous human beings and expressed the belief that Rosenberger found acid-fast para tubercle; bacilli which are frequently present in distilled water. This question which is of elinical importance is therefore, as yet, unsettled.

The muscles or flesh of tuberculous animals also contain bacilli only in rare cases. When tuberculous masses (lymph glands) are found between the muscles or when a general tuberculosis is present following an artificial intravenous injection, the bacilli which are found in the muscles after a few days are as a rule no longer virulent. The expressed juice from muscle, even of badly infected cattle, is infectious only in exceptional cases. Further, the demonstration of tubercle bacilli in such cases has been possible only by intraperitoneal or subcutaneous infection, while attempts to produce infection through feeding experiments have all produced negative results.

Perroncito injected muscle juice obtained from tuberculous animals into the peritoneal cavity of several hundred guinea pigs and rabhits with not a single positive result. Nocard demonstrated in this manner that the expressed muscle juice of only one cow out of 21 with general tuberculosis was infectious. Galtier 
found the expressed muscle juice of two ont of 15 tuberculous cows infectious. Kastners arrived at negative results in all eases as long as he confined his experi. ments to the muscle juice obtained from tuberculous cows at the abattoir of llunich that were passed for beef. However, when he turned his attention to 7 cows, 6 of which had been condenned on account of advanced generalized tuberculosis, he produced 5 positive results. According to this view, meat may contain bacilli when the internal organs are affected with cheesy, purulent foci, while dry or calcifiel lesions do not produce infection. Swierstra found bacilli in the muscle juice of 7 out of 18 cows and of 2 out of 8 swine affected with advanced tuberculosis. Bongert found the muscle juice virulent in 5 out of 13 eattle affected with softenei tuberculous foci. Hoefnagel arrived at negative results in cases of advanced chronic tuberculosis and Westenhöfer obtained only one positive result of acute miliary
tukerculosis.

It seems that the feeding of meat from tuberculous animals produced positive results almost exclusively at the time of the discovery of the tubercle bacillus (Gerlach, Giinther, Harms). More recently only the reports of Forster \& Brown give positive results, while those of Galtier, Nocard, MacFadyean \& Leclainche give results Similar to the earlier ones of Perroncito which had been negative without exception. fonable to infect pigs by means of feeding large quantities of muscle from animals affected with advanced tuberculosis, having produced only one positive result and in that case only by adding tuberculous organs and bones
to the muscles.

Natural Infection. Observations in regard to the occurrence and dissemination of tuberculosis point to the fact that under normal conditions tuberculosis is transmitted only after prolonged exposure to the contagion, or after repeated infection with the virus. It seems that a single light infection usually produces only a local tuberculous lesion which terminates in recovery and in addition increases the resisting power of the individual to the disease. In other words, it produces a certain temporary immunity.. (For further information see chapter on Pathogènesis.)

In the vast majority of cases infection occurs through the medium of ingested food or of inhaled air.

In alimentary infection the milk of diseased animals fed in bulk or suckled by the young is of the greatest importance. In addition to this the skim milk obtained from skimming stations also plays an important role in the dissemination of the disease. Skim milk is particularly infectious when the slimy residue which collects in the separator bowl is added to it, as this slime contains large masses of tubercle bacilli when infected milk is centrifuged (Kjerrulf).

The infectiousness of milk containing tubercle bacilli hears an intimate relation to the occurrence of tubereulosis in swine which are fed with skim milk furnished hy skimming stations.

For example, in North America where swine were at one time fed almost exclusively with grain, statisties for 1894-1895 show that of $13,616,539$ swine slaughtered in the larger abattoirs only 579 head were tuberculous, that is $0.0004 \%$. On the other hand in 1908 when skim milk from skimming stations came into general use for the feeding of swine, the percentage increased to $2.05 \%$. Of $35,113,077$ swine that were slaughtered 719,309 were tuberculous. Thus the perannual loss caused by tuberine has increased five thousand fold (Salmon). The annual loss caused by tuberculosis in swine in the United States exceeds $\$ 3,000,000$. in some abattoirs of Holland (Amsterdam, Rotterdam) the disease is observed in 15 the disease was formerly present in 15 to $18 \%$ of all swine; in Copenhagen $20 \%$ of all swine were infected (Pederson); in Germany some shipments averaged 50 to $60 \%$, and in Hamburg 
in one instance $90 \%$ of all slaughtered swine were found tuberculous. In Hungary the disease is becoming alarmingly prevalent with the increasing practice of feeding skim milk.

In an experiment conducted by the Bureau of Animal Industry at Washington, $87 \%$ of a number of swine that were fed for 3 days with tubereulous milk became tubereulous within 3 to 4 months. When tuberculous milk was fed for 30 days all animals in the experiment became infected with general tuberculosis within 50 days thereafter.

The danger in feeding milk containing bacilli to calves is shown by the common experience that tubereulosis occurs much less frequently in calves that are fed with sterilized milk than in calves that are permitted to suckle their infected dams.

Finally tuberculosis occurs more frequently also in horses in those countries where milk is used in the treatment of sick horses (MacFadyean in England), or for food (Bang in Denmark).

Other food stuffs also and drinking water may act as the carriers of the contagion when they are infected with the secretions and excretions of tuberculous animals. Abundant opportunity for such infection is offered in all infected herds.

The feeding of tuberculous organs from slanghtered animals and the sputum of tuberculous persons are effective means of transmitting the disease to carnivora and omnivora.

Infection by means of inhalation occurs most frequently in stables occupied by animals affected with pulmonary tuberculosis. Slowly expired air from infected animals probably contains no infection (Cadéac \& Malet, Cornet). However, when such air is expelled by coughing the minute particles of water which are expelled with it may contain tubercle bacilli and, similarly, particles of sputum that are thus forcibly expelled will soil and infect the surroundings of diseased animals (Johne, Bang, Fränkel, Flügge). Thus healthy animals standing in stalls adjacent to those infected with pulmonary tuberculosis are continnally breathing infected air while the danger to other animals in the same stables but farther removed from the infected ones, is much less. As a matter of fact it is frequently observed that, following the introduction of a diseased animal into a healthy herd, the animals in the immediately adjacent stalls are always the first to become infected.

Johne was the first to call attention to the importance of the small solid or liquid particles which are expelled in the act of coughing. More recently Flügge and his pupils have shown that tubercle bacilli which are expelled in the act of coughing, sneezing or even in the act of loud conversation, will infect guinea pigs when properly exposed (spray infection). The same results were obtained by Ravenel by exposing guinea pigs to consumptive eattle in the act of coughing. The results of numerous experiments made with sprays of pure cultures to prodnce infection by inhalation conform with the former in demonstrating the danger of the moist air of expiration. (See Pathogenesis.) On the other hand the danger of dust infection (Cornet) or of air containing merely dry particles of secretions is considerably less. Experiments with such material gave negative results. Thus Cadéac obtained only 5 positive 
results in 37 guinea pigs that were made to inhale dust produced from sputum which had dried in the dark. A similar experiment with 11 rabbits gave negative results, while 36 guinea pigs and 8 rabbits which were forced to inhale moist material in the form of a fine spray, all acquired tuberculosis. Analogous experiments by Peterson gave similar results, while investigations by Koehlisch showed that in inhalation of a dry spray (dust) at least 50,000 bacilli must be inhaled in order to produce tuberculosis.

The floor, food stuffs and air are liable to be infected with bacilli particularly in dark, poorly kept and crowded stables when the air is saturated with moisture which retards the precipitation of the droplets expelled by conghing. (Ostertag found that the air of a cattle stable contained a relative humidity of 80 to $95 \%$.) The danger of infection in such cases is in direct proportion to the number of animals that are housed together, because the larger the herd the greater the probability that diseased animals are in it. The generally observed fact that large herds of cattle are rarely free from tuberculosis is in accordance with this statement as is also the fact that a much larger percentage of small herds is found free from tuberculosis. And this is true in the face of the further fact that the sanitary arrangements, care, etc., of large stables is usually much better than that of smaller stables (see p. 503).

In the open air the danger of infection is in general very slight. The bacilli which are expelled with the expired air of diseased animals are rapidly disseminated and are destroyed in a short time after reaching the ground by the action of sunlight. Accordingly tuberculosis is almost inknown among animals that are continually on pasture. The disease spreads much more slowly in stabled animals if they are pastured a greater portion of the time.

Infection through the act of copulation may occur when the generative organs of either the male or the female are diseased, in males especially the epididymis causing infection of the seminal fluid, in females a tuberculous uterus causing infection of the vaginal secretion.

Tuberculosis of the uterus may be produced in guinea pigs by injection of cultures into the intact vagina. Observations, though rare, confirm the possibility of this mode of infection in domestic animals. Thus Franck observed tubereulosis of the vagina in several cows that had been served by a bull with a tuberculous penis. Similar observations are recorded by Zippelins, Haarstick, Eber. Goerig and Schmirlt record the development of a primary tuberculosis of the epididymis following copulation with infected cows.

The primary infection of the udder through the milk ducts can occur by the virus which is present on soiled litter gaining entrance into the milk cisterns through the milk ducts where it will propagate and disseminate (Bang, Modius, SchmidtMühlheim, Conte, Alessandro). 
Infection from cutaneous lesions is exceedingly rare and in such cases the disease usually remains localized or sprearls at most to the adjacent lymph glands (Lydtin, Johne). Much greater danger as far as the source of infection is concerned is presented by castration wounds, which are rather rare in cattle (Bongert) but more frequent in swine as a result of the application of milk or the infection of the litter with excretions. Sometimes infection may follow the application of sputum to the wound by a consumptive operator (Tempel, Jahn, Michael).

The mode of infection varies to a certain extent according to the species of animal. Cattle, it seems, are always infected by the products of diseased animals of the same species. Thus calves are usually infected by means of infected milk while older animals are infected by the ingestion of solid food or of contaminated drinking water or by the inhalation of infected air. In horses tuberculosis usually begins in the alimentary canal; occasionally this is produced by infected material deriveil from tuberculous fowls (Nocard), but in most cases by infectious material of human origin (Röbert, Röder).-In pigs tuberculosis occurs in a vast majority of cases as a result of feeding cows' milk or whey. Feces of diseased cattle are next in importance as a source of tuberculosis in swine, infection with this material usually occurring by way of the pharynx or the intestinal tract; a pig with diseased teats may infect its young while suckling; and finally the inhalation of tubercle bacilli is probably also an occasional cause.-Sheep and goats are probably always infected by diseased cattle.-The infection of dogs and cats usually stands in causal relation with tuberculosis in man. As a rule pet dogs become infected by licking the sputum of their consumptive owners or by inhaling infected air, while cats have hecome infected by the ingestion of infected milk. (The tuberculous dogs examined by Petit came, for the greater part, from poorly kept saloons and coffee houses which were much frequented by laborers.)

Susceptibility to tuberculosis varies according to the species of animal. Among domestic animals cattle and swine are most susceptible to artificial infection while other animals possess much more resistance. The natural resistance of any species of animal may be overcome by intensive and repeated infection. In reality, however, buffalo, horses, sheep, goats, dogs and cats acquire tuberculosis only rarely in spite of their frequent exposure to diseased cattle or himan beings.

The frequency of tuberculosis among the different species of domestic animals is shown in statistical tables furnished by slaughter house reports (see page 502). - Among buffalo the disease appears to be less common in Russia than it is in Hungary, where, at the abattoir of Budapest, it is sometimes found in $0.9 \%$ of all slaughtereil animals. Kanzelmacher found only 5 head infected among 2,715 buffalo slanghtered at Tiflis, i. e., 0.02\%.-Among asses Blane, Pleindoux and Nocard each observed one case of tuberculosis. The fact that this disease seems to be so rare in this species may be due to the small number of observations, at least. Stockmann, 
Chauveau and Arloing have demonstrated that asses may be readily infected by intravenous injection of tuberele bacilli.

Among dogs and cats tuberculosis is particularly freqnent in large cities. Thus Fröhner found 27 tuberculous dogs among 62,500 patients observed in the ambulatory elinies in Berlin, that is, $0.09 \%$. Johne and Eber in Dresilen found 11 out of 400 dogs tuberculous and but one among 100 cats. Jensen in Copenhagen found in the course of 2 years $5.7 \%$ of tubereulous animals among 28 dogs and 25 cats; Petit \& Basset in Alfort found, among 2,717 dogs examined, 152 infected with tubereulosis.

The breed of an animal may influence its susceptibility to tuberculosis. The fact that tubereulosis occurs more frequently in some breeds than in others is due in a great measure to the different conditions under which they may be kept or the uses to which they may be put. Cattle from the Russian steppes, for example, become infected just as readily as the more domesticated breeds when they are removed from pasture and fed in stables (working oxen!). On the other hand the young animals of the latter breeds on mountain pastures are much less frequently affected with tuberculosis than animals raised in barns. However that may be, a greater resistance to artificial infection can be demonstrated to exist in the cattle of the steppes and, according to Kitasato, in the Japanese breeds of cattle. The ohservation that the breeds of the lowlands are more generally affected than the breeds of mountainous regions is explained by the fact that the former, on account of their greater milk producing capacity, constitute the large dairy herds and are kept in stables of large estates and of dairies where they are milked as much as possible (Roeckl, Bongert).

In the numicipal abattoir of Budapest, in the years 1899-1902 the percentage of tuberculous cattle among the gray breeds of the plains did not exceed $0.23 \%$ and among the cows of the Hungarian breeds, which are also kept out of doors the morbidity was 4.0 to $6.1 \%$. The Hungarian working oxen, on the other hand, that had been kept in stables for several years and at work were tubereulous to the extent of $13.6-18 \%$, while of the cows of the colored breeds $14.8-17.2 \%$ were tuberculous. According to Kitasato the native Japanese eattle, under normal conditions, are free from tuberculosis while imported breeds and cross breeds frequently acquire the disease. The injection of highly virulent tuberculous material into 52 native calves and 19 calves from these cross breeds showed that the native cattle withstood the subcutaneous injections withont harm and that they conld be made seriously ill only by means of intravenous or intraperitoneal injections. Similarly, Hutyra found that calves of the Hungarian long horned breeds were difficult to infect by the inhalation of bovine tubercle bacilli in contrast to the easy infection of the calves of the ordinary colored breeds.

Individual susceptibility is not the same in all animals of the same susceptible breed. In large herds where the disease has been thoroughly established, certain animals are always found that may be perfectly healthy and live to a considerable age, although they are constantly exposed to the same danger of infection as their mates. In experiments of artificial infection also it is noted that among animals of the same breed and age some resist infection with large masses of virulent bacilli. This individual immunity may be congenital, or it may be acquired in the sense that the animals in question may have 
suffered a slight or passing attack of the disease and thus acquired a certain immunity which was later perhaps intensified by repeated infections at longer or shorter intervals of time.

The dissemination of tuberculosis was formerly explained by assuming special individual and a family predisposition. In human being's this was supposed to find expression in a lymphatic constitution; and in an improper development of the thorax (flat and norrow chest, habitus phthisicus). As far as our domestic animals are concerned there is no foundation upon which to base such a supposition and the great regularity with which we find the disease in all large herds points against the correctness of this riew. It is true that the disease is especially prevalent in certain families or strains but this may readily be explained by the uniformly farorable conditions for infection.

Formerly individual predisposition was supposed to be due to a slight power of resistance on the part of the hody to the tubereulous virus as well as to an unfavorable constitution and to hypoplasia of the hlood vessels. According to Baumgarten tuhereulosis of human beings is usually eongenital in the sense that the offspring is horn with tubercle bacilli in its system. where they remain latent until they develop and produce aetive disease perhaps only after years under the deleterious influenee of various ageneies. Tubereulosis of the lymphatic glands and of the bones especially was supposed to originate in this manner.

The existence of such long-eontinued lateney is eontradicted by the common observation that the human body is, especially in its earliest infaney, highly suseeptible to tubereulosis and that the disease then as a rule runs a rapid and fatal eourse. Aceording to Behring the predisposition to tubereulosis is attributed to an infection in early youth, resulting in disease of the lymph glands (scrophulosis) whieh, by the destruetion of certain lymphaties and their glands and the production of ehanges in the walls of hlood vessels. produces a hypersensitiveness to tubereulin, and that involvement of the thoracie lymph glands gives rise to an abnormal development of the thorax hy affecting the sternal artieulations. According to this view an individual predisposition would not be eongenital but rather acruired and eould he guarded against by the preventing of extra-uterine infection in early life. But this view must be objected to on the same grounds as the former. As a matter of fact, we ean not as yet offer a satisfactory explanation for individual predisposition, the existence of which eannot be denied.

After careful study of the herd registers of the estate Weidlitz, covering a period of 59 years, Hermann concluded that in all cases of tulereulosis in this herd one of the following predisposing causes could be established; late horn calves in large families, lack of fresh air and of proper exercise, inbreeding, tuberculous parents.

Heredity plays no very important role in predisposition nor in the dissemination of tuherenlosis in general. Although tuberculosis may be directly transmitted from the mother to the unborn calf, as has heen demonstrated by the actual observation of well developed disease in the newborn animal, such cases are very rare and oceur only when the uterus itself or the cotyledons are diseased. Aside from this, animals born 
tuberculous do not live and are frequently born prematurely. Calves several weeks of age are rarely found affected with tuberculosis and among slaughtered calves the percentage of diseased animals usually does not reach $1 / 2 \%$, while tuberculin tests even in badly infected herds rarely reveal more than 10 or $15 \%$ of infected animals under 6 months, in spite of the fact that these animals liave had abundant opportunity to become infected. The most convincing proof against the effects of heredity in the transmission of tuberculosis is furnished by the fact that the offspring of tuberculous cows remain healthy and free from tuberculosis if properly guarded against infection after birth.

The first ease of hereditary tuberculosis was establisher by Johne when he demonstrated tubercle bacilli and tubercles in the lungs and liver of a fetus eight months of age. Since then similar cases were reported by others (Csokor, Nocard, MacFadyean, etc.). Up to the year 1909 Albein had compiled reports of 107 similar cases, 91 of which were unquestionable. At a later date Höyberg reported 6 cases of congenital tuberculosis among 500 calves examined immediately after birth and Bergman reported 108 personally observed cases, among them 4 fetal; the others in ealves not exceeding three days of age. These were observed during the years $1904-8$ in the abattoir of Malmoe $(0.42 \%$ of all slaughtered ealves).

Among slaughtered calves up to five months of age, Klepp of Kiel found 0.64-1.18\% tuberculous. In a badly infected region of Jütland, in Aarhus, Knudsen reported $0.3 \%$ to he tuberculous. Stroh 's estimates for 1901 and 1902 gives for Bavaria the following figures: For every 100 tubereulous slaughterei cows, 6-7 tnberculous calves; for every 100 cows with advanced tuberculosis, twenty-five tuberculous calves, but some of these were evidently cases of extra-uterine infection (see statisties on page 502 ).

In the course of his tuberculin test work in Denmark, Bang found anong 24,267 calres under six montlis, $10.6 \%$ tuberculous. The anthor's own observations on 544 calves under six montlis showed 59 tuberculous, that is, $10.8 \%$. In one badly infected herd 15 out of 48 calves or $31.2 \%$ reacted. In calves of this age, however, the results of extra-uterine infection are already evident. because according to Nocard and Bang the percentage of reactions does not exceed $5 \%$ during the first few weeks of life, the authors themselves have observed that in one herd where $44.8 \%$ of all cows were infected only one out of 64 sucking calves gave a positive tuberculin reaction $(1.6 \%)$.

In congenital tuberculosis of calves the periportal lymph glands are always involved. Next in frequency are the liver, the mediastinal and the peribronchial glands, then the lungs and, in exceptional cases only. other organs (Klepp, Rievel). This localization points to the fact that infection takes place in a late stage of embryonic existence, through the placental circulation from the dam. (Bang is of the opinion that isolated tuberculosis of the posterior mediastinal glands has the same origin.) In regard to the rare occurrence of congenital tuherculosis compared with the comparative frequency of tuberculosis of the uterus of cattle, it is probable that infection of the foetus occurs only in far advanced tuberculosis of the uterus or if the cotyledons and chorion villi are affected (Kockel and Lungwitz have demonstrated tubercle bacilli in the villi of the chorion and Burgmann, demonstrated them in three instances in the placenta).

Without disease of the placenta or the fetal envelopes it would hardly be possible for the bacilli to pass from the mother to the fetus. Although Ravenel and Bucher each reported a case of congenital tuberculosis in the calf where the uterus of the dam was healthy, very slight tuberculous changes could certainly have escaped macroscopic ohservation. 
The possibility of a conceptional or germinative transmission of tuberculosis has never been demonstrated and is, in addition, highly improbable. Tubercle bacilli have never been found in a germinal vesicle or in the egg. Besides, infection of the egg would result in its destruction before it could mature. It is true that Maffucei, and later Baumgarten, found that in hens' eggs, that were infected artificially into the white with avian tubercle bacilli, normal cell division took place and occasionally the embryo developed completely. However, what has been found to be true for the meroblastic chick egg does not necessarily apply to the egg of the mammal because infection did not take place directly in the egg cell but in the surrounding allumen and consequently the tubercle bacilli would still have to pass through the area vasculosa, the analogue of the placenta, before they could reach the embryo (Wassermayer, Bongert). However, the chicks hatched from eggs thus artificially infected always died of tuberculosis after a short time.

While in tuberculosis of the urino-genital tract, and especially in tuberculosis of the epididymis tubercle bacilli are frequently present in the spermatic fluid, infection of the orum could occur only by the sinultaneous introduction of a bacillus with the spermatozoon into the ovum (bacilli have never been demonstrated in spermatazoa). These theoretical conelusions are in harmony with the results obtained from actual experiments. Gaertner, Cornet and Hansen never succeeded in their attempts to infect the offspring of rabbits or guinea pigs by the previously infected male.

The frequency of the disease bears a close relation to the age of the animal. Young animals are in general more susceptible than older ones, yet the disease is more frequently met with in the latter for the reason that they have been exposed to infection for a longer time. As a matter of fact the percentage of morbidity increases gradually from the suckling period, year after year, and in cattle more than $50 \%$ of all cases, as revealed by the tuberculin test or in the abattoir, are found in animals over six years of age.

In the abattoir of Budapest in the year 1899, 9,046 tuberculous cattle were classified as follows: 3 at 3 to 4 weeks, 1 at 5 months, 4 at 7 to 9 months, 12 at one year, 39 at 2 years, 81 at 3 years, 118 at 4 years, 326 at 5 years, 1,223 at 6 years, and 7,239 over 6 years (among the latter 5,173 Hungarian draft oxen). In Denmark, according to Bang, 40,624 eattle (1895-1904) gave the following results after first tuberculin tests: of 5,559 calves under six months, $12.1 \%$ reacted; of 7,744 eattle from 6 to 18 months, $27.5 \%$; of 5,047 head from 18 to 30 months, $38.6 \%$; of 10,350 head from $21 / 2$ to 5 years, $44.9 \%$, and of 11,924 cattle over five years, $43 \%$ reacted. Of the latter $79 \%$ were already infected at the age of two years. In Norway, according to Malm the tuberculin reaction in calves under 6 months reached 1 to $2 \%$; at 6 to 12 months, 1.5 to $3.4 \%$; in 1 to 5 year old eattle 4.3 to $7.9 \%$, and in still older animals 5.6 to $10.4 \%$ reacted. Naegeli reports similar conditions among human beings; 1 to 5 years gave $17 \%$ of reactions; 5 to 14 years, $33 \%$; 14 to 18 years, $50 \%$; 18 to 30 years, $96 \%$ of reactions.

The sex influences the prevalence of tuberculosis only in as far as it affects the conditions under which the animals may have been kept. The disease is most prevalent in dairies composed of cows of the more domesticated breeds. Among these we frequently find from 70 to $80 \%$ and more, of reactors. Steers and bulls of these same breeds, which are rarely allowed to reach the same ages, give a much lower percentage. Compared with these figures the percentage among the gray cows and bulls of the steppes which are kept mostly in the open air are much lower than in stable-fed oxen of the same breed that are worked for years (see p. 519).

In the German Empire the frequency of tuberculosis was found in the abattoirs as follows: Oxen $20.04 \%$, bulls $15.82 \%$, cows $27.13 \%$, yearlings and young cattle $6.2 \%$, ealves $0.30 \%$. In the abattoir at Budapest in 1903 the following results were 
obtained of the stepje-breeds: Bulls $4.40 \%$ tuberculous, oxen $16.84 \%$, cows $5.39 \%$; while the colored breeds gave the following results: Bulls $3.92 \%$, oxen $6.98 \%$ and cows $22.61 \%$.

External influences which reduce the vitality of the animals and the resisting powers of the tissues in general favor the development of tuberculosis. This applies particularly to damp and poorly ventilated dirty stables and to lack of exercise in the open air. These two factors alone tend to produce superficial breathing and catarrhal conditions of the respiratory tract and thus they produce a local predisposition, which is increased by the collection of secretions in the bronchial tubes. In addition to this close stabling affords more abundant opportunity for infection from diseased animals, especially when frequent purchases are made from suspicious sources.

The predisposing influence of eatarrhal conditions of the air passages is particularly striking in eattle that are kept in the vicinity of sulphur and arsenic mines and smelters. In these animals the inhalation of the smoke from the works, which contains arsenious acid, eanses a eatarrh of the air passages and then pulmonary tubereulosis (which used to be known as smelter smoke pneumonia) usually spreads very rapidly (Siedamgrotzky, Johne, Pfeifer). In this connection the experiments of ('adéac \& Malet deserve mention. They suceeeded in producing pulmonary tuberculosis in rabbits by the inhalation of dust contaminated with tubercle bacilli only in such eases where the respiratory tract was previously irritated with fumes of bromine; also the experiments of Plate according to which irritation of the intestinal mucous membrane of guinea pigs with croton oil favored infection of the intestines with tubercle bacilli in $80 \%$ of the animals.

Insufficient and improper feeding with food deficient in nutrients (distillery slops and factory refuse) and over-exertion or excessive milk production are also predisposing factors by weakening the organism.

Tuberculosis frequently develops after acute inflammation of the lungs. The diseased lung tissue and the incompletely absorbed exudate constitute a favorable medium for the development of tubercle bacilli. In other cases an attack of acute disease weakens the body and prepares the soil for a renewed and snceessful attack of an existing, but dormant, tuberculous process. Finally the shedding of the milk teeth probably favors in young animals, especially in pigs, infection through the buccal cavity and the development of tuberculosis of the cervical lymph glands.

Pathogenesis. The principal ports of entrance for the tubercle bacilli are doubtless the respiratory and digestive tracts. It is an open question, however, as to which channel is of greater importance in natural infection.

The fact that in man as well as in animals the lungs and the adjacent lymph glands are, in the vast majority of cases, affected either alone or in conjunction with other organs or parts of the body had 
always pointed to the apparently well founded conclusion that the lungs constituted the principal primary seat of infection. Infection was supposed to be brought about by the inhalation of air contaminated with the virus. It was assumed that the bacilli entered the finest ramifications of the bronchioles and alveoli, producing a broncho-pneumonia, or that they were taken up by the leucocytes from the mucous membranes of the larger bronchioles and transported to the bronchial glands through the lymph vessels where they gave rise to the development of tuhereles. From these glands they were supposed to pass into the lungs by way of the blood stream and produce interstitial tubercles.

This view of the possibility of an "inhalation tuberculosis" was based upon positive experimental evidence which, in fact was obtained by making small animals inhale sprays of tuberculous material (Cornet, Fluegge, Petterson, Baumgarten, Ribbert, Cadéac and Malet, and others). Similar results were obtained in cattle (Ravenel, Kesselmann, Nocard \& Rosignol, Kossel, Weber \& Heuss, and others); also in goats (Reichenbach) and dogs (Tappeiner). In all these experiments tuberculosis of the thoracic organs and especially of the lungs was produced. (See also page 508). During the past few years the value of these experiments has, however, been questioned.

In the first place it was pointed out that tubercle bacilli thus inhaled were precipitated on the mucous membrane directly posterior to the nasal openings or in the diverticula of the anterior portion of the respiratory tract, thus never gaining entrance to the deeper-lying portions of the respiratory tract and that the lungs, in these cases, had to become infected by absorption of the bacilli into the lymphatic and blood vessels which transported them to these organs. (See p. 526). This objection was met, partially at least, by Fluegge \& Heymann, more recently also by Bartel \& Neumann, as well as by Kuss who experimented with guinea pigs, and Weber \& Titze who used calves in their experiments. They showed that immediately after the inhalation of infected sprays even the most peripheral portions of the lungs contained tubercle bacilli (although only few). In calves they were demonstrated on one occasion each, in the middle and posterior lobes of the right lung. Similar results were obtained with bacillus prodigiosus by Hartmann \& Hartl and with mold fungi by Hildebrand and Ballin. Kuss showed that soot and fine particles of pigments (India ink) also reached the finest bronchioles by means of the inhaled air. Anthracosis is undoubtedly caused by direct inhalation, although this statement is opposed to the view of Vanstenberhe \& Grysez.

The important role played by inhalation is emphasized by the fact that minimum quantities of virus ( 50 bacilli in guinea pigs) are sufficient to infect experiment animals while for infection through the alimentary canal, masses a hundred thousand times as great are necessary (Findel). Recently Weber \& Titze showed the same to be true with calves, viz., that in- 
fection with tuberculosis resulted after inhalation of $0.01 \mathrm{mg}$. while infection through the alimentary tract required $10 \mathrm{mg}$. of tubercle bacilli culture.

In view of these results we can no longer doubt the possibility of inhalation tuberculosis. As to the practical importance of this mode of infection, we must remember that comparatively few bacilli reach the terminal ramifications of the bronchial tubes through the medium of ordinary infected air and also that the body is provided with natural means to protect itself against even considerable quantities of virus. Another fact that should receive due consideration in this connection is that it is only with difficulty that calves are infected by means of intratracheal injections, even of large masses (10 cc. bacillus emulsion) of virus.

The question as to whether an infectious inhalation produces pulmonary tuberculosis primarily by aerogenous infection, or secondarily by lympho-hematogenous infection is yet an open one. While some authors believe that the inhaled bacilli give rise to the development of the first tubereles in the nucous membrane and its lymph follicles (Ziegler, Ponfik, Schmorl, ete.), others (e. g., Orth, Ribbert and Baumgarten) helieve that the bacilli are immediately absorbed by the mucous membranes and deposited in the bronchial glands from where they reach the lung tissue and the walls of the bronchial tubes through the lymph and blood circulation. The tubercles and cheesy foci which here develop are then supposed to give rise to a tuberculous bronchitis and broncho-pneumonia.

This view is especially applicable to pulmonary tuberculosis of domestic animals. According to Bongert the first tuberculous changes in the lungs of eattle are observed in the posterior lobes to which places tubercle bacilli would be most apt to be carried first. This is due to the fact that the pulmonary artery passes in a straight direction from its point of entrance toward the base of the lung, while the artery for the anterior lobe branches off at an angle in a more or less opposite direction. In a direct infection from the inhaled air the anterior lobes would naturally become infected first because the origin of the bronchial branch supplying this lohe as well as that of the first branch of the main bronchus, according to Baerner, is situated at the lowest point of the main bronchus. Consequently material inhaled with the air, especially fluids, is drawn prineipally into the anterior lobes of the lungs. Pigs often aspirate food particles into the lungs, and hence the fact that the most pronounced lesions of tuberculosis are usually met with in the anterior lobes accords with the above explanation. (In human beings, for reasons as yet not satisfactorily explained, the superior apical lobes are affected most frequently.)

The great frequency of pulmonary affection does not in itself, however, prove that the infection takes place with the same frequency by way of the respiratory passages. Recent experiments have shown beyond question that the lungs as well as the thoracic lymph glands may be infected from the digestive tract. Chauveau was the first to call attention to this possibility (1868-1872). The latter fed tuberculous material to 
young cattle and produced exclusive infection of the bronchomediastinal lymph glands. Klebs, in 188t, supported this view when he referred to the meat and milk of tuberculous cattle as the commonest source of infection for human beings. Orth (1887) emphasized the importance of intestinal infection as a source of pulmonary tuberculosis. This question came into particular prominence through Koch's well known acldress at the Lonclon Tuberculosis Congress (1901) and more so through the position assumed by v. Behring (1903), according to which the principal cause of tuberculosis was intestinal infection of nurslings, while inhalation tuberculosis was of wholly secondary importance, and which asserted that when this form appeared to exist the infection usually resulted through the lymphatic ressels of the naso-pharyngeal or intestinal mucous membranes, thus being after all an intestinal infection. Following this a great number of experiments were conducted by Weleminsky, Ravenel, Kovacs, Calmette \& Guérin, Vallée, Weichselbaum and others which threw more light upon this mode of infection and led many to regard it as the most frequent.

Formerly a primary intestinal infection was not admitted unless the peripharyngeal lymph glands or the intestine and the mesenteric glands were the exclusive or at least the principal seat of disease. In direct contrast to this view experimental feeding of tuberculous material showed that tubercle bacilli, especially when introduced in small quantities per os, would pass through the intact mucous membrane of the intestine and, without necessarily lodging in the lymph glands along their path, would pass, in part at least, to the glands in distant regions of the body or even directly into the blood vessels and be finally carried to the lungs where they produced a primary pulmonary infection with all the appearances of an inhalation tuberculosis. The usual course taken by the bacilli is then, on the one hand, from the pharyngeal region through the cervical lymph vessels to the broncho-mediastinal lymph glands and on the other hand from the small intestine, especially the posterior portion of the ileum by way of the lymphaties to the mesenteric glands, through the latter to the thoracic glands or, through the thoracic duct directly into the pulmonary circulation.

Behring produced characteristic inhalation tuberculosis in guinea pigs by injecting cultures into the tissue of the tongue. The submental glands became affected first, following these, the cervical and then the mediastinal glands. Later, the virus reached the bronchial glands through the blood vessels. The subpleural ramifications of the pulmonary artery then carried the virus to the lung tissue where miliary tubercles developed.

Weleminsky and Kovaes fed young guinea pigs repeatedly with 0.1 $\mathrm{mg}$. of culture per $100 \mathrm{~g}$. body weight and after a few days found the bacilli on the one hand in the submental, submaxillary and cervical glands and, on the other hand, in the ileo-caecal, mesenteric and portal glands. These results agree with those obtained by Behring. Calmette \&. Guérin introduced tubercle bacilli $(0.1-1.0 \mathrm{~g}$.) partly through a tube, 
partly with the food, into the digestive tract of guinea pigs, goats and cattle. In the older animals, whose lymph glands, on account of their less dense structure allow an easier passage of the bacilli the thoracic organs were frequently found affected exclusively. Occasionally the bronchial glands also were involved. The younger animals showed affection of the mesenteric glands. In old animals bacilli could be found in the lungs twenty-four hours after alimentary infection had been produced, while in young animals they could not be demonstrated until after the fifth day. Vallée obtained similar results by feeding infected milk and by direct injection of the bacilli into the mesenteric glands. Orth \& Rabinowitsch got the same results from endorectal infection. DeHaan injected tubercle bacilli into the stomach of a heifer and three months later demonstrated a pronounced tuberculosis of the lungs and pleura, but found the intestinal mucous membrane and its lymph follicles intact.

The experiments of Ravenel as well as those of Nicolas \& Desco demonstrated the rapid transmission of tubercle bacilli from the intestine to the thoracic organs. They fed cultures in butter, warm water, pastry and in soups. Three or four hours later bacilli were present in the thoracic duct. Bisanti \& Panisset demonstrated the presence of tubercle bacilli in the blood five hours after they had been ingested. Orth \& Rabinowitsch injected an emulsion of bacilli into the rectum, Oberwarth \& Rabinowitsch injected the bacilli into the stomach of a pig and in both instances the germs were afterwards found in the circulating blood.

That tubercle bacilli may and do pass through healthy mucous membranes has been amply demonstrated on healthy animals by numberless feeding and inhalation experiments with material which was mechanically and chemically innocuous. The experiments of Dobroklonsky among others showed that the mucous membranes might remain perfectly unliarmed during this process (although Baumgarten, on the other hand, holds that the mucous membrane always suffers by the entrance of bacilli into its lymph follicles, especially in the intestinal mucous membrane).

Although there is no doubt that an infection of the respiratory organs may follow an intestinal infection, or result from one, the fact that the former are by far the most frequent seat of primary disease must be explained by their greater susceptibility. This is especially true of the lungs, thus explaining the fact that these organs, no matter what the mode of infection, are usually more intensely involved than any other part of the body. Occasionally we find the development of an isolated tuberculosis of the lungs following the injection of virus into the subcutis or into other remote organs. Thus we may justly consider the lungs as far as infection with tuberculosis is concerned, as a locus minoris resistentiae (Cohnheim, Orth, Baumgarten, Bartel). In regard to the frequent affection of the lymphatic glands it is significant that these constitute a close filter for corpuscular elements, especially in young animals, and that they arrest a considerable portion of tubercle bacilli which are in the lymph stream, and which then in spite of the bactericidal action of the lymph elements (Manfredi, 
Bartel, Fontes and others), can more easily give rise to the pathological process, on account of their great numbers, than is possible in other organs.

The great predisposition of the lungs for tuberculosis is further emphasized by the fact that aerogenic infection results exclusively in pulmonary affection. According to Neumann \& Wittgenstein this predisposition is explained by the ahsence of certain ferments which have the power of splitting or reclucing fat molecules. Baumgarten observed the development of pulmonary tuberculosis after the introduction of small quantities of bacilli into the bladder of rabbits. Bartel saw pulmonary tuberculosis develop after intraperitoneal infection. Schroeder \& Cotton produced pulmonary tubereulosis in cattle and swine by subcutameous infection at the tip of the tail. Vallée got the same result after injection of a culture into the milk cistern of a cow's teat. In the latter case not only the lungs but also the supramammary, the abdominal and the broncho-mediastinal glands became involved. It is important to note that intravenous or peritoneal infection of goats and rabbits eauses the development of isolated pulmonary tuberculosis with the formation of cavities, especially when the susceptibility of these animals was previously reduced.

Weleminsky attempts to explain the frequent primary affection of the bronchial lymph glands by the intimate relationship existing between these glands and the entire lymphatic system. These glands constitute a sort of "heart", into which lymph ressels from all directions pour their contents which are then carried through the broncho-mediastinal trunk into the subclavian vein. The correctness of this view has never been demonstrated as far as guinea pigs are concerned and, as to other animals, it is at best questionable. However, these glands are also readily infected following intravenous or intraarterial injections of virus (Baumgarten). It has been observed that in calves infection by these methods usually results in the development of pronounced lesions of these glands as well as of the lungs proper.

In regard to the transportation of tubercle bacilli from Waldeyer's ring Behring is of the opinion that they give rise to the first lesions in the superior cervical glands and then travel through the lymph vessels to the bronchial glands and the lungs. In opposition to this view, Bongert, Beitzke and Devrient point out that the lymphatic system of the head and neck is entirely separate from that of the lungs and its glands and that the bacilli from the diseased subparotideal and submaxillary lymph glands pass from the great lympathic trunk into the vena cava, thence, with the blood, to the lungs and the bronchial glands (especially common in swine). The tonsils play no important role in this process, especially not in cattle. With their abundant supply of lymphoid cells they would rather act as a protection. However, should the bacilli successfully pass through them they would cause changes ir the retropharyngeal glands (Devrient).

Isolated tuberculosis of the lungs and of the broncho-mediastinal glands in cattle is frequently observed in abattoirs. Thus, in the abattoir of Budapest in the years 1898-1901, of 46,092 cases of tuberculosis in eattle, 40,306 showed affection of the lungs only, including the peribronchial and mediastinal glands. In about one-third of the cases the lymph glands only were affected; tonsils and retropharyngeal lymphatic glands in only 102 eases. Bongert found the latter glands affected in 3 out of 1,200 eases, Devrient in 2 out of 1,400 eases. Rasmussen found the tonsils affected in 52 out of 4,708 cases $(1.1 \%)$ and the retropharyngeal glands in 228 cases $(4.8 \%)$. 
Of course any organ of the body may oceasionally be found to be particularly predisposed to infection and it seems that primary affection of the bones and articulations is thus explained (traumatic influences). The species of animal is also of importance. Thus subeutaneous infection of guinea pigs usually produces disease of the lungs, almost never of the spleen, while in rabbits, next to the lungs, the kidneys are particularly prone to disease, and in chickens the lungs are practically immune to infection with avian tuberculosis (Weber). Similar differences are observed among the domesticated animals, e. g., in the pig the spleen is particularly susceptible in contrast to other animals, in cattle the serous membranes, and in dogs the pericardium. Finally, the variety of tubercle bacilli is to be considered. Thus, in man, the bovine type usually causes disease only in the digestive tract and in its lymphatic glands.

From what has been stated it is evident that in tuberculosis the infection of any internal organ whatever may result through the respiratory apparatus (aerogenic infection) or through the digestive apparatus (alimentary infection). As to which of these modes of infection is the more common, views differ at the present time. It is not probable either that the solution to the problem will be an easy one. Bacilli carried with the inhaled air to the naso-pharynx may readily be absorbed through the alimentary mucous membrane. But, whichever view may be the correct one, there can be no doubt as to the danger of inhaling air laden with bacilli. The manner of infection depends no doubt, at least, in a great measure, upon the conditions alrearly discussed. Patients with open pulmonary tuberculosis, by virtue of the exhaled infected droplets constitute a source of either mode of infection; an open tuberculosis of other organs of the body constitutes a danger for alimentary infection almost exclusively.

The localization and character of the tuberculous lesion is determined to some extent also by the intensity of the infection. After infection with a large mass of virulent bacilli the morbid process usually, especially in older animals, begins its development at the point where the largest number of bacilli became lodged on the mucous membrane. At these points tubercles and nlcers are formed, such as are observer in the tonsils (especially in swine) and in the ileum. In the latter they usually begin in the lymph follicles of the mucous membrane. If the infection happens to be slight the mucous membrane remains intact and the lymph glands only are affected. This is because most of the bacilli remain lodged in these glands and the few that happen to pass through are either destroyed (phagocytosis) or, on account of their small numbers, produce lesions that are not observed until a later time. Thus it may occasionally be observed in cattle that the retropharyngeal glands contain large tuberculous foci, the upper, middle and lower cervicals and finally the mediastinal glands successively smaller and more recent foci (Brever). By this means we may often be enabled, in the beginning of the disease, at least, to

vol. 1-34 
recognize the mode of infection by the seat of the oldest lesion. In by no means rare instances the first lesions develop in regions or organs remotely situated from the point of infection, apparently with preference after an infection that is not intensive. In these cases the infection was transported by the blood or lymph circulation without producing changes along their path. This path cannot often be determined with any degree of exactness and consequently it is only in rare instances that an autopsy reveals evidence unon which reliable conclusions could be based.

The milder the infection and the greater the resistance offered by the body the slower will be the development of the ensuing disease. Thus it is possible to produce artificially a pulmonary phthisis characterized by excessive tissue destruction and the formation of cavities by the introduction of comparatively small quantities of bacilli of slight virulence (Baumgarten) or by inoculating animals which had been previously partially immunized. Under these conditions it may require months for the disease to develop in guinea pigs and two or three vears in goats (Behring \& Roemer, Nocard, De Jong). According to Calmette and Guérin a single intestinal infection of calves is said to produce a tubereulous process which usually heals in the course of three or four months and results in a certain degree of immunity. On the other hand a number of successive infections repeated at short intervals may result in a progressive tuberculous process.

According to Behring the mother's milk constitutes the principal source of pulmonary tuhereulosis in man. According to this author infection takes place from the gastro-intestinal tract, conditions for infection being particularly favorable at this period of life since, according to Disses, the epithelial layer of the intestinal mucous membrane is not eovered with a continuous layer of mucus during the first days of the infant's life. As a result, as shown by Roemer's findings, large undigested proteid molecules and also bacteria ean pass unchanged through the intestinal mucous membrane of new born colts, ealves and the small laboratory animals, while infection in adult animals is much more difficult and only possible either after repeated ingestion of virus or after a single ingestion of exceptionally virulent material in large amount. The bacilli thus taken up produce a scrofulous diathesis which constitutes a groundwork for the infantile infection to develop into manifest disease in the course of years or decades. This result is favored by additional infections which take place in later life, as well as by the exhausting effects of deficient nutrition, eatching cold, overexertion, long continued confinement in poorly ventilated houses, ete. This theory has also been applied to animals. However, just as its correctness in regard to man is generally questioned, its applieability to eattle is doubtful, the more so as tuberenlin reactions in calves are seldom positive and as the percentage of reactions increases only later, but then steadily.

The development of the tubercle. At the point of their lodgment and propagation the tubercle bacilli give rise to an inflammatory process resulting in tubercle formation. This 
is caused by a specific toxin produced by the bacilli and results in the characteristic product of the disease, the tubercle. Surrounding the bacilli are grouped round cells (according to Baumgarten these are derived from connective tissue cells and epithelial cells, according to Borrel they are large mononuclear lencocytes). These round cells become transformed into epithelioid cells, with vesicular nuclei, which enclose several tubercle bacilli. As a result of repeated karyokinesis which process the diseased protoplasm can no longer follow (Baumgarten, Weigert) or as a result of the confluence of several epithelioid cells (Metchnikoff) there develop in the center of the cell mass polynuclear epithelioid cells as well as true giant cells. These also frequently contain tubercle bacilli. Later, as a result of reaction of the surrounding tissue, mononuclear lymphocytes and polynnclear lencocytes migrate from the adjacent blood vessels, form a dense zone around this cell group and lodge themselves between the epithelioid cells while a fibrinous network of coagulated exudate is deposited between the cells of this group. As a result of the toxic action of the chemical products of the bacilli, perhaps also on account of the nonvascular condition of the nodules and therefore of insufficient mutrition (Virchow), the completed tubercle suffers regressive metamorphoses. The central cells die off, break down, and together with the scanty connective tissue and the intermingled exudate are converted into a granular, cheesy mass in which lime salts are eventually deposited.

In cattle and swine calcified and caseated tubercles may contain virulent bacilli, so that this metamorphosis does not indicate complete recovery. The virulence of these bacilli is, however, as a rule, reduced (Lubarsch, Schmitz).

The structure of the tubercle in different species of animals exhibits variations of minor importance, which nevertheless are responsible for the often characteristic microscopical appearance of the lesions in different species of animals. The tubercle of cattle is characterized by an abundant groundwork of connective tissue frequently containing giant cells but containing comparatively few snall round cells. These tubercles have a great tendency to become calcified. As a result of this dense connective tissue groundwork numerous adjacent tubercles frequently become confluent and form tumors resembling fibrosarcomata in appearance. The tubercles of sheep and goats have a similar structure. The tubercle of the horse is well supplied with cell substance and consequently readily undergoes softening. This softening process may, however, not set in until at a late stage of its development. Calcification is only rarely observed. The tubercle in swine is well supplied with small round cells with little connective tissue ground substance. They readily become caseated and not infrequently calcarious. The tubercle of the dog often undergoes mucoid degeneration, or softening, in its later stages. 
The term tuberculum was formerly applied to every possible and conceivable form of nolule or tumor. In 1794 Baillie and in 1510 Bayle applied this term exclusively to the small gray nodule which oceurs in tuberculosis. Later on Laïnnec regarded the eheesy substance as characteristic of tubereulosis and finally Virchow laid particular stress upon the fact that the tubercle was composed of small cells resembling lymph cells and that the structure was devoid of a rascular supply. He regaried the tubercle as characteristic of tuberculosis. Langhals called attention to the frequent occurrence of giant cells in the tubercle. Schueppel laid especial stress on the importance of the frequently observed epithelioid cells. Accolding to the latter a true tubercle consisted of one or several giant cells surrounded by one or more rows of large epithelioid cells which latter were again bound by a cone of small round cells. As a matter of fact, however, giant and epithelioill cells may be absent from true tubercles, and giant cells occur also in other pathological formations. Ziegler showed that the tuherele was the result of an inflammatory process, originating like ordinary inflammatory granulation tissue, that is, prineipally from migrated white blood corpuseles and that other tissne elements such as the endothelial cells of the lymph ressels played only a suborlinate role in the construction of the tubercle. According to him the true tubercle was characterizell hy its non-vascularity and by its teudency to undergo cheesy degeneration. Baumgarten maintains that the tubercle bacillus gives rise to a proliferation of the fixed connective tissue cells exclusively (epithelial as well as connective tissue proper) which produce the so called epithelioid and giant cells and that the peripheral zone of round cells consists of white blood corpuseles which emigrated subsequently. Finally, according to Metschnikoff, the epithelioid and giant cells are phagocytes of mesodermal origin which are attracted by the positive chemotactic action of the tubercle bacilli which they take up into their substance.

Nodules resembling tubercles will also develop around dead bacteria and around inorganic foreign borlies. These nodules however do not undergo cheesy legeneration, have no progressive tendencies and cannot be transmitted by inocilation!

In recent times it has been pointed out by various investigators, especially Weichselbaum and his pupils, that tuhercle bacilli may exist for a long time in a dormant state in the lymph glands without causing the development even of microscopical tubercles. Before the discovery of the tubercle bacillus Orth demonstrated by inoculation experiments that lymph glands of normal appearance may contain microscopic tubercles $(1876)$ ! The effects of the tubercle bacilli can manifest themselves only in lymphoid hyperplastic processes ("lymphoid stage of tuberculosis"-Bartel \& Spieler). This stage may have a variable duration, may even retrogress and possihly exert an immunizing effect. The process may, however, with or without subsequent infection lead to the development of specific tuherculous changes at that point or in remote parts of the hody. Microscopically, glands in the lymphoid stage of infection appear either entirely normal or show only a homogeneous swelling. Tuhercle bacilli can be iemonstrated by inoculation only. Joest doubts the occurrence of such a lymphoid stage at least in animals with generalized tuberculosis, especially in cattle and swine. He examined 27 such glands in cattle and 4 in pigs and found specific histological tuberculous changes in all of them (epithelioid tubercles with giant cells). Jonske found the same condition in five glands that produced inoculation tuberculosis. Smit failed to find histological tuberculous changes in seven swollen and hyperemic glands, and Rievel and Linnenbrink could not discover microscopical tuberculous lesions in three out of twelve such glands that had a normal appearance.

That lymph glands may be virulent without exhibiting microscopic tubercles has been proven by investigations of Frothingham, Calmette, Guérin \& Deléarde. These experiments were made in calves, but the inoculations with human tubercle bacilli proved that they may remain latent in the hody for months and, according to Lignières, even for a year. Virulent bacilli may be found in partially calcified lesions (Bamm- 
garten, Lubarsch), and according to Rabinowitsch and Schmitz they are also met with in completely calcificd lymph glands, although in these latter they have become more or less attenuated.

When inhaled into the finest ramifications of the bronchial tubes and the alveoli of the lungs, the bacilli produce a catarrhal inflammation resulting in a filling up of the alveoli with fibrin, lencocytes and large epithelioid cells. This cellular exudate soon undergoes cheesy degeneration, the intraalveolar septa break down and thus larger cheesy foci are formed, which are at first surrounded by inflamed catarrhal alveolar tissue and later on by connective tissue walls. Fresh tubercles are usually developed in the immediate neighborhood as a result of the migration of bacilli through the lymphatic spaces. Bacilli carried to the lings with the blood produce typical tubercles in the interalveolar connective tissue or in the bronchial walls where they produce fibrinous proliferation.

After the death of the tubercle bacilli the tuberculous process may come to a standstill in any stage of its development. Following this phenomenon a reactive inflammation results in the production of a fibrinons envelope around the entire focus (fibrous tubercle). In slightly advanced local affection this process may terminate in complete recovery from the disease.

Distribution of tubercle bacilli in the body. According to the foregoing explanations in regard to the modes of infection the bacilli pass along the lymph channels to the regional glands, then, to a certain extent at least, to remoter glands and finally into the blood stream. If they are not destroyed by the lymph elements, or otherwise, they prodnce specific tubercles at points where they happen to lodge. From the tubercle thus formed they pass through the lymph spaces ("the histological highways of the body" Cohnheim), into the neighboring intact tissue where they canse the development of new tubercles. Every new tubercle then becomes the source of other tubercles. As these undergo cheesy degeneration the infected focus becomes larger and larger, cavities are formed in the lungs and ulcers on the mucous membranes. In the periphery of these new tubercles make their appearance.

In their further course the bacilli follow the lymph spaces into the regional lymph glands and neighboring organs where they produce similar changes. From the affected organs they pass over to their serous membranes, become disseminated through the lymph plexuses, thus not infrequently giving rise to a diffuse tuberculosis of these membranes. The bacilli may also penetrate the lymph spaces of the diaphragm and the morbid process spread from the peritoneum to the pleura and pericardium. The development of tubercles on the serous membranes is not infrequently attended with a sero-fibrinous inflammation. 
From the tuberculous foci in the lungs the virus passes from the bronchioles to the larger bronchial tubes and the neighboring healthy alveoli. Thus the diseased area becomes larger and larger. Secretions containing bacilli are coughed up and lodge on the mucous membrane of the trachea and larynx, especially on the rentral surface immediately posterior to the attachment of the rocal chords. Thus the tuberculous process gains a foothold here. These secretions may also reach the mouth whence they reach the small intestine with the food, causing the development of tuberculosis of the mucous membrane, especially of the ileum. Tubercles and ulcers that are occasionally found here are usually produced in this manner. In a similar manner a metastatic process in the renal parenchrma may spread to the pelvis of the kidney and follow the course of the ureters downward. On the other hand primary infection of the uterus, the vagina, the epididrmis and the bladder may spread into the pelvis of the kidney and from there attack the parenchyma.

If the bacilli enter the ressels they are transported by the blood stream and, if not destroyed, produce embolisms in various organs from which tubercles are developed. This is comparatively frequent in the kidney and spleen. If the number of bacilli entering the blood stream is excessive, numerous tubercles may develop in several organs and give rise to an acute miliary tuberculosis (tuberculosis miliaris acuta). This happens most frequently when tubercles which develop in the walls of arteries or veins of an infected organ, degenerate or develop into ulcers from which the bacilli pass into the blood stream. This danger is particularly great if the ressel communicates by a direct opening with a nearby or adjacent cheesy focus. Exposed tubercles in the walls of the thoracic duct may also be the cause of infection of the blood stream. (Joest reported two cases of open tuberculosis of the thoracic duct in cows.)

If bacilli are discharged into the lumen of a peripheral vein or into a branch of the pulmonary artery most of the bacilli will lodge in the lungs and produce tubercles almost exclusively in these organs. On the other hand if tuberculous ulcers exist in one of the pulmonary vems the bacilli which they discharge may enter any organ of the body that are reached by blood from the left ventricle and there produce a miliary tuberculosis, most commonly in the spleen, liver and kidneys.

According to Roemers' experiments, which were confirmed by Hamburger, Courmont \& Lesieur and by Leber, the body of a tuberculous animal offers nore or less resistance to subsequent external infections. Such additional external infections are either successfully resisted or they have only a local effect; they may even have a curative effect, as had already been shown by Koch. On the other hand, after infection with large masses or virulent bacilli lung eavities may derelop instead of miliary tuberculosis. Calmette \& Guérin demonstrated such an increased resistance in tuberculous eattle. In these animals the injection of highly virulent bovine bacilli produced chronic processes only. This increased power of resistance is said to manifest itself also toward bacilli from the borly of the animal itself 
and not alone toward bacilli of a new strain, hence in metastatic autoinfertion. The fact that metastatic tuberculous infection occasionally occurs in human beings and animals with chronic tuberculosis (Joest) does not necessarily contradict this statement. Repeated or massive hematogenic infection from chronic foci may establish a new focus of infection eren in relatively immune tissues.

Toxins. Tubercle bacilli owe their activity to endogenic toxins as well as to toxic products of metabolism. Necrobiosis and caseation in the tubercle is the result of the endogenic toxins. Subcutaneous injection of dead bacilli results in a local sterile abscess, necrosis, caseation and cachexia. Alkaline, ether- and benzine-extracts of the bacilli will produce a similar result (Koch, Weyl, Auclair \& Barbier, Siegenbeck van Heukelom). On the other hand the fever which occurs in the course of the disease is caused by the products of metabolism which later also are to be found in the filtrates of cultures which, in a concentrated form. are known as tuberculin (Koch, Behring).

According to Marmorek the toxin that circulates in the blood of an infected animal and with which the bacilli themselves are also impregnated is not the same as that in tuberculin. This toxin is formed outside of the animal body in fresh cultures growing in leucotoxic serum, e. g., liver bouillon. When subcutaneously injected into horses it produces inflammatory edema. Doses of 5.0 ec. will kill guinea pigs. According to Calmette \& Bretton boiled cultures act similarly to tuberculin. According to Vallée, Martin \& Vaudremer bacilli which have had their fat contents removed act similarly to hyperactive tuberculin. Cantacuzino observer that the intravenous injection of large doses into guinea pigs rapidly produced death following hypothermia, degeneration of the kidneys and the heart and increase of eosinophiles in the blood. Small doses produce chronic disease. Maragliano recognizes two toxins of the tubercle bacillus, an exogenons thermolabile toxalbumin (destroyed by boiling), producing diaphoresis and hypertrophy and an endogenous thermostable toxoproteid (heat resisting) said to have a hyperthermic action. Koeppen on the other hand conld not discover any difference between these two groups. De Waele inserted collodion capsules filled with cultures of tubercle bacilli under the skin of experiment animals. A few weeks later they reacted to tubereulin. Moussu inserted clay tubes filled with cultures of bacilli under the skin of cattle anil produced the same result.

Mixed Infection. Tuberculous organs constitute a favorable soil for the growth of saprophytes as well as for other pathogenic bacteria. These are carried to open lesions by the air or the blood stream transports them to closed cheesy foci. They may multiply in these places and are then liable to modify the anatomical character of the lesions as well as the clinical course of the disease. It is also possible that pathogenic organisms produce certain primary lesions and thus prepare the soil for an easier infection by the tubercle bacilli. The pyogenic bacteria are particularly important in this respect, a purulent and soft character of tuberculous foci being frequently, though not always, an indication of such a mixed infection. Such a metamorphosis of a dry cheesy focus into a soft purulent mass favors the dissemination of the tubercle bacilli in the organism, if only because they are usually present in them in large numbers (Bongert) and furthermore the frequent development of tuberculosis following catarrhal affections and acute pneumonia points to the favorable influence of other 
pathogenic organism in preparing a soil for the tubercle bacillus. The pronounced intermittent fever observed in advanced cases of tuberculosis is usually an indication of an infection with pyogenic bacteria causing suppuration in tuberculous organs.

According to Oestern the bacterial flora in soft tuberculous foci is a very limited one. Besides tubercle bacilli there are chiefly staphylococci, especially staphylococeus albus; sarcina and bacteria resembling colon bacilli are rarely found; streptococei are absent. If only tubercle bacilli are present the mass is yellow and of a slimy watery consistency. Otherwise these soft foci are caseo-purulent or muco-caseous. Bongert has shown howerer that in cattle the purulent softening of the tuberculous tissue is caused almost exclusively by tubercle bacilli, especially when these bacilli are destroyed in considerable numbers.

Anatomical Changes. The tubercle which is the characteristic product of tuberculosis, is at first a gray and transparent nodule, just visible to the unaided eye, but soon becomes yellowish as a result of cheesy degeneration which commences in the center. Nodules of the size of millet seeds or larger are the result of fusion of a number of primary nodules. The continuation of this process of fusion or conglomeration leads to the formation of still larger, dry, vellow cheesy masses or foci. The tuberculous nature of these is indicated by the presence of characteristic tubercles in the tissue immediately surrounding them. Tubercle formation and caseation are, in general, the characteristic pathologic-anatomical changes in tuberculosis (but considerable variations may be present in different species as well as in different organs).

In cattle the thoracic organs, especially the lungs and the broncho-mediastinal limph glands are the chief seat of disease. In the tissue of the lings there are foci of all sizes, which may be firm and sharply circumscribed; the surface on section appears reddish-gray or yellow, their substance consists of a dry, crumbling, cheesy or mortar-like mass surrounded by tough connective tissue; on the other hand they may consist of soft yellowish-white masses of pus surrounded by a thin connective tissue capsule. The presence of such foci is immediately recognized by the nodular appearance of the surface of the lung or by palpation of the deeper seated nodules. Their size varies considerably. In advanced cases there are all transition stages from the small gray nodules just visible to the unaided eve up to tumors larger than a man's fist. In the tissue surrounding the large tumors there are almost always new-formed tuhercles resulting from the migration of bacilli through the lymph spaces. Foci of embolic origin are usually found in the interstitial tissue of an otherwise healthy portion of the lung. The pulmonary tissue between the various tuberculous tumors may be apparently normal, through the filling up of the alveoli with exudate they have a homogeneous reddish-gray or yellowish appearance. As a result of proliferation of the interstitial connective tissue certain areas become thickener and tough and partly or wholly devoid of air. According to the severity of the disease only a portion of the organ may be affected (in cattle usually the posterior lobes in swine the an- 
terior lobes) or tubercles of all sizes may be scattered through the entire substance of the lung.

The confluence of cheesy or caseo-purulent foci of all sizes leads to the formation of cavities which are surrounded $\mathrm{hv}$ dense connective tissue lined on the inside with suppurating granulation tissue which was formerly called the pyogenic membrane. The interior of these cavities contains strands of comnective tissue which are obliterated blood vessels. Some of these cavities communicate with bronchial tubes through which they discharge their contents to the outer world. As a result of exposure to the air the muco-caseous material which is retained in these open cavities decomposes into discolored ill-smelling masses (phthisis tuberculosa).

In young animals pulmonary tuberculosis frequently occurs exclusively in the form of catarrhal pneumonia. The diseaser parts of the organ appear uniformly firm, solid and devoid of air. The cut surface is reddish-gray, grayish-white or yellowishgray and presents to view a slight quantity of cheesy matter that can be scraped off witl a knife. Here too the cheesy degeneration of the exudate and the breaking down of the lung tissue results in the formation of dry or soft cheesy foci of various sizes. Tuberculosis of the superficial layer's of the pulmonary tissue is frequently attended with a productive inflammation of the pleura. This may lead to pleural adhesions or to the development of excresences which will be described below. In rare cases a sero-fibrinous pleuritis develops.

Besides the affection of the lung tissue proper there is a catarrhal condition in the bronchial tubes of the diseased areas. In severe cases all of the bronchial tubes may be filled with muco-purulent secretions. The nucous membranes are swollen and injected and frequently contain tubercles and ulcers; they may become detached from the bronchial walls and obliterate the lumen or cause bronchiectases. Ulceration of the bronchial mucous membrane is not infrequent. The tuberculous nature of the ulcers is indicated by their irregular form, raised borders and yellowish-red base, and by the fact that on and around the ulcers there are small tubercles to be seen. Sometimes dense masses of tuberculous growths are found in the larynx, especially in the ventral angle of the glottis. They mar completely cover the vocal chords or be fused into one large tumor. In exceptional cases the nasal mucous membrane may be covered with yellowish-red granulations containing numerous caseous foci (Joest).

Tuberculosis of the serous membranes is characterized in the beginning by grayish-red, succulent, soft, yet tough excresences in some of which vellowish nodules mav be found. In the later stages there are firm dense globular formations (pearl nodes) with cheesy or mortar-like foci in their interior. The smaller excresences may be attached by means of a broad base or may be pedunculated. They frequently become ad- 
herent, producing grape or cauliflower-like masses (Fig. 81). These growths may be present in enormous numbers on the serous membranes, and the membrane itself may be thickened to the extent of several centimeters. This is especially common in the folds of the pericardium which are frequently found in a state of adhesion, surrounding the heart in the form of a dense fibrous capsule several centimeters in thickness and

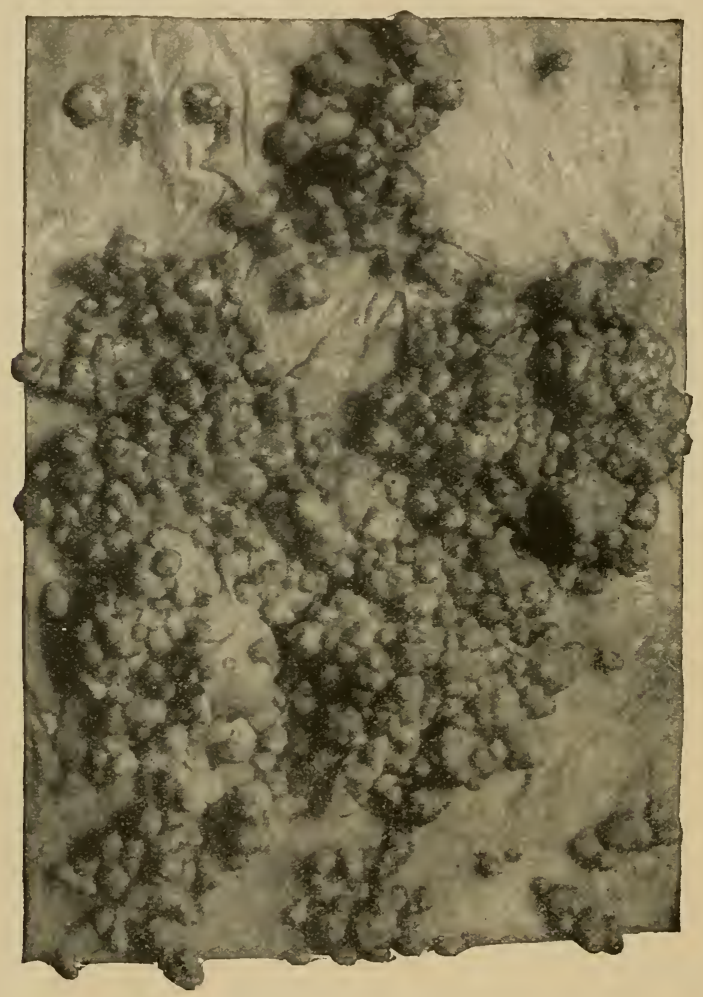

Fig. 81. Tuberculosis. Pearl Disease of the Pleura.

riddled with cheesy or mortar-like foci (so called armoured heart-Panzerherz). This capsule is either sharply separated from the heart, or white connective tissue septa may pass from it into the substance of its muscular structure. The heart substance itself may contain cheesy foci which may also be present where the epicardium is intact (embolism). Tuberculosis of the endocardium or the valves is very rare (Bergeon found miliary tubercles on the valves and on the intima of the aorta). 
It is usually possible to demonstrate tubercles with giant cells in fresh villosities. Some of these, however, do not show a tuberculous structure nor does their inoculation into guinea pigs produce tuberculosis. (Heymans found tubercle bacilli, virulent upon inoculation, in only 58 out of 70 exeresences examined.)

The lymphatic glands in the region of the diseased organs are always involved. On the other hand they are not infrequently, especially in young animals, the sole seat of the disease. At first they are only homogeneously swollen and enlarged and

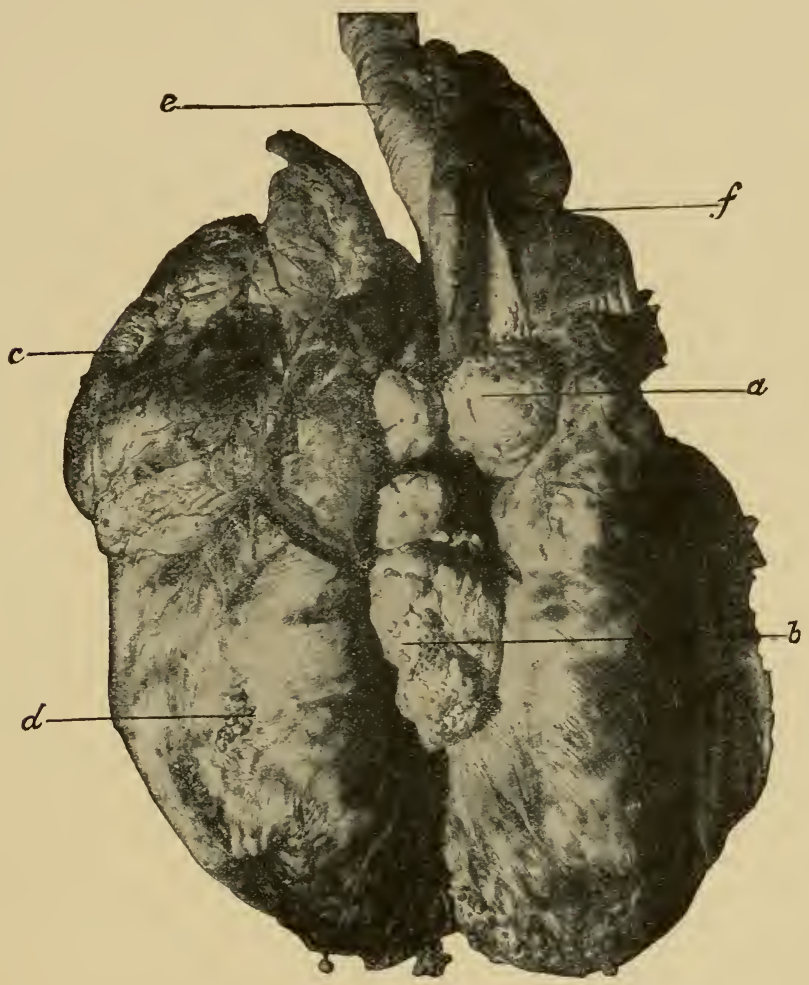

Fig. 82, Tuberculosis of Thoracic Organs of Cattle. $a$ and $b$ enlarged tuberculous bronchial and mediastinal glands; $c$ and $d$ pearly tumors on the pericardium and pleura; $e$ trachea; $f$ esophagus. Cheesy nodules in the lung substance.

more or less hemorrhagic; later on a section will show spotted turbidities in a grayish-red background. The turbidities contain tubercles or diffuse caseous masses. At a later stage the glands develop into large firm tumors consisting of grayishwhite fibrous tissue in which are imbedded large foci of a dry 
cheesy or mortar-like consistency. By confluence with other similar tumors they may become metamorphosed into fibrous sacks filled with a caseous, caseo-purulent or mortar-like mass.

Another picture of tuberculosis of the glands is presented by radiating caseation. Here a section of the hard dense gland has the appearance of a section of a radish, with strands radiating in all directions from the center. Cheesy sections alternate with viscid tissue strands (Bongert). In pulmonary tuberculosis the mediastinal and the peribronchial glands in particular may attain to enormous size (Fig. 82). In alimentary infection the retropharyngeal, the mesenteric and the portal glands become similarly enlarged.

Stroh found the small lymph glands of the museles affected in $1.4 \%$ of cases in oxen, $3 \%$ in bulls, and $22.9 \%$ in calves. In cattle the popliteal glands were most frequently affected $(27.1 \%)$. The prescapular glands were affected in $26.7 \%$ of eases, the precrural glands and the ischial glands in $17.2 \%$ and the axillary glands in only $5.5 \%$ of all cases. In calves the percentages were as follows: Prescapular glands $40.8 \%$, popliteal glands $23.8 \%$, precrural glands $22.3 \%$, axillary glands $6.9 \%$, ischial glands $6.1 \%$. The mammary glands were affected in $1.7 \%$ of all tubereulous cows.

In the course of the digestive tract cheesy nodules and ulcers may occur in the parenchyma of the affected tongue, sometimes just anterior to the eminence of the dorsum (in the tonsils of calves) and in exceptional cases in the œsophagus (May, Nicolas). On the mucons membrane of the small intestine, especially the ileum, and in the cecum isolated tubercles as well as ulcers of variable size may be found. For them, also, the infiltrated prominent borders of the ulcers surrounded with tuberculous nodules and the firm base covered with dry, cheesy material, are characteristic. They may extend to the submucosa or to the muscularis; are often elongated in form and lie at right angles to the long axis of the intestine (belted ulcers). In rare cases similar ulcers are observed in the mucous membrane of the fourth stomach (Walley, Kitt, F. Arloing, Zietschmann. Plate observed one case of fatal hemorrhage from such an ulcer). Johne observed ulceration of the mucons membrane of the abomasum. In exceptional cases the tuberculous process assumes the form of polypous growths on mucous membranes (Jolne), or of a swelling with ulcerating surface (such was found in the abomasum by Joest).

Chaussée deseribes three forms of intestinal tuberculosis. (1) Tleerating tuberculosis of the mucosa and submucosa, eausing necrosis and sloughing. (2) Hypertrophic tuberculosis in which tuhercles appear exclusively in the submucosa, the mucosa itself becoming hypertrophied, projecting in the form of a shallow plateau above the surrounding healthy mucosa. (3) The herpetic form of tubereulosis which occurs exclusively in the mucosa on the surface of which small shallow erosions are found.

In $1905,0.45 \%$ of all cattle slaughtered in the German Empire were affected with intestinal tubereulosis.

The substance of the liver may he dotted with small tubercles and cheesy foci or it may contain abscesses as large as 
a man's fist with contents of a mortar-like consistency or a soft muco-caseous mass. These abscesses are surrounded by dense connective tissue capsules and frequently give rise to enormous enlargement of the organ. In a case reported by Streitberg the liver weighed 64 pounds.

The spleen, especially of young animals, frequently contains numerous small tubercles. Sometimes only a few large cheesy foci are present.

The kidneys, on the other hand, are more frequently affected in older animals. The small firm tubercles may be distinctly seen in the brownish-red renal tissue. The large cheesy foci are usually embedded in tough connective tissue forming projections on the surface. By becoming confluent such foci may involve an entire lobule and upon rupturing discharge their cheesy contents into the pelvis of the kidney (nephrophthisis). The mucous membrane surrounding the point of rupture may contain snall tubercles or it may have been converted into a loosely adhering cheesy mass. Tuberculous foci of the kidneys may now and then have the appearance of lymphosarcomata. As a rule the ureters are indurated and their mucous membranes converted into a cheesy mass similar to the condition found when the process spreads upward from the neck of the bladder. Tuberculous processes in the mucous membrane of the urethra and likewise in the vagina are rare.

Of the male sexual organs the epididymis and secondarily the testicles which then consist of tumors with cheesy interior are most frequently affected; in females the uterus and the Fallopian tubes with or without involvement of the peritoneum. In slightly advanced cases small nodular indurations may be recognized by palpating the horns of the uterus. In advanced cases these changes become more pronounced, and firm solid masses enclosing large quantities of yellowish creamy or smeary and sometimes ichorous fluid are imbedded in the uterine walls. The mucous membrane contains at first small firm grayishwhite nodules which develop into tumor-like structures. Sometimes the mucosa becomes extensively ulcerated. These lesions may perforate the serous coat of the uterus and their ragged edges project into the peritoneal cavity. As a rule the Fallopian tubes, especially the abdominal third (Fischer), are involved and appear as thick solid strands. The ovaries are usually affected only in generalized tuberculosis of the genital organs; in nearly all of these cases both ovaries suffer, the morbid changes being limited to hyperplasia and the formation of villosities on the surface (Mayfarth), or to the formation of large tumors covered with villosities and enclosing cheesy foci. (Gergely observed one ease of extensive tuberculosis confined to the testicles following an injury which resulted in retraction of these organs into the pelvic cavity.)

Abattoir statisties of the German Empire show that $0.47 \%$ of all slaughtered cows were affected with tuberculosis of the uterus. According to Ostertag $65 \%$ 
of all cases of generalized tuberculosis involve the uterus, while Lungwitz found this organ involved in 152 out of 264 cases of pearl disease of the peritoneum. In far advanced cases the affected uterus may weigh 40 kilograms (Moebius). According to Fischer's investigations the majority of cases spread from the Fallopiau tubes to the uterus. This seems to be true even when the lesions of the uterus appear more adranced.

Tuberculosis of the udder is usually characterized by the presence of large firm tumors with cheesy contents and numerous small tubercles scattered through the intervening tough connective tissue (Fig. 83). As a rule either one or both posterior quarters and the adjacent regions of the anterior quarters are affected. The diseased part which is usually enlarged, but sometimes atrophied, is separated from the normal portion by a well marked sinnous line of demarkation. The walls of the

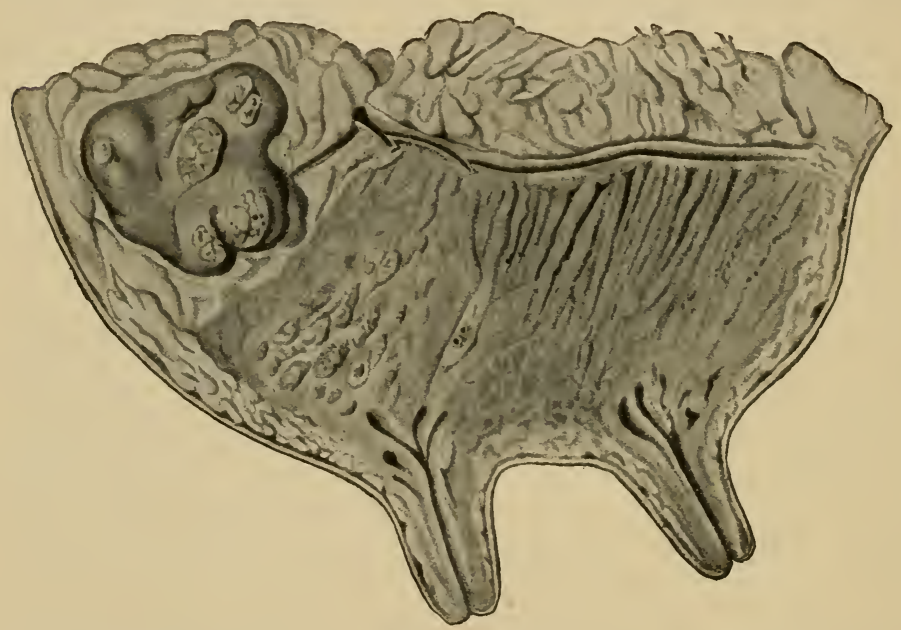

Fig. 83. Tuberculosis of the Udder. Tubercular foci in the glandular tissue of the hind quarter. Supermammary gland much enlarged with cheesy foci. Mossu.

milk ducts may contain tubercles or they may be enormously distended and enclose masses of cheesy detritus and flaky or curdled milk. Some of the veins of the ndder may be found obliterated with blood clots in which colonies of tubercle bacilli may here and there be demonstrated (Bang). In recent embolic infection the lobules of the affected quarters are swollen and their gray substance sprinkled with yellow points or streaks while the intervening connective tissue is riddled with minute tubercles and cheesy foci ranging in size from that of a lentil to a pea (Bang, Moser). In advanced cases the affected quarter may have been transformed into a firm tumor containing cheesy masses enclosed by tough fibrous connective tissue. The supermammary glands are always involved when the udder is af- 
fected, but they may also be diseased when tubercles cannot be demonstrated in the tissue of the udder, at least with the naked eye.

Of the central nervous system the brain is most frequently affected. There may be several large tumors (tyroma, tuberculum solitarium) or a number of smaller cheesy foci. These always involve the meninges and produce a tuberculous inflammation. In such cases recent tubercles can be found at the base of the brain, in particular in the region of the Sylvian fissure (meningitis basilaris tuberculosa). As a rule this is accompanied by acute internal hydrocephalus (very common in calves after protective inoculation-Hutyra, Weber, Titze and others). Similar affection of the spinal cord and its envelopes is far more rarely met with, and when present it is usually observed near tuberculous vertebrae.

Tuberculous lesions are observed also as follows: In the spongy substance of flat bones especially the ribs and vertebrae but in exceptional cases also in the bones of the cranium and of the pelvis; in long bones the marrow may be affected; in articular cartilages of the long bones; in the joints (exudative inflammation with induration of the capsular ligaments resulting in villous proliferation and destruction of the cartilages, according to Hamoir most common in the knee); on the nasal mucons membrane with occasional involvement of the cartilages, muscles and septum; in the muscles of the body (usually cheesy foci originating in the intermuscular lymph glands and occasionally foci approaching the size of a bean scattered between the bundles of muscle fibres); in the subcutaneous connective tissue (firm tumors arranged in rosary-like order and cheesy abscesses); very rarely in the skin, tendon sheaths of the extremities, penis, prostate gland and in the eve (tubercles in the iris followed by caseous panophthalmia, or fibrinous iritis without tubercles [Manleitner]).

On post-mortem examination, especially in meat inspection, we distinguish between primary and secondary tuberculosis of the various organs. The term primary is applied to lesions resulting from direct infection from outside and spreading into the surrounding tissue from the point of infection, either by direct growth or through the lymph spaces and lymph vessels, but not by means of the blood stream. Secondary lesions are those resulting hæmatogenically from infected embolisms of the blood vessels, and are consequently found in the interstitial connective tissue. They do not communicate with the outer world and therefore rarely suppurate (Ostertag). According to the discussion on page 530 the possibility of the occurrence of primary lesions of lymphogenic or even hæmatogenic origin in internal organs is not excluded.

The term local tuherculosis is applied to lesions that have spread or have become disseminated hy direct growth only or through the lymphaties or one of the lesser circulations (pulmonary or portal). If the greater circulation was the means of disseminating the virus the resulting affection is referred to as generalized tuberculosis (Weigert, Ostertag). In the latter instance, if the infection was slight the resulting 
disease assumes a chronic character, while if the infection was extensive, acute miliary tuberculosis develops. Generalized tuberculosis is atypical when the organs usually attacked escape an infection from the blood stream and localization of the morbid process occurs in remote organs (Poppe).

Breuer's statistics on the localization of tuberculous processes for 1900-02 (Budapest) give the following information: Of 28,968 eases of tuberculosis $98.6 \%$ were local, $1.4 \%$ generalized. In $91 \%$ of local affections the lesions oceurred in one thoracic organ only, in $0.8 \%$ in one abdominal organ, in $0.2 \%$ in the tonsils and the retropharyngeal glands, while in seven instances several organs were affected. Of 100 cases of generalized tuberculosis the lungs were affected in 92 , the liver in 76 , the intestines and mesenteric glands in 59, the kidneys in 57, the serous nembranes in 70 , the spleen in 40 , the udder in 22 and the uterus in 8 instances. (Ostertag observed tuberculosis of the uterus in $65 \%$ of all cases of generalized tuberculosis. Henschel found nterus affection in $6.4 \%$ of all cases of hæmatogenic origin.)-In young cattle under four years with generalized tuberculosis the spleen is nearly always affected while the kidneys are free from lesions. In older cattle the kidneys are attacked as frequently as the spleen (Ostertag). Meat inspection statisties of the German Empire for 1905 show that $0.6 \%$ of all tuberculous cows have tuberculosis of the udder, according to Bang, 2\%; Ostertag, 0.5 to $1.0 \%$; MacFadyean, 1 to $2 \%$; Williams, $3 \%$; Tallée and Villejean, 5.3 to $6.5 \%$; Stroh, $1.7 \%$; and according to Henschel, $2.6 \%$.

Acute tuberculosis (tuberculosis miliaris acuta) is confined either to single organs, particularly the lungs, or the entire body may be affected. This depends upon whether the virus was distributed through a ressel supplying a limited area only as would be the case with a peripheral artery or with the pulmonary artery, or whether it had gained access to the greater circulation or the pulmonary reins. This form of the disease is characterized by the presence of enormous numbers of recent tubercles of approximately the same age. They are usually distributed evenly through the tissues. The surrounding areas, contrary to what is observed in chronic tuberculosis, are usually normal in appearance. There may however be general hyperemia or indications of beginning inflammation. There is always acute swelling of the lymph glands and reddening of the cortical substance.

In buffaloes the tuberculous foci (mostly in the thoracic organs) are grayish-white, yellowish or entirely white and contain remarkably juicy cheesy masses. Softening is rare and calcification is an exceptional occurrence; pearl disease of the serous membranes is equally rare (Breuer).

In sheep and goats the tuberculous changes are in general the same as in cattle. The serous membranes may contain pearly tumors and the uterus may be the seat of disease just as in cows. Koch and Probst have observed the formation of large cavities in the lungs of these animals. Contrary to the usual observations in other animals sheep do not exhibit the progressive changes of necrotization, caseation and calcification. The lesions are usually calcified and surrounded with thick fibrous walls. Delmer ascertained the presence of generalized tuberculosis in a goat six months of age and Micucei observed primary intestinal tuberculosis in the sheep. 
In horses, especially in young animals, the mesenteric and retropharyngeal lymph glands are most frequently affected (phthisis mesaraica). The affected glands may be white and moist on section and resemble lymphosarcomata or their interior may consist of a soft muco-caseous mass. In exceptional cases there is calcification. They may represent masses of tumors firmly enclosing large vessels (e. g. the posterior vena cava and portal vein); they may involve the vessel walls or have grown into their lumen producing thrombi (Lustig), or leading to generalized tuberculosis. Cheesy lymph glands may communicate with the interior of the large intestine (Lothes). In some cases the intestinal mucous membrane contains numerous nodules of the size of a hazelnut, some of them ulcerated on the crest, also irregular ulcers with smooth or ragged borders, ranging in size up to that of the palm of a hand; their base may extend to the submucosa and contain small tubercles. The large intestines and the rectum alone may be thus affected (Rátz; Darmagnac observed a case of perforation of a rectal ulcer producing acute peritonitis). The liver may contain similar lesions but they are rare in the spleen. The former may reach three times its normal size and the latter has been observed to weigh $13 \mathrm{~kg}$. (Schulz) or, according to A. Mareus, dimensions of 74 by $581 / 2 \mathrm{~cm}$. (According to MacFadyean, Johne and Kitt tumors of this sort formerly described as lymphadenomata, which occasionally are almost tendinous in consistency are usually tuberculous in nature.)

The disease of the lungs is usually miliary in nature. Less frequent are tumors as large as a nut or larger, which section appear homogeneous, like a turnip, or with soft centers containing a dirty gray or yellow purulent or caseo-purulent mass; in some cases they are enclosed in a connective tissue capsule. The intervening connective tissue is either normal or excessively proliferated. Changes indicating acute inflammation, as are observed in glanders, do not occur in tuberculosis. The peribronchial lymph glands are usually considerably enlarged and contain yellowish tubercles. The mucous membranes of the respiratory tract may also show ulcers.

Tuberculosis of the serous membranes (peritoneum, plenra and pericardium) usually occurs in the form of typical pearl disease (Bang, Nocard, Felisch, Foelger, Rátz). Occasionally there is an accumulation of much sero-fibrinous exudate in the plenral cavity and the pericardium (Aubry). Tuberculous lesions may occur also; in the pharyngeal and cervical glands (Froehner), in the vertebrae and ribs (Wolff, Haendel, Town \& Hobday), in the heart-muscle (Foelger), in the subcutaneous connective tissue and in the muscles (Cadiot. Fally). Enders described the rare occurrence of complete calcification of the thoracic aorta where it lies adjacent to the dorsal vertebrae. Hansenkamp describes tuberculous lesions in the marrow of the lumbar vertebrae and in the adjacent membranes. Behr

Vol. 1-35 
describes calcified concave scaly lesions in the endocardium as large as a silver dollar, also lesions as large as a lentil on the nasal mucous membrane.

The tuberculous lesions usually contain tubercle bacilli in large numbers.

In pigs tuberculous lesions are most common in the digestive organs and their lymph glands. In some of the cases we find, in addition to tubercles in the mucous membrane of the pharynx and in the tonsils, enlargement of the retropharyngeal, cervical and mediastinal lymph glands (scrophulosis). These glands contain a thickish cheesy purulent mass. Tuberculous processes in some of the superficial glands may even perforate the skin. In other cases the mesenteric glands may be similarly affected. The lumbar glands may constitute an irregular, convoluted tumor mass under the spinal column. In these cases tuberculous nodules and ulcers occur also in the intestines, especially in the ileum and cecum. The liver and spleen may contain cheesy foci ranging in size up to that of a nut. In exceptional cases wedgelike or irregular firm foci extend from the surface of the liver or spleen toward the interior of the affected organ. On section these areas show a homogeneous structure, are grayish-white or yellowish-white in color and present no evidence of caseation or calcification (Junack, Sommer). In rare cases the peritoneum is covered with numerous small nodules. (The so called cheesy enteritis of pigs [page 133] may perhaps, in exceptional cases also develop upon a tuberculous basis.)

In the lungs the disease may manifest itself by the presence of a few or of exceedingly numerous small dry cheesy foci, or a correspondingly smaller number of larger centers. Or there may be a caseous or cheesy pneumonia with large areas of the lung devoid of air, firm and inelastic, the sections appearing grayish-red or grayish-yellow. The adjacent portions of the pleura are indurated, and sometimes covered with a layer of red fibro-granular connective tissue $1 / \mathrm{mm}$. thick and resulting in extensive adhesions (Schlegel). The same changes may occur in the pericardium. Small tubercles and small ulcers are occasionally found in the tracheal mucous membrane (Graf) ; in such cases the peribronchial, substernal and subspinal lymph glands are usually enlarged and caseated.

Tuberculosis of the central nervous svstem results from the spread of a tuberculous process of the inner or middle ear or of the petrous bone (Schuetz); it may also be produced metastatically in tuberculous disease of remote organs (Azary). Tuberculosis of the spinal meninges is very rare. It manifests itself in the appearance of numerous yellowish miliary nodules on the peripheral surface. Occasionally they form large conglomerate tumors which produce compression atrophy of the spinal cord (Vogt).

Tuberculosis of the bones is comparatively common, 
especially of the vertebrae, the ribs and long bones. Less frequent is tuberculosis of the mammary glands (Nocard). When present it is usually in conjunction with disease of the joints or of the muscles (Moulé, Stroese, Winter, Hasenkamp). In rare cases there is affection of the nasal mucous membrane which manifests itself in diffuse induration and the presence of tubercles and ulcers (Zschokke, Balás). Tuberculosis of the eye (Manleitner, Keil) and tuberculosis of the ovaries (Hasenkamp) is also rare.

Of 4,468 cases of tubereulosis in swine observed in 1902 in the abattoir at Budapest $97.5 \%$ had local, $2.5 \%$ generalized tuberenlosis. In $87 \%$ the local tuberculosis was confined to one organ; in $74 \%$ the tonsils and retropharyngeal lymph glands only were affected; in $13 \%$ several organs were affected in the same animal. The lungs were involved in $100 \%$ of all cases of generalized tuberculosis, the spleen in $97 \%$, the liver in $91 \%$, the bones in $43 \%$, the joints in $15 \%$, the museles in $3 \%$ (Brener).-According to Stroh the lymph glands of the muscles were affected in $14.4 \%$ of all cases, the prescapular glands being involved in $52.65 \%$ of these, the precrural in $34.1 \%$ and the popliteal glands in $13.3 \%$. In 6,107 cases of generalized tuberculosis studied by Hensehel the preseapular glands were affected in $35.8 \%$, the precrural in $3 \%$, the popliteal glands in $4.3 \%$, the ischial glands in $1.1 \%$, the pubie glands in $2.8 \%$, and the udder in $0.3 \%$.

American reports point to the extreme frequency of affection of the submaxillary glands which are involved in $93 \%$ of all cases of tubereulosis of swine.

In dogs primary tuberculosis may occur in the lungs or in the digestive tract. Particular attention has been given to this form of tuberculosis in the publications of Jensen, Cadiot, Froelner, Rátz, Petit and Basset. In the lungs we find sharply circumscribed tumors of variable size, which may consist of sarcoma-like tissue of an even white texture on section, or their interior may be a viscons mass of pus. They may also consist of dry, cheesy or chalk-like masses. Sone of these tumors may have developed into cavities, perforated a bronchial tube or communicate with the abdominal cavity through the visceral portion of the diaphragm (Fiebiger). In other cases there may be broncho-pneumonic areas with small soft foci in their gravish-red substance. The mucous membranes of the bronchial tubes and the trachea may occasionally contain tubercles or villous proliferations. The peribronchial and mediastinal lymph glands are as a rule considerably enlarged, resemble sarcomata on section, are grayish-white in color or their substance is dotted with cheesy foci or masses of viscous pus. If the bronchial glands are much enlarged they may result in an upward and backward displacement of the heart. In exceptional cases the tuberculous process is limited exclusively to the latter glands or to these and the cervical glands while the lung tissue remains normal in appearance or reveals only recent changes. The pleura may contain tuberculous nodules or indurated areas, sometimes also papilloma-like proliferations or tumors resembling pearly nodules (Wenzel, Joest). Sometimes there is an accumulation of reddish purulent exudate in one or both pleural sacs, or there may be more or less extensive adhesions. Affection of the pericardium usually results in ad- 
hesion of the pericardial folds, but there may be an extensive accumulation of sero-fibrinous or hemorrhagic exudate. Cheesr nodules are occasionally found in the muscle of the heart. In exceptional cases the aorta, surrounded by tubereulous lymph glands, becomes indurated. Portions of its walls may become necrotic (Eber) or a tubereulous nodule mar perforate the latter and project into the lumen of the ressel (Rátz).

The mesenteric glands are frequently considerably entarged and present the same characteristics as the affected thoracic glands. Frequently the peritonem is also involved, especially its visceral laver. A limited area mar be covered with a few large nodules or numerous small tubercles may extend over great expanses of this membrane, while the omentum is transformed into a thick irregular cord. On the mucous membrane of the intestine, especially the dnodenum and ilemm, only few cheesy nodules or nlcers are occasionally found. The liver is frequently the seat of cheesy foci, some of them as large as an apple, the larger ones causing depressions on the surface of the organ. Their marrow-like substance contains gravishwhite, white, soft, purulent or crumbling foci. In rarer instances small or large tuberculous foci are found in spleen or kidneys; these occasionally perforate into the pelvis of the latter organ and set up nlcerating processes (Jensen). Ball observed isolated tuberculosis of the mucous membrane of the pharrnx in the form of a granular pharyngitis.

Finally tuberculous changes occur in rare instances in the sexual organs, pancreas, bones, certain joints (Cadiot), subcutaneous connective tissue (Rátz observed two cases of tuberculosis of the thoracic lympl glands that extended to and involved the overlying skin). Tubereulosis may also occur in the cerebral meninges as a tubercular meningitis (Nocard, Cadiot, Rátz, Petit), etc.

Acute niliary tuberculosis has also been observed in dogs and Cadiot describes a case where small tubercles in the swollen parenchymatous organs were risible with the maided eye while the tissues contained numerous tuhercle bacilli (tubereulous septicemia).

In 32 cases described by Petit and Basset the pleura was affecterl in 19 instances, the pericarlium in 15 . the peritoneum in 10 , the myocardium in 1 , the endocardium in 2, the intestine in 3, the mesenteric glanis in 5, the liver in 15 , the spleen in 1 , the kidneys in 9, the lmngs and bronchial lymphatic glands in 17, the bronchial lymphatic glands alone in 1, the lungs alone in 2, and the mesenterie glands were affected in one case where the large and small intestines were intact.

In cats tuberculous changes are similar to those observed in dogs. The abdominal organs and especially the mesenteric lymph glands are affected most frequently. The disease mar have its seat in these organs exclusively or the respiratory organs may also be involved. Jensen observer one case of tuberculosis of the uterus, one of the testicles, one of the joints and two of the subcutaneous connective tissue; Hobday, one case of generalized tuberculosis. 
Symptoms. In keeping with the extraordinary variability of the pathologic-anatomical changes that oceur in tuberculosis, the symptoms of the disease vary not only in different species of animals but to an almost equal extent in individuals of the same species. The insidious and long drawn out course of the disease gives rise to additional variations.

In advanced stages of the disease the body temperature is at times temporarily elevated. The course of the fever is intermittent or remittent, with evening exacerbations and morning remissions; sometimes the typus inversus is observed. In the chronic conrse of the disease febrile attacks lasting several weeks alternate with free intervals of the same duration. Near the termination of the disease the fever is usually high and continuous. The same is observed when an acute miliary tuberculosis develops from a chronic affection.

Tuberculosis of Cattle. The period of incubation, after severe artificial infection, is rarely longer than two weeks. In natural infection it is nuch longer; sometimes months and even years may elapse before the appearance of the slightest indications of disease become manifest to ordinary observation.

According to Nocard and Rossignol artificial infection by inhalation requires 19 to 32 days and alimentary infection 32 to 48 days hefore the animal will react to tubereulin. In experiments conducted with calves by the authors symptoms of fever appeared eight days after the intravenous injection of $0.02 \mathrm{gm}$. of eultures of tubercle bacilli.

Pulmonary tuberculosis, the most frequent form of the disease, manifests itself by a short, dry, vigorous congh. At first this occurs only when cold or dusty air is inhaled or when the circulation is accelerated by the sudden rising of the animal or by drinking cold water. In this stage the appetite is usually good but symptoms of exhaustion may be observed after prolonged exercise.

Gradually, usually after the lapse of months, symptoms pointing to pulmonary affection become more pronounced. The cough becomes more frequent and painful, may be dry and coarse or feeble and almost soundless. From time to time the animals discharge a ropy, muco-purulent, yellowish-gray secretion from the open mouth; as a rule however this secretion is swallowed as may be demonstrated by observing the left esophageal grove immediately after an act of coughing. It may also be retained in the space anterior to the soft palate between the tongue and last molar teeth where it may be reached by the hand (Ostertag). Sometimes the bronchial secretion is discharged through the nose; in this case the presence of pus corpuscles, alveolar epithelium that has undergone fatty degeneration, fat crystals, elastic fibres and tubercle bacilli may be demonstrated.

As the cough increases in frequency the type of respiration begins to change; it becomes more frequent and laborious, inspiration assumes more of a costal type and expiration, which 
may be attended by groans, becomes abdominal. These symptoms are most pronounced after exercise or during work.

A physical examination of the thorax rarely reveals characteristic changes. Vesicular respiration may be increased, diminished or imperceptible. In slight affections there may be dry rales, observable locally or over the entire extent of the thorax, caused by the presence of more or less viscous or fluid secretions or by accumulations of mucus in the bronchial tubes. The percussion sound may be normal or changed only slightly. If lesions are of considerable size and located near the surface the sound becomes dull in circumscribed areas, and in such cases bronchial sounds may be recognized, while large contiguons areas of dullness hardly occur. Cases where tympanitic resonance points to the presence of large cavities are rare, but an odor of decomposed bronchial secretion and necrotic lung tissue, perceived in the expired air, points to this condition.

Where auscultation gives unsatisfactory or negative results it is advisable to exercise the animals by running them around for a time, a procedure which usually produces more or less distinct rales in animals whose lungs are affected. In similar cases Roebert and Ellinger recommend closing the nasal openings with the hancls while auscultating. Nocari suggests the injection of 0.2 to $0.3 \mathrm{gm}$. of pilocarpine. Krautstrunk suggests arecolin, $0.08 \mathrm{gm}$. Ostertag, however, observer that the injection of $0.0 .5 \mathrm{gm}$. of arecolin in a strong heifer, producel staggering, falling and great dyspnoea. It should be noted here that the injection of the above substances may now and then produce rales in perfectly healthy animals.

Affections of the trachea and larynx usually produce catarrhal symptoms only. However, where there is suspicion of tuberculosis it is safe to make a probable diagnosis of laryngeal tuberculosis when we find the region of the larynx sensitive, the cough very painful and spasmodic and deglutition diffienlt. When tuberculous tumors exist in the larynx the latter may be enlarged and distinct vibration may be felt at each inspiration. At the same time the respiration becomes stertorous, the animals lolding their heads stretched forward and avoiding lateral movements, and sometimes attacks of suffocation are observed. On account of the incomplete closing of the glottis, particles of food may enter the trachea during the act of deglutition resulting in attacks of coughing and if only for this reason, the affected animals nsually eat sparingly. Holterbach noted aphonia in a case of isolated tuberculosis of the larynx in a cow with good nutrition. When attempting to low, the cow would only emit a faint blowing somnd. According to Friedberger tnberenlosis of the respiratory organs involves the larynx in only $0.13 \%$ of cases.

In rare cases the nasal mucons membrane is affected. Near the nostrils the mucous membrane contains firm nodules up to the size of a pea which are usually yellowish in the center. Ulcers may also be visible, or they may be sitnated higher up and detected by palpation only. This condition is always attended with a muco-purulent nasal discharge. The pharyngeal glands are enlarged and contain cheesy foci (Jensen, Gutbrod). 
The alae of the nostrils may be indurated and the lower jaw enlarged (Liénaux).

Tuberculous inflammation of the pleura is not frequent but usually occurs simultaneously with the affection of the lung. It may be the cause of soreness of the thoracic walls which causes the animals to flinch or cough when pressure is applied to the part. Pleuritic friction sounds may indicate roughness of the pleura or the presence of pearly tumors, and large masses of the latter may produce a dull sound upon percussion, while in fully developed cases the friction of the rough pleural surfaces may be felt with the hand. As a rule, however, especially when the lung is extensively affected, it is impossible to diagnose pleuritic involvement.

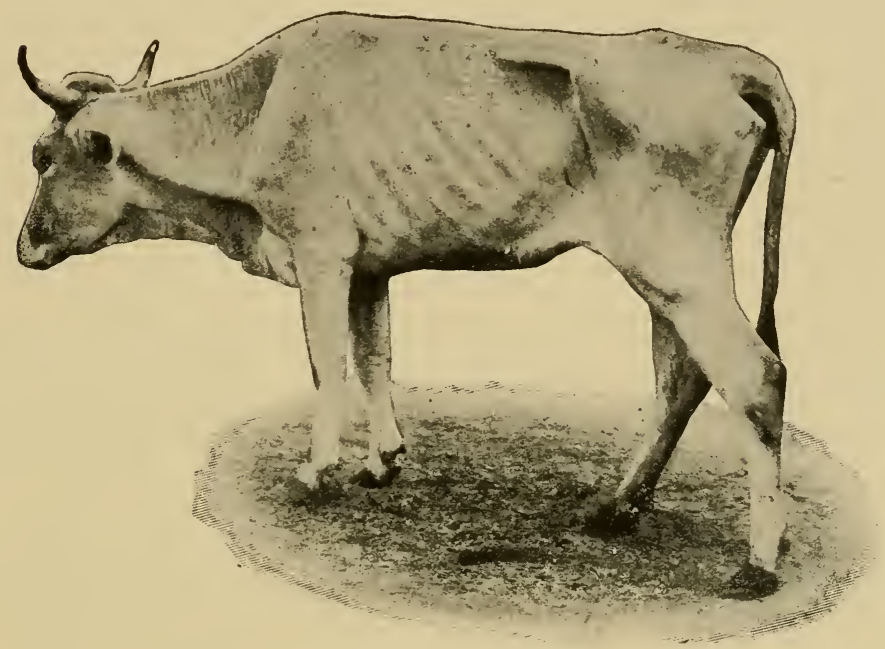

Fig. 84. Cow with Adranced Pulmonary Tuberculosis.

The general nutrition of the animal suffers in direct proportion as the disease of the respiratory organs advances. Symptoms of oligemia are indicated by paleness of the mucous membranes and increasing emaciation. The skin loses its elasticity and adheres more firmly to the underlying structures, the animals become "hidebound," the tuberosities and angles of the bones of the pelvis become prominent. The skin, when raised into a fold returns reluctantly to its hormal position and the liaircoat becomes staring and loses its luster (Fig. 84). The facial expression indicates exhaustion and sickness, while the eyes lose their natural luster and are withdrawn into their sockets. The appetite is at first variable but finally becomes poor; while in addition attacks of diarrhea persisting for days 
weaken the patients. The digestion is usually more or less disturbed and the condition is aggravated by periodical attacks of bloating, caused by compression of the esophagus by the enlarged peribronchial and mediastinal lymph glands which interrupt or prevent eructations of gas and interfere with rumination. In case of suspected animals this iatter condition always indicates the probable presence of tuberculosis! (Johne), it is however, usually not ohserved until srmptoms of pulmonary affection have manifested themselves. Distention of the jugular vein, which is occasionally observed, may he clue to the same cause.

Cardiac srmptoms are usually observed only when the pericardium is involved. There may be pericardial friction sounds and more or less pronounced distnrbance of the heart action. But since the pericardial sac rarely contains any considerable amount of fluid these sounds are usually absent. The heart somds are clearly audible and in rare cases only may cardiac dullness be noted. Swelling of the skin in the anterior thoracic region is rare as is also a jugular pulse (contrast with trammatic pericarditis). In advanced stages of the disease the pulse is subject to considerable variation in character as well as in rate. In advancerl cases it is much accelerated and emptr.

In one case ohserved by Liénaux there was displacement of the heart toward the right side, and great distention of the jugular vein as a result of compression by the anterior lobe of the left lung which was indurated and contained numerous cheesy and calcified foci. In another case he succeeded in demonstrating the presence of tubercle bacilli in the pericardial fluid which was obtained by aspirating the pericardial sac.

Toward the termination of the disease the respiratory difficulties become increasingly great. The affected animals stand without moving from the spot, with elbows projecting outwarr, the neck held low and the head stretched forward, groaning and gasping for breath while their forcerl respiratory movements cause the whole hody to tremble. The congh is weak, painful and frequent and is easily provoked hy slight pressure exerted upon the back. Purnlent or ichorous secretion which is discharged from the nostrils is no longer "licked away" hy the animal. As the animals become weaker and more anil more emaciated they lie down continnously, being no longer able to stand. In the meantime the abdominal organs mar liave liecome involved; there is a continnous diarrhea, and finally, after long suffering the end approaches without spasms.

Tuberculosis of the abdominal organs which in regard to frequency of occurrence is second to pulmonary tulserculosis levelops in an equally insidions manner. Tuberculosis of the peritonenm may reach an advanced stage of development before it manifests its presence ly any outward indications. In cows, 
however, when the process spreads to the serous coats of the sexual organs there is musual excitement during the period of oestrum. If served by a male they either do not conceive or they are apt to abort at about the middle of gestation. In later stages of the disease this excitement becomes continuous (nymphomania) and may then also exert an unfavorable influence upon the nutrition of the animal. Otherwise this form of tuberculosis does not necessarily interfere serionsly with nutrition and animals may grow fat even with advanced and extensive disease. The presence of the latter is often unsuspected until on post-mortem examination after the animals are slaughtered. Still, in the advanced stage of the disease the affection of other organs, especially the liver and the spleen and also the mesenteric lrmph glands will exert a disturbing influence on the organs of digestion and gradually anemia and emaciation become more and more evident.

Large tumors on the peritoneum may now and then be accurately located. The presence of large pearl nodes on the left side of the paunch or on the opposing peritoneal membrane may be palpated by applying the hand to the left flank. Tumors in the pelvic cavity, especially when located near the internal sexual organs may occasionally be palpated through the rectum. Any large tumors with irregular surface, or masses of smaller structures of liard consisteney, when present in the pelvic cavity, may be looked upon as tuberculous excrescences. These structures do not take finger impressions and the small ones if loosely imbedded in comnective tissue or if they are pedunculated ustrally slip from the grasp (Storch). Enlarged sacral, lumbar, medial iliac and mesenteric glands may also be palpated.

In the course of the digestive tract tuberculous changes may, in rare cases, be met with in the mouth, viz., the mucous membranes of the pharynx, the hard palate and the fauces. Here there are irregular ulcers with firmly adhering grayishyellow, cheesy deposits, sometimes knobby borders. The surface of the tongue, especially at the point just anterior to the eminence of the dorsum of the tongue may have similar ulcers. Its substance may contain firm nodules or be uniformly indurated which condition interferes more or less with the movements of this organ also mastication and deglutition (Moussu).

The symptoms of intestinal tuberenlosis are very indefinite. Digestive disorders which are hardly ever absent in advanced tuberculosis are not infrequently caused by an involvement of the intestines but, on the other hand, digestive disorders may be entirely absent even in advanced disease of these organs. The most significant symptoms are colicky pains and constipation alternating with diarrhea. The intestinal evacuations may be quite fluid and not infrequently mixed with mucus and pus. Sometimes there are streaks or even clots of blood. Occa- 
sionally microscopic examination will reveal the presence of pus corpuscles and red blood cells when its general appearance would not cause us to suspect them.

Tuberculosis of the liver and spleen can be recognized as such only in cases where considerable enlargement of the organs has taken place. There may be dullness upon percussion in the overlying region or their posterior irregular nodular borders may he palpated behind the costal arch.

Among the sexual organs tuberculosis of the testicle may be recognized by the presence at its superior and posterior border, corresponding to the position of the epididymis, of a firm painless swelling. The testicle itself does not become enlarged until at a considerably later date and then, becoming

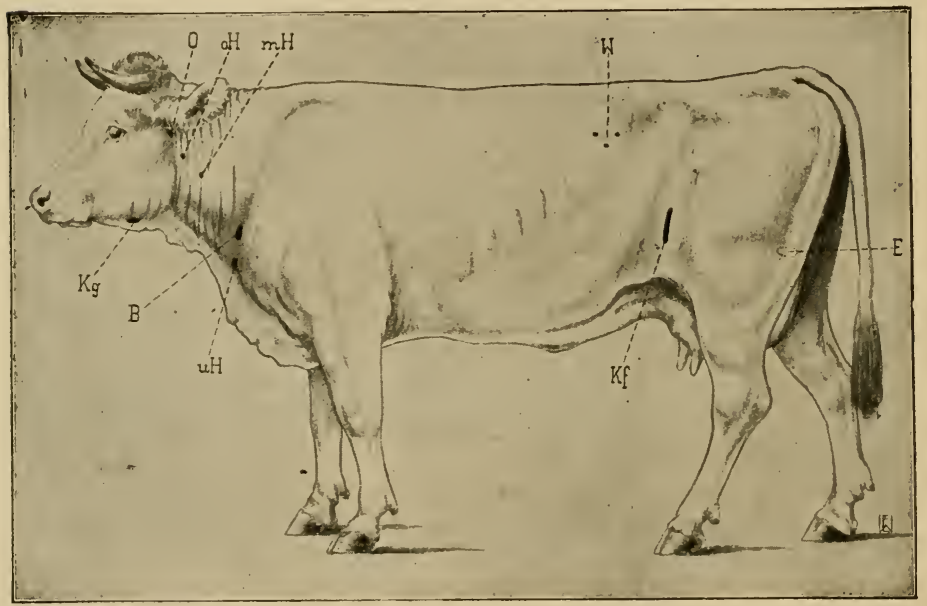

Fig. 85. Superficial lymph glands of the ox. $K q$. Submaxillary gland; $O$ parotid gland; $o H$ superior, $m H$ middle, $u H$ inferior Cervical glands; $B$ Prescapular glaud; Kf Precrural glands; WV Inguinal glands.

confluent with the epididymis, the two organs constitute one tumor. Usually there is simultaneous hydrocele. In a few cases fistulae perforate the skin above the epididymis and discharge yellowish pus. In tuberenlosis of the penis, which is very rare, we find firm nodules at the end of the organ and occasionally induration of the edges of the prepuce (Eber, Trotter). In tuberculosis of the uterus a turbid, mucous or muco-purulent, yellowish, or rather ichorous and ill smelling vaginal discharge is a constant symptom. Occasionally this discharge contains cheesy fragments and, in exceptional cases streaks of blood. Rectal examination may, in some cases reveal the presence of enlarged sacral lymph glands or the horms 
of the uterus which have become transformed into knotty structures the size of a man's arm. The Fallopian tubes may present twisted strands as thick as a finger and occasionally the uterus is transformed into a large firm ball (Hess). Cows thus affected do not conceive but are frequently in heat, although sexual desire vanishes as the disease adrances. Tuberculosis of the vagina and of the vulva, which is very rare, manifests itself in the appearance of small yellowish nodules and erosions (Fischer) or tumors as large as peas and ulcers with raised borders. There may also be observed a purulent discharge, hypersensitiveness and swelling of the lips of the vulva and enlargement of the perineal lymph glands. Hess observed a case of tuberculosis with thickening of the labia of the vulva resembling

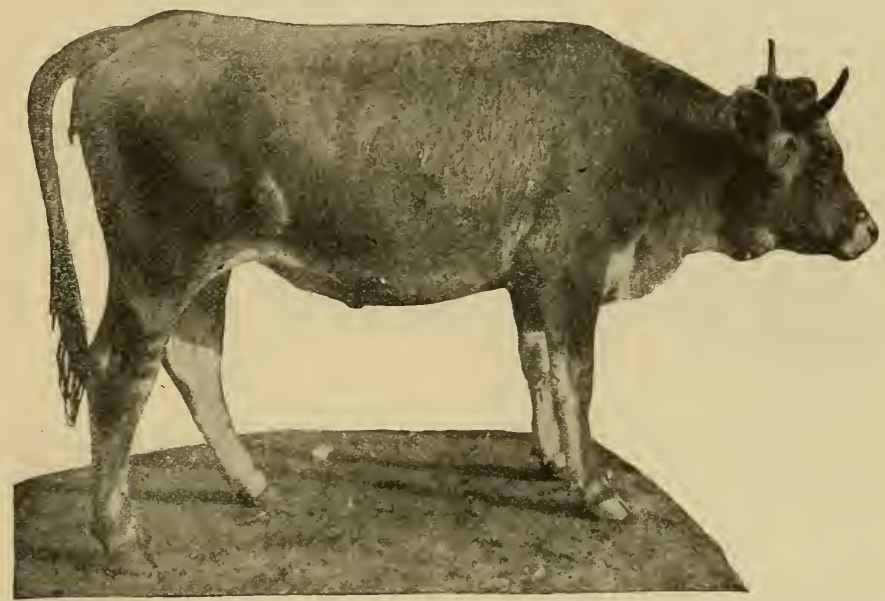

Fig. 86. Tuberculosis of the submaxillary parotid and precrural lymph glands.

elephantiasis. Fischer referred to this form as lupus hypertrophicans of cattle.

Since the superficial lymphatic glands (Fig. 85) in the pharyngeal and cervical regions become primarily infected and the remaining glands become involved in most cases as a result of tuberculosis of neighboring organs the determination of infection of the former glands is of special importance in the diagnosis of this disease. Large tumors, attaining to or exceeding the size of a man's fist are now and then found in one or several of the following locations, viz., intermaxillary space, behind the ascending portion of the inferior maxilla in the subparotid region, on the side of the neck above the jugular furrow, anterior to the scapula, at the inside of the elbow, in the precrural and inguinal regions, in the flank, at the superior border 
of the mammary gland. These tumors are usually only slightly movable, round or oval in form, the very large ones usually nodulated, firm, painless and not adherent to the overlying skin (Fig. 86 and 87). In calves they may be elastic and fluctuating, if incised they evacuate a white creamy pus. In rare cases inflammatory processes develop in and around these tumors causing them to adhere to the overlying skin, rupture and discharge their contents outwards. There then remains a fistula with pale red granulating borders. Very greatly enlarged lymph glands may interrupt the functions of neighboring organs; thus enlargement of the glands of the throat may interfere with deglutition or respiration while that of the glands of the axillary region and the groin occasionally produces lameness.

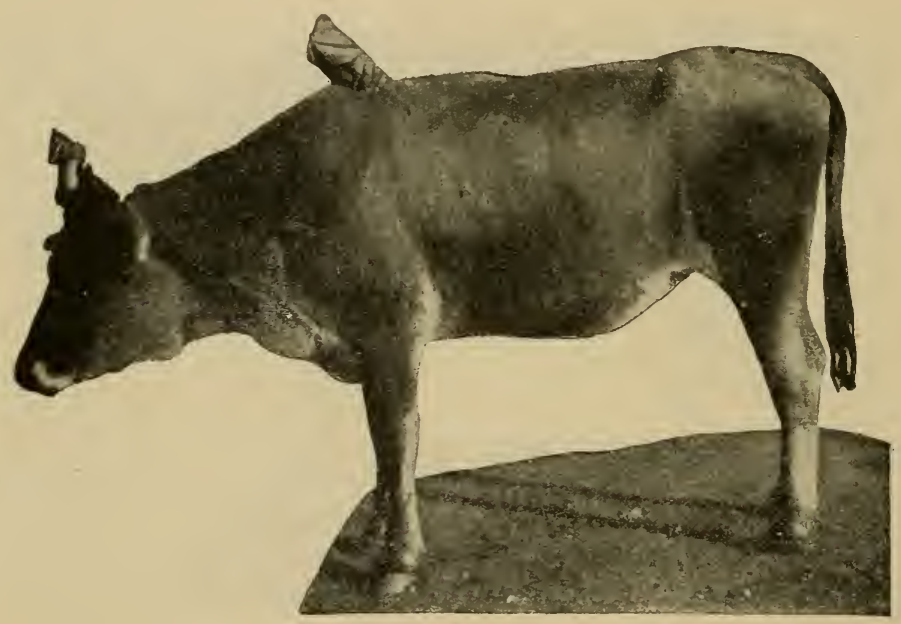

Fig. 87. Tuherculosis of the submaxillary, prescapular and inguinal lymph glands.

Tuberculosis of the udder begins with a rather diffuse painless induration of one or both posterior quarters without local hyperthermia. The process occasionally spreads to the adjacent quarters and gradually develops into an exceedingly hard and nodular tumor which may approach the size of a child's head and cause atrophy of the remaining parts of the gland (Bang, Fig. 88). In other instances we may observe, especially after the udder has been milked dry, several nodules, or larger nodes in the otherwise miformly elastic glandular tissue of one or more quarter's of this organ. These tumors are firm, painless, do not show an increased temperature and may exist singly or as a conglomerate mass with nodular surface, affecting a part of or an entire quarter. This may cause the teats on one side of the udder to assume an irregular direction and 
become parallel or converge instead of diverging (Ostertag, Fig. 90). At the upper border of the udder either on the affected side or on both sides the enlarged lymphatic glands may be located by palpation (Fig. 89). In exceptional cases the supermammary lymph glands may be enlarged and the udder itself present a normal appearance. In these cases, however, it may be assumed that in all probability the udder itself is also infected. In very rare cases the affection begins with increased local temperature and moderately painful swelling: of one quarter but in the course of a short time the above de-

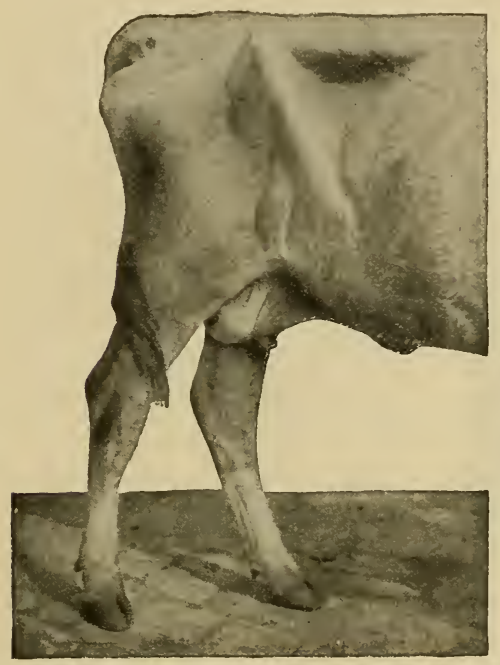

Fig. 88. Tuberculosis of the right hind quarter of the udder with pronounced atrophy of the glandular tissue. Enlargement of the precrural lymph gland.

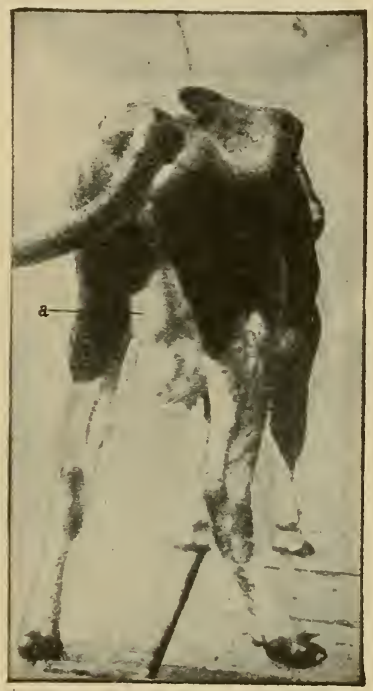

Fig. 89. Tuberculosis of the left hind quarter of the udder with extensive enlargement of the left supermammary gland (a). (Ostertag.)

scribed characteristics appear. In other rare cases chronic conditions will become acute, the diseased quarter becomes more tumefied and painful, there is increased temperature and the neighboring lymph glands show acute enlargement (Lucet).

In regard to milk production, contrary to what occurs in other inflammatory processes of this organ, no interruption occurs for some time in the course of tuberculous disease. As the morbid processes advance, however, the secretion gradually diminishes until it ceases entirely in the affected quarters. In the meantime the milk, which is at first normal in appearance, becomes thin and greenish-yellow in color (Fig. 92). Later, 


\section{white flakes and curdled masses appear and finally the secretion assumes a watery appearance.}

According to Stroh the milk obtained from the tuberculous quarters is frequently alkaline in reaction, contains an increased amount of salts of sodium, chlorine, lime and phosphorus, the fat is diminished and the milk sugar present in only small quantities or absent altogether. The nitrogenous contents consist of paraglobulin and albumin instead of casein and albumin as in normal milk. Monvoisin also found the acidity considerably reluced (from 1.4 to $2.0 \mathrm{~g}$. of lactic acid-normal contents per liter-to $0.80,0.24$ and even $0.12 \mathrm{~g}$.) which is in contrast to ordinary inflammations of the udder where the acid content is usually increaseil on account of decomposition of lactose. The percentage of fat may be relucerl from the normal (being instead of $3.5-4.5 \%$ only $1.5 \%$ ). Milk sugar may be almost entirely absent. The freezing temperature remains about the same.

According to Ostertag the examination of the mammary lymph glands is best accomplisher by grasping with the index, midile and ring fingers the skin of that portion of the lateral faces of the udiler which lie opposite the inner sides of the thighs, beginning at about the middle of the udiler and pushing upward, when

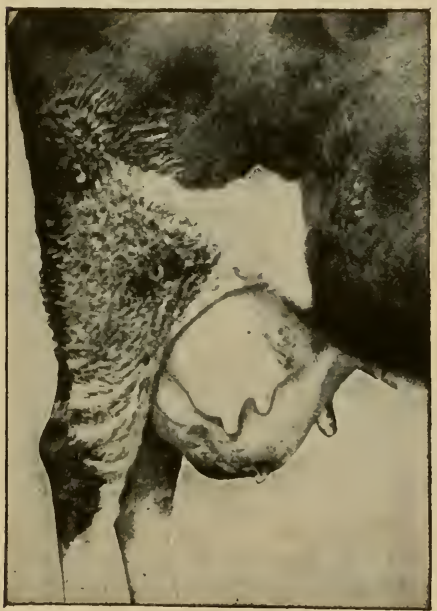

Fig. 90. Tuberculosis of the greatly enlarged left hind quarter of the udder. (Ostertag).

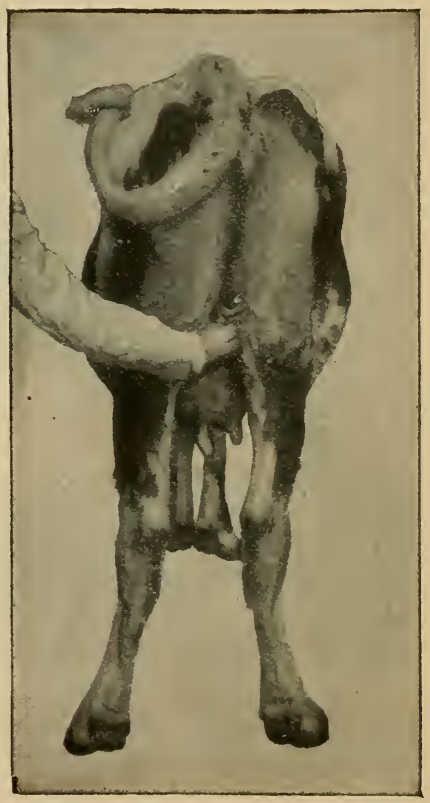

Fig. 91. Palpation of the mammary lymph glands.

the lymph glands may be palpated from without and from below (Fig. 91). These glands lie in the angle which is formed by the upper and posterior border of the udder. As a rule the posterior border of these glands does not project beyond the posterior border of the udder.

To confirm the diagnosis it is advisable to cleanse and disinfect the udder with soap and water, rinse with alcohol and, with the aid of a harpoon, remove a portion of tissne from the affected region for bacteriological examinations (see lp. 571 and 574).

Tuberculosis of the kidneys or more properly speaking, tuberculosis of the pelvis of the kidneys, is indicated by the 
pronounced ammoniacal odor of the urine and the presence of albumin in the turbid urine. Microscopically, pus cells, varionsly formed epithelial cells, irregular detritus, connective tissue fibers and tubercle bacilli (Iro) may be demonstrated.

Tuberculosis of the central nervous system manifests itself in two forms. Solitary tuberculous foci, according to their location and size, either in the brain or in its nerve trunks, produce disturbance of function. The clinical picture is the same or similar to that produced by other tumors and for that reason a specific diagnosis can be made only by the determination of tuberculous disease in other organs.

The symptoms of cerebral affection on the other hand are: fearfulness, unsteady swaying gait, constrained or forced movements, occasional clonic spasms which may involve the whole body in the form of epileptiform attacks, partial or complete paralysis of regions supplied by certain cranial nerves (incoordination of the extrinsic muscles of the eyes [eyes awry] and impaired or total loss of vision, comparatively frequent); slow and frequently interrupted mastication, holding the head to one side, desire to push forward or upward, hemiplegia, arythmic and slow pulse; if the spinal cord is involved, stiffness of the hind quarters and tail, paraparesis or paraplegia. These symptoms not infrequently appear suddenly in animals that were apparently healthy, rapidly become more aggravated and pronounced and terminate in death after one or two days. (In regard to localization of foci, see Vol. II.)

The symptoms of acute inflammation of the cerebral meninges

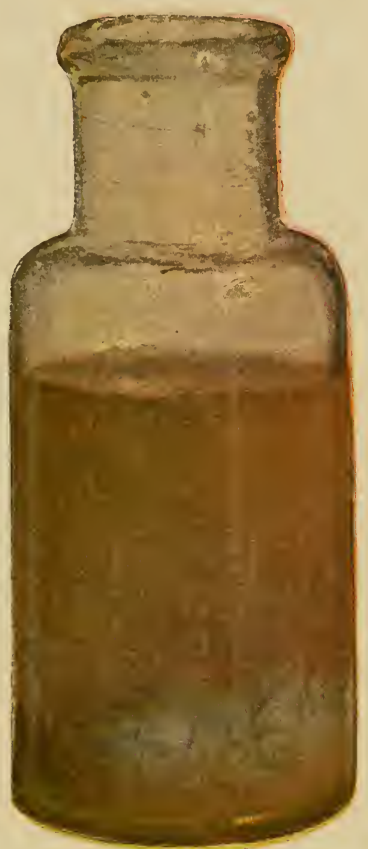

Fig. 92. Milk from a tuberculous quarter of the udder. Turbid, greenish yellow fluid with flaky cheesy sediment. may follow the symptoms of a local affection or they may make their appearance without previous warning The principal symptoms are: increased reflex excitability and fearfulness, great restlessness, delirium or raving madness; unsteady gait, muscular tremors, nystagmus, jerking of certain muscles, opisthotonus or spasms of the entire body; symptoms of paralysis in the region of certain cranial 
nerves, especially incoordination of the extrinsic muscles of the eyes and unequal size of the pupils. Later there may be extreme stupor and finally complete unconsciousness; the animal gets down, is completely helpless and occasionally makes forced involuntary movements with the extremities. In the meantime respiration and heart activity are subject to great variation, not infrequently the Cheyne-Stokes type being observed.

In tuberculosis of the eye, which is very rare, we may observe tubercles up to the size of a hemp-seed in the bulbar conjunctiva (Priewe), in the substance of the iris (Winter), or on the posterior surface of the cornea (Schmidt). 'The development of the affection is attended, either from the begimming or not until a later stage, with turbidity of the cornea and symptoms of an acute iritis. The process usually terminates in panophthalmitis and atrophy of the bulbus.

In localization of the tuhereulous process in the posterior portions of the ocular globe, e. g., the posterior face of the lens, a diffuse iritis and turbidity of the lens may be observed (Spoerer). Winter observed nodules in the sclerotic in the external eanthus of the eye in one case and Lottermoser observed a tumor-like swelling, as large as a hazelnut, in the lower nasal quadrant of the bulbus. The granular structure of this growth could be observed through the overlying yellowish cornea and sclerotic. There were observed, also, minute yellowish nodules in the iris which latter was adhered to the turbid cornea.

Manleitner found the eye affected in five per cent of all cases of generalized tuberculosis. The choroid is most frequently affected $(70 \%)$ although elinical symptoms are usually absent while tuberculosis of the iris begins with symptoms of iritis and leads to the formation of visible nodules.

Of the bones we most frequently find a few ribs involved which become thickened in circumscribed areas, cold abscesses and fistulae developing in the surrounding parts. In disease of the vertebrae symptoms pointing to compression of the spinal cord is observed and usually sensitiveness to pressure of the affected region of the spinal column.

Haug observed stiffness of the hind quarters and tail and contraction of the muscles of the loins and croup in tuberculosis of the first two lumbar vertebrae. Hamoir and Stenstroem observed inability to stand and occasionally loss of sensation in cireumseribed areas of the spinal column, also lameness and genu-flexion. The general condition of the animal often remained good. Wyssmann observed mydriasis in one ease of tuberculosis of the occipital condyles, also opisthotonus, heavy gait and subsequently dullness. In a ease observed by Gueldra an animal with disease of the last two cervical vertebrae could not move its head laterally and in another case tuberculosis of the twelfth dorsal vertebra interfered with mastication and locomotion.

Tuberculosis of the joints, most frequent of the knee, less common of the carpal or tarsal articulations (Guillebeau) manifests itself in subacute inflammation, moderate swelling and disturbance of function of the affected joint. Upon puncture a 
ropy, caseo-purulent exudate is usually discharged. The tendons in the affected region are usually irregularly enlarged and very sensitive. According to Hess the tendons of the M.ext. carpi radialis and of the M.ext.digit.pedis longus are most frequently involved.

In the skin and in the subcutaneous connective tissue there occur, in rare instances, firm tumors the size of a hazelnut containing cheesy or mortar-like masses in one or more locations. Lazace observed a case in which 30 such nodules occurred in the shoulder and arm while the remaining organs of the body were healthy. These nodules may break spontaneously, leaving fistulae which heal only with the aid of surgical treatment and then leave shiny radiating scars (according to Vigadi this affection resembles tuberculosis cutis colliquativa hom. in appearance). In exceptional cases a considerable nodular enlargement of the tail results from tubercular infection (Moule \& Nocard).

Acute miliary tuberculosis runs its course attended with severe febrile and general symptoms, the true cause of which can not usually be recognized except in animals already known or suspected to be tuberculous. The symptoms consist of extreme dullness, rapid emaciation, continuons or remittent but invariably high fever, increased heart activity and weak but rapid pulse also respiratory difficulty without recognizable percussion dullness in the lungs. Animals thus affected usually die in the course of a week. In a few cases symptoms of acute meningitis are observed (incoordination of the extrinsic muscles of the eyes, nystagmus, unequal pupils, opisthotonus).

Tuberculosis of goats and sheep. Thus far only few clinical cases have been observed. Mathis and Leblane observed, in a goat four years of age, emaciation, coarse cough, accelerated and labored breathing, bloody mucous discharge from the nose, bronchial breathing and rales upon auscultation of the lungs, and pericardial friction sounds. Edgar described a similar case. In a goat that became sick one month after parturition, Hess observed loss of appetite, frequent, moist, short and painful cough, abdominal respiration, rapid decrease in flow of milk but normal temperature throughont. In a goat with well developed pulmonary tuberculosis Schroeder observed that the udder was also affected and transformed into a nodulated painless tumor as large as a man's head and as hard as a stone. Nocard saw a similar case.

Tuberculosis in sheep always runs a chronic course and usually manifests itself in the form of a gradually progressing pulmonary affection. As a result of enlargement of the thoracic lymph glands bloating is common. Other superficial lymph glands also become occasionally involved (Mayer).

Tuberculosis of Horses. The first symptom of pulmonary tuberculosis in horses is an occasional dry, or slightly moist,

Vol. $1-36$ 
short, weak cough. Later on the animal is easily exhausted after work, and symptoms of "heaves" make their appearance. In some cases there is slight mucous or muco-purulent nasal discharge which may also be intermittent. The discharge may be streaked with blood or there may be epistaxis. Sometimes it may be very profuse and consist of almost pure pus. In the act of coughing similar secretions and occasionally necrotic particles of limg tissue are discharged from the mouth. Percussion of the thorax usually reveals nothing abnormal and auscultation usually reveals nothing except rough vesicular breathing or, in addition, toward the end of expiration, fine rales. Occasionally areas of dullness or an extensive but subdued tympanitic percussion sound without respiratory bruits or with bronchial bruits may be recognized in the posterior borders of the lungs (Goedecke, Zuern). In rare cases strmptoms may be observed that point to breaking down of lumg tissue and cavity formation. Sometimes a pleuritis with a tendency to latent development accompanies the affection of the lung proper. There are friction sounds and fibrinous exudate which later becomes serous. At a later stage there is loss of appetite and coincident with this a gradual or rapid development of extreme anemia and emaciation.

In tuberculosis of the pharrngeal lymph glands Stiennon observed dysphagia, moist cough, muco-purulent nasal discharge, sensitiveness of the pharyngeal region and rattling expiration.

Tuberculosis of the intestines and mesenteric glands is more frequent, especially in colts, but presents no characteristic symptoms. The affected animals are in a bad state of nutrition, develop poorly, the flanks are drawn in, the abdomen is pendulous or, on the contrary, "tucked up." There may be occasional slight symptoms of colic; defecation is irregular, constipation alternating with diarrhea, the latter finally becoming chronic and leading to exhaustion of the patient. 'The feces, especially when large intestinal ulcers are present, are thin in consistency, yellowish and ill smelling. Rectal examination may enable us to demonstrate the greatly enlarged lvmph glands which appear as tumors the size of a man's fist, firm, nodular and movable. Similar structures may be felt below the spinal column near the kidneys as well as in the liver and the spleen (Zuern), a form of so called tabes mesaraica.

According to Nocard and Goedecke a comparatively conmon symptom, especially of tubereulosis of the abdominal organs, is that of polydypsia and polyuria. The quantity of urine may, for weeks at a time be increased four-fold and then resemble that of the carnivora in having an acid reaction. Uric acid is present in large quantity while hippuric acid is diminisher or disappears entirely. During febrile attacks the urine contains also albumen. 
In a case of tuberculosis of the abdominal organs Portet found the urine to contain $4.5 \%$ of urea and $9.2 \%$ of uric acid. Although the urine contained no albumen its sediment was rich in homogeneous, light-refracting, easts and some epithelial casts. Schindelka also observed polyuria in one case of tuberculosis of the lung, while Knipscheer demonstrated albuminuria in a similar case.

The lymph glands near the surface of the body are occasionally also enlarged. Thus in a case described by Rabe the subparotideal lymph glands formed a mass 3 to $5 \mathrm{~kg}$. in weight, but hardly underwent any changes in the course of a year. In a case observed by Jolme \& Siedamgrotzky and in one by Roebert the prescapular glands were greatly enlarged, in the last case forming a tumor weighing $25 \mathrm{~kg}$. In a case described by Davis the trachea, esophagus, blood vessels and nerves were involved but the lungs were free from tuberculosis! Eberhardt observed foci of thick pus in the enlarged submaxillary lympl glands three years before the death of the animal.

In very exceptional cases tuberculosis of the nasal mucous membrane may he met with, which manifests itself exchsively in the appearance of firm gray nodules with reddenerl borders (Behr) and in size approaching that of a lentil. Or there may be defects in the mucous membrane surrounded with raised borders while nodules as large as small peas are seen in the surrounding area. Eventually the commencement of the morbid process is announced by intermittent epistaxis and enlargement of the intermaxillary glands into painless and not sharply defined tumors as large as a hulled walnut. The overlying skin is not adherent (Gerspach). Again, the nasal mucous membrane may contain only a scar while the firm intermaxillary glands are studded with miliary, or transparently fibrous, or partly calcareous nodules (Joly). The latter glands may also consist of a mass of nodules with cheesy or partly calcareous interior (Nocard).

Tuberculosis of the udder is very rare. Parascandolo \& Meis observed a case in the "dry" udder of a mare. The lesion consisted of a large tumor containing a thick cheesy purulent substance.

Tuberculosis of the skin and subeutis is equally rare (Schindler, Goedecke). Firm, painful tumors approaching the size of an apple appear in numerous regions of the body, the surrounding areas are edematous but the lymph vessels do not become prominent. Some of these tumors may break open and the lesions are then covered with dark brown scabs.

The temperature is from time to time elevated from $2^{\circ}$ to 3 F. Near the termination of the disease it ranges between $103^{\circ}$ and $105^{\circ}$ F. (Siedamgrotzky). Schindelka and also Batt observed morning exacerbations and evening remissions with comparatively slow pulse. Tabusso observed chills and profuse sweating during the exacerbations.

Coincident with tuberculosis of the abdominal organs the development of miliary tuberculosis of the lungs is frequently 
observed. This is characterized by high fever, excessive dyspnea and by a rapidly fatal course. The lungs give a tympanitic percussion sound and vesicular bruits upon auseultation (Goeclecke).

Tuberculosis of Swine. During life the disease can be definitely recognized only when the lymph glands are involved (scrophulosis). In pronounced cases the submaxillary, pharyngeal and cervical glands are excessively enlarged and, throngh confluence, constitute firm nodular tumors as large as a fist. They are very sensitive to pressure and more or less adherent to the surrounding tissues. This process is attended with the development of a diffuse swelling in the parotid region which interferes with the free movement of the head and lower jaw. These tumors may extend along either side of the neck in rosary fashion and additional tumors may be present in the prepectoral region. Swelling of the lymph glands of other regions of the body, especially those of the flexor surfaces of the extremities may also be present. Some of these tumors may become fluctuating, break open and discharge a thick purulent or cheesy mass. This results in the production of a fistula which discharges cheesy-purulent secretions for a long time. Spontaneous healing does not take place.

Tuberculosis of the abdominal organs, especially as a primary affection in young pigs manifests itself in slowly developing but steadily increasing impairment of digestion. The affected animals gradually lose their normal vigor and liveliness, their tails lose the curl, the back is hmmped, they stand around listlessly or bury themselves under the bedding for hours at a time. The appetite is at first impaired, the animals selecting only choice morsels and later refusing all food. Constipation and diarrhea alternate and during the latter ill smelling semifluid feces mixed with crumbly material are discharged. Now and then there is bloating caused by the accumulation of gases in the intestines. Later on the flanks become hollow, the abdomen becomes pendulons and the lower portion increased in its transrerse diameter. If the animals are handled they evince signs of pain, the enlarged scrophulous lymph glands, and the loops of intestines which have become adherent by inflammatory process may occasionally be felt as firm irregular swellings. The animals become more and more emaciated, the mucous membranes become pale and the eyes are drawn into their sockets. Here and there the skin is covered with brown crusts. The weakness constantly increases until exhaustion leads to death after several months of illness.

In tuberculosis of the lungs there is at first a dry, short, suppressed congh as well as moderately accelerated and difficult breathing. In the course of time the cough becomes more frequent and distressing and is sometimes attended with vomiting. In the further course of the disease the respiratory difficulties become excessive, the animals sit on their haunches and, with 
their forelegs spread out and extended breathe spasmodically with the aid of the accessory muscles of respiration. The nutrition suffers from the beginning except in a few cases where the disease takes a chronic course. The animals emaciate rapidly, the mucous membranes become pale, and death results from exhaustion after a few months or sometimes in three or four weeks.

Solitary tubercles in the brain produce, at first, clonic spasms and forced involuntary movements that may be limited to certain groups of muscles or affect the whole body. Later there may he paralysis of certain cranial nerves, or hemiplegia. These symptoms are particularly frequent in young pigs. Vogt observed paraplegia and prolapsus of the rectum in tuberculosis of the fourth lumbar vertebra as a result of pressure on the spinal cord.

Tuberculosis of the eye manifests itself in turbidity of the cornea and accumulation of a plastic exurlate in the anterior chamber with simultaneous affection of the posterior regions of the ocular globe. Keil saw both eyes of a pig thus affected. Tubercles in the background of the eye may also be recognized with the ophthalmoscope (Azary).

The bones and joints are particularly liable to become affected in general tuberculosis. In addition to individual ribs and vertebrae the articular surfaces of the bones of the extremities may become involved. The corresponding joints swell excessively and impede locomotion. Now and then the process extends to the skin from which point the accumulated cheesypurulent masses are discharged. Tubereulosis of the petrous portion of the temporal bone causes vertigo and disturbanee of bodily equilibrium. Occasionally granulating growths are observed in the external auditory canal when the latter affection exists.

Acute miliary tuberculosis is, as in other species, characterized by high fever, dyspnea, diarrhea, rapid emaciation and death after a short time.

Tuberculosis of Dogs. The clinical picture of this disease in dogs has heen described most minutely by Cadiot and by Froehner. The first symptoms, though usually not very conspicuous, are as follows: Capricious appetite, slight emaciation, exhaustion on running, difficult breathing, general weakness, etc. In some cases the clinical evidence of the disease is for a long time limited to the aforementioned symptoms which are little characteristic. In the majority of cases, however, symptoms of tuberculosis of the lungs and abdominal organs make their appearance.

Pulmonary tuberculosis begins with a short dry cough which may, in some animals lead to vomiting. Later on the cough becomes more frequent, the patients discharge mucopurulent secretions, there may be an occasional bloody discharge from the nose and respiration becomes more and more 
labored ( 25 to 40 respirations per minute). Dry or moist rales may be heard on auscultation, and percussion gives in spots a dull or empty sound. In these areas the respiratory sounds are either absent or they are bronchial in character. In rare cases there may be indications of cavities (tympanitic or amphoric sounds, succussion and amphoric respiration). Sensitiveness of the thoracic wall, or continuous areas of dullness which may extend over the entire field of percussion, together with the absence of respiratory sounds may, in frequent instances point to the existence of a pleuritis. If this occurs on the left side, the beats of the heart, which organ has been lisplaced to the right, may be felt on the right side. In rare cases there may be symptoms of pneumothorax but these are always attended with accumulation of large quantities of serous exudate (dullness and muffled heart sounds). In the advanced stage the greatly exhausted and emaciated animals are tortured by a frequent spasmodic cough. The secretions discharged on coughing are purulent, gray or greenish in color and, if cavities are present, have a bad odor. Not infrequently there is a nasal discharge which has similar characteristics. "The exhaled air, also, has a disagreeable odor.

Tuberculosis of the pericardium occurs in the course of the disease in the lungs or the pleurae or independently of the involvement of any other organ. In either case it may lead to the accumulation of large quantities of serous exulate and manifest itself by the usual symptoms of an exudative pericarditis. The nature of the affection may therefore be recognized or suspected only by the insidiousness of its course or by microscopical examination of aspirated pericardial fluid. If the thoracic glands are much enlarged the heart sounds may in exceptional cases become less distinct, the heart may be dislocated upward and backward, and there may be strmptoms of compression of the esophagus (Marek).

Tuberculosis of the abdominal organs is indicated in a general way by impaired nutrition as a result of which the affected animals become emaciated and anemic. If the lrmph glands are much enlarged they may be felt through the abdominal walls. The enlarged liver may be recognizert in the same mamer, also by the increased percussion dullness or by the nodular border which may be felt behind the costal arch and the xyphoid appendage.

In the course of the disease the temperature is periodically elevated $\left(1^{\circ}\right.$ to $2^{\circ} \mathrm{F}$. or more). Toward its termination the pulse becomes soft and rapid, the heart beats throhhing. Hydrothorax and ascites are comparatively frequent. The appetite is diminished, thirst increased and the mucous memhrane of the mouth is drv. Constipation alternates with persistent diarrhea during which the intestinal evacuations contain much mucus and occasionally also blood. Finally, after becoming extremely emaciated the animals die, the temperature finally falling very low. 
In one ease Mueller observed, on the side of the neck, a deep ulcer with a sinuous border and torpid granulating base. The neighboring lymph glauds were enlarged. Cartiot observed a fistula of the withers and chronic arthritis of tuberculous origin.

Acute miliary tuberculosis rums a rapid course attended with symptoms of an acute infectious disease (Petit). According to Cadiot tuberculosis in dog's may also assume a septicemic character.

Tuberculosis of Cats. The clinical appearance of this disease is as yet not well known. Since the pathologico-anatomical changes are very much alike, the clinical symptoms are probably similar also.

Thus, Lellmamn observed the following in two angora cats: Excessive emaciation and anemia, anemic heart brnits, labored breathing and accelerated, weak pulse; in one case the abdominal cavity contained a milky fluid with tubercle bacilli, there was also pronounced lencocytosis; in the other case there was dullness upon percussion on both sides of the chest and bronchial hreathing; a tumor was present in the abdominal cavity, under the spinal column. Hobday \& Belcher, in a case of general tuberculosis, found the subparotideal, the submaxillary and the prescapular lymplı glands enlarged and purulent. Finally, Petit \& Coquot described a case of tubereulosis of the skin on the face in which the bridge of the nose, one cheek and the corresponding lower eyelid were transformed into one ulcerating surface.

Course. Tubereulosis always has an insidious beginning, develops in a chronic manner and usually preserves its chronic character until its fatal termination. The development of the disease is usually so slow that years may elapse before morbid symptoms point to its presence and when observed in mature animals it is usually found to have had its beginning in early youth. The tuberculous process, in its slow and chronic course, usually does not exert a harmful influence on the general condition of the animal until after the lapse of considerable time, which explains that animals in well nourisher, or prime condition and in apparently perfect health may frequently exhibit extensive tuberculous changes when slanghtered. This is particularly true, in a general war, with regard to tuberculosis of cattle and especially in cases of pearl disease of the serous membranes. In many cases this form of tuberculosis, even when far advanced, does not in the least affect the general condition of the animals. In other animals, especially in young animals, the disease usually runs a less insidious course. In young pigs that are infected by milk from tuberculous cows the course of the disease may be so rapid that the progressive changes may be noted from week to week.

In the course of this naturally chronic affection, especially in its later stages, acute exacerbations occur from time to time, 
during which previously healthy areas become involved by the process. Bacillary emboli give rise to the development of new tuberculous foci in remote organs or an infection with pyogenic organisms complicates the morbid process. The chronic course of the disease may be accelerated by influences that exert a weakening action on the body like catching cold, railroad transportation, overwork, etc. Intercurrent acute disease and parturition may have the same effect (Froehner observed a chronic case of tuberculosis of the horse become acute after an attack of pleuro-pneumonia and Guenther the same in a cow after abortion). Exacerbations of this sort are announced by elevation of temperature, symptoms of general indisposition and occasionally more frequent coughing. After a few weeks former conditions again prevail. In the advanced stage of the disease these temporary improvements become less and less frequent. Again, in other cases, especially in young animals and after an intensive injection of virus, the disease may become steadily progressive and lead to complete exhaustion.

Following the entrance of numerous bacilli into the blood stream the simultaneous development of numerons tubercles or rapid increase of virus in the blood may lead to a fatal termination within a few weeks. Symptoms of acute miliary tuberculosis, which is an acute febrile disease, attend this condition.

Tuberculosis of the cerebral meninges also runs an acute course, while solitary tubercles developing in the substance of the brain may remain unobserved for a long time or only cause functional disturbances of the nerves, especially spasms and paralysis, until they finally reach the meninges and there set up acute inflammation.

In large herds the disease usually spreads very gradually. Since the development of the disease in a single animal may extend over a course of years, large herds may, in spite of infection, be kept up for years or even decades. The prompt removal of affected animals which show physical indications of the disease and their replacement by younger stock may retard a rapid deterioration of the apparently fair general condition of the herd. However, in herds of this sort, especially if they are dairy herds, the disease will continne to spread irresistibly. In the course of time the age limit at which the first clinical symptoms of disease make their appearance is gradually reduced and accordingly more and younger animals must be ranged out every year. Finally the malady will make its appearance as a clinically observable disease in quite young animals, viz., in the form of tuberculosis of the lymph glands, of the lungs and of the bones.

Diagnosis. Even if we exclude the early stages of tuberculosis in which no clinical symptoms whatever are observable it is only rarely possible even in advanced stages of the disease 
to recognize it with absolute positiveness. The symptoms simply point, in a general way, to affection of certain organs, but hardly enable us to form an opinion as to the nature of the disturbance. Although the diagnosis is often suggested by a suspicion of an infection, this of itself is not sufficient to justify the positive diagnosis of tuberculosis. Symptoms pointing to the existence of tuberculosis are as follows: Irisidious development of the disease, gradual running down in condition, stunted growth in young animals, periodical or occasional attacks of fever without apparent cause and a gradually progressive affection of the lungs. Great importance should be attributed to enlargement of the lymphatic glands. While the latter may occur as an independent affection it is usually an indication of tuberculosis of the corresponding organs. On the other hand enlarged lymph glands may, by exerting pressure on neighboring organs, produce disturbances of function (bloating, venous pulse, lameness) that would justify the conclusion that deeper lying organs are affected.

In the great majority of cases, however, an exact diagnosis is possible only by the aid of special diagnostic methods, among which microscopical examination of morbid tissues and secretions, inoculation of experimental animals and the tuberculin test are of practical importance.

Post-mortem examination of slaughtered animals, or of animals that died from the effects of the disease is usually the easiest method of determining the tuberculous nature of existing lesions, but even in such cases a microscopical examination is almost indispensable to enable us to differentiate with certainty between tuberculosis and other morbid conditions which occasionally show a remarkable similarity (actinomycosis, pseudo-tuberculosis).

Microscopic Determination of Tubercle Bacilli. The determination of true tubercle bacilli in a tissue or secretion absolutely clinches the diagnosis of tubereulosis. Negative results of an examination for tubercle bacilli do not, on the other hand, justify the exclusion of the disease because tuberculous lesions, especially cheesy foci, may contain very few tubercle bacilli. If tubercle bacilli are present in large numbers, however, they are comparatively easy to demonstrate. But in making a diagnosis it should always be kept in mind that there are other bacteria with morphological and staining characteristies (acid-fast hacteria) that give them a remarkable similarity to tubercle bacilli (see p. 571). However, these acid-fast bacteria, except in paratuberculous chronic enteritis of eattle, are not known to be pathogenic. They oceur merely as harmless saprophytes which have been earried from the outside into exposed eavities and canals and their secretions. It is only exceptionally that they are found in pathological tissues and thus it is comparatively safe to regard all acid-fast bacilli that are found in tissues and secretions not communicating with the outer world (unopened lymph glands, deep cheesy foci, tissue of the udder) as tuherele bacilli while similar findings under opposite conditions ean 
not be looked upon as conclusive evidence. Strictly speaking, this is also true in the examination of milk because paratubercle bacilli may gain entrance into the lactiferous ducts through the excretory canals (De Joug). Nevertheless this is only of rare occurrence. (Ostertag, in his extensive investigations along these lines never found acid-fast bacilli in freshly drawn milk, while Bang failed only 26 times in 2,174 cases in which tuberculosis was diagnosed on the basis of an examination of the milk.) Accordingly, the demonstration of acid-fast bacilli in milk drawn after the first fow streams from the udder have been discarded may, though not with absolute certainty, be looked upon as in all probability indicating the presence of tuberenlosis. In regard to other secretions, especially those of the bronchial tubes, the possibilities of contamination are much greater and consequently greater caution must be exereised in making a diagnosis on the strength of a bacteriological examination. But even in these cases the demonstration of acid-fast bacilli, slender in form and irregularly stained, point with great probability to the presence of tuberculosis while, on the other hand, the occurrence of these forms in the vaginal secretions is of less definite value and their occurrence in the feces has slight significance only.

In the living animal a resected subeutaneous lymph gland or other nodule, the purposely remored contents of an abscess, aspirated exuctate from the thoracic earity or from the joints, suspected tissue from the udder removed with a harpoon or nilk drawn under aseptic precautions are materials best suited for examination. Ejecta from the lungs, nasal discharge, pus from an already open abscess or joint, secretions from uleers, urine, spermatic fluid and raginal secretions may also be examined. Since animals seldom discharge bronchial secretions directly into the onter world (although expelled particles are now and then to be found adhering to the wall immediately in front of the animal's head, in the stall) these must be obtained by specially derised artificial methods. Holiling a cloth over the mouth and nose and then forcing a congh impulse by compression of the nostrils will enable one to obtain particles of mueus which lodge on the cloth (Leeuwen). The same results may he obtained by foreing the animal to congh against a board wall or a linen cloth held immediately in front of it. After the animal is through coughing, masses of mueus are usually lodged between the tongue and molar teeth where they can be removed by hand. The mueus which remains lodged on the pharyngeal membrane may be removed hy means of a small wad of cotton or a sponge attached to the end of a flexible rod (Nocard, Greffier, MacFadyean). According to Ostertag the use of a small spoon with handle about $45 \mathrm{~cm}$. long is more practical. Previous injections of pilocarpine have no value, because they simply increase the quantity of secretions, the purulent admixture containing relatively fewer tubercle hacilli. Hasenkamp constructed a special instrument for this purpose ("Lungenschleimfaenger" pulmonary muens collector) consisting of an elastic rod with a pear-shaped cup attached to one end. The cup end is inserted into the esophagus, the animal forced to cough and, after swallowing, the "mucus collector" is withdrawn, filled with mucns! Poels recommends tracheotomy at the lower end of the trachea and the removal of secretion from the posterior tracheal wall with a cotton swab. Neuhaus uses Dieckerhoff's venous trocar, inserting the same into the trachea and then, by means of small chicken feathers passed through the canula, causes the animal to cough, whereupon the expelled mucus is caught up by the feathers.

Forcing a cough impulse while the tongue is mechanically pulled part way out of the mouth prevents the animal from swallowing and thus permits the collection of expelled masses of mucus; bending the head forward and downward while the animal is coughing may enable one to collect the discharged saliva on a plate and then examine the particles of mucus which it may contain (Klauwers).

Microseopic examination of discharges from the lungs does not always give positive results even in open pulmonary tubereulosis Negative findings, therefore, do not necessarily exclude the presence of tuhereulosis. Among 77 cases attended with bronchial rales, Ostertag found tubercle hacilli in only 27. In 11 cases he found bacteria resembling tuherele bacilli in appearance and in 11 other eases short thick acid-fast rods were present while the examination gave negative results in 29 instances. He succeeded only once in demonstrating tubercle bacilli in open tulierculosis withont rales. 
Milk must always be drawn under aseptic precantions (p. 558) and allowed to settle in a conical vessel (protected from exposure) for 24 hours, or centrifuged and the sediment examined.

Methods of Staining. The acid.fast tuberele bacilli may be demonstrated by various means which are all based on staining coverglass preparations, fixed in the usual manner, with anilin dyes, the staining properties of which liave been intensified by the addition of a mordant and then treating with a mineral acid or with alcohol. The methods in common use are as follows:

1. Method of Ziehl \& Nelsen. Stainiug solution: $1.0 \mathrm{gm}$. fuchsin, $10.0 \mathrm{gm}$. absolute alcohol, 90.0 gm. $5 \%$ carbolic aciıl solution; stain a few minutes uniler the action of heat; decolorize a few seconils in $5 \%$ sulphuric acis, wash in alcohol and re-stain in aqueous methylene blue solution. (The prepared staining solutions keep well.)

2. Galbet's Method. Stain in hot carbol-fuchsin as above then place for a few seconds in mixture of 1 to 2 parts of methylene blue and 100 parts of $5 \%$ sulphuric acid to decolorize and double stain; rinse thoroughly in water. After staining and drying the stained coverglasses are mounter in Canada balsam and examined under a power of 500 to 600 diameters, preferably with an oil immersion objective.

Material supposed to contain only small numbers of bacteria may be treated with antiformin hefore staining, as follows: Mix 20 to 30 parts of the material to he examined with 15 parts antiformin and 55 to 65 parts of water, shake and let.stand for 1 to 2 hours. The antiformin will dissolve tissue cells and all nonacid-fast bacteria but will not affect tubercle hacilli or other acir-fast lacteria which accumulate in the sediment of the vessel (Uhlenhut and Kersten).

Much's non-acid-fast granular rods and granules may be stained by Gram 's method, but this requires 24 to 49 hours at hoily temperature. The Herman-Caan method is more rapid, viz. Staining fluid: 3 parts of a $1 \%$ ammonium carbonate solution in distilles water and one part of a $3 \%$ crystal violet solution in $96 \%$ alcohol; stain with the aid of heat; decolorize with $10 \%$ nitric acid, then with $96 \%$ alcohol.

Spengler and Betegh recommend special staining methors for the examination of sputa by means of which, it is claimed, human and bovine types may be differentiated. These methods undoubtedly produce beautiful pictures but whether they are of value for the purpose indicated, or in determining the spore characteristic of the granules which they bring to view, is as yet a question.

Acid-fast Paratubercle Bacilli. The property of the tubercle bacillus which enables it to retain stains with which its tissues have once become impregnated, even after treatment with mineral acids, is not an exclnsive characteristic of this bacillns. Soon after the discovery of the tubercle bacillus it was found that, aside from the human leprabacillus, the smegma of the genital organs and the cerumen of the external auditory canal contained saprophitic bacilli that had the property of retaining stains much like the tuberele bacillus, though not as tenaciously. Spina, as early as 1883 , demonstrated acid-fast bacilli in decomposing blood, in sewage and in the sputum of healthy human beings. This property of the tubercle hacillus, however, was until very recently regarded as a distinct characteristic in the examination of pathological secretions until Petri (1896) and later Rabinowitsch found bacilli in butter and milk that resembled true tubercle bacilli. There is no doubt that these forms had previously frequently been mistaken for true tubercle bacilli. Since that time hacteria with similar charaeteristics have been discovered in raw animal products, food stuffs and in human and animal secretions and excrements. The differentiation of these various forms from true tubercle bacilli becomes the more difficult since some of them produce lesions in experiment animals that are very similar to those of tuberculosis.

The bacteria of this class are without exception saprophytes that may oceur in soil, water and dust, decomposing substances, food stuffs, manure, etc., occasionally gaining access to raw animal products, particularly milk, butter and oleomargarine. Occasionally they are also found in the bronchial secretions, intestinal evacuations and urine of 
man and animals and, in exceptional cases, in pathological tissues, e. g., pearly nodules of tubereulosis.

Of the acid-fast bacilli now known the following may be mentioned: The Petri-Rabinowitsch butter bacillus which was found in $52.9 \%$ of butter samples and in $6.3 \%$ of milk samples by Petri, in Berlin, and by Rabinowitsch in $33 \%$ of butter samples in Berlin and in $26 \%$ of butter samples in Philadelphia; Korn's bacillus friburgensis, once found in butter; Moeller's milk bacillus, found once in a culture made from butter; Moeller's Bacterium Phlei, found by the discoverer on timothy grass and later also on other grasses; Moeller's grass bacillus and dung bacillus found in the dust of dwellings and in manure. Later on the dung bacillus was also found in the excrement of cattle, horses, mules and swine;-Moeller's pearl disease bacillus, which Moeller cultivated from pearl growths of cattle and swine;-an acid-fast bacillus which Preisz found in the nasal secretion of an ox suspected of having pearl disease;-Binot's butter bacillus;-Beck's Bac. tuberculoides I, from milk and Bac. tuberculoides II cultivated from the tonsils of a tuberculous woman, etc.-In addition to these, acid-fast bacilli were demonstrated: By Cowie in the smegma of the horse, ox and dog as well as in the epithelium of the udder of the cow; by DeJong in the milk of cows with udder tuberculosis and by others in various substances, comparatively frequently in sputum of human beings with pulmonary tuberculosis or pulmonary gangrene.

Acid-fast bacilli have certain common characteristics which make it possible to differentiate them from tubercle bacilli. According to the comparative investigations of Aujeszky these characteristics are as follows: Morphologically the paratubercle bacilli in general resemble the tubercle bacillus and like the latter they constitute transition forms leading to the streptothrix species. The great majority is less acid-fast than the tubercle bacillus and they are also easily stained by the simplo anilin dyes; some of them are acid-fast only and not alcohol-fast, others show the same tinctorial characteristics as the tubercle bacillus; in cultures on artificial media, in contrast to the tubercle bacillus, they grow rapidly and, with few exceptions, grow luxuriantly also at room temperature; their cultures are moist and shiny and have a hrighter color than tubercle bacillus cultures, often a yellowish-red color; on the surface of bouillon a massive membrane forms within a few days and they frequently emit a pungent odor suggesting decaying vegetation or ammonia.

Most paratubercle hacilli exert a slight pathogenic action on experiment animals. This action, however, usually does not manifest itself unless the cultures are administered in conjunction with some "enveloping" substance like sterilized butter or oil. If thus administered intraperitoneally they usually produce an exudative peritonitis which often leads to massive accumulation of deposits and to adhesions; in addition to this, pseudo-tubercles make their appearance which differ from true tubercles in that they do not become caseous, or only rarely so, but have more a tendency to become purulent or become organized; in exceptional cases only do they contain cells of Langhans; finally-and this is perhaps the most important difference-inoculation with these forms produces only local inflammatory processes which do not progress from the primary foci. (The bacillus of chronic paratuberculous enteritis of cattle seems to occupy a unique position in this respect which is true also of its cultures.)

In spite of these important differences between these bacteria and 
the true tubercle hacilli there exist certain points of close relationship which find expression in peculiarities referred to as group reactions. These are, aside from morphological resemblances, as follows: The formation of tuberculin-like products in cultures as well as their mutual agglutinating properties when treated with sera from animals that have been treated with tubercle bacilli or other acid-fast bacilli; also their property of immunizing animals against the effects of true tubercle bacilli, though this is true only in a slight degree. These and many others of the above mentioned common characteristics are doubtless indications of a phylogenetic relationship and hence arises the possibility that tubercle bacilli represent acid-fast saprophytes that have become pathogenic in the course of time, or that the latter are tubercle bacilli that have been attenuated through the influence of external agencies.

In view of the fact that the term pseudo-tuberculosis has already been applied to certain other morbid processes it is suggested that the acid-fast bacilli under discussion be referred to as acid-fast paratubercle bacilli.

Diagnostic Animal Inoculation. These often give a positive result when the presence of bacilli in material to be examined can not be demonstrated with the microscope on account of their small numbers. The demonstration of the infectiousness of milk, in particular, is frequently possible in no other way. In other cases diagnostic inoculations of animals are necessary when a microscopical examination is by itself insufficient to determine beyond question that the suspected tubercle bacilli are genuine.

With a diagnostic inoculation as a basis the diagnosis of tuberculosis is justifiable only when true tubercles (in a histologic sense) have been demonstrated in the inoculated animal or when tubereulous processes have spread from the point of infection to a neighboring lymph gland and to internal organs, liver, spleen, lungs (see above: Pathogenic action of acid-fast paratubercle bacilli).

The guinea pig is, above all, adapted for this purpose. It is so extremely susceptible to tuberculosis that negative results from the inoculation of suspected material may, in practice, be looked upon as positive evidence that the material was free from tubercle bacilli. This does not, of course, exclude the possibility that material taken from the same animal at another time may contain the bacilli.

If the probability of contamination is exeluder (specially drawn milk, pus from a closed abscess, tissue juices from internal lymph glands) the material may be injected into the peritoneal cavity without further preparation, or after being merely diluted with water. However, if contamination is probable, or suspected (nasal discharge, bronchial or ulcer secretions), it is advisable to inject a small quantity subcutaneously in the abdomen or on the inner surface of the thigh or into the muscles of the latter. In such cases the foreign bacteria will cause a-local inflammation resulting in an abscess which will break and discharge its contents to the exterior and if tubercle bacilli were also present the characteristic pathological changes will develop as usual. (According to Ostertag the intramuscular method is just as reliable as the intraperitoneal but preferable to the latter because results are obtained quicker. Laurier recommends intramammary inoculation.)

In from 15 to 20 days after the intraperitoneal injection of virulent material the peritoneum, spleen, liver and lymph glands contain tubercles and the liver which assumes a mottled appearance undergoes fatty degeneration. After subcutaneous inoculation an ulcer, which has little or no tendency to heal, develops at the point of inoculation; simultaneously with the development of the ulcer the neighboring lymph glands become enlarged, and later on the process spreads to the internal organs, especially the peritoneum, liver and spleen, and in these numerous tubercles may be found from the 25 th to the 30 th day after inoculation. After intramuscular inoculations the neighboring lymph glands may be recognized (frequently as early 
as the tenth day) as hard, firm, painless, listinctly circumscribed tumors the size of small peas or larger (Ostertag). Later on the internal organs are also attacked. The enlarged lymph glands may be resected and examined under the microscope, or the inoculated animals which in positive cases gradually emaciate and whose fur becomes rough, may be killed after three or four weeks for post-mortem examination. After intramammary inoculation tubercles appear in the mammary glands as early as the fifth day and the milk contains numerous tuberele bacilli.

Allergic Tuberculin Reactions. Tuberculous infection produces certain changes in the animal body as a result of which it reacts to a second infection or to the injection of toxins of the tubercle bacillus in a different manner than the body of an animal that has not yet been infected. This condition, referred to by Pirquet as allergia, i. e., altered power of reaction, manifests itself in increased resistance toward the virus in question (see pp. 530-535) and in hypersensitiveness to its toxins (a kind of anaphylaxis). This latter manifests itself in more or less pronounced symptoms of inflammatory reaction following the administration of very small quantities of the toxins which would have no effect on healthy animals.

This hypersensitiveness is particularly evident with respect to the tuberculin which Koch prepared from glycerin bouillon cultures and which was recommended as a curative agent in tuberculosis. Expectations along this line have, however, been realized only to a limited degree but later experiences and observations have confirmed Koch's declaration that, on account of its specific action on a tuberculous animal, tuberculin is admirably adapted as a diagnostic agent. For years the general febrile reaction which usually follows the subcutaneous administration of tuberculin was exclusively depended upon for this purpose. Recently however the local ophthalmic and cutaneous reactions also have attained great diagnostic importance.

Tuberculin and Its Preparation. Koch prepared tubereulin by growing tubercle bacilli at $37-38^{\circ} \mathrm{C}$. for eight weeks in real bouillon containing $1 \%$ pepton and $5 \%$ glycerin. The culture was then eraporated on a water bath to one tenth of its original volume, sterilized at $100^{\circ} \mathrm{C}$. and then passed through a clay filter. Crude tuberculin thus produced is a clear brownish-yellow oily fluid which, in its concentrated form, will keep unchanged for months but loses its activity in dilute solution (according to Jansen light and heat does not affect the activity of the concentrated tubereulin). By repeated treatment of the solution with $60 \%$ alcohol a white powder may be obtained, the so called purified tuberculin, of which 0.1 $\mathrm{gm}$. produces the same effect as $0.5 \mathrm{gm}$. of crude tuberculin. This powder is readily soluble in water but aqueous solutions are not stable. In solution with $50 \%$ glycerin and water it keeps a long time and is not destroyed even at a temperature of $160^{\circ} \mathrm{C}$. (Brieger and Proskauer).

Tuberculin adapted for diagnostic purposes may be prepared in other ways also. Thus Helman prepared it from a glycerin and water extract of cultures grown on potatoes treated with alkaline serum-glycerin (this has only one-fourth the strength of Koch's tuberculin but a similar action). Bujwid makes an extract of glycerin agar cultures by repeated treatment with water, sterilizes the finid at $100^{\circ} \mathrm{C}$., filters through clay and then concentrates on water bath (action identical with Koch's tuberculin). Nalm prepares tuberculin by the use of an artificial culture medium that is free from albumen (the tuberculin thus prepared nevertheless contains an albumose-like substance).

In actual practice the tuberculin prepared according to the original method of Koch is generally used. Tuberculin prepared from human tubercle bacilli is effective in animals as well as such that is prepared from cultures of the bovine bacilli. Kanda found howerer that tuberculin prepared from borine cultures acts 
more promptly and more energetically than that prepared from bacilli of the human type. But the experiments of Weber and Dieterlin on cattle and guinea pigs, agreeing with the assertions of Koch, DeJong, Wolbach and Ernst, showed that there was no difference in the two kinds of tuberculin, provided that both have the same titre. However, since this is not ascertained in all preparations it is adrisable, in order to be safe, especially with ruminants and swine, to use tuberculin exclusively that has been prepared from bovine tubercle bacilli. Tuberculin may also be prepared from avian tubercle bacilli but this has a considerably weaker action (Borrel).

Later Koch made other tuberculin preparations from aqueous extracts of dried tubercle bacilli or from dried bacilli finely ground in an agate mortar. The whitish opalescent fluid obtained by centrifuging the first named extract represented the preparation known as "T. O.;" the fluid obtained by repeatedly mixing the residue with water and centrifuging produced the tubereulin known as "T. R." The latter contains only substances contained in the bodies of the bacilli, and none of their products of metabolism. Koch ascribes to it a special immunizing action, but since it produces no reaction in tuberculous animals it has no value for diagnostic purposes.

The tuberculol of Landmann is prepared by extracting the fat from tubercle bacilli, making further extracts by heating at various temperatures in physiological salt solution and then concentrating the extracts in a vacuum. This preparation is more toxic than other tuberculins.

Finally there are also the simple eulture filtrates of Denys and Detre which have however not as yet been used in veterinary medicine.

Standardization of Tuberculin. According to Koch 0.5 ec. of tuberculin injected subcutaneously into a guinea pig that was infected four weeks before nust be sufficiently active to kill the animal within 30 hours, producing intense inflammation at the periphery of the tuberculous foci. Von Behring indicates the unit of toxicity of tuberculin by the minimum quantity ( $\mathrm{L}+=$ Limeswerth) which will kill the test animals in 24 to 30 hours. The toxicity of the Marburg tuberculin is sufficient for $0.2 \mathrm{gm}$. to kill a guinea pig weighing $400 \mathrm{gm}$.

\section{Subcutaneous Tuberculin Test (Thermal Tuberculin} Reaction). The characteristic thermal reaction for tuberculosis consists in a gradual rise of temperature beginning from the 6 th to the 12th hom after the subcutaneous injection of the tuberculin, reaching its maximum from the 12th to the 21st hour, thereupon falling: with slight interruptions until the normal is reached at the 24 th to the 40 th hour. In some cases a second rise of shorter duration is observed (see Fig. 93). The difference between the maximum temperature

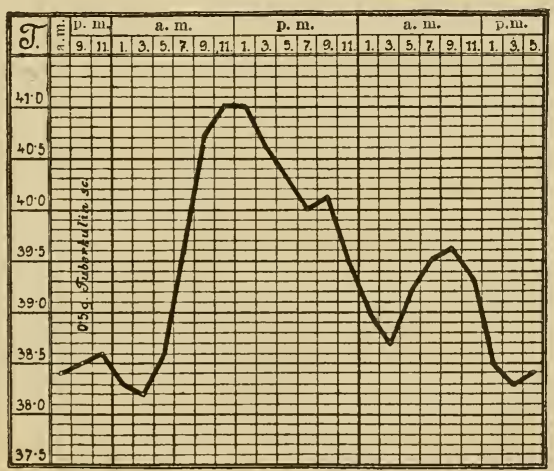

Fig. 93. Typical Tuberculin Reaction. after injection and the temperature recorded before injection is usually from $2^{\circ}$ to $4^{\circ}$ or $5^{\circ} \mathrm{F}$. In rare cases the fever curve just described may show variations in that it may begin sooner or much later (14th to 18th hour), and then, particularly in the latter case, reach the maximum at a later hour also. 
- Simultaneously with the elevation of temperature the pulse and respiration often become accelerated, but not infrequently they remain practically normal. Great depression and loss of appetite are equally frequent and, in addition, usually from the 6th to 8th hour, muscular tremors are observed (organic reaction). The milk secretion is usually somewhat diminished during the period of reaction and on the following few days, owing to the fever and the diminished appetite.

The decrease in the quantity of milk in reacting cows varies according to Zschokke from 3.2 to $8 \%$, according to Eber 3.4 to $6.1 \%$, according to Bohm the average is $9.2 \%$, according to Eber \& Fehsenmeier the maximum is $15 \%$, according to Martel $3.1 \%$. According to the investigations of Tiraboschi the milk secretion decreases during the first 24 hours after injection and again becomes normal on the third day. Non-reacting cows, however, also show a slight decrease (average $1 \mathrm{~kg}$. as compared with $2 \mathrm{~kg}$. [15\%] in reactors).

The intensity of the reaction and the extent of the lesions bear no relation to each other unless this is an inverse one in the sense that extensively tuberculous cows, lience emaciated and weak animals, usually react with less intensity than robust individuals in which the tuberculous process is in its first stages, or more or less localized. In greatly advanced cases reactions may not take place at all.

As a rule the tuberculin test does not perceptibly influence the course of the disease or the health of the animal. In advanced tuberculosis the elevated temperature (fever) may persist, though to a slight degree only, and this may, to a certain extent, excite the tuberculous process to greater activity and hasten a fatal termination. Virchow explained the unfavorable action of tuberculin by the supposition that the thermal process mobilized or stimulated the bacilli to greater activity, causing them to multiply faster in the congested areas surrounding the tuberculous foci. In the lungs the increased bronchial secretions were supposed to play an especially important part in the dissemination of these organisms.

As a matter of fact tuberculin exerts a peculiar action on tuberculous tissues without destroying the bacilli which manifests itself, in some cases, in hyperemia and increased juieiness of the tissues as seen in post-mortem examinations. This local effect explains to a certain extent why tuberculin injections occasionally result in the development of acnte inflammations in areas surrounding old tubereulous foci, sometimes causing cicatrization and "healing" of the lesion. In other cases, however, fresh tubercles will develop in this area and the tuberculous process is fanned into reneweo activity.

Healthy animals are not affecter by as large or larger doses as will produce violent reactions in tuberculous animals; there may occasionally be arr increase in temperature not exceeding $2^{\circ} \mathrm{F}$. but general febrile symptoms are still less common. According to Malm intravenous injections of $25.0 \mathrm{gm}$. of tuberculin produce no toxic symptoms. The same indifference to the action of tuberculin is observed in animals affected with other disease than tuberculosis, reports to the contrary are for the most part based upon incorrect interpretation of 
the reaction or of the post-mortem findings, or possibly atso on mistakes in conducting the test.

Tuberculin is therefore an extremely valuable diagnostic agent, especially in the detection of latent tuberculosis. A positive reaction to the tuberculin test is a certain indication of the existence of tuberculosis while the absence of a positive reaction justifies the exclusion of latent tuberculosis.

In interpreting a tuberculin reaction the following principles are safe to follow (according to the authors):

Animals over 6 months of age may be regarded as tuberculous, (a) if the highest temperature indicated in the course of a typical reaction exceeds the highest recorded temperature before the injection of tuberculin by $2.7^{\circ} \mathrm{F}$. or more or if the rise was at least $.9^{\circ} \mathrm{F}$., or higher than $104^{\circ} \mathrm{F}$.; (b) if the temperature is elevated 1.8 to 2.5 or exceeds 103.1 with symptoms of an organic reaction.

Calves under six months of age must show an increase in temperature over $104.9^{\circ}$ or $105^{\circ} \mathrm{F}$. to indicate a positive reaction.

The animal may be considered free from tuberculosis if the rise of temperature was not more than 2.7, and did not exceed 103.1 and if the animal showed no organic reaction, provided that a systematic clinical examination revealed no evidence upon which to base a suspicion of the disease.

If the results of temperature records and clinical examinations are interpreted according to the above principles they will be found to agree with post-mortem findings in about $98 \%$ of all cases, thus possible errors will hardly exceed $2 \%$.

However, the rules variously laid down to be followed in the interpretation of results of tuberculin tests are at present not as uniform as might be desired.

According to Nocard a reaction is to be considered as positive if the highest temperature after injection exceeds the highest pre-injection temperature by 2.7 , while an increase of 1.5 to 2.5 indicates suspicion only. In the latter case the animal should be re-tested after one month and given an increased dose of tuberculin.

According to Eber pre-injection temperatures not exceeding 103.1 followed by post-injection temperatures of 104 or more, or at least exceeding 103.1 and in all eases indicating a rise of at least 1.8 must be regarded as positive reactions.while post-injection temperatures lower than 104 and exceeding 103.1, but not representing a rise of 0.9 to 1.8 must be regarded as pointing to suspicion of tuberculosis.

According to Ostertag post-injection temperatures exceeding 103.1 and representing a rise of at least 0.9 above the highest pre-injection temperature points to the existence of tuberculosis. In calves under six months of age post-injection temperatures exceeding 104 and representing a rise of at least 0.9 above the highest pre-injection temperature indicate tuberculosis.

According to the decision of the International Veterinary Congress held in Budapest all eattle showing pre-injection temperatures not exceeding 103.1 and post-injection temperatures exceeding 104 must

Vol. $1-37$ 
be regarded as tuberculous while all post-injection temperatures above 103.1 but not exceeding 104 must be regarded as doubtful and be considered individually.

In interpreting the results of a tuberculin test the highest temperature recorded after the injection of the tuberculin is of paramount importance because the existence of a reaction is based upon the presence of a distinctly febrile condition. The difference between the highest pre-injection and the highest post-injection temperatures is next in importance though of less value. The highest pre-injection temperature must of course always constitute the basis of our considerations.

Tuberculin reactions will occur in very early stages of tuberculosis. Nocard and Rossignol observed reactions from the 32 nd to the 48th day after alimentary infection, from the 19 th to the 32nd day after inhalation infection and on the 13th day after injection of the virus into the udder, while experiments made by the Royal Agricultural Society showed that reactions occurred on the 8th day after the subcutaneous injection or the alimentary administration of infectious material. According to MacFadyean the maximum time elapsing between infection and reaction to tuberculin is 51 days.

In some cases repeated injections of tuberculin result in immunity to its action. Thus some cattle that are injected subcutaneously with the usual dose, will fail to react, or will react only slightly to a second application of tuberculin. Accordingly it is only possible to judge the results of a tuberculin test correctly when it is positively known that the animal in question was not treated with tuberculin at any time during the preceding four weeks.

Nocard's pertinent experiments showed that of 24 reacting cows only $33 \%$ reacted to a test applied 24 to 48 hours later, $50 \%$ reacted after one week, $60 \%$ after two weeks and nearly all of them reacted after the lapse of one month. According to results obtained at the Prussia sea-quarantine stations, however, eattle previously treated with tuberculin will always react if five times the ordinary dose is administered, and Vallée's experiments have shown that cattle will react to double doses administered 36 to 48 hours after a previous injection of tuberenlin. In such cases, however, the reactions set in earlier (4th to 9th hour) and are of shorter duration, hence the necessity of recording post-injection temperatures at two-hourly intervals beginning immediately after the injection of the tuberculin.

Malm, Lignières, Mettaru and Lueders report similar experiences. In experiments made by Klimmer and Kiesig $88.3 \%$ of cows receiving an ordinary dose of tuberculin reacted to a double dose administered after one to three weeks. The second reaction usually began at or after the 7 th hour, but earlier in $18.5 \%$ of the eases and usually lasted only two hours, occasionally only one hour and the maximum temperatures were lower than in the first reactions. On the other hand, Zabala observed that injections of the usual dose, repeated several times at two - days' intervals would prevent reactions to subsequent excessive doses. According 
to Storch the simultaneous administration of an antipyretic (e. g., $30.0 \mathrm{gm}$. acetanilid) will prevent a thermal reaction.

A positive typical reaction to tuberculin indicates the presence of tuberculosis with a great degree of certainty, even in the initial stages of the disease. If the evidence revealed at a post-mortem examination does not confirm the diagnosis it is more than probable that the lesions were so minnte, or restricted to so small an area that they escaped detection. In some instances the morbid process is actually limited to a single focus as large as a pea in a lymph gland, or possibly to a diffuse swelling of some lymph glands (lymphoid stage, see p. 532) or a spotted reddening of their tissues (Hottinger). (Bang, in the abundant material at his disposal, found three cases only where reacting animals showed no lesions of tuberculosis on post-mortem examination.)

In the past the lymph glands, which are not infrequently the sole seat of the tuberculous process, receiver little attention and this we have an explanation of the many apparent mistakes in diagnosis. Even in those cases where post-mortem examination of reacting animals revealed the presence of some other disease instear of tuberculosis (actinomycosis, botryomycosis, abscesses in internal organs, verminous bronchitis, distomatosis, caseated echinococeus cysts, etc.) it would be difficult to eliminate the possibility that tuberenlosis did not exist simultaneously in some organ of the body. In this connection the fact should be noted that nearly all animals upon which observations were made, or which served as a basis for statistics on this subject, where slanghtered for meat and that minute examinations of all organs, bones and joints were out of the questions for economic reasons.

The absence of a tuberculin reaction loes not have the same decisive diagnostic value because some animals with advanced disease and suffering from disorders of nutrition will not react, at least not during the usual period of observation. This fact, however, is of little practical importance because cases of this kind can be detected by their clinical symptoms or they will at least be suspected. In some of these cases postmortem examination has revealed calcified foci in which living hacilli could not be found.

It is possible that in many of the cases referred to reactions actually occurred but were not observed during the usual perior of observation (12th to 21 st hour after injection). In experimental tuberculosis of eattle and goats the reaction may set in as early as the $2 d$ to 5 th hour and reach its maximum at the 6 th to 9 th hour (Arloing). In cattle that have previously received injections of tuberculin the reaction may pass over sooner than normally and in advanced disease it may set in very late. With these possibilities in mind a careful clinical examination should be made in all cases where the least suspicion may exist of the possible presence of the disease (chronic cough, barrenness, intestinal, uterine or uller affection, ete.).

How important, aside from the use of active tuberculin, a correct interpretation of the reaction and the experience of the observer or veterinarian really is shown by the results of tuberculin tests in Bavaria (1895-1899) according to which errors in positive diagnosis fell from $13.5 \%$ to $2.6 \%$ in four years and those of negative diagnosis from $11.1 \%$ to $5.7 \%$.

According to Bang tuberculosis may be recognized in $96 \%$ of all cases sub,jected to careful and accurate tuberculin test. Malm gives as his estimate under the same conditions $2 \%$ of failures in recognizing the disease. According to Jensen's compilations, of 468 tuberculous animals $90.8 \%$ reacted, $4.9 \%$ failed to react and $4.3 \%$ gave doubtful reactions; of 290 non-tuberculous animals $14.5 \%$ reacted, $81.7 \%$ did not react and $3.8 \%$ gave doubttul reactions. According to 
Eber's statisties corering 563 cases post-mortem finlings confirmerl $86.9 \%$ of all results of tests and failed to confirm $13.1 \%$. Carini reports still less favorable results: In 361 cattle tested, post-nortem findings failed to confirm $17 \%$ of diagnoses based upon the tubereulin test. In a test made by the authors and covering 156 cattle, only $2 \%$ of the diagnoses failed to be confirmed at post-mortem examination. Schnetz reports results of accurately controlled tests including 75.5 eattle in which T. A. was used on 255 and T. O. on 500 animals. Only $2.9 \%$ of unconfirmed diagnoses were noted. Finally, of 24,784 positive reactions in cattle observed in the United States from 1903 to $1908,98.9 \%$ were confirmed on postmortem examination (Nohler).- In echinococeus disease and in actinomycosis Nocard never noted positive reactions. The authors never saw positive reactions in actinomycosis.

In the practical application of the tuberculin test the fact should always be considered that positive reactions indicate tuberculous infection merely and neither the degree nor the seat of the tuberculous process. It is a notable fact also that a positive reaction is not necessarily the result of a clinically manifest lesion which may be present, but that it may have been brought about by an otherwise unimportant and inconspicuous lesion in another organ of the body (of especial importance in the diagnosis of tuberculosis of the udder).

In other species of animals the tubereulin test has, in most instances, given similar results. Bang found the test reliable in the horse; Eichhorn obtained accurate results in three goats; in swine the test proved reliable in six cases reported by Bang, in 58 cases by Mohler and Schroeder, while Thiro found 21 tuberculous swine among 24 reactors. According to Thiro a rise of temperature above $103.7^{\circ}$. providing it exceeds the pre-injection temperature by at least $1.8^{\circ}$, and according to Schroeder and Mohler a rise of $1^{\circ}$ should be regarded as a positive reaction. In dogs the results seem to be less reliable. Although Froehner noted a marked elevation of temperature $\left(1.2^{\circ}\right.$ to $\left.3.7^{\circ}\right)$ in eight cases, a number of tuberculous dogs did not react and in one positively infected animal there was a drop of $0.7^{\circ}$. In other diseases, excepting one case of generalized sarcomatosis, no reactions were noted. Poenaru observed positive reactions in only 6 out of 13 dogs affected with tubereulous exudative pleuritis. Finally, a tuhereulous monkey tested by Froehner, receiving $0.01 \mathrm{gm}$. of tuberculin, gave a reaction of $4.5^{\circ} \mathrm{F}$. Hoch also observed prompt reaction in one ease.

The nature of the action of tuberculin is as yet only imperfectly understood. The fact that healthy animals may receive comparatively large doses (guinea pigs $2.0 \mathrm{gm}$., dogs $1.0 \mathrm{gm}$., eattle $25 \mathrm{gm}$.) without reacting or being otherwise injuriously affected, and that tuberculons animals will react upon the application of much smaller doses but only after a certain time has elapsed after its application, points to the prohability that the reaction is due to some substance which is present in the body of an infected animal and with which the tubereulin comlines or in the presence of which the tubereulin forms a new substance.

Wassermann and Bruck advance the theory that the tuberculous foci become charged with body fluids and migrating blood cells under the influence of tuberenlin and that as a result the diseased tissue undergoes a breaking down process. Certain tubercle bacilli in the tuberculous foci die off, are dissolved and extracted by the neighboring cells and body fluids, their tuberculin is taken up by the surrounding fluids and the production of antituberculin is the result. Injected tuberculin is attracted to these foci by virtue of its avidity for its antibody; this coubination causes complement fixation or concentration of complement in the tuberculous tissue and as a result albumin is dissolvel or digesterl. The resulting products gain 
access into the circulation and, together with the pyretie action of the tuberculin, bring about the general elevation of temperature. As a result of injecting tuberculin, antiborlies develop in the blool and when further injections of tubereulin are made these antibodies neutralize its effect before it ean reach the tubereulous foei and thus no reaction takes place. In very recent cases large quantities of antituberculin are present in tuberculous foci, but in old lesions where the cells are completely destroyed or easeaterl antitubereulin can no longer be formed even though tubereulin is present, hence the absence of reaction in such eases. According to this theory then, reaction is the result of the attraction of tuberculin by its antibody into the diseased centers and the concentration of the tissue-destroying forces of the borly at these points. Immunity to the action of tuberculin injections results from the production of antibodies following the first injection which then prevent later injections from reaching the diseased foci before the tubereulin is neutralizerl.

Weil and Nakayama as well as Dieterlen also demonstrated antitubereulin in tubereulous organs while, on the other hand Morgenroth and Rabinowitsch failed in their researches to find either antitubereulin or any other specific antiborly in the serum or tissues of tuberculous animals. Moussu showed that eattle that received intraperitoneal injections of cultures of tuhercle bacilli filtererl throngh porcelain tubes, also reacted when treated with tubereulin, thus proving that the reaction is due to the products of metabolism of the bacilli and not to the bacilli themselves.

According to Eber's view the increased physiological activity of the cells causes tnberculin to produce tuberenlopyrin and under the action of the resulting pyretic condition of the body the antotulereulin alrealy present in the borly of the tubereulous animal produces additional quantities of tubereulopyrin. Accorling to Marmorek tuberculin stimulates the bacilli in the bolly to the production of toxins which, passing into the circulating fluids of the body, cause the resulting fever. And since the bodies of animals with extensive foci of infection alrearly contain these toxins in large quantities a slight additional amount has no appreciable effect and hence no reaction follows. Accorling to Behring the active prineiple of tuberculin consists in tubereulinic acid (a combination of phosphorus free protamine and phosphoric nucleic acill) the effect of which, on guinea pigs is 100 times as violent as that of tubereulin. Arloing and Bancel found that the injection of serum from a tuberculous cow or the expressel juice of large masses of tubercles did not produce reactions in tuberculous eattle, goats, dogs or guinea pigs.

Technic of the Subcutaneous Tuberculin Test. Tuberculin used for diagnostic purposes is usually dilutel with nine parts by weight of a $5 \%$ solution of carbolic acil. The ordinary dose of common tuberculin (tubereulin A) is as follows: Adult cattle, 0.30 to $0.50 \mathrm{gm}$. according to size, yearlings $0.20 \mathrm{gm}$., ealves under six months $0.10 \mathrm{gm}$., the doses for horses are the same as those given for eattle; goats and sheep, 0.02 to $0.03 \mathrm{gm}$.; swine, 0.05 to $0.10 \mathrm{gm}$; $\operatorname{dogs}, 0.05 \mathrm{gm}$. The fluid is preferably injected at the sile of the neck into the subcutaneous connective tissue and not into the muscles. The hair should be elipped and the skin disinfected over a small area before injection is made.

The test should be comlucted in well ventilated stables that are not kept too warm. The animals to be tested should be perfectly rested and under normal conditions of feed, shelter and surroundings. A pre-injection temperature should be ascertained immediately before injection and, if possible, twelve hours previous to this time, preferably in the morning. (A more practical and reliable procedure is to recoril at least three pre-injection temperatures at intervals of three or four hours, but not less than two hours, the last pre-injection temperature to be taken immediately before the tuberculin is injected.-Translator.)

In animals affected with fever the test does not give reliable results, it is therefore advisable to wait with such animals until they regain their normal condition. It is best to make the injections late in the evening or some time during the night, reactions that may oceur can then be observed early the following morning or some time during the day. Animals should be kept at perfect rest and under accustomed conditions of feeding, stabling and watering. After injection the temperature, pulse and respiration should be recorded, if possible, every two hours, but at least every three hours. Where eireumstances do not permit this arrangement the animals should be under observation at least from the 6 th to the 21st hour after injection. The uninterrupted observation of the animal between the 6th and Sth hours is of special importance hecause museular trembling and other general symptoms usually appear at this time. Since reactions may sometimes set in at an unusually late hour animals must be kept under observation and have their temperature recorded after the 21 st hour, if the temperature shows a tendency to rise at that time unless, of course, a decided reaction has already taken place. The recording of the temperatures should, in such cases, be continued 
until it legins to drop again, the object of our observations being to determine the highest temperature reached after the injection of the tuberculin which is possible only by following this rule. In order to obtain aceurate results for comparison of temperature, the same thermometer should always he used for the same animal throughout a test. Animals should not be watered immediately before taking the temperature.

In sucking calves the results of tests are less reliable for which reason it is best to defer the test until they are six months of age. Animals with climical symptoms of tuberculosis, very old and very emaciated animals may give very unreliable or unexpected results and for practical purposes might as well be excluled from the test.

Malm and Kitt recommend the intravenous injection of tuberculin on account of the earlier appearance of the reaction. If for example the tuberculin is injecterl from $7: 00$ to $9: 00 \mathrm{~A}$. M. the reaction may set in between $12: 00$ noon and 5:00 $\mathrm{P}$. M.

(The dose of American commercial tuberculins is usually indicated in the instructions furnished by the manufacturers.)

2. The Ophthalmic Tuberculin Test (Ophthalmoreaction. Conjunctival Reaction). Trolff-Eisner (1907) and almost simultaneously Calmette also observed that a few drops of tuberculin applied to the conjunctival sac of tuberculous persons produced an inflammatory reaction manifesting itself in reddening ancl swelling of the conjunctiva. During the same year Vallée demonstrated that a similar reaction takes place in cattle and subsequent investigations of other authors have shown that this test is quite valuable for diagnostic purposes, especially when concentrated bovine tuberculin is used. A pronounced positive reaction to this test may be considered as almost conclusive evidence of the presence of tuberculosis. However, since animals known to be affected with tuberculosis may not very infrequently give negative reactions to this test, the absence of a positive reaction does not necessarily exclude tuhereulosis.

In eattle the conjunctival reaction usually sets in between the 6th and 9th hour or a little later. It manifests itself in watering of the eyes, reddening and edematous swelling. Later, hetween the 12th and 24 th hour there is a production of purulent secretion which accumulates under the nasal canthus of the eye in the form of an elongated mass (see Plate III) which finally drops off or dries up to form a yellow crust. The reaction continues until the 36 th to 48 th hour or even three or four days. To be positive the reaction must consist of a purulent conjunctivitis (polynuclear leucocytes in turbid yellow exudate). A catarrhal inflammation attended only with profuse watering of the eyes or mucous exudate, especially if concentrated tuberculin has been used, may follow in perfectly healthy animals. The severity of the conjunctivitis is of course no indication of the extent, or stage, of the tuherculous process. A simultaneous, or a previous subcutaneous injection of tuberculin has no effect on the conjunctival reaction, except that in some instances the conjunctivitis is even more pronounced. Occasionally when the subeutaneous test is applied to tuberculous animals that have previously reacted to the conjunctival test the ophthalmic srmptoms may again manifest themselves. As a result of hypersensitiveness of the conjunctiva following the eye test a repetition of the latter may result in a more pronounced reaction than before. The conjunctival reaction, even if excessive or severe, does not affect the general condition of the animal nor reduce the secretion of milk. 

Plate III.

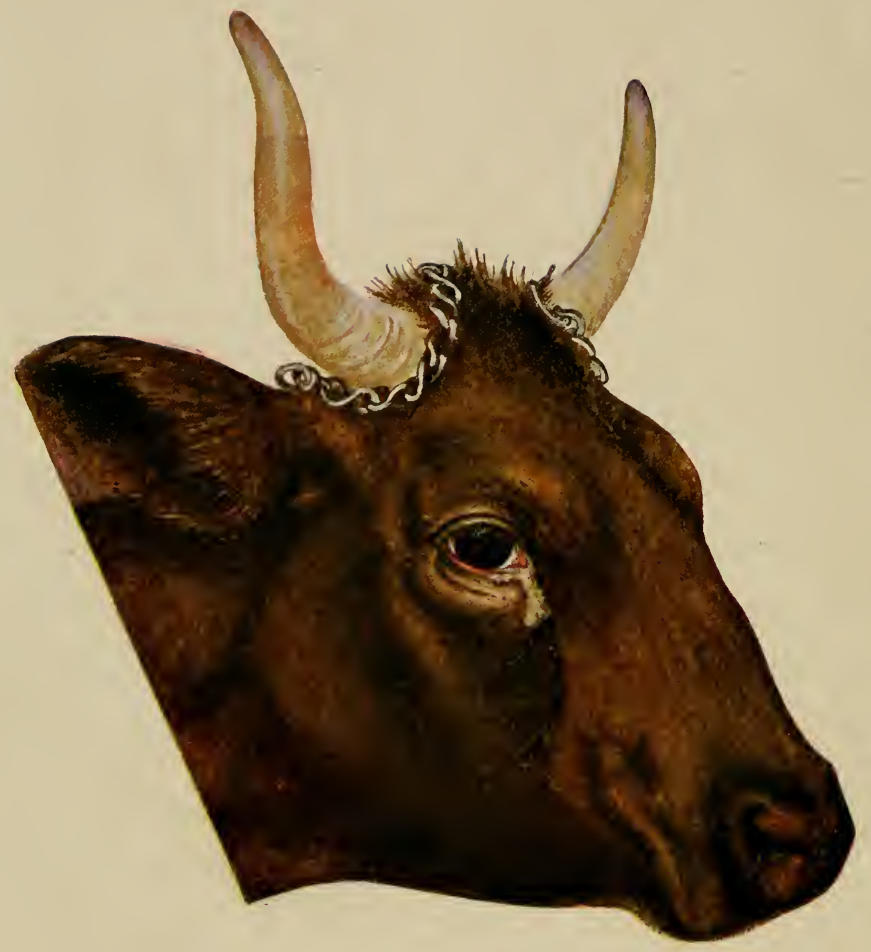

Conjunctival Reaetion in Tuberculosis.

12 hours after the instillation of erude tubereulin. 
The first conjunctival tests that were made on cattle did not give very uniform results, probably because they were made with weak solutions of tuberculin. While Vallée, Lignières, Guérin and Delattre reported very satisfactory results, Arloing, Vanderheyden, Grátz and Reinecke would not admit any practical value whatever for this test. Subsequently, however, Garth, Kranich and Gruenert showed that although dilute solutions of tuberculin gave unreliable results, concentrated material produced distinct and specific reactions. By using $50 \%$ tuberculol they succeeded in demonstrating the presence of the disease in $96.1 \%$ of reactors at the time of slaughter.

Since then nearly all eye tests have been conducted with concentrated bovine tuberculin with results correspondingly more favorable. Thus Sekyra reports $15.9 \%$ of errors in the diagnosis of 82 . cattle, Richter $14.7 \%$ of doubtful results and $16 \%$ of positive errors in 75 eattle, Opalka and Duering had $10 \%$ of negative reactions in 66 tuberculous eattle, while on the other hand Klimmer and Kiessig found $83.3 \%$ of reactors tuberculous at post-mortem examination after slaughter, and of $14^{\circ}$ non-reactors all were found to be free from the disease at slaughter; of 121 eattle that were tested by both the conjunctival and the subcutaneous methods $87.6 \%$ gave the same results for both tests, while $12.4 \%$ gave different or opposite results. Similarly favorable results are reported by Marinescu for 820 cattle, Meyer for 150 and Bellini for 200 test cattle. Basing his conclusions on 487 conjunctival tests of 412 Danish quarantine eattle and 66 tests of that number of inland cattle Foth found that the test gave pronounced and positive results in about 505 of such cattle as had ceased to react to the subcutaneous test on account of previous applications of tuberculin.

Technic of the Ophthalmic Test. With the head of the animal secured by an assistant a few drops of tuberculin are dropped into the conjunctival sac by means of a pipette, or the tuberculin may be applied with a camels' hair brush on the lateral portion of the bulbus, whereupon the closed eyes are slightly massaged for one-half to one minute.

The material used should be $50 \%$ of concentrated bovine tuberculin. In Germany bovotuberculol D Sol. I and $50 \%$ tubereulin A Dohna has been found effective. Foth however obtained good results also with a $5 \%$ solution of dry tuberculin. It seems that tuberculins containing ground or pulverized bacilli (endotoxins) are better adapted for local tests.

The result of the test is determined between the 12 th to 24 th hour after application of the tuberculin. The untreated eye serves as a check or control. Particular attention should be paid to the character of the exudate and to the appearance of the membrana nictitans. In positive reactions the latter is intencely reddened and its normally ${ }^{-}$sharp border is swollen or thickened. In exceptional cases the reaction may persist for three or four days.

Other mucous membranes besides the conjunctiva have been found to be hypersensitive to applications of tuberlin in tuberculous animals, Richter calls attention to the special adaptability of the vaginal mucous membrane for this purpose. After application of the bovotuberculin to the accessible portion of the mucous membrane of the vagina, the reaction, if positive, will manifest itself in redness, swelling, and the production of a glairy, whitish mucous or sometimes purulent exudate. Although the results are equally as accurate as those of the conjunctival test (20\% errors) this method is hardly to be considered as practical on account of the possibility of secondary infection which might interfere with the diagnosis.

3. Tuberculin-Cutaneous Tests (Dermo-reaction, intra(lermal reaction). Since Pirquet (1907) has shown that the inoculation of minimal quantities of tuberculin into the upper layers of the cutis of tuberculous persons produced local reddening and swelling at the point of inoculation, Vallée applied this new diagnostic method in horses and cattle with the same satisfactory and positive results. The scarified area, into which tuberculin had been rubbed, usually showed a distinct reaction in tuberculous animals while healthy animals showed slight inflammatory reddening at the border of the area only in exceptional cases. Since then other investigators have verified these results and thus proved the specific value of the cutaneous reaction. The technic of the method has been variously modified 
and reactions were brought out in different forms, but all are based upon the hypersensitiveness of the cutaneous tissue of tuberculous patients. In all of these methods the reactions are due to the same underlying principles and the results are rather uniform especially in the fact that distinct positive reaetions have a considerably greater diagnostic value than negative results. Cutaneous reactions differ from the conjunctival reaction in that simultaneous subcutaneous injections of tuberculin, or injections made a short time before, may prevent the development of the former.

The cutaneovs reactions vary according to the method of application of the tuberculin. In all cases, however, there is local inflammation and serous infiltration of the tissues, the symptoms usually becoming pronounced on the second or third day, and they may persist until the fifth day. When the first test gives a doubtful result it is advisable to repeat it with equal or double doses of tuberculin. This frequently brings out a decisive reaction.

(a) Cutaneous Reaction. According to Vallée an area about the size of a silver dollar, on the shoulder or chest wall is shaved perfectly smooth and the cutis scarified with a sharp scalpel (incisions may be parallel or intersect each other) whereupon a $50 \%$ or concentrated tubereulin is applied with a brush to the slightly bleeding surface.

If the reaction is positive there will be a distinctly visible and palpable infiltration of the border of the wound and an edematons infiltration of the area surrounding the field of operation. This persists from one to three days and may occasionally result in a separation of the epidermis. In negative cases the borders of the wounds made by the sealpel show only a slight swelling (mainly traumatic reaction) which rapıdly disappears. As a control or check it is advisable to prepare a similar scarified area to be left untreated with tuberculin.

Vallée, Moussu and Lignières, as well as Pirquet and Schnuerer obtained results of definite diagnostic ralne with this method while Vanderheyden, Garth, Kranich and Grunert as well as Reinecke express themselves unfavorably in regard to results obtained. Klimmer and Kiessig who applied this nethod on the udder report $42.9 \%$ of failures in diagnosis.

(b) Dermoreaction. Lignières applies the tuberculin to the cleansed, and shaved surface of the skin, without previous scarification. In case of a positive reaction the area in question becomes reddened, painful and edematous after about twenty-four hours. Occasionally there are small pustules.

According to Lignières from 15 to $20 \%$ of tuberculous eattle fail to react to this test. Vallée succeeded in obtaining a reaction in 13 out of 18 tuberculous animals while Klimmer and Kiessig record $37 \%$ of negative results in known tuberculous cattle.

(c) Intradermal (intracutaneous) Reaction. This consists of a circumscribed edematous swelling of the skin following the injection of 0.1 ce. of $50 \%$ tubereulin into the tissue of the eutis. According to Moussu and Mantoux the folds of the skin betwreen the base of the tail and the anus, stretched tense by elevating the tail are best adapted for this test. In positive reactions the fold which received the injection of tubereulin swells up to the size of a hulled walnut or more in the course of twenty-four hours and forms a sharp contrast with the 
opposite thin fold (Fig. 94). In some animals a small hemorrhage occurs at the point of inoculation. As a rule the overlying epidermis dries up into a brown crust or scab.

A swelling appearing immediately after the injection of the tuberculin but disappearing after a few hours and which, according to Vallée is due to glycerin and salts contained in the tuberculin must not be regarded as a positive reaction.

Lignières as well as Roemer and Joseph inject the tuberculin on the side of the neck and as near as possible to the cutis. In this case a positive reaction consists in the appearance, beginning on the following day, of a painful edematous swelling around the point of inoculation.

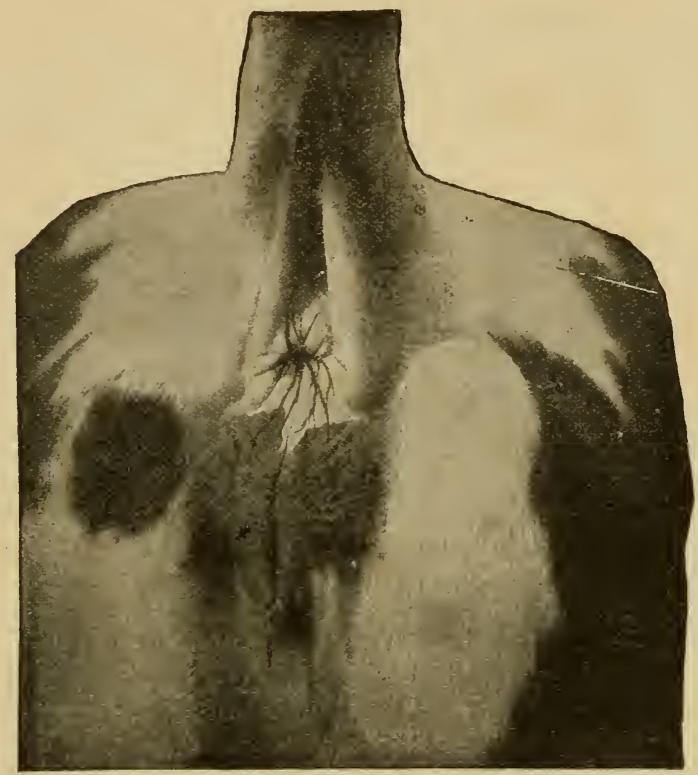

Fig. 94. Intradermal Tuberculin Reaction. Edematous swelling of the right anal fold following injection of $0.1 \mathrm{ec}$. of $50 \%$ tuberculin into the tissue of the cutis.

For an objective determination of a reaction the thickness of a fold of skin about $5 \mathrm{~cm}$. wide is accurately measured by means of micrometer calipers immediately before, and then again three or four days after the injection of the tuberculin at that point. If, during that time the fold has increased in thickness to the extent of $0.4 \mathrm{~cm}$. or more, the reaction is to be regarded as positive, if the increase is only 0.2 to 0.3 $\mathrm{cm}$. the reaction is to be looked upon as doubtful and the test should be repeated with 0.1 ce. of concentrated tuberculin applied on the opposite side of the neck.

By means of their method (0.1-0.2 ce. of $10 \%$ tubereulin) Moussu and Mantoux obtained in $\tau 0$ head of eattle reactions corresponding in all respects 
to the results obtained by Koch's subcutaneous test. Cattle free from tuberculosis but affected with other diseases (Metritis, Cancer, ete.) did not react to the test. The results of tests by Valiée were equally satisfactory, 494 cattle in 521 tests giving the same result for each method (12 cattle with positive thermal reactions gave no cutaneous reaction). Foth succeeded in detecting about one-half of the tuberculous individuals among the Danish quarantine eattle by this method. By the method of Roemer and Joseph (0.1 ce. of $50 \%$ tuberculin) Joseph records only one error in 126 cattle as shown by subsequent post-mortem examination, viz., one animal out of 79 tested, while no reactions occurred in 27 healthy animals tested. Zschokke found in his experiments with 300 cattle, which were for the most part slaughtered afterwards, that neck and tail inoculations were of about equal value and that both were subject to about $15 \%$ error, only $85 \%$ of known tuberculous cattle giving positive reactions.

The intracutaneous method is applicable also for swine, the tuberculin being injected into the skin at the base of the ear near the median border on the dorsal surface. If the animal is tuberculous a flat swelling with central reddened zone will develop after twenty-four hours. In some cases there may be a hemorrhage, the size of a silver dollar, in the center of the swelling, which may lead to necrosis of the overlying skin.

This method, recommended by Moussu and Mantoux, was tried by Zschokke on 266 pigs with the result that $90 \%$ of the tubereulous animals gave positive reactions.

(d) Local Subcutaneous Reaction. According to Tallée and Fernandez dead bacilli with their fat contents removed, mixed with four parts of finely pulverized glass and injected in amounts of $1 \mathrm{mg}$. into the subcutis of the ear produce, in tuberculous cattle, in addition to the edematous swelling which occurs simultaneously with the thermic reaction in the subcutaneous method, a very painful, edematous swelling which is valuable for diagnostic purposes. This condition results from the slow absorption of the injected mass at this point. This method has not yet been used in actual practice.

Comparative value of the various tuberculin tests. According to collected experiences the best results are obtained by Koch's subcutaneous method. In this test a positive reaction is a certain indication of tuberculosis while a negative reaction indicates with the greatest probability the absence of the disease. A great advantage offered by this method consists in the possibility of recognizing the presence or absence of a febrile reaction by the maximum temperature recorded after injection of the tuberculin. The intradermal or intracutaneous test is next in value to the subcutaneous method. This produces with the use of $50 \%$ tuberculin good and clear results in both positive and ${ }^{\circ}$ negative cases. The ophthalmic test is of less diagnostic importance, errors being much more frequent than in the foregoing methods, especially in that even tuberculous animals not infrequently give no reactions whatever. The cutaneous test has the least practical value, errors occurring much more frequently than in any of the others; this is due, in part: to the fact that the positive reaction is frequently impossible of differentiation from a simnle traumatic reaction.

Under these circumstances it is advisable, in all cases where an accurate diagnosis is the first consideration, to use the sub- 
cutaneous test. It cannot be denied, of course, that the application of this method is attended with certain objections or at least inconveniences, viz., the long time required to make the observations, the repeated recordings of temperature required, and finally the fact that positive reactions reduce, temporarily, the yield of milk from the animals in question.

Since none of these disadvantages are presented by the various local tests, it is advisable, in practice, where large herds are to be tested, to apply a local test first, preferably the intradermal (intracutaneous) or the ophthalmic test. Thus. after the great majority of tuberculous animals have been recosnized the remainder of the herd may be subjected to the subcutaneous test and the examination of the herd be completed. Such a procedure is the more admissible since the occurrence of a thermic reaction is in no way prevented or interfered with by the previous local test.

(The above arguments would hardly apply, with equal force, to conditions as they exist in America at the present time.-Translator.)

Agglutination Test. According to the investigations of Arloing \& Courmont the blood serum or pleural exudate of tuberculous persons or animals when actiled to homogeneous liquid cultures causes clumping of the tubercle bacilli and their precipitation to the bottom of the vessel, leaving the previously turbid fluid clear and transparent (agglutination) while the blood serum of healthy individuals loes not possess this property. On the basis of these observations these authors recom mended the agglutination test as a practical means of diagnosing latent tuberculosis. In one of their series of experiments the blood serum of thirty healthy calves did not, in any ease, agglutinate a culture when added in proportions of one to five while the serum from 70 mature cows, with only one exception, agghtinater cultures when added in volumetrie proportions of one to ten or even thirty.

Other authors (Beek \& Rabinowitseh, Panisset) could not confirm the diagnostic value of this method nor has the same been found of any practical importance in human medicine.

Precipitation Reaction. According to Bonome the blood serum of tuberculous persons or animals produces a nore or less conspicuous clouding and precipitation when added to a filtered emulsion made of fresh cheesy tubercles or eultures of dried tubercle bacilli mixed with $5 \%$ glycerin. The serum of healthy individuals either does not possess this property at all or only to a slight degree. It is claimed for this test, also, that when pure cultures of tubercle bacilli are used it is possible to differentiate between infection with human tubercle bacilli and infection with the bovine type.

Calmette \& Massol, Vallée and also Jousset found that the serum of highly immune animals contained a thermolabile substance which produces deciled precipitations in solutions of tuberculin as well as in non-concentrated tuberculosis bouillon and in bacillus extracts. In the bodies of tuberculous animals, howerer, it seems to be present in such small and variable quantities that precipitation experiments give too irregular results to be of value for diagnostic purposes. In corresponilence with this statement the results obtained by Zwick in his experiments with the serum of a cow with advanced tuberculosis were negative throughout.

Complement Fixation and Opsonins. Vallée was able to demonstrate antibodies in the serum of highly immunized horses (Hennepe the same in the serum of calves treated with bovo-vaccine) which had the power to fix guinea pig complements with strains of the tuberele bacilli that had been used in the immunizing process as antigen. Bach, however, found that sera from cattle free trom tuberculosis, as well as that from slightly or extensively affected eattle manifester the same pecnliarities in complement fixation experiments and that this action was not specific. At present, therefore, this method has no more diagnostic value than the opsonic index of the blood of tubereulous animals, the study or investigation of which latter has as yet received very little attention in veterinary medicine

Strubell \& Felber have conducted a series of investigations along this line with 80 beef cattle, without however getting clear or decisive results. Of the tuberculous cattle $83.3 \%$ showed tuberculo-opsonic indexes to human tubercle bacilli ranging 
between 0.90 and 1.10 of the normal, $57.8 \%$ giving the same resuits with bovine tubercle bacilli, while $21 \%$ of the healthy animals had indexes abore the normal. Moderately subnormal indexes $(0.80-0.90)$ were observed in $8 \%$ of diseased as well as in $5 \%$ of the healthy animals. Marked subnormal indexes were observed in such eattle only as had been experimentally infected.

Differential Diagnosis. Of the diseases resembling tuber(ulosis in clinical symptoms or anatomical changes the following are of most importance.

Contagious plemro-pneumonia of cattle in its chronic or subacute form: In this disease solidification. of large continuous areas of lung tissue mar be recognized by the dullness revealerl upon percussion, bronchial respiration or the entire absence of respiratory sounds, the pulmonary affection is always attended with an acute pleuritis indicated by pain upon percussion, friction bruits, weak, suppressed and painful cough. There is less secretion from the respiratory passages and the disease takes a less insidious course than that observed in tuberculosis. In very chronic cases a differential diagnosis is hardly possible from the clinical symptoms alone, irrespective of the fact that suspicion of the existence of pleuro-pneumonia does not exclude the presence of tubereulosis, or that both diseases may exist at the same time.

Lung-worm disease in ruminants and swine. This is usually an affection of younger animals, the congh is strong, spasmodic and occasionally attended with attacks of choking; there is rarely an elevation of temperature, auscultation reveals fine rales and friction bruits, and the general condition of the animals is usually little affected even after the otherwise pronounced symptoms of disease have continuerl for some time. Finally, the parasitic filaria may be demonstrated in the secretions of the lungs and nostrils.

Actinomycosis in cattle and swine. In this disease the tumors of the udder and in the pharyngeal region are frequently firmly attached to the overlying skin; if the tumors rupture the characteristic ray fungus may be rearlily demonstrated under low magnifying power without previous staining. In actinomycosis of the tongue the sublingual and pharyngeal glands are not enlarged. Post-mortem examination reveals vellowish granules imbedderl in soft foci which are surrounded iy an abundant connective tissue.

Ecchinococens disease of the lungs. In this affection the congh is weak and coarse, febrile symptoms absent; the affected animals may be in good condition in spite of the respiratory troubles which may have persisted for a long periocl. Finally, the disease is most frequently observed in roung animals.

Traumatic pericarditis. In contrast with tuberculous pericarditis, symptoms of accumulation of fluids (friction bruits, dulled heart sounds in later stages, splashing and weak cardiac sounds) are rarely absent. There is usually, also, more or less swelling in the anterior thorax. 
Leucemia and psendo-lencemia. On account of the cachexia and the enlarged lymph glands which occur in the course of these diseases they may be confused with tuberculosis. In them, however, the lymph glands are enlarged to an equal degree throughout the body and an examination of the blood in lencemia will reveal the abnormal increase of the white blood cells.

Chronic meteorism resulting from non-tuberculous affections of the abdominal organs. When due to tuberculous changes, meteorism does not develop to the same intensity and after each temporary attack the accompanying digestive disturbances immediately subside (Albrecht).

Glanders in horses, especially that form which is confined to the internal organs. The presence of large tumors in the abdominal cavity contraindicate glanders but otherwise a differential diagnosis must be based upon the results of a mallein or tuberculin test. Chronic enlargement of the submaxillary glands does not in itself justify the exclusion of tuberculosis (see p. 562).

Distemper may be confused with tuberculosis when it is localized in the internal lymph glands and assumes a chronic character. In the clinical examination the anamnesis alone may enable us to make a differential diagnosis. At post-mortem examination the presence of abscesses only and the absence of sarcoma-like structures eliminates tuberculosis.

Hog cholera. In chronic cases the morbid picture of this disease is very similar to that of tuberculosis. The anamnesis, however, especially if this shows that the animals in question have previously suffered from acute pulmonary or intestinal affections, will indicate hog cholera as the probable disease while the existence of chronic enlargement of the lymph glands or inflammation of the bones and joints will point to the tuberculous nature of the affection. At post-mortem small gray or yellowish nodules in the neighborhood of cheesy foci or in the lymph glands indicate tuberculosis. The simultaneous affection of a large number of pigs that have been fed with milk or whey is in itself a probable indication of tuberculosis.

Rachitis, especially in young swine. In this disease the epiphyses of the bones are enlarger generally while the joints themselves show no symptoms of inflammation. In tuberculosis of the joints the affection is confined to a few joints and there is also swelling of the capsular ligaments.

Aside from the above named diseases suspicion of tuberculosis may be aroused in other chronic affections that gradually produce cachexia, especially chronic lung affections. Finally, tuberculous meningitis has many symptoms in common with inflammation of the cerebral meninges from other causes and, similarly, cerebral tuberculosis with neoplasms of the brain, or with coenurosis. The same may be said with reference to acute miliary tuberculosis and severe acute infectious diseases.

In contrast with inflammatory affections of the udder from 
other causes in tuberculosis of that organ, the milk retains its normal appearance for a long period. When in the later stages (changes do oceur in the physical appearance of the milk, they are characterized by the absence of pus and mucus (sliminess). Irregular nodules in the tissue of one or more quarters of the ndeler and enlargement of the super mammary glands indicate the tuberculons nature of the affection. Actinomycosis and hotriomycosis prodnce similar swellings in the tissue of the udder but are apt to be firmly adherent to the overlying skin, they frequently break open and discharge their contents, and the mammary glands are unaffected or only slightly and evenly enlarged. In inflammation of the udder caused by streptococeus infection the firm swelling bears a close connection with the inciurated base of the teat while tuberculous disease usually occurs higher up in the tissue of the udder. In the former case also the milk is purulent from the beginning, and while the mammary lymph glands may be swollen or enlarged they are never irregular or nodular in form. Finally, the secretion of the udder contains streptococci. Milk cysts may be differentiated from tuberculosis by their elastic consistencr. I definite diagnosis of tuberculosis of the udder, especially in the less pronounced cases, cannot be made without a hacteriological examination and demonstration of the tuhercle bacilli in the milk (see p. 558) or in the tissue of the udder (p. 570).

For the anatomical diagnosis of tuberculous processes the presence of small gray transparent or yellowish, firm nodules (tubereles), which usually oceur either exclusively within or in the neighborhood of cheesy foci and ulcers may be accepterl as definite evidence. Accepting this as a basis, other processes that resemble tuberculosis (actinomyeosis, botriomycosis, caseated parasitic nodules, chronic inflammations of the lungs, etc.) are easily differentiated from tuberculosis. In all cases, however, the demonstration of the tubercle bacillus is necessary as final evidence of the tuberculous nature of any morbid product or tissue.

Prognosis. Local morbid processes brought ahout by natural infection may, under favorable conditions, not infrequently remain restricted to a definite area or end in recovery throngh an inflammatory reaction in the surrounding tissue. Healthy adult animals are almost always met with even in badly infected herds and it seems that their condition of health is due, not to a natural individual immunity but rather to repeated infection with tuberculosis followed by recovery and consequent immunity to later infections. The encapsuled and calcified cheesy foci which are not infrequently met with on inspection of slaughtered animals, in certain organs, especially lymph glands, point to such a favorable course of local infections. The results of tuberculin tests, especially in the case of roung animals frequently point to this possibility, as in certain cases where the 
animals in question permanently cease to react to the tuberculin test.

Although the possibility of recovery from tuberculosis has thus been demonstrated the course or final termination of the disease cannot be predicted even with a degree of probability. In general, favorable surroundings, especially pure fresh air, good food and care, moderate exercise and middle age have a favorable influence on the course of the disease. Not infrequently, however, the disease makes rapid strides toward a fatal termination in spite of favorable external conditions. Under opposite conditions a gradual spread of the morbid process is the rule and the unfavorable effect of hard work in this respect is well demonstrated by the frequency of advanced tuberculosis among the otherwise naturally tough working oxen of the range breeds of Europe. The unfavorable course of the disease in these cases is no doubt also associated with the fact that these animals, when kept with infected herds, are almost continually exposed to infection and reinfection, resulting in aggravation of already infected centers and in the production of new areas in healthy organs.

According to the intensity and the frequency of reinfections the disease advances with increasing certainty to its fatal termination. When clinical symptoms have made their appearance, recovery may be considered as entirely out of the question.

Treatment. Since the incurability of tuberculosis of do. mestic animals, after clinical symptoms have made their appearance, has been recognized from time immemorial, serious attempts to bring about recovery are hardly ever made in recent times. The immediate use of evidently affected animals for food purposes has now been generally accepted as the best method of dealing with this problem. In human medicine, also, in spite of numberless attempts in this direction, reliable curative methods are unknown. On the other hand, careful observations made in recent years have shown that favorable hygienic conditions, especially living in pure fresh air, nutritious food and physical rest may check the progress of the disease in the early stages and not infrequently bring about a cure. Similar conditions would probably have the same favorable effect in animals. Among these, however, favorable opportunities for systematic treatment along the lines suggested would rarely be offered while, on the other hand, the successful treatment of an occasional individual bears no comparison with the beautiful results that are obtained by prophylactic measures in the suppression of the disease. Nevertheless the proper regulation of hygienic conditions in infected herds, and especially the restriction of stable life to the minimum, as well as cleanliness in stables where these are a necessity, are of immense importance in the solution of the problem of the suppression of tuberculosis.

The systematic treatment with tuberculin, in spite of the 
undeniable favorable results occasionally obtained along this line in human medicine, is of course out of the question with animals both on account of the attending expense and the impracticability of the treatment.

Hauptmann claims to have obtained curative effects in tuberculous cattle by treatment with iodipin (50 to $100 \mathrm{gm}$. of $25 \%$ iodipin repeatedly injected subcutaneously) while Jacob, Bongert \& Rosenberg observed good results following intrabronchial injections of tubereulin and creosote $(500 \mathrm{gm}$. of a 0.1 to $0.4 \%$ solution or $0.1 \%$ solution intratracheally). These apparently favorable results, however, require further confirmation. The same may be said of Burow's assertion that 500 cattle, treated with bacterial preparations of unspecified character, showed "definite improvement" with few exceptions, in the course of a few weeks. The preparations made from tubercle bacilli (Tulase, tulaselaktin, tulon, tuberculase, ete.) and reconmended by v. Behring for the treatment of human tuberculosis have thus far given no tangible results. Under the direction of Roemer extensive experiments condueted in Argentine with tulaselaktin as a curative agent for bovine tuberculosis gave uniformly negative results. Arloing also found tuberkulase inert in his cattle experiments. The same is probably also true with respect to the use of attenuated or avirulent tubercle bacilli.

Prevention. The establishment of the most farorable external conditions plays an important role in the control of tuberculosis. Out of door life, especially on pasture, diminishes, on the one hand the chances of infection and on the other hand prevents the tubercle bacilli from obtaining an easy foothold in the bronchioles and alveoli by thorough ventilation of the air passages and therefore by preventing the development of chronic catarrhal conditions. The customary healthy condition of young cattle in hilly and mountainous countries and the great infrequency of tuberculosis in range cattle is due to the conditions just referred to.

In order to prevent the too rapid spread of tuberculosis in already infected herds it is advisable to keep them ont of doors as much as possible during the day time and, when the weather permits, at night also. In addition the stables shonld be equipped with modern systems for ventilation and thoroughly cleaned and disinfected at least every two weeks. Stable arrangement whereby cattle are compelled to face each other, and continuous watering troughs and mangers should be avoided as these conditions favor the dissemination of the disease. Above all, animals with clinical symptoms of tuberculosis or symptoms of chronic pulmonary affections, chronic diarrhea, chronic discharge from the uterus, emaciation, etc., that point to the probable existence of open tuberculosis should be slaughtered or otherwise disposed of at once in order to check at least the principal disseminators of tubercle bacilli. Among such animals those affected with udder tuberculosis are of course the most dangerous. (Where attempts are made to control the disease according to the rather conservative plan here suggested it should ever be borne in mind that animals without clinical symptoms of disease and otherwise in apparent perfect health may be suffering with advanced tuberculosis and distribute tubercle bacilli in enormous numbers through the medium of their solid excrement.-Translator.) 
In this comnection care should be taken that the young offspring from such herds are not fed on infected milk. This is accomplished by heating all skim milk to $85^{\circ} \mathrm{C}$., or better, to the boiling point, before feeding to ealves or swine. In all cases, however, the milk from evidently infected udders should be destroyed.

In feeding sucking calves it is all important that the milk be free from tubercle bacilli. Infection is most successfully prevented by having young ealves suck dams that are known to be free from tuberculosis as indicated by physical examination and tuberculin tests. If for economic reasons this arrangement is impracticable the calves should be fed with fresh raw milk obtained from healthy cows with every possible aseptic precaution. Milk sterilized by heat should be used only as a last resort. Milk thus treated has lost the ferments so important for digestion and for the prevention of abnormal gas production in the intestines. For this reason every possible precaution should be observed that the vessels used and the hands of the persons in charge of this work are kept scrupulously clean. Heat sterilization is of course always vastly preferable to the use of formalin (see p. 155) or of hydrogen peroxide, because milk thus treated (perhydrase milk, budderized milk), aside from the fact that it may contain virulent tubercle bacilli, is unsuitable for sucklings on account of its low fat content $(2 \%)$.

By careful compliance with these instructions it is possible at least to check the too rapid progress of the disease and to prevent for years the occurrence of very great losses. The measures referred to are however recommended for consideration only in cases where for economic or other insurmountable reasons the complete extermination of the disease is out of the question.

Extermination of Tuberculosis. Investigations showing that tuberculosis is an infectious disease, transmissible from animal to animal, and demonstration of the fact that heredity plays only an unimportant role in its transmission has placed the warfare against tuberculosis upon a new and safe basis. The aim and object of this warfare now is to protect healthyborn animals from infection during life; if we are successful in this effort the animals will remain free from tuberculosis, irrespective of whether they are the offspring of diseased parents or otherwise.

Upon the basis of this principle Bang has worked out a plan for the extermination of tuberculosis from dairy herds which has been put into successful practical operation since 1892. The very satisfactory results already obtained have demonstrated not only the correctness of this theory but also its practical worth.

Bang's Method of Extermination. This consists, in effect, in the destruction of clinically affected animals, the separation and isolation of reactors to the tuberculin test which are otherwise elinically of healthy appearance, and the artificial feeding of calves from the latter with milk free from tubercle bacilli. The details of the method are as follows:

Vol. $1-38$ 
Every animal in the infected herd is subjected to careful examination and each animal which shows clinical evidence of tuberculosis, but particularly those animals with symptoms of pulmonary, intestinal, uterine or mammary disease are condemned and slaughtered, to be used as food, subject to inspection under the laws of the country, or condemned as food as the case may be. The remainder of the herd consisting of clinically healthy appearing animals is then subjected to the tuberculin test. All nonreactors which are free from all other symptoms that might possibly point to the existence of tuberculosis are then separated and isolated from the remainder of the herd. Each herd is now placed in an entirely separate stable, the quarters for the nonreactors or healthy portion of the herd having previously been thoroughly cleaned and disinfected. In the absence of separate buildings a single building may be utilized by separating the quarters of the two herds by means of a tight board wall and providing separate exits for each herd. Young stock, calves, and working oxen should have quarters of their own. Each herd or group must have separate attendants that have absolutely no contact whatever with the other herd. If an animal in the reacting herd at any time develops clinical symptoms of tuberculosis it must be removed at once and disposed of preferably by slaughter. The remaining animals in the reacting herd may be used as before for breeding and dairy purposes, providing they exhibit no elinical evidence of tuberculosis. Yearlings and other young stock reacting to the tuberculin test had best be discarded for breeding purposes at once and for all time. Where special reasons exist for desiring to breed certain reacting young heifers this may be permitted but only on the condition that they be quartered with the reacting portion of the herd. Reacting calves under six months of age should always be destroyed or disposed of by slaughter.

The calves dropped by the healthy "non-reacting" cows should remain with their dams while those from the reactors, after being permitted to suck for twenty-four hours so that they may get the colostrum, are removed from their dams and quartered with the healthy portion of the herd and provided with nurse cows or fed by hand with fresh milk from nonreactors or with milk obtained from other sources, but sterilized by heating to at least $85^{\circ} \mathrm{C}$. Particular attention should be paid to the cleanliness of utensils used as well as to the hands of the attendants in charge of the feeding of these young animals. It is self-evident, of course, that the quarters where these calves are kept should be kept scrupulously clean and occasionally disinfected. Wherever it is possible to do so, the calves might be kept in separate quarters for the first few weeks after birth. If any of the calves hecome affected with diarrhea, they should be isolated at once and the former quarters thoroughly cleaned, disinfected and treated with a hot solution of lye.

As soon as weaned the calves should be subjected to the tuberculin test and all reactors disposed of (the loss in this process hardly ever exceeds one or two per cent). After this they may be added to the free herd or kept in separate calf quarters or with the other young stock. Until used for breeding purposes they should be kept out of doors or on pasture as much as possible and under no conditions may, they be allowed to come in contact with the reacting portion of the herd. The young heifers, after being bred, should be retested with tuberculin and if found free from disease, added to the free herd, otherwise isolated with the reactors. (It is only occasionally that it is found necessary to add an animal to the reacting herd.) 
The free herd should be retested with tuberculin every year so that animals that were infected and failed to react at a previous test, or that have become infected since that time, may be removed and added to the quarantine herd. Newly acquired animals should under no circumstances be added to the free herd unless they have been found free from evidences of tubereulosis by physical examination and tuberculin test made immediately before.

Males used for breeding purposes should, if at all possible, be free, not only from every suspicion of tuberculosis, but should otherwise be in perfect health. If unavoidable, a reacting male may be used but such animals should at least be free from clinical evidence of disease. Such an animal must, of course, be kept under constant supervision during the act of coitus and never be permitted to run with the free herd.

$\mathrm{By}$ this method, the nonreacting or free portion of the herd will constantly increase in numbers while the reacting herd may be correspondingly reduced in size without interfering with the amount of business transacted with the products. Eventually the reactors can be entirely eliminated and a herd free from tuberculosis remain in place of the original infected herd. This can be accomplished with comparatively light expense, and with proper attention and occasional retests with tuberculin the herd may then be kept free permanently from tuberculosis.

The extermination of tubereulosis after the method of Bang has found its widest application in Denmark, Sweden and Norway where government support is liberally extended and favorable results have been quite general. As a result of the personally conducted campaign of Bang, in Denmark, the average percentage of reacting animals in $1893(40 \%)$ has been reduced in 1908, to $8.5 \%$. Among calves the average percentage in $1893(15.5 \%)$ has been reducen to 10.6 in 1898 . Up to the year 1904, 66 badly infected herds comprising 1,045 reactors and 780 healthy animals have been entirely freed from disease, the 66 herds consisting at that time of 1,896 healthy animals. In Norway, according to Malm, the percentage of reactions has been reduced from $8.4 \%$ in 1896 to $4.9 \%$ in 1903 , and the proportion of inspected herds that were found infected was rednced from $26.1 \%$ to $13.6 \%$. In Sweden, where, in the beginning of the campaign of extermination (in 1897-98) 11,089 out of 33,000 animais reacted to the tuberculin test, i. e., $33.6 \%$, reports for the year 1904 showed that among 34,996 cattle tested only $1,664, \mathrm{j}$. e., $4.7 \%$ reacter. During this interval 214 herds comprising 8,205 animals were freed from the disease (Regnér.) - In Finland reactions reached $25 \%$ in 1894 while they were reduced to $10.1 \%$ by 1900 (Hójer). In Hungary there exists now a number of large dairies in which the percentages of reactors have been reduced in the course of a few years to 2 or 3 per cent without any reduction in the size of the herds. Thus on the Mezoehegyeser government estate the first tuberculin test which was conducted in the spring of 1898 in a herd of 329 cows revealed $44.8 \%$ of reactors while $26.6 \%$ of the total number tested (647) reacted. In the fall of the year 1903 a test of 502 cows on the same estate gave only $2.8 \%$ of reactors and a test of all of the cattle on the estate $(1,132)$ gave $1.8 \%$ of reactors. During this period of five years the herd on this estate was increased $75 \%$ in numbers by addition of its own offspring, while the prevalence of tuberculosis was reduced $88 \%$ (Hutyra).

The favorable results of feeding calves with sterilized milk are illustrated by the following: In Sárvár $93.8 \%$ of the calves thus reared gave no febrile reaction to Behring's protective inoculation while only $42.1 \%$ of the calves reared under usual conditions responded similarly. (Behring's protective inoculation, when applied to tuberculous animals, gives a reaction similar to that of tuberculin.) On several German estates, where similar experiments were conducted, the proportions were $74.2 \%$ and $48.1 \%$ respectively.

Modification of Bang's Method. In only slightly infected herds where the question of expense is not the first consideration the quickest results may be obtained by slaughtering all reacting animals at once. In this way the necessity of maintaining two herds, one healthy and 
the other diseased, and the artificial rearing of the calves on nurse cows or with sterilized milk is obviated. The herd may then be kent permanently free by replacing the reactors with newly purchased animals that have been subjected to the tubereulin test, and repeating the test annually in order to remove any affected animal that may have gained an entrance to the herd.

Where the separate maintenance of two herds and the artificial rearing of the calves from the infected dams meets with insurmountable objections a modified form of Bang's method may be applied. Thus, if a herd is so badly infected that it is known in advance that only a comparatively few animals would be found free from disease, the tubereulin test of the adult animals may be dispensed with but applied to the young stock and the calves reared with the same precautions prescribed under the regular Bang method. As the healthy young heifers drop their calves and come into milk they may be used as a nueleus to build up a new free herd to which nothing but healthy cows are added, while the number of infected animals in the original herd may be correspondingly reduced.

Ujhelyi provides healthy nurse cows for the newly born calves or, where necessity demands, leaves the ealves with their infected dams and subjects them to the tuberenlin test when they are weaned, slaughtering all reactors. (In this method the loss is somewhat greater than in the former.) In this case the Bang method is strictly adhered to in so far as separation of the herd into a free and an infected group is concerned and in so far as all animals with clinical evidence of disease are removed at once and slaughtered. This modification of Bang's method may he practiced where the rearing of ealves with sterilized milk meets with insurmountable difficulties. It has the advantage that calves remain free from diarrhea even when under the care of less careful attendants.

By means of this methor Tihelyi has succeeter in bringing about a considerable reituction in the percentage of reactions in a number of large herds. Thus on the estates of Vaál, Szent-László anł Martonvásár in March, 1899, of 1,031 adult cattle S.st reacted $(\$ 5.7 \%)$ of 626 head of yearlings $333(53.3 \%)$ reacted, while in September, 1903 , of 898 adult cattle only 37 or $4.1 \%$ and of 710 yearlings only 19 or $2.6 \%$ reacter to the tuberculin test. On eight other estates, at the beginning of the campaign (1898-1902), of 1,715 cattle tested 1,011 , i. e., $59 \%$ reacter while a few years later (1904 and 1905) only 63 head, $3.0 \%$, reacter.

Method of Ostertag. Ostertag abandons the tuberculin test entirely for adult animals and consequently also the separation of the herd into reactors and nonreactors. He lays special stress upon the importance of an early disposition of all cattle with so called open tuberculosis and the rearing of all ealves with milk sterilized at $85^{\circ} \mathrm{C} . \mathrm{or}^{\circ}$ raw milk from cows free from tuberculosis. When the calves are weaned they are subjected to the tuberculin test and the reactors are slaughtered. In order to dispose of all cases of open tuberculosis at the earliest possible opportunity affected herds are subjected every six months to examination by a veterinarian and all suspicious cows are removed at once. In addition to this the milk (of the individual cows as well as the mixed product from the entire herd) is subjected to periodical bacteriological examination as are also any morbid secretions that may become evident.

This method, which has been made obligatory in 192 herds of the Fast Holiandish-Prussian Herd-Book Association since 1900 and since then has been introduced into other German districts, can hardly be expected to bring about a 
complete extermination of the disease. By removing the necessity of maintaining two separate herds it is less objected to by the owner but the results achieved are correspondingly unsatisfactory. F'rom 1900-01 to 1903-04 among 15,000 arlult cattle 1,071 eases of open tuberculosis were diagnosed, during which time the percentage was reluced to $1.3 \%$ but since that time and up to 190 s-09 it has been reduced ouly to $1.2 \%$ (Mueller, Ostertag). In the herds of the East Prussian Herd Book Association for Mottlecl Red Cattle the percentage has been reduced from 3.2 to 1.24 in the course of six years; in Pommerania, in the course of five years, from 2.93 to $0.39 \%$; in Brandenburg, in the course of five years, from 2.28 to $0.7 \%$ (in the first 20 heris, including 2,131 animals, which were subjected to this method, the percentage was reducerl from 4.1 to $0.23 \%$ ). In Schleswig-Holstein, in four vears the reduction was from 2.5 to $1.47 \%$ and in the Province of Saxony, in four vears, from 3.6 to $1.15 \%$ (Ostertag). These percentages, ot course, all refer to open tuberculosis. Whether or not the total number of all cases of tnbereulosis were correspondingly diminished or whether there may have been an actual increase is not recorderl! At ail events the figures furnished show that the results obtained l.ear no comparison whatever with those obtained by the Bang methorl.

In infected herds of swine the disease may be successfully stamped out by eliminating the affected animals upon the basis of the ontcome of the tuberculin test, subsequent thorough disinfection of quarters and the observation of the necessary precautions to prevent the reintroduction of the disease (Thiro).

Immunization. Basing his observation upon the specific identity but varying pathogenic action of hmman and bovine tubercle bacilli, v. Beluring, in 1901, declared that by treating cattle with the less virulent human tubercle bacilli they would be protected against the more actively virulent bovine tubercle bacilli and thus also agaiust natural infection with tubereulosis. On the basis of this assertion several methods of immmization for cattle lave been recommended and actually carried out in practice. More or less satisfactory results however were ol)tained in such cases only where hygienic and prophylactic measures, effective in themselves, were instituted at the same time.

Double Immunization with Human Tubercle Bacilli (Immunization with hovo-vaccine; Behring's method). The immunizing material consists of nearly full-virulent human tuhercle bacilli, dried in a vacuum and injected into the blood circulation of ealves. The first tests conducted after these treatments showed that two intravenous injections of bovo-vaceine, or of any fresh culture of the human type of tubercle bacillus into cattle resulted in a considerable and immediate increase in their power of resistance to artificial infection. Calves thus treated would, as a rule, resist successfully four months later intravenous injections of virulent bovine bacilli in doses that proved invariably fatal in untreated calves. Further experiments, however, showed that resistance thus artificially increased was of short duration, suffering considerable reduction at the end of one year and disappearing entirely six months later. On the other hand, the results of the practical application of the method in herds showed that it had no noticeable effect on the improvement of the health of infected herds. Since immunization produces no harmful effect upon previously healthy calves it might he possihle to prolong their immunity indefinitely by annual vaccinations. The ohjection to this plan, aside from its attendant trouble and expense, lies in the fact that the hacilli introduced by the act of vaccination remain alive and active in the bodies of the animals 
for a long time (according to Lignières, von Weber \& Titze for two years) thus making the slaughter of such animals for food a procedure of doubtful propriety. In addition to this objection, there is the possibility that the bacilli, circulating freely in the blood stream, may lodge in the tissue of the udder and set up local tuberculous processes (Weber is Titze).

Control experiments conducted by Hutyra showed that intravenous injections of 2 to $2.5 \mathrm{cg}$. of highly virulent bovine tubercle bacilli (doses which wonld invariably kill control animals within two months) made in calves that had been immunized two months before, usually produced only a very few tuberculous foci confined almost exclusively to the lungs and showing a tendeney to encapsulation and healing. On the other hand, injections of the same doses made subcutaneously resulted in lesions confined to the area of injection. Administration of the same virus by the way of the alimentary canal to animals previously immunized resulted in rather extensive affection of the tonsils. Similar results were obtained not only with Behring's original hovo-vaccine but also with culture of human tubercle bacilli 4 to 6 weeks old. When the resistance of the vaccinated animals was tested $71 / 2$ and 17 months later the control infections resulted in extensive and partly in fatal general tuberculosis.

Eber tested the resistance of four animals immunized by Beliring's method by repeatedly exposing them to animals with experimental tuberculosis. Two years later, when they were slaughtered they were without exception found to be affected with focal tuberculosis of a more extensive nature than that affecting the control animals.

In experiments conducted with 20 vaccinated animals by Rossignol \& Vallée in Melun, with the same number of controls, while giving gratifying results three inonths later when 13 animals of each group were subjected to control infection, further observation disclosed that two of the vaccinated animals became tubercufous and another died within 50 days from the effects of subsequent intravenous infection. Furthermore, experiments carried out by Degive, Stubbe, Mullie \& Liénaux in Belgium and under Mazzini's direction in Italy, gave more cr less unsatisfactory results. Finally, in an experiment made by Weber \& Titze with 12 calres treated according to Behring's instructions and which were exposed to infection from $21 / 2$ to 9 months later by subeutaneous or intravenous injections by inhalation and alimentary administration of bovine virus, and in three instances exposed to natural infection, only one animal, and that of the last group mentioned, remained free from tuberculosis.

On experiences in practice, Roemer (1905) reports on statistics covering 5,576 animals with the conclusion that raccination resulted in a considerahle decrease in the prevalence of tuberculosis in the herds treated. Very evidently, howerer, this favorable showing must be ascribed to the improved hygienic conditions that were established because other reports have been almost unanimously unfarorable. Thus Hutyra failed to discover any improvement in the course of one to three years in three herds that had been subjected to the Belring treatment. Eber, in 1907, in testing 148 animals that had been treated strictly according to the prescriberl Behring method, found 56 reactors, i. e., $37.8 \%$. Tuberculin tests conlucted during the two following years on seven estates gave $52.2 \%$ of positive reactions among 90 inmunized cattle. The percentages increased with the age of the animals in the same proportion as is usually observed in extensively infected herds (among 55 vaccinated animals $30.9 \%$ reacted at the first test, two years later, at the second test, $58.2 \%$ reacted $)$. Of 36 of the vaccinated eattle that dier in the interval or were slaughtered, 16 were tubereulous.

The results obtained on four estates in Galicia by Nowack were similarly unsatisfactory. Of 105 vaccinated cattle $44 \%$ reacted at the ent of the first year and of 138 vaccinated cattle $68 \%$ reacted at the end of the second year. Thirteen of the reactors were slaughtered and all found tuberculous.

In Swerden Regnér and Stenstroem conducted raccination experiments since 1904 in 8 infected herds enforcing no other hygienic measures. Of 142 animals vaccinated $61.2 \%$ reacted to a subsequent tuberculin test. After deducting 33 animals that were possibly infected at the time of vaceination $49.5 \%$ of the remaining 109 animals gave positive reactions, while $59 \%$ of the 61 control animals gare positive reactions to the tuberculin test. On one estate $57 \%$ of 16 animals vaccinaterl reacted to a subsequent test while only $20 \%$ of the 15 control animals reacter. In a few cases, however, raccination seemed to have a favarable influence and eren produced curative effects in already tuberculous animals.

Finally, Weber \& Titze report on the raccination of 206 cattle located on 6 different estates. The results are summarizer by the statement that while some of the animals became more resistant by the treatment the experiment was by no means a conspicuous success. In one herr that hal previously been entirely freed 
from the disease by the Bang method the disease spread just as rapidly among the raccinated as it had spread among the unvaccinated animals at the time when the Bang method was introduced.

A favorable report is made by Strelinger with reference to his experience of fice years on the Domain of Sárvár in Hungary. During this time the percentage of reactors was reduced to $9.6 \%$, however, not without the simultaneous enforcement of general prophylactic and hygienic measures.

Pearson \& Gilliland as well as de Schweinitz \& Schroeder immunized cattle in the United States by the intravenons injection of human tubercle bacilli. Detailed reports of the practical results obtained have, however, not yet been published.

2. Single Vaccination with Human Tubercle Bacilli (Vaccination with Tauruman; method of Koch \& Schuetz). According to the experiments of Koch, Schuetz, Neufeld \& Miessner it is possible to immunize cattle against highly virulent bovine bacilli by means of a single injection of 1 to 3 eg. of human tubercle bacilli (one dose of Tauruman contains $0.01 \mathrm{gm}$. of virulent human tubercle bacilli in emulsion). The resistance of animals thus immunized has been tested after a period of at least 169 days in only three cases. If we consider that a more durable immunity can hardly be expected from a single intravenous injection of human tubercle bacilli than from a double injection of even larger doses of the same bacilli and further, that Tauruman bacilli may remain active in the body of the treated animal for a period of $21 / 2$ years the results of experimental investigations above referred to as to the practical value of bovovaccine would probably apply with equal force to the practical value of Tauruman.

Although Tauruman has been used for vaccination since 1905 extensive reports on the practical results of its use are not obtainable. Eber reports on the vaccination of 48 cattle on four different estates; after about two years 10 out of 21 of the vaccinated animals reacted to the tuberculin test. Weber \& Titre raccinated 63 calres on two estates and achieved apparently good results which, however, were brought about by the fact that simultaneously with the introdnction of the vaccination one cow, affected with mammary tuberculosis was removed from the herd and all calves were thereafter reared upon boiled milk. The only temporary character of the resistance following treatment with Tauruman is shown by an experiment of Hutyra, in which a vaccinated calf received eleven months after treatment an intravenous injection of $0.02 \mathrm{gm}$. of bovine bacilli and lied 18 days thereafter of acute miliary tuberculosis.

3. Immunization with Avirulent Human Tubercle Bacilli (Dresden Method of Klimmer). According to Klimmer cattle may he immunized for a period of one year either with human tubercle bacilli that have been attenuated by heating at $52^{\circ}$ to $53^{\circ} \mathrm{C}$. or with pure cultures of avirulent tuhercle bacilli that have been passed through salamanders (originally virulent human tubercle bacilli repeatedly passed through salamanders) in the same manner as with virulent human tubercle bacilli. Both of the viruses referred to are said to be nonpathogenic for animals (even guinea pigs) and do not regain their pathogenicity by simple passage through animals. On account of this nonpathogenicity and the fact that bacilli introduced subeutaneously into the bodies of cattle are soon destroyed and completely removed, this method of vaccination may be repeated every year without jeopardizing the health of persons that consume the meat of animals disposed of by slaughter at any time, and thus the neriod of immunity may be prolonged indefinitely and at will. In addition, vaccination by this method is said to exert a curative influence upon already infected animals.

Cattle twice treated with these viruses successfully resisted experimental infection with virulent bovine bacilli after a period of from 2 to 9 months. No difference was noted in the protective value of the two forms of virus. According 
to Klimmer's reports about 10,000 cattle were treated successfully by this methoi (strict prophylactic and hygienic measures, however, were always enforced-rearing calves with pasteurized milk or the nilk of healthy nurse cows, removal of all cows with open tuberculosis, isolation of raccinated animals as much as possible, ete.). In the course of this work 43 vaccinated animals were destroyed and found free from tuberculosis on post-mortem examination. Gloeckner also reports favorable results from the raccination of 23 cattle, four of which were slaughtereil at a later date, one of these a non-reactor before vaccination was found to be free from tuberculosis, anil the other three reactors at the time of vaccination, showed post-mortem evidence of the curative effect of the treatment.

On the other hand Eber is of the opinion that the good results obtained are to be attributed to the simultaneous prophylactic ani hygienic measures. Weber \& Titze hold the same opinion. The latter exposed two vaceinated anil two unvaceinater control calves to infection from a cow with pulmonary tubereulosis, after three months all were slanghtered and found affected with tuberculosis in approximately the sanie degree. These experimenters are inclined to regari the bacilli contained in the avirulent vaccine as so called piscine tubercle bacilli or reptilian bacilli.

\section{Immunization with the Products of Metabolism of Tubercle} Bacilli (Heymans' Method). Heymans introduces human or bovine tubercilli, enclosed in the membrane of reeds under the skin of cattle, assuming that the bacilli will continue to vegetate and diffuse their products of metabolism through the membrane, whereupon they pass into the circulation, and immunize the body of the animal thus impregnated with them even exerting a curative effect upon already existing tuberculous processes. In the effort to maintain an effective degree of immunity by annual repetition of the immunizing process it is found that the degree of sensitiveness to tuberenlin is gradually diminished and that 50 to $60 \%$ of the animals will cease to react to the third test. With the aid of this method it is claimed to be possible to exterminate tuberculosis without resort to other hygienic measures, in $80 \%$ of the herds treated. In badly infected herds, however, this is possible only with the aid of other prophylactic measures.

As to the possibilities of this methor which has been applied to over 20,000 cattle in Belgium, thus far only Heymans' reports have been available. Accoriling to these the percentage of reactors in the vaccinaterl herds has been considerably reduced year after year. Thus, for example, of 18 herds comprising 188 eattle tested in 1907, and retested in the two following years, the percentages of reactors were $45.32 \%$ and $21 \%$, respectively. Only $50 \%$ of the reactors of the first test responding positirely two years later at the third test. That the tuberculin test retains its diagnostic value after the subjection of animals to this treatment is evidenced by the fact that on post-mortem inspection more than 1,400 treated cattle, which had given negative reactions to the test, were found free from tuberculosis.

Experiments with 18 vaceinated and 13 control animals showed that raccinated animals possessed $\bar{a}$ higher degree of resistance to subcutaneous as well as to intestinal infection than not vaceinated eattle. Moussu, however, found that four cattle that had clay tubes containing eultures of tubercle bacilli imbediled in their tissues for some time, were affected just like cattle not thus treated when subjecter to intravenous infection with virulent bacilli. The sermu of similarly treaterl experiment animals showed no eridence of possessing any immunizing properties. However, animals with culture tubes of bacilli imberded in their tissues will react to the tuberculin test. After the bacilli have died off, or after their remoral from the body, the animals cease to react.

Technic of Immunization. In the first two methorls disenssed the virus or vaceine is injected into the blood stream while in the third and fourth methorls it is applier subeutaneously.

1. Behring's Method. The rirus (bovoraceine) consists of cultures of human tubercle hacilli of a certain strain drierl in a varum. "'he powder-like sulustance thus obtained is prepared for use in quantities sufficient for immeriate 
requirements by triturating in a mortar with sterilized, lukerrarm normal salt solution, to form an emulsion. The emulsion should be prepared in two strengths. That used for the first vaccination should contain $0.004 \mathrm{gm}$. of dried culture per 2 ce. of fluid, while that for the second vaccination should be double this strength or contain $0.004 \mathrm{gm}$. per one ce. of fluid ( $1 \mathrm{I} . \mathrm{U} .=0.004$ of dry raceine). These emulsions are used in 2 ec. doses, i. e. $1 \mathrm{I}$. $-\mathrm{U}$., for the first treatment and in 5 ec. loses, i. e. 5 1 I.-U., for the second treatment, in each case injection being made into the jugular vein.

The most suitable age for vaceination is from one to four months. Calves otherwise in good health are not injured in any way by the treatment. If the calves are tuberculous at the time of the first treatment a reaction follows, similar to that produced by tuberculin, while the second treatment gives rise to a febrile reaction in healthy calves as well. After this reaction has subsicled the animals continue to develop and increase in weight in a normal mammer. Unfarorable results are obtained only in animals affecter with some disease at the time of treatment, especially so called calf-pneumonia. Roemer recommends that, in addition to the vaceination of calres, as soon as possible after birth all calves should be fed with milk free from tubercle bacilli, and that all cows with open tuberenlosis should be remored from the herd at once. Tallée \& Rossignol recommend, also, that calves should not he exposed to infection from the first raccination or untii 6 weeks after the second vaccination.

Later. Behring introduced, or recommended, a new method, Tauro-vaccination, in which an agent with anti-tubereulous properties (?) is added to the vaceine, resulting in marked attenuation of the latter which is injected subcutaneonsly insteal of intravenonsly. Accoriling to Lignières oily emmlsions are better adlapted for vaccination than aqueous emulsions. These methods hare not been applied in practice.

2. Method of Koch \& Schuetz. Tauruman, a ready-for-use emulsion of bacilli, ( $0.01 \mathrm{~g}$. virulent human tubercle bacilli in emulsion). after breaking off the point of the glass tube containing the vaccine, is drawn out with a hypodermie syringe and injected into the jugular vein in a manner similar to that follower in the use of bovo-vaccine. A second application is not made in this methorl.

What has been stated in regard to immmization with boro-raccine might also be applicable in this case. Since both vaccines contain virulent tuberele bacilli the greatest cantion should be observed in their manipulation (Moeller reports repeated serions infection of his assistant with Taurmman).

3. Method of Klimmer. In the "Dresilen Method of Immunization to Tuberculosis., emmlsions of attennated human tubercle hacilli (TH) or avirulent tuberele bacilli (AV) (the latter especially for milk cows) were formerly useit. More recently, howerer, avirulent material only, known to the trade as Antiplny. matol, has been in nse. Of this material doses of 5 ce. are injected subcntaneously on the side of the neck. The raccine must be preserved in a cool dark place and used within three weeks. Cattle may be treated from the age of three days to 18 months, or older animals that are free from tuberculosis. Vaceination must be repeated after three months and anmully thereafter (young animals suspected of being tuberculous are allvantageously treated four times during the first year at intervals of three months). It is recommended to protect animals from exposure to infection and to feed calves previous to immunization, and for two months after with milk that is free from any infection. All animals should also be subjected to the ophthalmic tuberculin test before treatment. The non-reactors should then be placed in the most farorable positions in the stables, particular eare being observed that healthy animals are not stabled so as to face zeactors.

4. Heymans' Methor. The raceine consists of dried hmman tubercle bacilli enclosed in gelatinized recd sacs. The process of raceination eonsists in the introduction of one of these saes under the skin of the croup or back by means of a specially constructed trocar.

Cattle of either sex or of any age may be vaceinated whether they are free from tuberculosis or infected, but in order to obtain permanent results the vaccina. tion must be repeated every year. Special hygienic measures are not necessary.

Other Methods of Immunization. Aside from the very numerous, more or less successful laboratory experiments in the immunization of small animals for which purpose varionsly attenuated or dead tubercle bacilli as well as toxic substances and secretions have been chiefly used, a few other methols that have been recommended for actual practice will be briefly mentioned.

Lignières introdnced subcutaneous vaccination with living human tubercle baeilli into practice in Argentine. (The hmman tubercle bacilli were either in. 
emulsion or homogeneous liquid eultures). Baumgarten had then already shown that a single subentaneous vaccination with human tubercle bacilli increased the resistance of eattle to a marked degree. Hutyra had also shown that cattle thus raccinated would be protected for a time against infection by intravenous injection of $0.04 \mathrm{gm}$. bovine tuhercle bacilli but that such immunity diminished noticeably after six months. According to Arloing's experiences subcutaneous vaccination loes not produce as high a degree of resistance as the intravenous administration of vaccine.

2. Friedmann reconmended a strain of tubercle bacilli for vaccination of eattle which be bal cultivated from a turtle, and successfully protecterl rabbits against bovine bacilli. After Libbertz and Ruppel and also Orth failed to confirm the favorable results clained by Frieimann, Weler \& Titze showed that while the repeated intravenous administration of large masses of bacilli ierived from cold-blooded animals, or of acid-fast grass bacilli, no loubt might increase the resistance of cattle to infection with bovine bacilli to a slight extent, the effect is so slight that the practical application of the method is of questionable value.

3. Accorling to Behring as well as Calmette \& Guérin it is possible to imnunize cattle against intestinal infection by the administration of a small quantity of bovine lacilli per os. Roux \& Vallée obtained good results by two administrations, per os, to young calves, at intervals of three months, of $0.20 \mathrm{gm}$. doses of equine tubercle bacilli or of viruent bovine bacilli. Animals thus treated were fouml, in some cases to possess greater resistance to subsequent infection than those immunized by the intravenous method. The advantage of this method of immunization is claimert to be that bacilli thus artministered are completely absorbed in a period not longer than 7 months. On the other hand, alimentary infection occurring during or immediately preceding the period of preventive treatment will give rise to a progressive tubereulous condition. Even Vallée himself admits that attenuated human tubercle bacilli, the alimentary administration of which gives similar results, cre perhaps more suitable for this purpose.

4. Arloing uses homogenized cultures of human or bovine bacilli grown ou $6 \%$ glycerin bonillon for the immunization of cattle. These cultures do not prorluce microscopic tubercules in small experimental animals or in calves but only microscopic changes of the Yersin type. The cultures are administered intravenously, subcutaneously or per os. Accorling to the first methorl young calves receive $0.5 \mathrm{ec}$. for the first dose; two months later $1.5 \mathrm{cc}$. of a culture one month old is injected into the jugular vein (older animals receive 1.0 to $1.5 \mathrm{cc}$.). In the subcutaneous method both the first and second applications are made subcutaneously ( 2 ce. of culture being injected each time in the side of the neck or in the flank). According to the third method the first dose consists of 15 ec. and the second dose, two months later, of $20 \mathrm{cc}$. of eulture carefully injected into the mouth so that the animal will swallow the same. In all three methods it is necessary to follow up the treatment with annual subcutaneous injections of 2.0 cc. of culture in order to maintain an effective degree of immunity. These treatments always result in tuberculin-hypersensitiveness which, however, gradually leads in about six or eight months to the opposite condition, that is a tolerance. Moderate fever of short duration follows the intravenous as well as the subentaneous administration of the virus and in the latter method a local tumor with friable cheesy contents develops at the point of injection. This usually disappears in the course of several months.

Results of the vaccination of 60 eattle ranging in age from 4 to 12 montls, with 30 control animals in the experiment, were only partly successful. After exposure to infection only about $50 \%$ of the vaccinated animals remained healthy; of the infected animals scme were only slightly affected but others were extensively diseased. Nevertheless the results as a whole were in favor of immunization. The fact that the cultures used were harmless for eattle, as well as for monkeys, also, deserves favorable consideration of the nethod. The intravenous method gave the lest results, the ingestion method came second and the subcutaneous method last in efficacy.

5. Vallée immunized horses by intravenous injections of successively increasing doses of, first, equine bacilli avirulent for guinea pigs, which then were followed liy human tubercle bacilli slightly virulent for guinea pigs. The injections produced no tuberculous processes whatever. After treatment for one or two years the serum of these horses, though possessing only slight agglutinatiug power, contained protective substances that could he demonstrated by the complement fixation method, and which in doses of 100 to $200 \mathrm{cc}$. protected young cattle against experi- 
mental infection with tuberculosis. Tallée expresses the hope that such serum may be used for therapentic purposes, in fact some humlreils of persons have already been treated with this material but reports on results have not as yet heen available.

Veterinary Sanitary Police Measures. The extraordinary prevalence of tuberculosis, especially among the improver breeds of cattle, and the consequent enormous losses have been the motive of government interference for the purpose of the controlling, and, as may be possible, completely exterminating the disease. On the very account of the great prevalence of the disease howerer, measures of protection and extermination that have proved successful for other animal plagues can hardly be applied to tuberculosis. With the exception of states and countries where the disease has as yet made little lieadway, general measures for compulsory slaughter can not be considered because such a procedure, aside from the enormous financial sacrifice, would otherwise most seriously affect the best interests of the live stock industry of the country and of other interests generally. Tnder existing conditions, therefore, and for a long time to come, govermment activity must be confined to gradually enforced measures of repression and the extension of every possible aid to such owners of live stock whose earnest endeavors are aimed at the extermination of the evil.

In order to reduce the danger of exposure and infection official regulations should require, above everything else, that animals with open tuberculosis, and particularly cows with tuberculosis of the udder, be excluded from trade by rigid quarantine and destroyed as soon as possible, subject to reasonable compensation to the owner from government appropriations or from funds provided by a system of obligatory live stock insurance. To aid in the enforcement of this measure it should be made the duty of meat inspectors to report every case of tuberculosis to the proper anthorities whereupon it would devolve upon the latter to make an official investigation of the herds from which the diseased animals originated and cause any and all evidently diseased remaining animals to be disposed of by slaughter subject to proper inspection.

The permanent professional supervision of all herds, but particularly and primarily of dairy herds, is justified by the importance of an early recognition of clinically affected or suspected animals so that the milk from these animals, which is dangerous to the health of human beings, shall not be put on the market unsterilized. Milk from infected herds should not be permitted to be sold unless previonsly heated to at least $80^{\circ} \mathrm{C}$., and the product of animals with affection of the udder should under no circumstances be permitted to be sold, given awav or otherwise utilized. In addition, official regulations should provide that all skim milk and other by-products from creameries and skimming stations must be heated to at least $80^{\circ} \mathrm{C}$. before they may be returned to the producer 
or to other persons. Further, governments should offer premiums, and otherwise aid in the acquisition of such male animals only as have been shown to be free from tuberculosis br the tuberculin test or by protective inoculation. This would have the additional advantage of stimulating the breeders to make every effort to produce healthy animals because of the greater value of the young animals. Finally, a properly organized and effective system of meat inspection, aside from its value in locating centers of infection, would add to the success of the whole undertaking by insuring the destruction of badly infected animals or their diseased organs, and preventing their sale as food.

The gorernment may assist or support efforts on the part of private individuals by paring the expenses of professional advice or supervision where the Bang system for the extermination of the disease is attempted, and by furnishing the necessary tuberculin free or at cost of production. Such aid, however, should be extended only on condition that the owners of the herds carry out to the letter all necessary measures to insure successful prevention of infection of healthy animals, dispose of all clinically affected or suspicious animals by slaughter within a specified time and exclude all reactors from trade. In this connection it is advisable and necessary to provide proper means of identification of all reacting animals because without this the tuberculin test may merely serve to assist certain owners to dispose of their diseased animals by sale and thus bring about a still wider dissemination of the disease. For the same reason the manufacture and sale of tuberculin and its importation from other countries should be controlled by the government. Finally, the dissemination of information by the state or gorermment in regard to the nature and control of tuberculosis would be of great aid in a campaign of suppression directed against this disease.

Since 1909 the live-stock sanitary laws of Germany require owners to report to the proper anthorities all clinically recognizable forms of the disease affecting the lungs, udder, uterus or intestines. Provision is made for the official condemination and destruction of such animals or their safe quarantine, disinfection of infected premises and the marking of quarantined cattle for future identification. The milk from such animals must be sterilized by heat before being sold or given away and in case of tuberculosis of the udder it is absolutely excluded from use as food for human beings.

Austria has enforced very similar laws since 1909 , requiring the notification of all cases of clinical tuberculosis to the authorities, isolation and marking of animals thus affected, disinfection of infected stables, compulsory slaughter of calves (!) from diseased cows and government support for the control ani externination of the disease.

In Hungary, since 1910, eattle with tuberculosis of the udder are confiscated l,y the government and immediately slaughtered. In addition, the use of nilk for himan food from cows with open tuberculosis is forbidlen and all skim milk from rreameries and skimming stations must be pasteurizerl before it may be sold or given away.

The most snccessful results in the control and extermination of tuberculosis are to be found in the northern states of Denmark, Norway, 
Sweden and Finland, where individual efforts on the part of owners have for years received the support of legislative measures (see also statistics on page 502).

The endeavors of the Danish Government for the extermination of tuberculosis, which are worthy of emulation, began in 1903 when annnal appropriations of 50,000 erowns were maile for the support of the work. These appropriations were later increased to 100,000 crowns. At first tubereulin was furnished free of expense for young animals only, later it was furnished for all cattle upon the same basis, and beginning with 1599 the government assumed all expenses connected with the control and extermination of the disease. The laws of 1898 preseribe obligatory Pasteurization at $85^{\circ} \mathrm{C}$ for all skim and butter-milk intended for feeding animals. This resnlter in an immediate reduction in the percentage of tuberculosis in calves and even more so in swine. In 1904 the preseribed temperature of $85^{\circ} \mathrm{C}$ was reduced to $80^{\circ} \mathrm{C}$. The laws of 1898 also require the compulsory slaughter of all cows with tubereulosis of the udder and provide compensation to the owners. This resulted in the annual withdrawal from trade of 600 cows with udder tnberculosis out of a total of about one million cattle. For seren years (1898-1904) the annual losses were as follows; 407, 592, 610, 584, 615, 583 and 689 respectively.

In Sweden methods of extermination inaugurated in 1897 along lines similar to those upon which Danish operations were based gave equally happy results. While, for example, tuberculin tests of 1370 herds comprising 48,576 animals gave 14,225 reactors $(29.3 \%)$ in the beginning of the campaign, tests of the same herds in 1908 , then comprising 57,660 animals gave only 1765 reactions $(3.1 \%)$. This result was obtained at a cost to the government of 830,000 crowns.

On account of the difficulties or obstacles above discussed the compulsory official destruction of all animals affected with tuberculosis has not given satisfactory practical results. Thus, in Belgium, in 1895, all animals affected with clinical tuberculosis, as well as all reactors to the tuberculin test were ordered killed within a preseribed time. As a result, of 2,905 herds comprising 19,004 cattle in 1896, 9280 were slanghtered. The surprisingly enormous expense of this procedure and the opposition offered by the owners brought about a modification of this order so as to apply to elinically affecter animals only. Even with this restriction the enforcement of the order resulted in the destruction of 10,269 eattle in 1902 at a cost to the state, in indemnification expenses, of about one and one half million franks. In the North American State of Massachusetts a similar radical attempt inangurated in 1895 was abandoned at the end of the first year.

The official tuberculin testing of import animals before they are permitted entrance within the loorders of a country, a practice in force in a number of states has not been fonnd effective except from a purely sanitary police point of view. This is due to the fact that injections of tuberculin made immediately before shipment (plugging) robs the tubereulin test subsequently applied of its diagnostic valne. A measure of this kind is in effect along the North German horder for all imported cattle. While $30.8 \%$ of the 74,813 quarantine cattle reacter to the tuberculin test during the first year of the enforcement of this measure the percentage of reactions has fallen off heavily in the following years although a considerable percent. of the animals were found tuberculous at the time of slanghter. Thus, of 772,037 cattle subjected to the tuberculin test in 1899 to 1908 only 9,197 , i. e. $1.2 \%$ reacted, although 176,554 tuberculous animals were found at the subsequent slaughter of 748,628 nou-reactors, i. e. $22.2 \%$, and of these 2407 were affected with generalized tuberenlosis (!)

Literature. Villemin, Rec., 1867. 5. - Chauveau, Ibid., 1869. 202; Rev. gén., 1907. IX. 497. - SeMMer, D. Z. f. Tm., 1876. II. 209. - Virchow, B. kl. W., 1880. Nr. 14. - Косн, B. kl. W., 1882. 221; Mitt. d. G.-A., 1884. II. 1; D. m. W., 1897. 207. - JoHNE, D. Z. f. Tm., 1883. IX. 1 (History, with Lit.). Bang, Ibid., 1885. XI. 45; 1890. XVI. 353; Z. f. Tm., 1902. 81. - Hess, Schw. A., 1889. 155; 1896. XXXVIII. 210. - JENSEN, D. Z. f. Tn., 1891. XVII. 255 (Lit. on tub. of dogs and eats). - САDiot, La tnb. du chien. Paris. 1893 (Lit.); Monh., 1894. V. 97. - CADÉAC, J. vét., 1894. 723; 1905. 577; 1907. 65. - Kiтt, Monh., 1896. VII. 216; 1905, XVI. 267; 1907. XVIII. 385 (Review). - Malm, Rev. de la Tub., 1898. 337. - Nocard, Diet. de méd vét., 1892. XXI. 426; A. P., 1892. VI. 44; Rec., 1090. 721. - ARloing, C. R., 1898. CXXVI. u. XXVIII; Rev. gén., 1909. XIV. 781. - ERnst, Monh., 1900. XI. 229. (Review on Law in Milk). - FlẗgGe, Z. f. Hyg., 1901. XXXVIII. 1. - Behring, Beitr. z. exp. Ther., 1902. 1; D. m. W., 1903. 689; Tuberkulose, 1905. IV. 371. - OstertaG, Z. f. Hyg., 1901. XXXVIII. 45; Z. f. Infkr., 1910. VII. 1. - EBER, Z. f. Tm., 1905. IX. 81; 
Cbl. f. Bakt., 1097. XLIV 463; 1909. LiI. 389. - Calmette \& Guérin, A. P., 1905. XIX. 601; 1906. XX. 353 u. 609. - Ostertag, Breidert, Kaestner \& KrautStrunk, Arb. d. hyg. Inst. 1. tieräratl. IIsch. Berlin, V. H. 1905 (Lit. on Diagnosis). - Pearson \& Gilliland, J. of comp. Tath., 1905. XVIII. 207. - Koch, Sch'̈tz, Neufeld \& Miessner, A. f. Tk., 1905. 545. - Motssu, Rec., 1905. 777; 1907. 701. - Hermans, Arch. internat. de pharmacodynamie, $1905-1910$. XVII - XX. Meyer, Z. f. Tm., 1906. X. 241. - Fischer, Ibił., 1906. X. 82 (Lit. on Uterustub.). - Hutro.1, Z. f. Tm., 1907. XI. 2+1. - Pirquet, Vakzination u. vakz. Allergie. Tien 1907. - WeBer \& Titze, Thk.-Arb. d. G.-A. 7., 9. u. 10 H. 1907, 1908 u. 1910 (Lit. on Immmization). - TALléE, Bull., 1907. 180; Rev. gén., 190s. X1. 318; 1909. XTII. 355; A. P., 1909. XXVII. 555. - Lignières, Bull., 1908. 112 u. 517; 1909. 146. - Mohler \& Washburn, An. Ind., 1907. 215. - Schroeder \& Cotron, An. Ind., Bull. Nr. 99. - Much, Beitr. z. Kl. d. Tb., 1907. VIII. S5; 1968. XI. 175. - Wrssmann, Schw. A., 1908. L. 90 (Lit. on Tub. after Contraetion). - Titze \& Weidanz, The.-A. đ. G.-A., 9. H., 1908. - MAyer, Diss. Bern. 1908 (Lit. on sheep tub.). - Calmette \& Gúkrin, A. P., 1908. XXII. 1. - Klimakr, Z. f. Tm., 1908. XII. 81. - Klimmer \& Kiessig, Monh., 1908. XX. 97 ; Z. f. Tm., 1909. XIII. 313. - Römer, Z. f. Infkr., 1909. VI. 393, - Joest. Ibid., 1909. V. 224. VI. 257; 1910. VII. 131. - Albien, Z. f. Tm., 1909. XIII. 109 (Lit. on Concenial Tub.). - Bongert, A. f. Hyg., 1909. LXIX. 263. - Forster, Cbl. f. Bakt., 1909. LI. 417 (Lit. on sterlization of milk.). - Hensches, Diss. Bern. 1909 (Lit. on anatomical statisties). - WolfF-EISNER, Frïhdigan. u. Tbe.-Imm. Wiirzburg 1909. - Richter, Z. f. Infkr., 1909 V. 243 (Lit. on local reaction). - JosePH, B. t. W., 1909. 847. - GoEdecke, Tbe. d. Pferdes, Hannover, 1909. — FlïgGE, Verbreitungsw. u. Bekämpfg. 1. Tub. Leipzig. 1908. - NowaK, Z. f. Infkr., $19(19$. VI. 313 (Lit. on immunization).

Tuberculosis in Man. At the present day tuberculosis is the most destructive disease of mankind, estimates showing that one-seventh of all deaths, and one-third of all deaths occurring during the "working' age" of human beings, are the to this disease.

In the majority of cases it manifests itself as a chronic destructive affection of the lungs (consumption, phthisis pulmonum). In cases where the disease appear's primarily as an affection of the peripharyngeal and cervical lymph glands, the digestive organs or the bones, the development of pulmonary tuberculosis is usually only a question of time. In a certain number of cases the disease confines its entire course to the abdominal organs (abdominal lymph glands, especially in children). In exceptional cases the disease terminates as a generalized acute miliary tuberculosis.

As has been found to be the case in animals, man also with few exceptions acquires the disease in extra-nterine life. Infection is caused as a rule by the human type of tubercle hacillus which is disseminated in large quantities by diseased human beings, particularly through the medium of expectoratel secretions from the lungs. The bacilli are then inhaled or ingested by persons that come into contact with the patients, particularly when they live with them in the same close, dirty and poorly ventilated dwellings.

The virus of animals, and especially that of bovine tuberculosis plays a less important role in the infection of man. Experimental investigations of recent years have shown, however, that man is susceptible to infection with bovine virus. This has been demonstrated by well anthenticated cases where human beings became affected after consumption of milk from 
tuberculous cows or after injuries acquired when making postmortem examinations of tuberculous animals. Even more positive in this respect has been the evidence furnished by the fact that tubercle bacilli, agreeing in their pathogenic properties in every particular with those of the bovine type, have been isolated from the lesions of tuberculous persons, especially children (see p. 623).

If, in spite of this, bovine tuberculosis plays only a secondary role in the dissemination of tuberculosis in man the cause for this, may in part at least, be found in the fact that human beings do not live in as close association nor come in as intimate contact with tuberculous cattle as they do with their fellow beings.

Pulmonary consumption, the commonest form of human tuberculosis, appears to be caused almost always by the human type of the bacillus. Koch only recently emphasized the fact that up to that time the bovine type of bacillus had not been demonstrated in a single ease of human pulmonary tuberculosis. Indeed Kitasato could find only the human type of bacillus in 152 and Dieterlen in 50 examinations of sputum from consumptives. But Mohler \& Washburn as well as Beitzke report each one case in which they succeeded in obtaining pure cultures of the bovine type from such material. In general the bacilli of the bovine type seem to possess only a slight tendency to spread in the human body, consequently they produce, in the majority of cases, only local lesions in the region of the point of infection (peripharyngeal, cervical and mesenteric glands).

The danger which threatens man in the direction of bovine infection demands the maintenance of every measure of protection by means of which this danger may be averted.

In this respect all raw animal products, and particularly milk, demand adequate consideration. There seems to be no question as to the justification of the demand that all cows with open tuberculosis and such as have affected udders be unconditionally excluded from dairy purposes and that all milk offered for sale to the public should be subject to professional or expert supervision. Herds supplying ereameries and skimming stations should be subjected to particularly stringent regulations for the reason that a single infected herd or animal may be the means of contaminating the entire product of a large station. Control of this character should confine itself not merely to the exclusion of diseased animals but also to enforcement of general sanitary regulations in the production and handling of the milk, thus insuring the healthfulness of the various other dairy products, butter, cheese, etc.

Since suitable measures of control of this kind are at present enforced in very few localities, it is incumbent upon every individual to observe certain measures for his own protection. Uncertified milk should not be used as food without previous boiling or at least heating to $80^{\circ} \mathrm{C}$. Children, in 
particular, should be fed exclusively on pasteurized milk on account of their susceptibility to infection.

The possible danger attending the consumption of meat from tuberculous animals, though of much less consequence than that attending the consumption of dairy products, may be entirely overcome by a general system of obligatory meat inspection. Asicle from the destruction of tuberculous organs, all tuberculous meat should be condemned and all suspicious carcasses should be sterilized by heat before they are permitted to be placed on sale.

Since human beings are exposed to the danger of contracting disease in infected stables, attendants should not remain in them for longer periods than necessary and under no circumstances use such stables as dormitories. In dwellings, adequate measures should be taken to protect residents from possible infection by tuberenlons dogs and birds (parrots).

Finally, persons employed in the care, or otherwise, of tuberculous animals and in the examination of tuberculous carcasses should always be on their guard in the observation of ordinary precautionary measures.

Literature. S. Cornet, Nothnagels Hanlbueh, 1900. XIV.

\section{(b) Tuberculosis of Fowls. Tuberculosis avium. Avian Tuberculosis.}

Historical. After Panlicki had reeognized the similarity between this disease in birds and tuberenlosis of human beings and mammals even before the discovery of the tuherele baeillus, Koch showed in 1882 that a disease of fowls existed which was caused by a hacillus similar in its morphologieal and staining characteristies to the tubercle hacillus. Koch as well as Ribbert looked upon the disease as identical with mammalian tuberculosis and occasional observations that fowls apparently became infected from the expectorations of siek persons (Joline and others) seemed to confirm this view. Rivolta (1888) however could not succeed in his efforts at experimental infection of fowls with mammalian bacilli and therefore declared tuberculosis of mammals and of birds to be etiologically distinet diseases. Sinee this time avian tuberenlosis has been the subject of numerous investigations which, however, have not led to uniform results and the question of the identity or relationship of the two forms of disease is still an open one. While Strauss and others, also the pupils of the former, Gamaleia and Wuertz, and more recently Koch also stood for the non-identity of the two diseases on account of the difference in virulence of the bacilli, Maffucei took the same stand on the basis of the morphological and cultural differences; more recently Weber \& Bofinger (1904) have expressed themselves as accepting the same view but Cadiot, Gilbert \& Roger, Fischel, Hueppe, Johne, Nocard, Behring, Roemer as well as M. Koch \& Rabinowitsch have taken the opposing position in claiming specific identity for the two forms of bacilli (see p. 617).

Occurrence. Avian tuberculosis occasionally appears enzootically among fowls and pigeons, causing enormons losses 
(Zuern found tuberculosis in $10 \%$ of deaths in these animals). Among water fowls the disease, though generally much less prevalent, also appears as an enzootic (Huss). Birds kept in captivity are also affected (M. Koch and Rabinowitsch found 118 tuberculous subjects among 459 birds that died in the Zoological Garden in Berlin).

Etiology. The bacillus tubereulosis avium obtained from tissues is usually shorter and stains more evenly than the bacillus of mammalian tuberculosis, but otherwise agrees with the latter in staining characteristics (see Fig. 95).

Cultivation. On glycerin blood serum the bacillus thrives much better than the mammalian variety, glassy, transparent, roundish colonies being visible on the surface of the nutrient medium as early as the 8 th and sometimes even the 5th day. Later they become confluent and form a grayish-white or yellowish-red, moist, slimy, stringy, smeary scum that is easily emulsified in water. The bacillus thrives best between $30^{\circ}$ and $40^{\circ}$ C., but will grow also at $25^{\circ}$ and at $45^{\circ} \mathrm{C}$. In cultures grown at a high temperature we find aside from short rods also longer forms that stain uniformly or exhibit a granular appearance, some of them thick and club-shaped or even branched (Maffucei). On potatoes they form a grayish-white, blackish or reddish, moist, smeary, crumpled layer (Matzuschita; see Plate II); in glycerin houillon granules develop on the floor and wall of the vessel while only some of the strains form a dry wrinkled membrane on the surface of the

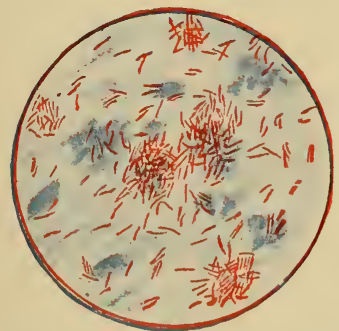

Fig. 95. Bac. tuberculosis avium. Articular exudate from chicken; Stain according to Ziehl-Nelsen. liquid (Weber \& Bofinger). On some media, e.g., glycerin chicken bouillon, egg yolk, etc., occasionally also on ordinary media, the cultures of some strains present an appearance similar to that of mammalian tubercle bacilli.

Not all tubercle bacilli obtained from birds will show the above culture characteristies, but the greatest variety of transition forms occur, including the dry sealy cultures of mammalian bacilli. (M. Koch \& Rabinowitsch).

Tenacity. The bacillus of avian tuberculosis is on the whole more resistant to external influence than Koch's bacillus. Thus a culture two years old may still contain living bacilli. Temperatures of $70^{\circ} \mathrm{C}$. require 15 minutes to destroy them. (Maffucei).

Pathogenicity. Experimental infection of fowls is most certain by introduction of the virus into the blood stream. One or two months later, upon the appearance of symptoms of marasmus, small tubercles are found in the spleen and liver, while the blood vessels of these organs are filled with masses of bacilli. Intraperitoneal or subcutaneous inoculation give less certain results. These are followed by local foci only, but the affected animal may die from the toxic effects of the lesion. A single injection of culture masses suffices as a rule for infection. The fowls die after one or two months 
with characteristic symptoms of abdominal tuberculosis. Geese and ducks may be infected in the same manner (TVeber \& Bofinger). Pigeons possess a much greater power of resistance to experimental infection. Articular tuberculosis is, however, readily produced by injection of the bacilli into the articulations.

Rabbits are very susceptible to experimental inoculation, and the feeding of cultures to these animals produces strmptoms very similar to those following infection with bovine tuberculosis (intravenous administration of the virus is frequently followed by tuberenlosis of the joints and of the adjacent hones and tendon sheaths). On the other hand, guinea pigs offer a somewhat greater resistance to such infections; althongh they get sick after treatment with rather large amounts of virus, morbid changes (nodules and ulcers) usually develop only around the masses of bacilli found in the body and which always develop rather slowly (much the same as lesions occurring around other foreign bodies). A progressive tuberculosis is a rare occurrence. Intraperitoneal infection, however, produces death from tuberculous septicemia ("Type Yersin"') (O. Bang). The virulence of the bacilli is frequently completely destroyed in the bodies of gninea pigs (M. Koch \& Rabinowitsch). Of the larger mammals, cattle and goats respond to subcutaneous injection much like guinea pigs. Intraperitoneal administration causing death from tuberculous septicemia. Animals of mature age are very resistant to the alimentary administration of avian tuhercle bacilli while very young animals (calves, kids and colts) develop acute tuberculosis which affects the intestines and mesentery with particular severity, occasionally also the lungs, and which terminates fatally (O. Bang). Swine seem to be only slightly susceptible, dogs and cats not at all, while mice and rats are readily infected through the alimentary tract.

Natural infection of healthy fowls is brought about by taking up the droppings of infected birds. When intestinal ulcers are present such droppings contain enormous masses of bacilli. Still more frequently is infection hrought about by permitting healthy fowls to have access to tuberculous cadavers that have been consigned to the manure pile. The liver and spleen of such cadavers contain large masses of bacilli.

Infection from tuberculous persons. In view of the very slight susceptibility of fowls to infection with mammalian tuberculosis (which has been demonstrated experimentally) infection from the sputum of human beings probably occurs only in such rare cases in which the lung secretions of consumptives contain avian tubercle bacilli (see p. 618). Infection from tuberculous mammals is undoubtedly also a rare occurrence (parrots, however, are an exception in this respect. See p. 615). On the other hand, the possibility exists that 
spontaneously infected mice or rats may be the means of spreading the disease (Rabinowitsch).

Infection may also take place through injuries of the mouth or of the skin; the possibility of an infection through the air passages by means of inhaled air containing the bacilli must also be admitted.

Heredity plays only a subordinate role in the dissemination of the disease. Maffucei demonstrated that the embryo of experimentally infected eggs is capable of development and the possibility also exists that they may become infected through tuberculous ovaries. According to Lichtenstein, who found that all the progeny of an infected cock had the disease, there exists also the possibility of a germinative infection through the male parent. Chicks however that have been hatched from infected eggs live at most only a few months before they succumb to the effects of the disease. On the other hand the plague may be introduced into healthy flocks through the means of infected eggs the albumen of which oceasionally contains bacilli (Nohler \& Washburn). Rabinowitsch observed the hatching of 8 chicks from 32 experimentally infected eggs. Of the eggs infected with avian tuberculosis the embryos died in $90 \%$, of those infected with guinea pig tuberculosis $70 \%$ and of those infected with bovine tubereulosis $40 \%$ died.

Pathogenesis. The bacilli, which usually gain entrance into the alimentary canal with the food, usually pass through the solitary and agminated lymph follicles of the large and small intestines into the intestinal walls and thence, with the blood of the portal vein, enter the liver or they gain access into the general circulation and lodge in other organs and portions of the body, thus in the spleen, lungs, joints and tendon sheaths, causing the development of specific lesions. The intestinal mucous membrane may remain perfectly intact or small nodules may develop in its tissue. The overlying villi may present no changes in the beginning but ulceration follows at a later time. These ulcers may heal with the formation of granulation tissue (Schern). The nodules occurring in the intestinal walls under the serosa develop from the lymph nodes in the peripheral layers of the intestinal walls, or between the serosa and muscularis of the cecums, or from the agminated follicles in the mucous membrane without causing any observable changes in the intestinal mucous membrane which, however, they perforate at a later stage and then communicate with the lumen of the intestine (M. Koch \& Rabinowitsch).

The symptoms of cachexia observable in diseased fowls (emaciation, anemia, marasmus) are a result of the toxic action of the chemical products of the bacilli. These toxins, which kill guinea pigs with the same symptoms as those observed in chickens, may be produced from artificial cultures, particularly those grown on liquid glycerin serum (Maffucci, Héricourt \& Richet). The death of dogs and guinea pigs 
following the administration of large masses of virus is ascribed to the same cause since no tubercles develop in the body. The same is true of the excessive irritation, fever, suppuration at the point of injection and the temporary swelling of the regional lymph glands which are observed after the subcutaneous administration of arian tubercle bacilli in cattle (Kossel, Weber \& Heuss).

Anatomical Changes. On post-mortem examination the liver and spleen are most frequently found affected and this is not rarely the case when the intestine and peritoneum are entirely free from changes. As a rule there are nodules ranging in size from that of a lentil to that of a pea, and sometimes approaching that of a hazelnut, with colorless, gelatinous or yellowish, friable, cheesy and occasionally calcified contents and surrounded or enclosed by a connective tissue capsule. In rare cases minute gray or pale yellow points, here and there collected into groups under the serosa, are scattered throngh the tissue of the liver. The diseased liver, especially in chronic cases, is considerably enlarged and may be ratler friable on account of fatty degeneration. Now and then hemorrhagic areas occur near the surface of the organ.

Affection of the intestine, especially near the cecums, is comparatively common. The mucons membrane is covered with small yellowish nodules among which funnel-shaped ulcers with finely nodulated or raised borders may be present. On the serons surface there may be firmly attached coarsely nodulated roundish tumors that may approach the size of a hazelnut. These may also occur in the mesentery (Fig. 96). These tumors or nodes may have a wide or narrow base or they may be pedunculated, but they are always clearly differentiated from the surrounding tissue and covered by peritoneum. The interior of the small norlules consists of a homogeneous grayish-yellow, tough, almost hornlike or cartilaginous tissue, while in the larger nodules, which are otherwise of similar structure, there are also cheesy foci, or the nodules may consist throughout of a yellowish, crumbly substance. The interior of these nodules may communicate, at the apex, with the lumen of the intestine by means of a fistulous canal (Volkmann found, at the end of the left cecum, a cheesy tumor of the size of a hen's egg; the remaining portion of the cecum was thickened and covered with norlules). The abdominal lymphatic glands are much enlarged and caseater. In a few cases the risceral layer of the peritoneum is covered with nodules.

In tubereulosis of the bones and joints, which is rather common, the articular carities are filled with cheesy masses; the cartilaginous covering of the enlarged epiphyses shows erosions while the bone substance and, occasionally the periarticular connective tissne contains cheesy foci.

Tuberculosis of the lungs is much less frequent. These may present the picture of a miliary tuberculosis or their 
tissue may contain large, yellowish-white, cheesy foci. The pericardial sac may contain much fluid while the surface of the heart may be covered with flat, grayish-red, raspberry-like or almost wart-like proliferations (Tietz). Among the other organs, we find, now and then, tuberculous caseous foci on

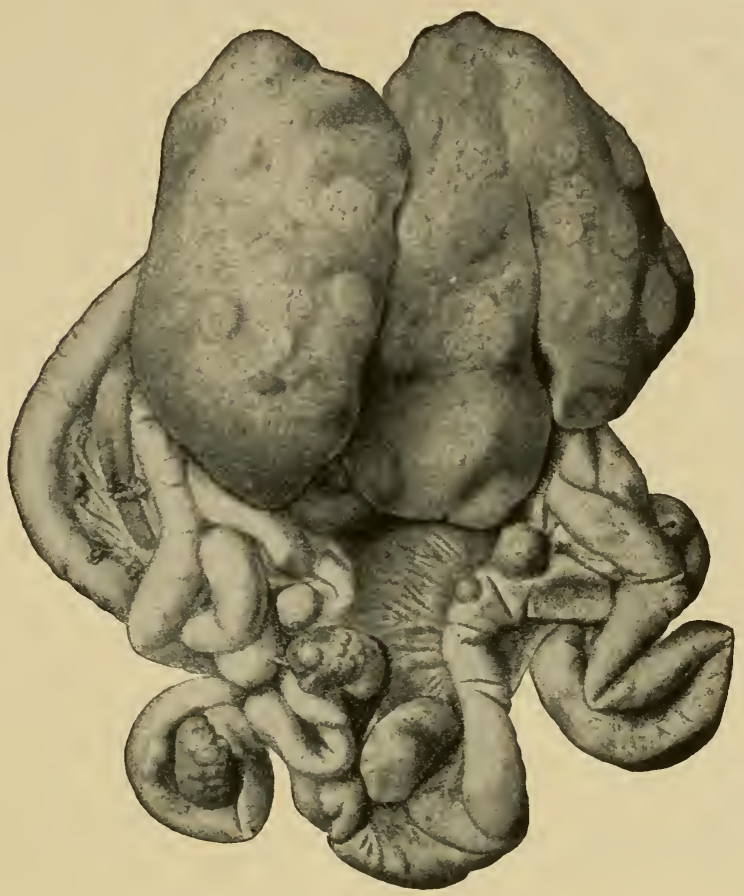

Fig. 96. Avian Tuberculosis. Caseated foci in the liver and nodules on the intestinal wall.

the peritoneum, in the kidneys, in the ovaries, in the testicles, in the pericardium and the muscle tissue of the heart, in the air cells, in the gizzard, as well as under the skin and the balls of the feet.

The histologicai structure of the avian tuberele is similar in character to that of the mammalian tubercle with the unimportant exception, that by far the greater part of it consists of large epithelioid cells of the foreign-body giant-cell type containing tuberele bacilli and disposed in radial or wreathlike scaly fashion (as seen in section) around the central necrotic mass. The degenerative process of the tubercle also corresponds more to the hyaline type. Tubercle bacilli are always present in large numbers in the fresh tubercles as well as in the cheesy nodules and in the contents of the involved joints. They are readily demonstrated by means of the usual methods of staining (See p. 569). 
Symptoms. Tuberculosis of the internal organs results in gradual emaciation without attendant characteristic s!mptoms of specific disease. This condition, which is readily recognized by palpation of the pectoral muscles, progresses in spite of the good appetite that exists in the beginning until the symptoms of anemia, evidenced by the paleness of the mucous membranes, wattles and comb, become more and more pronounced. At a later stage the appetite is affected, the animals become listless, are easily captured, etc. Finally the onset of diarrhea leads to complete exhaustion.

The symptoms of tuberculosis of the bones and joints are more characteristic. The tarsal, knee and shoulder articulations are most frequently affected. They begin to swell gradually and palpation reveals enlargement of the epiphyses. Pain and attendant disturbance of function (lameness, and drooping of the affected wing) is slight at first, but soon becomes more conspicuous if for no other than mechanical reasons. Occasionally the articular swelling breaks open and discharges a cheesy secretion filled with bacilli.

In rare instances small painless nodules develop in the subcutaneous connective tissue. At a later stage they may ulcerate. Finally, large caseous masses may collect in the cella infraorbitalis, causing enormous distension of the same and attendant pressure atrophy of the eye ball.

Tubereulosis of the internal organs ean hardly be differentiated from ehronie disorders of nutrition due to other causes, particularly worms. As a rule the disease is suspected only upon the ground of the apparent infectious nature of the malady. Tuberculosis of the joints is very similar in appearance to rheumatic arthritis or to that form of arthritis which is met with in chronic ehicken cholera. These three diseases, however, may readily be differentiated by microseopic examination of the contents of the joints which are easily procured in sufficient quantity by making an incision into the tumefactions.

The body temperature has no value from a diagnostic point of view since it does not vary beyond the normal limits $\left(39.5^{\circ}\right.$ to $41.5^{\circ} \mathrm{C}$.) even in tuberculous animals (Klimmer \& Saalbeck).

The tuberculin test is also not applicable because tuberculous fowls will not give specific reactions to tuberculin, no matter whether this is prepared from avian or mammalian bacilli or whether injected subcutaneously, dropped into the eonjunctival sac or applied to the scarified skin (Babes, Weber \& Bofinger, Moore, Saalbeck).

Prevention. Since medicinal treatment is practically out of the question and segregation of the diseased animals from the healthy ones is hardly possible on account of the insidious character of the disease the most practical and economical method of procedure is to kill the entire flock as soon as the disease is recognized. All animals in good flesh may be utilized as food. The diseased products and organs as well as the entire carcasses of badly affected animals should of course 
be destroyed. The poultry yard as well as the houses, coops, etc., should be thoroughly cleaned and disinfected before new stock is acquired.

Literature. Roloff, Mag., 1868. XXXIV. 190. - Paulieki, Ihi九., 1872. XXXYII. - Zuern, Krankh. d. Gefluegels. Weinar 18s2. 197. - Ribbert, D. m. W., 1883. 413. - Maffueci, Z. f. Hyg., 1892. XI. 445. - Wolffhuegel, Monh., 1904. XV. 457. - Weber \& Bofinger, Tbk.-Arb. 1. G.-A., 1904. 1. H. 112 (Lit). Schern, Z. f. Tnı, 1905. IX. 419 (Lit.). - M. Koch \& Rabinowitseh, V. A., 1907. CXC. Beiheft (Lit.). - Teetz, B. t. W., 1906. 244. - Moore, Z. f. Infkrkh., 1906. I. 333. - Volkmann, W. f. Tk., 190s. LII. Nr. 20. - O. Bang, Cbl. f. Bakt., 1908. XLTI. 461. - Saalbeck, Diss. Leipzig 1909 (Lit.).

Tuberculosis of Parrots. Since Cadiot \& Roger found tubercle bacilli in the organs of a parrot that were pathogenic for guinea pigs, others also have shown (the authors in five eases) that tubereulosis of these animals (though they are susceptible also to avian tubereulosis) is, in most cases, eaused by the human type of tubercle bacillus. These reports confirm the view entertained long before that parrots become infected in the dwellings of consumptive persons, and conversely justify the assumption that persons may be infected by tubereulous parrots. Infection, however, usually proceeds from injuries in the skin of the head or of the mucous membrane of the mouth cavity ; primary affection of the lungs is much less frequent and intestinal infection is only occasionally observed. The bones or joints may sometimes become involved through metastasis.

After M. Koch \& Rabinowitsch demonstrated by experimental infection with pure cultures that parrots are susceptible also to the virus of avian tuberculosis, Weber, Titze \& Weidanz showed, by more extensive experiments, that parrots could be fatally infected with intravenous injections of $0.001 \mathrm{gm}$. of pure culture of any of the three types of tubercle bacilli. A single ingestion of human or bovine bacilli resulted in infection just as readily while it required from 10 to 13 ingestions of avian tubercle bacilli to produce fatal generalized tubereulosis.

Tubereulosis of parrots is a compraratively common dicease. Thus Froehner found that 170 of the 700 parrots treaterl at the Berlin Klinik and Poliklinik from 1856 to 1894 were tubereulous, i. e. $24.3 \%$.

Tuberenlosis of the skin and subeutaneous eonnective tissue is manifested by the appearance of globular or oval, rather firm nodules and tumors with smooth surface, ranging in size from that of a pin head to a hen's egg. In the course of time these become caseous and the overlying epithelium becomes horny and forms thick scabby layers, sometimes growing into structures several centimeters long (Eherlein). Some of these nodules, especially those of the palpebral conjunctiva, are soft and bleed upon the slightest provocalion. When they develop near the angle of the mouth they interfere with the movements of the bill, while those in the region of the angles of the eyes may push aside or dislocate the eyeball; those developing on the top of the head may perforate the skull (Cadiot). If these nodules are broken by scratching. ulcers covered with cheesy masses form in their place.

Similar nodules, though usually smaller and of softer consistency, are comparatively frequent in the mouth cavity, especially on the tongue, near the frenum, on the hard palate, more rarely also in the nasal cavities. According to their seat or their size they may in- 
terfere with the acts of deglutition, respiration or the movements of the tongue (speech) (!).

The symptoms of tuberculosis of the bones and joints are similar to those observed in other birds and an affection of the internal organs is manifested only by emaciation and anemia.

The diagnosis of the disease which offers no particular difficulties is definitely confirmed by demonstration of the tubercle bacilli in the nodules and in the secretions of the ulcers in which they are usually quite numerous.

When the affection is localized on the skin or in the anterior portion of the mouth eavity, it is possible, in a portion of the eases, to bring about definite recovery by extirpating the swellings. Not infrequently however relapses oceur and the surgical treatment must be repeated. Cauterization of the ulcers is seldom followed by satisfactory results.

Canary birds are also susceptible to infection with mammalian tubercle bacilli; while M. Koch \& Rabinowitsch obtained positive results in two instances by feeding human tubercle bacilli, Weber, Titze \& Weidanz succeeded only with bovine and avian bacilli in their attempts to infect canary birds per os. The first named investigators succeeded also in obtaining cultures of the human type of tubercle bacillus from 118 birds of different species furnished by a zoological garden, from two birds of prey and from one sparrow.

Literature. Cadiot, Gilbert \& Roger, Soc. biol., 1890. 542. - Cadiot, Bull., 1894. 197; 1898. 254. - Eberlein, Monh., 1894. V. 248. - M. Koch \& Rabinowitsch V.-A., 1907. CXC. Beiheft (Lit.). - Weber, Titze \& Weidanz, Tb.-Arb. d. G.-A., 1908. 9. H. (Lit.).

\section{(c) Etiological Relations Between Human and Animal Tuberculosis.}

The practical similarity of anatomical changes produced in rabbits and guinea pigs with tuberculous material obtained from consumptive human beings or from cattle affected with pearl disease in itself points to the close relationship of these two diseases. The correctness of this view has been emphasized by the successful production of lesions very similar to those of human tuberculosis by feeding tuberculous organs of man to cattle, typical pearl disease having indeed thus been produced (Klebs, Bollinger), and further, by the fact that the feeding of tuberculous material from cattle will produce tuberculosis in other animals, horses, swine, dogs, eats (Chauveau, Guenther \& Harms, Gerlach, Klebs, Bollinger, Toussaint, Puetz, etc.). After Koch had demonstrated the presence of morphologically similar bacilli in the tuberculous organs of man, monkeys, cattle, horses, swine, goats, sheep and fowls, as well as in rabbits and guinea pigs, and after he had failed to recognize any material differences in their cultural or pathogenic characteristics, the etiological identity of the tuberculous affections of the various animal species was generally accepted as a demonstrated fact. More recently, however, the correctness of this view has been questioned from several sources.

Tuberculosis of Mammals and Fowls. The continued and 
persistent investigation and study of Koch's tubercle bacillus has at least cast doubt upon the supposed relationship of mammalian and avian tuberculosis. It has, for instance, been found that the bacilli causing these two morbid processes possess marked cultural differences, that the diseased organs of tuberculous mammals, as well as the cultures of bacilli obtained from them (with rare exceptions) possess only slight pathogenicity for fowls, and that, on the other hand, the bacilli of avian tuberculosis have a different effect upon mammals than the bacilli of mammalian tuberculosis.

On account of these facts some authors (Rivolta, Maffucei, Strauss \& Gamaleia, more recently also Weber \& Bofinger) regard these two forms of bacilli as distinct species and consequently also look upon mammalian and avian tuberculosis as two etiologically distinct diseases. On the other hand there is weighty evidence pointing to a close relationship between these two forms of bacilli.

In this connection the cultural differences between the two organisms are of slight significance for the reason that cultures of hacilli from the lesions of tuberculous fowls frequently resemble those obtained from mammals. Thus Weber \& Bofinger occasionally obtained dry crumpled cultures from fowls, a culture that had been kept up for years could not be distinguished from cultures of human bacilli, but after a single passage through a fowl or a mouse this characteristic was lost. And again 0 . Bang has observed many strains of avian bacilli, grown on glycerin potato medium, to produce dry, mneven masses, while one bovine strain observed by this author, when cultivated on glycerin serum resembled an avian culture in appearance. In addition to these observations it is known that some avian strains when cultivated on bouillon ean hardly be differentiated from bovine varieties. Among 95 strains obtained from 70 different birds Rabinowitsch found occasional strains that exhibited characteristies of growth, on solid as well as on liquid media, very similar to those of mammalian hacilli. Three of these strains, however, belonged undoubtedly to that group (pathogenic for guinea pigs, nonpathogenic for fowls). Finally, it is possible, by means of special methods, to obliterate these differences. Thus Fischel obtained a material by eultivating human tubercle bacilli on egg medium and on boracic-acid-glycerin-agar that would produce moist smeary cultures on the ordinary media. Nocard cultivated human tubercle bacilli in collodion capsules filled with bouillon which he imbedded in the abdominal eavity of fowls. After several months these capsules were removed, the bacilli transferred to solid culture media and cultures produced that resembled avian cultures in every respect.

The marked differences in the pathogenicity of the two forms of bacilli are of vastly greater importance. It is, for instance, impossible to infect any of the various species of fowls by feeding with organs of tuberculous mammals. Straus and Wuertz have fed chickens for months with tuberculous organs and sputum of tuberculous persons, and although some of them received as much as $58 \mathrm{~kg}$. of such material they remained healthy. Similarly negative results were obtained by Esser, Peroncito, Nocard and others. Since, however, tuberculosis among fowls frequently occurred under circumstances pointing to in- 
Iection with sputum of tubereulous persons (Johne, Nocard, Roemer, Darmagnac, Siegel and others) and since Zschokke eultivated a hacillus from tuberculous fowls that had all the cultural characteristics of the bacillus of mammalian tubereulosis, the possibility that fowls may occasionally become infected with mammalian tuberculosis does not seem excluded.

Intravenous and intraperitoneal inoculation of eultures of mammalian tubercle bacilli does not, as a rule, produce tuberculosis in fowls. Contrary to the numerous negative results of experimental inoculations Cadiot, Gilbert \& Roger have obtained positive results in $10 \%$ in all cases where they simultaneously fed tuberculous organs or injected horse serum into the tissues of the fowls. Furthermore, Fischel olstained positive results with intraocular, and Courmont \& Dor by subcutaneous administration. Finally, O. Bang produced fatal tubereulosis in fowls in three eases by intravenous inoculation and in one case by subeutaneous inoculation of bovine strains of bacilli. He found also that 12 out of 18 strains of bovine bacilli were more or less virulent for fowls.

Mature mammals, with the exception of rabbits, guinea pigs and mice, are either only slightly susceptible or entirely immune to avian tubercle bacilli. Nocard, however, found bacilli in several cases of abdominal tuberculosis of horses that resembled avian tubercle bacilli in their cultural characteristics as well as in their pathogenicity while o. Bang demonstrated that very young mammals (colts, ealves, kids) are very susceptible to the avian virus, becoming affected with acute tuberculosis after intestinal infection (see p. 608). Mohler \& Washburn also suceeded in infecting swine with material obtained directly from chickens. Bacilli of the avian type have also been found in the sputum of tuberculous persons (Straus, Nocard, Kruse, Loewenstein; Rabinowitsch obtained cultures from a man that died from generalized tuberculosis) also in apes (Fischel, Rabinowitsch), in the tuhereulous lumgs of eattle (Sanfelice, Kruse, Pansini) as well as in the easeous lymph glands of a pig (Weber \& Bofinger), while Karlinski procluced tuherculosis of the tracheal glands, diaphragm and spleen of a goat after intravenous injection of avian material. On the other hand, Hueppe cultivated bacilli identical with human bacilli from a pheasant and from a fowl. Tuberculosis of parrots, however, is in most instances caused by human tuhercle bacilli.

The specific virulence of avian tuberele bacilli is rather constant and is not easily altered. Thus Weber \& Bofinger were unable to increase by passage through guinea pigs the virulence of bacilli from fowls sufficiently to approach that of mammalian bacilli. Similarly, the attempts of Kossel, Weher \& Heuss to transform avian tubercle bacilli into mammalian bacilli by continued cultivation or growth in the bodies of mammals were unsuccessful. On the other haud, however, Cadiot, Gilbert \& Dor produced a virus by oft repeated passage of avian bacilli through guinea pigs that would cause lesions very similar to those of human tubereulosis and was also virulent for dogs and no longer pathogenic for fowls. M. Koch \& Rabinowitseh also succeeded, by cultivation continued for years, in giving an avian strain the appearance of human bacilli. Nocard succeeded by three or four passages of human tubercle bacilli through chickens in transforming them into a strain that would produce disease in fowls. These results have recently been confirmed by careful experiments conducted by O. Bang who succeeded in transforming bacilli of the human and 
bovine types into the avian type. This was particularly evident when a fowl passage was preceded and followed by a guinea pig passage. Bang observed also that a bovine strain which had lost its virulence after long continued or oft repeated passage through fowls, regained its virulence by passage through goats.

These positive results of experimental investigations, even though not very numerous, cause doubts to arise in regard to the supposed specific differences between the bacilli of mammalian and avian tuberculosis. If we consider in connection with the foregoing that Héricourt \& Richet immunized monkeys and dogs against human tuberculosis with avian virus and that MacFadyean and Behring immunized cattle against bovine tuberculosis by the same means, and further that effective tuberculin may be prepared from avian bacilli (according to Maffucci cattle and sheep will react to human tuberculin, no matter whether they were infected in the first place with bovine or with avian bacilli), that mammalian and avian bacilli exhibit reciprocal agglutination characteristics, and finally that it is possible to produce, experimentally, avian tuberculosis in mammals and mammalian tuberculosis in birds, the conclusion that the avian bacillus is merely a variety of Koch's bacillus resulting from prolonged growth for numberless generations in the bodies of fowls is probably justified. Accordingly, avian tuberculosis might be looked upon as bearing a similar relation to mammalian tuberculosis as human tuberculosis bears to bovine tuberculosis.

Tuberculosis of Man and Tuberculosis of Mammals. These two diseases have in recent times been looked upon as etiologically identical notwithstanding the fact that conspicuous differences have been observed to exist in the pathogenicity of strains of different origins. Though Puetz, as early as 1882, doubted the identity of human and bovine tuberculosis because he was unsuccessful in infecting calves either by feeding or with intravenous or subcutaneous injections of human tuberculous material and Semmer (1893) expressed the opinion that pearl disease and miliary tuberculosis of man were not identical morbid processes, later authors assumed upon the basis of occasional negative results in the experimental transmission of the two diseases to other animals that this difference must be due to modifications in the virulence of an essentially identical virus and to the variable relative susceptibility of the different species of animals.

Th. Smith had observed certain morphological and cultural differences between human and bovine bacilli and demonstrated that the bacillus of pearl disease had a considerably greater and more constant pathogenic action on experiment animals than that of human tuberculosis. He believed, therefore, that the bacillus of bovine tuberculosis occupied a unique position among the various mammalian bacilli and 
that human beings were only slightly susceptible to the contagion of pearl disease; in view of all this, however, he did not regard the possibility of human infection with bovine bacilli (especially in children ingesting large quantities of bacilli with milk from cows with tuberculosis of the udder) as out of the question. Even before Theobald Smith, Sidney Martin, Frothingham and Dinwiddie had made similar observations in regard to the variability in the pathogenic action of these two types of bacilli, although Frothingham observed rather extensive morbid changes in the peritoneum and in the mesenteric lymph glands of calves that were infected with bacilli from a tuberculous child while Dinwiddie, on the other hand, found that in one case material obtained from a tuberculous cow proved avirulent for a calf. According to Dinwiddie the differences exhibited by the two forms of bacilli manifest themselves only in horses, cattle, dogs and cats, while the human as well as the bovine types of bacillus produce generalized tuberculosis in swine. Finally Vagedes succeeded in procuring human cultures that had pathogenic properties very similar to those obtained from cattle.

The question of identity or non-identity attracted renewed attention when Robert Koch announced it at the London Tuberculosis Congress in 1901 as his emphatic view "that human tuberculosis differed from bovine tuberculosis;" that the former was not transmissible to cattle and "that accordingly it did not seem necessary to take any measures whatever for the protection of milk, butter and cheese from tuberculous cattle against infection with the germs of this disease."

Koch based this view on the results of infection experiments that he had con ducted with the co-operation of Sehuetz. 19 calves were treated with the pulmonary discharges of tuberculous persons and with cultures of human tubercle bacilli which material was administered with the food, with the inhaled air, or the cultures were injected intravenously, subcutaneously or intraperitoneally; all of the ealves remained perfectly healthy, increased steadily in weight and, at post mortem examination sereral months later only caseous abscesses could be detected at the point of inoculation. On the other hand, six calves given subcutaneous, intraperitoneal or intravenous injections of bovine cultures of bacilli developed fever and became very sick. At post-morten examination tuberculons ehanges were found not only at the point of inoculation and in the regional lymph glands but there was extensive tuberculosis of the internal organs, especially in the lungs and spleen. Of six pigs that had been fed for 104 days with tuberculous sputum mixed with the food, small scattered tuberculous nodules developed in the cervical glands and in the lungs and mesenteric glands of two of the animals while the remaining pigs remained unaffected. On the other hand, of six pigs that had been fed for 70 days with cultures of bovine bacilli, all contracted extensive lesions of tuberenlosis. Subcutaneous, intraperitoneal and intravenous injections of human tubercle bacilli proved harmless in 5 pigs while the 6th aninal, which received an intravenous injection, developed miliary tuberculosis of the lungs and mediastinal glands. Two sheep were unaffected by subcutaneous injections of cultures of human bacilli while one, receivirg an intravenous injection, developed slight tuberculous changes in the lymph glands of the lungs. On the other hand, sheep treated in a similar manner with cultures of bovine bacilli became rery sick.

The large number of experimental animal inoculations that have been made since Koch's epoch making address, according to which the bacillus of human tubereulosis as compared with that of bovine tuberculosis is only slightly or not at all pathogenic for cattle, as shown by the investigations of Koch \& 
Schuetz, have, in a general way, confirmed this assertion otherwise, however they have not substantiated the claim for the specific differences between the two forms of bacilli, but rather that the difference in their biological characteristics was sufficient, at most, to establish them as varieties or types of the same species.

Transmissibility of Human Tuberculosis to Cattle. As a rule human tubercle bacilli are harmless for cattle or they produce local lesions only. In exceptional instances, however, they may produce grave and even fatal disease.

Thus Chauveau (1892) observed tuberculous foci in the intestinal mucous membrane, in the mesenteric glands, in the pharynx and in the lungs, in three eases following the alimentary administration of infected material from a human lung; Schottelius observed the development of a caseous pneumonia as well as tuberculous changes in the intestines, in the mesenteric glands and on the pleura after the ingestion of tuberculons sputum, while in three out of five calves similarly treated by Hamilton and MeLaughlan Young, the cervical and mesenteric glands were affected. Wolff observed typical pearl disease in a calf following the subcutaneous injection of human virus, Fibiger and Jensen, general tuberculosis in two calves, Hamilton and McLaughlan Young extensive tuberculous lesions in 7 out of 9 calves (in three the lungs also were affected and the spleen in one case); Stuurmann saw death follow from generalized tuberculosis in a ealf 56 days after injection with a culture obtained from the tuberculous wife of a peasant and Eber observed the same result from miliary tuberculosis 37 days after infection. Death in ealves following intraperitoneal infection has been observed by Prettner as occurring on the 46 th day, by Ravenel on the 27 th, by Orth on the 26th day; in a second case reported by Prettner and in one case observed by each of Delépine, Fibiger \& Jensen, extensive tuberculosis of the abdominal organs was noted; in one case of Eber's there was typical pearl disease of the pleura and peritoneum (Klebs and Bollinger each produced typical pearl disease in a cow before this time by intraperitoneal injection of tuberculous sputum and Bollinger, in addition, produced the same condition in a goat by intraperitoneal injection of material from a cheesy tuberculous lymph from a man). In one case each reported by Prettner and DeJong and in two cases reported by Ravenel intravenous infection eaused death in from 17 to 37 days as a result of generalized tuberculosis. Hamilton and MeLaughlan Young succeeded in producing extensive tuberculous pleuro-pneumonia in two calves by inhalation infection with human tuberculous material. A calf infected by Stuurmann by the intrapulmonary method died on the 31st day while Thomassen observed development of tuberculosis of the cervical and pectoral glands and of the lungs following intraocular administration.

The results of very extensive investigations along these lines that were conducted by special commissions appointed by the governments of Germany, England and Sweden afforded particular information upon the biologic and pathogenic peculiarities of those forms of tubercle bacilli which occur in man.

According to investigations by Kossel, Weber \& Heuss of the German Imperial Board of Health two types of mammalian tubercle bacilli could be obtained from the organs of tuberculous persons. These types, aside from their morphological differences (see p. 504) also possessed individual peculiarities with respect to this virulence in rabbits and cattle, the human type, which was less virulent for rabbits, did not produce progressive lesions in cattle, while the bovine type exhibited a pronounced pathogenic character in both rabbits and in cattle, causing generalized tuberculosis in both species of animals.

Among 67 cases of tuberculosis in human beings the human type of bacillus alone could be cultivated from 56 , the bovine type alone in 9 cases and both of these types in two cases. The 9 cases in which the bovine type alone could bo 
demonstrated were all children under eight years of age, three of them having generalized miliary tuberculosis. Among 12 cases in ehildren under 10 years of age five were due to infection by the bacillus of the human type alone, six to infection by bacilli of the bovine type alone and one to infection by bacilli of both types. (Up to April, 1907, pure cultures of the human type of bacillus were obtained from 117 out of 138 cases of human tuberculosis [Steffenlagen]). The bacillus of the bovine type was found exclusively in 11 tubereulous cattle, in seven tuberculous swine and in one tuberculous sheep.

From the above noted results the authors draw the conclusion that in those rare cases where tuberculous human beings ean discharge bovine bacilli they may be the means of infecting eattle and that, on the other hand, infection of human beings is caused primarily from man to man, but that the possibility must be admitted that human beings may be infected with meat or milk from tuberculous cattle (and swine). They further admit, or assume, that tuberculous cattle are always infected with bacilli of the bovine type only and that swine and other domestic animals while generally infected with this type may occasionally also be infected with bacilli of the human type.

The results of the experiments above referred to were augmented by Oehlecker who obtained 45 pure cultures of human bacilli and 5 pure cultures of bovine bacilli from 50 different surgical cases of tuberculosis.

The British Commission (Foster, Woodhead, Martin, McFadyean \& Boyce) arrived at similar results in so far as they found pure cultures of tubercle bacilli obtained from human beings to possess different degrees of virulence while in pure cultures obtained from cattle this variation was not as pronounced.

Pure cultures obtained from 60 cases of human tuberculosis could be classified into two general groups. Group I included 14 strains which agreed in that they did not grow readily on artificial culture media (dysgonic strains) and that all of them produced generalized and progressive (though not always equally pronounced) tuberculosis in cattle as well as in other animals. Group II included 40 strains that were characterized by their ready growth on artificial media (eugonic strains) and by the production, in cattle, of local disease only or, at most, isolated tuberculous foci of a retrogressive character and by not producing generalized tuberculosis even when administered in large amounts, nor being very virulent even for rahbits. Finally, this commission recognized six strains which, on account of the variable character of their virulence, could not be classed in either of these groups.

In 30 instances cultures which agreed in their cultural and pathogenic characteristics with the strains of Group I were found in cattle. All of these had a decided pathogenic action not only for cattle, but also for other mammals, including monkeys.

Among 60 cases of human tuberculosis there were 28 in which the clinical symptoms indicated that infection occurred through the intestinal tract and of these the bacillus of the Group I type was found in 13 ; of 9 cases of tuberculosis of the cervical glands 3 belonged to this group and of 19 cases of tuberculosis of the peritoneum 10 were caused by the bacillus of Group I.

In view of the existence of intermediary forms as well as the variable virulence even of strains classified in each group the commission has not found it advisable to recognize two distinct types of bacilli. Nevertheless Cobbett called attention to the fact that it is possible, even with only limited experience, to recognize in advance 
the virulence of a given strain in its first generation by its eultural characteristics alone. In its summary the report of the commission lays stress upon the fact that the bacillus of Group I differs from that of Group II not only in its lesser degree of virulence, but also in its manner of growth and that, in view of this two-fold difference it seems impossible to helieve that human tuberculosis (II) is one and the same disease as bovine tuberculosis (I). Finally, the practical conclusion is expressed that a certain number of cases of tuberculosis of man and especially of children is the direct result of infection by the bacillus of bovine tubereulosis.

Of other investigations mate along these lines the following may also be mentioned.

Hamilton repeated the experiments of Koch \& Schnetz, using the same number of eattle and was able to demonstrate tubereulous lesions in 15 animals infecteil with human material. Dammann \& Muessemerer did not suceed in making calves very sick by feerling sputum and pure cultures of human bacilli but they succeeder in producing progressive, though in most eases only slight tuhereulosis in 13 calves by the subcutaneous alministration of 8 cultures out of a total of 18 prepared from every possible affected organ. One strain killed a calf in 28 days and another, after intravenous injection, in 17 days. These authors could not recognize two distinct and separable types with transition forms of bacilli ancl believe rather that the different strains are merely varieties of the same species that have arlapterl themselves to conditions existing in the bodies of each species of animal.

Fibiger \& Jensen investigated seven cases of unequivocal primary tuluerculosis of the alinientary canal of children from 4 montlis to 12 years of age, They found five eases in which the bacilli possessed a very pronounced virulence for cattle (in three cases the children in question had for some time consumed raw milk from uninspected dairies). Other authors (Schweinitz \& Schroerler, Westenhoeffer, Lignières, Schweinitz, Mohler, Rabinowitsch) also have found bacilli from tuberculous children (especially from primary intestinal tubereulosis) highly virulent for cattle. Thus Mohler \& Washburn examined twelve strains from nian, one from monkeys, four from cattle, two from dogs and one from sheep and found that bacilli virulent for cattle may and do occur in human beings. They were of the opinion, however, that two types could be recognized although there were intermediate transition forms and that the individual types were not necessarily confined to definite species of animals. Finally, Behring maintains that there are human strains of tuberele bacilli that are more virulent than material obtained directly from eattle and Dorset, also, found many strains of bacilli of human tuberculosis that were just as virulent as those of bovine tuberculosis.

Eber tester the virulence of the bacilli in seven cases of intestinal tuberculosis of children and found only two cases in which they were only slightly or not at all virulent, two eases in which the virulence was of average intensity and three cases in which the bacilli were highly virulent for cattle. In later investigations only two out of 8 cases of human tuberculosis proved non-virulent for cattle while repeated attempts with the remaining 6 strains were uniformly successful in producing serious and in some eases fatal tuberculosis in cattle (especially after combined subeutaneous and intraperitoneal administration of the virus). Two strains exhibited only a medium degree of virulence for eattle but after being transferred to another animal they became highly virulent. Since both strains showed atypical characteristies from a morphological point of view also, as well as in their action on rabbits, Eber is inclined to look upon them as transition forms which seem to constitute a connecting link between the two extreme types and make the gradual transition from one form to the other seem possible.

Pathogenic Action of Bovine Tubercle Bacilli on Cattle. The inoculation of the pathologic products of tuberculous cattle or cultures of bovine tubercle bacilli is usually followed by the development of a severe tuberculous affection, which frequently ends fatally within 4 to 8 weeks. Cases are observed, however, in which local lesions only result.

Thus Arloing was able, in some cases, to produce only local changes at the point of inoculation or in the regional lymph glands by sub- 
cutaneous injection of bovine bacilli. In the investigations of the Imperial Board of Health, 1 out of 11 strains of bovine bacilli had a similar mild action (injected intravenously, however, it was quite virulent), while Dammann \& Muessemeier found the injection of $5 \mathrm{cg}$. of a culture of bacilli into a calf absolutely incffective. According to the report of the British Commission the subcutaneous injection of bovine bacilli oceasionally produces only local changes, which, when they become more general (as may sometimes oceur) are limited to occasional, small foci in internal organs usually in an arrested stage of development. Between the two extremes of rapidly progressive generalized tubereulosis and of well marked regressive forms, every possible intermediate form may be observed.

The feeding of bovine virus frequently fails to result in severe disease. Thus Nocard failed to get positive results in four attempts, Hutyra failed to produce serious infection of calves six months of age by feeding cultures of bacilli, and the British Commission observed tuberenlous changes only in the immediately adjacent regions of the intestinal tract following alimentary administration of milk containing tubercle bacilli. Schweinitz, Schroeder, Nocard and Eber have obtained bacilli from cattle that were only slightly virulent for calves, while Preisz produced in one ealf tuberculous changes in one peribronchial lymph gland only and failed to cause any observable changes whatever in a second ealf, both of which received intravenous injections of a mixture of six different strains of bovine bacilli (see also experiments of Kossel, Weber \& Heuss, p. 508).

Pathogenic Action of the Two Types of Tubercle Bacilli on Other Animals. More recent investigations have, above all, confirmed the observations of the past that the human type of tubercle bacillus is decidedly less virulent for rabbits and guinea pigs than the bovine type, and that it is frequently impossible to infeet rabbits at all with bacilli of the former type (Smith, Ravenel, Kossel, Weber \& Heuss). Similar observations have been made also with regard to sheep and goats (Dinwiddie, Ravenel, Beck) ; Karlinski, however, suecessfully infected pregnant goats with intravenous and intramammary injections, and kids by alimentary administration of human bacilli while Dammann \& Muessemeier observed slight tuberculosis of the lymph glands of lambs following feeding of human material and in one case fatal tuberculosis followed within 44 days after alimentary administration of $0.1 \mathrm{gm}$. of bovine culture.

In regard to the susceptibility of other species of animals the results of experiments have varied. While, for instance, according to Dinwiddie and Gratia the two forms of bacilli possess much the same action toward horses, swine, dogs and eats, Ravenel noted the same differences that are mentioned above. Aside from the fact that bovine bacilli are always present in the organs of tuberculous swine the virulence of this type of bacilli is abundantly demonstrated by the rapid and certain infection of large herds of swine that have been fed on skim milk. On the other hand Dean \& Todd infected swine by feeding tuberculous pus from man and Dammann saw a pig die within 42 days after subcutaneous infection with a culture of human tubercle bacilli. In the feeding experiments of Kossel, Weber \& Heuss, the result of infection was always a chronic general tuberenlosis of slow evolution. Th. Smith and Ravenel produced eultures of typical bovine bacilli from swine, the same from one horse and an intermediate form from a second horse. 
The susceptibility of dogs to the virus of human tuberculosis was demonstrated by the experiments of Tappeiner, Weichselbaum, Leudet \& Petit and F. Arloing who were almost invariably successful in infecting dogs by the alimentary administration, and occasionally also by inhalation of sputum or pure cultures of human bacilli. On the other hand Titze \& Weidanz found that dogs generally had a considerable resistance toward infection with any kind of tuberculous material, but that this could be overcome by administering excessive doses of both types of the bacilli. Th. Smith obtained cultures from three cats that agreed morphologically in every respect with bovine bacilli, but only one of them was moderately virulent for cattle.

Susceptibility of Monkeys to the Virus of Bovine Tuberculosis. Experiments relative to this question, so important because the results permit conclusions in regard to the susceptibility of man, have been rather uniform and almost entirely positive in their results. Years ago Imbach successfully infected monkeys by feeding milk from tuberculous cattle. Of the more recent investigations, besides those of Gruenbaum, Salmon, DeJong, Cipollino and others, the following in particular are worthy of mention: In two series of experiments Nocard fed four monkeys with bovine bacilli and two with human bacilli; all of the six animals became affected with a severe form of generalized tuberculosis, but the infections with bovine bacilli produced a more rapid development of disease (Schweinitz \& Schroeder observed a similar difference in the pathogenic action of the two types of bacilli). Gratia fed four apes with the organs of tuberculous cattle, four more received the tuberculous organs of guinea pigs infected with bovine tuberculosis, two were fed on organs from similarly infected monkeys and three were fed with milk from cows with tuberculous udders; of these 13 monkeys 12 died from generalized tuberculosis while one (from the first group) only contracted an intestinal catarrh. In MacFadyean's experiments five monkeys that were fed on organs from tuberculous human beings died between the $33 \mathrm{~d}$ and 65 th days, three others that had been fed on sputum died between the 30th and the 65 th days (all from tuberculosis) while the feeding of infected material from tuberculous cows resulted in death from generalized tubereulosis in a similar or in a still shorter period of time. Dungern \& Smith inoculated three gibbons with human, six with bovine cultures (each $0.01 \mathrm{gm}$.) and another lot of three received repeated decigram doses of cultures of both types; all of these animals became ill with the only difference that those receiving the bovine virus had intestinal ulcers and caseous mesenteric glands, while those receiving the human virus had a solitary caseous focus in one lung and caseation of the corresponding bronchial gland. The British Commission tested the virulence of bovine bacilli on over one hundred monkeys and all animals, whether they received subcutaneous or intravenous injections, or were fed upon cultures (in some instances only $0.001 \mathrm{gm}$.) contracted severe generalized tuberculosis. Experiments with anthropoid apes (chimpanzees) also gave positive results and Eastwood is of the opinion that their susceptibility to infection with tuberculosis is materially greater than that of eattle. Rabinowitsch found on examination of 33 monkeys, most of them affected with human tuberculosis, while only a relatively small number (3) had bovine bacilli in the lesions. In one case both forms were associated (one an avian culture) and in a few other cases there were transition forms of bacilli. Kraus \&

Vol. $1-40$ 
Grosz noted a considerable difference in the two types after infection of the searified skin, the human type producing changes limited to the area of inoculation and showing no tendency to become destructive while the bovine type resulted in the formation of local ulcers followed by involvement of the regional lymph glands and later of the internal organs also, as a rule, with fatal termination. (Cutaneous inoculation with the typus gallinaceus resulted in very slight local ehanges only.)

Susceptibility of Man to Tuberculosis of Animals. Inder ordinary conditions this question is, of course, beyond the possibility of experimental demonstration. Nevertheless, our literature, and especially that of more recent date, contains numerous reports of observations that seem to justify the assumption of such susceptihility without even taking into consideration of numerous instances in which typical bovine bacilli were found in the lesions of tubereulous human beings.

Tuberculous disease proceeding from injuries to the skin has repeatedly been observed in persons who had the care of tuherculous eattle or in veterinarians and meat inspectors who were employed in inspecting or making post-mortem examinations of tuberculous cattle. In the vast majority of cases (DeJong, Ravenel, Ostertag, Jensen, Gratia, ete.) an injury of the finger or hand under such conditions (in one instance injury of the face) resulted in the development of a tuberculous nodule or ulcer at the point of infection, very similar to those occurring from infection following injuries receired in the post-mortem examination of tubereulous human hodies. Occasionally (Johne, Krause, Troje) these local affections have heen followed by swelling of the lymph glands of the elbows and axillae. In a case reported by Weber the primary affection spread from the mucous membrane of the gums to the tongue and the submaxillary gland. Lassar calls attention to the fact that dermatitis verrucosa cutis is a particularly frequent occurrence among persons employed in slaughter houses, having himself observed 7 eases among 365 persons examined, while otherwise the rate of morbidity is only $0.03 \%$. In several cases repeated applications of milk to the face resulted in dermal tuherculosis (Grothan, Priester, Leloir, Salmon), while in one case 60 cold ahscesses developed on various portions of the body following an injury received during milking. In this ease there was also tuherculosis of the iris which resulted in destruction of the eyeball (Coppez). Infections of this character that have their origin in wounds of the articulations are particularly dangerous and infections of finger articulations have been observed to result in death from consumption (Pfeifer, Hartzell, Salmon, Rich).

On the other hand the experiments of an unknown physician are worthy of mention, who treated persons affected with earcinoma with cultures of bovine bacilli injected subcutaneously, with no ohservable injurious results (Baumgarten). F. Klemperer, in experiments performed on himself and on five tuberculous persons by repeated subcutaneous injections of $0.25 \mathrm{cc}$. of an emulsion containing many bovine bacilli, observed only local inflammatory symptoms but in no case tuberculous lesions, while the injected bovine bacilli disappeared from the tissues in a short time. Spengler also injected himself with $1 / 2 \mathrm{mg}$. of a bovine culture (subeutaneously) which resulted in an abseess that broke after 34 days: suppuration continued for 8 months and was sometimes quite profuse, hut the lesion remained localized and the regional lymph glands remained free. 
In general, bovine bacilli seem to have little tendency to spread in the human body, usually causing local disease at the point of infection and the neighboring tissues (mesenteric and cervical glands), but in some cases the disease may become generalized.

In regard to the frequency of primary intestinal infection and its relation to the ingestion of fuberculous milk, reliable data have as yet not accumulated in sufficient quantity. In the past too little attention has been paid to this form of disease and besides, there is no definite agreement as to the exact characteristics of primary intestinal tuberculous disease. While, for instance, some authors recognize only primary intestinal ulcers as such, others, and probably correctly so, regard the primary affection of the mesenteric glands with intact intestinal mucous membrane also as such. According to modern views in regard to the pathogenesis of tuberculosis (see p. 526) many cases of primary tubereulosis of the thoracic and cervical glands as well as scrofulosis must be looked upon as the result of primary intestinal infection. The variable conceptions of this question is a sufficient explanation of the conflicting statistical reports. While, for example, Koch regarded primary intestinal infection (intestinal ulcers) as very rare, and Baginsky noted only 10 such cases in the course of five years in the Charite Hospital in the city of Berlin, Heller reports $37.8 \%$ of primary intestinal infection in post-mortem examinations of tuberculous children in Kiel; Hueppe 25 to $35 \%$ in Prague; Councilman $37.1 \%$ in Boston; Wagener, Eder and Ipsen in Kiel, Berlin and Copenhagen, report 44 cases in 289 bodies of children examined. According to Ashby and Woodhead $70 \%$ of children that die of tuberculosis show affection of the mesenteric glands.

Weber, in a statistical report authorized by the German Imperial Board of Health, shows that among 360 persons, among them 151 children, who had consumed raw milk and dairy products from 69 cows affected with tuberculosis of the udder the presence of "pearl disease" could be demonstrated in only two of the children (tuberculosis of the cervical glands). This result by no means harmonizes with the fact that the numerous authenticated cases of tuberculosis in human beings, demonstrated to be caused by bacilli of the bovine type have in most instances been tubereulosis of the glands in children that had evidently been infected by the milk of tuberculous cattle.

Constancy of Virulence of Tubercle Bacilli. The virulence of the different types of bacilli seems to be very constant. It has always been a difficult matter to change this artificially. Although according to Orth, Hamilton, Karlinsky, Hamilton \& if'Laughlan Young the passage of human bacilli through cattle, according to v. Behring, Karlinski, DeJong and Dammann \& Muessemeier the passage through goats and according to Mohler the passage through cats and rabbits increases the virulence for cattle, experiments made by the German Imperial Board of Health gave negative results throughout. Thus Weber, in particular, was unsuccessful in increasing the virulence of the human type of tubercle bacilli by five or eight consecutive passages through goats, the passage of 15 strains through pigs for a period extending over 300 days, and by four consecutive passages through cattle for a period of 685 days. This type also fully retained its cultural characteristies. Janesó \& Elfen in their experiments with 18 strains of human bacilli to increase their virulence for chickens by passage through fowls, or their virulence for rabbits by passage through the latter animals, obtained negative results. 
The negative character of experimental attempts does not, of course, exclude the possibility of a modification in the course of many generations under favorable natural conditions. This possibility has recently. been demonstrated by Eber who succeeded in producing in three instances an acute fatal tuberculosis in cattle with bacilli of the human type obtained from phthisical sputum. The bacilli, after first a passage through guinea pigs followed by intraperitoneal injection in cattle, produced only a circumscribed peritoneal tuberculosis, but cultures obtained from this affection and which had all the characteristics of bovine bacilli, produced death in from 21 to 34 days.

Experimental investigations thus far carried out have therefore not resulted in the discovery of fundamental differences between the causes of human tuberculosis and tuberculosis of the various domestic animals that would justify any modification of our former views as to the specific identity of all tubercle bacilli.

The differences in the form or structure of bacilli of various origin are least constant because they usually disappear within a very short time when grown on artificial media.

Their cultural differences, however, are more constant. These may remain unchanged through many generations, even after passage through the bodies of other species of animals. In this respect, however, gradual variations are observed which make it impossible at times to classify certain strains of bacilli in their proper group.

The remarkable difference in virulence of strains of bacilli of different origin is still less adapted for the determination of their specific character. Great variation in virulence, especially with respect to different species of animals, with otherwise great constancy of character, has also been observed in other pathogenic bacteria (very marked in the bipolar bacilli of hemorrhagic septicemia) while their specific identity has never been questioned on that ground.

The inadmissibility of such a view is further emphasized by the fact that variations ranging from the highest degree of virulence (with respect to certain species) to complete avirulence may be artificially produced by successive animal passages or by artificial cultivation and, on the other hand, by the fact that strains of the same type, obtained from different individuals of the same species may show variations in virulence of no inconsiderable degree.

The similar form and the similar staining characteristics of the various strains of tubercle bacilli, the general resemblance of their cultures, the identity of the pathological changes caused by them, the gradual differences observed between the different strains as well as the occurrence of transition forms, the similar effect of tuberculins prepared from strains obtained from different species of animals, the fact that cattle may be immunized with strains of bacilli obtained from human beings or fowls and, finally, the fact that the 
bacilli from one species of animal (at least in exceptional cases) may produce local or even generalized tuberculosis in other species, all point to the conclusion that tubercle bacilli obtained from various sources all belong to the same species, bacillus tuberculosis, and only represent varieties of "habitat" which differ from each other in unimportant characteristics. These characteristics are evidently the result of prolonged growth, generation after generation, in different hosts and may, under favorable conditions, again be obliterated.

The pathogenic action of the bacilli of human tuberculosis, pearl disease and tuberculosis of fowls is always most pronounced for the species of animal from which they have been respectively obtained, but none of them are harmless for other species. In addition to guinea pigs and rabbits the virus of human tuberculosis is pathogenic, in general, for all carnivora, slightly so for swine and for many species of birds (birds like canaries and parrots kept as pets in dwellings, in particular). The bacillus of bovine tuberculosis is quite virulent for swine and is, in general, more pathogenic for other domestic animals than the human type. The bovine bacillus is also occasionally the cause of fatal generalized tuberculosis in human beings, especially in children. The bacillus of avian tuberculosis is, in general, only slightly pathogenic for mammals, but may occasionally produce tuberculous disease in man, in the ox and the horse.

In view of the experimentally demonstrated possibility of the transmission of tuberculosis from one species to the other, including man, the fact that this occurs in part only occasionally is of subordinate importance. Neither does the fact that bovine bacilli that have been the cause of tuberculous lesions in the human body (or avian virus found in lesions of the horse, or human virus found in parrots inhabiting the dwellings of human beings) have retained for a time their original characteristics, detract from the force of the argument. As a matter of fact, the vast majority of medical and veterinary medical investigators admit the etiological entirety of tuberculosis in general and the specific identity of the various forms of tubercle bacilli and to this day accept Koch's original point of view, viz.: "In spite of the anatomical and clinical differences observed in the course of the disease, tuberculosis of animals, and particularly bovine tuberculosis, must be regarded as identical with tuberculosis of man on account of the identity of the parasites that are the cause of the disease.

For practical purposes the recognition of a typus humanus, a typus bovinus and a typus gallinaceus may be justified in order to designate the morphological, cultural and pathogenic characteristies of the different groups of bacilli, providing the fact is kept in mind that these types represent merely habitat varieties of the same species and that the occurrence of transition forms be kept in mind. 
Literature. Smith, Transactions of the Assoc. of Amer. Physicians, 1896; J. of exp. med., 1898. III. - KocH, D. m. W., 1901, 549. - SzekelY, Cbl. f. Bakh., 1902. XXXII. 167; 1903. XXXIV. 161 (Review). - Gratia, Tub.-Kongr. Brüssel, 1904 (Review). - Kossel, Weber \& Heuss, Tub.-Arb. d. G.-A., H. 1 (Lit). Dammann \& Müssemeier, Bez. 2w. d. Tub. d. Menschen u. d. Tiere, Hannover 1905. - Eber, Cbl. f. Bakt., 1906 XXXVIII. 449; B. t. W., 1910. 317. - RabiNowitsch, B. kl. W., 1906. Nr. 24. - Fibiger \& Jensen, Ibid., 1907. 4. — Second int. Rapport of the $R$. Comm. appointed to inqu. into the relations of human and animal tuberculosis, Vol. 1-4. London 1907. - Mohler \& Washburn, An. Ind., 1907. Bull. 96 (Lit.). - O. BANG, Cbl. f. Bakt., 1908. XLVI. 461. - Zwick, Z. f. Infkr., 1908. IV. 161 (Lit.).

Tuberculosis of Fish and Reptiles and Other Cold Blooded Animals. The acid and alcohol-fast bacilli that Silby obtained from a garter suake, Bataillon, Dubard \& Terre from a carp, Moeller from a hazel worm (Anguis fragilis), and Friedmann from a turtle, are regarded by these authors as elosely related to Koch's tubercle bacillus. The two first named, however, grow only at room temperature, like saprophytes, but the turtle bacillus grows also at $37^{\circ} \mathrm{C}$., produeing cultures very similar to mammalian cultures. It seemed also, that Friedmann's experiments in successfully immunizing rabbits against human tubercle bacilli (see p. 599) as well as Moeller's success with the Anguis fragilis bacillus point to a close specific relationship with mammalian tubercle bacilli. Weber \& Taute, however, concluded from the results of their experiments that the so called reptilian tubercle bacilli belonged to the group of acid-fast saprophytic bacilli which gain access into the bodies of cold blooded animals and produce local disease. (True tubercle bacilli inoculated into frogs remain virulent for a long time and produce nodules of tubercles similar to those produced by dead tubercle bacilli in other animals.)

The bacilli of piscine tuberculosis are nonpathogenic for warmblooded animals. Nevertheless Dubard was successful in making the fish bacillus very virulent for guinea pigs and Aujesky, by growing the same bacillus at a temperature of $37^{\circ} \mathrm{C}$. and by repeated passage through the bodies of guinea pigs succeeded in producing a strain that differed in no essential from the bacilli of guinea pig tuberculosis.

The transformation of mammalian tubercle bacilli into reptilian or cold-blood bacilli has, as yet, not been accomplished (the reported positive results of Sorgo-Suess were not confirmed by Tsukiyama).

Literature. WEBER \& TAUTE, Tub.-Arb. d. G.-A., 1905. H. 3 (Lit.). Aujeszky, Orvosi Hetilap., 1906. Nr. 8. - Sorgo-Suess, Wien. klin. W., 1907. Nr. 38. - Tsukiyama, Diss. Gieben 1908.

\section{Pseudotuberculosis.}

Since the discovery of Koch's bacillus such pathological processes only are classified under the head of tuberculosis as can be demonstrated to be due to this bacillus. Changes that resemble those of tuberculosis from an anatomical point of view and especially those that are associated with the formation of nodules which bear a resemblance to true tubercles, but are due to the pathogenic action of other bacteria are occasionally observed as occurring in the internal organs. Sometimes, also, such pathological changes are due to spirochætae or animal parasites. Such cases are usually classed 
under the general name of pseudotuberculosis. Preisz, however, suggested that this term be limited exclusively to morbid processes that are produced by bacteria other than Koch's bacillus. In this sense the subject will be discussed below.

Malassez \& Vignal (1883) found tubercle-like nodules following subcutaneous administration of a nodule obtained from a child that died with tuberculous meningitis. The nodules in the guinea pig, however, contained zooglœea masses composed of cocci and short hacilli instead of Koch's bacillus. Since then other nodular structures, some of them the results of experimental inoculation of animals with various substances, others accidentally discovered, have been described. After a careful study of the literature up to 1891 and a comparison of the bacteria found by Nocard, Pfeiffer, Parietta and Zagari, Preisz designated the following three species of bacteria as independent causes of pseudotuberculous processes:

1. Streptococcus Pseudotuberculosis Rodentium Dor; a thick, short, immotile bacillus with blunt round ends; frequently form long chains in tissue as well as in artificial media, particularly in bouillon; easily stained with aqueous aniline dyes, but not according to Gram's method; does not liquefy gelatin, forms an iridescent membrane on agar and emits a disagreeable odor; on potatoes yellowish-brown colonies develop, the medium taking on a greenish-gray color in the surrounding areas. Inoculation of pure cultures produce pseudotuberculosis in rabbits and guinea pigs with fatal termination in 8 to 10 days.

This bacillus is identical with the bacillus described by Malassez \& Vignal, Charrin \& Roger, Pfeiffer, Zagari \& Parietti under various names, tuberculose zoogléique, pseudotuberculose bacillaire, tubercuose streptobacillaire, etc., and to the action of which various morbid processes in guinea pigs and rabbits have been ascribed. It is also very similar to Bact. pseudotuberculare orchitophlogogenes recognized hy Cagnetto as the cause of a certain guinea pig enzootic. This bacillus seems to be widely disseminated in nature; Chantemesse found it in the dust of dwellings, Grancher \& Ledoux-Lebard found it in garden soil, Lignières in hay infusion, Parietti in milk. According to Nocard it occasionally causes epizootic affections of fowls (emaciation, tubercles in the lungs). Nocard also found this organism in the nasal discharge of a cow while Pfeiffer cultivated the same from the organs of a horse affected with glanders. In addition to this it is probable that the bacilli found by Labrazès in subcutaneous cheesy foci in the neck of a pigeon, and by Galavielle and t'Hoen in nodules of a cat's liver, are identical, forming chain-like structures in bouillon and cansing pseudotuberculosis in eats and guinea pigs. Those mentioned by Galavielle also produced pseudotuberculosis in the testes of guinea pigs.

2. Courmont's Bacillus. Up to the present time this has been found only in nodules resembling those of pearl disease observed on the pleura of a cow. This is a short, thick bacillus, actively motile, does not form chains in cultures. It grows well on artificial media, does not liquefy gelatin and forms long threads at $40^{\circ} \mathrm{C}$. These latter contained broken plasma masses. Fresh cultures eause the development of pseudotuberculosis in rabbits while in guinea pigs acute 
septicemia results. On the other hand cultures of three weeks' growth produce pseudotuberculosis in guinea pigs and are non-pathogenic for rabbits.

3. Bacillus Pseudotuberculosis Ovis. Preisz cultivated this bacillus from nodules in the kidney of a sheep (described on page 633). According to later investigations this bacillus is identical with that causing ulcerous lymphangitis in horses (see this) and probably also to that causing contagious acne of horses (see Vol. 2). Kitt demonstrated its presence in broncho-pneumonia of cattle, Sivori in bronchopneumonia of sheep (see p. 636), Nocard in catarrhal pneumonia of calves (see p. 165) and Carré \& Bigoteau in mal rouge of sheep (see p. 637), Sérés \& Guillaume in swine, and finally Lignières found this organism in rabbits and guinea pigs.

In addition to the three above-named bacilli more recent investigations have resulted in the discovery of other bacteria as causes of pseudotuberculous processes. Some of these are as follows:

4. Bacillus of Pseudotuberculosis of Calves described by Vallée (see p. 638).

5. Bacillus Pseudotuberculosis Murium found by Kutscher in a mouse that died of pseudotuberculosis ; it is immotile, sometimes staining according to Gram 's method, forms club-shaped growths in cultures and is pathogenic for mice only.

6. Corynethrix Pseudotuberculosis Murium described by Bongert as a short rod, Gram positive in old cultures, its products are violently toxic.

Structure of the Pseudotubercle. Nodules developing in the internal organs, on serous membranes or in subcutaneous connective tissue and, on being viewed by the unaided eye, bearing a perfect resemblance to true tubercles, but having a different internal structure, are characteristic of pseudotuberculosis. While degeneration and necrosis of the tissue clements does not occur in true tubercles except at an advanced stage of their development, the tubercles of pseudotuberculosis undergo cheesy degeneration at an early stage. Pseudotubercles develop more rapidly and consist throughout of necrotic detritus encased by a narrow peripheral layer of round cells. Viewed with the unaided eye even the smallest nodules appear white or yellowish white; in addition to this they are of rather soft consistency, the larger ones usually being creamy or mushy. Lime salts are occasionally deposited in the substance which is encased by a gray, translucent, comparatively thick, tissue capsule.

Pseudotuberculons processes in the sense above referred to manifest themselves, according to location, in the most variable morbid pictures. The PreiszNocard bacillus in particular produces affections in horses that have long been looked upon as well characterized specific forms of disease. For practica? reasons they will be discussed under separate headings (See lymphangioitis ulceros $\mathbf{w}$. below and acne pustulosa contagiosa in Vol. II 
Literature. Pfeiffer, ¿̇b. d. bazilläre pseuds tub. bei. Nagetieren, Leipsic 1889. - Preisz, A. P., 1894. VIII. 231. - Bongert, Z. f. Hyg., 1901, XXXV. 449 (Lit.). Cagnetto, A. P., 1905. XIX. 449 (Lit.). - Glässer, A. f. Tk., 1909. XXXV. 471 (Lit.).

\section{(a) Pseudotuberculosis of Sheep. Pseudotuberculosis ovum.}

\section{(Cheesy Broncho-pneumonia; Llymphadenitis caseosa ovum.)}

Occurrence. The disease occurs in some flocks in the form of an epizootic. Thus Turski observed it in 44 sheep in a herd of 150 in western Prussia; von Zeeb in the abattoir of Langensalza, in small herds frequently every animal being affected. The disease has also been reported from the provinces of Hanover and Silesia. Cherri \& Bull observed the disease in the abattoirs in Melbourne, Australia, in from 15 to $70 \%$ of all slaughtered sheep. According to Nörgaard \& Mohler it is very common in the western United States of North America and according to Sivori it is very frequent in adult sheep in the Argentine Republic (the last named authorities have furnished exact anatomical and clinical descriptions of the disease).

Etiology. The disease is caused by the Bacillus pseudotuberculosis ovis (bacillus of Preisz and Nocard). This appears as a delicate immotile rod (Fig. 97), which is non-sporulating and easily stained with aqueons aniline dyes as well as according to Gram's method. In cultures the bacilli are somewhat thicker and longer and have a tendency to develop oval, club-shaped and pear-shaped forms which appear cross-striated when stained.

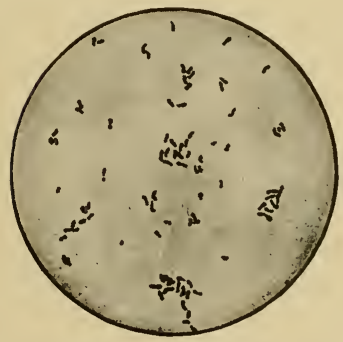

Fig. 97. Bac. pseudotuberculosis ovis. Agar culture ; Gram's Stain.

Cultivation. This facultative aerobic bacillus thrives only at body temperature (according to Carré \& Bigoteau, also at room temperature); on agar it forms gray or yellowish-white colonies which resemble minute scales and which are not readily emulsified; in the deep portions of the medium they form a white streak, rather thick above and attenuated below, gradually ending in minute white points; in bouillou small granules develop on the bottom of the vessel while the surface of the liquid is covered with a white, rigid membrane; on coagulated blood serum the areas surround. ing the colonies liquefy and become yellowish in color; no growth takes place on potatoes; it causes fermentation of grape sugar, does not produce indol or phenol. Cultures remain alive and virulent for months.

Pathogenicity. In sheep the subcutaneous inoculation of the pure culture may produce only a local cheesy focus which will break, discharge its contents and heal, or there results extensive swelling of the regional lymph glands, whereupon 
excessive emaciation gradually sets in; at post-mortem examination cheesy foci are found in the lungs and occasionally in the liver. Intravenous infection is followed by a development of numerous cheesy foci in the lungs and extensive swelling of the bronchial lymph glands. According to Nocard and Glässer it is possible also to infect lambs and kids by inhalation or ingestion of pure cultures. In both instances the morbid process may become localized exclusively in the lungs. Swine succumb to the effects of intravenous infection in 4 to 10 days, 10 intraperitoneal infection in 8 to 15 days; post-mortem examination revealing many cheesy foci, particularly in the lungs and in the liver, while the lymph glands are found to be greatly enlarged and partly caseated (Nocard \& Mohler). Intraperitoneal infection may also result in nodule formation on the peritoneum and in the lungs, while subcutaneous infection causes nodule formation and destruction of the tisshs at the point of inoculation (Noack). In guinea pigs and rabbits subcutaneous inoculation results in the formation of cheesy nodnles at the point of inoculation, while administration of the virus into the abdominal cavity is followed by the formation of caseopurulent foci in the lymphatic glands, the spleen, the liver and the mesentery; occasionally small psendotubercles will also develop on the peritoneum. In male guinea pigs we have, in addition, an inflammation of the enveloping membrane of the testes with the production of a purulent, fibrinous exudate, but the inflammation is nsually not as extensive as that following infection with glanders (Preisz, Nocard). Pigeons and fowls are not readily infected experimentally, and passage through their bodies causes attenuation of the virus.

According to Carré \& Bigoteau the bacillus produces toxins in peptone bouillon in the course of 5 or 6 days. The filtrate of such cultures injected subeutaneously into sheep in doses of 1.0 ce. produces a diffuse subeutaneous edema, extravasation of bloody serous fluid into the body eavities, swelling of the spleen and intestinal hemorrhages; following acute injections into the milk dncts there was swelling of the udder, serous exudate in the pleural cavities and bronchial hyperemia; intravenous administration is followed by excessive congestion of the lungs and death within 8 hours. The toxin possesses a similar action on rabbits and guinea pigs, while injection of large quantities of dead bacilli produces abseesses and cachectic symptoms in gninea pigs. Goats are less susceptible and dogs and cats are immune to the action of the toxin, while in horses and eattle it produces only a local inflammatory edema. Sheep that have been immunized against the toxin may he infected with live bacilli. Dassonville demonstrated in cultures the presence of a toxin resembling diphtheria toxin (see also p. 637).

Noack believed to have discovered a certain relationship existing letween the form of the bacillus and its virulence in the sense that short oval rods such as occur in the soft cheesy nodules and in other cultures, exert a much stronger pathogenic action than longer and more slender forms, which are found in the dry cheesy and caleified broken down foci. However, these various forms may occur side by side in the same lesions. 
Dunkel, in his comparative investigations, arrived at the conclusion that the bacillus pseudotuberculosis ovis and the bacillus pyogenes bovis et suis are varieties of the same species because they are reciprocally agglutinated by their respective immune sera; because mice, after treatment with one bacillus become immune to the other, and further, because the Bacillus pyogenes after being passed through the rabbit assumes the appearance of Bacillus pseudotuberculosis when inoculated into sheep. Glässer also suspected a elose relationship between the two bacilli and in addition is inclined to look upon the bacillus of pseudotuberculosis of sheep and that of rodents as varieties of the same species. Finally, according to Nocard, Vallée, Panisset \& Loiseau a certain relationship existed between the pseudotuberculosis bacillus and the bacillus of diphtheria of man, while Dunkel, on the other hand, doubts the identity of the pseudotuberculosis bacillus and the bacillus of ulcerous lymphangitis of horses, basing his belief upon an experimental inoculation of a horse. As a result of these various views the proper classification of the bacteria referred to is in such an unsatisfactory state that a thorough revision of this question is very desirable.

The natural mode of infection is as yet not definitely known. Probably it occurs most frequently through the intestinal tract, and possibly also through the respiratory passages. The bacilli may also gain an entrance through wounds of the skin and through the navel of newborn animals. In older lambs infection may occur following amputation of the tail, the germs entering the lymph channels of the subcutis. The droppings of infected sheep seem to be a prolific means of disseminating the disease, Carré having found large masses of pseudotubercle bacilli in the droppings of apparently healthy sheep.

Anatomical Changes. In the majority of cases the morbid processes even in animals that have died with symptoms of cachexia is confined to the intrathoracic and the external lymph glands, or to the latter only, while the mesenteric glands are only very rarely affected. The glands appear to be considerably enlarged and contain circumscribed foci, or they have become transformed into greenish-yellow, sticky, smeary, cheesy masses which may consist of concentric layers resembling the structure of an onion, and surrounded by a thick connective tissue wall with smooth inner surface. Subsequently lime salts are deposited in this caseous mass giving it a grayish-white color and a mortar-like or gypsum-like consistency.

In some cases small gray, or grayish-green nodules or even large tumors of a structure similar to that of the cheesy glands are found scattered through the lungs.

In addition we may occasionally find diffuse, lardaceous, indurated, lobar and lobular pulmonary lesions with soft, greenish cheesy foci. This condition is usually attended with an adhesive pleuritis and normal appearance of the regional lymph glands (Sivori, Noack).

Noack mentions, as a rare observation, oval groups of nodules the size of a pea arranged in a manner resembling coral, in the walls of the large and small intestines. Finally, the liver, the spleen and the kidneys may occasionally contain similar cheesy or caseo-calcareous nodules. 
According to compilations made by Noack including 150 cases, the lungs were affected in $98.3 \%$, the lymph glands in general in $80.6 \%$, the muscle lymph glands in $33.3 \%$ and the liver in $17.2 \%$.

Symptoms. If the morbid changes are limited to the internal organs the disease is nsually not discovered until the animals are slaughtered. Such animals may, however, appear perfectly healthy or show only indefinite symptoms of gradually increasing anemia or cachexia. In other cases, on the contrary, the affection of the lympl glands may be recognized during life by the presence in various regions of the body of painless tumor's as large as a man's fist. These tumor's occur most frequently in the prescapular and precrural regions. The general health of the animals is affected only in so far as the enlarged lymph glands may produce more or less conspicuous disturbance of locomotion. In some animals, however, cough and marked dyspnea develop in the course of the disease, attended with progressive emaciation and anemia, thus indicating the existence of subacute or chronic broncho-pneumonia.

The disease always develops very gradually and fatal results have as yet not been observed in adult animals.

Carré \& Bigoteau report an enzootic among lambs a few weeks of age, which is evidently identical with psendotuberculosis. In some of these animals post-mortem findings were absolutely negative, while in others multiple abscesses were found in the connective tissue and sero-fibrinous inflammation in the carpal and tarsal articulations. In addition, the incompletely healed navel of some of the animals contained pus, the urine always contained albumen. The contents of the abscesses consisted of a creamy, greenish-yellow pus from which the Preisz-Nocard bacillus could be obtained in pure culture.

Bridré described a disease among lambs which proved fatal at about the age of three weeks. Post-mortem examination revealed abscesses, principally confined however, to the liver and the lungs. A small hacillus similar to the one mentioned above, could be cultivated from the abscess contents. This bacillus would curdle milk, liquefy coagulated blood serum, was non-pathogenic for rabbits and guinea pigs but caused local abscesses when injected suhcutaneously into sheep, and was followed by death within 15 to 20 days when pure cultures were injected into the navel of newborn lambs.

Prevention. Since the active cause of this disease seems to be widely disseminated in the litter or bedding, or on the ground where infected herds have lived, infection per os can hardly be effectively prevented, the disease can only be checked by careful aseptic or antiseptic treatment of the navel after birth and of the wounds resulting from amputation (docking) of the tails. By this method (washing of the navel with Lugols' solution and treatment with $1 \%$ iodine collodion) Carré reduced the percentage of deaths in one herd from 50 to $0.3 \%$. Bridré also achieved good results by this method. 
Immunization. Carré reports very favorable results following the treatment of young lambs in a badly infected herd, as follows: Two vaccines were used, one of which produced only local edema in guinea pigs, the other occasional suppuration only, while in sheep no morbid changes whatever followed its administration (the manner of preparation was not indicated). As to results: In herds where the losses of lambs had formerly reached $70 \%$, the treatment of 318 lambs was followed by abscess formation only in 5 .

Literature. Preisz \& Guinard, J. Vet., 1891. 563. - Zeeb, Z. f. Flhyg., 1893. XIII. 117. - Turski, Z. f. Flhyg, 1897. VII. 178. - Cherry \& Bull, The Vet., 1899. 523. - Nörgaard \& Mohler, Án. Ind., 1899. 638. - Sivori, Rec., 1899. 657. - Bridré, Bull., 1905. 358. - Carré \& Bigoteau, Rev. gén., 1bid. 369. u. 433 (Lit.). - Noack, Diss., Bern 1908 (Lit.). - Glässer, A. f. Tk., 1909. XXV. 471 (Lit.). - Carré, Rev. gén., 1910. XV. 65.

Mal rouge. Carré \& Bigoteau made a bacteriologieal study of a disease of sheep that had been described in 1876 by Teissier, and since then by several French authors, especially Delafond, and generally elassed with anthrax. The disease, known in the infested regions as pourriture aignë, mal de Sologne, mal ronge, eaux rousses, jaunisse, etc., is prevalent in southern France and eauses losses that may reach $10 \%$ in infected flocks.

The symptoms consist of dullness, paleness of the mucons membranes, muco-serous, and later bloody, nasal discharge, increased thirst, bloody diarrhea and hematuria. Toward the termination of the disease there is edematons swelling of the intramuscular region and of the extremities. The disease terminates fatally after 2 or 3 or, sometimes, after from 8 to 15 days. Occasionally death occurs quite suddenly.

At post-mortem examination we find hemorrhagic and serous edema in the subcutis and mesentery, excessive hyperemia of the membranes of the stomach and intestines, marked acute swelling of the spleen and lymph glands, dark red, almost black discoloration of the kidneys, hemoglobin in the urine of the bladder and finally there are usually large masses of bloody fluid in the serous body cavities. The disease may be differentiated from anthrax by the paleness of the muscles, the comparative emptiness of the small blood vessels, the pale color and the coagulated condition of the blood and finally the negative results of experimental inoculations.

Microorganisms can be demonstrated in the body fluids by means of baeteriological examination, althongh in somewhat protracted cases small abscesses may be found in the pharyngeal and retropharyngeal lymph glands. These contain the pseudotuberculosis bacillus of Preisz and Nocard which may be obtained from them in pure culture. Carré $\&$ Bigotean are inclined to regard the disease as an intoxication cansed by the toxic products of metabolism of the bacilli in question.

In animals of considerable power of resistance the bacillar toxins found in the lymph glands are supposed to produce a chronic intoxication resulting in the familiar clinieal condition known as eachexia aquosa, and it is further assumed that the weakened condition, the anemia and hydremia observed in any distomatosis is not caused by distoma but rather by baeterial toxins (?). Aceording to this view cachexia aquosa is merely a chronic form of the above disease.

Finally Carré is of the opinion that many cases of colic in horses, especially that form descrihed as congestion intestinale by French authors, which is char- 
acterized by rery severe pains, rapid course, extreme hyperemia and hemorrhagic serous infiltration of the intestines with acute swelling of the spleen, may be aseribed to the toxins of the bacillus of pseudotuberculosis.

Literature. Carré \& Bigotean, Rev. gén., 1908. XI 369 u. 433 (Lit.). Carré, C. R., 1910. ('L. 350 .

\section{(b) Pseudotuberculosis of Calves.}

\section{(Pseudotuberculosis vitulorum.)}

This disease was observed by Vallée on an estate which had for a number of years suffered serious losses among calves. They usually became infected one or two weeks after birth, showing symptoms of great weakness, complete loss of appetite; death following without exception in the course of 8 to 10 days. Post-mortem examination revealed in all cases numerous tubercle-like, gray, and partly confluent nodules in the liver, while other organs as well as the portal lympli glands were apparently healthy.

Many other bacteria and a very small immotile non-sporulating bacillus which readily stained according to Gram, have been cultivated from the liver. This latter bacillus does not liquefy gelatin and produces minute translucent colonies on coagulated blood serum, agar and potatoes. Cultures in bouillon cause cloudiness of the medium and accumulation of a slimy sediment does not cause fermentation of sugar nor coagulate milk. Intravenous injection of the pure culture is fatal to guinea pigs, rabbits and dogs in which cases small tuberclelike norlules may be found in the liver and occasionally also in the lungs. Guinea pigs become affected after their administration per os or intraperitoneally. Pigeons are affected only after intravenous injection of cultures.

The natural mode of infection has as yet not been satisfactorily explained, probably it results through the digestive canal (possibly from infected milk).

In the livers of calves slaughtered in an apparently healthy condition in which post-mortem inspection revealed swelling of the spleen, punctiform hemorrhages of the kidneys and occasionally bronchial catarrh, Langer found numerous minute, grayish-white nodules which contained a facultative aerobic Gram negative bacillus of the paratyphoid group, and the cultures of which were agglutinated by typhoid serum (Bac. nodulifaciens bovis). Pure cultures produced no toxins but on administration to experiment animals resulted in the formation of nodules in the liver, in rare cases also in the lungs and kidneys. Pitt, who observed one case in which the navel and the umbilical cord were considerably enlarged and edematous the mesentery glands swollen and of a marrow-like consistency, recognized as the causative agent ? bactllus belonging to the group of Gärtner's Enteritis bacilli.

Literature. Vallée, Rec., 189s. 400. - Langer, Diss., Gieszen 1904, - Iitt, (b). f. Bakt., 1909. XITX. 593. 


\section{Specific Paratuberculosis of Cattle.}

(Enteritis paratuberculosis bovis specifica. Johne's disease [English]; Enteritis chronica bovis pseudotuberculosa s. specifica; Enteritis chronica hypertrophica bovis.)

History. In 1895 Johne \& Frothingham observed a peculiar affection of the intestinal mucous membrane in an ox which manifested itself in extensive maculate swelling and reddening, and a profuse secretion of mucus; tubereles and ulcers were absent. On account of the presence of numerous masses of acid-fast tubercle bacilli they regarded this affection as a special form of intestinal tuberculosis, possibly brought about by infection with avian bacilli. More recently this disease has also been observed in other countries, occasionally in the form of an enzootic, and has been made the object of special investigation by Markus, Liénaux \& van der Eeckhout, Bongert, Bang and von Mieyer.

The name enteritis paratubereulosis bovis specifica is probably best adapted for designating this disease, since other names that have been suggested also apply to other enterites of eattle and because the term paratuberculosis is generally understood to refer to acid-fast bacteria which must be differentiated from true tubercle bacilli, thus clearly indicating the non-identity of this disease and tubereulosis (better than psendotuberenlosis).

Occurrence. Up to the present time the disease has been observed in Germany, Denmark, Holland, Belgium, France, Norway, Sweden, Hanover and North America. It occurs principally in young cows, but also in calves and heifers as well as in older cattle, usually sporadically but not infrequently as an enzootic. In Switzerland $80 \%$ of the cases of chronic gastro-intestinal catarrh might be ascribed to this disease, while losses occurring in Denmark probably approach $10 \%$.

Etiology. The disease undoubtedly bears a certain relationship to the presence of small acid-fast bacilli which exist in large numbers in the intestinal mucous membrane, since, however, it has not yet been possible, in spite of numerous attempts, to cultivate the bacilli and produce specific affection with their pure cultures, the views expressed in regard to this matter are rather divergent, while for example Johne \& Frothingham, Bourgeaud and Stuurman regarded them as avian tubercle bacilli, Liénaux \& van der Eeckhout, and also Bongert looked upon them as attenuated mammalian tubercle bacilli. More recently Liénaux considered them as a saprophytic variety of bovine bacillus, while Markus, Bang, MacFadyean, Meyer \& Miessner expressed the opinion that it is a matter of a specific disease caused by acid-fast bacteria of an entirely different nature than the true tubercle bacilli. It was impossible to produce a true progressive tuberculosis in experiment animals (guinea pigs, rabbits, goats and fowls)' by means of material obtained from naturally infected animals. The results of inoculations consisted merely in the development 
of local cheesy foci or abscesses, and in most cases they were of a completely negative character. This disease occurs occasionally also in herds that are entirely free from tuberculosis. Many of the animals do not react to tuberculin and upon postmortem examination are found absolutely free from any tuberculous lesions whatever (Bang).

Recently Stmman reported that he has suceeeded in producing pure eultures of the organism from guinea pigs and rabbits that had been infected with material from naturally infected animals. These eultures were similar in many respects to those of the avian tubercle bacillus. The results of alinentary administration of pure cultures were however negative.

The fact that the disease is contagious was demonstrated by Bang when he succeeded in infecting six calves by feeding the scrapings of the intestinal mucous membranes of infected cows in large quantities (300 to $1,500 \mathrm{gm}$.). Miessner also succeeded in infecting cattle in this manner as well as by intravenous administration. These positive results point to the probability that natural infection results from ingestion of contaminated feces. However, the pathogenicity of the disease is also demonstrated by the observation of its introduction into herds previously free from the same.

Anatomical Changes. At post-mortem examination we find the following: The cadavers are frequently excessively emaciated, the walls of the small intestine considerably thickened, either miformly through its entire extent or in local areas only. These changes may extend also to the cecum and colon. The surface of the intestines is smooth and pale. The mucous membrane of the affected regions is increased to from 4 to 5 times its normal thickness, being transformerl into irregular folds running longitudinally or transversely so as to resemble the convolutions of the brain (Fig. 98). These areas are covered with a dirty looking, grayish-white or grayishgreen, sliny fluid resembling meal soup. At the crest of the folds the mucous membrane is reddened in spots or dotted with hemorrhages, and usually smooth on the surface; between the folds it has a delicately warty appearance (no nodules) as though gnawed by fine teeth. The mesenteric glands and Peyer's patches are slightly swollen.

Meyer found specific changes in the fourth stomach in $50 \%$ of the cases exanineil. Of the various regions of the intestines, the duodenum was macro. scopically infecter in $13 \%$, the jejunum in $65 \%$, the ileum in $82 \%$, the ceeum in $36 \%$, the colon and rectum in $32 \%$, the mesenteric glands in $60 \%$. Microseopical exanination of the hypertrophied areas of the mucous membrane revealed extensive, diffuse, cellular infiltration, the villi having assumed irregular and clumsy forms. The iliseased cell masses frequently contained great numbers of large epitheloid as well as giant cells and great masses of small acid-fast rods.

These rods are occasionally found also in great numbers in the feces in which they are easily demonstrated by staining processes adapted for tuberele bacilii (the bacilli also stained according to Gram's method and with earbomethylene blne). When the rectal mucous membrane is affected the diaguosis can readily be marde intra vitam by removing a small portion of the nucous membrane with the aid of the fingernail or with a curette and examining it microscopically. 
Symptoms. The symptoms of this disease consist in gradual emaciation unattended with fever or pain, occasional diarrhea (when present usually persistent), the evacuations being thin or watery, frequently containing gas bubbles. The affected animals, though very anemic, do not always show symptoms of exhanstion even when emaciation has become excessive. On the other hand, the appetite is quite variable, milk production diminishes, and the disease terminates fatally after a condition of collapse often lasting several days. In rare cases diarrhea is absent but in spite of this, the disease terminates with symptoms of excessive emaciation and anemia.

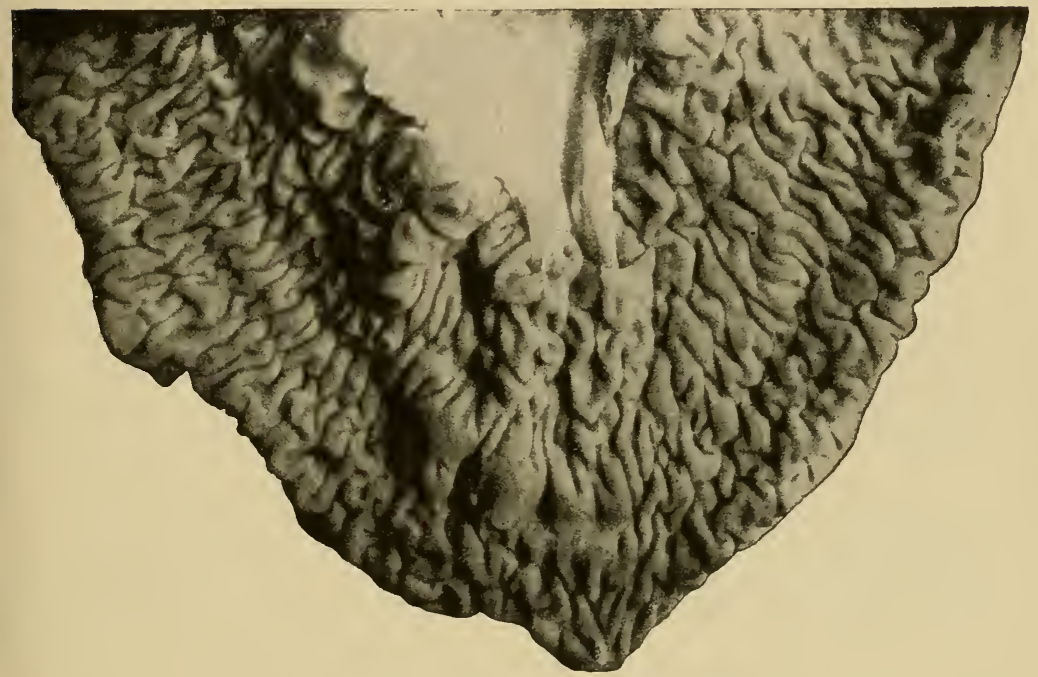

Fig. 98. Enteritis chronica parutuberculosa. Hypertrophy of the Mucous Membrane of the Small Intestines.

The course of the disease always extends through several months. Excessive feeding of root crops, and parturition exert an unfavorable influence on its course, while pasturing has a beneficial effect.

Diagnosis. In some cases where the peculiar intestinal evacuations arouse suspicion of the disease, the diagnosis may be made by a microscopical examination of the intestinal contents or of portions of mucous membrane (see p. 639 ; Meyer obtained positive results in 60 to $70 \%$ of such cases). The tuberculin test is also of value in this connection, although tuberculin prepared from avian tubercle bacilli must be used.

Vol. 1-41 
O. Bang found (except in adranced cases) that tuberculin of this kind when applied subcutaneously, would produce an elevation of temperature up to $41.0^{\circ} \mathrm{C}$., frequently attended with great depression, diminished milk vield, chills and diarrhea. While tuberculous cattle always react to this treatment the condition may be differentiated by an application of mammalian tuberculin either before or after the use of the avian material. Howerer, this disease, especially in its more pronounced form, is hardly ever associated with tuberculosis.

The suspicion expressed by Bang that the disease in England is frequently confused with gastro-intestinal strongulosis seems, according to Meyer, not to hold with respect to so called cold gangrene (Kaltbrändigkeit) observed in Switzerland and the presence of filaria in the stomach and intestines does not necessarily exclude paratuberculous enteritis since these two diseases, particularly in the more serious affections, may co-exist.

Prevention. Since all attempts to treat the disease medicinally have led to unsatisfactory results, and since means for immunization are unknown, the best course to pursue with our present state of knowledge is the isolation and destruction of aftected animals as soon as possible after recognition of the disease, thorough cleansing of the stables and raising the offspring on new or uninfected pastures. This is usually attenderl by more or less success.

Literature. Johne \& Frothingham, D. Z. f. Tm., 1895. XXI. 438. - Markus. Z. f. Tm., 1904. VIII. 68, - Liénanx \& Van der Eeckhout, Ann., 1905. LIV. 65; 1906. LV. 84. - Liénanx, Ibid., 1905. LIV. 598. - Bourgeaud, Sehw. A., 1905. XLIV. 221 - Bongert, D. t. W., 1906. 231. - Bang, B. t. W., 1906. 759. Mac Fadyean, J. of comp. Path., 1907. XX. 4s - Meyer, Arb. d. Seruminstituts Bern 1908. - Stuurman, Hiessner, Kongr. Haag 1909.

\section{Actinomycosis. Lump Jaw, Big Jaw.}

Actinomycosis is a non-contagious infectious disease of cattle, occasionally affecting other domestic animals and man, which manifests itself either in the appearance of connective tissue tumors or in chronic processes of suppuration and is caused by the Ray fungus, Streptothrix actinomyces.

History. The Ray fungus has been known since the middle of the last century and demonstrated by a number of authors; in man, in the contents of chronic abscesses (Langenbeck, 1845; Lebert, 1848; Laboulbène, 1853; Robin, 1871), in diseased maxillae (Davaine, 1850; Rivolta, 1868; Perroncito, 1875), and in woody tongue in cattle (Hahn, 1870). Its etiological significance, however, was not demonstrated until 1877 (Bollingers' work on Actinomyeosis of the Jaw of Cattle). After the botanist Harz demonstrated the fungus nature of the cause of this disease, the latter assumed greater prominence through the works of Israel (1878) and Ponfick (1880) who found this fungus also in 
the morbid products of man, and Ponfick demonstrated the etiological identity of this disease in man and animals.

The literature of recent years (Johne, Bang, Harms, Azary, Jakob) has added much information concerning the great variability of the clinical appearance of this disease. On the other hand, Wolff, Israel, and more particularly Boström, Lignières \& Spitz have added much to our knowledge of the etiology of the disease, while Thomassen (1885) discovered an effective internal treatment.

Occurrence. Actinomycosis is of frequent occmrence in cattle, particularly in young animals, especially in low, swampy, damp regions, or regions subject to periodical flooding, and occasionally appear's as an enzootic. However, it is in general more frequently met with in stable-fed animals than among those kept on pasture.

The disease in general is more common in regions where the live-stock industry is conducted on an extensive scale (as in the western states of North America) than in regions where this industry is carried on uniler opposite conditions. In some regions the disease appears almost annually among young stock in the form of peripharyngeal tumors.

The disease is very common in Russia, especially in southern Russia where, according to Ignatjew $10 \%$ of all cattle are attacked by the disease, affecting principally the lips. It is common also in certain regions of Demmark, England, Holland (according to Schouten $10 \%$ of all cattle) as well as in North America. The disease is much less frequently met with in France and Germany but is occasionally quite prevalent in western Prussia (Preusse) and in the mountainous regions of Bararia (Imminger). Ollendorf observed the disease in 76 annals in a herd of 329 cattle in western Prussia, and according to Franck it was so common in certain regions that it was formally included in the list of diseases which under the German laws were considered sufficient when present, to annul a contract of sale. Piot assumes that $3 \%$ of all cattle in the Nile Valley of Egypt are affected with this disease.

According to statistics compiled by Jelenevski the morbidity as established in the various abattoirs from 1896 to 1911 was as follows: Berlin 3.1, Vienna 0.1, Moscow 33.4, Kief 6.7, Warsaw 6.5 per thousand, respectively (the mild form of tongue actinomycosis is evidently not included in these statistics).

In horses, swine, sheep and goats actinomycosis is rarely observed, and occurs only sporadically.

\section{Etiology. The Ray fungus (streptothrix actinomyces} Rossi Doria, actinomyces bovis Harz) according to the latest views, is a thread-like schizomycete belonging to the group of streptothrix (Fig. 99). It forms branching growths, propagates by sporulation and under favorable conditions produces characteristic colonies (granules) consisting of numerous individuals (Fig. 100).

Morphology. The size of these colonies varies from $0.15 \mathrm{~mm}$. to $0.75 \mathrm{~mm}$. Under the microscope colonies $0.01 \mathrm{~mm}$. in diameter may be observed. On the other hand many small colonies may unite to form characteristic large granules $2.0 \mathrm{~mm}$. in diameter. The more recent and usually the smallest colonies are translucent gray, with a greenish tinge, resembling a globule of gelatin or mucus or a granule of sago, soft and smooth upon the surface. The older colonies are whitish-gray 
and subsequently assume different shades of yellow and blackish-gray (commonest form), varying from light yellow to yellowish-brown, and in rare cases, a blackish-gray. These granules or colonies have, as a rule, an irregular or warty surface; the older structures unite to form masses which are visible to the unaided eye and bear some resemblance to a blackberry. They are firm, not easily crushed, and sometimes hard as a result of calcification.

In each granule these may usually be recognized by a central portion consisting of filaments, rods and spores and a peripheral portion composed of the club-shaped ends of the parasite.

The individual filament consists of a membrane enclosing a body of protoplasm and shows a distinct tendency to dichotomous branching, each branch in its turn possessing the same habits. Transverse partitions divide these filaments into short rods and the latter seem to be divided by similar partitions into coceus-like structures, the so called

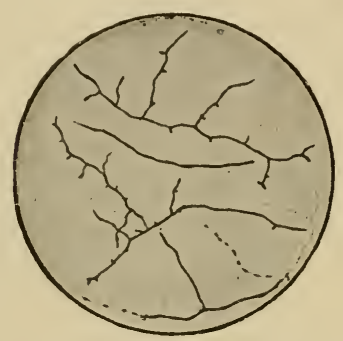

Fig. 99. Streptothrix Actinomyces. Agar culture in dilute fuchsin solution.

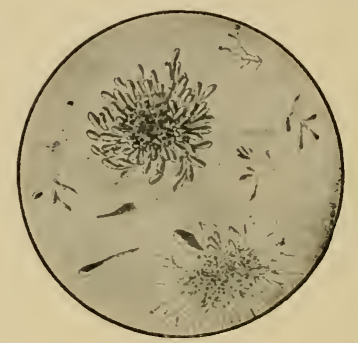

Fig. 100. Actinomyces colonies and single club-shaped indiviluals. From tumor of maxilla ; examined in caustic potash solution.

spores which, however, may also be found in the interior of the filament (according to Domec, at the ends only), thus producing torula-like structures. (These spore-like bodies, unlike the spores of bacteria or those of some mold fungi, e. g., those of the Oidium species, are readily stained with aniline dyes.) The so called spores, after separation from the filament, develop into rods which finally form new branching filaments.

Filaments thus developed intertwine and produce mycelium-like colonies, on the periphery of which the radially-arranged club-shaped ends project. In the old colonies in particular, these club-shaped ends constitute a continnous envelope surrounding the central mycelium, thus giving the colony the characteristic mulberry appearance. In colonies obtained from infected tissue the envelope referred to is usually broken and interrupted at some point, from which a thick strand of filaments projects from the central mass and to a certain extent penetrates the surrounding tissue walls (radical filaments). The club-shaped ends which are not infrequently constricted so as to resemble the fingers of a hand were formerly looked upon as buds (gonidia) (Harz, Bollinger, Johne, Ponfick). At present, however, the view of Boström is probably looked upon as more correct. According to this view these so called gonidia are the result of a specific hyaline degenera- 
tion and swelling of the external membrane of the filaments. (These club-shaped ends may be entirely absent when growth is prolific.) When the conditions for development are unfavorable, granules of lime salts are deposited in the colonies, sometimes also granules of iron sulphate.

The typical form of colony thus described which consists of a central mass of filaments with a peripheral envelope or mantle, is usually found well developed only in the actinomyces of human beings, while those found in cattle usually consist in the main of masses of club-shaped, swollen filaments.

According to the investigations of Lignières \& Spitz the property of forming dichotomous filaments is characteristic of a number of species of bacteria which may be referred to as actinophytes, while the diseases cansed by their presence are known as actinophytoses (See page 667). Among the actinophytoses we have 1. the classic Actinomyces bovis or Streptothrix actinomyces; 2 . the Streptothrix Israeli and 3 . the actinobacillus.

Staining. The filaments and spores stain readily by Gram's method or by that of Gram-Weigert. The elub-shaped ends may be stained with carmine, eosin, ete.

Cultivation. The Ray fungus thrives on the usual artificial media in the presence of oxygen, hut only moderately so under anaerobic conditions (cultures will not develop unless the colonies are crushed and the detritus thus produced transferred to the culture medium). On gelatin or agar, but still more beantifully on coagulated blood serum, it forms small points resembling dewdrop, which gradually become white and project above the surface like buds. In gelatin they sink to the bottom of the medium as a result of liquefaction of the latter. Subsequently the granules assume a yellowish or reddish (the latter especially in blood serum), occasionally also a decided brick red color, are rather hard and, being provided with radical filaments they become firmly attached to the bottom of the vessel; finally they become confluent and form large, dry, wrinkled and proininently projecting colonies. When not too thickly sown on potatoes they form gravish granules which finally develop into prominent, pitted colonies with crumpled borders and depressed centers, usually having a sulphur yellow, reddish-yellow or moldy color (Fig. 101), while on the other hand, when the material is thickly sown, a continuous growth occurs from 1 to $2 \mathrm{~mm}$. thick, delicately folded on the surface, yellowish-gray in the beginning, and later hecoming reddish-yellow. In houilion whitish granules occur which form a membranous mass on the surface of the fluid, or confluent masses imbedded in a slimy substance at the bottom of the vessel, while the fluid itself always remains clear. In fluid serum or in pleural exudate a capsule is formed around the filaments (Wright). The Ray fungus will grow also on eggs, in sterilized milk, in water and in other media.

Tenacity. Cultures are quite resistant to the effects of drying; if kept at ordinary room temperature they remain active for a year or more. The spores are not destroved by boiling for 14 minntes; $5 \%$ carbolic acid solutions are harmless in their effect; corrosive sublimate solutions of 1:000 will kill them in five minutes (Liebmann). Dry spores remain active for six years and withstand continuous exposure to the sunlight for 238 hours (Berard \& Nicolas). According to Nocard the addition of $1 \%$ potassium iodide to the medium does not retard the derelopment of the fungus, but according to Rajewsky the addition of $1 / 2 \%$ of potassium iodide completely checks development. 
Pathogenicity. Thus far it has been impossible to produce absolutely positive results in the transmission of the disease from man to animals. These experiments have usually consisted in the subcutaneous administration of colonies of the

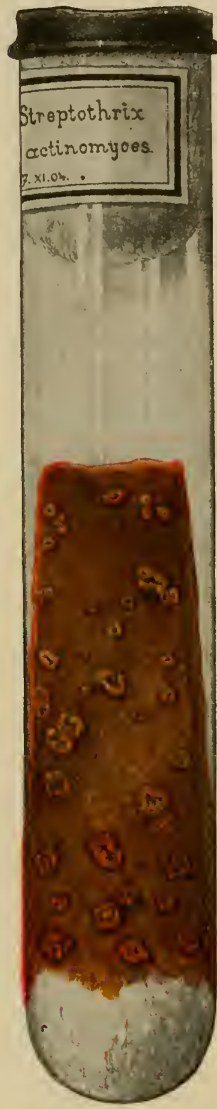

Fig. 101. Potato Culture of Streptothrix actinomyces six weeks old. parasite from man to animals. Submucous membrane inoculations and administration into the abdominal cavity or the anterior of the eye have also been tried. Johne, Ponfick, Israel, Rotter and Hanau believed to have obtained positive results in calves and guinea pigs in which nodules, ranging in size from a pea to that of a hazelnut, developed on the serous membranes. These lesions, however, were invariably of a benign and never of a progressive character. (Similar nodules are observed to develop when other foreign bodies had been introduced into the serous cavities.) Affanassjew, and subsequently also Israel and Wolff attempted experimental infection with pure cultures which, however, were also without convincing results, although Wright observed growth and club formations in the filaments found in the center of the resulting local abscesses.

Biagi succeeded in detecting an endogenes toxin in cultures, which prodnced marasmus in animals while Poncet, Lacomme \& Thévenot failed to recognize the presence of soluble toxins.

Natural infection takes place through the mucous membranes, usually that of the mouth, through the medium of gramineae, particularly the awns of the heads of barley (Johne). Boström found in the examination of 5 cases of liuman actinomycosis portions of awns, only $0.2 \mathrm{~mm}$. in length, imbedded in the more recent portions of the tumors, in the center of masses of lencocytes and granulation tissue, which in turn were surrounded on all sides by mycelium and characteristic fungus colonies. In one case he demonstrated that the fungus had originally existed in the air spaces of the awns where it had proliferated, penetrated the walls of the air spaces and found its way into the surrounding tissue elements.

This result of Boström's microscopical examination explains the manner of infection in these and similar cases. Awns of grasses which accidentally come in contact with the mucous membrane of the upper digestive tract force themselves through 
the mucous membrane by means of a peculiar boring motion, particularly by way of the excretory ducts of the salivary glands or through the depressions on the surface of the tonsils into the deeper tissues where they are firmly held by numerous fine barbs, the contraction of the neighboring muscles serving to draw them still deeper into the tissues. The characteristic tissue changes may be observed to develop along the path of invasion of the awn by subsequent irritation through the fungus contained in the latter (this explains the frequently observed metastases of the actinomycomata from the base of the mouth cavity to the region under the skin of the intermaxillary space. The Ray fungus exists as a saprophyte on grass and especially the various species of grains and according to Boström has a special preference for the air tubes in the stems of these plants. Its propagation is favored by damp soil and moist atmospheric conditions, while the considerable resisting power of the spores insures the perpetuation of the species under unfarorable climatic conditions. Infected plants, especially barler, when fed drr, may produce injuries of the mucons membranes and thus afford opportunity for the entrance of the fungus into the tissues. The disease is in fact most frequently observed in winter, and in spring, corresponding to the seasons of dry feeding. On the other hand a similar entrance may be effected on pasture, particularly in stubble fields, and give rise to infection at the lips.

Brener observed actinomycosis during the latter portion of winter and early part of the spring months (period of dry feeding) in $33 \%$ of adult animals, while during the summer months only a frequency of $16 \%$ is recorded. Liebmann observed the development of the Ray Fungus on wheat and barley straw that had been grown on infected soil. Berestnew observed the development of the fungus on the straw and hearls of grains that had been kept moist for some time, while accorting to Bang they have been observed to thrive on grains and straw, particularly barley. According to Brazzola the fungus thrives most luxuriantly on a certain species of wild barley.

The enzootic development of the disease following feeding with barley or barley straw points to the intermediary role played by the awns of barley in the dissemination of this disease. Thus on an estate near the Danish seacoast the majority of a herd of cattle became affected with actinomycosis after having been fed for some time with straw and grain that had been grown on a drained area of the seacoast. At a subserfuent date when similar material was fed, the disease appeared anew (Jensen). According to Nicolaus the disease occurs in regions of Silesia where barley is grown extensively, apparently as a result of the practice of feeding barley straw, especially chaff, without preliminary steaming or scalding.

Barley awns have been found in the diseased tongues of cattle as well as in the tonsils of swine and in a few instances (Johne, Neuwirth, Piana, Korsak) it was possible to demonstrate the presence of the Ray fungus on the surface or in the interior of the awns as well as in the surrounding tissues.

Contrary to the opnerally accepted view that the Ray fungus is a saprophyte or facultative parasite, Wright has expressed the opinion 
that the cause of actinomycosis of man and animals is an obligatory parasite which will not grow at room temperature and exists as a permanent inhabitant of the digestive tract from which it occasionally penetrates the underlying tissues. Foreign bodies, according to the latter's view, play a part in this process only in so far as they assist the parasite in gaining an entrance into the tissue substance. According to Wright the saprophytic fungi that are found on grains, etc., should be differentiated from the true actinomyees as belonging to the group "Nocardia."

Infection is farored on the other hand by the soft and swollen condition of the mucous membranes of the mouth and gums or by injuries such as occur during the shedding of the teeth (Roger, Imminger, Schouten), or in the course of foot and mouth disease (Faletti). On the other hand infection is favored by the anatomical structure of the tongue. The remarkable frequency of actinomycosis of the tongue in mature cattle bears a certain relationship to the fact that the formation of the so called "groove" of the tongue begins at that time as a result of atrophy of the filiform papillae on the crescent-shaped surface of the tongue, causing the mucons membrane to become thimner and drawn into transverse folds. Hard particles of food become lodged in this groove and are, by the muscular contraction of the tongue, forced into its interior (A. Bremer). According to Henschel, Falk and Nicolaus particles of plant food are retained especially in young animals by the lorn-like filiform papillae, and are thus afforded a favorable opportunity for entering the pocket-like depressions which are located just anteriorly to the dorsal prominence of the tongue. In the more posterior portions of the digestive tract this method of infection is not as frequently observed, which is explained by the fact that food particles when they reach these regions have been moistened with the saliva and become softer in consistency. In spite of this fact infection occasionally occurs in the esophagus and even in the intestinal wall, especially in the region of the cecum where the feces are dryer and are retained for a longer time.

Infection by means of the inhaled air may be assumed in sucl cases only where the lungs are the primary seat of the disease.

The skin affords an effective protection and consequently infection is possible only through injuries. Castration wounds in horses, more rarely in cattle and swine, constitute the most frequent ports of entrance for the contagion. So called scirrhus cord is the result at least in a part of the cases of contact with infected straw (Johne). The development of actinomycomata in other regions of the body as a result of superficial or deeper injuries of the skin are much more rare (Gooch observed them in cattle treated with setons, Liénaux after rumenotomy). Finally, this parasite may gain entrance into 
the udder through the milk ducts thus causing the comparative frequency of affection of this organ in swine.

Of the domestic animals cattle are most susceptible to infection. The disease occurs much less frequently in swine and only rarely in horses, sheep, goats and dogs (according to Schonten it is quite prevalent among the goats in the Netherlands). Breed or sex have no influence on the development of the disease, although the seat of the morbid process is determined by the age of the animal. Thus in young cattle actinomycosis of the pharyngeal region is quite common while actinomýcosis of the tongue is usually observed in mature cattlè only.

Pathogenesis. As a result of the inflammatory reaction, nodules composed of small round cells form around the parasite. Some of the mycelia penetrate the tissue cells which become enlarged and develop into epithelioid cells which, in their turn, by division of their nuclei, develop into giant cells. The cells in the interior of the nodule, which resembles a tubercle, degenerate and thus the center of these nodules are composed of cell detritus, nucleated and free fat globules. These primary nodules are surrounded by gramulation tissue on the periphery of which new nodules develop which are again enclosed by newly formed connective tissue. In this manner large spherical and lobulated tumors so called actinomycomata (Johne) are developed. These may resemble in structure either a soft sarcoma or have the appearance of a dense fibroma. Accordingly a section of the tumor may appear homogeneous, grayish-white, marrow-like, or fibrinous and reddish-gray. In this ground substance there may be found pale gray or yellow foci of loose consistency in which are imbedded grayis!. or sulphur colored actinomyces imbedded in a mucous or purulent substance and having the appearance of grains of sand. These foci may become confluent and thus give rise to fistulous canals filled with granulation tissue of a gelatinons consistency and penetrating the substance of the tumor

Actinomyces which have gained access through the mucous membrane of the tongue become disseminated by means of the lymph channels and give rise to the development of small nodules surrounded by an area interstitial inflammation. These nodules gradually increase in size, finally breaking abruptly throngh the mucous membrane from which they project like a mushroom or cauliflower. In the so called groove of the tongue, on the other hand, simultaneous infection with pyogenic bacteria may give rise to deep nlcers.

When these tumors develop in the interior of flat bones the granulation tissue causes destruction of the bone lamellae. At the same time the production of new bone tissue proceeds from the periostemm, while on the other hand the development 
of abundant fibrinous tissue may cause the destruction of the actinomyces and thus bring about spontaneous recovery.

Occasionally in loose connective tissue generally, especially in swine, but also in man, there occurs emigration of large numbers of leucocytes from the surrounding vessels as a result of the irritation produced by the parasite. Following this, large areas of the tissue surrounding the nodules undergo purulent softening, become confluent with other similar areas and thus produce large abscesses or fistulae which are always surrounded by a thick and usually very firm connective tissue wall. Occasionally the suppurating process is induced by pyogenic bacteria which are met with most frequently in the pus of the larger abscesses.

The mycelium of the parasite may penetrate the cells of the blood vessels and be transported to other regions of the body, thus giving rise to the development of metastatic tumors or abscesses such as are observed quite frequently in spongy bones, but also in the brain and other organs.

Anatomical Changes. The seat and character of the anatomical changes vary with the species of animal.

In cattle the tongne is most frequently affected, the maxillae coming second; tumor's of the skin in the region of the hearl and neck as well as larynx are also quite common, while the internal organs are only rarely affected.

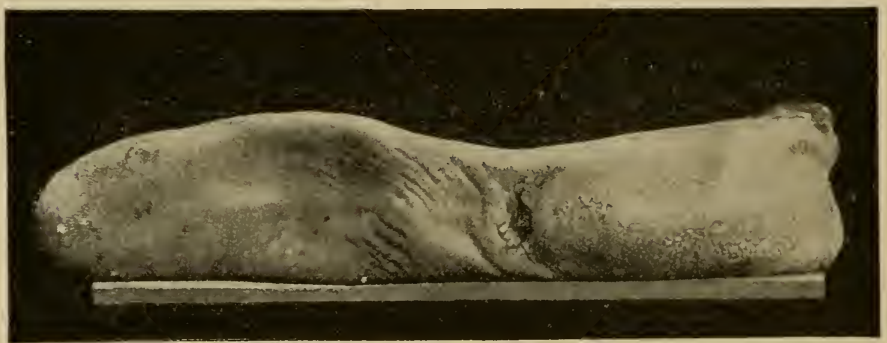

Fig. 102. Actinomycotic Ulcer on the Dorsum of the Tongue (food cavity) in an $0 x$.

Actinomycosis of the tongue frequently takes the form of an ulcer situated immediately anteriorly to the dorsal prominence of the tongue (so called "food cavity"). In this condition there are one or more ovoid lesions, or transverse bands, consisting of loss of tissue substance; the bases of these lesions are surrounded by raised borders and are covered by numerous small depressions with ragged outlines. Hairs, or masses of vegetable fibers project from the depths of these depressions, sometimes in such quantities that they hide the entire lesion from view (Fig. 102). Thus in a section of the tongue there 
may be seen whitish-gray spots penetrating to a certain extent below the surface of the mucous membrane; when subjected to pressure these will discharge greenish-yellow masses of plant food imbedded in a tough enveloping substance. The border's of these spots are surrounded by yellowish-gray, firm nodules which contain a cheesy or pus-like mass in which are found gray or brownish actinomyces colonies as large as grains of sand. In addition to this, fistulous canals may occasionally penetrate the substance of the tongue. These canals are surrounded by a firm connective tissue wall from which hairs and small nodiules project, the latter being filled with decomposing food particles mixed with a cheesy, fatty mass emitting a highly disagreeable odor (Breuer).

According to Breuer's statisties this form of actinomyeosis of the tongue occurs in over 20 to $25 \%$ of all eattle one year of age, or more, and in the great majority of Hungarian draft oxen slaughtered in the abattoirs of Bulapest. Un the other hand Nicolaus found in Silesia (Glogau) $88.5 \%$ of affecter cattle to be under 4 years of age and only $19.5 \%$ above 5 years. This difference is possibly due to the fact that Hungarian draft oxen are usually not stable fed until after they are 4 years of age.

In some cases nodules and tumors are found, varying in size from a millet seed to that of a hazelnut, scattered throughout the mucous membrane and in the muscle substance of the tongue, especially the lateral portions of this organ. These tumors may be visible as prominences under the mucous

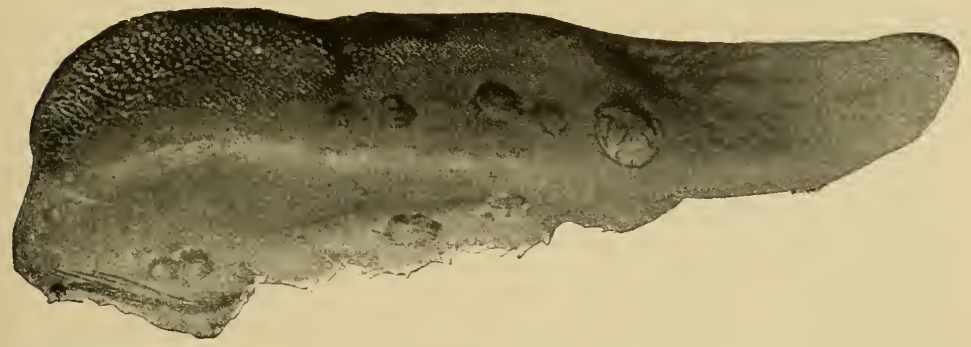

Fig. 103. Actinomycosis of the Tongue with Pyramidal Tumors on the Surface.

membrane of the tongue or project in the form of a fungoic mass through the opening in the mucous membrane, with characteristic, sharply defined borders resembling holes made by a punch (Johne; Fig. 103).

In a more advanced stage of the disease the interstitial connective tissue of the tongue is much proliferated and usually contains numerous nodes that have undergone softening while the consistency of the organ which is much enlarged is very firm and almost wood-like, hence the name "woody tongue.", The upper surface of the tongue may contain cicatricial con- 
tractions which occasionally cause marked deformity of the organ, especially an abnormal upward curvature and a contrac. tion of the whole organ (Axe).

In the maxillary bones the disease usually begins in the marrow cavity or periosteum. In the first instance the spongy bone substance is replaced by a soft sarcoma-like tissue resulting in breaking down of the bony trabeculae and distension of the lamellae. The affected bone appears much enlarged with the external lamella considerably thickened as a result of osteoplastic inflammation (periostitis ossificans), eventually becoming perforated. This is followed by the projection of soft canliflower-like proliferations under the skin, into the month or into the pharynx.

In the interior of the tumor the bony substance has been reduced to an open network composed of thin trabeculae, as may be recognized upon section by its white color and hard consistency. The soft tissue proliferation surround also the roots of the teeth and may cause them to be forced out of their alveoli. Thus teeth with healthy crowns may be found to have their roots imbedded in the soft substance of a tumor.

The granulations which project from the gums consist of hemispherical, irregular masses varying in size from a pea to that of a walnut. Sometimes they perforate the gums or mucous membranes and project into the mouth cavity. Frequently they present an ulcerous surface.

After maceration the affecterl maxillary bone appears to consist of a more or less perforated external bony lamella and an internal open spongy structure. This condition recalls vividly to mind the deformity formerly designated as "spina ventosa" resulting from myelosarcomatous invasion. It is significant that actinomycomata usually develop near the molar teeth and are only exceptionally met with on the toothless portion of the gums or in the region of the incisors (Moussu).

Tumors originating in the periosteum of the maxillary bone are usually much firmer and tougher in consistency while upon section they are found to contain fewer and smaller centers of softening. They occur on the external or internal face of the maxilla, occasionally also near the incisors and are attached only loosely to the skin or mucous membrane, but are firmly united with the periosteum. In exceptional cases they may give rise to the development of so called "dermal", horns with bony bases (Johne).

Tumors of the superior maxillary bone are apt to sprearl to the subparotideal region and to the fossa pterygo-palatina. In exceptional cases they force themselves through the natural foramina, perforate the flat basal bones of the cranium, and enter the cranial carity, affecting the meninges and even the brain itself. On the other hand they may extend from the alveolar process to the antrum of Highmore. The disease may also develop primarily in the latter (Bang). In such cases after filling out the antrum and breaking down the bony tissue, 
the tumor may perforate the skin of the face in the temporal region, or the hard palate, and project into the oral cavity.

When occurring in the pharynx, actinomycomata consist of mushroom-like polypous tumors, slightly pedunculated and occasionally filling the entire pharyngeal space. On the other hand they may be observed as more or less prominent, irregular, or lobulated tumors under the skin on either side of the throat. In some instances these retropharyngeal tumors which consist almost wholly of granulation tissue containing soft, grayishyellow foci, may also contain large abscesses which may perforate to the interior or to the exterior, as the case may be.

Actinomycosis of the esophagus is very rare. Siedamgrotzky \& Johne observed small flat subepithelial nodules, the larger norlules containing a yellowish transparent focus. The confluence of the smaller nodules gave rise to larger irregular groups which projected into the esophagus. In rare instances large polypi may be observed, or roundish tumors with smooth surface may occur on the esophageal wall near the larynx (Joest).

In exceptional cases actinomycosis has been observed in the omasum (Bollinger), in the reticulum (Johne), in the abomasum and small intestine (Perroncito, Jensen, Axe), oceurring either in the form of tumors resembling fibromata or sarcomata, roundish in form or lobulated, and containing soft foci in the interior, or in the form of morbid changes resembling tuberculous ulcers. In nearly all cases adhesions had occurred in the affected portions of the intestine.

In the affection of the abdominal organs we occasionally find the peritoneum covered with numerous actinomycomata of various sizes, the latter also being frequently present between the tough pseudo-membranes which unite the affected organs and loops of intestine. These tumors are frequently suppurating or ichorous. The liver often contains metastases. Whether or not actinomycosis of the peritoneum ever occurs (as is assumed by Jensen) is as yet an open question.

Actinomycosis of the respiratory organs is of much less frequent occurrence. Kitt observed involvement of the nasal mucous membrane in the form of grayish-white nodules as large as millet seeds and containing yellow centers. Bragadio observed excessive proliferation and Röders, in one instance, saw a tumor which developed near the opening of the nostrils and almost completely obliterated them. Stadie and Kutsera each found in one instance diffuse infiltration of the mucous membrane of the nose and its sinuses, the mucous membrane was firm in consistency, irregular on its surface and in some places $5 \mathrm{~mm}$. thick. Its substance contained numerous grayishyellow nodules. These nodules, some of which were as large as peas, were frequently confluent, forming folds and ridges or more extensive prominences (Kutsera referred to this form as actinomycosis confluens). Similar nodules may also occur 
on the epiglottis (Johne), while sessile pedunculated tumors as large as a hen's egg, of spongy or firmer consistency, may form in the larynx.

In the lungs the disease is manifested by numerous gray or yellowish tubercle-like nodules or tumors which are present in large numbers in the lung tissue and contain a thick, mayonnaise-like pus in their center's. Now and then an atelectatic or marbled area of lung tissue may be riddled with foci consisting of pus or tissue detritus. In such cases the pleura is thickened, occasionally covered with pedunculated tumors, or the visceral and parietal layers are adherent. In exceptional cases there may be hydropneumothorax (Moussu).

The lymph glands are rarely affected and as a rule only as a result of disease of the neighboring organs. They then form firm tumors containing white or yellowish, glistening, non-caseated nodules surrounded by a connective tissue capsule and imbedded in the tough connective tissue of the substance of the gland. The submaxillary, pharyngeal, subparotidial and mediastinal glands are most frequently affected.

In contrast to the not infrequent occurrence of this disease in the lymph glands of cattle, actinomycosis of these organs has never been observed in mau (Ponfick, Israel, Boström, ete.). Schuhewitsch has observed the disease in the lymph glands and its dissemination throngh the medium of the lymph vessels in from 15 to $20 \%$ of all cases observed in cattle at the abattoir of Moscow.

Actinomycosis is occasionally observed also in the following organs: In the liver (Rasmussen observed 20 cases in the course of one year in the abattoir of Copenhagen; in a case described by Balas infection was produced by a piece of wire in the reticulum), in the spleen, in the kidneys, in the brain, in the basal meninges, in the diaphragm, in the testicle (Kowalewsky observed one case in which this organ was extensively affected), in the nterus, in the vagina, in the bladder, in the bones and in the muscles. In the muscles the disease appears in the form of fibroma- or sarcoma-like masses and in the form of pus foci containing yellow, sandy formations and enclosed by means of a thick, tough connective tissue capsule.

Actinomycomata in the skin appear either as primary lesions or as a result of the extension of lesions in the underlying tissues. This is particularly common in young animals in which they occur below the ear and behind the lower jaw. They are much more rarely met with in the subcutaneous connective tissue, for instance, near the larynx where they appear in the form of firm nodules, or in other regions of the body where they appear as whitish, soft, but firm, foci. Infection of the lips manifests itself by thickening and induration of this organ.

Primary actinomycosis of the udder is rare, but when present, it appears in the form of nodes varying in size from a bean to that of a walnut, enclosing soft purulent contents and 
imbedded in one or more lobes of the gland. In rare cases the gland is enlarged; section of the udder shows enlargement of the affected lobules which contain small, yellow points in their centers so that the cut surface appears granulated and suggests the picture of miliary tuberculosis (Jensen).

Generalized actinomycosis is very rare. In such cases metastases are present chiefly in the lungs, in the liver, in the kidneys, in the brain, in the subcutaneous connective tissue, in the udder and, finally, also in the internal as well as external lymph glands.

Localization of the lesions of actinomycosis seems to vary according to its geographical occurrence. In the compilations made by Mitteldorf for Bavaria, including 3621 cases, the head and neck were affected in $75.5 \%$; the tongue in $23.3 \%$, the abdominal organs in $0.77 \%$, the udder in $0.05 \%$. Imminger found the head and neck affected in 55 to $90 \%$, the tongue in only 4 to $8 \%$ of all cases observer. In the abattoir of Burlapest on the other hand, actinomycosis of the tongue is much more frequent than disease of the maxellae (Brever). In Russia, according to Jelenenszki, disease of the lips is conspicuously frequent, thus $37.9 \%$ of all cases observed in Moscow, $85.9 \%$ in Tiflis, $81.6 \%$ in Jelisawetgrad. Kowalewsky also found the head affected in $99 \%$ ( 1465 eases) of which number $57 \%$ were affections of the submaxillary lymph glands $6 \%$ of the retropharyngeal lyniph glands, $5 \%$ of the lower lips. According to Mari (Moscow) on the other hand, the skin is the most frequent seat of the disease (271 out of 541 eases), next in order being the parotid gland, 177 cases, and last the bones of the head, 117 cases (this author classifies tumors extending from the skin or from the glands to the maxillary bones according to the original point of infection). According to Schouten the tongue is the most common seat of the disease in England, Germany and Italy, the maxillary bones in France and the lips in Russia, the liver in Denmark, the submaxillary and sublingual glands, the lungs and the maxillary bones in Holland. In the abattoir of Amsterdam 33 to $65 \%$ of all cases were affections of the udiler.

In horses actinomycomata develop most frequently in the inguinal canal where they may attain the size of a child's head and may extend into the abdominal cavity. Their tough connective tissne contains yellowish gelatinous foci in which are imbedded the actinomyces colonies (the anatomical structure is much the same as that in botryomycosis of the spermatic cord). Actinomycosis has also been observed in the superior and inferior maxillae and in the submaxillary lymph glands (Schmidt), in the cervical and bronchial glands, in the tongue, the lips, the urlder, the bones and the muscles (Struwe, in generalized form), in the lung, liver and spleen (Bruschettini) as well as in the skin and in the subcutaneous connective tissue.

In swine primary actinomycosis of the mammary glands is the most frequent form of the disease (Rasmussen observed in Copenhagen 52 cases in the course of 3 months). The mammary glands become partially or wholly transformed into a firm swelling approaching the size of a man's head, and on the surface of which the teats seem shrunken and not infrequently gangrenous. The main mass of the tumor in these as in other animals consists of a very tough connective tissue structure, lardaceous in appearance and in it are imbedded small or large abscesses filled with greenish-yellow pus or foci consisting of soft, marrow-like tissue. These foci frequently 
contain minute yellowish points and small cavities which suggest on cross section the appearance of a sieve or a sponge. Tumors occurring in the region of the elbow or under the abdominal wall have a similar structure.

Actinomycosis of the tonsils is also frequent (Johne). This may be followed by abscess formation in the peripharyngeal connective tissue. The maxillary bones, the larynx, the lings and occasionally other bones may also become affected in swine. Metastatic infection may occur in various internal organs, the pathologico-anatomical changes being similar to those observed in cattle, with the difference, however, that actinomycosis of swine, like the disease in human beings, frequently manifests itself in the form of extensive suppurations which may extend to the subcutaneous retropharyngeal and prevertebral connective tissue, causing a breaking down of the tissue elements and leading to the ultimate formation of abscesses and fistulous canals under the serous membranes and the glands. This destructive process may extend from the prevertebral connective tissue to the vertebrae themselves. As a result the periosteum of the latter is destroyed. They become rough and soft from loss of tissue, and their spongy substance contains small pus foci. In this manner the process may spread from the retropharyngeal connective tissue, following the course of the prevertebral comnective tissue along the back or to the mediastinum, and after destruction of the bodies of the vertebrae into the vertebral canal itself.

In actinomycosis of the tongue its parenchyma contains abscesses approaching a hazelnut in size and consisting of coagulated masses of pus containing actinomyces colonies, or of a conglomeration of actinomyces colonies $6.0 \mathrm{~mm}$. or less in length and surrounded by a connective capsule (Hollandt observed such lesions in 11 out of 170 tongues).

In the generalized form of actinomycosis there are now and then metastatic abscesses in almost any of the organs, including the brain, the bones, the lymph glands and serous membranes. The primary focus may have its seat in the mucous membrane of the digestive tract or in the skin (Knoll, Carl, Zietschmann, Assmann).

In sheep and goats actinomycosis is very rare, but when present, it is usually found in the tongue. In exceptional cases the lips, the jaws and the lungs may also be affected. (Berg observed three cases among 400,000 sheep. Zala-Szent-Gróth observed the disease in the tongue of three sheep in a flock of 42.) The pathological changes are similar to those in cattle.

Fumagalli observed the disease in a dog in the form of a firm swelling of the gums (epulis) about the size of a walnut, while Rossi found numerous nodules in the lungs of two cats 40 days old. The lungs of one of these contained a mass of firm connective tissue studded with actinomyces colonies, some of which were calcified. 
Symptoms. The pathological changes described above may be recognized intra vitam as far as they are accessible by external examination.

Actinomycosis of Cattle. In actinomycosis of the lips which is quite common in some regions, there are to be found round or ovoid, firm tumors that have a tendency to undergo softening in the center. These tumors occur in the submucous connective tissues of one or both lips and vary in size from a pea to that of a walnut. In exceptional cases excessive proliferation of fibrinous connective tissue is noted in the surrounding region, as a result of which the upper lip is transformed into a firm and rigid mass sometimes $56 \mathrm{~cm}$. in diameter (Fig. 104).

The first stage of actinomycosis of the tongue, when it occur's in the form of a dorsal ulcer as already described, is usually unobserved. When the muscle substance becomes affected to any extent, the movement of this organ, and consequertly also mastication, are interfered with. The animals lick their noses, saliva flows from the mouth while closer inspection reveals the presence of the above described ulcers on the rigid and painfully sensitive tongue. The secretions of the ulcers contain colonies of actinomyces. In more serious or advanced cases the

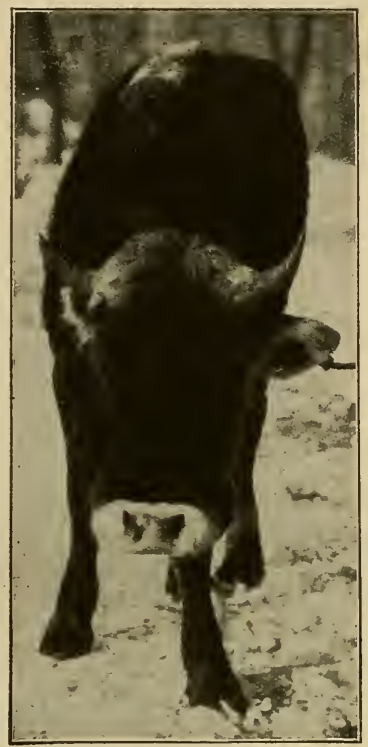

Fig. 104. Actinomycosis of the Upper Lip. Cured by internal application of potassium iodide. tongue becomes swollen and as a result is forced out of the mouth, its tip usually being ulcerated and covered with a foul secretion emitting a disagreeable odor. The surface of the tongue is coarsely bosselated and the organ itself is firm, almost wood-like in consistency.

Actinomycosis of the jaw bones (Figs. 105 and 106) is usually not observed until the head of the affected animal presents more or less of a deformity. Myelogenic actinomycomata produce only a uniform swelling of the body of the jaw bone, which in itself, however, may prevent mastication. If the process extends from the bone to the masseter muscles mastication may be seriously interfered with and the condition may even result in trismus, which is, however, in some cases, only

Vol. 1-42 
temporary. The general mutrition of the animal may be interfered with as a result of the soft pathological tissues in which the teeth are now imbedded. In some cases tissue masses project through the external bony plate of the maxilla and may there be palpated under the skin as lobulated ulcers. In other cases the skin may be ulcerated at one or more points, resulting in the formation of a purulent secretion containing actinomyces colonies and covering the surface of the canliflowerlike mass of granulation tissue. The submaxillary lymph glands may be enlarged, firm, slightly movable and eventually ulcerated.

In the skin or subcutaneous connective tissue (Figs. 107 and 108) there is usually a firm, elastic tumor posterior to the angle of the jaw, below the ear, on the cheek or at the neck. This firmly unites with the overlying skin, which it usually

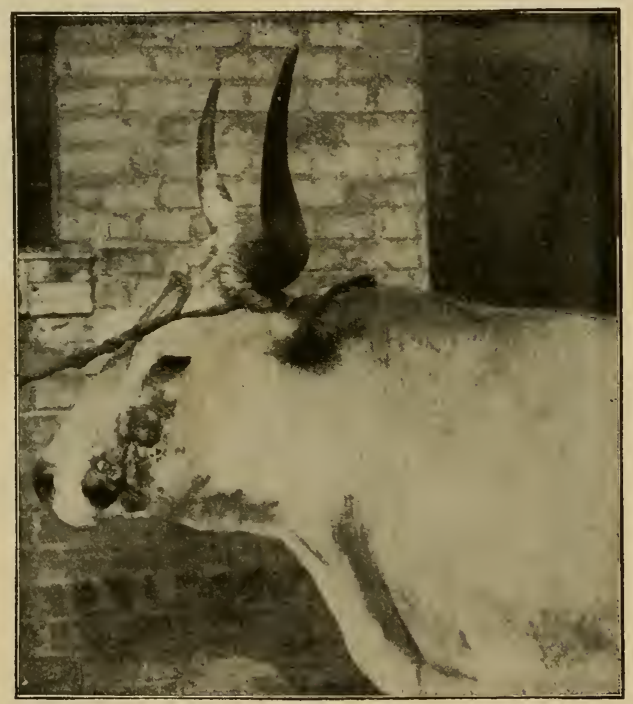

Fig. 105. Actinomycosis of the Upper Jaw.

perforates, and discharges a thick creamy pus or pus-like mass containing tissue detritus and coagulated pus elements. In other cases the cavity thus formed becomes filled with granulation tissue which may project beyond the surface of the skin in the form of a cauliflower-like tumor, the surface of which is covered with crust. Upon removal of the scab the surface is bright red, the slightest contact causing bleeding. Numerous minute yellowish points from which small drops of pus may. be pressed out may also be observed. In some cases pus foci 
may appear in a number of places and discharge their contents, while the surrounding skin and subcutaneous connective tissue is swollen and edematous, thus producing conspicuous deformity of the affected region of the body (in a case observed by Moussu the head had the appearance of the head of a hippopotamus, as a result of diffuse infiltration of the skin and subcutaneous connective tissue of the face and the entire maxillary space). In rare cases the process is arrested spontaneously, whereupon the tumor gradually recedes, swelling subsides, the tenseness of the surrounding tissue disappears and finally recovery takes place.

Large tumors may also occur on the extremities, Liipke observed an infection of the foot beginning in the interdigital space and resulting in a condition resembling elephantiasis. In a similar case reported by Gulyás the leg was enlarged from the middle of the carpus down to three times its natural size, its surface being wrinkled and covered with nodules, the flexor surface of the pastern containing a granulating ulcer as large as a dinner plate. The calloused subcutaneous connective tissue and the underlying muscles contained numerous nodules ranging in size up to that of a hazelnut, the lymph vessels were much enlarged and numerous nodules were scattered along their course to the sacrum. The popliteal glands formed an extensive cauliflower-like mass.

Actinomycomata developing in $\mathrm{t}$ h $\mathrm{e}$ wall of the pharynx or in the neighboring areas will extend in the subparotideal region, and interfere with deglutition. As a result rumination is interrupted, animals take less food, there is more or less salivation, but in spite of this, the general nutrition may not be seriously affected for several months. Respiratory difficulties a re more pronounced, the act of respiration being attended by $10 \mathrm{ud}$

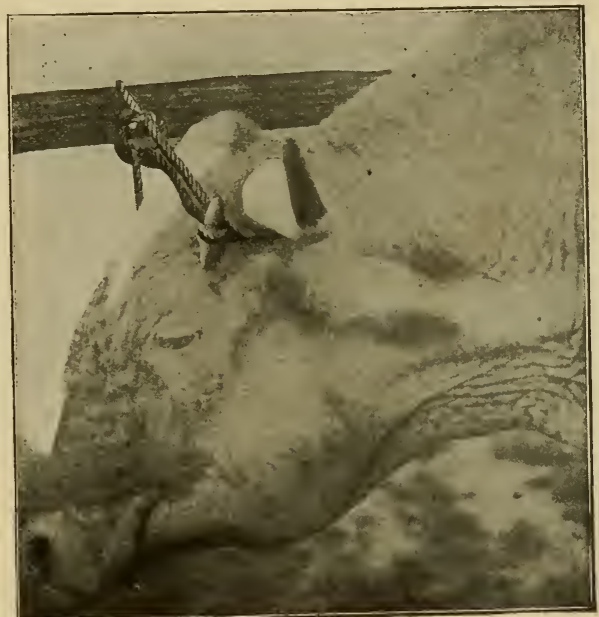

Fig. 106. Actinomycosis of the Lower Jaw. whistling or rattling sounds which are very audible when the head is depressed. When the head is extended tumors in the subparotideal region may be palpated with the fingers either from the exterior or by inserting the hand through the animal's mouth. Animals may 
also cough from time to time, especially while feeding, sometimes also in the act of swallowing, the cough is strong, dry and whistling.

Sometimes large numbers of calves in the same herd will be found affected in the region of the larynx as manifested by the appearance of firm tumors as large as a man's fist in the perilaryngeal connective tissue. The presence of these tumors interfere with respiration much the same as tumors within the larynx, the presence of which may be recognized by the whistling, rattling or stenotic sounds produced during inspiration.

Affection of the esophagus manifests itself in difficult deglutition (dysphagia) and other symptoms of esophagusstenosis. The cause of this trouble which develops very gradually is usually not disclosed until after the death of the animal, although it may be suspected during life when actinomycotic tumors are present on other parts of the body, especially the neck. (In one case reported by Ujhelvi an affection of the peribronchial and mediastinal lympli glands produced chronic meteorism.)

The symptoms of actinomycosis of the lungs are not very characteristic. In cases thus far observed only symptoms of chronic pulmonary disease have been noted, the nature of which was not disclosed until post-mortem

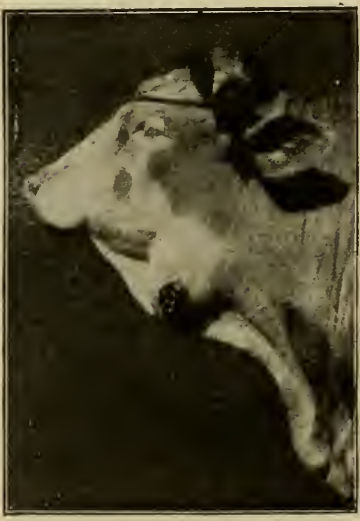

Fig. 107. Actinomycosis of the skin.

(According to Plósz). examination. The bronchial secretions in such cases might possibly contain actinomyces and their demonstration would establish the diagnosis with certainty.

Actinomycosis of the udder manifests itself either in a firm enlargement of the whole organ or in the appearance of firm, sliarply circumscribed, somewhat painful nodes as large as walnuts, which may be scattered throughout the gland, but particularly through the upper portions of one or more quarters. In case these nodes should discharge their contents into the milk ducts, the milk of the corresponding milk cistern will contain pus and be of a ropy consistency; in some cases there is merely a slight discharge of a caseo-purulent mass. In some instances we may be able to recognize actinomyces colonies in the milk with the unaided eye, though better with a microscope (Klepzow). The supramammary lrmph glands are not enlarged as they are in tuberculous disease.

Actinomycosis of the cerebral meninges manifests itself 
by the appearance of symptoms of meningitis consisting at first in disturbance of consciousness, unsteady gait, falling down, climbing into the manger and finally, pronounced coma (Assmann).

Görig observed one case in which the testis was considerably enlarged, very firm and sensitive. The skin of the scrotum contained an ulcer as large as a five cent piece, which discharged a caseo-purulent secretion. A similar case has been described by Petit. Disease of the scrotum exclusively is mentioned by Hell.-Poes observed one instance in which an actinomycoma penetrated the spinal canal in the region of the seventh cervical vertebra, obliterating the greater portion of its lumen up to the fifth vertebra. This was attended by symptoms of general muscular weakness, fear, difficulty in deglutition, attacks of mania and finally spasms of the muscles of the anterior extremities.

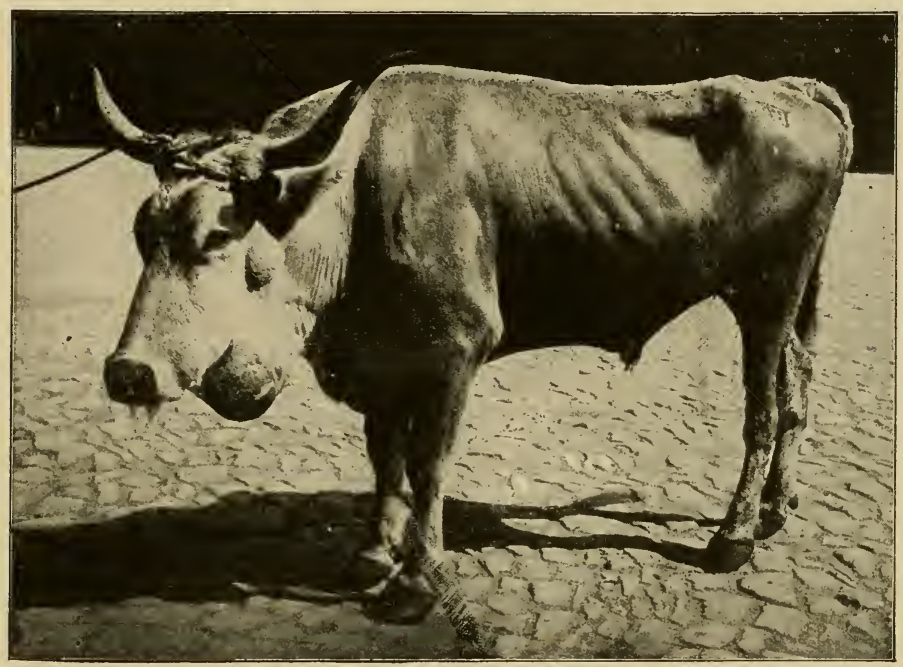

Fig. 108. Actinomycosis of the Intermaxillary Space and the Subparotideal Region.

Among horses this disease is quite rare, but when present, usually affects the spermatic cord (fistula of the spermatic cord). Affection of other organs produces symptoms similar to those produced in the corresponding organs of cattle.

Affection of the intermaxillary lymph glands is of particular interest. It may be primary and bear some resemblance to glanders. However, an actinomycotic tumor is more liable to suppurate, and the retropharyngeal and the superior cervical lymph glands are usually also affected in this disease (Schmidt, 
Roettger). Tumors on the neck may attain an enormous size, sometimes interfering with the lateral movement of the head (Bonchet).-Actinomycosis of the superior maxilla was observed in a mule by Aubry, affection of the spinous process of the superior maxilla by Rousselot, while in a case described by Hamburger the clinical picture of actinomycosis of the bone resembled that of rachitis.

In swine actinomycosis of the mammary glands usually appears in the form of a nodulated, painless swelling and induration of the base of the teat. Subsequently this process spreads to the neighboring teats and glandular substance, thus producing the deformity of the mammae already described. Affection of the tonsils usually does not attract attention. In rare cases, however, they may become much enlarged and then are accessible to palpation in the region of the pharynx. Cold abscesses which occur in the pharyngeal region and frequently attain the size of a child s head are nearly always of an actinomycotic nature. In actinomycosis of the ear the deformer, firm concha may attain a weight of $3 \mathrm{~kg}$. (Junack).

Course. The course of actinomycosis is always chronic and may extend over months and even years. Especially when it lias its seat in the interior of bones, the disease develops at first very insidiously, and in such cases it is usually not recognized until conspicuous deformity of some external organ of the body has developed, or, in case of affection of the nuaxillae, disturbance of nutrition or respiration become evident. In spite of the presence of the disease of the maxillae or the tongue, affected animals may nevertheless remain for a long time in good condition, in fact the presence of pharyngeal tumors which may interfere with deghutition as well as respiration, may not prevent the affected animal from taking on flesh. Finally, however, such conditions will exert an injurious influence, the affected animals may no longer be able to feed properly, they lose flesh, become emaciated and finally die from exhaustion. In rare cases polypous tumors in the pharrnx may close the lumen of the larynx and cause suffocation.

The presence of tumors in the skin and in the subcutaneous connective tissue may not interfere with the general condition of the affected animal for a long time, it is only when they are very extensive that they disturb the functions of neighboring organs or tissues. On the other hand tumors in the region of fhe larynx and especially when they occur within this organ may produce serious disturbance of the function of respiration before they become very large.

The course of the disease is unattended by fever as long as it remains of a local nature; when suppuration becomes more profuse (mixed infection) as well as during the development of numerous metastases a rise of the body temperature is noted. 
Diagnosis. In doubtful cases the diagnosis of actinomycosis may be confirmed by the demonstration of actinomyces in the tissues or secretions. The fungus colonies which are usually rellowish and of the size of a poppy seed, may be seen by the unaided eye. Under the microscope, however, they are readily recognized in unstained preparation by their glistening, clubshaped structure (in case of existing calcification treatment with hydrochloric acid is necessary). When stained according to Gram's method the central mycelial mass may be recognized, and in the same manner branching threads of mycelium may be found in the purulent secretion. The presence of the latter is sufficient evidence upon which to base a diagnosis.

In regard to the differential diagnosis of superficial tumors, sarcomata, lymphomata and botryomycomata are of particular importance. In affection of the tongue, other inflammatory conditions of this organ as well as tuberculosis should be kept in mind. The latter disease should also be taken into consideration in the occurrence of subcutaneous tumors, although tuberculous lymph glands usually do not in mature cattle become adherent to the skin, and consequently do not break open and discharge their contents. There is also no diffuse infiltration of the surrounding tissues in the latter disease. As a matter of fact, however, the clinical pictures of tuberculosis and actinomycosis, especially in affection of the udder, bones and the lungs liave much in common, and a positive differentiation between the two diseases is possible only by an exchusion of the presence of secondary affection of the regional lymph glands as well as the exclusion of similar changes in more remote organs. The positive results of microscopical examination of secre'ions or tissue masses are of course always final. In regard to other affections of the udder, the differentiating characteristics are similar to those referred to under tuberculosis of the udder (see p. 591). The enlargement of the regional lymph glands and the absence of yellowish granules in the secretion of the ulcers of the tongue are sufficient to differentiate between actinobacillosis and actinomyeosis.

Prognosis. The prognosis may be considerer as favorable in those cases in which the seat of the disease is superficial and accessible to surgical treatment. Actinomycomata in the subparotideal or cervical regions may in exceptional cases terminate in spontaneous recovery (Bang, Jensen). In such cases the tumor becomes encapsuled in tough, fibrous tissue, which checks the further progress of the disease (cicatricial contractions so frequently observed in the tongues of cattle are evidently, for the greater part at least, the result of actinomycotic lesions). Since the introduction of the potassium iodide treatment for actinomycosis, the pregnosis of the affection of the internal organs ean be made much more favorable. However, the prognosis of the disease in bones is always unfavorable. 
Treatment. The quickest and most certain results are obtained by the total extirpation of the actinomycotic tumor, but such treatment is to be considered only when the tumor is sharply circumscribed and surrounded by healthy tissue, thus permitting easy removal (pedunculated actinomycomata of the pharynx may be removed through an opening in the subparotideal region [Harms, Mayer]). Very large cutaneous tumors are treated most satisfactorily by deep incisions followed by thorough curetting and the application of gauze or tow dipped in tincture of iodine. A very practical method and one which frequently proves successful, consists in inserting a trocar and canula into the apex of the tumor, permitting the purulent contents to escape after withdrawal of the trocar, irrigating the abscess cavity with water and then inserting with the aid of forceps a lump of arsenious acid 1 to $2 \mathrm{~g}$. in weight. This is followed by necrosis of the tumor mass, whereupon sequestration from the surrounding tissues and recovery follows (Heine, Koványi). Actinomycosis of the tongue, if not too advanced, frequently terminates in recovery after simple scarification and application of tincture of iodine or iodovasogen $(10 \%)$.

Moussu has succeeded in bringing about recovery in actinomycosis of the jaw by removal of the superficial tumor, curetting the interosseous tissue and internal iodine treatment.

In cases where these methods are impracticable or inpossible, parenchymal injections of antiseptic remedies frequently produce good results. Tincture of iodine, Lugol's solution (10 to $15 \mathrm{gm}$.$) and iodovasogen (8 to 12 \mathrm{gm}$. of a $10 \%$ solution) are well adapted for this purpose. External applications of caustics, e. g., ung. arsen. (1:8) or cantharides ointment with iodide of mercury $(8: 1)$ are also recommended. After suppuration has resulted in breaking down of the tumor mass, the latter should be incised and the abscess thoroughly irrigated.

The internal iodine treatment, recommended in 1885 by Thomassen on the basis of results obtained from 80 cases treated by this method, is very effective (a special commission investigated the merits of this treatment in the United States and reported 131 complete cures among 185 cases treated). The treatment is recommended particularly in such cases when surgical treatment is for some reason impossible and when the affected animals are still in good physical condition. Affection of the tongue or larynx, as well as of the skin and subcutis, is very responsive to this treatment, while actinomycosis of the bones is much less so.

The treatment consists in the internal administration of potassium iodide, young cattle receiving 2 to $4 \mathrm{gm}$., adult cattle 5 to $10 \mathrm{gm}$. daily in their drinking water. The treatment is continued from 2 to 4 weeks or until the complete disappearance of the tumor and ulcers. In ease symptoms of iodism (nasal and conjunctival eatarrh, cutaneous 
eruption, loss of harr, emaciation) appear, the daily dose must be decreased or the treatment must be suspended for 5 or 6 days. The effect is increased by external application of tincture of iodine to the tumor, particularly to the affected tongue, or by the injection of this solution into the tissnes of the tongue. If the heart action is affected it is recommended that sodium iodide be administered instead of the potassium jodide. Prietsch observed good results from the internal administration of iodovasogen in doses of $10 \mathrm{gm}$. of a $10 \%$ solution administered at 5-day intervals, while Blume and Dorn recommended, in addition, the injection of the tumor with the same substance. On the other hand Mitteldorf obtained very favorable results even in advanced cases from the direct injection into the tumor of iodipin (40 to $100 \mathrm{gm}$.) and the administration of similar doses per os. Franz recommends this remedy for both external treatment $(25$ to $50 \mathrm{gm}$. of a $25 \%$ solution injected into the tumor or applied to its surface) and internally $15 \mathrm{gm}$. per day.

Prevention. In regions where the disease is enzootic it is recommended that hay and straw from low, damp soil be excluded from feeding. Young cattle should not be permitted to graze on low or damp pastures. In a number of instances it has been found that green feed which had been known to be dangerous could be fed with impunity after being scalded or steamed. The dangerous character of certain pastures and meadows may, however, be ameliorated by proper cultivation and drainage.

Literature. Bollinger, D. Z. f. Tm., 18i 7. III. 334. - Johne, Tbid., 1881. VI. 141; 1889. XIII. 140. - Ponfick, D. Actinomykose d. Menschen, Berlin. 1882. - Bang, D. Z. f. Tm., 1884, X. 233. - Thomassen, Echo, vét. belge, 1885. XV. 409, - Boström, Beitr. z. path. Anat., 1890. IX. 1. - Rasmussen, D. Z. f. Tm., 1891. XVII. 455; 1894. XX. 299. - Saluon, An. Ind., 1891 u. 1893. - Jensen Monh., 1893. IV. 166. - Imminger, Monh., 1899. XI. 129. - Brever, Z.f. Flhyg., 1901. XI. 103 - Assman, D. t. W., 1904. 63. - Mitteldorf, ibid., 1904. 402. Lignières \& Spitz, Bull., 1905. 64. - Hollandt, A. f. Tk., 1905. XXXI. 417 (Lit.). - Wright, Publice of the Massachusetts Gen.-Hospital, 1905. I. 1. - Nicolaus, Diss. Bern, 1908 (Lit.). - Kowalewsky, Hyg. de viande, 1909. III. 204.

Actinomycosis of Man. This rather rare disease is caused by the same actinomyces which produce the disease in cattle. The localization of the morbid processes is also very similar to that observed in actinomycosis of cattle. The morbid changes which are most frequently observed in the gums, in the lungs and the maxillae, consist either of small, firm tumors or more frequently of larger cold abscesses or fistulous ducts surrounded by a very tough lardaceous connective tissue. As in the case of animals, infection usually occurs through the medium of plant tissues (awns of barley) either in the gums, the dental alveoli (especially in the presence of defective teeth) or in the mucous membrane of the mouth or larynx, the esophagus or intestine. In much rarer instances infection may take place from the skin. Direct transmission of the disease from cattle to man has not been established.

Literature. Korányi, Nothnagels Spez. Path. u. Ther., 1897. V. Bd. I. T

Actinobacillosis. Under this name Lignières \& Spitz (1902) described a disease of cattle which oceasionally assumed an epizootic 
character in Argentine, sometimes $50 \%$ of all animals in a herd becoming affected in the course of a few weeks. As a rule, however, the disease occurs more or less sporadically. It has also been observed in a Rambouillet ram by the same authors, while Nocard and Petit observed it in France, Bongert in Germany and Higgins in Canada among cattle.

The clinical symptoms and anatomical changes are almost precisely the same as those of actinomycosis bovis. As in the latter disease the most frequent lesions consist of large, firm swellings gradually undergoing softening in the center, occurring in the skin and in the subcutaneous connective tissue of the pharyngeal region; so called woody tongue and polypous swellings of the pharynx constitute the most frequent lesions. The jaw hones, the udder and the lungs are much more rarely affected. On the other hand, however, the lymph glands and occasionally some of the salivary glands are involved. Simultaneously with the development of the local changes the animals suffer in their general nutrition and when tumors are present in the pharynx or in the pharvngeal region, the disturbance of nutrition and respiration may hecome so great that the affected animals finally suceumb to the effects of inanition.

In the purulently softened foci of the tumors are found granules varying in size up to that of a millet seed, readily visible to the unaided eve, but in contrast to the colonies of actinomyces they are grayishwhite in appearance. Slightly yellowish and oceasionally also calcified granules oceur exclusively in the sarcoma-like tumors of the maxillae. Fresh pus examined under the microscope contains club-like structures which are branching at the larger end, the pointed ends converging to form radiating colonies which, however, contain no mycelium centers. These structures are easily stained with acid dyes or with picro-carmineglycerin. On the other hand they do not take Gram's stain nor can threads of mycelium be demonstrated in the pus.

Pus triturated in a mortar and inoculated in alkaline food media, when kept at body temperature for 24 hours, will produce small, glistening colonies of a small bacillus hardly exceeding that of fowl cholera in size, forming long ehains in bouillon and not staining after Gram. Cultures on agar resemble those of the typhoid bacillus. This bacillus acidifies grape sugar agar and milk, but does not coagulate the latter. The indol-reaction is not pronounced. Fluid cultures contain toxins which, when injected into cattle, produce an elevation of temperature of from 1 to $2.5^{\circ} \mathrm{C}$. persisting for several hours, trembling and loss of appetite. The cultures are only slightly resistant to the influence of external agencies. Simple drying or heating to $52^{\circ} \mathrm{C}$. destroys them rapidly. Grown at $37^{\circ} \mathrm{C}$. in the thermostat they are gradually attenuated.

Bacilli injected under the skin of eattle collect in small groups in the bodies of the leucocytes from which they subsequently escape and develop into characteristic actino colonies. The center of these colonies, the "germinative zone" consists of small bacilli which are held together by a sticky substance while the outer zone consists of club-shaped projections which continue to grow and undergo digitate branching ("vegetative zone").

Pathogenicity. In guinea pigs intraperitoneal injection of bouillon cultures produces purulent peritonitis with characteristic colonies in the exudate, while subcutaneous injection is followed by the formation 
of ahscesses which do not contain these structures. In horses, asses and cattle intravenous injection is followed by phlebitis and thrombus formation while subcutaneous injection of these animals as well as of sheep is followed by abscess formation. In cattle and sheep these abscesses contain the characteristic colonies while in horses only a few bacilli are present. In these animals as well as in dogs, eats and swine intravenous injections are followed by symptoms of intoxication, otherwise, however, cultures are only slightly pathogenic for the last named species of animals. They are non-pathogenic for rabbits, white rats and mice.

Although feeding experiments have not given positive results it seems that natural infection results from the digestive tract. The bacteria are probably aided in their entrance into the tissues by particles of vegetation to which they adhere. In general, the contagiousness of the disease is only very slight and extensive outbreaks occur only under certain favorable conditions as, for instance, after an outbreak of foot and mouth disease.

Iodine treatment has been found effective in many cases of soft tumors. Preventive treatment consists of the destruction of badly infected animals and isolation of the milder cases. Recovery from the disease does not produce immunity to a second infection.

Literature. Lignières \& Spitz, Actinobacillose. Buenos Aires, 1902; Bull., 1902. 450. - Nocard, Bull., 1902. 695. - Higgins, Canad. Dept. Agric. Biol. Labor. Bull., No. 1. - Petit, Bull., 1905. 268.

\section{Streptotrichoses.}

\section{(Atypical Actinomykoses; Pseudoactinomykosis, Actino- phytosis.)}

The term streptotrichoses is usually applied to morbir processes produced by fungi that resemble actinomyces, in so far as they consist of thread-like filaments undergoing true branching, as well as in their wavy growth, and their propagation by means of division as well as spore formation, but which differ from actinomyces in that they do not form actiniform colonies.

The morbid processes belonging to this group are generally characterized by chronic suppuration.

Literature. s. in Petruschky, HIb. f. p. MI., 1903. II. 832.

\section{(a) Farcy of Cattle. Lymphangioitis Farciminosa Bovis. (Farcin du boeuf [French].)}

This is a chronic infectious disease of cattle cansed by a species of streptothrix and characterized by purulent inflammation of the superficial lymph glands and lymph vessels.

History. Lymphangioitis farciminosa bovis was first described by Sorillon (1829) and was formerly observed frequently in France. In 
more recent times, however, it has become more rare, while it has as yet never been observed in other parts of Europe. On the other hand it is a common and malignant disease in Guadeloupe and on Moritz Island. Its etiology was cleared up by Nocard, while Maillet furnished an accurate description of the clinical symptoms. It is evidently the same disease that Holmes observed in oxen in Calcutta.

Etiology. The cause of the disease is a streptothrix (Str. farcinica), the branching filaments of which are readily stained by Weigert's method, but do not stain according to Gram. They thrive in artificial media in the presence of air at a temperature between 30 and $40^{\circ} \mathrm{C}$. On agar or on coagulated blood serum, yellowish white finely granular colonies appear which coalesce and form a membrane; on potatoes the colonies are dry, crumpled, and pale yellow in color; in bouillon white granules appear in the depths of the medium, and membranes resembling congealed drops of fat form on the surface of the fluid.

Pathogenicity. In guinea pigs the subcutaneous injection of a pure culture is followed by a purulent inflammation of the lymph vessels and glands, in the course of which the purulent foci become ulcerous. After evacuation of the purulent contents the animals which have in the meantime become much emaciated, gradually recover. Intraperitoneal infection is followed by the development of numerous tubercle-like nodules in the peritoneum, while intravenous infection causes similar changes in the parenchymatous organs. Injection of pure cultures into the circulation of cattle or sheep produces similar results, while infection of superficial wounds of the skin produces chronic lymphangitis.

Natural infection probably takes place by the entrance of secretions containing streptothrices into superficial wounds of healthy animals.

Symptoms. The morbid changes almost invariably develop on the extremities and especially on the median surface, where firm, painless strands and nodes are to be noted along the course of the superficial veins. Subsequently these nodes become soft and fluctuating and when incised discharge a whitish, odorless mass resembling soft cheese; local healing follows within 5 or 6 days. In exceptional cases these abscesses may break spontaneously, but as a rule they remain intact, becoming firm and hard and consisting of white or grayish tough lardaceous connective tissue. The regional lymph glands become enlarged and form firm, painful tumors.

The disease progresses for 12 to 18 months without necessarily disturbing the general condition of the animal to any serious extent. Affected animals do not even get lame but are exhausted by work. In the later stages, however, gradual emaciation is observed, which finally terminates in extreme cachexia. 
The disease may be confused with tuberculosis of the lymph glands, although a microscopical examination will readily differentiate these affections.

According to Vryburg a special form of bovine farcy occurs on the Island of Sumatra; this begins with chronic abscess formation of a lymph gland, whereupon the process spreads to the neighboring lymph glands and also along the course of the lymph vessels, producing abscesses which break, discharge a thick, ereamy, odorless, rarely, viscous, pus and become transformed into torpid ulcers. The prescapular and inguinal glands as well as the lymphatic vessels of the pectoral and abdominal region are most frequently affected. The disease which appears occasionally as an enzootic, runs its course in from 1 to 9 months. About $90 \%$ of affected animals recover.

The pus found in the abscess contains an immotile aero-anaerobic, Gram negative bacillus which produces indol in cultures. It is said that animals may be protected against natural infection by treatment with attenuated and then by virulent cultures or by treatment with a mixture of cultures and immune serum.

Liénaux described a disease of cattle under the name of Lymphangioitis pseudofarcinosa which is supposed to be caused by the bacillus of pseudotuberculosis (see p. 633).

Treatment and Prevention. Extirpation of the tumors and cauterization of the surrounding tissue, if resorted to before the disease becomes advanced, will occasionally check the local process, but even in these favorable instances recurrences are frequent.

To prevent the spread of the plague it is recommended that all diseased animals be isolated, their morbid products destroyed and all contaminated premises and utensils thoroughly disinfected.

Literature. Maillet, Rec., 1829. 631. - Nocard, A. P., 1888. II, ะ93. Liénaux, Ann. vét., 1905. 297. - Vryburg, Rec., 1907. 171. - Holmes, Trop. Vet., 1908. III. 289.

Other Streptotrichoses in Ruminants and Horses. According to Luginger cattle are subject to endocardites as a result of hematogenic infection with a form of streptothrix which is said to constitute a specific species (Str. valvulas destruens bovis). When injected into the blood stream it produces purulent pleuritis as well as pseudotuberculosis in sheep.

Berestneff observed a delicately branched streptothrix (coccobacillus pseudoactinomycosis polymorphus) in a tumor from the lip of an ox which he designated as pseudoactinomycosis. Bonvicini isolated a species of aerobic streptothrix from a similar cutaneous affection from a steer, which had been transmitted to several cows. Finally Zschokke isolated and cultivated a fungus from a goat's tongue which Silberschmidt described as Str. caprae.

Zschokke described an affection under the name of actinophytosis which manifested itself by the appearance of a tumor as large as a child's head in the mucous membrane of the upper lip of a horse; in another instance there was a similar tumor as large as a man's fist 
on the intermaxillary bone. The small soft eenters contained mieroorganisms which were characterized by a radial structure and by giving rise to destructive ehanges in their periphery. Their exact nature was not determined. Complete recovery followed extirpation of the tumors.

An affection of a horse, deseribed by Cuille, which developed with symptoms of a distemper-lymphangitis and numerous abscesses, might possibly also belong to this group. The pus foci openel spontaneously, one after the other, the creamy pus contained in part small, white, transparent granules or larger yellowish masses of Gram positive intertwined mycelia. Artificial cultivation was possible only in the vacuum. The cultures thus obtained produced abscesses in horses as well as in guinea pigs. The horse referred to showed symptoms of improvement for the time, but finally died with symptoms of eachexia. A post-morten examination was not made. A similar species of streptothrix was observed by Dean in a hard nodule at the commissure of the mouth of a horse.

Carougeau described an epizootic disease of mules and horses in Madagascar under the name of sporotrichosis which is characterized by its chronic and unfavorable course and by the development of tumors in the most varied portions of the body. These tumors are at first firm, but subsequently they suppurate; they do not involve the regional lymph glands. In exceptional eases nodules and chancre-like ulcers develop on the nasal mucous membranes. The purulent contents of the nodules contain Gram positive mycelia with club-shaped ends, vacuoles in their protoplasm and also spore-like Gram positive granules. The parasite (sporotrichum equi) is easily grown on artificial media, propagating by means of exospores. Horses and mules have been suc cessfully infected with sporulating cultures. Intermal treatment with potassium iodide has been found very effective.

Literature. Luginger, Monh., 1904, XV. 289. - Berestneff, Aktinomykose u. ihre Erreger. Diss. Moskau, 1897. - Silberschmidt, A.·P., 18s9. III. 841. Zschokke, Schw. A. 1903, XLIV. 303. - Cuillé, Rev. vét., 1905. 816. - Dean, Transact. of the Jenner Institute, 1899. II. 17. - Carougeau, J. vét., 1909. 8.

\section{b) Streptotrichosis of Dogs. Streptothrichosis canum.}

This disease, which has been observed only occasionally, is caused by a specific form of streptothrix. It is characterized by purulent inflammation of the serous membranes and by sulicutaneous chronic abscesses.

History. In 1888 Rabe demonstrated that the "granules" found in some of the pleural and peritoneal exudates consisted of the interwoven mycelium of a fungus described by him under the name of Cladothrix canis. Subsequently this fungus was minutely investigated hy Bahr (1904).

Etiology. The virus of the disease (Streptothrix s. actinomyces canis) produces long, branching filaments (Fig. 109), which stain by the Gram-Weigert method and form occasional club-shaped terminals in the course of their growth. 
In some respects they are similar to those found by Wolf \& Israel in subcutaneous abscesses and fistulae as well as in exudates of serous cavities in man.

Culture. On artificial media the fungus will grow only at body temperature, at first only anaerobically, but after repeated transfers to new media it will thrive also aerobically. In the deeper layers of the agar medium white, mulberry-like colonies appear in the course of 3 or 4 days, which consist of long branched threads with club-shaped terminals, or of short rods and spore-like structures, easily stained after Gram in bouillon, granules as large as pin heads develop in the course of 4 to 6 days, the medium remaining perfectly clear. Milk is not coagulated although its reaction becomes distinctly acid.

Pathogenicity. The intraperitoneal administration of pure cultures in mice produces pus foci as large as peas on the peritoneum, while subcutaneous inoculation of rabbits is followed by the development of nodules approaching the size of hazelnuts, consisting of a fatty or calcareous substance surrounded by fibrous tissues. Similar treatment of dogs is followed in the course of a few days by the development of firm nodules which subsequently disappear

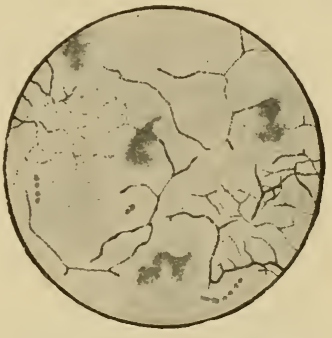

Fig. 109. Streptothrix canis. Pleurae exudate; Fuchsin Stain. or become converted into cold abscesses, the slimy, reddish-gray odorless pus of which contains streptothrix mycelia. Guinea pigs, calves and monkeys resist experimental infection.

In the brain of a dog that had died after several weeks of illness Trolldenier found numerous light grayish-white foci ranging in size to that of a pea, some of which contained purulent centers. One of the bronchial lymph glands was greatly enlarged and contained a slightly purulent substance in its interior. Mycelia similar to those described could be demonstrated in the cerebral nodes and in the affected bronchial lymph gland. These mycelia would grow on artificial media at room temperature under aerobic conditions. On agar the colonies subsequently developed yellow centers, while their periphery remained white (actinomyces bicolor). These cultures were pathogenic for mice, guinea pigs, rabbits and dogs. Jochim described the same fungus in a culture obtained from a dog with a phlegmonous condition in the cervical region and numerous nodules in the internal organs.

Symptoms and Anatomical Changes. The disease usually manifests itself by the appearance of a peculiar inflammation of the pleura or peritoneum, in the course of which purulent or reddish-gray, cloudy exudate accumulates in the respective body cavities. Pronounced symptoms of fever are absent. White granules of the size of pin heads may be recognized in this exudate by the mnaided eye, while the pleura is covered with fibrous masses; the lungs occasionally contain firm, gray nod- 
ules as large as peas, which may be soft inside. In some cases inflammation of the serous membranes is a sequence to external local affection; thus, for instance, a torpid, phlegmonous inflammation or abscess of the extremities, in the subparotideal region or neck, or a fistula in the vaginal wall or in the subperitoneal connective tissue of the pelvis. These abscesses contain a turbid, gray or reddish-brown, thick, muco-purulent substance, which also contains the granules referred to. The inflammatory process which occasionally develops as the result of an injury, e. g., of the toes (Rabe) or side of the chest (Rivolta) gradually progresses, attended with only slight pain, and without producing conspicuous disturbance of function. After the abscesses have discharged their contents and healing has occurred, new abscesses will occasionally develop in other regions of the body, or the pus in the old abscesses may burrow downward along the course of the loose connective tissue. Inflammation of the lungs and pleura may also develop very gradually, attended with only a moderate elevation of temperature, until finally physical examination of the greatly emaciated animals reveals the accumulation of exudate in the pleural cavity and consequent compression of the lungs. Inflammation of the peritoneum produces a similar effect, the exudate distending the walls of the abdomen to a greater or less extent. In other cases the presence and exact nature of the exudate may be definitely determined by aspirating the contents.

At post-mortem examination other organs also (spleen, liver, kidneys, heart muscle) may be found to contain firm or softened nodules. Now and then pus accumulations occur in some of the joints.

Treatment. In some cases the surgical treatment of primary nodes may bring about a complete recovery (Gohn). As a rule, however, new nodules will develop subcutaneously in other regions of the body, and the emaciated animals finally succumb to the disease (aspiration of the pleural or peritoneal exudate has in no instance proved effective).

Literature. Rabe, B. t. W., 1888, 65. - Bahr, Z. f. Tm., 1904, VIII. 47 (Lit.). - Trolldenier, ibid., 1903.' VII. 81. - Jochim, Diss. Bern, 1909 (Lit.).

\section{Botryomycosis.}

Botryomycosis is a chronic infectious disease of horses which may, in exceptional instances, occur in cattle. It usually results in the formation of local tumors resembling fibromata, and occasionally in the formation of metastases in the internal organs. It is caused by the Botryomyces equi.

History. Bollinger was the first to observe the cause of the disease in the nodules of a horse's tongue (1869); subsequently it was observed 
by Rivolta \& Micellone (1879), Johne (1884) and by Rabe (1886) in fistulae of the spermatic cord of horses, as well as in tumors of the withers and pectoral region. Since then the frequent occurrence of this disease has been confirmed by numerous observations. In more recent years Kitt, de Jong, Galli-Valerio and Parascandolo \& Meis made special bacteriological studies of this organism.

Etiology. The botryomyces equi, Bollinger (Discomyces equi, Rivolta; Micrococcus botryogenes, Rabe; Micrococcus ascoformans, Johne; Botryococcus, Poncet \& Dor) appears in the diseased tissues in the form of mulberry-like or grape-like clusters, $1.0 \mathrm{~mm}$. in diameter. The individual elements in these clusters are surrounded by a homogenous gelatinous capsule of sharp contour, thus forming zooglœa masses (Fig. 110 and 111).

Staining. These cocei stain readily in aqueous aniline dyes as well as according to the Gram-Weigert method. The gelatinous capsule does not take the stain. The organisms are very resistant to the action of alkalies and acids.

Culture. On gelatin the broken zoogloa give rise to the development of colonies, at first silver gray but subsequently grayish-yellow, with a metallic luster, while a white streak may he ohserved to develop

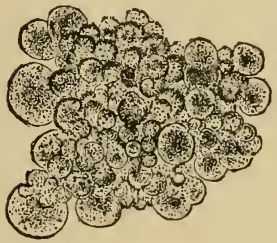

Fig. 110. Botryomyces equi. Zoogloea mass from a soft focus. Not Stained. Mag. ea. 100 diam. (according to Rabe).

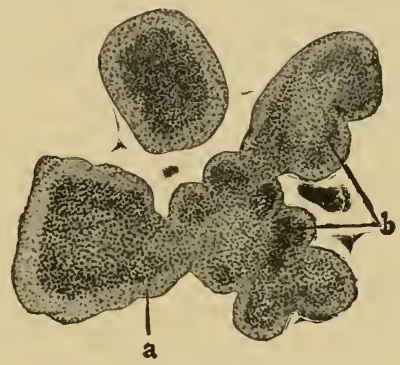

Fig. 111. Boctryomyces equi. Section of Zoogloea. Stained according to Gram. $a$ Ground Substance; $b$ Cocei. Mag. ca. 500 diam :

along the needle puncture in the depths of the medium, gradually causing the gelatin to liquefy along its course when the colonies sink to the bottom. On potatoes a pale yellowish, hoar-like coating is formed. The cultures emit a peculiar odor recalling that of strawberries. The colonies are composed of micrococci without, however, any characteristic grouping and without hyaline capsules.

Pathogenicity. Mice are not susceptible in infection; infection of guinea pigs with pure cultures produces fatal septicemia. Inoculation of sheep and goats is followed by an inflammatory edema occasionally followed by necrosis of the surrounding area of the skin. In horses inoculation of pure cultures produces 
also an inflammatory edema which disappears in from 3 to 10 days, but is replaced in the course of 4 to 6 weeks by the gradual development of lobulated boils, in the interior of the soft foci of which groups of cocci with hyaline capsules occur, just as after natural infection (Rabe).

According to Rabe as well as Poncet \& Dor, a hyaline capsule which envelopes the individual groups of cocei is the product of a reactive inflammation of the surrounding tissues, while according to Johne it is a product of the cocei them. selres.

On the other hand, Ernst looks upon the capsules as a degeneration product resulting from the mucoid degeneration of the cocei in the periphery of the groups under the influence of lytic or agglutinating immune bodies which cause their destruction, the growing central mass causing the peripheral cocei to be compressed into a scaly or striated structure. The eapsule thus formed is supposed to be distended and ruptured here and there as a result of central growth, the regetating cocei finding their way through these openings to the periphery and forming new, smaller encapsulated structures. The repetition of these processes at various points results in the formation of the morula like structures.

Very frequently it is possible to cultivate organisms from botryomycomata that resemble staphylococcus pyogenes aureus in every respect and for this reason de Jong as well as Galli-Valerio regarded the Botryomyces as identical with, or at least as a variety of this Staphylococeus. According to de Jong these cocei, when retained in primary abscesses as a result of incomplete discharge of their contents, suffer a check in their vitality which results in the formation of capsules and the characteristic zooglœa forms; the latter thus constitute a permanent irritant in the tissues. As an argument against the identity of the forms above mentioned, Parascandolo cites experiments in which blood serum obtained from rabbits treated with staphylococei failed to agglutinate botryomyces, and he further claims that reciprocal agglutination on the part of these two parasites could not be demonstrated. It has also never been possible to produce typical botryomyces with cultures of staphylococeus aureus.

Kitt and Bodin have also succeeded in obtaining the $\mathrm{S}$. aureus, Hell the S. albus, Mari the S. aureus as well as the S. citreus in cultures from botryomycomata and these authors as well as Sabazès \& Laubie and MacFadyean are also inclined to look upon the botryomyces as a variety or latent form of the cocei referred to, viz., S. aureus, albus and citreus. On the other hand Poncet \& Dor as well as Parascandolo regarded this organism as a specific variety of bacterium. Letulle assumes a unique position claiming that the individual elements of this organism are identical with intestinal amebae which have enveloped leucocytic nuclei.

Natural infection usually occurs through bruises or incised wounds of the skin (in exceptional instances following amputation of the tail, docking). The virus is usually rubled into superficial wounds and into the excretory ducts of the cutaneous glands by a tight-fitting harness. When the tumor thus resulting breaks, portions of the discharged viscous secretions arlhere to the harness and are thus transmitted to other animals on the first opportunity (Wester). In exceptional cases botryomycomata may also occur on portions of the body that do not come in contact with harness (for example, in the udder, under the 
abdomen). In such cases infection evidently occurs through the medium of previously existing wounds. Infection of the spermatic cord doubtlessly occurs in this manner, the contagion probably being transmitted from the bedding, and finding a fertile soil for development and propagation in the necrotic end of the cord and in the retained products of inflammation (Chaussée).

In the internal organs botryomycotic changes develop as a rule as metastases resulting from superficial botryomycomata on some other part of the body. In rare cases, liowever, they may constitute primary lesions. Thus, in a case described by Bollinger, the lungs and the bronchi only were affected, indicating infection through the respiratory organs. In another case (Rabe) a pelvic tumor communicated with the bladder by means of a fistulous canal, possibly caused by catheterization. Finally the possibility that botryomyces, like actinomyces, may gain entrance into the tissues from the lumen of the intestine and thence pass to other abdominal organs, does not seem excluded.

Pathogenicity. The botryomyces constitute a tissue irritant with a similar action to that of the actinomyces. They cause the emigration of round cells which subsequently become transformed into epithelioid and giant cells, although the emigration of leucocytes is more rapid in infection with botryomyces, consequently the formation of pus is earlier evident. Connective tissue proliferation also occurs in the region surrounding the pus foci and leads to the formation of firm, fibromalike tumors. Finally this fungus may be distributed through the lymph spaces, in exceptional cases also through the blood vessels, and give rise to metastases in remote regions of the body.

Anatomical Changes. Botryomycomata consist of tough, firm, fibrous connective tissue, grayish-white or pale red on section and lardaceous in appearance, containing yellowish-red or brownish-yellow gelatinous foci which project above the cut surface and in which whitish-gray granules are embedded, the latter even visible to the naked eye. Very large tumors may now and then contain large cavities or fistulous canals filled with a muco-purulent mass. Their tough walls are lined with yellowishred soft granulation tissue.

These tumors are found, as a rule, in the subcutaneous connective tissue and in the skin, occasionally also in the muscles. In addition they are found quite frequently in the end of the spermatic cord of geldings, in which cases they spread through the inguinal canal to the peritoneum and may give rise to secondary tumor formation in the anterior portions of the abdominal cavity (Schimmel).

In the rare cases observed in the lungs, the latter organs may contain one or more tumors that may have attained the size of a man's head, and occasionally very numerous smaller tumors approaching the size of a fist; these are sharply circumscribed 
but firmly adhere to the pulmonary tissue; they are firm, tough, tumor-like nodes, sections of which contain the soft foci above described, or they may be homogenously fibrous, white or grayish-white in color; the milky fluid which may be expressed contains fine granules. The surrounding pulmonary tissue usually shows inflammatory changes. Lesions on the pleura or the pericardium resemble tuberculosis of the serous membranes, with the exception that the nodules are softer in their interior and "sandy" (Piper).

Similar tumors occur now and then in other internal organs, usually in conjunction with involvement of the spermatic cord; thus in the kidneys, the suprarenal glands, the liver, the spleen, the uterus, the maxillary sinuses, the udder, as well as certain bones (maxillae and ribs), the spongy tissue of which is destroyed by the tumor mass and the external lamella here and there perforated. The regional lymph glands may be affected, but as a rule they remain entirely free.

In exceptional cases the morbid process is simultaneously observed in a number of organs in a generalized form (Rieck, Fröhner, Kofler, Türnau).

Symptoms. Botryomycomata nsually develop very gradually and without pronounced acute inflammatory symptoms. They may occur on any portion of the skin as roundish or lobulated, occasionally pedunculated fibroma-like tumors which now and then attain a considerable size (collar boils and shoe boils belong to this category) without affecting the movements of the animal to any great extent, if at all (Fig. 112). In some cases, however, the tumor becomes more diffuse, causing the formation of extensive, very firm enlargements almost wood-like in consistency, or of shield-like formations, from the surface of which fistulous canals pass into the deeper tissues. Innumerable additional nodules may develop in rapid succession in the tissue of the skin. These consist of lardaceous connective tissue with one or more purulent, cheesy, mortar-like foci; the neighboring lymph vessels remain intact, although the regional lymph glands are occasionally enlarged, firm and bosselated and contain small soft foci. The nodules may become transformed into cratershaped ulcers with prominent borders and discharge a tough, muco-purulent, yellow secretion.

Another frequent form of the disease is the (improperly) so-called fistula of the spermatic cord which in most instances is a result of infection with botryomyces (Johne). The process develops very slowly and may extend over a period of 4 years before the peritoneum is affected (Plósz); the tumor may attain the size of a man's head and extend over the lower portion of the abdomen.- In affection of the udder (quite common, according to Sand) this organ becomes enlarged and firm nodes are formed in its tissues. These nodes subsequently break and discharge pus through fistulous canals. (In a case described by Unterhössel the udder of a mare attained the size of that of a cow.) In exceptional cases the disease appears also on the 
external mucous membranes; thus Fröhner observed it in the form of a soft polypous proliferation, the size of a walnut, near the left nostril. Patrick observed the same in the form of a tumor of the size of a hen's egg on the upper jaw, while Gutbrod described a case in which numerous small nodules occurred on the inner surface of the upper eyelid, causing this organ to appear much swollen.

Liénaux saw one case in which the right intermaxillary gland attained double the size of a man's fist, while about 20 nodes the size of a hazelnut could be observed in the course of the right facial erest.

In one case small boils with muco-purulent, yellowish-red contents were found to develop after the operation of docking; there was simultaneous thickening of the skin and complete alopecia; subsequently the cutis of the head and particularly in the region of the lips became enormously swollen, numerous fistulae were formed and there was slight enlargement of the intermaxillary glands.

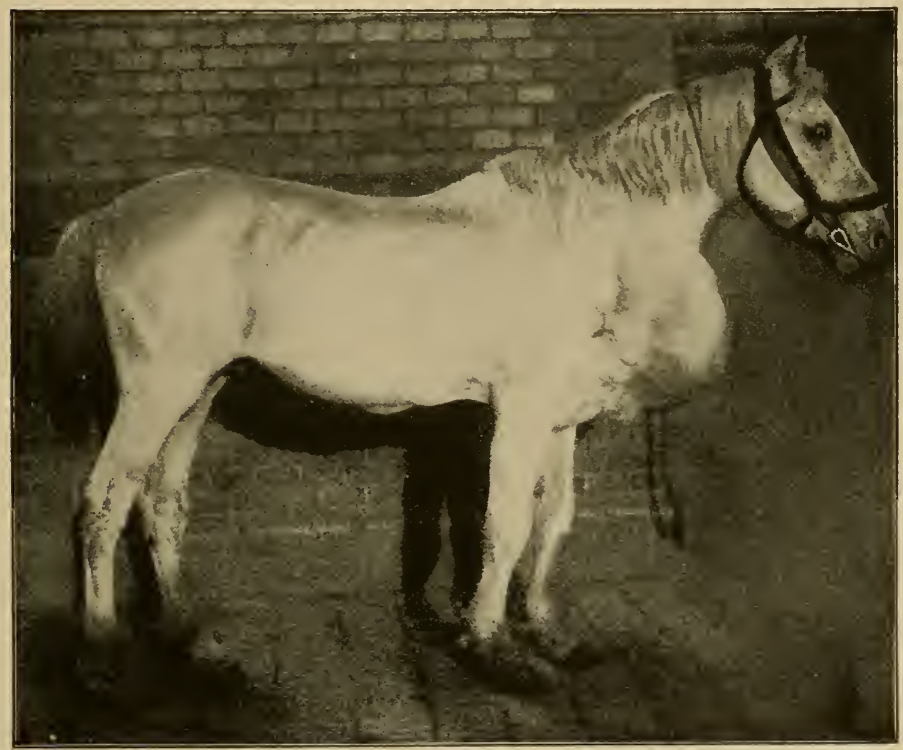

Fig. 112. Botryomyces of the prepectoral Region. (Plosz.)

In cattle the disease is much less frequent; Csokor and Immelmann observed the same as an affection of the udder, Reali in the form of tumors of the subcutaneous connective tissue of the shoulder and croup, Bollet as small subcutaneous nodes of the cervical region, extirpation of which was followed after two years by the appearance of very numerous nodes in the sub- 
cutaneous and intermaxillary connective tissue of the thorax and of the abdominal region.

In swine Vilbrandt observed the development of botryomyces in the spermatic cord within six weeks after castration, Petit \& Cozette the same within three months after this operation. The grayish-yellow pus contained granules which consisted of coccus-zooglœa but formed no staphylococcus groups.

Diagnosis. The symptoms of botryomycosis are similar to those of actinomycosis, and the two conditions may be differentiated with certainty by means of microscopical demonstration of the characteristic morula-like colonies. As a rule botryomycosis is most frequent in the horse and actinomycosis in the ox.

In occasional instances, especially when diffuse swellings exist, glanders or farcy may be suspected. These swellings, however, are considerably firmer in botryomycosis, while the peculiar node-like enlargements of the lymphatic vessels are not present in botryomyces. The neighboring lymph glands are rarely involved, and ulcers that may exist show a tendency to luxuriant granulation. Horses affected with botryomycosis will not react to mallein.

Treatment. The treatment for superficial botryomycomata is purely surgical (extirpation). Iodine treatment for affection of the spermatic cord as recommended by Thomassen has not been effective either in this disease (Fröhner, Winter) or in affection of the udder (Vennerholm).

Literature. Bollinger, V. A., 1870. XLIX. 583; 1887. XIII. 176. - Johne, S. B., 1884. 40; D. Z. f. Tm., 1885. XII. 73; 1886. XII. 204. - Rabe, Ibid., 1886. XII. 137. - de Jong, Diss. Giessen, 1899. - Kitt, Cbl. f. B., 1888. III. 177; Monh., 1890. I. 71. - Jensen. D. Z. f. Tm., 1892. XVIII. 433. - Galli-Valerio, Cbl. f. B., 1902. XXVI. 508 (Lit.). - Glage, Hb. d. p. M., 1903. III. 795 (Lit.). - Chaussée, Rev. gén. 1905. V. 425. - Parascandolo \& Meis, O. M., 1805, XXXI. 433. - Ernst, Cbl. f. Bakt., 1907. XLV. 121. - Letulle, J. de phys. et path. gén., 1908. X. 256

\section{Glanders. Malleus. Farcy.}

(Malleus humidus et farciminosus; Rotzkrankheit, Hautwurm [German]; Morve, Farcin [French]; Morvo, Farcino [Italian].)

Glanders is a contagious and usually chronic disease of Solidungula. It is characterized by the development of nodules that have a tendency to break down or degenerate, and by ulcers resulting from the latter process. These changes occur in the tissues of the mucous membranes, the cutis and the internal organs and are the direct effect of the Bacillus mallei. In exceptional cases carnivora become affected. Human beings are susceptible to infection by the virus.

History. Glanders is a disease that has been known since the remotest times, having been mentioned by Vegetius 400 years before 
the birth of Christ (the name, malleus, owes its origin to Aristotle, being derived from the Greek word $\mu \eta \lambda \iota s=b a d$ disease or epidemic). Its transmissibility was recognized by Apsyrtus and Vegetius in the fourth century and in the seventeenth and eighteenth centuries it was generally recognized as an infectious disease. In 1784 the French government enacted stringent veterinary sanitary police laws for the prevention of the spread of the disease. At that time Viborg (1797) maintained that glanders and farcy (nasal glanders and cutaneous glanders) were identical. But before Viborg's time Abilgaard had expressed the same view and had experimentally demonstrated by inoculation the infectious nature of nasal glanders. Toward the middle of the last century, however, the Alfort school, on the basis of unsuccessful inoculation experiments, denied the infectiousness of glanders and in spite of the fact that the School of Lyons took a firm stand against this view, this new doctrine which was defended even by such men as Renault and Bouley, found many adherents. Under their influence the former regulations of protection and extermination were for the greater part suspended, which resulted in an extraordinary dissemination of the disease. This was in itself a convincing argument against the correctness of the new conception and when Rayer (1837) and Le Blanc (1838) again demonstrated the transmissibility of the disease by faultless inoculation experiments the former, more correct view, gradually gained the ascendency, and at the same time the claims of Dupuy that the disease was the same as tuberculosis, those of Bouley. Hering and Funke that it was merely a pyemia, and the claims of others that it was a general dyscrasia, diphtheria, ete., were permanently abandoned.

After the contagious character of the disease had been generally acknowledged, the belief still persisted that the disease now and then developed spontaneously, or as the result of a degeneration of some other morbid process, e. g., distemper or strangles, until Gerlach (1868) and Bollinger (1874) demonstrated, by means that eliminated the last doubt, that glanders resulted from the mediate or immediate contact exclusively with diseased animals or their pathologic products.

The investigations of more recent times have demonstrated the exact nature of the contagion. After Chauveau had shown that the removal of the cellular elements from glanderous secretions by means of filtration destroyed their infectious properties and thus demonstrated that the infectious principle was in some manner fixed to organized elements, a number of authors directed their search for the virus to the microscopical examination of the secretions. Thus Babes (1881) observed in the pus and in the walls of an ulcer of a man affected with glanders, small rod-like structures with thickened ends. Rózsahegyi observed straight or slightly bent, homogeneous, immotile, slender rods in the contents of a pustule of a diseased man. Bouchard, Charrin \& Captain (1882) produced bouillon cultures from the morbid products of diseased persons and horses which produced glanders in guinea pigs and asses in the fifth generation, but it remained for Loeffler, with the cooperation of Schuetz (1882) to isolate and grow pure cultures of the characteristic rod-like bacterium which he demonstrated indisputably by means of exact animal experiments (1886) to be the etiological factor at the bottom of this disease.

Since that time the chief object of researches has been the determination of the exact modes of infection and the perfection of methods of diagnosis. For the latter purpose, aside from the experimental 
inoculation of laboratory animals, mallein in particular had attained considerable importance. Helman in St. Petersburg and Kalning in Dorpat produced this in 1890 independently of each other, both recognizing its diagnostic value. In more recent times MacFadyean, Jensen, Schuetz \& Miessner, Schnuerer and others added the agglutination test while Schuetz \& Schubert as well as Miessner \& Trapp enriched our diagnostic methods by the complement fixation test.

On the other hand the view concerning the exact manner in which infection actually takes place, as wcll as those bearing upon the pathogenesis of the disease have been directed into more correct channels by the investigations of Nocard, Schuetz, Riegler and Hutyra.

Occurrence. Glanders is at this time still quite prevalent among Solidungula. In former times the disease was extensively prevalent everywhere, independent of climatic or soil conditions, and was the cause of enormous losses. Thus, for example, $5.1 \%$ of all horses in the French army were destroyed on account of glanders every year from 1835 to 1845 . In the Hungarian Stud Mezöhegyes about 20,000 horses were killed on account of glanders in the first half of the 18th century. In recent times, however, conditions have much improved in those states and countries in which proper measures for protection and extermination are rigidly enforced.

Since the introduction of mallein as a diagnostic agent it has been found that the disease spreads much faster in large stables than was formerly suspected when only manifest or clinical cases of the disease were taken into consideration. At the same time the fact was recognized that the disease frequently confines itself to the internal organs, in which form it not infrequently terminates in recovery.

Statistical records, especially those of former years, usually include manifest elinical eases only.

According to Krabbe's compilations for 1857 to 1873 the number of glandered horses per 100,000 was as follows: Norway 6, Denmark 8.5 , Great Britain 14, Württemberg 77, Prussia 78, Servia 95, Belgiuın 138, France 1130, Algiers 1548, annually.

In Germany a considerable decrease in the number of cases reported has been noted in recent years. Thus, for instance, while in 1890, 1,417 cases occurred in 355 parishes of 267 districts, the number had decreased by 1908 to 418 cases in 141 parishes of 96 districts.

In France the disease was formerly widely prevalent. Since 1900, however, the number of eases has been reduced from 1,365 in that year to 398 in 1908 (in the Department of the Seine the number was reduced from 1512 in 1895 to 179 in 1903. In 1895 the disease was so prevalent in the Compagnie générale des voitures of Paris that 586 out of 1,200 horses had to be destroyed on account of it, and in the following two years 607 more had to be killed (Blane \& Drouin; see also mallein test).

In Great Britain the number of cases increased from 1,385 in 1898 to 2,443 in 1903, in addition to which 77 glandered English horses were reported from the abattoirs of Belgium. In 1901 there were 2,370 cases, of which 1,828 were reported from London, $67 \%$ of the latter coming from permanently infecter stables (Hunting). In 1908 there were 789 outbreaks of the disease, of which 495 were in London and 198 in the Metropolitan counties; 2,433 horses were killed on account of the disease. 
In Austria the number of cases ranged from 400 to 550 from 1891 to 1900 . In 1896 there were 647, and in 1898 there were 1,043 cases (among these wer 519 horses slaughtered for food in Lower Austria). In 1908 the weekly average of infected parishes ranged from 7 to 41 , most of the infected parishes being in the provinces of Galicia, Lower Austria and the Kingdom of Bohemia, while Upper Austria, Tyrol and Vorarlberg were free from outbreaks.

Russia is at present badly infested; about $4 \%$ of all horses are infected with the disease, and over 10,000 horses are officially destroyed every year. In 1900 the loss in the Cherson Government reached 2,000 , in Tauris 2,067, in St. Petersburg alone 469, in Odessa 722 glandered horses (Samborski). In 1908, 15,693 cases were reported in 7,828 parishes.

In Hungary the annual number of cases has for years ranged between 700 and 950 , although in 1895 the number reached 1,177, and in $1898,1,683$ cases. In 1908 , 946 cases were reported from 261 parishes, mostly based upon the results of mallein tests. The largest number of cases were reported from the southern districts.

The following official figures are available for 1908: Belgium 18, Netherlands 26, Italy 535, Roumania 200, Switzerland 4 cases, while Denmark reported 21 infected stables, Bulgaria 132, and Norway and Sweden are free from disease.

The disease is prevalent also in Asia (India, Japan), in Africa (Algiers, Egypt and South Africa; in the latter country only since recent times), as well as in America (United States, especially in the northwestern States). Australia has thus far remained free from the disease.

\section{Etiology. The Bacillus mallei is} a straight, or slightly bent, slender, immotile, non-sporulating rod with rounded or somewhat pointed ends; its length is about $1 / 3$ to $2 / 3$ of the diameter of a red corpuscle $(2-5 \mu$ long, 0.3-0.5 ${ }^{\mu}$ in diameter; Fig. 13). In tissues the bacilli are usually found in pairs or in larger sheaf-like bundles, while in artificial cultures they may occasionally form filaments or branched threads. (Marx and Conradi therefore classify them as Streptothrixes.)

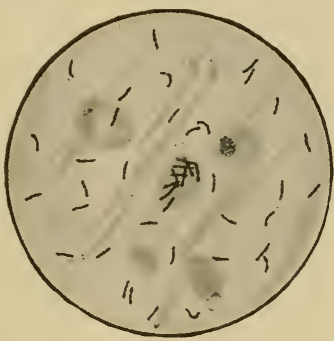

Fig. 113: Bac. mallei. Pus from testis of Guinea Pig; Stained with methylene blue.

Staining. The bacilli stain slightly with aqueous anilin dres more readily in staining solutions containing caustic potash or earbolic acid, often imperfectly or irregularly in diseased organs. They are decolorized by Weigert's or Gram's methods. In bacilli from eultures, stained with carbolfuchsin or methylene blue, we may observe onc or two more intensely stained granules or bodies (usually at the ends).

Cultivation. The bacillus of glanders is an obligate parasite; on artificial media it thrives only at temperatures above $20^{\circ} \mathrm{C}$., best at body temperature; between $41.5^{\circ}$ and $43^{\circ} \mathrm{C}$. its growth is very slow and ceases above $45^{\circ} \mathrm{C}$. Neutral or slightly acid, as well as glycerin media are best adapted for its growth. The cultures have a peculiar smeary or slimy consistency and thrive only in the presence of oxygen. On potatoes there appear, on the second day, small honeydrop-like, transparent colonies that subsequently coalesce into a fawn or chocolatecolored, thick, shiny, ropy mass, while the culture medium in the adjacent area becomes bluish or greenish in color (Fig. 114). On agar there develop gray, transparent, slimy, ropy colonies with smooth outlines, subsequently becoming dark gray and more tenacious in consistency. On coagulated blood seruin pale yellow, transparent colonies 
develop that subsequently become milky white. Bouillon is evenly clouded but at a later stage a white, ropy, slimy sediment accumulates on the bottom of the vessel. Milk cultures remain neutral in reaction but cause the medium to coagulate in the course of ten to twelve days.

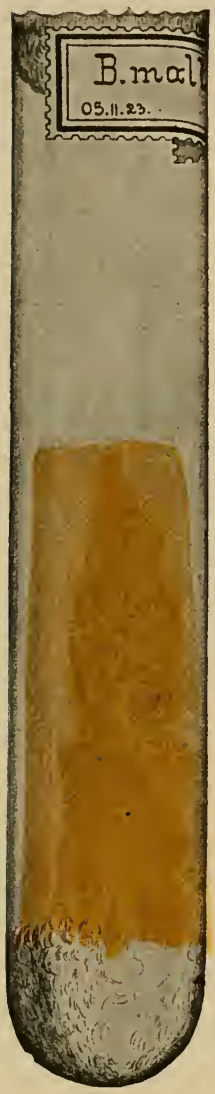

A

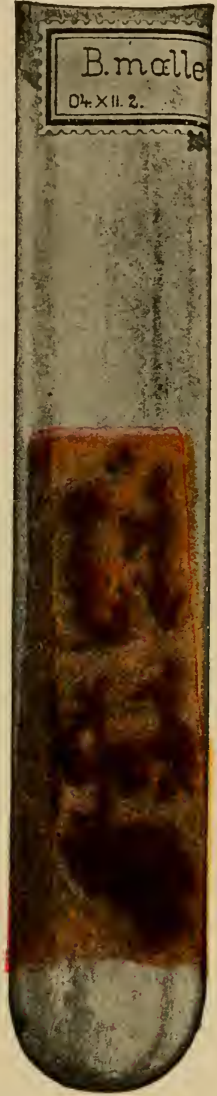

$B$

Fig. 114: Potato Cultures of Bacillus mallei. $A 4$ days, $B$ days of age.

which small nodules develop affection, in the course of which small nodules develop at the point of inoculation and subsequently also in more remote regions of the body. These nodules ulcerate and the lymph vessels and lymph glands in the neighboring tissues become enlarged, which condition is followed by rapid emaciation and deatl. Post-mortem examination reveals similar nodules in the internal organs, especially 
in the lungs. Subcutaneous inoculation also is followed by the development of symptoms of nasal glanders.

The alimentary administration of small quantities of virulent culture of glanders bacilli (0.01 to $0.02 \mathrm{gm}$.) usually results in the development of miliary interstitial nodules of glanders in the lungs, especially near the pleural envelope, subsequently these may develop into broncho-pneumonic foci or diffuse lesions of glanders (Nocard, MacFadyean-Riegler, Hutyra). In some, but by no means in all cases, nodules may also develop in the abdominal organs, especially in the liver. The mesenteric and the intermaxillary lymph glands may also become affected. The feeding of large masses of virus is followed by the formation of glanderous lesions in the intestinal mucous membrane, in the lymphatics, in the lungs as well as in other organs also (Schuetz).

Inhalation of an atomized bouillon culture produces a diffuse inflammatory swelling, attended with the formation of nodules and ulcers in the lower portion of the nasal cavities, also pneumonic areas in the lungs, which symptoms are soon followed by a fatal general infection.

According to Johne, the character of the malleous process is dependent upon the virulence of the infectious material; highly virulent bacilli producing acute lesions with tendency to ulcerous degeneration, while attenuated bacilli give rise to connective tissue, new formations (fibroid glanderous cieatrices, proliferations) as well as to transparent nodules that subsequently become fibrous.

Among other animals that are very susceptible to infection with glanders may be mentioned guinea pigs, cats and young dogs (see diagnosis), as well as species of arvicola and field mice (Kitt); mice die within three or four days after subcutaneous infection, post-mortem examination revealing small pus foci in the spleen, liver and occasionally also in the lungs (house mice and white mice, on the other hand, are only slightly susceptible, while rats are immune). Sheep and goats may occasionally be infected experimentally (Csokor, Penchu), also swine (Cadéac \& Malet, Saccharow), while camels are very susceptible (Petrowsky) and cattle practically immune. Fowls also resist experimental infection.

According to Sacharow, Aruch \& Petrini, as well as according to Galtier \& Nicolas, subcutaneous inoculation of virulent glanders bacilli in cattle is followed by local abscesses, while intravenous alministration produces respiratory symptems and temporary swelling of the joints. In a cow that had repeatedly been treated with dead cultures, Riegler succeeded in producing a fatal disease by the intravenous injection of a virulent culture. Nodules and ulcers were found in the lungs, mucous membranes of the nose and pharynx as well as in the lymph glands. On the other hand, Prettner observed transitory illness in a calf following intravenous injection of $26 \mathrm{gm}$. of a culture of the bacilli.

The virulence of the bacilli is attenuated by passage through the bodies of Spermophilus citillus or through rabbits (Gamaleia), while serial inoculations into the central nervous system of susceptible animals (Tedeschi) and passage through young eats (Foth) increases their virulence. According to Bulloch \& Twodt virus cultivated from lesions from man are extremely virulent. 
Natural infection is brought about through the medium of the secretions of diseased animals. In diseased horses, except in animals with acute glanders, in which case the entire body is infected, the virus is present only in the affected organs and their secretions. The nasal discharge and the secretions of the cutaneous ulcers are particularly virnlent, while the saliva is virulent only as a result of admixture of secretions from the lungs; the same mar be said in regard to the feces, although they may become infected from intestinal ulcers also. In affection of the kidneys the urine may be virulent. Bacilli that gain access to the outer world by these means, though they are incapable of reproduction under ordinary conditions (obligate parasites) may retain their vitality for some time, especially in damp, dark places, where they are protected from drying out, and may then, even after the lapse of several days, gain access to the bodies of susceptible animals in a virulent condition.

In the majority of cases infection of horses takes place through the digestive tract by the ingestion of food or water contaminated with the infected nasal secretions of a diseased animal. The food as well as the water may also have been contaminated by infected mucus that has been conghed up out of the lungs or by the secretions of cutaneous ulcers, the feces or the urine. Accordingly infection is most apt to occur when healthy horses feed out of the same manger or drink from the same trough to which glandered horses have access. Thus, in a large stud of horses, where the animals are not tied up, but are all permitted to feed and drink out of the same common racks or troughs, a single glandered horse may be the means of infecting a large number within a very short time. Under ordinary conditions, on the other hand, the disease usually spreads much more gradually. On the range or pasture affected animals may contaminate the grass with their nasal secretions, and thus infect healthy horses.

According to positive results of feeding experiments, infection may also take place through the intact mucous membranes; the presence of wounds or injuries of course favors the process. Thus Cadéac \& Malet found that horses and cats are very easily infected if the virus is rubbed into the superficially scarified mucous membrane of the mouth, while the same effect is produced in asses by the simultaneous administration of fodder contaminated with prickly vegetation. Catarrhal conditions of the intestinal mucous membrane also favor infection.

Infection rarely takes place through the uninjured mucous membrane of the respiratory tract. Aside from the fact that primary nasal glanders is very rare, the observation that experimental infection of the nasal mucous membrane nearly always produces acute glanders argues against the theory that natural infection usually takes place through the nasal mucous membranes; glanders resulting from natural infection usually runs a chronic course. Primary pulmonary glanders also evidently 
develops only in exceptional instances as a result of inhalation of the infection; this mode of infection can be assumed only when the morbid process manifests itself exclusively in bronchopneumonic foci. An infection after this manner can in fact occur only through the medium of fresh particles of secretion that are forcibly expelled from the lungs in the act of sneezing or snorting; dried virus loses its virulence or vitality within a very short time.

The air normally expired by glandered horses is not infectious. This is at least the conclusion reached in their experiments by Renault, Cadéac \& Malet. The latter investigators forced healthy horses to inspire the same air that was expired by glandered horses by means of linen tubes passing from the head or nostrils of one animal to the other, but in no instance did they observe the transmission of the disease to the healthy animals. They also failed to produce the disease by the subcutaneous injection of the precipitate of moisture from the expired air of glandered horses. On the other hand Nocard failed to get positive results in any case where he injected virulent cultures, that had been admixed with albuminous substances and dried without the application of heat, into the peritoneal cavity of guinea pigs, or where he blew the dried material into the nostrils of asses. Similarly, Hutyra found that virulent nasal seeretion, dried either in the presence of light or in complete darkness, was ineffective in producing glanders by insufflation in horses. Finally Cadéac \& Malet report positive results from intratracheal injections of 10 to 20 ce. of virulent cultures in asses in only $50 \%$ of the cases.

The disease may also be produced experimentally by infection through other uninjured mucous membranes. Thus guinea pigs may be infected by the application of a few drops of culture on the conjunctiva or the mucous membrane of the vagina (Galtier). Under natural conditions this mode of infection can of course be only of rare occurrence (Richter observed primary glanders of the conjunctiva of the horse and it is recorded that a Russian stallion transmitted the disease to a number of mares in Prussia by coitus).

A severance of continuity of the skin or at least of its horny epithelial layers is, as a rule, necessary before infection can occur. The domestic animals are, however, well protected against this possible mode of infection, since infectious secretions usually adhere to the hair coat, dry up and become harmless. This explains why primary cutaneous glanders is generally of rare occurrence as compared with metastatic affection of this organ. As a rule the virus is rubbed into lesions of the skin by means of the harness or combs and brushes previously used on affected horses, or infection may take place through larger cutaneous wounds, or through bites (Lothes).

Babes observed the development of the disease in guinea pigs following rubbing of virulent cultures into the previously shaved skin, as had also been observed long before by Viborg. However, when Nocard repeated the experiment with asses and 15 guinea pigs he obtained positive results in only three of the guinea pigs. Probably the infection was in all cases brought about through abrasions in the superficial layers of the skin, but nevertheless the possibility that the bacilli may pass through the hair follicles of the intact skin and from these into the neighboring lymph spaces, can not be excluded.

The disease is usually introduced into uninfected stables through the medium of affected horses. As a rule the horses 
in the stalls immediately next to that of the affected animal become infected first, although sometimes more remotely located animals are attacked while the intervening ones remain apparently perfectly healthy, which, however, does not necessarily exclude the possibility that the latter were not also infected. The disease may merely not have developed as yet to the same degree or may have been completely arrested. In some cases the horse that was the means of introducing the disease may remain in apparently good condition for months and at the same time infect other horses by its expectorated lung secretions or its infected feces. If such cases are not recognized in time they may result in the infection of the greater part of a large stable full of horses, and thus give the disease a firm foothold for years after.

Diseased horses disseminate the virus of glanders in various ways. Horses helonging to express drivers, transportation companies and miners and those handled by horse traders and gypsies, in particular, play a very important role in the dissemination of the disease. Livery stables and country inns often constitute permanent centers of infection, and horses that have been kept in such places over night, or that have been merely fed there or watered may transport the contagion to other places. Finally it is possible also that feed or hay may be the means of transporting the infection from one stable to the other, although infection through these means is necessarily a rare occurrence on account of the slight degree of tenacity possessed by the virus.

Of all Solidungula the ass is the most susceptible to glanders. This animal is very easily infected and usually develops the acute form of the disease. The horse, which is usually affected with the chronic form of the disease, is less susceptible and may recover from its effects. The mule seems to occupy an intermediate position in this respect. Among animals kept under similar conditions and exposed in the same manner a few may be hard to infect, others become lightly infected and recover, while a few individuals seem to be immune. Horses with reduced vitality as a result of other diseases or hard work, or animals in a poor state of nutrition, are more frequently affected than others, but the common occurrence of glanders among horses of this character is in a great measure due to the fact that they are more frequently exposed to infection.

Camels are easily infected by cohabitation with glandered horses. Symptoms of the disease, which usually point to affection of the lungs, the nasal mucous membrane and the lymph glands, may occasionally appear on the 6th day after exposure.

The hereditary transmission of the disease is possible but seems to be rare in horses (Valentini observed one instance in which the fetus in a mare was affected in the 9th month of its development), while it is comparatively frequently observed in small experiment animals. This is probably explained by the fact that the bacilli are 
rarely found in the blood of diseased horses (as a rule only during a brief period immediately after infection or in the acute form of the disease) while in some experiment animals, especially cats and guinea pigs this is much more frequently the case. Bonome, however, has demonstrated that intrauterine infection may occur not only as a result of hemorrhage but through the intact placenta as well. The extra-uterine disease of the horse has as yet never been demonstrated positively to be the result of intrauterine infection.

Pathogenesis. The discovery that the lesions of the disease, in clinically healthy appearing animals that have been killed on account of positive reaction to the mallein test, are found almost exclusively in the internal organs, especially the lungs, but also the liver and spleen (sometimes only in the latter) while the nasal mucous membranes are hardly ever affected until the morbid processes in the internal organs have become quite advanced, point to the conclusion that the nostrils are usually secondarily infected or are attacked simultaneously with the internal organs. After Renault (1851) had successfully produced infection by feeding infected nasal secretions, Nocard demonstrated that horses could be infected with food or water that had been contaminated with only slight quantities of infected secretions and that the intestinal mucous membranes remained intact while lesions of the disease in the form of small transhucent "tubercles" were found in the lungs, liver and spleen. Following this the disease continued in its usual chronic character and gradually spread to other organs. Nocard's view in regard to the intestinal origin of glanders has been fully confirmed by MacFadyean, Riegler, Bonome, Hutyra and others. The results of Schuetz's experiments are also in harmony with these conclusions in so far as they showed that the feeding of large masses of virus resulted within a short time in extensive affection of the lungs. The possibility of intestinal infection is further supported by those post-mortem observations in which lesions of glanders are noted in the posterior sections of the small intestines and in the cecum; finally also by the observation that carnivora in zoological gardens usually become infected from the ingestion of the organs of glandered horses.

Nocard fed virulent eultures mixed with turnip leaves, or bread, or drinking water to 20 horses, asses and mules that had had water withheld from them for some time and then gave them free access to all the water they would take. All of these animals reacted to mallein within three to eight days and in all of them small hyaline nodules or large cheesy foci developed in the lungs; in some animals the submaxillary lymph glands, the epiglottis, the turbinated bones and the nasal septum also developed glanderous lesions (some of them in 8 days). Schuetz administered large masses of cultures enclosed in gelatin capsules to three horses this was followed within 11 to 13 days by serious affection of the lymph glands and of the lacteals of the 
mesentery and the formation of soft nodules varying in size from a pea to that of a hazelnut in the liver and lungs. In one of the horses there were two nodules in the lungs, hyaline, yellowish-red in appearance, the size of hens' eggs and one as large as a man's fist. In a horse that had received $1 / 10$ of a needle-loop dose of culture on 14 consecutive days, the lungs contained glanders nodules ranging in size from pin head to that of a pea, besides similar lesions in the liver and in two mesenteric glands; after feeding smaller masses of virus small nodules were found in the lungs. Miessner \& Trapp report similar results obtained from feeding cultures en masse to three horses. As a matter of fact Sadowski had some time ago demonstrated glanders nodules in the lungs as a result of feeding infectious nasal secretions. Dedjulin, who administered glanders cultures in gelatin capsules to cats per os, found lesions of glanders in the lungs exclusively. MacFadyean found typical lesions of glanders in the lungs of all horses that had been fed potato cultures of glanders bacilli while in one horse the lungs were affected exclusively. Bonome produced nodules in the lungs in a similar manner. Riegler observed, after intestinal infection of three horses, nodules in the lungs and affection of the bronchial glands in every case. In one case the lesions were limited exclusively to these organs while in one of each of the others either the intestine, the mesenteric glands or the liver were also involved. In Hutyra's experiments with 13 horses that had been fed glanders cultures enclosed in gelatin or keratin capsules in doses down to $0.01 \mathrm{gm}$., providing the animals survived only ten days, this author observed disseminated nodules in the lungs, but in those cases in which the animals were not killed until the end of the third week after infection, there were large typical nodes of glanders of broncho-pneumonic foci.

According to the experiments referred to there can be no doubt that glanderous infection may proceed from the intestinal canal; but it is as yet an open

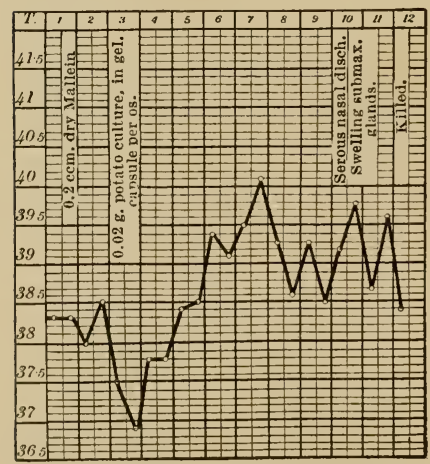

Fig. 115. Glanders. Intestinal infection with $0,01 \mathrm{~g}$. potato culture. Postmortem: miliary nodules in the lungs, acute swelling of the lymph glands. question whether the affection of the lungs is to be looked upon as primary or secondary. While Schuetz defends the position that affection of the lungs is always a sequel to primary infection of the mesenteric glands and that primary pulmonary glanders of horses, if it ever occurs at all, is one of the rarest diseases of the horse, all other authors mentioned, except Meissner \& Trapp regard pulmonary glanders of the horse as a primary morbid process. The correctness of this latter view is supported by the fact that in those experiments where intestinal infection by means of small amounts of virus was successful, the occurrence of rather extensive lesions of the lungs is not attended with changes of the 
lymph glands or of other abdominal organs; there may be, at the most, slight swelling of the lymph glands. In addition to this, Hutyra showed that an alimentary infection was always followed immediately by a distinct febrile reaction (see Fig. 115), that the bacilli of glanders may be present in the circulating blood on the 4th day after intestinal infection, and finally that hematogenic miliary nodules of glanders (see p. 690) will develop within seven days following such an infection.

Since the inhalation of virulent cultures results in infection of the lungs, the possibility of natural infection taking place through the respiratory organs can not be denied. As a matter of fact, however, experiments of this kind (inhalation of bouillon cultures reduced to a spray immediately in front of the nostrils of a horse) result in the formation of nodules and ulcers in the lower portions of the nasal cavities only, while the upper regions remain perfectly intact (Fig. 116) and the lungs may contain, at most, iso-

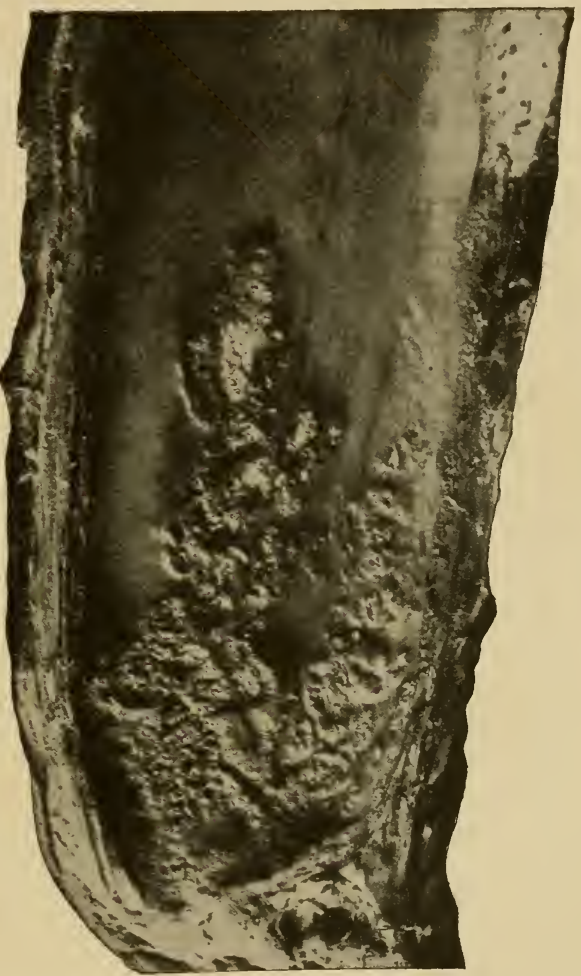

Fig. 116. Glanders nodules and ulcers on the lower portion of the nasal septum 16 days after inhalation of a bouillon culture of glanders bacilli-36 hours' growth. observation suggests that moist air, charged with bacilli, loses most or all of them as a result of the whirling air currents immediately behind the alae of the nostrils and that at most only a few bacilli ever reach the deeper portions of the lungs in this manner. If we consider in connection with this, that the virus of glanders is rapidly destroyed after reaching the outer world and that consequently the inspiration of dry air plays no role in the process 
of infection and, further, the fact that normally expired air contains no glanders bacilli and that direct transmission of the virus from animal to animal through the act of coughing or snorting is only exceptional, one is necessarily forced to the conclusion that aerogenic infection plays a very secondary role in the development of glanders.

As a rule the disease is a result of the ingestion of food or water contaminated with the secretions (glanders bacilli) of infected horses. The bacilli enter the lymph vessels of the intestinal walls through the intact mucous membrane and then pass into the mesenteric lymph ressels; during this process, although it is undoubtedly a rare occurrence, they may give rise to inflammatory changes in the follicles of the intestinal mucous membrane as well as in the mesenteric glands. Following the course of the lymph stream they enter the thoracic duct and are discharged into the blood with the contents of the latter. This results in a general blood infection, attended with febrile elevation of body temperature, inflammatory swelling of the lymph glands (including those of the intermaxillary space), and of the nasal mucous membranes, as the first stage of the disease. A part of the bacilli which circulate in the blood are deposited in the capillaries of the lungs, the organ preeminently susceptible to infection, while others may lodge in other organs-thus, also in the nasal mucous membrane-and exert their pathogenic action; in part, howerer, they are evidently destroyed in the blood and in the lymph glands. However, as Lothes had already noted in his practical observations, the possibility exists that the virus may enter the lymphatics of the neck and thorax directly from the pharyngeal region.

After lodging in the small blood and Jymph vessels the bacilli of glanders give rise to a proliferation of the surrounding endothelial cells and of the extravaseular connective tissue cells which results in the formation of nodules composed of epithelioid cells; subsequently white blood cells pass from the periphery and lodge between the cells of the nodules, as a result of which the latter become softer in consistency and finally degenerate into a purulent mass which contains, in addition to well preserved pus corpuseles, large masses of degenerated cells and cell- and tissue-detritus (Baumgarten). The nodule which is at first hyaline, assumes a white or yellowish-white color, while the surrounding hyperemic tissue which is infiltrated with small round cells forms a red area. As the nodules enlarge and the cellular elements rapidly break down larger foci composed of detritus and pus cells and surrounded by a red area are formed. If the nodule developed in the mucous membrane or the skin, the resulting superficial necrosis is followed by gradually progressive ulceration.

When only a few bacilli are present or when the tissues possess unusual resistance the process may be interrupted in its initial stage, or it may not be interrupted until considerable connective tissue has accumulated around the glanderous foci; following either condition definite recovery may result, the ulcers cicatrizing, the purulent centers becoming dry, and, in exceptional eases, calcifying.

In other eases the bacilli find their way into the surrounding tissues through the lymph channels, the lymph vessels of the subcutis forming thick, knotty strands and the regional lymphatic glands becoming enlarged.

If, as a result of hematogenic infection, the bacilli have accumulated in large masses in the capillaries, they may cause emboli and hemorrhages and edematous infiltration of the sur- 
rounding tissues. These serous infiltrations occur particularly in the lungs, both in the perivascular connective tissue and in the bronchial walls; the bacilli may then pass through to the surface of the mucous membrane and be discharged to the exterior with the catarrhal secretions (Nocard \& Leclainche). On the other hand they are aspirated into the alveoli with the secretions where they give rise to catarrhal processes which are followed by the development of broncho-pneumonic foci which, as a result of the glanderous nature, undergo early central softening.

According to the investigations of Schnetz glanders nodules develop in the lungs as miliary fibrinous inflammatory foci, in which the chromatin of the nuclei breaks up into fine granules as the process of necrosis advances (Unna's nucleus degeneration or cluromatotexis), while calcification is said never to take place $(?)$. Similarly, Csokor, in larmony with the views of other authors (Roloff, Rabe), is of the opinion that the primary glanders nodule is the result of swelling, coalescence and subsequent fatty-granular degeneration of the alveolar endotheliun following aerogenic infection. According to Nocard, on the other hand, the primary pulmonary lesions of glanders consist in the development of hyaline tubercle-like nodules, as had alreaily been observed by Loeffler and Schuetz in the lungs of horses that had been infecter experimentally by subcutaneous and intranasal arlministration of cultures: viz., fresh, gray nodules varying in size from a millet seed to that of a hemp seed and surrounded by a red area. More recently these nodules have been regarded as of particular importance, especially through the experimental investigations of Nocard, who frequently demonstrated them to be present as the only pathological lesions in naturally infected horses that were condemned and killed upon the evidence of a reaction to the mallein test, and in horses that had been infected experimentally by the alimentary administration of small quantities of virus, in all cases the glanderous nature of the nodules having leen demonstrated by experimental inoculation of laboratory animals. Coremans and Priesz express similar opinions in regard to the nature of these nodules, having demonstrated the presence of glanders bacilli in them by microscopical examination as well as by culture. On the other hand Schuetz as well as Olt and Angeloff deny the glanderous nature of these norlules. According to their views they are the result of infection with embryonic filaria (nodules of this character consist for the most part of eosinophile cells which absolutely do not occur in glanders norlules); in addition to this the aforementioned anthors maintain that the eneapsulated and frequently calcified foci are also due to the presence of these parasites. Kitt and Johne, however, have sueceeded in demonstrating virulent glanders bacilli in partially calcified foci, and it seems, therefore, that the many conflicting observations and riews on this question are evidently due to the fact that different investigators have investigated structures of a different nature.

According to Hutyra's histological investigations of the lungs of infected horses and guinea pigs, nodules due to hematogenic infection are caused by bacillary emboli, followed by the formation of thrombi and the development of an almost simultaneous vasculitis and perivaseulitis in the peribronchial and interlobular connective tissue which cause the formation of sharply circumscribed groups of round cells which appear macroscopically as glassy, hyaline nodules (in a few instances glanders bacilli could be demonstrated within these nodules, but in no instance could filaria be found). As the cell groups enlarge, the inflammatory process extends to the neighboring alveolar walls, which may, however, be involvei from the beginning as a result of the lodgment of bacilli in the interalveolar capillaries. In either case a miliary fibrinous inflammation is the result. Finally the nodules which develop in the walls of the small bronchioles may perforate their lumen and discharge their contents into them, producing an inflammation of their mucous membranes, which is followed by bronchopneumonic processes. The coalescence of neighboring pneumonic foci results in the formation of larger nodes, and the fusion of diffuse inflammatory areas in the interlobular septa results in the formation of so-called glanderous growths. In both instances thrombus formation in the bloodvessels of the affected area goes hand in hand with the inflammatory process.

In regard to local susceptibility, the various organs show considerable difference. After the lungs, the mucous membranes 
of the respiratory organs, especially of the nose, are most predisposed to infection. The latter is the seat of the glanderous lesions so frequently observed in the later stages of the disease and which are probably the result of infectious emboli or, though only occasionally, of infection with bronchial secretions charged with bacilli (the acute exacerbations of the morbid process which manifest themselves in nasal lesions are undoubtedly due to embolic origin). 'The lymph glands are also very susceptible to affection and the same is true also of the lymphatic plexuses of the skin and the subcutaneous connective tissue (the nodes found in these localities are also usually of embolic origin), following these the spleen, the liver, testes, bone marrow and periosteum are next in order of susceptibility, although lesions are much less frequently met with in these organs.

The susceptibility or resisting power of the various tissues is also influenced by previously existing other morbid processes. Thus Cadéac \& Malet succeeded in infecting swine, the vitality of which had been reduced by other causes (tuberculosis, prolapsus of the rectum, mammary abscess). A horse that had just recovered from an attack of pneumonia but in which a pulmonary adhesion persisted, was fed with eultures of glanders by the authors; autopsy showed that glanderous lesions were present in the immediate neighborhood of a small eavity only, and therefore were confined to the locus minoris resistentiae.

When large masses of glanders bacilli gain an entrance to the circulation and thus lodge in the capillaries of a number of organs at the same time, the development of glanders nodules will be observed in numerous places, but a? vays in greatest abundance in the nasal mucous membrane and the skin. The resulting generalized infection is then manifested in appearance of grave acute symptoms which however are probably caused in part by pyogenic bacteria. (According to Sewsejenko, streptococei can almost always be demonstrated in the blood in the course of acute glanders.)

The bacilli of glanders exert their pathogenic action by means of toxins. These are formed also in artificial cultures, probably in the form of endotoxins as a result of dissolution of the bacilli, and constitute the active ingredient of mallein. Similar toxins evidently also circulate in the bodies of affected animals causing cell proliferation and cell emigration in the neighborhood of the bacilli. The degenerative processes that attack the cellular elements as well as the febrile reactions following infection with large masses of virus and finally the excessive emaciation and great exhaustion which are frequently entirely out of proportion with the extent of the morbid lesions are no doubt due to these toxins.

Toxins obtained from cultures have a disfase producing effect in rather large doses only; in small experiment animals they produce spasms, paralyses, symptoms of congestion and occasionally edema at the point of injection (Finger, Babes). Dead bacilli have a similar effect. Bromberg succeeded, in his experiments with icats, in producing fatal acute affections resembling glanders following the injection 
of dead bacilli, while Cantacuzene and Riegler observed enlargement of the spleen, nephritis and swelling of Peyer's patches, and of the mesenteric and bronchial lymph glands in guinea pigs, following intrastomachal administration of dead bacilli of glanders.

Anatomical Changes. In those cases where the skin and subcutaneous connective tissue are affected, the skin may contain nodes varying in size from a lentil to that of a pea, of firm consistency and with soft centers, and round ulcers with ragged edges that have developed from the latter, while on the other hand (and more frequently) considerably larger abscesses and nodes are present in the subcutaneous connective tissue. Subsequently these may also ulcerate (see symptoms). The surrounding tissue is gelatinously infiltrated or lardaceous and glistening in appearance, firm in consistency and frequently contains small foci composed of yellowish-gray or reddish viscous pus. The lymph vessels between the nodes and ulcers are enlarged and filled with a thick, vellowish-white coagulated lymph; the regional lymph glands are much enlarged and firm and their interior tough connective tissue is filled with small pus foci.

The respiratory organs are found affected almost without exception in every case, sometimes the morbid process being limited to the lungs while in other instances the upper air passages and the nasal cavities are also involved.

In the lungs the initial morbid changes are represented either by small tubercle-like nodules or by large lobar pneumonic areas. The nodules consist of gray, hyaline or grayishwhite, firm structures ranging in size from a pin head to that of a mustard seed imberded in the lung tissue, occasionally present in very large numbers and which are surrounded, in the later stages of the process, by a hemorrhagic area (see p. 691).

The catarrhal pneumonic form of pulmonary glanders (broncho-pneumonia malleosa) appears in the form of small atelectatic foci which are at first of an even brownish-red color, but subsequently, as a result of central softening, become transformed into a yellowish caseo-purulent mass and surrounded by a firm, red, inflammatory halo which, like its immediate surrounding area, has a peculiar yellowish gelatinous sheen.

In some of the more advanced cases large contiguous areas of the lungs have become transformed into sarcoma-like, firm though not very tongh, structures (so called glanderous growths). Upon section the surface is either uniformly gravishred or has imbedded in its structure numerous small purulent or cheesy foci, in either case the peculiar gelatinous character being evident. Glanderous foci of still greater age consist of very tough, almost cicatricial connective tissue with cheesy or mortar-like foci imbedded in its structure.

More extensive or more acute affections of the lungs are always attended with an acute bronchitis while the swollen 
and bright red mucous membrane is covered with a purulent secretion, occasionally studded with small nodules and ulcers, the bronchial walls thickened and here and there distended.

In affection of the superficial areas of the lungs the adjacent visceral layer of the pleura

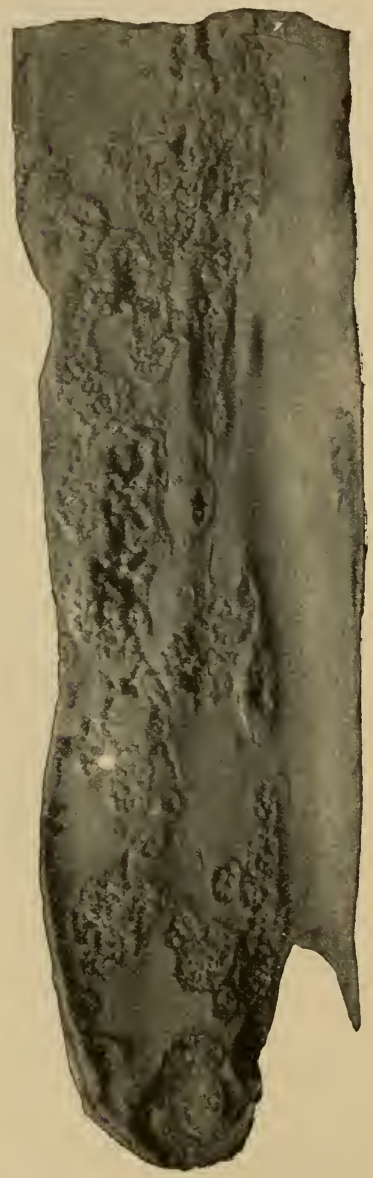

Fig. 117. Glanderous Ulcers on the Nasal Septum. is indurated while wide strands of yellow gelatinous tissue radiate between the pulmonary lobules. In very rare cases serous or serofibrinous exudate may be present in one or both pleural cavities.

. The lesions of glanders of the upper air passages and especially of the trachea, the larynx, the nose and the nasal sinuses are essentially similar. The beginning of the morbid process is manifested by the appearance of gray or yellowish, prominent nodules as large as millet seeds (rarely larger) and surrounded by a tumefied and brightly reddened area. The ulcers resulting from the breaking down of the nodules are at first round, regular, and smoothly bordered, but subsequently, as a result of progressive tissue destruction, they become irregular with raised borders, ragged edges, pale yellow lardaceous base covered with pus and detritus or, eventually, a brown scab. Larger ulcers are formed by the coalescence of small ones and these are usually surrounded by fresh nodules and ulcers (Fig. 117). On the other hand, ulcers that have "healed" are replaced by irregular starshaped, either smooth white or prominent reddish or white callous cicatrices, which may, in some rare cases, extend over the entire surface of one side of the nasal septum. In addition to this scar-like glanderous growths of connective tissue nature may form without previous ulcerative processes.

According to Johne, glanderous proliferations which may subsequently break down, completely or partially, are products of attenuated bacilli, the toxins of which are no longer able to produce coagulation necrosis of the proliferated con- 
nective tissue. That from such connective tissue new formations can actually levelop without the previous existence of lesions has been confirmed by Hahn by the demonstration of an intact mucous epithelium on their surface and by Rabe, who demonstrated the presence of the normal elastic basement membrane of the mucosa on the surface of the cicatrix.

In the trachea the above-described lesions usually occur on the ventral wall, in the larynx usually on the arytenoid cartilages, the lateral ventricle and epiglottis. In grave cases the trachea may be studded with its entire course with thickly strewn nodules, ulcers and cicatrices, the cartilages may even be exposed by some of the ulcers.

In the nasai cavity only a few nodules or ulcers are found, usually in the upper portions and on one side only, while the lower portion of the mucous membrane suffers no changes except catarihal injection and tumefaction. In cases that are less recent or are more acute in character the mucous membrane of one or both nasal cavities may be so studded with nodules and scars that it is almost impossible to find a healthy area of tissue; old scars from former attacks may also be found in the midst of acute lesions. In rarer instances the ulceration may have extended to the cartilage of the septum or the destructive process may have resulted in the perforation of the latter and formed one or more roundish holes. Finally, in cases that are equally rare, when the inflammatory process has extended to the periosteum of the neighboring bones (maxillary, nasal and muscles) bosselated enlargements and exostoses may occur on their surfaces.

Disease of the simuses is usually the result of an extension of the morbid process from the nasal cavities. The antrum of Highmore on the side of the affected nasal cavity is affected most frequently, the frontal sinus coming next. Here also the disease sometimes leads to the formation of nodes and ulcers, although in most instances the surface of the mucous membrane is smooth while its substance together with that of the submucous connective tissue forms an intensely yellow gelatinous layer about 1-2 cm. in thickness, the corresponding sinus containing much muco-purulent secretion. In rare cases cicatrices are found here also, or the mucous membrane is simply thickened, tough and bosselated on the surface. Similar morbid changes are occasionally found also in the guttural pouches and the Eustachian tubes.

The mucous membrane of the digestive tract is rarely found affected. In exceptional instances lesions are met with in the pharynx but only when the nasal cavities are likewise involved; here, too, the lesions consist of gelatinous infiltration of the mucous membrane and submucous connective tissue; occasionally there may be ulceration, but cicatrization is not the rule. Involvement of the intestine is still more rarely observed. In several horses from a badly infected stable the authors found the solitary follicles and Peyer's patches in the 
posterior sections of the ileum excessively tumefied, while the corresponding visceral peritoneum was raised into flat bosselations by the gelatinously infiltrated subserosa. (Vecchia observed glanderous nodules and ulcers of the mucous membrane in the cecum in four cases.)

The spleen and the liver quite frequently contain small hyaline nodules or yellowish-white nodes which are centrally softened and appear lardaceous upon section. More frequently, however, there are rellowish pus foci or mortar-like masses surrounded by a white, tough connective tissue capsule. In much rarer instances similar nodes are found in the kidneys. The testes are more frequently affected. These lesions are rare also in the brain where they occur as gelatinous hyaline or more or less purulent foci. Occasionally the muscles of the body also contain glanderous nodes, in a few cases they have been observed in the muscle substance of the heart.

Among the bones, the ribs are most frequently the seat of morbid changes while the vertebrae or the long bones are rarely affected. Sawed sections show pale yellowish, roundish or irregular cavities or fistulous canals containing a viscous substance, the bone substance in the immediate surroundings showing a denser structure. Affection of the ribs may be attended with an ossifying periostitis as a result of which the diseased section becomes enlarged to a remarkable extent, so as to be easily recognized by palpation; the adjacent pleura is reddishgray and contains gelatinous granulations.

The lymphatic glands in the neighborhood of affected organs are almost always involved; thus in nasal glanders the maxillary glands, in pulmonary glanders the peribronchial glands, in cutaneous glanders or farcy of the extremities the axillary or the inguinal glands, as the case may be, are acntely tumefied and juicy in recent cases, while in clironic cases they are firm and tough, adherent to the overlying skin or surrounding tissues, and frequently contain small, yellow muco-caseous or purulent foci.

In horses that have died of acute glanders we find, besides the lesions of a general acute infection (tumefaction of the lymph glands and the spleen, hemorrhages of the serous membranes) numerous small nodules and small superficial confluent ulcers on the tumefied and reddened mucous membrane of the respiratory tract, and nsually also older lesions of glanders; the lungs contain nodules, catarrhal pneumonic foci and hemorrhagic infarcts, there are also punctiform hemorrhages in the visceral pleura, miliary nodules in the liver and spleen, fibrinopurulent inflammation of the testes, and finally numerous nodes and ulcers in the subcutaneous and intermuscular connective tissue.

Statistics on the comparative frequency of the lesions in the various organs that have been pulblished in the more remote past (Roell, Haubner, Bagge) do not 
correspond to actual facts, because those investigators and authors did not recognize the initial stages of the disease in the internal organs. But in spite of this, the immense frequency of pulmonary glanders has always been recognized, the observations made in this respect since the general use of mallein, only making it more evident, while disease of the nose and skin is less frequently observed.

Thus, according to Roell, of 173 glandered horses dissected from 1869 to $187 \mathrm{~s}$ in Vienna, $145(84 \%)$ were affected with pulmonary glanders; of 216 glandered horses dissected at the veterinary school at Berlin only 10 failed to show lesions of pulmonary affection, in four eases it was present exclusively in the lungs and the nose was free in 32 instances. On the other haud, among 274 horses destroyed in Budapest in 1896, mostly on the basis of mallein reaction, 256 were affected with pulmonary glanders exelusively, 3 with nasal glanders exclusively, 12 with both pulmonary and nasal glanders, while 3 of the latter showed cutaneous lesions also.-According to Bagge's Danish statistics there were 1,336 eases of glanders and 167 of cutaneous glanders or farcy.-Nocard observed affection of the lymph glands of the cecum, the testes and the right kilney in one case in which all other organs were free.

Symptoms. The duration of incubation depends to a great extent on the manner and on the intensity of the infection. Following experimental infection from the skin or nasal mucous membrane, an elevation of temperature begins as early as the second day and local changes may be observed after from three to five days. The alimentary administration of small quantities of cultures is followed in three or four days by elevation of temperature, and in some instances swelling of the submaxillary glands and serous nasal discharge may be observed on the eighth day. After natural infection, however, several weeks and sometimes months may elapse before evident external symptoms (except elevation of temperature) manifest themselves. (In this sense Dieckerhoff assumes the period of incubation to be at least two weeks.)

From remote times it has been customary to distinguish pulmonary, nasal and cutaneous glanders. For practical purposes this classification may still be retained, provided it is always borne in mind that these forms merge into each other and that any or all of them may be present at the same time. However, this affection usually begins in the lungs, while disease of the nose as well as of the skin is usually secondary in nature.

Pulmonary glanders usually develops very insidiously (latent or occult glanders). Months may elapse before a horse that is affected with pulmonary glanders begins to show the first observable symptoms of lung trouble and these may be so little characteristic that they will for a long time excite no suspicion of the glanderous nature of the trouble; in the meantime the horse in question may have been the medium through which many others have become infected. In some cases attention is attracted to the animal's condition by sudden bleeding at the nose as the first symptoms of a destructive pulmonary process (Greve) or the animals may discharge bloody mucus in the act of coughing (Delamotte). However, the first symptoms (which in fact point to nothing specific except a chronic pulmonary disease) are generally manifested in a "run-down condition" and a dull, dry asthenic cough. This condition 
which simulates heaves may persist unchanged for a long time or it is gradually aggravated; percussion of the thorax reveals nothing abnormal and auscultation at most dry or moist rales. In rare instances the percussion sounds may be somewhat dulled in some regions or even quite flat as a result of atelectatasis, glanderous infiltration or extensive glanderous "growths" in certain areas of the lungs; upon auscultation the absence of the normal respiratory sounds is noted or there may be distinct vesicular or bronchial respiration admixed with rales; occasionally there may be bronchial respiration in the area in question. Respiration is of the costo-abdominal type and more or less labored. At the trachea large moist bubbling rales may then usually be heard while abnormal sensitiveness, painful spasmodic cough or excessive inspiratory dyspnea as well as stertorous or snoring respiration point to an affection of the larynx.

In some cases after these symptoms have existed for some time and while the animals have gradually become emaciated, more characteristic lesions of glanders make their appearance either on the nasal mucous membranes or on the skin or in both regions; previous to this an edematous painless swelling of the sheath or of the udder and of the extremities may be observed, which may disappear and reappear from time to time. In exceptional cases exclusive affection of the lungs may be attended with unilateral enlargement and abscess formation of the submaxillary glands (Eggeling).

Nasal glanders begins with inconspicuous symptoms of nasal catarrh. The mucous membranes are moderately reddened and there is a slight unilateral or bilateral serous nasal discharge which subsequently becomes more mucous. Later on it becomes more copious and purulent or may consist of a mixture of a greenish-yellow serous secretion with white masses resembling coagulated egg albumen; sometimes there are streaks of blood. The secretion adheres to the alae of the nostrils and forms dry crusts or is ejected in large quantity by conghing or snorting.

In the meantime the catarrhal injection and swelling becomes more pronounced and the veins are here and there distended. When the morbid process remains confined to the upper portions of the nasal cavities this condition may continue for weeks or even months without material change. After a certain time, however, gray or vellowish nodules of the size of hemp seeds or lentils make their appearance in the lower third of the cavities; these soon break down into small ulcers with rather smooth and sharply defined borders, they spread rapidly however and assume ragged outlines, raised borders and a lardaceous base. Their favorite seat is at the lower end of the turbinated bones and the opposite surface of the septum. They may finally extend to the edge of the nostrils 
where they coalesce into large irregular ulcerous surfaces (see Fig. 117 on p. 694 and Plate IV). Among the ulcers, or also in their absence, the regions in question may contain large or small star-shaped, bulging or flat, reddish or white, glistening scars. Isolated ulcers may terminate in such scars whereupon the nasal discharge subsides temporarily or permanently. As a rule, however, exacerbations occur with the symptoms just described, or with signs of some other localization. In extensive ulceration of the nasal mucous membrane the alae of the nose and the lip may become swollen and the skin may contain nodules as large as peas and which subsequently ulcerate. In exceptional instances a fluctuating abscess approaching the size of a walnut may develop on the nasal mucous membrane and occlude the nasal opening (Spassky).

The submaxillary glands are always enlarged on the side of the affected nasal cavity. At first the tumor is somewhat painful and indefinitely outlined, but subsequently it becomes painless, firm and knotty. (Fig. 118.) In the beginning the tumor is movable but soon becomes firmly united with the bone, the overlying skin and the surrounding tissues owing to the extension of the inflammatory process, and the overlying skin can then no longer be raised into a fold. Occasionally, and usually simultaneously with the appearance of fresh nodular eruptions on the mucous membrane of the nose, acute inflammatory symptoms manifest themselves in the tumor, such as increased pain and swelling, but they subside again within a few days. In rare cases a point in the periphery of the tumor undergoes softening, the overlying skin is perforated and a small quantity of viscous, yellowish muco-purulent secretion is discharged, whereupon the borders of the wound contract and gradually heal (Trasbot, Chardin).

Disease of the nasal cavity is frequently also attended by a catarrh of the corresponding conjunctiva. Richter observed. a case in which the cornea showed glanderous changes, the external border of the cornea being bluish-red and studded with gray or grayish-yellow nodules ranging in size from a millet seed to that of a pin head; in the course of 1 to 2 days they broke down and formed small nlcers. DeHaan observed an eye affection in a horse that resembled periodical ophthalmia, there was turbidity of the cornea and a tenacious fluid exudate in the anterior chamber.

In cutaneous glanders nodes and ulcers may develop in the skin or in the subcutaneous connective tissue. The cutaneous nodes, usually as large as peas or beans, soon break open and become converted into ulcers which subsequently either heal or extend into the surrounding tissue by the breaking down of their borders. The subcutaneous nodes usually appear on the extremities, on the sides of the thorax and under the abdomen; they attain the size of a walnut, are only slightly, if at all painful, may sometimes be fluctuating or surrounded by 
edematous tissue. Subsequently, after they become adherent to the skin, a small round opening forms and discharges a viscous, yellowish-grav, occasionally reddish pus. By progresion of the destructive process in the borders, rather deep crateriform ulcers form, with grayish-red, easily bleeding borders that show torpid granulations and similar but more lardaceous bases and discolored, purulent secretion (see Plate $V)$. They frequently continue to progress in spite of careful treatment, hut occasionally local healing occurs, often with scar formation.

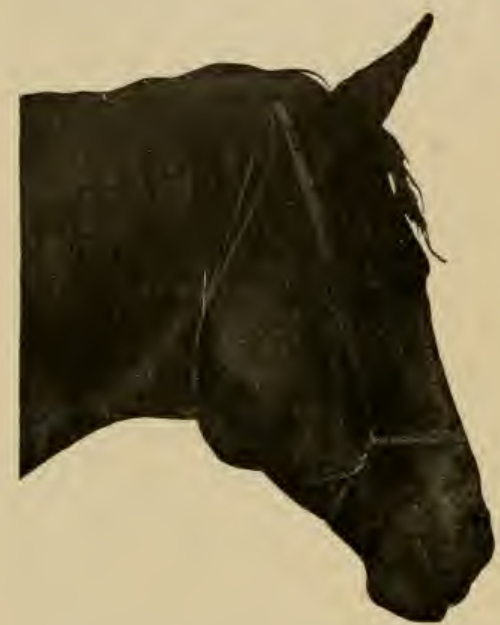

Fig. 118. Eularged Submaxillary Lymph Gland in Sasal Glanders.

In the region of the nodes and ulcers the centripetal lymph vessels finally become enlarged and are then palpable through the skin as slightly painful or completely insensitive tense strands which unite the nodes with the ulcers and with the neighboring enlarged lymph glands (rosary). Fresh nodes develop in the course of these strands and subsequently also nlcerate (Fig. 119).

In the meantime the edematous infiltration of the surrounding subeutaneous connective tissue progresses and spreads over a large area so that finally conspic u ou s, lloughy, painless swellings appear on the lower abdomen, the thorax or on one or more of the extremities. In some instances one or more of the legs become enlarged to such an extent that they resemble $\log$ s that are sharply set off at the hoofs (Elephantiasis malleosa); while nodes and ulcers may occur here and there in the tense skin. Enlargements of this character interfere seriously with the movements of the limbs, and sometimes large nodes, but especially enlarged lymph glands, may be the cause of locomotor disturbances by exerting pressure on neighboring nerve trunks; as a rule, however, these disturbances are not serious on account of the painlessness of the condition.

Nocard \& Leclainche described a special form of glanders under the name of lymphangioitis reticularis which manifests itself in diffuse swelling affecting in the course of a few hours one extremity or the lower portion of the body and is attended with increased heat and pain; after a few days the acute symptoms subside somewhat but the swelling 
persists while nodes and ulcers subsequently develop in the skin and subcutaneous connective tissue.

Glanders of the bones is rare and when observed it is usually in the form of spindle-shaped, painless enlargements of the ribs. deDoes observed the disease in one of the metatarsal bones, a painful swelling extending from the hoof to the tarsus having appeared after the operation of shoeing and becoming hard as stone in the course of a month, but it subsequently subsided and left only an enlargement the size of a

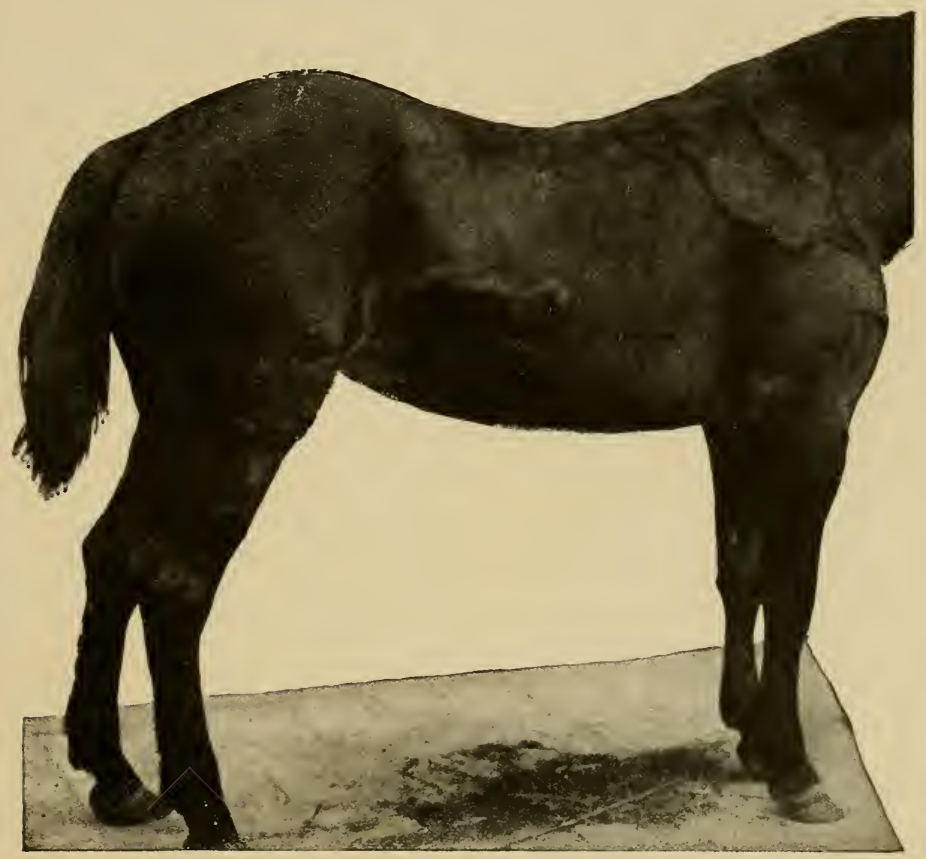

Fig. 119. Cutaneous Gilanders (F'arcy). Nodes and Strands of Lymph Vessels.

dollar. Affection of the joints with symptoms of moderate pain and diffuse swelling is equally rare.

In male animals a glanderous inflammation of the testes, in mares a similar inflammation of the udder (Schmidt) may be observed in connection with other symptoms.

In the line of general symptoms disturbances of nutrition usually make themselves manifest before the disease has advanced much; even in the latent stage of the affection. In other cases, especially in pulmonary glanders, the general nutri- 


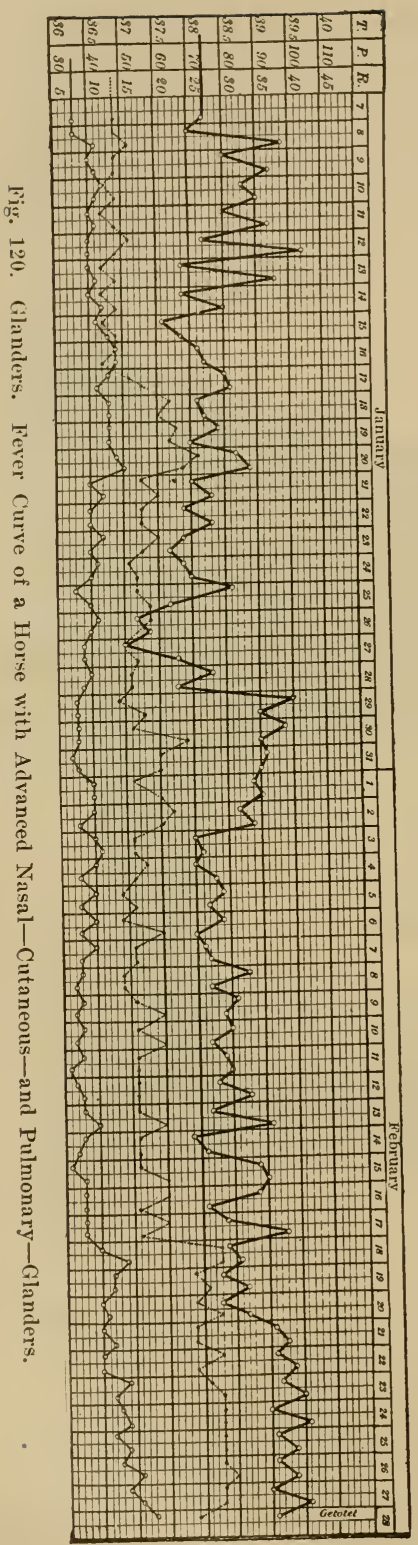

tion of the animals may be remarkably good for a long time. These disturbances manifest themselves in the loss of the normal gloss and smoothness of the haircoat, the elasticity of the skin, gradual emaciation and excessive anemia as indicated by the decided paleness of the visible mucous membranes.

The body temperature is subject to constant fluctuations. At times the temperature may rapidly approach or exceed $39^{\circ}$ to $40^{\circ} \mathrm{C}$., but it returus to the normal after a few days or weeks, again undergoing a rise after a few days, weeks, or months (see Fig. 120). These fluctuations are observed much more frequently in work horses than in animals that are permitted to rest much.

According to Eckert the amount of fibrin in the blood steadily increases toward the termination of the disease while the hemoglobin slightly diminishes, although it shows no fluctuations in the course of the disease. Mikrukow observed a reduction in the number of erythrocytes and an increase in the leucocytes, the proportion being $1: 125$ near the termination of the disease. The red cells showed no tendency to form rolls, they were paler than normal, greenish in color and of irregular form. Burnett \& Pearce also often observed lencocytosis.

In very sick animals heart weakness may develop, resulting in many forms of venous congestion (nasal mucous membrane) and being responsible, in part at least, for the edematons swellings of the extremities, the abdomen and the sheath. 
Acute glanders, which usually develops from the chronic form in horses, but frequently appears primarily in mules and asses is characterized by rapidly aggravated symptoms of a general acute infection. Attended with sudden elevation of temperature and other febrile symptoms large numbers of fresh glanders nodes and their consequent ulcers appear in the course of a few days, on any portion of the body, but particularly in the nose and under the skin. The nasal mucous membrane throughout its extent, may, in the course of 2 to 3 days, become thickly studded with nodules and ulcers; coincident with this condition is a profuse and often bloody nasal discharge; the respiration becomes wheezing as a result of the swollen nasal mucous membranes and of the submucous connective tissue, while the submaxillary glands become larger and sensitive.

Simultaneously with the appearance of these conditions acute edematous swellings with nodes or farcy buds in the surrounding cutis and subcutaneous tissue make their appearance; the nodes soon undergo softening, the resulting ulcers become confluent, producing extensive discolored, ulcerating surfaces. The superficial lymph glands undergo acute swelling and, in some instances, suppuration.

In the meantime the animals, in a constant condition of fever, emaciate rapidly, respiration becomes labored, and pulse accelerated and small; finally diarrhea sets in, whereupon death usually follows in the course of the second or third week of the acute affection.

Course. The course of this disease may be extremely variable, extending over a few weeks or over several years (Dieckerhoff was able to trace the course of one case back for seven years while Petrowsky reports a case of six years' standing). In the course of the disease, which is usually chronic in character, acute exacerbations alternate with periods of subsidence or of apparent complete arrest of the morbid process; near the end, however, the chronic cases usually assume an acute character and terminate with the symptoms above described. In rare cases the fatal termination is the result of gradual exhaustion or of a sudden pulmonary hemorrhage. The temperature elevations that are occasionally observed in the course of the chronic disease, are usually due to the development of fresh glanderous foci, particularly in the formation of glanderous lesions in the skin and subcutaneous comnective tissue.

The acute exacerbations may occur without external cause in the normal course of the disease, or they may appear as the result of an intercurrent acute affection, as for instance an influenza-catarrh of the upper air passages. In general the disease develops more rapidly in animals in a poor state of nutrition or in hard worked animals in which it assumes an acute character at an earlier stage than under favorable con- 
ditions. Auto-infection of animals by the contamination of their food and drinking water with their nasal and pulmonary secretions also bears a certain relationship to the gradual aggravation of the disease.

In a certain proportion of cases the disease terminates in recovery. The possibility of a local healing of the ulcers is proved by the presence of the characteristic cicatrices on the nasal mucous membranes and on the skin, as well as by the frequently observer dry, cheesy and, in some instances, partly calcified foci in the lungs and other internal organs the glanderous nature of which has repeatedly been demonstrated.

According to observations made in the warmer southern countries, for instance, in Southern Russia (Noniewicz, Semmer) the course of the disease is not infrequently benign in character, and horses that have already presented clinical șmptoms of the disease may occasionally recover (Semmer). Finally the possibility of recovery from the disease has been demonstrated by observations occasionally made in connection with mallein tests, according to which horses that have once reacted to the test cease to react to subsequent applications of this agent and remain free from disease thereafter, while post-mortem examination reveals, in various internal organs, encapsuled nodules that are in every respect similar to those of glanders. These observations point directly to the fact that even in northern countries (Middle Europe) the disease not infrequently results in recovery in its imitial, stages, when only a few nodules have as yet developed in the internal organs.

In the stables of the Compagnie générale des Toitures 2,037 out of 10,231 horses gave characteristic reactions to the mallein test in 1895 ; of these 687 subsequently dereloped manifest symptoms of glanders, while 338 horses ceased to react to later injections of mallein. A part of the non-reactors were subsequently killed for other reasons, and in all of them Nocard found fibrous or calcareous lesions in the internal organs.

The possibility of recovery from the disease should, however, be counted upon only in those cases that present no clinical evidences and that have been determined by the mallein reaction. On the other hand, all cases showing clinical signs of infection should, for practical purposes at least, be considered as incurable, although local processes may heal while new lesions develop in other parts of the body.

Diagnosis. Since the various symptoms do not present a characteristic clinical picture in the chronic form of the disease until the later stages, and since symptoms that are characteristic in themselves, such as nodes and ulcers in the nasal mucous membrane, do not occur until the disease is quite advanced, the definite diagnosis of glanders upon the basis of clinical symptoms alone is frequently a difficult matter

In stables where the disease has already been recognized 
in some of the animals, in other words, in stables that are known to be infected, mere temporary elevation of temperature in otherwise apparently healthy animals is in itself sufficient evidence upon which to base suspicion, especially when a temporary nasal discharge and slight swelling of the submaxillary glands has been noted and when the elevations of temperature recur.

Any nasal discharge of some duration, especially when it is unilateral and possesses the characteristics described above, and any chronic swelling of the submaxillary glands should direct suspicion to glanders. These symptoms are, however, not characteristic either alone or collectively, because similar symptoms are occasionally observed in simple nasal catarrh and especially in catarrh of the antrum of Highmore and of the guttural pouches. The above-mentioned symptoms may, however, be present for a long time without apparent change, especially when the patients are kept at rest and otherwise under favorable conditions of feeding. The nasal catarrh and even the swollen lymph glands may then subside to some extent and the ulcers undergo healing (cicatrization in the nasal mucous membrane!).

The absence of a pronounced acute character of the inflammatory changes and the torpid character of the ulcers point to the glanderous nature of skin lesions while any edematous swelling, nodular enlargement or ulceration on any part of the body, especially the ventral surface of the abdomen, on the sheath, the udder and the extremities, if not accountable for in some other manner must be regarded with suspicion.

The recognition of pulmonary glanders is attended with the greatest difficulties. For months symptoms of chronic lung affection may persist without any specific character whatever, such as occasional weak, dull cough, rapid exhaustion during work, poor condition, rough hair coat, now and then moderate fever, etc. These are the symptoms of heaves in general, and may therefore be brought on by other causes, since, however, they may have a glanderous origin as well it is always advisable, where the least opportunity for exposure has existed, to look upon such an animal at least with suspicion.

The difficulties that often present themselves in the recognition of glanders may be overcome by recourse to special methods of diagnosis.

Extirpation of the Submaxillary Tumor. The operation is usually performed easily on the standing animal and may be of service in those eases where no other symptoms exist than swelling and nasal discharge. In case the tumor is glanderous, small cheesy foci surrounded by a reddened area will be found in the tough, white, frequently lardaceous connective tissue. (According to Bistroumow immersion in 5\% carbolic acid solution brings out the foci more prominently above the surface of the section.) The presence of these lesions makes the diagnosis of glanders very probable, and the authors have confirmed this in

Vol. 1-45 
all eases by post-mortem examination. The foci in question are adapted equally well for microscopical examination and for the inoculation of experiment animals. According to Rudenko glanders bacilli may be found in lymph glands as early as two days after endo-nasal or percutaneous infection, while no other changes, except, at most, slightly increased juiciness of the tissues, are visible to the naked eye.

Microscopical Examination. The practical value of a microscopical examination is limited by the fact that the bacilli of glanders are not readily recognized as such in bacterial mixtures. Both the nasal secretions as well as the secretions of ulcers always contain a mixed bacterial flora and are consequently ill adapted for a microscopical diagnosis. Freshly incised nodes in the skin or subeutaneous connective tissue constitute an exception to this however, as do also the foci in the submaxillary lymph glands, but the bacilli are present in these in such limited numbers that their discovery is not always an easy matter. However, when bacilli that resemble those of glanders in their morphological characteristics and stain according to Gram's method are found in such material, the diagnosis of glanders is justified.

The technic of the examination consists in the staining of coverglass prepara. tions with aqueous or carbolized anilin staining solutions. Loeffler's methorl (Staining fluid: 30 ce. concentrated alcoholic methylene blue solution $+100 \mathrm{ce}$. of a 1:10,000 caustic potash solution or: anilin water, gentian violet or fuchsin and similarly diluted caustic potash solution or $1 / 2 \%$ ammonia $\overline{a a}$, followed by treatment with acetic acid) as well as the method of Sahli (Staining solution: $1 / 2 \%$ methylene blue solution and $1 \%$ Borax solution $\bar{a} \bar{a}$ ) are in no way superior to the ordinary methods.

Culture Method. This may oceasionally give positive results when the microscopical examination was fruitless. Sterile boiled potatoes or coagulated blood serum are specially adapted for this purpose. A small quantity of the contents of a suspected focus, obtained under aseptic precautions, is carefully spread over the surface of the medium by means of a platinum needle. If kept at a temperature of $25^{\circ}$ to $27^{\circ}$ C., distinctly visible colonies may be recognized after 3 to 4 days by their viscous nature and, on the potato medium, by their. honeyyellow color which subsequently becomes reddish-brown. On blood serum they have a honey-like opacity and are viscid like the potato cultures (on potatoes some strains of coli bacilli form similar masses, though are less distinctly reddish-brown, but more yellowish-gray and not viscid). If the material is applied thinly the bacillus may sometimes be obtained in isolated colonies from bacterial mixtures.

Portions of organs with suspected glanderous changes are best preserved in glycerin and sent to specially equipped laboratories for bacteriological examination. According to Galtier tissues that are infected with bacilli and immersed in $30 \%$ glycerin will retain their virulence for at least ten days, and frequently as long as 30 days.

Diagnostic Animal Inoculations. The inoculation of experiment animals with suspected morbid products may frequently give very valuable results, although they are decisive only when they furnish positive evidence. Such results are best obtained by the inoculation of material from the interior of encapsulated nodes or abscesses or from the base of fresh ulcers, while nasal secretion is less adapted for this purpose because it usually contains admixtures of other bacteria 
and may produce septicemia. (Aruch \& Savarese obtained positive results from the inoculation of aspirated pulmonary secretions). A negative result from such an inoculation does not, however, justify the exclusion of glanders because virulent bacilli are frequently absent in true glanderous lesions or secretions, and because, on the other hand, foreign bacteria, e. g., pus cocci may prevent the pathogenic action of the glanders bacilli by stimulating phagocytosis (Panisset). Aside from the solidungula experiment animals best adapted for this purpose are, above all others, guinea pigs, after which come the cat and the dog.

Of the solidungula the ass is the most suitable animal, although horses and mules may also be used. The application of glanderous material to the nasal mucous membrane or its subcutaneous administration is usually followed by the development of acute glanilers in three or four days and terminates fatally in about 10 to 14 days. It may be recognized with certainty long before its fatal termination by the characteristic symptoms (nasal glanders follows also the subcutaneous administration of the virus). Unfortunately the expense attending the use of these aninals for experimental or diagnostic purposes makes this method impracticable.

The inoculation of guinea pigs with glanderous material is also followed by the development of characteristic lesions of disease. If the material was applied subcutaneously (Trasbot, Loeffler), a swelling develops at the point of inoculation, followed by an ulcer with thick borders and purulent base and subsequently by suppuration of the regional lymph glands.-After intraperitoneal injection (Strauss) in males the testes begin to swell in from 2 to 4 days, though exceptionally not until the 12th day, becoming hot and painful; subsequently they rupture and discharge pus that contains large numbers of bacilli; at the same time the animals emaciate and usually die in about two weeks after inoculation. In some instances nasal glanders also develops (sneezing, wheezing respiration, nasal discharge) and one or more joints or extremities may become swollen. The animals may be killed upon the appearance of the ulcers or of the swelling of the testes or lymph glands; at post-mortem examination purulent exulate is formed between the tunies of the testes, small white points like needle pricks or somewhat larger may be found in the spleen, liver, testes, lymph glands, etc., and in these the bacilli of glanders can usually be demonstrated in large numbers, either microscopically or by cultural means. (Liénaux observed that periorchitis occasionally did not develop until after the 12th day, while Carléac \& Malet observed in exceptional cases a chronic affection following intraperitoneal injection of the virus, which persisted for 2 to 4 months; post-mortem examination revealed dry, cheesy foei in the internal organs, and the nasal mucous membrane contained deep and extensive ulcers. According to these authors, inoculation glanders in guinea pigs may, in exceptional cases, terminate in recovery).

The subsequent bacteriological examination of the exudate of the testes and of the metastatic foci of the internal organs must not be neglected for the reason that orchitis occurs also after inoculation of other bacteria. Nocard demonstrated this to be the case with the bacillus of ulcerous lymphangioitis of horses (see p. 731), Preisz in regard to the bacillus of pseudotuberculosis in sheep (see p. 633 ), Kutcher with regard to a bacillus found with the bacillus of glanders in a guinea pig that had been inoculated with the nasal secretion of a horse (see p. 632); these bacilli, however, in contrast to glanders bacilli stain according to Gram. According to Baruchello the Bac. Pyocyaneus may in guinea pigs occasionally be the cause of periorehitis, although this is not followed by ulceration of the swelling. Nicolas demonstrated an oval bacillus in a periorchitis following intraperitoneal injection of a guinea pig, with nasal secretion from a horse, and observed nodules resembling those of glanders in the internal organs. Finally, Lignières produced an exudative periorchitis in guinea pigs with the actino-bacillus Basset obtained the same result with a small Gram negative bacillus obtained in culture from a rabbit affected with psendotubereulosis, Cagnet with a bacterium obtained from pus foci in the abdominal organs of a guinea pig, Panisset \& Loiseau with the bacillus of human diphtheria, and Joly, Basset and Panisset with tubercle bacilli.

According to the observations of Russian experimentalists (Lisicyn, Malzew, Sacharow, etc.) cats are specially adapted for diagnostic inoculations. In animals that have been inoculated subeutaneously the temperature rises to $40^{\circ}-42.5^{\circ} \mathrm{C}$. beginning with the 2 nd or $3 \mathrm{rd}$ day and remains at this height; at the point of 
inoculation a circumseribed swelling develops, and $t$ to $i$ days thereafter an ulces appears; subsequently to this similar nodes and ulcers appear in other portions of the body, and finally the lesions of nasal glanders make their appearance. Following rapid progressive enaciation the animals die, with few exceptions, on the 5 th to the 12th day after inoculation. On post-mortem examination the lungs, spleen, testes, ete., are found to contain nodules; bacilli may also be demonstrated in the blood. (Young eats are particularly recommended as suitable subjects for inoculation.)

Dogs, especially young logs, are also adapted for experimental inoculation (Puetz, Galtier, Gruenwall, ete.). After the application (by rubbing) of the nasal lischarge of a glandered horse to the scarified skin at the forehead, ulceration may be observed on the $3 \mathrm{rd}$ to the $4 \mathrm{th}$ day; in the majority of cases recovery takes place, although in some instances general infection may result, leading to the formation of miliary nodules in the lungs, spleen, ete.

Finally fiel, mice and a certain species of arvicola may be used for diagnostic inoculations (Kitt), but only in cases where the inoculating material is known, beforehand, to le free from foreign admixtures. '

Technic of Inoculation. The most expedient' method of inoculating horses and asses is to insert the suspected material or secretion into the nasal mucous membrane by means of an inoculating needle or applying it directly to a portion of the mucous membrane that has previously been prepared by rubbing with a piece of coarse linen. Inoculation of the skin is less reliable because the resulting norlule not infrequently heals before definite results are obtained. The subeutaneous injection of a larger mass of secretion mixed with water is more apt to produce results.

If pure material is at our disposal, guinea pigs are best infected by intra peritoneal injection, otherwise (in the use of nasal secretion or material from a contaminated uleer) the subeutaneous or intramuscular method is better. Under the first named conditions a small quantity of the suspected material is thoroughly ground up in a mortar with distilled water, filtered through a sterile piece of linen and then injected with a hypodermic syringe into the abdominal cavity; in subcutaneous inoculation the hair is elipper from a small area on one side of the abdomen, an incision is made through the skin and a small pocket formed with the end of the scalpel handle, whereupon a small particle of the suspected tissue (as large as a lentil) is inserted and the skin firmly pressed into place; the intramuscular inoculation consists in the injection of an emulsion, prepared as above lescribed, into the muscles of the thigh (according to Aruch \& Savarese lesions are produced more rapidly by this method than by subeutaneous infection). If at all possible several animals should be inoculated, and preferably males.

Cats receive subcutaneous injections of the emulsion, while dogs may be infected by insertion of a seton saturated with the suspected material into the subentis of the back of the neck (Puetz, Galtier), or the skin of the foreheal may be scarified by means of superficial incisions and the material applied directly (Nocard). A more practical method consists in the injection of the suspected material, after dilution with sterilized water, into a joint, a serous eavity or under the skin (Balitzky).

Allergic Mallein Reactions. Infection with glanders produces a peculiar allergic condition of the animal body very similar to that observed in tuberculosis (see p. 574). This condition manifests itself by an increased susceptibility to the toxins of the disease-producing organism. This hyper-sensitiveness is made use of for diagnostic purposes by administering the toxin of the bacillus of glanders to the suspected animals in question, and the resulting positive or negative reaction is looked upon as an index of the presence or absence of infection, as the case may be.

Mallein constitutes the antigen or inoculating material. It is prepared from the cultures of glanders bacilli and is injected into the subcutaneous connective tissue or applied to the conjunctiva. 
Preparation of Mallein. Mallein contains the toxic substance, endo- and exotoxin, of the glanders bacillus cultivated in a liquid glycerine medium. It was first prepared by Helman and Kalning by extraction of potato eultures. Preusse as well as Preisz prepared the material as follows: Potato eultures that have been permitted to grow for several months are immersed in a mixture of equal parts of water and glyeerine and kept for several days in an incubator at $35^{\circ}$ to $37^{\circ} \mathrm{C}$.; the liquil is then sterilized by means of steam and filtered through porcelain (in order to insure greater uniformity of the product; Preisz adds bichloride of mereury). Roux eultivates highly virulent glanders bacilli in glycerine-pepton-boullion; at the end of 4 weeks the culture is sterilized, filtered and evaporated to 10 its original volume (a similar methor is recommended by Bang, also by Johne; the latter, however, does not evaporate the filtrate, but adds nine volumes of a $2 \%$ solution of earbolic acid). In Russia, also, mallein is used in its dilute form in 1.0 ce. doses. It is prepared by Kresling by eultivating a specially toxie strain of bacilli in $5 \%$ glycerine bouillon for a period of 8 months. Foth evaporates the original bouillon culture to $\frac{1}{10}$ its volume, then treats the same with absolute alcohol, and in this way obtains a white precipitate which subsequently becomes yellow; when ary this leaves a spongy mass, which is readily pulverized (dry mallein). Schweinitz \& Kilborne produce a pulverized form of mallein by a similar method, while A. Babes prepares his "'Morvin," which is used almost exclusively in Roumania, by eultivating the bacilli on bonillon-potato merlium; 5-7 weeks later this mass is washed on a Witt filter until the aldition of aleohol to the filtrate no longer produces a precipitate. Finally Schnïrer attempterl to produce an effective mallein by dis. solving glanders bacilli in $2 \%$ antiformin solution, evaporating to $\frac{x}{10}$ of its volume and obtaining therefrom an alcohol precipitate. This preparation, though it possessed properties similar to those of mallein, was only $1 / 15$ of the strength of Roux's erude mallein.

Crude mallein is a dark brown, syrupy fluid possessing a peculiar odor and having a neutral or sliglitly acid reaction, if kept in a cool place and protecterl from sunlight it retains its activity for months. When injecterl in large doses (20 to 50 ce.) into the blood of healthy horses it may produce death, preceded by symptoms of increased heart activity, respiratory difliculties and profuse sweat; the subcutaneous injection of doses of 1.0 to $2.0 \mathrm{gm}$. into healthy horses produces no norbid symptoms; on the other hand in horses affected with glanilers eren smaller doses will produce a characteristic reaction. Experimentally infecter guinea pigs react both locally and generally to injections of 0.2 to $0.5 \mathrm{gm}$., while the injection of $2.0 \mathrm{gm}$. into healthy guinea pigs produces only temporary elevation of tempera. ture (Pearson).

The effectiveness of mallein varies according to the virulence of the strain of bacilli userl as well as to the method according to which it has been prepared. At present we have no reliable methoils of standardizing this material, asicle from its practical application in testing glandered horses.

1. Subcutaneous Mallein Test (Thermic Reaction). Following the subcutaneous injection of a suitable, comparatively small dose of mallein glandered horses show a rise of body temperature which usually begins in the fourth to eighth hour after injection and rapidly increases for from 8 to 14 hours, in exceptional cases not until the sixteenth or thirtieth hour, until it reaches its maximum; after this the temperature gradnally returns to the normal, but frequently a second less prononnced elevation may be observed (Fig. 121). In the course of the thermic reaction other febrile symptoms may also frequently be observed (organic reaction); thus increased heart action and respiration, which, however, are not always synchronous with the change of temperature, muscular tremors, which, when they occur at all, usually appear between the sixth and eighth hour after injection; that is, in the beginning of the febrile attack; finally there may be depression, dullness and loss of appetite, as well as a more or less extensive inflammatory edematous swelling at point of injection (Fig. 122). (In the 
case of glanders of the cornea before referred to [see p. 699] Richter observed hyperemia of the corresponding region of the cornea after subcutaneous injection of the mallein. The number of visible nodules on the cornea became very numerous. On the other hand Walter observed conspicuous redness of the cutaneous ulcers during a reaction). In some cases the temperature remains permanently elevated after the appearance of the reaction and the disease, which, up to this time, has been chronic, takes an acute course, the general symptoms increase in intensity and the animal succumbs in the course of a few weeks. Healthy animals do not react to mallein; and in horses with other diseases a reaction, though occasionally observed, is of very exceptional occurrence (possibly in some cases of alveolar emphysema of the lungs?).

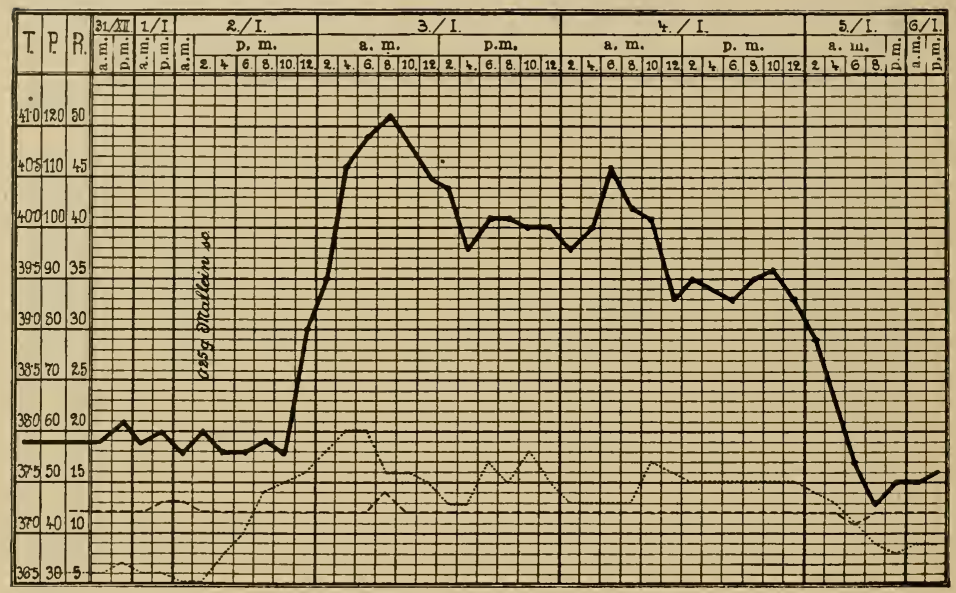

Fig. 121. T'ypical Thermic II allein Reaction.

In interpreting the results of the mallein reaction the following principles should be observed:

A mallein reaction is positive (typical) if the temperature after injection exceeds the preinjection temperature by $2^{\circ} \mathrm{C}$. or more and rises above $40^{\circ}$, providing the preinjection temperature did not indicate fever. The reaction is positive, also, if the increase in temperature is only 1.5 to $1.9^{\circ} \mathrm{C}$. or exceeds 29.5 to $39.9^{\circ}$ C., providing it is attended with a pronounced organic reaction.

The reaction is indefinite or doubtful if the rise in temperature equals 1.0 to $1.9^{\circ} \mathrm{C}$. and no organic reaction occurs.

The reaction is atypical if the rise in temperature, no matter how excessive this may be, is very rapid and subsides with equal 
suddenness, that is, if the febrile condition is of short duration only, four to six hours at the most.

The reaction is negative if the rise of temperature does not exceed $1.0^{\circ}$, or $39.0^{\circ} \mathrm{C}$.

A positive (typical) reaction justifies the conclusion that the horse in question is affected with glanders; on the other hand a doubtful reaction as well as an atypical reaction, justifies a suspicion only, in which case a final decision depends upon a repetition of the test in about four weeks; finally the absence of a reaction in a horse in good condition and not of great age, justifies the exclusion of the disease.

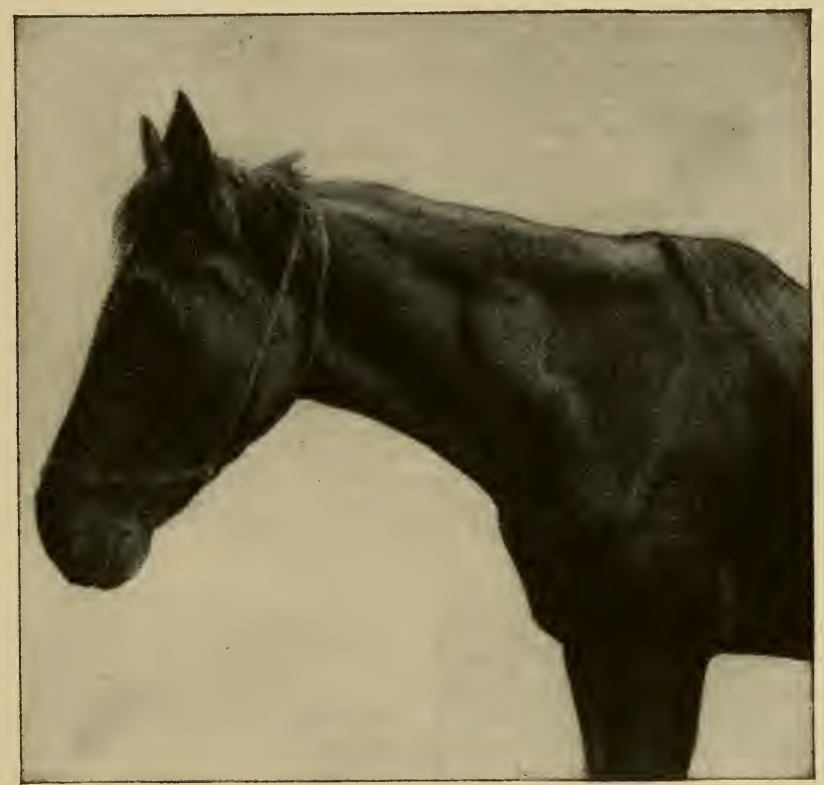

Fig. 122. Organic Ifallein Reaction: Edematous Swelling at the Point of Injection and the Afferent Lymph Vessels Following Subc itaneous Injection of Mallein.

In regard to the interpretation of mallein reactions the views are still quite at variance. When mallein was first introduced into practice a reaction of $1.0^{\circ} \mathrm{C}$. was considered as positive (Johne, Dieckerhoff \& Lothes). Subsequent experiences have, however, shown that higher and longer continued temperatures only may be regarded as such. Nocard regarded every horse which showed an organic reaction and an extensive painful swelling at the point of inoculation and showing a typical rise of $1.5^{\circ} \mathrm{C}$. of temperature or exceeding $40^{\circ} \mathrm{C}$. as affected with glanders, while an elevation of only 1.0 to $1.4^{\circ}$ simply 
pointed to suspicion. According to Schindelka a positive reaction consisted of an elevation of temperature of $2.0^{\circ}$ while an elevation of $1.5^{\circ}$ to $1.9^{\circ}$ simply indicated suspicion, an elevation of less than $1.5^{\circ}$ being regarded as of negative importance. Thomassen and MacFadyean also considered an elevation of $2^{\circ}$ as a positive reaction, but regarded an elevation of 1.0 to $1.4^{\circ}$ as pointing to probable or possible infection. Babes and Furtuna defined the term "specifie reaction" as a rapid increase of temperature of at least $2^{\circ} \mathrm{C}$. occurring within the sixth to eighth hour after injection and exceeding $40^{\circ} \mathrm{C}$. followed on the next day by a second rise above the preinjection temperature, with simultaneous local and general organic reaction (great typical reaction); such a reaction only justified the diagnosis of glanders while a similar elevation of temperature not exceeding $40^{\circ}$, but also with simultaneous organic reaction (small typical reaction), as well as pronounced elevations of temperature of short duration (great atypical reaction) indieated suspicion only; a similar but slight elevation of temperature (small atypical reaction) had no significance. According to Peter, Preusse and Foth an elevation of temperature of $1.5^{\circ}$ is to be regarded as positive. These authors, with the exception of Foth, lay special stress upon the importance of the organic reaction in the interpretation of the results of the mallein test.

In poorly nourished overworked as well as in old glandered animals a reaction may fail to take place or it may occur at an unusually late period; thus in a case observed by the authors the elevation of temperature did not begin until the twenty-first hour after injection, being typical however in other respects (Fig. 123).

Horses affected with other diseases do not give typical reactions to mallein. Thus Hutyra \& Preisz failed to observe reactions in distemper, sarcomatosis, phlegmonous conditions, pectoral influenza, morbus maculosus and botryomycosis, while Schindelka failed to observe reactions in distemper, nasal catarrh, as well as in emphyemia of the antrum of Highmore.

The diagnostic value of a mallein reaction has been confirmed by the practical experience of the last two decades. The varying results obtained during the first few years after the introduction of mallein into general use were due to the fact that the interpretation of the temperature reactions were based on different principles (an elevation of $1.0^{\circ} \mathrm{C}$. was looked upon as a positive reaction while the animal's state of nutrition as well as advanced age did not receive due consideration, etc.) and the fact that the primary lesions of glanders were at that time not as thoroughly understood. The lack of uniformity in the mallein preparations was also at fault. At the present time, however, it has been firmly established that mallein is an extremely delicate and reliable agent for the diagnosis of latent glanders. But in order to apply this agent properly and successfully in the extermination of glanders it is necessary that the test be made intelligently and systematically, further that the results of observations during the period of reaction be carefully interpreted and that all clinical symptoms receive proper consideration. In this respect as well as in the general technic of the test the instructions given under the head of 
tuberculin testing are equally applicable to the mallein test (see p. 577). There seems to be a difference, however, in regard to the immunizing effects of repeated injections of these two diagnostic agents in that horses will usually react typically to repeated injections of mallein made at short intervals. In some cases the reaction becomes even more pronounced (thus Galtier observed 11 positive reactions in a glandered horse in the course of a month).

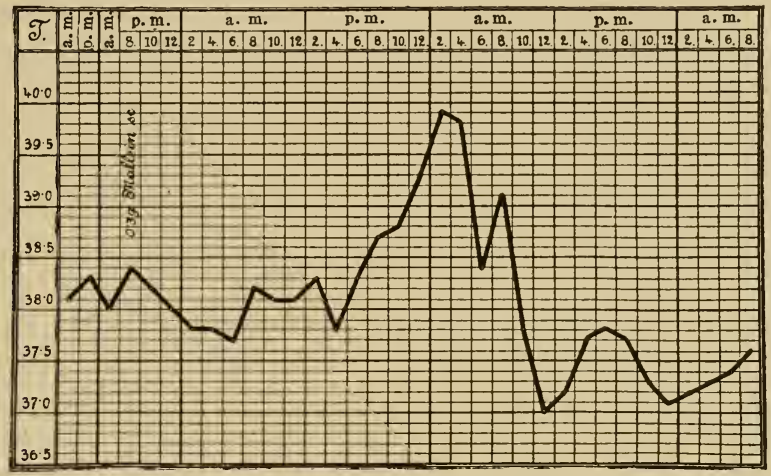

Fig. 123. Late Thermal Mallein Reaction.

Reports on the use of mallein on a large scale have with few exceptions been favorable in that post-mortem examinations as a rule confirmed the diagnosis of the disease. Thus according to Preusse's compilations of reports recorded in the literature up to 1897 , positive reactions were confirmed by post-mortem examination in $94.7 \%$, negative in $95.7 \%$ of all cases (Foth estimates the value of the reactions at $86 \%$ and $94 \%$ respectively, Rudovesky at $87 \%$ and $96 \%$ ).

According to the compilations of Hutyra \& Preisz, post-mortem examinations were held on 85 out of 410 horses tested with mallein in Hungary up to 1893 ; of these 14 gave a reaction of less than $1.5^{\circ}$, and 6 of them were found affected with glanders; 12 gave a reaction of 1.5 to $2.0^{\circ}$, and all of them were found affected; 59 gave a reaction of $2.0^{\circ}$ or more, and 58 of them were found affected with glanders; in one the post-mortem findings were negative, but this one gave an atypical reaction. In those cases where the temperature elevation was more than $1.5^{\circ}$ and exceeded $39.5^{\circ}$, all horses without exception were found affected.

Kitt (1901) basing his observations on reports from the Kingdom of Bavaria since 1892 showerl that mallein always produces a prompt reaction in glandered horses, and that reactions failed to appear in more than 120 other diseases (nasal catarrh, disease of the maxillary sinus, heaves, etc.). According to Feist 72 out of 73 reacting horses in Alsace-Lorraine were found affected with glanders, while on the other hand not one out of 287 non-reactors were found affected. Schindelka of Vienna maintains that, aside from glanders, only pulmonary emphysema will produce an occasional reaction to mallein, basing his statement on the results of 503 tests and 148 post-morten examinations. Jensen, of Denmark, collected data covering 184 cases; in 177 cases the post-nortem findings confirmed the diagnosis as based upon the text, in six cases the results were doubtful, and in one case the result was of an opposite character. Nocard (1897) on the basis of several thousand tests conducted by him in France, concluded that a positive mallein reaction justified the diagnosis of glanders. A negative reaction on the other hand justified the diagnosis of an absence of glanders, irrespective of the presence of any clinical symptoms that simulate this disease. Upon this basis he succeeded in differen- 
tiating several diseases from glanders which had formerly been confused with that affection. Thomassen in the Netherlands, MacFadyean and Wright in England, Wirtz in Holland, Heyne, Dieckerhoff \& Lothes, Johne, Edelmann, Piel and Schlegel in Germany, Kowalewski in Russia, Babes and Fortuna in Roumania, Schweinitz \& Kilborne in America, Hoogkaner \& de Haan in Lower India, and Schnürer in Austria have all expressed themselves definitely in favor of the diagnostic value of mallein.

The opposite view has been championed by Schïtz, who in 1884 conducted 54 autopsies on horses, of which 15 reacted to the extent of $1.5^{\circ}$ to $2.5^{\circ} \mathrm{C}$., while 7 gave a reaction of $1.0^{\circ}$ C. to $1.4^{\circ} \mathrm{C}$. (the temperature curve and the highest post injection temperature were not reported). In the high reactions, as well as in a portion of the others, post-mortem examination revealed only hard nodules, asicle from other immaterial changes, and in one ease cheesy masses, while in several others there were gray maculae (?), and in one yellow foci in the peribronchial lymph glands. In another instance, among 4 horses which reacted ont of a total number of 6, post-mortem examination in three showed calcified foei in the lungs, the liver and the peribronchial lymph glands (!) In one ease there was induration of the pulmonary tissue with muco-purulent foci. In 1898, 42 horses were tested with Preusse's mallein; 9 of these gave a reaction of $1.5^{\circ} \mathrm{C}$. or more (in two this was followed by a temperature exceeding $40.0^{\circ}$, in three ouly to $39.5^{\circ}$ ). In these horse glanders could not be demonstrated, while three horses that were affected with chronic glanders gave no reactions (the temperatures were recorded only up to the 14th hour. On the other hand the post-mortem diagnosis was confirmel by microscopical examination). In addition to the above, Olt, Robeis, Poetschke, Schoeneck and others have expressed themselves against the diagnostic value of mallein in practically all cases, basing their views on limited observation. (In Olt's experiments it is reported that nine glanderous horses did not react, while three healthy horses gave positive reactions.)

In Hungary (1897 to 1901) 570 horses were killed on account of the disease or suspicion of it and positive reaction to the mallein test; of these 536, i. e., $94 \%$, were found affected with glanders at post-mortem examination conducted under unfavorable conditions. In 1902 to $1908,1,385$ horses were officially destroyed on the strength of positive reactions to the mallein test and suspicion of disease, 1609 were destroyed on the ground of positive reaction to the test and suspicion of infection or exposure; in the first group 1,313, i. e., $94.8 \%$, in the second 1,447, i. e., $89.9 \%$ showed anatomical lesions of glanders at post-mortem examination.

Technic of the Subcutaneous Mallein Test. The mallein is prepared in a manner similar to that followed in the preparation of tuberculin (see page 582). Since the normal temperature of horses does not exceed $35^{\circ} \mathrm{C}$, it is recommended that the test be applied in such cases only where the body temperature does not exceed $38.5^{\circ}$ at the time. Fluid crude mallein should be diluted before injection (in case this has not been done by the manufacturer) with 9 parts of a $1 / 2 \%$ solution of earbolic acid.

The diagnostic dose of Preusse's mallein or of Preisz's potato mallein is 0.50 gm., that of Roux's condensed bouillon $0.25 \mathrm{gm}$, that of Johne's unconilensed $\left(10^{\prime} \times\right.$ diluted) mallein $5.0 \mathrm{gm}$., that of Foth's dry mallein. 0.10 gm., that of Russian mallein and Babes' morvin $1.0 \mathrm{gm}$. for each injection.

In positive reactions the temperature usually reaches its maximum at the 12 th to the 15th hour after injection; in view of the possibility of a late reaction (see page 712) temperature readings should be made up to or beyond the 21st hour. Tatray observed in 395 cases of typical reactions (his observations, however, extending only to the 21 st hour) that the maximum temperature occurred in two instances in the sixth hour; in 74 instances in the ninth hour; in 174 in the 12 th; 114 in the 15 th; 23 in the 18 th, and in 8 instances in the 21 st hour after the injection. According to Calinescu, the intravenous injection of the mallein $(0.02$ gm. morvin) results in a reaction in the 5th hour.

Although glanderous horses will, as a rule, react to repeater injections of mallein, it is recommended in cases where doubtful results have been obtained (in order to evade every criticism) not to repeat the test until the expiration of 4 weeks. In an emergency the test might be repeated after 15 days.

2. Ophthalmic Mallein Test (Ophthalmoreaction, conjunctival reaction). Following observations on the action of tuberculin on the conjunctiva and the skin of tuberculous persons and cattle (see p. 583) similar experiments were made on glanderous and healthy horses with mallein. The results of the latter experiments, however, can not as yet be judged prop- 



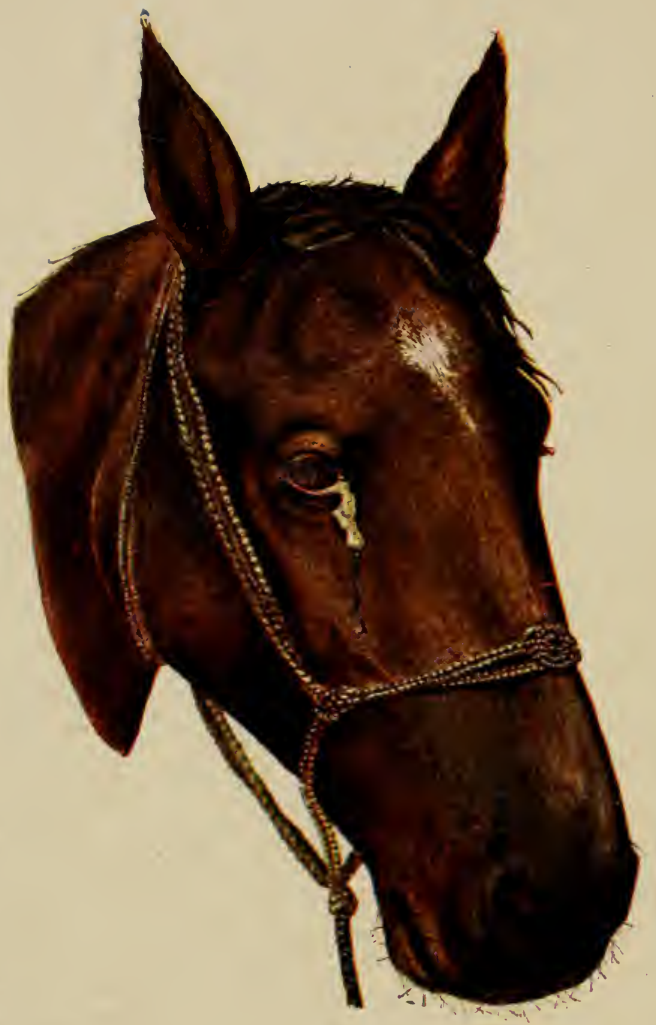

Conjunctival (Ophthalmo) reaction in Glanders.

8 hours after the instillation of crude mallein. 
erly on account of the comparatively small number of horses tested and the lack of sufficient post-mortem control work.

After the administration of a small quantity of dilute mallein into the conjunctival sac the ophthalmic reaction appears in the form of a conjunctivitis, swelling of the eyelids, intense redness of the conjunctiva tarsi et bulbi and a purulent secretion (see Plate VI). According to observations thus far recorded a pronounced reaction with purulent exudate seems to point to the existence of the disease, while a negative or doubtful reaction has only a circumstantial value since it is known that actually infected horses may sometimes react only slightly or not at all. (It seems that these tests not infrequently give negative results in the first stages of the disease.)

The ophthalmic test was first applied suecessfully by Choromansky; 15 glandered horses that were treated with $0.1 \mathrm{gm}$. of Russian mallein reacting by the appearance of a severe conjunctivitis, while 37 healthy horses gave a negative reaction to this, as well as to the subcutaneous test which was subsequently applied. Wladimiroff reports a similar result obtained in 12 affected and 20 apparently healthy horses; this author, however, obtained positive ophthalmo-reactions also in horses that had ceased to react typically to the usual subeutaneous test, consequently he did not consider a posi. tive reaction to the ophthalmic test as necessarily indicating the presence of disease. (An observation made by M a rte I agrees with this view; this author obtained a typical cutaneous reaction on his arm 14 years after he had been infecter with glanders). Selnnürer expresses himself very favorably in regard to the value of the ophthalmic test, having obtained only one negative result in 47 glanderous horses;

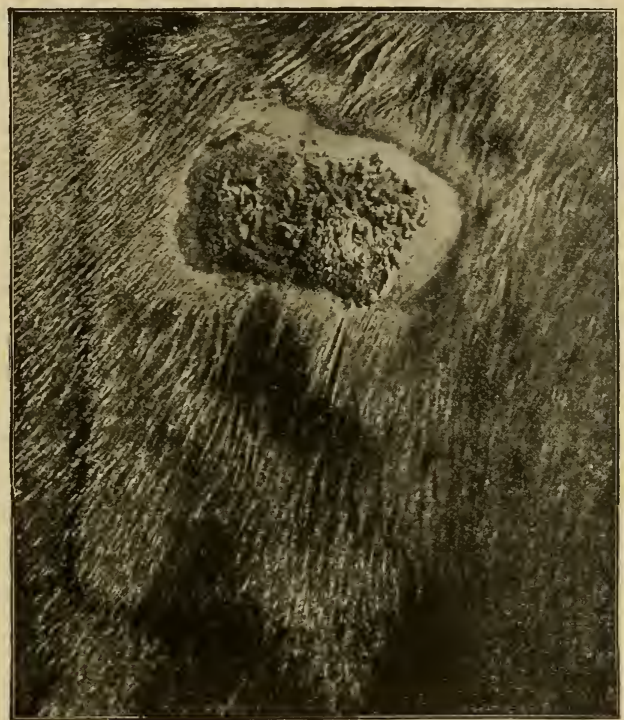

Hig.124. C'utaneous Reaction in Glanders. Lymph Vessels Enlarged as Result of Inflammation Radiating from Searified Area.

while the test on 353 suspected horses and on 16 horses affected with some other disease agreed with the results of the cutaneous and subcutaneous tests in the negative character of all of them.

The observations of Vallée in three cases, of Dietrich in 13 , of Putzey \& Stiennon in 7 , of Martel in 7 , and of Klimmer \& Kiessig in 9 glandered horses were less satisfactory. These authors obtained distinct reactions in only about one-half of their tests, which were marle with 10 to $20 \%$ mallein solution.

Technic of the Ophthalmic Test. Since the most satisfactory results have thus far been obtained from the use of concentrated mallein, it is recommended that 
this material be applied to the inner angle of the conjunctival sac by means of a pipette or camel's-hair brush. Before making the application the operator should satisfy himself that the eye contains no foreign bodies, and that no symptoms of conjunctivitis or periodical ophthalmia exist.

The reaction begins from the 4 th to the 6 th hour after the application, and may continue for 24 to 26 hours, or eren longer, the accumulation of pus helow the inner canthus of the eye as well as inflammatory hyperemia and swelling of the conjunctiva are readily recognized (the purulent exudate may drop off from time to time, or it may be removed intentionally by an attendant, so that the positive character of the reaction may not be observed at the time!). If, in case of some other disease or on account of suspected infection, the result of the reaction is negative or loubtful, the test niay le repeated on the following day, in which ease the reaction is in some horses decidedly more pronounced. In exceptional eases the reaction appears also in the untreated eye, and in very rare instances there is noted also a simultaneous slight elevation of temperature. Previous subentaneous injection of mallein may partly or wholly prevent the local reaction, for which reason this test should be applier after, but not before, the ophthalmic test.

3. Cutaneous Mallein Tests (Dermoreaction, intradermal reaction). These reactions manifest themselves by local symptoms very similar to those observed in tuberculosis (see p. $58: 3$, and Fig. 124). However, the small number of observations with regard to these tests which have thus far been reported constitute a very unsatisfactory basis upon which to form an opinion in regard to the value of these tests.

According to Vallée, the eutaneons test is unreliable because the thin and sensitive skin of healthy horses may react with equal facility and because the difference between reactions in healthy and infected horses is merely a matter of degree; Martel and Putzey \& Stiennon also expressed themselves unfarorably, but Schniirer considers the test as of diagnostic value. But even the last named autlor adnits that it is probably less accurate than the ophthalmic test. According to his observations the intradermal test is more satisfactory, the latter possibly leing of value also in standardizing the mallein, since the eilematous swellings ileveloping at the point of inoculation are usually in direct proportion to the amount of mallein injected.

Technic of the Cutaneous Mallein Test. The skin is carefully washed and then searifier until slight bleerling occurs, whereupon concentrated mallein is applied to the field of operation. Schnürer recommenils washing an area $10 \mathrm{~cm}$. by $5 \mathrm{~cm}$. at the side of the neck and then slightly scarifying three areas, thus \# \# \#. The first and third areas are treated with mallein, the middle one serring as a control for the tranmatic reaction.

The reaction begins at about the 6 th hour and increases in extent during the following 24 hours, whereupon it gradually disappears with the sulssidence of the inflamed area. The degree of the reaction is determined by the extent of the swelling $(15-50 \times 20-55 \mathrm{~mm}$. $)$ and the degree of its thickness (1-2 cm.). Application of the mallein to the scarified skin by rubbing with a rough eloth does not give so satisfactory results.

Serum Diagnosis. Under the influence of the toxin of the bacillus of glanders, as antigen, other antigen bodies besides immune substances are formed as prorlucts of reaction in the infected animal body. These may be demonstrated by suitable methods as agglutinins, precipitins and complement-fixing substances. Since these reaction bodies possess an affinity for their own antigen only (glanders toxin) and consequently are strictly specific in their nature, their presence in the blood of an animal justifies the conclusion that the animal in question is infected with glanders.

This constitutes the basis of a number of diagnostic methods all of which are conducted with the blood serum of the animal to be tested and the use of cultures of the bacilli of glanclers 
or of their extracts. None of these methods are infallible. They may, however, be supplemented so that doubtful results given by one are rectified by a positive result obtained from another method, thus the simultaneous application of several methods will reduce the errors to a very small percentage.

Since the methods of sermm diagnosis are all laboratory methorls, it is necessary for the practitioner to senil suspected material, properly preserved, to a suitable laboratory for examination. For this purpose from 30 to 50 cem. of blool are drawn from the jugular vein (technic as in ordinary phlebotomy) into a sterilized test tule. After supplying the tube with a cork to prevent infection, it is kept in a cool place for a few hours to permit separation of the serum from the clot, whereupon it may be sent to the laboratory for examination. As a rule, results may be reported within one or two days.

Since the results obtained in serum diagnosis may be influenced by previous subeutaneous malleinization, horses from which blood is obtained for this purpose must not be tested with mallein until after the withdrawal of the serum.

1. Complement Fixation Method. The method of Bordet \& Gengou, inaugurated in 1901, and adapted by Wasserman \& Bruck for the practical diagnosis of syphilis in particular, is based upon the demonstration of antibodies in the blood serum. These antiborlies, with the aid of their antigen, have the power of fixing the complements of fresh serum and thus divert them from hemolytic systems. This method which was worked out by Schütz \& Schubert for the diagnosis of glanders has alrearly given results of such an exact nature that it may be looked upon, at this time, as the best sero-diagnostic method for the determination of this disease.

If an experiment animal is repeatedly treated with red blood corpuscles from another species, the serum of the former develops the property of dissolving* the red blood corpuseles of the latter within a short time, even in very dilute solution. By heating for 30 minutes at $56^{\circ}$ to $58^{\circ} \mathrm{C}$. this property is destroyed. However, if fresh serum from any animal, not previously treated as above described, is added to a mixture of serum thus incubated (inactivated) plus red corpuseles, a solution of the erythrocytes (hemolysis) again takes place. This phenomenon therefore occurs as the result of the action of two substances: (1) A thermostabile substance which is found only in the serum of an animal previously treated with the blood corpuseles from another species, and which develops as a reaction product, or antibody for red blood corpuscles, i. e., as an antigen. (2) A thermolabile substance which is present in the fresh serum of any animal and which is designated as complement or alexin. According to Ehrlich's theory this process may be explained by supposing that an antibody possesses two affinities or haptophore groups (see p. 447) (hence the name: hemolytic amboceptor or mediating body); one of these combines with the red blood corpuseles as antigen, the other with the complement. The complement ean not combine directly with the antigen, but if anchored to it by means of the amboceptor it will act as a sort of digestive or proteolytic ferment and dissolve the red blood corpuscles. (According to Bordet's theory the red corpuscles are sensitized for the alexin by the antibody in a manner similar to the action of mordants in the dyeing of cloth, for which reason he designates the antibody as

*NOTE:-Strictly speaking there is no complete solution of the red blood corpuscles but only an action of the hemolysin on their stroma which permits the escape of the hemoglobin. 
substance sensibilisatrice or fixateur). Red blood corpuscles themolytic amboceptor + complement form a hemolytic system!

The hemolytic amboceptor is strictly specific, consequently it will combine by means of its eytophile group with its own antibody only (red blood corpuscles). On the other hand, the complement, which is present in all fresh serum, is not specific, hence it may be fixed by various amboceptors in suitable complementophile groups. Thus the complement will fix specific immune bodies that develop in the tissues of infected animals under the action of bacteria; these immune amboceptors attract the complement to the bacteria in question or to the dissolved elements of their substance. Hence, if we add fresh serum which contains the complement in suitable quantity, to a mixture of a suspension of bacteria or a bacterial extract+immune serum, the complement will unite with the amboceptor of the immune serum so that the free complement disappears from the fluids. Now, if we subserpuently add to this fluid a mixture of hemolytic amboceptor+red blood corpuscles hemolysis will not result, because the necessary complement has already been fixed by the immune amboceptors and the free complement no longer exists. In this ease, therefore, a fixation of the complement by means of an immune amboceptor has occurred; in other words the complement has been diverted from the hemolytic system. On the other hand if immune amboceptors were not present in the first mixture, the complement remains free and can therefore exert its corpuscle-dissolving properties on the subsequently formed hemolytic system. These two cases may be expressed graphically as follows :

1. Bacteria+Immune-Serum+Complement+Hemolytic Amboceptor+Red Blood Corpuscles (Antigen) (Immune Amboceptor)

$=$ No Hemolysis (Complement Fixation)

2. Bacteria+Normal Serum+Complement + Hemolytir Anboceptor+Red Blood Corpuseles (Antigen) (No Immune

Amboceptor)

$=$ Hemolysis (No Complement Fixation)

Consequently if hemolysis fails to occur, i. e., if the complement is fixed, we may conclude that the fluid contains an immune amboceptor; since, however, the latter can fix complement only with the aid of its antigen and since the antigen (bacterial suspension or extract) is known, the further conclusion is justified that the fluid contains an immune amboceptor which corresponds with this antigen and thus originates from an animal which is infected with the bacteria in question. For example, if glanders bacilli were used as antigen the complement fixation which followed indicates that the serum possessing the fixing property originated from a glanderous horse. Under opposite conditions, for instance, if hemolysis is not prevented, the conclusion is justified that the horse which furnished the serum is not affected with glanders.

According to the observations of Schütz \& Schubert, Miessner \& Trapp, the authors and others, the complement fixation method for the diagnosis of glanders has already proved itself very valuable. Its advantage consists in that the relative amount of immune amboceptor in the serum to be tested can be determined quantitatively and that the result of the test is very evident. If, for instance, earefully and progressively graduated quantities of serum are used with suitabic amounts of hemolytic amboceptor and complement it is possible to de- 
Plate VII.

Strongly positive Reaction.

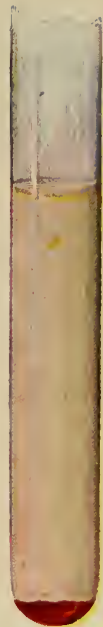

Horse serum 0,2 Hemolysis 0

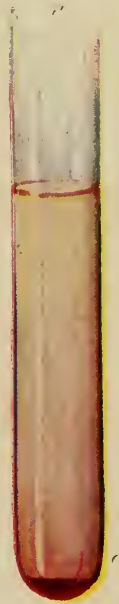

Horse serum 0,2 Hemolysis +

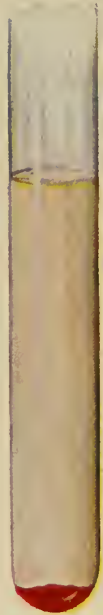

0,1

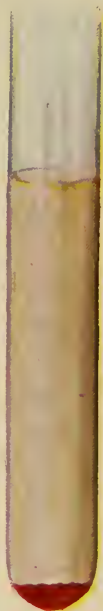

0,05

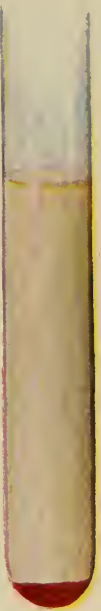

0,02
Doubtful Reaction.

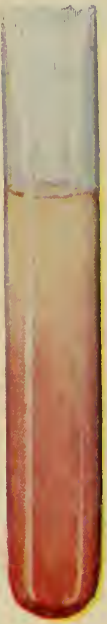

0,01
++

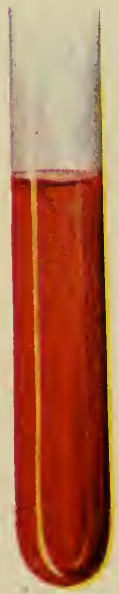

0,01

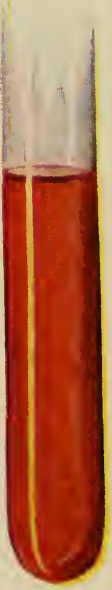

0,02
++++

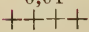

Complement fixation in Glanders. 

termine the smallest quantity of serum that will prevent hemolysis. The result is shown by the contrast between the clear serum following complement fixation and the distinctly red serum containing the hemoglobin in solution. Although errors may occur in this method of diagnosis in those exceptional-instances in which the serum of horses affected with chronic glanders will not fix the complement and in the still rarer cases in which an apparent specific fixation occurs in healthy horses, these errors are considerably less frequent than those obtained by other sero-diagnostic methods.

According to the official regulations of Prussia, which prescribe that the serum of horses which will fix complement in maximum quantities of 0.1 c.em. in the prescribed method of procelure (see below) are regarded as affected with glanders. Basing their work upon this standard, the results of tests made by Miessner \& Trapp in the examination of 549 healthy horses showed that the serum of only 7 $(1.27 \%$ ) showed a complement fixation power equal to $0.1-0.01$, while on the other hand the sera of 69 glandered horses showed a fixing power of 0.2 in two cases and a fixing power exceeding 0.2 in five cases. In one experimentally infected horse the fixing power, as well as the agglutination, began to decline on the sixth day, but in horses infected naturally with glanders the fixing power was retained for a longer time; previous malleinization also tended to increase the fixing power temporarily. Haan, de Bliek, and Valenti, as well as Klimmer \& Kiessig, also report favorably on the results obtained by this method, while Keyser found this method applicable to eadavers also. Sera of horses affected with other diseases gave no positive reaction.

Technic of the Complement Fixation Method. This consists essentially in mixing suitable amounts of glanders-bacillus-antigen and fresh guinea pig serum containing complement with the inactivated serum to be tested and then incubating for one hour, in order to hasten fixation. To this mixture are then added the inactivated hemolytic serum and the corresponding red blood corpuseles. This two-fold mixture, after again being incubated for two hours, is ready for the determination of results. If complement fixation has been complete all of the red blood corpuscles will have settled to the bottom of the test tube, while the supernatant slightly yellow fluid is perfectly clear. On the other hand, if hemolysis was complete all blood corpuscles will have been dissolved, so that the supernatant fluid, which is clear also in this ease, is of an even red or port wine color. Transition stages in which only a part of the red blood corpuscles are dissolved, may be observed between these two extremes, in which case the fluid is only slightly reddened, or reddened only in the lower part of the test tube, while a certain number of intact blood corpuscles, forming a correspondingly smaller mound, will have collected on the bottom of the vessel. For the sake of convenience the different degrees of hemolysis are referred to as 0 , trace, incomplete, nearly complete, and complete.

This method was first applied practically to the diagnosis of glanders by Schïtz \& Schubert, but subsequently perfected by Miessner \& Trapp. According to these investigators the test is conducted with the following reagents:

(a) The antigen usually consists of a normal saline solution of sterilized shake-extract, or of a suspension of dead glanders bacilli; antiformin extracts or mallein are less suitable for this purpose, although in some cases the authors obtained more pronounced results with these than with the first named antigens.

(b) A series of successively decreasing amounts of the serum to be tested, which must be inactivated by incubating for 30 minutes at $56^{\circ}$ to $60^{\circ} \mathrm{C}$.

(c) Fresh guinea pig serum, eontaining complement in the smallest amount (usually 0.03-0.04 c. cm.), in which it will produce complete hemolysis in a hemolytic system.

(d) Hemolytic inactivated rabbit serum (from rabbits previously treated with sheep blood corpuseles), in double the amount required to produce hemolysis.

(e) Sheep blood corpuscles, $5 \%$ suspension in physiological salt solution.

Reagents of which only small quantities are needed should be standardized by the addition of normal salt solution, so that equal volumes of each reagent enter into the mixture. By providing suitable control tubes we are enabled to determine that neither the antigen nor the serum under test have alone been the cause of the complement fixation, and also that the hemolytic system was properly formed. The successive steps in this test are illustrated graphically in the tables on page 720 . 


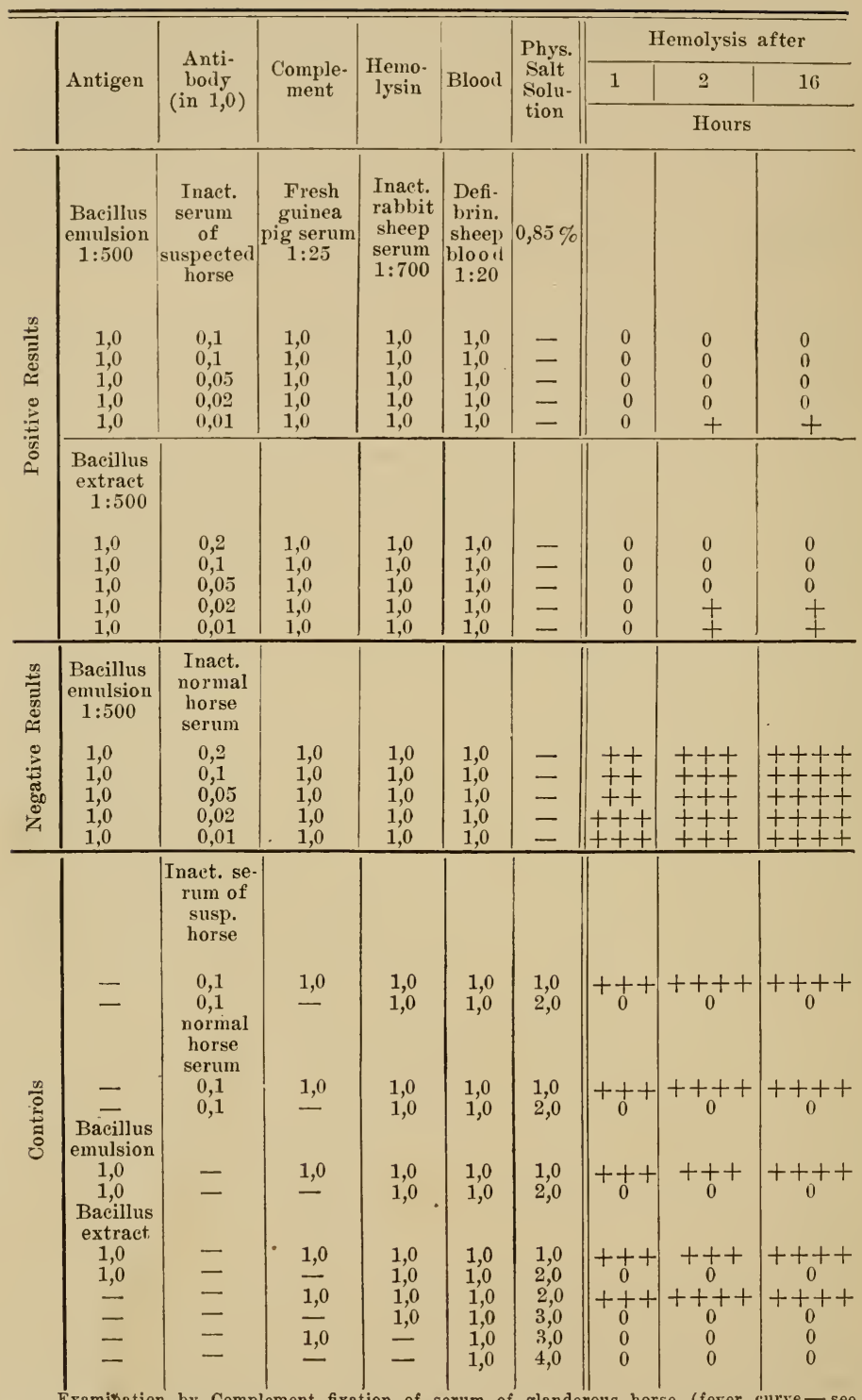

Examikation by Complement fixation of serum of glanderous horse (fever curve - see p. 702; Exam. Jan. 17).

Hemolysis: $0=$ no hemolysis, $t=$ trace, $++=$ incomplete, $+++=$ almost complete, $+++t=$ complete. 
2. Agglutination Test. This is based upon the fact that blood serum which contains agglutinating substances will cause the clumping of glanders bacilli in suspensions of potato or agar cultures, whereupon these clumps are precipitated to the bottom of the supernatant clear fluid.

The agglutinating action of blood serum was first observed by Dedjulin in human beings affected with glanders, by MacFadyean in horses affected with this disease. Subsequent investigations, especially those of Schütz and his co-workers, showed that the serum of healthy horses may also contain (normal) agglutinins in rather large quantities, but that, nevertheless, the reaction is specific in the sense that agglutination is brought about by the serum of infected horses in considerably greater dilutions than is the case with sermun of healthy horses. The following general rule has been found quite applicable and reliable, viz., agglutination, if appearing in dilutions of $1: 400$ or less, with few exceptions, indicates freedom from infection; on the other hand dilutions of 1:1000 or more, also with few exceptions, indicates the presence of infection, while agglutination in dilutions of $1: 2000$ or more indicates recent infection. However, in infected stables we usually find a rather considerable number of horses the serum of which will produce the agglutinating phenomenon in dilutions of $1: 500-800$; a titer of this kind has no diagnostic value in either direction. This fact which presents a considerable disadvantage to the practical application of this method of diagnosis is compensated for in a certain number of cases by the discovery that the agglutination titer of glanderous horses undergoes very considerable fluctuation from month to month, thus it may be very high (2000-4000 or more) a few days after infection and then grarlually drop, while exacerbations of the morbid process may again cause a pronounced upward tendency.

The practical value of the agglutination test is considerably reduced by the necessity, in a considerable number of suspected horses, of repeated serum tests at intervals of several weeks. Since healthy horses also occasionally show physiological fluctuations in agglutinating power of no inconsiderable degree, and since on the other hand these fluctuations can no longer be recognized in horses with chronic glanders, the practical value of this test must not be ranked too high and by no means above that of the subcutaneous mallein test. Nevertheless by giving due consideration to the possible negative results, careful attention to technic, and especially supplementation of other methods, particularly the complement fixation method, this test constitutes a valuable addition to our diagnostic methods (see p. 717), and may be of much value in doubtful cases. In recent infections it is quite reliable in itself.

The agglutination test was first recommendei for practical use by Bourges \& Méry, Pokschischewsky, Árpád, Rabieaux and particularly by Jeusen. The last named author ranks it above the mallein test because post-mortem examinations were Vol. 1-46 
negative in horses which gave positive or doubtful reactions to mallein, but the blood serum of which did not distinctly agglutinate glanders bacilli. Subsequent experiments showed that sera which agglutinated the typhoid bacillus, the Bacillus suipestifer, or the colibacillus, would not affect the bacillus of glanders.

In the experiments of Schïtz \& Meissner the agglutination titer of the sera of 1,911 horses which were free from glanders was 1:100-400 in 1,602 horses $(83.8 \%), 1: 500-800$ in 299 horses $(15.7 \%)$ and $1: 1000$ in 10 horses $(0.5 \%)$; on the other hand, among 298 glanderous horses the titer was $1: 400$ in $6(2.0 \%)$, 1:500-800 in 103 horses and $1: 1000$ in only 189 horses (63.4\%). If we regard titers of $1: 400$ as decisively negative and 1:1000 or more as decisively positive evidence of infection, the errors in diagnosis in these cases were only $0.77 \%$. Howerer, in $18.2 \%$ of all cases and in $34.6 \%$ of the infected horses that gave agglutination titers of 1:500-800 the diagnosis harl to be held in abeyance.

Schnürer places a similar value on the results of the agglutination test, although according to his opinion the average amount of normal agglutinins fluctuates between 400 and 600 . However this investigator, whose observations extend over 300 cases, recommends this method strongly for practical use, as do also Benome, Wlatlimiroff, Ferlorowsky, Langer, Moore, Taylor \& Giltner, Berns \& Way, etc., while Foulerton, Preusse, Stanciu and Riemer express themselves less favorably. The last named author observed agglutination powers of 1:500, 1:700 and $1: 1000$ in glanderous horses, while the titers in six healthy horses were $1: 900$ (in four cases), $1: 2000$ and 1:4000, respectively. In pleuro-pneumonia (Miessner) as well as in distemper (Sustmann) the serum seems sometimes to possess greater agglutinating power, it has also in the course of a long period of observation been found to be more uniform, while other diseases do not influence the agglutination titer (Langer).

After an experimental infection, the agglutinating power may rise to 1:2000 or more in 2 days (Bonome, Hutyra; according to Sustmann in 3 days; accorling to Schütz \& Miessner in 6 days) remaining at this point about 4 weeks, whereupon it gradually returns to the normal.

Subcutaneous malleinization raises the agglutination titer in healthy as well as in infected horses. Pekschischewsky saw the titer double in the course of a mallein reaction, and according to Pfeiler it sometimes reaches 4,000 in these eases, while Meissner as well as Fedlorowsky, Kleine and Bonome observed only a moderate but long continued increase of titer. Schïtz \& Miessner failed to observe any change in three horses. Árpád observed the titer increase in two days from 300 to 1,600 in healthy horses, but it dropped again to 800 in 7 days, while according to Miessner the increase does not begin until the 5 th or 7 th day, reaching its maximum in 14 days and not beginning to gracually return to the normal until after 4-6 days. According to Sustmann the elevation continued for more than 3 months.

Practical observations of Nevermann include statistics on 3,466 horses which were tested in the course of two years in several hunilred Prussian stables or studs. According to these statistics the temporary diagnosis based upon the agglutination test was confirmed by post-mortem examination in $79.2 \%$ of the negative results (titer $300-400$ ) and in $79.5 \%$ of the positive results (titer 1,000 or more, with fluctuations). Of $18.2 \%$ of the horses destroyed on the ground of suspicion (giving titers of $500-800$ ) lesions of the disease were present in $46.6 \%$. In more than one-half of the stables the test for some of the animals had to be repeated from 3 to 6 times.

Technic of the Agglutination Method. The test fluid, is prepared with a suspension of agar or potato culture of 2 to 5 days' growth prepared from a suitable strain of glanders bacilli and diluted with physiological salt solution. After killing the bacilli by heating at $60^{\circ} \mathrm{C}$. the test fluid is standardized by adding the necessary amount of normal salt solution. For each 2.0 ce. of this fluid in test tubes the serum to be tested is added in successively decreasing amounts (from $1: 300$ to 1:4000) whereupon the tubes are incubated for 36 hours. By keeping the fluid in the thermostat for one-half hour and subsequently centrifuging for 5 to 15 minutes (the time being determined by the number of revolutions), the result may be ohtained within one to two hours. positive reactions are indicated by the accumulation of agglutinated bacilli on the bottom of the tubes in the form of a circular film with serrated border, while the supernatant fluid has become perfectly clear. On the other hand a negative reaction is indicated by the cloudiness of the fluid, while the precipitated but not agglutinated bacilli have accumnlated on the bottom of the test tube in the form of a flat lise with a smooth outline.

King \& Houghton have worked out a method by means of which the test can easily be made in actual practice. Carefully measured amounts of properly prepared test fluid are placed in graduated test tubes whereupon square pieces of filter paper 
of varying but definite size, that have been saturated with serum, are placed in the fluit. In this manner varying dilutions of serum are produced in the test fluid, which is then incubated for 24 to 48 hours or allowed to stand near a warm stove. The agglutination titers may then be determined by comparing the results in the tubes with a properly tabulated guide.

3. Precipitin Reaction. In this phenomenon the serum of an immunized animal produces a fine flaky precipitate in the culture filtrate of the organism used in immunization. It was first observed by Kraus, subsequently by Wladimiroff, who noted that it occurred also in filtrates of glanders cultures after the addition of serum from glanderous horses. The latter's observation was confirmed by Pfeiler, Miessner and Müller, although the investigations of the latter showed that the sera of healthy horses also produced precipitates (normal precipitins) but in a less degree, thus causing more or less confusion in diagnosis. On the other hand they obtained better results when they used bacillary extracts or mallein solutions instead of culture filtrates and instead of mixing these with the serum gently pouring them on the surface of the same (contact or ring test). By this latter method a distinct gray band of precipitate which may be regarded as quite specific, will form within a few minutes at the line of contact of the two fluids. According to observations thus far recorded this method is giving satisfactory results in most instances while the fact that precipitins can be demonstrated also in chronic cases of disease presents an additional advantage.

Pfeiler, who uses extract of glanclers bacilli prepared with carbol-sodiumchloride solution or carbolized horse serum as a precipitinogenic fluid, tested 452 blood samples obtained from 306 horses suspecter of the disease or exposure thereto. He compared his results with those obtained by the agglutimation and complement fixation tests and found that they confirmed each other, particularly in recent cases of infection. In experimentally infected horses the precipitins could be demoustrated within 4 or 5 days after infection. Similarly favorable results were obtained by Miessner in 18 glanderous horses by the use of extract of glanders bacilli and $10 \%$ mallein solution made with Foth's dry mallein. In very old cases only were the results from this method less satisfactory. Finally, Mïller was able to determine the specificity of the reaction in his guinea pig experiments.

Other Diagnostic Methods. In former times the following were frequently resorted to: The inoculation of a suspected horse with its own secretions, a healthy portion of the boly being selected to reproduce the characteristic, glanderous lesions; trephination of the antrum of Highmore, in order to permit direct inspection of its mucous membrane; the artificial prorluction of a fever or of the acute form of the disease by subjecting the suspected animal to hard work or by the subentaneous injection of oil of turpentine (Cagny) or by the injection of streptococcus cultures (Jewsejenko). In any of these methods only a positive result had any value, and all of them have long been superseded by modern methods of diagnosis.

In more recent times Baldoni and Dieckerhoff recommended the intravenous injection of Credé's colloidal silver, which produces a febrile reaction in glanderous horses and consequently might be of service in the diagnosis of doubtful cases. Röder, Passau and Blome have also observed this action, although Poetschke and subsequently also Röder observed that a similar reaction occurred occasionally in the course of other internal and external diseases and even in healthy horses.

Carrozo recommends Ehrlich's diozoreaction (red coloration of the urine after the addition of hydrochloric sulphanilic acid, potassium iodide and ammonia), this reaction being much more pronounced in the urine of glanderous horses than in 
that of horses affected with other infectious diseases. The ralue of this method has not as ret been confirmed by others.

Fursenko tested the blood of 25 horses for opsonins and obtained 23 positive and two doubtful reactions. The results were better than those obtained with mallein or with the agglutination test.

Differential Diagnosis. From a differential diagnostic point of view the following diseases should be considered in connection with nasal glanders; chronic nasal catarrh may arouse suspicion of glanders when the nasal discharge persists for a long time and there is enlargement of the submaxillary glands; in this disease, however, the nasal discharge is always bilateral, the tumefied lobules of the submaxillary gland may be distinctly palpated, the nasal mucous membrane usually contains no nodules, catarrhal ulcers, which may be present, never exceed the size of lentils, are circular and regular in outline, have sharp borders and smooth, bright red bases, while proper treatment results in recovery. Although occlusion of the afferent ducts of the mucous glands in the course of simple catarrh may cause the formation of nodules as large as lentils, these persist for a long time without ulcerative degeneration.Croupous inflammation of the nasal mucous membrane is attended by acute symptoms, the nasal mncons membrane is much tumefied, highly reddened and appear's to be covered with extensive membranous masses, the hright red mucous membrane beneath bleeding at the slightest tonch; the submaxillary glands which are usually swollen show increased heat and pain.Rhinitis chronica proliferans, and so called rhinoscleroma are confined to the lower portions of the nasal cavity; in these conditions cicatricial induration of the mucous membrane as well as catarrhal ulcers mar he present, while the mucons memhrane above as well as that of the turbinated bones is normal. The submaxillary glands do not show the characteristic firm enlargement.- Ulicers and scars of traumatic origin are usually found near the nasal fossa on the septum; the ulcers have a red actively granulating base, the scars usually being elongated, or angular, flat, rarely bulging in character; the lymph glands show nothing abnormal.-Cauterization of the nasal mucous membrane with lime may produce extensive ulceration but the submaxillary glands are not involved.

Petropawlowski called attention to the fact that mineral- and coal-dust may produce an ulcerous inflammation of the nasal mucous membrane and Jewensko observed a severe inflammation of the nasal mucous membrane in the horses of a regiment of dragoons in Ismail, after feeding with dusty fodder, as a result of which 200 horses were destroyed on account of suspected glanders!

Very obstinate catarrhal conditions may also be produced by pentastoma and by gastruslarvae. In old horses chondromalasia may be confused with glanders. In this disease the nasal septum may contain ulcers with pronounced ragged horders and nodules of varinis sizes while the cartilage contains cavities filled with a glassy, mucous mass (Petropawlowski). 
In some districts injuries of the nasal mucous membrane and from them cicatrices which have been caused by quacks in an effort of treatment, are very frequent occurrences. Cicatrization may also occur after fractures of the nasal bones.

Chronic catarrh of the nasal sinuses often gives rise to suspicion of glanders, the nasal discharge in this disease frequently being unilateral and otherwise very much like that in glanders while the submaxillary lymph glands are usually also enlarged. However, the sensitive condition of the infraorbital nerve, the temporary, profuse nasal discharge, the fluctuating results obtained in percussion of the sinus, the soft and lobulated structure of, and the absence of adhesion in, the submaxillary glands as well as the absence of nodules and ulcers on the nasal mucous membrane, are usually sufficient to enable one to recognize the nature of the affection. In addition to this the results of trephination which would be therapeutically indicated in this case would decide the character of the affection, as would also the determination of any special cause of the trouble (tooth caries, bone necrosis, neoplasms, etc.). In chronic catarrh of the guttural pouches also there may be milateral nasal discharge, the origin of this, however, is at once indicated by the tumefaction in the subparotideal region.-Distemper or strangles might be confused with glanders but only in affections of a subacute character in which the intermaxillary abscess appears late in the development of the disease or fails to make its appearance, or in eases where the lymph vessels of the surrounding region become enlarged, and finally also when metastatic abscesses appear in remote regions of the body; but even in this case the morbid changes present a more acute character than those of glander's. There are no nodules on the mucons membrane while the interior of the swellings or abscesses contain only streptococci (ulceration of the mucons membrane does not necessarily exclude distemper since this may also be brought about by the streptococcus of strangles [Rabe]. See p. 373).- Stomatitis pustulosa contagiosa may excite suspicion of glanders when the lips and nasal alae become swollen and norlules and ulcers are present on the nasal mucous membrane; aside, however, from the acute and favorable course of the process the existence of similar changes on the oral mucous membrane in itself would exclude glanders.Finally ulceration of the nasal mucous membrane occur's also in the course of tuberculosis (see p. 550) as well as in epizootic lymphangioitis (see p. 737). In these cases chronic enlargement of the submaxillary glands may also be present (this, however, may also occur in actinomycosis), consequently a microscopical examination of the extirpated tumors or tissue portions alone would be confirmatory.

The following conditions may be confused with cutaneous glanders: Septic lymphangioitis, which, however, is accom- 
panied by continuous fever, extreme pain, a conspicuous disturbance of function, in addition to which the abscesses after perforation heal readily by active gramulation.-Botryomycosis, in those cases where subcutaneous tumors undergo suppuration attended with edematous infiltration of the surrounding tissue; in these cases there is also active granulation and the efferent lymph vessels are not involved.-In ulcerous lympliangioitis the cutaneous ulcers are very similar to those of glanders, but the regional lymph glands are not enlarged, a short bacillus which takes Gram's stain is present in the ulcerous secretions, while in epizootic lymphangioitis the presence of coccus-like structures which do not readily stain confirms the diagnosis; furthermore, no reactions follow the injection of mallein in this disease.-Urticaria is characterized by its sudden appearance and the equally sudden subsidence of the cutaneous swellings.-Contagious acne is characterized by pustule formation and the rapid, complete healing of the smail ulcers.-In morbus maculosus we have extensive swellings with abrupt contour. Cutaneous nodes caused by nematodes are characterized by their permanence and their hemorrhagic character.

Chronic inflammatory affections of the lungs in general and pursiness resulting therefrom lave so much in common with pulmonary glanders in their general symptoms, that their presence can never be definitely excluded in suspicious cases. Since these conditions are frequently attended by poor general nutrition the mallein test (and in protracted cases serodiagnostic methods as well) may not enable us to arrive at a definite diagnosis. It is, therefore, necessary that animals thus affected be kept under careful observation for some time so that any characteristic symptoms of glanders may be discovered at once. In this respect torpid edematous swellings, bloody bronchial secretions as well as cachectic symptoms in general demand consideration.

As far as the pathologico-anatomical diagnosis is concerned the following morbid processes are of particular importance: Peribronchitis nodosa multiplex (Dieckerhoff) s. Nodosis pulmonum; this is characterizel by uniformly gray, firm, frequently calcified nodules ranging in size from a poppy seed to that of a lentil, the surrounding pulmonary tissue slowing no evilence of reactive inflammation; the peribronchial lymph glands also are intact in this affection.-Peribronchitis diffusa and Pneumonia interstitialis chronica: the walls of the bronchial tubes are uniformly thickened, the inter-alveolar connective tissue is uniformly indurated in large surrounding areas; norlules are present and there is no gelatinous infiltration of the tissue nor enlargement of the lymph glands.- In pneumonia catarrhalis of roung horses the hepatized pulmonary areas are miformly red or grayish-red and without soft centers, although there is acute swelling of the peribronchial lymph glands they contain no caseous foei.-In tuberculosis we find as a rule in addition to gray hyaline, fresh nodules as large as poppy seeds, large sarcoma-like nodes as well as broncho-pneumonic foci, while the thoracic lymph glands are caseated.Norlules causerl by animal parasites (nematodes or their embryonic forms anil echinococei) are very similar to glanilers nodules, but all of them, even the smallest, are enclosed by a delicate capsule, in addition to which their peripheral layer is frequently livaline, while calcimm salts have been deposited in their centers; the capsule surrounding even the larger nodes is much more delicate than those enclos- 
Ing the cheesy nodules of glanders; the diagnosis can, however, only be confirmed definitely by microscopical examination (numerous eosinophile cells in parasitic nodules!). - In botryomycosis and in pneumonomycosis the morbid changes are generally more chronic in character, while the presence of botryomyces or mycelia and other fungi is readily determined by the microscope--Metastatic pus foci are freqnently very much like embolic nodules of glanders in their general appearance, especially those of acute glanders, but the absence of more advanced glanderous changes in the lumgs, as well as in other internal organs, is against their glanderons nature (pus bacteria may also be present in broken-down glanderous foci!). Metastases of new formations (sarcomata, carcinomata) may be recognized, aside from their characteristic macroscopical appearance on section, by the presence of primary tumors or by histological examination.

Finally, suspicion of glanders may be aroused in those instances in which new formations (rhinoseleromata, sarcomata, carcinomata, fibrous polyps, etc.) occur on the nasal mucous membrane or when metastases of malignant tumors, particularly melanomata, give rise to chronic enlargement of the lymph glands in different regions of the body (Bissonge and Feiler observed the submaxillary glands thus affected). In these cases an exact diagnosis is possible only by careful consideration of the anamnesis or by means of a histological examination of portions of the tumors. Observations of the past have shown that the mallein test gives negative results in these cases.

Treatment. Since, according to generally accepted principles of sanitary police control evidently glanderons horses must be destroyed at once, successful treatment of this disease is of no practical consequence. But this is true irrespective of this fact since all the many attempts to cure the disease that have been made in the past have proved failures. The internal administration of the most varied medicinal agents has been found just as ineffective as the more recently recommended intratracheal injections of Lugol's solution (Delamotte, Trinchera), even in human medicine favorable results have been obtained only now and then by the repeated cutaneous application of gray mercurial ointment.

The appearance of local reactions following the injection of mallein has given rise to the belief that repeated injections of this kind would have a healing influence on the glanderous process. Although the healing of ulcers and even definite recovery from the disease has been observed now and then to follow such treatment (Johne, Helman, Semmer, Pilavios, Choromansky, Sitschew) the possibility of spontaneous recovery in these cases could not be definitely excluded; even if we admitted the possibility, this mode of treatment would hardly be of practical value.

Immunization. Recovery from this disease does not protect against subsequent infection, at least not as far as experimental observations on dogs treated with virulent material are concerned (Charrin, Galtier). Experiments made with repeated mallein injections (MacFadyean, Schindelka) also gave no satisfactory results.

After Sadowsky had suceeded by the administration of eultures killed at $62^{\circ} \mathrm{C}$., in increasing the resisting power of a colt to such 
an extent that the inoculation of virulent cultures produced no harmful effect, Levy, Blumenthal \& Marxer obtained happy results in horses by the subeutaneous administration of $600 \mathrm{mg}$. or two successive injections of $300 \mathrm{mg}$. of bacilli that had been. killed in $80 \%$ glycerin or in $10 \%$ urea solution. Subsequently they prepared an immunizing powder from cultures treated in the manner mentioned and which they named "farase." This is applied in doses of $0.4 \mathrm{~g}$. followed in three weeks by $0.8 \mathrm{~g}$. injected subcutaneously. This substance which keeps well even at high temperatures is said to be absolutely harmless, treatment therewith not seriously affecting the general condition of the animal and producing an immunity of at least one year's duration.

According to Malzew, eattle serum in doses of 250 to 420 ce. injected subcutaneously protects horses against the action of virulent cultures of glanders. Nocari, Aruch \& Petrini, as well as Prettner, found that even the serum of cattle which had been injected repeatedly with virulent cultures was ineffective, and Galtier \& Nicolas observed that treatment with serum of this kind after experimental inoculation would at most prolong the course of the disease.

Veterinary Sanitary Police. The live stock sanitary laws of all civilized countries include glanders among those diseases which must be officially reported, and provide also for regulations requiring the immediate destruction of diseased and the quarantine of suspected and exposed animals, including thorough disinfection of infected premises. Public traffic with horses is subjected to careful supervision for the purpose of the prompt detection of new outbreaks. The strict enforcement of these regulations, especially in those localities where adequate compensation is provided for condemned animals, has resulted in a marked decrease in this disease, which is strictly contagious in its nature.

In recent years the systematic application of mallein tests and of serodiagnostic methods has added much to the success in the control and eradication of glanders; while formerly the presence of doubtful symptoms or the absence of pronouncer lesions merely pointed to suspicion of disease and consequently involved the necessity of a long period of observation while the animal was held in quarantine, modern methods of diagnosis make it possible to decide the nature of the malady at once, or within a very short time, and thus make possible the immediate isolation (or destruction) of dangerous individuals. These methods also enable us to remove at once such horses from infected stables as are healthy in general appearance, but yet may have been the cause of the dissemination of the disease. By isolation and further observation of such animals the healthy horses are protected against possible infection. This makes it possible, also, to dispense with many quarantine regulations that were formerly necessary. The advantages thus afforded to the owner of horses and to horse traffic in general is evident.

The application of the mallein tests and of serodiagnostic methods is recommended in all eases where individual horses show symptoms 
pointing to possible infection with glanders, or in such cases where the remaining horses in the stable are under suspicion of exposure. While differences of opinion still exist in regard to the value of allergic reactions and serum tests, nevertheless observations thus far recorded show that the exact and intelligent application of these methods produce very favorable results and that the supplemental use of several of these methods enables us to reduce errors to a minimum. While the newer methods and particularly the serum tests make the duties of the practicing veterinarian less burdensome, clinical examinations nevertheless are still of great importance and quite indispensable, especially for the reason that these tests will occasionally give negative results in very chronic and extensive (and consequently the most dangerous) cases.

Since 1910 the complement fixation method and the agglutination test iave been user in Prussia exclusively as official tests. All animals with a fixation titer of 0.2 or less, or with agglutination power of over 1,000 nust be destroyed; all horses with a fixation titer above 0.2 or an agglutination power below 1,000 are regarded as free from suspicion, provided that the examination is marle at least two weeks after the animal in question has been removed from exposure. From 1906-7 to $1907-8,648$ animals were destroyed on evidence furnished by the agglutination test; of these $395(60.9 \%)$ were found affecterl with glanders. The success of the method consisted in the fact that after completion of the blood test (which, however, had to be repeated in a number of cases) not a single glanderous animal was found in any of the stables investigated.- In Hungary all aninials which give typical, atypical or doubtful reactions to the subeutaneous mallein test and show clinical symptoms of disease, are destroyed at once. Horses that react to the test, but are otherwise in apparent health, must be isolaterl from other solidungula, but may with the consent of the proper authority be used under certain restrictions; thus for farm work, ete., contact with other horses must be avoided. These animals must be examiner every two weeks by a veterinarian, and in the event that any suspicions symptons of glanders make their appearance, the animal must be destroyed at once. Those animals which have reacted to the mallein test but appear otherwise in perfect health nay be used by their owners withont restrictions, except that none of these animals that have been in immediate contact with diseased horses may be sold outside of a parish before the expiration of 60 days nor without a second examination by a vetermarian. Exposed animals which have reacterl to the test are retested after 30 days; those animals that do not react at the second test and otherwise appear in perfect health are released from the restrictions, whilo the reactors are treated as above indicated (destroyed or continued in quarantine and again tested as the authority in charge may designate for each case).

Under these regulations (1902-1908) 1,385 suspected glanderous horses anit 1,609 horses exposed to glanders (a total of 2,994 actually glanderous horses, all of which were affected with glanders in the first stage of the disease) gave positive reactions to the mallein test and were quarantined ( $60.1 \%$ of all glanderous horses destroyed during this period). A similar method was followed in the extermination of glanders in the Compagnie générale des Toitures of Paris without serious interruption of the business of the company; while the disease was very prevalent in the stables of this company in 1895 and 1896 (see page 680), only 101, 40 and S5 horses, respectively, had to be destroyed on account of disease in 1897, 1898 and 1899. In 1903 only 18 reacting horses were found in all of the stables.

During the time intervening between the mallein tests and that of procuring the blood for serum tests the body temperature of those horses suspected of infection or disease should he recorded systematically twice daily. An elevation of temperature continuing for several days points to recent infection unless it can be ascribed to some other cause (see pp. 690 and 697 ), or to the continuance and the probable progression of already existing lesions.

Literature. Löffler \& Schütz, D. m. W., 1882. Nr. 52; 1883. Nr. 14. Löffler, Arb. d. G.-A., 1886. I. 141. - Johne D. Z. f. Tm., 1886. XTI. 321. Cadéac \& Malet, Rer. vét., 1886. 406; 1888. 581; Bull., 1894. 555. - Schütz, A. f. 
Tk. 1899, XX. 425; 1895. XXI. 382; 1898, XXIV. 1; 1908, XXXIV. 74. - Olt, A. F. Tk., 1895, XXI. 352. - Foth, Z. f. Vk., 1892. 169; D. Z. f. Tm., 1894. XIX. 437. - Bass, D. Z. f. Tm., 1893, XIX, 217 (Historical). - Nocard, Bull., 1893, 116; 1894, 89. 225. 367; 1896, 196; 1897, 781; 1901, 387; Rec., 1897, 675; 1898. 296. - Semmer, D. Z. f. Tn., 1894, XX. 59. - Hutyra \& Preisz, D. Z. f. Tm., 1894, XX. 369. - Olt, A. f. Tk., 1895, XXI. 352. - MacFadyean, J. of comp. Path, 1896, IX. 322; 1901, XIV. 265; 1904, XVII. 295. - Wladimiroff, Rec., 1897, 618; Hb., d. p. M., 1903, II. 707 and 1904. III. 1020 (Lit.) ; B. t. W., 1908. 50. - Jensen, B. t. W., 1901, 621. - Babès, Moyens à comb la Morve, Bucharest 1903. - Bonome, Patogenesi, ete., della Morva chiusa, Padova 1905. Riegler, Kongr. Budapest 1905, II. 338. - Schlegel, D. Rotzbekämpfung usw., Stuttgart 1905. - Schnïrer, Cbl. f. Bakt., 1905, XXXIX, 180; Z. f. Infkr., 1908. IV. 216; D. t. W. 1910. 65 (Lit. ou Allergy). - Feodorowsky, A. f. Tk., 1905, XXXI. 505. - Schiitz \& Miessner, 1905. XXXI. 353. - Hutyra, Z. f. Tm., 1907. XI. 1. - Levy, Blumenthal \& Marxer, Z. f. Infkrkh., 1907. III. 294 (Lit. on Immunization). - Sustmann, Diss. Zürich 1908 (Lit. on Aggl). - Schulz, A. f. Tk., 1908. XXXV. 198 (Lit. on Aggl.). - Schütz \& Schubert, ibid., p. 44. - Pfeiler, ibid., p. 323 (Lit. on Prezip.). - Miessner \& Trapp, ibid., p. 85 (Lit. on Pathogenese); Cbl. f. B., 1909. LII. 115 (Lit. on Fixation).

Glanders in Carnivora. After the ingestion of the meat and the organs of glanderous horses carnivora may also become infected with glanders. In zoological gardens actual enzootics are occasionally ohserved among the animals of the eat species from this cause, while animals of the dog kind are much more rarely the victims of these conditions.

In such cases the disease usually takes an acute course. As a rule the first symptoms consist of an excessive inflammation of the conjunctivae and of the mucous membranes of the air passages, the eyes and nose discharging at first purulent but later a greenish-gray or bloody secretion. Following this the respiration becomes very labored as a result of excessive swelling of the respiratory mucous membranes. These symptoms become rapidly aggravated, the tissues of the head become swollen, particularly in the nasal region, while metastatic nodules, which soon perforate the skin and form ulcers, make their appearance in the subcutaneous tissue of various regions of the body. Finally diarrhea sets in, whereupon the animal succumbs in the course of the first or second week after the appearance of the first symptoms.

Glanders in Sheep and Goats. Although sheep are susceptible to experimental infection with glanders they are not known to become infected from natural sources. The destructive disease described by Audum in Livland under the name of glanders, causing the death of 60 Merino sheep and the slaughter of 200 exposed animals, is evidently not the morbid process under discussion, experimental infection of dogs as well as of guinea pigs having been unsucessful. (Hemorrhagic septicemia was formerly described repeatedly as glanders of sheep; see p. 118.)

Ercolani, Karsten-Harms, Koch and Trashot observed glanders in goats in stables where glanderous horses had been kept for a long time. Of morbid symptoms were observed particularly profuse purulent nasal discharge and in one instance abscess of the udder.

Literature. Ereolani, II. med. vet., 1891. - Harms, H., Jhb., 1874. 88. Trasbot, Ann. d'Alf., 1876. 521. - Audum, Unters. üb. d. Schafrotz, Diss., Dorpat, 1888 . 
Glanders in Man. In man glanders seems to oceur most frequently when the virus gains an entrance directly into the lvmph spaces or into the subcutaneous and submucous connective tissue from lesions in the skin or mucous membrane, or when it enters the blood stream. directly. Aside from positive observations along this line this view is supported by the fact that persons who are frequently exposed to danger of infection (stable attendants) are comparatively rarely affected with the disease. Nevertheless the possibility of an intestinal infection must be admitted.

The course of the disease may be acute or chronic. In acute cases a small nodule or ulcer develops at the point of infection, the surrounding connective tissue is edematous and the afferent vessels and the regional lymph glands are swollen. Subsequently small nodules and bluish-red pustules appear on other parts of the body where they are in a short time transformed into ulcers; frequently there may be purulent inflammation of the articulations and of the corpus cavernosum of the penis. In the course of these changes there is febrile elevation of temperature with occasional attacks of chills, and the patient complains of severe muscular and articular pains. In some instances there is also an ulcerative inflammation of the nasal mucous membrane attended at first by a thin discharge which subsequently becomes purulent, reddish and viscid. The appearance of cough, hoarseness, difficult respiration and deglutition indicate affection of the larynx and lungs. These symptoms are attended with, or followed by, gradual exhaustion of the patient and finally terminate in death in the course of 2 to 6 wecks.

In some cases (intestinal infection?) the morbid picture is very similar to that of typhoid fever; the persistence of the fever beyond the third week as well as the subsequent appearance of eutaneous pustules alone make the final definite diagnosis possible.

The chronic form develops insidiously; while febrile elevations of temperature continue, nodules and small ulcers appear on various parts of the body; these may heal and the patients may be apparently well for months or even years; later, however, fresh nodules appear and symptoms of lymphangioitis and phlehitis set in and the process finally becomes acute, or the disease may run a chronic course throughout (in Bollinger's cases the disease continued for 11 years), although this form of the disease is said to end under proper treatment not infrequently $(50 \%)$ in recovery (superficial application of gray mercurial ointment).

Literature. Bollinger, Ziemssen's Handbuch, 1874. 399 (Lit.). - Korányi, Nothnagel's Handbuch, 1900. V. Bd. 1 T. - Strube, A. f. Klin. Chir. LXI. (Lit.).

\section{Ulcerative Inflammation of the Lymphatics of the Horse.}

\section{Lymphangioitis ulcerosa equorum.}

Ulcerative lymphangioitis is a chronic, contagious disease of horses in the course of which, usually without involving the regional lymph glands, there is developed a progressive inflammation of the subcutaneous lymph vessels with a tendency to ulceration. 
History. This disease was first described by Nocard (1892 and 1896) as a specific morbid process, this author having demonstrated the disease (in 59 horses suspected of having glanders) to be etiologically different from glanders. Following the latter author Mollereau and Tallée reported the disease from France, Darmagnac from Algeria, while Schwartzkopf observed similar diseases in the Philippine Islands. The identity of the disease, in the latter instance, has however not as yet been definitely determined owing to the absence of bacteriological evidence.

Etiology. The cause of the disease is identical in all of its characteristies with the bacillus of pseudotuberculosis of Preisz and Nocard (see p. 626).

Pathogenicity. Subcutaneous injection of the pure culture in a horse or ass is followed by local suppuration; the abscess thus formed will break in the course of 6 to 10 days, whereupon the contents are discharged and healing follows with the formation of a scar (Nocard observed in one case only the development of progressive inflammation of the lymphatics following such inoculation). Intravenous injection produces at the most a temporary febrile condition.

Injection of pure cultures or of pus obtained from nodes or nlcers into the abdominal cavity of a guinea pig is followed by inflammation of the tunica albuginea of the testes which is usually quite pronounced at the end of the $3 d$ to the 5th day and in the course of which abundant purulent, fibrinous exidate collects between the tunies of these organs. Occasionally death results by the end of the first week, post-mortem examination revealing an exudative peritonitis in addition to periorchitis. Highly virulent cultures may cause death in from 24 to 48 hours without involving the testes.

Natural infection apparently takes place from wounds or other solutions of continuity in the skin, and the fact that the disease usually develops on the limbs seems to point to the fetlocks as the ports of entrance of the infection, or the process may be induced by the kick of a horse. However, the disease does not usually spread direct from animal to animal, but the bacilli probably gain access into the lymph spaces and the cutis directly from the soil.

Symptoms. Attended with diffuse swelling of the extremities of the limbs, isolated, firm, sharply circumscribed, sensitive nodes appear on one or both hind legs; later these nodes rupture and become transformed into irregular ulcers with bulging but not thickened borders which at first discharge a creamy, but subsequently a thinner, viscid purulent secretion. These ulcers are not progressive in character and vield readily to antiseptic treatment. This is, however, soon followed by the appearance of fresh nodes in the neighborhood or in more remote regions 
of the body, which also become transformed into ulcers, while the comnecting lymph vessels form thick strands in the course of which new nodules and ulcers develop. In this manner the disease may continue for months, during which time it may spread from the posterior to the anterior limbs, the trunk, the neck, and even the facial portion of the head, and may finally terminate in the death of the animal. In some cases the disease may continue for years. In one case the nodes and ulcers appeared only during the cold season while the affected horse seemed perfectly healthy during the summer.

The lymph glands in the region of the ulcerous processes may occasionally undergo moderate swelling, but as a rule, there is no induration, while suppuration never occurs.

An unusual course of the disease is noted by Darmagnac inasmuch as the ulcerous process spread in spite of proper treatment from the inner surface of the thigh to the scrotum, and caused an extensive swelling of the entire lower portion of the abdomen, fresh nodes rapidly reappearing throughout its extent, some of them leading to the formation of indolent fistulae. The constant loss of tissue secretions finally caused the death of the horse, and post-mortem examination revealed innumerable pus foci scattered thronghout the firm lardaceous connective tissue of the swelling. In addition to this there was also simple hypertrophy of the inguinal and sublumbar lymph glands.

Cocu observed in a mare of advanced age a large paranephritic abscess, from the interior of which a fistulous canal communicated with the outer world in the lumbar region. The right kidney, which was surrounded by pus, contained in its otherwise normal parenchyma numerous abscesses ranging in size up to that of a pigeon's egg. Nocard demonstrated the above named bacillus in the pus.-The authors observed a similar paranephritic abscess as large as a man's head at the post-mortem examination of an ass, they could also demonstrate the hacillus referred to in a purulent secretion of an otherwise benign wound of the fetlock of a horse which healed perfectly.

Diagnosis. The disease resembles cutaneous glanders in many respects. The differences are that the small ulcers which develop from the nodes in ulcerous lymphangioitis heal rapidly, the lymph glands are hardly involved in the process, the nasal mucous membrane remains intact and the mallein test gives no positive reaction. In the pus of the nodes and in the secretion of the nleers short Gram positive bacilli can be demonstrated, which do not thrive on naturally acid potatoes. Finally, intraperitoneal infection of guinea pigs is followed more rapidly by periorchitis than is the case in infection with glanderous material, the bacilli in question are found in great masses in the purulent fibrinous exudate, while pus foci are found in the parenchymatous organs.

Epizootic lymphangioitis is in general a more malignant disease while the lymph glands also usually suppurate and the pus contains large globular schyzomycetes which do not stain readily.-Contagious acne is probably closely related etiologic- 
ally to the disease under discussion. It usually develops on portions of the body which come in contact with the saddle and harness.

Treatment. Application of antiseptic fluid to the ulcers usually brings about speedy recovery; if the aloscesses persist the nodes may be incised and treated with strong antiseptics. In exceptional cases, however, even heroic treatment does not check the progress of the disease.

In view of the similarity of the organisms at the bottom of this disease and Löffler's diphtheria bacillus, Vallée tried treatment with diphtheria antitoxin. Subcutaneous injection of the serum (40-50 cc. at four day intervals) as well as the local application of pulverized selum was followed by remarkably rapid healing of the ulcers and the checking of the morbid processes.

Literature. Nocaril, Bull., 1893. 116; A. P., 1896. X. 609. - Cocu, Bull., 1899. 232. - Schwarzkopf, Amer. vet. Rev., 1902. XVI. 111. - Dassonville, Bull., 1907. 576. - Darmagnae, Rev. gén., 1904. III. 303. - Vallée, Bull., 1907, 181.

Lymphangioitis Pseudofarcinosa. Liénaux observed a very chronic affection of cattle in Belgium which manifested itself in the appearance of large painless nodes on the legs, approaching the size of a fist and communicating with each other by means of greatly enlarged lymph vessels; the interior of these nodes contain pus foci which, however, do not break spontaneously; as a rule they become indurated. The regional lymph glands become swollen and develop into firm nodes with purulent centers. This disease which otherwise resembles glanders (see p. 668) is etiologically related to pustulous dermatitis, described by Liénaux. Treatment consists in early evacuation of the abscesses and surgical treatment of the tumors (Ann. 1905, LIV, 297; see also p. 670$)$.

\section{Epizootic Lymphangitis. Epizootic Inflammation of the Lymphatics. Lymphangioitis Epizootica.}

\section{(African Glanders; Japanese Glanders or Farcy; Saccharomy- cosis; Lymphangite épizootique, Farcin de rivière, Farcin d'Afrique, Farcin cryptococcique [French]; Linfangite farcinoide, Farcino cripto cocchico [Italian].)}

Epizootic lymphangitis is a chronic, contagious disease of Solidungula caused by a yeast fungus, Cryptococeus farciminosus; in exceptional instances cattle also are attacked. The disease is characterized by purulent inflammation of the subcutaneous lymphatic vessels and of the regional lymph glands.

History. The disease was first described by Italian and French veterinarians. Its cause was described by Rivolta (1873), while its 
etiology and symptomatology were studied and elucidated by Rivolta \& Micellone, Nocard, Tokishige, Marcone, Tartakowsky, Sanfelice, Pricolo, etc.

Occurrence. The disease was formerly observed only in southern Europe, particularly on the shores of the Mediterranean, quite frequently in Italy and in France. Recent reports, however, mention its occurrence in more northern localities (Finland and northern Russia, England). It seems also to be widely prevalent in Asia and Africa.

The disease was olserved by Lindquist in Finland, by Tartakowsky, Dedjulin and Awryinsky in the governmental districts of Olonetz and Novgorod in Russia, by Tokishige in Jajuan (rather common), by Pearson in Pennsylvania, Fiseher in Ohio, by Does \& Haan in Lower India and by Nockolds in the Philippine Islands, where it appears as a very frequent lisease with unfavorable course, on account of which large numbers of horses have in recent times been destroyed under suspicion of glanilers.

In 1902 it was transported with shipments of horses from India to South Africa and from there with English troops that returnell from the Transvaal war, to England in which latter country it gained wide prevalence in several garrisons and was subseqnently disseminateil by discariled army horses to private stables. This plagne became so prevalent that the English government formulated special regulations for its control, the success of which is evident from the report that only two cases of the disease were observer in 1906. - In 1901 a number of eases were observed in a German-Russian borter villages in the government district of Gumbinnen.

Etiology. The Cryptococcus farciminosus Rivolta (Saccharomyces farciminosus Tokishige) forms comparatively large, 3 to $4{ }^{\mu}$ long, 2.4-3.6 ${ }^{\mu}$ wide, doubly contoured, somewliat oval borlies usually either somewhat pointed at the ends or provided with bud-like protuberances. These organisms multiply by budding and in this respect resemble saccharomyces, although they are smaller and do not cause saccharine fermentation. They are not readily stained by the usual methods, even carbol-fuchsin staining the contents only slightly while the cell membrane remains unstained.

Culture. Artificial cultures are difficult to ohtain and growth on artificial media is slow. Thus on nutrient agar grayish-white granules appear only after about 30 days, subsequently they enlarge to form prominent colonies drawn into folds resembling the convolutions of the intestines. On gelatin bouillon yellowish-white sandy granules appear in the course of 56 days, on potatoes light brown masses appear in a somewhat shorter period while in pepton bouillon white flakes develop in the course of 17 days. Under the microscope the colonies are seen to consist of hyphae, spherical fungi and granules resembling spores (Tokishige).

According to Bridré \& Nègre the fact that immune bodies are present in the serum of affected horses which fit into complement fixation systems with ordinary yeast cells points to the blastomycetic nature of these parasites.

In contrast to the above view Ducloux, whose observations were subsequently confirmed by Thiroux \& Teppaz, is of the opinion that the eause of the disease is a protozoon (Leucocytozoon piroplasmoides). These investigators found round 
or pear-shaped structures in the pus of norles and ulcers. Some of these structures were enclosed by mono- and polynuclear leucocytes, in the cell substance of which karyosomes conld be demonstrated with eosin-methylene blue, and which suggested the appearance of Heliosoma tropicum, the cause of Oriental boil plague. Thironx \& Teppaz failed in their attempts to cultivate the parasite, nor could they reproduce the disease by inocnlation of the pus from the nodes. According to their view, the leucocytozoons may be transported to the internal organs by means of lencocytes and there set up their morbid process.

Pathogenicity. After the administration of pus taken from the nodes or ulcers into cutaneous wounds of solidungula, inflammation and swelling of the lymphatics may occur in rare cases in the course of 20 to 60 days. Along the course of the lymphatic vessels nodes develop which later form nlcers (Tixier, Ċhauvrat, Delamotte, Peuch). In rabbits experimental infection produces local abscesses only, while in guinea pigs swelling of the lips and the popliteal glands usually results. Other experiment animals are immune.

Natural infection probably oceurs from superficial wounds by immediate contact with infected objects (harness, litter, bandages, instruments, etc.), or from contact of diseased animals with healthy ones. (Thiroux \& Teppaz are of the opinion that infection is spread through the medium of insects). The ass is most susceptible, the horse and the mule less so while, according to Tokishige's observations, cattle are also susceptible.

Anatomical Changes. In the affected regions the skin and subcutaneous connective tissue become thickened (up to 5 or $6 \mathrm{~cm}$.) and firm; cross sections have a lardaceous appearance and contain pus foci of various sizes, while the distended lymphatics are filled with coagulated serum and pus, the walls of these vessels being intensely reddened and having a finely granulated appearance. The regional lymph glands are swollen and frequently contain small pus foci or cicatrices which have resulted from their presence. In the diseased joints we find large masses of serous or sero-purulent exudate and the synovial membranes are covered with villous proliferations; adjacent tendons are much enlarged showing serous infiltration and containing large pus cavities, in the internal organs (lungs, testes, intestinal wall) metastatic pus foci are found only in exceptional cases. The nasal mucous membrane is occasionally swollen near the nostrils and contains on its surface sharply circumscribed, flat, yellowish elevations as well as deep ulcers.

Symptoms. Inflammation of the lymph ressels begins most frequently on the limbs, especially the anterior ones, but is observed also on the scrotum or the udder, somewhat rarely on the abdomen and neck where they usually develop from a wound or a fresh scar. Such wounds refuse to heal and gradually form ulcers with red luxuriant granulations, or the re- 
sulting cicatrice is transformed into a firm painless node which may attain the size of a pigeon's egg, breaking spontaneously and discharging a thick, yellowish, gruel-like pus.

The local process is followed by swelling of the regional lymph vessels which form prominent, wavy and branching painful strands in the course of which develop firm tumors up to the size of a fist, subsequently these become elastic and finally fluctuating and contain the above described thick, yellow pus. The nodes also break and discharge their contents, whereupon they are transformed into fistulous ulcers with indolent, easily bleeding granulating borders. The ulcers frequently produce excessive granulations which lead to the formation of ugly ulcerous surfaces.

The regional lymph glands swell and form firm, tuberous or nodular tumors which do not adhere to the surrounding tissue; they also suppurate, whereupon the abscesses break spontaneously and gradually heal with scar formation. In some cases an excessive but uniform swelling of the ends of the extremities or the region of the joints may develop followed by ulceration of the overlying skin.

In other instances there may be in various regions of the body spontaneous development of flat elevations as large as a copper cent or of nodes approaching the size of a walnut, which break and, after discharging their muco-purulent contents, are transformed into flat ulcers which heal without scar formation. In rarer instances numberless minute furuncula appear which partly communicate with each other by means of strands with serous contents which later dry and form scabs.

In exceptional cases yellowish flat elevations or white nodules as large as lentils occur on the nasal mucous membrane. Some of the nodules may have become transformed into ulcers with projecting labiate granulating borders (Pricolo). The intervening mucous tissue is hardly swollen to an observable degree, though it is usually cyanotic; simultaneously with the appearance of these lesions the submaxillary glands become enlarged and occasionally suppurate. On the other hand nasal discharge is rarely observed. In a case described by Marcone the nasal mucous membrane was covered with numberless nodules and ulcers, while the turbinated bones were covered with pedunculated growths which had resulted in the partial destruction of the dental alveoli. The nasal alae were much enlarged, firm and studded with small pus foci covered with scabs, in addition to which a thick strand was observed to cross from the left nasal ala toward the facial crest. Cominotti observed a similar case except that the submaxillary gland was enlarged to the size of a small apple, only slightly sensitive, bosselated on its surface and not adherent to the surrounding tissue. Finally the conjunctiva and especially the membrana nictitans have in rarer cases been observed to be affected with nodule formation and ulcers 
(Carparini, Awryinsky), while deDoes observed in one instance glanderous-like enlargement of the testis, with numerous soft centers in its tissue (the weight of the testis was $1.5 \mathrm{~kg}$.).

In eattle Tokishige observed firm painless nodes as large as walnuts scattered through the subeutis in various regions of the body; these remained distinctly isolated, showed a slow growth and did not result in abscess formation nor in ulceration (see also p. 670).

The course is very protracted; the morbid process may under favorable conditions continue for from 4 to 6 weeks; under opposite conditions it may persist for six months; even after apparently complete recovery the symptoms of the disease may reappear after a certain time. The general nutrition usually remains good, although in malignant cases the animal may become emaciated. In the meantime exacerbation may appear with acute symptoms, elevation of temperature to $40.6^{\circ} \mathrm{C}$., rapid enlargement of the limbs, the lips and the nasal mucous membrane, eruption of small vesicles, etc. (Awryinsky). In a portion of the cases the disease progresses without interruption until the patient finally becomes exhausted, and the disease occasionally terminates with symptoms of hectic fever.

The mortality is about 7 to $10 \%$ (Tokishige, Pallin), although in tropical countries it is considerably higher.

Diagnosis. The differential diagnosis of this disease from glanders may occasionally be quite difficult. In recent and well marked cases, however, the flat and actively granulating ulcerous surfaces as well as the creamy pus in contrast with the crateriform ulcers of glanders and their viscid secretions, are sufficient diagnostic characteristics. In chronic cases, however, the nlcers of epizootic lymphangioitis may have a similar appearance. In such cases microscopical examination will rereal the nature of the affection, in epizootic lymplangioitis the bright parasites, partly enclosed in pus cells, are readily observed without staining under a magnifying power of $400-500$. In arddition to this, inoculation of rabbits-or guinea pigs with pus produces only local suppuration, while the mallein test gives a negative reaction. The good physical condition of the animal in the presence of ulcerating skin disease speaks against the existence of cutaneous glanders.

Ulcerous lymphangioitis takes a more benign course and the pus from glanderous lesions contains small bacilli which stain according to Gram's method and when injected intraperitoneally produce orchitis in guinea pigs.

Treatment. This consists, especially in the beginning of the affection and wherever possible, in the extirpation of the nodes and lymph strands and the neighboring lymph glands. The prompt incision of abscesses and careful antiseptic treatment of pus cavities and ulcers is of course always indicated. Thiroux 
\& Teppaz had favorable results in the treatment of mild cases with atoxyl, althongh this gave no results in severe forms of the disease.

Prophylaxis consists in the isolation of the diseased animals, destruction of morbid products and disinfection of stables with strong disinfectants.

Literature. Rivolta \& Micellone, Giorn, die Anat., Fis. et Patol., 1883. 143 - Nocard, Bull., 1897. 367. - Tokishige, Cb. f. Bakt., 1896. XIX. 105. Baruchello, Sul. fare. eriptoc., Torino 1898. - MacFadyean, J. of comp. Path., 1903. XVI. 379. - Martin, J. of comp. Path., 1905. XVIII. 81. - Sullivan, Vet. Rec., 1905. Nr. 870. - Sanfelice, Z. f. Hyg., 1906. LIV. 299. 一 Mrowka, Z. f. Vk., 1906. XVIII. 261 - Mareone, Rev gén., 1907. IX. 249. - Pricolo, ibid., 1907. X. 457. - Ducloux, Soc. biol., 1908. LXIV. 593. - Thiroux \& Teppaz, A. P., 1909. XXIII. 420 .

Malignant Hyphomycosis of Horses. Leeches (Hyphomykosis destruens equi.) In India as well as on the Sunda Islands a disease occurs among horses which is known by the name "bursattee" (burus=rain), and which has been described in particular by F. Smith and Stell, and more recently also by deHaan and Hoogkamer. According to Lyford and Bitting this same disease occurs quite frequently in North Ameriea among horses and mules where it is known as "leeches" while in Europe only a single ease has been observed by Drouin \& Rénon in Alfort.

The morbid symptoms consist in the appearance of very firm nodules under the skin of the lips, the nasal alae, the eyelids, the neck, the body and the limbs, finally also in the mueous membrane of the gums and the nasal eavities; these gradually enlarge and later become transformed into ulcers. The ulcers have uneven, ragged, easily bleeding granulating borders which communicate with deep, sinuous, fistulous canals which occasionally extend to or into the bones (perforation of the hard palate has also been observed). Within the ulcers and fistulous canals as well as in the interior of the nodes are found grayishyellow, oceasionally also caleified masses and cores which may approach the size of an egg and have a bosselated surface, these are firmly attached to the surrounding tissue by means of their ramifications. The lips, nasal alae and eyelids may be extensively swollen, while the month and nose discharge a bloody mucous mass. The affected regions of the body seem to eause excessive itching. In severe cases, which however do not involve the regional lymph glands, the nutrition of the patients suffers serionsly.

The grayish-yellow cores above mentioned contain branching mycelia and free spores of a mold fungus imbedded in hyaline connective tissue bundles and leucocytes; according to deIlaan these form a delicate sod on nutrient agar while in acid bonillon they form groups of delicate balls. Experimental inoculations have failed to give positive results.

The treatment consists in an early extirpation or eauterization of the affected portions of the cutis; deDoes observed healing of the lesions in the mucous membranes after internal iodine treatment $(10-30 \mathrm{gm}$. potassium iodide daily) while deHaan \& Hoogkamer found this treatment ineffective.

Literature. Drouin \& Rénon, Soc. biol., 1896. 425. - De Haan \& Hoogkamer, A. f. Tk., 1903. XXIX. 395. 


\section{Infectious Abortion. Abortus Enzooticus.}

\section{(Abortus infectiosus; Seuchenhaftes Verwerfen [German]; Avortement épizootique [French]; Aborto \\ enzootics [Italian].)}

Under the term "infectious abortion" are included those cases which occur in otherwise healthy mothers, as a result of external infection producing inflammatory lesions of the uterine mucous membrane and of the fetal membrane. It usually occurs in an enzootic form.

History. As long since as the beginning of the last century this disease was considered as contagions in several mountainous regions of England (Lawrence, Skellet), and since then other authors (Zündel, St. Cyr) have maintained that an infection was the causative agent in spite of the opposite view held by others (Hurtrel d'Arboval, Hering, Stockfleth). The correctness of this view was however only established by Franck (1876), and later by Lehnert (1878) and Bräuer (1880), by producing abortion artificially through the introduction of vaginal discharge and fetal membranes into the vaginas of healthy cows. Later Nocard (1885) called particular attention to the importance of the inflammatory changes in the fetal membranes. Bang (1896) with the cooperation of Stribolt, discovered the cause of infectious abortion of eattle, which was later also investigated extensively by Preisz (1902), while on the other hand Ostertag (1901) connected a streptococcus with the cause of infectious abortion of mares.

Occurrence. Infectious abortion occurs most frequently in cattle breeding establishments, more seldom in studs and in the breeding of sheep and swine. The stable-fed, finer bred animals are especially affected, but the infection may become widely spread in range animals (Kocourek, Keleti). The introduction of the disease into large cattle herds causes heary losses, because of the fact that a number of unviable calves perish, and because of the decrease of the milk production, moreover the cows conceive only with difficulty after abortion or not at all.

Infectious abortion is widely spreal in all sections in which intensive stock farming is in rogue. In Central Europe (Belgium, Denmark, Germany, France, Austria, Switzerlaud, Hungary) and in England it appears frequently, especially in cows, while in North America it appears that mares are especially affected.In England Mascall called attention to the disease as early as 1567, and during the last century it gained such a foothold in the dairies that in certain localities $50-60 \%$ of the cows slunk their calves (McFadyean \& Stockman recently could show the disease in 36 shires); besides the disease appears frequently in horse breeding and sheep raising establishments (Penberthy).

Etiology. In cattle abortion is caused by a small, short and comparatively thick bacillus (Corynebacterium abortus infectiosi) which is non-motile, forms no spores; in artificial 
cultures one end is frequently club-shaped, and sometimes branches (Fig. 125).

Staining. The bacillus stains easily but not uniformly with aqueous as well as with earbolized aniline dyes, giving the appearance of segments separated by clear spaces; the Gram and Gram-Weigert methods give negative results.

Cultivation. The bacillus of abortion grows especially well in a pure oxygen atmosphere, or in air with low oxygen contents, while in the total absence of oxygen it grows only with difficulty, and in ordinary atmosphere only after it has gradually become accustomed to such environment. Cultures made according to these conditions give particularly good results in or upon gelatin-agar, a combination of agar, gelatin and blood serum, as well as in glucose-agar. Upon the surface of such media, kept at body temperature there appears very fine, flat, conical, evenly rounded, on transmitted light, bluish transparent colonies which even

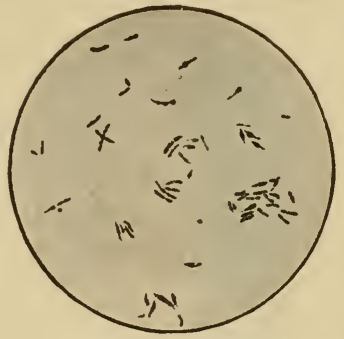

Fig. 125. Corynebacterium abortus infectiosi. Two-dayold agar cultures; carbol fuchsin. if thinly seeded reach at most the size of a pin head. When the material to be examined is mixed with the media there develop, at a fixed distance from the surface and downward, numerous similar colonies closely placed (Fig. 126). Coagulated calf serum becomes liquefied in the vicinity of the colonies. In peptone bouillon and in liquid blood serum, few small white granules form. Milk becomes coagulated. Potato cultures show a marked resemblance to a culture of the bacterium mallei (McFadyean \& Stockman).

Tenacity. In cultures the bacillus dies within two weeks (Nowak found, however, that agar cultures were even after two years still capable of development. A temperature of $55^{\circ} \mathrm{C}$. will destroy the organism in 3 minutes. It is destroyed by $0.05 \%$ corrosive sublimate solution in 15 seconds, by $1 \%$ carbolie acid in 1 minute, by $2 \%$ acetic acid in two minutes, by $1 \%$ acetic acid in 20 minutes (Preisz). On the other hand it may retain its virulence for many months in uterine secretions if preserved sterile, also if uncontaminated inside of the womb and in the dead foetus (Bang).

Pathogenicity. In Bang's investigations the injection of pure cultures into the vagina of pregnant cows and ewes produced in 8 to 10 weeks either abortion or premature birth; in these cases large numbers of bacilli were present in the vaginal discharge, in the exudate on the surface of the cotyledons and the chorion. Injections of 8 and $18 \mathrm{cc}$. of culture respectively into the circulation of pregnant ewes produced fever lasting several days, whereupon these animals gave birth after 7 and 12 days to small lambs. Bang's experiments, the results of which were subsequently confirmed by McFadyean \& Stockman, demonstrated that the feeding of cultures or exudate to cows, ewes and goats results in abortion after 1 to 2 months, with the characteristic changes in the fetal membranes and cotyledons. Finally it was also possible to produce. 
premature birth in a mare 28 days after the injection into the vagina of $25 \mathrm{cc}$. of culture and the chorion exudate contained mumerous bacilli. (Nowak caused abortion in guinea pigs and rabbits by subcutaneous, intravenous, or intraperitoneal injections of pure cultures.)

The bacilli are always found in large numbers in the exudate of the fetal membranes and of the cotyledons, either

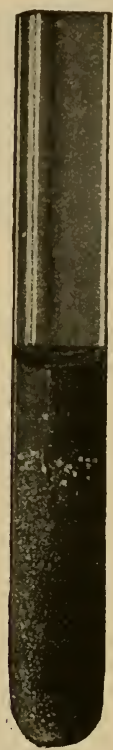

Fig. 126. Agar culture of the bacillus of abortion.

(After Preisz.) free in the serous exudate or enclosed in cells, besides these locations the organisms may also be demonstrated in the intestinal contents, in the blood, as well as in other organs of the aborted fetus.

In no case of infectious abortion of mares did Ostertag find the Bang bacillus in the fetal membranes, but in its stead he found short Gramnegative streptococci in the heart blood, pleural fluid, and in the stomach contents of the stillborn foals, as well as in the sub-chorial edema. They grow on serum agar in the form of fine deposits, which are hardly visible to the naked eye, in stab agar as a frail thread reaching from the surface to the bottom of the tube, while in serum bouillon after two days' growth there appears a uniform turbidity which after two more days sinks to the bottom. In seven cases the streptococci were obtained in pure culture from the bodies of the fetuses, in the other cases the streptococci were found in common with other bacteria on the surface of the chorion; in the chorion some were present in the epithelial cells.

One mare aborted twenty days after the intravenous injection of the culture, another one give birth after a vaginal injection at the normal time to a very weak foal; in the heart blood of the fetus from the first mare the streptococci could be demonstrated, and they were also present in the reddish-gray, thick deposits of the uterus of a mare killed immediately after having aborted.

Aside from the use of pure cultures the artificial production of abortion was repeatedly made possible in pregnant animals by the transference into their genital tracts of vaginal discharge and portions of fetal membrane from aborting animals of the same species, and this is not only true with reference to cows (Bräner, Lehnert, Trinchera), but also in mares (Ostertag, Guillerey); on the other hand the introduction of shreds of fetal membranes from mares into 10 cows and two goats failed to cause abortion (Ostertag).

According to Lignières the bacillus of tuberculosis zooglique may also cause abortion indirectly, when it enters the alimentary tract with food. By the elimina- 
tion of toxins it farors the penetration of other bacteria present in the intestines and other tubular organs or body cavities, into the blood curculation and other organs, thereby indirectly producing abortion. He produced abortion successfully in guinea pigs, rabbits and cows by feeding pure cultures.-Abortion can evidently be occasioned by various infectious causes, since it is quite frequently observed in the course of acute infectious diseases, but such cases should be interpreted differently than as the independent disease of infectious abortion.

Natural infection may take place from the external genitals, most frequently it is transmitted during copulation by males, who have previously covered infected females. On the other hand the straw and floors of stables become contaminated with the vaginal discharge during and after abortion, by the amniotic fluid and the fetal membranes, which contain enormous numbers of bacilli, whence the contagion may likewise penetrate the genital organs; at the same time the infection may be introduced by various objects, such as sponges and other utensils and also by attendants.

The possibility of an intestinal infection in cows, goats and sheep, by the ingestion of food and drinking water contaminated with amniotic fluid and afterbirtl, was proven beyond a doubt by the successful experiments of Bang; and this mode of infection should be considered as the natural infection more often than has heretofore been the case. Especially does it play an important part in cases where the malady spreads rapidily on a farm.

In cattle establishments free from the infection the virus is usually introduced by an already infected female, who during abortion infects the stable, and during copulation infects the male. The infection then spreads on this particular farm with every favorable opportunity, since the first cases of abortion or premature births are given little attention, as they are followed by new cases only after a considerable period, and consequently the necessary measures to eradicate the disease are omitted. The introduction of the disease can also be accomplished by means of animals that suffer from a chronic uterine catarrh subsequent to abortion, and also rarely by animals that in spite of their being infected, carry the fetus the full term. More seldom is the disease introduced by males that have recently been covering animals on infected premises, and likewise also by new-born animals from such farms.

The infection spreads as a rule only among animals of the same species, at times however animals of other species are attacked. As a matter of fact the Bang bacillus is not only capable of producing abortion in cattle, but also in horses, goats and sheep (Garcia \& Izcara found during an epizootic abortion among sheep, a bacillus in the exudate which morphologically corresponded with Bang's organism); on the other hand Ostertag's streptococcus has to the present date been found exclusively in mares. 
Pathogenesis. The contagion which reaches the vagina through copulation passes into the uterus, where it multiplies on the surface of the mucous membrane, and later also on the fetal membranes. The bacteria that reach the vagina dnring pregnancy are evidently capable of passing the mucous plug of the neck of the uterus, whereupon they multiply between the fetal and mucous membranes. The bacilli may also reach the mucous membrane of the uterus from the intestinal canal by means of the blood stream, either before or after conception; since it appears that this organ is an exceedingly favorable medium for their propagation.

The infective material produces an inflammation, as a result of which a fibro-purulent exudate forms between the mucons membrane and the chorion, which loosens the contact of the lavers of tissues, and finally causes a partial detachment of the fetal membranes. The inflammatory process extends in the meanwhile also to the layers of comnective tissue between the chorion and allantois, as well as to the navel cord, on account of which they acquire a gelatinous appearance, on the other hand the bacteria may also pass into the body of the fetus either through the circulation, or by means of the amniotic fluid.

In consequence of the loosening and detachment of the fetal membranes abortion may occur in various stages of gestation; at times, however, the nterine catarrh does not cause expulsion, but only the death of the fetus, in which case there is formed a gradnally accumulating exudate around the fetal membranes, enclosing a shrunken mummified fetus. Later this exudate becomes inspissated, assuming a tenacious gelatinous consistency and in this the abortion bacillus may remain viable even for nine months after the death of the fetus (Bang). The fact that the bacilli remain alive for so long a time in the nterus explains the etiological connection in the abortions that oceur in an advanced stage of pregnancy as a result of infections occurring during or even several weeks before copulation. It also explains the cases of abortion in females that have previously aborted but were not exposed to any new infection in the meantime.

Repeated abortion reduces the otherwise very great susceptibility to new infections. Cows abort quite frequently a second time, but a third abortion is exceptional. The reduced susceptibility of the animal, and possibly the decreased virulence of the virus is also evidenced by practical experience upon certain farms where abortion occurs in progressively later stages of gestation, and finally by the fact that the respective animals, if they conceive at all, carry their pregnancy to term in spite of the infected surroundings (Sand).

Symptoms. Cows are most likely to ahort in the fifth or sixth month of pregnancy, mares in the fourth to eighth month, 
sheep in the fourth month, and swine in the eighth to twelfth week; at times the abortion takes place in an earlier or later period of pregnancy.

Animals which are pregnant for the first time, as a rule abort at an earlier period of gestation than do older ones or those which have previously aborted. The Danish veterinarians observed that abortion would most often occur in cattle during the fifth or sixth month, but in the beginning of an outbreak, eases also occurred in the third or fourth month. In mares Konge saw it oceur in one-third of the cases in six months, in one-fifth in $7-8$ months, Guillerey as a rule in 4-7 months; Poijakow, on the other hand, reports most of the cases as occurring from the ninth to eleventh month of gestation. According to the experiments of Berger sows abort in the tenth to twelfth week of pregnancy; according to those of Fritsche in eight to twelve weeks.

The abortion is usually preceded by a catarrh of the genital passages. With the moderate swelling of the lips of the vulva the vaginal mucous membrane becomes reddened and reddish nodules, like millet seeds in size (swollen follicles) appear on its surface. At the same time there appears from the vagina a mucous or muco-purulent, exceptionally bloody, and always odorless discharge which in cows is reddish-gray or yellowish, in mares and sows whitish-gray in color. The milk production becomes simultaneously diminished, the milk having a colostrumlike appearance and coagulating on boiling.

Two to three days after the appearance of the first catarrhal symptoms the abortion usually takes place with moderate pains and with mild general manifestations. Should the abortion occur in an early period of gestation the fetal membranes are expelled together with the fetus. On the other hand in abortions taking place at a later stage of gestation the afterbirth is passed off subsequently to the fetus, and it is not a rare occurrence to have it retained for some time. Following the abortion a continuous vaginal discharge is always observed for one or two weeks. The secretion which is then dirty brown or reddish-brown, odorless, or somewhat fetid, may accumulate in the uterus, and from time to time be discharged under straining. Gradually the discharge becomes diminished, and finally it disappears entirely, still in spite of their otherwise healthy appearance the animals either do not conceive, or if they abort at an earlier or later stage of gestation, and in rare cases this may be repeated more than once. Abortion reappears as a rule in cases when copulation occurs shortly after the first abortion, or when the vaginal discharge was still present, whereas in cases where copulation occurs several months after the abortion normal births are more likely to follow.

Fetuses aborted at early periods of gestation are usually dead, those however that are expelled at a later period are not seldom born alive. Prematurely born calves often emit a loud prolonged bellowing, which reminds one of the howling of a rabid dog. Nocard attributes this to a diseased condition of the medulla. Poorly developed fetuses mostly die within 
one or two days, with manifestations of intestinal catarrh. The afterbirth shows a subchorial edema and a marked injection of the chorion, and a muco-purulent exudate exists between the tufts of the cotyledons.

Poljakow frequently found a rarifying ostitis in the beginning and towards the end of an outhreak in mares, whereas at the height of the outbreak he found manifestations of septicemia, such as a de. generation of the parenchymatous organs, hemorrhages in the serous membranes, and dark liquid blood.

Guillerey observed in the course of an outbreak in which 87 out of 97 pregnant mares aborted, the development of sequels, such as febrile nephritis lasting from 6 to 8 days (of 15 such mares one died as the result of it), arthritis (especially in the hock), tendonitis, acnte laminitis, hemoglobinuria, inflammation of the Vena saphena, finally pneumonia. Desoubry saw in mares after abortion a rise of temperature up to $40^{\circ} \mathrm{C}$., and with a marked depression of the sensorium catarrhal symptoms of the genital passages, which in exceptional cases increased to an inflammation of the uterus.

Bulls that have covered affected cows, exceptionally show a diseased condition of the genital organs. Thus Reindl found nodules on the penis of a bull similar to those observed in cows shortly before ahortion; Nohr also observed acute inflammatory manifestations on the penis in several bulls after the infectious coitus.

Diagnosis. The infectious character of abortion is indicated by the prodromal symptoms which appear 2 to 3 days before the expulsion of the fetus. These are manifested in a swelling of the vulva, vaginal discharge, as an indication of an existing catarrh of the genital passages. In other forms of abortion, excepting in infectious vaginal catarrh, the abortion occurs without such prodromal symptoms, or there may previously be observed disturbances of digestion and symptoms of general illness as in general infectious diseases or in poisoning. The infectious nature of abortion is further indicated by an existing vaginal discharge which continues subsequently for a long time, and by a large amount of fibrinous purulent exudate on the fetal membrane. Later sufficient ground is afforded in this respect by the rapidly following cases of abortion, when the diagnosis is further confirmed by the exclusion of other causes.

In young cows, sometimes also in mares, swelling of the vulva, a slight amount of mucous discharge, as well as debility and diminished appetite appear sometimes toward the end of the fifth month of pregnancy, but these symptoms disappear after 3 to 4 days, and then the pregnancy and parturition follow in a normal manner (Cagny).

In abortion of cows a microscopical examination of the vaginal discharge is very advisable; in this the fine, unevenly stained hacilli of abortion are found either exclusively or in predominating majority, but always in great numbers (the dried cover-glass preparations stain most readily with earbol-fuchsin; see Fig. 127). 
Sero-Diagnosis and Allergic Reaction. Recent investigations permit the assumption that it will be possible to deter-

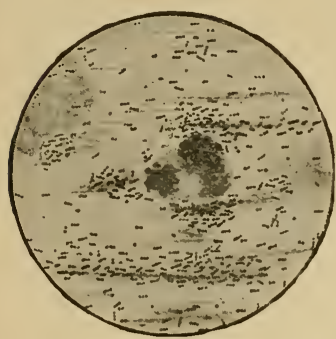

Fig. 127. Vaginal discharge with bacilli of abortion; carbol fuchsin. mine infection with the bacillus of abortion by bacteriological methods long before and after the abortion. If these results are confirmed the combatting of the disease will appear considerably more effective, as it will be possible to prevent the introduction of the virus by apparently healthy animals.

1. Agglutination Test. Experiments in this direction by MacFadyean \& Stockman show that cultures grown in serum-free media are agglutinated by normal serum as a rule in a proportion of not higher than 1:25, whereas serum of infected cows, but which have not yet aborted, shows frequently an agglutination value of $1: 100$ or over. These results, however, were not sufficiently uniform for utilization in practice. Better results were obtained by Holth with serum bouillon cultures, or suspensions of such centrifugalized cultures, as 37 out of 39 samples of serum from cows which had previously aborted gave an agglutination value of over 100, mostly over 500, while seven specimens from healthy heifers and cows failed to give an agglutination, even in dilutions of 1:50. Grinsted also found that in infected cows the agglutination value, even several weeks before abortion, rises to $1: 300$ and over, and after abortion it rises to $1: 2000-4000$, while in healthy animals it does not exceed $1: 300$.

2. Complement Fixation. MacFadyean \& Stockman obtained, although not in all cases, distinct complement fixation (see p. 718) with sera from cows which hat already aborterl, and a still more distinct reaction with sera of infected, still pregnant cows, while Holth showed that the complement fixation test corresponds with the results of the agglutination test.

3. Allergic Reaction. MacFadyean \& Stockman's experiments with the preparation named "Abortin", which they prepared from 4-6 weeks' old glycerin sugar bouillon cultures of the bacillus of abortion, in the same manner in which tubereulin is oltained, showed that infected, as well as cows which had alrearly aborted respond after several hours, with few exceptions, to an intravenous or subcutaneous alministration of the preparation $(5-10$ ce. $)$, with a rise of temperature to $40.5-41^{\circ} \mathrm{C}$., and some also with organie symptoms.

Course. Infections abortion often persists for years in infected premises when proper measures for its suppression are not taken. After the first case of abortion new cases appear as a rule only after several weeks, then however they follow each other in more rapid succession, and finally normal births occur only exceptionally. After a time however the cases of abortion again become more rare, as animals which have already aborted once or twice again give birth at the normal termination of pregnancy, and only the newly introduced young cows or newly purchased animals from healthy herds abort, until finally the disease dies out of its own accord after several years.

Prevention and Treatment. For suppressing the disease after it has appeared in a herd, first of all the change of the remaining apparently healthy female animals which have never 
aborted, to a place free from the infection is indicated. If this cannot be accomplished under existing conditions it is at least advisable to keep the already infected and the suspected animals in another stable, or at least in an isolated part of the same stable, and to supply special attendants for them. Subsequently it is necessary after removing the remnants of food and straw in these parts, to disinfect the entire infected stable thioroughly and repeatedly, the floor and walls as well as the stable utensils, in addition to which the external sexual organs of the pregnant animals should be washed daily with a nonirritating antiseptic fluid, and their vaginas should also be irrigated. In the meantime these animals should be subjectèd to careful observation, and those in which prodromal symptoms of abortion are noticed should be isolated immediately and their stalls disinfected. At the same time it must be taken into consideration that the vaginal discharge has possibly already contaminated the litter and food.

The expelled fetus together with the fetal membranes and the afterbirth should be burned, or at least scalded and then buried deeply. If remains of the fetal membrane were retained in the uterus it should be attempted to remove them soon, if possible in the course of two or three days.

Males which have covered affected females, or in which there is a suspicion in this direction, should be subjected to treatment by cutting the hair short at the opening of the sheath, the prepuce sack should be washed with a $1 / 2-1 \%$ lysol solution, $1 / 4-1 / 2 \%$ septoform, or $1 \%$ soda solution, and following each covering the washing should be repeated with pure lukewarm water or with one of the fluids mentioned. In sheep and swine herds where this is accompanied with difficulties it is advisable to suspend breeding for a certain time.

The treatment of animals which have aborted consists in irrigation of the vagina and uterus with lukewarm antiseptic fluids, by means of an irrigator, the soft rubber tube of which should be introduced deeply into the uterine cavity. For disinfecting fluids, which are also satisfactory for washing the surrounding parts of the vulva, freshly prepared corrosion sublimate in rain water $(1: 8000-10,000$, concentrated solutions cause severe straining!), 1-2\% creolin or lysol solution, 1:1000 permanganate of potassium solution, etc., may be used, of which large animals receive $3-4$ liters, small animals 0.5 liters each. This treatment should be continued at first 1 to 2 times daily, later every 2 or 3 days, until the discharge has ceased for several days (usually for two weeks). After the conclusion of this procedure it is advisable to keep the animals separated for several weeks, and to breed them again, not earlier than two months after the abortion.

Bräuer recommends as prophylactic measures, subcutaneous injections of $2 \%$ carbolic acid, in the fiftl to seventh months of pregnancy (10 cc. weekly). He, as well as Schrader, 
Tafin and others, observed a gradual reduction, and finally a complete disappearance of the disease from such treatment. Haubold, Schlag, Vollrath and others, however, found this treatment ineffective, and even in the favorable cases the disappearance of the disease was probably the result of a disinfection which was carried out simultaneously. The same probably would apply also to the internal administration of carbolized water (1-11/2 liters of a $1 \%$ carbolic acid solution once daily [Nuesch]), as well as the weekly prophylactic irrigations of the vagina of pregnant cows with $1 / 4$ liter of a $21 / 2-3 \%$ solution, with which Horlyck and Dolmer have recently obtained very good results.

In order to prevent the introduction of the disease from outside, newly purchased pregnant animals should be observed carefully, and should be kept isolated until calving; further animals with symptoms of a uterine or vaginal catarrh (leucorrhea!) should not be bred at least until completely recovered. The association with persons who are employed in infected premises should also be limited as far as possible. In male animals which have served on other premises, it is advisable to disinfect the prepuce and the penis before the act of covering. Furthermore, each case of abortion should be treated as if it were an infectious form of the disease, and particularly does this appear to be necessary in those cases where the abortion occurs under the above-described manifestations even in a herd which has been known to be free of the affection.

Females which have aborted one or more times should be disposed of only if they fail to conceive after a subsequent covering, provided that they have received suitable treatment. By keeping pregnant animals the disease will be brought to a standstill more quickly than if new susceptible material is continually added in their place (Bang).

If the described method is carried out accurately it almost invariably succeeds in suppressing the disease. In already infected animals abortion cannot be prevented, but the infection of healthy animals can be prevented. Authoritative measures which would prevent animals from infected herds being taken to markets, animal exhibitions and public pastures, would considerably aid in the prevention of the dissemination of the virus (such measures exist at present in Norway).

Immunization. Bang's most recent investigations indicate the possibility of immunization of eattle, sheep and goats against the infection. He found that several animals which repeatedly received intravenously living cultures (10 ec. each time) at least two months before conception, carried the fetus to normal time in spite of the fact that during their pregnancy they were infected per os with cultures or exudates. As however the intravenous injection sometimes disturhed the general health greatly, experiments were later undertaken on sheep and goats with subcutaneous injections of cultures (5 to 7 times, a 
total of 50-70 ce.), the results of which also proved satisfactory. Of five cattle which, a long time before breeding, were injected subeutaneously in eleven doses with $100 \mathrm{cc}$. of cultures of bacilli killed by Tuluol, and which during pregnancy were fed with cultures and exudates, two animals gave normal births. Of four eattle which received living cultures subcutaneously in increasing doses, from 4-40 ce., a total of $140 \mathrm{cc}$, one gave birth at the normal time, while one aborted shortly after the feeding of the infectious material, and two aborted later. Based on the results of encouraging laboratory experiments MacFadyean \& Stockman recommend immunization with 150 ce. of virulent culture 6 to 8 weeks hefore breeding.

Literature. Nocard, Rec., 1586. 669. - Bräner, D. Z. f. Jm., 18s8. XIV. 95; 1895. XXI. 455. - Sand, ibid., 1895. XXI. 195 (Lit.). - Berger, D. t. W., 95. 117. - Bang, Z. f. Jm. 1897. I. 241; A. f. Jk., 1897. XXXIII. 312. - Guillerey, A. F. Jk., 1903. XXIX. 37 (Lit.). - Ostertag, MIonh., 1901, XII. 356; Hl. d. p. M., 1903. III. 28i. - Preisz, Cbl. f. Bakt., 1903. XXXIII. 190. - Schöttler, A. f. Jk., 1904. XX. 329. - Penberthy, J of comp. Path., 1904. VII. 362. MacFadyean \& Stockman, V. J., 1909. 459. - Holth, B. t. W., 1909. 686. - Nowak, A. P., 1908. XXII. 541.

\section{Infectious Vaginal Catarrh of Cattle. Colpitis Granulosa Infectiosa Bovum.}

(Vaginitis infectiosa granularis s. follicularis, Taginitis verrucosa; Ansteckender Scheidenkatarrh der Rinder [German];

Vaginite granuleuse, infectieuse ou contagieuse [French].)

Infectious vaginitis is a chronic infectious disease of cattle, which is principally characterized by peculiar nodule formations on the catarrhal, swollen vaginal mucous membranes. A Gram negative streptococcus is at present considered to be its cause.

History. The disease was recognized generally only towards the end of the last century. After Isepponi (1887) and Ehrhardt (1896) had observed its occurrence in Switzerland it was described in the vears of 1894-1904 by German authors (Trommsdorf, R. Fröhner, Martens, Ellinger, Georges, Hecker, Ostertag, Raebiger and others), further by Jensen in Denmark, by De Bruin in the Netherlands, by Grátz, Keleti, Ladányi and others in Hungary, and by Saccani and Tosi in Italy. The eause of the disease has been described by Ostertag and Hecker, while knowledge concerning the mode of infection and symptomology was especially furthered by the inquiries of the "Association of Swiss Veterinarians" in 1902, and by the compilation of their results by Hess.

Occurrence. The disease appears to be quite widely spread on the European continent, and most of the cows in the infected herds usually become affected. As some of the affected animals are impregnated with difficulty, abort easily, and subsequently remain sterile, the disease causes considerable loss in cattle breeding, which in severe cases is also increased by a reduction in their nutrition and milk production. 
The disease is widely distributed in middle and northern Germany, and is still spreading. In eastern Prussia alone about 30,000 cows appear to be affecter (Müller), $90-98 \%$ of the cows being usually affected in the infected herds (Raebiger), and in the corporation herds also the frequency reaches $90 \%$. The disease existel in 1901 in Thüringia to such an extent that not a single township, indeed in many places not even a single stable, was spared (Pr. V.-B.). The disease was reported in 1903 from almost every government district, and about $90 \%$ of 30,000 cattle of the East Prussian-Netherlands Society were affected.

The general inquiry in Switzerland showed a very considerable distribution of the disease, as among 4,322 cattle examined in 344 stables, $60 \%$ were founc to be infected. In the western part of Austria one-half of the entire number of cattle are supposed to le affecterl (ReichI). In Hungary it is also widely spread. According to the investigation of Ladányi at the stockyards of Budapest, $43.8 \%$ out of 5,013 eattle showed symptoms of the disease; of the improverl breeds $58.9 \mathrm{c}$, and of the range eattle $43 \%$ proved to le affected, while all of the buffalo cows were found to be healthy. According to the origin of the animals the morbidity in cows from Hungary was $50.7 \%$, from servia $43.8 \%$, from Bosnia $57.8 \%$, from Croatia $52.3 \%$, besides which the disease has also been diagnosed in calves.

Etiology. The organism considered as the cause of the disease is a streptococcus chain consisting of 6-9 cocei, the individual cocei being enclosed in a fine capsule.

Staining. The cocci are easily stained by aqueous anilin dyes while the capsule remains unstained; by Gram's method even the cocei remain unstained.

Cultivation. The streptococei grow on the ordinary alkaline media in the presence of oxygen, at body or room temperature; luxuriant cultures grow especially on agar containing glycerin or urine. Gelatin and coagulated blood serum are not liquefied; in bouillon a diffuse turbidity develops; milk is not coagulated. Neither gas nor indol form in the cultures.

Tenacity. The streptococci are killed in cultures in one minute by corrosive sublimate 1:5000, silver nitrate $1 / 2 \%$, lactic acid $2 \%$, lysol or creolin solution $21 / 2 \%$ (Ostertag). One per cent tannin solution appears to destroy them after 20 hours, while a $1 \frac{1}{2} \%$ solution of bacillol has a rapidly destructive action (De Bruin). From fibrinous purulent discharge of the vaginal mucous membrane, dried on small wooden sticks, the streptococei may be grown on artificial media even after $3 \frac{1}{12}$ years (Heckelmann).

Pathogenicity. Pure culture or vaginal discharge introduced into the vagina of healthy female cattle produces in from 2 to 3 days a purulent vaginal catarrh, in the course of which the discharge at first (according to Thoms as early as from 16 hours on) contains exclusively streptococci, later also staphylococci and colon bacilli. A transmission to other animals is not successful (only DeBruin reports that he succeeded in transmitting it to horses, sheep, goats and hogs).

Following the investigations of Prowazek \& Halberstäiter on trachoma of man, Blahn found that the epithelial cells on the point of the follicular nodules contain very small bodies resembling the trachoma bodies, which sometimes were numerous, again few, and were enclosed in cells or free in great clumps. He considers these bodies, which sometimes have a sickle shape, and are invariably present only on one side of the nucleus, as evolution forms of a protozoon as yet unknown, kelonging to Prowazek's Chlamydozoa. According to his view the disease represents a specific affection of the epithelia, with degeneration of the adenoid layer of the mucous membranes, probably as a result of an obstruction of the glandular 
canals, and subsequently appearing follicular formations. This conception requires confirmation, as does also that regarding the etiological importance of the trachoma bodies (Pomayer already reports that he never observed the bodies in question).

The natural infection results as a rule during the act of coitus; more rarely, but also easily by contact of healthy cows with affected animals, or by straw contaminated with raginal discharge, ichor, or stable utensils, further by the contaminated hands of the attendants during the examination of the genital organs, as well as by instruments which are used in the treatment of affected animals.

Bulls may transmit the disease more readily, as sometimes they themselves may be affected, and besides the infection mar retain its virulence for a long time in the pelvic portion of the urethra, from the flexura sigmoidea to the neck of the bladder (Hess). As a matter of fact bulls infected by diseased cows may within a short time cause the appearance of the disease at different places, by disseminating the infection during service of cows led to them from other villages, and the infection then extends rapidly in the herds. Young cattle are espeeially susceptible to the infection, and the disease has been observed in calves only two weeks old. Young strong cows, and in general animals with tender, sensitive vaginal mucous membranes are more easily, more quickly, more severely and more typically affected than older animals with tougher, less sensitive mucous membranes. Accordingly the disease is observed only very exceptionally in cows over ten years of age, and animals with a chronic affection of the vaginal mucous membrane (prolapse of the vagina, nymphomania), are especially very resistant (Hess).

Female animals are generally more susceptible than males. The nutrition, the method of feeding, and the character of the utilization of the animals has no influence, likewise the period of the year, or the weather (the frequency of the disease in the spring and summer is the result of the breeding period which usually occurs at this time).

Pathogenesis. With the entrance of the streptococci into the mucous membrane of the vagina, they produce on one hand a chronic catarrh, and on the other a swelling of the lymph follicles. They may be demonstrated in the mucous membrane between the epithelial cells, and also in the papillary body. As the nodules characteristic of the disease occur also in the os, and on the mucous membrane of the uterus, and streptococci were demonstrated not only in the secretion of the uterus, but also in the contents of the degenerated cystic ovaries (Heckelmann, Raebiger), sometimes changes result which later render conception difficult, or altogether impossible.

According to Pomayer the inflammatory process always begins at the folds running backwards and forwards from the clitoris, as well as in the prepuce of the clitoris, and extends only later to the lateral and superior walls of the vestibule 



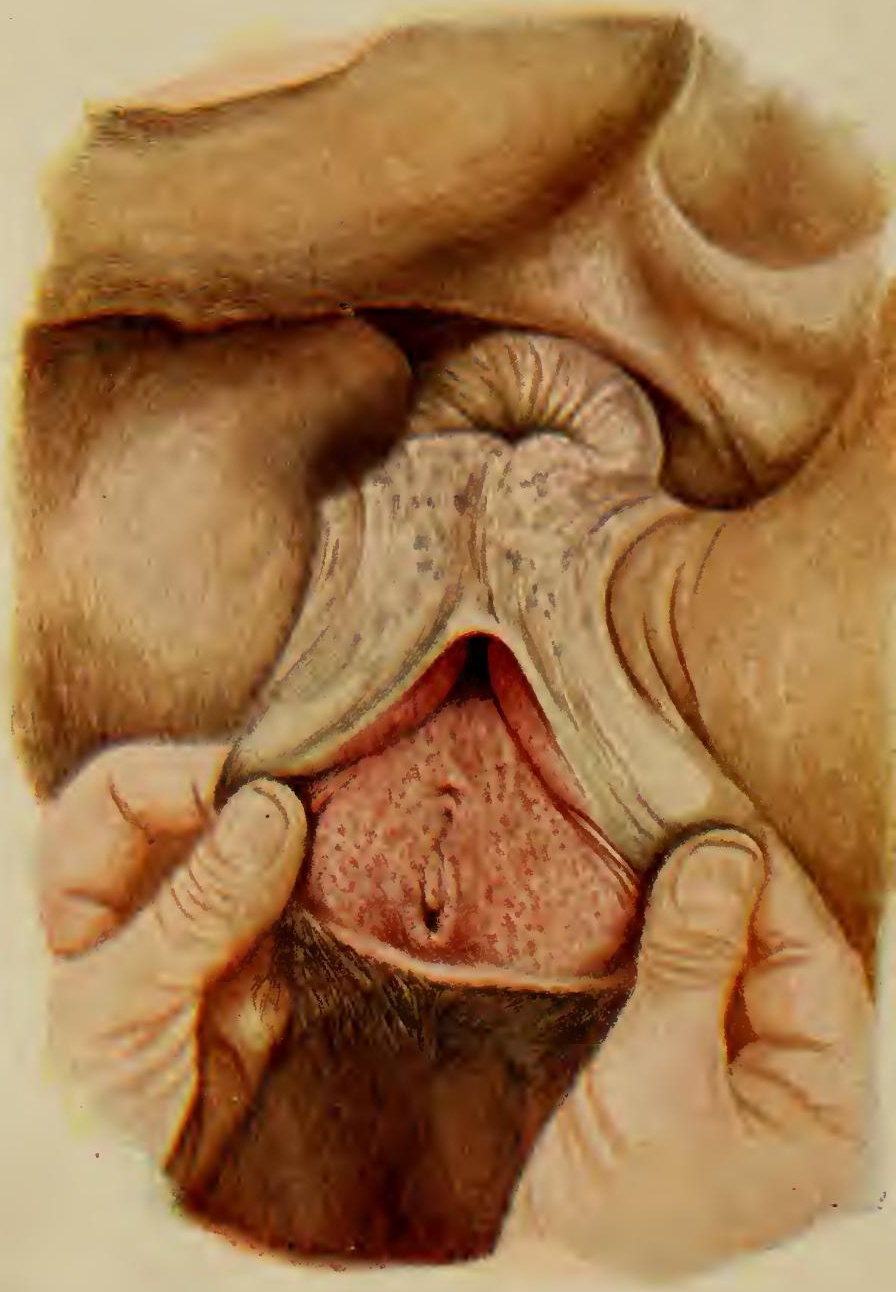

Infeetious Vaginal Catarrh of Cattle

Nodules on the swollen and redlened mucosa of the vagina of a cow. 
of the vagina. By pressure on the anterior wall of the elitoris a drop of yellowish, thick, odorless nueus may be squeezed out from the follicular eavity, which contains diplococei in pure cultures. The specific changes nerer attack the cervix or the uterine mucous membrane, and that affected animals frequently fail to conceive may be explained thus that during copulation the pressure of the penis on the hypersensitive nerve endings of the inflamed clitoris, and the vulva, causes by reflex action the os to be drawn upwards and to elose. Mostly, howerer, sterility, as well as abortion, is caused by a eoexisting uterine catarrh (fluor albus), which exists independently of the specific affection of the vagina.

According to the histological examinations of Thoms, the nodules of the mucous membrane, especially in the surrounding parts of the clitoris, represent principally elub-shaped, thickened papillae of the already normally strongly developed papillary body, while in other parts the nolules develop from one or more follicles, which cause a profusion of the surface of the mucous membrane. After recovery from the vaginal catarrh the follicles gradually retrogress, but they persist in part, although in somewhat less definite form. Therefore recovery from the disease is not necessarily connected with the entire disappearance of the nodules.

Symptoms. The incubation period of infectious vaginitis is, after artificial infections, according to Ostertag, 2 to 3 days, according to Hess 20 to 72 hours, after coitus usually 3 to 5 days, sometimes, however, only 24 hours (Raebiger).

The disease commences with a moderate swelling of the vulva, and diffuse spotted, or streaked reddening, further swelling, formation of folds and increased sensitiveness of the vaginal mucous membrane, when a muco-purulent secretion collects on the surface. In from 1 to 2 days millet seed to half of a hemp seed sized, more rarely even somewhat larger, smooth nodules at first dark red and easily bleeding, appear on the mucous membrane of the vestibule of the vagina, especially on the clitoris and on the folds of its prepuce, as well as in the immediate surroundings. They appear especially sharply prominent by side illumination, forming dense clumps in the vicinity of the clitoris, on the lateral walls of the vestibule of the vagina, however, they are arranged in sagittal rows (Plate VIII), and by stroking the mucous membrane with the fingers they are easily palpable. The tissue of the mucous membrane lying between the nodules shows a diffuse or pad-like swelling and a reddish-yellow discoloration as a result of edematous infiltration.

According to Hess the first changes on the vaginal mucous membrane consist in slightly elevated, red points, which change inside of 24-72 hours to millet-seed sized, pale gray, transparent vesicles. With a severe increase of the inflammatory manifestations they burst after an additional 24 hours, forming very small, regular, superficial, rapidly coalescing minute ulcerations. Meanwhile the mucous membrane is covered with a tenacious, muco-purulent discharge, and only after its disappearance do the highly reddened nodules become prominent on the mucosa, which in part is still bare of epithelia. The nodules therefore are not to be viewed as primary, but as the terminal stage of the process. The resicular state, however, may pass urnoticed on account of its short duration; on the other hand, the described stages may be altogether absent, and the affection manifest itself only in a diffuse catarrhal, or catarrhal purulent inflammation. On account of the derelopment of the process from primary vesicles and the protracted course, the author considers, in agreement with several Swiss veterinarians, that the disease is a severo form of vesicular exanthema. Nielsen is also inclined to accept the view that the resicular eruption represents only an acute form of follicular vaginitis, as he succeeded in producing an acute vesicle formation with the mucous fron cows with chronic nodular raginitis. Jüterbock, however, failed to observe in 4,000 cases, vesicles on the vaginal mucous membrane in a single instance.

Vol. 1-48. 
Simultaneously with the development of the nodules, a vaginal discharge appears in the majority of cases, which is at first very scanty, later somewhat more profuse, glassy, tenacious, and sometimes more muco-purulent in character. It is odorless, alkaline or neutral in reaction. The discharge soils the parts surrounding the vulva, where it dries to brown crusts; exceptionally a profuse secretion forms, which passes in long strings from the vagina.

The general health of the patient is usually undisturbed, and the body temperature is not elevated. The catarrh of the vaginal mucous membrane however causes frequent micturition, and sometimes also straining for action of the bowels. At the same time frequent shifting of the feet, swinging the tail, occasionally also standing with legs widely spread, a stiff gait, rubbing, kicking against the abdomen, and arching of the back is observed. Only in rare, severe cases are appetite and rumination absent, or is the milk secretion suppressed.

This clinical picture remains almost unchanged from 3 to 4 weeks, whereupon the process gradnally passes into a chronic stage, which is manifested by the nodules becoming pale, a disappearance of the discharge and of all acute, inflammatory symptoms, the mucous membrane appearing of a light yellowish shade. The nodules are then glassy transparent or yellowishgray, and no longer surrounded by a reddened zone. The process may remain in this form for weeks and months, until finally recovery ensues.

Heckelmann describes as a rare finding in the first eight days numerous yellowish-gray-white spots on the mucous membrane, which represent either erosions covered with broken-down epithelia or grayishyellow membranes adhering strongly to the mucous membrane, while dark red nodules appeared later. Probably similar findings eaused Ladányi to distinguish a special eroding form of the disease, which, according to his observation is manifested so that on the swollen yellowish mucous membrane, poppy seed to lentil-sized, always yellowishwhite, sharply circumseribed spots appear, which are not elevated over the surrounding parts, and which degenerate from the center, whereby the mucous membrane obtains a peculiar, reticulated appearance, until finally, in from 10 to 14 days, the superficial loss of substance heals without formation of nodules.

Regarding the influence of vaginal catarrh on pregnancy and subsequent conception, the views of the practical veterinarians vary widely. As nodnles were found in some cases on the mucous membrane of the uterus, apparently the result of an extension of the inflammatory process from the vaginal mucous membrane, and possibly also by aspiration of the infected semen during copulation, an unfavorable influence on the development of the fetus cannot be disregarded. In harmony with this many observers report on relatively frequent cases of abortion in affected cows, and still more frequent are 
the observations in which oestrum appears repeatedly in the animals, and conception only after repeated copulations, or not at all. On the other hand there are also numerous observations at hand according to which the disease has no unfavorable influence on either conception, or on the full development of the fetus.

These contradictory results of observations are probably due to the variations in the character of the disease. On the other hand the possibility is not exchuded that conception is prevented by a non-specific uterine catarrl, and the abortion to which Bang lias already called attention, is not brought on by the vaginitis, but hy "infections abortion." Both forms of the disease mar be confouncled with each other, as in infectious abortion, the follicles of the vaginal nucous membrane may also be swollen, hesides the animals affected with infectious vaginitis may very likely be affected also with the virus of the above-mentioned affection. As a matter of fact the observations in which most, or the total number of cows, or twothirds, and even $80 \%$ of the animals failed to conceive, as a result of the affection, can be hardly credited, in view of the nature of the somewhat mild local disease process. The same may be applied also to the supposed connection of raginal catarrh with the severe purulent, or septic inflammation of the uterine mucous membranes, as well as with affections of the ovaries. In consideration of the uncertainty of this, acenrate and especially bacteriologic examinations wonld be very desirable.

Aceording to Heckelmann's experience, out of 448 affected cows $65 \%$ con ceived with certainty, $14 \%$ probably, while $21 \%$ failed to become pregnant again. In the district of Olpe in 1902 only 48 out of 296 affected cows gave birth to living, well developed calves. According to Raebiger sometimes $68 \%$ of the affected aninals ahort, while Ellinger claims $50.70 \%$ of normal births. Swiss veterinarians also report on frequent sterility $(30-50 \%$, and even more) in affected animals, Nuesch claims infections vaginitis as the cause of non-conception in $80 \%$, Fasciati in 60-70\%; some veterinarians, however, have failed to observe such result in their practice, and accorling to Rusterholz conception fails only in a certain form of the disease.

Bad results in these directions have been olserved in Hungary only in recent times. Keleti established that in 20 herds, 17 ont of 503 affected cows remaineil sterile, and 7 aborted, while in the same locality out of 499 healthy cattle 1.5 remained sterile and $s$ aborteil. The percentage was therefore in both grours quite uniform $(4.8$ and $4.7 \%$ ). Likewise there was no difference in primiparae, whether affected or not. Korarzik failed also to observe had results, and Landler noterl the eonception of all affected animals in a herd of 240 , all of which gave birtb normally. Recently, however, he has not infrequently observed abortion and still more frequently sterility.

In regard to the publications on the economic losses from infectious vaginitis, Angstein states that the almost uniform sterility or abortion of the animals is by no means as extensive in practice as might be inferred from published reports; as a matter of fact, the disease appears very frequently in herds without the owners having the slightest idea of its existence, as they fail to observe any detrimental results.

Bulls which have covered affected cows become affected rarely and then usually but mildly. In such cases they show dullness and frequent micturition, which is sometimes asso- 
ciated.with pain; the penis is highly reddened and painful, exceptionally nodules develop on its surface and on the inner lining of the sheath a muco-purulent discharge being emitted from the opening of the sheath. Bleeding is sometimes observed during the act of covering. Swiss veterinarians further report nodules and vesicles on the mucous membrane of the penis, ulcerations on the orifice of the sheath, painful swelling of the scrotum, and even necrosis of the testicles, while Jiiterbock regularly found on slaughtered bulls pronounced follicular formations on the mucous membrane of the penis.

Course and Prognosis. The course is mostly very protracted. Although sometimes the acute inflammatory svmptoms, and with them the vaginal discharge subside inside of two weeks, in the great majority of cases they last from 2 to 3 months, and even longer. With the retrogression of the swelling of the mucous membrane the nodules become paler, yellowish, or grayish-transparent, whereupon they disappear entirely, or persist in this form for months. Their healing appears to be favored by pregnancy, but in such cases there is probably only an apparent disappearance of the nodules, as a result of the physiological swelling of the vaginal mucous membrane, as in some cases they become again more prominent after parturition (Hess).

The prognosis depends, aside from the character of the disease, first of all on the inaugurated treatment as well as on the existing hygienic conditions. In early and energetic treatment it is as a rule favorable, as under these conditions recovery of fresh cases ensues mostly in 2 to 4 weeks, chronic cases however are usually characterized by a marked stubbornness. Further the possibility of quite frequent recurrences must also be considered, which may result either after mechanical irritation (repeated copulation) or as a result of re-infection.

Diagnosis. For the cases running a normal acute course, aside from the epizootic character of the disease, the development of small nodules in association with inflammatory symptoms of the mucous membrane of the vagina, and especially of the vestibule is characteristic, as well as the fact that the nodules do not change into vesicles or pustules, and that they do not break and form ulcers. In the exclusive presence of nodules the diagnosis should be made with care, as the lymph follicles may also swell in infectious abortion, and from other inflammatory causes, as well as from simple traumatic irritation of the mucous membrane (Attinger, Jüterbock), (some authors even doubt their pathological nature). The diagnosis is especially difficult in pregnant cows, as nodules possibly present on the swollen mucous membrane in these cases are not, or only indistinctly visible, and appear only 10 to 15 days after parturition (Jüterbock). 
The vesicular exanthema with which the disease is frequently confounded, is associated with febrile symptoms, in which on the vaginal mucous membrane lentil to pea-sized vesicles, and only a small number of hard nodules develop within 3 to 4 days, which however change into vesicles, and later into ulcerations. Besides in this disease complete recovery follows in 2, more rarely in from 3 to 4 weeks. In infections abortion the nodules which are sometimes observed on the vaginal mucous membrane appear only several days after the expulsion of the fetus, and disappear shortly after. Moreover the usually profuse vaginal discharge contains the bacillus of abortion in great numbers. Of course animals may be affected with both diseases simultaneously. Profuse and especially fetid vaginal discharge in general indicates a complication with one or another inflammatory affection of the genital organs.

According to Schneider a vaginitis may develop in weakly or poorly developed cows after copulation by mechanical means, which is manifested by a marked reddening and frequently follicular swelling of the mucous membrane, as well as a mucopurulent rlischarge, in severe cases also in erosions and ulcerations.

Treatment. One of the most important requirements for satisfactory results consists in cleanliness, disinfection, and most painstaking care of the stables, also in the careful supervision of copulation, in order not to hinder the healing process by reinfection and fresh additional inflammation.

The most varied antiseptic and astringent remedies are recommended for treatment in various forms of application. The results however depend not so much on the selection of the remedies, as on the care and the accuracy of their application, as well as on the persistence of the treatment. In general it is recommended to repeat it in short (daily), later in longer (2 to 3 days) intervals, and to continue at least until the complete cessation of the discharge, if possible, even until the disappearance of the nodules, therefore on an average for 5 to 6 weeks.

For local treatment, which may be supported effectively hy cleaning and washing the external genital parts with lnkewarm soda solution or with $1-2 \%$ lysol or creolin solution, irrigation of the vagina with mildly acting disinfecting solutions is indicated, especially in acute cases, as in such treatment the remedies come in contact with all parts of the mucous membrane. In less acute and more protracted cases, tampons, painting, powdering, liniments, salves and suppositories come into consideration, all of which exert an intensive healing action at the point of application and in their immediate surroundings. Considering their easy application, as well as the fact that in this the remedy remains for a longer time in contact with the affected parts of the mucons membrane, these methods of treat- 
ment are especially advised in cases in which the daily application of irrigations meets with insurmountable obstacles, or in which the affection is found to be confined to the vestibule of the vagina.

The most varied disinfectants and astringents, further also cauterizing remedies have been recommended for treatment, thus especially for irrigation lysol and creolin (0.5-2\%), bacillol $(1.5-3 \%)$, potassimm permanganate $(1 \%)$, nitrate of silver $(0.5 \%)$, lactic acid $(2 \%)$, boracic acid $(3 \%)$, liquor cresolis compositus (1\%), alum (1\%), formaldehyde (1.5-1\%), protargol and ichthyol (0.5-2\% ; expensive), ichthargan (1:1000, expensive), and others. All may be used in similar concentrations, also for saturation of tampons for introduction into the ragina. For painting, iodide of glycerin $(1: 1)$, tincture of iodine with alcohol (1:2), further Lugol's solution may be employerl. Bacillol, ichthyol, chinosol, formaldehydum saponatum (septoform), and recently the hydrargyrum sozojodolicum (marketed as Bissulin, in the form of crayons), all in $10 \%$ concentration, are most frequently utilized as ointments. If they are to be applied in the form of suppositories, or so called vaginal balls, they are prepared with tallow, wax or cocoa-butter. Of dusting powders are recommended, four parts of sulphate of zinc or alum, and one part of boracic acid; bacillol with boracic acirl $(1: 30)$; boracic acid with flour or powdered oak-bark, $\bar{a}$ and others. Finally for superficial cauterization of the nodules $1 \%$ nitric acid, or $3 \%$ nitrate of silver solution may be used.

Technique of Treatment. The local treatment is inangurated by washing the vagina with a $1 \%$ luke-warm soda solution, in order to remove the secretion collecterl therein. If severe symptoms of irritation exist, it is allvisable to cover the restibule of the vagina, on the first day, with an ointment containing tincture of opium.

The irrigations are executed with the aid of a rather stiff rubber tube of an irrigator, or with a bulb syringe having a dull nozzle, a considerable quantity of fluid is to be introlluced, in order that it may penetrate up to the uterine os, and into all folds of the nucous membrane. Following this, a wad of cotton saturater with the same solution, and with a long string attached, may be introduced as leeply as possible into the vagina, where it remains until the following day, if not expelled before that time, when it may be removed by drawing it out by the protruding string.

For the application of ointments Raebiger's ointment syringe, constructed especially for this purpose is adapted, which makes possible the introduction of the remedies into the depths of the vagina and also the accurate measuring of doses. Besides a rolled rag may be covered with the salve, and placed in the vagina at such a depth that the front part touches the os uteri (Rusterholz); fiually the salve may be also used in gelatin capsules.

Taginal balls or bougies, such as are at the present marketed by many concerns and in varying compositions, are, after previous superficial warming in warm water or over a flame, introduced with the fingers or with a spoon forceps deeply into the vagina and allowed to remain.

Nale animals also require a treatment for which it is arrisable to remove the brush of hair at the opening of the sheath with scissors, whereupon the surrounding parts are washed with luke-warm soal water, and finally the sheath is irrigateil with a non-irritating disinfecting solution, or the extended penis during a copulation trial is covered with the salve.

To promote conception it is advisable to irrigate the vagina before copulation with a luke-warm $2 \%$ solution of sodium bicarhonate. 
Prevention. For preventing the spread of the disease it is advisable to segregate the healthy animals from the affected ones, to disinfect repeatedly and thoroughly the infected stables, and especially the stalls, as well as the draining gutters, and if possible to prevent the affected cows and bulls from copulation during the duration of the disease. This measure can be carried out in practice only at considerable expense on account of the resulting falling off in the number of calves. In bulls which are exposed to the danger of infection the brush of hair at the opening of the sheath should be cut short, and the sheath should be irrigated after every copulation with a mild disinfecting solution. Finally, in order to prevent the introduction of the disease, newly bought female animals shonld he carefully examined for the presence of the disease.

Literature. Isepponi, Schw. A., 1883. XXIX. 1. - Ehrhardt, ibid., 1896. XXXVIII. 80. - Hecker, B. t. W., 1900, 445. - Ostertag, Monh., 1901. X11. 533. - Raebiger, D. t. W., 1901. 454. - Zschokke, D. Unfruchtbarkeit d. Rindes, usw. Zürich, 1900; Schw. A., 1904. XLVI. 281. - De Bruin, D. t. W., 1905. 392. Attinger, B. t. W., 1905. 845. - Hess, Bericht üb. d. von d. Gesellsch. schweiz. Tierärzte veranst. Tntersuchung betr. d. Knötchenseuche. Bern 1905. - Keleti, Állatorvosi Lapok, 1905. 409; 1906. 229. - Larlányi, ibiıl., 1906. 97. — Thoms, Monh., 1906. XVII. 193 (Lit.). Casper, T. Z., 1907. 393. - Jüterbock, Z. f. Tm., 1909. XIII. 354 (Lit.). Pomayer, B. t. W., 1910. 173. 
Group VI.

\title{
INFECTIOUS DISEASES PRODUCED BY PROTOZOA.
}

\section{Piroplasmoses.}

\author{
(Babesioses, Malaria of Animals.)
}

To the group of piroplasmoses belong those diseases which sometimes show an acute, at other times a chronic course and are caused by a unicellular protozoa, the so called piroplasma (pirosoma, bahesia). The parasites are inoculated into the body fluids of mammals by ticks, and in penetrating the red blood corpuscles they produce their destruction, causing anemia, and frequently also hemoglobinuria, as well as icterus to rlevelop in the animals.

General Morphology and Biology of the Causative Factors. Piroplasmas are umpigmented, roundish, pear, ring or rodshaped protozoa with a nucleus which probably encloses a blepharoplast (centrosom), and are closely relater to the
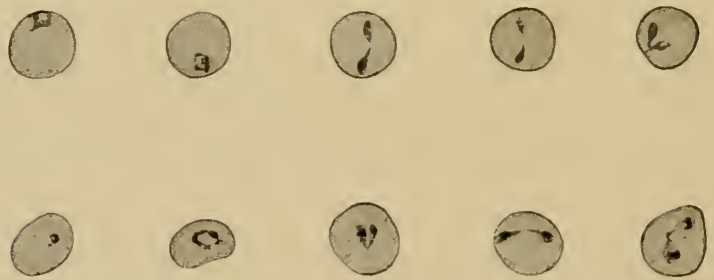

Fig. 128. Piroplasma bigeminum: Typieal ring and pear shapes. The upper row stained with alkaline methylene blue, the lower after Romanowsky. (After Kossel \& Weber).

hemosporidia. In the inside of the red blood corpuscles, where they are usually found, they are frequently present in pairs, in which the pear shapes meet eac's other with their pointed ends. In the fresh condition they show ameboid movements.

Their reproduction has, up to the present time, not been accurately investigated. In the blood of the host it results probably by the way of direct fission, or schizogony, in which 
the division of the plasma body is preceded by a division of the nucleus. Besides, according to the more recent investigations changes in generation appears to occur in the body of the ticks, as is indicated by the fact that certain species of piroplasma are transmitted to mammals only by certain ticks, and only by progenies of the ticks which liave sucked the infected blood. The nature and mode of this propagation however is not yet sufficiently clear, as the investigations along this line with various piroplasma gave variable results (further information is given under the various forms of the disease).

Staining. In cover glass dry preparations fixed in the air or in alcohol, the piroplasmas stain uniformly with methylene blue or other aqueous anilin dyes, while the preparations which are stained with one of the muclear staining methods by Romanoswky-Ziemamn, Laveran or Giemsa, disclose one or more chromatin granules in the blue stained plasma.

Cultivation. Attempts at cultivation in various ways, mostly in bouillon, have given only partially positive results, however, they were suceessful in keeping the parasites alive for a time ontside of the animal body, and in demonstrating in them morphologieal changes, which are considered by some observers to be stages of development (see p. 766).

The differentiation of various species of piroplasma depends partly on the characteristic form and size, and first of all on the fact that the individual species are strongly bound to certain mammals, so that they camnot even artificially be transmitted to other species.

Pathogenicity and Natural Infection. With few exceptions (coast fever, tropical piroplasmosis), the piroplasma nay be transmitted artificially with the blood of an affected animal containing the parasite, by subentaneous or intravenous inoculation, whereupon after an incubation period of several days, or even several weeks they appear in the circulating blood, and some species will produce the symptoms of their respective disease.

Under natural conditions all piroplasma are transmitted from affected to healthy animals by ticks, and not only in a purely mechanical way, but also in that not the tick which sucked the mammalian blood containing the piroplasmas transmits the disease, but its immediate or more remote offspring. 'This is possibly because the piroplasma undergo in the ticks in the meantime certain stages of development. Some piroplasma, as those of Texas fever, the piroplasma of $\log \mathrm{s}$ and of sheep, pass from the female ticks which suck the blood of affected animals, through the eggs to the larvae, and likewise also to the later forms, whereby the parasites are inoculated into the 
body of the mammals either by the larvae nymphs (Texas fever), or by the sexually ripe ticks (piroplasmosis of sheep and $\operatorname{dogs})$. Other piroplasma do not pass through the eggs, but are transmitted only by the nrmphs, which have sucked the infected blood as larvae, or by matured ticks, which sucked it as nrmmphs (tropical piroplasmosis, piroplasmosis of horses).

With a few exceptions, a species of ticks transmits only a certain species of piroplasma, while one certain piroplasma may be transmitted in different localities by different kinds of ticks.

The ticks are arthropodes, which as Ixodinae and Argasidae form the family of Ixodidae. As transmitter's of the piroplasma: the Ixodinae come exclusively into consideration, while ticks of the genus Argas transmit the infection in spirochetoses.

The Ixodinae are obligatory parasites and exist on blood which they suck from mammals by pricking the skin with the proboseis, more correctly with the hypostome. The females impregnated on the hosts fall to the ground, where they lay eggs on blades of grass. The hatched larvae look for a host in order to suck blood; in some species they then develop on the same animal up to the nymph stage or also to sexual maturity, while in others the larvae as well as the nymphs, or the nymphs alone, leave the host, in order to develop into the next stage. According to the nature of the development the Ixodidae may be combined into the following groups (Meuleman):

1. Species which pass through their development on the same host; here belong the species of the Boophilus (Margaropus, Rhipicephalus, blue ticks), namely B. annulatus, B. decoloratus, B. australis and B. argentinus (according to Neumanu only varieties of one species); all are transmitters of Texas fever, the B. decoloratus transmits also tropical piroplasmosis.

2. Species which leave the host as nymphs; here belong Rhipiceplialus evertsi (red tick: transmitter of East African coast ferer, and the piroplasmosis of horses), Rh. bursa (transmitter of piroplasmosis of sheep), and Hyalomma aegyptium (transmitter of Texas fever?).

3. Species which leave host as larrae and as nypuhs; here belong Ixodes ricinus s. reduvius (transmitter of piroplasmosis of eattle in Europe, probably also of piroplasmosis of (logs), Rhipicephalus appendiculatus (brown tick), Rh. nitens, Rh. simus (dottel black tick), anl Rh. capensis (transmitter of coast fever), Rh. sanguineus and Haemaphysalis leachi (transmitter of piroplasmosis of dogs).

Pathogenesis. Some piroplasma, as for instance, the ordinary piroplasma of cattle (Texas fever), of dogs, and of sleep, exert their pathogenic action by penetrating the red blood corpuscles and causing their destruction. According to the intensity of the affection a larger or smaller number of red cells is destroyed, as a result of which a more or less pronounced anemia develops, and simultaneously the hemoglobin of the attacked cells is eliminated into the blood plasma. If only a small number of erythrocytes is destroyed, only a hemoglobinemia results, the eliminated hemoglobin is then changed in the liver into bilirubin, and eliminated as such, while in a greater destruction of blood corpuscles a part of the hemoglobin is passed with the urine, and icterus sets in at the same 
time. The debris of the cells at the same time cause emboli in the various organs, especially in the kidneys, as a result of which hemorrhages develop, and parenchymatous inflammation is produced in the kidneys (Smith, Betegh), manifested in the presence of albumen in the urine, which exceeds the hemoglobin in quantity. The rapid diminution of the number of red blood corpuscles, and the disturbance in nutrition which is always present, lead finally to death from exhaustion in a large proportion of the cases.

According to the investigations of Barratt \& Yorke, the haemoglobinuria develops in piroplasmosis of dogs as a direct result of the haemoglobinemia only when the blood plasma contains as much hemoglobin in solution as $0.5 \%$ red blood corpuscles in proportion to the total volume of the plasma. At the same time the red coloration of the urine is pronounced only in a marked and rapid destruction of erythrocytes, while in chronic cases it is usually not observed, as in these the destruction reaches a greater proportion, but develops only gradually.

Contrary to the above piroplasmosis, hemoglobinuria is not observed in East African coast fever, or in tropical piroplasmosis, and only exceptionally in piroplasmosis of horses, although a great proportion of the erythrocytes contain the parasites. These affections are manifested in the acute cases by more or less pronounced symptoms of a general blood in-

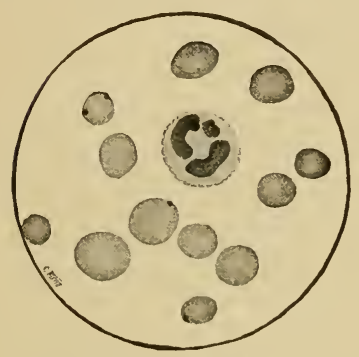

Fig. 129. Anaplasma marginale. Chromatin staining. (After Theiler). fection, and the anemia appears only in the more chronic cases. The cause of the difference in the pathogenic action is at the present unknown.

Animals which have recovered from an acute attack continue to harbor the respective piroplasma in the red blood corpuscles, even for years, in spite of the apparent recovery and satisfactory nutrition, although only in small numbers; they may also be present in animals which, probably on account of their high resistance, have never shown manifest symptoms of the disease. Such animals are constant virus carriers, and may be the cause of the infection of ticks, thereby aiding the spread of the disease among susceptible animals. At the same time they themselves are usually immune against later infection with the same piroplasma (cattle which have recovered from coast fever are incapable of transmitting the infection).

Anaplasma Marginale. In America and Africa, in localities in which the piroplasmoses are prevalent, the hlood of animals, especially of eattle, which pass through the disease, eontains erythrocytes with very small, round or oval, marginal bodies (Fig. 129), staining with the chromatin, the nature and importance of which is still in doubt. Smith \& Kilborne described them as peripheral, coccus-like bodies, 
Theiler as marginal points. These authors, as well as Knuth, Springfeldt and Spreull are inclined to consider them as special blood parasites, while Dschunkowsky \& Luhs consider them as piroplasma spores and are of the opinion that they produce the chronic form of tropical piroplasmosis. Theiler named them recently anaplasma marginale, as they consist only of chromatin substance, and he proved that after inoculation with blood which contained such bodies, they appeared in the blood of the inoculated animals, sometimes also causing severe febrile affections. According to his most recent experience he considers these parasites as the cause of the so-called gall sickness which is prevalent in Africa (see p. 783).

Literature on piroplasmas and piroplasmosis in general: Lïhe, $\mathrm{Hb}$. d. Trop. enkrkh., 1906. III. 193. - Hennig, ibid., page 744. - Sehilling, Hb. d. p. N., Ergänzb., 1906. 75. - Panisset, Rev. gén., 1906. VII. 113. - Kaestner, D. tierpathog. Protozoen, Berlin, 1906; Ergeb. d. allg. Path., 1907. XI 1. 496 (all with literature). - Rickmann, Tierz. u. Tierkrkh in. D. Südwestafrika. Berlin 1908. Doflein, Protozoenkunde. 1909, 653. -- Knuth, Z. f. Infkr., 1910. VII, 141 (more recent literature). - Theiler, Bull. Soc. Path. exot., 1910. III. 135.

\section{(a) Piroplasmosis of Cattle. Piroplasmosis Boum.}

(Texas fever, Reduater, Blackwater, Tick fever, Southern cattle fever, Haemoglobinuria s. Babesiosis boum, Haemo-

globinaemia enzootica, Malaria boum; Piroplasmose

der Rinder [German]; Piroplasmose du boeuf

Mal de brou, Mal de bois [French];

Ematinuria, Piscia sangue [Italian];

Tristezza [Spanish].)

This febrile infectious disease, which is usually observed in enzootic extension and in the course of which hemoglobin appears in the urine as a result of the breaking down of red blood corpuscles, is caused by the piroplasma bigeminum, which is in various localities inoculated into the bodies of cattle by various kinds of ticks.

History. The disease was first studied bacteriologically in Roumania by Babes (1888), who considered as its cause a bacterium which usually was present in the red blood corpuseles (Haematococcus bovis); he has also proved that the disease may be transmitted with the blood and kidney substance from affected cattle to healthy animals. Shortly after him (1889), Th. Smith, later in association with Kilborne, established for the Texas fever which is prevalent in North America, the fact that the parasite represents a hemosporidium, and that the disease, as already claimed by Reverley in 1881, is spread by a tick, the Boophilus bovis, in such a manner that the tick inoculates blood containing the parasites from affected animals into the bodies of healthy cattle.

The publications which have followed each other rapidly since that time, have established the occurrence of a disease which is identical with Texas fever, or at least very closely related to it, in many different localities. Thus Krogius \& Hellen established it in Finland, Kragerüd in Norway, Celli \& Santori in Italy, Nicolle \& Adil-Bey in Turkey, Jackschath and Zieman in Germany, Lignières in France, Gasille and de Jong in 
Holland, Hutyra in Hungary, Nocard, later Stockman in England, Bettencourt in Portugal; further Koch in East Africa, Theiler and Hutcheon in South Atrica, Stockman in India, Sanarelli and Lignières in South America, finally Pound and Tidswell in Australia.

Kossel \& Weber (1899) showed further for Finland, and later in association with Schütz \& Miessner (1903) for Germany, that the infection in Europe is transmitted by the Ixodes reduvius. These same authors, as well as Smith \& Kilborne, and also Lignières before them, have suggested a satisfactory method of immunization for the practice.

Occurrence. The disease occurs usually in the form of an enzootic in the spring and in the summer in certain localities, especially in marshy and forest pastures, whereas in the cold periods, and during stable feeding it occurs only exceptionally. The so-called forest disease (hemoglobinuria), which was formerly attributed to the ingestion of various plants, especially to those containing turpentine, and which was accepted as an intoxication caused by resinous substances (turpentine), is identical with the disease is question.

According to general experience older cattle become usually affected, and the disease sometimes causes very decided losses among the improperly wintered cattle of poor owners.

The disease causes great losses in Germany, especially in those localities in the northern part, where, as a rule, all animals are pastured, and among these in unfavorable years an emphatic decimation of the animals may result. It oceurs especially frequently in Prussia anıl Oldenburg, then in Bavaria, Würtemberg, Baden, Hessen, Mecklenburg, Braunschweig and in the imperial domains, but it is also observed as a permanent disease of spring and summer in other localities in marshy and moorland pastures.

In Rommania it occurs most destructively in the inundated territories of the Danube Delta, where the disease in some years causes a loss of 30,000 to 50,000 cattle.

In Hungary the "forest disease", prevails, especially in the mountainous regions of northern Hungary and Transylvania and occurs there in some localities almost every spring; it has also been observerl in the southern part of the country.

Texas fever has for a long time been known in America around the sea-girt portions of Mexico; in the middle of the last century the disease was introdnced to the United States with herds driven from Texas, and while it showed in its original home a very mild course, in the North it caused enormous losses among the native cattle, the money value of which has been estimated in some years as $\$ 2,000,000$. In the northern part of South America it also causes periodically great losses, especially among imported cattle. It also oceurs as a dangerous disease in India (Schein observed it there also among buffaloes), in Central ancl South Africa, further in Australia, where it was introduced by American cattle.

Etiology. The cause of the disease, the Piroplasma bigeminum (Babesia bovis, Pirosoma bigem., Apiosoma bigem., Ixidioplasma bigem.), occurs in the blood of febrile cattle, mostly inside of red bloor corpuscles, more rarely on their surface, or free in the blood plasma. The parasites are found eitler singly as roundish bodies of $1-2 \mu$ diameter which in a fresh state show ameboid movements, or in pairs in which case the somewhat larger, non-motile, and pear-shaped parasites (pirum = pear) frequently hang together with their elongated ends in a straight line, or at an angle (Fig. 128 on p. 760 and Fig. 130). Smaller diplococcus-like, as well as 
rod and lancet forms are found rarely, and the presence of more than two parasites in one blood corpuscle is also infrequent.

The cultivation and propagation on artificial media lias not yet been successful with a certainty.

According to Lignières and Miyajima they are supposed to multiply in bloodbouillon for a time, and here, according to the observations of the Japanese investigator, forms containing flagellae similar to the trypanosomes are supposed to appear after 3.4 days; the propagation reaches its height on the 10 th to 14 th day; on the 45th day, however, the culture died (it is possible that they represented mixed infections).

According to Smith \& Kilborne, as well as Laveran \& Nicolle, their propagation results by fission, or schizogony, while according to Theiler's former view, which was also accepted by Koch, the parasites assume, in certain stages of development, rod- and ring-shapes, and then appear similar to the cansative factor of African horse fever (Fig. 131). Such forms are usually found in the blood of cattle that are immune against Texas fever, and they also appear in the blood of calves, which have been infected with the blood from such cattle, during or after the second reaction. Recently, however, these forms were described by Theiler as a new variety, (Piroplasma mutans), (see p. 782).

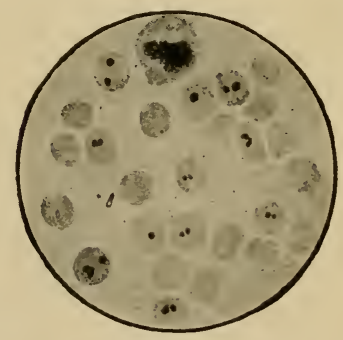

Fig. 130. Piroplasma bigeminum. Spherical and pear shapes. Cattle blood; stained with methylene blue.

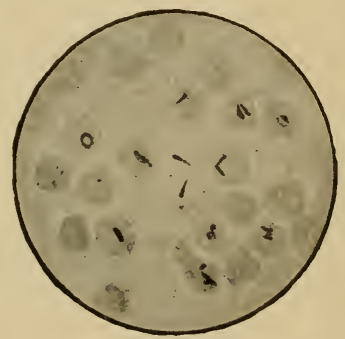

Fig. 131. Piroplasma bigeminum. Rod and ring shapes. Blood of a cow in a stage of commeneing convalescence; stained after Laveran.

Koch examined material from ticks which sucked themselves full of blood from affected animals, and found that, after leaving the blood corpuscles, the parasites become elongated to club shape in the intestines of the ticks and develop lancet-like projections, which may be slowly drawn in and extended (morning star shapes). Later the extensions become shorter, and diminish in number, whereupon the parasites change into round or pear-shaped bodies. Such bodies are found, also, in the eggs of infected ticks, but as they are 3-4 times the size of the piroplasma in the blood of cattle, it is possible that between the two there are still other transitory forms. (Kleine observed star forms also in dog piroplasma, which he placed in strong salt solution. Hartman, however, considered them as manifestations of degeneration.) 
Tenacity. Defibrinated blood kept at $8^{\circ} \mathrm{C}$. is still virulent after 42 days, while in the blood of cattle which have died or have been slaughtered by bleeding the parasites remain alive for nine days. In the meat of slaughtered animals they die at latest after twelve hours (Kossel, Schütz, Weber \& Miessner). Their rapid death may possibly be due to lack of oxygen, as well as to the acid reaction of the media which acts destructively on the piroplasma (Jackschath).

Pathogenicity. The disease may be transmitted to healthy cattle with blood containing piroplasma of affected animals. After an intravenous, subcutaneous, or intramuscular inoculation of 5-10 ce. of defibrinated blood, the parasites appear usually in from 8 to 9 days in the blood of the inoculated animals. At approximately the same time the body temperature becomes elevated, whereupon, during the reaction which lasts for 7 or 8 days, the number of red blood corpuscles diminishes, and in some of the cases hemoglobin appears in the urine. After 25 to 30 days another febrile reaction follows, nsually with milder symptoms. In some animals the artificial disease terminates in death, in the majority of cases however recovery takes place in one to two weeks. In the blood of such recovered animals the parasites may be demonstrated for a long time, although in smaller numbers (in one case the blood was still infectious 531 days after an artificial infection, and according to Schroeder \& Cotton the parasites may persist for 10 to 12 years). The disease may also be produced, although with less certainty, by the injection of blood containing piroplasma into the serous cavities, lungs, brain, further by rubbing upon the scarified skin; feeding is, on the other hand, always ineffective. To an infection which causes a severe affection among older cattle, cattle under one year of age usually react only with an elevation of temperature lasting for several days. Other species of animals are not susceptible to the infection.

In America, Africa, Australia and Eastern Asia, as well as probably in Sonthern Europe, the natural infection is transmitted by the boophilus species, in middle and Northern Europe, on the other hand, by the Ixodes ricinus (I. reduvius).

According to Salmon \& Stiles, the carrier of the parasite is the boophilus annulatus, in North America, and, according to Motas, also in Roumania; in South Ameriea, Cuba, Porto Rieo, in the Philippines and Australia, the B. australis, in South Africa the B. decoloratus (according to Theiler also the Rh. appendiculatus), act as carriers, while of the sub-family ixodiae, the Ixodes ricinus has only been known with eertainty to transmit the disease in Europe (according to Kragerüd the I. hexagonus acts in a similar way in Norway).

The piroplasmas of eattle transmitted by various ticks in different localities do not appear to be entirely identical, but probably represent varieties of one species, and Lühe even separates them into two species, apparently on account of the differences in size, namely, into the small, Babesia bovis, which is found in Europe and North America, and the larger, Babesia bigeminum, which is prevalent in America, Australia and South Africa. Their difference appears also to be substantiated by the fact that animals immunized against one of the varieties are not always immune against the other varieties. This has been established 
by Lignières for the European and Argentine piroplasmosis, by Theiler for the English and South African redwater; (on the other hand, eattle from Texas and from Queensland were immune against the South African redwater).

Lignières describes a special species, the piroplasma Argentinum, which is considerably smaller, usually only found singly in the erythrocytes, and produces in Argentine, Uruguay and Paraguay, an atypical form of Piroplasmosis, which is frequently characterized by a protracted and fatal course, and by nervous symptoms.

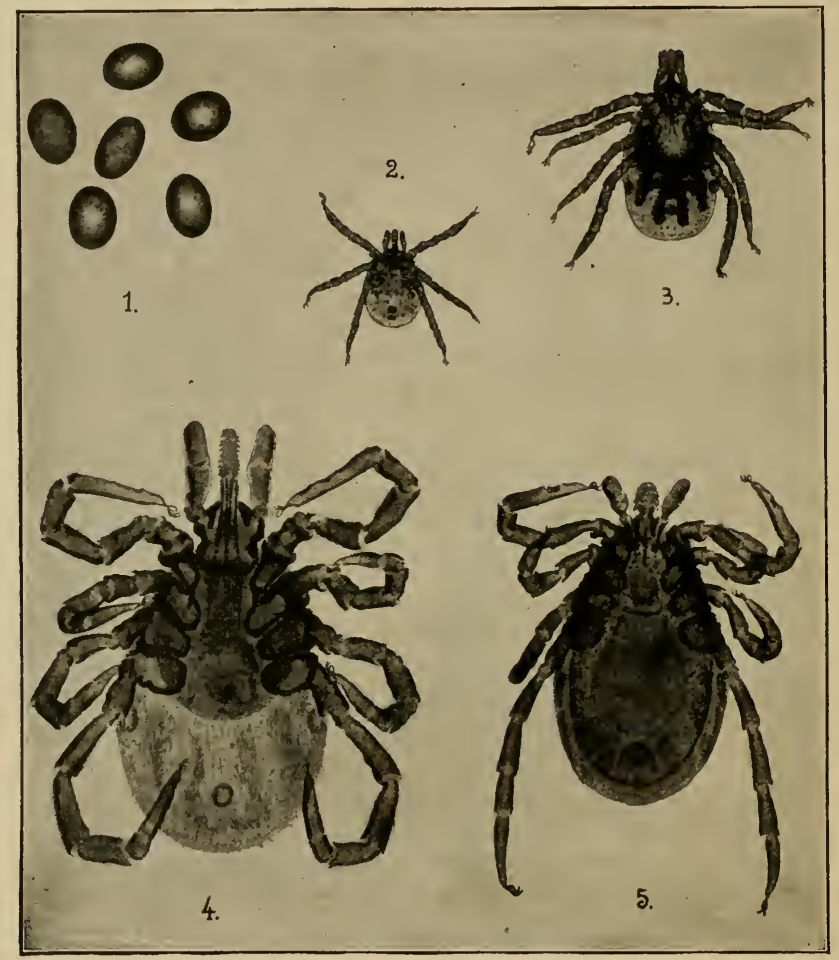

Fig. 132. Ixodes reduvius. 1. eggs; 2. larvae; 3. nymph, several days old; 4. sexually mature female; 5. male. Enlarged 16 times. (After Kossel, Schütz, Weber \& Miessner.)

The parasites of East African coast fever, and of tropical piroplasmosis represent a decidedly independent species (see p. 779 and p. 782 ).

Development of the Ticks. The Boophilus annulatus (Margaropus s. Rhipicephalus annulatus, Boophilus bovis) transmits, according to the investigations of Smith \& Kilborne, the infection in the following way : 
The larvae migrate from the grass onto the bodies of eattle, and suck their blood. After about eight days they change into pupas, and after another eight days into nymphs, which after another molting develop into sexually mature ticks. The nymphs, as well as the sexually mature ticks nourish themselves with the blood of the host animal, following im. pregnation however the female drops from the body of the cattle and lays from 2000 to 4000 eggs in the grass, from which the larvae hatch out in 3-4 weeks. They remain for a time in the grass, occasionally how. ever they crawl on the skin of the pasturing cattle, and if the female tick from which they originated has sucked infected blood during its life, the larvae, and subsequently also the nymphs, inoculate the virus into the body of the new host. Larvae and nymphs may also pass over directly from affected animals to healthy eattle, in which case they transmit the blood parasites in a direct mamner.(?)

The Ixodes ricinus s. reduvius transmits the infection, according to the investigations of Kossel, Schütz, Weber \& Miessner, in a singular manner; the female tick dropping from the body of cattle lays in the grass 100-1000 eggs, from which the larvae hatch out after an average of six weeks. If the larvae attach themselves to the skin of cattle or of other animals, they suck themselves full of their blood, and drop off. after 3-6 days, whereupon they change to nymphs on the ground inside of four weeks. These attach themselves agam to the skin of a mammal, from which they drop to the ground in from 3-5 days, where they develop in eight weeks to sexually mature ticks, which again look for a. host, where they suck the blood, and copulate. Therefore the total development from the laving of the eggs until the dropping off of the impregnated female takes about nineteen weeks, provided that the ticks in their different stages of development always meet a suitable host and that the weather conditions are favorable for their development. Accordingly the Ixodes distinguish themselves from the Boophilus among others, in that they leave the host twice during their development, whlle the Boophilus passes through all the stages of its development on one and the same host.

The eggs of the Txodes reduvius are $0.50-0.5 \mathrm{~mm}$ long, and 0.27 to 033 $\mathrm{mm}$. broad, of oval shape, and brownisli-yellow to brownish-red in color. The larva is $0.7-0.8 \mathrm{~mm}$. long, $0.42-0.50 \mathrm{~mm}$. broal, has a mite-shaped body, and three pairs of legs; filled with blood it attains a length of $1.25 \mathrm{~mm}$. and a breadth of $0.8 \mathrm{~mm}$., when it takes on a blackish-brown color. The nymph is $1.3 \mathrm{~mm}$. long, $0.74 \mathrm{~mm}$. broad; the body resembles the larva, but possesses four pairs of extremities; sucked full with blood it is about $2.0 \mathrm{~mm}$ long and $1.25 \mathrm{~mm}$. broad and of a dark grayish color. The developed female is $3-4 \mathrm{~mm}$. long and $1.5-2.5 \mathrm{~mm}$. broad; impregnated and sucked full with blood it reaches a length of $10-15 \mathrm{~mm}$, and a brearth of $5.8 \mathrm{~mm}$.; it is then blue or ash-gray in color, sometimes slightly brownish or yellowish, and has four pairs of extremities. The male is similar in appearance, although somewhat smaller (Fig. 132).

The larvae, nymphs, and the sexually ripe ticks may, with sufficient moisture, remain alive for months outside of the animal body, and they also withstand the cold of the winter without harm. In cold weather they are benumbed, but as soon as warm weather appears they liven up, and if conditions are otherwise favorable they continue in their development. They are harmed however by direct sunlight.

Experiments of infection conducted with both species of ticks showed that when larvae, hatched ont from an infected female in a glass container, are placed on the skin of healthy 
cattle, the piroplasma appear in the blood in about eight days, and in some cases fever and hemoglobinuria sets in (Fig. 133). The results of experiments conducted with nymphs which as larvae sucked blood from affected cattle, are similar, and the disease may be also produced with larvae, which have wintered in the open (the infectionsness of the sexually mature ticks of the species described here is as yet questionable).

According to these experimental results, which correspond with practical observations, the natural infection of cattle occurs usually on previously infected pastures in which one of the mentioned species of ticks are present, and in which the larvae and nymphs originated from females which had the opportunity to suck blood from affected animals or in which the nymphis themselves in a previous stage of development sucked infected blood. As these ecto-parasites subsist as a

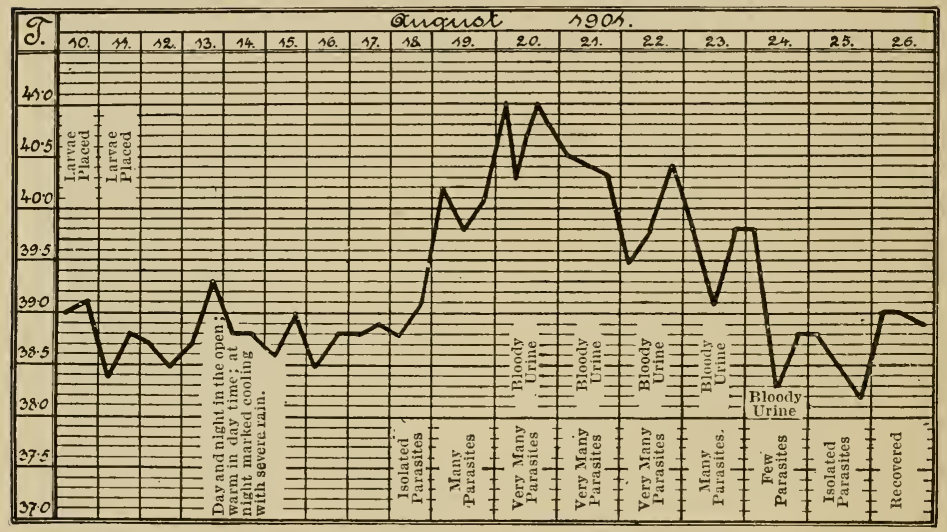

Fig. 133. F'ever curve in piroplasmosis of cattle. After placing sereral thousand infected larvae on the animal. (After Kossel, Schütz, Weber \& Miessner.)

rule in marshy places, especially in forest pastures or near forests and bushes, the disease usually affects cattle driven to such pastures. On the other hand its annual reappearance may be explained by the facts that the virus passes from the impregnated female ticks to their progeny, and that they are capable of offering considerable resistance to unfavorable weather conditions, especially the cold of winter. If at the onset of warmer weather the larvae have already hatched from the eggs, the first cases of disease among cattle driven to infected pastures usually appear after two weeks and, in case the respective pasture was badly infected the previous year, a great number of the animals may become affecterl within a short time. With stable feeding, however, the disease is observed only very exceptionally, and only in cases in which 
infectious ticks were introduced into the stables with the grass or foliage from infected pastures.

The introduction of the disease into localities which had been free from the infection, may be brought about by infected animals, and the more so as piroplasma may be present in the blood of cattle years after their recovery from the disease. In such cases the disease may be artificially produced with the blood of such apparently healthy cattle. If ticks which are suitable for the transmission of the virus are present in these localities, they suck blood from the cattle introduced into that locality, and pass the piroplasma to their progeny, which then infect healthy animals. Affected or healthy cattle may carry ticks about on their bodies, and these will continue to propagate in the new locality, provided the requirements for their development are favorable, where they may then infect fresh animals. In both cases the pasture, formerly free of the infection, becomes a new source of infection, in which the disease may possibly recur from year to year.

The extensicn of the disease was strikingly observed in America, where in the $60 \mathrm{~s}$ and $70 \mathrm{~s}$ of the last century it was introduced by eattle driven from Texas to the north, to the states of Indiana and Illinois, whence it extended later in a similar manner almost through the entire territory of the United States up to Lake Michigan. After the trailing of the cattle from the sonth, as a rule in from 30-50 days, which period corresponds to the eycle of development of the Boophilus tick, the native eattle became affected with severe symptoms (Reverley, Smith \& Kilborne). The disease was introduced into Australia by American cattle, with the Boophilus ticks, which harl been nnknown there up to that time, and it causes there great loss from year to year. It also spread recently in Finland to territories which harl heretofore been free from the affection (Krogius \& Hellen).

Although the disease develops exclusively through infection by ticks, certain accessory conditions have an influence on its appearance, and more so on its course. Thus as a rule very young animals show a greater resistance against the infection than older cattle. Poorly nourished, as well as generally weakened animals usually become affected with severe manifestations; unfavorable weather conditions, especially damp, cold weather, also exert an unfavorable influence on the course of the disease. This explains the experience that the affection is often observed in a severe form in the damp, cold spring periods, and especially among improperly wintered animals of poor owners (Kragerüd observed it repeatedly in such a form in cows 12 to 14 days after parturition).

Native cattle raised in affected territories usually become affected under much milder symptoms than animals brought from uninfected localities. The greater resistance of the former may be explained by the supposition that they became infected while young, and thereby acquired for their later lives a relative immunity, which was still more increased by infections developing in the meanwhile. Such animals, however, may also carry the virus in their blood in spite of their healthy appearance, and this may sometimes multiply rapidly on ac- 
count of harmful influences, and produce a severe affection (such acute outbreaks of the previously latent disease have frequently been observed after artificial infection with rinderpest blood) (see p. 255).

Anatomical Changes. Animals dead from the disease show changes in the digestive organs and in the spleen. The mucons membrane of the abomasum shows a catarrhal swelling and manifests, especially in the pyloric region, numerous small hemorrhages and erosions. Similar changes are present on the mucous membrane of the intestines and especially on those of the rectum, the contents of which is colored reddish on account of the numerous and extensive hemorrhages of the mucous membrane. The liver is enlarged, the substance flabby, lusterless, and covered with yellowish streaks and spots on a dull reddish-brown base. The gall bladder frequently contains great quantities of thickened bile. The spleen is always strikingly enlarged, its pulp is rery rich in blood, brownish-red, or brownish gray-red, and frequently softened, almost liquefied, the capsule extended or even ruptured (Witt). The bladder contains a great quantity of pale or dark-red urine, its mucous membrane is sprinkled with red discolorations.

The autopsy findings are further: Hemorrhages in the serous cavities, sometimes also in the brain substance and bone marrow, cloudy swelling of the kidneys, frequently with punctiform hemorrhages, serous infiltration of the subcutaneous and subserous connective tissue, hypostatic hyperemia of the lungs, exceptionally also catarrhal pneumonia with sero-fibrinous pleurisy. The blood is pale-red and thin, the red blood corpuscles of the spleen and liver contain piroplasmas in a proportion even up to $80 \%$. In some cases icterus is also present. (According to Piot the temperature in the abdominal cavity rises after death to $44^{\circ}$ and over.)

Symptoms. According to experimental investigations with larvae of ticks, the period of incubation ranges from 8 to 10 days.

The disease commences with a rapid rise in temperature, and usually with pronounced dullness and debility, accelerated respiration and heart action. The temperature remains for several days at $40^{\circ}$ to $42^{\circ} \mathrm{C}$., whereupon in mild cases it gradually returns to normal, while in severe cases it rapidly drops below normal before death.

In pasture the animals rest frequently, fall back behind the herd and ruminate with long interruptions; exceptionally symptoms of irritation, involuntary movements, and even delirium are observed at the onset of the disease. At first there is constipation, later the animals show srmptoms of colic and tenesmus, dry yellowish-brown, later thin, mashy feces, mixed with mucous or blood coagulum. The milk secretion 
is immediately diminished; the milk drawn in small quantities is sometimes yellowish and has a bitter odor.

In a later stage the animal manifests a staggering gait, and at times may knuckle over with the hind feet. Muscular trembling may be observed in some parts of the body, especially on the upper arm, shoulders, and croup. There is profuse lachrymation, the flanks are sunken, the skin feels dry and hard. The lymph glands in the knee folds swell early in the disease to a great extent (Schmidt). The mucous membranes are at first reddened, later they become pale, and show symptoms of icteric discoloration.

The blood drawn from a vein is more or less pale red and watery; after coagulation the separated blood serum also shows a red discoloration. The number of red blood corpuscles drops in several days from 7 to 8 millions per cmm., to one-half or one-third, in fatal cases even one-fifth to one-sixth of the above number. Within them piroplasma may be found in varying proportions, in severe cases from 5 to $58 \%$ (Fig. 130 and 131). The hemoglobin content may drop to $12 \%$ (Fleischl).

The microscopical demonstration of the parasites may lie successful eren in fresh bloor specimens, where they appear in the blood cells as pale, shining, on a warm object stage somewhat motile, coccus-like borlies (easily mistaken for the plasma extensions of the crenaterl blood cells!). A certain result is olstained from dry specimens fixed in absolute alcohol, or in a mixture of alcohol and ether $\overline{a a}$, and stained with a $1 \%$ aqueous methylene blue solution. The parasites on such slides appear colored blue. For the demonstration of the finer structures, anil especially of the chromatin bodies, Romanowsky's methor of staining or one of its modifications (Giemsa stain!) must be used. The red blood corpuscles proper show indications of a poikilocytosis and in their interior basophilic granulations.

The urine contains even in the early stages, albumen in various quantities; even when normal in color it has a high specific gravity, later, sometimes as early as on the second day, it becomes of a reddish or greenish color, as a result of the appearance of methemoglobin, probably also of bile pigments. The color gradually becomes more intense and brownish to blackish-red; at the same time in shaking much foam forms on its surface, while on boiling it coagulates to a jelly-like mass. On standing a great amount of sediment appears, in which numerous yellow granules, likewise also kidney epithelia and casats may be demonstrated, while red blood corpuscles are found only very exceptionally. The passing of the urine is always accompanied by severe straining.

In the cases with infarorable termination the patients become weakened after 3 to 4 days to such an extent that they constantly lie down, the respiration is very labored, and from time to time a painful grunting may be heard. At the same time convulsions may appear in certain groups of muscles, tears ooze out from between the swollen eyelids, and saliva drops out of the month, until finally under a rapid sinking of the body temperature death ensues, which however may 
occur suddenly under symptoms resembling those of anthrax (de Jong), or even without any previous indication.

In mild cases the fever disappears about the middle of the first week of illness, at the same time the urine clear's up, and the appetite as well as rumination returns. The animals which in the meantime have become greatly emaciated, do not regain their former condition for several weeks.

In some cases, especially in young animals, only a moderate fever which lasts but a few days, somewhat accelerated respiration and pulse, diminished appetite, as well as evident dullness are observed. The color of the urine does not however undergo any change, and the moderate destruction of the red blood corpuscles is only indicated by the paleness and slightly yellowish discoloration of the mucous membranes. Such patients recover very rapidly after the disappearance of the described symptoms.

Sexually mature ticks may be found on the body surface of affected animals, frequently in very great numbers in the parts surrounding the vulva and the rectum, on the udder, on the inner surface of the thigh, and in the inguinal region, while the larvae and the nymphs lodge on the head, in the vicinity of the muzzle, on the eyelids and ears, as well as on the udder.

According to Dschunkowsky \& Luhs a piroplasmosis of the type of Texas fever occurs in Trans-Caucasia, which runs a very rapid course of 2-3 days. It is always associated with hemoglobinuria and characterized by a high mortality. No parasites are found in the peripheral blood, while almost all erythrocytes of the parenchymatous organs are infested.

Course and Prognosis. If affected animals continue to remain on infected pastures, they sometimes die with a rapid aggravation of the symptoms as early as from 4 to 7 days, but the course may also extend over a longer time. If they are however removed immediately after the appearance of the first symptoms to an uninfected place, recovery follows in most cases. Damipness and cold, likewise very hot weather, influence the course of the disease unfavorably. Accordingly the mortality varies within wide limits ( 5 to $50 \%$ ), and is usually higher in southern warmer localities than in the temperate zone.

Rapid sinking of the body temperature to $37^{\circ}$ and below, during continual excretion of hemoglobin with the urine, indicates threatening danger, while a gradual diminution of the febrile symptoms with strong pulse, and clearing of the urine, indicates improvement. Sometimes however the improvement is only temporary, and may later, as a result of a reinfection, or under the influence of unfavorable weather conditions, again become aggravated. Cattle which have passed through an attack of the disease are usually immune against later infections. 
Diagnosis. Hemoglobinemia and hemoglobinuria which appear rapidly under febrile symptoms, characterize severe affections, and in cattle on known infected pastures a red discoloration of the urine is sufficient for a well foumded suspicion of the disease. If however the last-named symptom is absent, the presence of the disease may be suspected on the ground of febrile symptoms, only when at the same time other animals in the herd are affected with characteristic symptoms, in which the probability of the diagnosis is considerably increased by the presence of ticks in various stages of development on the body of the animals. Absolute certainty is obtained by the demonstration of piroplasmas in the red blood corpuscles on microscopical examination (see p. 773), or by artificial production of the disease in healthy animals $(10 \mathrm{cc}$. of fresh or defibrinated blood subcutaneously or intravenously).

Anthrax and hemorrhagic septicemia may be confused with the acute cases of piroplasmosis, as in those diseases the febrile symptoms are sometimes also accompanied by a red-colored urine, but their course is more rapid, no anemia develops, the urine contains red blood corpuscles, the examination of fresh blood shows long rods, or small bipolar bacilli between the blood corpuscles. The disease is distinguished from hematurias hrought on by other causes, by the absence of red blood corpuscles in the urine, from the so called "chronic hematuria" it is distinguished by its less chronic course, and the much more rapid development of anemia.

On autopsy the above-mentioned diseases should also be kept muder consideration, and especially anthrax, on account of the acute swelling of the spleen, and the numerous hemorrlages which may be present.

Treatment. After an outbreak of the disease in a pasture the herd should be removed immediately from the infected locality to a moderately cool, shady place, and most appropriately to stables where they can be given careful attention. At the same time suitable and sufficient feeding (good dry food, with the addition of potatoes or beets), is of great importance. In addition to this, the ticks should be picked from the body of the patients, or destroyed by washings with creolin, lysol or salt solutions.

Internal treatment has so far been of but little ralue. Salines may be administered against constipation, and the diarrhea treated with slimy gruels, combined with astringents or opiates. In great weakness stimulating treatment (rubling with alcohol, internally, alcohol or black coffee) is indicated. The quinine treatment (10-20 gm. of qumine hydrochlorate per os), which lias been recommended (Padovani, Hellens, Kröning), probably on account of the similarity of the disease with malaria in man, has not given uniform results. Kragerüd recommends intravenous injections of formalin or collargol (100-500 gm. of a $1 \%$ solution), and later carbolic acid or lysol internally 
(Acid. carbol. or lysol 10.0, Spir. frumenti 100.0, Aqua 500.0; one tablespoonful every hour until clearing of the urine).

Evers warmly recommends damholid (a hemoglobin preparation). The treatment should be introduced with the administration of $50 \mathrm{gm}$. (three times daily) per os. If no improvement results or if even an aggravation follows, the remedy should he injected subcutaneously or even into the blood circulation (200 gm. damholid is dissolved in one liter of $1 / 4 \%$ itrol solution, and of this 500 ec. or more is injecterl subeutaneously, or of a $10 \%$ solution 100-250 ce. is injected into the jugular; after the subcutaneous injection of simple watery solutions malignant edenia has repeatedly been observed). Westermann and Grabe have obtained good results with this treatment.

Recent experiments of Nuttall \& Hadwen, which have since been confirmed by Stockman, appear to indicate that the piroplasmas are greatly influenced by trypanred. In five artificially infected cattle in which the blood already contained numerous parasites and the urine was red colored, a marked improvement followed immediately after an intravenous or subcutaneous injection of $130-200 \mathrm{cc}$. of a $1.5 \%$ solution, the urine cleared rapidly, and the number of blood parasites diminished to a great extent. Later the blood was entirely free of parasites for 5-18 days, they again reappeared, although in moderate numbers; but all of the five cows remained alive, whereas one of four controls, of which only two were affected with hemoglobinuria, died. The trypanblue solution should always be prepared fresh with distilled water. It stains the body tissues blue.

After the disappearance of the symptoms in the presence of marked anemia, subcutaneous or intravenous injections of physiological salt solution (1-2 liters daily), and the internal administration of iron preparation $(5-10 \mathrm{gm}$. iron sulphate daily), is indicated.

Prevention. The disease may be prevented absolutely by keeping the animals from infected pastures and exchuding grass and foliage fodder from such localities for food purposes. On the other hand such pastures may become harmless by draining their swampy areas. Such means of prevention however frequently camnot be carried out on account of the lack of funds of the owners. As some animals show very little resistance to infection, as a result of improper wintering, care should be taken that weakened cattle are not driven to low forest pastures in the spring and early summer (according to Lignières alfalfa is supposed to keep the ticks away from pastures).

In order to prevent the introduction of the disease to previously uninfected territories, it is aimed in America and Australia to free the suspected animals of ticks. For this purpose animals in the southern part of the United States are dipped in vats containing 20\% petroleum or Beammont oil, while in Australia washing with various insecticide fluids (lysol, petroleum) is employed for such purposes. In order to destroy the ticks farmers in South Africa usually spray the animals in 12 to 19 day intervals, with a mixture of oil and water $(10-25: 100)$ (Lounsbury). For the same purpose a fluid is also suitable consisting of $0.2 \%$ arsenic and $0.2 \%$ wood tar. 
In the United States and North America the destruction of ticks was inaugurated on a large seale in 1906 . On this occasion 220,780 cattle were infested with ticks in 29,315 examined herds, and 328,064 were found to be free (Steddom). The procedure consists in placing the herds for three weeks in enclosed pastures free of ticks, until the young ticks become mature anu drop to the ground. Then the animals are driven to another uninfected pasture, and their former stands are disinfected. It is also aimed to clear the cattle pasture from ticks by keeping the cattle away for about six months, when the ticks are destroyed by starvation, or large pasture areas are burned over in the spring.

Immunization. As cattle recovering from the disease acquire a long-lasting immunity in North America (Schroeder) and Australia (Gray), cattle brought from non-infected territories into affected localities are infected artificially with blood of calves born in infected territories or of recovered cattle, which, as a rule contain piroplasma sparingly. After such inoculations the animals become sick after 8 to 10 days, sometimes with symptoms of anemia, hemoglobinuria, frequently also with bloody diarrhea, and at the same time the number of red blood corpuscles, some of which may contain typical piroplasma, diminishes $10 \%$ or more. This symptom disappear's after 8 to 10 days; after a month, however, a second reaction usually appears, which lasts 8 to 10 days, and the red blood corpuscles contain only atypical piroplasma in very small numbers (Theiler). If cattle so treated are driven to infected pastures after the lapse of about two months, a number of animals resist the natural infection, in the others, however, fever appears again as a result of the tick bites. In most of the animals recovery finally takes place, but a certain percentage succumb to the disease (Francis). Inasmuch as this percentage is considerably smaller than the loss observed from non-inoculated animals under the same conditions, the inoculation appears to be advantageous in strongly infested localities, in spite of the fact that the inoculation itself causes disturbance of nutrition, and sometimes even direct losses.

Blood from older cattle which have recovered shortly before from the disease is also suitable for immunization, and gives similar results.

The immunizing action of the blood from animals shortly before recovered has been confirmed through experiments, by Smith \& Kilhorne, also Schroeder in North America, Francis in Texas, Hellens in Finland, Koch in Africa, Theiler in Kossell, Schütz, Weber \& Miessner in Germany.

In Germany the immunization is carried ont with blood of artificially infected ealves, and has recently been employed extensively in practice; until the end of 1909, 6,153 cattle were immunized, principally in Prussia. According to the reports on 4,261 inoculated cattle, $1.97 \%$ became mildly and $0.49 \%$ severely affected, and recovered, and $0.09 \%$ died or were slaughtered. Besides, during pasturing $2.98 \%$ became mildly, $0.54 \%$ severely affected and recovered, $0.54 \%$ died or were slaughtered. The percentage in young stock amounted to only $0.04 \%$, in adults $1.46 \%$. In 10 herds of the immunized animals $4.14 \%$ were mildly, $0.46 \%$ severely affeeted; of those not immunized $19.63 \%$ mildly, and $7.48 \%$ severely affected (Schmitt). Satisfactory results are reported by Graffunder and Bugge. Less satisfactory 
were the immunizations in Texas, where up to the end of 1901 out of 1,251 immunized cattle 116 or $9.2 \%$ died later; likewise in Australia (Queensland, where inmunization of 35,000 cattle caused a loss of $3-4 \%$, and besides, of 17,960 immunized animals 679 , that is $3.6 \%$ succumbed later to the natural infection. But in one experiment, out of 95 previously immunized animals all remained healthy after attaching ticks to them, while 30 non-immune cattle became affected as a result of it.

Technique of Immunization. For the inoculation, blood is taken from the jugular vein of eattle which have passed through a natural or artificial affection about two months previously. The blook is defibrinated, and either nsed immediately or kept in the ice ehest until the inoculation. According to Kolle, bloor containing parasites from calves reared in infected localities is lest arlapterl, accoriling to Sichütz blood from artificially infected calves which have recoverert at least within 83 days. Three ce. of the defibrinated blood are injected subcutaneously, and if possible the operation should be undertaken during the winter or at the beginning of spring. It is best to undertake the inoculation on calves. Cows in advanceil pregnaney, poorly nourished, or in general animals which are not entirely well should not be immunized.

The immunized animals should be kept in the stable for three weeks and fed moderately before they are driven to pasture.

Other Methods of Immunization. Dalrymple, Morgan \& Dodson also obtained satisfactory results in America, by taking from the body of affected animals ticks sucked full with blood, grinding them, and inoculating cattle subcutaneously with the material, while Connaway \& Francis, in America, recommended the application of infected larvae or nymphs (the first time 20-50, after a month again 200-400) on the skin of healthy animals.

Lignières produces vaccine by cultivating (?) the piroplasma in defibrinated blood from affected animals, which produce in healthy eattle only a very mild affection at the same time immunizing them (the immunization requires three inoculations. The experiments carried out in Bnenos Aires and Alfort gave satisfactory results, as immunized cattle resisted artificial as well as natural infections, but there are no reports available relative to its application in practice. The method of preparing the vaccine is likewise $m$ mnown.

Blood serum of eattle which have recovered from the disease is not suitable for the immunization of cattle.

Literature. Babes, C. R., 1888. CVII. 692; V. A., 1889. CXY. 81. Smith \& Kilborne, An. Ind., 1893. Bull. Nr. 1. - Theiler, Schw. A., 1595. XXXVII. 3; Fortschr. ․ Vhyg., 1903. I. 133; Y. J., 1910. 99; Bull. Soc. Path. exot., 1910. III. 135. - Lignières, La tristeza, etc., Buenos Aires 1900 ; Arrh. de paras., 1903. VII. 398. - Kossel \& Weber, Arb. đ. G.-A., 1900. XVII. 460. - Techn. Dep. f. 1. Tet. Wesen. A. f. Tk., 1901. XXVII. 41. - Kragerüd, Z. f. Tm., 1901. V. 244. Kossel, Hb. d. p. M., 1903. I. 840 (Lit.). - Kossel \& Schütz, Weber \& Miessner, Arb. d. G.-A., 1904. XX. 1. - Schmidt, A. f. Tk., 1904. XXX. 42. - Knuth, Diss. Leipzig 1905. - Korh, Z. f. Hyg., 1906. LIV. 1. - Evers, B. t. IV., 190S. 45S. Witt, ibid., 190^. 628. - Nuttall \& Hadwen, ibid., 1910. 38.

\section{(b) East African Coast Fever.}

(East coast fever, Rhodesian reduater, Rhodesian tick fever; Ostafrikanisches Küstenfieber [German].)

This dangerous form of piroplasmosis in cattle is distinguished from the previously described affection especially in that it cannot be transmitted from animal to animal by the blood. Its cause is the Piroplasma parvum. 
The nature of the disease was deternined by the investigations of Theiler in the Transval, and of Koch in Buluwayo.

Occurrence. The disease formerly existed in a latent form along the East African coast, a severe outbreak however occurred in 1900 when some very susceptible cattle were brought to the Portuguese territory, to Beira (Koch; Creutz believes that the Australian cattle introduced the virus). With the severely affected cattle it was then spread into the interior of Africa, especially to German East Africa, Natal, the Transvaal and Rhodesia, where it caused great loss among the cattle herds.

In 1905 about 500 farms were infected in the Transval, and inside of a year about 50,000 eattle succumber to the disease.

Etiology. The Piroplasma parvum (Babesia parva), the smallest of piroplasma known at the present, may be seen in its characteristic form as a fine rod-shaped intracellular body, which on one end contains a chromatin granule, and frequently appear arranged in cross or willow leaf-shaped form; ring and dise shapes also occur, while pear-shaped twin forms are never present (Fig. 134).

The disease cannot be prockiced artificially by the inoculation of blood containing the parasite. Healthy cattle withstand subcutaneous, intravenous or intraperitoneal injections of such blood without harm, only when they are inoculated two weeks later for the second time, a rise in temperature sets in immediately, and after 10 or 12 days more a mild form of the disease appears, wherempon parasites may be demonstrated in the blood. Meyer obtained positive results by introducing large pieces of spleen from affected animals into the abdominal

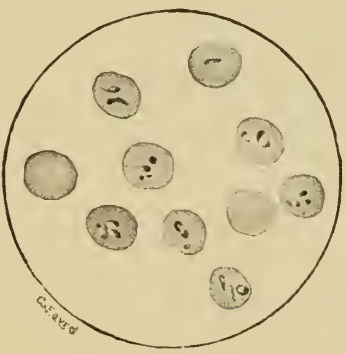

Fig. 134. Piroplasma parrum. Cattle blood, chromatin stain. (After Theiler.) cavity of healthy cattle, whereupon after 12 days the parasites appeared in the blood with a simultaneous rise in temperature.

The natural infection is transmitted by varions ticks, especially by nymplis of the sexually mature forms of Phipicephalus appendiculatus, further by sexually mature forms of Rhip. evertsi, Rhip. simus, Rhip. nitens, and Rhip. capensis, which in the earlier stages of their development have sucked blood from affected cattle. The parasite does not pass through the egg of the tick as is the case with the piroplasma bigeminum, neither is it transmitted from immune animals to health cattle (Theiler, Lichtenheld). Its development in the body of the 
tick is similar to that of the piroplasma bigeminum (Koch see p. 766$)$.

Stockman succeeded in producing the disease in cattle in England with nymphs of the brown ticks sent there from Africa.

The infection always occurs during pasturing, especially easily after the appearance of the rainy season and during the high grass season when the ticks, which are present in great numbers, easily jump from the high grass blacles to the cattle. As a inatter of fact, previously healthy cattle become affected with a dangerous form of the disease after they are pasturerl in the same field with animals from infected localities, or if they are driven over infected pastures.

In the endemicly infected territories on the coast and in lowlands cattle under one year of age usually become affectecl. In these the loss usually amounts to 60 to $90 \%$, while older animals are immme if they have already passed through the disease. The infection in the highlands is less severe, as there hardly more than $15 \%$ of the increase in stock die of the disease. For this reason older cattle in these localities are immune only to a slight extent, and a certain number of them fall victims to the disease (Lichtenheld).

Contrary to the described conception on the etiology of coast fever, Ollwig, as well as Fiblleborn is of the opinion that the cause is possibly ultra-microscopic, as it does not produce anemia, is not directly transmissible, and that the disease leares an absolute immunity. Piroplasma mutans and Piroplasma parvum are, according to their riews, not only morphologically but also etiologically identical, and both types probably produce the piroplasmosis which extends in all tropical and sub-tropical localities, and which shows variations only in its manifestations. This conception is also substantiated by the experience that eattle which have recorered from coast ferer are no longer infections for ticks, and therelyy they are apparently free of the virus.

Anatomical Changes. The antopsy reveals punctiform hemorrhages in the subcutis and the subserous comnective tissue, medullary or hemorrhagic swelling of the lymph glamds, edema of the lungs and of the mediastinum, liyperemia, or ulcerative inflammation of the intestinal mucous membrane and of the ahomasum; further grayish-white spots in the liver suhstance, and as a specially striking lesion hazelnut-sized, wedgeshaped infarets in the cortex of the kidneys. The spleen is not swollen.

The specific bodies, so-called plasma spheres (Koch), are present. in the swollen lymph glands, in the spleen, further in the spots of the liver and in the infarcts of the kidneys, as well as in the horders of the nleers of the mucous membrane, likewise also in the blood. By Giemsa 's stain they appear as sharply circumseribed, blue-colored spheres, containing point or comma-shaped chromatin granules, or they are covered by such.

Colland considers the formation of infarcts to be due to an injury of the endothelia of the ressels by endocellular toxins of the niroplasma. Similar toxins are supposed to be also prodnced by the Piroplasme bigeminum, only that they attack the erythrocytes. 
Symptoms. The incubation period is 10 to 12 days (Theiler, Kleine), visible symptoms however appear usually only 20 days after the time of exposure in infected pastures. These consist in high fever, difficult respiration sometimes associated with a cough, salivation, passing of very dry or bloody, tar-like feces, marked swelling of the submaxillary lymph glands, emaciation and weakness of the hind quarters. Contrary to Texas fever the appetite remains normal for a long time, and anemia, icterus and hemoglobinuria are only seldom observed. At the height of the fever parasites may be demonstrated in the red blood corpuscles, sometimes infesting 80 to $90 \%$. At the same time the destruction of blood corpuscles is only slightly evident. Cattle infected with Piroplasma bigeminum may later become affected with coast fever, in which case the symptoms of Texas fever are also pronounced, and the parasites of both diseases may be found side by side in the blood.

The disease is very destructive, as in previously noninfected herds $95 \%$ of the animals die, whereas in native cattle on the coast of East Africa deaths are rare.

Prevention. The infection may be kept at least temporarily from healthy herds by the careful segregation of suspected or affected animals (fenced in!). Dipping and spraying for the destruction of ticks, after fresh outbreaks, and driving the cattle into non-infected fields are advantageous preventive measures. In severely infected pastures the ticks may be infectious even after eight months, and even when there have been no cattle pastured there in the meantime. The danger of the pasture is eliminated after $151 / 2$ months, if during that time it is pastured only by horses, goats or sheep.

Immunization. (Koch). Animals which have recovered from the disease are protected against a second attack even if remaining in an infected pasture, repeated injections of blood containing the parasites likewise produce a lasting immunity. For this purpose eattle are injected subeutaneously at two weeks intervals for 4-5 months with 5 ec. of defibrinated blood from cattle, which have recovered from a severe attack of the disease. Cattle treated in this manner remain healthy in infected pastures, and even their offspring are supposed to resist a later infection after they have recovered from a mild form of the disease.

Theiler \& Stockman failed to obtain favorable results from Koch's method, and the conference of South African veterinarians, held in Cape City in 1907, also denied its practical value. According to Gray the method has been employed in the Victoria District on 5,700 cattle without any results, as the animals became spontaneously infected, even after 11 to 12 inoculations.

Cattle which have recovered from Texas fever are immune against the coast fever, while animals which have recovered from the latter are not immune against Texas fever.

I.iterature. Koch, A. f. Tk., 1904. XXX. 281 u. 586; D. med. W., 1905. 1867. - Theiler, Fortschr. d. V.-Hyg., 1903. I. 133; 1905. II. 256; J. of the Roy. Army Med. Corps, 1904; Comp. Path., 1907. XX. 1; 1909. XX. 115; Rep. of the Gov.-Bact., 1906-1908. - Theiler \& Stockman, Comp. Path., 1904. XVII. 3. Gray, ibid., page 203. - Kleine, D. mer. IV., 1905. 912. - Collaud, Diss. Zürich 1906. - Soulié \& Roig, C. R., 1908. CXLVII. 192. - Lichtenheld, A. f. Hyg., 1908. LXI. 261; 1910. LXT. 378. - Neyer, Comp. Path., 1909. XXII. 213. 
Tropical Piroplasmosis of Cattle. Dschunkorsky \& Luhs observed in southern Russia a disease of eattle resembling coast fever, in which in the acute cases, up to $80-96 \%$ of the red blood corpuscles contained a ring and punctiform, also a rod-shaped piroplasma, which could not be reprodluced artificially by inoculations of blood containing the parasite, (up to several liters were injected subcutaneously, intraperitoneally, or intravenously). On the other hand, according to more recent experiments, larvae of females of the Boophilus decoloratus, which later suck the blood of infected cattle caused, aitter 10-15 days, a fever lasting for several days, mostly however without any parasites in the blood. The symptoms were fever up to $40^{\circ}-41^{\circ} \mathrm{C}$, at intervals of 8-12 days, then continuous at $40^{\circ}-42^{\circ}$ C., symptoms of nervous irritation, sometimes with attacks on persons, accelerated heart's action and respiration, general icterus and pronounced emaciation, frequently bloody diarrhea, and shortly before death sphineter paralysis. The urine was only rarely reddish-yellow, the number of erythrocytes diminished to as few as 800,000 per cubic millimeter.-The autopsy revealed large hemorrhages in all organs, ulcerations corresponding to the hemorrhages, in the abomasum and small intestines, and acute swelling of the spleen. The disease also runs a more chronic cachectic form when only $10-40 \%$ of the erythrocytes contain the parasite, and on autopsy striped and variegated lesions of the mucous membrane of the abomasum are found with an ocher-yellow or dark base. (Cbl. f. Bakt., 1904. XXXV. 486.).

A similar disease was observed by Kowalewsky in Taschkend, only it ran a more rapid course, and in some cases grayish-yellow deposits developed on the mucous membrane of the lips and cheeks, and also on the tongues. (J. vet., 1907. 330.)

Further it appears that the disease observed by Penning in Java is identical with the above. There it attacks buffaloes, and a transmission to sheep with larvae of the Boophilus australis was successful. (Tierärztl. Bl. f. Niederl.-Indien, 1906. XVIII. 102.)

Pseudo-Coast Fever. Lichtenfeld designates by this name a disease of African eattle which manifests itself with fever up to $41^{\circ} \mathrm{C}$.,

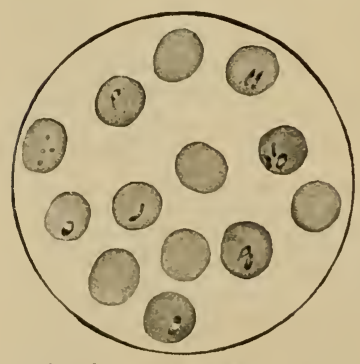

Fi:. 135. Piroplasma mutans. (After Theiler.) emaciation, diminished appetite, and which sometimes causes death. The blood appears watery, and up to $10 \%$ of the erythrocytes contain a small rod or ring-shaped parasite resembling closely the Piroplasma parvum, which had been found previously in sparing numbers, by Theiler in the blood of eattle, usually simultaneously with the Piroplasma bigeminum, and described as Piroplasma mutans. (Fig. 135.) After the inoculation of such blood the piroplasma appears after an incubation of 10-25 days in the blood circulation of the inoculated animal, and produces anemia with poikilocytosis, but never hemoglobinuria. The natural mode of infection is still unknown.

Theiler formerly considered the parasite as an immune form of the Piroplasma bigeminum, while Koch was of the opinion that such cases were nixed infections of eoast ferer and Texas ferer. Recently, however, Theiler showed that 
the Piroplasma mutans occurs also in localities free from coast fever, on the other hand he proved that cattle immune against Texas fever may be infected with it; therefore it could not be a special form of the piroplasma bigeminum.

Parasites resembling the Piroplasma mutans were also found by Miyajima \& Shibajama in Japan, by Martini in China, by Does in Netherlands-India, by Schein in Indo-China; moreover, the parasites seen by Dreyer in Egyptian fever, and by Springefeldt in Camerun in eattle, appeared to be iclentical with them. Lichten. held observed their appearance in ealves 32-91 days after birth, although the animals showed no symptoms of disease. He, as well as Broden \& Rodhain, succeeded in propagating them by the injection of blood containing piroplasma.

Literature. Theiler, Rep. of the Gov.-Bact., 1905/06-1907/08; Comp. Path., 1909. XX. 115. - Schein, A. P., 1908. XXII. 730. - Lichtenheld, Z. f. Hyg., 1910. LXV. 378 .

Gall Sickness. (Galziekte). Under this name the farmers of South Africa designate in general those diseases in the course of which a discoloration and thickening of the bile, as well as an icteric condition of the tissues develops. With the more accurate study of the diseases it was found that the most varied diseases are grouped under that name, (rinderpest, coast fever, Texas fever without red urine, heart water, and poisoning by plants). Nevertheless there are enzootic infections of cattle which cannot he classed with any of the known types of disease, and which are also designated by professional men as gall sickness, in the restricted sense.

The etiology of these affections has not yet been established satisfactorily. While Hutcheon, who in 1897 described them first as jaundice or biliary fever, attributed them to a primary affection of the liver from an unknown cause. Spreull identified them as Pasteurellosis with the Lamziekte (see p. 109), Eddington with heart water (see p. 257). Theiler was formerly inclined to connect it with Trypanosoma theileri, later with Piroplasma mutans; according to his recent investigations however he determined that they are produced by the anaplasma marginale (Fig. 129 on p. 763), in which the blood corpuscles are attacked and destroyed, whereby an oligocy themia is produced, which is associated with high fever, and which later leads to a degeneration of the large parenchymatous organs.

According to Theiler's conception the disease would be identical with the mild form of Texas fever described by Smith \& Kilbourne, which is observerl especially in North America in the fall, as a second attack in eattle that recovered from the acute affection in the summer. According to Knuth's observations it occurs in a similar manner also in South America, and the eachectic form of piroplasmosis deseribed by Dsehunkowsky \& Luhs in Trans-Caueasia is supposed also to belong to it.

The disease named Anaplasmosis by Theiler attacks cattle exclusively, and especially when they are brought from highlands into lowlands, or from less infected localities into more infected territories. African cattle are less susceptible, as they have mostly passed through the disease as calves, whereas fresh. imported animals usually become affeeted severely.

The parasites may be transmitted to healthy cattle with the blood of affected and recovered animals, whereupon in the first case they appear in the blood after 27-32 days, in the second after 16 days, and may be demonstrated during the febrile period, exceptionally also for some time after. Animals which have recovered from the disease show a great resistance against new infections, but they serve as virus reservoirs for the Boophilus decoloratus tick, which according to Theiler is supposed to transmit the natural infection in a way similar to Texas fever. 
Mixed infections occur especially frequently with redwater. According to Theiler's view, Texas fever, as it lias been formerly described, constitutes two different diseases, namely the Texas fever in the restricted sense (piroplasmosis, redwater), and the gall sickness (anaplasmosis). As the Piroplasma bigeminum and the Anaplasma marginale occur very frequently simultaneously in the same animal, the development of redwater with piroplasma in the blood is first observed after an inoculation of blood; when the animal has recovered and the time of incubation for the anaplasmosis has elapsed a new disease results, which was formerly considered as a relapse of redwater. Mixed infections oceur also with Piroplasma mutans, and with Spirochaeta theileri.

The symptoms of gall sickness are high fever with great debility, labored respiration, and very frequent heart's action. Constipation or diarrhea, further moderate swelling of the eyelids, with lachrymation. Edematous swellings may also appear in different parts of the body ; in the later course, anemia and icterus is observed, when the animals rapidly become emaciated. Some animals die within a few days, others in $1-2$ weeks; the greater part of the patients, frequently even $80-90 \%$ recover, but outbreaks with losses up to $50 \%$ may occur (Leipziger).

According to the course of the disease a more or less rapid multiplication of anaplasma, with a corresponding reduction in the number of erythrocytes is found in the blood at the beginning of the fever. Paleness of the remaining blood corpuscles is observed, and in less acute eases polychromatophilia, and basophilia, still later the appearance of normoblasts.

The anatomical changes consist in pronounced anemia and icterus, moderate gastro-intestinal catarrh, diffuse swelling of the lymph glands, acute spleen tumor with reddish-brown, soft but not liquefied pulp, small hemorrhages and degeneration of the heart muscles.

For the prevention of the disease it is aimed to keep animals from marshy pastures, especially from overgrown banks of rivers. If the disease has already appeared it usually subsides after the herd is driven to a dry elevation.

Literature. Edington, Comp. Path., 1905. XVIII. 155. - Theiler, ibid., 1907. XX. 1; Z. f. Infkr., 1910. VIII. 39. - Spreull, ibid. 1908. XXI. 193. Leipziger, D. t. W.. 1910. 150.

\section{(c) Piroplasmosis of Horses. Piroplasmosis Equorum.}

\section{(Biliary fever; Babesiosis equi; Pferdemalaria [German]; Piroplasmose équine [French].)}

History. Guglielmi (1889) in Italy found in the red blood corpuscles of horses parasites resembling the piroplasma bigeminum, but the disease has become better known only since the study of the South Afriean horse sickness (see p. 285). Although Rickmann still considered the blood parasites as the cause of this affection, Theiler proved that in South Africa there exists another disease of horses which may frequently be complicated with the latter; the same author also established more closely the symptomatology, and pathological anatomy of piroplasmosis of horses, and recently showed also its identity with a similar affection of mules and donkeys. The parasites were also studied by Laveran, Koch and Marzinowski, while Italian, English, French and Russian veterinarians contributed especially descriptions of its spread and symptomatology. 
Occurrence. The disease appears to occur frequently in Italy and Russia, in localities with forest and marshy pastures. Native horses as a rule become affected only when young, while animals brought from uninfected territories into infected localities are affected at all ages, and almost exclusively in the warm season (in Russia it is wrongly termed "Spring disease"'). The disease occurs also frequently in Africa and India.

In Germany only one case has been diagnosed by Ziemann in Oldenburg, while in Sweden Brickmann attributed a disease of horses to an infection with piroplasma.-In Italy Baruchello \& Pricolo, also Nori are inclined to islentify piroplasmosis with catarhal influenza, while Perrucei connects it with purpura hemorrhagica. This supposition is based principally on the similarity of the symptoms and evirlently does not apply to other localities.- In Russia the disease was observed in various localities after the demonstration of piroplasma in horses by Michailof (1902), also ly Bielitzer, Marzinowski, Feinschmidt, Michin and Yakimoff, especially frequently, however, in southern Russia (here, also, in donkeys), causing sometimes losses up to. $50 \%$.

It is widely spread in Africa, where it has been diagnosed and carefully studied in the south by Theiler, in the German protectorates by Koch, also by Roger as well as by Lafargue, Lussault \& Savary in Algeria, by Piot in Egypt, by Dupuy \& Pierre in the Sulan and in Senegambia, by Thiroux in Madagascar. Lingard \& Jinnings, Axe and Williams reported on its occurrence in India.

Etiology. The Piroplasma equi (Babesia equi) is a small blood parasite resembling the Piroplasma parvum. It is $0.5-2.5 \mu$ in size, of coccus, ring, spindle, rod or also pear shape. In the red blood corpuscles it is present singly, in pairs, or in fours in rosette form, which latter probably originated by direct division of the single individuals. In the blood plasma they are found only singly. By the Giemsa stain a clump of chromatin granules and sometimes also a second chromatin granule (Blepharoplast?) may be in their body.

Marzinowski \& Bielitzer observed in the intestinal contents of ticks which previously had sucked blood from infected horses, transitory forms similar to those described by Koch in piroplasma of cattle, and by Christophers and Kleine in piroplasma of dogs. They are also inclined to consider oval and worm-shajed bodies with large granular eytoplasm and small nueleus as one form, and those with a still smaller nucleus and paler cytoplasm, as other sexual forms from which new, worm-like actively motile forms (Ookinetes?) develop, whicl occur in great numbers in the saliva of the ticks with which the eggs are moistened. The demon stration of parasites in the egg of the ticks was not successful, but various stages of development, especially the worm forms, were found in the larvae in considerable numbers.

Marzinowski also reported successful cultivation experiments in bloor containing $10 \%$ sodium citrate solution, in which the parasites passed through similar stages of development as in the intestines of ticks. Their multiplication was only moderate, and they died in the third generation.

Theiler succeeded in producing by subcutaneous or intrarenous inoculations of blood of immune horses a febrile affection in mules and donkeys which developed after an incubation of 5 to 9 days, and in the course of which the parasites appeared in the blood. The first attack was followed by a second one, during which the number of the red blood corpuscles diminished to one third, and which frequently resulted in death. The disease was likewise reproduced in horses with the blood of immune mules

vol. $1,-50$. 
and donkeys. Positive results, also with the blood of affected horses, are reported by Perrucci, Pricolo, Edington and Bielitzer.

According to Theiler the natural infection is transmitter in Africa by the Rhipicephalus evertsi, in Russia according to Marzinowski \& Bielitzer by the Dermacentor reticulatus, that is by sexually mature ticks which have sucked blood containing piroplasma as larvae or nymphs. As hor'ses which have recovered from the disease laarbor piroplasma for years in their blood, such animais may spread the virus by transmission to ticks. Zebras and quaggas appear also to serve as virus carriers.

In infected localities native horses, donkeys, and their bastards, become affected in later periods of their lives only when their acquired resistance is reduced by overexertion, poor nutrition, or by intercurrent affections to such an extent that their latent piroplasma again rapidly multiply. Such relapses occur especially in affections of horse sickness, the comrse of which frequently becomes severe because of such relapses.

Animals of the horse genus which are brought from localities free from piroplasma to infected localities are very susceptible; thus during the Boer War in South Africa great losses were sustained in horses brought there from Europe and Anstralia, and in India horses from Australia become especially affected.

Anatomical Changes. On autopsy the icteric discoloration of the subcutaneous and of the subserous connective tissue, as well as of the internal organs is conspicuous, after a somewhat protracted course a general anemia is present which is also manifested in the watery consistence of the blood. The lymph glands and the spleen always show pronounced acute swelling, the pulp of the spleen being moderately softened but not blackish red. Numerous hemorrhages are present on and in the serous and mucous membranes, as well as under the endocardium, the intestinal mucous membranes show inflammatory swelling, that of the large intestine in some cases also contains follicular ulcerations. The lungs are edematous, the kidneys may be sprinkled with punctiform hemorrhages, or only anemic and filled with serous fluid, the liver lobules are yellow, on their borders greenish colored. The muscles, including those of the heart show cloudy swelling, in places also fatty degeneration. The urinary bladder frequently contains more or less reddish colored urine.

Symptoms. After an average incubation of about two weeks the disease commences with slow or rapid rise of the borly temperature, whereupon the fever continues for a long time remittent in character. The first visible symptom is a pale-yellow, or reddish-yellow coloration of the mucous membranes, the intensity of which is proportionate to the severity of the affec- 
tion. In the severe cases, hemorrhages occur later in the conjunctiva in varying number and extent. At the same time the patients look dull, tears ooze from the half-closed eyelids, the animals droop their heads, lie down frequently, and in general show great dullness, which is very pronounced, especially in cases with a chronic course, so that the disease greatly resembles chronic encephalitis. During the fever the heart's action is in the acute cases, greatly accelerated and weak, the respiration is of the abdominal type and markedly labored. In some cases urticaria, and exceptionally herpes labialis is observed in the meantime.

The appetite is depressed from the onset, while thirst is increased; at first or through the entire disease there is constipation, the feces are passed sparingly in small balls, which are covered with a yellowish mucus; later diarrhea appears in some of the cases, sometimes with colicky symptoms. The urine shows icteric or reddish-brown coloration, and is passed in large quantities. The animals rapidly emaciate, their legs swell, the tissue at the same time showing a very slight resistance against tranmatic influences, accidental injuries healing very slowly.

Blood drawn from a vein coagulates rapidly; the coagulum separates immediately in an upper intensely yellow, and a lower red layer, while the serum appears brownish yellow.

According to the physico-chemical examinations of Frei in Pretoria, the blood shows during the disease a continually diminishing volume of red blood corpuscles, and a reduction of conductivity, as well as of viseosity, which correspond with the progress of hemolysis; these ehanges set in very rapidly, sometimes $24-48$ hours after the infection, and therefore even before the rise of temperature. In the urine a diminution of the osmotic concentration, of conductivity, and of specifie gravity may be shown, changes which appear to be dependent on the condition of the serum, and on the polyuria which is usually present, and with which in some eases an increase of the total quantity of the eliminated solids is associated.

Stazzo found in one instance only $2 \frac{1}{2}$ millions of red blood corpuscles per cubic millimeter of blood.

In some cases the course is very mild, inasmuch as the disease is manifested only by a slight icteric discoloration of the mucous membranes, and by febrile manifestations, recovery following in a few days. In severe cases death ensues with a sharp drop of the temperature, usually in 2 to 5 days, while in a protracted course the disease lasts from 2 to 4 weeks, and may even extend over several months.

In chronic eases the disease is manifested by the presence of an almost continuous fever, in gradual emaciation, edematous swellings, anemia and the discharge of a brownish urine (Stazzi).

In the disease observed by Brickmann in Sweden ("Vesterboltenslau"), which also usually followed a chronic course, the symptoms consisted in fatigue and dullness, itching, nodular skin eruptions, perspiration and yellowish discoloration of the conjunctiva. The disease rarely terminates in death.

In mules icterus is rarely present, while discoloration of the urine never occurs; on the other hand frequent urination is almost regularly 
observed (Eassie frequently observed a luxation of the patella as a result of a relaxation of the thigh muscles). In donkeys icterus is also rarely present, while the urine frequently shows a red coloration; a vesicular eruption is also observed in some cases on the back, less frequently also on other parts of the body, and is followed by loss of the hair.

The autopsy reveals a large quantity of pale yellow fluid in the abdominal cavity and in the pericardium.

Piroplasma may he demonstrated in the circulating blood in variable numbers. While in severely acute cases and at the height of the fever they may be found in $50-60 \%$ of the red blood corpuseles, and some of these may contain as many as 5-6 parasites, in chronic eases hardly $1-2 \%$ are infested.

Course and Prognosis. Cases with serere onset, especially great debility, high fever, numerous hemorrhages and hemoglobinuria, likewise cases of a chronic character, usually rum an unfavorable course, whereas cases which are acute, but without severe symptoms, promise a favorable termination. In these cases the improvement sets in after about eight dars, whereupon recovery progresses slowly (according to Rickmann the benign forms are as a rule relapses).

Diagnosis. The disease differs from hor'se sickness principally by the presence of marked icterus, and on the other hand by the absence of extensire edematous swellings. A positive diagnosis is however only possible by the demonstration of piroplasma in the red blood corpuscles, or then by the infectionsness of the filtered blood in horse sickness, as well as in pernicious anemia. The microscopical examination makes a positive differentiation of pirolasmosis from trypanasomiasis possible aside from the fact that this disease is less acute, and does not show icterus and such marked debility. Besides this the symptoms of piroplasmosis to which Italian observer's have repeatedly pointed, closely resemble also those of influenza and purpura hemorrhagica.

Treatment. Horses which are attacked in the pastures should immediately be placed in stables or in dry shady places; where they should be freed from ticks, and nourished as freely as possible. The threatening heart weakness is combatted with digitalis or camphor, the constipation first with calomel, later with salines. Arsenical preparations are recommended for the after treatment.

The specific treatment has so far failed to give uniform results. Formerly large doses of quinine (10-20 gur.) alternating with ammonium chloride were given, whereas recently mereurial and arsenical preparatious are preferred. Thus Baroni recommends the Hydrarg. biiodatum injected into the muscle, and Lafargue, Lussault \& Savary also obtained good results from this treatment (1 gn. Hydrarg. biiod., $2 \mathrm{gm}$. iodide of potassium, $100 \mathrm{gm}$. aqua; $10-20 \mathrm{gm}$. daily). saikowitsch and Marzinowski \& Bielıtzer prasse intramuseular injections of corrosire sublimate (10 ec. of a $2 \%$ solution daily, 3-5 times in all), and according to Michin \& Yakimoff it is possible to reduce the mortality by this treatment to $20 \%$. The subeutaneous application of arsenious acid, and of atoxyl has not proved effective. 
Prevention. This consists in keeping non-immune animals from infected pastures especially in the warmer periods when ticks are numerous. Solipeds from countries free from piroplasma should be imported only while young, and during the winter.

Immunization. Based on the experiences on immunization against piroplasmosis of eattle with infectious blood of calves, Theiler recommends the immunization of horses with $1 \mathrm{ec}$. of blood of artificially infected donkey colts, from the fourth generation upwards. Horses thus inoculated remain healthy. Pregnant mares and run-down animals should not be inoculated. The inoculation was also well borne by horses, mules and donkeys imported from Argentine.

Literature. Guglielmi, Clin, vet., 1899. 220. - Theiler, Schw. A., 1901. XLIII. 233; D. Z. f. Tm., 1904. VIII. 382; J. of comp. Path., 1905. XVIII. 229 ; 1906. XIX. 283; Rep. of the Gov. Bact., 1905/06-1907/08. - Laveran, Soc. biol., 1901. 385. -Rickmann, B. t. W., 1902. 4. - Roger, Bull., 1906. 120. - Baroni, Clin. vet., 1906. 1033. - Perrucei, ibid., 1907. 159. - Stazzi, ibid., 1907. 46. Axe, Comp. Path., 1906. XIX. 222. - Williams, ibid., 1907, XX. 23. - Lafargue, Lusssault \& Savary, Rev. gén., 1908. XII. 489. - Marzinowski, Z. f. Hyg., 1909. LXII. 417. - Marzinowski \& Bielitzer, ibid., 1909. LXIII. 17. - Michin \& Yakimoff, Z. f. Infkr., 1909. VI. 265. - Frei, ibid., 1910. VII. 105. - Bielitzer, ibid., 214 .

\section{(d) Piroplasmosis of Sheep. Piroplasmosis ovum.}

\section{(Malarial catarrhal fever; Haemoglobinuria s. Ictero-IIaema.} turia ovum; Babesiosis ovum; Carceag, Roumanian.)

History. In Roumania a disease occurs among sheep, known as "Carceag," which was first observed by Mazureano (1884), and in which Babes (1892) found similar bodies in the red blood corpuseles to those occurring in the infectious hemoglobinuria of cattle. He also succeeded in infecting healthy animals with pulp of the spleen from affected animals. Since then the disease has been found by Bonome in northern Italy, Leblanc \& Savigne in France, Dschunkowsky \& Luhs in Trans-Cancasia, Laveran \& Nicolle in the vicinity of Constantinople, Ziemann in Venezuela and the West Indies, Eggebrecht in China, and Paschen believes that it occurs also in Germany. Its etiology was studied in detail by Motas in Roumania.

Occurrence. The disease is distributed in Roumania in the flats of the Danube and especially in the islands of the Danube which are frequently exposed to inundations, to such an extent that in some years up to $20 \%$ of the sheep die. It is observed in infected localities almost exclusively during the warmer season, especially in the low, swampy pastures, more rarely in mountainous pastures. Native sheep are considerably more resistant than animals brought from territories free from the affection, which succumb in great numbers under conditions farorable for infection.

Etiology. The piroplasma ovis (Babesia ovis is similar to the piroplasma bigeminum, only somewhat smaller, 1-1.8 ${ }^{\mu}$ ) is usually found in the red blood cells, singly or more rarely in 
double pear shapes, also in rod and cross shapes (Fig. 136). The disease may be transmitted with the blood of affected ani-

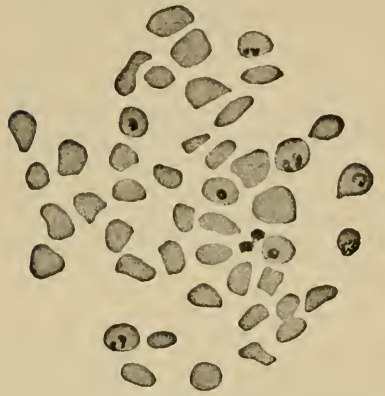

Fig. 136. Piroplasma ovis. Stained with carbo-thionin. (After Motas.) mals artificially to older sheep, but still more easily to lambs. The inoculated animals show, after 8 to 10 days, febrile symptoms, and at the same time parasites may be found in their red blood corpuscles.

The disease is transmitted under natural conditions by sexually mature ticks (Rhipicephalus bursa), which as larvae or nymphs have sucked the blood of affecterl sheep (the infection also succeeds artificially with such females, if they are placed in the fleece of lambs [Motas]).

Dsehunkowsky \& Luhs have ohserved the disease also in goats, and according to their observations in Trans-Caucasia these animals are affected simultaneously with sheep, although they are of the opinion that the piroplasma of goats (Ppl. hirei) differ from those of sheep, in that they are only one-half the size of the latter $(0.43-1-71 \mu \mathrm{long})$. Similar bodies were observed in goats also by Panse, in East Afriea.

Anatomical Changes. The autopsy findings disclose hemorrhagic inflammation, sometimes also superficial necrosis of the mucous membrane of the pyloric half of the abomasum, of the duodenum and rectum; further an acute swelling of the spleen, parenchymatous degeneration of the liver and kidneys (their dark brownish-red coloration frequently shows a greenish hue), finally a gelatinous or gelatino-hemorrhagic infiltration of the subcutaneous and the mediastinal connective tissue. Approximately $5-6 \%$ of the red blood corpuscles in the pulp of the spleen contain piroplasma.

Symptoms. The disease commences, after an incubation of 8 to 10 days, with a rise in the body temperature to $40-42^{\circ} \mathrm{C}$., weakness, loss of appetite and muscular trembling. Soon difficulty in respiration, painful bleating appear, and 1 to 2 days later indications of anemia, icterus, and lumbar weakness. The initial constipation is followed by diarrhea, in which the animals pass bloody feces under symptoms of colic. In some cases the urine is red colored, as a result of oxyhemoglobinuria, frequently however also through hematuria. The number of erythrocytes in the blood diminishes to $11 / 2$ millions per cubic millimeter, under the microscope they contain the small, mostly roundish or oval parasites, which are also found on their surface and in the blood plasma. About $50-60 \%$ of the affected animals, mostly older sheep and 3 to 4 months' old lambs, die in 2 to 5 
days, while the others recover after a convalescence of several weeks. In some cases however the affection manifests itself only by fever lasting 2 to 4 days, slight intestinal catarrh, and symptoms of anemia.

Recovery from the disease conveys to the animals an immunity of long duration against new infections.

Treatment and Prevention. Leblane \& Savigné recommend for the treatment internal administration of sulphate of quinine (0.5 gm. twice daily), and Glauber salts (30-60. gm.). Prevention consists in avoiding affected pastures.

Immunization. According to Motas the inoculation of blood containing only a few parasites produces a mild affection, after recovery from which the sheep withstand an injection of $16 \mathrm{ec}$. of virulent blood, 5 cc. of which would otherwise be fatal. Blood serum of hyperimmunized animals has no parasiticidal properties (in vitro), and at best protects animals only against mild subsequent infections, while bile is supposed to be effective against simultaneous and also against subsequent infection. Immunization with a mixture of blood containing parasites and hile, in which the blood corpuscles are destroyed and the piroplasma eliminated, are supposed to produce an active immunity, although no febrile reaction appears either after the inoculation or after a later injection of virulent blood, and no parasites appear in the blood of the immunized animals.

Literature. Babes, C. R., 1892. CXV. 359. - Bonome, V. A., 1895. CXXXIX. 1. - Laveran \& Nicolle, Soc. biol. 1899. 800. - Hutcheon, Vet. Ree., 1902. XIV. 629. - Motas, Soc. biol., 1904. 1523; Kongr. Haag. 1909. - Paschen, Hyg. Rundschau, 1905. XV. 545. - Dschunkowsky \& Luhs, Kongr. Haag. 1909.

\section{(e) Piroplasmosis of Dogs. Piroplasmosis canum.}

(Malignant protozoon jaundice, Malignant malarial fever; Bösartige Gelbsucht [German]; Fièvre bilieuse, Jaunisse maligne [French].)

History. After Piano \& Galli-Valerio (1895) had first discovered the blood parasites in Italy, the disease was observed in South Africa, where it is one of the most dangerous diseases of dogs, hy Purvis, Duncan, Hutcheon, Lounsbury and Robertson, in East Africa by Koch in Senegal by Marchoux. In France it was diagnosed by Nocard \& Almy at the clinic of Alfort, principally among hunting dogs, and was later studied more closely by Nocard \& Motas. In addition to the work of these authors the biology of the parasites was studied by Nuttal \& Graham Smith, Kinoshita and Christophers, their artificial cultivation by Kleine and Nuttall, while Nuttall \& Hadwen obtained remarkable results with the specific treatment of the disease.

Occurrence. The disease appears to occur with special frequency in tropical countries, and particularly in imported dogs it runs a very severe course. On the other hand in moderate zones it is considerably more rare, and usually runs a 
chronic course. In these locations hunting dogs usually become affected, which have previously hunted in the forests.

In Europe the disease has been diagnosed, besides in Italy and France, by Wetzl in Hungary, by Ljubenetzkij anc by Dschunkowsky \& Luhs in Russia ; Holterbach believes that a disease of dogs which he observed since 1907 in German Offenburg, should from its clinical picture be considered as a piroplasmosis. Of the tropical countries, excluding South Africa, India and China are especially infecter.

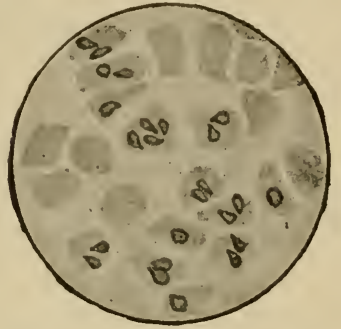

Fig. 137. Piroplasma canis. Stained with methylene blur.

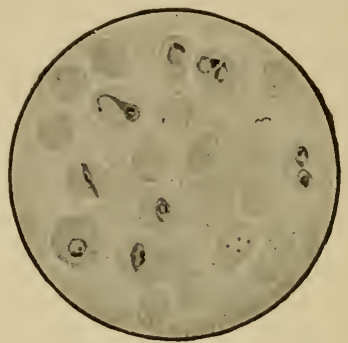

Fig. 138. Piroplasma canis. Stained after Laveran.

Etiology. The piroplasma canis (Babesia canis) is morphologically closely related to the piroplasma bigeminum (Figs. 137 and 138), only that the parasites found in the red blood corpuscles and those in the blood plasma are relatively larger $\left(.7-3.6^{\mu}\right)$, and that single blood cells contain more of the parasites, sometimes as many as 16 individuals. The extracellular ones are roundish, those inclosed in cells are pear-shaped or poly-angular. While living they show an active motility, in which they send out very fine pseudopodia. The homogeneous plasma encloses a spherical or oval mucleus. Propagation, which is especially prolific during the duration of the fever, is, according to Nocard \& Motas, supposed to occur by direct fission, in which the splitting of the nucleus precedes that of the plasma body.

According to Nuttall \& Graham Smith propagation is also supposed to oceur by direct fission. They observed free pear-shaped parasites in the blood plasma, actively swimming around, apparently propelled by flagellae, and subsequently to penetrate red blood corpuscles. In these they assumer a spherical shape, sent out pseudoporlia, and divided into two pear-shaped bodies, which subsequently separated, slipped out of the blood corpuscles and looked for new erythrocytes. If they failed to find them they died. Kinoshita saw similar phenomena, but believes that the individuals resulting from fission should be differentiated into male, possessing flagellae, and female forms without flagella.

If the defibrinated blood of affected dogs is mixed with equal parts of physiological salt solution and is kept at a temperature of from $24^{\circ}-27^{\circ} \mathrm{C}$., the piroplasma remain alive for four days, they show similar stages of development (star shapes) to those described by Koch for the piroplasma of eattle in the intestines of tieks (see p. 766) (Kleine, Nuttall). Similar forms were observed by Christophers in the intestines of nymphs and of mature ticks, besides the pear shapes are supposed to penetrate the cells of the salivary glands and the eggs in the body of the tick, where they break up into sporozoites (?), which then leave the body of the tick with the saliva. 
Pathogenicity. The disease may easily be transmitted to healthy dogs by subcutaneous or intramuscular inoculation of blood containing parasites. These appear in the blood after 2 or 5-6 days, and the infected animals usually die in from 3-5 or 9-11 days, sometimes however the disease may extend even to 60 days, or exceptionally recovery may take place. Other animals are not susceptible to the infection.

Tenacity. Blood containing piroplasma remains infective for 25 days when kept in a cool and dark place. At summer temperature, however, it becomes inactive after 14 days; the parasites die at $44^{\circ} \mathrm{C}$., in $1 \frac{1 / 4}{4}$ hours, at $50^{\circ}$ in a half hour.

In France the natural infection is according to Nocard \& Motas transmitted by the Dermatocentor reticulatus (possibly also by the Ixodes ricinus). In South Africa, according to Lounsbury \& Robertson, by the Haemophysalis Leachi, in India according to Christophers and Nuttall by the Rlipicephalus sanguineus. The two last named species of ticks pass their development as larvae and nrmphs on three hosts, possibly also on other animals than dogs. Females which have sucked the blood of healthy dogs transmit the virus by their eggs to the larvae, in which, as well as in the nrmphs it remains latent, and only the sexually mature ticks are capable of again transmitting the piroplasma to dogs (Lomnsbury, Theiler), whereas the infection does not succeed with

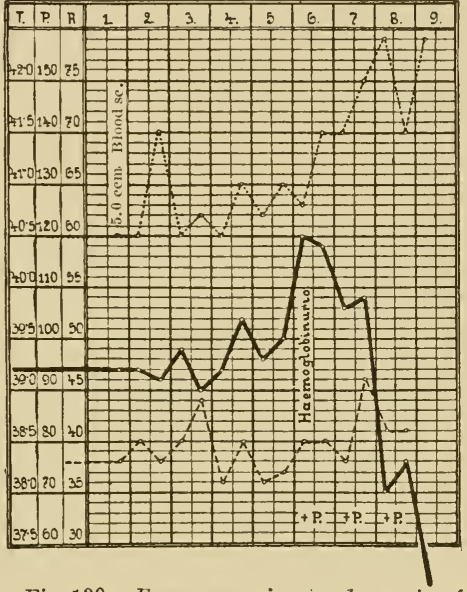

Fig. 139. Fever curce in piroplasmosis of dogs. After subeutaneous injection of virulent blood; two-months-old dog; fatal termination. (After Wetzl.)

mature ticks taken from affected dogs. Ticks capable of infection may carry virulent piroplasma in their bodies even for seven months, and transmit them in the meantime to dogs (Nuttall).

Young dogs are much more susceptible to the infection than adults.

It is questionable whether the piroplasma of dogs are iilentical in different parts of the world. MacFadyean and Nuttall found dogs, which had recovered from the piroplasmosis of India, to le still susceptible to infection with the African parasites.

Anatomical Changes. The autopsy of dogs dead with the disease shows pronounced acute swelling of the spleen, with a dark, bluish-red, somewhat soft pulp, hyperemia of the liver, 
kidneys, and the bole marrow. In these, and also in the lungs and pericardium small hemorrhages, and in some cases acute eatarrh of the stomach and duodenum, with bloody contents in the intestines may be found, and in many cases also general icterus. The changes are generally less pronounced in very acute cases, while in chronic cases icterus is frequently absent, on the other hand a severe anemia is present. Piroplasma may le demonstrated in great numbers, especially in the kidneys and in the spleen.

Symptoms. After an incubation of 7 to 10 days, a rise of the body temperature which inside of 2-3 days reaches $40^{\circ}-43$ C., and which is associated with languor, is observed in the acute cases as a first symptom. The visible mucous membranes appear at first a pale red, later cyanotic, and in about half of the cases also icteric. The pulse and the respiration are accelerated, the latter is moreover very labored and panting. The patients refuse food, but drink a great deal. They move listlessly and stagger, and towards the end they can scarcely stand on their feet.

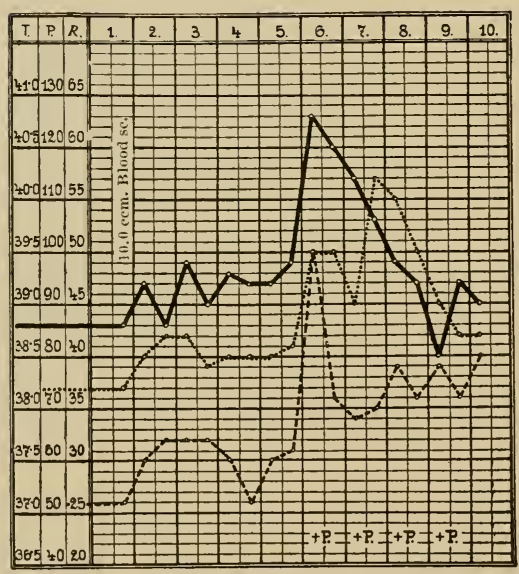

Fig. 140. Fever curve in piroplasmosis of dogs. After subcutaneous injection of virulent blood; 7-year-old dog; recovery. (After Wetzl.)
The urine contains albumen from the onset, and in some of the cases up to $31 / 2 \%$ hemoglobin, sometimes also bile pigments; for this reason it shows a more or less intense red coloration with a greenish hue. According to Maignon the quantity of urea increases at each febrile attack (up to $95 \mathrm{gm}$. per liter of urine), and sugar also appears in the urine.

The blood is pale red, the blood serum also reddish or greenish-red in color. The number of erythrocytes diminishes to $1 / 2$ to $1 / 3$ of the normal or even more, while the lencocytes appear to be increased. Piro-

plasma are found at first exclusively in the red blood corpuscles, later also in the blood plasma. In the later comrse of the disease their number increases, so that finally even $30 \%$ of the erythrocytes may be infested with them. With their increase the percentage of the red blood corpuscles diminishes, and an increase of hemoglobin in the plasma occurs. 
In the chronic cases, which frequently result in recovery, fever is observed only in the first days of the affection or may be entirely absent. In rare cases intermittent fever may be present. Un account of the severe anemia the animals are listless and languid, their mucous membranes are strikingly pale, while icterus is usually absent. The appetite diminishes, and the patients become greatly emaciated. The urine frequently contains albumen, while hemoglobin is only exceptionally present, and even then only in small quantities. The number of red blood corpuscles in the blood diminishes up to $1 / 4-1 / 5$ of the normal while a simultaneous and marked increase of the colorless cells, especially of the polynuclear leucocytes occurs.

After the disease has lasted for 3 to 6 weeks the symptoms of anemia gradually disappear, and finally complete recovery takes place. Such recovered animals however harbor the parasites for a long time in their bodies, as their blood proves infectious even after one year (Theiler), and sometimes even after $21 / 2$ years (Robertson). If fever is produced in such animals, the parasites reappear in great numbers (Marchoux).

Diagnosis. This can be established with certainty only by the demonstration of piroplasma; in those cases especially in which no hemoglobinuria and icterus but only anemic symptoms are observed. There exists in dogs a disease which in its clinical manifestations corresponds completely with piroplasmosis (anemia with disintegration of red blood corpuscles transition forms and hyperleucocytosis, albuminuria and hemoglobinuria), which probably develops as a result of the ingestion of putrefierl meat. In this disease however piroplasma cannot be demonstrated microscopically or by animal inoculation (Szoyka). Nevertheless the suspicion of piroplasmosis is justified in the presence of the symptoms mentioned, especially if it concerns hunting dogs and if ticks are found on their bodies. In the case of negative microscopical findings a positive result may sometimes be obtained by inoculation of blood into very young dogs, for which especially intravenous or intraperitoneal inoculations are adapted; for after this mode of infection the disesse usually develops in a few days.

Treatment. Nuttall, Graham Smith \& Hadwen showed by accurately controlled laboratory experiments, that trypan red, and trypan blue, have a specific destructive action on the prroplasma of dogs. In dogs which already have harbored numerous parasites in their blood, they disappear from the circulating blood inside of 20 to 96 hours with the simultaneous subsiding of the fever, after a single subcutaneous injection of 5-6 cс. of a $1 \%$ solution of one of these substances. At first the pear-shaped subsirle later the roundish ameboid forms, although they reappear after 9 to 12 days, but the animals show no symptoms outside of a short rise in temperature, and finally they recover 
entirely. If the trypan blue is applied 24 hours after artificial infection the affection is aborted. The treatment has only thisdisadrantage that by these remedies the skin and the mucous membranes become strongly colored for a time, and that a painful swelling develops at the point of injection, which sometimes also results in abscess formation.

The recovery is of course hastened by good nourishment and careful nursing.

The authors mentioned obtained negative results from treatment with hydrochlorate of quinine, tartarus stibiatus, methylene blue, B- naphthilamin, adrenalin, arsazetin and soamin. Gonder also obtained unsatisfactory results from atoxyl. Holterbach recommends damholid (20\% solution, tablespoonful doses), without however proving the nature of the disease in the recovered patients by the demonstration of parasites in the blood. Stahn olserved the recovery of a log from treatment with potassium arsenate.

Immunization. According to the investigations of Nocard \& Motas, dogs which have recovered from the disease are still immune against a virulent infection after six months. Blood from such animals destroys the piroplasma, as a subcutaneous injection of a mixture of virulent blood and 3-5 times the quantity of blood serum proves entirely ineffective. The action of such serum may be increased by inoculating the recovered dog several times with blood containing the parasites. Serum of such hyperimmunized animals protects in quantities of 3-5 cc., against the pathogenic action of 3-5 drops of virulent blood, which otherwise produces a fatal infection in 5-6 days. Twenty ce. of serum even renders ineffective an infection indertaken 24-42 hours previously. If, however, parasites have already appeared in the blood the serum treatment no longer produces any results. Heating the serum to $56^{\circ}-57^{\circ} \mathrm{C}$. for half an hour, destroys its parasiticidal action but does not influence its protective properties. Theiler confirmed the immunizing action of the blood serum of immune dogs; he found however that blood from which such serum separates is virulent for young dogs, when fresh or defibrinated, and therefore contains parasites, but by the addition of serum of the same serum-producing dog, it loses its pathogenic action.

Literature. Piana \& Galli-V'alerio, Il. mol. Zooiatrio, 1S95. 165. - Hutcheon, The Vet.-J., 1899. XLIX. 398. - Marchoux, Soc. biol., 1900. 97. - Nocard \& Motas A. P., 1902. 257. -Lounsbury, J. of comp. Path., 1904. XVII. 113. Wetzl, Z. f. Tm., 1906. X. 369 (Lit.). - Kleine, Z. f. Hyg., 1906. LIV. 11. Kinoshita, A. f. Protk., 1907. VII. H. 2. - Christophers, Brit. med. J.. 1907: J. of comp. Path., 1907. XX. 175. - Nuttall \& Graham Smith, J. of Hyg., 1907. V. 232. - Nittall \& Hadwen, Parasitology, 1909. 1I. 156.

Piroplasma in Man. Leishman (1900) and later Donovan (190:3) found in the vicinity of Calcutta, in the blood of persons affected with febrile manifestations double nuclear bodies which resembled the piroplasma of cattle, also in leucocytes and in the tissue fluids of the bone marrow and the spleen, more rarely also in the blood. These have since heen recognized as the Leishman-Donovan bodies, (Leishmania s. Piroplasma Donovani), producers of the tropical splenomegaly, a fehrile cachexia, which in India is known under the name Kala Azar) (=Black disease), and occurs also in China, North Africa and Arabia. Their classification has recently again become questionable, as Rogers \& 


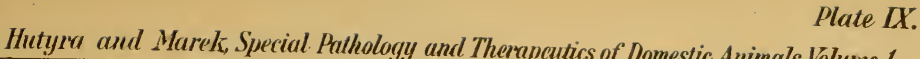

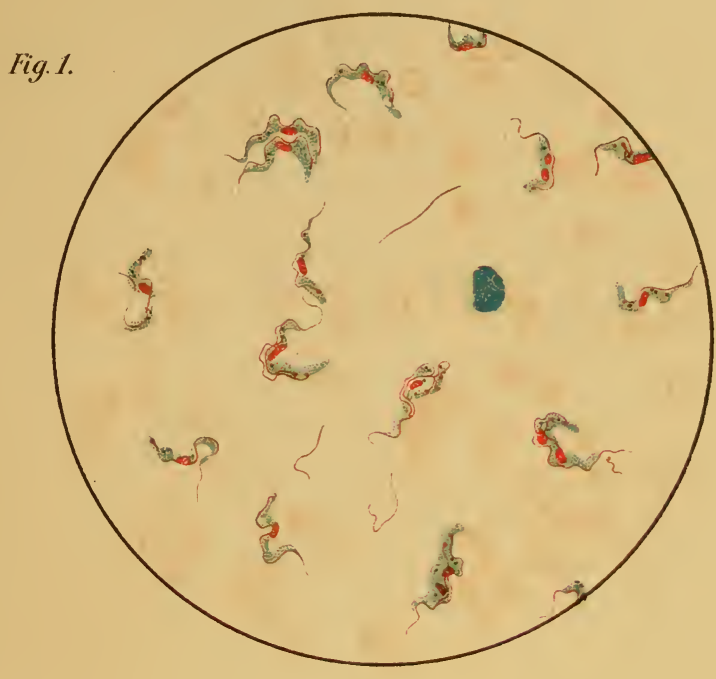

2

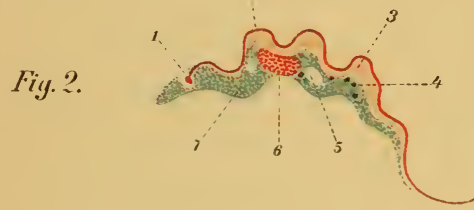

Fig. 1. Trypanosoma equiperdum. Blood of a Mouse.

Fig. 2. The same. 1. Chromatingranule, 2. Flagellum 3. undulating Membrane, 4. Granulations, 5. Vacuole, 6. Vucleus; 1. Cell Plasma.

Stained after Romanowsky. 

Leishman have observed their transformation into trypanosome-like flagellates in blood mixed with sodium citrate, and for this reason Donovan himself placed the parasites under the genus Herpetomonas. Blood-sucking bugs are supposed to transmit the natural infection to man. Similar bodies were found by Nicolle \& Comte in a dog, and Nicolle is also supposed to have succeeded in infecting dogs artificially with splenic blood from man; Patton and Donovan however had absolutely negative results. (Mathis \& Léger also report a leucocytozoon in a Chinese $(\log )$.

The parasites of endemic boil disease, (Delhi or Aleppo boils), discovered by Wright and named Helcosoma tropicum, later as Leishmania tropica, are similar to the Leishman bodies.

Literature. Lïhe, Handb. d. Tropenkrankh., 1906. III. 202. - Leishman, ibid., S. 521. - Donovan, J. of trop. Med., 1909. XII.

Malaria of Man. This disease, which occurs in all parts of the world in marshy localities, and in an especially severe form in the tropies, is cansed by a blood parasite the malaria pasmodium, discovered by Laveran (1880), which differs entirely from the piroplasma of animals. According to the duration of their asexual development, on which also the appearance of the recurring attacks of fever depend, Tertiana, Quartana and Perniciosa parasites are distinguished, which correspond with each other in that besides their multiplication in the red blood corpuscles of man by schizogony, they also multiply sexually in mosquitoes (Anopheles-species). By the inoculation of young forms (sporozoites) of the parasites, with the saliva of mosquitoes into human blood, where they continue to propagate, a brief febrile attack sets in after each development of a generation of the parasite which is manifested by chills, then fever, and perspiration. As at each attack a number of red blood corpuseles are destroyed, an anemic condition develops gradually which in severe cases increases to a pronounced eachexia; in the tropies however febrile attacks may result in early death under manifestations of hemoglobinuria, (black water fever). The disease is combatted successfully with preparations of quinine. (Ziemann, Menses Mandh. d trop.. Krankheiten, 1906. III. 269;Ruge, Hb. d. p. M., Ergzsbd., 1907. 85.)

\section{Trypanosome Diseases.}

\section{(Trypanosomiases, Trypanoses.)}

Investigations during the last three decades liave proved that various tropical diseases of domestic animals and also of man are caused by blood parasites of the flagellate class of protozoa, the so-called trypanosomes or trypanozoa. In the thorough investigation of these parasites in various localities as well as in different species of animals, and in various diseases, certain differences, though of slight significance, have been established in their morphology, and especially in their pathogenicity, which led to the classification of a number of not always distinctly characteristic forms of trypanosomes or 
rarieties, and of several diseases. Considering the marked resemblance of the morphological characteristics of most of the parasites belonging to this group, there exists a possibility that the diseases discussed in the first part (Nagana, Surra) represent a pathological unit, while the unimportant differences roted in the others depend chiefly on the variability in virulence of the sereral kinds of trypanosomes, likewise also on the geographical and climatic conditions, whereby the differences in the mode of natural infection, as well as the course of the disease can be explained readily. In deference to the general conception, the diseases considered at present as independent affections will be treated separately, after previously explaining these points of view.

Besides the pathogenic trypanosomes there exists a great number of others which occur as harmless blood parasites in domestic animals and in other species of animals.

\section{General Morphology and Biology of the Causative Agents.} The trypanosomes are unicellular, animal microorganisms, containing a nucleus, a blepharoplast, and a flagellum. They propagate by longitudinal division, possibly also sexually. The parasites known to be prodncers of disease possess slender, spindle-shaped bodies, the posterior ends of which are more or less blunt; they are enclosed by a periplastic membrane. The parasites are mostly $21-35 \mu$ long, and $11 / 2-3 \mu$ broad, in the middle portion they contain a spherical or oval nucleus and close to the posterior end a shining nucleolus (centrosoma, blepharoplast, flagella root, trophonucleus) (see Table IX). From the latter a fine flagellum rums out, which extends forward on the outside borders along the undulating membrane, which is present on one side of the body, and terminates in a free portion at the anterior point of the body. In the fine granulated protoplasm there are distributed one or more vacuoles, and also various sized granules in varying numbers.

By means of lashing movements of the flagellum and spiral contraction, the trypanosomes are capable of lively motions in fluid as well as in the blood between the blood corpuscles, usually in the direction of the flagellated extremities.

On the addition of certain substances, especially of immune serum, the trypanosomes unite in pretty rosette shapes (agglomeration), by approaching each other, usually with the posterior ends, in radiating arrangement and executing whip-stroke movements with the free flagellated extremities directed outwardly.

Propagation occurs thus that first the centrosome, then the flagellum and nucleus split longitudinally in two parts, and finally the plasma body breaks into two halves from front to back. Sometimes the division of the body does not follow that of the centrosome and the flagellum immediately, so that irregular 
ameboid forms result, which contain two or more chromatin substances, and only later separate into more individuals.

Besides this simple mode of multiplication, a sexual propagation, or a special development is considered possible in the transmitting insects as intermediary hosts (Ziemann, Prowazek, Koch, Schaudin, Stuhlmann, Kleine and others).

This opinion is based principally on the investigations of the intestinal contents of stinging flies (Glossinae), infected with trypanosomes, in which besides typical, broad forms rich in plasma, very thin forms are found containing only a small quantity of plasma with elongated nucleus, and sometimes without flagella. According to Koch the former are considered as females, the latter as male trypanosomes, and they are supposed to copulate with each other. According to Kleine's investigations of sleeping sickness, the development is supposed to extend over several weeks, so that infected flies may be capable of causing infection for that period.

According to the experimental results of Battaglia, obtained by a study of the multiplication of the Trypanosome Lewisi and the Trypanosome Brucei, a multiplication by sporogeny occurs also in the blood of the same animal, inasmuch as after an infection with small motile, intra-globular ameboid forms, first similar extra-globular forms develop, later larger, roundish or pear-sliaped unflagellated forms, and only from these finally result the fully developerl forms, and these then multiply by longitudinal division. This conception is supported by the experience that it is possible to transmit the disease artificially with filtrates of infected blood, after passing it through Berkefeld (not Reichel or Chamberland) filters.

Staining. The trypanosomes are even with slight magnification easily recognizahle by their peculiar form, and by their lively snakelike movements. They stain uniformly with the ordinary aqueous anilin dyes, while the structure may be brought out by the use of Romanowsky's or a similar stain by whieh the nucleus, the Blepharoblast and the flagella appear pale red (chromatin stain) and the plasma pale blue.

Recently Giemsa's stain is used almost exclusively with the azure prepared by him in pure form. Staining fluid: to each ce. of distillerl water, one drop of Giemsa's solution, always to be freshly prepared. Staining of the smear prepara-

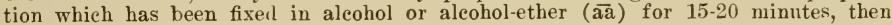
washing in running water.

Cultivation. Novy \& MeNeal succeeded in eultivating the trypanosomes of rats and later also those of nagana on agar to which had been added about $50 \%$ of blood, at a temperature of $25^{\circ}-34^{\circ} \mathrm{C}$. In the cultures non-motile forms develop 1-2, up to 50-60 $\mathrm{long}$, and the first of them may pass through porous filters. Trypanosomiasis was produced artificially in rats with Berkefeld filtrates of cultures. Since then the cultivation of other disease-producing trypanosomes was successfully carried out in a similar manner (Irikura eultivated them in blood houillon of $1 / 2-2: 10)$.

Tenacity. The parasites can be kept alive outside of the animal body for several days, but only in defibrinated blood, to which pure serum is added. Cool temperature usually lengthens their lives, while against higher temperatures $\left(43^{\circ}\right.$ and over) and chemical influences they are in general very sensitive.

The differentiation of the various types depends, aside from marked deviations in the form of the trypanosomes, almost ex- 
clusively on their occurrence in certain species of animals and in well-defined localities, further on the variance in their pathogenicity towards the different species of animals; and also on the fact that animals immunized against one type are still susceptible to others. However these differences are not constant, as it is often possible to increase the virulence of one strain of trypanosomes for otherwise slightly susceptible or apparently immune species of animals by passage-inoculations. Morphologically the trypanosomes of surra, nagana, and dourine, as well as of sleeping sickness, correspond absolutely, and those of Mal de Caderas are supposed to be different from them only in that their blepharoblast is smaller, and that they are more difficult to stain.

Distinetly different types are: the relatively large $\left(50: 3.5-4.0^{\mu}\right)$ Trypanosome Theileri, the even much larger Trypanosome giganteum some non-flagellated trypanosomes, thus especially the Tr. dimorphon (see p. 837), further the Tr. Lewisi occurring in the blood of rats, which has been most thoroughly studied, and in which the nucleus is not in the center, but in the posterior third of the plasma body.

Pathogenicity. Artificial transmission to susceptible animals succeeds most readily by subcutaneous, intraperitoneal, or intravenous inoculations, with less certainty by applying blood containing trypanosomes to fresh wounds. After a certain time the parasites appear in the blood in large numbers, when in certain species of animals, especially in dogs, cats and rabbits, fever appears as a usual symptom, in addition to edematous swellings on different parts of the body, resulting sometimes in the loss of hair, emaciation, sometimes also inflammation of the cornea and of the eyelids, whereupon the anemic animals emaciate rapidly and succumb. If virulent blood is inoculated into an animal of the same species from which it originated, a severe or mild disease results, which is identical with the original affection.

Dourine may be produced artificially by applying material containing trypanosomes on the uninjured mucous membrane (Vrijburg obtained in one case positive results in a horse with surra material).

The virulence of the trypanosomes varies in different species of animals, and may be increased for certain species and reduced for others by passage experiments. Generally native animals are less susceptible in infected localities than those brought to such places from non-infected countries.

Natural infection in the domestic animals, exclusive of dourine in which it occurs by coitus, usually results from the stings of flies, namely, Glossinae, Tabanidae and Stomoxys species, they transmit the trypanosomes mainly in a purely mechanical manner by sucking blood from affected animals and inoculating it to healthy individuals. If, however, the trypano- 
somes may undergo sexual propagation in the insects as intermediate hosts, as appears probable from recent investigations (see p. 799), there would be a possibility that they may harbor the parasites in their bodies for a longer time, and in the meantime transmit it to susceptible mammals.

The infection results apparently only exceptionally through fresh wounds in the skin and mucous membranes, whereas an infection through the uninjured mucous membrane of the digestive tract, as it was accomplished experimentally by Yakimoff \& Schiller, has not been observed.

Pathogenesis. As trypanosomes are always present in the blood plasma and the tissue fluids, they apparently exert their pathogenic action only indirectly by the production of toxic products, which principally canse the destruction of the red blood corpuscles, and thereby anemia. They probably influence the metabolism of the host in other liarmful ways. The fever, the marked emaciation (with good appetite!), as well as the affection of the nervous system (neuritis, later degeneration) can be explained only by a toxic action, but it has so far not been possible to demonstrate the supposed toxins of the trypanosomes objectively (Laveran, Mesnil, Mayer and others). The fact however that the parasites multiply outside of the animal body only on media containing a great amount of hemoglobin appears to indicate that they require hemoglobin, and the destruction of the erythrocytes may be explained by the fact that they abstract this substance from them.

Following the infection a marked increase of trypanosomes in the blood occurs periodically in the animals, and this is usually accompanied by an elevation in temperature. Then again they disappear rapidly, only to return again in great numbers after a longer or shorter time, having probably matured meanwhile in certain organs (spleen, bone marrow?) from the young forms. Their disappearance is according to the investigations of Massaglia and Rodet \& Vaillet, supposed to be clue to trypanolytic substances, which appear in the blood of infected animals and the quantity of which increases until the crisis. To what extent the lencocytes participate in this has not yet been determined; as a matter of fact their number greatly increases during the disease simultaneously with a hyperplasia of the spleen, and a great number of leucocytes which contain in their bodies broken-down products of trypanosomes are to be found in the spleen tissue, as well as in the bone marrow.

General Symptoms. They appear after a longer or shorter incubation period, and excluding the acute and peracute cases, which are manifested under severe febrile symptoms, consist in general in intermittent or recurrent fever, transitory or constant edema of the lower parts of the body, and progressive

Vol. 1-51. 
anemia as well as emaciation, with usually a good appetite. Nervous functional disturbances, especially peripheric paralyses, are also frequently observed. Death is usually preceded by a comatose condition.

Treatment. While in former years the trypanosome affections have been treated mainly with quinine, corrosive subl:mate and iodine preparations, althongh withont apparent results, Lingard obtained in surra, and Bruce in nagana, noteworthr results with arsenious acid, inasmuch as with this treatment the number of the blood parasites was sometimes reduced very markedly. The improvement was however usually only temporary, as after a certain time the trypanosomes reappear in the blood, so that the final results, at best, showed only a prolongation of the course of the disease.

More recent investigations, above all the great laboratory experiments of Ehrlich and his school, show that certain clienical substances have a specific destructive action on trypanosomes in the infected animal bodies, or at least exert an inhibitive action on their development, and in suitable application may even result in permanent recovery from the disease. Such substances, excluding some of the basic triphenylmethan dyes (parafuchsin, methylviolet, pyronin), are certain azofar stains, such as the trypan red, the trypan blue, the trypan rosan, and the trypan violet, certain arsenical lerivatives, especially atoxyl, arsazetin, the arsenophenyglyzin, and the dioxydiamidoarsenobenzol (Ehrlich-Hata 606), also some antimony preparations, such as tartar emetic.

Special study was given to atoxyl (sodium arsanilate) first recommended by Thomas and Breinl, and Mesnil \& Nicolle. It was found that this remedy, different from the basic stains mentioned, which are effective even in test-tube experiments, does not even in concentrated solution affect the trypanosomes harmfully in vitro, while in the animal body it causes the disappearance of the parasite even in a dilution of $1: 120000$. Ehrlich believes that the action of the atoxyl in vivo is due to the fact that the substance is reduced to paraaminophenylarsenoxyd, which kills the trypanosomes also in the test tubes, even in dilutions of $1: 1000000$. This is corroborated by the experimental results of Levaditi, Yamanouchi \& Brimont, as these authors have found that the atoxyl is reduced in the animal body (to a hypothetical trypanotoxyl); although later they supposed that the reduction product in such a reduced condition, combines with proteid molecules, to form a toxic arsentoxalbumin.

Breinl \& Nierenstein however believe that the atoxyl combines with the serum proteids, whereupon arsenic is set free through oxydation of the atoxyl in the serum, and this, in statu nascendi exerts a destructive influence on the parasites. The correctness of this conception appears to be substantiated by the experimental results of Loeffler, who obtained the best and most lasting recoveries in nagana-guinea pigs and eats from the administration of atoxyl, together with arsenious acid in subtoxic doses (3-5 mg. arsenic per os., and $315 \mathrm{cg}$. atoxyl subcutan. 
eously). Similarly Holmes observed excellent curative results in horses affected with surra, after the use of arsenious acid. However in order to make the recovery complete all trypanosomes, even the latent forms in the internal organs must be destroyed (therapia sterilisans magna), therefore the remedy should be used in as heavy doses as possible, that is in sub-toxic doses (as formerly the arsenic has been used in too small doses, and the parasites which were not destroyed were capable of causing relapses). The good results obtained with the auripigment can also be explained by the fact that it usually contains free arsenious acid.

In the experimental treatment it was further observed that the trypanosomes are capable of adapting themselves to the remedies under discussion, for instance trypanosomes which, after atoxyl treatment with incomplete recovery, remain alive in the animal and later again multiply, are no longer influenced by the same remedies, and likewise not by other derivatives of phenyl arsenious acids. This resistance is transmitted by subsequent passages through the same species of animals from generation to generation. This condition may become objectionable in practice, inasmuch as from animals which have been subjected to such specific treatment and which have not completely recovered, flies may later transmit to other animals atoxyl- (arsenic-, trypanblue-, fuchsin-, etc.) resisting trypanosome strains. Such animals then resist treatment with the same or with a related remedy. Most recently however Ehrlich succeeded in producing new arsenical preparations, the arsenophenyglycin, and the above-mentioned arsenobenzol, which, with a slight toxicity for mammals are capable of attacking even arsenicresisting strains of tryponosomes and which have also otherwise proven most satisfactory for trypanosome affections ont of the great number of chemicals that have been tested.

Ehrlich aimed to explain the mechanism of the specific therapentic action through certain groupings (chemoceptors) in the protoplasm of the trypanosomes, which possess for certain chemical substances, for instance for the trivalent arsen as it occurs in arsenical acids, a special affinity, anil are capable of anchoring such to the plasma. The resistance of alrearly treated strains may result from the fact that the respective chemoceptor experiences a reduction of its affinity to the applied remedy, therefore its resistant strain is destroyed only by a greater quantity of the remedy, or by a more strongly avidious remedy, for instance the arsenophenylglycin.

Based on these experimental results, which have repeatedly been substantiated especially by Uhlenhuth, Hübener \& Woithe for the treatment with atoxyl of experimental dourine, by Loeffler with arsenic in experimental nagana, by Levaditi and his co-workers for the action of atoxyl on various strains of trypanosomes, arsenical preparations are used in practice for the treatment of these diseases. They are employed either combined with eacl other, or alternately; further antimony preparations, especially tartar emetic, and with these treatments satisfactory results are generally obtained, or at least encouragement for further therapentical experiments. In general, the rule should be observed to administer the remedy as soon as possible after the infection, in a single, or at most in two subtoxic doses, as only in this manner can relapses and the development of hypersensitiveness be prevented. (For details see the individual affections.) 
Prevention. Excepting dourine, which can be controlled by comparatively simple measures in the supervision of breeding and may be entirely eradicated, the prophylaxis of the trypanosome diseases consists above all in the eradication of the flies which play a part as transmitters, and probably also as hosts for the virus. The procedure must of course be suited to their habits of life and their modes of derelopment. In the glossinae and tabanidae, which confine themselves to certain forests and marshy localities, and probably also to certain wild species of animals, the eradication in practice succeeds at least in small territories, by cutting of the bushes, and draining of the meadows. Besides regular cultivation of the soil, the destruction of the game, or keeping it from the roads of traffic, or confining it to certain reservations would aid in the elimination of the source of infection. The species of stomoxys which exist in close proximity to living houses and stables, may be reduced in numbers by cleanliness, as well as by the destruction of all vegetable refuse.

As the flies do not accompany the cattle herds for great distances, and as they do not swarm during the night, threatened herds may be protected from infection by driving them at night to non-infected localities, avoiding rivers, thus keeping them from the virus carriers.

In practice the control of trypanosomiasis is rendered more difficult by the fact that not only affected, but also apparently perfectly healthy, and especially the animals which have recovered from the disease harbor trypanosomes in their blood for years. Such virus carriers constantly produce virulent material for flies in infected territories, and may, on the other hand, disseminate the virus into previously non-infected territories. By the destruction of all such virus carriers the source of the infection for the flies could be destroyed, but such a procedure could only come into consideration in small, freshly infected territories.

For countries which are free of certain trypanosome affections careful quarantine measures, or even the prohibition of the importation of susceptible species of animals from infected territories, comes into consideration.

Immunization. Immunization experiments with serum of recovered animals and those repeatedly treated with virulent blood (Ziemann), or with blood containing parasites and subjected to high or low temperatures, have not yet given manifest results (Uhlenhuth, Gross \& Bickel). The same applies to the experiments which have been carried out by passage of the virus through various species of animals (Koch, Schilling, Martini). In the efforts to work ont a method for active immunization the fact must be considered that the immunized animals harbor the parasites for years, and these may later become virulent, and sometimes may be transmitted to healthy animals in such a condition.

The fact that animals acquire, through recorery from certain trypanosomiasis, a lasting although not an absolute immunity against the same and not against other 
trypanosomes, indicates that strictly specific immune bodies develop in the affected animals. Such, however, have not yet been demonstrated with certainty in an objective manner. In this regard the complement fixation method, in which blood containing parasites or material from organs was used as antigen, gave uncertain and varying results (Weber, Manteufel \& Woithe). Better results were obtained by Levaditi \& Mutermilch, when they carried out experiments with trypanosomes separated from blood corpuseles. They could always demonstrate complement fixing bodies in the serum of guinea pigs treated with trypanosome extract or in the course of infection. These substances were specifie for the genus trypanosoma, but not for the individual species and varieties, therefore they could not be differentiated by this method. Further, the complement fixing substances proved different from the trypanolysines, which form in great numbers at the height of the infection (see p. 801), and exert their dissolving action also in test tube experiments.

According to Laveran \& Mesnil, human blood serum has a similar destructive action on the trypanosomes of nagana, surra and mal de caderas, as the arsenic acid. Serum of animals, even of those which have recovered from the disease, or which have been repeatedly infected, has no such action, and on the other hand the human serum is harmless for the trypanosomes of sleeping sickness.

Literature. Nocard \& Leclainche, Maladies microbiennes, Paris 1903. II. 575. - Laveran \& Mesnil, Trypanosomes et trypanosamiases, Paris 1904. -Lühe, Handb. d. Tropenkrkh., 1906. III. 92. - Sander, ibid., 690. - Nocht \& Mayer, Hdb. d. p. M. Ergänzgsb., 1906. 1. - Sauerbeck, Ergebn. d. allg. Path., 1906. X. 305. Kaestner, ibid., 1907. XI./1. 455. - Ehrlich B. kl. W., 1907. Nr. 9-12. — Manteufel \& Woithe, Arb. d. G.-A., 1908. XXIX. 452. - Koch, Beck \& Kleine, ibid., 1909. XXI. 1. - Levaditi \& Yamanouchi, Soc. biol., 1908. LXV. 23. - Levaditi \& Mutermilch, Z. f. Immf., 1909. I1. 702. - Kleine, D. m. W., 1909. 924 u. 1257. - Doflein, Protozoenkunde, II. Aufl. 1909. 350. (Mostly with literature.)

\section{(a) Nagana.}

\section{(Tsetse disease.)}

History. The disease was deseribed as a destructive cattle disease by Livingstone (1857) during his voyage in Zambesia, and was connected by him with the sting of the native tsetse fly. Its cause was discovered by Bruce in 1894, in the territory of the Zulu Kaffirs, (nagana=without strengtl, debilitated), and the first detailed descriptions of the clinical symptoms were also made by him. Shortly afterward the disease, as well as its cause was investigated thoroughly by Kanthack, Durham \& Blandford and by Plimmer \& Bradford, while Theiler deserves special credit for the epidemiology. The problem of the propagation of the nagana trypanosomes was studied by Koch and recently by Kleine and Battaglia, while MICNeal, and Laveran \& Mesnil were successful in their artificial cultivation.

Occurrence. The disease used to be spread widely through the entire Central and South Africa, where it annihilated at times the cattle of entire districts. Recently it disappeared from the south, so that at present it occurs only in some outof-the-way localities of Zulu land. The infected territory was limited partly by the driving northwards of the game, principally however by the invasion of rinderpest in 1896 and 1897 , as at that time besides cattle, numerous game animals and buffaloes fell victims, and at the same time the tsetse fly also disappeared from those localities (Theiler).

Trypanosome affections oceur also in Northern Africa, but the elassification of these, with the exception of dourine, has not been cleared 
up with certainty. Some enzooties have a great similarity to nagana, while others are more closely related to surra (see also p. 837 and p. 812).

Etiology. The Trypanosoma brucei, recognized as the cause of nagana, is on an average $25-33{ }^{\mu}$ long, $1.5-2.5^{\mu}$ broad, and possesses a central oval nucleus, a blunt, Indian-club-shaped posterior end, slightly developed undulating membrane, and long flagellum (Fig. 141). Under the microscope it exerts a lively snake-like movement, but without pronounced locomotion.

Pathogenicity. Inoculation of blood containing trypanosomes, under the skin or into the blood circulation of horses,

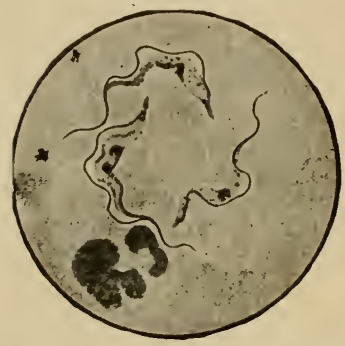

Fig. 141. Trypanosoma Brucei. Guinea pig blood. Stained after Romanowsky-Ziemann. causes after from 2 to 6 days a marked elevation of temperature and in the first case also a painful swelling at the point of injection. About the end of the first week the fever diminishes but reappears later at certain intervals, and during the duration of the febrile attack trypanosomes may be - demonstrated in great numbers in the blood. In the meantime edematous swellings appear on the lower abdomen, and in the vicinity of the external genital organs; the animals emaciate in spite of continuous, and often ravenous appetite; marked muscular atrophy and also pronounced anemia develop. Sometimes turbidity and ulceration of the cornea results with subsequent iritis. Finally a condition resembling lumbar paralysis sets in, whereupon the animals die from 15 days to 4 months after the appearance of the first symptoms. In donkeys and mules the clinical picture is similar, although sometimes with a still more protracted course after artificial infection; the zebra is also susceptible to artificial inoculations (Martini).

In cattle, also in sheep and goats the inoculation usually results in a chronic disease.

Dogs and cats are very susceptible to infection, and likewise become affected under symptoms of recurrent fever, edamatous swelling of head, chest, gradual emaciation and loss of strength. Guinea pigs, rabbits, rats and mice, are very easily infected artificially, while hogs show considerable resistance to artificial inoculation. Monkeys are very susceptible and usually die a few weeks after the infection. Of fowls only geese (Schilling) and chickens (Goebel) could be infected artificially, while other birds showed no symptoms of disease.

Horses and other solipeds, then cattle, more rarely sheep and other ruminants, become most frequently affected as a result of natural infections. 
The infection is transmitted by the so-called tsetse fly, which somewhat resembles the house fly although it is somewhat larger, principally by the glossina morsitans (Fig. 142), in. such a way that it sucks blood from the infected animal, and then carries the parasites into the body by its bite. Freshly molted flies are harmless, if however they have alighted upon an affected animal only a single time they may transmit the disease and trypanosomes may be demonstrated in their proboscis and stomach shortly after sucking the blood. By the transportation of affected flies the disease was successfully produced in previously non-infected localities, while on the other hand animals driven during the day in infected localities became affected even when they had not partaken of food nor water while en route. Furthermore, the extension of the infected territory corresponds accurately with the geographical distribution of the tsetse fly (between the 10th deg. north, and 30 deg. south latitude), where its appearance in great numbers is usually followed by severe outbreaks.

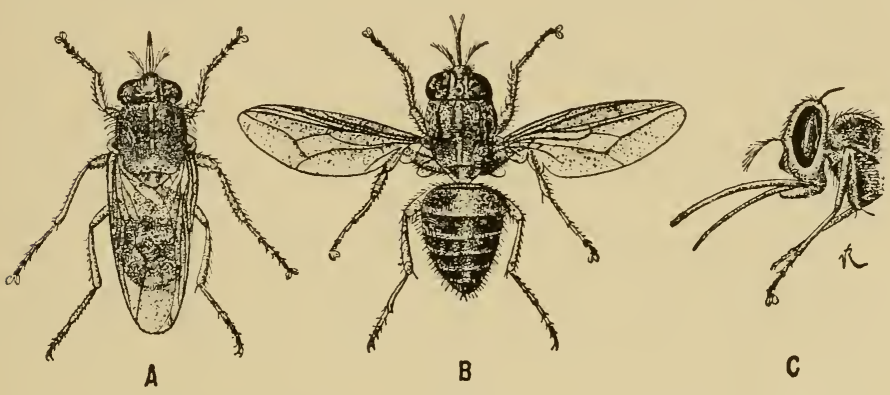

Fig. 112. Glossina morsitans. A with closed, $B$ with spread wings, $C$ anterior part in profile. $A$ and $B$ enlarged 2.5 times. (After Bruce.)

There affected animals are present or those which have passed through the disease within a short time, the fly is afforded ample opportunity for taking up trypanosomes; flies may also obtain the infectious material from wild animals (buffaloes, antelopes, hyenas, zebras and quaggas), in the blood of which it may oceur as a harmless parasite. The tsetse follows these animals everywhere; with the appearance of the summer season the infections material is then taken from them by the young flies, and transmitted to domestic animals, while with the eradication of the game the tsetse fly also disappears, so that infected territories may thus become free.

According to Koch's experience in western and northern Africa, where the glossina morsitans is unknown the disease is transmitted by the closely related Gl. fusca. According to Brumpt the Gl. pallidipes, 
the Gl. longipennis, the Gl. palpalis and the Gl. Decorsei are also capable of transmission.

According to former viens the tsetse fly transmitted the infection only mechanically, and as trypanosomes could be demonstrated in their intestines 48 hours after sucking the blood, it was supposed that after that time healthy animals could beeome infected only when the flies had in the meantime again alighted on infected animals. Aceording to the newer results of investigations on the generation changes of the parasites (see p. 799), the possibility must be considered that the flies may transmit the parasites for weeks.

In accordance with the part played by the insects in the transmission of the disease this usually rages in low-lying localities with damp soil, especially during warm periods and rainy seasons, the animals being exposed to the infection especially during the day, as long as they are in the vicinity of marshes, lakes, water canals and rivers. The transmission of the disease from animal to animal is also possible by contamination of fresh wounds on the skin or mucous membrane, with blood containing parasites, while the possibility of natural infection through the uninjured mucous membrane of the digestive tract has not yet been proven.

Anatomical Changes. The autopsy usually rereals pronouncerl anemia and emaciation. The subcutaneous connective tissue shows in many places gelatinous infiltration, and in the serous cavities transudate is found in variable quantities. Small hemorrhages may be present in the serous membranes, in the mucous membrane of the stomach and intestines, as well as in the kidneys. If the animal dies during a febrile attack the spleen shows acute swelling, whereas otherwise a chronic splenic tumor is usually present; sometimes however it may have an entirely normal appearance. The lymph glands are moderately swollen. In some cases hemorrhages and catarrhal pneumonic areas are present in the lungs. No trypanosomes can be demonstrated in the blood or in the internal organs 24 hours after death.

Symptoms. The manifestations of the disease are similar in their nature in the different species of animals.

In horses the temperature rises rapidly after an incubation of 2 to 10 days, according to the severity of the infection, to $40^{\circ}$ to $41^{\circ} \mathrm{C}$., and at the same time other febrile symptoms, such as languor, accelerated and weak pulse or diminished appetite make their appearance. The mucous membranes are reddened, there is profuse lachrymation, and at the same time edematous swellings form on the extremities and lower abdomen, especially around the genital organs, also in the submaxillary space, and on the eyelids.

The febrile condition remains unchanged for 2 to 3 days, sometimes for a week, then the temperature drops rapidly 
to normal or below, whereupon the animals become livelier and the other symptoms diminish or may disappear entirely. If the fever has lasted for a longer time the patients have in the meantime become emaciated in spite of the fact that the appetite has not been impaired much. After a certain time the afebrile period is again followed by fever, and from then on several fever days alternate with fever-free intervals, only that the rises of temperature in the later course are lower than in the first attack.

Under such variations of temperature the animals become emaciated, although their appetite continues very good, and a pronounced anemia usually develops in which the hemoglobin content may drop to $25 \%$ of the normal, while the number of red blood corpuscles may diminish to $21 / 4$ millions per cubic $\mathrm{nmm}$. with a simultaneous moderate increase of lencocytes (Schilling; Nierenstein found in experimental nagana an increased acidity of the blood, probably due to the production of amido acid). Accordingly the mucons membranes appear pale and in the later stages frequently icteric in color.

The edematous swellings which develop on different parts of the bodies vary in the first period in their intensity and may sometimes disappear entirely; in the later course, however, they remain constant. Urticaria sometimes appears especially at the begimning of the affection, but soon disappears again.

With the progressive emaciation and anemia the animals become steadily weaker, their motions are incoordinated and staggering, the animals knuckle frequently, and gradually a condition resembling lumbar paralysis develops, so that the patients, in which difficulty in respiration has appeared in the meantime, remain continually lying down until they die, greatly emaciated and completely exhausted.

After artificial infection of dogs, Spielmeyer observerl recent degeneration of nerves in the posterior roots of the spinal cord, in the roots of the sensory trigeminus, and of the optic nerve.

In some of the chronic cases an opalescence or an ulcerative breaking down of the cornea results later or a hemorrhage follows into the anterior chamber of the eye, which becomes associated with an iritis, and finally leads to blindness.

The urine periodically contains albumen in varying quantities. The blood contains trypanosomes in great numbers during the first febrile attack, while later they are found more sparingly, usually only during the febrile attack. In the very acute cases they usually occur constantly, and their number may amount to 70,000 per cubic centimeter of blood. They may also frequently be demonstrated in the serous fluid of the swellings.

The course usually extends to $11 / 2$ or 2 months, but may sometimes last for 4 or 5 months. In some cases, which for 
a time show a chronic course, a sudden aggravation of the condition may develop during a febrile attack, whereupon the animals die in a few hours (cerebral embolus). Some cases may be very acute and in these almost continuous fever is present, the animals emaciate very rapidly, and death ensues after 2 or 3 weeks.

Recovery has so far been observed only very exceptionally (most likely in donkeys).

In cattle the symptoms, which consist of emaciation and anemia, periodical fever and edematous swellings (especially on the dewlap), sometimes do not become manifest for several weeks, or even months after the infection, and even later they become aggravated only very gradually. The eyes are rarely affected. The disturbance in their movements is only slightly pronounced, while the emaciation may reach a very high degree. Trypanosomes are present in the blood only in very small numbers, and frequently can only be demonstrated by inoculation of the blood into susceptible animals. Acute cases are observed only exceptionally. Recovery is more frequent than in horses.

The disease in sheep and goats manifests similar stmptoms to those exhibited by cattle. Parasites are also found only in small numbers.

In the advanced stages of the disease in dogs, emaciation, labored labial respiration, acute swelling of the lymph glands, shifting edema, especially on the extremities, edematous swellings of the feet, incoordination of movements, keratitis and iritis, as well as general falling of the hair may be observed.

Diagnosis. The sudden and rapidly changing edema associated with febrile manifestations, in the later stages the emaciation, anemia and muscular weakness in association with recurrent fever which progress in spite of good appetite, offer in infected localities a sufficient basis for a probable diagnosis of the disease. Absolute certainty however is only ohtained by the demonstration of the trypanosomes in the blood and in the fresh edematous swellings.

For the examination of trypanosomes a drop of blood should be taken from a superficial vein, mixed with several ec. of physiological salt solution, and the mixture examined in fresh, unstained or stained specimens (see p. 799).

The inoculation of blood into small test animals (also dogs) is valnable in the establishment of a positive diagnosis, especially since it accomplishes its purpose not infrequently, even in cases of negative microseopical findings.

Montgomery \& Kinghorn recommend puncturing of the swollen lympl glands, as, according to their experience, trypanosomes may frequently he demonstrateil in the glandular juice thus obtained, even when the examination of the bloor gave negative results.

Treatment. At present the reports on the results of Ehrlich's chemo-therapy (see p. 802), which has been employed in nagana of domestic animals in Africa are quite insufficient (in sleeping sickness of man which is also prevalent in Africa, 
atoxyl has been used successfully for several years). Ziemann mentions that atoxyl acts favorably in horses in chronic cases, while Schilling recently warmly recommends arsenophenylglycin (dose for hor'ses: $0.05 \mathrm{gm}$. per $\mathrm{kg}$. body weight) (see also the treatment of surra on p. 814).

Bruce fom that affected horses and donkeys treated with arsenical preparations lived longer than those which were not treated, and Laveran \& Mesmi also obtained favorable action from arsenious acid in experiments on animals artificially infected with nagana trypanosomes (rats, mice and dogs). According to Bruce \& Theiler, also Schilling, the arsenical treatment is useless in nagana of cattle.

Thiroux \& Teppaz recently reported lasting recoveries with auripigment and atoxyl, in the closely related trypanosomiasis of horses in Senegambia (Tryp. Timorphon, Cazalboui and Pecaudi). The treatment consisted either in the administration per os of 7-12 doses auripigment of $20-30 \mathrm{gm}$. each at 3 days' intervals, or alternating with atoxyl at 2 days' intervals (12-25 gm. auripigment per os; $5 \mathrm{gm}$. of a $1 \%$ atoxyl solution subentaneonsly).

According to Diesing the serum of byper-inmunized donkeys is supposed to improve the general condition of artificially infected horses and cattle, inasmuch as the number of blood parasites considerably diminishes after each injection.

Prevention. In addition to general prophylactic measures (see p. 804), attempts are made in infected localities to protect the animals from the infection by keeping the flies away from the animals by smoke and also by inunetions with disagreeably smelling salves or oils (creolin, petroleum, also stomach and intestinal contents of ruminants, etc.). Limiting pasturing to the night-time also rednces the cliance of infection. (In Uganda it was found that citronella grass keeps the dangerous flies away.)

Literature. Bruce, Further rep. on the Tsetse-fly disease or nagana, London 1897. - Theiler, Schw. A., 1901. XLIII. 97. - Schilling, Cbl. f. Bakt., 1902, XXXI. 452. - Laveran \& Mesnil, A. P., 1902, XVI. 1 u. 785 (Lit.). - Koch, D. m. W., 1905. 1865. - Samerbeck, Z. f. Hyg., 1905. LI. 31. - Schilling, A. f. Schiffs- u. Trophyg., 1909. Nr. 1. - Battaglia, Chl. f. Bakt., 1910. LIII. 113 (Lit.). — See also the general Lit. on page s05.

\section{(b) Surra.}

History. Griffith Evans found in 1880 in the territory of Punjab, in the blood of horses, mules and camels affected with the disease known there under the name of surra ( $=$ spoiled), a flagellated parasite, the etiological importance of which he immediately succeeded in establishing by successful inoculation experiments. Several years later the parasite was recognized as a trypanosome. English physicians and veterinarians, especially Lingard have studied the disease in detail. Rogers was the first to discover the agency of the insects as transmitters of the virus.

Occurrence. The disease occurs in eastern and western India, also in the adjoining Chinese territories. It has also been established on the coast of Persia, on the Mauritian, Malayan and Philippine Islands, and in Java and Sumatra (it appears that it has only recently been introduced to these islands). It exists everywhere in marshy localities, especially during the warm season, and causes great losses among solipeds 
and camels, while cattle and dogs are affected less severely. According to some authors (Laveran, Pease and others), Mboire and the El Debab of the camels in North Africa (see p. 838) is supposed to be identical with surra, having been introduced with camels from India. Surra appears to occur also in northeastern Africa.

In 1906 the disease was brought to New York with an importation of eattle from India, but was suppressed by the slaughter of the infected, and prolonged quarantine of the other animals. Camels have introduced the disease from India to Australia.

Etiology. The Trypanosoma Evansi is morphologically aımost identical with the producer of nagana. As an only, although not constant difference is given a somewhat more slender form, somewhat longer flagella, a more pointed posterior part of the body, and a smaller proportion of chromatin granules, and finally more active motility.

The possibility of differentiating surra from nagana consists at the present time principally in the fact that goats and eattle immunized against the usually more virulent nagana virus, are still susceptible to surra (Laveran \& Mesnil, Nocard \& Vallée), which although a differ. ential sign, is declared by Koch to be not conclusive.

As Trypanosoma vivax, Ziemann described in Kamerun a trypancsome which is eharacterized by very lively motility and resembles the virus of surra in other particulars.

Relative to the pathogenicity between the trypanosomes of surra and those of nagana, the only difference is that in artificial infection experiments the nagana trypanosomes are usually slightly more virulent.

The natural infection is transmitted according to Rogers and Musgrave \& Clegg by horse flies, namely, the Tabanus tropicus, the $\mathrm{T}$. lineola and the Stomoxys calcitrans, according to Leese also by the Hamatopa fly. Carnivora may infect themselves also by the ingestion of blood from affected, or of meat from dead animals, also through losses of substance in the skin and mucous membranes (Lingard; Vrijburg succeeded in infecting a mare by smearing the urethral secretion of an infected stallion upon the mucous membrane of the vagina).

Cattle and zebus are generally considered in the first place as virus carriers (virus reservoirs), although they are only slightly susceptible to the disease and usually become affected but mildly; nevertheless they may harbor trypanosomes in their blood for years. In the second place camels, when recovering from the disease, may likewise carry the parasites in their blood for a long time. Wild animals (foxes, jackals, hyenas) may also supply the virus to stinging flies.

For all stinging flies which have hitherto been considered as transmitters of the natural infection of surra, it is generally accepted that they transmit the trypanosomes entirely in a mechanical way; at least the experiments carried out failed to show that the surra trypanosomes pass through any stages of development 
(Leese) in the bodies of the flies, which latter are infectious only the first day after sucking the blood.

The question as to the mode and nature of natural infection cannot yet be considered as solved, and only recently Holmes, among others, expressed himself negatively on the exclusive transmission by horse flies, pointing out that the disease rages sometimes in the months from October to December inclusively, whereas the flies usually fly only in the months of June to August.

Anatomical Changes. Besides marked emaciation and changes indicating anemia and cachexia, a yellow, gelatinous infiltration of the subcutis is present in different regions of the body, also small hemorrhages in the serous and mucous membranes, serous exudates in the pericardium and in the abdominal cavity, more or less pronounced acute swelling of the spleen (especially in acute cases), and swelling of the lymph glands; in some cases superficial ulcerations also appear on the tongue and on the gastric mucous membrane.

Symptoms. These resemble in general those of nagana. In solipeds, which are the most susceptible animals to natural infection, the disease commences after an incubation of 4 to 13 days (Lingard) with fever (rise of temperature up to $40.5-41^{\circ}$ ), great languor and weakness, whereupon petechiae soon develop on the mucous membranes, especially on the conjunctiva. Urticaria-like eruptions appear on the skin, and edematous swellings on the extremities, the genital organs, in the submaxillary region, and on the lower abdomen.

A fatal termination may result even in the first days of the disease, usually however a marked improvement sets in after several days, but the febrile attack returns after a short time. The febrile stages usually last for four days, and alternate with fever-free periods lasting for several days, whereby the animals become more and more weakened, the paleness, and later also the icteric discoloration of the mucous membranes becomes more pronounced, the superficial lymph glands swell, the respiration becomes accelerated and labored, the pulse frequent and small. In spite of good appetite the animals become greatly emaciated, their gait is staggering, finally they lose the use of their hind parts and die with symptoms of severe dyspnea.

Of other symptoms there may be observed hemorrhages in the anterior chamber of the eye, diffuse keratitis, petechiae in the mucous membrane of the vagina, albuminuria during the febrile attacks, continuous erection of the penis (formation of thrombi in the corpus cavernosum?), etc.

Trypanosomes are found in the blood especially at the beginning of the febrile attacks, otherwise their number fluctuates in the course of the disease within wide borders.

The course extends usually from 1 to 2 montlis, more rarely from 1 to 2 weeks, or on the contrary for 3 to 4 months, the febrile attacks becoming gradually weaker in the later stages. 
The disease appears almost without exception to terminate in death.

In cattle the disease occurs much less frequently and is manifested by less pronounced intermittent febrile symptoms, becoming apparent only with the gradual development of anemia and emaciation, which sometimes reach a very high degree. In most cases ultimate recovery takes place.

There occur however outbreaks in which the disease appears in numerous cattle with severe manifestations, such as high ferer, reddening of the mucous membranes, diarrhea and hemorrhages, and runs an unfavorable course, although even in such instances recovery may follow.

Buffaloes rarely become affected, and then usually only in very chronic form, although their blood frequently contains the parasites.

In camels the disease nsually has a fatal termination, and as in solipeds it may rum an acute and severe course, or it may oceur in a ehronic form. Thus Pease \& Gaiger observed two camels affected for four years, during which time they noted more than 100 febrile attacks.

Of dogs, animals used in hunting become affected most frequently. The symptoms consist in a periodical fehrile temperature, reddening of the conjunctivae, swelling of the head, the throat region and extremities. also incoordination of movement. In some cases there is a bloody serous infiltration in the joints, keratitis, and falling out of hair. Among imported hunting dogs in Indo-China the disease is severe, while native animals sometimes recover (Blin).

Treatment. Lingard recommended arsenious acid in daily doses of $0.8 \mathrm{gm}$. for the treatment of horses affected with surra, Holmes recently reports continuously good, permanent results in ten ponies after the use of considerably larger, namely, subtoxic doses, at the height of the febrile attack. For ponies of $150 \mathrm{~kg}$. weight, $1 \mathrm{gm}$. is recommended, while for horses of 450-500 kg. weight, $3 \mathrm{gm}$. should be given in the form of bolus, or electuary immediately after feeding, 5 to 10 doses being administered with intervals of one day. It is not recommended in solution either per os, subcutaneously, or intravenously. Somewhat less satisfactory were the results from Löfler's treatment with arsenious acid and atoxyl, inasmuch as out of 16 solipeds thus treated, four failed to recover (alternately. $4 \%$ atoxyl solution subcutaneously and arsenic in bolus form with one-day intervals; the dose of the atoxyl solution is $30-50$ cc. for ponies, $100-150$ cc. for horses and mules; of the arsenic 0.5-1 gm. for ponies, $1.5-3 \mathrm{gm}$. for horses and mules; 5 doses in all).

Thiroux \& Teppaz found auripigment effective in experimental surra; they used it either alone, in doses of 15-25 gm. every 3-4 days, or alternately with tartar emetic (1.0-1.2 gm. intravenously), eontinued for weeks.

Gaiger, Hallot and Holmes obtained only a temporary disappearance of trypanosomes from the blood with atoxyl, and likewise little satisfaction was obtained from the treatment with corrosive sublimate, soamin, atoxyl and tartar emetic (Holmes), with atoxyl and corrosive sublimate (Fraser \& Simmonds), also with methylene blue (Gaiger). 
Prevention. In this general principles apply (see p. 804), also the precantions advised for the control of nagana (see $p$. 811). In fresh outbreaks it is advisable to select the infected animals with the aid of taking their temperature, blood examinations, and inoculation of mice and rats, and subsequently their isolation or destruction. In this way it was possible in Java, among other places, to eradicate the disease which had been introduced there not very long before.

Literature. Evans, Vet. J., 1881. 1.; 1882. 97. - Lingard, Rep. on HorseSurra, Bombay, 1893. - Rogers, Proc. of the Roy. Soc., 1901. LVIII. 163. Vrijburg, Bull., 1907. 293. - Ziemann, Cbl. f. Bakt., 1905. XXXVIII. 307. Schein, Trop. Vet., 1908. III. 191. - Leese, ibid., 1909. IV. 107. - Holmes, ibir., 1910. V. 1. - Thiroux \& Teppaz, A. P., 1910, XXIV. 220 u. 234. - (See, also, general Lit. on page 805.)

\section{(c) Dourine.}

(Exanthema coitale paralyticum, Polyneuritis infectiosa; Beschälseuche [German]; Maladie du coit [French]; Marbo coitale maligno [Italian].)

Dourine is the name of a usually chronic, contagious infectious disease of breeding horses, which commences shortly after an infective coitus and is at first characterized by a local inflammatory affection of the external genital organs, with which subsequently symptoms of paralysis become associated as a result of an affection of the peripheral nerves and the intervertebral ganglia. It is caused by the Trypanosoma equiperdum.

History. The disease was first described by Ammon, based on his experienee (1796-1799) in the Prussian stud at Trakehnen. At the beginning of the last century it had already been recognized that it is most effectively spread during coitus, and the disease has frequently been confounded with coital exanthema of the genital organs. At the same time, however, it was also assumed that the affection is identical with syphilis in man, with which it doubtlessly has much similarity. This view was also prevalent long since in Algeria, where the Arabs, in their helief that syphilis can be cured hy copulation with asses, followed such unnatural intercourse whereby the disease was supposed to have been transmitted from the affected female asses to the stallions (Daumas; lience also the old designation of the disease: Lnes venerea equi, which has been introduced by Veith into the literature of this subject.) Laquerrière aimed to prove this view in France, in 1883, by theoretical considerations; its incorrectness, however, was esablished by Knauert \& Haxthausen (1837), and the negative results of experiments for the transmission of syphilis to domestic animal also proved these conceptions conclusively as erroneous. The difference of dourine from coital exanthema of the genitals was established by Hertwig in 1842, and still more conclusively in 1847 , and he, also Mares and later Prince \& Lafosse, proved further that the disease results exclusively through specific infection.

In the second half of the last century it was observed especially by French veterinarians in Algeria (Signol, Viardot), and by Rodloff in 
Russia. In Hungary the Department of Agriculture appointed in 1877 a special commission for the study of the discase, at which occasion Thanhoffer (1882) examined histologically the organs of more than 30 affected horses, while Azary, besides making artificial experiments of transmission, thoroughly studied the clinical manifestations.

Rouget (1894) found in Constantine in the blood of a stallion affected with dourine, a trypanosome in which he immediately suspected the cause of the disease. This finding and its etiological importance was later confirmed in Algeria by Schneider \& Buffard, in Netherlandish-India by Does, in India by Pease, in Hungary by Marek, and in Germany by Kleinpaul \& Neumann, and Lorenz.

Artificial experiments of infection were recently carried out by Schneider \& Buffard, Marek and Zwick \& Fischer, while careful clinical investigations were undertaken by Marek and Fröhner.

Occurrence. Dourine was fairly prevalent in Europe previous to the midlle of the last century, and caused heavy losses by its frequent enzootic extension, especially in the horsebreeding establishments. The veterinary police measures subsequently adopted resulted in a rapid diminution of the disease, so that at present it prevails only in some southern and eastern states, whereas in other parts of the world it occurs more frequently.

In Germany the disease was formerly widely disseminated, especially in the northern territories (Hanover, Silesia, Posen), but has been eradicater since the inauguration of the infectious disease law. After a decade of freedom from the disease it was, through a mare imported from Russia, introduced again in 1906 into the districts of eastern Prussia, Angerburg and Lotzen, from which it spread into the districts of Johannisburg and Lyck. By the end of 1908, 82 horses had been affected on 62 premises, in 40 townships.

In Hungary the disease was first observed in 1819 at Mezohegyes, and later by Pillwax (1833) in the stud of Babolna. As a result of the measures adopted in 1877 the disease has been observed only sporadically since the year of 1890 , and after the eradication of two local outbreaks in the counties of Abauj-Torna and Szaboles in 1896, the entire country has remained free since 1901. In 1905, however, the disease was imported from Croatia into the county of Zala, where it spread to 16 townships. In this, and in the following year, a total of 129 horses were affecter, but in May of 1907 the entire country was free.

In Croatia dourine was widely prevalent at the end of 1901 in nine townships of three districts (26 horses had to be destroyed during the year). In Austria it was first observed in 1813 at the veterinary school of Vienna, the last time in 1878 in Bohemia. In France it has recently always been confined to small territories, occurring as a result of importation from Algeria and spain, and par. ticularly in the Department of Basses-Pyrénées cases oceur almost annually. Spain appears to be infected considerably, likewise Russia, where the disease was diagnoserl by Tschernogoroff in 1897-1902 among 37 stallions in the stud of Oldenburg. Iu Roumania, where it was found by Motas in 1598, it is also widely distributed (Pav. losevici).

Dourine ( = dirt, unclean copulation) is widely disseminated in North Africa, and especially in Algeria, where it causes an annual loss of at least 150,000 franes, it likewise occurs in Asia Minor, Syria and Persia.

In America the disease was recently encountered repeatedly in the States of Illinois and Nebraska, and also in Chili; the principal focus of infection, however, exists on the Indian reservation of South Dakota, where in 1903, on the occasion of the examination of the horses, 511 were found to be affected, 277 suspected, and 1,889 stallions had to be castrated. The energetic measures appear to have heen crowned with good results, as since 1906 no other cases have been noted.

Etiology. Dourine is caused by the Trypanosoma equiderdum, which strikingly resembles the trypanosome of nagana. 
According to Laveran \& Mesnil the only distinguishing signs are the sometimes pointed, at other times blunt, or sometimes split posterior extremity, further in the parasites originating directly from the horse, the absence of granulations is mentioned.

Pathogenicity. Material containing trypanosomes taken directly from horses is always virulent for horses, while it appears to possess for other animals a varying virulence, while in Algeria, rabbits and dogs are usually infected very easily, and a virus constantly effective for rats and mice was obtained by further inoculations, similar material from horses in Humgary proved pathogenic only for solipeds. During the last invasion in Prussia, similar experiences were recorded, although in some instances mice were successfully infected. Zwick \& Fischer then cultivated a strain by further passage throngh mice with which they succeeded in infecting not only small test animals, but also dogs, cats and sheep, and likewise goats and cattle.

Mares covered by stallions, the urethral secretions of which contains trypanosomes, develop after about 2 to 4 weeks, sometimes even later, an acute inflammation of the vagina and an edematous infiltration of the surrounding tissue, while later "dollar-spots" and still later symptoms of paralysis appear which continually become more intensive until the animal dies. During the existence of the inflammation of the vagina, trypanosomes may be demonstrated in the vaginal discharge, in the edematous swellings and in the dollar-spots, much less frequently also in the blood. An affected mare may also infect the stallion, in which case the effects are first manifested in an edematous swelling of the sheath and an accumulation of mucous secretion in the urethra which contains trypanosomes. The disease may also be produced artificially by smearing such secretion or blood containing trypanosomes on the mucous membrane of the vagina or urethra, as well as by dropping it into the conjunctival sack.

The injection of an emulsion of the spinal cord substance from an affected horse, into the anterior chamber of the eye of a healthy animal, is also effective (Nocard; Hutyra observed in a pony in such an instance the appearance of dollar spots as early as in one week, and subsequently a rapid development of paralysis.)

After the inoculation of virulent blood under the skin of a horse an edematous swelling appears at the point of injection in 7 to 12 days; after 3 to 6 weeks transitory swellings of the skin appear on different parts of the body, especially in the vicinity of the genitals; after six weeks, sometimes not until after five months, a paralysis of the hind parts develops with knuckling of the hind legs, until the animal, which has become greatly emaciated in spite of constantly good appetite, succumbs with symptoms of a lumbar paralysis. During the course of the disease intermittent fever is observed. In some cases

Vol. 1-52. 
periods of improvement are obserred in the course of the disease or complete recorery may take place.

In rabbits a subcutaneous or intravenous injection results sometimes in acute, septicemic, at other times in a chronic affection. In the latter an inflammatory swelling of the genital organs, falling of the hair, necrosis of the skin, crustaceons eczema on the forehead, purulent conjunctivitis, or panophthalmia is observed, while copulation of an affected with a healthy animal produces in the latter a gangrenous inflammation of the genitals. Infected guinea pigs remain apparently healthy in spite of the periodical appearance of the parasites in their blood, and suddenly succumb months later.

In dogs a warm and painful swelling appears at the point of inoculation, also extensive edematous infiltration of the abdomen, in the parts surrounding the genitals and in the inguinal region; also acute balanitis; in bitches inflammation of the vaginal mucons membrane with a profuse discharge is observed. The back is sensitive, round flat swellings of the size of the palm of the hand which again disappear within 2 to 3 days appear on the skin, in some cases an inflammation of the joints, purulent conjunctivitis, and ulcerative keratitis may be present. In the later stages rapid emaciation, in spite of good appetite, stiffness of the hind parts, general hyperesthiesia, and in the last stages complete inappetence without fever, marked emaciation, as well as very laborions respiration are noted.

In dogs that are paired with affected animals an acute inflammation of the external genital organs develops, in which the discharge contains trypanosomes, and similar results are obtained from an injection of blood containing trypanosomes into the vagina of the bitch.

In sheep plaques develop in different parts of the body, which leave in their places large bare spots; besides this an inflammation of the genitals develops while the general condition remains undisturbed for months. In cattle a positive result of the infection can be only established by the demonstration of trypanosomes in the blood. Fowl can be infecter only exceptionally (positive results are reported by Yakimoff \& Kohl).

The natural infection is usually transmitted by coitus. Dourine affects almost exclusively stallions and breeding mares, and the disease has repeatedly been reproduced intentionally by allowing affected mares to be covered by healthy staliions, and healthy mares by affected stallions (Prince \& Lafosse, Trasbot, Azary, Marek). In studs it is, as a rule, mares covered by certain affected stallions that bccome affected, and only much later are other stallions infected $a z$ a result of an infection through affected mares, and these may in their turn infect many mares.

During coitus the healthy genital organs are infected hy the secretion of the urethra or of the raginal mncons mem- 
brane. Trypanosomes at times in rarying numbers are present in these for months even before the appearance of the symptoms, and also in the later course of the disease, even when the animals otherwise show no symptoms or at most only a very moderate swelling of scrotum or vulva. During the periodical aggravations of the local manifestations, an increase of their number may usually be demonstrated.

In the rare cases in which the disease appears in horses not used for breeding, sometimes even in gelding's, the infertion is probably conveyed by sponges or by the hands of the attendants.

The possibility of transmission by stinging flies cannot be discarded entirely. Lingard has already indicated such a possibility, and Sieher \& Gonder have observed the infection in a horse which stood in a stable alongside of an artificially infected animal. Schubert \& Kuhn also succeeded in an intentional transmission with the aid of the stomoxys calcitrans. Further than this Rabinowitsch has infected laboratory animals with dourine trypanosomes through lice and fleas, while Uhlenhuth \& Woithe, Manteufel, also Zwick \& Fischer, have proved in rats the possibility of infection through the intact skin. As however trypanosomes occur at least in northern countries, only rarely in the blood of horses, and even then but few in numbers, stinging flies have only slight opportunity for taking up the parasites (most likely from the fresh plaques). Therefore such a mode of transmission is apparently of very minor importance as is also confirmed by experience in practice (according to Sieber \& Sender the trypanosomes remain viable only for three hours in the stomachs of flies).

In former times an important part in the extension of the disease was attributed to heredity (Roloff, Jessen). With the experience acquired in its eradication, and with the establishment of the etiology of the disease, this conception is no longer tenable.

In general the susceptibility of solipeds for infection is considerable, but it varies individually, as not all mares covered by an infected stallion become affected. This may however be explained by a temporary disappearance of the trypanosomes from the secretions of the genitals.

According to Roll about $66 \%$ of mares which are exposed to the infection become affected. Rodloff in Sierakowo observed in all of 107 mares which were covered by an infected stallion, mild or severe symptoms occurring later on ( 54 of these died), while in the experiments of Prince \& Lafosse only 10 out of 15 mares became infected under similar conditions. Kern reports 24 cases in 105 mares which were covered by an affected stallion.

Pathogenesis. The trypanosomes which penetrate into the mucous membrane of the external genital organs first multiply at the point of entrance where they produce a serous and in part a cellular inflammation. Later they enter the blood and with it pass to distant parts of the body. This mode of dissemination is indicated not only by the possibility of demonstrating at times the parasites in the blood, but also by the fact 
that in some cases paralysis develops in the region of some of the peripheral nerves (Facialis!) even in the early stage of the disease, and further that in the affected tissues the round cell infiltration is always observed in the immediate vicinity of small blood vessels.

The intermediate attacks of fever appear to be produced by periodical invasions of large masses of trypanosomes into the blood stream, or by their temporarily rapid multiplication in the blood. Their disappearance appears to be the result of the development of trypanolytic substances. The trypanosomes apparently exert their pathogenic action by the production of certain toxins, which have not yet been demonstrated; these on the one hand produce a serous inflammation of the walls of the vessels, on the other hand they principally attack the substance of the peripheral nerves, and the intervertebral ganglia. In these a cellular infiltration of the nerve tissue develops (neuritis interstitialis), whereupon the nerve fibres, as well as the nerve cells, degenerate and finally disappear entirely. The sensitive nerve bundles become involved in great numbers, and in a more severe form than the motor fibres, the affection of which results in a degeneration of the corresponding muscle fibres. The disturbances observed in the movements and in the sensibility of the living animals are the direct result of the inflammation and degeneration of the peripheral nerves, while the emaciation indicates the action of a toxin circulating in the blood.

In cases in which the parasites disappear entirely from the body a complete recovery may result even in cases in which conspicuous nervous symptoms have already appeared, and such instances are not rare in northern regions. As long as the motor cells of the medulla and the spinal cord are intact, new nerve fibres may form from the nerve cells or from the stumps of their prolongations of the axis cylinder. This is proved by the microscopical examination of cases in which the disease process showed improvement, when the nerves will be found to show fine pale stained, new nerve filaments (Marek).

The dourine trypanosomes have a pronounced predilection for the mucous membrane of the genitals, for they may be demonstrated in these even after subcutaneous, intravenous or conjunctival infections. They are evidently capable of passing out of the blood vessels, producing exudation of blood plasma and a cellular infiltration on the irritated place, and thus cause among other symptoms an urticaria-like eruption of the skin, the so-called dollar spots.

Anatomical Changes. Carcasses of animals dead îrom dourine are extremely emaciated, and show on the prominent parts of the body sores resulting from continuous lying down. The subcutaneons connective tissue is poor in fat, and in places shows gelatinous infiltration.

In stallions the connective tissue of the scrotum is in many 
cases edematously swollen; the layers of the tunica propria are adherent by tough connective tissue in their entire extension or only in parts, or they may be covered by shredded granulations, while the cavity contains a clear or slightly clouded serous fluid. In rare cases small purulent foci are also found in the tissues of the testicles or between the pseudo-membranes, otherwise the tissue of the testicles and the epididymis appears unchanged, although the cord may show gelatinous infiltration. In mares the tissue of the udder and the vulva may sometimes show an edematous infiltration or sclerotic thickening. On the glans penis, also on the nucous membrane of the urethra near the outside orifice, in mares on the mucous membrane of the vaginal opening and the vulva, spots freed from pigments may be observed, as well as fine or thick radiating shrunken cicatrices. The lymph glands, especially those in the region of the genital organs, are swollen, soft, and show on the cut surface a moist glistening appearance.

The muscles, especially those of the croup and of the posterior extremities are pale red, in places intermixed with butteryellow stripes and hemorrhages, or with blackish-red or yellowish-red areas. The capsular ligaments of some joints are reddened, the cartilages of the epiphyses are ulcerated, while the synovial fluid appears cloudy and reddish-gray in color. The intra- and intermuscular comnective tissue shows serous infiltration or may be changed in places into cicatricial tissue.

The internal organs show pronounced anemia and edematous infiltration, sometimes a hypostatic pneumonia or manifestations of a general septic infection. In cases which have had a rapid course, an acute swelling of the spleen and of the lymph glands may also be present. Fröhner describes a thrombosis of several peripheral arteries by masses of leucocytes as a rare lesion.

According to the descriptions of older authors portions of the spinal cord, especiallyl in the lumbar section, contain softened areas in the gray substance, besides small punctiform hemorrhages; as a matter of fact however changes visible with the naked eye may be entirely absent, even in cases with fatal termination. The meninges of the spinal cord may in parts contain smeary, friable masses. The larger nerve branches, especially those of the extremities, are either serously infiltrated or changed into connective tissue cords, which are strongly adherent to the intra- or intermuscular comnective tissue.

The histological examination of the nervous system (Marek) in the chronic cases shows in the extra-spinal nerve trunks, and most pronounced in the nerves of the posterior extremities, sometimes also in some of the cerebral nerves (V., VII. pair) a cellular infiltration, degeneration and atrophy of some of the nerre fibres, and an increase of nuclei of the endoneurium. These changes reach the highest degree near the sensory ganglia and in the facial nerve in the Fallopian canal. The nerve cells of the inter-vertebral ganglia in general, especially in the lumbar portion of the spinal canal, show an atrophy, chromatolysis, sclerosis, and peripheral displacement of the nuclei, while the connective tissue of the nerve fibres, which shows round cell infiltration, has partially disappeared. The peri- and endoneural 
nuclei, however, are increased. The cellular infiltration extends also to the sensory roots of the entering zone of implantation, while the degeneration of some of the nerve fibers may be followed into the posterior trunks of the spinal cord, and up to the nuclei of the medulla. The other trunk, as well as in general also the nerve cells, are intact in the spinal cord, as well as in the medulla, otherwise, however, the chronic cases which have been examined nuanifested no changes, with the exception of some perivaseular, inflammatory foci in the gray substance of the spinal cord. This, however, does not exclude the occurrence of intensive inflammatory changes in other cases.

A fatty degeneration of the musele fibers, also perivascular cellular infiltration of the intramuscular connective tissue, the intensity of which varies in different parts of the borly, is present in the muscles. It is most pronouncerl in the muscles of the croup and the posterior extremities (Fig. 143). Marek failed to find special changes either in the corium, or in the subcutaneous connective tissue, corresponding with the location of the dollar spots, whereas he met a focal cellular infiltration under the mucous membrane of the vagina, even in chronic cases.

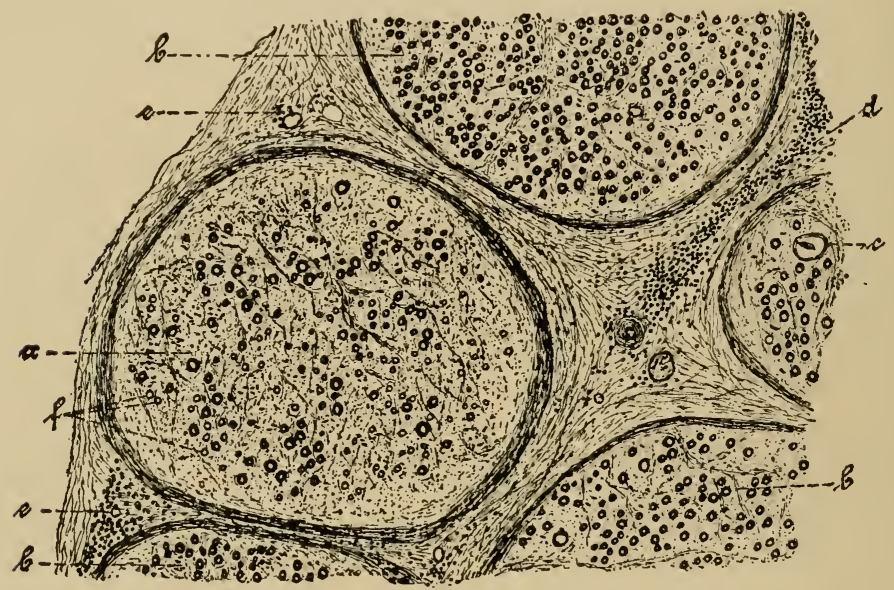

Fig. 143. Transverse section through the $\mathrm{N}$. cruralis of a horse affected with dourine. $a$ pronounced, $b$ slight absence of nerve fibres; $c$ distended nerve sheath; $d$ and e epineural cell infiltration. (Marek.)

Symptoms. In the appearance of the symptoms two stages must be distinguished, for which essentially different symptoms are characteristic, and which moreover are separated from each other by shorter or longer, apparently normal periods. The symptoms of the first stage affect principally the genital organs and differ from each other according to the sex of the animals, while in the second stage the symptoms predominate which point to an affection of the nervous system and which are not influenced by the sex of the patient.

The characteristic symptoms of the first stage develop subsequently to the infectious coitus, after an incubation of from 5 to 6 days up to one month, sometimes however apparently after several months, as in some cases the disease is manifested only by moderate febrile attacks which may be unnoticed. 
Prince \& Lafosse observed after the infectious coitus an incubation of 7-60 days, Hertwig 2-8 days (?), Rodloff 8-14 days. Schneider \& Buffard, also Baldrey, observed recently in experimental infections the development of the first symptoms, or at least the first febrile attacks after 8-20 days, Buoy after 40-45 days, Marek after 5-33 days.

Trypanosomes may sometimes be demonstrated in the urethral or vaginal secretion as early as 4-5 days after the infection; in spite of this, howerer, excepting the rise of temperature, the local symptoms may not develop for months (according to Kern sometimes only after 9 months).

In stallions the disease usually commences with an edematous infiltration of the sheath, which gradually extends from one place orer the entire skin of the part, and especially to the anterior pendulous portion. The swollen sheath does not

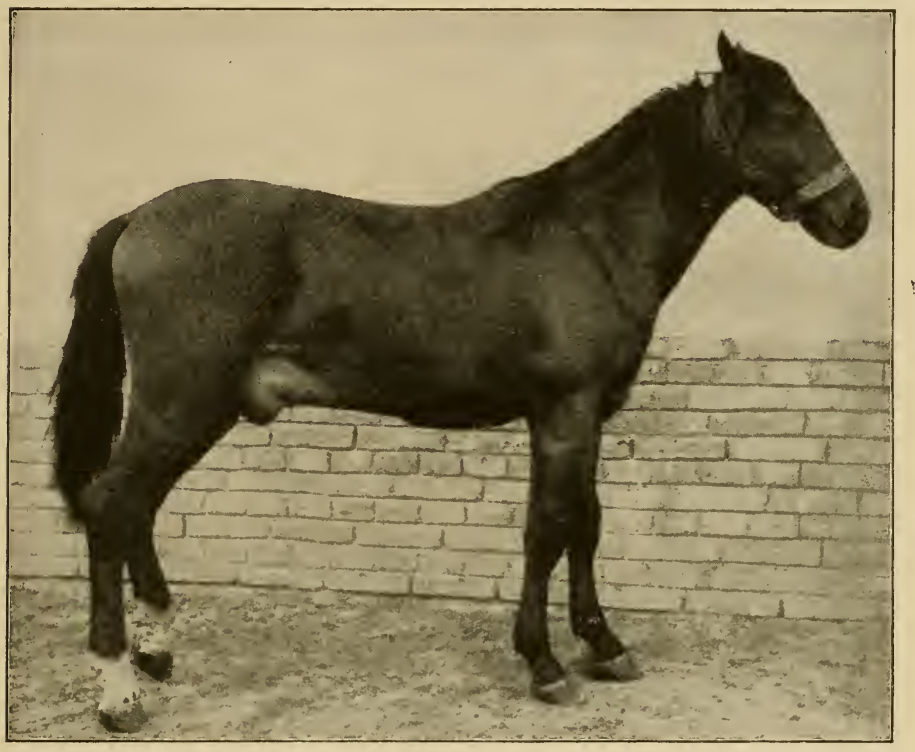

Fig. 144. Dourine. Swelling of the sheath and the lower ehest; plaques on the croup and on the neck of a stallion.

feel warmer and is not painful. It is of doughy consistence, resembling somewhat a fat bag, only that the skin is stretched and not wrinkled (Fig. 144). The swelling spreads along both folds which pass from the orifice of the sheath to the abdomen and forwards and backwards to the scrotum. At the same time the glans penis also swells and the animal protrudes a longer or shorter portion of this organ from the sheath. The glans proper shows hardly any perceptible changes, excepting the edematous swelling which however may also be very slight, and a grayish transparent, or yellowish-gray mucus oozes out 
from the urethra. In infrequent cases the swollen glans appears of higher temperature and more sensitive. The border of the urethral opening is also frequently swollen, slightly reddened and the mucous membrane protrudes slightly (especially in jacks). Later lentil to pea-sized, yellowish-red nodules on an infiltrated base develop, sometimes singly, at other times in groups, on the mucous membrane of the urethra in the vicinity of the urethral opening, also on the glans and on the body of the penis, and change into round, flat ulcers in 12 to 36 hours; these usually heal after a time, leaving behind roundish white spots. In some cases however they may persist for a long time, and even enlarge, after which their base is lardaceous, their borders raised, and on final healing prominent scars remain.

During the development of these changes the animals manifest a continnous desire to urinate, but they pass only small quantities of urine and nervously raise one of the hind legs or arch their backs. Besides this frequent erections of the penis are observed and the animals usually show an increased sexual desire.

In mares the first manifestations consist in an edematons swelling of the vulva, discharge from the vagina, also reddening and inflammatory swelling of the vaginal mucons membrane. The labia of the vulva usually appear swollen on both sides, frequently however not uniformly; at the same time they are dense, elastic, not painful, and frequently covered with non-pigmented spots, similar to those sometimes occurring in the vicinity of the rectum (so-called toad spots). Sometimes the clitoris is swollen, so that it protrudes from the lower gaping opening in the vulva. The mucous membrane of the vagina is loosened, shows dark red spots on a reddish-yellow hase and is sometimes thickened into gelatinous, quivering, longitudinal pads. The mucous follicles are also swollen, especially in the superior commissure, and in the vicinity of the clitoris gelatinously translucent, and may be felt as milletseed-sized, rarely larger nodules. The nodules may later break down, whereupon in their place small round ulcers, with sharp borders and yellowish bases appear. The ulcers finally heal, either without leaving any trace, or in their places light yellow spots may remain for a time (proliferated epithelium) (Fig. 145). Besides the nodules, pea-sized vesicles may develop exceptionally on the mucous membrane of the vagina and on the rulva, in rare cases also in the surrounding portions of the skin, these contain a yellow serous fluid, and similar small ulcers develop after they burst.

In serere cases the ulcerations increase in size, their bases becoming covered with necrotic tissue and the dark red borders showing an elevated swelling. They heal very slowly, and in their places round, smooth scars remain for a long time. Meanwhile the lischarge from the vagina increases; first it 
is white or yellowish and slimy, later more tenacious and finally purulent, dirty yellow, or reddish, dries on the lips of the vulva to dirty brown crusts and glues the hairs of the tail together. The edematous swelling may extend to the ndder and the abdomen, as well as to the thighs.

During the development of the local changes the mares manifest oestrum-like behavior, they shift their feet restlessly, swing their tails, strain frequently to urinate, sometimes passing only a little mucus and at other times a small quantity of urine after which they arch the back.

The symptoms do not always reach so high a degree that attention may be attracted to their presence. Cases are relatively frequent in which the characteristic changes are only observed in the later stages, when by subsequent careful examination the traces of the preexisting ulcerative processes may be found on the genitals in the form of white scars. If however nodules and ulcers develop only on the mucous membrane of the urethra, as sometimes occurs in stallions, not even traces of the local affection may be found on the genital organs. The initial symptoms are sometimes unnoticed, especially in mares in breeding establishments,

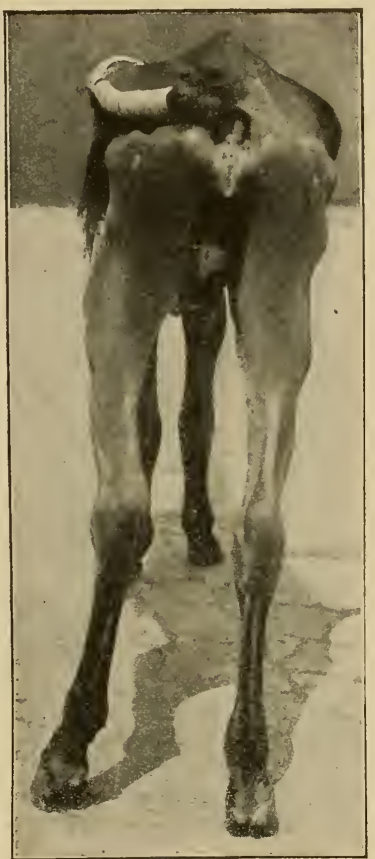

Fig. 145. Dourine. Unpigmented spots on the lips of the vulva; pronounced emaciation. but carefully watched stallions may direct the attention to the affection by the appearance of the nervous symptoms.

The ulcers on the vaginal mucous membrane are described by all authors, with the exception of Rodloff, who never observed them. IIares observed a discharge from the vagina only in 17 out of 197 affected mares.

In the second stage, which, as a rule, follows the first after several weeks, and sometimes after a temporary improvement, exceptionally however without any period of transition, symptoms appear which partly indicate an affection partly of the skin, partly of the peripheral nerves and the intervertebral ganglia.

The so-called dollar spots appear first, usually on the sides 
of the chest and on the croup, more rarely on the neck, shoulder, withers or on other parts of the body (Fig. 1t6). They are either round or semi-circular, or again irregular in shape. The former have a diameter of $4-8-15 \mathrm{~cm}$., and may be recognized in that their periphery is formed by a ring $1-3 \mathrm{~cm}$. broad, over which the hair stands erect or disarranged as a result of an edematous infiltration of the capillary bodies, on account of which the ring appears darker and less glistening. Within the ring the skin is not swollen, the hair is smooth, and therefore this portion appear's sleek and glistening like the skin ontside of the ring. The second

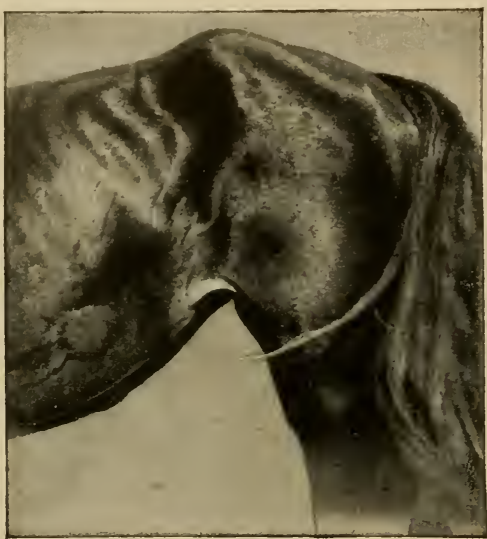

Fig. 146. Dourine. Plaques on the left side of the abdomen. category of the dollar spots is a very irregular, zigzag-shaped form, frequently extending 15-20 cm. in one direction, and covered with roughened hair. Besides these spots, lentil to bean-sized norlules derelop on the skin, over which the hair is also rough; such nodules are mostly observed on the inner surface of the thighs, in the flanks, and more rarely on the sides of the thorax and on the shoulders. The fourth form resembles urticaria and the designation of "dollar spots" is really appropriate only for this form of eruption. Sometimes on the inner surface of the thigh, sometimes in the flanks, lentil to dollar-sized, uniform protuberances appear, over which the hair appears rough and dull. The number of the dollar spots may be considerable, even exceeding one hundred. They usually persist for 1 to 8 days, when they disappear without leaving a trace, whereupon however fresh plaques soon appear on other parts of the borly. All forms of eruptions are painless, are not of a higher temperature, and are not associated with itching.

Strausz in Mezohegyes observed besides the dollar spots a severe vesicular and crustaceous eczema on the abdomen, on the chest, also on the croup, and at the base of the tail. The intensely itching eruption ("itch disease") which eaused the patients to rub and scratch severely, resisted all treatment, but after a time it was arrested of its own accord, and finally healed! later however hair failed to grow on the hairless spots, and if in these places the pigment of the skin had disappeared the affected part of the body showed a variegated appearance. 
Kern observed in some cases persistent perspiration and subsequently alopecia, Fröhner saw white hair appear on the rump of a stallion.

Hyperesthesia either over the entire body surface or only in the region of certain nerve trunks, may be established either simultaneously with the appearance of the dollar spots or later.

On account of the increased sensitiveness of the skin, sometimes a slight touch or a slight prick on the skin causes the animal to offer opposition, while stroking the back with the fingers causes the patients to groan and depress the back.

At the same time an increased sensitiveness of certain nerve trunks may be ascertained; thus the animal manifests sharp pains on pressure or stroking over the $\mathrm{N}$. ischiadicus and peroneus, the Nn. intercostales, the N. medianus and infraorbitalis. As the contraction of certain groups of muscles press or stretch the nerves lying between them a disturbance in their movements results owing to their sensitiveness. The walk of the patients is stiff, they hold one or the other leg more flexed, especially on trotting and galloping, also during quick turns and therefore evince lameness. If the animal stands still it periodically raises a foot spasmodically, as if tortured by lancinating pains, and holds it high for a while trembling. Similar manifestations are observed when the animal starts to move and during passage of the bowels. These spasms occur more frequently during nighttime than during the day and are sometimes observed when the animal is lying down. In some animals a sudden lameness sets in, which however disappears after a few days. On account of the sensitiveness of the intercostal nerves the respiration is accelerated and superficial, while owing to irritation of the vagus nerve, the patients cough periodically.

The increased sensibility gradually passes into a hyperesthesia, exceptionally into a complete anesthesia, and as a rule paralysis of certain motor nerves appears in the meantime. This is observed in almost every ease in the hind extremities, quite frequently in some of the facial nerves, especially the $\mathrm{N}$. facialis, while the nerves of the anterior extremities, and those in other parts of the body are but rarely affected.

The symptoms of paralysis are usually not even on both sides of the body. On the head they are almost invariably unilateral. As a result of paralysis of the nerves on the posterior extremities, the animals show a staggering gait, which is noticeable while walking in a straight direction, but more so while walking in a circle. On trotting they hold the hind legs spread apart or drag them with the flexed hock on the ground; when halted they usually knuckle (Fig. 147). The paralyses of certain nerves of the head are manifested in corresponding disturbances of motion and in distortions. According to Lorenz and Fröhner a peripheral recurrens-paralysis 
occurs frequently sometimes in spite of its long existence without leading to a demonstrable atrophy of the posterior muscles. The sphincter muscles of the anus and the bladder are not paralyzed.

The superficial reflexes are increased over the region of the hyperesthesia, but they become weaker after the appearance of the muscular paralysis and finally disappear entirely. The tendinons reflexes, corresponding to the affected nerves, are diminished from the beginning or entirely absent, especially the patellar reflex. The electrical irritability of the nerves and muscles is likewise reduced in the regions of the paralysis or a partial and even a total degeneration reaction may be established (Marek).

The srmptoms of paralysis diminish sometimes considerably after sereral weeks and may disappear entirely, even

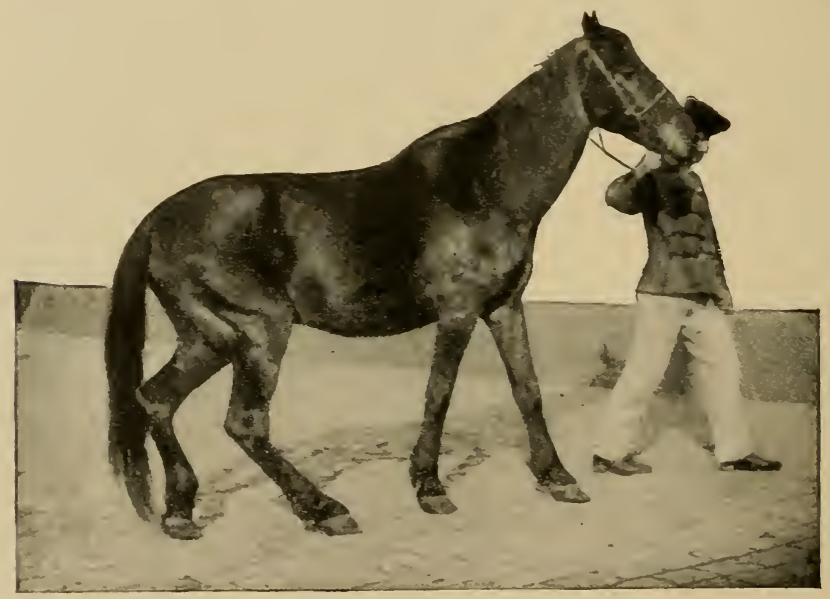

Fig. 147. Dourine. Paraparesis in an affected mare. (Snapshot.)

when a degeneration reaction had previously been ascertained. In other cases they become more aggravated, the withering muscles become markedly atrophied, and finally the paralysis of the hind parts reaches such a degree that the animals are no longer capable of rising from the ground.

Frequently affections of certain joints and tendons become associated with the nervous simptoms. The hock joint, more rarels the tarsal joints, or some articulations of the front legs, swell from day to day, together with the corresponding tendinous sheaths. The swellings are produced by the accumulation of a large quantity of fluid in the srnorial capsule or in the tendinous sheath, but at the same time there are no inflammatory symptoms perceptible. On palpation ther 
show fluctuation, at the same time they are not warmer or painful. Besides they may retrogress as rapidly as they develop and recur again after a certain time.

The respiration is quiet and slower than normal, but even after short exercise it becomes very much accelerated, calming down only gradually. Toward the end of the disease a catarrh of the air passages or broncho-pneumonia may develop. The heart action is mostly normal, only the heart is more easily excited, as even after short exercise acceleration, and sometimes also an arrhythmic pulse may develop.

The superficial lymph glands, most frequently the inguinal, sometimes also the submaxillary glands, occasionally become swollen, when they feel soft, elastic and painless or only moderately sensitive.

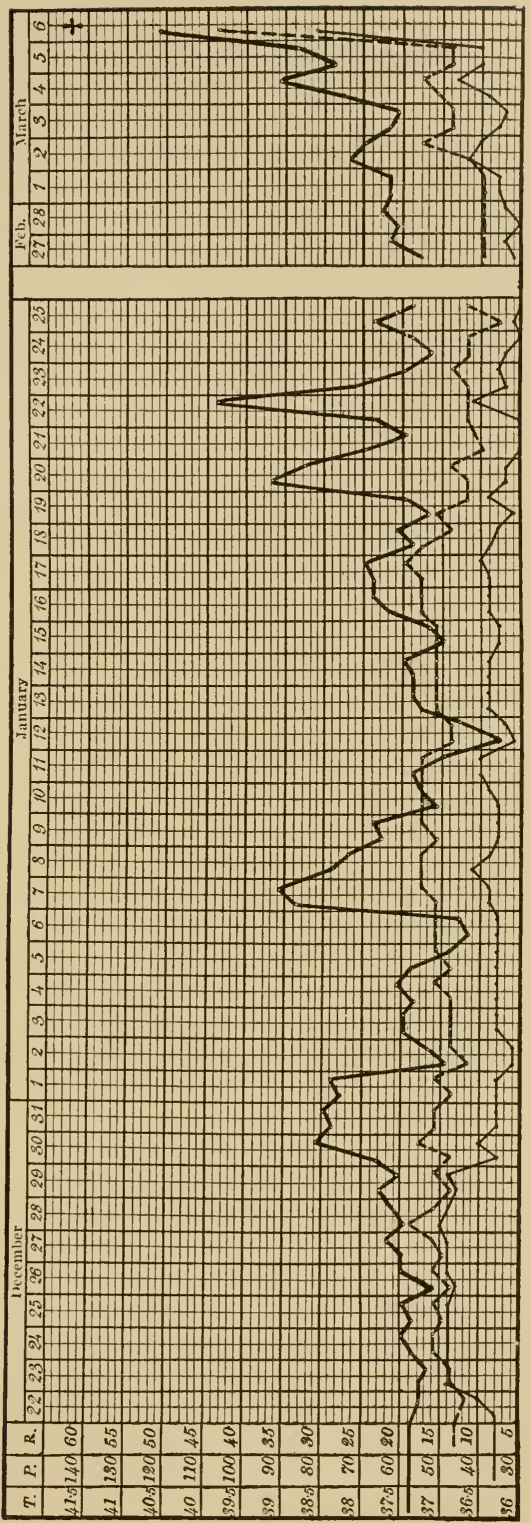

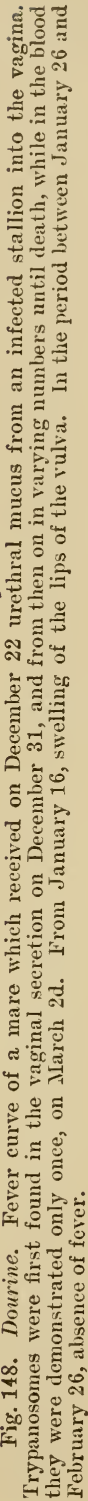


The number of red blood corpuseles in the blood may diminish to one-tenth of the normal, while white corpuscles may increase 10 to 30 times. In many cases a pronounced eosinophilia may also be demonstrated (Marek, Fröhner).

The daily quantity of the urine fluctuates within normal limits; its specific gravity is 1,023 to 1,060 and on an average it is lower than in healthy horses. Of the normal constituents the quantity of phosphoric acid is markedly increased in about $70 \%$ of the cases (up to $4-5 \mathrm{gm}$.) ; besides the quantity of urea is also increased (Liebermann; according to Roger the urine contains glucose).

In the eyeground the papillae and their surroundings appear congested in some cases, and in time blood pigment is leposited in the tissues surrounding them. These changes may later reduce the vision and even result in blindness. Conjunctival catarrh is a frequent symptom, in some cases however also an exudative iritis develops which finally leads to atrophy of the bulbus. Fröhner observed in one case a unilateral contraction of the pupil.

The body temperatures show a periodical rise of remittent character (Fig. 148). In the later course a continuous fever may set in, probably produced in part by suppurative processes, especially in the genital organs, but towards the termination of the disease also by the gangrene of the sores from constant lying down. With the temporary febrile attacks, fresh plaque eruptions develop frequently, but the course of the disease may continue without fever even for months.

Mares which are impregnated by an infected stallion frequently abort during the second or third montl of pregnancy.

The affected animals become greatly emaciated during the paralytic stage of the disease, although the appetite remains good almost to the end. Besides the paralysis, the weakness brought on by the emaciation contributes to the inability of the animals to rise in the later stages of the disease. The intmediate cause of death is either a hypostatic pneumonia or a septicemic affection in association with the gangrene caused by the pressure sores.

Course and Prognosis. In northern regions dourine usually runs a chronic course. After the local affection of the genital organs, several weeks or even months usually elapse before the nervous symptoms are markedly perceptible, while the dollar spots may appear earlier, sometimes simultaneously with the swelling of the sheath. After the appearance of the nervous symptoms the disease may in unfavorable cases take a rapid course, so that the animal succumbs in 3 to 4 weeks, completely exhausted, or - and this is more frequently the case-the disease extends over several months, frequently from 1 to 2 years. In such cases more or less pronounced improvement may be noticed periodically, which is however only temporary, and 
finally the disease passes into the aggravated condition which leads to a fatal termination.

In southern regions the disease appears to run a more acute course, so that the patients may die after 1 to 2 months and even inside of 6 to 8 days (Monod). At the same time the febrile attacks are more conspicuous following each other in more rapid succession, and the paralytic symptoms also develop more rapidly, therefore there are no marked, and especially no chronic interstitial changes of the nerves in the dead animals.

The termination of the disease varies in different outbreaks. While sometimes about half of the affected animals die (in India, according to Pease, the mortality amounts to $70-80 \%$ ), in other cases the loss is much smaller (thus for instance in A.-Vadász and vicinity, out of 30 mares in which the disease was established with certainty only two died). Recovery is possible even after the appearance of the nervous symptoms, dollar spots and paralysis, and such animals may then perform hard work for years. It is probable that in some cases only the local changes of the genitals develop after an infection, which definitely heal without the later development of nervous symptoms.

Some authors are of the opinion that the disease develops more rapidly in stallions and that it runs a more unfavorable course than in mares, and in Algeria it is supposed to limit itself in asses in the majority of cases to a local affection of the genital apparatus.

Diagnosis. In northern regions where dourine apparently represents the only trypanosome affection of solipeds, the diagnosis is definitely established by the demonstration of trypanosomes; but this is associated with considerable difficulty, as the parasites are present but rarely in the blood, and even then only in very small numbers. Their occurrence in the serous fluid of the dollar spots, especially immediately after these appear, is more constant, as is also the case in the edematous swellings in the vicinity of the external genital organs, but even here the examination requires great patience. Their demonstration in the mucous secretion of the urethra and vagina is much easier (Fig. 149), but even there it is to be remembered that they may disappear periodically from the secretion.

A sufficient quantity of secretion is obtained from the vagina by scraping the mucous membrane with a sealpel or with a glass slide. In stallions the penis is drawn out with the left hand, and then a long-handled, bean-shaped, oval spoon with dull edges is introduced into the urethra, with which the mucous membrane is scraped off.

In doubtful cases blood may be injected subcutaneously into dogs. In the swellings which subsequently develop trypanosomes are always present in great numbers (Buffard \& Schneider). Mice may also be used for this purpose. Since the material is frequently non-pathogenic for test animals, even from horses positively affected with dourine (see p. 817), only a positive result of the inoculation has a diagnostic significance. 
As the ring and semi-circular dollar spots have been observed in northern regions only in dourine, they may be considered as characteristic for the disease, or at least accepted as suspicious in a high degree. Their repeated appearance for weeks and months, and frequently also their persistence for several days, distinguish them from the ordinary urticaria.

In tropical regions the differentiation of dourine from nagana, surra and Mal de caderas may evidently cause difficulty, but as according to the reports so far available, trypanosomes in the secretions of the genital organs are found only in dourine, their presence there would clear the diagnosis; likewise an infection which may be shown to have been transmitted by coitus speaks for dourine.

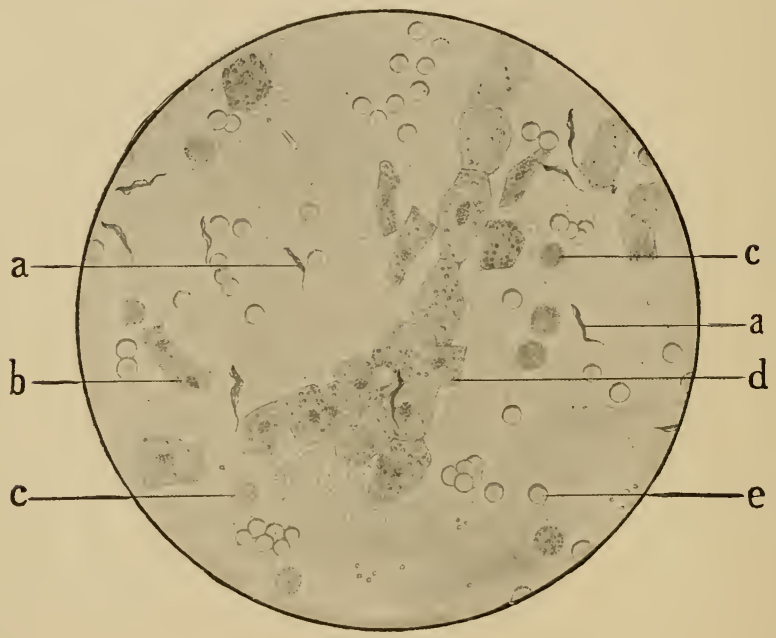

Fig. 149. Vaginal secretion of a mare affected with dourine. a trypanosomes; $b$ epithelial cells from the deeper, $d$ from the superficial layer of the mucous membrane; c lencocytes; $e$ erythrocytes. After a fresh, unstained preparation.

According to Baldrey the disease may he distinguished from surra in that the latter usually occurs in epizooties, geldings and unbred mares become affected, the body temperatures reach high degrees and last longer, and trypanosomes are present in the blood in great numbers at the beginning of the fever. According to Davison, Bursattee (see p. 739) also resembles dourine as it also is associated with a swelling of the genitals and inflammation of the skin; these however are very painful.

At times where there exists no suspicion of infection, the initial stage of dourine is sometimes mistaken for a traumatic inflammation of the genitals; however, the traumatic origin may 
easily be excluded by the absence of a visible injury, or on the ground of the torpid character and persistent presence of the edematous swelling. The coital, vesicular exanthema can readily be taken for dourine, as both affections develop after coitus with quite similar symptoms. In the coital exanthema however the eruptions disappear much more rapidly and are almost invariably benign; further the vaginal mucous membrane is reddened, the affected parts are painful, the usually numerous ulcers heal within a few days and with this the disease terminates, that is, no nervous symptoms develop with the local affection. Glanders may also come into consideration, as in its later course edematous swellings may result on the sheath and on the abdomen, but a mistake is prevented by the other symptoms, which are usually present. The swelling of the submaxillary lymph glands may readily be distinguished by its soft elastic consistency from the glandular swelling of the glands.

The weakness of the hind parts is only characteristic of lourine when associated with the other nervous symptoms. From other diseases in which similar weakness of the hind parts occurs, the paralysis of the musculus quadratus lumborum muscle, and the tearing of the lumbo-sacral articulation should be mentioned, in which however the animal is not capable of backing up at all, while a horse with dourine is capable of performing this function, although with difficulty and staggering. Paralysis of the facial nerves from other causes may be distinguished from dourine by the absence of edenatous swelling of the genitals and the plaques as well as other symptoms of paralysis.

Besides frequent accessory conditions, the affection of several animals in association with coitus, and the multiplicity of the symptoms in different animals are as a rule sufficient points for establishing the diagnosis of the disease in question.

Experiments by Thlenhuth \& Woithe, Manteufel, also Zwick \& Fiseher, with the complement fixation, the precipitation and the agglomeration, further the cutaneous and ophthalmo reactions for the diagnosis of lourine, gave negative results thronghout.

Treatment. Already formerly arsenical preparations had been used frequently in treatment, Trélat having obtained satisfactory results from the internal administration of arsenious acid (3-6 gm. daily), Arkhangelsky \& Novikoff from subcutaneous injections (0.01-0.05 gr. in increasing doses). Thlenhuth \& Woithe found atoxyl very effective on small animals in laboratory experiments. In horses the conditions for such a treatment are rather unfavorable, as the large doses required at the beginning of the disease are badly tolerated by these animals, also on account of the presence of the parasites in the secretion of the genitals, in the edematous fluid of the swellings and in cavities of joints, where they may elude the destruc-

Vol. $1-53$ 
tive action of the remedy. Nevertheless there are reports which appear to indicate the possibility of a recuvery of the disease processes following such treatment.

Thlenhuth \& Woithe sncceerled in keeping an artificially infected horse in good strength for one year by increasing doses of atoxyl (0.3-5.0 gnı. intravenously), its blood, however, contained trypanosomes throughout the entire period in varying quantities, and it suldenly died from acute septicemia with extensive intestinal hemorrhages. Rennes obtained better results in an artificially infected horse, which he injected at intervals of 3-4 days, alternately with $4 \mathrm{gm}$. of atoxyl subcutaneously and $3 \mathrm{gm}$. kalium tartarieum intravenously; after the administration of 32 gm. atoxyl and $21 \mathrm{gm}$. kal. tart. the horse was permanently cured. Yakimoff treated 10 horses by injecting atoxyl ten times in succession, either subcutaneously (4-8 gm.) or intravenously (0.5-3.0 gm.), in increasing doses, and this treatment was repeated. The results were good, as a relapse was observer in only one ease. Monod also is supposed to have succeeded in permanently curing an affected stallion with sinilar treatment (first $0.5 \mathrm{gm}$. intravenously, then every second day an increase of $0.1 \mathrm{gm}$. until the maxinum dose of $1.0 \mathrm{gm}$. was reacheil).

The atoxyl solution must be freshly prepared at each application by hoiling on a water-bath.

Of other arsenical preparations, Miessner found in one case arsenophenylglycin very effective, in which the nutritive condition and strength of an already greatly weakened animal was materially improved by two injections of the remedy (dose?) and the edematons swellings retrogressed. Fröhner, on the other hand, not only failed to observe an improvement in one case after an intravenous injection of $24 \mathrm{gm}$. atoxyl, but even noted severe symptoms of intoxication, such as marked psychic irritation, severe colic and on the second day nephritis. Finally Monod reported lasting results in three stallions, which in part were treated with arsen-trisulfid alone (15-30 gm. per os), the others alternately receiving atoxyl.

The local treatment of the swellings with astringent washes and the suppuration of the testicles or lymph glands in accordance with the rules of surgery are only of subordinate importance.

Prevention. Stallions and mares should be examined before coitus as to their health, and horses with inflammatory changes on the genital organs should be excluded from coitus on general principles. Besides this it is advisalble to wash the penis of the stallion after every covering with a sponge dipped in clean water.

Veterinary Police. The veterinary police measures depend on the principle that horses affected with dourine should be excluded from breeding once and for all. Such measures appear to be justified in spite of the possibility of a complete recovery, by the frequent relapses and also by the fact that trypanosomes may be present for months in the genital organs of apparently recovered animals. Accordingly affected stallions should be castrated, while affected mares should receive a distinctive branding. The relatively late development of the symptoms 
characteristic of the disease, as well as the possibility of a healthy stallion transmitting the disease from an affected mare to a healthy animal, appear to justify an action requiring that healthy stallions which have covered mares affected with dourine should be considered the same as those affected.

Literature. Hertwig, Mag., 1842. VIII. 269; 1847, XIII. 373. - Rouget, A. P., 1876. 717. - Schneider \& Buffard, Rec., 1900. S1; J. vét., 1902. 144; A. P., 1905. XIX. 715. - Marek, Z. f. Tm., 1900. 1V. 401; D. t. W., 1909. 121. - Kern, Z. f. Tm., 1905. IX. 259. - Lingard, Cbl. f. B., 1904. XXXVII. 537. - Baldrey, J. of comp. Path., 1905. XVIII. 1. - Sieber \& Gonder, A. f. Schiffs- u. Trophyg., 1908. XII. 246. - Thlenhuth \& Woithe, Arb. d. G.-A., 1908. XXIX. 403 (Lit. on chemotherapy). - Fröhner, Monh., 1909. XX. 385. - Zwick. D. t. W., 1910. Nr. 9 Beibl.

\section{(d) Mal de Caderas.}

History. The etiology of the disease (Mal de eaderas=hip disease) was cleared up by Elmassian, when in 1901 he found trypanosomes in the blood of horses at Argentine. Voges, Zabala and Lignières confirmed the finding, and furnished further contributions on the etiology as well as symptomatology of the disease.

Occurrence. Until the present time the disease has been diagnosed exclusively in the sub-equatorial countries of South America: Brazil, Argentine, Bolivia and Paraguay, as well as on some islands of the delta of the Amazon River.

According to Lacerda the disease is supposed to have been introduced from the islands of Marajo, westward and sonthward, and resulted in some localities of Brazil in a complete annihilation of the horses. There are marshy localities in Argentine in which up to $25 \%$ of the horses die, and it is stated that a cavalry regiment lost 100 out of 600 newly purchased horses within half a year (Voges).

Etiology. The trypanosoma equinum (Th. Elmassiani) is similar to the parasite of nagana, with the only difference that its blepharoplast is very small and can be stained only with difficulty (Lignières; Fig. 150).

Ruminants and hogs are only slightly susceptible to artificial infections; other animals have about the same susceptibility as for the organism of nagana. In horses infected subcutaneously with blood disturbances in locomotion develop in the later stages of the disease in a striking manner (similarity with dourine).

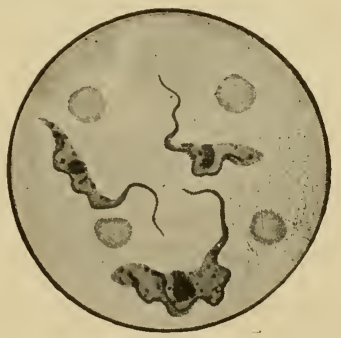

Fig. 150. Trypanosoma equinum s. Elmassiani. Guinea pig blood; stained after Romanowsky-Zieman.

The disease cannot be reproduced, even in susceptible animals, by feeding fresh blood containing numerous parasites (Zabala). Animals immunized against nagana or surra are still susceptible to caderas and vice versa. 
The natural mode of infection has not yet been elucidated. At first it was thought that stinging flies were the transmitters of this disease, especially the stomoxys brava (Mosca brava) and tabanidae as well as possibly also mosquitoes (Lignières \& Zabala, Voges); according to the observation of Elmassian \& Migone, howerer, horses which are separated from affected animals only by a fence remain healthy in spite of being swarmed by stomoxiys flies.

Later the last-named authors connected a rodent with the appearance of the disease (Hydrochoerus capibara, rulg. Carpincho), observing that these animals sometimes die in great numbers, and that hunting dogs which have eaten their flesh also become affected, whereupon the disease soon breaks out among horses.

Anatomical Changes. These consist usually in an acute swelling of the spleen and the lymph glands, accumulation of sero-fibrinous exudate in the serous body cavities and in the joints, hemorrhagic nephritis, parenchymatous degeneration of the internal organs, besides hemorrhages and serous infiltration of the muscles of the croup.

Symptoms. A weakness of the hind parts is noticeable from the onset of the disease, as the animals show a staggering gait, bringing forward the hind legs, dragging them on the ground, and frequently knuckling in the tarsal joints. With the rapidly progressing emaciation, the paraparesis reaches a very ligh degree, so that the patients rise from the ground with difficulty, after which they stand with legs spread apart, and can not be made to move. Finally they are unable to rise, and then the sphincter ani becomes relaxed and the rectum fills with balls of feces or defecation becomes involuntary.

The body temperature shows a febrile eleration atypical in character, urticaria-like swellings appear on the skin which become covered with small crusts; subentaneous edemas are observed but rarely, and then only on the lower abdomen, while swelling of some of the joints is frequently observed. The urine contains albumen and frequently some blood, the latter sometimes in great quantities. Inflammation of the conjunctivae and cornea is a frequent complication. The appetite remains good until the end.

The course is always unfavorable. After the appearance of the disturbances in locomotion the animals usually succumb in 1 to 2 months, but there is also a chronic form of the affection (known in Paraguay as Baacy-poy=slow emaciation), which develops very gradually, so that the emaciation and weakness of the hind parts becomes apparent only after months.

Trypanosomes are found in the blood only in acute cases in considerable numbers, while otherwise their demonstration is successful only by inoculation of test animals (mouse, rat).

The disease rims under similar manifestations in mules and asses. 
Treatment and Prevention. Ehrlich \& Shiga found trypan red effective in experiments on mice against the trypanosomes of Mal de caderas. For practice however no satisfactory method of treatment is known.

In consideration of the fact that the mode of natural infection is still unknown, no certain preventive measures can be established. Nevertheless keeping the solipeds from marshy pastures, and the destruction of affected horses, may prove beneficial.

Literature. LImassian \& Migone, A. P., 1903, XVII. 241 u. 1904. XVIII. 587. - Voges, Z. f. Hyg., 1902. XXXIX. 323. - Lignières, Bull., 1903. 51.

Other Trypanosome Diseases. Besides the affections considered above other diseases of domestic animals have been described, the classification of which however is at present still doubtful. The most important will be set forth briefly in the following:

1. Gambia Fever of Horses. This is caused by the trypanosome dimorphon discovered by Dutton \& Todd in Senegambia. It occurs in the blood of horses shortly after infection in a short tadpole form, and in the latter stages in a longer, more slender form, in both of which the plasma extends almost to the anterior end of the flagella. The clinical symptoms resemble those of nagana, only subeutaneous edema is absent (for treatment see p. 802).

The transmission of the parasite is supposed to oecur by the Clossina palpalis. In recent times the same trypanosome has been demonstrated in different localities of Africa, not only in horses, but also in other domestic animals, as the cause of an affection which is mostly chronic (Laveran \& Mesnil, C. R. 1904. CXXXVIII. 732).

2. Zousfana. Observed by Szewczyk and Rennes in Algeria in solipeds, and attributed to a particular trypanosome similar to the Tr. sudanense. The disease runs a chronic course, with symptoms of anemia and hemoglobinuria, but without edema. Possibly identical with nagana cr surra. (Szewczyk, Bull, 1903. 220.-Rennes, Ibid., 1907. 298.)

3. Soumaya (Souma). Described by Cazalbou as a disease of horses in the French Sudan and supposed to occur also among mules, eattle and sheep in the upper valley of the Niger and the adjoining territories. Its cause, the Trypanosoma Cazalboui, was described as a new species, its nucleus being placed near the rounded posterior end. Dogs and small test animals are not susceptible. According to Buffard it is also virulent for cattle, and is transmitted by stomoxys flies. Symptoms in horses are anemia, swellings of the skin and weakness of the hind parts. The course is sometimes acute, eovering about 50 days, at other times it may extend over a year. Thiroux \& Teppaz reported good results from treatment with atoxyl and auripigment, or with auripigment alone. (Cazalbou, Acad. de méd., 1904.-Laveran, A. P., 1907, XXI., 325.-Buffard, Soc. hiol., 1907, 71.-Thiroux \& Teppaz, C. R., 1908, CXL, 651.)

4. Baleri. Oceurs quite frequently among solipeds in certain localities in the Sudan, with symptoms of fever, infiltration of the skin, anemia, emaciation, and muscular weakness. Its course is from 3-4 
months. The Trypanosoma Pecaudi, named by Laveran, resembles the Tr. dimorphon, and is, according to Buffard, said to be transmitted by the Glossina palpalis. Dogs are also susceptible. Thiroux \& Teppaz found the auripigment effective. (Cazalbou, Rev. gén., 1907, IX, 564.Bouffard, A. P., 1908, XXII, 1.)

5. Mbori and El Debab of Camels. (Maladie de la mouche.) The Sergent brothers in Algeria, and Cazalbou in Timbuctu, have deseribed this as an inclependent affection, but according to Laveran it is supposed to be identical with surra. The camels become affected with symptoms of anemia and emaciation, also turning the head and neck to the side, while edema and disturbances in locomotion are not usually observed. About $10 \%$ of the dromedaries are supposed to be affected in Algeria. (Laveran, Acad. de méd., 1904.-_Sergent \& Ledoux, Soc. de Path. exot., 1908, 22.)

Other Trypanosomes. Besides those described, other species of trypanosomes have recently heen found in domestic animals; some of these will be briefly described in the following:

Trypanosoma Theileri; first demonstrated by Theiler in cattle in South Africa, later by Schilling in Togo, Szewezyk \& Rennes in North Africa, Sander on the Island of Mafia of the German-East African protectorate, Valladares in Madras, Luhs in Trans-Cancasia, Pease found it in India also in buffaloes. It is characterized by its considerable size (30-70 $\mu$ long, 2-5 $\mu$ broad, and a long flagella. First it was considered by Theiler as the cause of gall sickness (see p. 783), but it appears to be a harmless habitant of the blood of cattle. (Theiler, Comp. Path., 1903, XVI, 192.-Mayer, Z. f., Infkr., 1909, VI, 46 [Lit.].)

A similar trypanosome was found by Frank in great numbers in the blood of German cattle in Stein-Wingert (Wiesbaden), and named by Frosch Tryp. Frank. Further Knuth, Rauchbaar \& Morgenstern succeeded in demonstrating trypanosomes of the same type by inoculation of defibrinated cattle blood into sterile beef houillon; in the District of Oberwesterwald in 7 out of 25 , in another stable 10 out of 17 , and in a third 6 out of 9 otherwise healthy cattle, which originated from various localities of Germany. (Z. f. Infkr., 1909, V. 313.-B. t. W., 1910, 539 u. 610.)

Also very similar is, according to Lingard, the Tryp. himalayanum and the Tryp. indicum, further the Tryp. muktesari, this however somewhat smaller, and with shorter flagella (C. R., 1908, 452), also the Tryp. americanum, which was by Crawley found frequently in healthy cattle in North America (An. Ind. Buil., 119, 1909).

Broden described the Tryp. congolense as a special species, which frerfuently causes disease in the Congo especially among horses, but also among other domestic animals and has also been found in the northern part of Rhodesia. It is smaller than the nagana trypanosome, has no free flagella, and otherwise resembles the small form of the Tryp. dimorphon. (Laveran, A. P., 1910, XXIV, 81.) This closely resembles the Tryp. nanum, which was demonstrated by Theiler in Zambesia and in Limpopo in cattle, and in Zuzuland in a horse. (Soc. de pathol. exot., 1909.)

Mathis \& Léger ohserved a trypanosome in a chicken, which they named Tryp. Calmettei. (Soc. biol. 1909, LXVI, 452.)

(See also Lühe. IIandb. d. Tropenkrkh., 1906, III, 124, n. Doflein, Lehrb. d. Protozoenkunde, 1909, 390.) 
Trypanosome Affections in Man. By the findings of Nepveu (1898), Dutton (1902) and Castellani (1903), as well as by numerous subsequent confirmations, it was established that the so-called sleeping sickness of the negroes is caused by a blood parasite, the Trypanosoma gambiensis. It greatly resembles the parasite of nagana, and is also transmitted into the healthy body of man by the tsetse fly, especially by the Glossina palpalis. The disease, which occurs in tropical Africa, manifests itself in the first stage (Trypanosome fever) in irregular fever, transitory edema, and erythema, also swelling of the lymph glands and spleen. If the patients survive, symptoms of the sleeping sickness proper develop in a few months. Fatigue with frequent headaches, subcutaneous edema, diminution of the intelligence, and finally symptoms of a severe meningitis, especially conspicuous somnolence, whereupon the patients die in a deep comatous condition. Good results were obtained in the treatment with arsenical preparations, especially with atoxyl. (S. Mense, Handb. d. Tropenkrankh., 1906, III, 617.Koch, Beck \& Kleine, Arb. d. G.-A., 1909, XXXI, 1, H.)

Chagas found in a child in Brazil a trypanosome (Tr. Cruzil), which supposedly is transmitted by the Cinorrhinus insect. The symptoms were similar to those of the African trypanosome fever. (A. f. Schiffs, u. Tropenhyg., 1909, XIII, 351.)

\section{Spirochaetosis of Fowls. Spirochaetosis avium.}

\section{(Spirillosis or Spirochaetosis of Chickens; Spirillosis gallinarum et anserum.)}

Spirillosis represents usually a fatal septicemia of chickens, geese and ducks, caused by a screw-shaped blood parasite, the Spirochaete gallinarum.

Eistory. Spirillae were first demonstrated as disease producers by Sakharoff (1891) among geese in Trans-Caucasia, later by Marchoux \& Salimbeni (1903) in Brazil. At first it appeared as if two different diseases were concerned, but Williamson (1908) and Galli-Valerio (1909) have established the identity of their causative agents. In the meantime Levaditi, Borrel \& Marchoux, Neufeld \& Prowazek and Nuttall have contributed to the etiology, Uhlenhuth and his co-workers, then Levaditi \& McIntosh, Dschunkowsky \& Luhs to the atoxyl treatment of the disease.

Occurrence. The disease occurs frequently as a dangerous plague among chickens in the most varied localities, while in geese and ducks it has been found only sporadically.

Ontside of Brazil the chicken spirillosis has been met with to the present time in Bulgaria (Gareitschnoff), in Roumania (Mezinescu), in Cyprus (Williamson), in Egypt (Dreyer, Balfour, Bitter), in Tunis (Galli-Valerio, Comte \& Bouquet), in South Africa (Johnsohn, Bevan, Mohr), in Australia (Cleland), and in India (Greig, Montgomery, Rainey); the spirillosis of geese, outside of Trans-Caucasia (Sakharoff, Dschunkowsky \& Luhs) only in Tunis (Ducloux). 
Etiology. The causative agent of the disease (Spirochaete gallinarum, Sp. anserum) is a blood parasite, probably belonging to flagellated protozoa, which closely resembles the cause of

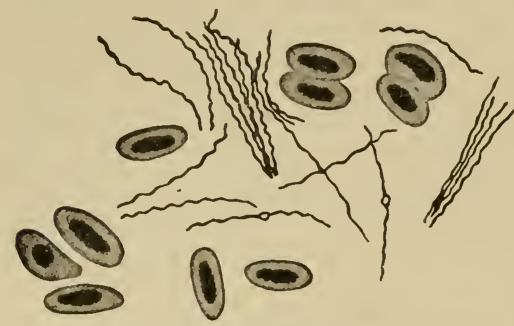

Fig. 151. Spirochaete anserina. Goose blood. (After Cantacuzene.) recurrent fever of man (Spirochaete Obermeieri) which has been known for several decades. It occurs in fine threads, $10-20 \mu$ long, formed similarly to the spiral thread of a screw, supplied with one or more cilia (Borrel) which move actively between the blood corpuscles in the blood of living animals affected with the disease (Fig. 151). They disappear from the blood shortly after death and in drawn blood they remain alive at best only for 2 to 3 days.

Dschunkowsky \& Luhs are reported to have succeeded in cultirating the spirochaete of geese in blood plasma of geese by means of reed sacks in the body of a rabbit up to the third generation; later indications of degeneration became apparent on the threads, but the fluid possessed a protective action against artificial infections.

The disease may be produced easily by subcutaneous injection of blood containing spirillae from geese to geese, from ducks to young chicks, but only with difficulty to grown chickens, while the transmission from affected chickens to chicken species, as well as to water fowl is readily accomplished (it was not possible to infect monkeys with the spirilla of chickens). After a subcutaneous infection in chickens a considerable number of parasites appear on the second day independently of the quantity of inoculated blood which continue to increase until the fifth day, and in the meantime clump together, forming great agglomeration masses. On the sixth day after the temperature has fallen rapidly, they disappear from the blood, whereupon death ensues or the animal gradually recovers.

The artificial infection is also successful by placing infected ticks upon the birds (see lower down), further by feeding blood containing spirillae, as well as by cohabitation of affected with healthy chickens.

Brumpt described the Sp. Nicollei from Tunis, also the Sp. Neveuxi as rarieties of the chicken spirochaete, which are also transmitted to chickens by the Argas persicus; between this and the chicken spirochaete only immunizing differences exist.

The natural infection is in chickens transmitted principally by the Argas miniatus tick, more rarely by the Argas persicus and reflexus, further by the Ornithodorus moubata. These ticks exist freely in the bushes at the borders of forests, etc., attach 
themselves at night to chickens and suck their blood. If the blood contains spirochaetes, they are found for 3 to 4 days in the digestive canal of the ticks, whereupon they disappear from there, but they may remain infectious for a longer time, according to Nuttall even after six months. If they are kept at $35^{\circ} \mathrm{C}$., the parasites multiply in their bodies, and after 4 to 5 days they may be found in the excretory duct of the salivary gland of the ticks (Borrel \& Marchoux).

Anatomical Changes. In birds dead with the disease the spleen is considerably enlarged and swollen, the liver swollen, showing fatty degeneration and focal necrosis; the heart muscle also shows at times fatty degeneration and the epicardium is covered with fibrinous membranes. No spirillae are found in the blood.

Symptoms. The incubation is in chickens 7 to 9 days after placing infected ticks upon them; spirochaetes may however be demonstrated in the blood as early as from the fifth day on (Marchoux \& Salimbeni, Uhlenhuth \& Gross).

The symptoms consist in inappetence, rise of temperature as high as $42.5-43^{\circ}$ C., marked weakness and somnolence, paleness of the comb, diarrhea and emaciation, with which in some cases paralysis of the legs becomes associated. (Mohn observed, even before the appearance of spirochaetes in the blood, the feet swollen and the toe turned under and backwards.)

After the somnolence has reached a high degree, the crisis sets in 1 to 2 days later, the comb taking on a bluish-red coloration, the temperature falls, and soon death follows, usually under severe convulsions or recovery commences. Sometimes however this is only apparent, as the paralysis of the legs increases and gradually extends to the wings, the birds become greatly emaciated, and finally die with a few rare exceptions (chronic form).

According to Nuttall the spirochetes are totally or mostly destroyed by bactericidal and agglomerating substances after having multiplied for a time in the blood. The more resistant, and therefore the surviving parasites are then the cause of the supervening chronic affection. Serum of birds which have recovered from one attack, destroys in test tube experiments the spirochetes of the first attack, but not those of later attacks. The appearance of parasiticidal substances in great quantities in the blood before the crisis is reached has also been demonstrated by Gabritschewsky and Uhlenhuth.

By microscopical examination spirochaetes may be demonstrated in great numbers in fresh bloor preparations made with physiological salt solution until the appearance of the crisis, but later, and also in animals dead of the disease, they are no longer present.

The duration of the disease in the more frequent acute cases is mostly 4 to 5 , in the chronic cases 8 to 15 days. 
Treatment. According to the experiments of Uhlenhuth \& Gross, which were later confirmed by Levaditi \& McIntosh, three subcutaneous injections of $0.05 \mathrm{gm}$. each, likewise two administrations of $0.1 \mathrm{gm}$. atoxyl per os, protected chickens against a simultaneous or an immediately preceding or subsequent subcutaneous virulent infection, and chickens treated in this manner apparently acquired a lasting immunity.

Affected chickens, which have already harbored spirochetes in their blood for 2 to 3 days, but which are not yet severely affected, recover from a single intra-muscular injection of 0.05 gm. atoxyl, when the parasites disappear from the blood after 34 hours and the animals survive. In the internal application of the remedy double doses (0.1 gm. per os) appear to have the same action.

Uhlenhuth \& Manteufel found atoxylate of mercury more effective than atoxyl; by its intra-muscular application (in suspension with olive oil and gum arabic), in doses of $0.1 \mathrm{gm}$., it produced recovery even at the height of the blood infection, and in earlier stages it prevented a fatal termination even in doses of 0.04-0.06 gm. Such recovered chickens remain immune against later infections.

Dschunkowsky \& Luhs obtained good results in geese with 2 or 3 subcutaneous injections of $0.1-0.15 \mathrm{gm}$. each of atoxyl; per kilogram of borly weight.

Finally, according to the favorable results in the treatment of the human recurrent fever and syphilis, Ehrlich's amidoarsenobenzol may also prove effective.

Immunization. Blood drawn from infected chickens is no longer infectious after 48 hours, and if injected subcutaneously into healthy chickens produces an immunity against a virulent affection. Fresh blood heated for five minutes to $55^{\circ} \mathrm{C}$. has a similar action, whereas when heated for 10 minutes at that temperature it no longer produces immunity (Marchoux \& Salimbeni). An active immunity may further be produced in geese with emulsion from organs of geese dead of the disease, also with atoxyl and a simultaneous injection of virulent blood, as well as with blood from infeeted geese which have been kept on ice for some time (Dschunkowsky \& Luhs). Blood serum from geese and chickens, which after recovery from the disease were treated with blood containing spirillae, protects in quantities of 2 ec. against virulent infection. Finally horses produce serum after repeated injections with blood containing spirillae which in a quantity of 3-5 ce. renders ineffective in geese an inoculation made 24 hours previously (Sakharoff).

Injections of immune serum in the blood circulation of affected birds results in leath, as it produces an agglomeration of the spirochaetes and thereby thrombosis of the blood vessels (Levaditi).

Literature. See also literature in Uhlenhuth \& Gross, Arb. d. G.-A., 1907. XXVII. 231. - Further: Sakharoff, A. P., 1891. V. 564. - Gabritschewsky, Cbl. f. Bakt., 1899. XXIII. 365. - Cantacurène, A. P., 1899. XIII. 529. - Borrel \& Marchoux, Soc. biol., 1905. 362. - Borrel, ibid., 1906. - Levaditi, A. P., 1906. XX. 924. - Levaditi \& MeIntosh, Soc. biol., 1907, XLII. 1090. - Nenfeld \& Prowazek, Arb. d. G.-A., 1907. XXV. 494. - Th'enhuth \& Mantenfel, Z. f. Immf., 19ns. I. 10.. - Dschunkowsky \& Luhs, Kongr. Haag 1909. - Brumpt, Soc. path. exot., 1909. JI. 
Spirochaetes in Mammals. Parasites resembling the spirochaetes of fowls morphologically occur sometimes also in mammals, their etiological part however is at present not yet cleared up, as they were met with either in healthy animals or in such as are infected with piroplasma or trypanosomes. Transmission experiments have not given conclusive results. The available findings are therefore described only very briefly in the following:

1. Spirochaete (Spirillum) Theileri. First demonstrated by Theiler (1902) in the Transvaal in febrile animals together with piroplasma and trypanosomes. They are 20-30 $\mu$ long, spiral-shaped bodies. The first experiments of transmission were without results, later however Theiler reported that he succeeded in transmitting the parasite from cattle to cattle and to sheep, and Dodd also found that they may be transmitted from animal to animal.

According to Theiler the natural infection is transmitted by the Boophilus decoloratus and the Rhipicephalus Evertsi, and Laveran \& Vallée succeeded in infecting a cow at Alfort, by placing larvae of the first-named species, which originated from ticks taken from cattle in the Transvaal. In this case spirochetes appeared in the blood between the fifteenth and the nineteenth day without the development of perceptible symptoms. Nevertheless Theiler believes that these parasites are independently capable of producing a disease, the autopsy findings of which resemble those of piroplasmosis.

Evidently the same spirochetes were found by Ziemann in Kamerun in a calf, by Heanley in two Chinese buffalo ealves, and by Koch in Daressalam in cattle.

2. Spirochaetes Tschischir, demonstrated by Djatschenko in Kuban (Russia) in the blood, spleen and liver of eattle which were affected with symptoms of an infectious hemoglobinuria. The parasite has the form of a comma or of an " $\mathrm{S}$ " and can ie cultivated artificially" (Vibrio?). Transmission experiments were negative.

3. Spirochaete Ovina, found by Martoglio \& Carpano in Erythtaea simultaneously with endoglobular parasites in a sheep affected with febrile symptoms. It forms threads $10-20 \mu$ long, with $4-10$ spiral windings; artificially they could not be transmitted.

Theiler also reports finding spirochaete in sheep in the Transraal, and Ziemann in Kamerun (Dodd considers them illentical with the Sp. Theileri).

4. Spirochaete Suilla, found by Dodd in Pretoria in the tissue of superficial ulcers of the skin, which extended over the entire body of hogs (10-14 $\mu$ long, with 2-6 spiral windings). Material from the ulcers rubbed into the scarified skin of healthy hogs produced a similar skin affection, which under progressive emaciation usually led to death. Similar parasites were found by Cleland in West Australia in fibrous nodules of castration wounds.

5. Spirochaete Equina. Stordy in East Africa found spirillae in very great numbers in the blood of a horse which showed a fatal affection with symptoms of high fever, swelling of the subcutis and emaciation. A similar finding is reported by Stordy in a severely 
affected pony, whose blood transmitted the parasite to horses, eattle and sheep, and he considers them identical with the Sp. Theileri.

Literature. Theiler, J. of Comp. Path., 1904. XVII. 47; Bull. P., 1905. II. 617. - Djatschenko, Cbl. f. Bakt., 1904. XXXV. 737. - Martoglio \& Carpano, Ann. d'Tgiene sperim., 1904. XIV. 577. - Laveran \& Vallée, C. R., 1905, CL. 1515. Ziemann, Cbl. f. Bakt., 1905, XXXVIII. 447. - Dodd, J., of Comp. Path., 1906. XIX. 318. - Heanles, ibid., S. 322. - Stordy, ibid., S. 226. - Titre, Z. f. Infkr., 1908. IV. 139. (Review with literature). - Knuth, ibił., 1910. VII. 149 (recent lit.).

Spirochaetosis in Man. Recurrent fever (Febris recurrens) is known as a spirochetosis and is caused by the Spirochaete recurrentis s. Obermeierei discovered by Obermeier (1868). The affection is manifested by severe febrile attacks lasting from 3 to 12 days, and recurring after 2 to 11 days. In most cases lasting recovery results after several febrile attacks. The natural transmission appears to result through the agency of blood-sucking arthropodes, possibly also by insects and lice. According to the most recent observations of Iversen in Russia the disease may be cured quickly and permanently with Ehrlich's Amidoarsenobenzol.

A similar aftection in the tropics, the African recurrent fever, or "tick fever," is produced by the Spirochaete Duttoni (according to Koch identical with the above), and is transmitted to healthy man by a tick (Arnithodor.us moubata). (Schilling, Mense's Handb. d. Tropenkrankheiten, 1906, III, 668.)

Since the discovery by Schaudinn of fine spirochaetes (Sp. pallida, Treponema pallidum) in the syphilitic organs, syphilis is also considered as a spirochetosis. This view has received material support by the splendid results of Ehrlich's chemotherapy. Besides a spirochete (Sp. pertenuis) was found hy Castellani, as a probable cause of the disease in Framboesia tropica, a skin affection of the tropies which clinically resembles syphilis. (A. f. Schiffs- u. Tropenhyg., 1907, XI, 22.) 


\section{Diseases of the Blood and Blood- Producing Organs}

\section{Anemia. Anaemia.}

The name anemia designates the absolute diminution of the hemoglobin in the blood without a visible affection of the blood-producing organs and without symptoms of abnormal lencocytes in the blood. These pathological conditions may depend either upon a more or less uniform diminution of all of the constituents of the blood (Oligemia), on the diminution of red blood corpuscles (Oligocythemia), or on a simple diminution of the blood-coloring matter in the presence of otherwise normal numbers of red blood corpuscles (Oligochromemia). In the majority of cases Oligemia and Oligocythemia are present simultaneously, and in severe cases the water contents of the blood is considerably increased at the expense of the other constituents (Hydremia).

Etiology. After profuse losses of blood a severe anemia may develop in a short time, sometimes even in a few minutes (Anemia acuta); thus after phlebotomy, epistaxis, after injury of larger blood vessels, after bursting of aneurysms, after very profuse parenchymatous bleeding (hemoglobinuria, intestinal hemorrhages, etc.), also after severe hemoglobinemia.

In the majority of cases the development of the anemia occurs slowly and gradually (Anemia chronica). Thus repeated losses of blood may in time produce anemia. A continuously insufficient nutrition has an important part in the etiology of anemia. The unfavorable action of the food may become manifested either as a result of an insufficient quantity by feeding foods deficient in proteids or in iron. The effect of a qualitatively insufficient nutrition may be assumed in anemia of sheep (the so-called chlorosis, Cachexia aquosa), which develops when the animals are pastured in marshy moorland pastures, or after failures of crops if the animals are fed in winter insufficiently on poor food-stuffs such as beet leaves, etc. In cattle a continuous feeding on food lacking in nutritive material may also cause severe anemia (Hydremia). Thus the 
affection results with relative frequency in cattle kept near alcohol distilleries or sugar refineries (Jost, Hildebrandt, Arloing), where the principal food consists of the very watery (up to $95 \%$ ) slops, beet shavings or the syrup-like fluid remaining after the refining of the sugar. The affection attacks preferably working oxen, which must utilize a part of the otherwise insufficient nutritive substances for the execution of their work; also because they have less opportunity after satiating themselves with food substances abounding in water, to partake of additional solid fodder (Roloff). Horses, sheep and goats become affected from the same cause much more rarely. In this form of anemia toxic substances and acids which form during the storing of these foods apparently have an accessory action. (Arloing attributes the development of the disease exclusively to the action of these substances.) Werner observed anemia and hydremia in hogs in connection with the feeding of watery food.

The anemia of sheep and cattle previously mentioned is considered by various authors as an independent affection under the name of chlorosis of sheep, or as hydremia of eattle. This does not appear well founded on account of the fact that these conditions are differentiated from the other forms of anemia only by special etiological factors, the pathological changes corresponding with those of other forms.

The investigations of Carré \& Bigoteau appear to show that the chronic intoxication of sheep produced by the bacillus pseudo-tubereulosis ovis (Preisz) may result in a hydremic condition of sheep.

The keeping of animals in poorly ventilated places as well as lack of exercise, may in itself produce anemia, as is sometimes observed in fattening hogs, in milch cows, house dogs, etc.

Anemia may develop as a regular symptom in chronic affections with disturbances of appetite or of digestion, or in affections with great loss of proteids (long lasting suppuration, albuminuria, persistent diarrhea, effusion of copious transudate, exudate, helminthiasis, etc.), or in increased splitting up of proteids (fever, action of bacterial toxins in various chronic infectious diseases, mineral poisons, malignant tumors).

Blood parasites may be mentioned as another cause of anemia (piroplasma, trypanosomes, Filaria immitis).

The anemia of sucking pigs and young hogs, which has been observed by Braasch in Schleswig-Holstein and which caused great losses in some localities, is not yet sufficiently cleared up. This very severe anemia which appears already in the first weeks of life, may be the result of an unnatural keeping of the breeding hogs, but the action of an infectious substance (bacillus pyogenes) cannot be excluded with certainty.

Guittard reported on a disease in ehicks which appeared in some localities of France as an infectious anemia with symptoms of severe anemia and weakness, developing soon after hatehing, and resulting in death in a few days.

The occurrence of chlorosis in animals is not yet proven. In human medicine a disease is designated by this name, which usually occurs during the period of 
puberty, especially in girls, and is associated with a diminution of the hemoglobin contents of the red blood corpuseles. It subsides after a systemic administration of rron. The affection of sheep designated under this name is, as already mentioned, not identical with this disease.

Anatomical Changes. All organs of animals which have died as a result of hemorrhage are characterized by a conspicuous paleness and by the absence of blood; this is most readily recognized in the lungs, which appear pale pink in color. At the same time the heart and the large blood vessels contain only small amounts of loose blood coagulum. In chronic anemia blood is frequently found in nearly normal amount in the heart and in the blood vessels, but it appears paler, more watery, stains the hand only slightly and contains only little loose gelatinous coagulum. Fatty degeneration of the parenchymatous organs, especially of the heart muscles and of the kidneys is not infrequent. Dropsical development is almost constantly found in chronic fatal anemia.

Symptoms. The symptoms of acute anemia develop in accordance with the severity of the hemorrhage, sometimes very rapidly, at other times somewhat more slowly. The animals become much depressed, perspire profusely, stagger and sway in walking. The mucous membranes become gradually more and more pale, finally even porcelain white; the heart action is throbbing, the pulse at first frequent, tense and small, later however soft and empty. The respiration is accelerated and labored; a conspicuous cooling off develops in the peripheral parts of the body; there is also muscular trembling. If the animal has lost great quantities of blood within a short time its entire behavior expresses great anxiety, it sways, finally falling to the ground, becomes unconscious and dies in convulsions if the bleeding continues.

The symptoms of chronic anemia develop much more slowly and persist for a longer time. The mucous membranes appear very pale, pink colored, in severe cases almost milky or porcelain white. There is languor, easy fatigue even on quiet walking; after prolonged exercise the animals commence to sway and sometimes even fall to the ground. At rest they appear somnolent and indifferent and lie down a great deal. The frequency of the heart action increases considerably, even on very slight external causes. The heart sounds sometimes become imperceptible or indistinct owing to the false sounds; the pulse is at the same time weak. In severe cases slight hemorrhages appear in various organs. Further symptoms of severe anemia are diminished appetite, irregular movement of the bowels, accelerated and superficial respirations, lusterless hair, in sheep dryness and dullness of the wool. The nutrition is at first, and sometimes also in the later stages satisfactory, the anemia however which is caused by organic affections or insufficient feeding causes emaciation. 
Corresponding to the intensity of the affection the blood appears lighter, its hemoglobin content diminished (in the determination with Fleischl's or Gower's hemoglobinometer under $70^{\circ}$, in very severe cases even between $30-40^{\circ}$ ). The number of red blood corpuscles is diminished and may fall to $3,000,000$ to $2,000,000$ per cubic millimeter. Besides in severe cases the red blood corpuscles may show a poikilocy tosis; that is they are smaller or larger, angular, elongated, supplied with projections, chub or biscuit-shaped. Not infrequently, especially in an anemia which has developed rapidly nucleated red bloor corpuscles may be found (erythroblasts) in suitably stained preparations (with hemotoxylin-eosin), further in some of the red blood corpuscles basophilic granules, the remains of brokendown nuclei of the erythroblasts are observed. The number of white blood corpuscles is, as a rule, increased on account of the increase of polynuclear lencocytes (in one of the authors' cases 180,000 white blood corpuscles could be counted per cubic millimeter of blood). Through these, also as a result of the diminution of the red blood corpuscles, the numerical relation of the two becomes much closer (in one case of anemia caused by the Filaria immitis, the authors observed the relation of red blood corpuscles to the white as $18: 1)$, so that the examination of unstained blood preparations may cause confusion with leukemia.

As the clinical methors of blood examination gire only relative values of a certain volume, the degree of anemia eannot always be established from the number of red bloor corpuseles or from the hemoglobin content, especially so since the proportional values may be normal in spite of the present anemia, for instance in the oligemia which occurs exceptionally without qualitative changes of the constituents of blood, as well as in all those cases of anemia in which the animal lost either too much water or had not taken any water, and in which a thickening of the blood resulted in consequence (Wetzl).

After a time edematous swellings appear on different parts of the body, on the lower abdomen, lower chest, in the submaxillary space, at the lower part of the extremities, being not sharply circumseribed, but flat swellings of doughy consistence. The eyelids may show a pad-like swelling and an effusion may take place into the body carities. The edematous swellings are especially extensive and prominent after feeding very watery food stuffs, thus in anemias of cattle in sugar factories the swelling may increase to such an extent that it renders the movement of the animal entirely impossible. In this stage of the disease the animals are usually affected with diarrhea, by which they are weakened still more, finally they are unable to stand up and succumb.

Jost, Hildebrandt and Moussu have described other peculiar manifestations in hydremia of eattle in sugar factories. Sometimes the affected animals suddenly collapse during walking, others die after a sickness lasting but a few days in the course of a few hours as if struck by apoplexy. Some are entirely incapable of passing feces as a result 
of the weakness of the abdominal and body muscles. There is polyuria, in which a very pale watery urine of low specific gravity is passed; sometimes profuse salivation is present. In acute cases the autopsy may reveal a gastro-intestinal inflammation.

Course. Great losses of blood may produce death inside of a few hours, or even in a few minutes, while the duration of chronic anemia may extend to several months and even years. This occurs especially in anemia which develops in association with chronic diseases. Ascites of sheep and cattle lasts at least from 3 to 6 months or even longer, but some animals may die in a shorter time. The last mentioned short course may however be only apparent if the first indications escape detection.

Diagnosis. The recognition of anemia causes, as a rule, no difficulty. It may be confounded, especially on a single examination, with pronounced weakness of the heart and a general collapse of the blood vessels which result also in a conspicuous paleness of the peripheric parts of the body. Simple anemia is distinguished from psendo-lenkemia or from leukemia by the normal condition of the blood-forming organs, and also by the condition of the white blood corpuscles. Further the milder cases may be distinguished from pernicious anemia by the normal appearance of the blood corpuscles, on the other hand from the severe cases by the absence of gigantocytes and gigantoblasts, as well as by the severe general affection. In horses infectious anemia should also be taken into consideration.

In all cases of anemia however it should be aimed to determine the fundamental affections, the recognition of which is of great value from a prognostic and therapeutic standpoint.

Prognosis. The acute anemia which develops after great losses of blood terminates with suitable nutrition of the animal almost without exception in a relatively short time in recovery. By the ingestion of large amounts of water the blood attains within a few days its original quantity, the salts and the proteids. being rapidly replaced from the food. Only the replacement of the red blood corpuscles requires a longer time, up to several weeks; after exceedingly large losses of blood the recovery may require even several months, and may not follow at all even after suitable treatment. If the animal has lost at least half of the normal quantity of its blood within a short time, there exists no longer any hope for its improvement.

The prognosis of chronic anemia depends on the nature of the fundamental affection. In the independent forms however the prognosis depends, not only upon the deg'ree of anemia, but also upon the condition that the cause of the disease is capable of remoral, although under farorable conditions the disease usually terminates in recovery, the course of an already

Vol. $1-54$ 
existing dropsy can no longer be influenced in spite of suitable care and treatment.

Treatment. In acute anemia, if bleeding still continues, the hemorrhage should first of all be arrested; in external hemorrhage by surgical measures, internal bleedings by the administration of hemostatic remedies. Most satisfactory are Ergot (15-25.0 or $0.5-2.0 \mathrm{gm}$. of the extract; $5-10.0$ or $0.2-1.0 \mathrm{gm}$. per os; the extract may also be used subcutaneously in half doses), Ergot in (1-2.0 or 0.02 to 0.2 gm.) Extractum hydrastis (10-15.0 gm. subcutaneously for horses) ; very good results may further be obtained by intravenous injections of adrenalin or suprarenin (1-5 ce. of a solution of 1:1000 per $100 \mathrm{~kg}$. body weight). The transfusion of blood from a healthy animal of the same species can hardly be accomplished in practice, while the infusion of physiological salt solution into the veins (combined with adrenalin or suprarenin), or into the rectum is frequently carried out and may be a life saver. In cases developing less suddenly an increased partaking of water may increase the fallen blood pressure, the ingestion of water should however be permitted only after the cessation of the hemorrhage.

Berger always obtained good results by infusions of salt solutions into the rectum (10-20 liters of a $0.6 \%$ solution for horses and eattle). The infusion should be made slowly and the rubber tube of the irrigator should be introduced far into the rectum.

Flatten, Dorn and Frick constructed suitable appliances for the application of intravenous injections of physiological salt solution, but the infusion may be carried out in emergeney with the aid of a simple rubber tube and funnel. "The solution should be sterilized by boiling and then cooled down to body temperature.

Albrecht repeatedly injected defibrinated blood drawn from healthy horses under the skin of affected horses. The blood, which was kept at $40^{\circ} \mathrm{C}$., was injected in quantities of $80 \mathrm{cc}$. with the aid of a syringe of suitable size into various parts under the skin of the abdomen and facilitated the absorption by massaging the place of injection. In this manner he succeeded in injecting $11 / 2$ liters of blood under the skin of the abdomen in a short time.

In all cases of anemia special care must be taken in regulating the diet. The necessary substances for the formation of blood (proteids and mutritive salts) should in the first place be given in increased amounts. Best adapted are nutritious food stuffs (oats, barley, legumin, good hay, meat, etc.), in very weakened animals yolks of eggs, milk, strong bouillon mixed with eggs, in the absence of appetite the animals should be drenched with this food. In primary anemia with normal digestion the purely dietetic treatment alone frequently produces an improvement progressing from day to day and even complete recovery may result in a relatively short time. The food should be given in small rations but frequently. In anemia brought on by feeding food stuffs lacking in nutritive qualities, above all substances saturated with water, recovery may be obtained in such cases which are not very severe, by a suitable change in food, particularly by the substitution of dry foods. An edema which may be present does not require any special treatment. 
Of the medicinal agents iron is first to be considered. The selection of the iron preparations is considerably influenced by its price, otherwise the iron preparation should be administered for a long time and in not too small doses. Of the numerous preparations employed Ferrum pulveratum (2-5 gm. for large animals, 0.5-1.0 gm. for sheep, 0.02-0.1 gm. for dogs, should be given for a dose three times a day), Ferrum sulfuricum (in same doses) may be mentioned. It is advisable to administer both with stomachics and salines. For drinking water the water in which the red hot pieces of iron are cooled in blacksmith shops, may be given. For small animals Ferrum hydr. reductum (0.005-0.01 gm. with sugar in powder), Ferr. lacticum (0.02-0.1 gm.), or Tinet. Ferri pomati (10-20 drops), are suitable. In the presence of inappetence a small quantity of hydrochloric acid should be added to the drinking water. Arsenic (of Fowler's solution 10-30 gm. for large animals, 2-10 drops for small; 0.1-0.3 gm. or 0.001-0.005 gm. of the arsenious acid) may also be administered, as according to experience it aids metabolism. In small animals the Tablettae ferri peptonati cum arseno sec. Bleyer may be conveniently administered (daily 1 to 3 tablets). Evers treats anemia with Damholid (100 gm. of a fresh solution subcutaneously for large animals, for small animals the same amount per os).

Literature. Arloing, C. R., 1892. 776. - Berger, B. Nit., 1902. 8. Braasch, Monh., 1891. II. 59. - Carré \& Bigoteau, Rev. gén., 1908. XI. 433. Dorn, B. t. W., 1906. 433. - Dralle, A. f. Tk., 1886. XII. 71. - Evers, B. t. W., 1905. 201. - Flatten, Ibid., 1906. 697 - Frick, D. t. W., 1904. 253. - Guittard, Pr. vét., 1897. 32. - Hildebrandt, Pr. Mt., 1857/58. 114; 1858/59. 120. - Jost, Ibid., 1857/58. 114. - Petzold, S. B., 1863. 86. - Pflug, W. f. Tk., 1857. 413. Plate, B t. W., 1906, 713. - Pr. Vb., 1902. II. 34. - Werner, W. f. Tk., 1897. 186. - Wetzl, Klinische Blutuntersuchungen, Diss. Budapest 1908.

\section{Infectious Anemia of Horses. Anaemia Infectiosa Equorum.}

(Swamp fever of horses, Malarial fever of the horse, River bottom disease, Loin distemper; Infektiöse Blutarmut der

Pferde [German]; Anémie infectieuse, Anémie pernicieuse, Typho-anémie infectieuse du cheval

[Carré \& Vallée], Anémie pernicieuse

progressive, Anémie épizootique

[French].)

Infectious anemia is a specific infections disease of the horse species which is manifested sometimes as an acute, at other times as a chronic septicemia, and causes a destruction of great numbers of red blood corpuscles. It is caused by an ultra-microscopical microorganism.

History. Originally it was without a doubt confounded with the idiopathic simple anemia. As an independent disease it was first described by Lignée (1843), and almost simultaneously by Charlier (1843) 
and Dénoc (1843), and was associated with conditions of feeding, the more so as later Delafond (1851) failed in its artificial transmission. Its infectious nature was first indicated by Anginiard (1859). Later Zschokke (1883) reported several cases of pernicious anemia observed by him in Switzerland, which in part developed as an outbreak, and clinically as well as anatomically completely corresponded with the infectious anemia, the same as the cases ohserved somewhat later by Fröhner (1886) and Ostertag (1890) in Germany. As a matter of fact Fröhner considered the pernicious anemia of horses even at that time as an infectious disease. The nature of the disease was cleared up in all details by Carré \& Vallée (1904-1906); further contributions on the disease were furnished by Ostertag (1907), Marek (1907) and Hempel (1909).

Ekwall (1895) described an enzootic anemia of horses in Sweden, which also corresponded with the infections anemia clinically and anatomically, but its identity with infectious anemia cannot be established positively for the reason that Brickmann recently (1904-1906) demonstrated parasites in the blood in a similar affection of horses.

Occurrence. The disease is observed among hor'ses, although other solipeds may be infected artificially, and it is probable that they may here and there become also affected by natural infection. It appears in more or less circumscribed localities, in the first place in marshy places, usually as a disease confined to single or several adjoining premises, and causes considerable economic loss because the disease as a rule leads sooner or later to death. Sporarlic cases of the disease may also be observed, namely, through the introduction by newly purchased affected or infected horses, which then under certain conditions may alone become affected, while the other animals of the premises may be spared.

In France infections anemia prevails in the northeastern territories, above all in the entire valley of the Mense, further in the districts of Anbe, Marne, Côte-d'Or, Meurthe-et-Moselle, and was recently observed by Dupas also in Châlons. In some of the localities it causes very great losses, which according to Vallée are estimated for the Department at 200,000 franes annually.

In Germany the disease occurs less extensively than in the adjoining territories of the infected French districts, especially in Lorraine (Köpke, Jarmatz, Ostertag), further in the district of Trier. Hochstein observed it in a horse stable in Bavaria.

In Hungary the disease was established by Marek upon three premises in three different counties.

From what was already said it appears that the disease occurs also in Switzerland and probably in Sweden. It was likewise observed in Mexico.

To all appearances infectious anemia of horses has a far greater geographical distribution than appears from the data mentioned, only it is frequently confounded with other internal diseases. Thus in certain cases the so-called skalma is probably as a matter of fact an infectious anemia.

Etiology. The fundamental investigations of Carré \& Vallée, the results of which were later fully confirmed by Ostertag and Marek, showed that the contagion of infections anemia belongs to the ultra-microscopical microorganisms. The virus passes the porous (Chamberland-Berkefeld) porcelain filters and cannot be demonstrated by staining methods or by cultivation. 
The virus is present in the blood, in the urine and in the feces of the affected, also of the apparently recovered animals. The saliva has no infectious properties (Ostertag). Whether there exists any kind of a relation between infectious anemia and horse pest has not been decided (see p. 286).

Tenacity. The virus loses its virulence entirely when heated to $58^{\circ} \mathrm{C}$, dry. ing at room temperature does not influence it; thus dry blood serum was found harmless only after seven months (Carré \& Vallée). In storing the blood without lrying it loses its infectiveness only after three months. The virus resists putrefaction for a long time, even in strongly ammoniacal substances (urine, manure). Thus Carré \& Tallée found urine of an infected horse, kept in a manure pit, virulent even after $21 / 2$ months.

Pathogenicity. The disease may be produced artificially in horses by intravenous or subcutaneous administrations of virulent blood or blood serum, immaterially whether larger quantities (up to $750 \mathrm{cc}$.) or very small quantities (1 cc.) are injected (Carré and Vallée). The disease of the test animals is indicated by a febrile rise of the temperature, which according to Carré and Vallée always occurs within 5 to 9 days; in one of Marek's cases however the incubation lasted for 18 days. The disease in test animals rarely develops with the same characteristics as in animals from which the blood has been procured. To produce a successful infection per os, larger quantities (at least $15 \mathrm{cc}$.) of virulent blood or urine are necessary according to Ostertag, and in such cases the incubation also extends over a longer time, 15 to 24 days. The incubation period may however be shortened by the repeated administration of great quantities of virulent material. Donkeys cannot always be infected (Carré \& Vallée, Marek), while other domestic animals, also small test animals are entirely resistant (Carré \& Vallée, Ostertag, Marek). Man also resists the infection.

The experiments of Ostertag and Marek proved that, after an intestinal as well as subcutaneous or intravenous infection, the disease in test horses not infrequently runs a chronic course, and aside from periodical rises in temperature, for a long time canses no noticeable disturbances, a conclition which deserves notice in transmission tests.

Natural infection may occur indirectly through the ingestion of food or drinking water contaminated by urine or with feces of affected animals. As the urine and feces always contain the virus, but especially during the febrile attack, and as it is very resistant against outside influences, there is an ample opportunity afforded for indirect infection. The virus is taken up either with the bedding or with the grass in the pasture, or the food may become contaminated by attendants or in other ways, especially in insanitary stables. With the ichor, or more directly with feces or urine, the virus may contaminate the drinking water, for instance by draining from the manure pit or in the pasture. On the other hand a direct transmission does not appear to occur. Carré \& Vallée failed in demonstrat- 
ing an intermediate action by any parasitic insects. Contrary to this Ries asserts that the infection is transmitted by the larvae of gad-flies or mosquitoes. He failed however to substantiate his contention by experimental proof.

Infection only results when either large quantities of virulent material are taken at one time, or from the taking of smaller quantities at frequent times. Accordingly the occasional ingestion of a mouthful of contaminated hay or straw does not appear at all dangerous (Ostertag).

W'hether the affection which sometimes occurs in the sucking foals of affected mares, results from an intrauterine contamination or an infection through the milk cannot be decided.

The introduction of the disease in previously uninfected premises invariably occurs through newly bought, affected or apparently recovered horses, whose blood however continues to retain its infectiousness (Marek). As long remissions are by no means rare in the chronic forms of the disease, and as in the cases of slow course only very few apparent symptoms are generally noticeable, it happens not infrequently that horses suffering with the disease are offered for sale.

Anatomical Changes. The infectious anemia of horses is characterized by the anatomical changes of an acute or chronic septicemia, with visible changes of the blood, in which the changes depending on the duration of the disease may show very pronounced variations.

The spleen in acute cases, or in animals which have died during a relapse of the disease, appears considerably enlarged, sometimes double or four times its size. Its capsule is tense and covered with hemorrhages, the pulp is blackish-red, distended, mushy, sometimes even liquefied, as in anthrax. Sometimes in the otherwise normal or more or less enlarged spleen, various sized nodulated protuberances are found, which represent softened areas of a blackish-red color. The slower the course the less marked is, as a rule, the swelling of the spleen, while in a very protracted course it may be entirely absent, but in such cases the consistency of the organ is rather somewhat increased. All of the lymph glands of the body show acute swelling and a hemorrhagic condition is noted in cases in which hemorrhages have occurred in the corresponding organs; in chronic cases however the lymph glands either fail to show an edematous swelling or it is but slight. Subserous hemorrhages are usually found. In chronic cases however they are not prominent. Hemorrhagic epots up to the size of a silver dollar are especially numcrous under the peritoneum of the cecum and colon, they occur also, but in smaller numbers in other parts of the peritoneum. A vellowish or reddish serous fluid may be present in small quantity in the abdominal cavities. Usually numerous hemorrhagic spots are seen in acute cases under the capsnle of the more 
or less enlarged liver, the substance of which is usually yellowish, discolored, and may be so brittle that a rupture may result (Carré \& Vallée). In the intestinal mucous membrane either single, roundish hemorrhages appear, or the mucous membrane, especially in the large intestines, may manifest diffuse hemorrhages, when a more or less bloody consistency of the intestinal contents is noted. Hemorrhages are less frequent in the stomach, but they may sometimes occur. The heart, with the exception of the very slow cases, shows subperior subendocardial and intramuscular hemorrhages, their number and size being usually in an inverse proportion to the duration of the disease (in very acute cases the heart appears as if sprinkled with blood). The kidneys and the lungs contain small hemorrhages only in acute cases, at the same time there is always a parenchymatous inflammation of the kidneys, heart muscle, and the liver substance. The mucous membranes of the urinary bladder sometimes show hemorrhagic spots of recent or older origin.

Conspicuous and constant changes are present in the bone marrow. In the proximal long bones of the extremities, especially so in the bones of the upper thigh or upper arm, the bone marrow is changed, either for its entire length or only in parts, to a clark brownish-red or blackish-red mass (Fig. 152). In the cases with slower course only various sized circumscribed areas are found in the fat marrow or this may appear normal. At the same time however the spongy bone marrow shows the above changes or at least a reddish discoloration. The

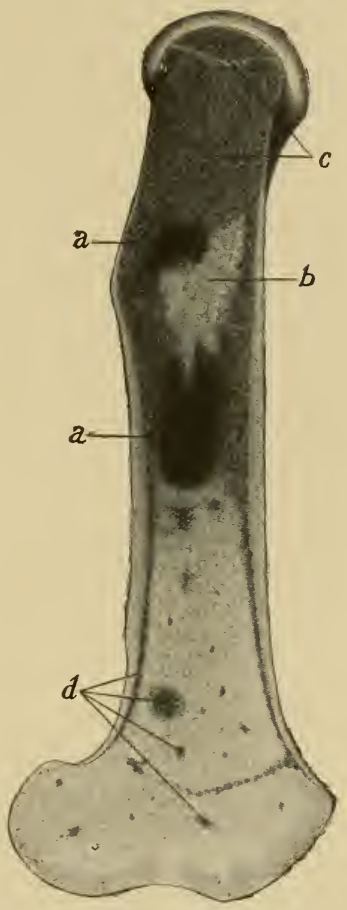

Fig. 152. Infectious anemia. Femur of a horse. Longitudinal section; $a$ dark red area in fat marrow; $b$ normal yellow bone marrow; $c$ diffuse dark red discoloration of the spongy marrow; $d$ small dark red foci. spongy bone marrow of the vertebrae, ribs, etc., manifests similar changes. Even in the most chronic cases at least small hemorrhages are found, which however appear of a peculiar inkblack color if of earlier origin. This condition of the bone marrow does not result from hemorrhages, but represents, as in older losses of blood in general, only a regenerative process, and consists in an increased activity of the blood-forming elements of the red marrow areas, the normal occurrence of which, 
in the fat or gelatinous marrow, especially in the femur and humerus was already indicated by Skiba.

In. acute cases of the disease the blood shows no peculiar macroscopical changes with the exception of slower coagulation, while in the more chronic eases indications of an anemia appear. This is increased in proportion to the duration of the disease. Moderate edematous infiltrations of the subcutaneous, perirenal, as well as in the intermuscular connective tissue between the mesenteric lavers, further icteric discoloration of the tissue are not infrequent occurrences, while marked emaciation is frequently observed.

Symptoms. The period of incubation after a subcutaneous or intravenons infection varies between five and nine days, and may also be the same after an intensive natural infection. Ordinarily however it probably lasts for 2 to 3 weeks, as was noted in the experiments of Ostertag and Marek (see p. 85t). With respect to the duration and the symptoms, two clinical forms of the disease may be recognized to good advantage, between which however no sharp line can be drawn, and consequently Carré \& Tallée have even described a subacute form.

The acute form appears quite suddenly with distinctly pronounced symptoms. A conspicuous depression and general weakness develops, as a result of which the animals rapidly tire, and sometimes even fall down while in motion. Then they rise only with great difficulty or can regain their feet only

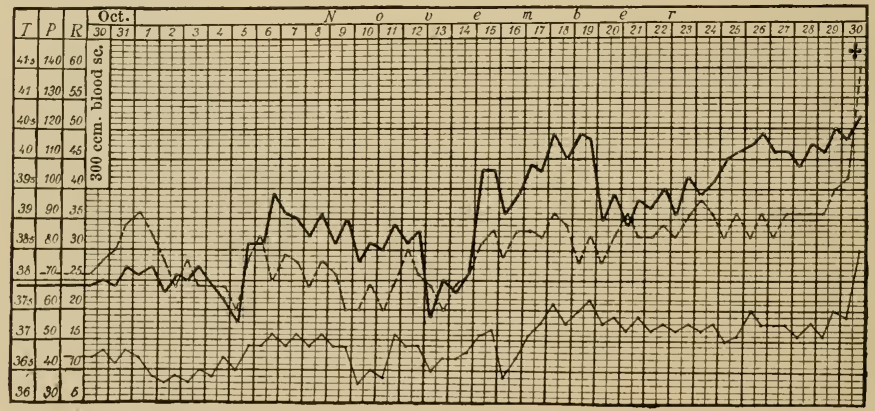

Fig. 153. Fever curve in the acute form of infectious anemia of horses.

with human assistance. At the same time the weakness, as in all general infectious diseases, is distinctly noticeable in the hind parts. Frequent tripping, or an uncertain nse of the hind leg may also be observed quite frequently. Sometimes the position of the body is similar to that in laminitis.

Fever sets in at the same time (see Fig. 153), and after a gradual rise of the temperature, reaches its height on the second or third day $\left(40-40.5^{\circ}\right.$, sometimes even $42^{\circ}$ [Carré 
$\&$ Vallée]), and then it may remain at the same height, at least intermittently, until the fatal termination of the disease. Sometimes intermissions of 1 to 2 days are observed (see Fig. 153). The number of pulse beats increases up to 60 to 90 per minute, the pulse appearing weak and soft, the heart's action however stronger. Exercise of even short duration produces a considerable increase of frequency of the heart's action and a temporary irregularity of the pulse.

The conjunctivae appear somewhat swollen and of a uniform washed-out red color with a somewhat yellowish tint, sometimes showing hemorrhages up to the size of a dime, irregular in shape (principally in the membrana nictitans). At the same time slight lachrymation is present. The nasal mucous membrane also appears reddened and shows punctiform hemorrhages, especially on the nasal septum. Not infrequently there is a scanty serous, sometimes also reddish colored nasal discharge, in some instances a cough is also observerl.

Diarrhea, with passing of broken halls of feces or fluid excrement, which may be stained reddish or covered with blood coagulum is frequently present. The urine is passed at short intervals, and contains albumen, which may reach $1.5 \%$ in amount but is usually found in much smaller quantities. In the sediment of the urine granular or epithelial casts may be found.

The appetite is depressed from the beginning. At the same time a rapidly increasing emaciation is noticeable which may progress so rapidly that the animals may lose one-fourth to one-third of their body weight in a few days (Carré \& Vallée), or they may emaciate inside of two weeks almost to skeletons. Very slight edematous swellings in the distal parts of the extremities, the lower chest and the lower abdomen, are also not infrequently observed.

Coagulation of the blood is retarded, the separated blood serum appears darker yellow, or even somewhat greenish and dichroic. The number of red blood corpuscles diminishes in proportion to the duration of the disease, so that after 10 to 15 days a diminution of $1,000,000$ to $2,000,000$ may be demonstrated. Immediately before the fatal termination however the number may be reduced to one-half (Carré \& Vallée). As the relative number of the red blood corpuscles depends also considerably on the existing water contents of the blood, there may be decided variations in this respect, in spite of the fact that a considerable reduction of the absolute quantity of the red blood corpuscles exist (see p. 848).

The determination of the relative quantity of $\mathrm{r}$ ?d blood corpuscles with a test tube as first recommended by Zschokke, is particularly suitable for practical purposes. Any desired test tube is filled with blood drawn from the veins, then is allowed to stand in water at a temperature of $10-12^{\circ} \mathrm{C}$. for a half hour; then the length of the sections of the test tube measured are those which contain the blood plasma and the layer of the deposited red blood corpuseles. Finally the ratio between the two is ealculated from these measurements. (Normally the length 

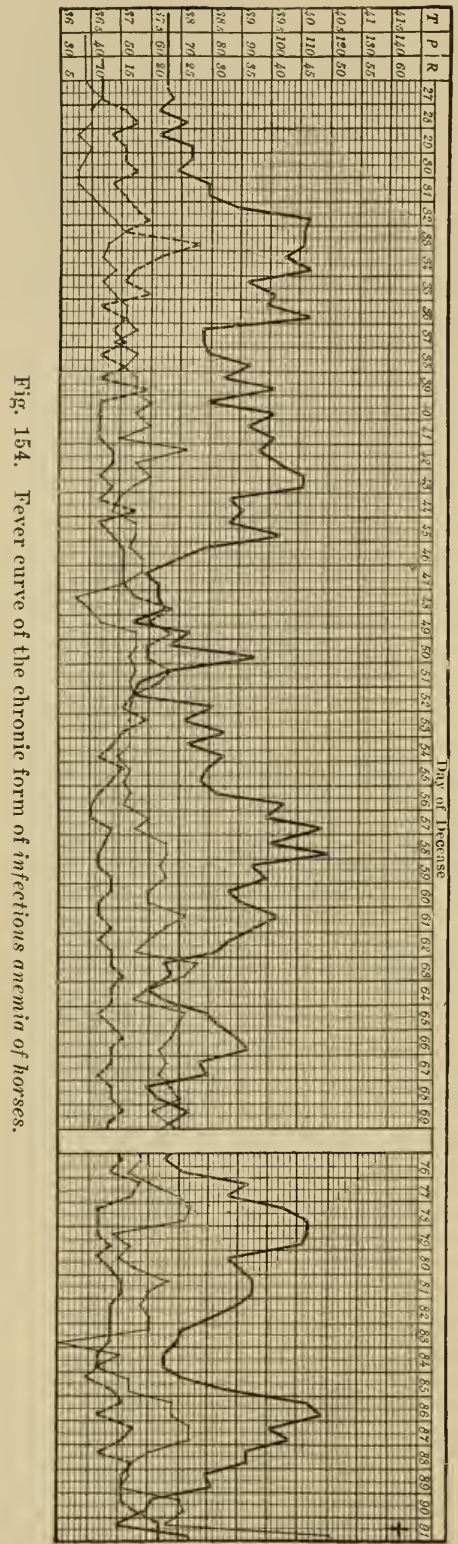

of the bloor plasma in the tuke, compared with that of the layer of the red blood corpuscles, is in a ratio of $6: 4$.) It is advisable to employ specially graduated test tubes for this purpose.

The red blood corpuscles appear pale, and in their cell bodies basophilic granules (fragments of the cell nuclei) may be recognized, which may be mistaken for endoglobular parasites (Carré \& Vallée). Contrary to the observations of Carré \& Vallée, Ostertag and Marek found no changes in the form of the red blood corpuscles.

According to the view of the authors the apparent poikilocytoses are frequently only artifacts. If, for instance, the blood is smearel onto a cover glass in a somewhat thicker layer or in a moist place, the red blood corpuseles assume the most varied changes of form because of the somewhat slow drying.

Under gradual loss of the strength, finally an apparent paralysis of the hind parts develops; not infrequently however the animals are capable of standing almost until the time of death. In pregnant mares abortion results almost invariahly.

The duration of this acute form, according to Carré \& Vallée, is from 5 to 15 dars, on an average one week, but according to Marek's observations the disease may in young foals last only 1 to 2 days, and in adult animals, on the other hand, not infrequently for 3 to 4 weeks (see Fig. 154).

The chronic form of the disease manifests itself with periodical febrile attacks and with indications of anemia, which is not recognizable in the acute form, on account of the peculiar diffuse red coloration of the mucous membranes. The febrile attacks appear at 
intervals of several days, up to several weeks or even longer, exceptionally even after months (Carré \& Vallée). They develop without any obvious course, and last from 2 to 3 days or even for several days. The rise and also the fall of the temperature always occurs gradually (see Fig. 154). During the febrile rise of temperature the pulse is also more or less increased, sometimes however only very slightly. In cases which are not too chronic a moderate increase in pulse exists also in the afebrile periods which becomes well marked even on unimportant external influence, and then appears weaker. Other febrile symptoms, such as diminished appetite, depression, muscular trembling are absent in very protracted cases, so that if no systematic temperatures are taken, in these cases they can only be recognized in the advanced stage of their development.

The mucous membranes appear at the beginning either normal in color or slightly reddened, in the later stages however they become continuously paler in color, while during the febrile attack, or during a temporary improvement of the condition, they may present normal or at least nearly normal color. Hemorrhages are rare in the visible mucous membranes, but they may nevertheless occur.

The urine usually contains small quantities of albumen in the not very protracted cases, otherwise however it may only appear periodically, especially during the febrile attacks. Not infrequently a periodical fetid diarrhea sets in, and here and there colicky symptoms are observed.

Edematous swellings of the extremities, or of the lower parts of the rump or the head (on the head especially in pasturing animals) are a not infrequent occurrence.

The blood coagulates only slowly also in this form, so that hemorrhages resulting from external causes can be stopped only with difficulty. The number of red corpuscles diminishes considerably, even to 2,000,000 per cubic millimeter of blood (Carré \& Vallée). The number of blood corpuscles however varies not a little in accordance with periodical improvement or aggravation, and their diminution may in very protracted cases persist continuously moderate; or on the contrary, after partaking of food and water there may be present a relative increase in red blood corpuscles (see p. 848). Changes in the form of the red blood corpuscles are missed also in this form of the disease in suitably prepared blood specimens.

The nutrition may be affected from the onset of the disease, or on the contrary in a slowly progressing case it may remain satisfactory for weeks or even for months. Finally however emaciation becomes noticeable even in these cases. It does not progress uniformly, but at times may come to a standstill or the nutritive condition of the patients may improve for a certain time.

In this form the duration of the disease is from one to 
several months; not infrequently however it may even extend over several years (Marek).

Course and Prognosis. The acute form of the disease usually leads to a gradual aggravation of the symptoms, death ensuing without any struggle. Sometimes however it passes into a chronic form. Death may occur unexpectedly in the acnte as well as in the chronic forms. The chronic form develops from the acute, with a gradual diminution of the severe general symptoms, or the disease may have developed from the beginning with a chronic character, when periodical improvements or aggravations of varying duration may be observed. The fatal termination results either after the development of the cachectic condition or because the disease assumes an acute course.

Recovery is observed only exceptionally (Carré \& Vallée, Ostertag, Marek). One should not be deceived by the sometimes very lasting improvement in the chronic form, as later a relapse, or a progressive anemia witl emaciation usually occurs.

In a large stur in Hungary with a great number of horses, in which the disease prevailed for five years, there were several horses which originally were affected visibly, but for several years had showed no symptoms whatsoever, but even in those rare cases recovery occurred only in a clinical sense, as in one case blood from one of the apparently recovered animals proved infectious (Marek). A similar occurrence had previously been olsserved by Carré \& Vallée.

The epidemiology of the disease varies greatly with the duration of the individual cases. Usually, in connection with the introduction of new horses, only isolated affections occur at first, the disease taking a rapid course from its onset, while in very chronic cases the disease is only recognized when a large number of the horses have already become infected and affected because the symptoms are for a long time very obscure. If in such cases no preventive measures are introduced, their number increases from week to week and the animals beconc attacked without reference to age or breed. In stables however, which have been infected for a long time, the newly introduced animals become affected first and most severely. For this reason the disease is usually acute in newly infected stables (Carré \& Vallée). At the same time animals in other stables, or of the same stable kept in other pastures and watered in separate places, remain temporarily free from the disease at times even for several years.

In a stud in Hungary the disease did not appear among foals, which had been kept in an isolated stable or on a separate pasture, until four years after apparently recovered mares had been placed among them.

Diagnosis. In infected localities or in infected stables, the clinical symptoms, the course of the disease, and the negative 
findings in the various organs, usually afford sufficient indications for a diagnosis. On the other hand, in localities which have been free from the infection the disease is recognized with certainty only on autopsy or from animal inoculations, while the clinical symptoms arouse only well founded suspicion. Infectious anemia is indicated especially by changes of a septicemic character, such as hemorrhages in the serous membranes or in the mucous membranes, further by the regular discoloration of the bone marrow of the bones of the upper extremities (not to be mistaken for the normal inclosures of red marrow!), degeneration of the parenchymatous organs, swelling of the spleen and edematous infiltration, without other changes in the organs. The complement fixation has no diagnostic value (Hempel).

From the standpoint of differential diagnosis the eatarrhal form of influenza barely comes into consideration, in spite of the fact that its symptoms resemble the acute form of infectious anemia not a little. Nevertheless it can always be easily excluded, above all on account of its rapid extension in the affected stables and by the fact that its course is almost always rapid and favorable; in influenza the eatarrhal symptoms predominate while hemorrhages in the mucous membranes are absent. The disease mav be mistaken for anthrax in the acute form of infectious anemia in the living animal as well as on autopsy. Contrary to infectious anemia however, in anthrax the rise of temperature is rapid, and at the same time circumseribed, rapidly extending inflammatory edemas appear not infrequently on certain parts of the body, also colicky pains are not infrequent, the conjunctivae are cyanotic, there is dyspnea, and the disease usually terminates in 1 to 3 days. If death occurs later than after 10 days anthrax may be excluded on post-mortem on this ground alone, aside from the slight anemic indications in the subcutis, and in certain internal organs, which in anemia are already perceptible after such a duration. On the other hand in cases of death resulting within a short time, the bacteriological examination of the blood or test inoculations are confirmatory. Enzootic spinal meningitis, the nature of which cannot be yet considered as definitely eleared up (see Vol. II), corresponds as described in its original by Schlegel, clinically and anatomically with anemia to such an extent that it can be excluded with certainty only by careful bacteriological examination or by animal inoculation. The piroplasmosis of horses is differentiated by the distinct icteric discoloration of the visible mucous membranes, but especially by the microscopical demonstration of piroplasma in the blood. At the same time however it is advisable to be cautious, as in the acute cases of infectious anemia fragments of nuclei resembling piroplasma may be present in the red blood cells; besides this, the piroplasmosis of horses cannot be transmitted to healthy animals. Sclerostomiasis in its 
chronic form (Glage) runs an afebrile course contrary to chronic infectious anemia, and attacks colts exclusively, while in its more acnte form (Schlegel) only the autopsy or the demonstration of sclerostome larvae in the intraabdominal hemorrhages is decisive. At the same time however the presence of a few sclerostome larvae in the subserous or submucous tissues must not he misleading. Simple anemia is distinguished by its afebrile course and by the demonstration of dietetic errors of some primary affection or indications of some outside influence.

Treatment. A treatment of infectious anemia which promises positive results is at present unknown. The remedies used so far, such as quinine, collargol, arsenical preparations, have either proved powerless or at best only result in temporary improvement, the same as intensive feeding and keeping the animals from work. Nevertheless the arsenical preparations appear to have a favorable influence on the disease, at least in certain cases, thus Ostertag obtained at times a striking inprovement from atoxyl (0.5-1.0 gm. intravenously in physiological salt solution; according to Loewenthal up to $3 \mathrm{gm}$. of atoxyl are well tolerated). Marek observed similarly good results from the cheaper natrium arsenicosum, which he administered in gradually increasing doses, from 1-4 gm. per os for two weeks, and then suspended for a period.

Prevention. In order to prevent the introduction of the disease the greatest eare should be exercised in the purchase of horses. Horses which are in any way suspicious, and especially those which without any apparent cause are poorly nourished, anemic, or easily fatigued, or in which the frequency of the heart increases considerably from insignificant outside causes, and in which at the same time the urine contains albumen, should not be placed in the horse stable until after systematic isolation and observation extending over three montlis (for instance in the cattle stable). Every newly purchased horse in infected localities should be subjected to a quarantine of three months. At the same time the sanitary conditions of the horse stables should be given full consideration.

In already infected premises the attempt shonld be made first of all to isolate all affected horses by systematic temperature measurements and then to disinfect the stables thoroughly. The excrements of the affected animals are best disinfected by packing them for at least one month in piles of about one cubic meter (Ostertag). At the same time the food and drinking water should be prevented from coming in contact with the feces and urine of horses. If the disease appears among pastured horses the apparently healthy animals should be driven to a pasture which has not yet been used by horses, and the affecter animals should be stabled. Finally efforts 
should be made to remove all affected horses as soon as possible. This is usually carried out easily in sporadic occurrences of the disease or in localities where horse insmrance is maintained.

Immunization. The experiments which have been undertaken by Carré \& Tallée along this line with the blood of apparently recovered animals, also with blood from donkeys or eattle which have been inoculated repeatedly with virulent material, were unsatisfactory; likewise Marek's experiments with the blood of an apparently recovered horse or with blood exposed to various high temperatures, or which had been treated with a trichloride of iodine solution.

Literature. Brickmann, D. t. W., 1907. 724. - Carré \& Vallée, Rev. gén., 1906. Tlll. 593; 1907. IX. 113 (French Lit.). - Ekvall, Tidsskr., 1895. 208. Francis \& Marsteller, Texas Agricult. Exper. Station, 1908, - Fröliner, A. f. Tk., 1886. XII. 382. - Hempel, Z. f. Infkr., 1909. V. 381 (Lit.). - Hochstein, W. f. Tk., 1907. 145. - Jarmatz, Z. f. Vk. 1904. 68. - Köpke, Z. f. Vk., 1901. 356. Meier, Z. f. Tm., 1906 X. 1. - Mohler, The Vet. Journ., 1909. 395. - Ostertag, Monh., 1890. I. 127; Z. f. Infkr., 1907. III. 1. - Ries, Rec., 1908. 11. - Vallée, Bull, 1907. 526. - Zschokke, Schw. A., 1853. XXT. 11.

\section{Progressive Pernicious Anemia. Anaemia Perniciosa Progessiva.}

By the name of progressive pernicious anemia is designated in human medicine a severe and gradually progressing anemia, with the appearance of gigantocytes and gigantoblasts in the blood (Ehrlich) or with a change in the proportion between the hemoglobin content and the number of blood corpuscles in favor of the blood coloring matter (Tallquist), in which the form of the red blood corpuscles undergoes conspicuous changes. The disease appears withont any apparent cause (cryptogenetic form of pernicious anemia) or in connection with certain organic diseases (secondary form of pernicious anemia).

History. The disease was first described in human medicine in 1868 by Biermer, but was mentioned previously already as idiopathic anemia by Lebert and Addisson. Since that time the disease has been observed in a large number of cases in certain localities, especially in Switzerland, England and Sweden. There exist several publications relative to the occurrence of pernicious anemia in animals, but most of them probably allude to the infectious anemia of horses, which is a specific infectious disease, and according to the blood findings does not correspond with the progressive pernicious anemia (see p. 851). Regarding the few remaining cases it is undecided whether they should be considered as pernicious anemia, because in these cases the diagnosis was based only on the severity of the anemia and on the demonstration of a poikilocytosis, which however cannot by any means be considered as a proof of the pernicious nature of the anemia, since the poikilocytosis may represent artifacts (see p. 857). At any rate it remains for thorough hematological investigations to decide how frequently true progressive pernicious anemia occurs in domestic animals.

Etiology. Nothing positive can be said at present relative to the cause of primary cryptogenetic pernicious anemia. Human physicians frequently associate the origin of the disease 
with certain gastro-intestinal distmrbances, at the same time accepting the action of absorbed toxic substances from the intestines as its cause. This hypothesis recently received a substantiation by the investigations of Tallquist, which proved that certain lipoid substances which develop in the intestines during qualitative or quantitative changes of the splitting of the fat and are then absorbed, possess a distinctly hemolytic action. Berger \& Tsuchipa found in fact a lipoid substance in the gastric and intestinal mucous membrane of such patients, which could be extracted by ether, and showing about a ten times stronger hemolytic action than the lipoid substances of normal mucous membranes.

The development of the disease as a result of a lipoid action was established experimentally also in secondary pernicious anemia by Tallquist, which disease sometimes develops in man in the presence of the Dibothriocephalus latus in the intestines. A similar origin of the disease is further suspected by Tallquist in those cases in which the disease develops in connection with malignant growths in the gastro-intestinal canal. logical part in man.

From the veterinary standpoint severe anemias are frequently included in this affection, which are caused by gastrointestinal parasites (dochmiasis, strongylosis), blood parasites (trypanosomiasis, piroplasmosis), even those which are produced by chronic suppurations or other exhausting diseases.

To what extent this broadening of the conception of pernicions anemia is justified ean only be determined when in such eases the characteristic changes of the blood in the presence of pernicious anemia are established by thorough hematologic examinations. It is probable that all of these affections belong to the simple secondary chronic anemia, which in severe cases may be associated with poikilocytosis, although in some cases the pernicious nature of the affection cannot be disputed without further consideration.

Many human physicians count only those eases as pernicious anemia which at least apparently develop independently (Grawitz).

Pathogenesis. Ehrlich explains the development of the disease in man in such a manner that the unknown causative agent produces on the one hand a destruction of the red blood corpuscles, and on the other a degeneration of the bone marrow, thereby producing the formation of megaloblasts or megalocytes in the bone marrow. In opposition to this Tallquist lays the principal stress on the exhaustion of the regenerative power of the bone marrow, while he conceived the formation of megaloblasts only as a result of the increased functions of the bone marrow.

Anatomical Changes. The autopsy shows outside of the changes of the blood yet to be mentioned, in general the same 
changes as in severe simple anemia. The bone marrow appear's to be replaced by a raspberry-like mass or sonetimes resembles embryonic bone marrow; it contains fat cells sparingly, but on the other hand numerous different kinds of red blood corpuscles with one or two nuclei, which are either very small or very large (Babes, Rindfleisch). Not infrequently a relatively large layer of fat is conspicuous under the skin and surrounding the internal organs.

Gróhé found iron in large quantities in the spleen and the liver, Quincke in the spleen and in the kidneys; it was demonstrable by ammonium sulphide, and probably resulted from the great destruction of red blood corpuscles. Quincke found $2.1 \%$ of iron in the ash.

Symptoms. The disease always commences insidiously. Ready fatigue, somewhat disturbed appetite, and as a result a failing of the nutrition as well as a decrease of the circumference of the abdomen, first direct attention to its presence. At the same time the paleness of the mucous membranes continually increases. Difficulty of respiration appears during work or even during slow walking. The heart's action is accelerated, now and then throbbing, the pulse is later threadlike, the heart sounds sometimes appear indistinct.

The blood is pale red in color, sometimes with a rellowish tint, it coagulates slowly, its specific gravity, as well as its hemoglobin content, is lower (Lindquist found in one case the hemoglobin content diminished to $60-80 \%$, Friedberger to $60 \%$ ). In affected man the hemoglobin content of the blood is always found to be too high in proportion to the existing number of red blood corpuscles. The number of red blood corpuscles diminishes considerably, and they show changes in form. First of all their non-uniformity in size is conspicuous, inasmuch as some appear much larger and at the same time are frequently pale (macrocytes, gigantocytes), others again are very small, and of an intensely yellow-red color (microcytes); in addition, a variable number of nucleated red blood corpuscles may be seen (erythroblasts). At the same time the red blood corpuscles form small money-roll-like groups and some appear peculiarly wasted, angular, elongated, containing one or more projections, club or biscuit-shaped, and sometimes entirely shrunken (poikilocytosis). Friedberger observed in one case an increase of white blood corpuscles and blood plaquelets. In blood of affected man large or sometimes nucleated red blood corpuscles are sometimes found, the diameter of which may reach up to $20^{\mu}$, and the presence of which is, according to Ehrlich, supposed to possess a high diagnostic value; this is however disputed by some authors. As the pernicious anemia of man and animals should not show any marked differences in the blood findings, more attention should be attributed to the presence of gigantocytes or gigantoblasts in the diagnosis of pernicious anemia in animals, and also to the relation beVol. 1-55 
tween the hemoglobin content of the blood and the number of red blood corpuscles.

The examination of the blood may be carried out by placing a very small drop of blood on a cover glass from which the fat has been carefully removed by washing with ether, and whose borders have previously been covered with a layer of fat, while a drop of physiological $(0.9 \%)$ salt solution is placed on one of its surfaces. The blood which is placed into the salt solution is immediately diluted suitably and is then placed upon a elean slide with the surface containing the blood drop. The fat-covered borders of the cover glass enclose the blood air tight, so that the evaporation of water and consequently the shrinking of the blood corpuscles is prevented.

If it is lesired to stain the blood corpuscles, several thoroughly clean cover glasses are smeared with a layer of blood by placing a small drop of blood upon the middle of the border of the cover glass, and then this cover glass is drawn over another at an angle of $45^{\circ}$, so that the drop of blood is spread in a thin layer over the lower glass. The specimens are dried in the air and fixed by dry heat $\left(107^{\circ}\right.$ to $\left.110^{\circ}\right)$ for $1 / 2-2$ minutes, or in alcohol and ether ( $\left.\overline{\mathbf{a} a}\right)$ for two hours, or in methyl-alcohol for 2-3 minutes. They may be stained with a mixture of eosin and hematoxylin, or more suitably with Ehrlich's triacid mixture, especially when finer details of the white blood corpuscles are desired. This is prepared as follows: Concentrated aqueous solutions of Orange G., acid fuchsin and methyl green are prepared in separate containers, and cleared by allowing them to stand for some time, then 13-14 ec. of the orange solution, 6-7 ce. of the acid fuchsin solution, 15 ce. of distilled water, 15 ec. absolute alcohol, and $12.5 \mathrm{ce}$. of the methyl green solution are mixed in the order given; after the addition of the methyl green solution the mixture is thoroughly shaken, and 10 ce. of alcohol and 10 ec. of glycerin are added during further shaking. (The mixture may be obtained ready for use.) The cover glasses are laid with the smeared surfaces upon the mixture, which should not be filtered. After about five minutes they are washed in running water and dried. In staining with the triacid mixture the cell nuclei appear pale green, the red blood corpuscles either orange, yellow or red, the acidophilic granules of the lencocytes copper red, the neutrophilic granules of the leucocytes bluish red, while the basophilic granules remain unstained. Instructive pictures are further obtained by Romanovsky's method, with its modifications by Giemsa or May \& Grünwald (the necessary stains for these methods may be obtained ready mixed).

In the further course of the disease edematous swellings develop on the lower abdomen and on the extremities. Not infrequently hemorrhages from the nasal and buccal mucous membrane may be observed, hematuria or intestinal bleedings may also occur. The urine sometimes contains albumen and, as in man, probably also urobilin and more iron.

The temperature shows in some cases variable elevation.

The sensorium appears disturbed from the onset of the disease. The nutrition always suffers, in some cases however the emaciation is not especially pronounced in proportion to the severity of the affection.

Course. The disease either develops acutely so that the animals die in 6 to 8 weeks after the appearance of the first symptoms, or it runs a chronic course and extends over several months. In a more protracted course remissions and exacerbations are observed. The animals die either from exhaustion or a sudden hemorrhage terminates the disease within a short time.

Diagnosis. The recognition of the disease is always associated with considerable difficulty. Of importance in this 
regard is the very marked diminution of red blood corpuseles, with which the falling of the hemoglobin content is not proportionate; the striking poikilocytosis and the presence of large erythroblasts are further indicative of the disease. The intercurrent febrile manifestations which may be present and the relatively good appearance of the animals also deserve full consideration.--Leukemia and pseudo-leukemia may be excluded by the absence of swelling of the lymph glands.-In infectious anemia of horses the blood examination reveals indications of a simple anemia, moreover the appearance of the disease in connection with the introduction of newly purchased horses, as well as its infectious nature, points to that disease. It appears to be advisable to eliminate the suspicion of infectious anemia only when the non-infections nature of the disease has been established on the ground of long continued observations, history, animal experiments, and by accessory conditions.Although sub-acute encephilitis, and cerebral meningitis are associated with similar depressions, in these conditions the blood shows no important changes. Special stress should be laid upon excluding septicemic affections of slow development, which can usually be accomplished when the blood and the other organs are carefully examined and all accessory conditions are considered. The form of influenza of horses which was named "Skalma" by Dieckerhoff often produces a longlasting severe anemia.

Prognosis. The prognosis is always unfavorable. In human medicine there is not a single unobjectionable case known which did not terminate fatally. The recoveries observed in animals must be judged carefully, since confusion with other diseases cannot be altogether excluded.

Treatment. According to observations made hitherto, even a rational treatment can at the best only prolong the course of the disease. A suitable regulation of the diet, keeping the animals out of doors or in well-ventilated stables and moderate exercise, should first of all be considered. Of medicinal remedies iron and arsenical preparations (arsenic, Fowler's solution, atoxyl, see p. 850), natrium kakodylicum (for $\operatorname{dogs}$ 0.1-0.3 gm. subcutaneously), may be employed. In human medicine intravenous infusion of blood and of salt solution, as well as subcutaneous injections of blood give relatively satisfactory results (Benczúr). Of these, especially the last-named method could occasionally be utilized also in animals.

Literature. Ehrlich, Lazarus \& Pinkus, Die Anämie. Wien, 1898.--Grawit7, D. m. W., 1904. 1092. - Johne, S. B., 1884. 60. - Tallquist, Z. f. klin. Med., LXI. 427. 
Progressive Pernicious Anemia in Cattle. Imminger (W. f. Tk. 1886, 137) described under this name a disease of eattle which first occurred around Kemnath in Bavaria sporadically, but in the years of 1883-1889 in an enzootic form. It affected cattle mostly at an age of from $3 / 4$ to 2 years. The disease occurred more frequently in stabled animals or in those fed on dry feed than in pastured cattle, or during the time of green feeding.

The disease commences with a gradual emaciation in spite of satisfactory appetite. From the fourth to tenth week a severe diarrhea, edematous swelling of the dewlap, paleness are observed, and at the same time yellowish discoloration of the visible mucous membranes, and of the unpigmented parts of the skin (especially on the ears), tightness of the skin and staggering gait. The pulse is thread-like, accelerated (70-80 per minute), and the systolic sound of the heart is frequently accompanied by a hissing murmur. The blood shows poikilocytosis. In the further course the respiration is accelerated and a cough appears. The temperature usually remains normal, very rarely it rises toward the fatal termination of the disease or it falls on the contrary to subnormal. After 2 to 3 weeks the appetite also becomes suppressed, and sometimes hemorrhages occur in the retina. With the appearance of the inappetence the loss of strength makes rapid progress, and the animals soon succumb. Sometimes, especially however in older animals or after parturition, the disease takes a rapid course. The mortality amounts to about $50 \%$.

The autopsy reveals a severe general anemia, here and there hemorrhages in the muscles, moderate hydropic signs, and a reddish discoloration of the otherwise softened bone marrow.

The treatment consisted in the administration of iron and arsenical preparations (see p. 850 ).

Anemia of Sheep and Goats in Punjab. Walker \& Baldrey (J. of trop. vet. science, 1906, 410) observed an enzootic affection in sheep and goats which is known to the natives as "Gillar" (swelling of the neck). The disease is confined to the swampy lowlands and the territories of the Punjab, which are frequently flooded, it attains its highest extension during the months of December to February and rapidly disappears during the summer months. Its causative agent is supposed to be some parasite, but the investigations earried on in this direction have given negative results.

The symptoms of the disease are languor, separation from the herd, tenacions, sticky nasal discharge and a hoarse cough. After about one week an edematous swelling of the submaxillary space appears, which may in severe cases extend to the breast bone and which usually disappears during the night. After three weeks a bloody diarrhea and increased thirst appear, the visible mucous membranes become pale and yellowish. In the meantime ascites develops. The animals die from loss of strength after 3 to 4 weeks; less frequently death ensues only after six weeks or even later. The mortality amounts to $90 \%$.

The autopsy reveals, in addition to the manifestations of a severe anemia and ascites, inflammatory areas on the intestinal mucous membrane.

Treatment is without effect; a change of pasture, with the administration of iron sulphate and salt is recommended. 


\section{Leukemia. Leukaemia.}

\section{(Leucocytemia; Leukämische Lymphadenie [German]; Leuco- cythémie, Lymphadenie [French].)}

\section{(a) Leukemia of Mammals.}

Leukemia of mammals is a severe and general systemic affection or proliferation of the lymphoid or myeoloid tissue, as a result of which swellings of the blood and lymph-forming organs develop and the number of white blood corpuscles increases more or less, partly at the expense of the red blood corpuscles. According to whether the increase affects the lymphocytes or the lencocytes, two forms of leukemia are distinguished, namely, the lymphatic leukemia and the myeologenous lenkemia (í. myeloides).

History. The first descriptions of the disease were given independently of each other in 1845, by Virchow in Berlin, and Bennett in Edinburgh. By Virchow it received the name by which it is known at the present "Leukemia" ( nection between the affection of the spleen or the lymph glands and the increase of white blood corpuseles was early recognized by Virchow, the part of the bone marrow however was first established by Neumann in 1899.

Leisering was the first to recognize in 1858 the disease in animals, but greater attention was paid to it only in the last two decades, so that at present quite a number of positive cases are known. Siedamgrotzky (1878), Johne (1879), Nocard (1880), Fröhner (1885), Wolff (1892), de Jong (1903), have earefully studied the disease, likewise Hutyra and Marek, whose case was not published in detail.

Occurrence. Leukemia occurs most frequently among dogs, more rarely horses, cattle, hogs and cats are affected, whereas nothing is known of leukemia in sheep and goats. Some of the cases recorded in literature very probably do not belong here, but to pseudo-lenkemia or to tuberculosis.

Sommer reported 46 cases up to 1889, of which 22 occurred in dogs, 10 in horses, 7 in eattle, 5 in hogs and 2 in cats. Among the horses of the Prussian army 2-4 cases occur annually.

Etiology. The leukemia of mammals is probably of a toxic-infectious origin, although nothing positive is known relative to this at the present time. The course of the disease would correspond with this view, as well as its localization in the various organs, but to date it has been impossible to produce the disease artificially in mammals by feeding affected organs (Nocard), or by subcutaneous or intravenous inoculation of emulsions from organs (Mosler, Bollinger, Cadiot, Roger, Gilbert, Marek). The bacteriological examination of 
the blood of an infected dog was also unsuccessful (Stockmann). On the other hand the infectious nature of chicken leukemia ( see p. 877) should be considered as proven, and in addition several observations are known in human medicine, which indicate the transmissibility of leukemia to healthy man (Ellermann \& Bang).

Löwit clains to have found protozoa (Haemomoeba leukaemiae) in the white blood corpuscles of man affected with myelogenous leukemia, with which he succeeded in producing a marked leucocytosis in animals, but without an affection of the blood-forming organs. Protozoa (H. leukaemiae vivax) were demonstrable in the blood-forming organs of lymphatic leukemia. Other authors have not substantiated this finding and consider the borlies observed by Löwit as fragments of cells. Pawlowsky found bacilli in leukemic blool, with which he failed, however, to produce the disease. Likewise negative were the attempts at inoculation of Ferni, Delbet and Lucet.

Pathogenesis. Ehrlich's thorough hematological examinations have thrown some light on the development of the disease. According to these, the hyperplasia of the lympladenoid tissues develops in some cases under the influence of a still unknown cause, whereby the blood becomes flooded with the lymphocytes which are formed in much greater numbers (Leukaemia lymphatica), whereas the number of true lencocytes remains unchanged. The hyperplasia of the lymphadenoid tissue affects not alone the lymph glands, but also the spleen, the bone marrow, the intestinal wall, and usually also other organs (liver, lungs, kidneys, glands, etc.), which under normal conditions contain only microscopically small lymph follicles. Exceptionally however the hyperplasia of the lymphadenoid tissues occurs only in a few, or even only in a single organ (for instance in the bone marrow).

In other cases of leukemia the causative factor effects a proliferation of the myeloid tissue, when the leucocytes migrate from the blood stream in great numbers, at the same time however young unripe lencocytes as well as numerous ripe lencocytes migrate from the lencocyte-forming bone marrow, and flood the blood (Lenkaemia myelogenes [s. myeloides]). Besides the bone marrow, other blood forming organs also become affected, more particularly a hyperplasia of the spleen, lymph glands, liver, and the development of areas of tissue-like bone marrow results in these organs.

This elassification of the forms of leukemia by Ehrlich appears, considering the morphology of the white blood corpuscles, much more appropriate than the designation of leukemia in accordance with the blood-forming organs, which are found alone or most strikingly affected on macroscopical examination, as on one hand all blood-forming organs are in most cases more or less affected in both forms of leukemia, while on the other hand the lymphatic and the myelogenic leukemia probably represent even etiologically two different diseases. As the white blood corpuscles formed in the spleen do not appear to have any part in the lenkemic cases of 
Hutyra and Marek, Special Pathology and Therrupattics or Domestic Animals: Iolume 1.

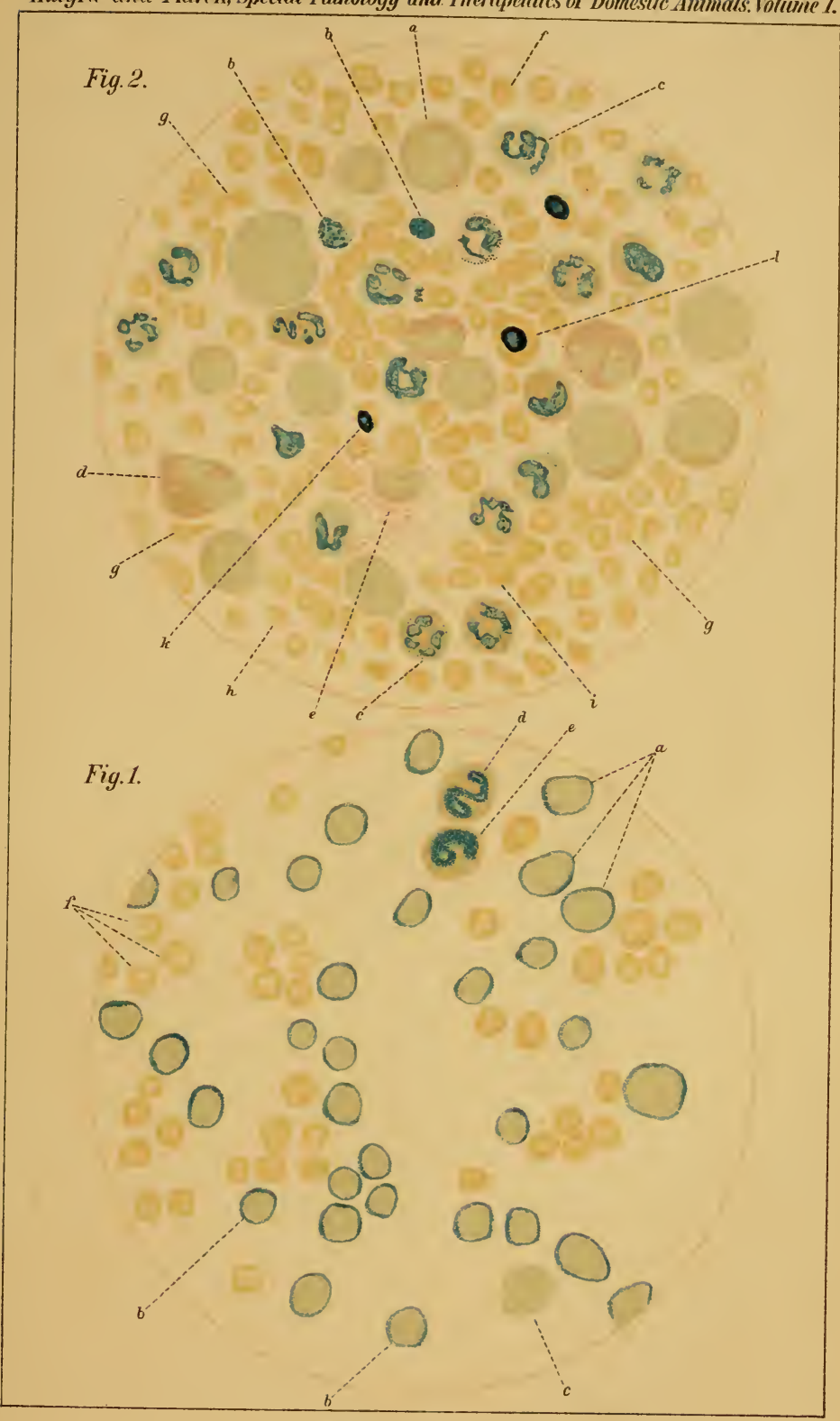




\section{Explanation of Plate X.}

Fig. 1. Lymphatic leukemia. (After a blood specimen from the dog illustrated in Fig. 155, stained with Ehrlich's tri-acid solution.) a, langeytes with umdifferlarkly stained cytoplasm; $b$, small lynclear leucocytes (neutrophile); $e$, transition entiated protoplasmic body; $d$, (Enlarged about 925 times.)

Fig. 2. Myelogenic leukemia. (Human bloocytes; $c$, polymorphonuclear (nentro tri-acid solution.) $a$, myeloeytes; $b$, lycocytes; $e$, disintegrated eosinophile cell; $f$, phile lelcocytes; $d$, eosinophile poikilocytes; $h$, microcytes: $i$, nacrocytes; $h$, phile) leucocytes; $d$, eosinoles; $g$, poikilocytes; $h$, minged 925 times. After Weichselbaum).
normal red blood corpusel 
which have been observed, a differentiation of "Leukaemia lienalis" does not appear justified.

In view of the fact that in the designation of the forms of leukemia by veterinary authors, the macroscopical changes of the blood-forming organs were almost exclusively taken into consideration, it cannot be decided how frequently the two forms of leukemia occur in animals. In the cases observed by Wolff, de Jong, Nocard, and Hutyra \& Marek, the examination of the blood reveals the lymphatic nature of leukemia. In all probability the lymphatic form of leukemia occurs in animals much more frequently than the myelogenic form.

Anatomical Changes. In both forms of leukemia the pathological changes visible to the naked eye greatly resemble each other.

The spleen appear's considerably enlarged, and exceptionally its surface is nodulated, its tissue is mostly dense, but at the same time somewhat friable, sometimes, on the contrary, very soft (in dogs and sometimes also in hogs). The cut surface appears usually pale brownish-red resembling the color of raspherry jelly, somewhat dry, and with a dull luster, it is not infrequently permeated by bluish-white, hard or soft, hemp-seed, pea, or up to walnut-sized areas (enlarged follicles), and also by dark red hemorrhages. Sometimes the connective tissue trabeculae form a conspienous network, and at the same time the capsule is thickened.

The spleen may at times reach enormous dimensions. Its weight may sometimes in horses reach 46 kilograms (Cunningham), in hogs 3.7 kilograms (Hellinger), in dogs 1.7 kilogram (Sierlamgrotsky), in cattle up to six times its normal size (Mauri). In one case of Zell the spleen hall a diameter of 89 centimeters. The cases, however, in which the spleen is so enormously enlarged were probably in most instances of pseudo-leukemic origin.

The lymph glands are very frequently enlarged. The glands miay attain a size from a lhazelnut to a walnut and coalesce with the neighboring glands, forming large nodular bodies. They are either denser or on the contrary softer than normal, their cut surface is uniformly white or grayish-white, in exceptional cases however they are permeated by red points, and a cream-like fluid may be scraped off.

The bone marrow appears either grayish-red, of jelly-like transparency, softened and entirely filling the marrow cavity, or pale gray and in this case almost of a pus-like consistency. The marrow tissue is changed to a lymphadenoid tissue, which is very rich in white blood corpuscles. Sometimes the bone tissue also becomes rarified and then the spaces appear filled with numerous lencocytes.

In abont half the cases the liver is found to be enlarged, somewhat harder, and on its grayish-brown or grayish-red cut surface pale gray points and a similarly colored network, or lymphona-like nodules appear sometimes between the lobules. 
The kidneys are also considerably enlarged on account of a cliffuse infiltration with white blood corpuscles and on account of the presence of larger or smaller lymphomas. In the intestinal wall, especially in the posterior part of the small intestines, various sized lymphomas are found, which protrude into the lumen of the intestines, and may contract the same considerably. In one of Olt's cases a chylusthrombosis developed in a $\log$. On the serous membranes and in the lungs nodules consisting. of lymphadenoid tissue may sometimes be found and the interlobular comnective tissue of the lungs may contain extensive areas of infiltration. In the heart, the peri- and endocardium of which may be permeated by white areas of infiltration or small lymph nodules, a great quantity of very soft blood coagulum may be present. The blood shows changes as described below. Similar cellular infiltrations may frequently be found also in other organs, thus on the cerebral meninges, in the uterine walls, in the various glands, as well as in the connective tissue of any other of the organs. Hemorrhages are not infrequently present in some of the organs.

Symptoms. Leukemia always develops insidiously. Rapid tiring is observed, especially during work, the animal perspires easily and shows in general the symptoms of pulmonary (mpliysema. Soon indications of languor and depression appear also during rest, the appetite becomes capricious and later entirely depressed, while thirst is not infrequently increased. The pale discoloration of the mucous membrane becomes more and more conspicuous, so that the mucous membranes finally take on a porcelain white color. The heart's action is acceleraterl and throbbing, the heart sounds being sometimes obliterated by secondary sounds, the pulse accelerated and small. Gradually emaciation becomes perceptible; edematous swellings appear on different parts of the body. The hair becomes lusterless and scanty.

In most cases a chronic swelling of the lymph glands appears symmetrically on both sides of the body. Enlargements which vary in size, dense, roundish, not painful, are found in the submaxillary space and in the vicinity of the pharynx, on the neck, along the jugular groove, on the chest, in the axillary region, and in both inguinal regions, as well as in all places where lymph glands occur. The skin covering them is normal and easily raised. Because of the enormous enlargement of the lymph glands locomotion is interfered with, either from purely mechanical causes or by compression of the neighboring nerve trunks. In infection of the internal lymph glands functional disturbances of some of the organs may result. Thus the compression of the bronchi or of the vagus nerve may cause difficulty in respiration, roaring, and even attacks of asphyxiation. At first this may appear only during work, later however also during rest. The compression of larger blood 
vessels, especially of the veins causes edematous swellings and indications of heart weakness. The affection of the mesenteric and other lymph glands of the abdominal cavity may result in ascites or a chylus-thrombosis, when the enlarged lymph glands may in large animals be palpated through the rectum, in small animals through the abdominal wall. Exceptionally the thyroid or the thymus glands may also appear enlarged.

The enlargement of the spleen may be ascertained in horses by rectal exploration, in small animals by palpation of the abdomen. The considerably enlarged spleen of the ruminants produces a dull sound running parallel with the posterior border of the lungs, between the lungs and the rumen. Exceptionally the left side of the abdomen may be distended downward and outward by the enormously enlarged and heavy spleen.

The sensitiveness of the bones to pressure, which might be considered as an indication of a simultaneous affection of

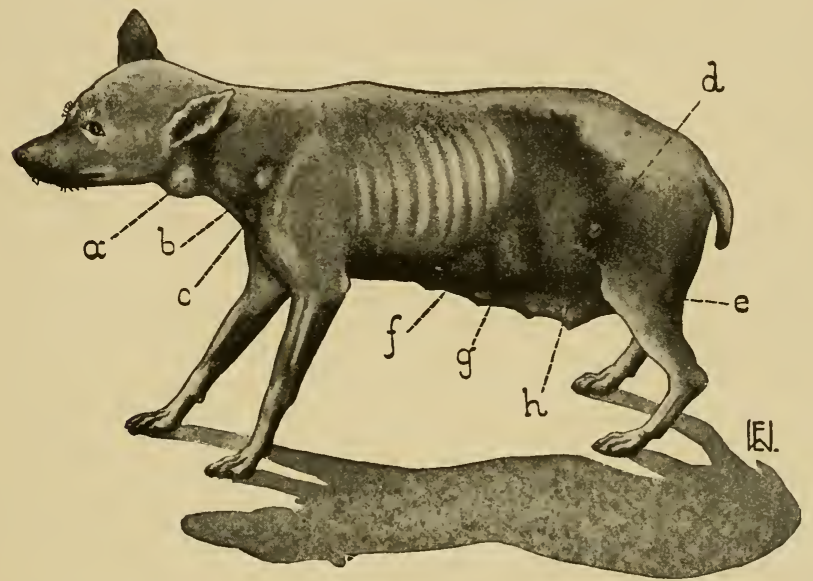

Fig. 155. Lymphatic leukemia in an old bitch. $a$ enlarged parotid gland; $b, c, d, e$ enlarged prescapular, inferior cervieal, precrural, and poplietal lymph glands; $f$ distension of the abdominal wall by ithe enlarged liver; $g$ distension of the abdominal wall by the enlarged spleen; $h$ enlarged mammary gland.

the bone marrow, was only reported by Wolff, who observed in a calf sensitiveness in the region of the lower ribs and attributed the same to lenkemic hyperplasia of the bone marrow. The disturbances in locomotion, which sometimes appear in the course of leukemia, may also depend to a certain degree on the painfulness of the bones.

The enlargement of the liver may be recognized in small animals from the increased area of dull sounds over the liver region in the anterior half of both costal arches and still more certainly by palpation of the epigastrium. In ruminants the 
dullness over the liver increases backwards and downwards to a level with the point of the shoulder; in case of very great enlargement, the posterior upper border of the liver may be felt in the anterior angle of the right flank. In horses the dullness over the liver appears first in the region of the last intercostal space on the right side. The recognition of a possible enlargement of the mammary or salivary glands causes no difficulty.

The blood appears pale red, sometimes chocolate brown, stains less intensively, coagulates slowly, causing on the upper surface of the coagulum a grayish-white laver or an adhering gravish-white deposit consisting of white blood corpuscles. The blood of leukemic horses separates on standing into three lavers, a lower violet-colored consisting of red blood cells, an upper formed by somewhat transparent rellowish fibrine, and a middle grayish-white consisting of white blood corpuscles, the lavers may appear of different heights in proportion to the number of white blood corpuseles. The blood of other leukemic animals separates on eoagulating into two layers, of which the lower, violet-colored, is formed by red blood corpuscles, the upper grayish-white, milk-like being formed by fibrin and white blood corpuscles.

An inerease of white blood corpuseles may always be shown. While normal blood contains at most 4 to 6 white blood corpuscles in a field, they are seen in lenkemic blood in much greater numbers, sometimes nearly as many or even as many as the number of red blood corpuseles. On counting, the number of white blood corpuseles is found considerably increased, as compared with the normal $(8,000-12,000)$ and the numerical relation between the white and red blood corpuseles (in normal conditions $1: 400-800$ ) becomes much less, as low as 1:5 (Berndt) and may be even less (in man the relation of 2:1 has even been observed [Sticker]). A diminution of red blood corpuseles may also always be demonstrated, and their number per cubic millimeter of blood may diminish from 7.5 millions to two millions (Nocard).

The establishment of the morphological condition of the white blood corpuseles in stained preparations is of especial value (see p. 865). In lymphatic leukemia lymphocytes are found to be increased (Wolff, de Jong, Marek), while the number of true lencocytes remains normal or comparatively so.

The lymphocytes appear uniform in size or slightly and sometimes even considerably larger than the red blood corpuscles; their large roundish meleus is stained less with basic dyes (methylene blue, methyl green) than the protoplasm surrounding the nuclens in the shape of a ring; in some large examples, however, both are sometimes stained uniformly pale (Plate X, Fig. 1-a, b).

In the myelogenic (myeloid) leukemia, on the other hand, the number of lymphocytes remains unchanged, and the lencocytes are considerably inereased; there also appear in the blood unripe, mononuclear forms, which possess granulated 
protoplasm, although there exists as yet no positive observations in regard to their occurrence in leukemia of mammals. The entire uniformity of white blood corpuscles of domestic mammals with those of man permits the supposition that the unripe forms of leucocytes occur also in the myelogenic leukemia of domestic animals. Accordingly besides the considerably increased number of normal cells in the blood there should be found neutrophiles, a few acidopliiles and probably also basophilic polynuclear leucocytes, also mononuclear neutrophilic leucocytes (marrow cells, myelocytes) in varying numbers and sizes, also monomuclear acidophilic lencocytes (Plate X, Fig. 2).

The red blood corpuscles in lymphatic leukemia show, as a rule, no changes in form, while in myelogenic leukemia poikilocytosis is frequent, and they are frequently nucleated (Plate X, Fig. 2). A diminution of the hemoglobin contents in the blood can always be demonstrated.

In some cases the temperature shows only a slight rise, or it may rise to $39-40$ C.; on the other lianrl, towards the termination of the disease, a subnormal temperature is not infrequently observed.

Fröhner found in the eye of an affected horse, near the inner border of the papilla, a lentil-sizerl, triangular, sharply circumseribed white spot (Retinitis leukaemica?)

Towards the termination of the disease hemorrhages occur not infrequently, especially on the mucous membranes (gums, conjunctivae, intestines, ete.), further on the skin and in the muscles, sometimes even in the brain. At the same time parenchymatous hemorrhages are very stubborn.

Course. Leukemia is usually a chronic affection and extends over several months and even years. An acute leukemia develops only exceptionally, principally in young animals which may succumb to the disease in a relatively short time, sometimes in a few weeks (Nocard observed in one case a termination of the disease in less than one week). At the same time chronic cases sometimes are arrested for a varving space of time, or an apparent improvement may even occur. By improper keeping or by using the animals for work the course is always influencer unfavorably. Frequently however the animals are destroyed before the occurrence of natural death; only rarely do they die from complete exhaustion or from internal hemorrhage, and still more rarely the fatal termination results unexpectedly or without apparent cause (Siedamgrotzky).

Diagnosis. The affection of the blood-forming organs, and the corisiderable increase of the white blood corpuscles, offer together sufficient indications for the correct recognition of lenkemia. Without a knowlerge of the morphological charac- 
teristics of the white blood corpuscles, the srmptoms described do not by themselves justify the diagnosis of a leukemic affection, as the enlargement of the blood-forming organs without a simultaneous increase of the white blood corpuscles may occur in pseudo-leukemia, on the other hand, however, an increase of polynuclear leucocytes without an affection of the blood-forming organs occurs also in leucocytosis. It cannot however be denied that the leucocytosis which develops in the course of infectious diseases, in severe simple anemia, in various other internal affections, and during pregnancy, is usually associated with a slight increase of the white blood corpuscles; but in some of the leucocytoses, as Marek's observations proved, the relation between white and red blood corpuscles may become as close (Marek found in one case the relation $1: 18$ ) as in the severe forms of leukemia. On the other hand it is to be considered that in the early stages of leukemia the increase of the white blood corpuscles need not necessarily be marked. For the determination of the form of leukemia, staining of the blood corpuscles is necessary.

Chronic swelling of the lymph glands may also be caused by glanders, tuberculosis, or by malignant new-formations. The symmetrical arrangements of the swellings on both sides of the body and their presence in all places where lymph glands are located, further also the involvement of the other bloodforming organs, usually leaves no doubt as to the nature of the disease. For the positive exclusion of tuberculosis, however, the examination of the blood corpuscles is indispensable, as tuberculosis may in some cases produce similar changes in the blood-forming and parenchymatous organs, for instance, lymphatic pneumonia (Nocard), or a pronounced symmetric enlargement of all the lymph glands may exceptionally result from tuberculous infection (observed by Anger in a dog, by Marek in a cow).

Prognosis. Up to the present time neither in man nor in animals has a single recovery been recorded in a correctly diagnosed case of leukemia. Accordingly the prognosis is always unfavorable.

Treatment. Treatment appears only indicated when for special reasons it is desired to prolong the life of the animals. In such cases the nutrition should consist of foods rich in proteids, the appetite should be stimulated by tonies, exertion should be avoided, and fresh air should be plentifully supplied to the animals. Of medicinal remedies, iron, quinine (may be given combined and always in large, full doses), further arsenic (Fowler's solution, in doses increased every 1 to 2 weeks, is most appropriate), and phosphorus; they must however be administered systematically and for a long time. In special eases transfusion of blood could be attempted, as it is supposed 
to have now and then produced a lasting improvement in leukemia of man. In human medicine relatively the best results have so far been obtained from arsenic and with the Röntgen rays or with both combined.

Literature. Berndt, A. f. Tk., 1889. XV. 300. - Bollinger, Schw. A., 1871. XXIV. 272. - Cadiot \& Weil, Rev. gén., 1905. V. 571. - Ehrlich, Lazarus \& Pinkus, Die Anämie, Wien 1898. - Güttlich, Pr. Mt., 1850/81. 19. - Johne, S. B., 1879. 24. - ile Jong, V. A., 1903. CLXXIII. 511. - Leisering, S. B., 1858. 35; 1861. 45; 1865. 29. - Lellmann, B. t. W., 1904. 699. - Löwit, Kongr. f. innere Med. in Karlsbad. 1899. - Lucet, J. rét., 1891. 570. - Nocard, De la Leucoeythémie chez les animaux dom., 1881 (Lit.); A. d'Alf., 1882. 361. - Olt, D. t. W., 1899. 197. - Pr. Mil. Vb., 1899-1908. - Siedamgrotzky, S. B., 1876. 21; Vortr. f. Tzte., 1878. H. 10 (Lit.). - Stockman, J. of Comp. Path., 1S93. 65. - Wolff, B. t. W., 1892. 121. - Zell, T. R., 1888/89. 49.

\section{(b) Leukemia of Chickens.}

Leukemia of chickens is an affection of the blood-forming organs, caused by an infection, and is associated with a considerable increase and infiltration of large mononnclear lencocytes, atrophy of the tissues of the bone marrow, and an increase of the myeloid tissue in the liver. The circulating blood frequently shows a diminished number of red blood corpuseles, and also a variable increase of white blood corpuscles.

History. Although Moore had already in 1896 described "an infectious lenkemia of chickens," these cases represented undoubtedly. a bacterial, acute infectious disease with a simple hyperleucocytosis, which is frequently met in various infectious diseases of chickens. The cases described by Butterfield (1905), and Yutaka Kon (1907), are more likely to belong to leukemia, although the blood of the affected birds was not examined. Ellermann \& Bang (1908) were the first to recognize and study the disease as such. Valuable studies relative to this disease in its clinical aspect were furnished by Hirschfeld \& Jacob (1907), also by Skiba (1909).

Occurrence. The disease occurs exclusively among chickens, and among these in an enzootic and even an epizootic form. The disease was discovered by Ellermann \& Bang in Denmark, and it has also been observed in Germany (Hirschfeld \& Jacoby, Eber).

Etiology. The disease is produced by some kind of an infectious substance, the nature of which is still unknown. Only this much is known from the investigations of Ellermann \& Bang that the cell-free filtrate from organ-emulsions may transmit the disease, and therefore the etiological role of an ultra-visible microorganism does not appear excluded. Hirschfeld \& Jacoby however up to the present always obtained negative results with filtrates through Berkefeld filters of organ emulsions of leukemic chickens.

The virus is present in all affected organs, the spleen, liver, bone marrow, etc. The infectious properties of the organs, however, are lost in a few days. 
The bodies resembling protozoa seen by Yutaka Kon were also found by Ellermann \& Bang, and these also observed in the bone marrow round bodies resembling paresites; the authors, however, refrained from expressing an opinion as to the nature of these bodies. Hirschfeld \& Jacoby, on the other hand, found peculiar long bacilli, which produced a temporary anemia with lymphocytosis in an experiment chicken; still they do not yet desire to consider this bacillus as the cause of the disease.

Pathogenicity. The disease may be transmitted to chickens by intravenous or intraperitoneal inoculations of affected organs, whereas other species of birds, likewise guinea pigs and rabbits, are not susceptible; subcutaneous injection, on the other hand, is negative even in chickens. After an incubation of 1 to 2 months about $40 \%$ of the inoculated chickens become affected, and then about one-half of the birds show indications of leukemia, the other half those of pseudo-leukemia.

Pathogenesis. The irritation of the virus produces a great increase in the production of white blood cells in the capillaries of the bone marrow, liver and spleen, whereby in these places an accumulation of white blood cells results, especially of large mononuclear cells, and also the appearance of myelocrtes. The active production at these places of white blood cells is indicated by extensive mitosis. The tissue of the bone marrow atrophies with the local increase of the white blood cells, while the myeloid tissues of the liver on the contrary increase. Temporarily the predominating intravascular process remains confined to the blood-forming organs without flooding the blood with white blood cells (according to Ellermann leukosis aleukaemica, pseudo-leukemia). In about one-lialf of the cases, however, the circulating blood becomes flooded with the newly formed white blood cells (according to Ellermann \& Bang, Leukosis leukaemica), probably for the reason that there are no agglutinines present at the places of formation. On the other hand, in some cases a local increase of cells occurs very strikingly in the tissue of the peritoneum (according to Hansen, so-called multiple Sarkomatosis of the peritoneum). Hand in hand with the leukemic process an anemia also develops, either as the result of a check in the formation of red blood cells, or then, owing to an increased destruction of these cells under the action of hemolytic toxins.

In opposition to the abore-described conception of Ellermann \& Bang, and Hirschfeld \& Jacoby, Skiba maintains on the ground of his observations in chickens which died of other infections diseases, that on one hand chicken leukemia differs from the similarly named affection of mammals, and that on the other hand it probably represents a disease which is associated only with a marked leucoeytosis. Chickens are supposed to have in general a great tendency to responi? with a marked leucocytosis to infections, or to other harmful influences (insuffieient exercise, improper keeping). The principal difference between lenkemia of chickens and that of mammals is supposed to consist in the main, in that in chicken leukemia the tissue proper of the blood forming organs remains unchangerl. Schridde maintains further that a disease simulating chicken leukemia may also be produced by intravenous injections of organ emulsions of healthy chickens, which, however, was not substantiated by the experiments of Hirschfeld \& Jacoby. 
Therefore the views in regard to the nature of chicken leukemia still differ, and a decided position in this connection is all the more difficult, as the morphologic and biological relations of the white blood cells and their part in the different diseases of birds have not yet been investigated sufficiently. The qualitative changes of the white blood cells demonstrated by Ellermann \& Bang, Hirschfeld \& Jacoby, namely, the appearance of abnormal cells in great numbers, establish very nearly the leukemic nature of the disease. The study of chicken leukemia also opens a prospect for the determination of the nature of leukemia of mammals.

Anatomical Changes. Most striking is usually the enlargement of the spleen and liver, also the grayish-red discoloration of the bone marrow. However, it occurs not infrequently that the enlargement is insignificant, and the color of the bone marrow does not always deviate noticeably from the normal. Now and then a rupture of the greatly increased spleen or liver, and fatal bleeding in the abdominal cavity is observed. Otherwise fine white points or stripes may be noticed in the liver tissue, even with the naked eye. Histological examination rereals a considerable increase of the leucocytic, and a diminution of the ervthroblastic tissues in the bone marrow, further very marked perivascular leucocytic proliferation in the liver, the capillaries of which appear plugged with leucocytes, while in the spleen numerous pale leucocytes are present and strings may be noticed, formed by capillaries filled with leucocytes which contain small nuclei. In all of these organs numerous cells are undergoing mitosis. Only Eber reports an enlargement of the lymph glands on the neck and in the mesentery, but the diagnosis in that case was not based on blood examination with consideration of the morphological relations of the white blood corpuscles; the autopsy findings are completed by the finding of anemia, while the blood proper manifests the changes to be described below.

Symptoms. After an incubation of 1 to 2 months indications of anemia become noticeable, inasmuch as the comb appears pale, its turbidity is diminished and the blood obtained by pricking shows a pale red color. In stained blood specimens several small or large, round, nucleated and mostly polychromatophilic red blood cells are noted (normoblasts and megaloblasts). Simultaneously, a relatively large increase of lymphocytes may also be observed. At the same time the number of the red blood corpuscles, which under normal conditions, according to Ellermann \& Bang, amount to $3,000,000$, according to Skiba however, $6,000,000$ per cubic millimeter, gradually diminishes to $1,100,000$. Likewise a diminution of the hemoglobin content is noted, inasmuch as the normal value of $50-65^{\circ}$ in roung chicks, frequently only $40-50^{\circ}$ (according to Sahli), drops 
to 15 . During the entire course of the disease there exists a hemorrhagic diathesis, as a result of which numerous and frequently severe hemorrhages result in some of the patients, and the wounds inflicted on the comb for drawing blood give rise to hemorrhages which are hard to control (Hirschfeld \& Jacoby). In this stage the clinical appearance of the disease corresponds to that of pseudo-leukemia.

As already mentioned the pronounced leukemic picture of the blood appears only in about half of the patients. This frequently develops suddenly, and sometimes the birds die quickly, the lenkemic changes of the blood being then recognized only at autopsy. The increase of white blood corpuscles is usually so pronounced that instead of the normal number of about $30,000,100,000$ to 600,000 white blood cells may be counted in the different cases, and the proportion between the white and red blood corpuscles (1:100; according to Skiba $1: 250)$ may fall to $1: 2$ or $1: 3$. At the same time the percentage of the different cells has changed to such an extent that the large mononuclear cells, instead of the normal percentage of 23 , are represented in a percentage of $60-94$, while the polynuclear leucocytes and the smaller lymphocytes are forced into the background, and at the same time the granulated polynuclear leucocytes contain spherical granules instead of the usual rodshaped eosinophilic granules (Hirschfeld \& Jacoby). In contrast to the normal blood round nuclear cells, also with granules, are seen in quite large numbers which may be considered as myelocytes, and sometimes their granula may attain the size of a cell nucleus.

Course. The disease usually lasts only 8 to 14 days, sometimes however 1 to 3 months, and unexpected deaths are apparently not exceptional. Spontaneous recoveries are very rare (Ellermann \& Bang observed such recoveries in two, Hirschfeld $\&$ Jacoby in one case).

Diagnosis. This can be established only on the ground of microscopical examinations, if a leukemic condition of the blood already exists, while otherwise the disease can at best only be indicated by determining the relative increase of lymphocytes by the infectious character of the disease, and still more positively by the autopsy findings. The greatest difficulty may be the exclusion of simple leucocytosis, which occurs very frequently in chickens and which may at times be very severe. In this the increase of the white blood corpuscles may reach such a degree that the relation of the white blood corpuscles to the red may fall even to 1:26 (Skiba). Leucocytosis occurs especially in chronic infectious diseases of chickens, most noticeably in tuberculosis, and may, as shown by Skiba, produce changes even in the blood-forming organs which resemble those of leukemia. A careful examination of the morphological 
conditions of the white blood corpuscles in stained specimens frequently affords sufficient grounds for a differential diagnosis. A relatively uniform increase of all representatives of white blood corpuscles and the absence of unripe forms of cells are met with in simple leucocytosis. The mononuclear lencocytes however appear to gain the upper hand even in certain cases of lencocytosis, which was indicated by the observations of Skiba.

Treatment. Very few experiments have been attempted in this respect. Ellermann \& Bang observed recovery in one case after treatment with arsenic; it cannot however be decided whether this did not represent an accidental spontaneous recovery. The Röntgen treatment proved unsatisfactory in another case.

Literature. Eber, Leipz. B., 1907/08. 54. - Ellermann \& Bang, Cbl. f. Bakt., 1908. XLVI. (Orig.), 595 (Lit.); Z. f. Hyg., 1909. LXIII. 231. — Hirsehfeld \& Jacoby, Z. f. kl. Med., 1909. LXIX. 1 (Lit.). - Skiba, D. t. W., 1909. 405 (Lit.).

\section{Pseudo-Leukemia. Pseudo-Leukaemia.}

(Hodgkin's Disease, Aleukemic Lymphadenia, Sublymphatic Leukemia [Helly]; Lymphadenia [Virchow]; Psendoleukaemia lymphatica [Pinkus].)

By pseudo-lenkemia is understood a chronic affection in which the blood-forming organs show the same changes as in leukemia. The increase of white blood corpuscles in the bloor is however absent.

Occurrence. Up to the present eases have been observed in horses, dogs, cattle and in a cat (de Does). The disease occurs more frequently than leukemia for which it is frequently mistaken. Leukemia of chickens is in about half of the cases also manifested by the symptoms of pseudo-leukemia (see p. 877).

Etiology. The absence of an increase of white blood corpuscles constitutes the only important difference between leukemia and pseudo-lenkemia; otherwise the anatomical changes, as well as the clinical symptoms are almost identical. As a matter of fact the lymphocytes may be present in a higher percentage than normally, although the total number of the white blood corpuscles remains comparatively normal. This phenomenon occurs in pseudo-lenkemia of man, was also observed by A. Zimmermann in a cow, by Jaeger in a dog, and by Hirschfeld \& Jacoby in the psendo-leukemic stage of chicken leukemia.

Based on this correspondence the view is greatly supported, according to which psendo-leukemia is held to be identical in 
its nature with leukemia and depends on a general affection of the lymphatic hematopoetic tissue (Rouvier, Jaccoud, Cohnheim, Nocard, Hirschfeld \& Jacoby). According to this view pseudo-leukemia would therefore represent only a form of leukemia, in which however the flooding of the cirenlating blood with white blood corpuscles is absent, as agghtinines are possibly present in the parts producing white blood corpuscles (Ellermann \& Bang), or there occurs no irruption of the proliferating lymphoid or myeloid tissue into the blood stream (Marchand, Neumann). In addition to this exceptional transitory stages of pseudo-lenkemia were observed in lenkemia of man, whereas in chicken lenkemia, as already mentioned, abont half of the birds manifest simple psendo-lenkemic changes in the presence of the same anatomical lesions in the blood-forming organs and in the liver. As a matter of fact Pinkus accepts pseudo-leukemia as identical with lymphatic lenkemia, or at least

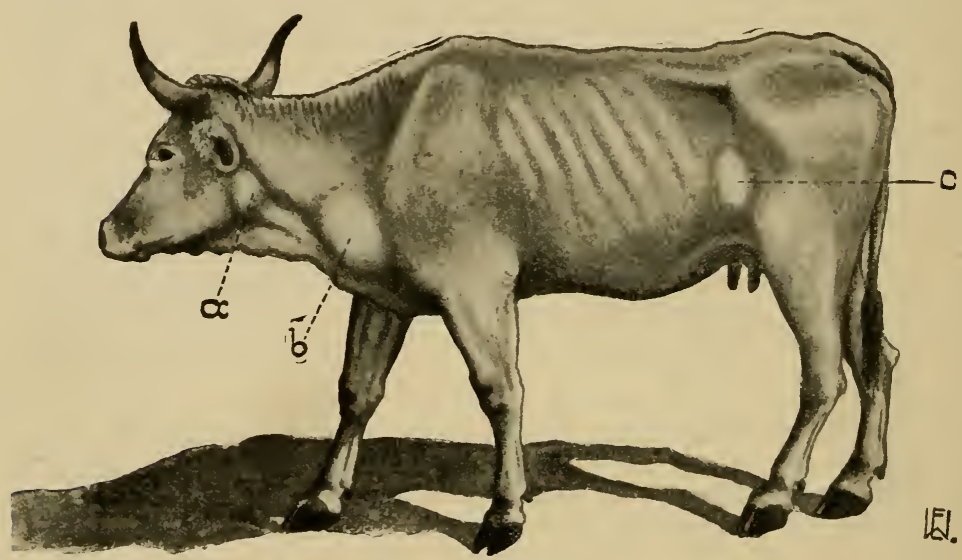

Fig. 156. Pseudo-leukemia: a enlarged retropharygeal gland; $b$ enlarged prescapular lymplı gland; $c$ enlarged precrural lymph gland.

as a rery closely related affection. However recently pseucloleukemias of myeloid types were also noted in man, and the pseudo-leukemic form of chicken lenkemia very likely also belongs to this group.

In opposition to this, other anthors, even at the present time, are supporting the view of the first recorder of psendoleukemia (Hodgkin), according to which both diseases would not be etiologically identical. In this they seek support in the fact that psendo-lenkemia hardly ever passes into lencocytemia.

At the present time various diseases are comprised under the name of psendo-lenkemia. Excluding the pseudo-leukemia proper which 
depends on a hyperplasia of the lymphoid and myeloid tissue, the infectious granulomata which in domestic animals are usually of tuberculous origin and which apparently oceur frequently, are also considered as pseudo-leukemia. Thus Nocard succeeded in demonstrating tuherele bacilli in all affected organs of the cases which corresponded to pseudoleukemia, and accordingly explained also the eases deseribed by him formerly as lymphademia to be tubereulous in nature. The same finding was also claimed by Brentano \& Tangel in a case of pseudoleukemia in man, the authors expressing the view that pseudo-leukemia is probably only a peculiar form of tuberculosis. These processes, however, of course, do not belong etiologically to pseudo-leukemia proper, although elinieally and in their gross anatomy they bear a marked resemblance to pseudo-leukemia, the same as the lymphosareomatous swellings of the glands (Kundrat's lymphosarcoma), which originate from malignant growths, but clinically greatly resemble pseudo-leukemia.

Symptoms. The symptoms and also the autopsy findings simulate greatly those of leukemia, while the course is, as a rule, somewhat shorter. The general hyperplasia of the lymph glands and the indications of a severe anemia strongly suggest pseudo-leukemia, but the nature of the disease can be cleared up only by the examination of the blood. Although the relation of the red to the white blood corpuscles is not found to be greatly changed, the red blood corpuscles may sometimes be distorted and smaller (poikilocytosis); at the same time their number is always diminished, while the lymphocytes are always present in a higher percentage than in normal blood. Sometimes flat, roundish, soft eruptions may develop on the skin, over which the hair falls ont (Liénaux).

Diagnosis. From the standpoint of differential diagnosis, leukemia, tuberculosis, glanders and malignant growths of the lymph glands come into consideration.

Prognosis. Up to the present time not a single case of recovery has been recorded.

Treatment. Comparatively the best results were obtained in man from a systematic administration of iron, iodine and arsenical preparations. On suitable occasions the treatment with Röntgen rays may be attempted.

Literature. Cadéac, J. vét., 1907. 266. - de Does, Holl. Z., 1890. 158.Dorst, Z. f. Vk., 1906. 437. - Ehrlich, Lazarus \& Pinkus, Die Anämie. Wien 1898. - MeFadyean, J. of Comp. Path., 1903. 379. - Fröhner, W. f. Tk., 1885. 245. Haffner, Z. f. Flhyg., 1906. XVI. 418. - Hodgson, J. of Comp. Path., 1903. 382. - Jaeger, B. t. W., 1907. 563. - Liénaux, Ann., 1901. 478. - Lothes, B. t. W., 1891. 9. - Lustig, Hann., Jhb., 1879/80. 54. - Nocard, De la Lencocythämie, 1891 (Lit.); Bull., 1885, 45. - Rottke, B. t. W., 1906. 712. - Siedangrotzky, S. B., 1876. 23. - Zimmermann, Vet., 1898. 473. 


\section{Hemoglobinemia. Haemoglobinaemia.}

Hemoglobinemia represents an affection of the blood in which the blood plasma contains dissolved blood-coloring matter.

The paralytic hemoglohinemia, which etiologically as well as from a symptomatological standpoint represents a special form of disease. will be treated in a later chapter.

Etiology. The simple hemoglobinemia of domestic animals is principally caused by blood parasites, especially by piroplasma (see p. 762), more rarely by trypanosomes (see p. 801). Besides these, althongh much more rarely, certain chemical substances or toxins may be responsible for a dissolution of the red blood corpuscles. Of the known chemical substances, chlorate of potassium, phenacetin, creolin, naphthol, naphthalin, antifebrin, and various acids (by insect bites formic acid enters into the body [Albrecht]) may exceptionally produce hemoglobinemia if they are arministered in large quantities. (Siedamgrotzky observed the disease in a horse which was given a handful of naphthalin.) The disease sometimes develops also in the course of acute infectious diseases (influenza of horses, distemper of $\operatorname{dogs}$ ), under the influence of specific infectious substances, or more often as the result of secondary infections (with streptococci). Szilagyi observed numerous cases of hemoglobinemia in horses, with intestinal hemorrhage, after the ingestion of very moldy clover. Hemoglobinemia sometimes appears also in connection with a nonspecific septicemia, extensive burning of the skin (Fröhner, Plosz). Whether the action of cold or the cooling of the body is capable of producing hemoglobinemia in domestic animals remains at present undecided. Although Saur, also Leech observed the affection in dogs, and Utz in a hog, occurring after the action of cold, another origin of the disease cannot be positively excluded in these cases. If solution of the blood corpuscles resulted from the action of the cold itself, hemoglobinemia would appear much more frequently after colds. In the paroxysmal hemoglobinemia of man hemolytic substances were found in the blood serum of the patients, which, when cold are anchored to the blood corpuscles in the fashion of an amboceptor, and when warm are supposed to produce solution of the blood corpuscles with the aid of the complement (Moro \& Noda).

Szoyka observed hemoglobinemia in two dogs, and Marek in three horses, and in enzootic extension in sheep also without being able to prove a hematozoal origin of the affections, either by examination of the blood or through injections or transfusions of the blood into animals of the same species. 
Pathogenesis. The development of the disease commences either with the breaking down of red blood corpuscles, or with the elimination of the blood coloring matter from the blood cells, but not infrequently with both processes together; the eliminated hemoglobin is then dissolved in the blood plasma and deposited mostly in the spleen and in the bone marrow. A considerable part however is worked up by the liver into bile coloring matter. If the blood plasma becomes suddenly flooded witl blood coloring matter, or if the quantity absorber by the blood plasma exceeds the sixtieth part of tlie total hemoglobin, a part of the coloring matter, as found by the experiments of Ponfick, is eliminated by the kidneys (hemoglobinuria), producing at the same time a degeneration, or even an inflammation of the kidneys. In severe hemoglobinemia, but according to Affanasiew only in hemoglobinemias with destruction of blood corpuscles, the liver forms bile pigments in large quantities from the blood coloring matter, and as the secretion of the fluid constituents of the bile does not keep up with it, the thickened bile accumulates, is partly absorbed, and thus the possibility is afforded for the development of icterus. Sometimes otler organs also become affected under the influence of the blood dissolving substances.

Anatomical Changes. The blood serum appears more or less reddish in color; in severe cases the blood may be even darker at the beginning, in the further conrse however it is lighter in color. The spleen shows an acute swelling on account of the taking up of blood pigments, its size corresponds with the intensity and duration of the disintegration of blood corpuscles. The spongy bone marrow appears more or less reddened, sometimes of a dark red color. In severe cases a general icterus may be noted.

Symptoms. Without a blood examination mild cases of the affection may pass unrecognized. In severe cases depression, icterus and hemoglobinuria are observed. Fever also appears, as a rule, and is brought on either by the causative agent of the disease, or by the febrile action of products from the disintegrated red blood corpuscles. As the coagulation of the blood increases hemorrhages occur probably as the result of a simultaneous destruction of white blood corpuscles in the mucous membranes and also in the other organs. The disintegration of blood corpuscles, as well as the substances causing the same, may further produce difficulty in respiration, disturbance of consciousness, also convulsions, weakness and accelerated pulse, and occasionally nephritis (renal epithelial cells, casts in the urine). The blood shows the same changes as in acute anemia (see p. 847), provided the blood serum contains hemoglobin.

Mild cases may terminate in recovery, whereas the severe 
attacks prodnce death in a few days or later. The animals which remain alive not infrequently show for a time the symptoms of simple anemia.

Diagnosis. I positive recognition of the disease is only possible by the demonstration of hemoglobinuria, or by the presence of dissolved blood coloring matter in the blood serum drawn with great care; a severe icterus appearing without any apparent organic affection should be considered as a suspicious indication of the disease. An attempt should always be made to determine the cause of the disease.

The paralytic hemoglobinemia is differentiated from the other forms of hemoglobinemia by the entirely different mode of onset, by the presence of the paralytic symptoms, and by the changes in the tendinous reflexes.

Treatment. Besides removal of the cause if it can be determined, and relieving the threatening symptoms, the treatment mentioned in anemia appears indicated (see p. 850).

Literature. Albrecht, Monh., 1892. III. 241. - Dieterichs, D. m. W., 1903. 293. - Marek, Z. f. Tm., 1904. VIII. 285. - Moro \& Noda, Münch. m. W., 1909. 545. - Siedamgrotzky, S. B., 1892. 16. - Szilágyi, A. L., 1907. 633. - Szoyka, ilisi., 1903. 1. - Ttz, B. Mt., 1885. 4.

\section{Paralytic Hemoglobinemia. Haemoglobinaemia paralytica.}

\section{(Azoturia, Haemoglobinemia rheumatica [Fröhner]; Haemo- globimuria toxaemica [Bollinger]; Lumbago [Dieckerhoff].)}

Paralytic hemoglobinemia is a specific acute affection of horses, characterized by severe disturbances in locomotion (mostly lumbar paralysis) as a result of a parenchymatous degeneration of certain muscles, and by the presence of hemoglobin in the urine.

History. Considering the nature of the etiologieal factors paralytic hemoglobinemia of horses must have been known since earliest times, although it might not have appeared as frequently as at present. Even in the 20 's of the last century, Coulboux, Charlot, Prévot and Bouley $\mathrm{jr}$. deseribed a disease as spinal apoplexy or congestion of the spinal cord, which etiologically and elinieally corresponds with paralytic hemoglobinemia, and which did not appear to be very infrequent at that time. Since the middle of the last century the reports of the disease continue to increase, especially in Germany. In former times "azoturia," "lumbago," "blackwater,", "rheumatic lumbar paralysis," "spinal meningitis," "Haemoglobinuria paroxysmalis," ete., were considered as different diseases. In 1852 however Hofer introdueed them in literature as identical. Since then until the most recent times, a great number of publications have been prepared on the subject, which however have not yet completely cleared the etiology and the nature 
of the disease. Studies on the nature of the disease were especially carried out by Bollinger (1877), Fröhner (1884), McFadyean (1888), Lucet (1889), W. Eber (1892), Dieckerhoff (1895), Lignières (1898), Cadéac (1899), Hink (1901), and Schlegel (1906). Schlegel at first strictly separated the affection from enzootic spinal meningitis (Meningomyelitis haemorrhagica), later however he considered them as identical affections. Furthermore paralytic hemoglobinuria has formerly frequently been confused with enzootic spinal meningitis.

Johne (1879) described the disease in a zebra.

Etiology. The observations of many years up to the present time have proved beyond a doubt that the development of the disease is favored by the cooperation of certain predisposing causes. The most conspicuous influence is the method of keeping the horses, inasmuch as the disease usually appears after a long rest with rich feeding at the same time. Accordingly it occurs especially after holidays of several days' duration. However, exceptions are not at all rare, as occasionally resting horses may become affected even in the stables, or horses that are hitched up every day may become affected during work, and again poorly nourished or even starving animals may become attacked by the disease (Hauptmann).

Brun olserved in a large stable, in which the horses were not worked for sixteen days and where the daily exercising was impossible, that eight eases oceurred inside of three days, while among the horses of another stable in which the animals were exercised every day only one, and that a slight case, was noted.

The influence of breed is sufficiently proved by general ohservations, in that horses of draft breeds become affected most frequently, while the disease appears more rarely in animals of the finer breeds. Among common Hungarian horses, as well as in the more hardened horses of poor men, the disease seldom oceurs.

Accorling to the tabulated statisties of Adam, among 63 affected horses $31 \%$ were heavy draft animals, $36 \%$ improved breeds of country horses, $20 \%$ wagon horses, and $9 \%$ helonged to higher breeds. Immediately before the affection five horses were stables for one day, 21 horses for two days, 11 horses for 3 days, 9 horses for 4-5 lays, four horses for 5-8 days. Out of 352 cases observed by Schindelka \& Höfling, $46 \%$ belonged to heavy draft, $19 \%$ of merium weight animals, and $35 \%$ to light weight breeds.

Horses usually become affected between the ages of five and eight rears, much more rarely at earlier or later ages. (Razsovits and Hutyra \& Marek each observed the disease in a foal of $11 / 2$ years of age; according to Kleinpaul even sucking colts are supposed to be susceptible?)

Excessive exertions may produce the disease even without a previous rest; besides cases are not infrequent in which the disease develops after parturition or in horses which have struggled considerably during painful operations (Bergstrand, Fröhner, Leipziger, Dagès, Pr. Mil. Vb.). Likewise horses 
may become affected which cross their foot orer the pole or the halter rope and strain their muscles (Hasse).

The arrangement of the stable is also supposed to have an unfavorable influence to a certain degree, inasmuch as horses kept in poorly ventilated, warm, damp stables are affected more frequently.

The influence of the season is manifested in that the disease generally occurs more frequently during cold weather. It may be attributed to this condition that cold has a more or less important, or even an exclusive etiological significance in the development of the disease. However in this respect there are numerous exceptions. Thus of the 91 cases observed during 19 years at the clinic of Budapest, 42 occurred in the first and fourth, 49 in the second and third quarters of the year. According to the statistical data of the Prussian Army in the last 10 year's 160 horses became affected in the first and fourth quarter, and 371 in the second and third quarter; in the second and third quarter, that is, in that half of the year in which there is less opportumity for the action of cold, the number of the affections were year after year considerably higher.

Numerous hypotheses were formed for the explanation of the true cause and nature of the disease, the more important of which only will be mentioned. Dieckerhoff accepted as the cause an autointoxication, claiming that the toxic substances (meat lactic acid and other substances) develop from the proteids that are not utilized during rest, and which are broken up under the influence of a supposed ferment. In Egypt, recently Bollinger also considered the disease as an auto-intoxication. According to this author a certain hemolytic toxin is supposed to develop from the proteids under the influence of muscular work or cold. Eber attributes it to the formation of a toxigenic substance which is supposed to change to a poison during muscular funetions. Hink accuses oxidation and decomposition products, which form rapidly during exertion of the muscles previously kept out of function for a long time; he thinks that under the cooperation of vasometer disturbances there results under a simultaneous attack of cold an accumulation of lactic acid and its compounds in the muscles, leading to a congealing of the muscle substance, paralysis of the muscles, and to a solution of the muscle coloring matter. According to Ohler the disease develops as a result of an excessive and rapid breaking down of the glycogen in the muscles, in such a way that the sugar formed from the glycogen produces a myositis and secondarily a nephritis. According to Siedamgrotzky \& Hofmeister the red blood corpuseles are supposed to be dissolved by the urea and by the extractive substances accompanying it which are formed in large quantities hy the muscle.

MacFadyean considers an excessive production of red blood corpuscles under the influence of rich feeding as the cause; the profuse, newly formed red blood corpuseles are then supposed to break down during subsequent work.

The greatest acclaim was given to the theory of cold. Thus Lucet attributed it to an acute nephritis brought on by cold and to subsequent uremic or other intoxications. A great number of French authors formerly accepted as the fundamental cause of the disease a hyperemia 
of the spinal cord, produced by cold and by rich feeding. According to the hypothesis of Fröhner the metabolism of the muscles is supposed to be increased to such an extent by the very intense irritation of the cold on the sensitive nerves of the skin, that it causes the contractile muscle substance to break down, and conseruently muscle coloring matter, which is identical with the hemoglobin passes into the blood circulation, and is eliminated by the kidneys.

Various authors assert an infectious origin of paralytic hemoglobinemia. Thus Cadiot believes in the possibility of an infection through the intestinal canal, while Cadéac considers a streptococcus infection probable. Lignières found Gram-positive streptococci in the subarachnoid fluid of the medulla, sometimes also in the kidneys, the inoculation of which into mice produced a red discoloration of the urine and increase of the reflex irritability; a horse which received 300 cc. of the culture intravenously could no longer get up from the sixth day on, and albumen was demonstrated in its urine. In contrast to this Nowak \& Hartel also found the streptococci, but could produce no disease with them; experiments carried out with the filtrates of the intestinal contents of affected animals were also negative.

Schlegel accepts as the cause of the disease the streptococcus melanogenes described by him, and identified the paralytic hemoglobinemia with the streptococcus of septicemia which is manifested by a paralytic weakness of the hind parts (enzootic spinal meningitis, see Tol. II).

Hutyra \& Marek believe it best to lold to the hypothesis of an auto-intoxication. According to their belief they claim to be justified in assuming that during the function of previously rested muscles or in muscles which have worked to excessive exertion, some substance is formed which exerts a toxic action and which produces a degeneration of the muscle fibers and an elimination of the blood-coloring matter from the blood corpuscles. This process occurs either while the blood passes through the affected muscles, or at a later stage, when the blood circulates in other organs. During rest and rich feeding the suspected toxin-forming faculty of the muscles may increase. It must probably be attributed to the last-named condition that heavy, phlegmatic horses, which, as a matter of fact relax their muscles much more completely during rest than horses of a livelier temperament, and young colts become affected more frequently; as a matter of fact colts are not used at all for severe muscular work. By the sudden action of cold the influence of the muscular functions could evidently be increased up to a certain extent, as the irritation of the cold stimulates the metabolism of the muscles (heat regulation). Of what nature however the suspected poisonous substance may be which develops during the muscular functions could only be determined by suitable investigations and experiments; simple theoretical deliberations do not lead to results.

Dispensing with detailed criticism of the above described hypotheses, only the more important factors in favor of the myogenic autointoxication will be brought out. The disease has, up to the present 
time, been observed without a doubt only in horses, and usually after the animals had been kept from work for a time, at the same time were well fed and subsequently were used for some hard muscular work, which, of course, may oceur also in the stable, as for instance after suddenly rising to the feet. Although sometimes the disease (levelops also without cooperation of these factors; in such eases an excessive exertion may be found to he originally connected with the development of the disease. At the same time it has frequently been observed that in horse stables in which the disease has occurred regularly it may he prevented by systematic exercising of the animals or by a considerable reduction in the quantity of nitrogenous food (Benjamin, Lavalard, Brun). The fact has further been supported by numerous observations that without exception the muscles usually become affected and the changes occur chiefly on that side of the hody, which has to perform the greatest muscular exertion in the execution of the work. These are especially the muscles of the hind parts, more rarely those of the anterior extremities. In animals which are lying down the muscles of the back may be involved.

Against the importance of the influence of cold the already mentioned observations may be quoted, according to which the disers occurs in continnous warm weather more frequently than in cold seasms (Pr. Mil. Vb., Höfling, Marek). Lassar \& Nassaroff succeeded by an excessive cooling of the body (the inner temperature of the animals falling to $18^{\circ} \mathrm{C}$.) only in producing a not especially severe degeneration in the muscles, and more markedly in the parenchymatous organs, which, exclusive of actual freezing to death, could not occur under natural conditions. On the other hand Dexler failed to produce the disease by strongly cooling the hind parts of a horse.

The signs of a nephritis which according to Lucet develops from cold and which is said then to produce the hemoglobinemia, are frequently absent.

Against the infectious origin of the disease it may be mentioned that the respective authors have failed up to the present time in producing the disease in its typical form artificially in horses, and that further the sudden appearance of the disease and its characteristic clinical picture is difficult to reconcile with a theory of infection, as is also the not infrequent sequel of an atrophy confined to certain muscles, which after a time again disappears. In spite of the position taken by Schlegel on this question, Hutyra \& Marek consider their non-committed standpoint in regard to the infectious origin of the disease the more justified since paralytic hemoglobinemia was originally separated by Schlegel himself from infectious spinal meningitis, and also because Zwick, who accurately deseribed a disease which appears to be identical with Schlegel's streptococcus septicemia, ohserved no similarity between infectious spinal meningitis and paralytic hemoglobinemia. It therefore remains for further investigations to elucidate the relationship of Schlegel's disease to paralytic hemoglobinemia and the nature of the last mentioned disease.

Against the myogenic origin of hemoglohinemia it may he said that in all cases investigated by Hutyra \& Marek the hemoglobin content of the blood was found to be somewhat diminished (on Fleischl's apparatus below $70^{\circ}$ against the normal of $70-80^{\circ}$ ). At the same time they never succeeded in finding an increase in the specific gravity of the blood, although it was tested very accurately with the pyknometer. On the other hand they established in all cases a diminution of red 
blood corpuscles (up to 6,450,000-7,200,000, against the normal number of $7,500,000$ to $8,000,000)$. MacFadyean and König demonstrated an increase of the red blood corpuscles. Schindelka and Höfling found frequently and König regularly, a very marked increase of the hemoglobin content of the blood, yet these findings cannot be considered as conclusive evidence without a simultaneous establishment of the molecular concentration of the blood serum. Mention should not be omitted of the fact that König found a comparatively strong increase of the hemoglobin content in the blood, but this finding may also have another explanation, as the increase of the hemoglobin content persisted for days after recovery, and for a long time after the disappearance of the hemoglobinuria. The experimental findings of Wetzl are interesting in this relation. According to these on one hand the hemoglohin content of the blood is subject to great variation, and on the other hand a considerable loss of water by the body produces a very significant increase of the relative percentage of blood corpuscles and the hemoglobin content, on account of the thickening of the blood. In paralytic hemoglobinemia there are ample opportunities for a decided loss of water (profuse perspiration, omission in watering the animals while lying down). The fact that in several cases examined by Schindelka and Höfling the dissolved blood coloring matter in the blood serum did not always correspond with the higher percentage of hemoglobin in the blood can only be explained in this manner. On the other hand the experiment of Camus \& Pagniez in dogs (injecting cold distilled water into the muscles of the thigh) by which they aimed to prove that the hemoglobin eliminated with the urine is muscle coloring matter, cannot be considered as conclusive as a solution of red blood cells around the point of injection appears far more probable under the influence of cold distilled water.

Anatomical Changes. The most striking changes are present in the muscles. Especially the lumbar muscles and those of the croup, as well as the psoas muscles, to a slighter degree the muscles of the hind quarters, sometimes those of the front extremities also, and other muscles appear either uniformly gray or yellowish in color, like fish-meat, or as if boiled and moistened on the cut surface. They tear easily or they may be firm, but they show on their cut surface, between the healthy brownishred bundles of muscle fibers, light gray or yellowish stripes, which convey to the basic color a more or less distinctly recognizable grayish tint. The degenerated parts sometimes stand out very sharply against the healthy tissue. The cross striation of the contractile substance can no longer be recognized under the microscope, the sarcolemma appears to be filled up by a mixture of fine albuminous granules, fat globules and coarse flakes (degeneratio parenchymatosa et adiposa, sometimes also Deg. cerea). Dexler demonstrated by Marchi's method the presence of fat globules arranged in longitudinal rows.

The kidneys are also affected with relative frequency, showing cloudy swelling or even severe parenchymatous or fatty degeneration, as well as diffuse necrosis of the epithelia (Johne). The spleen appears swollen in areas, more rarely the swelling is uniform. The muscle of the heart sometimes shows 
the same changes as the muscles of the rump, but always in a lesser degree, and probably as the result of a subsequent septic infection. (Dexler found in the lateral and posterior cornea of the lumbar, dorsal and sacral sections of the spinal cord, perinuclear chromatolysis of the motor, and in a lesser degree also of the other nerve cells; by Marchi's method he also demonstrated degeneration of certain nerve sheets of the lateral and anterior columns.) The bone marrow, especially in the fenur and in the lumbar vertebrae is hyperemic, permeated by small hemorrhages, sometimes showing a blackish-brown discoloration.

The blood shows no changes recognizable by the naked eye. The bladder contains mostly reddish or brownish-red urine.

Symptoms. The disease sets in without premonitory signs, almost invariably with disturbances in locomotion of the hind parts. After a certain time, sometimes as early as in 5 or 10 minutes, at other times only several hours after the animal has been taken from the stable and used for work, a pronounced stiffness is noted in the movement of the posterior extremities. There is staggering of the hind parts, the feet are raised only slightly from the ground, the horse knuckles at the pasterns, perspires profusely, and attempts to go on in spite of it until it finally is unable to stand up and collapses helplessly. The disturbance occasionally progresses so rapidly that the animal breaks down suddenly without the rider or driver even noticing the stiffness of the gait. It is only rarely that the horse is able to rise again to its feet, more often it is necessary to return it to the stable on a wagon. In the rare cases in which the disease develops in the stable, it is as a rule observed only when the animal can no longer rise from the ground in spite of its attempts. The collapsed horse then lies flat on one side, mostly with the hind extremities stretched somewhat backwards, at the same time it makes repeated attempts to rise; with the head raised it succeeds in standing on its front legs, but soon drops back again with painful groans. Sometimes it succeeds in half-way rising even on the hind legs and dragging itself for a short distance, but it soon falls exhausted and usually with the hind parts first (Fig. 157). In its repeated attempts to rise the horse strikes its head and feet against the ground or against the wall, thereby causing injuries to these parts. The numerous exertions, and the anxiety of the patient, which may be seen in the expression of its face and eyes, result in a profuse perspiration.

Sometimes the disturbances remain confined to only one of the hind extremities, affecting only the M. quadriceps fem. or the abductors, at other times they appear in one or both front extremities, especially in the triceps brachii. If the disturbances are only one-sided and not very extensive, the horse usually does not collapse, and remains perfectly quiet without perspiring. 
The muscles of the paralyzed parts of the body feel at first, and sometimes even later, very dense, almost as hard as a board; they are however not sensitive to pressure. At the same time the superficial muscles may show considerable enlargement. In the further course of the disease the muscles usualiy return to their normal consistency, or even become softer. The sensitiveness of the skin, as far as examination, because of the excitement of the animal, is possible is not changed; sometimes it appears somewhat reduced, but no great importance can be attributed to this finding, as the skin in the region of the croup or the thigh possesses a somewhat slighter sensitiveness, as compared with other parts of the body.

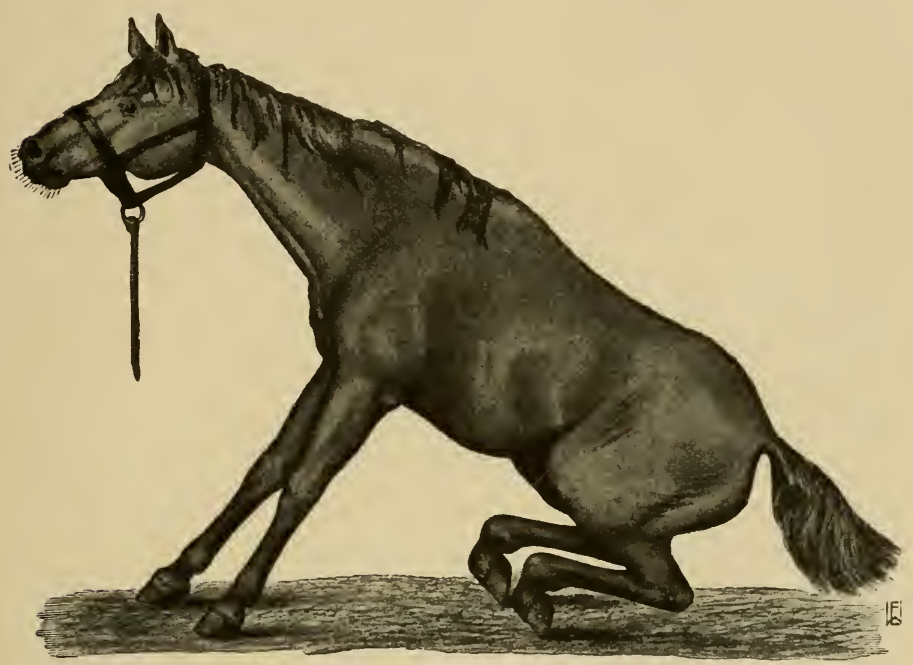

Fig. 157. I'aralytic hemoglobinemia. The horse rose on the second day of the affection, but immediately fell back to the position shown in the illustration.

Spontaneous indications of pain are however not infrequent. The passive resistance of the muscles is at first increased, later diminished. Dexler and Marek found an absence of tendinous reflexes in the region of the paralyzed muscles. As the M. quadriceps cruris is also involved, the patella reflex is, as a rule, absent either on one or both sides. Dexler found the faradic irritability normal in the affected muscles, the mechanic irritability on the other hand was absent in Marek's cases.

In mild cases as well as in the early stages of severe affections the temperature is not much elevated; but owing to the great restlessness of the animal a rise of $1 / 2$ to $1^{\circ} \mathrm{C}$. may exist from the beginning. In the later course of severe cases a considerably higher rise in temperature may be present, either 
resulting from an infection through decubital gangrene or from other complications, possibly also from the hemoglobinemia itself.

The heart's action, except in the mild cases, is from the beginning increased in proportion to the restlessness of the animal (50-60 beats per minute), at the same time however it is strong. After the development of complications it becomes more accelerated ( $80-100$ per minute), correspondingly the pulse which is at first tense and full, becomes gradually softer and finally tliread-like.

Frequently the passing of urine is retarded so that a considerable dilation of the bladder may develop. Excepting. in a few, mostly very mild and rapidly passing cases, the urine appears to be more or less intensely dirty reddish-brown or beer-brown to ink-black in color; and although it clears on filtration it retains its red color. The specific gravity is increased, but the reaction is mostly alkaline, more rarely acid; at the same time it contains more or less albumen, a large amount of phosphates, usually also uric acid salts, and less hippuric acid. 'The sediment with its pale gray or yellowish color stands out in contrast to the reddish-brown to blackish color of the urine, and offers a certain positive means of proof that the abnormal color of the urine originates from dissolver blood coloring matter. The sediment of the urine sometimes contains hyaline or granular casts, possibly also renal epithelial cells, sometimes white and red blood corpuscles in sparing numbers, which are present becanse of a simultaneous inflammation of the kidneys ; hemoglobin casts a re also frequently found. (Tatray observed in one case numerous hemoglobin crystals.)

For demonstration of the blood coloring matter in the urine the benzidine test of Schlesinger, or the guajac-oil of turpentine test of Schumm, is best adapted, while the spectral-analytic method or Heller's test is considerably less suitable, because of its sensitiveness (Mayer), when it is desired to demonstrate small quantities of blood coloring matter, which, however, is usually not the case in hemoglobinemia. The spectral-analytic method can, however, not be onitted for demonstrating the various combinations of the blood coloring matter, especially the oxy- and the methemoglobins. Two absorption bands are indicative of oxyhemoglobin, namely, one small dark band lying to the right of Frauenhofer's line D (on the border of the red and yellow color of the sun spectrum), and a lighter and somewhat broader band lying to the left from the E-line; the presence of methemoglobin is indicated by a band to the left of the $\mathrm{D}$-line (in the field of the red color). Frequently, however, all three bands may be seen simultaneously.

The quantity of albumen in the urine (according to Lucet $1-25: 1000$ ) is not always proportional with the intensity of the red color, but it is considerably greater in a simultaneous affection of the kidneys. The quantity of urea was found by Schindelka and Fröhner in some cases, and by MacFadyean always to be diminished.

Frequently the blood coagulates more rapidly, but the coagulum remains loose for a longer time, so that only a relatively small quantity of serum is separated. The blood serum appears reddish or of a pronounced red color at the onset, and 
in somewhat more severe cases, even later. The proportion of blood corpuscles and the hemoglobin content of the blood are subject to very great variation, as the diminished water content of the body is manifested in varying degrees from case to case. The observations of Marek, which were made after the cessation of the very frequent general perspiration and regular watering, showed a normal specific gravity of the blood (1.052-1.0544), a slight diminution of red blood corpuscles, and as a rule also a slight reduction of the hemoglobin content (under $70^{\circ}$ of Fleischl's or Gower's' scale). In contrast to this Schindelka, Höfling and König found an increase of one-lialf above the normal in the quantity of hemoglobin, especially König in those cases in which the percentage of the blood corpuscles appeared quite normal. Strangely enough the quantity of hemoglobin, although gradually diminishing, remained increased for days, even after the disappearance of the symptoms, and a considerable increase of red blood corpuscles is frequently also present, especially in severe cases (König found in one case $21,328,000$ blood corpuscles), probably owing to the reduction of water in the body (see p. 847).

The appetite remains good at the onset and in mild cases to the end of the disease. Consciousness appears at first undisturbed; the animals try to relieve themselves from the uncomfortable position, and make repeated efforts to rise until completely exhausted. Later however they lie exhausted on the ground. Sometimes svmptoms indicative of uremia may he observed, such as loss of consciousness and muscular convulsions.

Complications appear very frequently in severe cases. Among these gangrene of the skin from pressure should be mentioned in the first place, which appears on the prominent parts of the body after lying for two or three days and may develop under rapid extension into severe gangrene, resulting in a general infection. Similar complications may develop from deep tear's or bruises of the skin. In other cases the continuous lying on one side may cause a hypostatic hyperemia or later eren inflammation of the lungs. Now and then uremia develops, which manifests itself especially by deep coma and convulsions. When collapsing the animals may also fracture bones, especially in the pelvis or thigh.

Course. The disease is characterized by a very variable course. First of all there are cases where only a certain stiffness in locomotion is noted, which disappears within a few hours, or at the most after one-half to one day. In the great majority of cases however pronounced symptoms of paralysis appear, which persist for 2 or 3 days, sometimes even for a week, and then change either into a rapid or slower return to the normal condition, or on the contrary may lead to death under 
gradual aggravation of the general condition. Finally in rare cases there exists only a stiffness and weakness of the hind parts, as well as hemoglobinuria, for 3 to 5 days, and complete paralysis does not develop until later, as a result of which the animals collapse, and soon die (according to Utz there is a subacute form of the disease in contra-distinction to the ahove acute form).

In severe cases death may ensue on the third or fourth, exceptionally even on the first day, mostly however the animals do not die intil towards the end of the first week or at the beginning of the second. The immediate cause of the fatal termination is probably most frequently a general septic infection with its consequences, more rarely hypostatic pnemonia or uremia.

In the favorable cases the improvement makes either rapid progress, so that the gait of the animal becomes normal within a few days, or on the contrary a weakness of the hind parts persists for several weeks, although it gradually subsirles. Sometimes the weakness or the paralysis of one extremity may remain for months, even for 1 to 2 years, exceptionally even permanently, and meanwhile lead to a rapidly progressing atrophy of some of the muscles (the extensor quadriceps of the leg, the abrluctor of the thigh or the rump).

Repeated attacks also occur, either at shorter or at longer intervals, sometimes even after several weeks (Kronburger observerl a horse become affected three times within one week).

Diagnosis. The disease can be diagnosed with certainty only by the presence of its two cardinal symptoms, namely, the peculiar muscular affection and the hemoglobinuria. None of these symptoms however suffice in themselves as a firmer consistency of the muscles and their functional disturbances may be observed also in muscular rheumatism, but in this affection the tendon reflexes are normal. Hemoglobin may be present in the urine also in acute infectious diseases or intoxications, but in these pronounced symptoms of paralysis and reduction of the reflex irritability are absent.

A traumatic injury of the spinal cord or spinal meningitis can easily be excluded by the history of the case, in some cases by the evident sensitiveness of the vertebral column, by the complete insensibility of the paralyzed part of the body, by the disturbances of the functions of bladder and rectum, and by the action of the patellar reflex, which in contusions of the sacral cord is normal, in the presence of a lesion anteriorly to the lumbar cord appears to be normal or increased, while after injuries of the middle lumbar cord on the other hand it is not present. Moreover, in hemoglobinemia there also exists in most instances a hemoglohinuria which is absent in contusions of the spinal cord.-From the enzootic spinal meningitis (infectious spinal meningitis, Schlegel) the paralytic 
hemoglobinemia is distinguished by the history, the hard consistency and enlargement of the affected muscles, and by the hemoglobinuria, while in enzootic spinal meningitis, lematuria may also appear frequently. Sometimes a compression of the spinal cord (melanoma, exostosis) may also give rise to mistakes in cases in which they produce a sudden paralysis. They may be excluded in the same manner as contusions of the spinal cord. In unilateral paralysis a paralysis of the peripheric nerves must also be excluded on the ground of local examinations and with consideration of the presence of hemoglobinuria.

Fractures of the bones (pelvis and femur), as well as tearing of the Achilles tendon may result in clinical manifestations similar to paralytic hemoglobinemia. In such cases local examination would reveal the true cause; in a severe paralysis due to hemoglobinemia the urine almost invariably contains blood coloring matter, however as traumatic influences may simultaneously produce a hemorrhage in the urinary passages, the examination of the sediment of the urine determines in doubtful cases whether hemoglobinuria or hematuria is present. Swellings and a firmer consistence of the muscles of the croup, with absence of the patellar reflexes, have in such cases little significance, as enlargements of muscles are also observed in fractures of bones or tearing of muscles, with extensive hemorrhagic infiltration, and at such times the contractility of the muscle or percussion of the patellar ligament may disappear.

The thrombosis of the posterior aorta and its branches differs in that the animals are after a certain time again capable of standing up and walking normally; after more strenuous exercise however they again collapse. At the same time the presence of a thrombus or the absence of pulsation may be established by rectal examination of the iliac or of the femoral arteries.

Colicky affections can only be excluded by a somewhat careful examination.

Prognosis. The prognosis appears unfavorable in proportion to the completeness of the lumbar paralysis, the number of muscles showing functional disturbances, and to the pronounced character of the hemoglobinuria. If the animals cannot rise in spite of apparent health, or if they are not capable of standing up by themselves or in slings even for a short time, further if they remain lying for longer than three days, the urine containing at the same time elements indicating an affection of the kidneys, finally if high fever is present, there is only very slight chance for recovery, especially if extensive decubital gangrene becomes associated with the disease. A very accelerated and weak pulse has an unfavorable significance, according to König, also the marked increase of red blood corpuscles at the onset of the affection.

On the other hand there is always hope for an early reVol. $1-57$ 
covery in the presence of an incomplete and transitory paralysis, if at the same time the urine is pale red in color and the pulse strong; also in those cases in which the marked disturbances in locomotion rapidly diminish. In the prognosis however the possibility should be taken into consideration that sequels to the disease may develop, which diminish the value of the animal for a long time, or permanently. According to Thomassen and Thomson the paralysis of the muscles disappears in most cases inside of three months, not infrequently however only after 5 to 6 months. Marek observed a case in which a paralysis of the quadriceps, with a pronounced atrophy of the muscles, persisted for seven months, in another case even for 11 months; after that however it rapidly improved and disappeared together with the atrophy in the ninth and twelfth months.

The statistical data relative to the mortality vary greatly, as the severity of the affection in the various localities and at different seasons may show marked fluctuation. At the Budapest clinic in the years between 1890 and $1909,45=45 \%$ died out of 100 affected animals; the mortality, however, fluctuated considerably in the different years. According to Friedberger \& Fröhner the mortality at the Munich clinic amounts to $20 \%$, at the Stuttgart $40 \%$, while Bouley estimates it at $60 \%$, Stockfleth at $50 \%$, and Bay, based on 368 cases, at $70 \%$. Among the horses of the Prussian army the mortality varied in the different years between $26 \%$ and $46 \%$, and usually amounts to $32-36 \%$.

Treatment. First of all care should be taken that the animal be saved from all excitement, and especially from excessive muscular exertion. Accordingly horses which have broken down at a distance from their stables should be carried to shelter in wagons; further it appears advantageous to allow the animal to lie down for a half or a whole day on richly berded ground, and to turn it over at 3 to 4 hourly intervals if they show no inclination to rise by themselves. Besides they should be prevented from injuring themselves by continnous watching. After the lapse of the mentioned time an attempt should always be made to make the animals stand up, which in many cases may be accomplished with the aid of a girth placed under the rump, at the best however by the use of pulleys. It is a favorable sign if the animal bears more or less upon the feet. Frequently standing in the slings even for a short time is of great benefit. If in proper supports the animal places no weight on the extremities it should not be forced to stand, as it may asphyxiate; but attempts to get the animal to stand on its feet should later be repeated from time to time, about twice daily.

Spontaneous passing of feces should be favored by infusions of water, the passing of urine by moderate pressure with the hand in the rectum. If this procedure does not lead to results subcutaneous injections of eserin $(0.08-0.10 \mathrm{gm}$.), pilocarpin (0.20-0.30 gm.), or arekolin (0.06-0.08 gm.), or the administration of salines should be undertaken, and the bladder should be emptied with a catheter if possible every six hours. Flour and bran gruels, green feed and good fresh hay are best 
adapted for food. It is best to offer the animal drinking water as frequently as it is desired.

Rubbings repeated two or three times a day and wrapping the paralyzed part of the body with moist cloths may also be advantageous, while it is better to omit counter irritants, as they may contribute to produce an aggravation of the condition by causing restlessness. The frequently recommended bleeding was found ineffective by various authors. If heart weakness is present, stimulants (alcohol, black coffee, camphor [20-30 gm. subcutaneously], caffeine [2-5 gm. subcutaneously]), may be used to some advantage. If gangrene of the skin from pressure develops it may be treated with drying and disinfecting washes (alum and acetate of lead water [1:2:50], Goulard's extract), or with similarly acting dusting powders (charcoal powder containing corrosive sublimate, iodoform, dermatol, xeroform, etc.).

To increase the alkalinity of the blood, which is supposed to be considerably reduced, Dieckerhoff recommended the administration of alkalies in great quantities. He gave on the first day $150-300 \mathrm{gm}$. of bicarbonate of sodium, and $300-500 \mathrm{gm}$. of sodium sulphate, in three doses, and on the following days 50-200 gm. of sodium bicarhonate. The result was said to be satisfactory, but Hutyra and Marek failed to observe any particular results. Hink and Humann observed good results from the use of sugar $(1 / 2-1 \mathrm{lh}$. per os repeated several times), while Braund produced recovery in $95 \%$ out of 50 cases with iodide of potassium (15-25 gm. in $250 \mathrm{gm}$. of water on the first, and if necessary 10 $\mathrm{gm}$. additional on the following day), and subeutaneous injections of eserin. Atoxyl on the other hand was not effective (Rudolph). Finally the results of the veterinarians agree in that Lumbagin lacks any therapeutic value whatever.

After improvement has commenced, the rubbing should be diligently continued, and later the animal may be exercised quietly, then used at first for light, and finally for the ordinary work.

Prevention. The disease may be prevented with the greatest certainty by exercising the horses daily and reducing the grain food. In consideration of this, the horses should be exercised for a short time, and fed less even on days of rest and in bad weather. Sanitation and sufficient ventilation of the stable are also of importance.

In a large stable of horses in which year after year cases of hemoglobinemia appeared, new cases were avoided after the animals were only led out of doors after stable rest, if covered with blankets (Johne). Similar observations were made by Benjamin and Lavalard after a considerable reduction of grain food, by Brun from systematic exercising the animals by trotting on days of rest. Ohler orders washing the croup with cold water in the stable, after which it should be rubbed dry, shortly previous to the use of the horses; with this procedure he succeeder in preventing the appearance of the disease.

Literature. Benjamin, Bull., 1907. 240. - Bollinger, D. Z. f. Tm., 1877. III. 155; W. f. Tk., 1885. 439. — Brun, Bull., 1907. 237. - Cadéac, J. vét., 1 s97. 
465; Path. int., 1899. VI. 100. -Cadiot, Bull., 1907. 243. - Camus \& Pagniez, Ann., 1902. 621. - Dagès, Bull., 1901. 168. - Dieekerhoff, B. t. W., 1895. 1; 1896. 37; Spez. Path., 1904. II. 524. - Eber, Monh., 1892. III. 207; A. f. Tk., 1898. XXIV. 262. - McFadyean, Vet. Jhb., 1888. 71. - Fröhner, A. f. Tk., 1884. X. 296 (complete lit.). - Haase, B. t. W., 1897. 484. - Hauptmann, T. Z., 1898. 582. - Hink, D. t. W., 1901. 106. - Hofer, Ö. Vj., 1852. II. 151. - Höfling, T. Z., 1905. 373. - Humann, W. f. Tk, 1907. 546. - Johne, S. B., 1879. 94. Kleinpaul, B. t. W., 1904. 369. - König, Monh., 1909. XXI. 1. - Lavalard, Bull., 1907. 240. - Leipziger, Z. f. Vk., 1900. 389. - Lignières, Bull., 1898. 744. - Lucet, Rec., 1889. 96; 1899. 209. - Nowak \& Hartel, Vet. Jhb., 1901. \$2. Ohler, Münch. t. W., 1909. 829. - Pr. Mil. Vb., 1899-1908. - Razsorits, Vet., 1894. 559. - Schlegel, B. t. W., 1906. 463; Die inf. Rückenmarksentzündung oder schwarze Harnwinde, 1907 (Lit.). - Siedamgrotzky \& Hofmeister, S. B., 1878. 115. - Thomassen, Monh., 1902. XII. 305. - Thomson, Am. v. Rev., 1905. 309. Wetzl, Klinische Blutuntersuchungen, Diss. Budapest, 1908 (Hungarian).

Paralytic Hemoglobinemia of Cattle. There are a few publications from which it appears that a disease, resembling the paralytic hemoglobinemia of horses in its nature and symptomatology, occurs also now and then in cattle. (Leech is supposed to have observed several cases in dogs [D. t. W., 1899, 88].) As formerly all forms of hemoglobinemia were thrown together, and as a conspicuous weakness of the hind parts may also appear in other very frequent forms of hemoglobinemia (for instance piroplasmosis), a positive determination of the cases originating in former times is not possible. According to the present stand of knowledge however, it appears very probable that the observers usually had to deal with affections caused by piroplasma or by certain blood poisons, but not with cases of paralytic hemoglobinemia. A symptom complex which permits the acceptance of paralytic hemoglobinemia has been reported by Krug, Hartle, Saur, Notz and Baer \& Kurtz. These cases referred to affections which developed in work oxen either after several days of rest, or independently of it and also in other cattle supposedly after colds. The disease was manifested mostly by disturbances of gait in the hind parts or in weakness and stiffness (Bauer \& Kurtz), in enlargement and swelling of the muscles of the croup or thigh, further in an elimination of a brownish-red urine, which however showed no red blood corpuscles. Notz found at the same time in three cases, in an otherwise negative post-mortem, a bluish-gray discoloration, swelling, and softening of the muscles of the croup, back, shoulder and elbow.

Literature. Baer \& Kurtz, B. t. W., 1901. 48. - Härtle, W. f. Tk., 1896. 160. - Krug, B. t. W., 1893. 494. - Notz, W. f. Tk., 1900. 449.

\section{Hemophilia. Haemophilia.}

Hemophilia is a peculiar, as a rule congenital pathological condition, which manifests itself in that, even after very insignificant injuries or blood congestions, profuse hemorrhages appear which are very difficult or impossible to stop.

Etiology. Up to the present time the disease has been observed almost exclusively in horses, and especially in thoroughbreds (English thoroughbreds), but according to Zschokke an affection identical with hemophilia has not yet been demonstrated in animals. The cause of the hemorrhagic diathesis is still unknown; in man repeatedly an insufficient development 
of the vascular system was found (hypoplasia cordis et arteriarum), with which the tendency to hemorrhages is supposed to be inherited. In opposition to this Sahli pointed out the insufficient formation of thrombin in the walls of the vessel. As a matter of fact Morawitz \& Lossen found a deficiency of thrombin in a hereditary bleeder, probably as the result of a change of the chemical constitution of the cellular elements in the blood, which are to produce the thrombokinasis. In animals inbreeding appears also to have an influence on the development of a hemorrhagic diathesis in the progeny.

As incidental causes of the hemorrhages, rapid galloping, castration, rope pulling and other injuries of the skin or the mucous membranes, or even parturition may come into consideration (Stahn).

Symptoms. The hemorrhage is usually external, sometimes however it may be into a body cavity. The bleeding resulting from external wounds is usually parenchymatous, and can be stopped by the ordinary methods only with difficulty after several hours, or not at all, so that the animal may even bleed to death. Frequently the loss of blood is so great that it may result in acute anemia from which the animals recuperate only after several weeks. (Meyer observed in one case a diminution in the number of red blood corpuscles to $3,000,000$ in one $\mathrm{cmm}$. blood.) In other cases the bleeding may cease after a time, but may appear again later, and after frequent recurrences may produce death. In man hemorrhages are also frequent into the joint cavities and give rise to painful swellings of the joints.

Diagnosis. Before establishing the diagnosis of hemophilia all diseases which according to experience may cause hemorrhages must be excluded (acute infectious diseases, affection of the heart, aneurysms, kidney disease, new-formations).

Treatment. After the appearance of the hemorrhage the customary hemostatic remedies should be applied, likewise the borders of the wound, if present, should be united, compression should be applied on the bleeding place, and absolute rest of the body is required. If the hemorrhage continues in spite of these measures the blood must be coagulated by the use of concentrated chloride of iron solution, fuming nitric acid, or by the actual cautery on the bleeding surface. Internally ergot preparations may be administered. According to recent experiences in human medicine the subcutaneous injection of blood serum from other species might also be utilized to advantage. A resulting anemia should also be given appropriate treatment (see p. 848).

Zschokke succeeded in preventing the recurrence of hemorrhages in a horse in two weeks by the administration of $30 \mathrm{gm}$. of phosphorie acid daily.

Literature. Meier, Z. f. Tm., 1906. X. 1. - Stahn, Z. f. Vk., 1906. 214. Villemin, Ann., 1905. 154. - Zschokke, Ö. M., 1904. 433. 


\section{Animal Parasites in the Blood. Haematozoa.}

\section{(a) Filaria immitis.}

\section{(Filaria haematica.)}

Occurrence. The blood affection caused by filaria occurs in China and in Japan very frequently among dogs, but it has also been observed in other countries, although less frequently, especially in East India, Tonkin, Brazil and North America. In Europe dogs that were imported from Eastern Asia, or which were kept there for a time became affected mainly, but native dogs are not necessarily spared (Fröhner, V. Ratz).

According to Janson about $50 \%$ of dogs in Japan are affected with filaria, immitis, but about $75 \%$ of the affected animals show no symptoms of disease whatsoever for a long time.

Petropawlowsky in Charkow foumd 120 vagrant dogs infecter, almost witlout exception, while of 83 other dogs only 3 harbored filaria in the blood. GalliValerio demonstrated filariosis haematica in $18.5 \%$ out of 162 autopsies on dogs in Milan.

Etiology. The full grown filaria immitis is a long, white worm, of about 2 millimeter thickness. The male is 12-18 $\mathrm{cm}$. long, its posterior extremity shows a spiral bending; the female is $20-30 \mathrm{~cm}$. long and gives birth to living embryos.

The sexually mature filaria live especially in the right half of the heart, more rarely in the pulmonary arteries, very rarely in the vena-cava, and only very exceptionally in the left half of the heart or in the arteries of the large blood circulation. They are found at these places in varying numbers, sometimes up to several hundred, rolled up in a ball which is difficult to untangle, in which the females give birth to embryos about $1 / 4$ mm. long which may then gain entrance to the circulating blood in very large number's (Rieck estimated them in one case up to several millions). Not infrequently however the sexually mature filaria may be present at the same time also exclusively in the subcutis.

The mode and manner of the natural infection is not ret known with certainty. The infection is probably transmitted by impure swampy water, which the frequent occurrence of the disease in marshy localities of the warmer countries seems to substantiate fully. The dogs probably take the larvae of the parasite with their drinking water. These then reach the heart with the venous blood and there they mature. The embryos may leave the animal with some of its blood or sometimes with the excrement and the secretions of the air passages (Janson), principally however with the urine, and may then reinfect the drinking water. The artificial transmission of the disease by inoculation of blood containing filaria has never been snceessful (Fröhner). According to Janson and Galep \& Pourquier the embryos are supposed to pass sometimes to the 
fetus through the placental vessels. This however was not confirmed by Rosso in experiments with a bitch.

According to Manson the embryos of the filaria sanguinis hominis, a worm resembling the filaria of dogs, are taken up by mosquitoes (culex mosquito) with the blood of affected persons, in which they develop to larvae. When later the mosquitoes die in the marshes, after depositing their eggs, the young filaria are set free from their bodies, and may reach the stomach of new victims with the water, and from there gain the blood vessels of man. A similar development of the filaria immitis would also be expected, but Grassi proved that the majority of the embryos of the filaria sanguinis hominis die in the intestinal eanal of mosquitoes, and that the filaria immitis cannot live in fleas and lice of dogs. Grassi therefore believes that the embryos reach the water with blood from occasional wounds, and probably also with the urine, passing into the healthy animals directly with such infected water.

Pathogenesis. The sexually mature filaria appear to cause disturbances, especially in a mechanical way. They check the blood circulation more or less, depending on their numbers, either in the heart itself or in the blood vessels, with which of course a quite considerable thrombosis produced by the parasite usually becomes associated. After their infesting the heart cavities the filaria produces a chronic inflammation of the endocardium. The thrombi which develop in the heart cavifies or in the large blood vessels may again give rise to the dissemination of emboli. The formation of emboli occurs mostly in the branches of the pulmonary arteries, and may then lead to necrosis of circumseribed portions of the lungs or to abscess formation. Particles of the thrombi are exceptionally conveyed to the kidneys or into the brain.

The embryos of filaria produce first of all anemia with a pronounced leucocytosis, the severity of which depends on the number. These changes in the blood are probably brought about by the action of toxic metabolic products, and also by the utilization of the constituents of the blood. The purely mechanical action of the embryos produces on the other hand at most only insignificant effects, plugging of the capillaries, and occurs most frequently in the capillaries of the kidneys, more rarely in the other organs (lungs, brain).

Anatomical Changes. Sexually mature filaria surrounded with firm blood coagulum are found in the right half of the heart or in any branch of the pulmonary arteries, sometimes in the vena-cava, and exceptionally also in the left half of the heart (Hutyra found in one case almost the entire aorta filled up by firm masses of thrombi). At the same time chronic endocarditis, partial hypertrophy and dilation of the heart, possibly also rupture of the heart, with symptoms of blood stasis (hydrops, cirrhosis of the liver, etc.) may frequently be present. The lungs sometimes show hemp-seed to pea-sized nodules, each containing an embryo appearing as a red dot; besides these atelectasis, splenization, abscess formation or necrosis may be found. The kidneys may manifest a paren- 
chymatous, or not infrequently a chronic interstitial inflammation; they mar also contain punctiform hemorrhages. The subcutaneous and intermuscular comnective tissue may reveal sexually mature filaria free or encapsulated; in these parts they may be present even when the heart or blood vessels do not contain full grown filaria (v. Ratz). The autopsy finding is further completed by the presence of anemia and of the embryos in the blood. If accidentally only male filaria, or only unimpregnated females are present in the affected animal, the blood does not contain filaria embryos. On the other hand sometimes in spite of the presence of filaria embryos in the blood no sexually mature filaria can be demonstrated (Fröhner).

Symptoms. As long as the filaria and their embryos are present in the blood in only small numbers, their presence is not disclosed by disease symptoms. In severe cases however, after a month or even after a latent stage of years, more rarely immediately from the beginning, symptoms of severe anemia and gradually progressing emaciation develop; sometimes an abnormally increased appetite is also observed. Later hemorrhages appear in the internal organs, in the lungs, intestines and in the kidneys.

Besides these manifestations which are of general importance, local symptoms may appear in some of the cases, which are produced either by the thrombi developing in the heart or by embolic plugging of small blood vessels. As a result of these, various disturbances of the heart action may develop, which may even lead to paralysis of the heart, hydropic symptoms, sometimes venous or arterial thrombosis, further epileptic convulsions, cough and difficulty in respiration, symptoms of nephritis, hematuria, as well as weakness of the hind parts which may increase to complete paralysis. Rheumatoid pains were also observed (Fröhner). The blood shows, besides indications of anemia, also a pronounced leucocytosis. (Marek in one case found the relation between white and red blood corpuscles to be $1: 18$.) All of these symptoms develop slowly, and without fever, gradually reach a high degree, and the disease extends not infrequently over several years.

Exceptionally the disease manifests itself only in epileptic fits (Mégnin), which may lead to a fatal termination (Earl) or death ensues as the result of a rupture of the right auricle (Schneider, Guittard).

In certain cases a papular erythema, with the appearance of a diffuse reddening of the skin, and numerous small and hard nodules and vesicles develop, with which severe itching is associated. Otitis externa also occurs. In the contents of the nodules or vesicles, also in the eczematous or otitic products, embryos of filaria may be demonstrated.

Diagnosis. The recognition of the disease is usually only possible by microscopic demonstration of filaria embryos in the blood. A drop of blood placed between a slide and a cover glass without any special treatment, reveals even on slight 
magnification, fine, worm-shaped colorless embryos, which execute snake-like or circular movements between the blood corpuscles. (Hutyra \& Marek failed to find a variation in the number of embryos during the hours of the day and night, although it is claimed by Manson and Sonsino that filaria appear in large numbers in the peripheral vessels only during the night.)

Treatment. This promises results in strongly infected localities only in so far as some chronic cases without pronounced disturbances in nutrition and circulation, may be arrested (Janson). Good nutrition, avoiding exertion and new infection, as well as the administration of heart tonies or arsenical preparations come into consideration; Dorn recommends atoxyl, which produces in man a diminution of the filaria embryos. If a skin affection results this requires the application of antiparasitic and itch-preventing remedies (see eczema or scabies in Vol. II).

Literature. Cadéac, J., vét. 1897. 582. - Deffke, Monh., 1890. I. 323. Fettick, Vet., 1900. 245. - Fröhner, Monh., 1892. III. 494. - Grassi, Cbl. f. Bakt., 1888. IV. 615. - Janson, A. f. Tk., 1892. XVIII. 63. -Petropawlowsky, A. f. Tet.-Wiss., 1904. 484. - v. Rátz, Vet., 1898. 395. - Roger, Bull., 1906. 119. - Rosso, Mod. Zooiatrio, 1897. 185.

Filaria Sanguinis Equi. In former times repeated reports appeared relative to the occurrence of filaria embryos in the blood of horses (Wedl, Sonsino, Manzanti, Lange). The classification of the parasites found was never satisfactory, while their differentiation from trypanosomes was not attempted. Recently however Lingard proved by long continued experiments that filaria embryos do pecur in the blood and mature filaria in the blood vessels of horses.

The filaria sanguinis equi is a thread-shaped, thin, whitish worm, the males measuring 10-15 $\mathrm{cm}$., the females about three times that length. The caudal extremity appears rolled up and supplied with two uneven spicules which are bordered by a papillae. The worm is ovoviviparous; the length of the embryos amounts to $160-220 \mu$. The grown specimens live in the walls of the large arteries, especially in the anterior or posterior aorta, while the embryos circulate in the blood.

The worms were found by Lingard in India especially in the native horses while imported animals become affected only after long stays in the country. The mode and manner of infection is not yet known; the transfusion of blood containing embryos was negative in two cases. The symptoms of the disease probably only appear after a long period of latency, and consist of rapid fatigue, edematous swellings of the lower parts of the extremities and the prepuce, at the same time the respiration becomes much accelerated and small hemorrhages appear on the conjunctivae. Sometimes moderate fever is observed. The heart action is stronger and more frequent. The blood reveals embryos with a snaky and circular movement, their number increases in the afternoon and reaches its maximum in the evening. The embryos are especially numerous during the hot and rainy season, but now and then they may temporarily disappear entirely. 
The autopsy reveals, besides the already mentioned changes in the blood vessels and in the blood, numerous mustard-seed sized, caseous or calcareous nodules in the lungs and also in the liver, and in these are found embryos of the filaria. (Lingard, Rev. gén., 1906, VIII, 179 [Ref.].)

\section{(b) Other Animal Parasites in the Blood.}

Bilharzia crassa, B. indica, B. Bomfordi (Schistosomum crassum s. bovis, Sch. indicum, Sch. Bomfordi). These resemble the Schistosomum japonicum of man, which according to Tsuchiya is not identical with the Bilharzia haematobia (Distomum haematobium). The disease caused by them (Bilharziosis), occurs frequently in Egypt, India, Japan, Mozambique, Sudan, Abyssinia, Tunis, Cape Colony, Cochin-China, also on the Island of Martinique, and affects cattle, sheep, horses and donkeys. In Europe the disease was known until quite recently only in Italy in cattle and sheep, but recently a case was observed by Marotel in a cow in the vicinity of Lyons in France. Grassi \& Rovelli in Sicily founcl $75 \%$ of the examined sheep, Bertolini $9 \%$ of the cattle of Sardinia, affected with bilharziosis. The parasites were principally found in animals between two and four years of age.

Publications on bilharziosis were made by Sonsino (1876), Bomford \& Powell (1886), Grassi \& Rovelli (1888), Railliet (1889), Sanfelice \& Loi (1899), Montgomery (1906), Raja \& Peju (1907), Marotel (1908) and Bertolini (1908)'. Montgomery, Marotel and Bertolini described the cause of the disease very accurately.

The infection probably results from drinking stagnant water, which becomes infected with the eggs through intestinal excrements and through the urine of animals. This mode of infection is indicated by the fact that man, who, in infected localities drinks only filtered water, usually does not become affected.

The usual location of the bilharzia is the portal vein with its branches, in the region of which they produce manifestations of stenosis; Montgomery found them also in the liver, whereas Bertolini never observed them there. The worms are recognized, on placing the blood in a flat dish, as thin, white bodies about 1/4-1 $\mathrm{cm}$. long bent in a C-form. Not infrequently they are found in copulation, when the females are held by the males in groove-shaped depressions of their bodies.

The spindle-shaped eggs which are supplied at one end with spurshaped extensions, circulate in the blood and produce inflammatory changes or hemorrhages especially in the walls of the intestines, also in the urinary bladder. In eattle changes exceptionally develop in the intestines which may give rise to confusion with rinderpest. Small, wart-like new-formations develop in the bladder, rarely in the rectum of cattle.

The anatomical changes show certain variations in the different localities and in accordance with the number of parasites. Thus Bertolini for instance found only hyperemia of the small intestines and hemorrhages in the rectum, while the sections of the large intestines as well as the bladder were free of such lesions. At the same time nodules like sand-granules could be felt in the mucous membrane of the small intestines, which contained female bilharzia and their eggs; in 
the hemorrhagic areas of the rectal mucous membrane eggs of bilharzia were found massed together.

The symptoms are indicative in general of a subeutaneous or chronic eatarrh of the mucous membrane of the intestines and bladder, in which periodically hemorrhages may be observed, according to Bertolini in the first half of the summer. The disturbances of health are always slighter in horses than in ruminants.

A diagnosis would be possible during life by demonstrating the charaeteristic eggs in the urine, feces, or by the water-like new formations in the rectum which might be present.

Treatment is not promising of results.

Literature. Bertolini, Klin. Tet., 1908. 1 (Lit.). - Marotel, Bull., 1908. 119. - Montgonery, J. of trop. vet. science, 1906.138 (Lit.). - Raja \& Pejn, J. vét. 1907. 478 .

Haematozoon Lewis. Gruby \& Delafond found worms, among others in the blood of dogs, whieh resembled the embryos of the Fil. immitis with relative frequeney even in countries where the Fil. immitis rarely occurs (the mentioned two authors in Paris in every twentieth to twenty-fifth, Sonsino in Pisa, Lewis in Calcutta in every third dog). They cliffer from the Fil. immitis in that they attach their head ends to the cover glass, at the same time executing lively motions with the other parts of the body. They are remarkably resistant, so that ther remain alive for 10 days outside of the animal body at a temperature of $15^{\circ} \mathrm{C}$. They are found in the arterial as well as in the venous blood, and sometimes in such quantities that a drop of blood mav contain 3-5 and even 12-15 specimens.

Views differ in regard to the development of the parasite. According to Grassi they develop from larvae, which oceur quite frequently in fleas (Pulex serraticeps) and lice (Haematopinus piliferus) of dogs.

Dogs whose hlood harbors the embryos may appear entirely healthy; Gruby \& Delafond observed however epileptic fits in three dogs. The same authors have further ohserved that after transfusion of defibrinated blood, containing the embryos, into healthy dogs the embryos disappear from the blood of the injected animals in 8-40 days; in two eases however they remained alive for longer than three years, until the death of the dogs. A full grown worm corresponding with the embryo is still unknown. (Carougeau \& Marotel, Rev. gén., 1903. I. 447 [Lit.].)

Strongylus Vasorum. This is a fine, thread-shaped worm (the male is $14-15$, the female $18-21 \mathrm{~mm}$. long), which occurs in the right ventricle of the heart, and in the pulmonary artery and in its branches. In the intima of the vessel wall it causes the formation of small nodules or elongated cords, and in the small vessels thrombi. The eggs laid by the female remain lodged in the finer blood vessels, and lead, like the hatched embryos, to the formation of small, up to pinhead sized, sometimes quite numerous nodules. According to Laulanie the embryos migrate later into the bronchi, they are expelled by coughing, and are then occasionally taken up by other dogs.

The symptoms of the disease are not very characteristie. Symptoms of anemia, gradually progressing emaciation, frequent and irregular heart action, possibly hypertrophy of the right half of the heart, further a painful weak and dry cough are observed in severe cases, but the disease 
usually remains unrecognized. If it is suspected the small embryos may possibly be demonstrated in the expectorations.

Up to the present the disease has been observed in France especially in the vicinity of Toulouse (Baillet, Lafosse, Laulanié) and in Italy (Bossi) where it sometimes appeared in an enzootic form and caused considerable loss.

According to Lafosse it is possible to cure (?) the disease by the internal administration of oil of turpentine (2-4 gm daily for one week) (Neumann, Mal. parasitaires, 1892. 621 [Lit.].)

Strongylus Subulatus (Haematozoon subulatum) was found by Leisering (V. A., 1865. XXXIII. 111; S. B., 1864. 49) in the venous blood of the corp. cavernosa of the urethral penis and the glans, while in another dog the worms were present in nodular-shaped swellings of the lungs, the thyroid gland, and lymph glands.

In the walls of the large arterial branches there may further occur the Spiroptera sanguinolenta (see Vol. II), Filaria Blini and F. Evansi (see Inflammation of the Arterial Wall). 


\section{Diseases of the Spleen}

The diseases of the spleen in animals are known almost exclusively from the results of post-mortem examinations. This is due to the fact that in larger animals examination is very difficult owing to the unfavorable anatomical position of the spleen, so that in most cases only marked changes in form and size of the organ can be determined; furthermore diseases of the spleen usually produce only very indefinite disturbances in function. It must, however, be noted that in veterinary medicine a careful and systematic examination of the spleen has hitherto not received much attention.

In view of the very small number of clinical observations it is impossible for the present to afford a satisfactory description of the symptoms.

Acute Enlargement of the Spleen (Tumor lienis acutus). This occurs very frequently. Owing to its spongy structure the spleen contains, even under normal conditions, very much blood, the quantity of which may vary within wide limits according to different influences. Svoboda, for instance, found the spleens of healthy sheep, which had been given food and water just before being killed, enlarged two and threefold, and Lenkey frequently observed an enlargement of the spleen in food animals whose central nervous system was destroyed from brain to lumbar cord by means of a stick forced into the skull. In fact there is no other organ which, in a short time, increases so much in size through variations in its blood contents, while on the other hand the smooth muscular fibers and elastic elements of the spleen enable it to contract rapidly when the influx ceases, and to remove its surplus of blood.

A pathologically increased flow of blood to the spleen is sometimes produced by contusions or wounds of the splenic region, and is also likely to occur in the initial stages of inflammatory processes as well as in impediments to the portal 
circulation or to the splenic rein (as, for instance, in torsion). 'The most important swelling of the organ, however, is that which frequently occurs in acute infectious diseases. This form of acute splenic enlargenent is caused partly by the accumulation of bacteria in the pulp of the spleen, partly, and to a greater degree, by the increase in the number of lymph cells and the deposition of lencocytes containing bacteria as well as the deposition of disintegrated red blood cells which is quickly followed by an inflammatory process associated with swelling and increase of the cells and swelling of the connective tissue framework. (Splenitis hyperplastica acuta.)

The most striking pathogenic effect of bacteria and their toxins is ohserved in anthrax. In this affection the spleen may be enlarged as much as 5 to 10 times the normal size and the swelling may increase so rapidly that the capsule, being subjected to severe tension, sometimes tears. In other diseases the swelling is usually less in degree and it is probably least intense in such cases in which only bacterial toxins circulate in the blood. A marked splenic enlargement is met with in swine erysipelas, in acute glander's, and in acute tuberculosis (partly due to the formation of nodules), in pyemia, hemoglobinemia, in the acute infectious anemia of horses, while the various forms of hemorrhagic septicemia as well as croupous pneumonia are accompanied only by slight swelling, and this is entirely absent in purpura hemorrhagica or in tetanus.

In living animals, except in ruminants and in fat hogs and dogs, acute swelling of the spleen may as a rule be diagnosed: in horses by rectal exploration; in smaller animals by abdominal palpation. "The spleen is found to be enlarged, soft or more or less tense to the touch. A considerable swelling may be recognized by dullness on the left side, which is in horses parallel to the costal arch, in dogs and hogs in the direction of the last two intercostal spaces passing downward from the costal arch and which is 3 or 4 fingers wide. In ruminants the swelling is usually unnoticed because the spleen, covered by the lung, does not protrude beyond the posterior border of the lung even if decidedly enlarged. Nevertheless in severe cases careful percussion may show a dullness at the border between lung and rumen as was recently observed by Szathmáry in a buffalo affected with anthrax.

Literature. Jawein, Y. A., 1900. CLXI, 461. - Lenkey, Hússzemle, 1907. 65. - Szathmáry, A. L., 1909. 525.

Purulent Inflammation of the Spleen. (Splenitis apostematosa.) This occurs after the extension of a similar inflammation from neighboring organs or after the lodgement of infected emboli (as, e. g., in strangles, etc.). In a case reported by Arndt the embolus in the spleen came from a suppurating inflammatory focus which had been produced by a rusty needle 
in the hard palate). The suppurative foci may be isolated or numerous, and in case of metastasis several abscesses are usually formed. In exceptional cases gastrus larvae may produce extensive suppuration in the spleens of young horses (Kováts). Traumatic influences usually lead to the formation of only one abscess, but the entire organ may become subject - to the suppurative destruction. While an injury in the splenic region may be the immediate cause of such an inflammation it is far more frequently produced by foreign bodies which may get into the organ from the first stomachs of ruminants and, exceptionally, from stomach and intestine in other animals. The spleen may be enlarged to 4 or 5 times its normal size. According to Notz sucking calves often develop a purulent hemorrhagic inflammation of the spleen when they are stepped on by their mothers.

The symptoms of traumatic purulent inflammation of the spleen are very vague. Aside from the sensitiveness and enlargement of the organ which may be demonstrable, the clinical picture is composed more of general disturbances an explanation of which is possible only by reliable historical data to a certain degree. There are eases in which a large abscess is found in the spleen on autopsy of animals which presented no sign of disease during life. Möbius, for instance, found an abscess containing 3 liters of pus in the spleen of a wellnourished cow, and Kühnau reported a similar case. In other cases only digestive disturbances and colicky symptoms, showing little that is characteristic, are observed and are followed by a gradual or rapid emaciation (Knoll, Zimmermann; de Meestre ordered the animal to be killed in one case because he suspected lung plague). Fleischer observed, in purulent inflammation of the spleen in cattle, convulsive movements which recurred periodically. If the disease develops rather rapidly there exists a violent fever which may be continuous or remittent. Imminger is inclined to consider a continuous temperature of $40-41^{\circ} \mathrm{C}$, , which does not yield to antipyretic remedies, as characteristic of purulent splenic inflammation. In this he surely goes much too far, for in the presence of such symptoms the nature of the disease can be recognized only then when at the same time swelling and perhaps tenderness of the spleen may likewise be ascertained by percussion and palpation (van den Eeckhout found in a colt an area of dullness, five fingers wide, below the left costal arch, the anterior border of which passed in front and below, obliquely to the fifth rib and then turned vertically downwards; the enlarged spleen weighed $91 / 2 \mathrm{~kg}$.). Sometimes a large splenic abscess may be felt from the rectum and then it will be possible to determine fluctuation. Finally, purulent inflammation of the spleen may give rise to the development of peritonitis or pleurisy which will also occur after rupture of the abscess in which case it becomes purulent (Fröhner, Author's case). 
In an uncomplicated purulent inflammation of the spleen, surgical interference is to be considered, internal treatment affording no promise whatever of results.

Degive extirpated the spleen of a dog, which was adherent to the omentum, without any harm to the animal.

Literature. Arndt, B. t. W., 1889. 243. - Degive, Ann., 1888. 10. - van den Eeckhout, Ann., 1906. 619. - Fäustle, W. f. Tk., 1908. 362. - Fleischer, B. t. W., 1906. 922. - Fröhner, Monh., 1907. XVIII. 144. - Gmeiner, Monh., 1896. XVII. 510. - Imminger, WV. f. Tk., 1892. 229. - Kováts, Vet., 1895. 360. - de Meestre, Ann., 1887. 354. - Möbius, S. B., 1888. 71. - Notz, W. f. Vk., 1891. 280. Richter, Z. f. Vk., 1906. 390. - Zimmermann, B. t. W., 1894. 358.

Hemorrhage of the Spleen. (Haemorrhagia lienis.) Hemorrhages into the spleen are not infrequent, but can hardly be diagnosed clinically. (Rupp observed obstinate constipation in a dog' with a large splenic hematoma.) The cause of the hemorrhage may lie in active or passive hyperemia, or in acute splenic enlargement. Berg and other Danish veterinarians quite frequently observed diffuse splenic hemorrhage in cattle which suddenly became ill and died soon, so that anthrax was often suspected. Hemorrhage in the spleen not infrequently occurs after contusion of the splenic region, but is rare after the lodgement of non-infected emboli. The hemorrhage may cause the spleen to burst.

Literature. Berg, Maanedsskr., 1905. XVI. 441. - Bodon, Vet., 1899. 432. - Querrini, Monh., 1908. XX. 90. - Rupp, B. t. W., 1906. 44. - Witt, B. t. W. 1908. 625 .

Rupture of the Spleen. (Ruptura lienis.) A healthy spleen only very rarely tears from direct injury, but in rapidly increasing acute swelling this may occur without any traumatic effect, and internal fatal hemorrhages from such a canse are by no means rare; they are observed in anthrax with relative frequency. Sometimes here also a traumatic accident that may be slight in itself, such as falling down, rapid motion, cough, etc., may form the immediate cause of the rupture, but in other instances no external cause can be determined. Witt, who often observed sudden death, and hemorrhage into the abdominal cavity in cattle with ruptured spleen, took the disease to be malaria, as he had found white corpuscles, similar to parasites, in the blood cells. In a case of Darvas' the rupture of the spleen was due to the bursting of an echinococcus.

Rupture of the spleen produces in general only symptoms of internal fatal hemorrhage, the canse of which can at best be suspected on account of the sinultaneous colicky symptoms, the accumulation of fluid in the abdominal cavity, and still more on account of a splenic tumor that may be detected. (Drewien found on rectal exploration in a horse a large swelling in the left side of the abdomen.) (Literature under Hemorrhage of the Spleen.)

Torsion of the Spleen. (Torsio lienis.) Torsion of the 
spleen around the splenic ligament occurs not infrequently in hogs (Glage) and is observed occasionally also in dogs (Boston, Johne, Author's case). If the spleen has become heavier because of enlargement or swelling, and if the ligament has become atrophic (Glage), the organ may rotate on the ligamentum gastro-lienale during a forced motion of the body or yielding to the pressure of other organs. When the rotation has taken place, the veins passing through the ligament are compressed so that the return flow of the venous blood is impeded and a splenic tumor is produced which increases rapidly and enormously. Simultaneously stasis takes place in the gastric veins opening into the splenic vein, which leads to a venous hyperemia of the stomach; this is, however, usually not intense. Torsion may lead to necrosis of the spleen.

The affection is characterized by marked splenic enlargement and sometimes also by symptoms of gastric catarrh. Although a great swelling of the spleen, together with a rather soft consistency of the organ may suggest torsion, this cannot be definitely recognized by clinical methods.

Literature. Glage, Z. f. Flhyg., 1898. VIII. 4. - Zietschmann, S. B., 1903. 257.

Chronic Enlargement of the Spleen. (Tumor lienis chronicus.) Strictly speaking, this always develops in consequence of venous stasis, which by the copious offusion of nutritive material stimulates an increase in the connective tissue elements of the frame substance. As more frequent causes may be enumerated: Thrombosis of the splenic vein, chronic interstitial hepatitis, thrombosis or compression of the $\mathrm{Vv}$. portae by a swelling, by atrophy of the connective tissue or by a hematoma of the liver, sometimes also organic heart lesions.

In the diagnosis of chronic tumor of the spleen the symptoms described for acute swelling are deciding, but' with the difference that here the consistency of the spleen is firmer.

Chronic swelling of the spleen may include the following pathological conditions of the organ:

Splenitis chronica indurativa, which develops, according to Kitt, through the persistent influence of toxic infectious agents (for instance in the chronic erysipelas of hogs).

The leukemic splenic tumor can usually be clinically diagnosed with certainty. The nature of the enlargement is indicated by the characteristic blood changes; greater difficulties are encountered in the correct recognition of a pseudoleukemic splenic tumor.

Simple hyperplasia of the spleen (Hyperplasia lienis) is occasionally found on autopsy. The spleen is usually remarkably large (in the case of Bourret and Druille the spleen of a horse weighed $21 \mathrm{~kg}$; ; in that of Duschanek, 39; in that of Koch the spleen of a cow weighed $18.5 \mathrm{~kg}$.). During life gradual and progressive emaciation and respiratory difficulty was observed.

Vol. 1-58 
Literature. Bomret \& Druille, Rec., 1887. 300. - Duschanek, T. Z., 1894. 353. - Käppel, Z. f. Flhyg., 1904. XIV. 53. - Koch, B. t. W., 1893. 127.

New Formsiions in the Spleen. The most frequent of these are melano-sarcomas which oecur particularly in white horses (Deste) either in the form of fairly large metastatic growths which may become as large as a child's head, or in the form of diffuse infiltrations, and at times they become the cause of considerable enlargement of the spleen. Barrier observed in one horse first an enlargement of the circumference of the abdomen, the desire to eat being good and the ability to work satisfactory. Not until about three weeks before death did the animal begin to lose weight; the appetite was disturbed, the weakness increased progressively, the walk became staggering ard at times colicky symptoms appeared. On rectal examination a large swelling was found in the left half of the abdominal cavity.

Of similar clinical significance is the enlargement of the spleen in amyloid degeneration, in metastatic carcinoma or in the presence of large tubercles (especially in cattle and hogs, sometimes also in horses) or of echinococcus cysts. Direct examination can at most show the splenic enlargement which may occasionally cause respiratory distress (Barrier), the cause of the enlargement must be determined from other pathological signs. Laendler succeeded on rectal examination in a heifer in palpating the very knobby posterior border of the spleen which was increased about 5 times in volume through the presence of echinococcus.

Necrosis of the Spleen. This was found by Zietschmann and Poetsch (S. B. 1903, 259) in an apparently healthy heifer which had been killed for meat. As the cause of the necrosis a complete thrombosis of the splenic vein may probably be held responsible.

Atrophy of the Spleen. Mayer (Z. f. Flhyg. 1909, XIX, 251) found in a sow which had been killed for meat a far advanced senile atrophy of the spleen which was only $6 \mathrm{~cm}$. long and $4.5 \mathrm{~cm}$. wide. 


\title{
Diseases of Metabolism.
}

\author{
1. Obesity. Obesitas.
}

\author{
Fettsucht.
}

By obesity is understood an excessive deposit of fat which interferes in some way or other with the ability of the animals to work. If the increase of the fat deposit occurs while nutrition and inuscular activity are normal, it is called obesity in a limited sense of the word, in contrast to the alimentary obesity (fattening), which is the result of generous feeding or of inactivity.

Etiology. The cause of alimentary obesity lies in a disproportion between the intake and the utilization of nutritive units. This condition very frequently prevails if the animals receive more food than they need in order to maintain their bodily functions. It also occurs frequently enough that the intake of caloric units does not exceed the average amount, but that through insufficient activity the muscles of the body use up too small an amount of energy-units. Finally, both factors frequently combine in producing obesity.

A generous deposit of fat is promoted above all by the fattening of animals intended for food, but although strictly speaking a pathological condition, this does not usually form an object for therapeutic measures. Obesity is further frequent in breeding animals, especially in the males, which are, as a rule, not only fed generously, but are moreover spared all work, and are even not rarely prevented from taking the most necessary exercise. The same is true of pet and house dogs. In general, however, favoring factors are of essential importance. Above all a vigorous appetite comes into consideration, which apparently varies individually within wide limits. Then the nature of the food is important, food-stuffs which are rich in fat and carbohydrates producing a considerable fat de. posit. On the other hand the deposit of fat is promoted by all conditions which persistently diminish muscular activity. In this respect the phlegmatic temperament which may be in- 
herited is very important. Animals also become phlegmatic after castration (Lüthje) and then tend to put on fat. The same is true for older animals. By diminishing muscular activity anemia may favor the deposit of fat if it is not due to an organic lesion or to deficient feeding. It has for this reason become customary in many districts to promote the fattening of the animals by periodical venesection. The diminution of muscular activity finally becomes a favoring factor in producing obesity in many broken-winded horses.

In all these cases the obesity develops in consequence of an absolute or relative hyperalimentation. As to whether aside from this there also exists in animals a true constitutional obesity we do not possess any observations based upon examinations of the metabolism. This form of obesity might be expected to arise from a slowing of metabolism because in such individuals the weight unit protoplasm would transform less potential energy into living force, primarily into heat (v. Noorden). Is a matter of fact, v. Bergmann recently actually demonstrated the slowing of metabolism in persons affected with obesity. This diminution of the oxidizing function of the protoplasm may, according to $v$. Noorden, have its cause in a peculiar constitution of the protoplasm itself or it may be the consequence of abnormal internal secretions, especially those of the thyroid gland which exerts a stimulating action upon oxidation, while its function is regulated in its turn by other organs (pancreas, sexual glands, hypophysis, adrenals). v. Noorden thus explains constitutional obesity as a thyreogenic obesity.

Anatomical Changes. The fat is present in very large proportion, especially in the subcutaneous connective tissue whence it may enter between the muscles and even between the muscle fibers, also between the mediastinal folds, under the visceral layer of the pericardium and possibly in the heart muscle, in the omentum, the colon, in the region of the kidneys, etc. In severe cases individual fibers of the muscles which are permeated with fat, for instance in the heart muscle, take on a yellowish color owing to fatty degeneration. Of the internal organs it is particularly the liver in which there is a great accumulation of fat.

Symptoms. Obesity shows itself most strikingly in a peculiar alteration in the shape of the body by which the outlines of the body are rounded out and the skin forms thick, but soft and elastic folds. This change is most decided on the trunk, especially on the abdomen, then on the thighs, the shoulders and on the throat. The enlargud belly sometimes has an appearance as though the abdominal walls were forced apart by fluid or a swelling, but the actual cause can easily be determined by palpation and percussion and still better from the other signs of obesity. The skin feels soft and elastic; 
between its folds a superficial inflammation occurs not infrequently.

Another symptom is lassitude and rapid fatigue on motion which causes the animals to become dyspneic and to perspire. Respiration is frequent and superficial even at rest, because the diaphragm is forced against the thoracic cavity and because the contraction of the respiratory muscles is impeded. This may, however, be partly due to the weakened heart action through which the pulse becomes rapid and soft.

Later on digestive disturbances occur frequently because the motility of stomach and intestines is diminished by the masses of fat, which may also account for the frequent constipation. In breeding animals the ability to procreate diminishes proportionally with the obesity and eventually disappears entirely; furthermore, the increased weight and diminished muscle force renders coition difficult or impossible.

Course and Prognosis. With care and moderate feeding the diseased animals may be kept alive for a long time. But, unless the causes are removed, there develops a gradually increasing anemia leading to an aggravation of the respiratory and circulatory disturbances, and finally death occurs from heart failure or asphyxiation. Previous to the fatal termination there are often signs of blood stasis, such as cyanosis, dyspnea, edematous infiltrations, ete.

The prognosis is favorable insofar as a timely and proper regulation of diet will in most cases relieve the pathological condition.

Treatment. The method which is most certain to be successful consists in the diminution of the daily food ration and in systematic exercise of the animals. In order to prevent digestive disturbances the amount of the food should be reduced gradnally down to a daily allowance which corresponds to the species and size of the animal. Especially in dogs care should be taken to feed them only at certain times, once or twice daily. It is of course necessary to pay attention to the composition of the food-stuffs and especially the allowance of carbohydrates and fats should be limited as much as possible. On the other hand the limitation of the amount of drinking water has only the effect of assuring a freer motion of the animals and therefore a greater expenditure of energy.

It is advisable to attempt the systematic administration of Carlsbad water (in $\operatorname{dogs} 1 / 2$ to 1 tablespoonful once or twice before feeding), although in most cases this treatment is superfluons. Of excellent service are the thyroid gland preparations which stimulate a considerable oxidation of fat, but at the same time cause an increased disintegration of albumin, so that their administration must be supplemented by a more generous ratio of the proteids. The various preparations that may 
be used are Thyreoidinum depuratum (for dogs 0.2-0.3 to 0.75 gm., or 3 times daily 1 dessertspoonful of the $1: 1000$ solution); Thyreoidinum Poehl (3 times daily 0.3-0.6 gm.); Iodothyrin (0.1-0.2 gm.). If necessary the fresh thyroid gland of animals killed for food may be employed. If anemia is present this also requires appropriate treatment.

Literature. v.Bergmann, D. m. W., 1909. 611. - Krehl, Pathologische Physiol,, 1910. 430 (Lit.). - Lüthje, Areh. f. exp. Path. u. Phmk., 1903. L. 169. - v. Noorden, Ärtekongr. Bulapest, 1909. - Zimmermamn, Vet., 1901. 657.

\section{Diabetes Insipidus.}

Diabetes insipidus is a functional disturbance of the kidneys which causes the animal permanently to require large amounts of water for the elimination of the substances that are secreted with the urine, so that it voids large amounts of water'y urine free from albumen and sugar and having a low specific gravity.

According to this definition polyuria (Lauterstall), which is a transitory condition, is not to be classed with diabetes insipidus; except after excessive water drinking, it oceurs rather frecuently in the most varying diseases in which copions exudates are absorbed in a short time (pneumonia, pleurisy, ete.), then in certain chronic infections diseases (tuberculosis, glanders) and in certain affections of the central nervous system (tumors, hemorrhages, inflammations). That form of polyuria which occurs in the course of many acute or chronic kidney inflammations, usually together with albuminuria must also be considered only as a partial symptom of the diseases named and can therefore not be elassed with diabetes insipidus. The same is true for the transitory polyuria after the ingestion of irritating substances. In contrast to this transitory or symptomatic polyuria, diabetes insipidus occurs as an independent anomaly.

French medical anthors differentiate a diabetes insipidus rerus and a diabetes azoturiens. In the former are comprised those forms in which with an inereased total amount of urine the solid constituents are not, or only slightly, inereased, while in diabetes azoturiens the solid constituents are secreted in amounts far above normal. (If the amount of urine is normal and only the solids are inereased, the condition is designated simply as azoturia.) In veterinary medieine exact analytical examinations in this direction have yet to be made.

Distribution. As an independent disease in the meaning described above diabetes insipidus has been diagnosed witl certainty in animals only comparatively rarely (Hayne, Veith, Moiroud, Dammann, Perrin, Dieckerhoff, Schindelka), but from the point of view of the practician those far more frequent cases may be added to it which, while sccurring in consequence of improper feeding, take a chronic course. In veterinary medicine diabetes insipidus is generally understood in this sense.

Etiology. The disease is observed most frequently in horses, especially if these are fed for some time with mouldy feed. In this respect oats may be of importance, as it rapidly assumes a peculiar mouldy odor when it is stored up on the ground in large quantities without heing turned over from 
time to time. The feeding of such oats soon produces polyuria which may pass into a pathological condition similar to diabetes insipidus if the spoiled oats is continued for a long time. In former times when oats was often transported on ships on which it was impossible to turn it over at frequent intervals the disease was observed more frequently, and sometimes in an enzootic form. Todlay it occurs far less often because the oats is usually shipped in bags (Dieckerhoff). Other mouldy kinds of feed may likewise produce a similar effect; for instance mouldy hay, peas, vetches, etc., but these can not nearly as often be brought into connection with the appearance of the disease, perhaps because the coarse feed, even in large quantities, can be ventilated more easily than is possible with oats. Moreover it is not impossible that peculiarly toxic substances are formed in mouldy oats.

Certain plants are crerlited with the ability to eause diabetes. As such are considered the different species of Adonis and Anemone, and above all the Cynauchum s. Asclepias vincetoxicum, the latter especially since Veith succeeder in causing polyuria in sheep ly feeding it to them. According to Röll frozen fodder and slop are also at times the cause of diabetes.

Aside from transitory polyuria true diabetes is occasionally observed as a complication of affections of the central nervons system, but such cases are hardly known to have occurred in animals.

Other etiological factors are: The continued administration of diuretics, cold, traumatic accidents to the liver or to the vertebral column; some authors (Cagny, Cagnat) assume an infection as the cause of cases which occur in greater number's.

Horses which are used for heavy work and, according to Moiroud, especially stallions appear to be most disposed to diabetes insipidus, while age and race have no influence. The disease usually occurs more frequently in summer than in colder seasons.

The nature of diabetes insipidus is unknown. This much is certain that a transitory polyuria can be produced artificially by injuring a certain spot in the fourth ventricle near the Calamus scriptorius (Cl. Bernard). Later it was ascertained that injuries to other portions of the brain, especially the vermiform process of the cerebellum, as well as the division of the lumbar cord, or of the splanchnic nerve also produces similar results. These experiments permit the conclusion that diahetes insipidus perhaps also originates from nervous eanses, and according to this view the effect of mouldy fodder wonld have to be explained thus, that substances developed in it produce polyuria by the intermediation of the nervous system. It may be assumed that through disease of the vasomotor nerves in the kidneys a constant arterial hyperemia is maintained in consequence of which the amount of urine or its content of water is increased. Against this view stands the demonstration recently afforded hy E. Meyer and F. Müller that diabetes insipidus in man represents in fact only a secretory anomaly of the kidney's through which the organism requires very great amounts 
of water for the elimination of solid urinary constituents, primarily of urea and sodium chloride, or the kidneys have lost the faculty to reabsorb the water secreted into the glomeruli.

Anatomical Changes. The autopsy findings are sometimes entirely negative; in other cases changes are found in the central nervous system (in Holzmann's case the spinal cord of a dog was surrounded in almost its entire length by myxomatous tissue and several dural sarcomas) or signs of an earlier general affection, as well as secondary changes in the organ. The kidneys are either entirely normal or show only unimportant changes (cloudy swelling, hyperemia, etc.).

Symptoms. After feeding mouldy oats or other spoiled fodder the symptoms develop in a short time, sometimes within 3 to 5 days. In other cases the evolution is gradual so that the disease is only suspected from the striking emaciation and debility.

The most essential and important symptom consists in a decided increase in the amount of urine. The animals secrete large quantities of urine at brief intervals, the total amount in 24 hours being several times the normal daily output, so that horses may pass as much as 40 to 60 liters, dogs 3 to 4 liters. The urine is watery, without odor, of very low specific gravity which may be continuously around 1.001-1.002. Foreign constituents, especially albumen and sugar, cannot be found and microscopical examination in this direction is also negative. While the urine is usually voided easily, this may later on become somewhat painful because of catarrhal swelling of the urethral mucous membrane.

A second symptom, which is never absent, is a considerably increased thirst (polydipsia) which causes the animals to drink surprising amounts, horses as much as 100 liters of water, dogs up to 10 or 15 liters per day, and if the water is not given them in sufficient amounts, they will drink even bad and illsmelling water, as well as their own urine. The amount of urine corresponds to the amount of water ingested either alone or with the food, or it exceeds it at most only transitorily. (The idea that the skin and the mucous membranes of diabetics can absorb water from the air lacks all foundation.) Comparative researches have proved that with an equal ingestion of water the diabetic organism secretes more urine than the bealthy.

The appetite varies greatly. At first the animals take palatable fodder willingly, but later they refuse even this or eat only a little from time to time. The mucous membranes and the skin become dry, the hair dull and coarse. Schindelka observed in a dog cataract, abscess formation in the perianal glands and in the prostate. Sooner or later emaciation commences and may increase either gradually or rapidly. 
Course and Prognosis. The polyuria which develops after the ingestion of spoiled fodder usually improves soon and disappears if the fodder is changed in good time. But the longer the injurious factor has been active the smaller becomes the probability of complete cure and the longer will be the period of convalescence. In the neglected cases which have passed into actual diabetes the condition becomes gradually aggravated as far as the establishment of absolute cachexia, when the animals perish if a complication has not caused death in the meanwhile. The disease usually extends over months, in rare cases it lasts even more than two years (Grève, Dieckerhoff).

If the diabetes insipidus appears spontaneously or in consequence of a nervous affection it also usually takes a prolonged course with fatal termination.

Diagnosis. Simple polyuria is differentiated from diabetes insipidus by its transitory character, the polyuria of diabetes persisting in spite of appropriate regulation of diet, and being moreover accompanied by emaciation and debility of the animals. Diabetes mellitus is differentiated from diabetes insipidus by the increased specific gravity and the sugar content of the urine, chronic nephritis by the existing albuminuria.

On the basis of investigations by $\mathrm{E}$. Meyer and F. Müller true diabetes insipidus is diagnosed by determining the toleration for sodium chloride. This is done as follows: The daily amount of urine is ascertained for several days during which food poor in albumen and sodium chloride is given. Then $20 \mathrm{gm}$. of sodium chloride per day is added to the same kind of food for one or several days. If the amount of urine had previously been diminished and now shows an unduly great increase, the ease in question is one of true diabetes insipidus, since in polyuria the ingestion of sodium chloride has no particular influence on the amount of urine secreted. An exception is observed only in the polyuria of indurative nephritis which, however, is easily recognized from other symptoms (Lüthje).

Treatment. If the disease is due to faulty diet a change of fodder is requisite, in which the spoiled forder is replacerl by feed of unobjectionable character. If the disease has existed not more than a few weeks and the strength of the animal is still preserved the symptoms may be removed by a simple regulation of diet. If a change of food is not possible, the fodder at hand may be improved materially by frequent turning over or ventilating and by removing the badly spoiled portions. It appears, however, even more appropriate to feed small portions of the spoiled food mixed with such that is perfect. It is to be observed that a regulation of diet renders good service also in such cases in which the disease cannot be referred to inappropriate keeping of the animals. For horses the best feed is good, clean and dry oats and palatable hay, but in the warm seasons green feed should be given. According to 
experiences in human practice the food-stuffs should be as free as possible of albumin and table salt because the elimination of the latter and of the urea requires great amounts of water in this discase. In true diabetes insipidus a limitation of the water supply does not appear necessary, but it should be carried out in those cases which are similar to diabetes but must be classed with polyuria, which is always aggravated after the free drinking of water (Leblanc, Cagnat). In horses, for instance, the amount of drinking water should be reduced to 20 to 24 liter's per day. Finally, the animals must be kept from work for 1 to 2 weeks after the symptoms of the disease have disappeared.

The drug treatment of diabetes insipidus promises 110 results, although rasoconstricting remedies (Ergotin, Ext. Hydrastis) have always been employed.

Literature. Almy, Bull., 1899. 215. - Cagnat, A. d'Alf., 18s4. 168. Dammann, D. t. W., 1898. 125. - Gerlardt, Der Diabetes insip., Wien, 1906. Haase, B. t. W., 1898. 109. - Hertwig, Mag., 1859. 487. Holzmann, D. Z. f. Tm., 15s7. Xiı̈. 197. - Meyer, D. A. f. kl. M. 1905. LxxxilI. 1. - Pr. Mril. V.b., 1s99-1901; 1906. - Schindelka, Monh., 1893. IV. 135. - Sehlampp, D. Z. f. Tm., 1884. X. 133. - Siedamgrotzky, S. B., 187s. 57 ; A. f. Tk., 1895. XXI. 467. - Stockfleth, Rep., 1874. 60. - Veith, Rep., 1845. 261.

\section{Diabetes Mellitus.}

Diabetes mellitus is a chronic disease in which the blood, owing to peculiar changes in metabolism, contains an excess of grape sugar which is eliminated unchanged by the kidneys, so that the urine contains sugar either permanently, or at least temporarily.

The blood of healthy animals contains about $0.1 \%$ of sugar, and this proportion is maintained with only slight variations, an pxcess of sugar being promptly eliminated by the kidneys. By means of special methods it is always possible to find traces of grape sugar in specially heated large amounts of urine, particularly in cattle and sheep (physiological glycosuria). Certain drugs or poisons (morphine, chloroform, ether, phloridzin, chromic acid, adrenalin, etc.), also certain accidents to the organism (shock to the nervous system extensive convulsions, rabies, acute infectious bulbar paralysis, ete.) may even produce a glycosuria which ean be demonstrated with the usual methods. It has not been determined whether an alimentary glycosuria (after the absorption of very large amounts of sugar in the intestine) occurs in otherwise healthy animals. All these conditions with transitory elimination of sugar (glyeosuria, mellituria) cannot be ealled diabetes mellitus any more than the presence of milk sugar which appears in the urine of nursing animals (laetosuria) after a sudden suppression of the milk secretion, with particular frequency in the paresis of parturition.

History. The disease has long been known in human medicine (Dobson and Pool having demonstrated the presence of grape sugar in human urine in 1775), but was observed in animals in only com- 
paratively few instances. The reason may lie in the fact that urinary analysis did not receive proper attention in veterinary medicine until quite recently. Interesting cases of diabetes mellitus have been deseribed by Thiernesse, Rueff, Leblanc, Saint-Cyr, Haubner, Heiss, Dieckerhoff, Fröhner, Eher, Schindelka, Eichborn, Penberthy, Lindquist, Liénaux, Fettick, Sendrail \& Cuillé, Bohl, Krüger, Preller, Eisenmenger and others.

Occurrence. Diabetes mellitus is, as a rule, very rare in domestic animals, dogs being most frequently affected, horses far less often (according to Fröhner, one out of 10,000 dogs is diabetic; according to Eber, one out of every 1,000). Aside from $\operatorname{logs}$ and horses, the disease has heen observed only in cattle (Darbas, Girotti) and monkeys (Leblane), which does not, however, exclude the possibility of its occurring in other animals.

Ftiology. There is at present no doubt that diabetes mellitus is etiologically not a uniform disease. It can be produced by certain organic affections of the central nervous system, of the liver or pancreas, and occasionally occurs independently of any demonstrable organic disease. Recent investigations indicate that diabetes mellitus often develops on account of a pancreatic anomaly, especially of the islands of Langerhans, which may be demonstrated only with the microscope.

The influence of the central nervous system upon the sugar cleavage in the organism was shown by the well known experiment of Cl. Bernard in which it was found that an injury to a certain portion of the medulla oblongata, on the floor of the fourth ventricle near the vagus root ("sugar puncture," "pirqûre") leads to a transitory glycosuria. Other authors found later that the same takes place after lesions of other portions of the central nervous system (cerebellar worm, cervical cord) or of the cervical sympathetic. The central nervons system exerts its influence exclusively or mainly by the intermediation of the splanchnic nerve, at least the "picfurre" does not produce such results after the nerve is divided. The glycosuria occurs here evidently by the irritation of the puncture, causing the liver to discharge its glycogen suddenly so that the blood becomes charged with grape sugar. This agrees with some observations in diabetic human patients in whom these portions of the nervous system, the abdominal sympathetic, and principally the solar ganglion or the coeliac plexus were found altered. All these experiences indicate that certain parts of the central nervous system or of the sympathetic may be concerned in the origin of diabetes mellitus (neurogenic diabetes).

The liver is an essential factor in the formation of sugar, becanse in this organ the grape sugar which is ingested with the food or formed and absorbed during the digestion of carbohydrates is transformed into glycogen, and thus the blood is kept from being flooded with grape sugar during each period of digestion. For this reason it is possible, in case the function of the liver is disturbed, that a portion of the grape sugar which was absorbed in the intestinal canal is not 
changed into glycogen, but passes into the general circulation. While in eases of diabetes mellitus lesions in the liver only were repeatedly observed by several authors in human as well as in veterinary practice (Fröhner, Heiss, Storch, Penberthy, Eber, Fettick and others), this does not justify the assumption that in these cases the diabetes had actually been caused by the hepatic disease. Aside from the changes in the liver there may be slight alterations in the pancreas (Herzog), and on the other hand the liver disease may be a consequence of the diabetes. Especially the fatty liver which is frequently mentioned by veterinarians and considered by some as the cause of diabetes, is very probably due, as is fatty infiltration in general, to the deposit of fat from other parts of the body, without interfering with the functioning of the liver cells. The diabetes produced in dogs by extirpation of the pancreas is almost invariably followed by fatty degeneration of the liver (Naunyn).

Recently the experiments of $\mathrm{v}$. Mering and Minkowski have afforded the proof that a severe diabetes follows the complete extirpation of the pancreas, while it does not develop if at least one-fifth of the gland is left or has been transplanted successfully under the skin before the removal of the gland. If the entire gland has not been excised, only a slight diabetes develops, and especially after a generous ingestion of carbohydrates a glycosuria becomes noticeable but disappears again on insufficient diet.

These experiments were followed by a long series of control investigations and were responsible for a great many contributions to literature putting the theory of diabetes in an entirely new light. The occurrence of the disease in affections of the pancreas (pancreasdiahetes) was indeed shown to take place by older observers (Frerichs, Windle, Hansemann), hut the theory of pancreatic diabetes received a greater vogue only since more attention was paid to the behavior of the islands of Langerhans in the pancreas, to whose influence upon the carbohydrate metabolism Ebner had already called attention. Many authors (Ssobelow, Opie, Weichselbaum \& Stangel, Schmidt, Herzog, Sauerbeck, Halász) found alterations (simple atrophy, sclerosis, colloidal degeneration, disease of the vessel-walls) in the islands of Langerhans, even in those cases in which the secreting glandular tissue was entirely intact. The participation of the pancreas in the causation of diabetes mellitus in domestic animals is proved by cases of Liénaux, Sendrall \& Cuillé and of Bohl, in which a considerable atrophy of the gland was the only pathological finding. Eber also found atrophy in one case. Fettick observed carcinomatous degeneration of the gland in a case of general carcinomatosis, while Bohl noted diffuse parenchymatous degeneration in a dog, Preller ehronic inflammation in the pancreas of a horse. On the other hand diabetes failed to develop in a case of atrophy of the pancreas in a dog observed by Muiller. It is to be expected that diabetes mellitus will be brought more frequently into relation to pancreatic disease when this gland receives greater attention by veterinary authors. In man changes in the islands of Langerhans can not infrequently be determined simply by microscopical examination.

The views still differ as to whether pancreatic diabetes represents solely the consequence of some disease of the islands of Langerhans, or whether it may develop, on the contrary, through any diffuse alteration of the pancreas.

On the basis of his experiments, E. Pfliger has recently assumed that the antidiabetic action of the pancreas, which regulates the cleavage of sugar, is influ- 
enced by a nerve center situated in the walls of the duodenum. Even in earlier times an enterogenous or pancreato-enterogenous origin of diabetes mellitus hafl been assumed.

It appears thus from the observations and experiments eited above that a regular system of organs is concerned in the regulation of the cleavage of sugar. The action of the liver, by which the grape sugar, both that absorbed from the intestine and that formed in the organism from albumin and fat, is retained by being transformed into glycogen and yielded up again according to need, requires the cooperation of the pancreas. This probably exerts its action by an internal secretion, and substances are probably then formed which, immediately or through the intermediation of the nervous system, on the one hand influence the formation of glycogen in the liver, but on the other hand enable the body cells, and primarily the muscle fibers, to effect the cleavage of the grape sugar and finally to oxidize it. Besides these, however, other organs probably also belong to the system which regulates the blood-sugar, namely, the adrenals, the thyroid gland and the hypophysis cerebri, all these sugar-regulating organs being in communication through the nervous system. It follows that a prolonged functional disturbance in any one link of this system might give rise to diabetes mellitus, no matter whether the functional disturbance originated in a lesion that could be demonstrated anatomically or whether it developed without such a lesion.

Predisposition. In the great majority of cases that have so far been reported, the disease occurred in older animals. Schmitt observed a $\log ^{\circ}$ acquire it after swimming across a hroad river. Heredity also appears to be of importance.

Pathogenesis. If an excess of grape sugar passes from the liver into the blood, or if the cleavage of the grape sugar is incomplete a hyperglycemia follows (Preller found $0.521 \%$ of sugar in the blood of a horse). The excess of the grape sugar is then eliminated by the kidneys. Since the organism requires a certain amount of energy for the maintenance of the vital functions, it replaces the loss occasioned by the elimination of the sugar in such a manner that it uses up, at least to a certain degree, the sugar which is formed by the disintegration of the alimentary and of the organic albumin and which is probably more readily available, at the same time breaking up its own fat in a greater degree. To this fact are due the gradually increasing weakness and emaciation. In severe cases the sugar formed from the proteids and possibly from the fat is used up in always smaller amounts, which must lead to an increased combustion of fats without limiting the active disintegration of proteids. All these factors cause not only exhaustion, but also an accumulation of oxidation-products of fats (oxybutyric acid, azeto-acetic acid, acetone [Geelmuyden, Schwarz, Magnus, Levy]) in the blood and thus lead to an acid toxemia.

The diabetic disturbance in the metabolism easily leads to nutritional disturbances in the various tissues, especially in the lens of the eye and in the renal tissue. The insufficient tissue nutrition may further facilitate the localization of 
pathogenic bacteria, although the saturation of the tissues with sugar is here an important factor.

Anatomical Changes. The autopsy findings differ from case to case and may indeed be entirely negative except for secondary changes. In the central nervous system hemorrhages, new-formations, etc., are sometimes found in the medulla oblongata or in its immediate neighborhood. Fatty liver or an increase in the connective tissue of the liver are frequent in dogs, but less so in horses. In the pancreas there is at times connective tissue proliferation, atrophy of the glandular tissue or calculus formation. There are finally, as a rule, evidences of anemia and serous infiltration.

Symptoms. The clinical picture always develops insidiously. At first the animals show a degree of dullness of the sensorium, and fatigue; they perspire and easily tire whether at work or at exercise. Simultaneously a gradual emaciation becomes manifest in spite of a good appetite, and often in spite of the greedy eating of large amounts of food.

The most characteristic changes are found in the urine. The amount is, with few exceptions (diabetes decipiens), at first more or less increased and may rise to 3 to 5 times the normal daily output. It is discharged at brief intervals and without effort, but later micturition may become painful, after a catarrh of the external urinary passages or an eczema around the urethral opening has developed. The urine is pale in color, being almost like water if the polyuria is marked; of a peculiar sweetish odor, or somewhat like the odor of fruit or chloroform; it is always acid in carnivora, occasionally so in herbivora, and remains unchanged on standing for a relatively long time. The specific gravity varies between 1,040 and 1,060 , but is not rarely much lower in cases in which only small amounts of sugar are present in the presence of polyuria. The greatest importance must be attributed to the content in grape sugar which, however, may vary within wide limits, not only in different cases, but also in the same patient. In contrast to diabetes in man, where the sugar content is usually slight in the initial stage of the disease and only later increases gradually, in diabetic animals which usually come under the observation of the veterinarian only in advanced stages of the disease, large amounts of sugar are found as a rule (in horses $3.75-7.33 \%$; in $\left.\operatorname{logs}_{4} 4-10 \%\right)$. The sugar content is also decidedly influenced by the amount, and even more by the nature of the food. The more sugar or amylaceous food is ingested the more sugar is eliminated by the kidneys. On the other hand, especially in the first stage, the sugar may disappear even entirely from the urine after exclusive feeding with nitrogenous food-stuffs, during the abstention from food, during the course of intercurrent febrile diseases, after strenunus exertion. Preller found 
in the case of a diabetic horse that the urine contained more sugar in the morning than in the evening and that the amount was increased after psychic excitement, while hard work did not cause a diminution in the amount of sugar present. There are cases where, in spite of uniform feeding, the amount of the eliminated sugar does not remain constant, the urine being temporarily free from sugar (diabeces mellitus intermittens); if however the disease is far advanced, the glycosuria never ceases entirely.

For the practical demonstration of grape sugar the fermentation test is best adapted. The simplest way of making this test is as follows: A bit of yeast as large as a pea is added to a test tubeful of urine which is closed with a perforated cork, a U-shaped thin glass tube being inserted in the perforation. The cork is fitted so that no air bubbles remain on the surface of the urine. The test tube is then inverted and permitted to stand at room temperature, or better, near a stove for 24 hours. If grape sugar is present, the action of the yeast procluces alcohol, and also carbonic acid which collects in the upper portin of the test tube aul forces the urine downward. In order to make quite sure it is well to prepare two control test tubes, one of which is filled with normal urine, the other with a solution of grape sugar, to both being ariled a piece of yeast, in order to determine wliether the yeast itself produces carbonic acid and whether it is active. If the fermentation test is positive, grape sugar is surely present.

For a quantitative test with the fermentation reaction graduated glass tules or other apparatus must ke employed. None of these apparatus, not even Lohnstein's saccharimeter, is adapted for an exact quantitative test, but they are quite useable for practical work, especially for determining variations in the elimination of the sugar.

Preller found the reaction with aniline dyes sufficient for practical purposes: 10 ce. of urine are heated in a test tube to about body temperature; on alding a small amount of finely ground gentian violet or methylene blue, diabetic urine is colored blue immediately and without shaking.

A relatively easy test is that of Tronmer: One part of official potassium or sodium hylrate is added to three parts of urine; to this mixture a $10 \%$ solution of sulphate of copper is added drop by drop and shaken up until a trace of the precipitate remains on shaking. The upper layer of the mixture is then heated slowly, when in the presence of sugar a yellow or red finely granular precipitate occurs, even before the liquid commences to boil.-Porcher found a modification of Trommer's test useful: in this the reagent (Fehling's solution) and the urine are boiled in separate test tubes and then the urine is poured carefully over the reagent.

In human urine physicians have exceptionally found levulose, pentose, dextrin and inosite, kesides the grape sugar. There may also be found acetone, organic acids (formic acid, oxybutyric acid, azetoacetic acid) and albumen, which mostly appears in consequence of a complicating nephritis. (In the urine of a sick horse the authors found very much oxybutyric acid and azetoacetic acid; the same was observed by Preller.)

Another constant symptom is a striking increase in the sensation of thirst (polydipsia), and an abnormally increased appetite (polyphagia) is frequently observed. The mucons membranes of the mouth are dry, the gums at times loosened and sensitive.

The lens of the eye often becomes opaque (gray cataract; cataracta diabetica) commencing from the center, usually in both eyes and leading gradually to complete blindness; only in isolated cases may the opacity disappear temporarily if the disease is not too for advanced and the animals are fed suitably. Sometimes corneal opacity is met with, possibly together with ulcerations (Heiss), also inflammation of the iris, hemorrhage beneath the retina or a loosening of the latter. 
Sometimes the respiratory organs become affected secondarily with symptoms of laryngeal or bronchial catarrh, to which may later on be added pueumonia or even gangrene of the lungs. Krüger noted a sweetish odor of the exhaled air. In the terminal stage there appear symptoms of cardiac debility. In dogs an enlargement of the liver may be detected. Darras observed in a diabetic dog necrosis of the tip of the tail, Krüger saw in a horse furunculosis, but Preller found phlegmosis in the subcutis, which however healed completely in contrast to human experience.

Other organic diseases which may have a causal connection with diabetes are discovered only rarely during the lifetime of the animals. In this respect the case of Preller's is of interest, in which it was possible to diagnose the disease of the pancreas by means of the Cammidge test while the animal was alive.

In the further course of the disease the debility increases more and more; finally apathy and stupor appear; the amount of sugar gradually diminishes and sometimes the sugar disappears entirely. There is diarrhea, and, after complete exhaustion of the patient, death occurs with symptoms of convulsions or during coma.

By coma diabeticum or intoxicatio diabetica is meant in human medicine a peculiar pathologic eondition which develops toward the end of diabetes mellitus and is usually followed immediately by death. The respiratory movements suddenly become very deep, labored and hastened; at the same time or a little later the patient becomes unconscious, or symptoms of collapse appear, or great restlessness develops, followed immediately by death with symptoms of heart failure. In other cases the patients are tormented by headache, their motions are staggering and the end supervenes, the patients being in a stupor. The exhaled air then smells strongly of azetone. This condition is usually referred to the accumulation of organic acids (oxybutyric acid, azetoacetic acid) in the blood. Fröhner, Eichhorn, Liénaux have each observed a similar condition in a dog, and the authors themselves met with it in a horse.

Course. Diabetes mellitus always takes a chronic course. In the cases hitherto observed its duration varied between 1 and 12 months. It must however not be forgotten that the disease was always recognized only in a far advanced stage. According to experiences in human medicine the disease may persist for years, although in young animals its duration appears to be short.

Diagnosis. If the urine permanently contains considerable amounts of grape sugar the diagnosis of diabetes mellitus appears to be perfectly certain. The disease has otherwise no characteristic symptoms and can therefore be recognized with certainty only by means of regular urinalyses. Nevertheless there are a number of symptoms which should cause the affection to be suspected and should suggest a chemical analysis of the urine; such are particularly the increasing emaciation, 
the debility in spite of good appetite, then the increased thirst, polyuria, and the simultaneous occurrence of eataract. In the presence of polyuria with a clear, watery urine of high specific gravity the existence of diabetes mellitus is very probable.

Prognosis. In the cases that were observed so far in animals the disease showed a gradual progression, at most with transitory remissions, and always ended in death. Only Walley reports a cured case, but it is possible that either this was a transitory glycosuria or that the supposed cure was in fact but an intermission of the disease. The more sugar the urine contains, the sooner the fatal termination occurs. If the sugar content persists unaltered in spite of exclusive feeding with proteids and fats, the prognosis is much less favorable than if this diet leads to a diminution in the amount of sugar, or if this periodically disappears entirely without a simultaneous loss of weight. Complications always influence the course unfavorably and hasten the fatal termination.

Treatment. This consists essentially in limiting the supply of carbohydrates and in feeding with food-stuffs rich in fats and proteids. In severe cases even the albumins should be diminished to such a degree that it is just possible to maintain the N-equilibrium. The limitation of albumins appears indieated for the reason that in severe cases even that sugar which is formed from albuminous substances is eliminated unused. In order to determine the degree to which carbohydrates and albumins are to be limited, it would be necessary to test the toleration, that is, to determine the amount of carbohydrates and albumins borne without any sugar being eliminated with the urine. Of this method, which is quite customary in human medicine, no advantage has as yet been taken in veterinary practice.

Carnivorous animals are, in lighter cases, nourished most suitably with fat meat, eggs, bran-bread, or with oat meal, to which a generous amount of fat has been added; in severe cases the amount of meat must also be limited and the requirements of the organism for albumin must be supplied by the addition of vegetable albumins ("Roborat," rice-albumin, etc.). If acetone bodies appear in the urine in considerable amounts, it is necessary, according to experiences in human medicine, to limit the supply of albumin and especially of fat, and for a time to return to the carbohydrates (oats, bread, milk, possibly levulose). In herbivorous animals it is particularly difficult to carry out an appropriate dietary; probably the best foodstuffs for such animals are oats, good hay, bran and oil-cake. Preller caused a decided diminution in sugar in the urine of a horse by replacing the accustomed grain feed by oats, bran and oil-cake, but simultaneously a deterioration in the nutrition of the patient was observed. All sick animals should be spared exertion. 
Of drugs, sodium bicarbonate deserves mention and should be given regularly; in severe acidosis or diabetic coma large amounts are to be administered by mouth, or hypodermically dissolved in physiological salt solution. Carlsbad water or salt, also carbonate of ammonia are occasionally of use. Opium preparations in large doses (to dogs up to 0.5-1.0 gm. per day) have been recommended as they are capable of diminishing for a time the glycosuria. No results worth mentioning have been observed to follow the arsenic treatment.

Literature. Bohl, A. f. Vet.-Wiss., 1906. 569 (Lit.). - Cadiot, Études de pathol., 1899. 327 (Lit.). - Dammann, Hann., Jhb., 1876-77. 100. - Darbas, Rev. vét., 1890. 357. - Darras, Bull., 1906. 687. - Eber, Monh., 1898. IX. 97. - Eich. liorn, S. B., 1891. 184. - Falta, Z. f. kl. M., 1908. LXVI. 401. - Fettick, Vet., 1899. 277 (Lit.). - Fröhner, Monh., 1892. III. 149. - Girotti, N. Ere., 1896. 69. - Herxheimer, V. A., 1906. CLXXXIII. 228. - Krüger, Z. f. Vk., 1907. 488. - Liénaux, Ann., 1898. 190. - v. Mering \& Minkowski, A. f. exp. Path. u. Phmk., 1590. XXY. 371. - Minkowski, Ergebn. d. Path., 1894. I. 1. Abt., 69 (Lit. on pancreatic diabetes). - Müiller, Dresd. B., 1906. 162. - Naunyn, Der Diabetes mell., 1906. Penberthy, J. of Comp. Path., 1894. 184. - Pflüger, Pfliigers A., CIII. 1; CXYIII. 265. - Porcher, Bull., 1903. 409; Rev. gén., 1905. VI. 49. - Preller, Über Diab. mellitus beim Pferle, Diss. Bern, 1908 (Lit.). - Prietsch, S. B., 1902. 63. Sauerbeck, Ergebn. d. Path., 1902. VIII. 2. Abt., 538-697 (eomplete literature on diab. mell. in general). - Schinlelka, Monh., 1893. IV. 132. - Schnitt, W. f. Tk., 1863. 65. - Sendrail \& Cuillé, Rev. vét., 1906. 229. - Walley, J. of Comp. Path. 1893. 70 .

\section{Diseases of Metabolism which Particularly Affect the Osseous System.}

(a) Rachitis. Rickets. Rhachitis.

(Gliederkrankheit; Englische Krankheit; Zwergwachs [German]; Rhachitism [French].)

Rachitis is a disease of young animals which is due to a disturbance in metabolism and is characterized by the persistence of new-formed bony tissue in the osteoid condition, by proliferation of the cartilaginous tissue, and by an irregular advance of the lines of ossification, the result being manifold deformities of the skeleton.

Occurrence. Whether the disease oceurs in all parts of the globe or, as is the case in man, is almost exclusively limited to the temperate latitudes, cannot be ascertamed by any information in veterinary literature. The affection is frequent in all countries of the temperate zone.

Almost without exception the young animals become affected only during the first year of life and most frequently when only a few months old or after being weaned. The animals which acquire the disease most often are young pigs and dogs, lambs and kids, much more rarely calves and rabbits; the disease is observed quite often in wild carnivora which are raised in captivity, also in monkeys; of fowls, chickens are affected 
most often, less so pigeons and water fowl (Zürn), when they are $1 / 4$ to $1 / 2$ year old or even younger. (Haubold observed the disease in eight-weeks-old geese which had been confined for fattening. Blooded and large breeds or such as mature early or grow rapidly are in general more apt to acquire rickets than breeds of animals that are mostly kept out doors and are hardened. Among hogs the disease affects relatively frequently the English breeds and their crossings, among the dogs the Newfoundlands, the Danes and the setters. In animals living in herds, especially in pigs and lambs, rachitis usually reaches an enzootic distribution and this canses a very decided economic loss. On the other hand the disease is not observed in wildiiving animals.

Etiology. In the production of rickets in domestic animals the deficiency in lime of the organism is undoubtedly the most important factor. This may be concluded above all from the fact that the disease commences most frequently ir: the breeds which mature early, at the time of the most rapid development or after the nursing period is over, that is in that period in which there is most likelihood that the ingestion of lime is insufficient in proportion to the bony growth. Moreover numerous incontrovertible observations directly established the relation of rickets to food which is poor in lime. Finally a considerable number of positive experimental results in this direction are at hand.

A deficiency of lime in the organism will probably occur most frequently through an insufficient calcium content in the food. In nurslings rachitis develops, comparatively rarely, through an insufficient calcium content of the mother milk, when the mother animals are nourished with food poor in lime. (According to Dammann healthy young sucking animals become ill if they are put to a mother animal whose young is affected by rachitis.) Moussu observed a similar symptom complex as that seen in rachitis of roung pigs in sncking lambs, whose mothers were fed generously with cut carrots, but he looks upon it as an asthenic condition. It happens very rarely that the artificial raising of young animals with alien milk is of importance in this respect, although this milk may become injurious in so far as it may contain less lime than the homogeneous milk, and further the lime salts of such milk may be used up less completely (Uffelmann, Brüning) hecause the presence of alien enzymes may lead to an insufficient assimilation of the milk.

The calcium content of milk varies rerr considerably in the different animal species. Cow's milk contains an average of $1.7 \mathrm{gm}$. of lime and $1.8 \mathrm{gm}$. of phosphoric acid; goat's milk, $1.9 \mathrm{gm}$. of lime and $3.0 \mathrm{gm}$. of phosphoric acid; sheep's milk, $3.1 \mathrm{gm}$. of lime and $3.0 \mathrm{gm}$. of phosphoric acid; mare's milk, $1.2 \mathrm{gm}$. of lime and $1.2 \mathrm{gm}$. of phosphoric acid; and hog's milk, $4.3 \mathrm{gm}$. of lime and 4.0 gm. of phosphoric acid per liter (Stutzer, Kellner), while the corresponding values in dog's milk are $3.01 \mathrm{gm}$. and $4.11 \mathrm{gm}$. (Wedemeyer). 
Concerning the assimilation of the lime salts of the milk, Uffelmann found that in infants 78 per cent of the lime of the mother's milk is absorbed, but only 25 per cent of the lime of cow's milk. In calves 97 per cent of the lime of cow's milk is assimilated according to Soxhlet. Brüning left two out of four young dogs of the same litter with their mother and fed the other two from the sixth day on with cow's milk, one of them receiving the milk cooked, the other raw. The first two dogs developed quite normally, but the other two remained back in their development; their belly seemed enlarged, the ends of the costal eartilages thickened; the dog fed with raw eow's milk was also feeble, and the fontanelles hail remained open. Similar results were obtained in dogs fed with goat's milk, as well as in rabbits, guinea pigs and kids fed with alien milk.

An opportunity for a deficiency in lime to occur in the organism is afforded more especially in weaned animals. EXperience shows that rachitis develops not infrequently in carnivora if they are put on an exclusive vegetable diet, and aside from the small calcium content of vegetable food, the fact is here of importance that the lime salts of plants are used up much less completely than those of animal food (Bunge, Bertram). On the other hand a strict meat diet from which the bones are excluded may also give rise to the development of the disease. In herbivora the principal blame attaches to one-sided feeding with non-nutritious kitchen refuse, potatoes, mealy food substitutes and cow's whey, ground meat, barley and bran, which are known to have a low calcium content. In colts especially the exclusive feeding with bran causes rickets (the so-called bran-disease) while in pigs exclusive feeding with potatoes, barley or maize is a factor. Under the influence of unfavorable conditions of soil and weather (see brittle bones) of course even those food stuffs which ordinarily contain a sufficient amount of lime may become deficient in it. The varying calcium content of drinking water, and a relatively low calcium content of drinking water is probably of importance only in combination with other causes. Nevertheless an abnormally high content of water in sulphuric acid may become injurious by causing the formation of calcium salts which are only slightly soluble.

By considering the calcium content of the food on the one hand and the calcium requirements of the growing animals on the other, which vary greatly according to the species and breed, it is always possible to determine whether in a given case a deficiency in lime could occur in the organism.

Content in calcium and phosphoric acid in the most important food stuffs, for each 1,000 parts, after Stutzer: Coarse feed-Lucerne, 25.2 parts of lime $(6.5$ parts of phosphoric acid) ; red clover, 20.1 (5.6) ; bastard clover 13.6 (4.1); esparsette, 16.8 (4.6); vetch, 16.3 (6.2); peas, 15.6 (6.3); lupine hay, 8.8 (5.8); meadow hay of good quality, 9.5 (4.3); of poor quality, 5.4 (2.3); second crop ("grummet"), 5.1 (4.1); wheat straw, 2.7 (2.0); barley straw, 3.3 (1.8); oat straw, $4.3(1.5)$; cornstalks, $4.9(3.0)$; pea straw, $15.9(3.5)$; retch straw, 15.6 (2.7); wheat chaff, 1.7 (4.0); oat chaff, 4.0 (1.3); barley beards, 12.5 (2.4). Green feed-Lncerne, 8.5 (1.6); red clover, 4.5 (1.5); bastard clover, $2.9(0.9)$; esparsette, 4.0 (1.1); meadow feed, 2.8 (1.2); rich grass, 2.6 (1.9); green corn, 1.4 (1.0); carrot leaves, 1.5 (1.0). Tubers and roots-Potatoes, 0.3 '(1.2); beets, $0.3(0.6)$. Grain-Oats, $1.0(7.0)$; barley, $0.1(6.6)$; corn, $0.3(5.7)$. Seeds of legumes-Soy beans, 1.5 (12.1); peas, 1.1 (10.0); vetch, 2.2 (9.9). Waste from flour mills-Wheat bran, $1.5(26.9)$; oat bran, $2.1(34.4)$; barley bran, $1.9(9.1)$; 
barley feed flour, 0.7 (10.8). Waste from sugar factories-Beet-chips, 1.1 (0.2); beet treacle, 3.1 (0.5). Waste from oil mills-Linseed cake, 4.3 (16.2); rape seed cake, 7.1 (20.0); ground-nut cake, 1.6 (11.6). Animal food stuffs-Meat (without bone), 0.3 (4.2); ground meat flour, $3.6(6.9)$. Of the lime contained in vegetable food stuffs at most one-half is made use of (Kellner).

Calcium requirements of growing animals. According to C. Voit the calcium requirement in growing dogs of smaller breeds, weighing $1.5-2.8 \mathrm{~kg}$. is $0.128 \mathrm{gm}$. daily; in dogs of large breeds (weight $3.2-4.5 \mathrm{~kg}$.) $0.769 \mathrm{gm}$. daily; in pigs $1-240$ days old $2.8 \mathrm{gm}$; in pigs over 8 months old $1.7 \mathrm{gm}$; calves require in the second or third week $14.5 \mathrm{gm}$. daily, in the fifth month $13.3 \mathrm{gm}$. (Lehmann), one-year olds require $21 \mathrm{gm}$. of lime (Kellner). In lambs the ealcium requirement for $50 \mathrm{~kg}$. body weight is, according to Weiske, as follows: In the first month, $3.2 \mathrm{gm}$.; at 5.5 months, $3.8 \mathrm{gm}$; ; at 8.5 months, $3.2 \mathrm{gm}$.; at 11.5 months, 2.7 gm., while in the 16 th month of their lives they require only $0.6 \mathrm{gm}$. of lime.

The injurious effect of food which is deficient in lime was proved by numerous animal experiments.

Chossat (1842), Milne-Edwards (1861), Guérin (1862), Roloff (1866), E. Voit (1877), Lehmann (1877), Baginsky (1881), and recently Miwa \& Stoeltzner succeeded in producing a clinical picture which was in its symptoms and in its gross pathology quite similar to rachitis in young pigs and dogs that is due to food containing very little calcium. In these experiments they observed swelling of the epiphyses, softening and various eurvatures of the bones, while other authors, especially Weiske and Wildt (1873), Tripier (1874), Pütz (1875), Delcourt (1899) failed to cause the disease in this manner. The cause of their failure may lie on the one hand in the too short duration of the experinents, on the other, and mainly, it was probably to be found in a very far-reaching withdrawal of lime or of all nutritive salts. It must, however, be admitted that in most of the positive results cited the proof of the rachitic nature in the anatomical changes was not afforded by microscopical examination; only E. Voit and Baginsky described histological changes which corresponded with those found in rickets. Neither Miwa \& Stoeltzner, nor Reimer \& Boye were able to demonstrate such tissue changes in their experiment animals which appeared elinically to be rachitic, as would have agreed with those found in true rachitis. (For the interpretation of their results see Pathogenesis.)

Miwa \& Stoeltzner observed in a dog that was fed with fresh horse meat and bacon, and received distilled water to drink, a waddling walk as early as the 10th lay, and a moderate epiphyseal swelling on the fore-feet was apparent already 10 days later. At the end of the fourth week the dog disliked to move about, or crouched down, whining after taking only a few steps; nor was he able to climb upstairs. The swelling of the epiphyses gradually inereaserl, on the distal ends of the ribs strongly prominent enlargements became noticeable, which were arranged in rosary fashion, while the long bones were bent outwards. The motor disturbances did not continue to progress from the seventh week on, and the dog was killed at the end of the eighth week. At the autopsy the authors found the compact portion of the bones forming a network of wide-meshed bone lamellae and hollow spaces of different sizes; the cambium layer of the periosteum was enlarged; periosteal osteophytes were on the enchondral zones of ossification between epiphysis and diaphysis; the lines of ossification were rather irregular; the layer of proliferating cartilage extended; the columnae of cartilage cells in irregular arrangement and the cartilage cells themselves enlarged. The preliminary calcification of cartilege was, however, quite normal, as was also the bony substance which contains no lime, and which surrounds the lime-containing bony lamellae everywhere in very delicate traceries. In contrast to this autopsy result in place of the lormal delicate deposits which contain no lime, there were found in true rachitis thick plates of tissue entirely without lime which surround on all sides the limecontaining portions or which, in very severe cases, replace a considerable portion of the bony lamellae. A further distinction from rachitis was found in the normal zone of ossifying cartilage. Therefore the authors explain the process as a generalized osteoporosis with rachitis-like changes in the periosteum and in the uncalcified proliferating cartilage, and they suggest that the bony changes observed after withdrawal of lime by other authors had been similar to theirs.

In the experiments of Reimers \& Boye and of Götting the compact bone substance was found very small, the spongiosa with extremely wide meshes; the extension of the proliferating cartilage was only slight, a proliferation of the osteoid tissue had not taken place at all. 
As a further cause of lime deficiency in the organism an improper chemical composition of the food-stuffs may be mentioned even though the calcium content be normal. Above all the relation between the amounts of lime salts and of phosphoric acirl appears to be of importance (Klimmer \& Schmidt, Ingle). The intestinal contents being alkaline, it may perhaps be supposed that in such a case a mutual precipitation of these salts takes place in the intestinal canal, or that an excess of free phosphoric acid is formed and, owing to this, a proliferation of the osteoid tissue (Pütz, M. B. Schmidt, Ingle). On the other hand an abnormally high content of the food in potassium salts is to be considered. The effect of food-stuffs rich in potassium may be explained thus, that the chlorine of the $\mathrm{HCl}$ of the stomach is bound by the potassium salts and that therefore the formation of hydrochloric acid is reduced, which would again lead to an insufficient solution and absorption of the lime salts that are present in the food principally in insoluble form. According to H. Aron and Delcourt, however, the potassium salts tend to impede the deposit of lime into the new-formed bony substance. In food-stuffs which contain much sulphuric acid the lime salts help to form gypsum which dissolves with difficulty, and they are therefore absorhed less readily in the intestine. With this assumption, the observation of Haubner would agree, namely, that in the surroundings of metal factories near Freiberg animals acquired rachitis and osteomalacia, because the smoke contains arsenic, lead and sulphurous acid. When the sulphurous acid was collected in condensators both diseases ceased to occur.

Digestive disturbances of whatever origin are also capable of producing a deficiency of lime in the organism, either by interfering with the formation of $\mathrm{HCl}$ in the stomach (Seemann, Zander) or with the intestinal absorption; but also, and possibly very decidedly, by increasing the normal elimination of the calcium compounds in the intestine, a possibility that must be considered especially in protracted intestinal catarrh.

A second etiological factor of rickets is found in intoxication. Wegener (1872) who produced rachitis in animals by long continued feeding of phosphoric acid, withdrawing the lime at the same time, attributed to phosphoric acid an irritating action upon the bone-forming tissues. A similar view was expressed later by Pütz (1874). But Kassowitz (1878) showed that the administration of slightly enlarged amounts of phosphorus suffices of itself to produce a disease similar to rachitis. On the basis of his experiments Kassowitz assumed the action of a substance circulating in the blood which is similar to phosphorus. Lehnerdt caused similar changes with strontium, but held them to be different from rachitis because, in contrast to rachitis the new-formed tissue is capable of taking up lime salts. The intoxication theory received further support in the results of experiments undertaken by Ostertag and Zuntz (1907) 
concerning the licking disease of cattle (q. v.), according to which any toxic substance present in meadow plants interferes with the bone-formation, an action which cannot be prevented by the addition of lime salts to the food. Stoeltzner considers it probable that rachitis develops through a functional insufficiency of an organ which is analogous to the thyroid gland.

The importance of infection as an etiological factor was frequently assumed, but only through the investigations of Morpurgos $(1900,1902)$ did this hypothesis gain a more solid foundation. These results are supplemented by those of Moussu (1903), and also Liénamx (1907) supposes that rickets has an infections origin.

The experiments of Morpurgos were made on white rats which harl dereloped osteomalacia and which belonged to a breed that har been raised in his institution for seven years. In the bones, cord, spleen and liver of the affected animals he fomm Grain positive diplococei, the enltures of which, on subcutaneous injection into healthy rats of the same breed, produced, accorling to the age of the animals, symptoms of trpical osteomalacia or of rachitis. In adult animals the period of inoculation was 8 days, but often weeks anil occasionally months, while young rats inoculated inmediately after birth did not become ill until they were two or three montlis olit.

It must be mentioned that Piana (1889) already deseriberl an infectious rabbit rachitis and that he obtained positive results in young rabbits, after inoculation with the microorganism which he had isolated. Later, however, he disclained the correctness of his obscrvation because he failed later on to find the supposed virus in the affected bones and because animals kept in the same place also lecame ill without being inoculated.

Moussu watched a little pig become ill which was kept and fed together with one suffering from snuffles; another pig fell ill two and one-half months after being put in the stall of an affected pig, which had not been cleaned previously. By subeutaneous inoculation of the bone marrow taken from pigs in the initial stage of the disease, he succeederl in producing a similar disease in pigs, goats and later in rabbits. Liénaux also saw two healthy pigs become ill three and fire weeks after having been brought together with an affected pig of the same age.

It is supposed that the lime salts in the reacly formed bone substance undergo solution owing to an excessive formation of lactic acid in the intestinal canal (Heitzmann) or in the intermediary metabolism, or then under the influence of an acid that develops in the bony substance itself and exerts a local action (Vierordt). In the former case it is assumed that the alkalinity of the blood is diminished and therefore the calcium of the bones is dissolved, while in the supposed local acidulation the fixation of the lime salts, which circulate in the blood, is impeded while in addition to this the already formed bone substance is decalcified.

While according to Heitzmann animals acquired rachitis or osteomalacia after feeding with excessive amounts of lactic acid, Siedamgrotzky and Hofmeister found only an increased porosity of the bones, without rachitic changes, in young kids which, in aldition to the acid, received an appropriate amount of lime salts with their food.

Heitzmann's results cannot be admitted as proof, because he limited the supply of lime and because, moreover, other authors always got negative results from feeding lactic acid. Nor has it been possible to demonstrate the presence of consirerab?e amounts of lactic acid in rachitic bones. In fact, Virchow found the reaction of fresh rachitic bones to be always alkaline. 
Finally an insufficient assimilation of lime in the bones is often supposed to be a cause of rachitis, the osteoid tissue being incapable of making use of the lime salts carried there in the circulation and of retaining those that have been deposited. The abnormal condition of the osteoid tissue is believed to be shown by the fact that the bone substance contains only glutein instead of ostein. No positive proofs have as yet been afforded for the correctness of this hypothesis.

Not infrequently the influence of predisposing factors can be shown. It is commonly known that an absence of sunlight, but preferably confinement in damp stables or in similar places (cellar's, dark, damp rooms) promotes the development of rachitis; up to a certain degree a like influence may possibly be attributed to insufficient ventilation or exercise (Hansemamn). The possible influence of digestive disorders has already been mentioned. It is also probable that there are individual differences in predisposition. The state of nutrition or the amount of fat deposited in the tissue may insofar be of importance as, other things being equal, fat animals of much greater body weight become ill much sooner and also more severely.

The influence of predisposing factors may perhaps be explained thus that they affect adversely the metabolism in the organism and indirectly the calcium content there or in the bones, and that they also encourage the occurrence of an intoxication or infection. It is also probable that in some species the want of earthy ingesta is to be considered.

Although a predisposing role has often been attributed to heredity its influence is probably at most an indirect one. If in a given herd the disease appears more frequently and in several generations, the cause must, as a rule, be looked for in improper conditions of maintenance, also in close inbreeding or in disease of the mother animals. Under such circumstances weaker and less resistant individuals are born whose metabolism is easily disturbed by insignificant influences.

For the present it still appears questionable whether Rhachitis foetalis occurs in animals. Marchi made the observation that in the Chiana valley in Italy the cows give, in damp years, birth to somewhat smaller ealves whose extremities show remarkable curvatures, the bones being twisted and short, the epiphyses comparatively long, the diaphyses long. Histological examinations were not made. In this valley the conditions of the soil are unfavorable and the feed consists principally of sour grass; since the soil has been eultivated appropriately, the disease has become much less frequent. Similar observations were reported by Sivieri, Hansen, and before them by Siedamgrotzky. The cases are however probably instances of abnormal development (Osteochondrodystrophia foetalis) (Zschokke, Bourdelle \& Dubois).

Hardly anything is known in animals about rachitis which commenees soon after birth (rachitis congenita). Only Hansen mentions a few eases of rachitis in colts which were affected when about one month old. However they were all sired by the same stallion and came from mares that had already had colts with congenital rachitis. 
In a large herd of eattle in which many of the cows were affected with osteomalacia, only poorly developed calves were born with very delicate bony structures, which however presented no rachitis symptoms; these symptoms appeared only after the calves were weaned and when they received similar food as the cows (authors' observation).

From what has been said it is above all evident that the etiology of rachitis cannot by any means be said to be completely explained. This much may be asserted that the same factors do not always participate in its development and that therefore the disease does not represent an affection of uniform etiology. There is no doubt that in domestic animals the most frequent cause of rachitis lies in the deficiency of lime in the organism from whatever cause, no matter whether the disease is sporadic or epizootic. Whether the lime deficiency causes the bony changes immediately or perhaps only creates a foundation on which the rachitis develops through the intermediation of a resulting disturbance in metabolism cannot for the present be decided definitely. On the other hand a toxic or infectious origin cannot be denied for certain cases, especially for those with excessive proliferation of the osteoid tissue, but the investigations which have been undertaken in this respect are not yet sufficient to justify a definite position being taken in this problem.

Pathogenesis. If a deficiency in calcium occurs in the organism the calcification of the developing osteoid tissue remains insufficient and this leads to a lessened firmness of the bones. The more the firmness of the bones diminishes, the greater is the mechanical irritation exerted upon the boneforming tissues by the motion of the body or of parts of the body, and by the coincident jolts and shocks. In consequence there occurs an increased new-formation of osteoid tissue with proliferation of the epiphyseal cartilage and bone marrow tissue, that is, a process similar to a productive inflammation. The severity of the mechanical irritation depends not only on the degree of lime deficiency but also on the body weight of the animal, and especially upon whether, and in what degree, the animal is permitted to move about or does move about of its own accord. As a consequence of the lime deficiency in the organism there occurs a partial or even a complete cessation in the ossification of osteoid tissue, through which the solidity of the bones becomes less and less in spite of the increased function of the bone-forming tissue. It is as a further consequence of the lime deficiency, that we may consider the abnormally increased absorption of bone tissue which had been formed before the disease (osteoporosis).

Although the disturbances in the bone formation occur in various parts throughout the body, they reach a considerable degree only in certain parts under the influence of the constantly changing mechanical irritation. 
It also appears thinkable that, according to the degree of lime deficiency and to the external influences, either the proliferating process or then the osteoporosis is more prominent, the latter being indeed sometimes alone present (so-called jurenile osteomalacia). It might be assumed that when the affected animals are prevented from motion, or when the animals aroid all vigorous motions, because the bones are sensitive in consequence of their lessened resistance, the process of proliferation is far less intense than in animals in which the mechanical influences can manifest themselves fully. It need not be settled in this connection whether the proportions of the other salts contained in the food have any influence.

These considerations may perhaps explain why some authors produced typical rachitis by withirawal of lime, while with similar methods of experimentation others could only cause osteoporosis and marked proliferation of the epiphrseal eartilage, and still others only osteoporotic changes.

If the rachitis is based upon an intoxication or infection, an inflammatory process in the bone tissue will probably be primary and in such a case the bone salts may be dissolverl and absorbed, causing the bones to lose their firmmess. In the further course, however, similar reparatory processes would occur in the bones as take place in lime deficiency under the stimulation of the mechanical influences mentioned.

The process which takes place in the bones causes in them various changes in form. These are partly due to the proliferation in the bones and are therefore dereloped especially in those portions of the body in which the bones are more exposerl to mechanical influences, during motion or during the chewing of hard food-stuffs, or in rooting; this is true especially for the epiphyses of the long bones, the maxillary or fare hones, also for the ribs which are in constant motion during respiration. These external influences, which differ according to species and breed and according to the care of the animals, may produce the most varied combinations and forms in the bony changes, and especially in pigs the maxillary or face hones are often strikingly altered. The diminished firmness of the bones leads on the one hand to curvatures, moler the weight of the body or from muscular traction, and on the other to fractures.

The diminished firmness of the hones is further responsihle for dragging upon the sensitive nerves in the periosteum and in the bony tissue which in their turn cause pain in the bones on motion. The proliferating osteoid tissue may even compress certain nerve trunks (e. g., the nervus infraorbitalis [Hintze]).

A high degree of lime deficiency in the organism may of itself canse disturbances in metabolism because the calcium is necessary for the binding of certain metabolic products. In this respect, however, a diminution of the other food salts which is often coexistent with lime deficiency in the organism is 
probably of far greater influence. This diminution of the food salts in general may perhaps be held responsible for the increased cerebral irritability which is a prominent symptom in certain cases. If an intoxication or infection is the cause of the disease, the presumable toxins or infectious substances may cause disturbances in metabolism.

Anatomical Changes. The epiphyses of the long bones are thickened, club-shaped, the diaphyses are shortened and also thickened (Fig. 158). The bony tissue is porous and softer

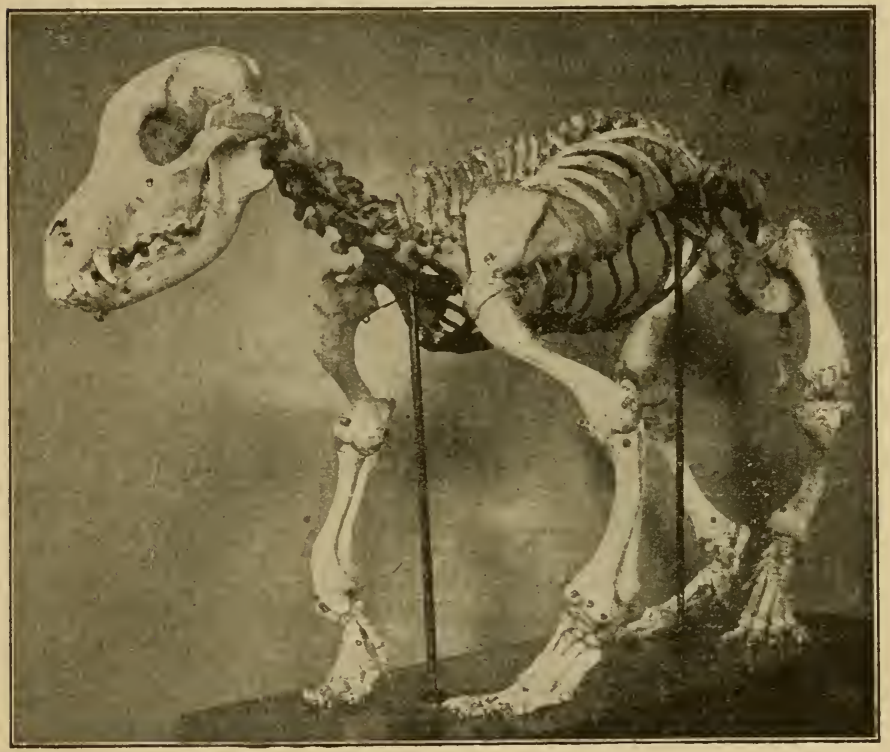

Fig. 158. Rachitis. Skeleton of a setter.

than normal, sometimes so much so, that it can easily be cut with the knife. Some bones show bends and even breaks (see Symptoms). On the cut surface of the bone, which has been cut lengthwise, there is visible, between the periostem and the bone tissue, a vascular, soft, spongy layer which in part remains adherent to the bony substance when the periosteum is peeled off. This osteoid layer is especially thick at the insertions of large tendons or muscles, and at these places exostoses are frequently formed. The epiphyseal cartilage is not separated from the diaphrsis by a bluish-white or yellowish double band of barely a few millimeters wirth, but there de- 
velops instead a spongy, soft, red tissue, certainly more than a centimeter wide, which passes into cartilage and bony tissue without any sharp border or in irregular streaks. The connection between epiphysis and diaphysis appears loosened, so that they can often be separated with slight exertion; this may even take place during the life of the animal and then the epiphysis will lie laterally to the diaphysis. The yellow marrow of the long bones is more or less reddened and gelatinous if the animal is notably emaciated. The spongy bone substance is also reddish in color and shows a rarification of the cancellous portion. The joints present, not infrequently, signs of inflammation and of ulcerative destruction of the cartilages.

The flat bones, especially the upper bones of the skull and the pelvic bones, are thickened, sometimes enormously bulged out, and changed to a lardaceous, grayish-red to dark red, firm tissue, which can be cut and which is covered only in spots by a flexible and porous cortical substance, while in other places it passes into the adjacent soft parts and contains only isolated bony islands. Owing to the bulging of the bones the nares and the maxillary sinuses are small or even obliterated, and in pigs the palate furthermore bulges into the oral cavity. Sometimes the proliferating tissue of the maxillary bones contains cysts which have developed from hemorrhagic foci (Hintze, Willies). The teeth are placed rather deep and lie loose in the proliferating tissue, without any alveolar borders; their enamel surface in the pig is uneven, pitted, honeycombed, or in exceptional cases the enamel layer may be entirely absent (Preuss). Sometimes the skull bones are soft and moderately thickened, in exceptional cases they may have become so rarified that in spots only a thin, connective tissue-like membrane (the single or the double periosteum) remains visible (Craniotabes), and in such cases there are usually also signs of hydrocephalus internus (Schütz). The other flat bones, especially those of the pelvis, may also be bent in various directions and present similar changes in their substance as those in the skull bones.

The microscopic changes in the long bones (Fig. 159) may be summe $\tilde{i}$ up as follows: Starting from the periosteum there develops a cellular and vascular osteoid tissue containing comparatively large osteoblasts and bone cells in varying numbers. In the diaphyseal part of the cartilage the cartilage cells are larger, arranged in wavy streaks, and similar eartilage-islands extend toward the marrow. From the marrow numerous vessels, with thin walls and surrounded by cellular tissue, enter deeply into the cartilage tissue, opening the cartilage cell columns, often in an oblique direction. Similar vessels may proliferate into the interior of the cartilage tissue, even from the perichondrium and loosen this in their vicinity which becomes softened. The layer of ealcification between bony and cartilaginous tissue is either entirely absent or there are only isolated foci of calcification; instead there is a very wide layer of osteoid tissue which is similar in structure to the layer starting from the periosteum, and is later partly changed into more solid, bony tissue.

In the so-called snuffles or maxillary rachitis of pigs a transitional zone with smaller decalcified bony laminae is joined to the zone of normal bony tissue, upon which follows a tissue similar to sareoma, consisting basically of spindle cells or cells of various shapes, rich in protoplasm and containing large nuclei. In this proliferating tissue there are seen numerous osteoid laminae which gradually 
become more sleniter and finally represent a veritable lattice work of splinters. Finally multinucleated giant cells are deposited around the laminae and cause their disappearance by lacunar absorption (Hintze).

In addition to the changes in the bones, symptoms of anemia, gastro-intestinal catarrh, or catarrhal affections of the respiratory organs are often observed; in the latter organs broncho-pneumonia is frequent.

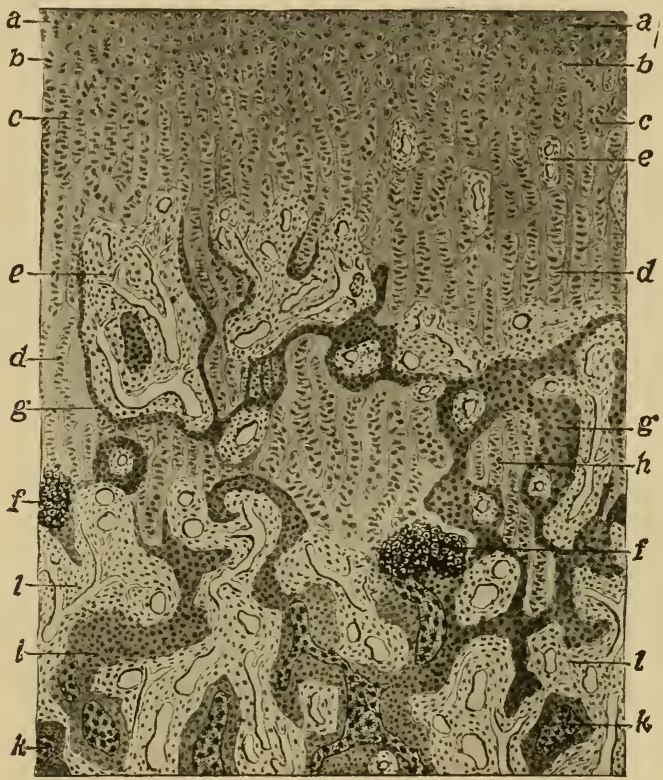

Fig. 159. Rachitis. Longitudinal section through the line of ossification of the upper diaphyseal end of the femur. $a$. unchanged lyyaline cartilage; $b$. cartilage in the first stages of proliferation; $c$, zone of proliferated cartilage columns; $d$. columns of proliferated hypertrophic cells; $e$. vascular marrow spaces: $f$ calcified cartilage tissue; $g$. osteoid tissue; $h$. remnants of cartilage tissue between osteoid tissue; $i$. tra. beculae of osteoid tissue; $k$. trabeculae of osteoid and developed (lime containing) bone tissue; $l$. vascular, cellular-fibrous marrow spaces. (Magnif. $50 \times$. After Ziegler.)

Symptoms. The development of the rachitic manifestations proper is sometimes preceded by disturbances in nutrition. The animals seem weak, their desire to eat is diminished, the belly somewhat bloated, and at the same time obstinate diarrhea makes its appearance. Sometimes there may be symptoms of pica (licking disease), gnawing the walls or the wood of the stable, drinking of manure water; these occur not only in the initial stage, but also in the further course of the disease. In some cases, especially in colts, the second dentition 
is retarded and irregular. All these symptoms may be unnoticed, so that attention is not attracted to the disease until the motor disturbances commence. The aching in the bones, which occurs before the changes in shape, canses the animals to step about restlessly, while at the same time they move gingerly and their walk is stiff or lame; they lie much on the ground and dislike to rise. Especially pigs, less so kids and lambs, and at times other animals do not nse their posterior extremities, or indeer, all extremities, at all. They seem paralyzed, and on heing forced to rise they crawl ahout squealing aloud. (The eases of paralysis in chicks, which Wilke diagnosed as poliomyelitis anterior, probably had a similar origin.) Smaller animals often assume a kneeling position (Fig. 160). These motor distmbances increase with the progress of the disease and when the shape of the hones commences to be altered.

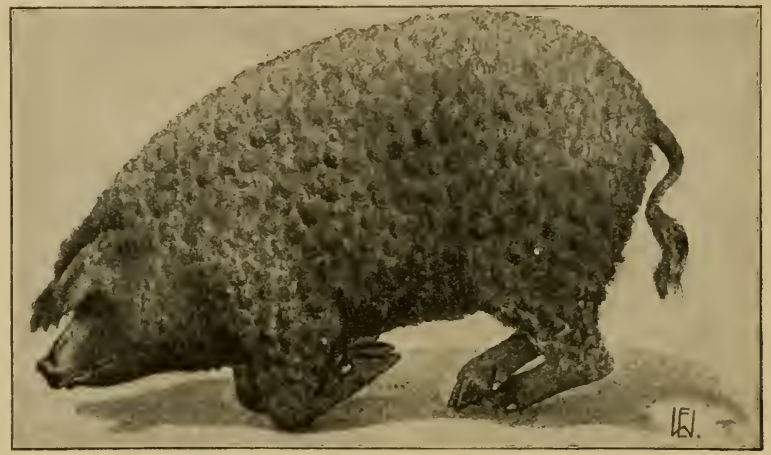

Fig. 160. Rachitis (of pig). Aside from the motor disturbances the pig showed slight snuffling.

In certain cases the disease becomes apparent through severe nervous disturbances, as was pointed ont by Klimmer \& Schmidt for osteomalacia and as the authors observed frequently in calves and pig's. The affected animals suddenly become somnolent, they stagger and even fall down, and occasionally develop generalized tonic-clonic muscular convulsions, which however may also be produced by external influences. Such attacks either terminate, in some 6 or 8 hours, with the death of the animal or are repeated a few times in the course of some days or weeks before death results, or then they do not appear at all, but the typical symptoms of the disease become evident at once.

The changes in form are, at least in the beginning, most striking in the long bones of the extremities. The epiphyses are enlarged and it looks as though the joints themselves were 
swollen, but the true nature of the swelling is easily ascertained on palpation (Fig. 161). Similar enlargements are to be seen on the sterno-costal articulations where the button-shaped cartilages form two concave lines rumning parallel with the sternum (rachitic rosary). These bony swellings are, especially at the beginning, often somewhat sensitive to pressure. Circumscribed exostotic enlargements are also frequent, especially in the ribs and the extremities (Fröhner observed a ringbone develop in rachitis).

The weight of the body and the traction of the muscles ranses the bones to bend and become crooked. These distortions are most frequent and striking on the extremities which

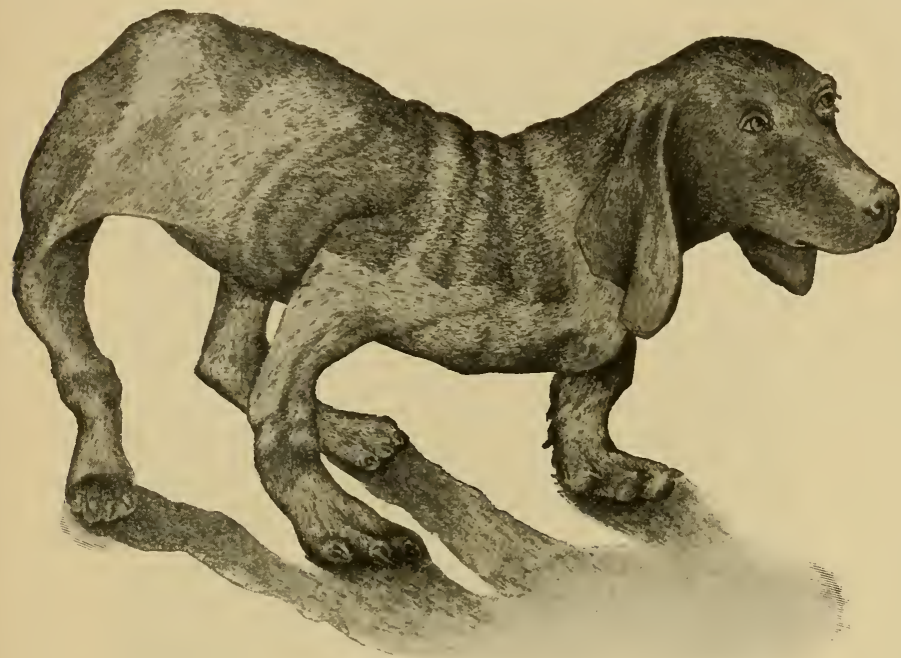

Fig. 161. liachitis. Setter. The skeleton of this $\operatorname{dog}$ is shown in Fig. 158,

assume various abnormal shapes, such as "dachshund" legs, knock-knee, bandy-leg, legs bent backward, legs like bear's paws (Figs. 161 and 163). The thinner bones, especially those of the metacarpus and the metatarsus are sometimes bent double and may break transversely (Schütz). Liénaux points out that the Achilles tendon is sometimes torn from its attachment, and this may be the first or even the only symptom of the disease. The muscles of the extremities either lose their tension or some of them become contracted and thus cause much pain.

The pressure of shoulders and elbows, and also the air pressure cause the middle portions of the ribs to be bent inward, giving rise on both sides of the thorax to a longitudinal 
shallow groove and to a shortening of the transverse diameter of the thorax. The inward bend of the ribs causes the sternum to recede from the vertebral column, while its borders are approximated, so that the sternum forms an acute angle or protrudes (chickenbreast, pectus gallinaceum, s. carinatum). When the animals lie down much, the antero-posterior diameter of the thorax is diminished (Fig. 161), because its anterior wall which rests on the ground is forced inward. In fowls the sternum shows in such cases a single and often a double inward bend (Zürn). The limited motility of the thorax is apt to cause the development of a bronchial catarrh or of catarrhal pneumonia.

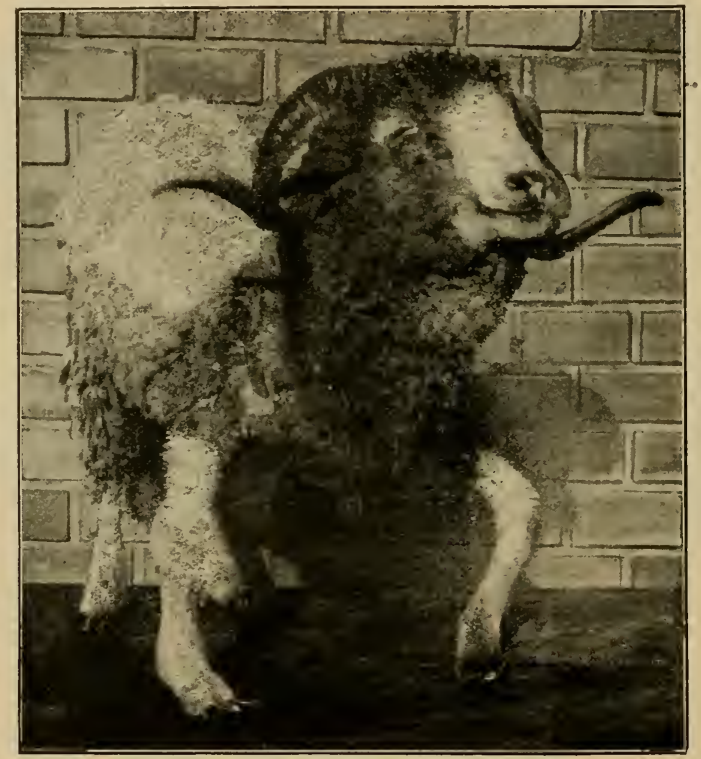

Fig. 162. Rachitis in a lamb.

In the pelvis the acetabulum is pressed upward and inward by the heads of the femurs, while the symphysis is forced downward and forward; in consequence the pelvis is narrow transversely, as is already indicated by the position of the hind feet which are turned inward.

The vertebral column presents various deformities (hence the name rachitis, viz., $\zeta \delta$ x $s=$ vertebral column). It is curved either laterally (scoliosis), or upward (kyphosis), or downwart? (lordosis). In most cases there are two or several antagonistic 
curvatures, for instance lordosis in addition to kyphosis, or the scoliotic vertebral column is bent both to the right and to the left (Figs. 158 and 161).

The deformities in the trunk and extremities may alter the entire body conformation (dwarfism) and naturally influence the motions of the animal, which is clumsy, uncertain, and may reel or stagger in walking even if the bones are not painful.

The skull bones either show certain changes, like thickening and deformity of the rami of the inferior maxillary, on close observation only, or they are so much altered that their

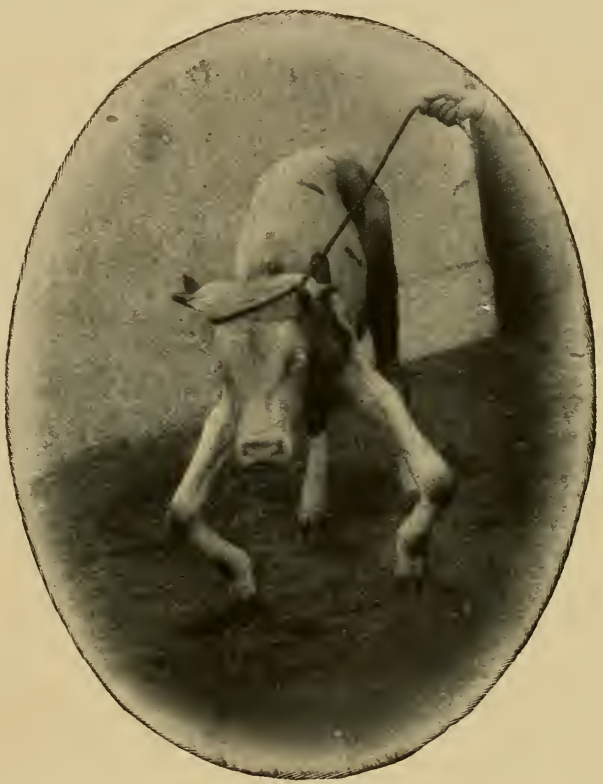

Fig. 163. Rachitis in a calf.

affection forms a prominent symptom of the disease. Sometimes early, more often later the respiration becomes sniffling and difficult with occasional attacks of asphyxiation, and as this increases the facial bones bulge out more and more, so that the head may in the course of 6 to 8 weeks become enormously large. The bulging of the bones commences mostly in the maxilla and gradually involves the contiguous bones and the inferior maxilla. The romer protrudes into the oral carity which therefore becomes smaller, so that the ingestion of food is rendered difficult and even impossible (Fig. 164). 
The teeth then seem shortened and sometimes barely stand vut from the gums; they are loose and may fall out; the enamel is perforated in places. The bones of the skull may be thickened in parts, or they may be thin and even membranous; the fontanelles, especially between the frontal and parietal bones, often remain open (Schütz).

The form of rachitis in pigs in which the facial hones bulge out strongly, has always been designated as snuffles (French: "reniflement"). With it were classerl other diseases characterized by a sniffling respiration, especially the infectious nasal inflammation of pigs ( $\mathrm{Vol}$. II). Since, however, the suffling respiration is only a. symptom of quite heterogeneous diseases, and has all too often given rise to misunderstanding, the name snuffles should be abantoned altogether. The same is true for the so-called bran rlisease of horses, which also represents only a form of rachitis or osteomalacia, eansed by the exclusive feeding of bran.

Course and Prognosis. After the appearance of clinical s.rmptoms rachitis runs a chronic course lasting for months,

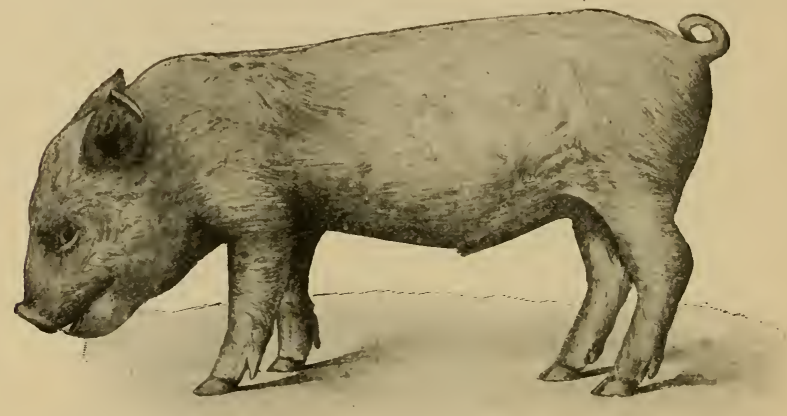

Fig. 164. Rachitic bulging of the maxillary bones and molrusion of the hard palate into the mouth, in a jig with rachitic changes in the other bones.

except in those cases in which, as already mentioned, the onset is associated with severe nervous disturbances, or in which death is hastened by complications. If the conditions are unfavorable and if the disease is not treated, it gradually progresses accompanied by increasing emaciation of the animals whose development is noticeably retarded. Spontaneous recoveries are not infrequent in large herds, but are observed only in animals. which are less severely affected, or in nearly mature animals in which, of course, growth is less rapid. By proper regulation of the diet and by prompt treatment many, even severely affected, animals are cured, or the bone deformities, which are not yet considerable, are made to disappear. But deformities which have become very decided persist, the narrowing of the pelvis and the thorax being of particular importance. Under unfarorable conditions, or if the disease is 
far advanced and has given rise to emphatic nutritional disturbances, death always follows, either through exhaustion or through some complication. The ultimate healing of the process is not rarely prevented by ulcers formed in the course of the disease in the articular cartilages.

Diagnosis. The changes which occur in the skeleton, especially in the bones of the extremities, in the ribs and in the bones of the skull are so characteristic that rachitis can be recognized withont difficulty, especially if the slow development and the early age of the animals are considered. Pyosepticemia of the new-born is differentiated by its acute onset few days after birth, and by the inflammatory symptoms which dominate the clinical picture. In articular rheumatism also the acute inflammatory eliaracter of the disease is diagnostic; moreover, the swollen joints are painful, the swelling is most intense in the middle portion of the joints, it is tense and fluctuating. In muscular rheumatism, which is of extremely rare occurrence, bony swellings or deformities are absent. Tuberculosis may produce similar clianges in calves or young pigs as they are observed in the initial stage of rachitis, but in this affection the articular ends of the long bones remain unaltered for a long time and the further course of the disease is entirely different. In all the diseases just mentioned only one or few joints are involved, as a rule, and these not with almost equal intensity as is the case in rickets. New-formations occur only in some bones, while in rickets the entire skeleton is diseased more or less.

Treatment. Above all the diet should be regulated; the animals are to be kept in moderately warm and well-ventilated places, or, still better, in the open air when the weather is favorable; they shonld be enabled to move about freely and receive food that is rich in calcium. Nurslings are properly left with their dams which are to be given nutritious food containing much calcium; the rachitic nurslings may be given milk to which lime water has been added, or if necessary they are put to a healthy nurse. Weaned animals and the dams of nurslings receive food containing much lime, in carnivora meat with the addition of a sufficient amount of bones, in birds fish (Merz), in herbivora first of all timothy hay, good upland hay, straw of leguminous plants, possibly waste of oil factories or certain leguminous foods being best adapted to their needs (page 932) ). Pasturing or green feed not rarely exert a favorable action, but often the disturbances in motion are increased at first when animals are put out to pasture, if they were not accustomed to move about freely. The treatment of gastric and intestinal catarrh, which may be present, also requires close attention in order to insure undisturbed intestinal assimilation.

The decided lime-deficiency in the organism is to be cor- 
rected by the administration of lime preparations, and here, of course, the lime requirements of the particular species and the probable lime content of the food must be considered. In many cases chalk (calcium carbonate), which is cheap, is appropriate; colts and calves may be given 10-20 gm.; lambs, pigs and dogs 2-10 gm. daily with their food, perhaps shaken up with milk. In order to prevent digestive disturbances in consequence of the $\mathrm{HCl}$ being bound in the stomach (an occurrence that was not observed, however, in the experiments made on rams by Volhard), Klimmer \&'Schmidt suggest to replace the chalk partially $(1 / 3-1 / 2)$ by calcium chlorate (not to be confounded with chloride of lime!). The prepared bone-meal (phosphated food-lime; food-bone-meal; precipitated calcium phosphate) renders excellent service in doses similar to those of chalk, and should be administered especially in those cases in which the administered food is deficient in phosphates (page 932). Feeding experiments made in this direction by Köhler showed that almost all of the ingested tri-calcium phosphate was absorbed, and about one-half of bone-ash or of bone-meal from which the gelatinous portions had been removed. In the experiments of Frese in dogs, organic calcium compounds were not found superior in their effects to the anorganic lime. The preparations containing lime, respectively phosphates, should be given at intervals of from 2 to 3 days (Klimmer \& Schmidt), or alternately at different feedings, in order to prevent their precipitating each other in the intestine. If phosphorus is deficient as well as lime, sodium phosphate may be given alternately with the chalk. In human medicine phosphated codliver oil is particularly well thought of as a remedy (colts and calves $0.02-0.05 \mathrm{gm}$. of phosphorus in $30-100 \mathrm{gm}$. of codliver oil daily; pigs $0.002-0.005: 20-40$ gm.; dogs 0.001-0.002:5-15 gm.; birds $0.0005-0.001: 2-5 \mathrm{gm}$.). Hansen \& Schabad have shown experimentally that the remedy produces an increased retention of the lime and phosphorus of the food and also an increased assimilation of the lime which is administered separately. The systematic administration of salt (rock salt) or of $\mathrm{HCl}$ may enhance the favorable action of the medicinal treatment just described. (Harms, Klimmer \& Schmidt.)

In a far advanced stage of the disease, especially when the deformities of the bones are very marked, it appears best to slaughter the animals early.

Prevention. The disease can be prevented almost with certainty when the animals are kept and fed properly. Care should be taken that the animals receive sufficient amounts of rock salt, and of course that the dams are suitably fed. In localities where the disease is frequent or where food-stuffs poor in lime are fed, it is advisable to administer systematically chalk or bone-meal in addition to the food. In this manner it has been found possible to eradicate the disease, for instance in 
swine herds in which it had, year after year, taken large toll. Colts should not be fed with bran at all, or only in small amounts. Moreover, the young animals should be protected by cleanliness and proper ventilation of their stables against acute diseases which may establish a predisposition to rickets, and they should be kept as much as possible in the open where they can move about freely. All these precantions are called for especially in pure-bred or high grade herds in which the greater or lesser degree of inbreeding of itself reduces the resistance of the animals.

If there is a suspicion of infectious or tcxic rachitis, the isolation of the diseased animals and the disinfection of their stables, as well as a change of feeding, is indicated.

Literature. Albu \& Nenberg, Physiol, u. Path. đ. Mineralstoff wechsels, 1906. - Aron, Pflügers, A., CVI. 91. -- Basset, Rec., 1907. 167 (Lit.). - Bourdelle \& Dubois, Rev. vét., 1907. 511. - Brüning, Z. f. Tm., 1906. X. 198. - Frese, Verwertbarkeit d. Nahrungskalkes, etc. Diss. Giessen. 1908. - Fröhner, Monh., 1904. XV. 211. - Hansen, Maanedsskr., 1908. XX. 553. - Hartenstein, S. B., 1894. 132. - Haubold, ibid., 1906. 77. - Hintze, A. f. Tk., 1909. XXXV. 535 (Lit.). - Kassowitz, Jahrb. f. Kinderheilk., 1882. 430. - Klimmer \& Schmidt, Monh., 1906. XVII. 481 (complete Lit.).-Liénaux, Ann., 1907. 93.-Loos, W. f. Tk., 1905. 421. - Marehi, Clin. vet., 1889. 349. - Miwa \& Stöltzner, Beitr. z. path. Anat., 1898. XXIV. 578. - Morpurgo, ibid., 1900. XXVIII. 620; Cbl. f. allg. Path. u. path. Anat., 1902. XIII. 113. - Moussu, Bull., 1903. 303; Ree., 1908. 559. Ostertag \& Zuntz, Z. f. Infkrkh., 1907. II. 449. - Pfaundler, M. m. W., 1903. 1577. - Piana, Clin. ret. 1899. 307. - Pommer, Unters. üb. Osteomal. u. Rhachitis, 1885. - Preuss, A. f. Tk., 1909. XXXV. 561. - Reimers \& Boye, D. t. W., 1905. 496 (review). - Rievel, Ergebn. d. Path., 1905. XI. 2. Abt. 597 (Lit.). - Roloff, V. A., 1866, XXXVII. 433; A. f. Tk., 1875. I. 189. - Schmidt, Ergebn. d. Path., 1897.' IV. 531 (complete Lit.). - Sivieri, N. Erc., 1902. 89. - Stöltzner, Jahrb. f. Kinderheilk., 1899. 268; Path. 11. Ther. d. Rhachitis, 1904 (complete Lit.). - Stutzer, Mentzel \& Lengerkes, Landw. Kalender, 1906. - Vierorit, Rhachitis u. Osteomal., 1896. - Voit, Z. f. Biol., 1880. XVI. 55. - Wedemayer, B. t. W., 1906. 703. - Wegner, V. A., 1872. LV. 11. - Wilke, D. t. W., 1909. 697. - Willies, A. f. Tk., 1908. XXXIV. 623. - Zschokke, Z. f. Tm., 1899. 268.

Barlow's Disease. In a young dog, which during life had shown signs of rickets, rheumatoid pains and debility, and which had often cried out in pain, Stöltzner found rosary-like thickenings of the cartilaginous ends of the ribs, a large subperiosteal hematoma of the scapula, a considerable swelling of the proximal epiphyseal end and of the adjoining diaphyseal portion of the humerns, with a large double hematoma. The lower two-thirds of the radius were thickened on account of a subperiosteal hematoma; where the swelling was largest, both in the humerus and in the radius, the corticalis was entirely absent. Histological examination showed that there was not a bone fracture, but that the cortical portion, and in places the spongy portion also, had become rarified; osteoid trabeculae and osteoblasts were present in only small numbers, the epiphyseal marrow contained fat cells and the diaphysis showed a spleen-like structure. Definite rachitic changes were not ascertained.

The author emphasizes justly the great similarity of the clinical picture with Barlow's Disease, which oceurs in children together with rickets and which leads to hemorrhages in bones and elsewhere, but he nevertheless does not feel justified in identifying the affection with Barlow's Disease. (Schmorl, Beitr. z. path. Anat., XXXX, 215.Stöltzner, V. A., CLXXVII, 466.) 


\section{(b) Osteomalacia. Brittleness of Bones.}

\section{(Bone softening; Halisteresis ostium, Osteopsathyrosis; Cachexie osseuse [French].)}

Osteomalacia is a disease arising from general nutritional disturbances, which causes an increased absorption of lime salts from the bones and therefore softening and manifold deformities; fractures of the bones are not infrequent.

Occurrence. Osteomalacia affects most frequently cattle, especially milk cows, less often goats and swine, and still more infrequently horses or mules, also sheep and buffalo; dogs and birds appear to acquire the disease only in exceptional cases.

The disease occurs preferably in regions with unfavorable conditions of soil, and in such regions it assumes, especially in dry years, an enzootic distribution and causes then very emphatic economic losses.

Osteomalacia has been ohserver in various parts of Belgium, Germany, France, Austria (especially in Moravia [Rudofsky]), then in Hungaria, and here more especially in the regions of the upper Danube as well as in certain mountainous regions. In other countries in Europe it has also marle its appearance, and further in many countries of Asia, Africa, North America and Australia.

Quite extensive epizootics of the disease oceurred as follows: 1778-1783 in the province of Brandenburg; 1816-1817 in Barlen; 1820-1822 in Anhalt; 1828 in Switzerland; 1837 in Krain; $1832-1838$ in Rhenish Hesse; $1833-1834$ in the government districts of Coblentz, Trier and Aachen; 1863-1866 in Bohemia; 1868 1869 in the grand duchy of Weimar; 1893 in Belgium and France; 1893-1894 in Baden, the Rhenish countries, etc., 1895 in the district of Cologne, in the region of Chemnitz and Grossenhain; 1904-1905 probably all over Germany, certainly in the kingdom of Saxony (Klimmer \& Schmidt). During the last-mentionerl period the disease was epizootic in Moravia (Rudofsky), and also in one county of upper Hungary on the Galician borders. In the years 1908-1909 many cases occurred in several parts of Hungary.

Osteomalacia (the so-called osteoporosis) is widely distributed in Asia, Africa and Australia, occurring especially in Tonking, India, Japan, Madagascar, Camerun (called by Ziemann disease of the maxillae of horses and mules), the Transvaal and in the Cape Colony; in these regions it is an annually recurring enzootic affecting the horses, mules and asses.

In America, also, enzootics were observed among horses in many regions.

Etiology. As in rachitis, so in brittleness of the bones of animals, a deficiency of lime in the organism is undoubtedly of the highest importance. This is in perfect agreement with the constant occurrence of the disease in localities with unfavorable conditions of soil.

In these regions the soil is usually very poor in mineral substances, especially in phosphoric acid and lime; the subsoil consists of porous bog- or peat-strata, or it is exposed to frequent floods. If in addition the cultivation is primitive, and particularly if the deficiency in salts is not corrected artificially by appropriate fertilization, the food plants growing on such a soil are likewise deficient in mineral substances. This may be true to an increased degree in dry years, when the salts 
contained in the superficial strata of the soil cannot be dissolved and absorbed sufficiently. Food grown under such conditions is to be compared in regard to its food value to the sour grasses growing in very wet, boggy and swampy regions, which are not only poor in proteids and in mineral substances (Morgen), but are moreover difficult to digest and easily produce gastric catarrh through irritating substances which they contain.

The, ralcium content of the food varies within wide limits, according to the conditions of soil. Germain found, for instance, in 1,000 parts of hay, of a region in which osteomalacia was frequent, only 2.97 parts of lime, while in another hay of gool quality the amount of lime present was 24.05 parts. Both kinds of hay contained like amounts of phosphoric acid. Karmrodt found 6.77 parts of lime and 2.25 parts of phosphoric acid; Morgen 3.7 and 6.7 parts of lime and 2 resp. 2.6 parts of phosphoric acid; Direks found in three samples of Norwegian forest lay only $1.85,2.79$ and 2.78 parts of lime, and $1.55,1.43$ anil 1.29 parts of phosphoric acid (Kellner). (For the normal percentage of lime and phosphoric acid of the foort-stuffs, see page 932.)

A deficiency of lime in the organism may, however, also occur under favorable soil and meteorological conditions, when food-stuffs, which naturally contain only small amounts of lime, are taken largely and for a long time; such food-stuffs are ('arrots, potatoes, grains and their waste products, grain-straw, slops, molasses, whey. Usually the unfavorable influence of soil or meteorological factors and of food poor in lime become manifest simultaneously since such food-stuffs are fed in considerable amounts in place of better feed when the latter is not available in sufficient quantities.

In regard to the insufficient absorption of lime salts to the improper chemical composition and, perhaps, to the digestive disturbances which may act as causes of a deficient lime content in the organism, the same is true that has already been said in the chapter on rachitis (see p. 933). Haubner and Röll have observed osteomalacia especially after the feeding of food that had been exposed to the smoke from smelters.

Sometimes the deficiency of lime in the drinking water appears to be of importance, at least Kasparek saw the disease, which had become stationary on an estate, disappear after the introduction of hard drinking water.

Further factors in depriving the organism of its lime are pregnancy and especially lactation, since in these conditions very considerable amounts of lime are withdrawn from the organism (see lime content of the milk, p. 931).

With the exception of females during pregnancy and lactation the lime requirements of adult animals are much less in proportion to the body weight than in growing aninals, although in these also the lime requirements usually diminish rapidly toward the end of the first year of life (see page 931). While a calf from 2-3 weeks old and weighing $50 \mathrm{~kg}$. needs a daily supply of $14.5 \mathrm{gm}$. of calcium and of $13.8 \mathrm{gm}$. of phosphoric acid (Lelumann), this need is only 21 and 19 gm., respectively, in a calf one year old (Kellner). The calcium requirements of a pregnant cow are $51 \mathrm{gm}$. per day and $25 \mathrm{gm}$. of phosphoric acid (Boussingault), those of a milk cow with a daily milk production of 20 liters are 200 
gm. of lime and $100 \mathrm{gm}$. of phosphoric acid (Kellner), those of a resting ox are 76-78 gm. and $36 \mathrm{gm}$., respectively (Henneberg \& Stohmann), the figures in the two last cases being calculated to $1,000 \mathrm{~kg}$. body weight. A ram 2 years old needs daily only $0.57 \mathrm{gm}$. of lime and $0.05 \mathrm{gm}$. of phosphoric acid for his $50 \mathrm{~kg}$. of weight (Weiske) compared to a need of $3.2 \mathrm{gm}$. and $2.3 \mathrm{gm}$., respectively, in a lamb 1 month old.

For the content of food-stuffs in lime and phosphoric acid see chapter on rachitis, page 932.

The etiological importance of lime deficiency in osteomalacia was likewise proved by some animal experiments, and the same significance must be attributed to the numerous observations according to which the disease may be relieved by a suitable supply of lime.

Chossat (1842) and later Gelpke (1891) succeeded in producing osteonalacia in pigeons by feeding them exclusively with oats, while Forster (1881) obtained positive results in dogs. Roloff $(1566)$ in a goat and a sheep. Stilling \& Mering (1889) saw in a bitch which liad been fed from the commencement of gestation with food-stuffs deficient in lime, softness of vertebral column and pelvis, and microscopically thick layers of osteoid tissue upon the bone trabeculae.

Further an intoxication was assumed as causative of osteomalacia, in so far as some irritating substance circulating in the blood was supposed to produce an inflammatory condition in the bones, similarly as in rachitis (p.933).

The infectious origin of osteomalacia is finding more and more adherents, since Morpurgo and Moussu published the results of their experiments which were mentioned above (p. 935), and even before them some authors (Kehrer, Zürnn) had pointed out the possibility of an infection. Moussu, Liénaux, among others, even assert that osteomalacia always arises in consequence of an infection, at most a predisposing significance being admitter for the lime deficiency of the organism.

Petrone (1892) assumed that Winogradsky's nicrococeus nitrificans was the virus of osteomalacia, but the experiments of Tschistowitsch (1893) in this direction had a negative result. Morpurgo found the diplococei mentioned on page 935 . Pécaud (1904) in Tonking also demonstrated diplococci, in a horse affected with osetomalacia and claims to have producer the disease with the blood of affected animals in a horse and in a guinea pig. But on the other hand, Moussu and Liénaux did not suceed in discovering any microorganism whatever. (Concerning the nicroorganisms found by Piana in rabbits affected with osteomalacia, see page 935.)

The osteomalacia (osteoporosis) of equides which is met with outside of Europe was considered by Theiler (1907) to be a specific infectious disease in spite of the fact that the inoculation experiments of Elliot and Robertson, and also Theiler's own blood transfusion riclded entirely negative results. Theiler assumed that the microorganism, which is perhaps ultramicroseopic, is transmitted by insects. But Ingle showed that the food-stuffs, which were supplied to the osteoporotic animals, were deficient in lime salts and contained relatively much phosphoric acid, and he concludes, therefore, that the cause of osteoporosis must be looked for in food-stuffs in which the phosphoric acid content is relatively very high in proportion to the ealcium content. Ingle refers to some animal experiments of Weiske's in which, after the addition of sodium phosphate to the oats, the bones of the experi- 
ment animals became thinner and fragile, while such changes did not occur if at the same time calcium carbonate was also ad"ded to the oats. Finally Lane saw during an enzootic the disease disappear when the food was made richer in calcium and was mixed with bone meal. The assertion of Elliot, according to which the extra-European osteoporosis is a climatic disease which oceurs principally in wet regions, was not confirmed by experiences in the Transvaal.

The liypothesis that the bone salts suffer solution because of the absorption of lactic acid from the intestinal canal (Heitzmann) or owing to the accumulation of carbonic acid in the blood (Rindfleisch) has at present hardly any adherents (p. 935).

Trophoneurotic disturbances were attributed a causal relation to osteomalacia by Fehling (1890). According to this author an increased activity of the ovaries produces, by reflex action, a hyperemia of the bones, which in its turn would lead to softening of the bony substance.

The hypothesis is based upon the favorable results obtained in women ill with osteomalacia by extirpation of the ovaries and also upon the demonstration of a so-called "physiological osteomalacia" in pregnant women which was made by Hanau (1892). This author repeatedly found newly formed osteoid tissue in the bones and in the Haversian canals of pregnantand lying-in women whose bones were otherwise healthy. But it must be noted that Lüthje failed to find any changes in the phosphorus- and calcium-metabolism in castrated female animals, and that Gracsányi saw recovery occur equally in six affected cows, although only two of them were castrated, all being fed alike.

Hönnicke (1906) is inclined to assume that osteomalacia occurs on the basis of a thyroid gland affection and that the disease represents a form of hyperthyreosis. (In two of his cases in horses the thyroid gland was much enlarged.)

In a rabbit that was fed with normal thyroid substance during, and for two months after, pregnancy, Hönnicke found a decided softening of the pelvic bones and morlerate cachexia, while two control animals, which were also pregnant, remained well.

Among predisposing causes pregnancy and lactation merit first consideration (see p. 951), while racial differences are of importance only in so far as the calcium requirements of different species vary. In regions where osteomalacia occurs enzootically, recently imported animals are more apt to acquire the disease than the native ones.

It is evident from these considerations that there are many problems still to be solved in the etiology of osteomalacia, and that osteomalacia is not a disease with a uniform etiology. Especially the experiences in European countries do not admit any doubt that calcium deficiency of the organism may be responsible for osteomalacia, but for certain cases another origin cannot be denied (see p. 937). 
Pathogenesis. The commencement of the pathological process in the bones is probably similar to that in rachitis (see p. 937), whether the trouble be due to a lime deficiency of the organism or to another canse. But since in this disease the process occur's in fully dereloped bones, the later tissue changes differ more or less from those in rachitis. In osteomalacia the peculiar proliferative processes are absent which are noted in rachitis at the borders between the epiphyses and the diaphyses of the long bones, and on the other hand the decalcification (osteoporosis) predominates over the newformation of osteoid tissue, at least in many cases. Still, exceptions are observed not very infrequently, in so far as a decided proliferative process may set in under the influence of certain mechanical factors (see p. 937) and of other agencies

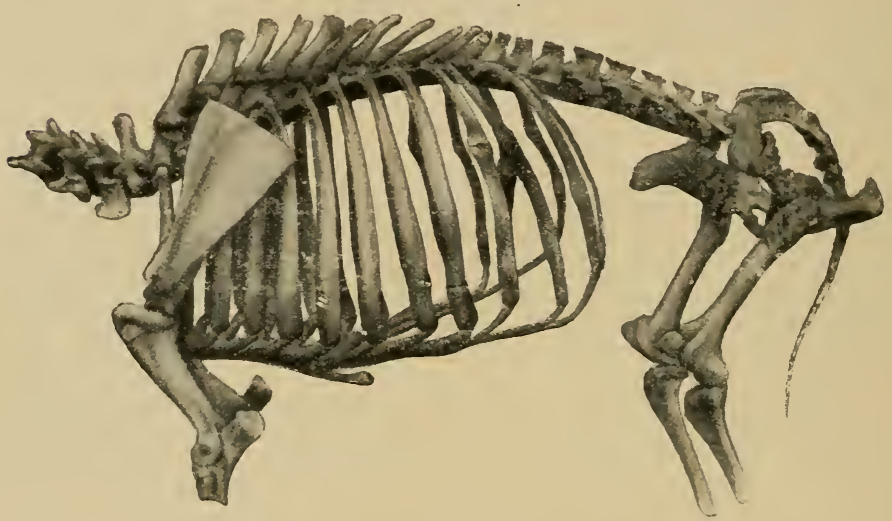

Fig. 165. Skeleton of the cow with osteomalacia, illustrated in Fig. 168. The rils are bent forward, show multiple fractures, and callus formation. Both ilia are fractmed, the fractured ends being erowded into one another. Enormous callus formation, moderate lordosis of the lumbar vertelrae; callus formation at the base of the spinal processes of the anterior dorsal vertebrae.

that are not fully known. In other cases the new-formation of osteoid tissue may be entirely lacking.

The further consequences of the diminution in the firmmess of the bones and of the disturbances in the metabolism are the same as in rachitis (see p. 937), except that in osteomalacia the bones are more often brittle rather than pliable.

Relations of Rachitis to Osteomalacia. While Virchow and others differentiated rachitis sharply from osteomalacia, still other authors declared both diseases to be closely related, or identical. Indeed the pathogenesis and the etiological ageneies of hoth affections agree in many points, and it appears as though the anatomical or microseopical differences were dependent solely upon the difference in the age of 
the affected animals, the more so, as both diseases may occur simultaneously. According to this viewpoint rachitis and osteomalacia would form processes which are identical anatomically, but which might possibly be due to different causes. But since many points are still disputed concerning the etiology and pathogenesis of both disease forms, the identification of rachitis and osteomalacia as one clinical entity of like anatomy and etiology does not appear justified for the present.

The relation of rachitis and osteomalacia to osteoporosis in ge eral (rarification of bony substance in favor of the marrow spaces) may be described as follows: Both in rachitis and in osteomalacia osteoporosis is a constant symptom, and it is either emphasized very slightly compared with the proliferative process,

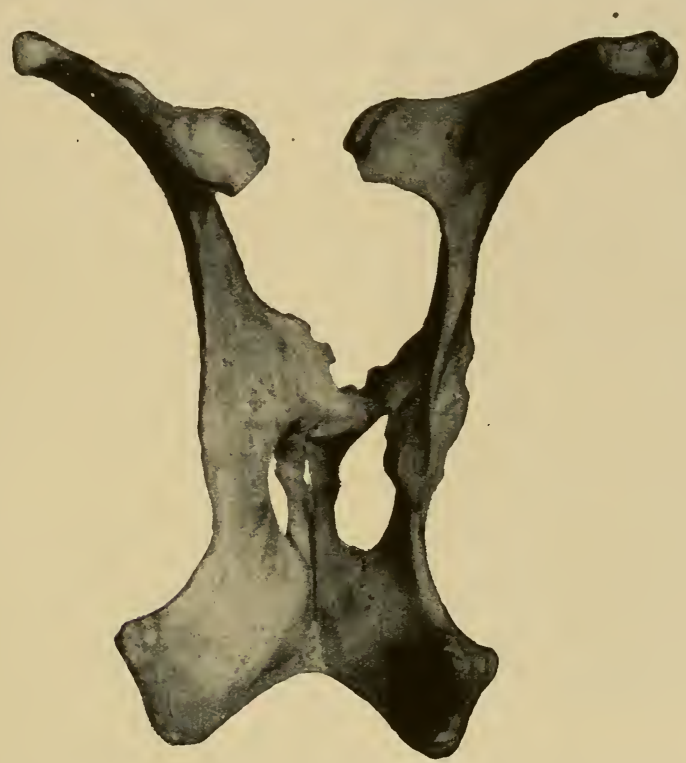

Fig. 166. Pelvis of a cow with osteomalacia. The pubes and ischium are bent, the foramen obturatum being distorted. The left internal angle of the ilium is lowered.

dependent upon the age of the affected animal, on the cause and on the variable mechanical influences, or on the contrary it predominates, at times so decidedly that it appears to exist alone. It is therefore not proper, at the present stage of information, to classify general osteoporosis (with the exception of senile bony atrophy) as an independent affection apart from rachitis or osteomalacia, as was recently proposed by Miwa \& Stoltzner, Elliot, Theiler and others.

Anatomical Changes. In well marked cases of the disease the marrow spaces of the long bones are dilated, the bony cortex is thin and spongy, brittle, or then so soft that it may be cut with a knife. In very severe cases the cortical portion of the long bones is barely a few millimeters thick, the bone 
marrow much reddened and studded with punctiform hemorrhages; if cachexia already exists the bone marrow is pale, gelatinous, even watery (marrow-fluid). The flat and short bones are also brittle or flexible; owing to the dilation of the marrow spaces the spongy structure of these bones is emphasized and may contain fair-sized marrow spaces. The sound of the bones is duller than that of normal bones.

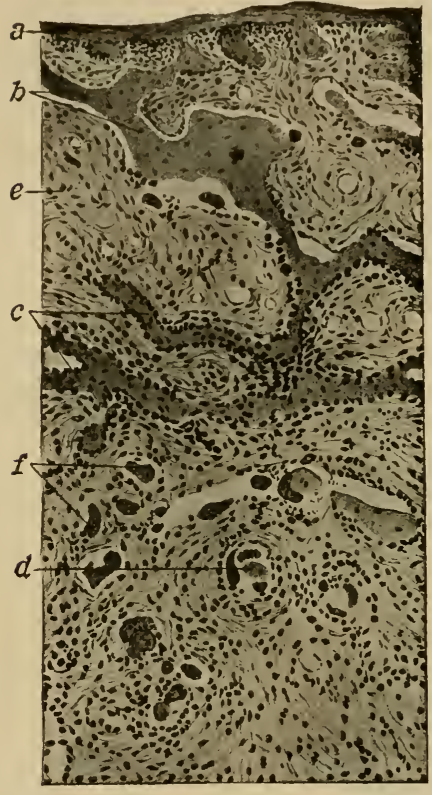

Fig. 167. Osteomalacia. Superior maxilla of a goat 20 months old. $a$. perionteum with numerous osteoblasts in the deeper layer; $b$. trabeculae becoming gradually more narrow; $c$. narrow trabeculae surrounded by numerous osteoblasts; $d$. remnants of trabeculae in the cellular-fibrous tissue; $e$. vascular cellular-fibrous tissue; $f$. giant cells, partially in the vicinity of remnants of trabeculae. Magnif. 100. After Bassett.

are most striking in the pelvis, the acetabula of which appear pressed inward, while the ilia are much bent (Figs. 165 and 166); on the rertebral column scoliosis, kyphosis and lordosis are to be noted and the depression of the ribs causes the thorax to become narrow and the sterum to protrude. The pelvic bones, and still more the long bones and the ribs often show recent or old fractures, callus formation, and circumscribed and hogs a considerable bulging of the skull bones is also often observed. It is less frequent in goats and dogs. All skull bones appear enlarged symmetrically on both sides and the periosteum is easily pealed off. The surface of the bones is reddened, at times discolored dark- or blue-red and contains numerous small holes which are often confluent; the outer lamella of the bones is soft and easily cut. These changes are particularly striking in the superior maxilla which may bulge out so much that the molars are imbedded in the reddish, rather fibrous bone mass down to their masticating surfaces. The weight of the macerated head is usually much diminished (in horses 400-1160 gm., as compared with the normal weight of $4000 \mathrm{gm}$. [Marcone]) ; only if the bulging is excessive is the normal weight maintained after maceration. The bone cavities are small or entirely obliterated.

In consequence of atrophy and softening of the bony tissue the bones are subject to all sorts of deformities. These all sorts of deformities. These
the acetabula of which appear is 
bulging; in other cases the tendons are loosened from the bones and the articular cartilages have lost in substance.

On histological examination the spaces between the bony trabeculae and Haversian canals are dilated and bulging, while the trabeculae show the structure of bony tissues almost only in the middle, their marginal portions, which are bordering on the narrow spaces, assuming the form of a homogeneous transparent tissue or of a cellular, non-calcareous tissue which may be finely or coarsely granular (Fig. 167). The finer trabeculae consist in places exclusively of this tissue. The marrow spaces are filled with a highly vascular tissue which is at first embryonal and fibrillary and later contains more fat. The transformation and absorption of the bone substance proceeds from the surroundings of these vessels in which multinuclear giant cells appear (so-called osteoclasts).

According to $\mathrm{O}$. Weber the specific gravity of osteomalacic bones is low, the fat content increased, the amount of inorganic constituents much diminished; the gelatinous substances are also diminished.--Keuscher found of mineral constituents only $321 / 2 \%$ instear of 60 in a thigh bone; in the ribs $30 \%$ instead of $571 / 2$; in the vertebrae and the pelvic bones $26 \%$ instead of 57 . The quantity of these substances is therefore reducel to one-half, the soft tissue increasing in like proportion. Landois found in the bones of a horse with osteomalacia $15.85 \%$ of fat; 12.59 of ossein; $47 \%$ of water, and $22.57 \%$ of lime salts, a percentage that was quite similar to that of healthy bones. Hebrant \& Mosselmann found the mineral constituents diminished on an average by $10 \%$, the ossein by $8-9 \%$; the water was increased by $3-4 \%$, the fat by $8-9 \%$. Ingle showed also a marked diminution of the ash constituents of the bones.

Finally there are found changes suggesting anemia and cachexia, principally edematous infiltration. The muscles which are attached to the affected bones show atrophy and fatty degeneration; in the joints and tendon sheaths inflammatory changes are frequent, as are also ulcers in the articular cartilages.

Symptoms. The signs which are characteristic of the disease are preceded, frequently in cattle, less so in the other animals, by digestive disturbances and by symptoms of licking disease. Snch disturbances are observed also later after the changes in the bones have become manifest. At first the animals simply lick the manger, the iron fastenings and the walls, later they gnaw them and swallow portions of them. They lick and gnaw preferably objects which contain lime, such as mortar, sand, bits of stone and brick, earthen vessels, etc., also woodwork, the latter especially if it is rotten or covered with soot. Later on a predilection for objects which have a disgusting smell and taste becomes more and more marked; those portions of the stable which are soiled with feces and urine are sought out and eagerly devoured, and all sorts of foreign substances are swallowed. At first the patients behave in this strange manner only between feeding and eat the fodder which is put before them readily, but later the appetite becomes gradually worse, the animals become capricious, eat reluctantly, slowly, and steadily smaller amounts. Still later they refuse good fodder entirely and their taste is perverted to such a degree that they may prefer urine or ichor to pure water. Meanwhile emaciation becomes manifest, the hair loses its gloss and becomes rough, the skin becomes harsh and dry, rumina- 
tion is irregular or suppressed, the feces are thin, mushy and foul smelling.

Klimmer \& Schmidt observed the nervous disturbances described in the chapter on rachitis in several instances in such cattle herds in which osteomalacia was diagnosed (p. 942).

The bone disease causes pain so that the animals lie down, dislike to rise, and move only when obliged to. On standing they arch the back upward, stand with their feet spread apart, trip restlessly here and there, lift one and the other foot and groan while discharging urine or feces. The pains are often intermittent, sometimes paroxysmal and change, similarly to

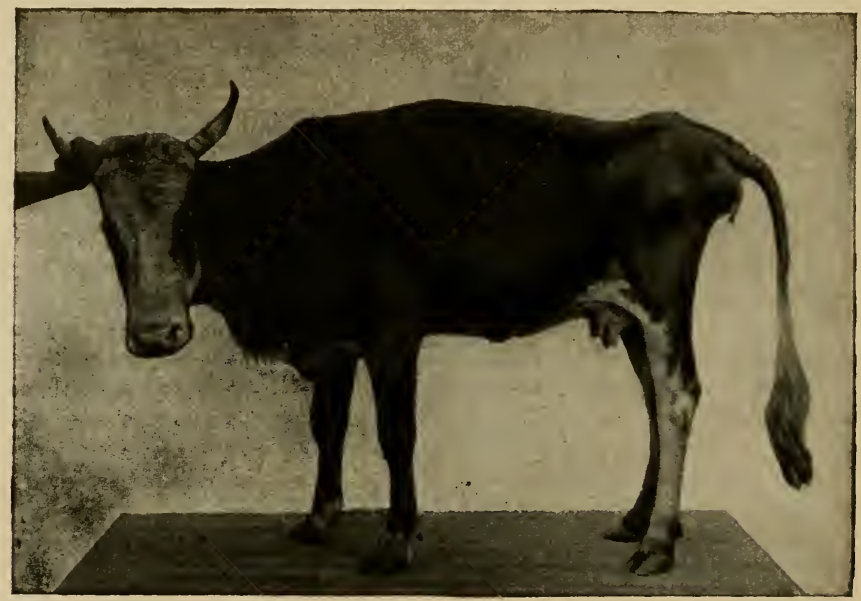

Fig. 168. Osteomalacia in a cow. Emaciation; lordosis of the lumbar vertebrae: the sagittal diameter of the pelvis is diminished (compare Fig. 165).

rheumatic pains, from one part of the body to another, becoming often so severe that horses, for instance, roll on the ground. The animals move carefully, their walk is stiff and a decided lameness is evident in one or the other, sometimes in all four feet. On walking, and also on rising, a peculiar eracking sound is perceptible, probably owing to the loosening of the articnlar capsules. In such cases the bones, still more the joints, the vertebral column and the pelvis are sensitive to pressure. The lower joints of the feet, also the tendon sheaths of the flexor tendons, are frequently subject to inflammatory processes with swelling of the respective parts of the body. According to Liénaux the Achilles tendon is often loosened from its attachment.

Meanwhile the solidity of the bones is lessened more and 
more so that they become bent or fractured on sudden motion, especially on rising or lying down, sometimes even for apparently very insignificant causes. The ribs and the transverse processes of the lumbar vertebrae can at times be depressed easily and even retain finger impressions. In goats the pliability of the skull bones may reach such a high degree that the anterior portion of the inferior maxilla may be bent or turned in an angle of $90^{\circ}$ (Richter). Fractures occur most frequently in the pelvic bones near the acetabulum, in the ribs, and in the proximal long bones of the extremities, also, especially in horses, not infrequently in the vertebral column. Multiple fractures or bulgings are observed fairly often in the ribs and on the pelvis. The fractures do not appear to cause much

Fig. 170.

Fig. 169 .
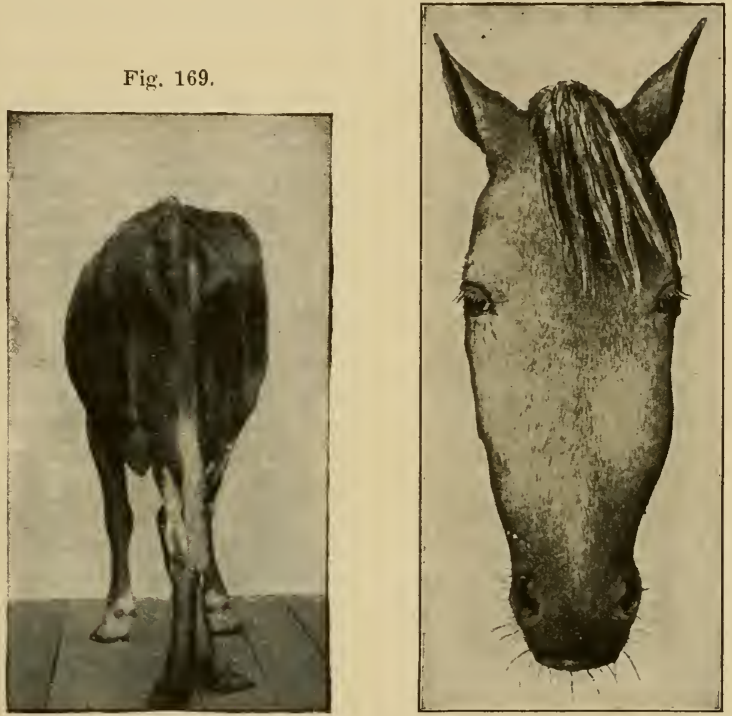

Fig. 169. Osteomalacia in a cow, whose skeleton is illustrated in Fig. 165. Asymmetry of the pelvis; bulging of the left hip: the posterior extremities are crossed.

Fig. 170. Osteomalacir. Bulging of facial bones in a horse. (After Moussu.)

pain, but they further diminish the power of motion which has already been reduced by the spontaneous pain, because they usually either heal not at all or imperfectly.

Deformities occur relatively most frequently in the bones of the extremities, the ribs and the vertebral column. In the later course of the disease, bulging of the skull bones ("big" head") is observed exceptionally in cattle, frequently in horses, 
hogs and goats, sometimes also in dogs; these may also occur in pelvic bones. The head appear's much larger, the roof of the mouth bulging into the oral cavity which therefore becomes smaller, or it becomes impossible to close the mouth so that the appearance of maxillary paralysis is produced (Figs. $16 t$ and 170$)$. In such cases there is blood stasis in the tongue, mastication is difficult or even impossible. The bulging of the bones causes the nasal passages to become narrow, respiration becomes difficult and accompanied by a sniffling or snoring sound; even attacks of asphyxia may occur. The bulging bones are elastic and easily depressed.

Moussu found the alkalinity of the blood diminished, the urinary phosphates increased five-fold; Haubner \& Siedamgrotzky, as well as Marcone, likewise demonstrated a considerable increase in the phosphorus content of the urine.

The emaciation of the animals gradually increases. The muscles become flabby and in some groups of muscles there occur at times fibrillary twitchings. With the severe pain in some bones there is associated a surprisingly high muscular tension in the corresponding region (Siedamgrotzky, the authors). The temperature is usually normal, but is raised to $40^{\circ} \mathrm{C}$. during very severe pain, and also in case of extensive decubitus-gangrene. The secretion of milk diminishes steadily and finally ceases. On the dry skin an eczematous eruption may develop. Finally the animals, which persistently lie prostrate, become totally exhausted and perish.

Course. Osteomalacia always takes a very chronic course. Although the first symptoms usually do not become manifest and the disease is recognized therefore only in a fairly advanced stage, several months usually elapse from the time of its recognition to that of complete exhaustion. Only the very rare cases with severe nervous disturbances usually lead to death in a short time. At times the disease may be arrested or there may be improvement, but in such cases relapses may occur. For the latter gestation, parturition and lactation are predisposing factors and rapidly progressive aggravations usually occur during such times. Sometimes the disease has been latent for some time and becomes manifest only through such an aggravation. With temporary improvements and relapses the disease may thus extend over several years.

Diagnosis. As long as there are no signs of bony softening, the disease cannot be recognized positively in sporadic cases. Licking disease may occur in the course of other affections, especially in gastric catarrh, while pain in the limbs is observed also in muscular and articular rheumatism. In the presence of these symptoms the suspicion of osteomalacia is always well founded if the fodder is poor in lime salts. The possibility of its existence must also be considered when milch 
cows become ill after repeated pregnancies. The licking disease which occurs in the course of gastric catarrh is not continuous; rheumatic affections, especially articular rheumatism, appear in acute attacks with febrile symptoms, and affect usually the higher joints of the feet, while callus formation is absent, especially on the ribs.-Actinomycosis of the facial bones is differentiated by being mostly unilateral and limited in extent, also by the fact that the other parts of the body are free from disease. Rachitis is already excluded by the different age of the animals. (According to Marcone a systematic quantitative determination of the urinary phosphates may afford diagnostic information, but must be made with reference to the quality of the fodder.)

In regions where the disease is enzootic already the very first symptoms give certain indications for a diagnosis, but this becomes positive only when softening of the bones can be demonstrated.

Prognosis. As long as extensive deformities or fractures have not occurred, the animals are not too exhausted and an appropriate regulation of their hygienic conditions can be carried ont, the symptoms may be expected to retrogress, but under other conditions a gradual aggravation of the trouble must be expected. When the animals can no longer rise or when fractures or considerahle deformities have occurred, improvement cannot be obtained by any treatment.

According to Krabbe 281 out of 3,240 sick cows, or $8.6 \%$, were killed in Norway in the year 1877 as incurable. According to Bongartz, the frequency of eures is $20-25 \%$, while Rudofsky observed about $16 \%$ of losses during an epizootic in Moravia (1905).

Treatment. The removal of the affected animals into another region with favorable conditions of soil can be carried out only in the rarest cases, although this procedure seems adapted to secure healing of the disease in cases which have not progressed too far. It is also only rarely possible to arrange for a complete change in feeding; it will rather be necessary to limit endeavors to a partial change of feed, in which the daily amount of food-stuffs which in spite of unfavorable conditions of soil contain comparatively much lime, must be increased considerably (see p. 933). In this respect the following must principally be considered: Clover hay, good meadow hay, esparsette hay, bean-, pea-, lupine-, buckwheatstraw, perhaps some waste from oil factories (see p. 933), especially when there is a deficiency in phosphorus in the feed. Green feed or pasturing on good pastures also influence the course of the disease favorably, as has been observed in the extra-European osteomalacia of equides (Grandmougin, Sourrel). Pasturing has the disadvantage that the motor disturbances increase at first, and that in some animals they only

Vol, 1-61 
then become evident, also that sometimes an increase in the frequency of fractures is observed. The supply of hard drinking water should also be considered, wherever the local conditions permit it.

Lime salts should always be added to the food. Among them the same preparations come first into consideration as are nsed in rickets (see p. 947), but they must be given in sufficiently larger doses; for instance, for large animals, according to weight and employment, and to the probable lime content of the food, $30-80$ to $100-200 \mathrm{gm}$.; for smaller ruminants and hogs, 10-20 gm. in three portions with each feeding. If there is no deficiency in phosphoric acid, chalk is efficient and may be partially $(1 / 3-2 / 3)$ replaced by calcium chlorate, which corresponds to about two parts of chalk, or hydrochloric acid may be given with the drinking water at the same time. In coexisting phosphoric acid deficiency "opened" bone meal is indicated and is, in accordance with Klimmer \& Schmidt, given suitably in alternation with the chalk, thus that the animals receive for 2 or 3 days a calcium preparation and during the next 2 or 3 days a preparation of phosphoric acid, for instance sodium phosphate (tablespoonful 3 times daily). The other preparations of lime (see p. 948) are less suitable. The systematic administration of $\mathrm{HCl}$ or of $\mathrm{NaCl}$ is also indicated in such cases in which calcium carbonate is not given. From phosphorus (0.01-0.05 per day in oil) Klimmer \& Schmidt saw no results. If symptoms of licking disease appear, apomorphine hydrochlorate $(0.10-0.20 \mathrm{gm}$. subeutaneously $)$ is indicated according to Lemke, who claims that after three doses the licking disease will be relieved for 3 to 4 months (Hafner saw no improvement follow this treatment). In cachectic animals Klimmer \& Schmidt obtained transitory improvement in the general condition by the administration of pilocarpine and caffeine.

The suggestion of Roloff not to milk the affected cows empty and to exclude them from breeding during the duration of the disease, deserves consideration if the economical condi. tions permit it. Klimmer \& Schmidt believe it more appropriate to diminish the nitrogenous food-stuffs in order to reduce milk secretion, or to permit cows in advanced pregnancy to stand dry for 6 to 8 weeks. Finally the extirpation of the ovaries might be considered, especially if a proper regulation of the diet is difficult. Very ill animals should be killed as soon as possible.

Prevention. Experience has shown that the disease can be prevented by proper feeding, especially of the cows during pregnaney and lactation, and by suitable cultivation of the soil. Prophylactic measures merit particular consideration in regions where osteomalacia is already enzootic; with appropriate fertilization, especially after the use of fertilizers which contain phosphorus, the disease usually becomes less prevalent in such 
places. (A chemical analysis of the soil is always advisable for the purpose of improving it deliberately.) If the feeding of fodder poor in lime cannot be avoided, the animals should systematically receive lime preparations in the amounts indicated.

If there is a suspicion of an infectious origin of the trouble, isolation of the diseased animals and disinfection of the stable are required.

Literature. Basset, Rec., 1907: 167 (Lit.). - Collard, Bull., 1893. 431. Elliot, J. of Comp. Path., 1908. 206. - Grandmongin, Rev. gén., 1905, V. 500. - Hoennicke, D. m. W., 1906. 116. - Immendorff, Fortschr. d.' V.-Hyg., 1905. 11. - Ingle, J. of Comp. Path., 1907. 35. - Klimmer \& Schmidt, Monh., 1906. XVII. 481 (complete Lit.). - Lüthje, A. f. exp. Path. u. Phmk., 1903. L. 268. Marcone, O. M., 1903. 481. - Martin, Rev. gén., 1905. V. 499, 501. - Pécaud, Rev. gén., 1904. III. 1. - Richter, Z. f. Tu., 1909. XII. 394. - Rudofsky, O. M., 1905. 193. - Schmidt, Ergeb. d. Path., 1897. IV. 531 (complete Lit.). - Sourrel, Rev. gén., 1906. VII. 233. - Theiler, Monh., 1907. XVIII. 193 (Lit.). - (See, also, literature on rachitis.

\section{Licking Disease. Allotriophagia.}

\section{(Nagekrankheit. Lecksucht, [German].)}

The licking disease consists in a morbid tendency, produced by disturbances in metabolism, to lick, gnaw and devour various objects, and carries in its train chronic digestive and nutritive disturbances as well as nervous symptoms.

According to this definition the morbid perversions of taste which are often observed in gastric catarrh, in rabies and in other diseases are not classed with licking disease.

Occurrence. Licking disease occurs only in certain regions and affects almost exclusively cattle, more particularly cows and weaned calves or heifers. In the affected regions the disease is stationary in certain premises, but occur's enzootically after failures of crops, causing considerable losses. The symptoms become manifest in winter and their intensity is the higher, the longer the winter has lasted. The disease has not infrequently been observed in regions in which osteomalacia is prevalent (Roloff, Roll, Diem, Bräuer, Hutyra \& Marek).

In Germany the following parts of the country are visited by the disease: the Saxonian Erzgebirge, the Bavarian Wald, the Western Allgäu, the Badish Black Forest, the Swabian Alp (Ostertag \& Zuntz). More recently it occurred with particular violence on the Johannisburg Heide (in Eastern Prussia), especially in calves and heifers. This greater prevalence of the disease coincided in time with the melioration of the peat fields.

In France cases of the affection are observed distributed fairly evenly throngisout the whole country (Moussu).

Etiology. Regarding the licking disease, which occurred in the vicinity of Johannisburg, the investigations of Ostertag and Zuntz showed that the causative factor is a foor poison, 
contained in peat hay and which is not known exactly. It occurs in greater quantities in the hay of meliorated meadows than in other meadow hay, is entirely absent in meadow plants before they are in bloom, also in the hay of the first cut before blooming time and in the clover hay of meadows which are otherwise dangerous. The second crop of hay is less dangerous than the first. The poisonous substance is destroyed entirely or partially by steaming, heating to $80^{\circ} \mathrm{C}$. and during the preparation of "brown hay." It has no harmful effects on horses.

The presumable poison is present in the injurions hay in comparatively small amounts and therefore it produces gradually increasing disturbances of metabolism through a eumulative action and only after the hay has been fed for a long time. These disturbances cause a defective formation of blood and bone tissue and eventually give rise to a morbid tendeney to gnaw and to lick. The elimination of $\mathrm{Na}, \mathrm{Ca}$ and of $\mathrm{P}$ is greater than the intake.

The chemical composition of Johannisburg peat meadow hay is insufficient also in so far as the hay is poor in Na and Ca salts and rich in $\mathrm{K}$ salts. Although the quantity of the ashes was found insufficient, the cause of the disease ean, according to Ostertag and Zuntz, not lie in a mineral defieiency of the fodder, because the addition of $\mathrm{NaCl}$, of ealcium-, phosphorus- and iron-preparations to the hay did not influence the toxic action and because this could be destroyed by heating.

How far the results of Ostertag \& Zuntz may be applied to licking disease occurring in other places cannot be determined at present. For those cases, indeed, in which licking disease develops in connection with osteomalacia or simultaneously with this disease, the same causes are probably active. It appears that particularly the deficiency in nutritive salts is of essential importance in this respect.

As other causes of the disease contagion, hererity, imitation, ball care, have been mentioned.

From what has been said it is evident that a close relation exists between licking disease and osteomalacia, since in both diseases certain changes in the bones develop under the influence of disturbances of metabolism. The most important difference between the two is seemingly that licking disease oceurs apparently independently and leads to severe cachexia even before manifest softening of the bones has developed. But since in such cases the bones have not been examined microscopically, the possibility cannot be excluded that changes corresponding to osteomalacia are indeed present, only not in such a degree that the softness of the bones could be determined by the coarser methods of examination; and this is evident from the observations of Ostertag \& Zuntz. It is thus thinkable that licking disease actually owes its development to osteomalacia, but that 
in its course the debility progresses much more rapidly than the pathological process in the bones.

Anatomical Changes. In far advanced stages of the disease there occur severe anemia and emaciation, with compiete atrophy of the fatty tissue, muscular atrophy and atrophy of glandular organs. Ostertag \& Zuntz also demonstrated atrophy and rarification of the compact bony tissue, with otherwise normal chemical composition of the bones, also a brittleness of the ribs.

Symptoms. At first the desire to eat is diminisher, rumination is slow, defecation arrested. The aberrations in taste or the peculiar characteristic phenomena appear only after these symptoms, increasing gradually and assuming the form which lias been described in the chapter on osteomalacia (page 957). Together with the lessened desire to eat, emaciation, timidity and an increased sensibility become manifest in the animals. Pregnant cows often abort during the disease. The symptoms persist for 2-4 months, according to the severity of the disease, lead gradually to cachexia and finally the animals die of starvation.

Course and Prognosis. The affection always takes a chronic course and extends over few to several months, sometimes even a year and more, during which time temporary remissions in the symptoms may take place. If no change is made in the hygienic conditions, fatal termination is the usual outcome, but otherwise licking disease may be cured, except in very severe cases with marked cachexia.

Treatment. $\Lambda$ positive result can be expected only from pasturing, although the improvement will make only slow progress. In several instances a favorable influence was observed from a change of feed, the addition of linseed or linseed cake, or feeding with slops, carrots, molasses (Kleinpaul) causing the symptoms to diminish. On the other hand, Ostertag \& Zuntz found the admixture of highly nutritious foodstuffs or of nutritive salts to the injurious hay useless, and also apomorphine, which, however, was employed by some veterinarians (Hackl, Reindl, Lemke) with lasting success, by Kleinpaul with only transitory benefit. The remedy was given hypodermically; to adult cattle and to calves in doses of $0.10-0.20 \mathrm{gm}$. daily three times, for three days (Kleinpaul; to horses three times daily with 3-day intervals (Hillebrand). (After the injection calves must be put in a closed place in order to prevent their injuring themselves, because apomorphine produces in them a very decided excitement [Kleinpaul]).

If the disease is associated with osteomalacia, the treatment described for this disease is indicated (page 961). 
Prevention. If the disease has developed after feeding of peat meadow hay, its occurrence may be prevented permanently, according to Ostertag \& Zuntz, by using the peat meadows for pasturage, by "brown hay" in place of the ordinary hay, or by sowing clover on the peat meadows. On the other hand, the harm is only diminished if the hay is treated with scalding water or if it is steamed; although animals may be carried through the winter fairly well on hay treated in this manner. The poisonous effect of the hay may also be reduced by fertilizing the meadows with sodium nitrate. A further preventive measure consists in feeding cattle in the affected regions only with second crop hay, or with the first crop which has been cut before flowering.Bräner and Lippold observed favorable effects from draining the pastures.

Literature. Bernitt, P. Vb., 1902. II. 30. - Bräner, S. B., 1895. 63; 1590. 80 ; 1893. 121. - Hillebrand, W. f. Tk., 1906. 367. - Kleinpaul, B. t. W., 1903. 1. Leibenger, W. f. Tk., 1906. 542. - Lemke, D. Z. f. Tm., 1882. VIII. 102. Lippoli, S. B., 1890. 80. - Ostertag \& Zuntz, Z. f. Infkr., 1907. II. 409. - Weigel, S. B., 1893. 121.

\section{Wool Eating.}

Occurrence. Wool eating is observed in the finer breeds of sheep only during wintering, especially toward the end of winter, and causes considerable losses in wool, sometimes even death owing to the formation of hair balls in the stomach.

Etiology. Apparently wool eating is often only a bad habit which is possibly formed in consequence of the tedium of the young animals when they are constantly kept in the stable, and may be assumed to spread by imitation. It is, however, very probable that in certain cases insufficient feeding or an unsuitable chemical composition of the fodder, especially a deficiency in nutritive minerals is a factor.

Symptoms. In a drove of sheep a few lambs begin, usually already in early winter, but mostly not until later, to gnaw the wool of their mothers, preferably on the thighs, on the abdomen and the tail, that is principally on those parts of the body which are soiled with urine or feces. More and more of the lambs follow the example, and eventually not only their own mothers, but other sheep as well are attacked, the lambs gnawing at them together. Among yearlings or older sheep also, one or at most a few animals begin to gnaw the wool of another one, but presently more and more animals acquire this vicious habit. At first the gnawing sheep attack the same animal, choosing a new victim only when they have eaten all the wool off the first one. In this manner the evil assumes a constantly increasing extension, until finally all sheep have acquired the habit and the wool eating has become general in the drove. 
Usually the animals appear to be in good health, only of the lambs a few perish, exceptionally many (Moussu, Richard), because wool balls have formed in a compartment of the stomach and occlude the openings of some of the compartments or the lumen of the intestine. In older sheep at most slight nutritional disturbances are observed, although in exceptional cases symptoms of anemia and emaciation may occur.

Diagnosis. The fact that wool eating is indulged in preferably, and at first exclusively, in day time and in the intervals between feeding, and also the fact that the animals gnaw only the wool of their fellows, never their own, is sufficient to distinguish this vice from other diseases which are accompanied with itching and also from trembling disease.

Treatment. The habit can usually be removed by isolating the wool eaters as well as their victims. It is advisable, even in winter, to turn the animals out of doors for a certain time during the day, when the weather conditions permit it; moreover it is necessary to provide proper food and a sufficient mineral content of the food. If the ewes give little milk, the lambs should receive cow's milk to make up the deficiency. Lemke obtained excellent results from the subcutaneous administration of apomorphine hydrochloride (0.1-0.2 grn., a dose for three days).

Literature. Körte, Tierzucht, 1892. 218. - Lemke, D. Z. f. Tm., 1882. VIII. 102.

Feather Eating and Feather Pulling in Birds. This vice is observed most frequently in eaged birds, especially in parrots and eanaries, but not infrequently also in domestic fowls, especially in chickens.

The causes of feather eating are probably various. Klee considers as the most frequent eause the deficiency in certain nutritive substances in the hody, which is due to one-sided feeding of animals which are at the same time confined permanently in narrow quarters, so that they have no opportunity of picking up insects and worms, chalk or greens. Other authors take feather eating to be mainly a bad habit originating in play or in tedium, when the animals have but little opportunity to move about in the open air, and although their food is unobjectionable. Feather eating may also develop in consequence of itching skin diseases and owing to the itching sensation when the young feathers break through during moulting (Klee). In this manner the feather eating which occurs, although rarely, in well-kept fowls may be explained.

Symptoms. In feather pulling the animals pull out either their own feathers or those of their fellows. Among chickens the most beautiful and valuable laying hens often first acquire this bad habit. At first only occasionally isolated feathers are pulled out, but later even the newly growing feathers are torn out so that the body becomes disfigured by the bald spots which are produced. 
Feather eating differs from falling out of feathers in various skin diseases or in nutritive disturbances, in that in the latter feathers are never pulled out.

The treatment is adjusted to the eause. In most cases a change of food and, when the weather permits, the opportunity for sufficient exercise in the open air are indicated. Klee and others saw good results from the administration of the blood of slaughtered animals, which was cooked and mixed with bran or curds. In addition the animals should receive green feed or cooked earrots, also chalk (powdered egg-shells, burned shells, bone meal). In general the principal stress should be laid upon as much variety as possible in feeding. Parrots, which are freed only with difficulty from the vice may be given sepia or bitter chocolate. If there is itching, daily sprinkling with water or with a $5 \%$ solution of thiol may be effective.

The feather eaters should be kept isolated and if possible in a dark place. Sometimes apomorphine hydrochloride is useful, injected subcutaneously in doses of $0.0005-0.001 \mathrm{gm}$. or in drinking water 0.05:100 (Friedberger \& Fröhner, Klee). Inunctions with remedies having a disagreeable odor or taste (tincture of aloes, veratrum, asafetida, gentian, rancid animal oil, earbolic acid) do not accomplish the purpose and may in fact be injurious.

\section{Gout.}

(Gicht, [German] ; Goutte, [French] ; Gotta [Italian].)

Gout depends upon the storage and deposition of urates in the tissues of the internal organs (visceral gout), or at the same time also in the joints (arthritis urica) and is based upon disturbances in metabolism.

Occurrence. Gont is, almost without exception, observed only in birds, in which it is of rather frequent occurrence. Among domestic fowls chickens are, according to Klee, affected with preference, less so water fowl, and most rarely pigeons. (Hartenstein observed visceral gout enzootically in a large goose establishment, but described it under the name of uremia.) Pheasants are affected not infrequently. But the disease is met with most often in the birds of prey of the zoological gardens, less often in ostriches. It develops only rarely in birds kept in the house, and bircls living at large do not appear to become affected at all. Among mammals only a few cases were observed so far in older dogs (Spinola, Bruckmüller), although Vogt claims to have seen a gouty affection also in a horse. Finally the disease has been noted in reptiles (alligator, snakes) (Kitt). Virchow and Mendelsolm recorded deposits of guanin in the joints in two hogs. Pflug found a gmanin deposit in a ham. In a cow which during life had shown poor appetite, inefficient nutrition and frequent epistaxis, Joest demonstrated deposits of xanthin in the spleen, the lymph glands and the kidneys. 
Etiology. Without a doubt gout stands in close relation to the diet, since it develops preferably on generous feeding with substances containing much albumin, especially nucleoproteids. Such substances are especially the thymus glands, the liver, kidneys, brain, pancreas, and the flesh of animals in general which, although poor in nuclei, yet contains much hypoxanthin; young germinating plants also contain much of nucleoproteids (Kionka and Bannes produced typical gout in birds by feeding them for weeks and months exclusively on horseflesh). That certain chemical substances have an etiological importance is indicated by the experience that in man the disease may occur in connection with chronic lead poisoning, and v. Kossa pointed out the gout producing action, upon chickens, of chromic acid, oxalic acid, carbolic acid, corrosive sublimate, cane sugar, aloine and acetone. According to earlier observations smut fungi (Ustilago maidis) also are said to be capable of causing gout in birds. Finally the disease probably develops in birds in certain cases in connection with disease of the kidneys or ureters, for Ebstein and others succeeded in producing gout artificially in birds by ligating the ureters, and v. Kossa proved in cases of toxic gout that nephritis must be considered as a cause for the deposition of urates in the tissues.

Favoring factors are want of exercise or confinement of the animals. Of birds, especially the more carefully bred and kept large breeds are inclined to the disease (cochin-china, brahma), then adult and especially older animals. Male animals are affected more frequently than females. Lastly it appears that individual peculiarities are of essential importance for the predisposition.

Pathogenesis. The mode of development of gout is at present not entirely cleared up. This much may be concluded from investigations or animal experiments that have been made that, under the influence of the enumerated causative factors, an increased production of uric acid occurs, and also a toxic nephritis, the latter either under the influence of the eliminated toxins or also in consequence of the excess in uric acid. The increased formation of uric acid, together with the inhibition (owing to the existing nephritis) of the elimination of urates, lead to a storing of urates in the blood (Benion, Kionka, v. Kossa) and under certain conditions to the deposit of urates in the tissues. According to Brugsch \& Schittenhelm, the ultimate cause of gout may, however, lie in a deficient transformation of the purin bases which are formed from the nucleoproteids (first of all of the hypoxanthin) into uric acid, and in retarded combustion of the uric acid. owing to the absence of certain ferments.

According to Ebstein the urates which are eliminated from the blood produce necrotic foci in the tissues, especially in places where the circulation is very slow, and are only later deposited in the necrotic tissue in the form of erystals. Around 
these foci a secondary inflammatory reaction ocenrs with emigration of white blood cells. Almagia found that cartilage tissue of itself decidedly attracts uric acid.

In disturbances of the renal function from other causes, or in a mechanical impediment to the flow of urine, the storage and deposition of urates occurs even without a previous increase in the formation of uric acid.

The source of the uric acid are the albumins of the body, both those ingested with the food and those that have disintegrated; in mammalians the nuclein bases are here especially concerned, in birds all albuminous substances. The uric acid content of the blood is therefore probably increased for the reason that the animal ingests an excess of albuminous substances. The fact that anong domestic animals birds which even normally eliminate very much uric acid are affected almost exclusively, indicates the predisposing influence of the presence of uric acid in large amounts. Nothing positive is known about the cause for the elimination of the urates of sodium and calcium which are in solution in the blood. The fact that the serous membranes and often the joints of the extremities are usually affected, might in part stand in relation to their being constantly subjected to mechanical irritation, and to the very slow blood circulation in the joints as well as in the serous eavities, although it might also be the consequence of some metabolic disturbance of these parts of the body.

Anatomical Changes. In visceral gout, which is by far the more frequent form, the serous membranes of the thorax and the abdominal cavities, especially the pleuræ or the peritoneum, the membranes of the air sacs, the pericardium, also the surface of the heart, and the liver are covered more or less densely with masses that are dust-like or chalky, and mortar-like; the kidneys are dotted white and the ureters are sometimes filled with white plugs.

Articular gout, which is much less frequent, affects preferably the articulations of the feet, less often also those of the wings, leading to swelling of the involved joints, in and around which white chalky swellings or deposits are seen which are like peas to cherries in size. Similar foci are also found in the adjoining bone tissue and bone marrow, in the articular ligaments and tendons, less often in the cartilages of the larynx, in the vessel walls or under the skin.

In the chalky masses great numbers of erystals which consist of sodium urate are seen under the microscope, especially after the addition of some acid. These erystals may sometimes be demonstrated also in organs which present no microscopic changes.

Symptoms. In articular gout the joints that are affected most frequently are the metatarsal, tarsal and phalangeal joints, and on the wings the elbow joints. There is at first aching, the affected animals moving about only unwillingly and going lame; they remain in one place or stand on one foot in order to ease the pain. If the wings are affected these are kept as quiet as possible, and when the animals are disturbed they flap at most a few times with their wings. At the same time the joints swell (Fig. 171), and while the swellings which are soft and sensitive are at first not sharply circumscribed, later on isolated nodes (tophi s. Noduli arthritici) are more and more 
sharply demarcated from the surrounding tissue and form firm, not very painful, tumors which are closely united to the cartilage, less often freely movable upon it. The nodular swellings are as a rule of from hempseed to lentil size but may, in exceptional cases and in the larger birds, become as large as pigeon eggs. The development of such thickenings on several joints causes very striking deformities. The toes are spread apart by modes in their joints, are bent toward the soles and thris apparently shortened.

The motility of the affected joints becomes limited more and more and may develop into complete ankylosis. Sometimes s of t e $\mathrm{ning}$ occurs later in some joints, the skin over the softened places becoming thinner and finally necrotic; throngh the resulting opening a soft, crumbly, y e $110 \mathrm{w}$ gray substance, like gypsum, is discharged. The borders of the ulcers which form in this manner are irregular and bleed easily; their base is formed by cartilage or by exposed bone tissue, or the probe may

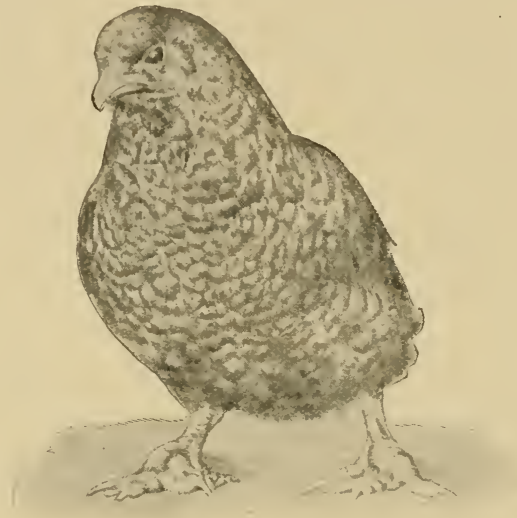

Fig. 171. Articular gout in a hen. (After KionkA.) even enter an opened joint. These ulcers may form in sucession and become confluent.

Smaller hard nodes are sometimes found in the tendons, skin and subcutis, and may even surround the base of the quills.

With the further progress of the disease nore joints are constantly involved, and the nutrition of the animals is interfered with. The feather coat becomes rough, some feathers fall out, the comb and wattles become pale, the animals are finally hardly able to move from place to place, and perish of exhanstion and emaciation.

In dogs nodular thickenings were observed on the lower articulations of the feet and on the lower ends of the ribs.

In the supposed gout of a horse Voigt noted painful swellings on the bones of the extremities near the joints, and lameness. Various parts of the extremities were affected alternately, giving rise to paroxysins. The appetite was diminished and the urine contained uric acid crystals. The disease terminated in recovery in the course of a year. 
Visceral gout is, in its ordinary course, manifested by similar nutritive disturbances as the articular variety; but unless the joints are likewise affected, the actual cause of the disturbances cannot be recognized during life.

Course and Prognosis. The course of gout is almost without exception chronic, only Hartenstein observed the disease in an acute form in geese. In most cases only one extremity is affected at first, and other joints not for several months, usually the corresponding joints on the other side; in the meantime the acute signs in the joint that was first affected have diminished, and the general condition of the patient has improved. The involvement of other joints is usually introduced by acute symptoms which, however, later go down.

The prognosis is unfavorable, since complete healing is rarely observed. This may be looked for at most in domestic fowl that live in the open; in birds kept in captivity the disease progresses steadily and leads the sooner to complete exhaustion.

Diagnosis. For the positive determination of gout in the living animal the microscopical or chemical demonstration of uric acid or of urates is absolutely necessary.

For the microscopical examination the material to be examined is rubbed up on a glass slite with pure or slightly acidulated water, after which the crystals of sodium urate are recognized as very fine needles which form sheaf-like groups or radiating balls. The chemical test for uric acid is properly made with the Murexid-reaction. The secretion is mixed in a porcelain dish witl a few drops of concentrated nitric acid and is warmed slowly until the mass is dried; if ammoniac is added to the onion red residue after this has become cold, it assumes a purple color, while the addition of sodium hydrate produces a violet coloration which disappears on heating.

Tuberculosis of fowls or the chronic forms of fowl cholera may cause similar changes in the joints, and if other indications of these diseases are absent, or if no information is afforderl by outside conditions, the exact nature of the disease can be determined only by the microscopical or chemical examination of the secretion. Purulent arthritis and periarthritis, and also articular rheumatism are differentiated, at least in a part of the cases by a more chronic course.

Treatment. The diet of the sick animals should be reg. ulated in such a manner that they receive only limited rations of nitrogenous substances, especially such as are rich in purin bases or nucleoproteids. Alkaline salts (sodium bicarbonate, Carlsbad salt, etc.) may be added to the drinking water. The patients should be enabled to exercise freely, especially in pure dry air, a desideratum which is usually not feasible in captive birds. Experiments in birds having permitted the conclusion that the deposit of urates is prevented when piperazine is present in the blood, this remedy might be tried in the 
sick animals (chickens 0.5-1.0 gm., pigeons 0.40-0.50 gm. daily, broken up in frequently repeated doses and administered for 4-5 (lays). Human physicians observed in acute gout analgesic effect from tincture of colchicum, and this remedy might also be tried in the articular gout of birds (2-5 drops).

If symptoms of acute inflammation are present, the affected joints are wrapped in salicylated cotton, which may previously be dipped in alcohol or carbolic acid. Later on the operative removal of the well circumscribed nodes may be attempted, especially of those in the skin and in the subcutaneous connective tissue, or the softened nodes may be opened and curetted, after which the opened joints are irrigated with a weak disinfectant solution.

It will be best to fatten and kill animals, which are intended for the table, immediately on the appearance of even moderately severe symptoms.

Literature. Bannes, vet. Jhb., 1901. 251. - Blanchard, ibid., 1891. 190. F'riedberger, W. f. Tk., 1879. 38. - Hartenstein, Vet. Jhb., 1899. 237. - Hébrant ¿ Antoine, Aun., 1909. 321. - Johne, S. B., 1877. 89; 1879. 39; 1881. 66. Kionka, A. f. exp. Path. u. Phmk., 1900. XLIV. 207. - Kitt, Monh., 1893. IV. 497. - Klee, Geflügelkrkh., 1905. 51. - Kossa, Közl., 1899. III. 65; 1907. VII. 101. - Pflug, Mag., 1871. 197. - Siedamgrotzky, S. B., 1872. 89. - Vogt, D. t. W., 1906. 357 .

\section{Scurvy. Scorbutus.}

Scurvy is a peculiar form of the hemorrhagic diathesis which is manifested by bleeding and ulceration of the gums, also by hemorrhages in the most various organs of the animals and which, in severe cases, causes debility.

History. In former times all diseases, in which the tendency to hemorrhage predominated in the clinical picture, were designated generally as scurvy, but more recently the large group of hemorrhagic diatheses was divided into several independent clinical pictures and, in addition to scurvy, Barlow's disease (p. 949), purpura, petechial disease, Werlhof's disease and hemophilia are differentiated. It is not possible to draw sharp distinctions between these diseases and, indeed, several investigators consider scurvy, Barlow's disease and Morbus maculosus Werlhofii only as different manifestations of the same affection.

Seurvy in man has become known especially since the great wars of the middle ages and since the sailing trips around the world, and at that time it caused occasionally great losses. When the hygienic conditions improved, the number of eases diminished steadily, and at present only sporadic cases are met with, at least in western and central Europe. Veterinarians also paid at first little attention to the differences between the various representatives of the hemorrhagic diathesis, and in consequence cases of purpura, especially in eattle, hogs and dogs, or cases of ulcerative stomatitis in lambs have often been described as scurvy. Some clinical descriptions by Spinola, Siedamgrotzky, Pringle, Arnous and others suggest that seurvy does 
occur among. domestic animals, especially dogs, although the disease only rarely reaches its complete development. According to the observations of Weber, Schupp and Wolf, the scurvy of hogs does not really correspond with the actual scurvy, but rather with purpura.

Etiology. In the occurrence of scurvy in hogs the benign urticaria appears to be of essential importance. Weber noted the disease in about 5\% of all pigs, from 3-6 months old, which he examined, almost all of which had passed through an attack of benign urticaria about four weeks previously, probably after an infection with erysipelas bacilli, and also in the cases of chronic erysipelas of hogs which have been described by Cornevin, Hess and Eisemmann. Predisposing factors are excessive attempts at improving the species, one-sided feeding especially with spoiled fodder, bad hygienic conditions, such as confinement in damp, badly ventilated, dirty stables, insufficient exercise; a severe and damp climate also seems to be of importance.

In dogs the disease develops occasionally after continued feeding with spoiled food-stuffs, especially spoiled meat.

It is thus probable that the disease of domestic animals clescribed as scurvy has a toxic-infectious origin.

Anatomical Changes. Punctiform or striated, or then, more extensive hemorrhages are particularly noticeable in the skin, the subcutaneous and even the intramuscular connective tissue, in the mucous and serous membranes, in the joints, within the skull, etc., while the gums show destructive ulceration. The parenchymatous organs show evidences of fatty degeneration. The spleen is often much enlarged and softened. The lymph nodes, especially of the mesentery, are swollen and hyperemic. The blood is not infrequently pale red and thin fluid.

Symptoms. In hogs the disease commences in some cases with the signs of gradually progressing anemia and emaciation, the animals being less lively, moving reluctantly, eating but little while they drink much water. In other cases the trouble begins, without any preliminary symptoms, with bleeding (Weber). In the skin there appear hemorrhagic spots which may be as large as a one-cent piece and which become more and more numerous, so that at the height of the disease the animals look as though sprinkled with blood. Extensive hemorrhages occur in the skin and subcutis of the ear, even on slight external causes such as taking hold of the ears of the animals. The bristles become loose and are easily torn out, the root-ends being surrounded by a drop of blackish blood (bristle-red). This symptom seems to occur less frequently or not at all in the improved breeds of pigs with fine hair; for instance, it was absent in the cases observed by Weber and Schupp. In the further course the bluish-red hemorrhagic spots in the skin may change into nodes, resicles and even ulcers. Simultaneously with the changes in the skin hemorrhages occur also in the mucous membranes, 
although a special involvement of the gums cannot be ascertained. The gums show macular reddening and swelling, they bleed easily and finally ulcers form upon them. In consequence the teeth become loosened, they fall out easily and a carrion smell comes from the mouth of the animals. Weber and Schupp usually noted hemorrhages also in other mucous membranes, for instance in the orbital cavity, the eye ball being forced outward, epistaxis, severe dyspnea due to hemorrhage in the air passages or in the lungs, also bloody discharges from the intestines and bloody urine. In very severe cases the joints may be swollen.

In this stage of the disease the animals are languid, those portions of the skin which are free from hemorrhages are pale, emaciation and debility progress rapidly and finally lead to death. Usually, however, the animals are killed earlier or die shortly after the appearance of the first manifest symptoms, in consequence of severe hemorrhage in lungs or intestines.

In the cases of scurvy which have hitherto been observed in dogs the disease commenced with symptoms of anemia, to which the changes in the gums, and hemorrhages were added later on. The gums are discolored bluish-red in the region of the incisors, and soon afterward around the molars; they become swollen, sensitive and bleed easily even on the lightest touch and during the mastication of coarse food (kernels, bones).

Later on the mucous membrane becomes necrotic, and gradually the picture of ulcerative stomatitis (Vol. II) develops. Hemorrhages occur also in the conjunctivae and in the skin, less frequent are epistaxis, intestinal hemorrhages, vomiting of blood, bloody urine, and in exceptional cases hemorrhages may occur in the anterior chamber of the eye and in the retina (Fröhner). Sometimes isolated joints are swollen. Death is due either to exhaustion or to severe loss of blood, or then to some complication (general septic infection, pneumonia).

Diagnosis. Ulcerative stomatitis of dogs differs from scurvy in its strict localization, the absence of hemorrhages, and also by the fact that it is not associated with cachectic symptoms until after the disease has lasted a very long time. In Purpura (Werlhof) the changes in the gums are absent, especially the loosening, necrosis and ulcerative destruction of the gums.

Prognosis. In the incipient stage of the disease the prognosis is rather favorable, provided the harmful influences can be removed. If, however, the symptoms of ulcerous stomatitis and hemorrhages have developed healing can no longer be expected.

Treatment. Scurvy ean be prevented almost certainly by suitable feeding of the animals if care is also taken to insure 
fresh air and sufficient exercise in the open. In the treatment of scorbutic animals the best possible results are also obtained by appropriate regulation of the diet. Hogs are best fed with grain with which acid or bitter substances may be mixed, such as acorns, horse chestnuts, calamus or gential root, powdered oak bark, etc.; for dogs good fresh meat, bouillon and milk are better adapted. In severe anemia it is proper to administer preparations of iron (p. 851) and bitter remedies, to dogs also wine and alcohol as well as the customary bitter tinctures. The disease of the mouth demands local treatment.

The sick animals should be isolated and their stables disinfected.

Literature. Arnous, Monh., 1892. III. 193. - Gundelach, B. t. W., 1907. 490. - Hébrant, Ann., 1903. 597. - Siedamgrotzky, S. B., 1878. 14. - Siedanıgrotzky \& Hofmeister, ibid., 1878. 121. - Weber, D. t. W., 1909. 297. - Wolf, Der Skorbut d. Sehweines, Diss. Leipzig 1909 (Lit.). 


\title{
Diseases of the Urinary Organs
}

\author{
Section I. \\ DISEASES OF THE KIDNEYS.
}

\section{Albuminuria.}

Etiology. Albuminuria is observed principally in those diseases of the kidneys in which the glomeruli or the epithelial cells are affected; affections of the epithelia of the tubuli uriniferi may also lead to albuminuria by causing the serum albumin of the surrounding tissues to pass into the lumen of the tubules. Albuminuria represents therefore an almost constant symptom of inflammatory and degenerative processes in the kidneys.

In like manner other nutritional disturbances of the glomerular epithelia may cause albuminuria; disturbances in the circulation of the blood and also changes in the composition of the blood in the various blood diseases act in this manner.

The immediate cause of the albuminuria which occurs in connection with skin diseases, with certain diseases of the nervous system (cerebral hemorrhage, inflammation of the spinal cord, convulsions) and with some very painful affections, has not yet been determined definitely. Possibly the cause lies in disturbances in the innervation of the renal vessels and in the nutritive disturbances in the epithelial cells which occur in consequence.

The glomeruli retain only serum albumen, while other albuminous substances, e. g., egg albumen injected directly into the blood or hemoglobin liberated through the destruction of red blood cells, easily pass into the urine.

Traces of albumen are sometimes found in the urine of healthy individuals (physiological albuminuria); in man especially after generous meals, severe muscular exertion, sexual excitement, copious perspiration, in depressed conditions of the mind and during pregnancy. Simader examined the urine of several hundred healthy animals by Posner's method, and found that minute quantities of albumen form a normal constituent of animal urine, and that even a fairly considerable quantity of urine, up to $0.01-0.03 \%$ in the urine of dogs and hogs, less in the urine of ruminants and very rarely in that of horses is devoid of any pathological significance. The albumen which occurs under normal conditions in the urine seems, however, to be mostly nucleo-albumen and mucin. Fettick has shown that in the dialyzed urine of healthy animals acetic acid does not cause cloudiness,

Vol. $1-62$ 977 
so that an existing precipitate has not been formed by serum albumen, but by another substance which is as yet unknown. Henn also doubts the occurrence of serum albumen in the urine of healthy animals.

Except in the true or renal albuminuria, albuminous substances also occur in the urine when blood serum, blood, pus, ete., is mixed with the urine somewhere in the urinary passages after it has left the renal tissue, or derived from the genitals (vagina, prostata). This constitutes spurious albuminuria.

Diagnosis. The recognition of albuminuria depends upon the chemical demonstration of albuminous substances in the urine; but since albuminuria is only a symptom of several diseases, clinical examination must discover also the underlying organic disease.

Demonstration of the Albumens which are Preformed in the Blood. (Serum albumen anil serum globulin.) For this Heller's ring-test is especially adapted and as small an amount as $0.02 \%$ of albumen can easily be demonstrated with it (Hammarsten); it is made as follows: A small amount of the urine, which has previously been filtered, is carefully poured unto a test tube containing concentrated nitric acil, so that it "over-lies" the acid. At the point of contact of the two liquids a sharply defined white ring forms, the depth of which varies according to the amount of albumen present.

For the boiling test a few cubic centimeters of urine are heated to boiling in the test tube and are then acidulated by the addition of 5 to 10 drops of concentrated nitric acirt. A precipitate which has been produced by earthy phosphates or earhonates will then dissolve, but one due to coagulated albumen persists.

The acetic acid-ferrocyanide of potassium test is marle thus that up to about $2 \%$ of acetic acid is added to the urine and then a $5 \%$ solution of ferrocyanide of potassium, the latter drop by drop and avoiding an excess. If albumen is present a decided turbidity or a floceulent precipitate appears. This test may be macle like Heller's test, a mixture containing a few cubic centimeters of dilute acetic acid and a few drops of ferrocyanide of potassium solution being made to over-lie the urine.

Spiegler's test seems to be particularly sensitive and is said to be exact for a minimum of $1: 350,000$. A test tube is filled one-half with a mixture containing 8 parts of mercuric bichloride, 4 parts of tartaric acid, 20 parts of glycerin and 300 parts of water; the urine is then allowed to run clown the wall of the test tube drop by drop, when a white ring is formed at the point of contact.

In the salicylic sulfonic acid test of Roch a few erystals of salicylic sulfonic acid are added to a few cubic centimeters of urine, when turbidity results.

The Metaphosphoric acid test after Hindenlang is marle by adding an aqueous solution of metaphosphoric acid to the urine; the result is opalescence or cloudiness.

Esbach's reagent, prepared by Merck in tablet form, is also adapted for practical purposes.

In the trichloracetic acid test after Raabe a small crystal of trichloracetic acid is added to a cubic centimeter of urine; at the point of contact a sharply defined turbid zone oceurs.

Henn recommends the following tests for albumen: the boiling test, the acetic acid ferrocyanide of potassium test, the metaphosphoric acid test (for dog urine), the salicyl-sulfonic acid test (for horse and cattle urine), the trichloracetic acid test (for horse and cattle urine), Spiegler's test (for cattle urine).

For the quantitative determination of albumen Esbach's albuminometer is adapted for the requirements of practice. Acidulated urine is poured in to the sign $U$, the reagent up to $R$ (1 part of picric acid, 2 parts of citric acid, and 100 parts of water); the tube is closed with a rubber stopper and slowly turned over severa] times without shaking; after standing at room temperature for 24 hours, the precipitate has settled and may be read off. The figure indicates the proportion of albumen per mille. Urine which contains very much albumen must first be diluted with water and the indicated figure must then be multiplied with the figure of the dilution.

If serum albumen has been demonstrated in the urine with the customary chemical tests, it remains to be determined 
whether the albumen is derived from the kidneys (albuminuria rera) or from the urinary passages or from the near-by genitalia (albuminuria spuria). If the urinary sediment contains no organic form elements, and if the presence of dissolved blood coloring matter may be excluderl, if further the organic sediment consists exclusively of form elements which are derived from the kidneys it can only be a question of renal albuminuria. On the other hand, in the presence of copious organic sediment a small quantity of albumen (less than 1\%) speaks against renal albuminuria. If the urine contains, in ardlition to pus cells, also epithelia from the urinary passages or, in addition to red blood corpuscles, comparatively much (several per cent of) albumen, the indications point not only to a spurious albuminuria, but it may be inferred that the urine contains albumen on leaving the kidneys. (In the presence of pus cells, at least 50,000 pus cells in a cubic centimeter of urine correspond to an albumen content of 1:1000 [Goldberg].)

Significance of Albuminuria. The importance of albuminuria lies in the fact that a portion of the albuminous substances which circulate in the tissue fluids of the body leave it unused, which necessarily leads to disturbances in nutrition. Accordingly a persistent albuminuria leads of itself to a gradual weakening of the organism. In a given case the amount of albumen present and the duration of the albuminuria are of importance, aside from the basic disease.

Treatment. As albuminuria is only a symptom of several different diseases, the treatment must be adapted to the underlying pathological condition.

Literature. Fettick, Z. f. Tm., 1899. III. 329 (Lit.). - Goldberg, Cbl. f. med. Wissensch., 1893. 36. - Henn, Die Albuminurie, ete. Diss. Giessen 1909 (Lit.). - Rievel, D. t. W., 1901. 151. - Senator, Albuminurie, 1852.

\section{Hematuria.}

Etiology. Blood or red blood corpuscles may become mixed with the urine either in the kidneys already or only in the urinary passages, and they may also get into the urine from the contiguous genital organs. Hematuria presupposes some disease of the blood vessels in the kidneys or in the urinary passages, which makes it possible for the red blood cells to extravasate by diapedesis or by rhexis. Thus renal hemorrhage may occur in consequence of certain forms of acute, less often of chronic renal inflammation, after injury to the kidney, in severe blood stasis, after rupture of the renal artery if this shows an aneurismal dilatation (Liebetanz, Voltz, Hackbarth), also in acute infectious diseases (purpura, anthrax, swine plague, hog cholera, etc.), and likewise in hemophilia and in cachectic 
conditions in general. Of the diseases of the urinary passages preferably the injuries produced by calculi or by parasites (Eustrongylus gigas) and deep-seated inflammations, also certain new-formations, e. g., angiomata (Schmidt) or papillomata may give rise to hemorrhages.

Of special importance is the so callerl hematuria of eattle ("Weiderot") pasture-red) which is, however, a hemoglobinuria produced by piroplasmas (p. 764) and as such is not included in hematuria proper.

Symptoms. The urine is colored more or less red, sometimes contains coagula and, in contrast to urine which contains only blood coloring matter in solution, the sediment is light or dark red and red blood corpuscles may be found in it. In all forms of hematuria the urine contains as a rule hemoglobin in solution, in addition to red blood corpuscles, owing to the destructive action of the urinary constituents upon the blood corpuscles. There usually also exist signs of the underlying disease, and after severe loss of blood the clinical picture of acute or chronic anemia develops.

Diagnosis. Hematuria is recognized with certainty only by the demonstration of coagula, of blood-red sediment or of red blood corpuscles in the urine. Except in the paralytic hemoglobinemia of horses, in which motor disturbances are noticeable from the beginning, jaundice usually occurs in hemoglobinuria. In piroplasmosis ticks are found on the body of the animals and piroplasmas may be seen in suitably stained blood specimens. Epizootic occurrence of the cases suggests piroplasmosis as the most probable diagnosis.

The clinical investigation must determine whether the blood has been added to the urine in the kidneys, the urinary passages or outside of the latter. In renal hemorrhage there are usually also form elements (epithelial cells, casts) which come from the kidneys; by means of palpation it may be possible to demonstrate some anomaly in the kidneys and the red blood cells are sometimes disintegrated (Gumprecht); in profuse hemorrhages from the kidneys or from the renal pelvis the blood coagulates in the ureters in the shape of thick cylindrical formations which are discharged with the urine (Ostermann. Author's case). In hemorrhage into the urinary passages the urine frequently contains irregularly shaped coagula and epithelial cells from the urinary passages; it is moreover not infrequently possible to demonstrate a disease of the urinary passages by means of a local examination. In case of bleeding from the genitals the urine removed from the bladder by means of a catheter contains no blood, and it is usually possible to show an abnormal condition of the genital organs.

Treatment. The mode of treatment depends upon the underlying condition. In copious bleeding hemostatic remedies 
may be employed, such as secale cornutum and its preparations, suprarenin or adrenalin (of the $0.1 \%$ solution $1-5$ ce. per 100 $\mathrm{kgm}$. of body weight, intravenously in physiological salt solution); also astringent or styptic irrigation of the bladder with a $0.5 \%$ solution of tannin, a $1 \%$ solution of alum or a $0.01 \%$ solution of suprarenin.

Literature. Liebetanz, Monh., 1907. XVIII. 454 (Lit.). - Schmidt, B. t. W., 1905. 426 .

Haematuria Vesicalis. (Stallrot der Rinder, German; Hématurie chronique des bovidés, French.) This is a chronic hematuria of eattle which oceurs in Baden (Hink, Anacker) and elsewhere in Germany, also in certain parts of France and Italy (Moussu), in Belgium (Liénaux), and in Hungary, and causes in some regions considerable losses. The trouble occurs after exclusive dry feeding in the stable, and more particularly in older cattle.

As canses of hematuria have been suggested: insufficient feeding (Anacker), bacterial infection (Detroye), coccidiosis of the bladder (Arnold), filaria or distomata (Lydtin), the action of irritating poisons (Galtier), and Hink considers stasis in the territory of the posterior vena cava as the probable cause. The latter view in a somewhat modified form has recently been shared by Liénanx, who referred the development of the disease to the periodical compression of the bladder by the rumen when it is filled unduly with voluminous food, and to venous stasis in the walls of the bladder produced thereby. The circulatory disturbances produced in this manner would then result in similar dilatations in the capillaries, in portions of the bladder, as they are found in teleangiectasia maculosa hepatis. The consequent periodical hemorrhages produce, according to Liénaux, a secondary proliferation of the connective tissue and moreover prepare the soil for secondary hacterial infections of the bladder. On the other hand, Götz maintains the view that certain irritations which are probably not specific but mechanical, chemical, mykotic or toxic, eause at first an inereased discharge of urine which is associated with tenesmus, in consequence of which the epithelia and the tissue of the mucosa are caused to proliferate, while the eapillaries and smaller veins are permanently dilated. In this manner he assumes a gradual varicose dilation of the blood vessels in the mucous membrane of the bladder, and from these hemorrhages into the tissue of the mucous membrane and into the lumen of the bladder. For this reason Götz believes the disease to be a eystitis verrucosa.

The anatomical changes consist at first only in the occurrence of dark red spots in the mucous membrane of the bladder; in a more advanced stage red or hrown, sometimes yellowish or gray varicosities are visible as large as hempseeds, and sometimes between these cellular proliferations, as large as nuts or hen's eggs and up to $2 \mathrm{kgm}$. in weight, roundish or lobulated, like cauliflower (Hink's round-cell sarcoma). Ulcerous destruction of the vesical mueosa or perforation of the bladder wall may also be present and occasionally unilateral or hilateral nephritis or hydronephrosis. The bladder contains bloody 
urine and sometimes coagulated blood. In far advanced cases there are evidences of general anemia.

Symptoms. The urine is light or dark red-brown in color; it furnishes a copious precipitate or red blood corpuscles and coagula, with which are mingled white blood cells, bladder epithelia, and rarely also renal epithelia if catarrh of the bladder or nephritis have occurred secondarily. The formation of good-sized coagula, and the obstruction of the urethra by them, frequently causes temporary retention, more often in male than in female cattle. The general condition is disturbed only later in consequence of anemia or through perforation of the bladder which oceurs oceasionally, likewise more frequently in male animals.

The course is always chronic, extending over weeks, months and even years. It is not rare to observe intermissions of variable duration (especially in cows far advanced in pregnancy the condition is strikingly improved after parturition [Anacker]). Permanent cures are the exception; usually the animals must be killed.

Treatment consists in vigorous feeding; if possible the food should not be very voluminous. In cows irrigation of the bladder with astringent and styptic solutions may be considered.

Literature. Delacroix, Ann., 1905. 244. - Götz, Schio. A., 1906. XLVIII. 1 (Lit.). - Liénaux, Ann., 1905. 185.

\section{Uremia.}

Etiology. In the course of severe, acute or chronic inflammation of the kidney, also in consequence of certain diseases of the urinary passages (obstruction of ureters, arrestment of a urinary calculus in the neck of the bladder or in the urethra, bilateral hydronephrosis, paralysis of the bladder, stenosis of the urethra, etc.) in which the discharge of the urine from both kidneys is impeded, and thereby the function of the kidneys is disturbed, there develops a peculiar train of symptoms which is referable particularly to an involvement of the central nervous system and of the digestive organs.

The nature of uremia is not known definitely. It is generally assumed that it develops owing to the retention, in the blood, of all or most of the solid constituents of the urine.

For a long time such a pathogenic action was attributed especially to urea, but since it was not possible to produce the clinical picture of uremia artificially hy the intravenous injection of urea, Frerichs expressed the opinion that the retained urea is transformed by the action of a supposed ferment into carbonate of anmonia and that the intoxication is produced immediately by this substance. Toit on the other hand assumed the retention of all $\mathrm{N}$-containing urinary constituents, Landois believed that the extractives (especially creatinin) and salts were retained: according to the last named author the respective substances exert their harmful action immediately upon the easily irritated cerebral cortex.-Traube attempted to explain the occurrence of uremia upon a purely mechanical basis by assuming that in kidney disease the blood pressure is inereased and that the blood is diluted through a retention of water, in consequence of which erlema, anil after it anemia 
develop in the brain.-Bouchard \& Lépine found that the normal urine has toxic properties which are considerably diminished in uremia because toxic substances which are ordinarily eliminated are retained in the blood.-According to BrownSéquard the kidneys are, similar to other organs, believed to produce certain substances, by the agency of an internal secretion, which are necessary for the normal maintenance of organic functions; the absence of such substances would then furnish the foundation for uremia.

In an acute form uremia is not observed very frequently in animals, but chronic uremia is by no means rare, especially in dogs, and gives rise to less striking disturbances, which also admit of a different explanation.

Symptoms. Acute uremia occurs especially as a sequel of severe acute, and sometimes also of chronic kidney disease, also in consequence of complete retention of urine if the function of the kidneys has been interfered with to a considerable degree within a brief space of time. As a constant symptom there occurs stupor, sometimes preceded by staggering and vertigo and usually increasing to sommolence and to complete unconsciousness. Simultaneously the animals often show trembling or even clonic contractions, which are often limiter to single muscles or groups of muscles; these are soon followed by general epileptiform convulsions (Pflug observed opisthotonus in a cow), and on their cessation consciousness does not return, but the animals remain prostrate and unconscious. There are, however, isolated cases in which consciousness is preserved even during the convulsive attacks (Liénaux), and in some cases symptoms of excitement are observed between times.

Respiration is sometimes retarded (owing to the unconsciousness) and sometimes rendered difficult owing to the edema of the lungs which develops; occasionally Cheyne-Stokes' respiration is observed. The temperature may at first be considerably increased, but in the stage of stupor it falls even below normal. Sometimes strong perspiration is observed and the skin emanations as well as the exhaled air disseminate a urinous odor (especially after rupture of the bladder).

The digestive troubles are manifested in inappetence, suppression of rumination, in carnivora also in vomiting, and in all species by diarrhea. (The last symptoms are attributed to disintegration products of urea which are eliminated through the intestinal mucosa and cause inflammation.) The romited material sometimes also has an odor of urine.

Acute uremia either causes death in a short time, the fatal termination sometimes occurring after the first convulsive attack, or it is postponed for several days during which the paroxysms are repeated in longer or shorter periods. In animals acute uremia has hitherto always had a fatal termination with the exception of a case of the authors, in a horse with retention of urine, in which relief of the retention caused the uremic symptoms to disappear after a short time. 
Chronic uremia develops exclusively in chronic diseases of the urinary organs, especially in dogs, in consequence of chronic indurative nephritis, as also in incomplete retention of urine. The nervous disturbances consist sometimes in a certain sensory dullness, sometimes in periodical epileptoid attacks, but they are often absent. The respiration is sometimes rendered difficult by asthmatic attacks (Liénaux). The usual phenomena of chronic uremia consist in digestive disturbances which occur without demonstrable cause (symptoms of gastric catarrh, vomiting, diarrhea) and which may be temporary or permanent. Pruritus has also been observed occasionally.

Chronic uremia usually causes death sooner or later, in most cases after the supervention of an acute uremic attack.

Treatment. With the exception of the relief of the urine retention, which is usually operative and which sometimes removes the affection entirely, treatment is not very promising, especially in the presence of severe nephritis. The administration of diuretic remedies, and diaphoresis must be considered; in convulsions nareosis or subcutaneous injections of morphine may be employed.

Literature. Albrecht, W. f. Tk., 1901. 25. - Bédel, Bull., 1897. 266. Grobon, Rev. vét., 1906. 21. - Honigmann, Ergebn. d. Path., 1902. V111. 1. Abt. 549 (Lit.). - Liénaux, Ann., 1894. 65. - Otto, S. B., 1905. 82. - Pflug, Krankh. d. uropoët. Systems, 1876. - Senator, Erkrankung d. Nieren, 1 s $\overline{\text { S }}$.

\section{Hyperemia of the Kidneys. Hyperaemia renum.}

Etiology. Arterial hyperemia of the kidneys develops if irritating chemical substances are carried to them with the blood and are then eliminated. Many diuretic remedies (squills, turpentine, especially cantharides, the latter even on cutaneous application) exert their action in such a manner that they increase the arterial blood in the kidneys. The action of many meadow plants (Ranunculaceae) becomes manifest in a similar manner, and especially young animals frequently show symptoms of renal hyperemia after the ingestion of such plants. In this category belongs also the polyuria (p. 919) occurring in horses after the ingestion of spoiled, musty fodder. Williams observed polyuria in two horses after drinking a small quantity of sea water. This form of renal hyperemia also occurs often in acute infectious diseases in which it is produced by bacterial toxins circulating in the blood.

Venous hyperemia usually accompanies diseases of the circulatory and respiratory organs, when the outflow of the venous blood from the kidneys is difficult. This is most frequently the case in organic heart trouble and valvular disease, also in pulmonary emphysema, in chronic interstitial pneumonia, 
in peritonitis and in edema of the thoracic organs, finally in exceptional cases, owing to compression or thrombosis of the posterior vena cava.

Anatomical Changes. The arterial hyperemia is manifested by livid redness of the renal substance, in which the congested glomeruli often stand out sharply and appear as dark red spots on the cut surface of the cortical substance.

In venous hyperemia the kidneys are dark blue-red, somewhat enlarged and firm; the tense capsule is easily peeled off, whereupon the smooth surface of the cortical layer, which is traversed by venous plexuses (Stellulae Verheyenii) becomes visible. A somewhat prolonged blood stasis leads gradually to an increase of connective tissue which causes the kidneys to become very firm (Induratio cyanotica renum), but later their volume diminishes with the atrophy of the newly formed connective tissue, the surface becomes irregular, and the renal capsule adheres in places to the substance of the kidneys (Atrophia cyanotica). In stasis-hyperemia there is no cellular infiltration in contrast to inflammatory atrophy of the kidneys, but red blood corpuscles lie in small clumps between the tortuous urinary tubuli (Kitt).

Symptoms. In arterial hyperemia of the kidneys an increased amount of arterial blood flows in a given time through the kidneys, in consequence the quantity of urine increases and its specific gravity becomes lower. Aside from polyuria (see p. 918), which is usually accompanied by an increased sensation of thirst, a certain sensitiveness in the region of the kidneys and a stiff gait were noted in many cases.

In venous hyperemia of the kidneys the slowing of the blood stream results, as an immediate consequence, in a diminution of the quantity of urine. If the stasis of the blood is prolonged, the nutrition of the epithelial cells suffers and there occurs an albuminuria which is usually only slight; on microscopical examination red blood corpuscles and hyaline casts may sometimes be found in the urine. The specific gravity of the latter is increased and it contains, in carnivora, large quantities of acid urates which form, on cooling, a reddish sediment on the bottom of the vessel and disappear when the urine is heated.

Diagnosis. For the determination of arterial renal hyperemia it is necessary to exclude all other diseases for which polyuria is also characteristic.-Diabetes mellitus is distinguished by its high specific gravity and the content in grape sugar, chronic indurative nephritis differs by the amount of albumen present in the urine and by the long duration of the disease.-From diabetes insipidus arterial hyperemia can 
be differentiated almost only on the strength of its acute course.

Venous hyperemia differs from renal inflammations by the fact that the urine contains only little albumen and no real form elements, and also by the existence of some basic disease which has produced stasis of the blood in the kidneys.

Treatment. Arterial hyperemia disappears in a short time after the removal of its cause, especially after an appropriate regulation of the feeding. The administration of irritating remedies should be avoided as much as possible, especially in acute febrile diseases.

The relief of blood stasis in the kidneys is possible only by suitable treatment of the underlying disease, and it is especially those remedies which raise the blood pressure that enter into consideration (see chapter on valvular diseases).

Literature. Augner, Mag., 1847. 95. - Dammann, D. t. W., 1898. 125. Friedberger, Z. f. pr. Vet.-Wiss., 1874. 292. - Pflug, Krankh. d. uropoët. Systems, 1976. - Williams, Vet. Journ., 1901. 263. (See also Lit. on Diabet. insipidus.)

Hemorrhagic Infarct of the Kidneys. The formation of infarcts in the kidneys is always a consequence of obstruction of the arteries, by emboli earried from elsewhere or by thrombi which form at the location of the infaret. Infarct formation is frequent after obstruction of smaller branches of the renal arteries by emboli, especially in valvular disease, but this is not to be discussed here because it cannot be diagnosed elinically, or at most presents the elinical picture of chronic indurative nephritis if numerous infarets have formed in succession. Those infarcts need also not be considered here which develop after the arrestment of pyogenic bacteria (see the chapter on purulent nephritis). It is intended to discuss here only the hemorrhagie infarction which oceurs after complete occlusion or in marked stenosis of the renal artery. It occurs probably only in the horse because thrombosis of the larger blood vessels (usually produced by larvae of sclerostoma) occurs with few exceptions only in this animal. It appears to be not infrequent, although it is usually mistaken for other diseased conditions of the kidneys. The authors have observed four cases within a short space of time and several eases are reported in literature (Cadéac, Lustig, Ostermann), which permit the diagnosis of a similar kidney disease.

The eause of hemorrhagic infarct lies in the occlusion or marked stenosis of one of the renal arteries, which causes the blood pressure to fall in that kidney. Arterial blood then flows into the kidney through the few anastomoses between the blood vessels of the kidney and those of the capsule and the ureters; on account of the considerable size of the region which is excluded from the circulation the blood remains under low pressure for a time or permanently. The great diminution in the arterial blood pressure causes a stasis and extravasation of the blood. (Compare Vol. II.)

The effect of an occlusion of the renal arteries was elucidated by Littén in animal experiments. If the vascular obstruction was not complete or if it was relieved after a short time, the circulatory disturbances retrogressed; but if in 
the meantime the nutrition of the renal parenchyma had suffered, a new-formation of connective tissue was stimulated in places.

The thrombosis is probably only rarely situated in the renal artery proper, but usually in the abdominal aorta immediately before the origin of the renal arteries or even in the anterior mesenteric artery, in which case the thrombus projects into the limen of the aorta. Such a thrombus may proluce an occlusion or stenosis of one or the other renal artery thus that it continues as far as its origin, or that the thrombus, which is freely movable in its posterior or upper enil, happens to be forced against the opening of the renal artery by the circulating hlood.

The most striking symptom is a profuse renal hemorrhage, the hlood extravasating from the capillaries entering in part the tubuli uriniferi or the pelvis of the kidneys and being then discharged. The animals usually pass light or dark red urine at short intervals, at times cylindrical clots, which are often regular casts of the ureters, are mixed with it. The urine contains an amount of albumen corresponding to the quantity of blood present, red blood corpuscles are found in the sediment, sometimes arranged in the form of tube casts, and also other renal form elements. On rectal exploration the enlargement of the one (left) kidney, which is not very sensitive, may be ascertained.

If the hemorrhage continues unchanged, death may occur in the course of a few days (authors' case), but in most cases such attacks (lisappear after a short time, although they may recur several times (the authors observed such repetitions in all their cases).

The treatment must be limited to the administration of remedies which increase the blood pressure, to intensive feeding and possibly to the administration of iron.

\section{Amyloid Kidney. Degeneratio Amyloidea Renum.}

Etiology. Amyloid degeneration of the kidneys occurs far less frequently than it does in the liver (Vol. II), although it develops upon a similar basis. The disease may be limited to the kidneys, but occurs more often simultaneously with a similar affection of the liver and spleen. The process has been observed in dogs by Rabe, Rivolta, Kitt, Dörflinger; in a cat by Mathis; by Rabe not very rarely in horses and cattle.

Anatomical Changes. In well marked cases the kidneys are enlarged, firm and rigid, anemic. The cut surface is very pale and shows a waxy or lardaceous luster (for the amyloid reaction, see amyloid of the liver).

In the kidneys it is usually also possible to find proliferation of the connective tissue and fatty degeneration of the epithelia. Amyloid kidney ean only oceur in association with chronic nephritis, and in this ease small punctate, waxy, somewhat transparent and glistening granules, the degenerated glomeruli are noticeable on the cut surface (Dörflinger).

Symptoms. The clinical picture of amyloid kidney is known only slightly. Rabe observed emaciation and debility of the hindquarter in his cases, also symptoms of uremia, 
especially vomiting, profound stupor and lowered temperature (down to $35.9^{\circ}$ C.). In two cases he saw edematous infiltration of all four extremities, and in one case ascites which, however, was probably due to liver disease.

The symptoms have great similarity with those of chronic non-indurating nephritis, but with the difference that the amount of urine is not diminished and that only little sediment is formed in spite of the large amount of albumen present.

Diagnosis. The disease is suspected only when in a chronic organic disease or in poor nutrition and anemia the urine, which is voided in approximately normal amount, contains rather much albumen, but furnishes only little organic sediment with cells that give the amyloid reaction, or hyaline casts. The probability of the diagnosis is much increased if at the same time enlargement and firmer consistency of liver and spleen are found to be present.

Treatment. Treatment does not promise particularly good results. In suitable cases the treatment of the hasic condition may be supplemented by intensive nutrition and by the administration of iron and iodine.

Literature. Ball, Arch. f. Vet.-Wiss., 1905. 362. - Dörflinger, Vet., 1597. 349. - Rabe, Hann. Jhl., 1882-83. 86. - Ries, Rec., 1903. 629.

\section{Acute Inflammation of the Kidneys. Nephritis Acuta.}

Acute nephritis is an inflammatory disease of the kirlners which is manifested either by nutritional disturbances of the renal epithelia and an only slight involvement of the interstitial tissue (Nephritis acuta parenchymatosa), or which in addition to changes in the parenchyma of the kidney produces very decided inflammatory changes also in the connective tissue, without, however, causing the formation of a purulent exudation (Nephritis acuta diffusa).

The elinical data concerning nephritis in veterinary medicine are as yet rather incomplete, probably partly because systematic urinary analysis does not yet oceupy its proper place in veterinary practice. In contrast to the many various forms of disease which have heen ascertained by post-mortem examination and still more by histological investigation in the various inflammatory processes in the kidneys, clinical examination is able to differentiate only few clinical pictures with the methods at its disposal.

In the following paragraphs an attempt will be made to classify the inflammations of the kidneys according to the system which is generally accepted in human medicine, on the basis of observations reported in literature and of the authors' own investigations. This is fully justified by the pathologieal anatomy of nephritis in animals which has been studied earefully, especially by Kitt. 
Bright included in the disease, which was later called after him "Morbus Brightii," all affections of the kidneys which are usually associated with albuminuria and symptoms of dropsy. Later authors extended the meaning of the term "Bright's Disease" to all hematogenous, non-purulent inflammations of the kidneys. The comprehension of all renal inflammations under this one common name does, however, not appear to be appropriate in consideration of the fact that these inflammations represent forms of disease which differ materially, not only clinically, but even more in their anatomical and pathological characteristics.

Occurrence. Acute inflammation of the kidney is a very frequent disease of animals because it develops mostly from infectious diseases which occur very often in domestic animals (Semmer found nephritis repeatedly in chickens).

Etiology. Acute nephritis may occasionally be based upon traumatic influences on the region of the kidneys or upon the kidneys themselves; as such are designated particularly straining, dull force (kicks, blows) upon the region of the kidneys, being run over, falling down, too abrupt stopping during sharp trotting or galloping, etc. In other cases the inflammation occurs in connection with cold (on crossing a cold river or on transportation in open railroad cars). The manner in which traumatic or thermic agencies exert their pathogenic action is beyond the limitations of direct observation; but the possibility is not excluded that they merely reduce the resistance of the renal tissue to the actual causes of inflammation (infectious substances).

Vegetable and mineral poisons are capable of giving rise to an inflammatory process if they are introduced in considerable quantities. Such substances are cantharides, oil of turpentine, squills, colchicum, extract of felix mas, also carbolic acid and preparations of coal tar in general; of mineral substances phosphorus, arsenic, corrosive sublimate and the mineral acids. Godbille observed acute inflammation of the kidneys occurring in cows after the ingestion of acorns. Many acid substances even cause inflammation in the kidneys when rubbed on the skin, for instance, oil of mustard, styrax, eantharides, etc. A similar action is also attributed to certain caterpillars which, after being swallowed with grass or leaves, are said to irritate the kidneys with their acrid substances which have been absorbed in the intestine (Cruzel). Finally insects (wasps, bees, Kolumbáes gnats) may inoculate substances by their stings which irritate the kidneys and thus produce inflammation.

In by far the most cases acute nephritis develops undoubtedly in the course and in consequence of acute infectious diseases. The bacteria which circulate in the blood may become wedged in the glomeruli and also in the intertubular blood vessels and may injure the tissue in their immediate vicinity. But they may also leave the blood vessels and be eliminated with the urine later. In this manner nephritis occurs in anthrax, swine erysipelas, glanders, hog cholera, and swine 
plague, and in septicemias in general. However, in the majority of cases the bacterial toxins exert an immediate pathogenic action upon the kidneys. In passing through the cellular elements of the kidneys they produce, in them and in the vessel walls, degenerative processes and subsequently an inflammation of the kidneys. The various toxins do not, apparently, exert a like action in this respect, but this much may be maintained in general that the development of nephritis is to be anticipated the more surely, the more intense the infection has been. This is the explanation of the development of nephritis in those cases in which no bacteria circulate in the blood and in which they cannot later be shown in the renal tissue, but where other symptoms of intoxication, especially fever, permit the suspicion that toxins are present in the blood; this is especially the case in influenza of horses, purpura, distemper, tetanus, footand-mouth disease.

In similar manner every extensive inflammation of the gastro-intestinal eanal and of the peritoneum may give rise to a nephritis. As a matter of fact every extensive organic inflammation may, under certain conditions, also supply the impetus for the development of nephritis. Finally infectious substances and their toxins are of etiological importance also in that form of nephritis which sometimes develops after the ingestion of spoiled food-stuffs.

The kidneys are likewise irritated by hemoglobin which happens to be in solution in the blood plasma, a fact which explains why in severe cases of hemoglobinemia also a degeneration or an extensive necrosis of the epithelial cells (Johme) develops, or even a true nephritis. In such cases, in addition to the blood coloring matter, the agencies upon which the hemoglobinemia is based or certain products of disintegration are probably of essential importance. It is evidently in the same manner that nephritis occurs after extensive burns of the skin.

The nephritis which occurs not infrequently accompanying certain skin diseases (eczema, mange) probably owes its origin to the injurious action of toxic substances which are formed in the inflamed skin, but which are not known exactly.

Anatomical Changes. The anatomical changes are either easily recognizable to the naked eye or can only be demonstrated by microscopical examination. According to Kitt, who was the first to attempt a systematic classification of inflammations of the kidneys on a histological basis, the following clinical pictures belong to the group of acute nephritis.

Nephritis Parenchymatosa Acuta (cloudy swelling, edematous, albuminous degeneration of the kidneys). The disease is essentially an affection of the epithelia of glomeruli and tubuli uriniferi, it is 
due to nutritive disturbances and leads to cloudy swelling and necrosis of these cells. The kidneys are enlarged only slightly or not at all, fairly firm or slightly softened, their capsule is easily peeled off, the configuration of the cut surface looks washed out, while the glomeruli, overdistended by blood, stand out clearly in the form of red dots; the cortical substance is grayish-red, perhaps traversed by grayishyellow spots and stripes; the medullary substance is colored intensely red; the tissue is sometimes moist with serous fluid, and turbid fluid which contains uninjured and degenerated epithelial cells, detritus, threads of fibrin, and perhaps white and red blood corpuscles may be scraped from the cut surface. In many cases the disease is most marked in the glomeruli (Glomerulonephritis); while in others the tubuli uriniferi appear to be involved more severely (Tubulonephritis).

Sometimes, most markedly in swine erysipelas, numerous small hemorrhages are found especially in the cortical substance (N. par. haemorrhagica).

Microscopical findings: The blood vessels are filled; fibrinous exulation, perhaps slight cellular infiltration between the tubuli uriniferi and fibrinous exudation in the capsules of Bowman, the epithelial cells being granular, swollen, partly degenerated and in process of breaking up; the tubuli contain hyaline and epithelial casts.

Nephritis Acuta Diffusa. The kidneys are decidedly enlarged, soft and friable, the capsule is soft, easily peeled off from the moist and glistening surface, the cortical substance is also moist throughout, very broad; the gray cut surface shows numerous yellow spots and streaks, often small or (especially in swine plague) large hemorrhages, a large amount of a thickish fluid may be scraped off from the cut surface (large, mottled kidney).

Microscopical findings: The blood vessels are distended; interstitial hemorrhages; lencocytes and epithelial casts in the tubuli uriniferi; cellular and serous infiltration of the connectire tissue; degenerative processes in the epithelial cells, exudation in the capsules of Bowman.

According to Cagny and Nocard the epithelial cells of the convoluted and straight tubuli disintegrate even in previously healthy kidneys during a prolonged severe attack of parenchymatous or even fatty degeneration and become loosened on the walls of the tubuli. In consequence a chylus like liquid which looks like pus is, on pressure upon the kidneys, discharged from the tubuli uriniferi; it contains numerous epithelial cells and free fat globules. A similar liquid is found also in the renal pelvis and in the upper portions of the ureters.

In cases of severe acute nephritis post-mortem examination usually reveals also dropsical changes in the body cavities and in the subcutaneous connective tissue.

Symptoms. Acute parenchymatous nephritis is the most frequent form of acute renal inflammation and preferably accompanies febrile acute infectious diseases (febrile albuminuria). It is accompanied by moderate albuminuria, the albumen of the urine usually amounting barely to $1-2 \%$. The quantity of urine is either normal-and this is more frequently the case-or it is somewhat diminished, mostly in consequence of the cardiac weakness produced by the basic disease; only in exceptional cases is the amount of urine slightly increased. The urine either furnishes no organic sediment or this consists only of few renal epithelia, sometimes of hyaline, granular or epithelial casts, also occasional red or white blood corpuscles. 
Parenchymatous inflammation alone does not cause any functional, particularly generalized, disturbances.

Acute diffuse nephritis occurs much more rarely than the preceding form, and either develops as such from the start or out of the parenchymatous inflammation.

The rapidly increasing inflammatory infiltration causes above all pain in the kidneys. The animals stand with feet spread apart or bunched below the belly, they arch the back and dislike to move; on moving they drag the hind legs and show in general a certain stiffness. Pressure upon the region of the kidneys causes the animals to depress the back. The sensitiveness to pressure on the part of the kidneys may be ascertained in large animals by rectal examination, in small animals by external palpation. Palpation from the hollow in the right flank may in cattle also be successful. Horses often show symptoms of colic, trip back and forth, look around toward the abdomen, at times they lie down carefully but soon rise again. These symptoms are not constant and are supplemented by depression, general debility, inappetence (in dogs also vomiting) and fever.

In a portion of the cases there is also pressure of urine, and in stallions there may occur incomplete erections. Sometimes the animals void only little urine, in spite of repeated efforts, perhaps only a few drops. This so-called strangury appears especially in the nephritis produced by cantharides or oil of turpentine. The diminished quantity of urine is one of the most striking symptoms and in some cases the elimination may be suppressed entirely for several (even 5 to 7 ) days. (Funk, Friedberger.)

The appearance of oliguria finds a sufficient explanation in the reducerl elinination of water in the kidneys, owing to the retarded blood flow in the capillaries of the glomeruli, also in the compression of the glomeruli and the obstruction of the capillaries by numerous desquamated endothelia, further in the impeded outflow of any secreted urine because the tubuli uriniferi are obstructed by renal epithelia and easts or compressed by the interstitial infiltration.

The urine is, especially at first, dark in color, reddish or even brown-red, clondy but without floceules; the specific gravity is increased. The urine contains variable amounts, usually one or several per cent, of albumen. The quantities of the other nitrogenous substances (urea, uric acid) are diminished, also that of $\mathrm{NaCl}$ and of the phosphates, because of the involvement of the epithelia of the tubuli uriniferi. The sediment is copious and consists of crystalline salts (uric acid, acid sodium urate, calcium oxalate and carbonate), of granular renal epithelial cells, hyaline, granular or epithelial casts, also not rarely (always in the nephritis produced by sharp substances) red blood corpuscles and leucocytes; sometimes it contains even bacteria. (Thomassen found numerous bacteria in the urine of calves affected with septicemia [see p. 157].) 
The renal epithelia (Fig. 172) are larger than the red blood corpuscles, rounder or quadrangular, containing a moderately large nucleus around which the protoplasm is usually granular and covered with fat globules. The epithelial cells are sometimes united into elongated cylindrical shapes (epithelial casts; Fig. 173.2). The other casts are either quite homogeneous (hyaline casts) or unevenly granular (granular casts), perhaps covered with fat globules, red or white blood corpuscles, sometimes with small crystals (Fig. 173, 174). In the urine of horses the calcium carbonate granules are also united into cylindrical formations, but are readily differentiated because they disappear with the development of gas after the adilition of acids.

In severe cases edematous swellings sometimes develop in the subcutaneous connective tissue, on the lower chest and abdomen, on the eyelids, in the larynx, on the extremities, etc., and sometimes serous effusion may be demonstrated in the body cavities.

The nutrition of the animals always suffers more or less; anemia develops after a comparatively short time the more so as the appetite is diminished, and vomiting occurs readily in carnivora. In very severe cases the debility increases rapidly; the pulse is more and more accelerated and feeble. $\mathrm{Fi}$ nally pulmonary edema or acute uremia (p. 983) terminate the case.

Course. Recovery from acute parenchymatous nephritis usually occurs in a few days or in 1 to 2 weeks, provided the basic affection itself does not endanger the

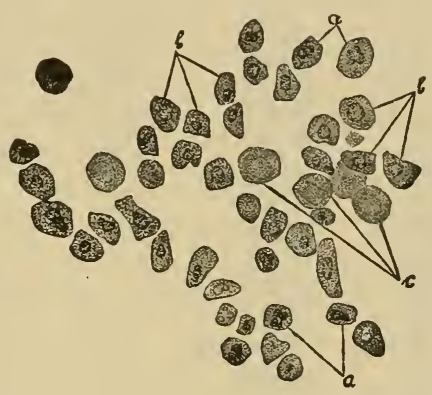

Fig. 172. Urinary sediment of a horse with acute diffuse nephritis. a renal epithelia with broken up, $b$ the same with uninjured nuclei, $c$ mononuclear white blood corpuscles. life of the animal; the albuminuria diminishes rapidly and disappears completely with the recovery of the animal. Only rarely the disease passes into acute diffuse nephritis, but it sometimes probably prepares the soil for the later development of a chronic nephritis. Generally acute parenchymatous nephritis in itself only indicates the intensity of an infection or intoxication, but does not in itself immediately endanger the life of the animal.

On the other hand acute diffuse nephritis lasts 1 to 2 weeks on an average, and ends in death, possibly in recovery, or gradually passes into a chronic inflammation. The commencing improvement is indicated by the increase in the amount of urine, which is at the same time lighter in color; its specific gravity becomes lower and the albuminuria gradually disappears. In the cases with fatal termination the quantity of urine is permanently diminished, it contains much blood, the appetite is lost entirely, the temperature persistently high;

Vol. 1-63 
finally the animals lie down constantly, a condition of stupor or coma develops in which at times muscular trembling or eclamptoid muscular convulsions are observed, the body is bathed in perspiration and death finally occurs in deep coma or with convulsions.

Diagnosis. Nephritis can be recognized positively only on the basis of urinary analysis, because often only the urine shows a deviation from the normal, while pain is observed also in purulent nephritis or in pyelitis. Of importance in this re-

Fig. 174.
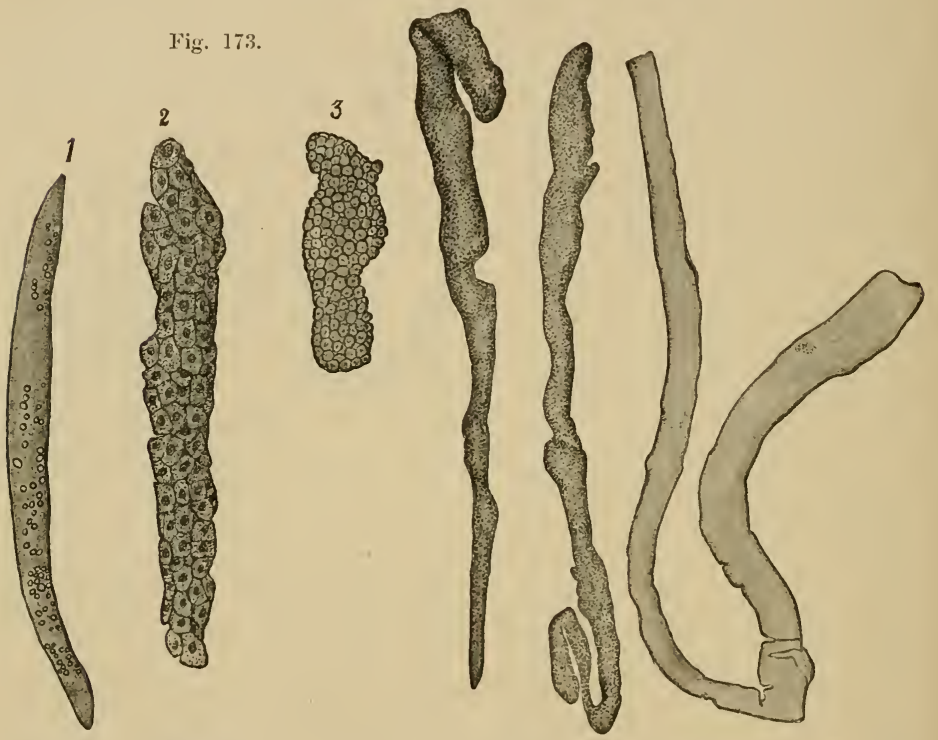

Fig. 173. Renal casts. 1 hyaline east, granular; 2 Epithelial east; 3 blood corpuscle cast.

Fig. 174. Renal casts. To the left two gramular casts, to the right a cylindroid.

spect are the quantity of albumen and in the sediment the presence of renal epithelia, casts, red and white blood corpuscles. The demonstration of the form elements and of the quantity of albumen is of particular importance, and it is not sufficient simply to ascertain the existence of albuminuria, because this may exist also in chronic nephritis, in severe passive hyperemia of the kidneys and in general nutritive disturbances, and because it affords no information as to the acute or chronic nature of the nephritis.-Albumen may also be found in the urine in affections of the urinary passages, even in affections of the immediately contiguous genital organs (prostate, vagina), but 
here the quantity corresponds to that of the pus or blood which is present in the urine. Further the presence of epithelial cells from the pelvis of the kidney, from the bladder or urethra gives information on the localization of the disease, while rectal exploration may often make it possible to ascertain immediately the abnormal condition of the organ.-In renal hemorrhage (infarct, injury, rupture of the renal artery) the quantity of the urine is unchanged, the sediment contains almost only red and a few white blood corpuscles, the blood often coagulates in the ureters to thick cylindrical shapes.

An exact designation of the various forms of acute nephritis is often possible if the quantity of albumen, the quantity and nature of the renal elements, and possibly the sensitiveness of the kidneys to pressure are considered. Small amounts of urine of high specific gravity, copious organic sediment, a high percentage of albumen, sensitiveness of the kidneys, perhaps also edematous infiltrations point especially to acute diffuse nephritis. On the other hand the slight and transitory albuminuria, which is of frequent occurrence in acute infectious diseases, and the presence of but few form elements as a rule permit the diagnosis of parenchymatous degeneration or inflammation of the kidneys.

Prognosis. Except for the less severe parenchymatous inflammation there are only few data available on the course of acute nephritis in animals; but this much seems to be fairly certain that even severe cases are capable of complete retrogression (Trasbot). During the course of the inflammation it is impossible to make a definite prognosis, because even relatively slight cases may later become aggravated or the disease may pass over into the chronic form. A favorable outcome may be expected in those cases of febrile diseases in which the urine contains only small amounts of albumen and very few or no organic form elements. But in the presence of decided oliguria or of complete anuria, or if the urine contains much albumen and many red and white blood corpuscles, the prognosis is very unfavorable; uremic symptoms always are an indication of approaching death.

Treatment. Of particular importance is the regulation of the diet in so far as the animals should receive easily digested food-stuffs which contain but small amounts of albumen and of salts, and which are neither sharp nor irritating. Herbivorous animals will therefore receive, if possible, fresh green feed or good hay, tuberous vegetables, drinks made with flour or bran, while grains should not be permitted; carnivorous animals are most appropriately put on an exclusive milk diet, milk may be given even to large animals if mixed with a decoction of oats or linseed, because it stimulates diuresis. It is advisable to keep the animals in an evenly warm place, and well covered. 
In case of marked oliguria the animals must be made to drink as much as possible, especially of carbonated water, in order to facilitate the process of filtration in the kidneys and to prevent the obstruction of the urinary tubuli by desquamated epithelia and by casts. The quantity of water must, however, be limited if dropsical symptoms already exist (Köresi \& Roth-Schultz). In order to increase the elimination of water through the kidneys mild diuretic remedies mav be employed, for instance, acetate of potassium (of Liq. acet. Potass. 50-100.0 or $1.0-2.0 \mathrm{gm}$. daily 3 to 4 times), potassium nitrate (10-15.0 or 0.2-0.5 gm.), also Diuretin (for $\operatorname{dogs} 0.1-1.0 \mathrm{gm}$. given to advantage with digitalis), theozin, theophyllin.

For promoting the elimination of the products of metabolism through other paths, diaphoresis is particularly appropriate, and may be produced in small animals by warm water- or steam-baths, by subcutaneous injections of pilocarpine hydrochloride (0.2-0.5 or $0.005-0.02 \mathrm{gm}$.) or of arecoline hrdrobromide (0.06-0.08 gm. for horses). In addition to these, laxatives are indicated, either neutral salts or better "Vienna drench," or castor oil, also aloes by which means not only a portion of the urine-forming solid substances (urea and according to Kövesi \& Roth-Schultz preferably phosphates). Uremic symptoms, which may have appeared already, are to be counteracted in the manner discussed farther back (p. 984).

In an inflammation of the kidneys which has developed upon an infectious basis, especially if the action of pyogenic bacteria may be assumed, an attempt may be made to prevent their multiplication by the administration of bactericidal remedies, of which sodium salicylate $(20-40.0 \mathrm{gm}$. or $0.5-1.0 \mathrm{gm}$.), and salol (15-20.0 gm. or 0.5-1.0 gm.) are appropriate.

The cardiac action must always be watched and any existing weakness counteracted with digitalis, camphor or other heart remedies (see valvular diseases).

In a dog with artificially produced inflammation of the kidneys Pericone produced healing by decapsulating the kidney.

Literature. Albrecht, W. f. Tk., 1901. 145. - Friedberger, Z. f. pr. Vet.Wiss., 1874. 171. - Gebhardt, W. f. Tk., 1904. 709. - Godbille, Rev. gén., 1906. VI. 655. - Jacob, B. t. W., 1904. 398. - Kitt, Monh., 1893. IV. 433 (Lit.). Köresi \& Roth-Schultz, Orv. H., 1904. 92. - Pericone, O. M., 1907. 49. - Pflug, Krankh. d. uropoët. Systems, 1876. - Roquet, J. vét., 1909. 385. - Schmidt, Die Cystitis und Nephritis usw. Diss. Giesszen. 1909 (Lit.).

\section{Chronic, Non-Indurative Nephritis. Nephritis Chronica Non-}

\section{Indurativa.}

(Chronic parenchymatous nephritis; Nephritis mixta [Kitt]; Nephritis chronica mixta [Marcone].)

Chronic non-indurative nephritis is a diffuse nephritis with slow course in which considerable degeneration of the parenchyma takes place, with cellular interstitial infiltration, edema, 
and new-formation of connective tissue, while atrophy of the new-formed tissue does not occur.

The differentiation of ehronic non-indurative nephritis from the chronic inflammation, with which atrophy of the new-formed tissue is associated, appears justified for the reason that these two forms of renal inflammation differ essentially not only pathologically but also clinically, and because the chronic indurative nephritis not infrequently develops from the commencement as such.

Historical. The reports in veterinary literature concerning chronic non-indurative nephritis are very seant and the available information on this form of inflammation of the kidneys is less complete than is the ease in other forms. Hable, Verheyen, Thomassen, Zimmermann, Nareone, Dell'Acqua, Boudoire, have reported cases belonging in this category.

Etiology. In certain cases the chronic nephritis develops out of the acute form and is therefore caused by the same injurious influences (see p. 989). Similar causes are of importance, however, also in those cases in which the chronic inflammation develops as such from the start, except that they exert their injurious action with slighter intensity but for a longer period. Here also irritating substances must therefore enter into consideration in the first place; and among these especially bacterial toxins which are formed in certain protracted infectious diseases (chronic endometritis, chronic infectious bronchitis, pneumonia, pleurisy, intestinal ulcers, tuberculosis, glanders), and which are eliminated by the kidneys after their absorption into the blood. Chronic nephritis may alsó develop in the vicinity of purulent foci in the kidneys (Kitt). Finally it can hardly be denied that repeated taking cold also will occasionally lead to chronic inflammation of the kidneys.

Anatomical Changes. As a rule the kidneys are found enlarged and on pealing off the capsule, the internal surface of which is more or less mottled, small shreds of renal substance adhere to it. Both the outer and the cut surfaces of the kidney contain red, yellow and gray spots, and likewise occasional small hemorrhagic foci. The substance of the kidney is moist, and if the disease has lasted for some time it is firmer than normal, the medullary substance is poorly defined, wine-red, dirty red and full of blood and fluid.

Under the microscope the same changes may be ascertained as in acute diffuse nephritis (see page 991), but with this difference that a new-formation of nucleated connective tissue is often met with in the territory of the cellular infiltration.

In the majority of cases general anemia and emaciation are present, also serous effusions into the body cavities and the subcutaneous connective tissue, and sometimes hypertrophy of the heart muscle. 
Symptoms. If the chronic disease has developed from the acute form, the clinical picture of acute nephritis gradually changes, its severe manifestations become less marked, and finally the symptoms of chronic non-indurative nephritis are established. In the great majority of cases, however, the chronic nephritis develops from the start, its onset is insidious and the symptoms of the disease are quite uncertain in character, so that a suspicion of the existence of the disease is aroused only in a farther advanced stage of the process.

In working animals attention is first attracted to the diminution in the ability to work; the patients are easily fatigued, out of sorts and languid, although on careful observation these symptoms may be recognized also in the other animals. As the disease progresses emaciation becomes evident in spite of the fact that the appetite remains good or is but slightly disturbed; at the same time the mucous membranes become more and more anemic. In some of the cases edematous swellings form on the lower abdomen, on the chest and about the feet or the thighs.

Simultaneously with these symptoms, or even in their absence, a diminution in the amount and an increase in the specific gravity of the urine may be noticeable, and the urine contains relatively much albumen, many renal elements like hyaline, granular and possibly epithelial casts, fatty renal epithelia and occasionally few red blood corpuscles.

The daily amount, the specific gravity and the relative quantity of albumen in the urine show great variations in the different eases. If the inflammation or the edema retrogress for a time, the specific gravity may become nearly or quite normal and the quantity of the urine may even be greater than normal. If the chronic indurative inflammation approaches the stage of atrophy, the amount of urine also increases, and in this case permanently, while the quantity of albumen at the same time becomes relatively lower. (In a case reported by A. Zimmermann the amount of urine in a dog varied toward the end of the disease between 310 and 110 cc., the specific gravity between 1010 and 1032 , and the urine contained albumen constantly $(0.05-0.1 \%)$, white blood cells and also amorphous detritus.)

Sometimes the sensitiveness of the organ is increased and it is enlarged, but as a rule examinations made in this respect are negative. Many animals rise with difficulty and the effort causes pain (Chouard).

Occasionally inflammation of the serous membranes and of the lungs supervenes and may have a fatal outcome. In the absence of such complications symptoms of heart weakness occur in the later course of the disease, or even from the onset; they may consist in pounding heart beat, soft, rapid pulse, and finally the animals perish with signs of pulmonary or laryngeal edema or of uremia.

Course. The disease always runs a protracted course and usually lasts several months. The clinical picture often shows variations, and aggravations are observed especially after over- 
exertion and after taking cold. In other cases the edema which develops in a certain stage of the disease disappears, the apparent improvement being accompanied by an increase in the quantity of urine voided. Eventually the clinical picture of chronic indurative nephritis may develop.

Diagnosis. Chronic nephritis can be recognized only by means of urinary analysis, all other symptoms pointing only to genera! nutritive disturbances. For differential diagnosis it is necessary to consider the change in the amount of urine voided, the presence of albumen in considerable quantity, and the presence of epithelia and of casts from the tubuli uriniferi.

Acute diffuse nephritis differs from the chronic form by its more rapid evolution, by the more considerable diminution in the amount of urine voided, and especially by the presence in the urine of numerous red blood corpuscles. It must, however, not be forgotten that acute exacerbations may occur in the course of chronic nephritis, which sometimes render a diagnosis extremely difficult, in case the history or, after the acute srmptoms have quieted down, the further course of the disease do not afford any information on the true nature of the trouble.-Amyloid kidney is very easily mistaken for chronic non-indurative nephritis, the more so as both processes mar exist simultaneonly (see p. 987). - In venous hyperemia of the kidneys the urine has a higher specific gravity and contains at most traces of albumen and few renal elements.In atrophy of the kidney there is polyuria, the urine is light in color, waterv, the specific gravity low, the percentage of albumen small and the sediment contains only few renal elements; hypertrophy of the heart is also frequent.

Prognosis. The course varies in different cases in so far as the animals may be kept alive for years under favorable external conditions, while severe exertion and insufficient nutrition render the course more rapid. Other things being equal, the prognosis becomes more unfavorable, the less urine is roided, and the more albumen and renal elements it contains, or the more prominent the cardiac weakness becomes.

Treatment. This is the same as in acute nephritis. First of all the diet must be regulated in such a manner that the patients, which are to be saved from all severe exertion, should receive only good non-irritating food (in carnivora an absolute milk diet is here also most effective [see p. 994]).

In the presence of oliguria and of dropsy cataphoresis and especially subcutaneous injections of pilocarpine repeated daily for weeks may have a good effect. Diuretic remedies are administered in order to increase the amount of urine (page 996 ), and in cardiac weakness heart remedies (see valvular diseases) are administered. The anemia also requires appropriate 
treatment (see page 850). The effectiveness or failure of the treatment can be ascertained from the quantity of albumen in the urine.

Literature. Dell'Acqua, C'lin. vet., 1906. 625. - Boudoire, Rev. gén., 1906. VII. 657. - Kitt, Monh., 1893. IV. 433 (Lit.). - Marcone, Rev. gén., 1906. VII. 345 (Lit.). - Thomassen, Ann., 1\$97. 9, 77. - Zimmermann, Z. f. Tn., 1s94. II. 372 .

White Spotted Kidney. (Nephritis maculosa alba. Nephritis fibroplastica.) This form of nephritis occupies a position between the acute and the chronic inflammation and develops usually in foci.

It is frequently found in calves but occurs also in arlult cattle. Blieck observer the disease enzootically almost exclusively in fattened ealves. In the abattoir in Budapest nephritis maculosa alba was found during the years $1900-1902$ in 2,323 ont of 291,573 slaughtered cattle $(0.8 \%)$, but in only 20 out of 343,012 ealves $(0.006 \%)$.

Etiology. Nephritis maculosa alba probably results from a cryptogenetic inflammation (Kitt, Kabitz), which appears to be acculired most frequently through the umbilicus. The disease is therefore probably embolic in origin. According to Rieck, however, the trouble is produced by the secretion of toxins which originate either through the influence of unknown microorganisms or without them. Fattening of calves with whole milk and confinement in dark, close and badly ventilated quarters favor the development of renal inflammation.

According to Vaerst and Guillebean the white spots represent foci of blastenas which persisted from embrvonal life and disappear eventually without leaving any traces. This view is contradicted by the undoubtedly inflammatory changes founi by other authors and also by the findings in the Burlapest abattoir.

Anatomical Changes. Numerous slightly prominent rounded spots are seen on the kidney, which are from hemp seed to a one-cent piece in size, smooth, grayish-white or yellowish. In exceptional cases they are confluent so that normal renal tissue may be discovered in only a few spots (Nephritis alba). The consistency of the organ is sometimes normal or somewhat soft, but usually rather firm. The cut surface also appears mottled, light gray streaks corresponding to the spots just mentioned, and standing out clearly from the healthy tissue which has a brown-red or more livid red color. Sometimes the white spots are softened in the center or show purulent disintegration (Kitt).

These changes may retrogress completely, but at other times assume a markedly chronic character.

On microscopical examination intense cellular infiltration is found, sometimes also fibrinous exulation between the tubules, and numerous fibroblasts or a considerable increase in connective tissue. The pathological alterations are sometimes arranged thus that the cellular infiltration is evident only at the borders of the islanils, while the center of the islanis consists of fibrous tissue in which only here and there portions of the tubuli are visible. The tubuli uriniferi and their epithelia perish apparently only secondarily by simple atrophy.

Blieck differentiates four forms of spotted kidney. In the first form the trouble is limited to localized white foci which vary in size, and either these foci are prominent above the surface of the kidney or the latter already contains cicatricial contractions. The second form is distinguished by a diffuse inflammation of the renal parenchyma and by the presence of white spots, the kidneys being always enlarged. In the third form hemorrhages occur in and around the white foci, and in the fourth form hemorrhages alone are present. 
Symptoms. The white spotted kidney usually causes no morbid symptoms. The investigations of Blieck showed that a moderate albuminuria $(1 / 4-1 \%)$ occurs only in those cases in which, in addition to the white spots, a diffuse disease of the parenchyma or multiple hemorrhages have developed. In other cases only nucleo-albumen can be demonstrated in the urine. The sediment consists of white blood cells which are sometimes arranged in groups, also of renal epithelia and hyaline or granular easts, which are much more numerous in urine that contains albumen. In severe cases the appetite is diminished, the animals are languid and may, rarely, suffer from diarrhea.

Treatment appears indicated only when the disease is well marked and must follow the same principles as that of chronic non-indurative nephritis.

Literature. Basset, Rev. gén., 1903. II. 582. - Blieck, A. f. Tk., 1906. XXXII. 225 (Lit.). - Brusaferro, Mod. Zooiatro, 1903. 436. - Fally, Ann., 1907. 463 (Lit.). - Guillebean, A. f. Tk., 1906. XXXII. 574. - Kabitz, Monh., 1901. XII. 4. - Kitt, Monh., 1893, IV. 433; Pathol. Anat., 1906. II. 493. - Panisset, Bull., 1905. 472. - Vaerst, A. f. Tk., 1901. XXVII. 110.

\section{Chronic Indurative Nephritis. Nephritis Chronica Indurativa.}

\section{(Granulated or atrophied kidney; Nephritis interstitialis chron- ica, Atrophia grannlosa renum.)}

Chronic indurative nephritis is a kidney disease which is associated with atrophy of the comnective tissue and takes a very protracted course. It either develops from acute or chronic non-indurative nephritis (secondary atrophic kidney) or occurs independently and slowly (primary atrophic kidney), or then it follows upon certain affections of the urinary passages (urogenic atrophic kidney). It always causes a destruction of the parenchyma.

Occurrence. Chronic indurative nephritis occurs in eattle and buffaloes fairly frequently. Dogs and hogs also are affected quite often, horses rarely, and the other domestic mammalians only exceptionally. Older dogs suffer from chronic nephritis comparatively frequently; but as personal observations of the authors show, the disease may also become manifest in dogs that are only a few weeks old. Horn found chronic nephritis in $0.32 \%$ of 7,000 slaughtered sheep; Semmer observed a case in a cat. In birds the disease may possibly develop upon a gouty hasis.

Etiology. The disease develops out of the chronic nonindurative nephritis in only an extremely small proportion of cases. This is indicated not only by the fact that the chronic non-indurative inflammation is observed far less often than the disease under discussion, but also by the other fact that this 
latter affection not infrequently becomes manifest without the severe symptoms of the non-indurative inflammation having been observed; moreover, atrophic kidney is sometimes discovered accidentally in animals which had always been healthy.

In regard to the primary atrophic kidney there is much to indicate that causes primarily similar to those in the chronic nonindurative inflammation are active (see page 996), but with this difference that they manifest their injurious influence less intensely but for a longer time. There is also a possibility that after an acute nephritis has run its course, a slowly progressive increase of the renal connective tissue commences. This would explain those cases in animals where the symptoms of inchurative nephritis become manifest a certain time after recovery from some infections disease. Frölner observed a case of chronic nephritis which formed a sequel of paralytic hemoglobinemia. Haase found a sequel of swine erysipelas in the kirlneys of hogs to have taken the form of isolated connective tissue foci containing few erysipelas bacilli.

In carnivorous animals the action of toxins must be considered which develop from the food-stuffs rich in albumins, during digestive distmrbances or in chronic skin diseases (eczema, mange); and in such cases confinement in dark yards is saicl to exert a predisposing influence. In birds the fact that the uric acid is formed in great quantities might have an unfarorable action, as is made strikingly evident in gont. Joest found chronie nephritis in a cow with urinary calculi of xanthin and with deposits of xanthin in the kidneys.

The influence of chemical poisons may be concluded firom the occurrence of the disease in animals which have eaten sonr plants on wet pastures, and in such cases chronic hepatitis usually develops at the same time (Vol. II). Such an action of chemical poisons is proved also by the experiments in which it was possible to produce a chronic neplnitis by the systematic ardministration of small amounts of lead-and copper-salts (Ellenberger \& Hofmeister, Heubel \& Meyer, Trashot).

A primary affection of the arteries only rarely is responsible for the production of chronic nepluritis. In isolated instances the disease was observed in aortic anemism (Carlóac, Marek) or in degeneration of the renal artery (Lustig), and in such cases the arrestment of small blood clots may contribute in producing the chronic inflammatory process. In dogs an inchurative nephritis is sometimes found simultaneously with valvular troubles, though probably not as a consequence of the heart lesion itself, but owing to the same cause which has given rise to the chronic endocarditis. On the other hand, repeated embolisms in the kidneys may also canse a process of atrophy (emholic atrophic kidney).

Mechanical irritation may prodnce chronic interstitial nephritis in the presence of urinary calculi or of worms in the renal pelvis. The same effect may probably be produced also 
$y$ periodical disturbances in the outflow of urine, and this factor probably is preferably active in ruminants and hogs.

The chronic nephritis which has been observed frequently in buffaloes in Hungary (Schoppelt, Vámos) is perhaps caused by renal gravel or by periodical interference with the outflow of the urine. The facts that the affection is often unilateral or that one kidney is involved far more extensively, and still more the atrophy with dilatation of the calyces which is observed in sections of the kidney that show only slight morbid changes, are certainly in favor of the urogenic origin of the process.

Anatomical Changes. Indurative nephritis is characterized principally by the increase of the connective tissue and therefore by a firmer consistency of the organ (Nephritis indurativa, Sclerosis renum). Later on the kidney becomes smaller and its surface uneven, in consequence of variable depressions.

On histological grounds Kitt distinguishes the following forms:

Nephritis fibrosa multiplex s. Sclerosis maculata. This occurs in eattle, in hogs and dogs and is characterized by yellowish or graywhite furrows on the surface of the kidney, and by white wedge-shaped foci in the cortical substance, which consist of fibrous connective tissue, round cells, and isolated urinary tubules with disintegrating epithelia. These changes are probably due to embolism and may sometimes develop out of nephritis purulenta disseminata. Degen, for instance, showed by careful examinations that purulent nephritis which in hogs usually is hematogenic, generally leaves the evidences of nephritis fibrosa multiplex on healing.

Nephritis fibrosa diffusa is an inflammation which is about evenly distributed over the entire kidney, which is very large, firm to the touch and, in the calf, sometimes calcified. White, yellowish-white and yellow islands alternate and any remaining healthy tissue is only indicated by hrown-red streaks. The ground substance consists of fibrous tissue which is poor in cells and in which few urinary tubules are imbedded; the latter contain only occasional normal epithelial cells but much granular detritus. The glomeruli and the vicinity of the thick-walled capsules show cellular infiltration.

The unequal atrophy of the proliferated connective tissue causes shallow depressions on the s'rrface of the kidneys, which therefore appears finely or coarsely granular (Nephritis granulosa) and at the same time the kidney becomes smaller (Atrophia granulosa). By the contraction of the connective tissue some urinary tubules are constricted in places, others are obstructed by masses of disintegrated epithelia and by casts, and in consequence the urine is dammed up above the constricted or obstructed places, leading to dilatation of the tubules and of the corresponding capsules of Bowman, which then are gradually transformed into small retention eysts (Nephritis fibro-vesiculosa).

Symptoms. Chronic indurative nephritis has only rarely been observed clinically, and it is therefore as yet impossible to deseribe its clinical picture exhaustively. The want of complete clinical knowledge is particularly great in the indurative nephritis of ruminants and hogs. The urinalyses made by Vámos in 
affected buffaloes permit the assumption that the urine is frequently altered in the course of the disease in ruminants, and evidently also in other animals, although the animals appear otherwise healthy. Other observations and those of the authors even indicate that the very light forms do not noticeably impair the health of the animals, even in bilateral disease, and do not lead to alterations in the urine. In the great majority of cases, however, morbid phenomena are observed during the life of the animals.

The development of the clinical picture proceeds in exactly the same manner as in chronic non-indurative nephritis (see page 997). Nutritional disturbances, diminished ability to work, listlessness, rapid fatigue, languor, diminished appetite, in dogs recurring vomiting are observed frequently, but may occasionally be absent for a long time and even to the end of the disease.

The most constant symptom is polyuria in which the animals void large amounts of urine at frequent intervals, and house dogs often discharge their urine in the room against their custom. The daily amount of urine is often several times the normal quantity, although great variations exist in this respect. The urine is watery or almost as clear as water, sometimes it has a greenish tinge, the specific gravity is very low, even as low as 1001. Small amounts of albumen are present, but may disappear entirely for brief periods. On standing, very little or no sediment forms, and occasionaly hyaline casts and epithelial cells from the urinary tubules may be found in it (Fig. 175).

Regenbogen observed lipuria in two cases of chronic nephritis in dogs associated with fatty degeneration of the renal epithelia; the urine looked like watery milk and maintained this appearance even on standing or after filtration; it contained $3 / 4-1 \%$ of fat, many fat globules and fatty casts.

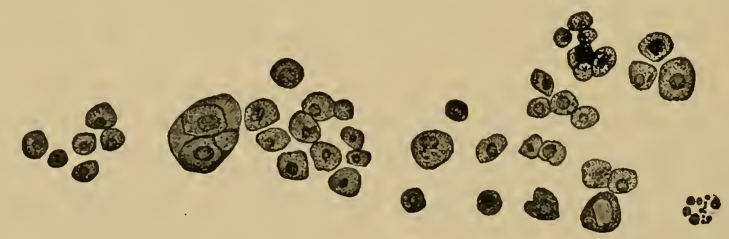

Fig. 175. Renal epithelia in the urinary sediment of a dog with chronic indurative nephritis.

The increased quantity of urine is due to several causes. It is characteristic, especially for the chronic inclurative form of nephritis, that more or less atrophied portions of the kidneys alternate with portions that are more or less unaffected and may be quite healthy; the latter assume the function of the diseased portions, owing to the power of accommodation of the renal tissue. For this reason the watery amount of urine does not diminish, in spite of the destruction of a portion of the renal substance. Since in the affected portions many arteries have become impassable, the remaining patent arteries of the portions of the kidney, which are still capable of excreting, dilate and this is the more likely to occur, as in many 
cases a hypertrophy of the left heart develops. Owing to the lasting hyperemia and increased circulation the functioning portions of the kidneys actually eliminate increased amounts of a dilute urine. On account of the raised blood pressure in the free renal arteries the urine passes much more rapidly through the urinary tubules and therefore a much smaller proportion of its water content is reabsorbed by the blood than under normal conditions. The functioning of the healthy renal portions, however, replaces that of the diseased parts only in regard to the elimination of water and not in regard to the elimination of most of the solid constituents. This, together with the arterial hyperemia in the excreting portions, is responsible for the lowered specific gravity of the urine and also affords an explanation why uremic symptoms may appear in this form of nephritis.

The sensation of thirst is increased in proportion to the increased elimination of urine.

In dogs the hypertrophy of the heart may often be recognized by the fact that the heartbeat is stronger, the cardiac dullness enlarged to the left and upward and the aortic sound accentuated; the pulse is strong and tense.

The causal connection between the cardiac hypertrophy and the chronic inflammations of the kidneys, especially the atrophic kidney, is not explained satisfactorily. According to Traube the hypertrophy of the heart is due to the occlusion of numerous renal vessels, but it may be objected to this hypothesis that the enlargement of the heart may occur also in the diffuse non-indurative chronic nephritis. A theory which was recently expressed by Senator and which had, in a somewhat similar form been suggested already by Bright, appeared more acceptable. According to this the metabolic products are retained in the blood in atrophic kidney, and produce, by irritation, an increase in the muscular elements of the heart and at the same time an increase in the vascular tone, which in its turn again increases the work of the heart.-Grawitz and Israel, also Lewinski, produced an artificial chronic indurative nephritis in dogs and rabbits by temporary ligation of the renal artery, and this was later followed by a hypertrophy of the heart.

Palpation sometimes shows enlargement, more frequently increased consistency, but occasionally a diminution and irregular contour of the kidneys.

The respiration becomes sometimes, especially in dogs, increasingly difficult in the further course of the disease and ultimately asthma may actually develop (Liénaux).

When the disease is severe, cardiac weakness eventually follows, manifested in pounding heart beat with feeble and rapid pulse, also in dropsical effusions; the amount of urine is then diminished, the specific gravity increased. At this stage emaciation progresses rapidly and finally the animals die of exhaustion, of pulmonary or laryngeal edema, possibly of intestinal hemorrhage or hemorrhagic enteritis. (Thomas observed epistaxis in a cow.)

The symptoms of uremia may appear in every stage of the disease. They usually correspond more to chronic uremia (disturbed appetite, vomiting, occasionally diarrhea, eventually epileptoid or asthmatic attacks) and often appear in repeated paroxysms. Occasionally an acute uremic attack closes the scene.

Course. The duration of the disease is always protracted. If it is recognized by urinary examination after the appearance 
of clinical symptoms, it has usually already existed for months and then is apt to continue for months or even years longer during which time frequent variations are observed in the intensity of the clinical picture. As has alrearly been mentioned, certain forms of this nephritis, preferably in ruminants and in hogs, run their course from beginning to end without interfering with the general health, and this may occasionally be the case also in other species.

Diagnosis. The existence of a chronic indurative nephritis may be assumed properly if the quantity of the urine is increased, the urine watery, the specific gravity low and when the urine contains only small amounts of albumen and at most few renal elements. These findings are often supplemented by the demonstration of cardiac hypertrophy and by symptoms of chronic uremia. The polyuria of active renal hyperemia differ's by a different history and by the constant absence of albumen in the urine. Certain difficulties may be encountered at most in the exclusion of that hyperemia which sometimes persists for a considerable time after acute inflammations of internal organs and which, on account of the simultaneous degeneration of the kidneys or of a catarrh of the urinary passages, is accompanied by slight albuminuria. In such cases the condition of the circulatory organs and the manual examination of the kidneys must decide.-In diabetes insipidus the urine is free from albumen, in diabetes mellitus it contains sugar and its specific gravity is usually high.- In amyloid kidney copious albuminuria is usual. Chronic non-indurative nephritis may enter into the problem in the terminal stage of atrophic kidney, when cardiac weakness is already present and has caused the development of edema, while the specific gravity of the urine is increased. But even in such cases the presence of atrophy of the kidney may be decided upon on account of the comparatively low content in albumen, the hypertrophy of the heart if present, and on account of the fact that the specific gravity of the urine does not correspond to the severe symptoms of urinary stasis.

Prognosis. In clinically well-marked cases the disease ends eventually in death, which is, however, in many cases postponed for years.

Treatment. A regulation of the diet is indicated in so far as the animals should be protected from all severe exertion and receive good, non-irritating food (see p. 995). In cardiac weakness heart remedies should be employed and an existing edema may be counteracted by diuretic remedies. For anemia the systematic administration of iron and of bitter remedies is indicated in addition to good nutrition. 
Literature. Albrecht, W. f. Tk., 1901. 25. - Ball, J. vét., 1906. 193. Degen Die hämatogene eiter. Nephritis d. Schweines. Diss Giessen. 1907. - Friedberger, D. Z. f. Tm., 1890. XVI. 188. - Fröhner, Monh., 1906. XVII. 137. Haase, B. t. W., 1908. 427. - Horn, Beitr. z. Kenntn. d. chron. Nierenerkrank. d. Schafes. Diss. Leipzig, 1908. - Joest, Dresdn. B., 1907. 170. — Kitt, Monh., 1893. IV. 433 (Lit.). - Liénaux, Ann., 1894. 65. - Mareone, Rev. gén., 1906. VII. 345 (Lit.). - Regenbogen, B. t. W., 1908. 499. - Schoppelt, Ung. Vb., 1901. 102. - Senator, D. m. W., 1903. 1. - Vámos, B t. W., 1905. 572.

\section{Nephritis Purulenta.}

\section{(Purulent Nephritis. Renal Abscess.)}

Etiology. Purulent nephritis develops through the influence of pyogenic bacteria which enter the kidneys usually by way of metastasis. The principal occasion for such metastases is afforded in purnlent diseases of other organs or pyemia, especially endometritis puerperalis, purulent inflammation of the udders, strangles, influenza of horses, gangrenous pnenmonia, purulent lung diseases (Horn found nephritis to have developed upon such a foundation in $0.1 \%$ of slaughtered sheep), also phlegmonous pharyngitis, purulent bronchial catarrh, pyemia due to umbilical infection. According to the experiments of Arenstein an embolic purulent nephritis may develop without the existence of purulent processes in other organs, for instance, owing to the absorption of colon bacteria from the intestine in severe obstipation. Degen, who found hematogenic purulent nephritis in about $0.5 \%$ of the hogs slanghtered in the Dresden abattoir, not infrequently found colon bacteria and bacilli of the enteritis group in the diseased portions of the kidneys, which could only have come there from the intestines. Nevertheless he never succeeded in determining the point of entrance of the infectious agents, among which a new variety of bacteria which he had discovered, the Bacillus polymorphus suis, was found frequently, and he considers therefore the hematogenic purulent nephritis of hogs as a disease arising from cryptogenetic and polybacterial infection. Haase finally saw occasionally purulent foci as sequels of chronic swine erysipelas, which contained few erysipelas bacilli.

Traumatic agencies, especially contusions of the region of the kidneys only in exceptional cases give an impetus for purulent nephritis by preparing the soil for the localization of bacteria which happen to be present in the blood.

Somewhat more frequently a urogenic origin may be recognized, inflammations of the urinary passages, especially those of the renal pelvis extending into the kidneys. (Ostertag saw in lambs repeatedly a purulent nephritis which had been continued from the urinary passages.) Only in exceptional cases do bacteria get into the kidneys from their vicinity (in the case of perirenal abscess).

Purulent nephritis is particularly frequent in cattle and hogs. 
Anatomical Changes. If the inflammation is metastatic, both kidneys are usually involved, in other forms usually only one and this may be affected only partially. The embolic purulent foci are found principally in the cortex.

According to Kitt purulent nephritis occurs in the following forms:

Nephritis purulenta (punctata, disseminata). The substance of the kidneys which are more or less enlarged contains numerous small whitish or yellowish dots and stripes which are surrounded by a narrow, dark red areola (hyperemia and hemorrhage) and from which thickish purulent masses may be lifted with the knife. The tissne around these places is changed in variable extension similarly as in the acnte diffuse inflammation. In hogs, less often in calves and cattle, tough white hands are seen between the purulent foci.

On microscopical examination conglomerations of pus cells are found inside of the small foci, and cellular infiltration containing pyogenic bacteria is noted in the surrounding tissue. The epithelial cells show fatty degeneration and are disintegrater, the glomeruli are cometimes surrounded by pus, while the convoluted eapillaries contain numerous leucocytes; in the urinary tubules casts are sometimes found which carry leucocytes and red blood cells.

The hematogenic purulent nephritis of hogs which has been studied more exactly by Degen belongs to this form. It commences mostly in the cortical substance with the formation of dark red foci, of from hemp seed to pea size. In these foci a central yellowish-white spot soon becomes visible as large as grits or up to birdshot in size. The foci eventually become tongher, their areola paler, the vellowish color of the center passes into a grayish-white and gray-white; sharply confined and slightly depressed cicatrices appear on the surface of the kidneys, continuing into the substance of the kidney in the form of wedges or strips. Abscesses are hardly ever formed.

On microscopical examination intense hyperemia and partial hemorrhagic infiltration is visible at the beginning; this soon gives way to a purulent infiltration which proceeds from the center, while the renal structure is obliterated and the parenchyna partially atrophied. Still later fibroblasts appear in the purulent infiltrated tissue, young granulation tissue is formed which, in the cicatricial stage of the disease is transformed into connective tissue.

Nephritis apostematosa (abscessus renis). In this form small pus foci become confluent and form larger abscesses or an abscess forms from an embolus; variable portions of the tissue of the kidney break down or the entire kidney is changed into a sac filled with pus (pyonephrosis). Larger pus foci occur, usually numerously, in horses by metastasis of stranges and in calves from unknown causes (possibly by umbilical infection). The connective tissue is increased around older abscesses but atrophies later, the inflammation of the surrounding tissue becoming chronic.

Symptoms. The clinical picture of purulent nephritis is characterized by its variability. It is evident that the symptoms will be different when the nephritis develops as a local affection and when it occurs as a part of general sepsis or pyemia. Variations also occur according to the duration, the number 
of inflammatory foci and in the involvement of adjoining tissue; the abscesses may or may not be in communication with the lumen of the urinary tubules or with the pelvis of the kidney; the changes may be unilateral or bilateral.

If the inflammation develops rapidly in both kidneys, the same objective symptoms become apparent as in acute diffuse nephritis (p. 992). Especially the sensation of pain in the kidney is quite marked; in horses slight attacks of colic are observed occasionally, also arching of the rigid back; while walking one or both hind feet are not advanced as far as usual (Benjamin observed paralytic weakness of one posterior extremity in a horse and is inclined to refer it to a compression of the plexus lumbo-sacralis by the inflamed kidney). In very rare cases the enlarged kidney or the purulent perirenal connective tissue produces a painful swelling above the lumbar region.

The voiding of urine is often disturbed in like manner as in the non-purulent acute nephritis. But the amount as well as the physical and chemical properties of the urine are not altered much, if only few abscesses are in course of slow evolution, without injuring the adjoining renal tissue noticeably and without communicating with the renal pelvis or the urinary tubules. In other cases, especially if the evolution progresses more rapidly, the urine shows the same or similar changes as in the acute diffuse inflammation (see p. 992). After the pus enters the urinary tubules or the renal pelvis the urine becomes cloudy, milky or mixed with floceules, it contains pus cells, renal elements; crystals of triple phosphates, and free ammonia. Exceptionally the sediment contains small portions of kidney substance (Leisering) and occasionally hemorrhage takes place in the kidneys.

If the hand is placed below the transverse processes of the lumbar vertebrae or into the rectum it is possible to determine pain, unilateral or bilateral enlargement of the kidneys and, in the presence of large abscesses, fluctuation which may be limited or may involve the entire kidney.

Edematous infiltrations are observed very rarely.

On rapid development of the inflammatory process or in septic pyemic infection severe general symptoms are observed, in other cases only temporary rises in temperature and gradually increasing emaciation. If the purulent inflammation is severe and affects both kidneys uremia may also follow.

In a slower evolution of the purulent process, especially if it is limited to one kidney, clinical symptoms seem sometimes to be entirely absent. The same is true for the hematogenic purulent nephritis of hogs (Degen).

Diagnosis. Purulent nephritis can be recognized with certainty only then when objective symptoms suggest this form of nephritis, when pain, fluctuation of the kidney, albuminuria

Vol. $1-64$ 
are present, or when the sediment contains also pus in addition to renal elements, while disease of the urinary passages can be excluded. An abscess of the kidney may be assumed to exist in those rare cases in which small portions of renal tissue are found in the sediment. In other cases the disease can only be suspected with more or less probability.

For differential diagnosis are to be considered especially acute and chronic non-purulent nephritis, catarrh of the urinary passages, purulent catarrh of the uterus, vagina and prostate (see p. 994).

Prognosis. The clinically manifest purulent nephritis ends sooner or later in death. If the inflammation is not very extensive, and especially if only one kidney is involverl the possibility of a relative recovery is not excluded.

Treatment. In case of unilateral disease the extirpation of the affected kidney (nephrectomy) is to be considered; the method of this operation in animals has been described in detail by Parascandolo. But as it is at present impossible in animals to make sure whether the function of the other kidney is undisturbed, there is always danger that after the resection of the one kidney the animal dies of nremia because the other kidney was also affecter. (Münnich has removed one kidney in a cow withont the desired result; Maksutow operated on a horse successfully). Punction of the abscess rarely gives good results.

In the clinic at Budapest experiments were made with the cystoscope which is nserl in human medicine. It appeared that in dogs the catheterization of the ureters was impossible on account of the anatomical relations of the ureteral openings, while it was carried out successfully. in horses.

If operative interference is not resorted to and other treatment is desired, the therapy described for acute diffuse nephritis (see p. 995) may be adopted.

Literature. Benjamin, Bull., 1905. 131. - Cocu, Rec., 1899. 232. - Colin, Rec., 1900. 20. - Degen, Die hämatogene eiterige Nephritis d. Schweines. Diss. Giessen. 1907 (Lit.). - Ellis, Am. v. Rev., 1901. 113. - Haase, B. t. W., $190 \mathrm{~S}$. 427. - Horn, Beitr. z. Kenntn. d. chron. Nierenerkr. d. Schafes. Diss. Leipzig. 1908. - Jolnne, S. B., 1875. 30, 32. - Kitt, Monh., 1893. IV. 433 (Lit.) ; Path. Anat., 1906. II. 487. - Leisering, S. B., 1872. 21. - Morel, Bull., 1904. 931. - Mïnnich, W. f. Tk., 1878. 17. - Parascandolo, Monh., 1903. XIV. 228. Schmidt, Maanedsskr., 1899. X. 179. - Siedamgrotzky, S. B., 1875. 29; 1891. 18. Stiegler, S. B., 1904, 172.

\section{Bacterial Inflammation of the Renal Pelvis and of the Kidneys. Pyelonephritis Bacterica.}

\section{(Pyelonephritis bacillosa s. diphtherica s. myotica bovum.)}

Bacterial pyelonephritis is an inflammation of the kidneys which usually rums a chronic course and is characterized by small softened foci in the cortical portion, diphtheritic disin- 
tegration of the papillae, involvement of the renal pelvis and usually also of the lower urinary passages. The disease is produced by various bacteria, among them the Bacillus s. Corynebacillus renalis (C. pyelonephritidis) bovis and occur's almost exclusively in cattle.

History. Originally bacterial pyelonephritis was included among the common purulent nephrites or pyelonephrites, but was separated, toward the end of the eighties of the last century, from the other inflammations of the kidneys as a special disease form. Siedamgrotzky (1875) demonstrated in one case the presence of hacteria in the affected portions of the kidneys, while Dammann (1877) had already noted the typical arrangement of the bacteria (which he called micrococci) in the urinary tubules. Hess (1888-1892), Bang (1889) and Schmidt (1890) studied the disease closely and called attention to the regular oceurrence, in the affected kidneys, of the Bacillus renalis, which was later studied at greater detail by Höflich (1891), and Enderlen (1891). Valuable investigations were made also by Lucet (1892), Mollereau \& Porcher (1895), Masselin \& Porcher (1895), Jensen (1896), Cadéac and Norot (1897), Albrecht (1900), Liénaux \& Zwaenopoel (1902) and Sommer (1906), while Ernst (1905) particularly studied the etiology and pathogenesis of the disease, emphasizing its polybacterial origin which had before him been pointed out by Lucet, Cadéac and Kit ${ }^{+}$.

Occurrence. In cattle pyelonephritis is a very frequent disease, cows being particularly affected, often shortly after calving, while calves or male animals (oxen, steers) acquire the disease much less often. In hogs it is also observed in exceptional cases (Jensen, Wyssmann) and Schmidt has seen it in a colt.

Etiology. Pyelonejhritis is a polybacterial disease in the cansation of which the Bacillus s. Corynebacillus renalis is concerned most often, while other bacilli also take part in the infection which are capable of producing suppuration or necrosis of the renal tissue.

The Corynebacillus renalis, Ernst (Bacillus renalis bovis [Bollinger, Enderlen, Hess], B. pyelonephritidis bovum [Höflich]) is characterized by its pleomorphic occurrence, it forms, according to Ernst, rods 2-3-4 $\mu^{\mu}$ long, 0.5-0.6 $\mu$ thick, which are often united to short thread-like groups and are irregular in form; sometimes they appear as slender rods, slightly larger in the middle and at the ends, where they also take the stain more deeply, sometimes as long thread-like formations which are wound into balls and nets.

Staining suceeds with the customary staius as well as by Gram's and Weigert's methods and shows either a division of the bacillary bodies into fields arranged in ladder-form, or then a homogeneous 
structure. The elongated thread-forms have club-shaped terminal enlargements and spherical bud formations, also bulgings, excrescences and often actual branching.

Liénaux \& Zwaenopoel, also Hutyra have shown that this bacillus is closely related to, if not identical with the $B$. pseudotuberculosis ovis, Preisz, which is active in several other diseases. This would agree with Ernst's elaim that the B. renalis is to be classed in the group of the Corynebacteria to which the B. pseudotubereulosis ovis belongs also.

Cultivation. The Corynebacillus renalis is aerobic. It hardly grows at room temperature, but kept on agar at the temperature of the body cultures are formed which are fine, whitish, dry, sandy or moist shining, or then dry, gray and scaly. In agar shake cultures two zones form, one about $2 \mathrm{~mm}$, the other $12 \mathrm{~mm}$ below the surface, where the oxygen tension is most favorable for growth. On blood serum tender, transparent whitish-gray dots grow which glisten like dew drops; in bouillon the cultures form a finely granular sediment. Ernst has shown that the bacilli grow very well in neutral or iaintly alkaline urine, in urine agar or in urine bouillon, and that in concentrated urine agar a deposit of crystals forms around the stab which consists of triple phosphates. Acids and indol are not produced. Certain cultural differences permit the assumption that there exist several slightly variable strains.

Pathogenicity. The injection of a pure culture under the skin of mice or chickens produces no results (Enderlen, Ernst, Masselin \& Porcher), while in rabbits and guinea pigs either a transitory swelling or an abscess develops (Enderlen, Künnemann, Ernst) or the animals are not affected at all (Lucet, Masselin \& Porcher). The same is observed after intraperitoneal or intrapulmonal inoculation of the culture (Enderlen, Ernst). An intravenous injection produced purulent-cheesy pneumonia in a rabbit (Ernst), intraocular inoculation often a passing iritis (Enderlen, Kitt). Intravenous injection preceded by ligation of a ureter was followed in rabbits by a process which was similar to pyelonephritis (Enderlen). In sheep and in cattle subcutaneous injections are usually without result or at most a temporary swelling develops at the point of inoculation (Ernst), while intravenous injections of cultures either remain likewise without result (Enderlen, Höflich, Künnemann) or Corynebacilli are eliminated with the urine for several days or weeks without other disturbances (Höflich, Ernst). Intravesical injections in cows do not as a rule cause any trouble (Hess, Enderlen, Höflich, Künnemann, Ernst) or they may exceptionally be followed by pyelonephritis (Masselin \& Porcher saw this in one case). A preliminary or simultaneous irritation of, or injury to the mucosa of the bladder enables the bacilli to localize in the bladder (Liénaux \& Zwaenopoel, Ernst), and according to Liénaux and Zwaenopoel a pyelonephritis may even follow.

Schmidt elaims to have found the Corynebacillus renalis also in a colt with bacterial pyelonephritis. 
Of other causes of pyelonephritis pure cultures have been found of the bacillus pyogenes (in cows and calves [Ernst]), pyogenic staphylococi (Cadéac \& Morot, Ernst), streptococci (Baillet \& Sérès, Ernst), bacillus pyocyaneus (Cadéac \& Morot), bacillus enteritidis (Sommer), bacilli of the colon group (Jensen, Kitt). Most of the enumerated bacteria were also often found together with the corynebacillus renalis, or several kinds of them at the same time, so that a mixed infection was present (Kitt, Ernst, Zschokke, Sommer). In two cases of kidney tuberculosis Ernst found similar ehanges as in pyelonephritis, althongh the tubercle bacillus was alone present.

The ability of the bacteria of the colon group to cause pyelonephritis after being introduced into the bladder was proved experimentally by Schmidt \& Aschoff, Savor, v. Wünschheim. On the other hand J. Koch produced proofs to show that saprophytic staphylococei are also capable of giving rise to a typical pyelonephritis in rabbits after subcutaneous or intravenous injection.

There exists therefore no specific virus of pyelonephritis in the domestic animals. Nevertheless, in consideration of what has been observed, it is not possible to agree with Ernst's view, according to which the Corynebacillus renalis has hardly anything to do with the genesis of the disease. Earlier observers and recently also Künnemann found pure culture of the bacillus in question in the diseased kidneys, and Ernst himself found it almost pure in a few eases. On the other hand, certain diseases (Pseudotuberculosis of sheep, ulcerating lymphangitis, pustulous dermatitis) prove that the bacteria belonging to the group of the Bac. pseudotuberculosis ovis possess pathogenic properties under certain conditions.

Although it is not determined with certainty how the natural infection is produced, it is at least very probable that the virus may be introduced into the kidneys or into the urinary organs in general in various ways. The general experience that the disease develops most frequently in cows, a certain time after parturition, if during the latter the birth canal had been injured or the placenta had been retained or if metritis developed, makes it very probable that the virus multiplies promptly in the diseased genital organs and may enter the urinary organs if opportunity serves. This can take place the more easily as most of the enumerated pathogenic germs are ubiquitous bacteria, and some of them (for instance the bacteria of the colon group) are regular inhabitants of the intestinal canal.

The dissemination of the virus may also take place from other organs which are diseased in some manner owing to a bacterial invasion. In a case described by Ernst the trouble developed in connection with an abscess in the region of the xiphoid cartilage, while in a case in a young steer observed by Hess the infection had probably proceeded from a paraurethral abscess. In newborn animals the umbilicus appears often to be the point of entrance of the bacteria, without it 
being necessary that the umbilicus is primarily diseased. The fact that male or young animals are also affected without other primary diseases has led Bang to suppose that the virus may possibly be introduced by way of the digestive tract. Bollinger considers also a cryptogenetic infection to be possible. This last hypothesis receives some support by the experiments of Ficker which showed that bacteria are absorbed by the uninjured intestinal mucous membrane if the animals are subjected to hunger or to excessive exertion.

Concerning the morle of invasion of the bacteria into the kidneys the views differ. Some authors insist upon a urogenic, others upon a hematogenic origin of the disease. Aside from Fnderlen's experiments in rabbits, which have already been mentioned and which were positive, the hematogenic invasion of the bacteria is supported by some observations of Ernst, in which the kidneys showed typical lesions while the urinary passages were free from alterations or only the bladder contained severe inflammatory lesions while the pelvis and ureters were normal. Of like significance is the observation of Scherzer where in a case of pyelonephritis the valves of the heart contained deposits in which the Bac. pyogenes, which was present in the kidneys, was found in pure culture. The above mentioned experiments of J. Koch afford further proofs (p. 1013) and also those of Albarden, Savor and Orth which demonstrated that bacilli of the colon group may produce the picture of pyelonephritis by being eliminated in the kidneys.

On the other hand the urogenic origin of pyelonephritis cannot be denied for all cases, the more so as, according to the experiments of Schmidt \& Aschoff, Savor and v. Wünschheim, colon bacilli may lead to the development of pyelonephritis on being introduced into the bladder.

Evidently, however, the urogenic origin is rather rare. It is always necessary to consider that the bacteria may enter the blood vessels of the bladder and then reach the kidnevs by the blood paths after the bladder has become affected in consequence of an artificial or a natural infection. This mode of infection may be thought of, especially since the experiences of human physicians in catheterization indicate that even superficial epithelial defects in the mucous membrane of the bladder may give rise to an absorption of bacteria into the circulation.

Favoring circumstances appear to be important factors for the origin of pyelonephritis. Aside from the frequent connection of the disease with the birth act or with inflammatory processes in the female genital organs, and possibly also in other organs, a functional irritation of the kidneys may have a predisposing effect, even after normal parturition, and still more the elimination of septic substances in the above mentioned organic diseases. The predisposing influence of minary stasis also deserves mention, whether it be due to pressure exerted by the pregnant uterus, especially if the embryo is excessively 
large, or by the full rumen or through some disease of the urinary organs. The influence of predisposing factors may indeed be so important, that without it the pyelonephritis would not have developed in spite of the bacterial invasion of the kidneys.

Pathogenesis. In the ordinary hematogenic origin of pyelonephritis the bacteria are arrested in the capillaries of some glomeruli in one, more frequently in both kidneys, and not rarely already in the small arteries; they then produce a local inflammation of the related portions of the cortex, or a thrombosis. A considerable portion of the bacteria is eliminated and is compressed into cap-shaped bunches in the capsules of Bowman, and partly transported with the nrine. The experiments of Orth and J. Koch, and also the microscopical examinations of Ernst have shown that the eliminated bacteria accumulate and multiply in the collecting tubules in the lower portions of the medullary portion", whereupon an inflammatory process commences in the immediate vicinity, also in the mucons membrane of the papilla, of the renal pelvis and in the ductus papillaris. In certain cases the deleterious action of the microorganisms which are werlged in the renal capillaries appears to become manifest only after their elimination in the collective tubules.

The further sequels of the inflammation differ to a certain degree with the nature of the virus and according to the presence or absence of a mixed infection. After the entrance of the corynebacillus renalis the more or less localized inflammation results in necrosis which leads to the formation of small softened foci in the kidner, while in the center of the papilla a sequestrum is formed, surrounded by healthy or at least by not degenerated tissue in which the collective tubules break up into fibres. Fventually caseation and perhaps calcareous incrustation of the necrotic tissue takes place and at the periphery the inflammatory process either progresses or the inflammatory focus is circumscribed by a zone of demarcation. If pyelonephritis has been produced by other microorganisms the process differs only in so far as the inflamed tissue undergoes softening and suppuration. The same is to be observed in mixed infection which occurs frequently. In the surroundings of the necrotic foci the connective tissue proliferates and then atroplies while the unaffected portions of the kidneys hypertrophy.

Sooner or later an inflammation of the descending urinary passages is added to the process in the kidney. Owing to the constant contact of the mucous membrane of the renal pelvis with the bacteria and inflammatory products which accumulate in the corners of the pelvis, the tips of the originally affected papilla and of other papillae, as well as the mucous 
membrane of the renal pelvis in general become involved in the diphtheritic and suppurating inflammatory process. Of the peripheral urinary passages the bladder is most endangered (and also the urachus if this is not yet obliterated) because the urine remains there for a longer time. Accordingly a very severe inflammation sometimes develops in the bladder. The affection of the descending urinary passages, not excepting the ureters, is greatly favored by the urinary stasis which necessarily occurs, at least at times during the disease, and also by the constant irritation by the disintegration products of the urine. Under these conditions the germs are packed into the collecting tubules and, after the destruction of the pelvic mucous membrane, the inflammatory process continues directly into the renal tissue. In this manner an ascending inflammation of the kidneys is added to the original process.

In the urogenic origin of the disease, which is undoubtedly infrequent, the inflammation of the kidney commences with the process that was just described, after the inflammation has existed for some time in the urinary passages.

It appears from what has been said that no sharp distinctions ean be drawn between embolic purulent nephritis and bacterial pyelonephritis.

Anatomical Changes. In well developed cases both kidneys or only one are enlarged more or less, $1-4 \mathrm{~kg}$. in weight (in a case of Cadéac's the kidney weighed $6.5 \mathrm{~kg}$.). The renal capsule is thickened, adherent to the cortex in many places and in variable extension, the surface of the kidney is multi-colored; on a red-brown ground yellowish-gray islands are prominent, and corresponding with them striae or wider wedge-shaped hands of similar color are visible on the cut surface. Within these islands and within the brown portions of the kidneys there appear yellowish-gray, turbid small foci which are suppurating and soft or friable and cheesy, each one being surrouncled by hemorrhagic spots. Hemorrhagic infarcts are rare. Later the consistency of the kidney increases, but in the initial stage the tissue shows more or less serous infiltration.

The dilated pelvis of the kidney contains a dirty-gray, gravish-rellow, yellowish-brown or brownish fluid which may be bloody discolored, clondy, flocculent and peculiarly milky and which contains pus, tissue shreds, blood clots and granular calcareous material. The mucous membrane is much swollen or thickened, in recent cases studded with hemorrhagic spots; in places it shows ulcerous destruction or dirty-gray deposits. The renal papillae are covered with a viscid purulent substance, they are reddened and terminate in dirty discolored tissue shreds so that they have an eroded appearance. Sometimes the places of the renal papillae are occupied by hollow spaces filled with purulent masses (Pyelonephritis cavernosa) or if the process progressed by multiple lobar extension, the entire 
kidney is transformed into a pus sac consisting of several compartments, 4-16 kg. in weight (Pyonephrosis) (Kitt).

The ureter is changed into a tube with thick walls, up to two fingers in width, the mucosa may present similar changes as that of the renal pelvis. The mucous membrane of the bladder also sometimes contains hemorrhages or a variable inflammation which may even be diphtheritic.

The microscopical examination of the affecter kirlney gives the following result, accorling to Ernst: Cellular infiltration in the cortex, in places assuming the form of snall or large purulent infarcts, the dilated urinary tubules are filled with anuclear epithelia, pus cells and casts, vascular thrombosis is frequent; in a nore advancerl stage connective tissue proliferation, necrotic or purulent foci especially in the papillae. Masses of bacteria are found in the small softener foci in the cortex, in the capsules of Bowman and in the thrombi of the blood ressels, while the collecting tubules appear as though filled up with bacteria.

The fluir in the renal pelvis and the ureter contains much tissue detritus, numerous pus cells, some red blood corpuscles, fibrin threarls, pavement and eylinIric epithelia and crystals of triple phosphates; there are also found masses of B. renalis, alone or more often associated with other bacteria.

Symptoms. It is impossible to indicate a definite period of incubation because the exact time of the bacterial invasion zannot be ascertained. It appears from the observations made up to the present that pyelonephritis may become manifest in from a few days to several months after the commencement of the primary disease.

In the more frequent chronic form the clinical symptoms are diminished appetite, suppression of rumination, emaciation, staggering gait, the urine is voided fairly easily. Pressure upon the lumbar region sometimes produces manifestations of pain. On rectal exploration the bladder is found half filled, sometimes the dilated ureter is felt above the pelvis, below the vertebral column, on both sides or only one, it feels like a firm rope passing to the kidneys and is usually thickened in its anterior portion. Palpation of the kidneys may indicate normal conditions or enlargement, sometimes fluctuation, or sensitiveness to pressure in one or both kidneys.

It is more frequent to find increased urination associated with painful exertions. Pressure on the sacral region causes pain, a thick purulent vaginal discharge soils the pubic region. Rectal examination gives similar results as in the preceding case, but on inspection catarrhal reddening or swelling of the vaginal mucosa is seen, especially around the urethral opening, and the mucosa may be covered with superficial ulcers. In male animals the prepuce may be inflamed (Hess). At times colicky symptoms appear, probably due to the temporary occlusion of the ureter's by masses of exudate, blood clots or sediment.

The urine is seldom clear and even then contains albumen, and on microscopical examination cellular elements and the bacillary clumps described above. In the great majority of cases the urine is turbid, muddy, sometimes bloody, strongly 
alkaline and contains as much as $2 \%$ of albumen, and also free ammonia. Under the microscope there appear pus cells, red blood corpuscles, epithelial cells, crystals of triple phosphates and often renal elements. By Gram's stain and even in unstained specimens of fresh urine clumped bacilli or other bacteria are seen (Fig. 176).

Toward the termination of the disease the animals are extremely emaciated and unable to stand. They die of exhaustion or of acute uremia unless they are killed. In the rare acute form symptoms of fever (up to $39.0^{\circ}$ to $40^{\circ}$ C.) and rapid pulse are observed. The local manifestations of

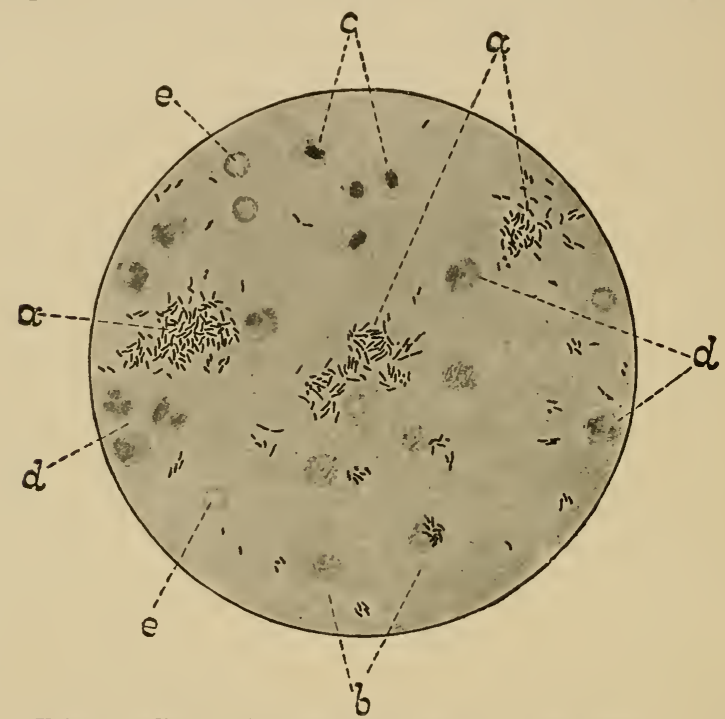

Fig. 176. Urinary sediment of a cow with bacterial pyclonephritis. a Clumps of Corynebacillus renalis. $b$ Squamous epithelium from the urinary passages. $c$ renal epithelium; $d$ pus cells; $e$ red blood corpuscles. (Magnif. about 1000.)

the chronic form are intensified and the urinary findings are similar. This form is probably due to a septicemic infection from the urinary organs or to a severe acute nephritis, it may lead to death within two weeks, but in exceptional cases the simptoms diminish in severity and the disease passes into the chronic form.

Recovery of a well defined pyelonephritis has never yet been observed, although the possibility of a clinical cure is not excluded if the disease is unilateral.

Diagnosis. As has been pointed out first by Schnidt, the disease may be recognized positively by the microscopic demon- 
stration of the Corynebacillus renalis which is usually present. Only upon this basis is it possible to differentiate the affection from the other forms of nephritis or from inflammatory affections of the uterus, vagina and bladder, and also from renal tuberculosis. Mistakes are the more easily possible as many of these diseases also frequently occur after parturition without simultaneous mephritis. If no symptoms exist pointing to these affections, and if some weeks after a delivery which was not quite normal, the urine contains pus, there exists a just suspicion of pyelonephritis which is further supported by the demonstration of a thickened ureter and enlargement of the corresponding kidney.

Treatment. Since all efforts at treatment have hitherto proved in vain it is advisable to slaughter the animals as soon as possible. In the begimning of the disease irrigation of the vagina and bladder and internal disinfectants like salicylic acid, Folia uvae ursi (25-59 gm.) may be tried.

Literature. Albrecht, W. f. Tk., 1900. 409. - Baillet \& Sérès, Rev. gén., 1903. I. 504. - Bartels, D. t. W., 1879. 303. - Cadéac \& Morot, J. vét., 1897. 65 - Dammann, D. Z. f. Tm., 1877. III. 265. - Enderlen, D. Z f. Tm., 1891. XVII. 325 (Lit.). - Ernst, Cbl. f. Bakt., 1905. XXXIX. 549; XL. 79 (Lit.). Friedberger, Münch. Jhb., 1899/90. 164. - Gillot, Rec., 188s, 159. - Hess, Schw. A., 1888. XXX. 269; 1891. XXXIII. 157; 1892. XXXIV. 70. - Höflich, Monh., 1891. II. 337 (Lit.). - Jensen, Ergebn. d. Path., 1895. II. 389 (Lit.). Koch, Z. f. Hyg., 1907. LVIII 287. - Künnemann, A. f. Tk., 1903. XXIX. 128. - Liénaux \& Zwaenopoel, Ann., 1902. 500. — Lucet, J. vét., 1892. 220. Masselin \& Porcher, Rec., 1895. 657. - Mollerean \& Porcher, Bull., 1895. 322. - Scherzer, B. t. W., 1903. 445. - Schmidt, Maanedsskr., 1890. II. 149; 1899. X. 179. - Sommer, B. t. W., 1906. 400. - Wyssmann, Schw. A., 1904. XLVI. 287.

\section{Inflammation of the Renal Pelvis. Pyelitis.}

Etiology. An inflammation of the nucous membrane of the renal pelvis arises usually in consequence of mechanical irritation, particularly in the presence of urinary calculi (in birds also when uric acid crystals are present) and of animal parasites in the pelvis of the kidneys, also in stasis of the urine in which fermentation is produced by bacteria. Infectious and poisonous substances which leave the kidneys with the urine may also exert a pathogenic action upon the mucous membrane of the pelvis. For instance, in acute infectious diseases the renal pelvis frequently shows catarrhal involvement when the renal tissue is also affecter; Dieckerhoff even observed a diphtheritic pyelitis in a case of influenza. Occasionally the bacilli of tuberculosis and of glanders produce specific changes in the renal pelvis. Of chemical irritants, especially cantharides, oil of turpentine and the severe diuretic remedies in general cause pyelitis if they are administered for a considerable time or in large amounts. Prolonged venous stasis in the kidneys may also give rise to changes in the mucous 
membrane of the renal pelvis which are similar to chronic catarrh. Finally the disease occurs in conjunction with severe, especially with purulent nephritis, and also with ascending inflammation of the bladder and of the ureters.

Anatomical Changes. Simple catarrh of the mucous membrane of the pelvis (Pyelitis catarrhalis) is characterized by increased redness and swelling, possibly small hemorrhages, in ehronic cases by slate-gray discoloration and thickening of the mucosa which is covered with thick mucus or pus and at times with a granular or lumpy deposit. In severe inflammation a great number of hemorrhagic foci are found in the mucosa and much blood within the pelvis (P. Haemorrhagica); in other cases there is ulcerous destruction of the mucosa (P. nlcerosa) or this is covered with membranous deposits (P. membranacea $\mathrm{s}$. diphtherica). The inflammatory process may be associated with a dilatation of the renal pelvis, its eavity being filled with urine (Hydronephrosis) or largely with pus (Pyonephrosis).

Symptoms. Usually the patients show symptoms of pain which are not, however, characteristic. In larger animals rectal exploration sometimes makes it possible to determine sensitiveness to pressure in one or both kidneys and, in some cases, a dilatation of the corresponding renal pelvis; in smaller animals pressure through the abdominal wall elicits evidences of pain. Some animals have frequent desire to urinate, only a small amount of nrine being voided at one time, in other cases micturition is normal.

The color and specific gravity of the urine are often normal, but the urine contains much mucus and more or less pus. The reaction is alkaline in herbivora, acid or alkaline in carnivora, the latter especially in ammoniacal fermentation. In any case the urine contains albumen corresponding in quantity to that of the pus. In consequence there is always a fairly copious organic sediment, containing pus cells, and also long-tailed, epithelial cells which look like molar's, have pointed projections at one or both ends, and are derived from the deeper epithelial layers of the mucous membrane of the renal pelvis; there are also pavement epithelia. At times the sediment contains red blood corpuscles, small blood clots and mucous shreds. If urinary calculi are present in the renal pelvis the urine contains whitish or yellowish sandy granules, if parasites are present the sediment contains eggs of worms.

Since inflammation of the renal pelvis is often accompanied by nephritis or by catarrh of the bladder, the clinical picture may be more or less obscured by the symptoms of these diseases.

Pyelitis is always of serious significance, because it is the 
sequel of dangerous primary diseases (calculi, parasites, nephritis) or leads of its own account to the development of nephritis.

Diagnosis. The disease can be recognized only by means of urinary examination. The presence of pus cells and of molar-shaped epithelial cells is particularly significant, but is characteristic only then when a catarrh of the bladder may be excluded, because long-tailed, molar-shaped epithelia may also be derived from the deeper layers of the vesical mucosa. The pus may also have entered the urine from other parts of the urinary organs, especially from the kidneys, from perirenal abscesses, from the bladder, the prostate, etc.; and it is often extremely difficult or impossible to ascertain its origin. It is finally to be considered that in the presence of a balanitis, of a uterine or a vaginal catarrh, pus may be mixed with the normal urine.

Treatment. For the purpose of disinfecting the renal pelvis the following remedies may be administered internally: Salicylic acid or its salts $\left(25-50^{\circ} \mathrm{gm}\right.$. for horses and cattle; 2-10 gm. for sheep, goats, hogs; 0.1-2.0 gm. for carnivora at each dose); according to Walther these preparations may, however, produce albuminuria and even nephritis by irritating the urinary passages and the kidneys. Salol in like doses, oil of turpentine (100-200 gm., or 5ं-25 gm., or 0.05-2 gm.), urotropin (5-10 gm. for large, 0.5-1.0 gm. for smaller animals, 3 times daily with much water [Gmeiner]), helmitol (6-10 gm. for large, $1-2 \mathrm{gm}$. for smaller animals, per dose, 3 times daily), Folia uvae ursi (20-50 gm. for large, 1-15 gm. for smaller animals).

\section{Kidney Stone Disease. Nephrolithiasis.}

\section{(Calculi renales.)}

Occurrence. Trinary stones are in domestic animals not infrequent and are found in various portions of the urinary passages. They oceur primarily almost always in the wider parts of the urinary apparatus, either in the renal pelvis, possibly already in the straight urinary tubes, or in the bladder. and are only later carried with the urine into other portions of the urinary passages. Stones are found most often in horses and cattle and are much more rare in the other mammals and in fowls. Their frequency is said to increase with the age of the animals (Möller). Sometimes the trouble occurs simultaneously in many animals among cattle, sheep and hogs.

In the rears 1862-1897 urinary caleuli were found in the Dresden pathological Institute in 12 out of 3,301 dogs $(0.38 \%)$, in 1 out of 4.50 eats $(0.22 \%)$, and in 11 out of 2,100 horses $(0.5 \%)$. 
Etiology. According to Klimmer the principal condition for the development of urinary calculi is that the urine contains an excess of salts or that slightly soluble or insoluble salts are formed in it, already within the minary passages. In both cases the eliminated salts are deposited upon any foreign body that may be present in the mrinary passages, and the small stone which is formed in this manner gradnally enlarges by the contimued deposit of salts. It is a question whether the presence of sticky substances, especially of albuminous or gelatinous borlies is required for the formation of stones. The nucleus on which the eliminated salts are deposited is usually furnished by some element derived from the urinary organs (epithelial cells, casts, blood clots, pus- and fibrin shreds, necrotic tissue shreds), it is rarely a foreign body which has entered the urinary passages from the outside (straw, gravel, etc.).

The amount of salts present in the nrine is materially influenced by the chemical composition of the food and of the drinking water, and therefore in a way also by the conditions of soil. Drinking water which contains much lime or many earthy constituents is of importance; so are food-stuffs that are mixed with earthy substances or which are rich in calcium. It can thus be explained why the disease is more frequent in herbivora in regions where the soil is calcareons (Láng observed on one estate the disease exclusively in those oxen which had lived, at least for a time, on a certain farm). Multiple cases may occur independently of the conditions of soil after inappropriate feeding. Especially feeding with very large quantities of potatoes, the waste of sugar beets, beets, and slop may be followed in cattle by the formation of silicate concretions, and a food rich in phosphorus (bran, grains) may give rise to phosphatic calculi (especially in sheep). Theis saw urinary stones in two mares which had for months received prepared bone meal.

Inflammatory diseases of the urinary passages, also in complete retention of mine, from whatever cause, may lead to the formation of calculi by causing the formation of slightly soluble or insoluble compounds, owing to the disintegration of the urine; they furnish at the same time a nucleus for the calculus. In this direction bacterial diseases are particularly dangerous.

The attempt has been made repeatedly to produce urinary calculi artificially. In Studensky's experiments salts were indeed deposited upon foreign bodies that had been introduced into the bladder, but actual calculi were formed only when the hladder was at the same time subject to eatarrhal affection. Ebstein and Nicolaier observed the formation of stones after the systematic administration of oxamide. According to these authors this substance is absorbed in the intestines, is then elim. inated in the kidneys and, on coming in contact with the mucosa of the urinary passages it causes a necrosis of the epithelial cells, so that the albuminous substance is supplier which is said to be required for the formation of calculus. Thomassen also found small stones in the renal pelvis of a small dog which had been fed with oxanide for two weeks. 
In calculating the time of development of the calculi their size and chemical composition must be considered, and also the external conditions (Klimmer). Pflug says that phosphatic concretions grow more rapidly than the other ealeuli. Rathke observed in eattle in the space of three weeks the formation of calculi of from pinhead- to bean-size.

Anatomical Changes. Kidney stones are sometimes quite small, covering the mucous membrane of the renal pelvis in the form of fine or coarse grits, and are there mixed with the urine, in exceptional cases they are imbedded in the renal tissue; in other cases they are good-sized actual stones and may fill out entire calyces and even the whole renal pelvis. The last variety of stone is sometimes round, lying free in the pelvis, or if present in numbers, they are polished smooth by mutual friction. Larger stones show irregular coral shapes with knobbed surfaces from having adapted their shape to the form of the cavity in which they formed. Sometimes each calyx is filled by one stone, in other cases the stones are connected by their extremities which point toward the ureters or they may fuse together to good-sized masses after atrophy of the calyces. As the stones grow, the renal substance gradually disappears and finally large stones may imitate the shape of the kidney, and are found surrounded only by a thick capsule.

If several stones are present, the mucous membrane of the renal pelvis is always inflamed, perhaps it contains hemorrhagic spots or ulcers, or is covered with diphtheritic psendomembranes. The renal tissue usually bears the signs of an acute or a chronic inflammation.

The urinary calculi of herbivora are grayish-white, brown or mottled and consist, according to examinations made by Fürstenberg, largely of calcium carbonate and magnesium carbonate which form multicolored layers around a nucleus consisting of organic substances, rarely of calcium oxalate. In cattle and sheep the stones are smooth, often small, yellowish or gray, spherical or angular, and have a peculiar metallic or mother-of-Iread luster, which evidently is due to the very fine arrangement of the layers, although Fürstenberg believes it due to the admixture of ferrous carbonate. Bourmer demonstrated $0.28 \%$ of iron in the renal stones of a cow. Some stones do not show layers and are more friable. They consist of a small amount of organic material, of calcium carbonate and of a small quantity of magnesia carbonate. In a cow which had probably suffered from leukemia or pseudoleukemia Joest found xanthin stones which usually are formed in case of considerable destruction of cellular nuclei and which had already been found by Weiske in a ram having presumably suffered with leukemia.

The calculi of earnivora contain ammoniacal magnesia phosphate and small quantities of calcium phosphate or carbonate, usually also uric acid and its salts. Cystin calculi are much less frequent and are always small, soft and of a greasy luster.

Symptoms. Renal calculi give rise to a clinical picture which is as a whole similar to that of pyelitis, except that the evidences of pain are more decided and are rarely absent. Nevertheless cases undoubtedly occur in which large stones are found on autopsy and perhaps total atrophy of one kidney, and in which the animal showed no signs of disease during life. The walk becomes stiff, the region of the kidneys sensi- 
tive to pressure. In many cases the animals (horses, cattle, sheep) are attacked by severe colic, either suddenly or after violent motions, after jumping, etc., and during these attacks repeated attempts are made to urinate. These severe colicky pains are observed especially when the ureter is occluded by a stone which has passed out of the renal pelvis, because both the mechanical irritation of the ureteral walls and the stasis of the urine cause pain until the stone is finally discharged into the bladder. During the attacks it may be totally impossible to void the urine if both ureters are occluded (Barthe), or if, in unilateral obstruction of the ureter, the function of the other kidney has been temporarily suppressed by reflex action (Morgagni). A prolongation of the anuria may lead to uremia. This "renal colic" usually recurs, because several small stones are present in the renal pelvis and because new ones are always formed which from time to time pass into the ureter.

The urine contains pus cells, perhaps epithelial cells from the pelvis of the kidney, and at times a sandy sediment or small stones. It is not rarely bloody, and on microscopical examination fine fibrinous coagulates are found in addition to red blood corpuscles. In cattle and sheep grit-like granules sometimes adhere to the hairs around the opening of the sheath.

The nature of the disease may at times be discovered by rectal examination or by palpation through the abdominal walls, that is, in cases in which the presence of one or several stones in the renal pelvis may be determined in addition to the increased sensitiveness of the kidneys and of the renal pelvis. If several stones are present a slight trembling is felt on palpation in the walls of the renal pelvis which is produced by the rubbing of the stones against each other. During the colicky attacks the stone, which is werlged in the ureter, may sometimes be felt as a swelling of pea to bean size, which is very sensitive to pressure.

Course and Prognosis. Kidney stones always cause prolonged disease, in the course of which attacks of colic and disturbances in micturition alternate in long periods. The disease develops slowly and may lead to gradual exhanstion of the animal. The pyelitis which has been produced by the calculi is followed by acute or chronic nephritis or the renal tissue is destroyed by atrophy so that the elimination of the urine is necessarily impaired, especially in bilateral disease. Occasionally necrosis develops in the wall of the ureter around the arrested stone and this leads to peritonitis or to purulent inflammation of the perirenal connective tissue. Kidney stones may also reach the bladder, may here increase in size and cause catarrh of the bladder, or they may later on be forced 
into the urethra where they produce retention of urine which may lead to death in a brief time.

Diagnosis. A sufficiently positive recognition of the disease presupposes the demonstration of pyelitis, or sandy or gritty sediment in the urine, or the direct discovery of stones in the renal pelvis by means of palpation. The disease may be mistaken for other colicky diseases, nephritis, paranephritic abscess, renal tumor, etc.

Treatment. If there is a suspicion of calculus formation, the diet must above all be regulated thus that the animals ingest with their food or drinking water as small amounts as possible of salts. In herbivora it is therefore necessary to withdraw especially bran and to limit grains; carnivora should receive only little meat; in addition, good soft water must be supplied. The drinking of large amounts of carbonated water is recommended to secure the solution of calculi and prevent their formation. In renal colic subcutaneous injections of morphine or the administration of chloral hydrate is indicated.

Lorge and Rubay have attempted the operative removal of kidney stones (nephrotomy) with satisfactory results. The resection of the diseased kidney (nephrectomy) may also be thought of.

Literature. Albrecht, W. f. Tk., 1900. 1. - Ball, J. vét., 1906. 193. Barthe, Rev. vét., 1906. 289. - Bourmer, Beitr. z. Nephrolithiasis usw. Diss. Bern. 1908 (Lit.). - Brun, Bull., 1902. 80. - Dammann, D. t. W., 1897. 435े. - Fréger, J. vét., 1907. 326. - Hébrant, Ann., 1902. 578. - Imminger, W. f. Tk., 1901. 553. - Klimmer, A. f. Tk., 1899. XXV. 336. (complete Lit. to 1899). - Prietseh, S. B., 1901. 45. - Rabieaux, Bull., 1902. 310. - Rathke, Pr. Mt., 1865-66. 129. Rubay, Ann., 1895. 413. - Theis, Bull., 1905. 255.

\section{Renal Dropsy. Hydronephrosis.}

\section{(Cystic Kidney.)}

Occurrence. Hydronephrosis is frequently found in cattle, hogs and sheep during meat inspection.

Lucks found the affection in $43(0.67 \%)$ out of 6,425 hogs examined, in female pigs almost 3 times as often as in males; in 29 animals the condition was unilateral (10 times on the left side, 19 times on the right side), in 14 animals it was bilateral. Horn saw hydronephrosis in $0.21 \%$ of 7,000 slaughtered sheep, usually unilateral, and due to kidney stones in $80 \%$ of the cases.

Etiology. The immediate canse of hydronephrosis is supplied by an impediment to the flow of the urine from one or both renal pelvis, in consequence of which the urine, which is steadily secreted, is dammed up behind the impediment with a constantly increasing pressure. The affected urinary passages gradually become dilated and the medullary substance of the 
kidneys atrophies, until finally even the cortex becomes atrophic; with this the process is arrested because no more urine is secreted. If the impediment is proximal to the bladder, only one kidney is usually affected; other impediments to the outflow of urine necessarily lead to bilateral hydronephrosis.

It is evident that only chronic or periodical abnormal conditions of the organs which discharge the urine are eapable of producing hydronephrosis, since its flevelopment requires a period of several months. Total occlusion of both ureters or of the urine-carrying organs from the openings of the ureters down is not followed by hydrouephrosis because the animal perishes of uremia or of rupture of the bladder in a comparatively sliort time. But even a unilateral total ocelusion of the ureter does not produce hydronephrosis if it is permanent, lecause then the pressure in the urinary passages rapidly inereases, and therefore the secretion of urine in the corresponding kidney diminishes just as rapidly and to complete cessation (Senator).

As to the individual causes, this much is certain that the following affections are, although comparatively rarely, of fairly equal importance in all animal species; inflammation of the bladder or of the ureters, obstruction of the ureter's by stones, secretions, coagulated blood (for instance in bacterial pyelonephritis), the obliteration of the ureter-opening into the bladder (evidently congenital [Kitt]), congenital valvular formations, constriction of the ureter by muscular fibers of the wall of the bladder (Lucks), stenosis of the urethra in enlargement of the prostate, compression through tumors, etc.

The most frequent causes are, however, of different character. In hogs, Lucks proved by the results of careful examinations in 30 cases that the cause of hydronephrosis usually lies in the fact that the openings of the ureters are congenitally placed too low in the neck of the bladder (on one or both sides) and that in the hog the attachment of the bladder is very loose. This makes it possible that the partially filled bladder, which then falls lower down, presses its neck, together with the caudal portion of the ureter against the anterior border of the pubis and thus periodically prevents the voiding of the urine. In cattle other causes are probably active, for instance, the occasional bending of the left ureter by the filled rumen, in which case the left kidney is pushed out of place (Breuer), also the compression of the ureter by the filled stomach.

In ehronic inflammatory processes in the kidneys some urinary tubuli are at times constricted in portions by the contraction of the connective tissue which is undergoing atrophy, or are filled with small stones or cell masses, so that the canals which are attached to the Malpighian bodies are dilated, the urine continuing to be secreted, and are thus transformed into retention eysts. Such small cysts are sometimes found in large numbers, the contiguous ones become confluent and the entire kidney is transformed into a collection of eysts of variable sizes (cystic kidney, hydrops eysticus). In this form the trouble is found especially in cattle, and occasionally as a congenital condition.

Anatomical Changes. The dropsical alteration of the kidney may be met with in various stages. If the process is far advanced, the kidney is changed into a large undulating 
bladder (in cattle it may be $1 / 2 \mathrm{~m}$. long and as wide [Kitt], in pigs it may attain a weight of $42 \mathrm{~kg}$. [Richter]). The wall of this bladder is formed by the thickened capsule of the kidney and only here and there isolated portions of the cortical kidney substance may be seen. The lobulated kidneys now are divided into several compartments and, if the impediment is in the region of the bladder, these compartments form the direct continuation of the ureter which occasionally may also be dilated excessively. In less severe cases the cross section shows a variable layer of the renal substance, which looks firm and anemic; in disease of slight degree, only the calyces and the pelvis of the kidney are dilated noticeably.

In the eystic kidney it is usual to find partial or total selerosis of the renal tissue and in addition watery eysts of from poppy- to hemp-seed size, or, if they have become confluent, vesicles as large as hazelnuts or larger, up to the size of a fist and even of a man's heall; they have a white-bluish luster and are wabbly. In eattle the separate renal lobes may thus be transformed into eysts.

Symptoms. One kidney may be altered very greatly, as above described, without eausing any particular disturbances in regard to the discharge of urine, because the other organ; which has become enlarged by compensation, is capable of attending to the elimination of the urine. In bilateral disease symptoms may become manifest much earlier, especially a permanent or intermittent diminution in the quantity of urine, which may even amount to complete, although transitory amuria, dependent upon whether the discharge of the urine is impeded continuously by the underlying canse or only from time to time. If the anuria persists for a long time, or in the case of a severe bilateral hydronephrosis, uremic symptoms are not long in making their appearance. On rectal exploration or on external palpation of the abdomen one or both kidneys are more or less enlarged and fluctuating but not painful. The dilated ureter may sometimes be palpated from the rectum. In smaller animals the enlargement of the kidney may lead to a considerable increase in the size of the abdomen and in such cases exploratory puncture of the swelling yields a urinelike fluid.

Richter observed, in a two-year-old pig, an enormous enlargement of the abdomen which almost touched the floor and showed distinct fluctuations; the motions of the animal were clumsy and staggering; it often assumed a sitting position like a dog.

In the majority of cases hydronephrosis does not seem to cause any particular disturbance of health, and none was ever observed in cystic kidney, which usually affects only portions of the kidneys.

Treatment. If the impediment to the flow of urine canno be removed only extirpation of the affected kidney (nephrec- 
tomy) can, in case of unilateral disease, be considered, if the enlarged kidney interferes with the health of the animal by compressing the abdominal organs. This procedure can only then be successful when the other organ is still capable of functioning.

Literature. Barrier, Bull., 1906. 683. - Basset, Rev. gén., 1903. I. 263. Horn, Beitr. z. Kenntn. d. chron. Nierenerkrank. d. Schafes. Diss. Leipzig, 1908. - Kowalewski, A. f. Vet.-Wiss., 1904. 1004. - Kukuljeviě, Ä. L., 1905. 539. Lucks, Monh., 1905. XVI. 254. - Richter, Z. f. Flhyg., 1907. XVII. 429. - Suffran \& Daille, Rev. vét., 1905. 612.

\section{Renal Tumors.}

Among new-formations in the kidneys there are found especially adenomas and carcinomas; primary sarcoma is very rare, but secondarily it occurs occasionally. In dogs villous proliferations sometimes develop in the renal pelvis and in the ureter, and impede the outflow of the urine, thus causing dilatation of the renal pelvis and atrophy of the substance of the kidney.

Tuberculosis of the kidneys is frequent in cattle and is also met with in dogs and pigs. Small tubercles or larger cheesy foci are disseminated mainly in the cortical portion where they originated through embolisms. As the cheesy foci enlarge they may finally perforate the walls of the renal pelvis and then the tuberculous process passes over into the mucous membrane of the latter. Tuberculosis may in this location sometimes also arise from urogenic infection.

New-formations in the kidneys are recognized only with difficulty and their recognition is only possible if one or both kidneys are considerably enlarged. The actual nature of the new-formation is sometimes indicated by external conditions, such as their presence in other organs or as the enlargement of inguinal glands (in carcinoma). If an enlargement of the kidneys exists or if the urine-carrying organs are not affected the diagnosis of renal tuberculosis depends upon the demonstration of tubercle bacilli in the urine.

The treatment can at best be symptomatic.

\section{Animal Parasites in the Kidneys.}

\section{(a) Eustrongylus gigas.}

Etiology. The enstrongylus gigas, which sometimes occurs as a parasite in the renal pelvis of dogs, horses, cattle; also in many wild living animals and in man, is a blood-red threaclworm about the size of a lead pencil; the male is $13-40 \mathrm{~cm}$. long, the female $20 \mathrm{~cm} .-1 \mathrm{~m}$. The eggs (Fig. 177) are oval in shape, brownish in color; their covering is thick and shows numerous round depressions. The eggs are 68-80 $\mu$ long and $30-44 \mu$ wide.

It is not known how the worm enters the renal pelvis. While it grows here it produces a severe purulent and hemorrhagic inflammation which in its turn causes a secondary in- 
flammation and destruction of the renal tissue. The kidney is eventually transformed into a thick-walled sac in which the worm is coiled up, imbedded in a purulent-bloody mass. In rare cases two worms are found together in one renal pelvis; Verney has even found four specimens in the kidney of a dog.

Symptoms. The symptoms which are caused by the worms vary from case to case, and it may even occur that in spite of their presence no disturbances

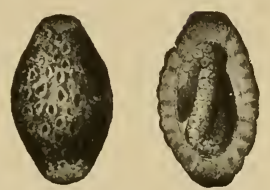

Fig. 177. Eggs of Eustrongylus gigas. (After RAILLIET.) in health are observed; especially in horses and cattle hardly any disease symptoms have been reported. On the other hand, dogs suffer severe pain, are restless and may even show mania and an inclination to bite, simulating rabies. Sometimes the vertebral column is eurved laterally, the concavity corresponding to the affected side. In other cases only general indisposition and emaciation are noted and may increase to complete exhaustion. The voiding of the urine may require an effort, it contains pus and blood and indicates the existence of pyelitis.

Diagnosis. The disease can be diagnosed only by the demonstration of the characteristic worm-eggs (Fig. 177) in the urine.

Course. In the presence of severe functional disturbances the disease usually ends in death. Lacoste saw in one case the worm pass out through the urethra after which the dog recovered completely. In exceptional cases the worm leaves the renal pelvis and is arrested in the bladder where it causes intense inflammation, or it passes on to the penal bone, causing a swelling in the region of the perineum which may become as large as a fist and in which the worm is coiled up (Leblanc, Mégnin). Uremic symptoms may also occur.

Treatment. The removal of the worm from the renal pelvis may be attempted by oil of turpentine (for dogs tableor coffee-spoonful). If the worm is arrested in the urethra it ean be removed by incising the swelling.

Literature. Leblane, Bull. de l'acad. méd., 1850. 640. - Mathis, Lyon méd., 1884. 499. - Mégnin, Ree., 1879. 223. - Neumann, Mal. parasitaires, 1892. 740 (Lit.). - Pease \& Smith, Vet. Journ., 1905. 200. - Railliet, Zool. méd., 1895. 419. - Rivolta, Med. vet., 1867. 1.

\section{(b) Other Parasites in the Kidneys.}

Of threadworms the larvae of the Sclerostomum are found in the kidneys of horses where they localize in the renal artery or its branches and may give rise to hemorrhage in the kidney (see page ...). Petit, 
Henry \& Germain (Rec. 1909, 493) found in a horse, that had been killed on account of repeated attacks of delirium, numerous tubercle-like foci, almost always in the cortical substance, which contained worm larvae similar to rhabditis. The Stephanurus dentatus oceurs in pigs (frequently in the perirenal connective tissue in American pigs). Rost found in $75 \%$ of 60 experiment animals nodules in the kidneys. which contained larvae of nematodes (D. m. W. 1906, 446).

Of tapeworms are found: Cysticercus cellulosae (in pigs; found also once in a dog [Railliet \& Trasbot]) ; Enchinococeus polymorphus (in ruminants, especially sheep; very rarely in other animals); E. multilocularis (found once by Ostertag); Taenia serrata (found by. Wolpert in both renal pelves of a dog). Finally Railliet \& Lneet have found small nodules containing coccidia in the kidneys of geese which had died with cachectic symptoms; Paechinger mentions a similar finding in horses and logs (Coccidiosis renalis).

Renal coccidiosis of geese is caused by the Coccidium truncatum (Eimeria truncata) and is manifested in progressive emaciation with extreme debility, owing to which the animals are found sitting almost minterruptedly. Many animals lie on their backs and spread their feet; if they are stood on their feet they take a few steps and again assume the sitting position. After a prolonged disease they usually lose the desire to eat and die. 


\section{Section II.}

\section{DISEASES OF THE BLADDER.}

\section{Catarrh of the Bladder. Catarrhus Vesicae Urinariae.}

\section{(Cystitis catarrhalis, Urocystitis.)}

Etiology. Catarrh or inflammation of the mucosa of the bladder is usually based upon an infection. Bacteria which get into the bladder find favorable conditions for multiplication especially in urinary stasis, for instance in stricture, compression or obturation of the urethra, or in paralysis of the bladder, sometimes in the voluntary retention of urine in house dogs. In such cases the bacteria enter the bladder from the urethra, especially in female animals in which the vaginal secretion may enter directly owing to the shortness and comparative width of the urethra.

An artificial infection may be produced by catheterization. Injuries of urethra and bladder, for instance during parturition, enable the bacteria to enter, and inflammatory processes in the neighborhood of the bladder may be continued to its walls or mucous lining. In this respect inflammatory diseases of the urethra, the prostate, the internal female genital organs, the kidneys, the renal pelvis and the ureters are to be mentioned.

Sometimes a bladder catarrh develops in general acute infectious diseases because bacteria have been carried to the mucosa of the bladder with the circulating blood. Bacteria may also reach the bladder from the diseased kidneys, or through the intermediation of healthy kidneys from distant organs (especially from the intestines [see Pyelonephritis]). Of first importance as pathogenic agents are the pyogenic bacteria but, especially in cystitis of intestinal origin, the colon bacteria and their related bacteria are important factors.

In many cases the occurrence of the disease is probably to be referred to taking cold, which reduces the resistance to the bacteria of the walls of the bladder.

Among irritating chemicals cantharides especially give rise to catarrh of the bladder which may, however, also appear after the internal administration of balsamics and of resinous remedies (oil of turpentine, etc.). It is not certain whether the inflammatory process is aroused immediately by the sub- 
stance circulating in the blood or only after it has, with the urine, entered the bladder and thus has come in contact with the mucous membrane (Hentrich observed eatarrh of the bladder in horses after feeding with beer slops which had become sour).

Vesical calculi can also give rise to inflammation in the mucosa of the bladder by mechanical irritation.

Anatomical Changes. In acute eystitis the mucous membrane is vividly reddened, swollen, perhaps studded with small hemorrhages, and covered with viscid mucus or with a purulent deposit; the submucous layers are soggy with serum. In more intense inflammation yellowish, croupous fibrin membranes are found, or dirty-gray diphtheritic deposits, or localized ulcerous destruction; it is also possible to find small or large abscesses in the walls of the bladder.

In chronic cystitis the mucosa is thickened, corrugated and not infrequently covered with branched-out villous proliferations. Salts are eliminated from the urine and form a coarse crusty deposit on the mucous membrane. The muscular layer usually hypertrophies when the disease exists for a considerable time.

Often inflammatory conditions are found also in the urethra, in the ureters and in the renal pelvis; in the chronic forms dilatation of the renal pelvis is not infrequent and is associated with inflammation or atrophy of the kidneys.

Emphysema of the bladder of cattle was observed in one ease each by Bunge and Malm and in two cases by Trolldenier. Outside of hemorrhages, thickening of the bladder wall was found with numerous air bubbles of various sizes in the latter. In Bunge's ease such air bubbles had also developed in the renal pelvis of the eow which was ill with pyelonephritis.

Symptoms. One of the most striking phenomena in acute catarrh of the bladder is the frequent and painful micturition (Tenesmus resicae, Stranguria, Dysuria). The animals make frequent attempts to urinate, during which they either void no urine or only a small quantity with manifestations of severe pain. The pressure to urinate and therefore the contraction of the bladder muscles may be repeated at such brief intervals that the urine is discharged almost uninterruptedly and in driblets, similarly as in paralysis of the sphincter.

In male animals there occur also frequent erections of the penis, the testicles are drawn up, and in horses signs of colic occur at times. Pressure upon the bladder causes severe pain and at the same time the organ will be found to be empty. Exceptionally there is retention of urine (ischuria), perhaps because the sphincter is contracted convulsively or because the mucosa at the neck of the bladder is much swollen, or the opening of the urethra is obstructed by exudation.

The urine is usually cloudy and dark in color; it contains variable, but comparatively small quantities of albumen 
(albuminuria spuria), occasionally white mucus- and pusfloccules, also gray pseudomembranes or tissue shreds. The reaction is alkaline in herbivora, but in carnivora either acid (in catarrh due to $\mathrm{B}$. coli) or more frequently also alkaline. Ammoniacal fermentation takes place already within the bladder and is recognized by the characteristic pungent odor and also by the fact that a moistened strip of red litmus paper turns blue when it is laid over the opening of a vessel which contains fresh urine. The sediment is always copious and consists of pus cells, red blood corpuscles, large pavement epithelia,

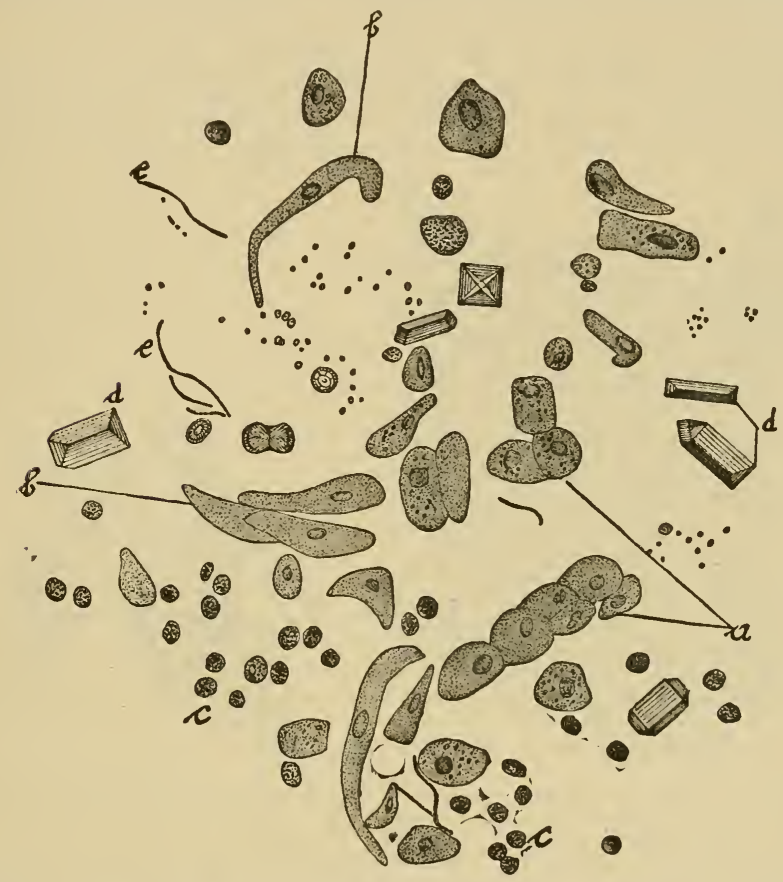

Fig. 178. Urinary sediment of a horse with catarrh of the bladder. $a$ pavement epithelia (superficial layer). $b$ pavement epithelia (deeper layer, tailed cells). c pus cells. $d$ triple phosphate crystals. $e$ streptococci.

also elongated, slender or molar-shaped epithelia (Figs. 178, $179,180)$. Together with ammoniacal fermentation numerous coffin-shaped crystals of ammonia-magnesia phosphate are found, and thorn-apple-shaped crystals of acid ammonia urate. Finally the freshly voided urine usually contains very many bacteria.

Simple acute catarrh is very rarely accompanied by high fever and lassitude, which is, however, frequent in inflammation 
of the bladder. In this case the appetite is suppressed and defecation retarded.

The clinical picture of chronic catarrh of the bladder is similar, although the symptoms, especially pain and pressure of urine are less severe and often absent (Gmeiner). The urine is also similar in character, but red blood cells are less numerous while on the other hand ammoniacal fermentation is more frequent. (Sivieri observed, in a mare with chronic bladder catarrh, rupture of the bladder after partial necrosis of its thickened wall.)

Course. Simple acute catarrh of the bladder is not rarely relieved by appropriate treatment, but in certain cases it passes over into the chronic form which is much more obstinate. Inflammation which is accompanied by copious pus-formation,

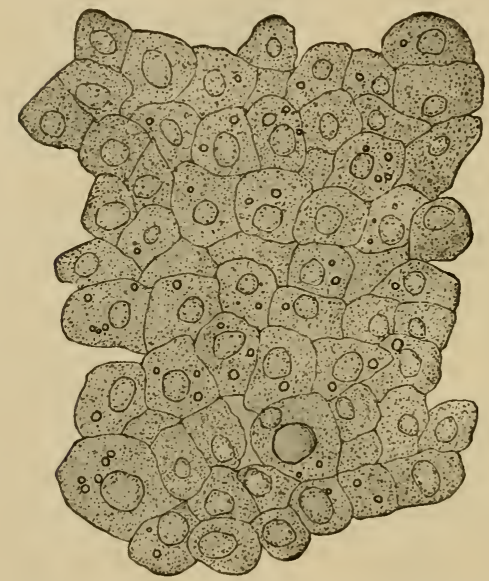

Fig. 179. Pavement epithelia (superficial layer) from the bladder of a horse with eatarrh of the bladder.

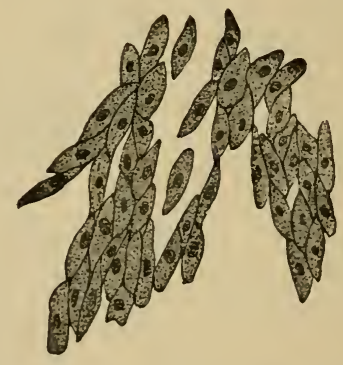

Fig. 180. Pavement epithelium (deeper layer) from the bladder of a horse with catarrh of the bladder.

hemorrhage, ulceration or necrosis takes usually an unfavorable course, because in such cases general infection is apt to occur, chemical changes in the urine give rise to stone formation; the inflammation may be transmitted to the pelvis of the kidneys and finally to the kidneys.

Treatment. Absolute rest, non-stimulating food and the drinking of as much water as possible are indicated in all cases.

In simple acute catarrh of the bladder healing is not infrequently produced by internal treatment only. For this the 
disinfectants are adapted first of all, for instance, according to Gmeiner and Schmidt, urotropin (5-8 gm. for large animals, 0.25-0.5 gm. for dogs, 2 to 3 times daily in much water) which exerts a disinfecting action after its absorption and elimination through the kidneys, owing to its ability to split off formaldehyde in the acid gastric juice. The remedy may be prescribed under the name of Hexamethylentetramin and is then much less expensive. In ammoniacal fermentation of the urine it is preferable, according to Uebele, to administer Helmitol (10-15 gm. for large animals, twice daily; 1-2 gm. for dogs, 3 times daily in powder or $10 \%$ solution), becanse it can split off formaldehyde in alkaline solution also and may therefore be given subcutaneously. Both remedies diminish ammoniacal fermentation and change the alkaline into the acid reaction. Salicylic acid and its salts are further to be considered, also salol, salipyrin, resorcin, benzoic acid, folia uvae ursi, etc. (see p. 1020).

In chronic catarrh of the bladder the same remedies are employed, and also balsamics, especially copaiba, and oil of turpentine. Here local treatment by means of irrigations with disinfecting and astringent solutions seems more useful (Gmeiner). According to Gmeiner oxycyanate of mercury in a solution of $1: 1000-5000$ is most appropriate, a small amount of $\mathrm{NaCl}$ may be added. Uebele advises irrigating the bladder with $2 \%$ solutions of helmitol. Other remedies are nitrate of silver $(0.1 \%)$, boric acid, tamnin, alum, lead acetate (1-3\%), resorcin $(3-5 \%)$, ichthyol solution $(1 / 2-2 \%)$, etc.

The bladder is irrigated as follows: After emptying the urine artificially, inkewarm water $\left(35^{\circ}\right.$ C.) or better, a $1 / 2 \%$ solution of $\mathrm{NaCl}$ is allowed to run into the bladder through a funnel which is connecterl with the eatheter by means of a rubber tube; in large animals the amount injected is about 300 ce.; in small animals as much as $50 \mathrm{ce}$. The water is allowed to flow out by lowering the funnel. Then the disinfecting solution is injected also lukewarm and likewise removed after a few minutes.

In certain cases of catarrh of the bladder operation may be considered.

Literature. Bunge, Z. f. Flhyg., 1898. TIII. 168. - Gmeiner, Monh., 1907. XVIII. 61. - Hentrich, Z. f. Vk., 1905. 59. - Malm, Monh., 1905. XVI. 254. Neneioni, N. Ere., 1903. 447. - Schmidt, Die Cystitis und Nephritis nsw Diss. Giessen. 1909 (Lit.). - Sivieri, N. Ere., 1908. 18. - Suffran, Rer. vét., 1909. 405. - Taylor, Vet. Rec., 1905. 75. - Trolldenier, Monh, 1904 XV. 5.

Twisting of the Bladder. This was observed by Strond and by Griveaux, each in one dog, and was manifested by pressure of urine, retention, enlargement of the abdomen; the dilated bladder could he felt through the abdominal walls and on rectal examination, which was painful. In Stroud's case there existed a paralytic weakness of the hindquarter and strong swelling of the perineal region; Griveaux met an impediment in front of the neck of the bladder on attempting catheterization. The trouble eaused death in a short time--(Griveaux, J. vét., 1906, 393.-Stroud, Ö. M., 1906, 219). 


\section{Paralysis of the Bladder.}

By paralysis of the bladder is meant the inability to void the urine voluntarily or to retain it.

According to this definition those cases may not be included in paralysis of the bladder in which purely mechanical factors have interfered with the voiding of the urine or in which the psychic functioning of the hrain has ceased to be active.

Etiology. A myopathic origin may be assumed for transitory paralysis of the Detrusor minae, which is observed occasionally in otherwise healthy animals when they were obliged to retain the urine for a long time on account of protracted labor or because they were confined in a room. In such cases the muscles of the bladder are stretched excessively by the urine which has collected in the bladder in considerable amount and thereby the power of contraction is reduced for a time. A myopathic paresis of the bladder is also accountable for the retention of urine which arises in exceptional cases in inflammatory conditions of the walls of the bladder or in deep-seated inflammations of the contiguous organs, especially the peritoneum. By passing to the muscularis of the bladder, the inflammatory process may diminish the contractility of the muscles. In such cases it is, however, probably more often an occlusion of the first urethral portion or a spasm of the sphincter that causes the retention.

A persistent paralysis of the bladder develops only in certain diseases of the nervous system, especially in injuries affecting the sacral and the posterior lumbar portion of the cord and their nerve roots and nerves. Such affections are: inflammation, contusion, hemorrhage in the conus medullaris, compression of the latter and of the related nerve roots in the anterior portion of the sacral canal, during intervertehral enchondrosis or pachymeningitis ossificans; exceptionally the trouble may occur in consequence of a new-formation or of an abscess. In horses it occur's rather often in inflammation of the cauda equina (combined paralysis of tail and sphincters). A lesion outside of the vertebral canal of the nerves which pass to the bladder has not been observed in animals nor disease of the centers for the bladder muscles in the sympathetic nerves of the pelvic cavity. In diseases of the spinal cord above the posterior lumbar section, paralysis of the bladder is found only in such cases in which the conductivity of the centripetal and centrifugal tracts has been diminished or destroyed. This may take place in contusion, inflammation and compression of the cord.

Pathogenesis. In addition to the ganglion cells within the bladder wall itself the motility of the bladder is influenced by sympathetic, spinal and cerebral centers. As an intimate functional reciprocity develops between these centers in the 
course of development, the disease of one of them or of the related tracts eliminates the function also of the other centers which may have remained healthy. As the spinal centers are connected particularly intimately with the sympathetic centers which immediately influence the motility of the bladder, disturbances in the bladder become striking and persistent, especially in lesions of the spinal centers which lie in the posterior lumbar and anterior sacral section of the spinal cord, the more so as in these cases the activity of the striped muscles of the urinary organs and the reflex action of the sensory nerves upon the urethra and the neighboring organs is necessarily eliminated. In all diseases of the nervous system above the sympathetic bladder centers there occurs more or less improvement in the disturbance, probably owing to the increased activity of the sympathetic centers.

In unconsciousness retention of the urine takes place in such a manner that the pressure of the urine is not felt or that the inhibitory action of the brain upon the sphincter is not exerted. The same mechanism may probably be assumel for the occurrence of that form of retention which is sometimes produced under the influence of certain intense psychic stimulations (excitement); and a similar origin is probably responsible for the retention which is frequently noted in the course of paralytic hemoglobinemia.

Symptoms. In paralysis of the Detrusor urinae the urine fails of being voided for a considerable time (Ischuria, Retentio urinae); owing to the constantly increasing tension of the bladder the animals become restless and attempt to urinate, provided the sensory conductivity is not interrupted as well. In spite of all efforts and of the cooperation of abdominal pressure they void only small quantities of urine in a thin and feeble stream. Soon, however, the sphincter is relaxed by the increasing tension, and dribbling of urine occurs. The bladder is found to be greatly dilated on rectal exploration, or in smaller animals by palpation through the abdominal walls and by percussion; pressure upon the bladder causes large amounts of urine to be discharged which ceases suddenly when the pressure is removed (Ischuria paradoxa). If a catheter is introduced into the bladder, the urine flows out without force.

In complete paralysis of the sphincter the animals cannot retain the urine at all or only for a short time, and in consequence this is discharged at very short intervals, in fact, drop by drop at the commencement of total paralysis, wetting the vicinity of the urethral opening or the pudenda (Incontinentia urinae); in incomplete paralysis small quantities of urine are voided at frequent intervals and without pain.

Usually the paralysis occurs in both muscles simultaneously and involuntary discharge is observed, or dribbling of the urine at first when the bladder is very full, later when it is only moderately filled.

In the further course of the disease disintegration of the urine commences already in the bladder, because bacteria easily enter it through the incompletely closed neck; they multiply 
readily in the dammed-up urine, and in a short time eatarrh of the bladder develops.

Diagnosis. In paralysis of the bladder due to transverse lesions of the spinal cord above the posterior lumbar segment, also in retention of the urine during loss of consciousness or after violent psychic influences, the urine is voided in a strong stream, at long intervals or after external mechanical influences upon the region of the bladder; the discharge of the urine does not cease immediately with the cessation of the pressure upon the bladder, and if a catheter is introduced into the bladder, the urine flows out in a vigorous stream. Inflammatory processes and mechanical impediments in the lower urinary passages can easily be excluded by local examination. Polyuria and frequent micturition might be mistaken for paralysis of the sphincter, but are readily differentiated by considering the other symptoms of the basic affection.

Treatment. In order to prevent the consequences of retention of urine as much as possible, the urine should be removed several times daily with a clean catheter, or the bladder should be made to empty itself by gradually increased pressure. It is well to apply a drying or disinfecting salve to the skin in order to keep the region of the urethra clean. In cases where no severe organic disease exists, the relief of the paralysis may be attempted by cold applications to the region of the sacrum, irrigation of the bladder with cold water, or friction on the region of the bladder. Faradic electricity (the positive pole on the sacrum, the negative on the pudenda in retention, on the perineum in dribbling) is powerless.-Parascandolo believes that certain cases of paralysis of the bladder may be relieved by operative treatment.

Literature. Luciani, Physiologie, 1906. II. 433 (Lit.). - Müller, Deutsche Zeitschr. f. Nervenheilk., 1902. XXV. 86 (Lit. on animal experim.). - Paraseandolo, D. t. W., 1901. 477.

Spasm of the Bladder. This is said to originate without local disease of the urinary tract, when the animals are obliged to retain urine for a long time on account of protracted work (Röll). But psychic influences, colicky pains and tetanus may also produce the trouble. In spite of repeated attempts the animals are not able to void the urine (Ischuria spastica), or at most a few drops are discharged at one time (Dysuria spastica). The bladder is greatly dilated and sensitive to pressure. The history as well as the absence of local pathologic changes (caleulus, swelling, etc.) afford sufficient diagnostic indications.

In horses the spasm usually disappears spontaneously if they are allowed to rest on clean straw or in a sheep barn. In horses and also in other animals sponging of the region of the bladder is indicated, also a gradually increasing pressure upon the bladder. Lukewarm enemas, chloral hydrate (25-50.0 or 1-2.0 gm.) per rectum hasten recovery; also morphine injections. If in spite of these measures no urine is voided, a eatheter covered with morphine ointment may be used, or puncture of the bladder may be required. 


\title{
Diseases of the Circulatory Organs
}

\author{
Section I. \\ DISEASES OF THE PERICARDIUM.
}

1. Pericarditis.

Occurrence. Pericarditis is not a very prevalent disease. It occurs most frequently in cattle in the form of traumatic pericarditis, inflammations of the pericardium from other causes being much more rare, and in all probability usually tuberculous in origin. Still more rarely the disease is observed in horses, in which it usually occurs as a sequel of croupous pneumonia. It occurs a little more often in dogs, usually on a tuberculous basis, less often in distemper or other diseases. In hogs pericarditis is not very rare, appearing most often in swine plague, less often in erysipelas. In fowls the disease is also observed occasionally, usually as a complication of cholera and chicken plague, less often in tuberculosis.

Etiology. The occurrence of pericardial inflammation is due, almost without exception, to the immediate influence of infectious substances, although these often become effective only if certain predisposing conditions are also present. Searching bacteriological examinations have not been made very often with respect to pericarditis in animals, but those that have been reported, and also the mode of onset of the disease, permit the assumption that the infectious substances mentioned below are particularly concerned in the production of pericarditis. The Bac. bipolaris may be active in this manner, not only alone but also in association with other bacteria ; in fact, there is hardly one of the diseases for which this bacterium is responsible that is not occasionally associated with pericarditis. Further, the pyogenic bacteria, the tubercle bacillus, the virus of swine erysipelas, chicken plague, contagious pleuro-pneumonia of cattle and of variola may also take part in the production of pericarditis. 
Jowett observed a pneumo-pericarditis which oceurred in turkeys in epizootic extension and was characterized by a copious fibrinous exudation; a bacillus bipolaris was found, both in the pericardial exudate and in the blood; chickens and pigeons which were kept in the same place remained healthy, but it was possible to infect them artificially. In Jowett's opinion the disease is a different affection from chicken eholera.

The point of entrance of the microorganisms may either be in an injury of the thorax, or more frequently the virus reaches the pericardium by way of the blood and lymph streams. The infection is most easily explained when traumatic influences affect the pericardium, and when pyogenic or putrefactive bacteria enter simultaneously with the injuring object or pass in subsequently through the opening. Penetrating injuries of the heart region are rather rare as a whole, and likewise rarely an effect of contusions of the praecordia is exerted without penetration of the wall of the thorax, so that bacteria which have entered the blood in another manner are carried to the pericardium and then proliferate in the contused tissue.

Those injuries of the pericardium are of greater frequency and of the highest importance in which foreign bodies have entered the pericardim from neighboring organs (Pericarditis traumatica). Pericarditis of such an origin occurs frequently in cattle, less often in goats and sheep and only exceptionally in the other animals. In a case observed by Henniges in a horse a needle, probably from the esophagus, entered the pericardium; in a case of Lewin's, from the stomach or duodenum; in a hog a needle came from an unknown part of the body (Teetz), or from the stomach (Nietschke), while in a dog seen by Petit the foreign body (needle) had probably passed with the blood of the posterior vena cava into the heart and had partially advanced into the pericardium after perforating the wall of the heart. In ruminants foreign bodies which have been swallowed usually pass from the second stomach toward the pericardium (Vol. II). The anterior surface of the reticulum is separated from the pericardium only by the thin diaphragm which is easily perforated even by comparatively short foreign bodies. In this respect needles are most dangerous because, after having perforated the mucosa of the reticulum they are constantly pushed farther by the repeated contractions of the muscles of the reticulum until the points reach the diaphragm; then the contractions of the latter aid in pushing the needles against the pericardium and in finally entering it. Of similar importance are other rigid bodies which are more or less pointed at both ends, especially thin wire (according to Collas parts of the wire which has latterly been used for packing pressed hay are responsible relatively often), nails, knife blades, etc. Together with the foreign bodies bacteria usually enter the pericardium as a matter of course.

The intermediation of infection by the blood- and lymphstreams must be assumed in those cases in which the affection 
develops in association with diseases of neighboring organs or $^{\circ}$ in the course of a generalized acute infectious disease. Aside from inflammations of the heart muscle or the endocardium which easily pass over into the pericardium which is connected with the intramuscular connective tissue, pericarditis occurs preferably as a sequel to pleurisy, pneumonia, pulmonary or pleural tuberculosis and is produced in these cases by the virus of the related diseases. Pericarditis may also develop directly, by continuity, after perforation of the gullet and in consequence of the mediastinitis due to it, also after suppuration of the peribronchial lymph glands, etc. Of the acute infectious diseases it is especially hemorrhagic septicemia, pyosepticemia neonatorum, less often strangles, variola, articular rheumatism, etc., which lead to pericarditis.

The bacillus bipolaris, the pyogenic bacteria and the tubercle bacillus do not necessarily exert their inflammatory action only after a previous affection of other organs or after a generalized infection (secondary or consecutive pericarditis) but may in many cases do so independently (primary pericarditis).

The pericarditis observed in epizootic extension in sheep by Trasbot, Anacker and Kowalewsky was evidently not primary, but a partial manifestation of hemorrhagic septicemia, and as a matter of fact Kowalewsky found, on autopsy, endocarditis and pneumonia in addition to pericarditis.

It is only exceptionally that chemical substances or mechanical irritants give rise to chronic pericarditis, the forner in bacterial diseases of other organs, in nephritis, etc., the latter by new-growths, parasites, sterile foreign bodies. (Borchardt saw serous-hemorrhagic pericarditis with nephritis and hepatitis in two horses which suffered from lysol poisoning.)

In infectious non-traumatic pericarditis certain predisposing causes like cold, over-exertion, railroad transportation, etc., are of importance although they are not capable, of themselves, of giving rise to an inflammation.

Anatomical Changes. In acute pericarditis delicate or thick fibrinous membranes are deposited on one or both layers of the pericardium which may, in larger animals, attain a thickness of 2-3 cm., and a fluid exudate accumulates in varying amounts in the pericardial cavity, which may be clear or very cloudy; it may contain floccules of fibrin and in traumatic pericarditis it has usually a foul odor. If fibrin is secreted copiously, the surface of the heart becomes uneven, villous (villous heart, cor villosum), and the fibrin masses may divide the pericardial cavity into irregular compartments (which are especially well marked in cattle). In many cases the exudation assumes a purulent character and if the inflammation is prolonged a proliferation of connective tissue is more manifest. The latter form of inflammation, and also pericarditis in its 
initial stage, is sometimes limited to portions of the pericardium (pericarditis circumscripta) in which the vicinity of the large ressels is affected preferably, less often that of the apex. In the majority of cases, however, the inflammation involves the entire perieardium almost uniformly ( $P$. diffusa), although also in these cases the visceral layer usually shows more intense changes. In proportion to the amount of exudate the pericardium is more or less distended and sometimes occupies a very considerable portion of the thoracic cavity. The quantity of exudate varies within wide limits and may amount to 36 (Friedberger) and even 40 liters in horses, 181/2 liters (Trasbot) in cattle, and $1 / 2$ liter in dogs. The connective tissue septa of the heart muscle are also infiltrated with sero-cellular exudate; if the amount of exudate is very great, compression and atrophy of the heart may result.

Finally there are evidences of stasis, such as marked fullness of the veins, passive hyperemia of the lungs and of the liver, edematous infiltration, etc.

In traumatic pericarditis of cattle the foreign bodies are not infrequently found in the exudate, either free or with one end penetrating the wall of the heart. It may also occur that the foreign body is not found because it has been pushed back into the reticulum, or because it had rusted and crumbled; in many cases it is entirely in the heart muscle, or exceptionally, in a ventricle. (It is possible for an abscess to form in the heart muscle and break into the pericardial cavity or into one of the ventricles [Cadéac].) A traumatic origin of the inflammation is suggesced by the presence of a firm connective tissue cord between pericardium and diaphragm, and also between the latter and the reticulum. This cord is either solid, or it contains several cavities, either side by side or communicating with each other, and filled with purulent or ichorous fluid; in rare cases a long canal is found in the center of the connective tissue cord, which connects the lumen of the reticulum with the pericardial cavity.

Chronic pericarditis is characterized by connective tissue proliferation leading either to simple thickening of the pericardial layers (tendinous spots, Maculae albidae s. lacteae) or to adhesions between them (P. fibrosa adhaesiva); sometimes, indeed, a complete union of the pericardial layer's results (Concretio pericardii). In tuberculous pericarditis cheesy foci may be found in varying numbers in the new connective tissue which may be very copious, but this form of pericarditis occurs, especially in dogs, also as a serous or hemorrhagic inflammation.

Symptoms. The clinical picture of traumatic pericarditis is usually preceded by prolonged digestive disturbances, which, 
according to Bongartz, last from 1 to 6 weeks; sometimes they lecome very severe and may be associated on motion with sensitiveness to pressure in the region of the reticulum, with superficial respiration and evidences of pain. Later, when the foreign body has reached the pericardium, the symptom complex of pericarditis follows immediately; although exceptions may be noted in so far as the signs of traumatic gastritis may disappear for a time the pericarditis becoming manifest only after their reappearance, or the trouble may develop without previous digestive disturbances. In similar manner a non-traumatic pericarditis of secondary origin is introduced by the symptoms of the basic affection.

The period between the ingestion of the foreign body and the appearance of the pericarlial symptoms depends upon the nature of the foreign hody, the manner in which the animals are kept and fed, and upon accilental factors (parturition, ete.).

In the initial stage of pericarditis the animals manifest severe pain, they avoid all motion, stand with head stretched forward, the elbows spread out, the hind feet drawn under the belly, the back arched, they frequently look back toward the abdomen and at times lie down carefully, only to get up again inmediately. Pressure on the region of the heart, on the chest or on the back; also percussion of the precordia increases the pain considerably; the animals groan, attempt to evade the pressure or depress the back deeply. They dislike to move, are surprisingly fatigued after brief motion, and then show shallow breathing, respiration becoming abdominal in type. The pain increases markedly, especially on going down hill. These symptoms are sometimes so mild as to fail to arouse attention, and the first expert examination, especially in cows which are not worked, often shows already a fairly advanced stage of the disease.

The lieart beat is hastened from the first by $1 / 2,2$ or 3 times the normal rate and may be arhythmical. The increase is sometimes aggravated further without any cause whatever, or through quite insignificant influences. Especially during motion the heart beat shakes the entire region of the heart, and the heart sounds are sometimes heard at a distance of 3 to 4 steps, being unusually clear and loud. (Leconturier describes them with the word "claque-claque"). Sonnenberg" observed bradycardia in a cow (26 heart beats to the minute), together with epileptoid convulsions and loss of consciousness, which ceased during periodical occurrences of increased heart's action (over 100 heart beats).

Friction sounds in the heart regions afford the most valuable symptom. These sounds are synchronous with the motions of the heart, but they do not follow as regularly as the endocardial sounds; they may be heard together with the systole or 
with the diastole, or then immediately before or after. They may be soft or rough, creaking, and always give the impression of friction, or if they are very short and intense they sound as though two liquid or solid bodies were beating against each other. These sounds are often felt with the hand placed on the cardiac region, and mar be increased on pressure upon the intercostal spaces. They are limited to the region of the heart, audible on both sides and are influenced by the respiratory movements at most by becoming less intense during inspiration, becanse then the lung has moved between heart and thorax.

Tn the presence of a fluid exudate, the friction sounds usually disappear entirely if delicate or few pseudo-membranes are formed separating the rough surfaces of the pericardium; in the presence of only small amounts of fluid the friction sounds may persist. If there are very copious masses of fibrin, some portions of the fibrin covered surfaces tonch even in the presence of much fluid and prorluce friction somnds to the end.

In traumatic pericarditis gases and fluid are not infrequently present together, and then splashing, gurgling and similar sounds are heard in addition to the friction somnds, also the sound of falling drops, etc., etc.

The heart beat, which is at first increased in intensity, gradnally becomes weaker and may at times be umnoticeable, but if much exudate is present, wave-like movements may be felt in the cardiac region. With the weakening of the lieart beats the intensity of the heart sounds diminishes and sometimes they disappear entirely.

An increased cardiac dullness may be ascertained only in the presence of much fluid, of very much fibrinons exudate or of tuberculous new-formations. The increase then gradually occurs upwards, forwards and hackwards; upward it mav reach as light as the shoulder line and may, in small animals, reach forward to the second intercostal space, backwards in all animals to the 6th or 7th intercostal spaces (Fig. 181). In the lateral position the upper border moves slightly down, because then the heart lies somewhat further away from the thorax. In ruminants an absolute cardiac dullness is at first noticeable, which later enlarges in the manner indicated. Any dullness on the right side is always less marked than on the left side. In some cases of traumatic pericarditis in which the gases of putrefaction form or in which gases from the reticulum have reached the pericardial cavity, a tympanitic and even a metallic percussion sound of varying extent may be elicited on the upper border of the dullness.

The pulse is accelerated from the beginning; at first fairly full and tense, often arhythmical, later it becomes very small, thread-like, owing to compression and possibly to involvement of the heart, and the arhythmia usually increases.

With the increase of the exudate the reins are constantly filled more strongly, and this is particularly noticeable in the 
jugular veins. The jugular veins, which are dilated like thick cords and stand out strongly, sometimes show undulations which are synchronous with the auricular systole and slightly precerle the heart beat (presystolic venous pulse). (In cattle, and often also in horses, even under normal conditions, undulation and $\mathrm{perh}$ a p a slight presystolic pulsation of the jugular veins may l.e noterl.) At the same time an edematous swe 11 ing levelops on the upper and lower chest and also in the anterior portion of the lower abdomen, further in the region behind the elbow, on the dewlap and in the trachea, where it may appear first, if the head is held low, and may be associater with e llema of the larynx.

Disturlances in respiration exist in all cases, the breathing bei $\mathrm{n} g$ more or less hastened and superficial, and labored on the slightest motion. From time to time a feeble and very painful c o $\mathrm{ugh}$ is heard. With the increase of the edema fluid accumulates also in the body cav-

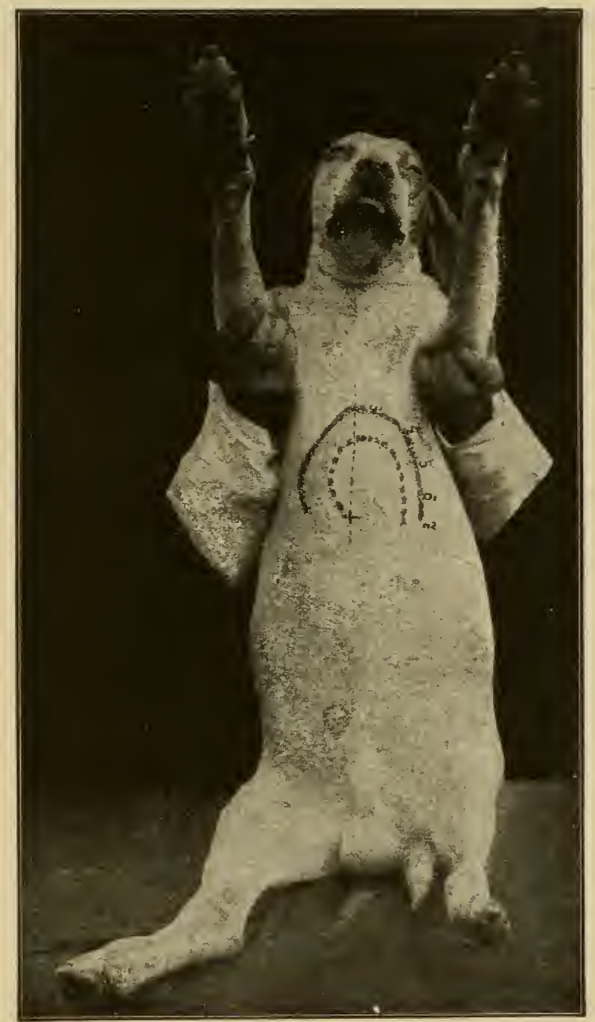

Fig. 181. Increased cardiac dullness in Pericarditis with fluid exudate. The dotted curved line shows the margin of the normal, the solid eurved line the margin of the increased dulluess. The straight dotted line indicates the middle line of the sternum, the figures give the ribs.

\section{ities and inter-}

feres still more with respiration, which is now labored even when the animals are resting, the more so as the symptoms of stasis constantly increase and may even give rise to pneumonia. The temperature is either raised from the beginning or 
a rise occurs in the later course of the disease ; it is not usually high, unless the underlying disease causes a considerable degree of fever. When the temperature rises within the body, chills and trembling are frequently observed and the temperature is unequally distributed over the surface of the body. In cattle traumatic pericarditis may in exceptional cases run an afebrile course.

With the development of the symptoms the general condition of the animals becomes constantly worse. Large animals avoid every movement as much as possible, and stand with neck stretched out straight, they lie down only rarely, very carefully and only for short periods; their eyes liave a plaintive expression; sinaller animals usually lie constantly on the ground. The desire to eat diminishes more and more, so that the patients become emaciated. Defecation is retarded, although in some cases, especially in traumatic pericarditis, cliarrhea occur's.

Chronic pericarditis usually arises out of the acute form, the condition of the animals improving after a certain time, but without complete recovery. It is more rare for the disease to develop insidiously from the commencement (mostly in the presence of tumors, tuberculons swellings, etc.), and in such cases the symptoms of the initial stage, like irregular heart action, rapid fatigue during work, may remain unnoticed for weeks.

The most striking symptoms are dyspnea and rapid fatigne on moving about and still more when working, then a decided increase and arhythmic character of the heart function. Enlargement of the cardiac dullness is either absent or examination gives the same result as in acute pericarditis with effusion. In the latter case the heart heat and heart sounds are weak. Friction sounds are usually absent. Another symptom is found in the fullness of the jugular veins. In the later stages of the disease edema may develop on the lower chest and in the vicinity.

Course and Prognosis. Acute pericarditis usually ends fatally, within the first week, in those cases in which purulent or ichorous exudate has collected in large quantities or in which the affection has occurred in connection with an infectious disease, especially pneumonia, pleurisy or hemorrhagic septicemia. In the majority of cases, however, the disease takes a longer course, 2 to 3 weeks and more, and shows at times transitory improvement. Ultimately, however, the permanent disturbance of the heart function or the degeneration of the heart muscle lead to death even in these cases, the end being preceded by symptoms of stasis, such as venous hyperemia and edema, sometimes hemorrhagic infarets in the lings, chronic catarrh of the respiratory passages and of the intestinal tract, cutaneous edema, thoracic dropsy, ascites, ete. The animals become much emaciated and exhausted and usually perish in this stage with 
symptoms of pulmonary edema. Sometimes death occurs suddenly at a time when the general condition of the animal is still fairly satisfactory. Recoveries are rare in cases which are clinically well marked and usually follow upon a period of convalescence lasting several weeks. However, even in those cases which take a comparatively favorable course, chronic changes remain which influence the heart action unfavorably. In extensive adhesions the heart beat is weak and the thorax is depressed in the region of the heart (Röll).

The course of traumatic pericarditis varies in the several cases, but is usually insidious, extending over weeks and months, and even longer, during which time the symptoms usually increase gradually or with transitory remissions. Eventually digestive disturbances become more and more evident and finally a soporose condition develops, accompanied by paralysis-like debility, probably due to the absorption of putrefactive poisons from the exudate (de Bruin). In many cases metastatic, purulent inflammatory foci form in various organs, for instance in the joints and tendon sheaths, and in such cases the disease may terminate with the clinical picture of pyemia. Other complications are pneumonia and pleurisy, gastric and duodenal catarrh, etc. Sudden death is not a very rare occurrence in any stage of the disease and follows either upon injury or perforation by the foreign body of the heart muscle or of the larger blood vessels of the heart, or through the influence of sapremia. On the other hand, protracted cases are observed occasionally, in which the well-marked symptoms gradually diminish in intensity, the mutrition improves and the animals may even be put to work (Mathis, Jensen). Sometimes spontaneous recovery occurs, the foreign body either breaking through to the outside or returning into the reticulum.

Diagnosis. During the initial stage the symptoms are not sufficiently characteristic to make a positive recognition of pericarditis possible, although the development of an acute periearditis is suggested by the very great sensitiveness of the precordia, and by the pounding, often arhythmic heart beat which is altered decidedly by even comparatively slight external influences. Later the friction sounds or the various splashing sounds, together with the decided enlargement and peculiar form of the eardiac dullness unite to make up a characteristic clinical picture, which is developed further by symptoms of blood stasis and by edema, and which it is almost impossible to mistake. It is, however, not to be forgotten that none of these phenomena are pathognomic in themselves and that some of them may be absent, not only in traumatic pericarditis but also in other inflammations of the pericardium.

For the diagnosis of a commencing traumatic pericarditis the high pulse rate (100-110 a minute) with a normal or at most maximal normal 
temperature (Meyer, Eber) is suggestive. According to Eber the question may be decided even in those rare cases in which the temperature rises more or less during the initial stage, the increased pulse rate remaining unchanged in spite of the lowering of the temperature by the action of antifebrin in doses of 50-100 Gm. per day. According to Otto a peculiar muscular trembling in the vicinity of the elbows is of importance in this respect, which occurs especially after the animal has been standing for some time.

Fibrinous pericarditis might be mistaken for fibrinous pleurisy when this has given rise to the so-called pleuro-pericardial sounds. These friction sounds are purely pleuritic and are characterized by being heard, together with the heart sounds, only during either inspiration or expiration ; moreover, they are heard synchronously with the respiratory movements during the heart-pause. Moreover, pleurisy is rarely limited to so small a space and therefore friction sounds, synchronous with the respiratory movements, are also heard more posteriorly. Still, it must not be forgotten that pleurisy and pericarditis may occasionally be present at the same time.-In rheumatism of the intercostal muscles the intercostal spaces are painful, but friction sounds are absent or at most dull muscular sounds are audible. If a fibrinous pleurisy should also be present, as is the case occasionally in the so-called pleurodynia of horses, the nature of the disease will be sufficiently cleared up by the history of the case (Vol. II.).-From the endocardial sounds in valvular disease the pericardial friction sounds are differentiated especially by the fact that they seem to develop immediately under the ear, are sometimes increased by pressure and become weaker on inspiration; finally by the fact that they are not quite synchronous with the heart movements.-At the very beginning general acute infectious diseases may have to be considered, in ruminants especially anthrax, but in such cases the temperature is usually decisive, since in pericarditis the temperature rises are usually slight unless the disease has developed upon a septicemic basis.

Pericarditis with effusion might give rise to confusion with pneumonia in those cases in which only the lower sections of the lungs, in the neighborhood of the heart, have become condensed and may therefore simulate an increased cardiac dullness. In such cases the character of the heart beat and of the heart sounds affords information because in pneumonia they are not weakened at all or only slightly so, in contrast to pericarditis with effusion.-In cattle, lung plague may canse a clinical picture which is somewhat like that of traumatic pericarditis, but in most cases sufficient points of difference are present, for instance, the preceding digestive disturbances, the comparatively small dullness, the striking weakness of the heart-beat, the complete absence of bronchial respiratory sounds, the characteristic pericardial sounds and slight fever or its absence.-In peri- 
tonitis with effusion an enlarged area of cardiac dullness may be simulated by an encapsulated exudation in the vicinity of the heart and, in larger animals, especially in cattle, by free fluid exudation. If free exudation is present in the thoracic cavity, the heart beat and the heart sounds are not weakened or only slightly, especially in smaller animals and in the sitting position; moreover, the dullness is limited horizontally.-An encapsulated pleural exudate or a swelling in the vicinity of the heart may be eliminated in sucl cases in which the heart has been displaced (see compression of the heart). A dullness due to enlargement of the heart (hypertrophy, dilatation) may have a similar shape, but hardly ever extends so far forward, the heart sounds are heard distinctly, friction sounds and sensitiveness of the precordia are absent.

About the nature of the exudate external conditions may offer information in so far as pericarditis occurring after cold, in consequence of croupous pneumonia, or of primary pleurisy leads to the formation of a fibrinous or sero-fibrinous exudate, while metastatic pericarditis is usually purulent and accompanied by high fever. In traumatic pericarditis of cattle the exudate may be sero-fibrinous, purulent or ichorous. The assumption of an ichorous pericarditis is justified by the greater severity of the general symptoms, by the tympanitic and possibly metallic nature of the percussion sound and by the presence of splashing sounds. Traumatic pericarditis is usually manifested by local and venous symptoms similar to those of the rather infrequent tuberculous pericarditis of cattle (Kläber, Liénaux, Wyssmann and others).

In doubtful cases Gmeiner recommends a test puncture of the pericardium, on the left side within the area of dullness, if necessary also on the right side.

Chronic pericarditis is very difficult of recognition without an exact knowledge of the history, because most of the symptoms are observed also in chronic pleurisy and in chronic interstitial pneumonia. Only an absolutely exact determination of the shape of the dull area, the nature of the heart beat and the characteristic symptoms of stasis may be of assistance. Chronic myocarditis cannot be differentiated with certainty from chronic fibrinous pericarditis.

Treatment. If treatment is justified by the fact that the prognosis is not absolutely unfavorable, complete rest must above all be assured. Any disturbance in defecation is to be relieved by small doses of mild laxatives, and the diet must be regulated appropriately. Herbivora should receive good hay, if possible mixed with fresh green feed, also flour- or bran-slop; carnivora are fed with gruel or broths, also milk. In persistent inappetence artificial feeding (Vol. II.) may become desirable.

The inflammatory process may be alleviated by cold compresses. A sac filled with snow or ice and fastened with a wide 
belt, or a compress made of tow or cotton, is applied to the region of the heart and cold water poured over it from time 10 time; large animals may be put under the shower. In mimary pericarditis preparations of salicylic acid may be tried in similar doses as in pneumonia ( ${ }^{\top}$ ol. II.) or in somewhat smaller ones, because their harmful action upon the heart is here more easily exerted. Lactophenin, salicylic acid, antifebrin, antiprrin and salipyrin may be administered in very high fever.

$\dot{\mathrm{F} O 1}$ weakness of the heart, cardiac remedies are indicated, digitalis (in large animals $2-4 \mathrm{gm}$. daily, in small animals, of a $2: 150.0$ infusion to which $5 \mathrm{gm}$. of potassium nitrate have been added, one tablespoonful every two hours). Caffeine is also of service (2-5.0 gm. or 0.2-1.0 gm. subcutaneously), camphor (camphorated oil 20-30 gm. or 2-3 gm. subcutaneously), possibly wine, alcohol, or ether.

To promote absorption diuretic remedies and mild laxatives may be tried. In excessive accumulation of fluid, with consequent compression of the heart, puncture of the pericardium should be considered. But in these cases puncture is not as harmless a proceeding as in pleurisy because a portion of the exudate may flow into the pleural cavity through the opening in the pericardium, after the trocar has been removed (Moussu, Gmeiner, author's case).

Puncture of the pericardium is done with a simple trocar immediately above the parasternal line, in the 5 th or 6 th intercostal space, but always within the area of dullness; after removal of the exudate the pericardial cavity nay be irrigated with lukewarm sterilized water or with a mild disinfecting solution. If the exudate is purulent it seems more advisable to open the pericardium by an incision and to wash out the cavity thoroughly. During this operation it may be possible to find and remove the foreign body (Bastin).

In traumatic pericarditis Meyer's operation may also be tried (Vol. II.). If this is not followed by improvement or if the disease is already far progressed slaughtering of the patients should not be delayed, because later on the flesh will become useless for human consumption on account of emaciation or sapremia or of general pyemic infection. In order to remove edematous infiltration and thus make the flesh fit for consumption Moussu recommends the removal of the exudate through a puncture from the direction of the xiphoid cartilage (Fig. 182). This procedure merits consideration also in non-traumatic pericarditis of cattle.

With the animal standing, the positions of the xiphoid cartilage, the linea alba and the left costal arch are ascertained. In the middle of the angle outlined by these landmarks, above $20 \mathrm{~cm}$. in front of the place where the milk vein runs toward the V. epigastrica cranialis, the abdominal wall is pierced, an incision is made through the skin about $20 \mathrm{~cm}$. long, parallel to the costal arch, through which a portion of the serous fluid which has accumulated in the subcutaneous connective tissue will escape promptly. The muscles in the bottom of the wound are then separated with the scalpel as far as the neck of the xiphoid cartilage, the subpericardial fatty connective tissue is forced apart with one or two fingers of the right hand until the pericardium is reached, as is shown by feeling the heart beats. The left index finger is now pushed up to the pericardium, and 
upon it a trocar, at least $2.5 \mathrm{~cm}$. long and $5 \mathrm{~mm}$. thick is earried forward with the right hand until the heart beat is communicated to it. The trocar is now pointed inward and forward and is forced with a sharp thrust about $3.4 \mathrm{~cm}$. into the pericardium, the fluid being allowerl to escape by remoring the stylet. A strip of iodoform gauze is placed into the wound, a compressing dressing is applied to the chest and renewed from time to time. The edema disappears, in the course of a few days, for a time, when the animal may be slaughtered.

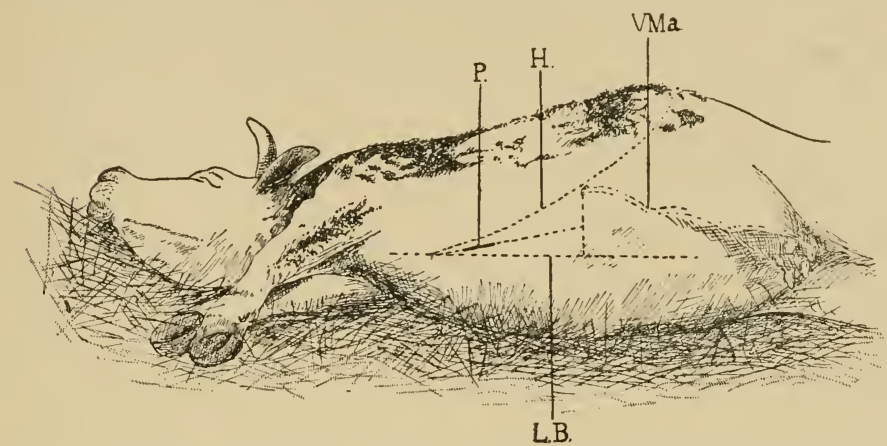

Fig. 182. Firld of operation for puneture of the pericardium from the xiphoid cartilige. $L B$ linea alba; $H$ costal areh: $T M a$ milkvein; $P$ point of incision. (After Moussu.)

Liénaux calls attention to the fact that in the presence of allhesions the operation fails just at this point of punction, or that the trocar may enter the rentricle, in which ca:e a stream of llood will escape through the trocar. This accident appear's however to be of no importance.

In order to prevent possible complications (phlegmons, malignant edema), Liénaux drains the pericardium, after punction, by introducing a rubber drain with lateral openings through the canula into the pericardial sac and securing it with sutures after removal of the canula. In a case treated in this manner the exulate disappeared permanently so that the animal could be fattened.

Chronic pericarditis can liardly be treated successfully. At best the animals must be saved from exertion and receive cardiac remedies. Animals which are intended for slaughter slould be killed as early as possible.

Literature. Albrecht, W. f. Tk., 1893. 259. - Aubry, J. vét., 1905. 355. Bragadin, T. Z., 1964. 420. - de Bruin, Monh., 1900. XI. 1 (Lit. on traumat. Pericarditis). - Döttl, W. f. Tk., 1908, 227. — Eber, Z. f. Tm., 1906. X. 321. Eisenmann, Monh., 1906. XVII. 17. - Gmeiner, B. t. W., 1906. 409. - Henniges, Mag., 184S. 514. - Jensen, Maanedsskr., 1905. XVIII. 35. - Jowett, J. of comp. Path., 1908. XXI. 324. - Kläber, B. t. W., 1901. 731. - Koppitz, T. Z., 1906. 537. - Leblanc, J. vét., 1905. 78. - Lewin, Z. f. Vk., 1894. 18. - Liénanx, Ann., 1905. 314; 1908. 311. - Mathis, J. rét,, 1904. 277. - Moussu, Rec., 1901. 465; Bull., 1905. 296. - Noack, S. B., 18s9. 74; 1890. 81. - Otto, ibid., 1899. 91. - Petit, Bull., 1901. 264; 1905. 281. - Schmidt, P. Mt., 1871-72. 162. Sonnenkerg, B. t. W., 1909. 171. - Teetz, Z. f. Flhyg., 1904. XIV. 61. - Wyssmann, B. t. W., 1905. 387 (Lit. on tuberk Pericarditis of cattle).

Compression of the heart (so-called pseudopericarditis). Aside from the compression of the heart which is produced by a copious pericardial exudate, the organ may be crowded or displaced by formations outside of the pericardium; this occurs especially in cattle, but some- 
times also in other domestic animals. Abscesses (mostly traumatic in origin) develop with preference subpleurally or subpericardially, and new-formations or the somewhat more frequent tuberculous swellings are found at the base of the heart or in the anterior mediastinum. Only rarely abdominal organs (especially the reticulum) compress the heart by prolapsing into the thoracic eavity; in other cases, which are also very rare, a pulmonary abscess or a very large echinococcus of the lung exerts pressure upon the near-by heart. If such formations are present, the diastolic dilation of the heart is more or less interfered with and at the same time the heart muscle is made to atrophy (Atrophia cordis). The large vascular trunks are often compressed at the same time, or they may be affected exclusively.

The clinical picture shows great similarity with that of pericarditis: especially similar symptoms of stasis are to be noted. On the other hand, the pulse rate is only slightly increased, intermissions are frequent and a displacement of the heart to the right, forward, backward or upward may he demonstrated by the character of the heart beat or of the heart sounds; pericardial sounds are absent. In certain cases the dullness is much larger than is usual in pericarditis. Sometimes an exploratory puncture affords valuable indications for diagnosis.

Treatment need he considered only in the case of subplenral or subpericardial abscesses or of cystic new-formations, in order to remove the fluid contents.

Literature. Bretagnier, Ree., 1909. 156. - Huynen, Ann., 1907. 144. Joest, Dresd. B., 1906. - Liénaux, Ann., 1905. 1, 121, 473. - Mathis \& Ball, J. vét., 1905. 653. - Moussu, Rec., 1903. 757. - Petit \& Delacroix, Bull., 1905. 133. - Seegert, Z. f. Vk., 1901. 126.

\section{Dropsy of the Pericardium. Hydrops Pericardii.}

\section{(Hydropericardium.)}

Etiology. With the exception of inflammatory processes, which do not belong here, an accumulation of serous fluid occurs in the pericardium either from stasis of the blood in chronic diseases of the heart muscle or of the valves, or in chronic pulmonary affections, and also in connection with hydremia. On a similar foundation dropsy of the pericardium develops in chronic inflammations of the kidneys, in chronic glander's, chronic anemia, in liver-fluke disease, lungworm disease, ete. The diseases which have been enumerated usually exert their effects also upon the veins of other parts of the body, and therefore symptoms of hydrops are in these cases observed elsewhere also.

Anatomical Changes. The pericardium of healtlyy animals contains at most a few cubic centimeters of a serous fluid, but in dropsy of the pericardium the quantity may amount to $100-200$ times the normal. The liquid is clear or slightly turbid (because of the admixture of desquamated and degenerated epithelial cells), it is thin fluid, sometimes bloody; the specific gravity is 
less than 1016, the content of albumen less than 3\%. Other changes are present which point to the underlying condition.

Symptoms. Dropsy of the pericardium is always observed in animals which have presented symptoms of the basic disease for some time. Aside from these the most important symptoms are an enlarged area of cardiac dullness of similar shape and extension as in pericarditis with effusion, feeble and dull heart sounds. If dropsy is present also in the thorax, the enlarged area of cardiac dullness is in small animals easily deternined while they are sitting or lying on the back, because then only the dullness due to the fluid accumulated in the pericardial cavity remains.

Symptoms of stasis, respiratory disturbances, albuminuria, feeble and small pulse beats also become evident, but these symptoms are largely due to the basic condition itself which indeed is of deciding influence upon the course and prognosis.

Diagnosis. Dropsy of the pericardium differs from pericarditis with fluid exudate by the absence of acute symptoms (pain, ferer) and of friction sounds, and by the presence of manifestations of hydrops elsewhere.

Treatment. As hydrops of the pericardium only represents a phenomenon of other affections, the treatment must be adapted to the nature of the underlying condition. It is sometimes possible to diminish the pericardial fluid by the systematic arlministration of diuretic and diaphoretic remedies and of laxatives. If the accumulation of fluid is excessive punction of the pericardium may be attempted.

Accumulation of Blood in the Pericardium (Haemopericardium). Hemorrhage into the pericardial cavity may occur from the cavities of the heart, from the blood vessels of the heart muscle or from the larger vessel trunks. As immediate causes of bleeding may be mentioned trauma or spontaneous rupture of the heart, rupture of the dilated coronary artery or of a large vessel trunk, especially of the aorta, if this is dilated above the valves. Finally bleeding may occur exceptionally in diseases with hemorrhagic diathesis.

Hemopericardium can only be recognized with a certain degree of probability in cases in which the bleeding is slow, because in rapid hemorrhage death occurs as soon as in $1 / 2-1$ hour. The diagnosis is based upon the demonstration of fluid in the pericardium (p. 1042), the symptoms of internal hemorrhage and the discovery of some disease which is known to be able to lead to hemorrhage in the pericardium.

\section{Gas in the Pericardium. Pneumopericardium.}

Etiology. An accumulation of gas in the pericardium occur's usually only in cattle, when a foreign body penetrating into the pericardium from the reticulum has formed a canal 
through which gases may pass from the reticulum into the pericardial cavity (see p. 1042); the same occurs in ichorous pericarditis in which putrefactive gases are formed. Atmospheric air enters the pericardium only in very exceptional cases, for instance after penetrating wounds of the pericardium by external agencies, by fractured ribs, by breaking down of a lung eavity which had been artherent to the pericardium.

Symptoms. An intense tympanitic percussion sound is noticeable in the region of the heart, over an area which is sharply limited at the periphery and corresponds to the cardiac area; this sound is sometimes metallic in character. In pure pneumopericardium the heart sounds are high and metallic, owing to the resonance of the pericardium. In most eases, however, the pericardium contains fluid exudate as well as gases, and this gives rise to loud splashing, elacking noises which are sometimes audible from a distance of several steps, and which are often compared with the noise made by a millwheel (bruit de moulin).

The elinical picture is supplemented by the more or less marked symptoms of pericarditis (p. 1043) which is usually present, or the signs of gas aceumulation appear cnly in a certain stage, for instance, at the beginning or toward the end of pericarditis. In making a diagnosis it should be remembered that a tympanitic sound in the region of the heart may also be produced by infiltration of the lung portions in the vicinity of the heart or by diaphragmatic hernia (portions of intestine in the pericardium [Pettit]).

Prognosis. Pneumocardium is always of very unfavorabie significance and a chance for recovery exists only in those rare cases of injury to the pericardium in which infection failed to occur.

Treatment. The same treatment is here indicated as in acute pericarditis, but the necessity for operation is greater.

Literature. Petit, Rec., 1902. 679 (see also Lit. on pericarditis). 
Section II.

\section{DISEASES OF THE HEART.}

\section{Palpitatio Cordis.}

\section{(Tachycardia, Hyperkinesis cordis.)}

A purely nervous palpitation which is independent of organic heart diseases appear's in domestic animals only very rarely, but it has been observed preferably in highbred and very vivacious horses and dogs, also once, by Brédo, in a fouryear-old cow.

The greater portion of eases reported under this name in veterinary literature must undoubtedly be classed with spasm of the diaphragm or with organic heart diseases. Older authors commonly considered spasm of the diaphragm as palpitation, and more recently organic heart diseases which, especially after external influences, also may give rise to similar spasmodic attacks, have not been duly considered in the interpretation of symptoms.

Etiology. An abnormally increased action of the normal heart muscle may be caused either by an excessive irritation or by an increased irritability of the N. sympathicus or by paralysis of the N. vagus. The first mode enters into consideration in those cases in which palpitation occurs after violent psychic irritation, after fright, in case of fire, on falling, after railroad transportation, or from over-exertion; similar conditions may however also lead to acute cardiac dilatation or to acute heart weakness. A congenital morbid irritability of the nervous system in nutritive disturbances, in hemorrhages, in Basedow's disease, must in certain cases also be brought into causal relation with palpitation. Then inflammation or degeneration of the $N$. vagus (in chronic lead poisoning) usually leads to tachycardia. Finally palpitation sometimes persists after influenza (Pr. Mil. Vb.).

Martius considers palpitation as a purely secondary phenomenon which is always based upon a paroxysmal acute heart dilatation, i. e., an organic heart disease. 
Symptoms. The entire behavior of the animals, especially their distressed expression, reveals great anxiety, the animals tremble, horses and cattle perspire. In the heart region thrills are seen which are synchronous with the heart beat and which are sometimes transmitted to the entire trunk and to the vertebral column. The heart beat is stronger, as a rule much accelerated and arhythmical in so far as a number of rapid lieart beats alternate with several which are less rapid, or as brief intermissions occur. The heart sounds are very strong and are sometimes audible from a distance of several steps. At the same time the pulse is usually feeble. These symptoms permit the assumption that a heart weakness was present in the cases in question, and this assumption is strengthened by other signs mentioned by several authors, such as intermittent pulse, systolic murmurs, greater fullness and pulsation of the jugular vein.

Diagnosis. The assumption of nervous palpitation presupposes the absence of any organic disease of heart or blood vessels which could produce similar disturbances in the heart function. Severe acute general diseases must also be eliminated.

Course. Palpitation always lasts only a short time, a few hours, or at most one-half to one day, after which the attack disappears either rapidly or quite gradually. In horses that are otherwise healthy, further attacks usually occur only if the external influences are repeated, but anemic and otherwise predisposed animals may have attacks in rapid succession. The tachycardia which is due to paralysis of the vagus is usually permanent, but as a rule it is followed in a few days by a foreign-body-pneumonia (vagus pneumonia).

Treatment. An attack of true tachycardia usually passes over without any treatment after complete rest is secured and the diet regulated. In nervous excitement the following drugs may be administered: morphine (for horses $0.30-0.50 \mathrm{gm}$., for dogs 0.02-0.05 gm. subcutaneously), chloral hydrate (20-40.0 or 1-2.0 gm. dissolved in lukewarm water, per os or per anum), possibly potassium bromide $(10-15.0 \mathrm{gm}$. or $1-2.0 \mathrm{gm}$. in the drinking water). Other narcotics may be given successfully. Cardiotonic remedies should be administered if cardiac weakness is present.

Literature. Brédo, Ann., 1897. 31. - Kroon, Holl. Z., 1899. 388. - Martius, Tachy-Kardie, 1895; Ergeln. d. Path., 1894. 1. 2. Abt., 59 (Lit. on tachycardia in general). - Nordheim, Z. f. Vk., 1890. 275. - Pr. Mil. Vl., 1900. 147. S.chmitt, B. t. W., 1897. 590.

\section{Slow Heart Action. Bradycardia.}

Slowing of the heart action depends upon a functional disturbance in the heart muscle without any demonstrable organic changes. 
Etiology. The cause of a decided slowing of the heart beat lies either in an excessive stimulation of the vagus or in certain affections of the heart muscle.

An excessive stimulation of the $\mathrm{N}$. vagus may follow upon a bilateral compression of this nerve (in the thorax cavity, in the medulla oblongata), it may be due to slight inflammation, to compression of the vagus center in certain brain affections, and finally, it may be a reflex effect from the stomach or intestine, from the peritoneum or from the skin (Albrecht). Certain chemical substances (e. g., tannates) are also capable of irritating the vagus. Certain diseases of the heart muscle frequently cause bradycardia, for instance, degeneration of the muscle in acute infectious diseases, chronic myocarditis, atrophy of the heart, affections of the coronary arteries, finally insufficient mutrition of the heart muscle in inanition.

From what has been said it is readily seen that bradycardia indeed oceurs as an accompaniment of various diseases, but that it must, nevertheless, be considered separately because in many cases a cause cannot be ascertained.

Symptoms. In horses a reduction in the number of heart beats has been observed to as few as 9 (Zimmermann), in most cases only to 12-20 (Dieckerhoff, Nordheim, Vogel, Zschokke), in dogs to 20-18 (Fröhner, Albrecht). The heart beat remains vigorous and the heart sounds are clear. The animals are apparently quite well; only in severe cases they show, aside from the symptoms of the underlying disease, languor, somnolence, lassitude, attacks of heart spasms or of heart weakness.

Diagnosis. Bradycardia is easily recognized and the cause may often be ascertained. It is possible to determine by subcutaneous injections of atropine (for large animals 0.02-0.05; for dogs 0.005-0.008 gm.) whether the cause lies in the vagus or in the heart, because bradyeardia which is dne to an excessive stimulation of the vagus disappears for a time after the administration of atropine. Symptoms of heart weakness, which may become manifest only on motion, point to an organic disease of the heart.

Treatment. Nerve stimulants are indicated in all cases, such as injections of atropine, alcohol, ether, also sponging, and the relief of any digestive disturbances that may be present.

Literature. Albrecht, W. f. Tk., 1895. 225 (Lit.). — Fröhner, Monh., 1891. II. 548. - Gutbrod, W. f. Tk., 1901. 493. - Nordheim, Z. f. Vk., 1890. 275. Sonnenberg, B. t. W., 1909. 171. - Vogel, Rep., 1888. 1. - Zimmermann, O. M., 1900. 289.

\section{Intermittent Heart Action. Actio Cordis Intermittens.}

\section{(Arhythmia cordis.)}

Intermittent heart action is a disturbance of the heart beats which may be produced by the total omission of one or several ventricular contractions or by additional systolic contractions.

Vol. $1-67$ 
Etiology. The omission of ventricular contractions occurs frequently in horses as a sequel of acute infectious diseases especially in the course, or during the convalescence, of influenza; in other animals it is hardly ever observed. As the actual cause a reflex or immediate excessive stimulation of the $\Lambda$. vagus may be assumed, since in the cases observed by the author the omissions conld always be relieved by atropine, which is known to paralyze the ragus endings. The stimulation of the vagus becomes manifest by the intermediation of the central nervous system when the omissions occur in brain affections, for instance, in case of increased intracranial pressure (chronic Hydrocephalus intermus, brain tumors, inflammation of the brain coverings, etc.). Digestive disturbances (enteric catarrl, obstipation) also sometimes give rise to transitory intermissions which are probably produced by (hemical substances that have been absorbed from the intestine, in such a manner that the poisons irritate the $\mathrm{N}$. vagus or its center either directly or reflexly. The ventricular systole may finally he omitted in certain cases in comnection with diseases of the heart muscle or of its nervous apparatus, for instance, in acute inflammatory processes in the heart muscle, in the pericardium or in the endocardium.

Intermissions of the heart beat which are produced by additional systolic contractions oceur in all animal species and always depend upon an abnormal stimulation of the heart. The abnormal stimulation consists either in an increased diastolic pressure in any one portion of the heart (in heart weakness), or it develops in acute inflammatory processes in the heart muscle, in the pericardium or endocardium. Sometimes poisons which have been absorbed in the intestines exert an abnormal stimulation upon the heart.

Pathogenesis. The physiological irritability or conductivity of the lieart is reduced by excessive stimulation of the N. vagus, and this occurs first on the points of transition between the auricles and the ventricles, where the compartments of the heart are connected only by small muscular bundles. In consequence single contractions of the heart muscle omit, according to the degree of disturbance either only in the ventricles or also in the auricles. The same occurs in diseases of the heart.

Inder the influence of an abnormal stimulation at the time when the respective heart segments are usually in diastole, an unusual contraction, an additional systole occurs following immediately upon a normal systole but preventing the occurrence of the next normal systole by reducing the irritability of the heart for a time. The sooner the additional systole follows upon the immediately preceding normal contraction, the less complete are the filling and contraction of the heart, the aortic pressure being as yet lowered but slightly, and in con- 
sequence the arterial pulse omits in spite of the arditional contraction of the heart.

Symptoms. In omission of the ventricular systole a pause corresponding to one heart beat can often be observed after every 2 to 5 heart beats. The intermissions are, however, often

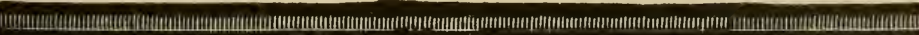

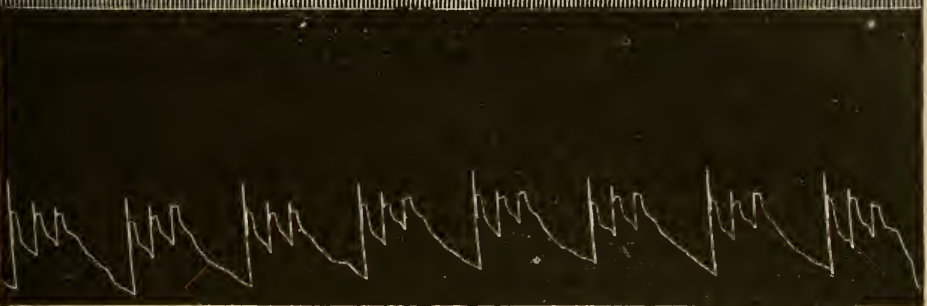

Fig. 183. Intermittent heart action in the horse. Sphygmograph of the abdominal aorta. Intermission of two successive pulse beats (Time indicated in $0.2^{\prime \prime}$ ).

much less frequent, only after every 10 to 15 heart beats, and occasionally no regularity can be observer. Usually only one heart beat is omitted, but there are cases in which 2 to 4 successive heart beats are missing (Fig. 183). In many cases a very feeble, dull, short heart sound is heard in the anterior

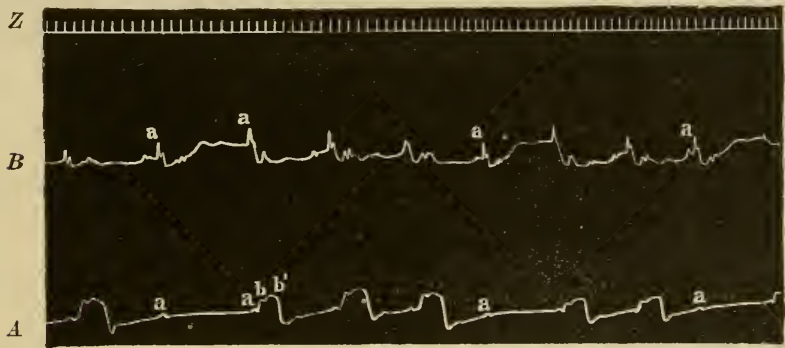

Fig. 184. Intermittent heart aetion and eontractions of the rentrieles, ocemring at different times, in a horse. I Cardiograpl, the small apices a correspond to the auricular contractions, the larger $b b^{\prime}$ to the ventricular contractions. The auricular apex persists during omission of ventricular systole. It corresponds with " of the Phlebigraph $B$ of the jugular vein (negative venous pulse). After each omission of the ventricular contraction the pressure in the rein rose, becanse the vein was not emptied. ( $Z$ time in $0.2^{\prime \prime}$.)

portions of the heart, which corresponds to the persisting auricular contractions, while the vigorous systolic heart sound which depends upon a contraction of the ventricles is omitted. The occurrence of the auricular contraction may be assumed positively because the negative venous pulse at the entrance 
of the chest can be felt even during the pause (Fig. 184). The heart beat following upon the pause is usually stronger, while the subsequent beats are either normal in force, or evenly weakened, or then gradually diminish in intensity. The pulse behaves similarly to the heart beat (true arhythmia of Caroni and Cadiot). During motion the number of heart beats is increased, the abnormal rhythm being usually preserved, although sometimes it changes in the same animal from time to time, so that the pauses occur at shorter, and again at longer intervals.

Extrasystolic arhythmia is recognized by the rapid succession of two or several heart beats, which are separated from the other, normal, heart beats by a pause, so that bigeminous, trigeminous, etc., heart beats are noted. The pulse beat corresponding with the additional systole is usually absent; only when this systole is separated from the preceding one by a longer interval, or if it occurs frequently a rather feeble pulse may be felt (Fig. 185). During motion the arhythmia becomes

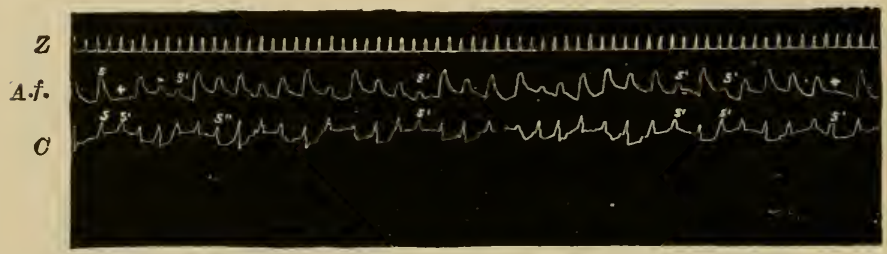

Fig. 185. Arhythmia of the heart due to additional systole, in a dog with cardiac dilatation and inability to close the bicuspid valve. $c$ Curve of heart beats. A.f. Sphygmograph of the Art. femoralis, $Z$ time in $1 / 5$ seconds; $s$ normal ventricular systole, the pulse is distinctly felt; $(s)$ in the sphygmograph; $s^{\prime}$ additional systole, to which at + in the sphygmograph not a beat corresponds, but at $s^{\prime}$ a nimimal rise; $s^{\prime}-s^{\prime \prime}$ crowded additional systoles, to which in spliygmograph elevations correspond which are in part minimal, are hardly or not at all to be felt.

still more striking, or if it had not been observed at rest it then becomes manifest. It may often be relieved temporarily by cardiotonics.

Course. If the intermission of the heart action occurs in the course of an acute disease, it disappears together with its removal; but if in the meanwhile lasting changes have developed in the heart, the arhythmia persists without interfering, as a rule, with the health of the animal. In chronic heart affections, however, the arhythmia disappears at most for a certain time and the animals show signs of being brokenwinded.

Treatment. Aside from the treatment of the basic disease, animals which show a considerable degree of arhythmia should 
be worked only with great eare. If the arhythmia depends upon organic diseases of the heart muscle, cardiac remedies are sometimes of value.

Literature. Cadiot, Bull., 1894. 188. - Caroni, Rec., 1894. 607. - Hering, D. m. W., 1906. 929. - Siedamgrotzky, S. B., 1886. 20. - Wenckebach, Die Arhythmie d. Herzens, 1903.

\section{Hypertrophy of the Heart. Hypertrophia Cordis.}

Hypertrophy of the heart consists in an increase in the heart muscle which arises from thickening and possibly from multiplication of the muscular fibers under the influence of an increase in function persisting for at least four weeks.

Etiology. During severe work the normal, or usually increased blood is forced against the normal or increased resistance at greatly shortened intervals, and if this is repeated time and again it is followed by hypertrophy of the heart muscle. In horses used for rapid motion, in hunting dogs, and exceptionally in pack-horses and draught-oxen, the relatively great size of the heart stands in relation to the increased muscular exertion. A comparatively large heart may be said to be a constant finding in some species of these animals, and it is not improbable that heredity may be of influence upon the dimensions of the heart. This physiological hypertrophy is always in proportion to the development of the muscles of the body and does not require any treatment.

On the other hand a pathological hypertrophy of the heart does not stand in direct relation to the development of the skeletal muscles or is limited to some portions of the heart. In persistent disturbances of the circulation the part of the heart which is connected with the place of the disturbance is forced to continuously increased exertion and this is eventually followed by hypertrophy of this portion of the heart; in diseases of the left venous ostium, however, the right ventricle also hypertrophies owing to increased work done. Pathological processes in the heart muscle itself, such as chronic myocarditis, tumors, echinococcic cysts, etc., also lead to an increase in the muscular tissue in those parts of the heart which are not immediately affected. A similar result is produced by adhesions of the layers of the pericardium because it necessitates an increased amount of work on the part of the heart. Acute cardiac dilatation may also form a basis for the development of hypertrophy if the heart muscle is comparatively unimpaired. (In chronic myocarditis the hypertrophy may at times be only apparent being simulated by the fact that the wall of some portion of the heart has become thicker owing to cellular infiltration or to simultaneous new-formation of connective tissue [E. Albrecht]). Finally hypertrophy of the heart often de- 
velops in older dogs in consequence of a chronic, especially interstitial, inflammation of the kidneys.

Senator sces the cause of nephritic hypertrophy, which nsually occurs in the left heart, less often in both halves of the heart, in a narrowing of the vessels which is produced by irritating substances retained in the blood, anl also in an irritation of the heart muscle by these same sulstances. In certain cases, which may le not a few, the enlargement of the heart probably occurs through the influence of the causes which are responsible for the inflammation in the kidneys.

The secondary hypertrophy of the heart which occurs in connection with chronic diseases of the heart, lungs, arteries or kirmeys is a phenomenon of these diseases and will therefore be considered in connection with them. Here only the so called idiopathic hypertrophy of the heart is to be discussed which arises from an acnte cardiac dilatation or is possibly produced by irritating substances that are not known exactly.

Many contributions on idiopathic hypertrophy of the heart may be found in reterinary literature; on the basis of their clinieal symptoms, however, most of them must be classed rather with chronic myocarditis or with cardiac dilatation.

Anatomical Changes. The determination of cardiac hypertrophy requires an exact knowledge of the physiological dimensions, the relations between heart and body weight and a consideration of the size of the respective heart cavities.

The relation of the weight of the heart to the weight of the living body in the horse is, according to Franck \& Martin, $1 \%$ on an average; according to Frey, $1.28 \%$; accorling to Schubert, $0.84 \%$; in cattle, according to Schmalz, $0.33-0.42 \%$; accorling to Schmeider, in the steer $0.442 \%$, in the ox $0.414 \%$, in the cow $0.492 \%$; according to Schubert, in the steer $0.436 \%$, in the ox $0.42 \%$, in the cow $0.52 \%$, and in calves $0.708 \%$; in the dog, according to Schubert, $1 \%$ of the body weight.

During moderate contractions the hypertrophy of the muscle may be noticeable even on external inspection, especially if it is only partial. The form of the heart is changed thus that with an increased thickness of the left ventricular wall the heart as a whole is slender, elongated, like a pointed cone, while in hypertrophy of the right ventricular wall the heart as a whole becomes broader and more flat, the right heart being involved in the formation of the apex; indeed, if the hypertrophy is very great, the right ventricle reaches beyond the apex of the left ventricle. The thickened muscle is usually firm and like rubber. The weight of the heart is also increased and may in well marked cases amount to twice the normal weight.

The customary classification of simple hypertrophy (walls thickened, cavity of normal width), concentric hypertrophy (walls thickened, carity smaller) and eccentric hypertrophy (walls thickened, cavity dilated) is well adapted for a concise description of the findings, but it has no particular significance, except that the last form indicates a considerable insufficiency of the previously hypertrophied heart muscle. 
Symptoms. Physiological hypertrophy of the heart does not give rise to any morbid symptoms. The symptoms of hypertrophy which develop in connection with organic heart diseases or arterial affections will be considered in connection with these diseases.

In the remaining eases the hypertrophy is characterized by an enlarged cardiac dullness, increased force of the heart lieat, accentuated diastolic heart sound and by a strong and tense pulse.

If the energy of the heart contractions is diminished later on, the symptoms of cardiac insufficiency appear which correspond with those of dilatation (p. 1064). At first they usually oceur only on motion, but may later be observed also at rest. There are then noticeable languor, feeble pulse, increased fullness of the veins, grarlually increasing emaciation, dropsical signs, sometimes vertigo and attacks similar to palpitation.

Treatment. The treatment will have to be limited to preventing, if possible, the exhaustion of the lieart miscle; this is done by careful use and proper feeding of the patients. The animals may, even for considerable periods, be used for uniform and quiet work, but exertion usually decidedly aggravates their condition, the circulatory disturbances are increased and sudden death may follow. In cardiac weakness the remedies mentioned for cardiac dilatation are applicable (p. 1067).

Literature. Köhne, Mag., 1870. 89. - Liénaux, Ann., 1904. 157. - Mauri, Rev. vét., 1s94. 121. - Sehrailer, D. t. W., 1902. 156. - Serhing, W. f. Tk., $18 s 8$. 205. - Sierlamgrotzky, S. B., 1887. 20. - Schubert, Beitr. z. Anatomie d. Herzens d. Haussäugetiere. Diss. Leipzig. 1909 (Lit.). - Vogel, Rep., 1S88. 1.

\section{Dilatation of the Heart. Dilatatio Cordis.}

The term "Carliac Dilatation" is used to designate a deficient emptring of the heart cavities rluring systole, a condition which is based upon a muscular force of the heart muscle which is relatively small in proportion to the resistance.

In accordance with its considerable power of adaptation, the heart is capable of taking up an amount of blood which is far in excess of the average amount, and to expel it during the next svstole, if its muscles are unimpaired. This is a eompensatory dilatation, purely diastolic, which develops in some forms of valvular lesions and leads, after a certain time, to hypertrophy. It will not be further discussed in this place.

Etiology. Dilatation of the heart develops acutely from an excessive increase of the blood pressure and usually appears quite early upon excessive and especially upon unaceustomed muscular exertion. Under such circumstances the heart muscle becomes incapable of responding to the excessive increase of the 
demands put upon it, and the less so as, owing to rapidly succeeding contractions, some muscular fibers may tear. i dilatation which has occurred in this manner either disappear's after a time, usually after a hypertrophy has developed in the meantime, or it persists and leads to death, rarely within a short time, although this has happened within a few minutes. The trouble usually occurs most frequently in army and race horses, and is designated by German anthor's as "Over-exertion of the Heart," or "Acute Cardiac Insufficiency;" by French authors, "Coeur forcé," "Coeur fatigué," "Surmenage." In cattle and hogs the disease is not infrequent if these animals are forced to do unusually severe muscular work (for instance, while being driven to market) after long continued confinement in the stable or after they are fattened. It is, however, to be noted that in a number of such cases the heart muscle possibly had previously not been quite sound. (According to le la Camp severe muscular work causes a dilatation only in a heart which is already diseased.)

In the majority of cases the basis for dilatation is provided loy a disease of the heart muscle or of the arteries, also by ralvilar anomalies, in which case the dilatation may develop either acutely or chronically. Extensive degeneration of the heart muscle may reduce the muscular force of the heart so much that this organ cannot even overcome the normal blood pressure. Of similar significance is myocarditis, which evidently enters as a factor even more frequently than degeneration of the heart muscle. Disease of the coronary arteries is, in domestic animals, probably only rarely a basis for dilatation. The same is true of tumors and parasites which may be found in the heart.

In the stage of insufficient compensation in valvular lesions, the section of the heart which lies in front of the diseased portion is dilated, while in diseases of the left venous ostium the right ventricle is also dilated. The cause of this dilatation will be discussed at greater detail under organic heart diseases.

It is also as a consequence of cardiac weakness that dilatation may occur as a sequel of cardiac hypertrophy associated with chronic nephritis.

Anatomical Changes. The affected cavities of the heart are larger than normal. The walls are flabby, so that the opening is not patent when an incision is made on autopsy. If the size of the heart was normal before the development of the dilatation, the walls are thinner in proportion to the legree of dilatation. If the disease has lasted for some time, there occurs an atrophy of the muscles, which may be so extreme that the visceral layer of the pericardium comes into opposition to the endocardium (Dilatatio passiva). If, on the other hand, the dilatation has developed in a heart that had previously become hypertrophied, the wall of the dilated 
cavity either remains of normal thickness, or appears to be even thicker (Dilatatio activa). In this case the presence of

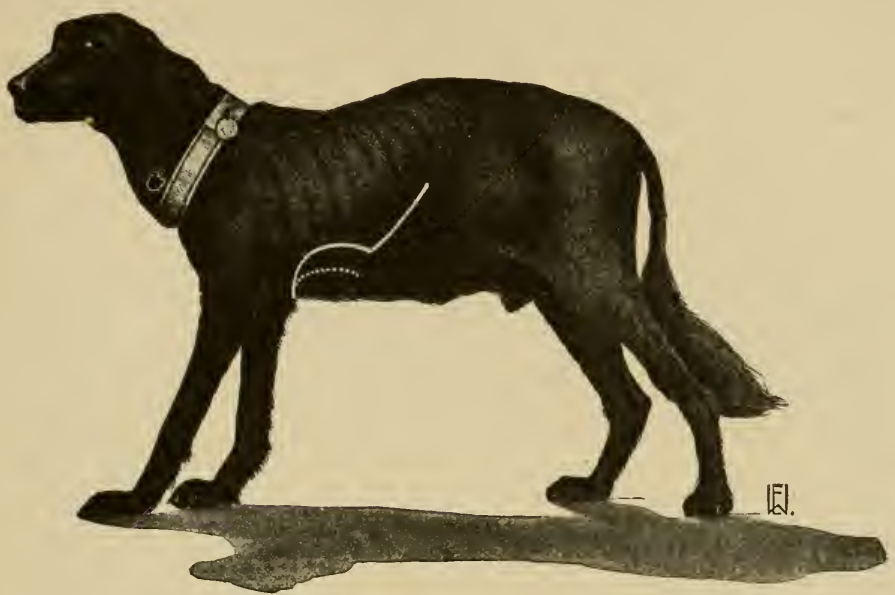

Fig. 186. Cardiac Dilatation. The dotted line gives the normal heart dullness, the solid line indicates the enlarged heart dullness (4th to 6 th intercostal space), the dullness passes backward into the anterior border of the liver dullness, which is also enlarged. (Same dog as in Fig. 187.)

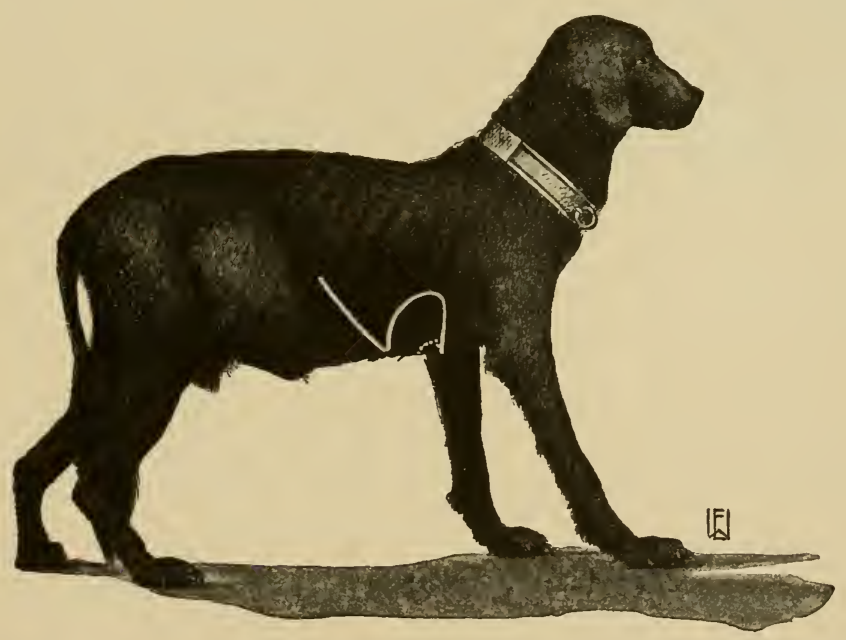

Fig. 187. Cardiac Dilatation. Enormously increased cardiac dullness on the right side. The dotted line gives the borders of the dullness, which oceurs normally in large dogs. (The same $\log$ as in Fig. 186.) 
dilatation may be surmised, aside from the diameter of the respective cavities, by the fact that the trabeculae on the inner surfaces of the ventricular wall are narrow and separated one from the other by wide spaces. At the same time the chordae tendineae are usually thin and elongated. The muscles almost always show signs of parenchymatous or fatty degeneration and also of atrophy. A dilatation of the auricles is as a rule observable at first glance and may be reeognized by the fact that the walls have become as thin as paper and that the septum has acquired larger dimensions.

In other organs, especially in the lungs, in the liver, spleen, kidneys, etc., there are symptoms of venous stasis and dropsical effusions due to the same.

Symptoms. In severe cases, enlargement of the cardiac dullness may be shown, which is sometimes very considerable, while in animals in which normally an absolute heart dullness is not found, above all the occurrence of such an absolute heart dullness is noticeable. In carnivora the dilatation of the left ventricle causes an enlargement of the cardiac dullness upward beyond the mamnary line (Fig. 186); in other animals, both upward and even more backward (Fig. 185). (n the other hand, the dilatation of the right ventricle is characterized by the occurrence of an absolute dullness on the right sicle, or by an enlargement of the dullness which is normally very sliglit in small-chested animals. (Fig. 187 and 189.) Considerahle clilatation of the right rentricle, howerer, causes an increase of the dullness even on the left side. It is possible, as shown by a case of the author's in a dog six weeks old, for the cardiac dullness in dilatation of the right half of the heart to extencl forward as far as the second intercostal space. It is not certain whether this is to be observed only in very young animals. The force of the heart beat is usually increased because a greater portion of the heart is in apposition to the chest wall. Sometimes the trunk receives a thrill synchronously with the heart beat. The beat appears weaker only when the heart weakness has become considerable. The systolic heart sound is increased in force like the heart beat, while the second sound is usually small or not at all perceptible. The action of the heart is not infrequently arhythmical, and the first, less often also the second heart somnds are dicrotic. Endocardial somds are caused by the relative insufficiency of the ralves which develops after a while in some cases.

In contrast to the increased leart beat, the pulse is always feeble. It may also be arliythmical and intermittent, corresponding to the lieart leat.

In the case of a prolonged acute, and in chronic dilatation, sometimes a renous stasis is observed, especially fullness and pulsation of the jugular veins. In a marked dilatation of the 
right heart a positive (systolic) pulse of the jugular veins may even be noticeable (relative insufficiency of the tricuspid). Further, cyanotic discoloration of the mucous membranes is observed; also attacks of vertigo; in more advanced cases, considerable dyspnea, sometimes pulmonary hemorrhage, edema, hydrops of the body cavities, diminution of the amount of urine, etc. Some of these phenomena are at first hardly noticeable when the animals are at rest, but they increase decidedly even on brief motion and sometimes to such a degree that the animal may die suddenly with symptoms of asphyxia.

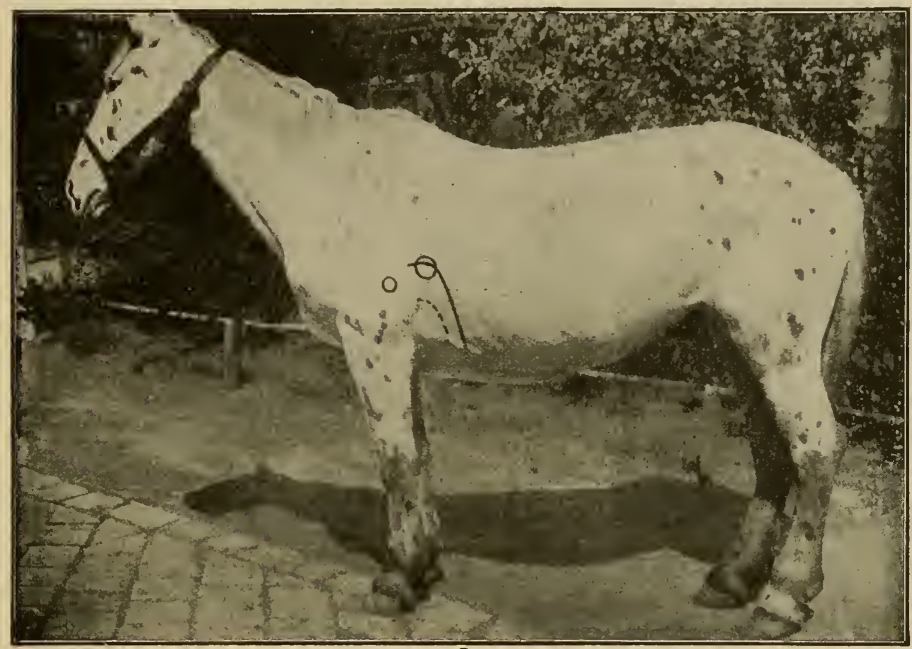

Fig. 188. Cardiac Dilatation. The dotted line gives the borders of the normal heart dullness, which appears in the illustration to lie too ligh, on account of the swelling of the lower chest. The solid line indicates the upper and posterior borders of the greatly enlarged heart dulhess. Owing to the dilatation, the punctum maximum of the systolic sounds arising in the left venous ostimm (large circle) and of the second pulmonary sound (small circle) is displaced upward. (Compare Fig. 189.)

Course. If the animals are cared for, the symptoms may, if they are not yet severe, diminish considerably, and in such cases the cardiac dullness will become much smaller. In acute dilatation a complete retrogression may be observer, which, however, usually leaves a hypertrophy behind.

Diagnosis. The characteristic symptoms of cardiac dilatation are: enlargement of the heart dulhess, increase in the force of the first heart sound, arhythmia of the heart action, feeble pulse, symptoms of stasis. The feebleness of the pulse differentiates the disease from primary hypertrophy of the 
heart. The absence of friction sounds distinguishes it from pericarditis or from hydropericardium.

Treatment. Permanent recovery may be hoped for only if a previously healthy heart has become dilated owing to very severe muscular exertion. In such cases the circulatory disturbances retrogress considerably or entirely under absolute rest and through the influence of repeated administration of cardio-tonic remedies, first of all digitalis. This improvement may occur in a short time. (For details see the chapter on

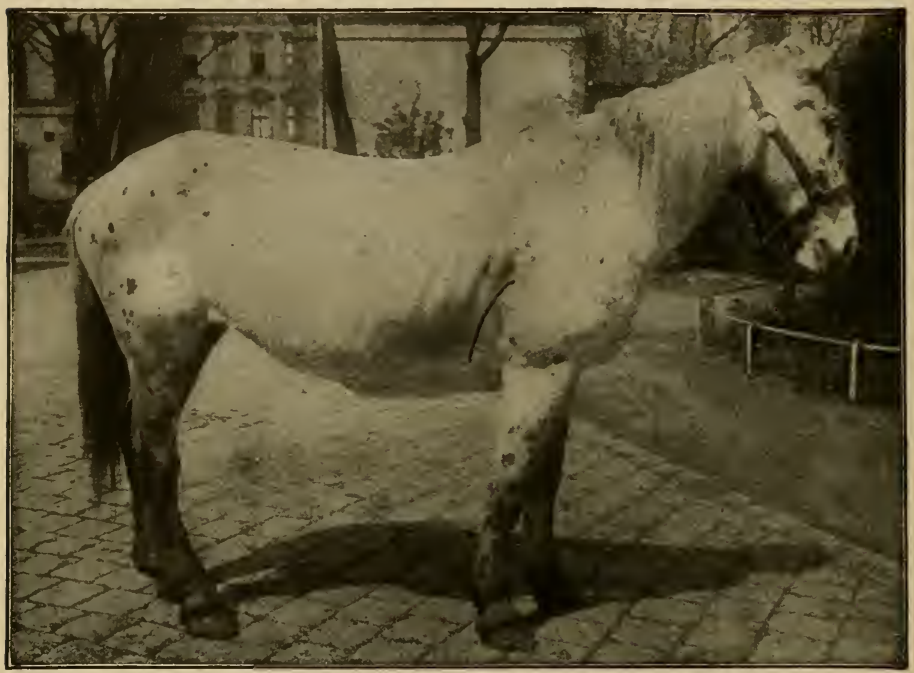

Fig. 189. Cardiac Dilatation. Heart dullness of the horse pictured in Fig. 188, on the right side, in the 4 th to 5 th intercostal space; in front the upper border reaches the scapular line.

valvular diseases.) Even dilatation which commences in the course of acute infectious diseases may sometimes be reduced by similar treatment; but if the heart had been diseased previously or if there are permanent impediments to the circulation of the blood, if, moreover. signs of degenerative processes exist, it may be possible to obtain an improvement that is at times quite considerable, but this will soon yield to an aggravation of the condition. Such patients ean be nsed for work only to a very limited degree. It is always necessary to insure nutritious feeding and to save the animals from work.

Literature. De la Camp, Z. f. kl. Med., 1904. LI. 1. - Goedecke, D. t. W., 1910. 205. - Pr. Mil. Vb., 1899-1908. - Trasbot, A. d'Alf., 1878. 204; Bull,' 1899. 206. - Zschokke, Sch. A., 1900. XLII. 211." (See also Lit. on hypertrophy of the heart.) 


\section{Inflammation of the Heart Muscle. Myocarditis.}

\section{(a) Acute Myocarditis. Myocarditis Acuta.}

There are two principal types of acute inflammatory processes in the heart muscle, namely, the parenchymatous myocarditis, which is characterized by a pathological effusion that may be very slight, and by a more emphatic degeneration of the muscular fibers, and secondly, purulent myocarditis, in which purulent softened foci are developed in the heart muscle.

In contrast to purulent myocarditis, the acute parenchymatous myocarditis cannot be sharply distinguished from parenchymatous or fatty degeneration. The essential point of differentiation is really only in the pathological effusion which is absent in purulent myocarditis (Kitt), but which, in parenchymatous disease, may be so inconsiderable that it is only possible by means of microscopical examination to decide whether the process is to be called an inflammation or a degeneration. If the gross anatomical appearance of the heart muscle does not always offer diagnostic information, the clinician is even less in a position to decide whether and for how long a time the process in the heart muscle is inflammatory or simply degenerative in nature, because both processes usually develop under similar conditions and are manifested by the same symptoms. It is therefore justified from a clinical point of view to consider the parenchymatous degeneration, together with acute parenchymatous myocarditis.

Nor does the consideration of acute parenchymatous and of purulent inflammation in separate chapters appear justified, because clinically they cannot be differentiated, and, moreover, the treatment of both diseases is similar.

Etiology. Aside from the inflammation, which, according to some authors (Friedberger \& Fröhner, Zschokke) develops primarily in exceptional cases in horses and hunting dogs owing to excessive exercise, and which probably belongs more properly to acute cardiac dilatation, acute parenchymatous myocarditis is a secondary affection, which occurs especially in acute infectious diseases and very rarely in certain intoxications. The toxins which, in the course of acute infectious diseases, circulate in the bloor, produce sometimes only a parenchymatous degeneration or at the same time a fatty degeneration of the muscular fibers, sometimes also a serous or cellular infiltration of the interstitial tissue, and frequently hemorrhages. This occurs especially in the septicemic diseases, for instance, in hemorrhagic septicemia, in swine erysipelas, anthrax, and acute glanders, while foot and mouth disease and especially variola, seem to be complicated by myocarditis only in their malignant forms. An inflammation which was observed by Dieckerhoff in connection with the so called Skalma and which was called by him "Myocarditis septica," undoubtedly 
levelops upon the basis of an influenzal infection. Since in infectious cliseases the heart muscle behaves like a parenchymatous organ, the frequency of myocarditis in infections diseases mar best be indicated br the comparatively frequent occurrence of renal albuminuria, whieh points to a parenchymatous disease of the kidney.

Of poisons, especially the heary metals (mercury, copper, antimony), also phosphorus and arsenic are capable of cansing a degeneration of the heart muscle.

Irocarditis, nsmally purulent in character, develops if infected emboli have been carried into the blood vessels of the lieart (nrocarditis embolica), which occurs most frequently in cattle. The emboli may be derived from any part of the body where suppuration or gangrenous disintegration of tissues is present, but most frequently a purulent or gangrenous inflammation of the lungs or of the intestinal tract, a disintegrating thrombus of the umbilical ressels, purulent inflammation of the hoof, puerperal endometritis, strangles, glanders or decubital gangrene are responsible for the affection. In cattle the necrosis bacillus sometimes gives rise to the formation of dry necrotic foci in the heart muscle (Kitt).

There is also a possibility in the case of ulcerating valvular inflammation that portions of the blood clots cleposited upon the valves together with pathogenic bacteria are arrested in the smaller branches of the coronary arteries. The transportation of bacteria into the blood vessels of the valves and into those of the heart muscle are probably simultaneous (see endocarditis), but the inflammation may be continued directly from the diseased valves to the heart muscle. The same is sometimes the case in purulent or ichorous pericarditis.

Injuries of the heart muscle produce a purulent or ichorous myocarditis most frequently in cattle and in the same manner as the inflammation of the pericardium which has usually developed earlier from the same cause (see p. 1040). It is much less frequent that foreign bodies which have penetrated the wall of the thorax and have entered the heart produce myocarditis, because such injuries usually cause death even before an inflammatory process could possibly be developed.

Anatomical Changes. A very variable number of muscular fibers is finely granular, swollen, and they sometimes contain fat globules. If the disease is extensive the muscular fibers disintegrate into larger or smaller clumps (Zschokke). The interstitial tissue frequently shows serous-cellular infiltration and sometimes contains red blood cells (Myocarditis parenchymatosa). The heart muscle is of a lighter brown-red than normal, or grayish-red. It is flabby and friable. The cut surface contains light gray or even butter-yellow dots and stripes (which are especially noticeable in animals dead of foot and mouth (lisease), sometimes also dark red dots. In the case of more 
extensire degeneration the muscle assumes an almost uniform gray-yellow color. These changes are not alike in all sections of the heart. Besides healthy or comparatively slightly changed portions there are parts of the heart, of various extent, which show very considerable disease.

Metastasis or immediate continuation of a purulent inflammation from the vicinity leads to the formation of smaller pus foci which form abscesses by gradual increase in size or by confluence (Myocarditis purulenta). Injuries of the heart muscle will also cause the formation of abscesses. The contents of an abscess may eventually be inspissated into a cheesy mass and eren become calcified. It may also break into the pericardial cavity or into a heart cavity. Occasionally ichorous pus is found in the abscesses. (In the heart muscle of calves which were apparently healthy Kitt found repeatedly a diffuse necrosis which was similar to the necrosis observed in logs in the skeleton muscles.)

Symptoms. The functioning ability of the heart muscle is diminished not only by the degeneration but also by the inflammation in direct proportion to their intensity and extension. Consequently, the symptoms correspond with the clinical picture of cardiac debility only if the affection is considerable. The clinical picture of myocarditis occurring in connection with acute infections diseases develops as a rule gradually. The number of heart beats increases three- or fourfold. The heart beat is at first, and sometimes permanently, increased and can be felt over a larger territory than nsual on account of the rapid contractions of the heart muscle which is otherwise weakened. In contrast the pulse is feeble, sometimes thready and often arrhythmical. To these symptoms are added signs of stasis, at first only in the form of a dark red coloration or cyanosis of the visible mucous membranes while at the same time the veins are filled more strongly. Later, however, the larger veins are more prominent. All these symptoms may eventually retrogress slowly, the number of heart beats becoming more and more normal, the pulse fuller and its tension greater, while the blood stasis disappears, and with the cure of the basic disease the cavity of the heart returns to the normal.

In severe cases, howerer, the disturbances increase, the heart beat becomes more and more rapid, the pulse more thread-like, irregular and intermittent, venous pulsation is noticeable, the respiration is hastened and more or less labored. Vertigo, trembling and debility become manifest, and finally paralysis of the heart terminates the disease. In exceptional cases the cardiac weakness develops so rapidly that the sick animals fall down as though of an apoplectic stroke, and die after a short time, for instance, in the malignant form of foot and mouth disease, and sometimes also in influenza of 
horses. Finally, sudden death may occur in animals that are apparently heaithy and in which autopsy shows that somewhere in the heart muscle an abscess had existed for some time and which had caused death by perforating into the pericardial cavity or into one of the heart cavities. Such cases occur preferably in animals that are continuously saved from all exercise (cows).

Diagnosis. Acute myocarditis may be mistaken especially for acute peri- or endocarditis, since in both these diseases the heart beat is usually much stronger and bounding. In the initial stage the differentiation, on the ground of clinical srmptoms alone, is hardly possible. Later on, however, these diseases may be eliminated by the absence of abnormal sounds. -The general vascular paralysis which is frequent in acute infectious diseases is characterized by the fact that the reins are not filled very strongly and by the pale blue discoloration of the mucous membranes. This affection is frequently associated with acute myocarditis.

The nature of the inflammation of the heart muscle can be ascertained only approximately by considering the basic disease or the morbid cause; especially a purulent inflammation can be assumed only then when other factors admit the possibility of metastasis. Aside from parenchymatous degeneration or inflammation of the heart muscle, the kidneys also become often similarly affected in acute infectious diseases, and the albuminuria which is due to such an occurrence may be made use of in making a diagnosis.

Prognosis. The significance of parenchymatous degeneration or inflammation depends primarily upon whether the basic disease permits a removal of its cause. If, however, the degeneration or the functional disturbances produced by it have passed a certain degree, healing becomes impossible. Of deciding importance in these cases is the tone of the vascular walls and also the degree of impediment to the blood stream; in general vascular paralysis, or in extensive infiltration of the lungs the circulatory disturbances assume a threatening character earlier, the heart changes being of like degree.

The pulse rate is of importance in so far as, with an increase to more than twice the normal, an equalization of the disturbances can hardly be hoped for. If the disturbances of the cardiac activity are less severe, the irregularity and weakness of the pulse are again of unfavorable import.-In purulent inflammation the prognosis is always unfarorable.

Treatment. The cardiac weakness may be counteracted by excitants or cardio-tonics. According to the intensity of the functional disturbances, the following remedies may be considered: Alcohol (in the drinking water); for smaller 
animals wine or brandy, digitalis (in powder form, as infusion, maceration or dialyzation), strophanthin subcutaneously, camphor internally, or camphorated oil subcutaneously, ether subcutaneously, etc. Gmeiner always observed good effects in myocarditis following upon acute infectious diseases from caffeine (large animals 6 to $8 \mathrm{gms}$., smaller animals 0.5 to 1.0 gms.) subcutaneously every 6 to 8 hours. In very severe cases the preparations of camphor are particularly indicated, and occasionally even in apparently hopeless cases the occurrence of heart paralysis may be prevented by subcutaneous injections, which are repeated every hour or every two hours.

Aside from this treatment good effects may be obtained from spongings and the removal of retained feces. It goes without saying that absolute rest and good nutrition of the patient must be secured.

Literature. Berton, Rec., 1898. 289. - Cadiot, Bull., 1893. 374. - Friedberger, Münch. Jhb., 1877-78. 58. - Gmeiner, B. t. W., 1906. 409. - Johne, S. B., 1878. 19, 21. - Trattner, A. L., 1904. 489 - Zsehokke, Schw A., 1900. XLII. 193; XLIX. 320 .

\section{(b) Chronic Myocarditis. Myocarditis Chronica.}

Chronic inflammatory processes in the heart muscle occur in domestic animals much more frequently than used to be assumed. A part of the cases described as idiopathic lrypertrophy or as dilatation of the heart was evidently due to chronic myocarditis.

Etiology. Chronic myocarditis not infrequently develops from the acute form. Further, abscesses developing in the heart muscle incite an increase in connective tissue, either in their immediate vicinity or in more distant portions. Occasionally chronic muscular rheumatism, chronic nephritis, chronic endoarteritis, may act as exciting causes. Tuberculous foci or abscesses with circumscribed myocarditis are sometimes seen in cattle and dogs, exceptionally also in hogs, not infrequently in chickens (Fölger). Chronic peri- and endocarditis is sometimes also followed by chronic myocarditis, either from a direct continuation of the inflammatory process or from the same cause as the peri- or endocarditis, and in fact the inflammatory process may occur simultaneously in the heart muscle. In a portion of the cases the disease undoubtedly develops idiopathically for reasons that are not known. Occasionally, perhaps, certain chemical substances or mechanical influences and agencies leading to increased arterial pressure may be of importance. Fleischer \& Lode have produced necrotic foci in the heart muscle in $59 \%$ of their experiment rabbits after combined injections of adrenalin and caffeine, and the necrotic foci were much smaller if adrenalin alone was injected intravenously.

Vol. $1-68$ 
A disease described by some Hungarian veterinarians (Schleiffer, Viasz, Horváth, László) as a sequel of foot and mouth disease is probably also based upon a chronie myocarditis which has developed out of the acute inflammation. The most striking morbid symptoms are dyspnea, attacks of asphyxia during motion, delayed change of the hair coat, bad nutrition and diminished milk secretion.

Anatomical Changes. The heart muscle is more solid, either throughout or, more frequently, in portions of various extension, and contains grayish-white spots or streaks (scars). (In extensive degeneration of the cardiac muscular fibers, the heart muscle may, however, become softer, even before the occurrence of an active tissue atrophy.) More extensive scars are loulged outward through the action of the blood pressure (aneurysma cordis chronicum fibrosum). The heart is enlarged in the case of extensive disease, both owing to a thickening of its walls and because of a dilatation of the cavities.

Symptoms. Smaller scars in the heart muscle sometimes (lo not cause any morbid symptoms. Since, however, larger portions of the heart are usually affecter, manifestations of heart weakness nevertheless appear sooner or later. A partial atrophy of the muscular fiber's may, in certain cases, be compensated by the increased functioning of the unaffected fibers, but this compensation lasts only for a time and finally the symptoms of heart weakness appear in such cases also.

In the initial stage of the disease, which occasionally lasts a long time, the cardiac insufficiency becomes evident only after severe exertion or excitement. The pulse is much increased, usually arhythmical and feeble. The heart beat is irregular and bounding; the systolic heart sound loud and high. The veins are filled strongly. There is vertigo, staggering gait, rapid fatigue, severe dyspnea, occasionally collapse. In the further course these symptoms become manifest even at rest, after a dilatation of the heart has been added in the meanwhile, owing to which endocardial sounds may be manifest. After variable periods of time serous fluid accumulates in the serous cavities and cutaneous edemata develop.

Course. The condition of the animals often varies considerably, especially under the influence of external conditions. In some cases, especially after over-exertion, death may occur suddenly even before the condition has become very serions. On the other hand, the disease may be recognized in auimals which are saved from excessive exercise only on the occurrence of symptoms of stasis (chronic bronchial catarrh, dropsy, gastro-enteric catarrh, etc.).

Diagnosis. Chronic myocarditis may be recognized only then when the development of the morbid condition out of an acute risease of the heart or of its linings can be followed 
exactly by continued observation of the animals. In making a diagnosis, chronic endocarditis, chronic pericarditis and chronic pulmonary affections must especially enter into consideration.

Treatment. In very ill animals which have become useless for work, no opportunity is offered for treatment, because such animals are usually disposed of otherwise. In other cases a similar therapentic proceeding is indicated as in dilatation of the heart (see p. 1068).

Literature. Ball, J. vét., 1906. 344. - Fölger, Monh., 1909. XX. 348. Horváth, A. L., 1906. 182. - László, ibid., 1906. 219. - Pr. Mil. Vb., 1900. 147. — Zschokke, Schw. A., 1900. XLII. 193.

Ossification of the Heart Muscle. This is an extremely rare disease, which so far has usually been observed only in older animals, exceptionally in younger animals (by Cadiot in a five-year-old pony) and in eattle (Marty, Joest). The eause of the condition is unknown. Stoss speaks of an extensive irritation produced by great variations in the blood pressure. The ossification begins almost without exception in the wall of the right auricle. At first comnective tissue proliferation occurs, then calcification and finally islands or platelets consisting of spongy bone substances. By union of these bone plates, which occurs later on, the wall of the auricle may eventually be transformed into a large connected bony plate. The process of ossification does not progress beyond the place where the veins open into the heart. The ossified auricle is usually considerably enlarged, full and tense.

In lighter cases the ossification of the auricle does not interfere noticeably with the heart action. If, however, the process is more extensive, signs of heart weakness become manifest during work, and later on also at rest. The canse of these signs cannot be determined during the life of the animal.

Literature. Joest, Dresdn. B., 1908. 175. - Krüger, B. t. W., 1892. 509. Nocard, Bull., 1884. 236. - Stoss, D. Z. f. Tm., 1888. XIII. 301 (Lit).

\section{Fatty Heart. Cor Adiposum.}

Etiology. In a general increase of fat deposits the thin fat layer, which is normally present underneath the serous covering of the heart, increases considerably, so that it covers the surface of the heart as a connected laver up to several millimeters in thickness, and may, moreover, form in places considerable fat deposits and polypoid nodes. This condition is observed preferably in obese animals, especially in fat house dogs; exceptionally a fatty degeneration of the heart muscles develops simultaneously with the degeneration of the protoplasm in intoxications and in infectious diseases.

The excessive deposit of fat becomes of importance because the fat accumulates not only on the outer surfaces of the muscles, but also between the muscular fibers which are then forced apart, at the same 
time compressed, and are finally caused to atrophy. Even the spreading apart of the muscular fibers alone renders the contractions of the heart muscle more difficult to a certain degree, and any occurring degeneration or atrophy reduces the strength of the heart muscle still more.

Symptoms. In an excessive degree of fatty heart, symptoms of heart weakness present, such as feeble heart beat, perhaps an increased cardiac dullness which, however, is difficult of demonstration in obese animals; also feeble heart sounds, possibly anorganic murmurs, feeble, small pulse; in severe cases vertigo, and finally cutaneous edema. The production of dyspnea and of vertigo is to a certain degree to be credited to the deposit of fat in the respiratory muscles. These symptoms become marked after severe exercise, or even during simple motion. Death occurs finally through paralysis of the heart, pulmonary edema or rupture of the heart.

Treatment. The treatment for obesity (p.915) is indicated in this condition. If signs of cardiac weakness are already present, cardio-tonic remedies must be considered.

\section{Rupture of the Heart. Ruptura Cordis.}

Etiology. The healthy heart muscles tear at most under the immediate influence of an injury (external injuries, falling down in cattle, from wounds caused by a foreign body entering from the reticulum). Dull pressure exerted upon the heart can hardly produce a rupture. Usually rupture of the heart forms a secondary occurrence which is observed when the resistance of the heart muscle has been reduced by degeneration or atrophy. This is most frequently the case in animals with valvular troubles, where, however, the permanent increase of the blood pressure in certain portions of the heart is also of importance. Rupture of the heart can, far less frequently, be brought into causal relation with occlusion of the coronary arteries or with abscess in the heart wall. In these conditions there usually exists already a circumscribed bulging of the diseased heart wall (aneurysma chronicum cordis) and this persists under special conditions. A bulging of the wall of the heart may occur exceptionally in acute myocarditis (aneurysma acutum cordis). As further causes for heart rupture may be mentioned tumors or parasites (especially echinococcus), in horses also aneurysm of the coronary arteries, which, however, is very rare (Walter, Gurlt, Bogmann, Cadiot, Piot-Bey).

In literature reports may be found of rupture of a sound heart muscle from nerrous causes, but this possibility has not been proved irrefutably in a single case.

The bursting of a heart which is already diseased occurs sometimes without any special cause, particularly under the 
influence of a normal blood pressure, but in most cases exciting causes may be demonstrated, such as severe exertion, straining on defecation, bloating, excitement during mating, during an operation, etc. As these exciting causes become active much more frequently in horses, heart rupture is observed more frequently in the horse than in other animals, with the exception of traumatic rupture of the heart in cattle.

Anatomical Changes. The pericardium contains more or less fresh blood (hemopericardium). A solution of continuity in the fibers of the heart muscle is often found in the walls of the ventricles, less often in the walls of the auricles. The tear is almost always small, its borders ragged and its path through the ventricular wall usually corresponds to a zigzag line, so that it is only with difficulty possible to introduce a probe into the ventricle. Sometimes the tear takes place in the interventricular septum (Dieckerhoff), and in such cases hemorrhage does not take place into the pericardium.

Symptoms. In general only symptoms of cardiac paralysis or of internal hemorrhage are evident, and these cause death rapidly because the heart is compressed by the blood or hindered in its diastolic dilatation, or then the action of shock upon tho heart becomes manifest. The occurrence of a rupture is usually announced by sudden signs of indisposition. In a part of the cases death occurs suddenly, at night, or so rapidly that hardly any symptoms are ascertained aside from collapse and convulsions.

Treatment. A rupture of the heart which has actually occurred cannot be treated. Its appearance, however, may sometimes be prevented by removing the exciting causes.

Literature. Berndt, A. f. Tk., 1893. XIX. 314. - Graux, J. vét., 1905. 231. - Maleval, Ree., 1904. 508. - Petit, Bull., 1905. 166. — Piot-Bey, Bull., 1906. 425, 430. - Vogel, B. t. W., 1906. 682.

\section{New-Formation in the Heart. Neoplasmata Cordis.}

New-formations which occur in the wall of the heart are of only slight interest, not only on account of their rarity, but also because their presence in the heart of living animals can rarely ever be ascertained with even a small degree of probability. According to the communications to literature, fibroma, fibromyxoma, myxoma, lipoma, sarcoma (in horses melanosarcoma) and fibrosarcoma, metastatic adenoma, carcinoma, exceptionally also lymphosarcoma, angioma, lymphangioma occur preferably in the ventricular wall and may, with the exception of carcinoma or sarcoma, be primary. The most frequent growths are fibrosarcoma, and in horses melanosarcoma. The new-formations which project towards the heart cavities or towards the pericardial cavity are not infrequently pediculated. 
The muscular tissue atrophies in the immediate vicinity of the swellings, hut the defect in muscular force is replaced by the uninjured parts, which later on become atrophied. A hypertrophy is more apt to develop as the swellings which bulge out into the eavities or against the openings of the heart, impede the free circulation of the blood. The size and location of the swellings is of influence upon the degree of compensation and therefore only unimportant disturbances or none at all are observed in some cases, while in others a severe clinical picture develops.

The morbid symptoms are very uncertain in character. In most cases sudden death was observed, or the swellings were found on autopsy of such animals which had not presented any symptoms of cardiac disease during life. Only rarely disturbances of the heart function were noticed; in horses dyspnea was often diagnosed, the basis of which had, however, remained obscure during the life of the animal. It is probably only in exceptional cases possible to refer disturbances in the cardiac function to the presence of a swelling in the heart wall with any degree of certainty, especially when the occurrence of a primary new-formation in the organs that are susceptible to immediate investigation permit the assumption of a metastatic swelling in the heart.

\section{Animal Parasites in the Heart.}

Of animal parasites eysticerci and echinococci occur most frequently in the heart muscle or under the epicardium (Neumann, Rev. Vét., 1905, 729, with literature). The cysticerci do not appear to produce any noticeable disturbances in the heart function, and echinococci are found, not at all rarely, in the heart muscle of apparently healthy cattle, as is proved by the findings in abattoirs. (In exceptional cases the echinococci occur also in horses, especially in the heart muscle or in the wall of the aorta.) If the echinococcus bladder is comparatively large, or if several of them are present at the same time (Morot found 20 in one ease) they naturally interfere with the heart action, but the cause of the disturbance cannot be determined even approximately. In a number of cases death occurs suddenly from paralysis or rupture of the heart, or from rupture of the echinococcus bladder into the heart cavity and the transportation of its contents into the pulmonary artery (Eggeling). The predisposing cause for the occurrence of sudden death may also lie in the presence of an infectious disease. In a case observed by Sequens, for instance, the rupture had developed in the febrile stage of foot and mouth disease.

Of other parasites the Sarcosporidia occur in the heart muscle of sheep without leading to any particular cardiac disturbances; also mature specimens of Filaria immitis in the right auricle and the Strongylus vasorum in the heart of dogs (see pages 902 and 907). Cocu found a living specimen of distoma hepaticum in the coagulum attached to the chordae tendineae of the right ventricle of a cow dead with symptoms of severe eachexia and dyspnea.

\section{Acute Endocarditis. Endocarditis Acuta.}

Occurrence. Acute endocarditis belongs to the less frequent diseases and occurs in horses usually in consequence of influenza or of pyemic affections; in cattle it develops most frequently in the course of pyemia, of articular rheumatism, rarely 
of tuberculosis. Hogs are usually affected by an erysipelatous endocarditis; less often the affection accompanies swine plague, and has only in exceptional cases another origin (Lüpke, Bang, author's case). In dogs the disease usually follows upon pyemia, septicemia, distemper, exceptionally also upon tuberculosis (Jensen observed 13 cases of ulcerating endocarditis among 3,240 sick dogs). In cats and birds acute endocarditis appears to occur only very rarely.

Etiology. Acute endocarditis develops as a rule through an infection, but the various infections substances concerned are at present only partially known. Comparatively often the pyogenic bacteria (cocci, streptococci, staphylococci, hacillus pyogenes) appear to be concerned in the production of the affection (Trasbot, Cadéac, Albrecht, Csokor, Nocard, Kitt, Bang, Lüpke, Fröhner, Joest, author's case). In hogs the erysipelas bacilli are usually of importance as etiological factors. In ulcerous endocarditis Jensen and Thomassen have found the bacillus coli communis in association with other bacteria; Kitt several times the botryomyces in horses; Luginger a streptothrix in cattle. The tubercle bacillus was found by Cadéac, Cadiot and Bergeon in cattle; the corynebacillus renalis hy Cadéac and Scherzer in the deposits upon the affected heart valves; the bacillus bipolaris also is probably frequently a factor. For instance, Jensen found oroid bacteria among other microorganisms in endocarditis of dogs, and Bang as well as de Jong observed entocarditis in swine plague (among 16,000 hogs dead of swine plague, 676 cases of endocarditis verrucosa were found in England). Acute endocarditis has also been noted in swine pest (de Jong).

In some cases an infectious endocarditis develops without an affection of the other organs and must then be considered as a primary disease. In the majority of cases, however, the trouble is secondary in nature and the bacteria reach the endocardium from inflamed organs (heart muscle, pericardium, mediastinum) by direct continuity or, much more frequently, from distant pathological foci with the blood stream. First in this respect are pyemic and septicemic diseases. Wherever in the body suppurating inflammations occur, like suppurating wounds, inflammation of the umbilical cord, variola (immediately before the suppurating stage or at its beginning), puerperal fever, strangles, fibrinous-purulent inflammation of the serous membranes, the malignant form of foot-and-mouth disease, etc., the opportunity for localization of pyogenic bacteria on the heart valves may be given. Sometimes seemingly quite inconsiderable losses in substance (ulcers at the tip of the tail or elsewhere on the skin of dogs, bites, pressure wounds in horses, etc.) or inflammatory foci in other organs (in the case of Blane's, inflammation of the bile ducts) afford a point of entrance to the pyogenic bacteria. The transmission of 
bacteria from the blood of the mother to the fetus is also possible, and this explanation must probably be accepted for cases that occur successively in several young of the same mother animal (Burke, Stephenson). Jensen and Fröhner even believe the transmissibility of the disease from animal to animal to be not impossible.

In contrast to man, articular rheumatism is not often complicated with endocarditis in animals; most frequently perhaps in cattle, and evidently also under the influence of pathogenic bacteria (streptococci or perhaps also staphylococci) which in all probability produced the articular rheumatism itself. Probably the endocarditis which sometimes follows upon rhenmatic founders of horses must also be explained in this sense. Inflammation of the endocardium must, especially in horses, undoubtedly be referred to the action of streptococci when it develops in connection with grippal or catarrhal pneumonia.

The bacilli of swine plague are deposited in the tissue of the heart valves after a slight acute general infection, or as Jensen, Lohnsee and Eisenmann have shown, after the erysipelatous urticaria has rm its course, and cause there an inflammation through which the apparently recovered animals perish in the course of a few weeks (see p. 68). Marek saw an acute endocarditis being produced by the erysipelas bacillus in two horses which had been employed for the production of erysipelas serum.

In many cases no bacteria are found in the territory of the inflammation, a fact that must be explained thus, that either the inflammatory virus had disappeared in the meanwhile, or that bacterial toxins absorbed from other organs had produced the disease.

Predisposing factors may be of import to such a degree that formerly many cases of endocarditis were referred to the immediate action of such factors. Among them are: cold (frequent in horses, according to Trasbot), over-exertion, traumatic influences upon the heart region, fracture of ribs, etc. The chronic form of endocarditis also predisposes to renewed acute infiammation (Fuchs).

Pathogenesis. The bacteria which circulate in the blood strean, probably rarely localize directly on the surfaces of the valves which are contiguous to the blood stream while this passes by them, less frequently on the parietal layer of the endocardium. Usually they are arrested in the capillaries of the valves, and also at the same time in the blood vessels of the heart muscle. The reason why the localization of the bacteria mentioned as the second contingency takes place also on that surface of the valves which is turned toward the lumen of the heart, lies, according to Eisenmann, in the peculiar arrangement of the blood vessels in the heart valves. The microorganisms, which have localized, and their toxins cause an in- 
flammatory condition in the tissue of the valves and in the wall of the heart. The surface of the valves becomes rough and masses of thrombi are deposited. These interfere mechanically with the circulation and at the same time the circulatory disturbances are much increased by the simultaneous myocarditis.

Anatomical Changes. In most cases the valves of the left half heart are affected, less often those of the right half as well, or even exclusively (Endocarditis valvularis); the pulmonary valves are involved in exceptional cases only. In this respect cattle make an exception in so far as in them disease of the tricuspid valves is more frequent. An inflammation of the endocardial lining of the muscular wall (Endocarditis parietalis) is rare.

Endocarditis occurs in two forms, one being designated as verrucous (Endocarditis valvularis verrucosa), the other as ulcerous (E. valv. ulcerosa); either one of them may pass into the other. In the verrucous variety nodules are found on those surfaces of the valves which face the blood stream, or at their margins; these are as large as poppy seeds or larger, they may later become confluent, forming larger nodes, and on the surfaces of the valves coagulated blood is deposited. It may happen that the inflammation does not progress further, the verrucous elevations gradually becoming smaller and leaving only a thickening of the valves. But in other cases ulcers with red, uneven borders develop, their bases covered with a grayish-red or yellowish deposit, or the inflammatory process is ulcerous from the beginning. Fibrin is deposited upon the ulcerating surface of the valves in thick, uneven masses which make the opening smaller.

In consequence of the ulcerous destruction the valve becomes thinner, in some cases it is bulged out by the blood pressure (Aneurysma valvulare acutum) and is eventually ruptured (A. valv. acut. ruptum). If the destructive process is rapid, irregular cleft-like openings form with swelled, gnawed borders (Valvula fenestrata) without aneurysmal changes. When the inflammation is continued to the endocardial lining of the heart, a purulent inflammation may arise in the heart muscle, giving rise to bulging and to rupture of the thinned muscular wall (Aneurysma cordis acutum). Occasionally the valve is even torn from the chordae tendineae or from its attachment.

Loose blood clots or loosened portions of the valvular tissue lead to embolism in various organs, which may be followed by purulent inflammation.

Symptoms. The clinical picture of acute endocarditis is characterized by its great variability. The varying nature of the inflammatory process and of the infectious virus, the 
variable degree in which the heart muscle is involved, the different localization of the inflammation and finally the presence or absence of a general infection which in itself may be very variable in nature, produce symptoms which differ in different cases. With respect to the last mentioned factor it must be emphasized that in many cases the blood remains free from microorganisms after the bacteria have been deposited upon the valves, while more frequently bacteremia exists from the beginning or develops later, in addition to the valvular inflammation.

The disease commences with more or less marked signs of indisposition. In this respect particularly deviations are possible according to nature and origin of the disease, only languor, lassitude, slight fever and fatigue becoming manifest iin some animals, while in others extreme debility, complete inappetence and high fever are observed, as they always occur in septicemic diseases. Between these extremes there are many transitions.

The heart beat is usually increased or bounding and may produce a tremor of the chest wall which can be noticed from

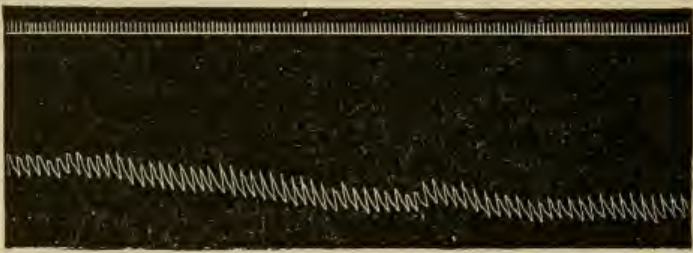

Fig. 190. Sthygmograph of the Art. femoralis in a dog with ulcerating endocarditis of the aortic valves. Pulse bounding and much accelerated. (Time indicated in $0.2^{\prime \prime}$.)

a distance. The rate of the heart heats varies; while it is increased by about one-half of the normal in light cases, it rises in severe attacks in large animals to 4 to 5 times, in small animals to 2 to 3 times the usual rate, and is frequently arhythmic. The cardiac dullness is not altered at first, nor in rapidly progressive cases through their entire course, but in more prolonged cases, in which the heart is dilated, the area of dullness is enlarged. The heart sounds may remain clear for a long time, indeed, to the end, except that they may become somewhat feeble and dulled, but in most cases they eventually become changed. Usually the two sounds run together into a single humming or roaring noise; at other times an endocardial murmur is heard instead of the first or second heart sound or in place of both, which is usually, soft, blowing or even has a certain resonance to it and is heard in varying intensity over different portions of the heart, just as in chronic endo- 
carditis. If the inflammation occurs in several portions of the heart, several sounds may be perceptible which rary in pitch, intensity and volume.

The pulse is alwars accelerated, although its frequency is extremely variable in different cases; it is frequently arhythmical, irregular and intermittent; the intermissions are sometimes so frequent that the number of heart beats is as much as twice that of the pulse beats. At the commencement of light cases, the pulse is strong and even vigorons in relation to the rate, but later, and in severe cases from the first, it is weaker and soft. The character of the pulse depends in part upon the localization of the disease (see valvular diseases); for instance in inflammation of the aortic valves it is bounding (see Fig. 190).

In correspondence with the intensity of the disease circulatory disturbances develop which are manifested by cvanosis, fullness of the reins, negative renous pulse, and by frequent or difficult respiration, and which may be aggravated into asphyctic attacks on the slightest motion or through external influences, or they only become manifest in such attacks, while they are not present at rest. Another consequence of the circulatory disturbances, especially of a

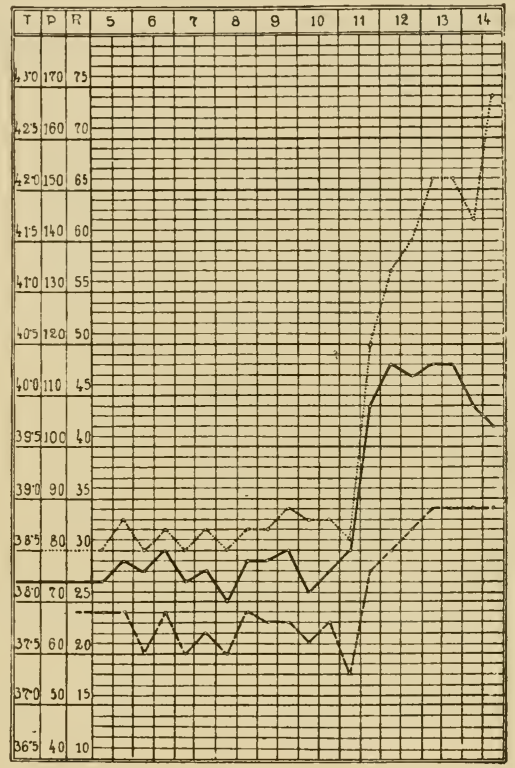

Fig. 191. Temperature curre in acute endocarditis. The disease had appeared in a Great Dane, brought to the hospital with an ulcer on the tail, and ended fatally in. four days. severe pulmonary hyperemia is in many cases a bloody discharge from the nose which may be accompanied by symptoms of pulmonary edema. Moreover, edemas sometimes develop quite early in various parts of the body, for instance, on the limbs and on the lower portions of the trunk. Occasionally, and particularly in hogs, a decided weakness of the hindquarter, or even of one posterior extremity, is observed, which is due either to a stenosis of the aortic opening, or to a general embolic obstruction of the arteries, or to the debility which follows upon the severe general disease. 
The temperature is raised in all cases (Figs. 191 and 192), but in slight attacks it is moderate, while severe cases are accompanied by high fever. There are, however, exceptions, and the great variability of the disease-causes makes it evident that the type of fever may be subject to numerous rariations.

Disturbances in the general condition, which are always observed, may be so insignificant in cases running a slow course that they are not observed at all for a time, especially in swine which are more indolent and live in herds. In other cases the attack commences with considerable indisposition or debility; in horses symptoms of colic are now and then apparent.

With the gradual increase of circulatory disturbances death occurs in some of the cases, while in others, especially

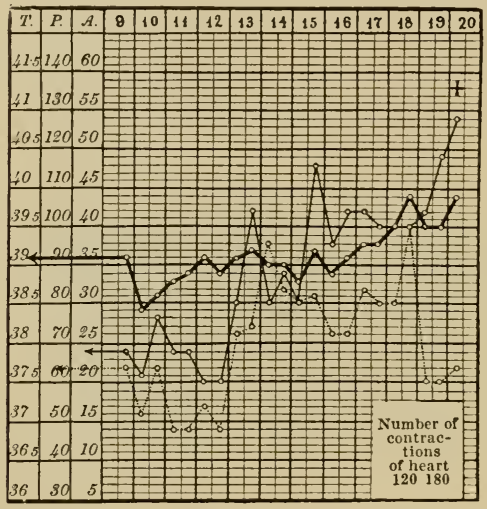

Fig. 192. Temperature curve in endoearditis of erysipelas in a horse. Two days before death the number of heart beats was twice that of the pulse beats. in those affected with the ulcerous form, symptoms of metastatic inflammation in various organs become evident. Such metastases may give rise to purulent pneumonia, or purulent nephritis sometimes accompanied by hematuria; to acute meningitis, purulent inflammation of $\mathrm{the}$ joints, etc. Similar causes must be attributed to the local hemorrhages which develop here and there, for instance, underneath the cornea.

In a very small percentage of cases a possibility of recovery may exist; but in animals which do not die the clinical picture of chronic endocarditis usually develops.

Diagnosis. The disease can only then be recognized positively when repeated examinations have demonstrated that valvular insufficiency or stenosis have developed in a previously healthy heart in a comparatively short time. A careful supervision of the heart action and repeated examinations of the heart are necessary the more, as the elimination of acute febrile diseases (anthrax, severe forms of influenza, septicemia, etc.), perhaps also of severe pulmonary diseases, is possible only in this manner. Of foremost importance is the character of the heart sounds and their transformation into murmurs, but even such findings do not afford absolute proof because 
similar phenomena may be observed in acute myocarditis and in cardiac dilatation. If the murmurs persist permanently, if to them are added circulatory disturbances and perhaps emboli, the nature of the disease may be assumed with sufficient certainty. Still it must not be forgotten that an animal ill with chronic endocarditis may become affected with another septicenic disease.

The differentiation of verrucous endocarditis from the ulcerous form is usually very difficult. In general it may be said that the more severe the symptoms, the higher the fever, and the more turbulent the circulatory disturbances, the greater is the probability of ulcerous endocarditis. Collateral factors may afford information in so far as the verrucous form usually develops in connection with swine erysipelas or with articular rheumatism, while endocarditis ulcerosa occurs in purulent or ichorous processes anywhere in the body.

Prognosis. Acute endocarditis is always a severe disease, especially in the ulcerous form. Even in the lightest cases there is little hope for complete recovery, because usually chronic changes remain which afford a basis for permanent valvular lesions and thus reduce both the ability to work and the mutrition of the animals.

Treatment. Absolute rest should be procured first of all. For the alleviation of the excitement, which is noticeable at least occasionally, cold or ice compresses upon the heart or cold sprays are appropriate. If the heart action is very rapid and irregular the cantious administration of digitalis or of its preparations or of other cardiac remedies is indicated (see valvular diseases). In heart weakness the danger of cardiac paralysis must be counteracted with stimulants, for instance, with alcohol, wines, black coffee, tea, caffeine, theine, camphor, Liq. Ammon. Anisatus internally; ether, camphorated oil, tincture of musk subcutaneously. In excessively high temperature the fever requires lactophenin, antipyrin, aspirin, antifebrin, etc. In endocarditis of rheumatic origin salicylic acid and its preparations may be tried.

Literature. Albrecht, W. f. Tk., 1893, 164. - Bang, D. Z. f. Tm., 1892. XVIII. 27. - Bergeon, Rev. rét., 1905. 320. - Coppol, Die chron. Klappenerkrank. beim Hunde, Diss. Leipzig, 1908. - Dasch, T. Z., 1907. 518. - Eisenmann, Monh., 1906. XVII. 97. - Fröhner, Monh., 1894. V. 171. - Hartl, Z. f. Tm., 1902. VI. 450. - Jensen, Maanedsskr., 1898. X. 65. - Joest, S. B., 1905. 300. - Joest \& Röder, Monh., 1908. XIX. 158. - Luginger, ibid., 1904. XV. 289 (Lit.). - Meyer, B. t. W., 1905. 740. — Trasbot, A. d'Alf., 1877. 921.

\section{Valvular Diseases. Vitia Cordis. Heart Troubles.}

The designation of valvular lesions includes the chronic organic diseases which cause functional disturbances of the heart valves and the cardiac ostia. They differ in their derivation. 
Occurrence. Since valvular lesions occur most frequently in consequence of chronic endocarditis which is most common in dogs, the valvular troubles are found most often in these animals. (According to Cadiot $5 \%$ of dogs over one year old suffer from valvular disease; among 793 dogs autopsied by Johme, chronic endocarditis was found in $4.3 \%$, and among 1,831 logs which were exanined in the pathologic-anatomical institute in Budapest $3.9 \%$ were found with evidences of endocarditis). Next in frequency are horses, and then, very much less often, the other mammalians, if the cases of acute endocarditis are not included. The observations of Larcher and Hartl show that valvular diseases are not infrequent in fowls, although they give rise to functional heart disturbances comparatively rarely.

Etiology. Valvular heart lesions may be caused by the following diseases of the heart:

1. Acute Endocarditis (see p. 1078), which in its less rapid cases takes a comrse presenting the clinical picture of valvular disease.

2. Chronic Endocarditis (Endocarditis valvularis chronica). This disease is the basis for most cases of valvular troubles and develops, probably principally, out of the acute inflammation; it either occurs thus that, after the acute endocarditis has run its comrse, a proliferation with subsequent atrophy of the connective tissue takes place, or thus that an acute inflammation, which is not very intense of itself, recurs periodically and produces proliferation and atrophy of connective tissue after each recurrence. Accordingly all causes and conditions which stand in etiological relation to acute endocarditis may occasionally also lead to a chronic inflammation. In this respect those factors especially enter into consideration which usually, or at least occasionally produce a mild acute inflammation. This is particularly the case in endocarditis following upon articular rhemmatism and upon crompous pneumonia, also in inflanmation due to tuberculosis and to erysipelas. In other case the inflammation is chronic from the begimning and is usually not noticed; and in such cases an infection may be a causative factor, although it does not give rise to acute symptoms. Further, chronic endocarditis develops in connection with chronic endoarteritis and arteriosclerosis, and in dogs frequently in association with chronic interstitial nephritis. Sonnenberg found the mitral valve of a cachectic tuberculous cow thickened and covered with a yellowish-gray deposit, while the endocardium of the left heart showed diffuse grayish-yellow discoloration. Hartl found in chickens repeatedly a chronic endocarditis the origin of which could not be determined. Lastly, severe exertion or the mechanical irritation of the valves which is necessarily exerted by it upon them, and also cold are said to produce the disease. 
3. Injuries to the valves are also capable of injuring their function. This occurs when a valve is perforated or when, during severe exertion, a semilunar valve is torn from its base or one of the bicuspid or tricuspid valves has been torn from the chordae tendineae by the force of the pressure which is periodically exerted upon the valves. (In a case of Lustig's the valve was torn off during severe work, in a case of Cantini's while the horse was jumping; similar cases were observed by Lüthens, Gerlach and Scliütz). In exceptional cases the simple over-distension of the aortic valves causes an inability to close (Zschokke).

4. Tumors and Echinococci may cause both insufficiency and stenosis if they grow from the annuli fibrosi or from the wall of a heart eavity toward an ostium.

5 . Diseases of the heart muscle may produce a valvular insufficiency even if the valves are not injured (Insufficientia relativa s. muscularis). Especially in the proper functioning of the auriculo-ventricular valves both the relaxation of the chordae tendineae and the contractions of the nuscles around the ostium cooperate to a considerable degree. If the contractions are not sufficiently energetic, the closure of the valves becomes insufficient. A similar disturbance results if one of the ostia has been dilated excessively in case of cardiac dilatation of high degree. Insufficiencies from this cause are by no means infrequent and are usually due to chronic myocarditis, sometimes they follow upon a cardiac weakness from other causes and usually affect the right heart. Finally dilatation of the arterial trunks close to the heart may result in an insufficiency of the semilunar valves.

6. Congenital heart lesions must also be included here. According to Preisz they are usually due to impeded, less often to faulty development or to intrauterine heart diseases. A comparatively frequent anomaly is the persistence of an opening in the upper anterior portion of the interventricular septum (Foramen interventriculare persistens) and according to Preisz the cases described by Csokor and Jolme under the name of Septum membranaceum persistens belong to this lesion. The Foramen ovale between the auricles remains open fairly often and also the Ductus Botalli. It oceurs only very rarely that only one auriculo-ventricular opening or a simple arterial trunk proceeding from the ventricle is found in addition to the above mentioned anomalies.

Anatomical Changes. Chronic endocarditis leads to newformation followed by atrophy of the connective tissue; owing to these changes the endocardium is thickened in varying degrees, verrucous, polypoid or cauliflower-like excrescences are formed on the borders of the valve which is in contact with the blood stream, and the chordae tendinae are thickened. The thickened ralves become rigid and the semilunar valves are 
transformed into thick flat pads, the separate portions of the bicuspid and tricuspid valves into thick, knobby formations; in exceptional cases contiguous parts of valves become adherent. The thickened chordae tendineae appear shortened. On the parietal layer of the endocardium changes are frequent but less striking and only rarely produce thickening.

If the atrophy of the valves or of the chordae tendineae has advanced to such a degree that the extended valves no longer tonch, the result is an insufficiency, but if polypoid or cauliflower-like deposits have formed on the valves or if the latter are very rigid, and their contiguous portions perhaps atherent, the result is a stenosis of the respective ostium. According to the nature and localization of the changes a stenosis is often present together with an insufficiency. The portion of the heart which is proximal to the diseased part (in the direction of the blood stream) is then dilated and more or less hypertrophic. During the stage of incompensation there exist symptoms of blood-stasis.

As regards the seat of the changes the disease affects in horses frequently the aortic valves (according to French authors this is the most frequent form of heart trouble in horses), more rarely the bicuspid valve. In cattle and goats the valves of the right heart are as a rule affected most frequently, in swine those on the left side of the heart, especially the bicuspid valve. In dogs mitral insufficiency is observed most often, then the simultaneous affection of bicuspid and tricuspid valves and about half as often disease of the tricuspid valve alone. According to Larcher and Hartl the valves of the right ventricle are usually affected in birds.

Symptoms. According to the localization of the morlid process the symptoms are so different that it appears proper to consider them separately.

\section{(a) Insufficiency of the Aortic Valves.}

In insufficiency of the semilunar valves of the aorta a slit-like opening persists between the valves during ventricular systole, in consequence of which a portion of the blood which had been forced into the aorta during the preceding systole flows back into the left ventricle, producing a murmur in so doing. At the same time blood flows into the left ventricle from the left auricle, and the ventricle, receiving blood from two directions, is dilated, owing to its power of adaptation, but forces out its entire blood content during the next systole, for which a stronger contraction is necessarily required. The continuous increase in the activity of the heart gives rise to a hypertrophy of the wall of the dilated ventricle.

The symptoms of insufficiency of the aortic valves are as follows: The stronger contraction of the hypertrophied and 
at the same time dilated left ventricle produces an increased lifting heart beat on palpation of the cardiac region, especially

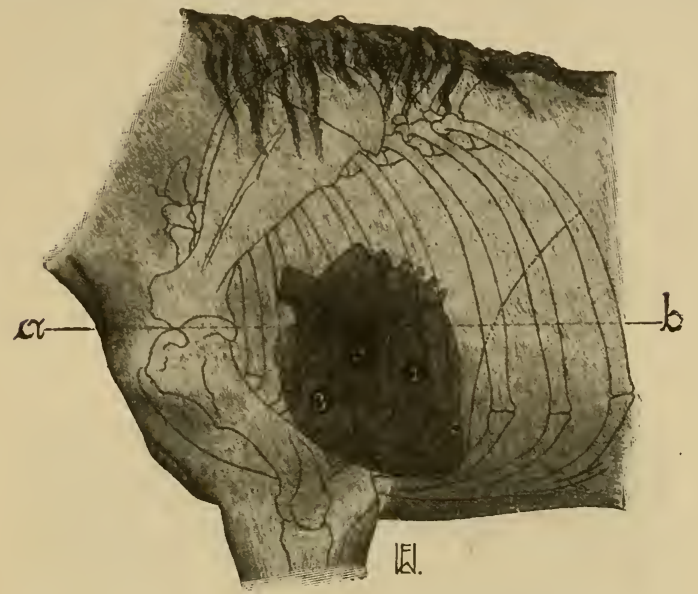

Fig. 193. Location of the greatest intensity of cudocardial sounds in the horse. $a$. b. shoulder line; 1 punctum naximum of the murmur originating in the left auriculo-ventricular opening; 2 in the aortic opening; 3 in the opening of the pulmonary artery. In the other species similar conditions may be shown.

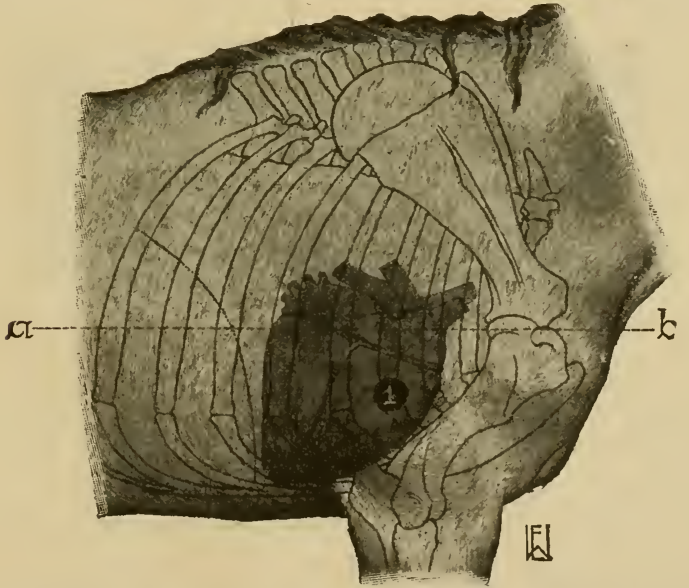

Fig. 194. Location of the greatest intensity of the murmur originating in the right auriculo-ventricular opening in the horse (1). The other murmurs the same as in Fig. 193.

after excitement, and a diastolic thrill of the wall of the thorax is noted on the left side. The area of heart dullness on the vol. $1-69$ 
left side is displaced in dogs about $3-5 \mathrm{~cm}$. or more upward, while in other animals it is displaced upwards and backwards and may reach as far as the seventh rib and beyond the middle of the lower third of the chest. The first heart sound is clear, but the diastolic sound is accompanied by a murmur which is usually loud, blowing or roaring, perhaps rough or ringing, prolonged, and which is separated from the systole by a rather short pause. Its greatest intensity is, in all mammals, in the left fourth intercostal space immediately below the shoulder line (Fig. 193.2), although it may be heard, a little less clearly, also in the same intercostal space on the right side. The purely diastolic sound which originates in the Art. pulmonalis can be heard in the region of the left ventricle in association with the murmurs just referred to, but near the origin of the

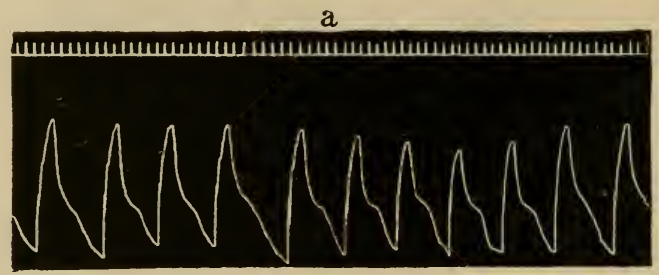

b

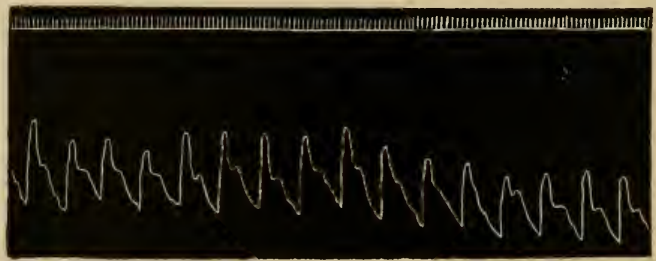

Fig. 195. Sphygmograph of the abdominal aorta in the horse. a bounding pulse in aortic insufficiency; $b$ pulse of a healthy horse (Time in $\left.0.2^{\prime \prime}\right)$.

pulmonary artery (left third intercostal space, lower half of the lower third of the chest [Fig. 193.3]) it may be heard almost alone.

The pulse is characteristic. The hypertrophied left ventricle forces an increased amount of blood into the arota and through it into the peripheral arteries in which the return flow of the blood during the preceding diastole had produced a very considerable lowering of the blood pressure in comparison with the normal. The increased amount of blood which is forced out with every ventricular systole, and the greater difference (compared with the normal) between the systolic and the diastolic blood pressure in the arteries produce a more vigorous 
and more rapidly increasing wave which disappears just as rapidly, because after the termination of the systole the blood can flow off rapidly toward the periphery and the heart. Accordingly the pulse is strong, large, rapid and bounding (Fig. 195a). A bounding pulse may be felt even in the smaller arteries, and indeed in the eye ground the pulsation of the arteries can usually be seen clearly. The larger, and often also the smaller arteries show systolic pulsation and sometimes a systolic pulsation of the liver may be observed. At the same time a clapping vascular sound may often be heard even over smaller arteries. Finally the mucous membranes may suddenly become reddened synchronously with each ventricular systole; this redness may be emphasized by producing previously a circumscribed pallor by the pressure of a finger tip. This capillary pulsation sometimes occurs in the severe cases of aortic insufficiency.

\section{(b) Stenosis of the Aortic Opening.}

In stenosis of the aortic opening the left ventricle is obliged to force the normal amount of blood through an opening which is smaller than normal, and this leads to the development of a systolic murmur and also requires an increased expenditure of energy which in its turn causes an increase in the thickness of the left ventricular wall.

The heart beat may therefore be heard over a larger territory and either more strongly or less strongly, because the socalled closure time of the heart function is omitted. Simultaneously with the heart beat a systolic thrill may usually be felt. The cardiac dullness is increased only inconsiderably. The first heart sound is covered by a very strong, prolonged systolic murmur, which is separated from the diastolic sound by only a brief pause; this murmur may be so strong that it can be heard in the neighborhood of the animal. Its maximal point is in the fourth intercostal space immediately below the shoulder line (Fig. 193.2). The systolic sound which originated in the right heart, as well as the transmitted murmur are heard on the right side also. The weakened aortic sound may be heard in the left fourth intercostal space, below the shoulder line, while the pulmonal sound is heard in fairly normal intensity in the third intercostal space in the lower half of the left lower third. The pulse wave is sluggish; its growth may be followed with the palpating finger, because owing to the aortic stenosis the systolic pressure of the arteries increases much more slowly than is the case under normal conditions. At the same time the pulse is remarkably small and presents a distinct contrast to the heart beat which is usually increased. The systolic murmur is transmitted even into the smaller arteries, for instance, those of the head and of the extremities. 


\section{(c) Insufficiency of the Bicuspid or Mitral Valve.}

If the two flaps of the expanded bicuspid valve do not tonch, a part of the blood in the left ventricle flows back into the left auricle at each ventricular systole, and the blood of the pulmonary veins is emptied into this auricle at the same time. This canses the pressure in the auricle to be increased, it becomes dilated and eventually hypertrophied, although the hypertrophy is usually not very marked. But since the auricular wall cannot expend the force sufficient to remove the increased pressure and since at each auricular systole blood is forced into the pulmonary veins which are not provided with valves, the stasis is continued upward into the pulmonary veins and from these through the comparatively wide pulmonary capillaries, to the pulmonary artery and the right ventricle, the muscular wall of which is hypertrophied in consequence. There occurs therefore an increase in blood pressure affecting the entire lesser circulation, which is compensated in the right ventricle. The persistent passive hyperemia of the limgs gradually leads to the development of the socalled brown induration of the lung tissne. The muscle of the left rentricle also is usually thickened, although not greatly, because at each systole it is obliged to force out a larger amount of blood.

The heart beat is usually normal on the left side, but increased on the right side; in horses a contraction is observed commencing immediately after the beginning of the ventricular systole, instead of the normal well-marked systolic rise in the region of the heart during systole. Simultaneonsly with the heart beat a systolic thrill may often be felt. The cardiac dullness is not infrequently unchanged, but at times it is somewhat enlarged on the right side, which may be seen by the fact that in the fourth and also in the fifth intercostal space on the right side a distinct cardiac dullness is manifest. On the left side the cardiac dullness is only rarely noticeably increased. Instead of the first heart sound a prolonged, blowing or rushing systolic murmur is heard on the left side, the punctum maximum of which is in the fifth intercostal space, in the middle of the left lower third of the chest (Fig. 193.1). In the intercostal spaces lying in front of it the systolic sonnd originating in the right ventricle may also be heard more clearly, and it is audible quite listinctly in the right 3d-4th intercostal spaces (Fig. 194.1), the transmitted and much attenuated murmur being also perceptible. The diastolic sound is clear, the second aortic sound usually normal in strength, the second pulmonic sound (in the left third intercostal space in the lower half of the lower third of the chest [Fig. 193.3]) much increased, clear and short, i. e., accentuated, because the blood column, which within the pulmonary artery is under an increased pressure, is thrown with greater force against the semilunar valves. The pulse presents 
no particular deviations, becoming weaker only in severe cases. The transmitted systolic murmur may usually be heard also over the larger arteries (carotis, first part of the abdominal aorta).

Owing to the continued increased blood pressure in the lungs superficial and accelerated breathing is noted, and also bronchial catarrh. The patients are inclined to pulmonary edema.

\section{(d) Stenosis of the Left Auriculo-Ventricular Opening.}

In a narrowing of the left auriculo-ventricular opening the entrance of the blood from the auricle into the ventricle during the ventricular diastole is impeded, so that a dilatation of the left ventricle is produced with subsequent hypertrophy. But as in spite of the greater work accomplished by the thin-walled auricle it is not capable of preventing the damming up of the blood, the stasis is continued to the right ventricle through the pulmonary veins and arteries. This leads in its turn to an increase of pressure in the pulmonary circulation and to hypertrophy of the right ventricle, while the left ventricle rather becomes smaller.

The heart beat and cardiac dullness are the same as in the preceding lesions. Of the heart sounds the first one is clear everywhere, but the second is accompanied by a diastolic murmur which is most marked in the left fifth intercostal space, in the middle of the lower third of the chest (Fig. 193.1). The murmur is not usually loud and either is heard through the entire diastole, being separated from the systole only by a short pause, or only at the commencement, eventually only near the end of the diastole (presystolic murmur). Aside from the murmur the second aortic sound may be, and the second pulmonic sound always is, heard distinctly, the latter being even more accentuated than in mitral insufficiency. The pulse is always small and soft because the left ventricle forces less blood into the aorta. The lungs show the same disturbances as in mitral insufficiency.

\section{(e) Insufficiency of the Pulmonary Valves.}

In this condition the right ventricle receives blood from two directions during each ventricular diastole, namely, the blood which regurgitates from the pulmonary artery and that which flows in from the right auricle; and accommodative dilatation of the right ventricle occurs in consequence of the excessive blood supply, which is soon followed by hypertrophy.

The ventricle does more work than normally and forces an increased amount of blood to a place where the blood pressure is lowered, the blood therefore flows more rapidly into the 
pulmonary artery and this will at times eventually dilate. As the expulsive force gradually diminishes, the right ventricle is finally dilated to such a degree that the right auriculo-ventricnlar opening also enlarges so far as to produce a relative insufficiency of the tricuspicl valve. The insufficiency of the pulmonary valves remains without effect upon the lungs and the left heart as long as it is compensated by the increased work accomplished by the right ventricle.

The heart beat and the cardiac dullness show similar conditions as in diseases of the left venous ostium. The first heart sound is clear everywhere; instead of the second sound a prolonged diastolic murmur which gradually becomes weaker in all directions is heard over the origin of the pulmonary artery (Fig. 193. 3), while the second aortic sound becomes constantly more distinct. No particular changes are noted in the pulse. The disease is always accompanied by respiratory disturbances.

\section{(f) Stenosis of the Opening of the Pulmonary Artery.}

The blood which is forced ont of the right ventricle against the pulmonary artery meets, in the diminished opening of the pulmonary artery, an impediment which increases the work of the right ventricle and leads to hypertrophy of its muscle. The blood pressure in the lungs is lowered permanently, and the amount of blood in the pulmonary arteries becomes less as soon as the energy of the right ventricle diminishes.

The heart beat and the cardiac dullness show the same deviations as in the preceding form. Over the origin of the pulmonary artery (Fig. 193.3) a loud, prolonged systolic murmur is heard, and more posteriorly also the first heart sound which originates in the left ventricle; the second sound is clear but weakened over the pulmonary artery. The pulse is small and feeble. Respiratory disturbances and cyanosis are noted from the first.

\section{(g) Insufficiency of the Tricuspid Valve.}

If the unfolded tricuspid valve does not close completely, a part of the blood in the right ventricle flows, during systole, back into the right auricle through a more or less narrow opening, the auricle is dilated and eventually slightly hypertrophied.

Since the auricular wall is capable of exerting only a slight force, and since the openings of the venae cavae are not closed by valves, the stasis of the blood is continued into venae cavae and into the other veins of the body.

The heart beat differs from the normal at most in so far as a systolic thrill is often felt simultaneously in the $3 \mathrm{~d}-4$ th intercostal spaces. The cardiac dullness is not altered. The first heart sound is heard in the left 5th-6th intercostal spaces, either 
quite clear or together with a very faint transmitted murmur; the systolic murmur reaches its greatest intensity in the right 3d-4th intercostal spaces toward the middle of the lower third of the chest. (Fig. 194.1.) The diastolic sound is either of normal intensity or frequently becomes weaker and in such cases the pulse becomes feeble and small. The veins are filled strongly; the jugular veins appear as thick cords and show a systolic (positive) pulsation following immediately upon the heart beat, which may, however, not be perceived owing to a long hair coat, especially in dogs. If the auricle could not remove its entire blood content, owing to its rapidly diminished functioning power a certain amount of blood is forced at each systole from

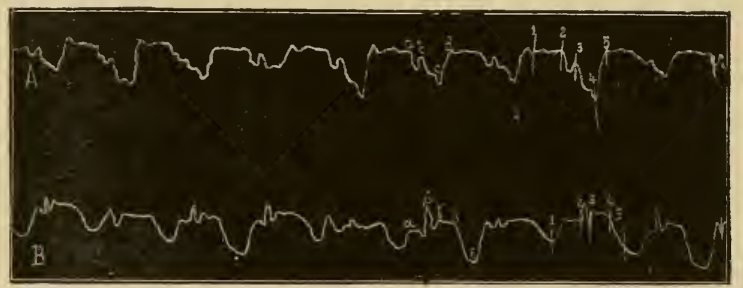

Fig. 196. Cardio-and Phlebigraph of a horse with insufficiency of the bicuspid and tricuspid valves. A Cardiograpl. Instead of the elevation which occurs normally at the begimning of ventricular systole, a descending line is noted $(a-c)$, due to the omission of the closure of the right ventricle; the line is interrupted by a small apex $(b)$ which corresponds to the systole of the left ventricle. At the beginning of ventricular diastole $(c-d)$ the pressure in the ventricles suddenly increases. $B$ Phlebigraph. a negative venous pulse; $\beta$ positive venous pulse; $\gamma$ carotid pulse; $\delta \epsilon$ rapid emptying of the jugular vein at the beginning of the ventricular diastole.

the right ventricle into the right auricle and from this into the vena cava. By the regurgitation of the blood into the venae cavae a systolic wave is produced and is continued to the jugular veins. The systolic venous pulse represents a pathognomic phenomenon of tricuspid insufficiency, but it must not be confounded with the presystolic (negative) pulsation, which also occurs in this heart lesion and precedes the heart beat, or with the pulsation of the carotis. Venous stasis leads soon to edemas.

\section{(h) Stenosis of the Right Auriculo-Ventricular Opening.}

A narrowing of the right auriculo-ventricular opening interferes with the flow of the blood from the right auricle into the ventricle, thus causing slight hypertrophy and at the same time a considerable dilatation of the right auricle. Owing to the latter cause blood-stasis occurs in the venae cavae from the beginning and is continued toward the periphery. On the other hand the other portions of the heart receive less blood and 
therefore become smaller. In consequence of the diminished pressure in the right rentricle and in the pulmonary artery thrombi easily form in them which may lead to embolism in the blood ressels of the lungs.

The heart beat is weak, a cardiac dullness can either not be demonstrated at all or it is diminished in extent. The first heart sound is clear, the second is accompanied by a presystolic or diastolic murmur (for similar reasons as in mitral stenosis) which is heard most strongly in the right $3 d-4$ th intercostal spaces in the middle of the lower third of the chest. The pulse is feeble and small. The veins are filled strongly and a presystolic (negative) renous pulsation, as well as undulation, may be observed. The characteristic signs of severe blood-stasis, especially marked dyspnea, possibly attacks of asphyxia, occur already early in the disease.

\section{(i) Congenital Heart Lesions.}

Clinical manifestations of congenital heart lesions have been described only rarely. The most decided stmptom usually consists in considerable weakness. The newborn animals are not capable of looking for their mothers. They stand with hardly a movement or cannot even keep their feet. Sometimes at rest, more often on motion, severe dyspnea and cyanosis are observed. Congenital valvular lesions give rise to the same local symptoms as the acquired forms, but murmurs are mostly absent in cardiac lesions which are due to faulty development, or they appear only in those cases in which there is a difference in the pressure in two communicating heart portions, provided the abnormal opening is not too large. In two cattle with persistent interventricular openings Huynen observed a systolic nurmur in the left heart region and an accentuater diastoiic heart sound.

The newborn animals usually die after a short time or are killed, as they are not fit to be raised. In rare cases they develop normally and may even live a fairly long time.

\section{(k) Combined Valvular Lesions.}

The clinical pictures of valvular and ostial affections are not always found as well marked as they have been described; in reality the forms which have been discussed separately often occur in combinations. The most frequent combinations are those in which a stenosis of the related ostium coexists with insufficiency of a certain valve. Since valvular insufficiency is, in a way, the opposite of stenosis, the individual components of the clinical picture lessen each other; an exception occurring only in the accentuation of the second pulmonic sound in combined disease of the left auriculo-rentricular opening. On this 
account the discernible deviations lose more or less in distinctness and are often confused into a clinical picture which is difficult to analyze. In insufficiency of the aortic valves and coexisting aortic stenosis, for instance, systolic as well as diastolic murmurs are discovered, while the pulse is either not bounding or only slightly so, because the variations in pressure in the arteries are not so considerable and less abrupt.

The clinical picture is even more complicated if two or several ostia and valves are involved simultaneously. Frequently only portions of one half of the heart are diseased, for instance, the aortic valves and at the same time the bicuspid valve, but sometimes morbid changes are found in the right as well as in the left halves of the heart, as in diseases of the left venous or arterial ostium simultaneously with tricuspid insufficiency.

As it is possible for two anomalies to occur in each of four places, i. e., insufficiency and stenosis, and as these eight individual forces may enter the most variable combinations with each other (a total of 247 combinations is possible), the variability of valvular lesions observed in the different cases is not astonishing, and at the same time renders descriptions of the clinical pictures suitable for all possible contingencies an impossibility.

Insufficiency of a valve or stenosis of an ostium disturbs the normal process of the circulation of the blood by producing a permanent rise in the blood pressure in the parts of the heart which lie proximally to the seat of the disease, and, in diseases of the left venous ostium, also in the pulmonary vessels. In order to maintain a normal, or at least approximately normal velocity of the blood current in the body and a normal blood pressure, certain parts of the heart are forced to accomplish continuously an increased amount of work. This causes their walls eventually to hypertrophy, and the heart is then enabled to accomplish the plus of work without constantly drawing upon its reserve strength. As long as the increased work of certain parts of the heart maintains the normal velocity of the blood current, and as long as therefore the functions of the various organs rest undisturbed in spite of the existing valvular lesions, with the exception of certain local disturbances which are constant accompaniments of some heart lesions: a compensation of the valvular lesion is said to exist. However, in this stage the function of the organs is usually undisturbed only in a condition of rest, in greater muscular exertions and still more in over-exertion transitory signs of incompensation may appear. Sometimes, especially in insufficiency of the aortic valves, the heart may be equal even to these excessively increased demands. On the other hand, in certain forms of valvular troubles the function of the organs is impaired to a certain degree, even while at rest, in spite of existing compensation; for instance, in diseases of the left auriculo-ventricular opening, the increased pressure in the lungs causes superficial and hastened respira- 
tion, while in aortic stenosis symptoms of cerebral anemia may exist.

The anatomical changes afford a satisfactory explanation why in diseases of the aortic ostium the symptoms of stasis generally become manifest later than in other valvular diseases. Not only is the most vigorous portion of the heart back of the affected portion in this case, but the weakening of the left ventricle causes a notable stasis only in the lesser circulation, which can moreover be compensated by the right ventricle. If, on the other hand, the right ventricle is obliged to compensate a heart lesion, the stasis continues, immediately to the right auricle and to the whole venous system, when the right ventricle becomes fatigued. Most unfavorable are conditions in diseases of the right venous ostium because the thin-walled right auricle is hardly capable of producing a compensation worth mentioning; in valvular diseases of the last variety a stage of compensation can therefore hardly be considered.

The cause of the permanent incompensation or decompensation which develops after a certain time can at present not be explained satisfactorily for all cases. In a number of cases the fact that the heart muscle is finally incapable of overcoming the constantly increasing difficulties must probably be attributed to the gradually increasing obstacles to the blood current. A similar effect results from frequently repeated severe muscular exertion, and from pulmonary edema which is not rare, especially in horses. In other cases the heart muscle is weakened by myocardial inflammation which developed simultaneously with or in consequence of the endocarditis. The strength of the heart muscle is also reduced by an extensive degeneration, while a slight degree of degeneration is not important in this respect. Finally, a functional disturbance of the heart muscle must be assumed, in a considerable number of cases, to form the basis of incompensation, which cannot at present be demonstrated histologically.

The incompensation is manifested by evidences of heart weakness, that is, by lowered arterial pressure and by venous stasis. The slowing of the blood current and the increased fullness of the veins become manifest in the appearance of cyanosis, especially a bluish-red discoloration of the mucous membranes and even of the umpigmented skin (especially in $\operatorname{logs})$; sometimes dilatation and perhaps an undulating course of the veins are also observed. The fullness of the jugular veins is particularly striking and is not infrequently associated with venous pulsations. Dropsical effusions into the body cavities and edemas are further occurrences. Edematous swellings appear first on the front and lower chest, on the lower abromen and in the lower portions of the extremities, although in the last location they usually do not attain a considerahle degree, owing to the tightness of the skin, especially in large animals. In the 
body cavities the effusion usually occurs rather early, first of all in the abdominal cavity.

The stasis in the brain and its membranes causes more or less striking disturbances in consciousness and attacks of vertigo, especially while at work, which are sometimes repeated very frequently, even as often as 10-20 times a day. During such attacks horses frequently fall on their knees while in motion (Katzke is inclined to consider this as a phenomenon which is characteristic of heart lesions).

The stasis of blood in the lesser circulation produces respiratory disturbances; foundering is in some of the cases due to a valvular lesion. Chronic bronchial catarrh is a frequent sequel of stasis in the pulmonary circulation. Rarely a bloody or saffron yellow nasal discharge occurs in consequence of pulmonary hemorrhage which, however, in exceptional cases causes death by asphyxia. Epistaxis occurs, though rarely. Toward the end of the disease pulmonary edema develops almost always as a terminal phenomenon.

The passive hyperemia in the digestive organs produces symptoms of chronic gastro-intestinal catarrh, and nutritional disturbances develop at the same time. The stasis in the renal veins leads to a diminution in the amount of urine, while the specific gravity is increased and the urine frequently contains albumen, and occasionally hyaline casts.

A constant symptom of incompensation is presented by the disturbed cardiac action, which finds expression in arhythmia and feeble pulse while at rest, and still more on motion.

Loosened portions of thrombi finally may give rise to embolism in various organs. In this manner emboli may get into the lungs from the right heart and into the other organs from the left lung. Cerebral emboli and also cerebral hemorrhages may be followed immediately by sudden death.

Course. Valvular disease is characterized by a very changeable course. Aside from the fact that its influence upon the circulation varies according to localization and intensity, the course is modified by external conditions and by work. While dogs which often pass their lives in quiet comfort not rarely live for years with valvular disease in animals which are employed for work, especially in horses, this becomes aggravated, relatively early, to such a degree that they become useless for exertion. For the same reason symptoms of stasis, the development of which requires considerable time, are observed in horses more rarely; they show instead signs of rapidly increasing dyspnea and usually die suddenly of pulmonary edema, cardiac paralysis or rupture of the heart, during work or while in motion. In dogs the gradual development of the sequelae of cardiac anomalies, up to severe edematous infiltrations, can be observer far better; in cattle and in hogs there is far less oppor- 
iunity because these animals are usually slaughtered before the occurrence of these symptoms.

The disease takes a more rapid course in very young and in very old animals, because both lack the strength which is required for a sufficient compensation. Congenital heart lesions lead to death, with symptoms of severe blood stasis, usually during the first few weeks or months of life, although occasionally the animals become older. Such cases have been observed by Thomas, Walley, Jolme, Hutyra, Liénaux.

Diagnosis. The recognition of valvular disease is only possible on the basis of an exact examination, which also considers the clinical pictures described above. In the interpretation of the results the following points should be considered, in addition to the conditions which have already been related.

Sometimes so-called anorganic murmurs are heard without valvular lesions and without any impediments to the circulation in the heart; these murmurs are heard more frequently during systole, only very rarely luring diastole. These murmurs may be eliminated if in addition enlargement of a portion of the heart or accentuation of the second sound can be demonstrated, or if a thrill can be felt ("frémissement cataire").

The absence of murmurs does not exclude the presence of a valvular lesion, because in case of slight insufficiency or stenosis, especially at rest, murmurs are not produced which are transmitted to the chest wall with sufficient intensity, and because in severe insufficiency the formation of murmurs may not occur, as in such a case the regurgitation of the blood does not take place through a narrow slit; finally, in the stage of incompensation too feeble contractions of the heart may canse the murmurs to disappear. In all these cases cardiac remedies and muscular exertion which have the effect of strengthening the contractions of the heart usually cause the murmur's to reappear. It appears therefore advisable in all cases in which a suspicion of heart disease exists, which includes all cases of dyspnea the nature of which is not quite evident, to examine the animals immediately after work and particularly to auscultate the heart region. Nor should it be forgotten that in aortic aneurysm murmurs are heard which correspond to the endocardial murmurs.

In domestic animals the recognition of the several valvular anomalies is always successful in the stage of compensation, sometimes also in compensated cases, if the character of heart beat, heart sounds and cardiac dullness is observed and the punctum maximum of the murmurs is ascertained; a task which is not particularly difficult in horses. A murmur which has originated in any section of the heart is naturally heard most strongly at that place where the related part of the heart is nearest to the chest wall; but a decided dilatation of the heart with displacement of the heart sections produces also a dis- 
placement of the puncta maxima. (Figs. 188, 189.) The nature of the pulse is of great importance for diagnosis; in fact, its significance is often decisive, and the same is true for the character of the arteries and veins. Finally the frequency of the several valvular anomalies must be considered on making a diagnosis.

With the determination of a valvular lesion the proof has not yet been afforded that this lesion is based upon a chronic endocarditis, although valvular disease depends upon this in a great majority of cases. The actual cause of a heart lesion can only then be ascertained with absolute certainty in living animals if the development of the disease could be traced from an acute endocarditis, in connection with an infectious disease or with chronic arthritis. On the other hand, the absolute disappearance of endocardial murmurs and of the symptoms of insufficiency, after the systematic administration of cardiotonic remedies and during pliysical rest admits the assumption of a muscular insufficiency.

Prognosis. Althougl the prognosis of chronic valvular lesions is always unfavorable in regard to the possibility of healing, the various forms of valvular anomaly require different interpretation with respect to their effect upon life and the usefulness of the animals. Stenosis of the ostia is in general a more severe disease than is insufficiency of the related valves, and disease of both valves of the heart is always more dangerous. Of comparatively the least dangerous significance are diseases of the aortic opening, because the strong-walled left ventricle maintains its functioning power for a fairly long time. Disease of the left auriculo-ventricular opening must be judged less favorably because the right ventricle is capable of less exertion and because in addition oxidation is interfered with by the persistently increased pressure in the lungs. The valvular lesions which are localized in the right heart are always of very unfavorable significance; that of the pulmonary opening because the lungs receive less blood, that of the right auriculoventricular opening because the auricle which is to produce the compensation is incapable of doing so.

The symptoms cis incompensation are always serious, and the more so the less they are to be referred to external conditions. Of course, the prognosis is the less favorable the less the animal can be protected against exciting and exhausting influences. If the disease has existed only a short time, the age and general condition of the patient must be 'considered.

Treatment. Since healing of valvular lesions is impossible of accomplishment, the treatment must attempt to postpone an aggravation of the fairly good condition by a proper regulation of the diet, as long as symptoms of incompensation have not 
made their appearance. The patients must therefore be protected from all influences which impose an increased amount of work on the heart, which suddenly change the blood pressure or irritate the heart in any way. It is therefore necessary to regulate the diet thus, that the animals receive no irritating substances with their food (which must be insisted upon especially in carnivora), that not too much food is taken in at one time, but that the usual daily ration is eaten in several small portions. They must be supplied witl pure air or led around in the open. 'The work done by the patients must be supervised very carefully. The animals are capable of accomplishing light, quiet and miform work in proportion to the mildness of their heart trouble. Experience has shown that, if these precautions are observed, animals with heart disease mar do satisfactory work even for rears, especially in agricultural employment. Finally the digestion must be watched becanse fecal retention increases the abdominal pressure, and therefore the activity of the heart. In such cases the administration of mild laxatives, especially of mineral salts, eastor oil, rhubarb or aloes is indicated.

In the stages of incompensation (and veterinary aid is usually requested only at this time) absolute rest must be insured and the action of the heart regulated. Above all digitalis does good service because the heart contracts more vigorously and less frequently under its influence, so that the muscles obtain more rest during the longer pauses. Larger animals should receive, if possible, powdered fresh leaves (horses 2-4 gm., cattle 4-6.0 gm. daily), dogs an infusion (1-2.0 gm.; 200.0 $\mathrm{gm}$. of water; $2-3$ tablespoonfuls daily), and the nitrate or acetate of potassium may be added to increase the diuresis. According to the investigations of Kunz-Krause \& Röder it appears better to employ Folia Digitalis dialysata (for larger animals 5-15 ec. per os) or the Digitalysatum Bürger (20-30 (lrops subeutaneously for smaller animals). Maceration of digitalis leaves (maceration in pure water for 12-24 hours) may also be given to advantage. Digalen has an excellent action and is to be recommended, especially if dangerous cardiac weakness is present (horses $5-15$ cc., cattle 20 cc., subcutaneously; or 15-30 ce. intravenously; dogs 5-15 ce. or 0.857 ce. per kg. of body weight), but if required for prolonged treatment it will be employed only in valuable or valued animals. Digitalis being rendered inactive in the stomach of the ruminants (Salvisberg), those preparations should be selected for these animals which are adapted for subcutaneous administration. Diehl observed abortion in pregnant cows after the use of digitalis. The tincture of strophanthus $(10-25.0 \mathrm{gm}$. for large animals, the same number of drops in smaller animals, daily) has been found unreliable in contrast to Strophanthinum depuratum, Merck, the good action of which is constant. It is given subcutaneously in aqueous solution (soluble in 40 parts of water) (0.002-0.003 $\mathrm{gm}$. for horses, 0.00025-0.001 for dogs). 
At the point of injection painful swellings occur and sometimes necrosis of the skin (Fröhner, Author's case). Caffeine also lias an excellent effect (caffeine sodium-benzoate or sodiumsalicylate $6-8.0 \mathrm{gm}$. for large animals; $0.5-1.0$ for small animals subcutaneously).

In pulmonary stasis, and also in general, moderate venesection may prove advantageous.

In case of distressing palpitation cold compresses upon the region of the heart will be found beneficial, also subcutaneous injections of morphine $(0.20-0.50 \mathrm{gm}$. or $0.01-0.03 \mathrm{gm}$.). Liénaux treats the annoying cough of coexisting bronchial catarrh successfully with apomorphine (0.005-0.05 for large, 0.003-0.005 gm. for smaller dogs, subcutaneously).

In edema of the subentaneous connective tissue calomel may be tried, since in human medicine a remarkable increase in urine and a rapid diminution of the edemas has repeatedly been observed after its administration (for dogs 0.05-0.10 gm. 3-4 times daily alone or with an equal amount of jalap. The other diuretic remedies (acetate of potassium, squills, etc.) may also be employed and at the same time the daily amount of drinking water must be reduced. Very effective is further agurin (for dogs daily 1-3 gm. dissolved in water), theocin (in like doses), theophyllin (in like doses). Together with the diminution of the subcutaneous edemas the transudations in the body cavities frequently also become less, and these may, moreover, be removed temporarily by repeated punction. If this is, however, followed immediately by a reaccumulation of serum, it should not be repeated.

Finally an existing anemia is treated by the systematic administration of iron and by appropriate feeding.

Literature. Bálint, Közl., 1897. II. 239. - Brunninger, A. f. Tk., 1895. XXI. 39 (Lit. on supposed heart lesions). - Cadiot, Rec., 1892. 568. - Coppel, Chron. Klappenerkrank. beim Hunde, Diss, Leipzig, 1909 (Lit.). - Fiebiger, Z. f. Tm., 1902. VI. 54. - Hartl, Z. f. Tm., 1902. VI. 450 - Húynen, Ann.,.1908. 391. - Korányi, Orv. H., 1906. 1019. - Kunz-Krause \& Röder, Monh., 1906. XVII. 344. - Liénaux, Ann., 1906. 325. - Lustig, Hann. Jhb., 1884-85. 53, - Malkmus, D. t. W., 1902. 389, - Marek, Z. f. Tm., 1906, X. 448. - Martius, Erg. d. Path., 1894. I. 2. Abt. 38. - Oppermann, D. t. W., 1905. 307. - Preisz, D. Z. f. Tm., 1898. XXIV. 169. (Lit.). - Regenbogen, Monh., 1904. XV. 418. - Salvisberg, Uber die Wirkung d. Digitalis usw., Diss. Bern., 1907. - Schütz, A. f. Tk., 1882, VIII. 139. - Sonnenberg, B. t. W., 1908. 213. - Trasbot, A. d'Alf., 1878. 210. Wilhelm, S. B., 1881. 77. - Zschokke, Schw. A., 1907. XLIX. 322. 
Section III.

\section{DISEASES OF THE BLOOD VESSELS.}

\section{Chronic Inflammation of the Arterial Wall. Endoarteritis Chronica.}

\section{(Endoarteritis chronica deformans. Atheromatosis, Arterio- sclerosis, Atherosclerosis.)}

Occurrence. Chronic inflammation of the arterial wall as an independent disease is apt to develop at a more advanced age; it is only rarely observed in animals, and then limited in contrast to man to the large arterial trunks. Only Lüpke saw four cases of periarteritis nodosa in axis-deer, which affects almost all arteries of the general circulation, not excepting the smallest blood vessels.

According to an inquiry of M. Schmidt an endoarteritis of the aorta could be shown macroscopically in $0.5 \%$ out of 2,069 horses autopsied in the pathologicanatomical institute in Budapest, according to Kitt a chronic inflammation is frequently met with at the origin of the aorta of older horses. Lyding found endoarteritic changes in the aortae of 35 out of 100 cattle, and out of 10 each of horses or dogs these changes could be ascertained in two each. These observations, and also those made by Sequens and Caparini, permit the assumption that on careful examination the disease may be found more often in domestic animals than has hitherto been assumed.

Etiology. Concerning the causes of the disease nothing positive is known. In a part of the cases the degenerative process probably arises from an insufficient nutrition of the tissues in advanced age. Whether severe exertion may be a factor, by raising the blood pressure repeatedly, is not certain.

In rabbits intravenous injections of adrenalin, continued for several weeks, give rise to a condition similar to arteriosclerosis, which however is limited exclusively to the aorta. Opinions are not yet in accord, as to whether the disease proceeds from the toxic action of the adrenalin upon the tissue elements of the media, or from the increased pressure in the arterial system which results from the administration of adrenalin (Erb jun., Külbs, B. Fischer, Scheidemandel, Korányi, K. Ziegler). Most authors take these changes to be arterionecrosis in contrast to arteriosclerosis. In this connection the experiments of Reinecke with barium chloride merit consideration, because the same changes were produced as by adrenalin, and even more the experiments of Philisophow, who succeeded in effecting the same process with salts of mercury, lead and zinc, that is, with substances which do not raise the blood pressure. A particular importance must finally be attributed to the experiments of Saltykow with saprophytio and killed virulent staphylococci, in which the changes in the aortic wall are claimed to correspont with those of arteriosclerosis. 
These experiments render it not impossible that the inflammation of the arterial wall usually develops under the influence of certain chemi 1 substances or bacterial toxins. In human medicine the exccsive use of alcohol and tobacco, gout, Bright's disease, syphilis and met lic poisoning are believed to stand in relation in this respect.

Circumscribed

barteritis very frequently arises in liorses under the influence of the Sclerostomum vulgare, preferably in the Arteria mesenterica anterior, not rarely in the aorta or in the arteries of the extremities, exceptionally also in the arteries of the other organs, including those of the heart (Cadiot, Piot-Bey, Magnin). A similar influence is exerted by the much less frequent Spiroptera sanguinolenta upon the aorta of the dog, the Filaria Poelsi (Vryburg) or the Filaria Blini (Carougeau \& Marotel) upon the aorta of buffalo in Sumatra, sometimes also upon that of cattle, without, however, causing disturbances in the health of the animals. In cattle the inflammatory process is sometimes produced by foreign bodies (Seiberth).

The virus of periarteritis nodosa in axis-deer could not be determined by Iüpke (the cocci which were repeatedly demonstrated in the blood and in the diseased organs failed to produce the disease in small experiment animals).

Anatomical Changes. In the incipient stage of the process small, elongated or round, white or pale yellow, shallow elevations are found on the intima of the artery, which are most numerous at those places at which the blood is thrown most strongly against the wall, for instance at the turns of main arteries, at places where branches are given off, etc. Corresponding to these spots there exists, in horses and cattle, a proliferation of the connective tissue, with a slight fatty degeneration and calcification, in dogs the media and intima are atrophied and there is fatty and calcareous degeneration. Cavity formation is rare, ulcerous destruction (atheromatous ulcer) is completely missing (Lyding). Eventually the arterial wall bulges and becomes rigid in consequence of diminished elastioity.

Calcification of the aortio wall occurs frequently in old birds, less so in horses and cattle; in the latter it is found, according to Lignières, as a sequel of the Argentinian disease known under the name of "Entéqué," not only in the walls of the aorta but also in those of the smaller arteries. In pigeons the Hypodeotes oolumbarum which exists as a parasite in the vicinity of large vessels may give rise to similar changes.

In periarteritis nodosa the arterial wall is thickened, consequently the vascular lumen is smaller or entirely obliterated. A nodose thickening of the arterial wall is observed in varying degree. The lumen is narrowed or obliterated or it is bulged out and often contains thrombi.

Symptoms. A generalized arteriosclerosis can, in a fairly advanced stage be recognized only if the vessels which are imme-

vol. 1-70 
diately accessible to examination are involved. The walls of the subcutaneous arteriosclerotic vessels give to the touch a peculiar rigid and sometimes uneven firmness, the pulse is sluggish but at the same time fairly strong because the left ventricle is usually hypertrophied. The aortic sound is sometimes accentuated in consequence.

The changes in the vascular walls may occasionally be recognized in the abdominal aorta and in its pelvic branches. In other cases the presence of the disease can only be assumed when the left ventricle becomes hypertrophic without manifest cause or when symptoms of thrombosis or embolism make their appearance.

Periarteritis of axis-deer is, according to Lüpke, manifested in gradually increasing digestive disturbances which show much similarity with the elinical picture of chronic intestinal catarrh. In spite of fairly goorl appetite a gradually progressive emaciation becomes noticeable which passes into a sickly condition lasting for weeks and months.

For the symptoms of circumscribed arteriosclerosis the following chapters should be consulted.

Treatment. The further development of an established inflammation of the arteries cannot be arrested in any way and can not even be retarded. In human medicine iodide of potassium is administered internally in arteriosclerosis. Korányi succeeded in obtaining a favorable influence upon the process, which had been produced in rabbits by injections of adrenalin, or with subcutaneous injections of iodipin, but Fleischer \& Loeb could not confirm his results.

Literature. Carougeau \& Marotel, Rev. gén., 1903. I. 447. - Erb, A. f. exp. Path. u. Phmk., 1905. LIII. 140. - Gulyás, A. L., 1906. 73. - Josué, Presse méd., 1904. 281. - Korányi, D. m. W., 1906. 679. - Lüpke, Verh. d. Deutschen Path. Gesellsch., 1906. 149. - Lyding, Z. f. Tm., 1907. XI. 359. - Philisophow, V. A., 1910. CXYO. 238. - Seheidemandel, V. A., 1905. CLXXXI. 363. - Saltykow, Beitr. z. path. An., 1908. XLIII. 147. - Sequens, Vet., 1892. 352.

\section{Dilatation of the Aorta. Aneurysma Aortae.}

Occurrence. The disease occurs relatively most.often in horses, mueh $\cdot$ less often in dogs and cattle; in other animals it is hardly ever' seen.

Etiology. The walls of the aorta and those of the arteries in general, with very few exceptions, become dilated only when their power of resistance has been reduced, for instance in the presence of chronic endoarteritis; especially when the blood pressure is lastingly raised. This evidently accounts for the fact that aortic aneurysm is observed most frequently in horses because these animals are often employed for hard labor.

It might be interesting to ascertain in how far the relation between the frequency of aneurysm to kind, species and employment of the animals is manifested. According to Tombari the severe exertions which hunters are obliged to 
undergo may be considered to stand in causal relation to the frequency of aneurysm, and according to Palat the same holds true for 'bus horses.

In exceptional cases the commencement of the derelopment of an aneurysm corresponds, as to time, with external influences, such as moving a heavy load, jumping, being thrown, or struck on the trunk, etc., or it may be refer'ed to such an accident with a degree of probability. In such cases the traumatic factor of a sudden excessive rise in blood pressure might cause a partial tear in the aortic wall, in consequence of which the weakened arterial wall eventually bulges out. On the other hand it is not impossible that even in such cases the wall of the vessel was not quite healthy before the accident.

Anatomical Changes. A portion of the aorta is either evenly dilated (Aneurysma cylindricum) or the healthy portion gradually passes into a dilated segment, which again gradually passes into the healthy part of the ressel ( $A$. fusiforme), or only a short segment of the vessel is sacculated (A. sacciforme). In the latter case the sacculation either affects the entire circumference of the vessel (A. sacciforme periphericum s. axiale) or only a part of it (A. sacciforme semiperiphericum). The sacculated bulging may reach in hor'ses the size of a man's head (Labat \& Cadéac), in dogs the size of a turkey's egg (Barrier). The wall of the aneurysm is usually thickened, although in places it may be thinned out, owing to compression by neighlboring organs or from other causes. The thickening of the wall may be aided by layers of fibrin, which are sometimes deposited on its inner surface, and these may entirely obliterate the lumen of smaller vessels.

The adjoining organs are crowded aside by the constantly increasing aneurysm or they are compressed. Usually the trachea or a bronchus are found compressed, one or more vertebral bodies atrophic, the Vagus or the N. Recurrens compressed. Loosened portions of fibrin are carried away with the blood and are usually arrested in the vessels of the posterior half of the body:

The bursting of an aneurysm almost always leads to fatal internal hemorrhage. The break may occur into the cavity of the thorax or into the abdominal cavity, or the blood may be poured into a contiguous organ to which the aneurysm had become adherent. In this manner hemorrhages may take place into the bronchi, the lungs, the stomach, the intestine or between the layers of the mesentery.

Symptoms. The symptoms of aortic aneurysm in animals have in the past not been observed with sufficient exactness. In the majority of cases the reports mention only increasing emaciation and weakness or sudden fatal internal hemorrhage.

Owing to the anatomical conditions only aneurysms which 
have formed on the abdominal aorta can be palpated, in larger animals from the rectum, in smaller animals through the abdominal wall. Below the vertebral column, slightly to the left, a pulsating swelling is felt, of variable size, elongated or spherical, firm or slightly elastic, which is continued into the normal segment of the aorta or into the two pelvic arteries. Sometimes the character of the swelling cannot be determined exactly; that is in cases in which edematous swellings or tough connective tissue have formed around it.

The pulsation of the swelling does not, of itself, prore the presence of an aneurysm, because tumors or abscesses connected with the aorta may show a similar pulsation, although it is then only by continuation. If it can however be determined by grasping the swelling, that it becomes liarder and larger with each pulsation, an anenrysm is undoubtedly present.

An important symptom is presented by the retardation and sluggishness of the pulse, which may, however, be observed only in case of a considerable thrombosis. The pulse is felt distinctly after the heart beat, distally to the dilatation, becanse the copious deposit of fibrin has usually made the lumen of the vessel narrower and has thereby impeded the flow of blood toward the periphery; in consequence the increase in the systolic blood pressure, which is recognized as a pulse beat, reaches its climax, in which it can be felt, later. The vessels in which this retardation can be felt depend, of course, urwn the situation of the aneurysm. If this is at the origin of the aorta, the retardation is noticed in all arteries of the body, an aneurysm in the posterior aorta causes a retardation of the pulse only in the arteries of the posterior half of the body It may also occur that the pulse is not synchronous in the two corresponding lateral halves of the body, viz., if an artery branching out from the aneurysm has become narrower.

Over aneurysms which lie superficially or which form on the aorta, vascular murmurs are usually heard, which may be perceived as either one or two murmurs or as'a continued roaring. Over a pulsating swelling in the sacral region Tombari heard a blowing murmur, which had more the character of rubbing, while over a dilatation of the first segment of the posterior aorta Schmidt perceived a humming sound which was synchronous with the heart beat. In aneurysms at the origin of the aorta the murmurs are heard over the base of the heart and even lower down.

The left half of the heart is hypertrophied only in those cases in which extensive arteriosclerosis is present at the same time as the aneurysm or in which the lumen of the dilated aorta has become much narrower on account of thrombi. A dilatation at the origin of the aorta may lead to insufficiency of the semilunar aortic valves.

In case of considerable stenosis of the vascular lumen the nutrition of the parts of the body which are distal to the dilata- 
tion is impaired. In almost all cases which have been observed for some time, weakness of the hind quarter could be determined, which was mostly noted only during motion in exceptional cases also at rest (Barrier). Finally there are symptoms of cardiac weakness (rapid fatigue, strong filling of veins, vertigo, sometimes epileptoid convulsions [Lustig]).

Nervous symptoms are observed only in exceptional cases, when a nerve trunk has been compressed by the aneurysm. In Vagus compression, for instance, the heart beat may be accelerated; compression of the N. Recurrens may cause laryngeal whistling. In a case observed by Schmidt the horse gave evidences of severe pain on lowering the head.

Course. A fairly long time is probably required for the development of aneurysms to that degree in which they give rise to decided morbid changes, probably several years (in Tombari's case the disease had existed at least three years). Death is due either to the compression of neighboring organs or to circulatory disturbances, unless the bursting of the aneurysm terminates life suddenly, possibly with symptoms of internal hemorrhage or with symptoms of pulmonary or intestinal hemorrhage.

Treatment. A successful treatment of aortic aneurysm is not possible. The stimulation of blood clots within the aneurysm by electro-puncture, injections of chloride of iron, the introduction of foreign bodies does not enter into consideration in animals. Nor do subcutaneous injections of gelatin promise results (in man 50-130 gm., injected in one dose, of a 2-3\% solution). It may be mentioned, by the way, that tetanus infection should be prevented by employing Gelatina sterilisata pro injectione (Merck). The treatment is therefore limited to saving the animals from exertion and regulating any existing eardiac disturbances. The administration of iodide of potassium also seems to be useless.

Literature. Barrier, Bull., 1894. 327. - Gerlach, Z. f. Flhyg., 1905. XV. 115. - Labat \& Cadéac, Rev.' vét., 1884. 105. - Petit, Rec., 1905. 273, 299 ; Bull., 1906. 104. - Piot-Bey, Bull., 1905. 170. - Schmidt, A. f. Tk., 18s9. XV. 295. - Tombari, Vet. Jhb., 1882. 74.

Aneurisms of other blood vessels. Even more frequently than in the aorta, a dilatation of the anterior mesenteric artery and of its main branches occurs in horses under the influence of the Sclerostomum vulgare (Vol. II.), exceptionally and also from other causes in swine (Koch, Leibinger). In the other arteries of the abdominal cavity aneurisms are formed much more rarely, but they occur for instance in the Arteria coeliaca, the Arteria mesenterica posterior or in Arteria hepatica. In other branches of the aorta dilatations are also very rare. In Cadiot's case in an ass, and in three cases reported by Piot-Bey in cattle a dilatation of the coronary arteries had caused sudden death. Steinmeier saw a dilatation of the carotis cause sudden death by bursting and Reimers observed in the sacral region in a cow a tense, faintly pulsating bulging 
which corresponded to a dilated Arteria ischiadica. Querippel described a dilatation of the crural artery, Straube one of the Arteria digitorum communis volaris, Blase one of the Arteria pharyngea in a horse 15 years old; in the last case the symptoms consisted in edema and ulcerations on the head and neck, swelling of the tongue and gums, salivation, and the usual signs of pharyngitis; the aneurism eventually burst into the pharyngeal cavity. Vogel observed a dilatation of the facial artery in a cow, Mouquet one in the cervical artery in a horse, Ravenel one in the brachial artery in a hen. Nocard found in a young dog an aneurism of the aorta and at the same time one of the pulmonary artery. Mouquet found in a horse a dilatation of the pulmonary artery; Chardin in a horse a dilatation of the hepatic artery, which weighed $14 \mathrm{~kg}$. (the horse had frequently stood still on trotting and disliked to gallop), Dupas found varicose dilatations in the territory of the mesenteric veins, and Fröhner in a horse a dilatation of the external thoracic - vein.

General diminution in the tonus of the small arteries. (General vascular paralysis). A reduction in the tonus of all small arteries may be produced by certain chemical substances, like amyl nitrite, alcohol, chloral hydrate, chloroform, if they have been introduced into the organism in considerable amounts. Romberg, Bruhns, Pässler and Rolly demonstrated in their experiments a similar action on the part of the toxins of various infectious substances. From a general relaxation of the arteries it necessarily results that the abdominal vessels, the innervation of which is derived from the N. splanchnicus, and which especially in herbivora form a very wide system of blood vessels, receive a very considerable portion of the total blood quantity, so that the amount of blood in the other organs is diminished. The diminution in blood pressure and the slowing of the blood circulation cause an acceleration of the contractions of the heart, the pulse becoming feeble and empty, because the retarded circulation and the overdistension with blood of the abdominal organs have produced a deficient filling of the heart. Cyanosis develops in addition, althongh the vessels of the mucous membranes and the other veins are not filled unduly, because the backflow of the venous blood toward the heart is not interfered with; in severe eases the bluish discolored mucous membranes are pale or livid. The anemia of the brain gives rise to disturbances in consciousness and in respiration, and at times the inner temperature falls below normal. Finally the slow current in the blood vessels reduces the functioning power of the heart, and heart weakness is added to the original circulatory disturbances. Severe general atony of the arteries causes death in a short time.

In fatal cases of colicky diseases in horses (especially after rupture of stomach or intestine), also in the course of general infectious diseases the authors have observed this clinical picture repeatedly. It probably occurs not quite rarely, either alone or associated with the symptoms of acute myocarditis.

The trouble is differentiated from cardiac weakness only by the fact that the veins are never filled strongly.

If atony is not yet complete its removal may be attempted by vasocontracting remedies (camphor, caffeine, barium chloride, eserine, adrenalin, suprarin or by intravenous infusions of a physiological salt solution with adrenalin or suprarenin, while vaso-dilating remedies (alcohol, ether) must be avoided.-(Pässler \& Rolly, Münch. Med. Woch. $1902,1737)$. 


\section{Obstruction of the larger arteries. Thrombosis et embolia arteriarum.}

Etiology. In exceptional eases the aorta and its larger branches may be obstructed by emboli which are derived from the heart or from an aneurysm of the thoracic aorta. Usually, however, the obstruction of a relatively wider vascular lumen occurs through a thrombus originating at a spot in the wall of the vessel which has become roughened or after an embolus has become wedged in, the thrombus becoming gradually larger by deposits of new layers of fibrin. For this reason all causes which are capable of producing an endoarteritis chronica deformans may also constitute the basis of thrombosis of larger vessels. This occurs most frequently in horses and is usually brought about by the larvae of the Sclerostomum vulgare (Scl. bidentatum); in dogs the Filaria immitis sometimes leads to extensive thrombosis. Exceptionally the vascular lumen may be obstructed when arteries are compressed by a tumor (Mack, Fröhner), by exostoses (Gassner, Pirl), which may also produce chronic inflammation, even to complete occlusion of the aorta. An acute inflammation, finally (Vennerholm) or contusion of the vessel wall ( $\mathrm{H}$. Bouley) may be the causes of occlusion of arteries.

Anatomical Changes. The thrombosis in horses which is usually produced by larvae of sclerostoma is found mostly in the anterior mesenteric artery, fairly frequently at the posterior end of the aldominal artery and in its pelvic branches (artt. iliacae, femorales, hypogastricae), from where the thrombus may become continued downward into the tibial arteries; in exceptional cases the vessels of an anterior extremity (art. axillaris, art. brachialis) or the first portion of the aorta (Bergstrand) is found obstructed. In a case of Filariosis haematica in a dog Hutyra found a large portion of the thoracic and abdominal aorta filled up.

The thrombus is usually attached to the roughened wall of the vessel; it is dry, tough, and usually arranged in layers. It may cover only a part of the vessel wall and thus make the lumen smaller (thrombosis parietalis), or it oceupies the entire periphery of the vessel wall, in which case there remains a patent canal in the direction of its longitudinal axis (Thr. canalisatus), or finally it obstructs the vessel entirely (Thr. obturans). The end or the outer surface of a thrombus which does not occlude the blood vessel completely is usually formed by layers of fresh fibrin, which reach into the circulating blood and perhaps into a lateral branch in the form of plugs, and are in all probability usually the result of post-mortem coagulation.

An obturating thrombus may in time disintegrate and be absorbed, but in the meanwhile a proliferation of connective 
tissue from the vessel wall takes place which may eventually fill out the vessel, the lumen of which is already narrowed, completely and transform it into a firm connective tissue cord (organization of thrombus). Such a change occurs, however, more often at the places where emboli have become wedged in, which is a fairly freguent occurrence in the smaller peripheral arteries.

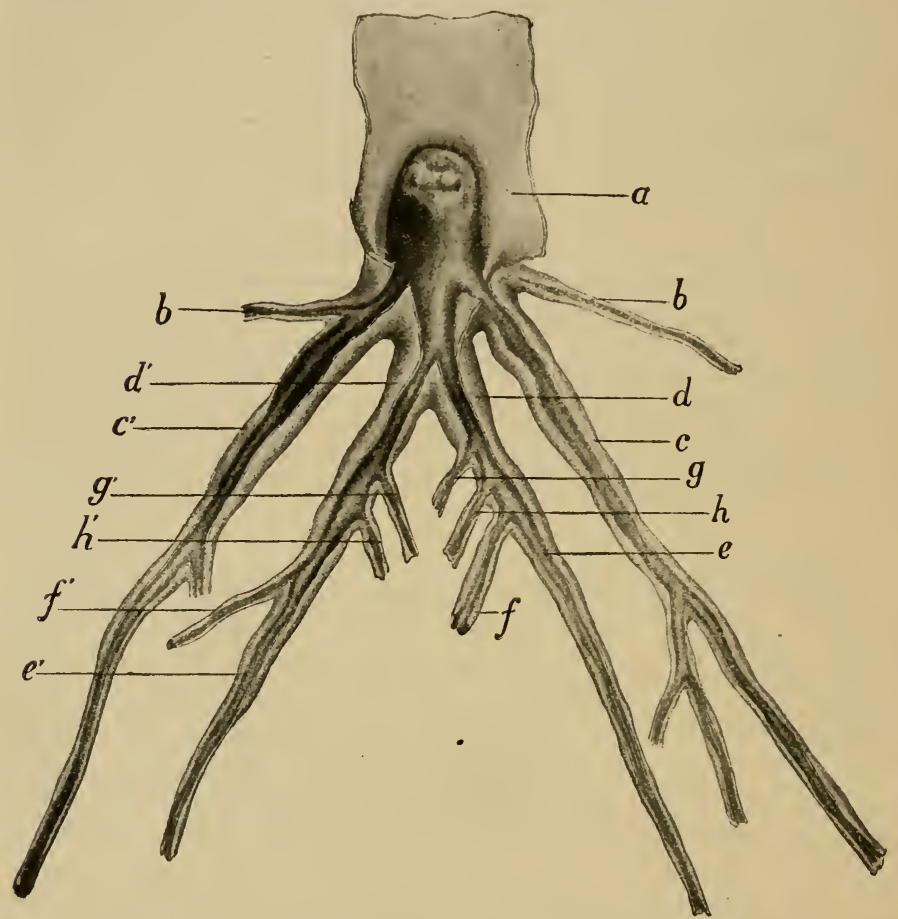

Fig. 197. Thrombosis of the posterior end of the abdominal aorta and of its branches, in a horse. a Abdominal aorta; $b b^{\prime}$ Art. circumflexa ilium prof.; $c c^{\prime}$ Art. iliaca externa; $d d^{\prime}$ Art. hypogastrica; $e e^{\prime}$ Art. obturatoria ; $f f^{\prime}$ Art. iliolumbalis; $g g^{\prime}$ Art. glutaea sup.; $h h^{\prime}$ Art. sacralis lateralis.

Pathogenesis. The obstruction of large arterial trunks proluces a peculiar disturbance in the function of the muscles. In the majority of cases the vessel is not completely obstructed by the thrombus, and even if this occurs, a collateral blood circulation is established in a short time by the intermediation of branches which pass off proximally to the thrombus. The part of the body which is to be nourished by the obstructed vessel receives also in future a certain amount of blood, sufficient to 
supply all tissues of the body part in question with the nutritional substances necessary for life, and which sometimes even enables in muscles to accomplish a certain degree of work. If, however, the animal accomplishes prolonged and severe muscular labor, the deficiency in oxygen causes an insufficient oxidation and therefore the development of insufficient force, and also a transitory accumulation of products of metabolism in the muscle, especially lactic acid and carbonic acid, which lead to a more rapid fatigue of the muscle. When the exercise is interrupted, the products of fatigne are gradually eliminated from the muscle, the feeling of fatigue and the disturbance in function are abated, but reappear on renewed prolonged muscular exertion.

Symptoms. The form and the intensity of motor disturbances depend upon the arteries which are obstructed and upon the degree of occlusion. In the rather rare thrombosis of the posterior portion of the aorta the trotting or galloping horse begins, after a variable time, to become uncertain in the hindquarter, it staggers, the feet are raised less high, and soon it can no longer move at all, remaining standing, bathed in perspiration and trembling. If urged further, it finally collapses and falls to the ground. While lying on the ground the animal gradually becomes calmed; it arises, after a time, of its own accord and may move at a walk.

In thrombosis of aortic branches, especially the pelvic or femoral arteries, the motor disturbance commences in a similar manner, but is limited to one of the posterior extremities. After a certain time, which may be only a few minutes, the horse raises the foot less high, it cannot move it very certainly, may interfere occasionally against the other foot; it hangs its head and if possible bears the weight of the body upon the front feet; soon the joints are bent less and less, the hoof scraping the ground at each step, and finally the affected foot gives out, the animal falling upon the diseased side of the body on being urged further. In bilateral thrombosis of the femoral artery the motor disturbances correspond as a whole with those of thrombosis of the abdominal artery, although the uncertainty in the sacral region is less striking.

Thrombosis of the axillary and brachial arteries leads to similar disturbances in motion in the one fore foot. Here also a gradually increasing muscular weakness becomes perceptible, the horse stumbling on the fore foot, knocking it against impediments and scraping the ground with the hoof, until the foot commences to tremble violently and is finally paralyzed.

Complete occlusion of the pelvic arteries causes paralysis of the sacrum, the tail, the bladder, and of the rectum; a partial thrombosis gives rise to motor disturbances, which occur only on motion and is similar to sacral paralysis.

The paralysis disappears after a certain period of rest but 
reappears on the next occasion, unless accidentally paralytic hemoglobinemia has been added, to which horses with thrombosis of the arteries of the extremities appear to be predisposed.

General symptoms are also present as a rule. The animal shows great anxiety, the heart action is remarkably excited and accelerated, the respiration is also accelerated and difficult, the mucous membranes show intense injection. Although a general perspiration takes place, the affected extremity remains dry (Humbert, Gál), it feels very cool to the touch (Palat). Friedberger \& Fröhner have observed no general symptoms in thrombosis of the axillary artery, in spite of existing motor disturbances.

Thrombosis may be recognized with absolute certainty, if it is possible on palpation to find firm blood clots or a compressing swelling in the related blood ressels. In many eases the hand, which has been introduced into the rectum, may ascertain the presence of the thrombus on palpating the aorta or the larger vessels on the sides of the pelvis; its presence may also be assumed, owing to the weakness or complete absence of pulsation or owing to a thrill which may be felt. If the thrombosis cannot be found within the pelvis, a total or partial obstruction of the femoral or axillary arteries may be assumed to exist from the fact that the pulse cannot be felt at all or only feebly in the vessels beyond the thrombus, on the metatarsus or metacarpus.

A partial thrombosis sometimes produces no disease symptoms whatever. For instance a horse with partial thrombosis of the posterior aorta covered a distance of $130 \mathrm{~km}$. two days before autopsy (Gratia).

Glöckner has observed difficult deglutition, whistling respiration, and later the symptoms of pulmonary emphysema in thrombosis of the carotid arteries. - Siegen saw epileptiform convulsions in thrombosis of the left carotid and the internal carotid; the convulsions appeared only after the horse had trotted for 20-30 minutes and lasted about 20 minutes.

Course and Prognosis. In a portion of the cases softening or contraction of attached thrombi occurs, so that the blood paths become more or less opened, or the obstruction persists, but the circulation in the territory concerned is equalized by the collateral circulation which has meanwhile been established. In both cases the affected part of a body may after a certain time (3-6 months) again receive a sufficient supply of blood for the muscles to accomplish even continued and severe work. A complete recovery in this meaning of the term is nevertheless very rare, since the supply of blood through the narrowed paths or through the new collateral tracks still remains insufficient, and since the existing thrombosis gives rise to the further production of fibrin or to embolism, which are followed by relapses and by aggravation of the condition. In exceptional cases thrombosis of the posterior extremities may be complicated eventually by thrombosis of the brachial artery (Schraml).

In other cases, an equalization of the circulatory disturbances does not take place, the function remains permanently im- 
paired, and the impediment to the circulation is even increased by the deposit of new masses of fibrin. In such cases the affected part of the body, usually an extremity, atrophies owing to insufficient nutrition, and the animal finally becomes unfit even for very light and slow work. In rare cases the obstruction may become so extreme that some organs receive no blood at all and die in consequence. Martin and Bouley saw, each in one case, gangrene of the muscles of the anterior extremity after obstruction of the axillary artery, Cadéac \& Malet observed the same in a posterior extremity in thrombosis of the femoral artery.

If a vessel has become obstructed by an embolis derived from the first segment of the anterior or posterior aorta, the fact must be considered in giving a prognosis that similar emboli might be carried into vital organs (brain, kidneys, etc.).

Treatment. In order to promote the collateral circulation, systematic exercise of the animals is indicated, the patients being led about daily at a walk until the first symptoms of paralysis appear (Bayer). It is sometimes possible to realize even complete improvement by continued and gradually increasing work (Bayer, Johne). It may also be attempted to massage a thrombus which is palpable in the pelvic cavity, with the hand in the rectum, for 5-10 minutes daily, which proceeding may also perhaps hasten the absorption of the fibrin coagulations (Collin, Beyer). Internal treatment which has repeatedly been proposed, especially the administration of iodide of potassium, promises no results.

Literature. Albrecht, W. f. Tk., 1901. 325. - Cadéac \& Malet, Rev. vét., 1885. 530 (Lit.). - Fröhner, Monh., 1903. XIV. 445; 1905. 553 ; 1907. XVIII. 136. - Gratia, Ann., 1906. 489. - Merkt, W. f. Tk., 1904. 261. - Schimmel \& Resser, Ann., 1905. 543. — Siedamgrotzky, S. B., 1896. 18.

Thrombosis of the Pulmonary Artery. The obstruction of the pulmonary artery is not very rare and is brought about by emboli from the right heart. The parts of thrombi may have originated in the right heart itself, or not infrequently they are carried there with the venous blood from distant parts of the body. In exceptional cases thrombosis forms in the pulmonary artery after the invasion of foreign bodies (hair which had been introduced on venesection from the jugular vein [Zschokke] or hroken hypodermic needles [Prévot]) or in the presence of parasites (Filaria immitis, Strongylus vasorum [Ferez]). In many cases the cause could not be determined, probably because distant organs had not been examined carefully. Käppel succeeded in $63 \%$ of the cases diagnosed by him to find the source of the embolism by careful and systematic examination of the organs of horses which had been slaughtered (he found thrombosis of the pulmonary artery in $9 \%$ of all slanghtered horses, which were mostly old animals); the embolisms originated in most cases in thrombotic veins in the posterior extremities and in the sheath.

Obstructions of smaller pulmonary vessels do not give rise to pathological disturbances, but when larger branches were ohstructed 
(Käppel found this to have been the case in 12 out of 38 instances), difficult respiration occurred, like dyspnea or sudden asphyxia which sometimes caused rapid death.-(Käppel, Z. f. Tm., 1904. VIII. 321.Prérot, Bull., 1908, 625.).

\section{Obstruction of the Large Venous Trunks. Thrombosis Venarum.}

Obstruction of the large venous trunks is rare and is usually due to compression (by enlarged lymph glands, tumors, abscesses), less often it occurs in connection with endophlebitis or slowing of the circulation.

Cases of thrombosis of the venae cavae have been observed especially in horses and cattle, once also in a dog. In the horse thrombosis of the portal vein has been known to occur.

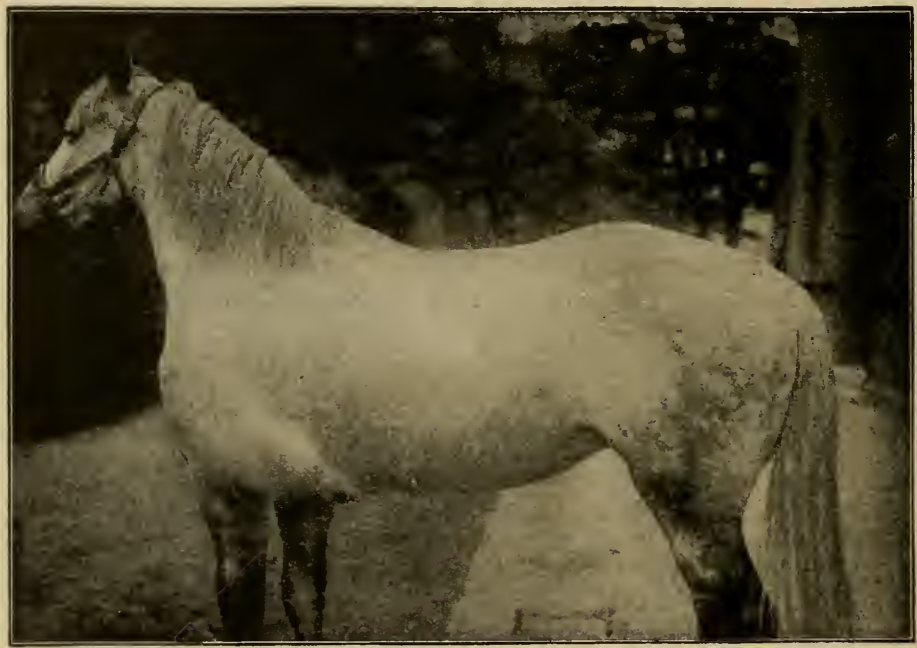

Fig. 198. Stenosis of the anterior vena cava. Edematous infiltration of the region of neck and shoulder; the swelling is sharply arrested near the xiphoid cartilage.

The symptoms are easily interpreted by the anatomical arrangement of the venous trunks, because stenosis or obstruction of one vena cava produces venous stasis in that part of the body from which the vein returns the blood to the heart. Thus thrombosis of the anterior vena cava produces strong. filling of the veins of head, neck, anterior extremities, and thorax, also edematous infiltration of these regions or dropsy of the chest (Fig. 198). If the obstruction is incomplete a negative pulsation may become evident in the jugular reins. 
Thrombosis of the posterior vena cava causes similar clianges in the hindquarter, with which abdominal dropsy is associated if the location of the obstruction is between heart and liver. Thrombosis of the portal vein impairs the motility of stomach or intestine (Vol. II.).

The circulatory disturbances may occasionally become much lessened, as was shown in a case of Albrecht's and in one of the author's.

Literature. Alkrecht, Z. f. Tm., 1902. VI. 428 (Lit.). - Barrier, Bull., 1906. 283. - Basset, Ree., 1905. 609. - Bretagnier, Rec., 1909. 156. - Chouleur \& Lebasque, Bull., 1908. 256. - Cuny, J. vét., 1906. 224. - Douville, Bull., 1907. 62. - Forgeot, J. vét., 1906. 362. - Friedrich, B. t. W., 1905. 4. - Petit \& l)elacroix, Bull., 1905. 133.

\section{Rupture of the Large Blood Vessels, Ruptura Vasorum.}

The walls of the large blood vessels rupture at times, even without previous disease. The first segments of the aorta and of the pulmonary artery are very thin at the level of the semilunar valves (the aorta of the hor se is in some places only $1 \mathrm{~mm}$. thick, while lower down the thickness is $5-7 \mathrm{~mm}$.), so that they are bulged out somewhat by the pressure of the blood, even under normal conditions (Sinus Valsalvae) and may rupture if a sudden rise in blood pressure occurs. Among causative factors, aside from immediate injuries, may be mentioned the pulling of heavy loads, severe exertion in galloping, vomiting, labor pains (Sigl) or other exertions. Similar causes may sometimes lead to rupture of the anterior or posterior vena cava or of the portal vein.

Usually, however, rupture is preceded by a disease of the vessel wall, although this may not be visible to the naked eye, as in a case of Caparini's. In most cases there is a selerosis with subsequent bulging of the vessel wall, which favors rupture. This cause is responsible for the rupture of vessels, especially in older animals. The same result is observed if an abscess or ulcerating process in the immediate vicinity of the vessel is transmitted to the vessel wall from an ulcerating valve, a carious bone, or from a breaking down new growth, causing erosion of the vessel. Finally animal parasites, in the horse the larvae of Sclerostomum vulgare, in the dog Spiroptera sanguinolenta may weaken the vessel wall so much in places that it finally tears under the influence of the blood pressure.

The following facts may illustrate the manifold etiological factors: Palat saw rupture of the aorta at the level of the valves without demonstrable external cause, Lustig saw it occur on galloping. Sequens found in three cases the point of rupture about $1 \mathrm{~cm}$. above the valves in the vicinity in which the vessel wall was diseased, but Cadiot could not find any pathological changes in two cases. In one case described by Vaerst hemorrhage occurred from a small aneurysm situated opposite the valves.-In a case reported by Prietsch the healthy pulmonary artery was ruptured while drawing a heavy load, Block saw a four-your-old stallion bleer to death after having been thrown; the pulmonary artery was torn at the 
bifurcation.-Ehlers observed rupture of the portal rein after a blow on the left side of the body.- Schrulle reports a case of fatal hemorrhage from rupture of the left axillary artery in a horse which had been thrown. On autopsy of a horse Bührmann found an abscess between the pillars of the diaphragm, which had ruptured into the aorta.-In Gassner's ease an exostosis on the 16th vertebra had injured the aortic wall, in Noack's case a sarcoma growing behind the kidney had done the same. Parasites may also be involved. Morgagni found in one dog, Mégnin in three dogs, the Spiroptera sanguinolenta in the wall of the aorta; it was present in the form of small whitish nodes one of which had caused rupture of the aorta. Durienx and Degive finally conld demonstrate in a horse that fatal hemorrhage had been due to destruction of tissue by the larvae of the Sclerostomm vulgare (in one case the aorta was apparently sound, in the other it presenterl in its entire length the picture of atheromatosis).

\section{The symptoms of rupture of a vessel indicate in general} only internal hemorrhage, the exact cause of which can usually only be ascertained on autopsy.

Literature. Bonnet, J. vét., 1905. 147. - Caparini, Arch. scient. d. R. Soc. ed Accad. Vet. It., 1903. 87. - Ehlers, B. t. W., 1889. 4. - Gamble, V. Journ., 1908. 345. - Lustig, W. f. Tk., 1877241 - Magnin, Rec, 1903 693. - Pécns, J. rét., 1902. 351. — Petit, Bull., 1905. 299; 1906 104. - Pr. Mil. Vb., 1899-1908. - Prietsch, S. B., 1881. 85. - Seqnens, Vet., 1892. 352. - Sigl, W f. Tk., 1905. 8. - Vaerst, Monh., 1893. IV. 185. 
Appendix.

\section{Lymphorrhagia.}

Etiology. In horses and cattle, and exceptionally in other animals swellings develop sometimes in a short time, in variable number and extent, containing a clear lymph-like fluid which may be mixed with dead tissue shreds. According to Hoffmann and Dratschinsky these swellings are due to tears between the skin and fascia or between muscle and fascia, produced by some considerable injury which has a pushing or pulling action. Into these tears lymph flows from the torn lymph vessels and is mixed with the torn tissue particles. (Astrachanzew found once in the lymph a worm $5 \mathrm{~cm}$. long, which he believed to be a kind of Filaria medinensis.)

Bayer has seen lymph effusions in a eat, in circular arrangement around the nipples; the lymph vessels were dilated.

Symptoms. Sometimes the swellings form successively in the course of several days and produce marked changes in form if they become confluent. In the case of a cow, reported by Zonda, which had broken down beneath an ox, swellings like those just described formed on the lower abdomen, on the chest and on the internal aspect of the thighs, all in the course of four days. Although the appetite is not impaired the animals rapidly become emaciated, owing to the loss of nutritive material. The swellings are not painful or warm, but they show fluctuation if situated superficially. After incision a clear, yellow odorless lymph flows ont, or sometimes a dirty yellow or reddish, illsmelling fluid which is mixed with tissue shreds. The quantity may be considerable.

Treatment. The swellings are usually treated by incision, removal of the lymph, irrigation of the cavities with disinfecting solutions or the application of disinfecting powders, finally the application of a compressing dressing. Sometimes continued irrigation of the cavity with lukewarm solutions of corrosive sublimate is of benefit (on opening such swellings there is always danger of septic infection [Johne]). If the skin has been loosened over a large extent it may have to be sutured to the underlying tissue. Small swellings disappear on the application of warm compresses and massage. The patients must be nourished generously.

Literature. Astrachanzew, A. f. Vet.-Wiss., 1893. 204. - Dratschinsky, ibid., 1904. 787. - Hoffmann, Z. f. Vk., 1897. 1. - Vennerholm, Srensk Vet.-Tidsskr., 1904. IX. 537. - Walley, J. of comp. Path., 1892. 360. — Zonda, Vet., 1894. 369. 



\section{Index, Vol. I.}

PAGi

Abortin .............. 747

Abortion, enzootic ......... 740

Abortion, infections ....... $7+0$

Abscess of kidney.......... 1008

Acetone ................ 39

Actinobacillosis .......... 666

Actinobacillus .......... 666

Actinomycosis ........... 642

Aetinomycosis, atypical ..... 667

Actinomycosis of the $\operatorname{dog} \ldots . .670$

Actinomycosis of $\operatorname{man} \ldots . . .6665$

Actinophytosis ....645, 667, 670

Actinomyces bicolor ........ 67 67

Actinomyces bovis ......... 643

Actinomyces canis ......... 671

Actinomyces cuniculi ....... 420

Actinomyces necrophorus ..... 420

Adenitis, equorum ......... 366

Adeno-carcinoma of the kidney 1028

Adenoma of the heart...... 1077

Adenoma of the kidney...... 1028

Adrenalin, action on bluod

vessels ................. 1104

Aerogenes bacillosis ........ 1.51

African glanders .......... 734

Agglutination in glanders.... 721

Agglutination in infections

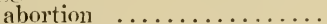

Agglutination in tubereulosis. .

Aggressins in anthrax.......

Iggressins of the bipolarbacillus .................

Agrressins in fowl cholera....

Agoressins in strangles......

Albuminometer, Esbach ......

Albuminuria .............

Albuminuria, physiological ...

Albuminuria renalis ........

Albuminuria, spurious ......

Albuminuria, true .........

Aleppo boils .............

Allergy in abortion..........

Allergy in glanders.........

Allergy in tuberculnsis. . . . . . .

Allötriophagia ...........

Amblyomima hebraeum ......

Ameisic acid in urine. .......
PAGit

Amyloid kidney ........... 98

Anemia .............. \$45

Anemia, acute ........... S47

Anemia, chronic ......... Sti

Anemia, infections of ehicks... S46i

Anemia, infectious of horses... \$ \$51

Anemia, progressive peruicions $\$ 603$

Anemia, progressive pernicious of cattle .............. stis

Anemia of the young pig..... $\$ \$ 6$

Anemia of sheep and goats in Punjab ............. $86 \pi$

Anaplasma marginale ...... 763

Anaplasmosis ............ 783

Anasarca, essential ......... 216

Anasarca, idiopathic ....... 216

Aneurism of the aorta....... 1106

Aneurism of the blow ressels 1109

Aneurism of the coromary arteries .............1076, 1109

Aneurism of the leart. acute

1076-10s1

Anenrism of the heart, ehronic 1076

Anemism of the heart, fibrons 1074

Anemism, rupture ......... 1081

Aneurism, valvular ......... 1081

Angina, Vol. II. . . . . . . . . . . .

Angina diphtl. vitulorum.... . 419

Angioma of the heart....... 1

Anopheles mosquitoes ....... 797

Anthraco-mucin ........... 12

Anthrax ............... 1

Anthrax, acute .......... 16

Anthrax, apoplectic ........ 16

Anthrax, bacterial ......... 24

Anthrax in man......... 31

Anthrax, intermittent ....... 18

Anthrax, subacute ......... 16

Antiphymatol ........... 85

Antistreptococeus ser'um . .231, 386

Aorta, aneurism of ........ 1106

Aorta, ealcification of ........ 1105

Aorta, rupture of ......... 1117

Aphthous eruption ........ 340

Aphthous fever of camivora.. 354

Aphthous ferer of fowls..... 354

A phthous ferer of the horse. . 354 
PAGit

Aphthous fever of man . . . . . 75.5

Apiosoma bigeminum ........

A poplexy intermeningialis ... 14

Argas miniatus .......... 840

Argas perspicus ........... 840

Argas reflexus ............ 840

Arion subfuseus ...........

Arteriosclerosis ............ 1104

Arteritis, acute .......... 1111

Arteritis, chronic .......... 1104

Artery, compression of. . . . . 1109

Artery, crushing of ......... 1111

Artery, plugging of ......... 1111

Arthritis urica ........... 968

Arythmatic action of heart, systolic ............. 1059

Arythmatic action of heart. true ............... 1057

Atropliy of heart......... 1052

Atropliy of kidney, evanotic. . . 985

Atrophy of kidney, granular .. 1001

Azoturia ............ 896

Babesia, see Piroplasma..... 760

Bacillus abortus infectio...... 740

Bacillus aerogenes .......... 148

Bacillus anthracis ... . . . . 3, 19

Bacillus anthracis asporogenes.

Bacillus anthracoides .......

Bacillus avicida ...........

Bacillus avisepticus .........

Bacillus bipolaris septicus. .79,

Bacillus bovisepticus ........

Bacillus bubalisepticus ......

Bacillus canariensis necrophorus

Bacillus canisepticus . . . . . . . .

Bacillus ehauveaui ..........

Bacillus cholerae columbarum. .

Bacillus cholerae gallinarum ...

Bacillus cholerae suum ......

Bacillus coli conmmunis. . . 146.

Bacillus euniculicida .........

Bacillus diphtheriae avium .424.

Bacillus diphtheriae columbarum ............424.

Pacillus diphtheriae hominis. .

Bacillus diphtheriae vitulorum.

Bacillus enteritidis Gaertner...

Bacillus equisepticus .........

Bacillus erysipelas suis. . . . 60.

Bacillus felisepticus ........

Bacillus filiformis ..........

Bacillus gallinarum ........

Bacillus gangraenae empliysem. ..............20. 39,

Bacillus gastromycosis ovis...

Bacillus mallei ............

Bacillus multocida .........
Bacillus necrophorus .......

Bacillus necrosis ..........

Bacillus nodulifaciens bovis...

Bacillus edematis aerogenes...

Bacillus edematis maligni...20,

Bacillus edematis sporogenes. .

Bacillus edematis thermophilus

Bacillus of Bradsot.........

Bacillus of calf noma . . . . . . .

Bacillus of Reindeerpest......

Bacillus ovisepticus .........

Bacillus paracoli ...........

Bacillus phlegmones emplysen.

................. 34 ,

Bacillus pneumoniae felis....

Bacillus pleurisepticus .......

Bacillus polyarthritidis .......

Bacillus polymorphous suis. . . 1007

Bacillus Preisz-Nocard ....... 633

Bacillus pseudoanthracis .....

Bacillus pseudotubereulosis commont .............

Bacillus pseudotubereulosis

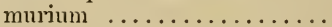

Bacillus pseudotubereulosis

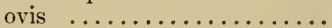

Bacillus pseudotubereulosis vitulorum $\ldots \ldots \ldots \ldots \ldots \ldots$

Bacillus pyocyaneus .........

Bacillus pyelonephritidis bovum 1011

Bacillus pyogenes bovis. . 144, 146

Bacillus pyogenes suis.... 143, 146

Bacillus renalis .......... 1013

Bacillus rhusiopathiae suis. . (i), 72

Bacillus sanguinarum ...... 99

Bacillus septicemia anserum exud. ............... 100

Bacillus septicemia murium.... 64

Bacillus suipestifer ........ 262

Bacillus suisepticus . .126, 139, 262

Bacillus tetani ........... 440

Bacillus tubereulosis .....5)4. 569

Bacillus tuberculosis. typus bovinus ............

Bacillus tuberculosis. typus gallinaceus $\ldots \ldots \ldots \ldots \ldots$.

Bacillus tuberculosis, typus lıumanus ............

Bacillus typli canariensis. . . .

Bacillus vitulisepticus ......

Backsteinblattern .........

Bacterium ehauveani ........

Bacterium phlei ...........

Baleri .................

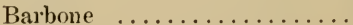

Barlow's disease . . . . . . . . .

Beschälausslag ...........

Beschälseuche ............

Big-head ...............

Bilharzia Bomforil ......... 
PALE

Bilharzia hematobia ........ 906

Bilharzia indiea ........... 906

Bilharziosis .............. 906

Biliary fever ............ 784

Blackleg ..............

Blacklegine ..............

Black quarter .............

Black water ..............

Bladder, eatarrh of.........

Bladder, irrigation of ..........

Bladder, paralysis of.........

Bladder, spasin of.........

Bladder, torsion of ...........

Blue tongue .............

Blood anemia .............

Blood cells, counting........

Blood coloring ............

Blood coloring matter in urine.

Blood diseases .............

Blood in pericardium........

Blood vessel, rupture of ...... 1117

Blood vessels, diseases of . . . . 1104

Bloody urine ....762, 979, 981, 987

Boil disease, endemic....... 797

Bollinger's Wild and Rinder-

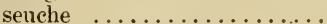

Boophilus annulatus .......

Boophilus Argentinus ... 762,

Boophilus Australis ....762,

Boophilus bovis ...........

Boophilus decoloratus .... 762 ,

Botryococeus ...........

Botryomyces equi .........

Botryomyeosis ...........

Bou-frida $\ldots \ldots \ldots \ldots \ldots \ldots$

Bovovaceine ............

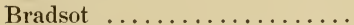

Braunschweiger bird disease...

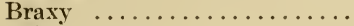

Bright's disease ...........

Broncho-pnemmonia of Anerican cattle.............105.

Broncho-pneumonia, caseous of

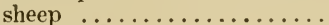

Bruit de Moulin... . . . . . . . . 10.54

Brustseuche .......... 170, 182

Brustseuche of rabbits....... 102

Buffalo disease ............ 113

Bulbar paralysis, infectious... 495

Bursattee ............739,

Butter bacillus ...........

Cachexia, aqueous ....... $\$+1$,

Cachexia, osseous ...........

Cachexia, pyemic .......14t,

Cachexia, tuberculous .......

Caderas, Mal de...........

Calculi, reual ............. 1021

Carbuncle ..............
C'arceay ................ 789

Carpincho .............. 836

Cataract, diabetic .......... 927

Catarrhal fever, malarial...... 418

Catarrhal fever, malignant.... 408

Catarrh of minary vessels.... 1031

Catarrh of vagina......... 750

Cattle plague ............ 234

Cement cough ............ 138

Cercomonas gallinae ........ 424

Chemotherapy .......... 802

Chicken cliolera.......... 87

Chlamydozoa in fowl pest.... 290

Chlamydozoa in fowl diphtheria 425

Chlamydozoa in pox......... 296

Chlamydozoa in rabies........ 466

Chlamydozoa in vaginal eatary 751

Chlorbaryum, effect upon walls of blood vessels........... 1104

Chlorosis .............. 846

Cholera of birds.......... 87

Cholera of ramary birkl..... . 99

Cholera of ducks............ 98

Cholera of fowl............ 87

Cholera of swine........... 257

Cholera of water birds. . . . . . . 98

Cholera vibrion ........... 101

Cholera-like gastro enteritis of birds ............... 101

Chromatotaxis .......... 691

Cimurro ............... 197

Cladothrix canis .......... 670

Clavelee ............... 302

Clostrydium sareopliysematous .20

Coccidiosis, Vol. II.........

Coccidiosis renalis ... . . . . . 1030

Coceidimm truncatum ....... 1030

Cocobacillus psendo actinomycosis polymorphous....... 669

Cocobacillus oznenae foetidus. . 199

Cocobacillosis ......146, 152, 157

Coma diabetic ........... 105

Complement fixation in influenza .............. 174

Complement fixation in glanders 717

Complement fixation in abortion

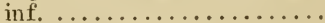

Complement fixation in tuberculosis ..............

Conjunctival reaction in glan-

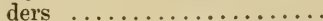

Conjunctival reaction in tuberculosis .............

Cornfodder disease ........ 105

Corynebacillus abortus inf..... 741

Corynebacillus renalis ....... 1013

Corynethrix pseudotubereulosis

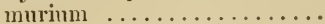

Coryza contagiosa equor.m. .. 
P'Atit

Coryza gangrenusa burum..... 408

Cow pox ...............

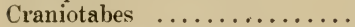

Croup of eattle............

Cryptocoecus farcinimosus ....

Culex mosquito ..........

Crstic kidner ............ 1025

Crsticereus rellulusase of the

kidney .............. 1030

Cystitis eatarhal .......... 1031

Cystitis vernueous .......... 980

Cystoscope .............. 1010

Cytorhyctes vaccinale ...... 29.5

Cytorhyctes variolae ....... 295

Debal ...............

Decompensation in heart valve deferts .............

Delhi boil ..............

Dengue fever ..............

Dermacentor reticulatus . . 786 ,

Dermo reaction in elanders....

Dermo reaction in tuberculosis.

Dextrin in urine..........

Diabetes azoturieus .........

Diabetes hepatogenic ........

Diabetes insipidus ..........

Diabetes insipidus verus......

Diabetes mellitus ..........

Diabetes mellitus decipiens....

Diabetes mellitus intermittent..

Diabetes nemrogenic ........

Diabetes pancreatic .........

Diamond skin disease........

Diathesis, hemorrhagic ......

Diarrhea neonatorum .......

Diazoreaction ............

Dikkopziekte .............

Dilatatio enrlis . . . . . . . . 1063

Dilatatio cordis activa . . . . . 106.j

Dilatatio cordis passiva . . . . . . 1064

Diphtheria of birds........ 424

Diphtheria of calves. . . . . . . 419

Diphtheria of mammalia..... 423 Diphtheria of man....... 437

Diphtheritic inflammation of the eyes of ducks.........

Diplococeus pnemmoniac ......

Diplococei in osteomalacia ....

Diplococei in rachitis........

Discomyces equi ...........

Disinfection of stock car......

Disinfection of skin........

Distemper in dogs...........

Distomum hematobium ......

Distomum lepaticum in the heart ...............

Dollar flecks .............

Dourine ................ i) 64

9

Hung bacillus ..........

Dunkoprickte ........... 285

Dwarfism ...........930. 945

Dysentery, Vol. II ..........

Dysentery of surklings....... 145

Dysentery of hens and turkeys 99

Dysuria ............... 103s

East coast ferer.......... 778

Echinocoreus in heatl...... 1078

Eehinocoreus in kidney...... . 10,30

Echinococens in liver. iol. il..

Echinococens in spleen....... 914

Eimeria trumcata .......... 10.30

El Debab ................ s3s

Emphysema gangrenosa . . . . . 39

Endoarteritis, elnonic . . . . . . 1104

Endoarteritis deformans ..... 1104

Endocarditis, acute ..... 107 S. 1086

Endocarditis, chronic ....... 10S6

Fndocarditis in errsipelas of swine ..............66.

Endocarditis, parietal ........ 1081

Endocarditis, ulcerous ...1079, 10s.1

Endoearditis, valvular ...... 1081

Endocarditis verrucous . . . . . 10 1081

Entéqué ............... 112

Enteritis hypertrophica bovum 639

Enteritis paratuberculons .... 639

Enzootic dysentery of fowls... 98

Enzootic pnemmonia of pigs... 138

Enzootic pneumopercarditis of turkeys $\ldots \ldots \ldots \ldots \ldots \ldots \ldots .99$

Ephemeral fever .......... 196

Epithelioma, rontagious ...... 424

Fpithelioma, contagions of men 428

Epitheliomyces crompogenes .. 425

Epizootic pneumoenteritis of turkeys $\ldots \ldots \ldots \ldots \ldots \ldots \ldots \ldots .99$

Erysipelas of swine........ 60

Erysipelotricheen .......... 65

Erythroblasts ........... Sts, $\$ 65$

Fustrongylus gigas . . . . . . . . . . 1028

Exanthema, eoital ........ 3.56

Exanthema, infections ... . . . . 295

Exanthema, vesicular ....... 351

Fxudative septiremia of geese. 99

Farein ............. $6 \pi$.

Farein eryptoeoesiculue ....... 734

Farcin d'Af fique .......... 734

Farcin de riviere......... 734

Farcin du boenf.......... 667

Farey ................ 678

Fat, alimentary ......... 915

Fatty heart ............ 107

Feather eatine and pulling... (iv)

Fever aphthoms ..........

Fever bilions ........... 791

Fever typhoid ........... 170 
Fibronra of heart......... 1077

Fibromyoma of heart........ 1077

Fibrosarcoma of heart....... 107

Filaria Blini .........908, 1105

Filaria Evansi ........... 908

Filaria hematica .......... 90.

Filaria immitis ........... $90^{\circ}$

Filaria Poelsi ............ 1105

Filaria sanguinis equi........ 905

Filaria sanguinis hominis..... 903

Fixed virus of rabies ........ 489

Flesh of tuberenlous animals. . 514

Foal paralysis ........... 15s

Foot-and-month disease ..... 328

Foot rot ............................. 346

Furest disease .......... 764

Formalin in milk ......... 155

Formic acid ........... 92

Fowl cholera ........... 8 T

Fowl diphtheria ......... 424

Fowl pest ............. 290

Fowl pox ............. t24

Fowl septicemia .......... 8 .

Fowl tuberculosis . . . . . . . . 510

Fowl typhoid ............ 290

Furunculin ............ 385

Gall disease inf. .......... $7 \mathrm{S3}$

Galloserin ............. 96

Gall sickness ............ 783

Gambia fever ............ 837

Gangrene in erysipelas of swine 68

Gangrene, emphysematous .... 39

Gangrene foudroyante ...... 34

Fas in pericardium ........ 1053

Gastro enteritis cholerica avium 101

Gastro enteritis hemorrlagica

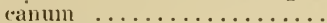

Gastronycosis $\ldots \ldots \ldots \ldots \ldots$.

Geel Dikkop .............

Gigantocytes ..............

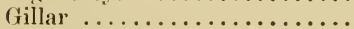

Girdle bacterium ..........

Glanders ...............

Glanders, African .........

Glanders bacilli .............

Glanders infection ..........

Glanders nodules ..........

Glanders nleers ............

Glomerular nephritis .......

Glossanthrax ............

Glossina decorsei ..........

Glossina fusca ............

Glossina longipernis ........

Glossina pallidipes .........

Glossina palpalis ..........

Glossina morsitans ........

Glyeosuria ..............

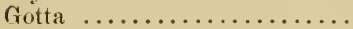

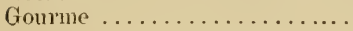

211
Gunt ................. 968

Grapes .............. 496

Grass bacillus .......... T.

(iregarinosis ............. t.

Grip ................... 170

(irips' bacillus ........... 142

Grouse disease ........... 101

Guanin formation .......... $96 \mathrm{~s}$

Gurmin ................ $38 \pi$

Heart, atrophy ......... 1052

Heart defects .............. 1055

Heart refeets, congenital ..... 10s

Heart dilatation ........... 1063

Heart fatty . . . . . . . . . . 1075

Heart hypertrophy .......... 106i1

Heart insulficieney ......... 1064

Heart muscle, calcifieation of. . 107.)

Heart muscle, inflammation of. 106!

Heart muscle, inflammation of, acute ............... 1069

Heart muscle, inflammation of, ehronie .............. 107:3

Heart muscle, inflammation of. parenchym. ........... 106!

Heart muscle, inflammation of , purulent .............. 1071

Heart valvular defects, compensating ................

Heart valvular defeets, nonconpensating .............. 1098

Heart water ............. 257

Hemameba leukaemiae ....... 870

Hemameba vivax .......... 870

Hematopa fly ............ 807

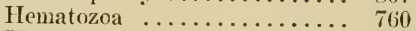

Hematozoon Lewis .......... 90.

Hematozonn subulatum ...... !n

Hematuria ............ 979

Hematuria resicalis ......... 981

Hemoaphthin ........... 349

Hemoglobinemia ......... s\&

Hemoglohinemia of eatte..... gn

Hemoglobinemia, enzontic .... Tr $\quad \boldsymbol{T}$ (4t

Hemoglobinemia, raralyt:e ... 896

Hemoglobinemia, rheumatic ... 886

Hemoglobinuria ........... 760

Hemoglohinuria, enzontic ..... 764

IIemoglobinuria, paroxysmal . 896

IIemoglobinuria, toxemic .... 886

Hemopericardim ........... 1053

Hemophilia ............... 900

Hemophysalis leachi .....762, 793

Hemorrhagie septicemia ..... 77

Hemorrhagie septicemia, of birds ..................

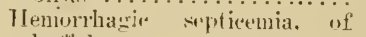
butfaloes ............. $11: 3$

Hemorrhagic septicemia. of eattle ................... 
PAGE

Hemorrhagic septicemia. of ducks ................

Hemorhagic sefticemia, of fowls ..............

Hemorrhagic septicenia, of

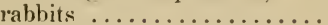

Hemorrhagic septicemia, of

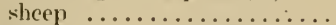

Hemorrhagie septicemia, of sucklings .............

Hemorrhagic septicemia, of

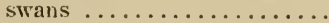

Hemorrhagic septicemia, of

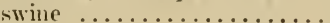

Hide bomind ............... 112

Hodgkin's disease .......... 881

Hog eholera ............ 257

Howf disease ............ $\$ 35$

Horse $p o x \ldots . . \ldots \ldots . . . . .324$

Horse sirkness ........... 25.5

Hyalomma . Eeypticum ..... 762

Hydremia ........... 845

Hydremia of cattle at singar refineries ...............

Hydrocephalus, Tol. II......

Hydronephrosis . . . . . . . . 1025

Hydropericardium ......... 1052

Hydrophohia ............ $\$ 63$

Hydrops ascites, Vol. II......

Hydrops pericardii ........ 1052

Hydrotetanin ............ $4+2$

Hyperemia renum ......... 984

Hyperglykemia .......... 9.95

Hyperplasia liensis ......... 913

Hypertrophy of heart muscle.. 1061

Hypertrophy, idiopatlic ..... 1062 Hypertrophy, nephritic ..1006, 1062 Hypertrophy, pathologie ..... 1061 Hypertrophy, plivsiologic .... 1061 Hypertrophy, secondary . . . . 1062 Hyphomycosis destruens . . . . . 739

Hypoplasia cordis et arterium..

Ietero-hematuria

Icterus, Vol.

Immunization, ........... inoculation.

Inoculation, diagnostic, of glan-

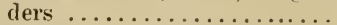

Inoculation, diagnostic, in rabies ................

Inoculation, diagurstic, in tuberanlosis

Tnoculation, p................ abortion inf.

Inoculation, protective, against

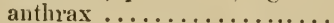

Inoculation, protective, against blacklor. buffalo disease ............

Inoculation, protective, against calf paralysis ...........

Inoculation, protective, against coast fever ..............

Inoculation, protective, against cholera, fowl ............

Inoculation, protective, against cholera, hog ............

Inoculation, protective, against diphtheria ............

Inoculation, protective, against diphtheria, of birds .......

Inoculation, protective, against distemper of dogs ........

Inoculation, protective, against dysentery of calves ........

Inoculation, protective, against foot-and-mouth disease .....

Inoculation, protective, against glanders ...............

Inoculation, protective, against lem. septicemia .........

Inoculation, protective, against hem. septicemia of sheep ...

Inoculation, protective, against horse sickness ...........

Inoculation, protective, against influenza ...............

Inoculation, protective, against lung plague of cattle.......

Inoculation, protective, against malignant edema .........

Inoculation, protective, against piroplasmosis ...777, 789.

Inoculation, protective, against pox ...............298,

Inoculation, protective, against rinderpest ............

Inoculation, protective, against sheep pox .............

Inoculation, protective, against spirochetosis ..........

Inoculation, protective, against swine erysipelas ..........

Inoculation, protective, against swine plague ........137,

Inoculation, protective, against tetanus ...............

Inoculation, protective, against trypanosomiasis .........

Incculation, protective, against tuberculosis ............ 5!7

Incontinence of urine......... 1037

Induratio eyauotica ......... 9.

Infarets, hemorrhagic ....... 986

Infectious disease of canaries.. $\quad 99$

PAGE

Infertions enteritis of fowls... 
Infectious enteritis of pheas-

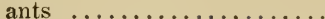

Infectious lenkemia of fowls..

Infectious necrosis of eanaries.

Influenza ...............

Influenza catarrhalis ....178.

Influenza of man ............

Influenza pectoralis .......178,

Infusion of salt solution, intravenous

Infusion of salt solution, rectal.

Inhalation, anthrax ......14,

Inhalation, tubereulosis .......

Inosite in the urine..........

Insufficiency, muscular ........ 108t

Insufficiency, relative ........ 1087

Insufficieney, valvular, bienspid 10!)?

Insufficieney, valvular, triens-

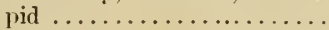

Insufficiency, valvular, semilu!!. aortae ............. 1083

Insufficieney, valvular, semilun. art. pulm. ........... 1093

Intermission of heart beats . . 105i

Intermittent paralysis . ...... 1113

Intoxicatio diabetica ........ 928

Ischuria paradoxa . . . . . . . 1037

Ischuria spastica .......... 1039

Ixidioplasma, bigeminum. . . . . 76.5

Ixodes, hexagonus .......... 769

Ixodes, reduvius ........... 768

Ixodes, ricinus . . . . . . . . 769

Japanese worm .......... 734

Jaswa ................ 3

Jaundice, protozoan ............ 783

Jaundice, malign .......... 791

Johne's disease . . . . . . . . 639

Joint evil ............. 158

Kala Azar ............. 796

Kyanolophiaea ........... 290

Kyphosis .............. 944

Lactosuria $\ldots \ldots \ldots \ldots . \ldots . \quad 922$

Lamziekte $\ldots \ldots \ldots \ldots \ldots \ldots \ldots \ldots \ldots \ldots$. 109

Latent infection in anthrax ... 9

Latent infection in blackleg. ... 54

Lead, effects of ........... 111

Lead chlorosis .............. 846

Leeches ................ 739

Leishmania Donorani ...... 796

Leishmania tropica ........ 797

Leukemia .............. 869

Leukemia, infectious ....... 877

Leukemia of fowls .......99, 877

Leukemia of mammals ...... 869

Leukemia lienalis .......... 871

Leukemia. Jymphatic . . . . . . 869

Leukemia, myelogenic ...... 869

Leukemia, sublymphatic ..... 881

Leukanthrakozidin ......... 12

Lencocythemia ........... 869

Leucocytozoon piroplasmoidcs.. 735

Lencosis, alenkenic . . . . . . 876

Lencosis, leukemic ......... 875

Leviulose, in the urine ....... 927

Licking disease ........... 941

lipoma of heart........ 1077

Loekjaw ................ 439

Loin distemper .......... 851

Lombriz . . . . . . . . . . . . 118

Lordosis ................. 944

Lues venera equi........... 815

Iumbago ............... 886

Lungs, infect. inflammation in goats .............. 124

Lungs, glanders in . . . . . . . 694

Lupine disease, Vol. II .......

Lymphadenia ............ . 869

Lymphadenia, alenken!i. ..... $\$$. 81

Lymphadenia, leukemic . . . . . . 869

Lymphadenitis caseosa orum.. 633

Lymphangioitis epizootica .... 734

Lymphanginitis f a r c $\mathrm{im}$ i nesa bovis .............. 667

Lymphangioitis malleosa .... 700

Lymphangioitis pseudofarcinosa ............... 734

Lymphangioitis reticularis ... $\quad 700$

Lymphangioitis nlcerosa ..... 731

Lymphangioma of heart..... 1077

Lymph, excess of ........ 1119

Lymphorrhagia ........... 1119

Lymphosareoma in heart..... 1077

Lymphosarcomatous swelling of glands ................ 878

Lyssa .................. 463

Macrocytes ............. 863

Maculae albidae ........... 1042

Maculae lactae ............. 1042

Malaria of animals ........ 851

Malaria of man .......... 797

Malaria plasmodia ......... 797

Malarial catarrhal fever ..418, 791

Malarial fever ............. 851

Mal de caderas ........... 835

Mal $\mathrm{du}$ coit............ 815

Malignant eatarrh ......... 408

Malignant edema ........... 32

Malignant malarial fever .... 418

Malignant protozoan jaundice. 791

Mallein ................ 708

Mallein reaction ........... 708

Mallein test, cutaneous ..... 709

Mallein test, organic ....... 709

Mallein test, thermic ........ 709 


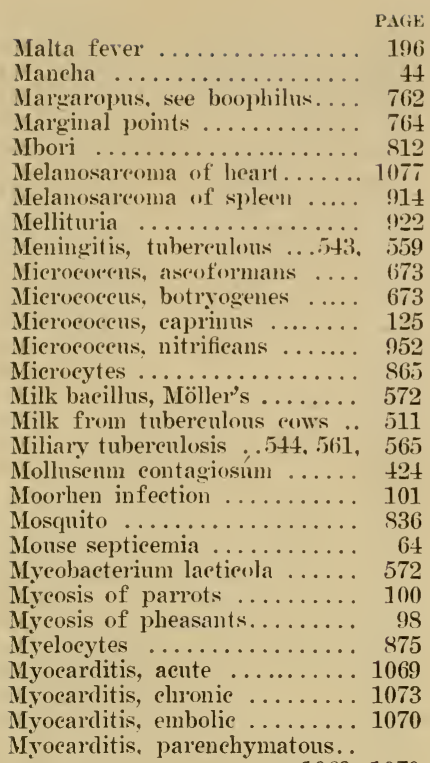

..............1069, 1070

Myocarditis, purulent ...1069, 1070

Myocarditis, septic ........ 1069

Myxomatous disease of rabbits. 102

Myxoma in the heart....... 1077

Nagana ................ 805

Navel ill ................ 158

Neerosis baeillus ........... 419

Necrosis, infectious of eanaries 100

Neplirectomy ............. 1010

Nephritis, acute .......... 988

Nephritis, alba ............ 1000

Nephritis apostematosa ..... 1008

Nephritis, chronic, indurativa. . 1001

Nephritis, diffusa ......... 991

Nephritis fibroplastica ...... 1000

Nephritis fibrosa .......... 1003

Nephritis fibro-resiculosial ..... 1003

Nephritis granulosa ......... 1003

Nephritis interstitialis elsronica. 1001

Nephritis maculosa alba..... 1000

Nephritis mixta ........... 996

Neplnitis mix. non-indurativa. 996

Nephritis mix, parenchymatosa. 996

Nephritis parenchymatosa hemorrhagiea $\ldots \ldots \ldots \ldots \ldots .986$

Nephritis purulenta ....... 1007

Nephrolithiasis ........... 1021

Nettle rash ............ 67
New born, diseases of ...... 145

Noeardia ............... 648

Noduli arthritiei ............ 970

Non-compensating heart valves. 1098

Obesity ................ 915

Oligemia ............. 845

Oligoeythemia ........... 845

Omphalophlebitis septica .... 158

Ophthalnic reaction in glanders 724

Ophthalmic reaction in tuber-

eulosis .............. 587

Opsonins in glanders ....... 724

Opsonins in trypanosomiasis.. - 833

Opsonins in tubereulosis..... 587

Oriental boil ............. 734

Oriental rinderpest ......... 234

Osteochondrodystrophia fetalis. 936

Osteomalacia ............. 950

Osteomalacia, juvenile ....... 938

Osteomalacia, physiologie .... 953

Osteomalacia, relation to osteoporosis .................

Osteomalacia, relation to rachitis $\ldots \ldots \ldots \ldots \ldots \ldots \ldots, 954$

Osteoporosis ….........937, 955

Osteoporosis simulating rachitis. 937

Osteopsathyrosis .......... 950

Onilbek ............... 418

Ovinatio ................. 314

Oxybutyric acid ........925, 927

Palpatio cordis .......... 1055

Panaritium ............. 346

Paracolon bacillosis ........ 151

Paralysis infectiosa ....... 495

Paralysis, vesicae urinariae ... 1036

Paratubereulosis bacilli ...5\%, 641

Paratuberculosis enteritis .... 639

Pasteurella ............. 79

Pasteurellosis ............ 77

Pearl disease ............. 498

Pectus earinatum ........... 944

Pectus gallinaceum ........ 944

Periarteritis nodosa ........ 1105

Pericarditis .............. 1039

Pericarditis eireumseripta .... 1042

Pericarditis diffusa ........ 1042

Pericarditis fibrosa adhesiva .. 1042

Pericarditis trammatica ....... 1042

Peripheral encens-like bodies. . 763

Peripneumonia ........... 389

Perisplenitis ........... 864

Perlsucht ........49, 539, 549

Perlsucht bacillus ........509, 623

Perlsucht hacillus, Möllep's ... 572

Pestis avium ........... 290

pestis bormm orientales...... 234

Pestis ermormm ........... 285 
Pestis gallinarum ........ 290

Pestis summ ............. 257

Petechial fever ........... 216

Pheasant epidemic ......... 98

Phlyctenular eruption ....... 356

Phosphorus content of milk... 931

Phosphorus in foods ........ 932

Phthisis mesaraica ......545, 563

Phthisis tubereulosa pulin .... .............4498,536.

Picotte .................

Piétin contagieux ...........

Pink eye ................

Piroplasma Argentinum ......

Piroplasma bigeminum ......

Piroplasma canis ..........

Piroplasma Donovani ........

Piroplasma equi ............

Piroplasma hirei ..........

Piroplasma horse typhus .....

Piroplasma hominis .........

Piroplasma of influenza ......

Piroplasma mutans ....766,

Piroplasma ovis ...........

Piroplasma parvum ........

Piroplasmosis ............

Piroplasmosis, atypical ......

Piroplasmosis of cattle ......

Piroplasmosis of the $\operatorname{dog} \ldots .$.

Piroplasmosis of the goat ....

Piroplasmosis of the horse ...

Piroplasmosis of man........

Piroplasmosis of the sheep...

Piroplasmosis, tropical .......

Plasmodium malariae .......

Pleuro-pneumonia, contagious, of eattle ...............

Pleuro-pneumonia, contagious, of the horse .............

Pleuro-pneumonia, infectious, of goats ...............

Pleuro-pneumonia, septic, of calves ................

Pneumo-enteritis of turkeys ..

Pneumonia, chronic, of sheep.

Pneumonia, enzootic, of pigs. .

Pneumonia, contagious, of

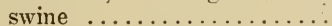

Pneumonia, multiple necrotizing ..................

Pneumo-pericarditis, infectious, of turkeys ..............

Poikilocytosis ...........45s,

Polmonera ...............

Polydipsia $\quad \ldots \ldots \ldots \ldots \ldots \ldots$.

Polyphagiat ..............

Polyneuritis infectiusa .......

Polyuria
549

302

346

170

768

766

792

796

785

790

218

795

174

782

789

778

760

768

764

791

790

784

796

789

782

797

389

170

406

111

99

407

138

131

98

865

389

927

927

515

918
Polyvalent serum in ealf dysentery ..................

Polyvalent serum in hen. septicemia ...............

Polyvalent serum in swine plague ............137,

Polyvalent colon sermm...... 156

Pommeliere ............ 498

Porcher's sugar test........ 927

Pox ................ 295

Pox, abortive ........... 309

Pox, cow ............... 318

Pox of fowls............ 424

Pox of the horse.......... 324

Pox of men ............ 323

Pox, pointed ............ 322

Pox of sheep ........... 302

Pox, stone ............. 322

Pox, swine $\ldots \ldots \ldots \ldots \ldots \ldots \ldots . .325$

Precipitine reaction in glanders. 723

Precipitine reaction in tuberenlosis ............... 587

Proteus bacillosis ......... 152

Proteus in $\operatorname{dog}$ typhus . . . . . 212

Protozoan diseases ......... 760

Protozoan jaundice ......... 784

Psendo acid-fast bacilli...... 572

Pseudoactinomyeosis . ...... 667

Pseudoantlurax bacilli ....... 20

Pseudoblackleg bacilli ....... 44

Pseudo coast fever ....... 782

Pseudocolon bacillosis ....... 148

Pseudoleukemia .......... \$s1

Pseudopericarditis ......... 1051

Pseudorabies ............ 49.

Pseudotuberculosis, baeillary .. 63.5

Psendotubereulosis, zooglique. . 631

Pseudotuberele ............ 633

Pseudotubercle bacilli ........ $\ldots \ldots \ldots \ldots \ldots \ldots 572,633,635$

Pseudotuberculosis ........ 633

Pseudotuberculosis of calves... 6.38

Pseudotuberculosis of eattle... 639

Pseudotuberculusis of sheep. .. 633

Pseudotubereular enteritis.... 641

Psittacosis .............. 100

Pulse, bounding . . . . . . 1091

Puncture of pericardium ..... 1050

Purpura hemorrhagiea ...... $\quad 216$

Pustula maligna ........... 31

Pustular inflam. of mouthe of horses ............... 354

Pyelitis ............... 1019

Pvelitis eatarrhalis ....... 1020

Pyelitis diphtherie:a ........ 1020

Pyelitis hemomhagien ....... 10.

Pyelitis membranace: $\ldots . . . . .1(12)$

Pyelitis ulcernsa .......... 10!20 
Pyelonephritis bacillosa ..... 1010
Pyelonephritis bacteritica bovum 1010

Pyelonephritis eavernosa ..... 1016

Pyelonephritis diphtherica ... 1010

Pyelonephritis mycotica ..... 1010

Pyemia of rabbits.......... 97

Pyemic cachexia .........141, 144

Pyemic joint inflam. of suck-

lings $\ldots \ldots \ldots \ldots \ldots \ldots$.

Pyobacillosis ...............

Pyobacillus bovis .......... 144

Pyobacillus eapsulatus cuniculi. 102

Pyobacillus suis ........... 141

Pyocyaneus bacillosis ....... 151

Pyonephrosis .........1008, 1017

Pyosepticemia of sucklings.... 158

Pyrosoma ............ 760

Quarter ill ..............

Quicksilver, action on blood ves-

Rabies

Rachitis $\ldots \ldots \ldots \ldots \ldots \ldots$

Rachitis, relation to osteomalacia ...............

Rachitis, relation to osteoporosis ................

Rachitis congenita ..........

Rachitis fetalis ...........

Red fever ...............

Red water ...................

Reindeer pest ................

Retentio urinae ........... 1037

Retinitis leukaemica ......... 875

Rhinitis fibrinosa bovum..... 415

Rhinitis gangrenosa bovum.... 408

Rhipicephalus appendiculatus ...............762,

Rhipicephalus bursa ...766,

Rhipicephalus capensis ...762,

Rhipicephalus Evertsi ...762,

Rhipicephalus nitens ....762,

Rhipicephalus sanguineus. .762.

Rhipicephalus simus .....762.

Rhodesian red water.....762,

Rhodesian tick fever.........

Rhusiopathiae suis $\ldots \ldots \ldots \ldots$.

Rickets ............... 930

Rinderpest .............. 234

Rinderpest in sheep ......... 256

Rinderseuche ............. 103

River bottom disease ....... 851

Roseola variolosa .......... 307

Roup .............. 427

Roup bacilliks ............ 427

Ruptura eordis .............. 1076

Ruptura lienis ........... 912

Ruptura vasorum .......... 1117

Saccharomyces farciminosus .. $\quad 735$

Saccharomycosis ........... 734

Salmonellosis of fowls ...... 100

Sareoma of the heart ........ 1077

Sareoma of the kidney ...... 1028

Sarcophysema gangrenosum .. 39

Sarcosporidia in heart muscle.. 1078

Scabrot .............. 302

Schistosomum Bomfordi ..... 906

Schistosomum bovis ........ 906

Schistosomum crassum ...... 906

Schistosomum indicum ...... 906

Schistosomum japonicum .... 906

Sclerosis renum ........... 1003

Sclerosis maculata .......... 1003

Selerostomum larvae in kidney. 1029

Seorbutus ............. 973

Scrofula ........499, 546, 564

Septicemia, apoplectic, of ducks 98

Septicemia, apoplectic, of fowls 101

Septicemia of canaries....... 99

Septicemia, erysipelatous ..... 69

Septicemia, gangrenous ..... 32

Septicemia of geese........ 99

Septicemia, hemorrhagic .... 77

Septicemia of mice......... 64

Septic joint infl. of sucklings.. $\quad 158$

Septic pleuropneumonia of calves ................ 111

Septizidin ............... 96

Sheep pox ............. 302

Sheep pox in man ......... 323

Side chain theory ......... 448

Sleeping sickness in fowls.101, 838

Sleeping sickness in man..... 839

Snuffling disease .......940, 946

Sore heels .............. 324

Souma .............. 837

Soumaya ............. 837

Southern cattle fever ....... 764

Spasmotoxin ............ 442

Spiegler's test ........... 978

Spirillosis of fowls........ 839

Spirillum, see spirochete.

Spirochete, anserina ....... 840

Spirochete, Duttoni ......... 844

Spirochete, equina ......... 843

Spirochete, gallinarum ...... 840

Spirochete, Obermeieri ....... 840

Spirochete, ovina ......... 843

Spirochete, pallida ........ 844

Spirochete, recurentis ........ 844

Spirochete, suilla ......... 843

Spirochete, Theileri ........ 843

Spirochete, Tschichir ...... 843

Spirochete, vaceinae ........ 296

Spirochete in influenza ...... 174

Spirochetosis of fowls....... 839

Spirochetosis of geese ...... 839

Spirochetosis of mammals ... 843 
PAGE

Sipirochetosis of man ....... 844

Spiroptera sanguinolenta ..... 908

Splenic fever .............

Splenitis apostematosa ......

Splenitis chronica indurativa. .

Splenitis hyperplastica ......

Splenomegalia ............

Sporotrichosis ............

Sporotrichum equi ..........

Staphylococei upon walls of

blood ressels ............. 1104

Stellulae Verheyenii ......... 985

Stenosis ostii aortae ... . . . . 1091

Stenosis art. pulm. ......... 1094

Stenosis art. ventr. dextri.... 1095

Stenosis art. ventr. sinistri... 1093

Stenosis ostii venosi dextri... 1095

Stenosis ostii venosi sinistri... 1093

Stephanurus dentata ... . . . . 1030

Stiff sickness ............ 196

Stomatitis aphthosa contagicsa. 328

Stomatitis pustulosa eontagiosa. 360

Stomoxys, brava .......... 836

Stomoxys calcitrans .......812, 819

Strangles .............. 366

Stangles in cattle ......... 388

Strangles in $\operatorname{dogs} \ldots \ldots \ldots . . . .388$

Strangles in swine ........ 388

Strangles, wandering ....... 390

Streptobacillus pseudotubercul. rodentium ............

Streptococcus capsulatus gallinarum ..............

Streptococeus of contagious abortion .............

Streptococeus equi .........

Streptococeus of inf. vaginal

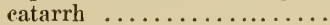

Streptococcus melanogenes ...

Streptococcus of pyogenes equi.

Streptotrichosis ..........

Streptotrichosis of $\mathrm{dogs} . . .$.

Streptotrichosis of horses ....

Streptotrichosis of rabbits ...

Streptotrichosis of ruminants..

Streptothrix actinomycosis ...

Streptothrix canis .........

Streptothrix caprae .........

Streptothrix emiculi .... 102.

Streptothrix farcinica ......

Streptothrix Israeli ........

Streptothrix necrophora ......

Streptothrix valvulae destruens:

Strongylus sublatus .........

Strongylus vasorum ........

Stuttgart disease of dogs .....

Sucholoalbumin ..........

Sucholotoxin ............
Suplagoalbumin .......... 128

Suplagotoxin ........... 128

Suptol ................. 135

Surmenage ............. 106i

Surra ................ 811

Susotoxin ............. 128

Susserin ............. 76

Swamp fever ........... 851

Swan disease ............ 98

Swine erysipelas $\ldots \ldots \ldots \ldots \ldots 60$

Swine fever ........... 257

Swine plaque ........125, 258

Swine pox ............ 325

Syphilis .............. 844

Tabanus bovinus ......... 333

Tabanus lineola .......... 812

Tabanus tropica .......... 812

Tabes dorsalis, Vol. II........

Tabes mesaraica ........... 381

Tachycardia ............ 105.5

Takosis of goats.......... 125

Tauruman ............. 601

Tenesmus vesicae .......... 1032

Tetanin $\ldots \ldots \ldots \ldots \ldots \ldots \ldots .442$

Tetanolysin ............. 448

Tetanotoxin $\ldots \ldots \ldots \ldots \ldots \ldots .142$

Tetamus .............. 439

Tetanus agnorum .......... 454

Tetanus antitoxin ........ 460

Tetan us cerebralis ......... 442

Tetanus dolorosus ......... 442

Tetanus idiopathieus ....... 439

Tetanus neonatorum ....445, 454

Tetanus partialis .......... 453

Tetanus psychicus ......... 439

Tetanus rhemmaticus ....... 439

Tetanus traumaticus ........ 439

Texas fever ...............764

Three days' sickness......... 196

Thrombosis of abdominal arteries ............... 1113

Thrombosis of axillary arteries. 1113 Thrombosis of the carotid arteries .............. 1114

Thrombosis of the iliac arteries 1114

Thrombosis of the portal veins .............. 1116

Thrombosis of the pulmonary arteries .............. 1115

Thrombosis of the rena cava. . 1116

Thrombosis of the reins ..... 1116

Thrombosis with embolismi of arteries . . . . . . . . . 1111

Thrombus canalisatus . . . . . 1111

Thrombus obturans ........ 1111

Thrombus parietalis ....... 1111 
Tick fever ...............

Tolerance tests in diabetes insipidus ................

Tolerance tests in diabetes mellitus

Tophi arthritici ............

Torsio lienis ...............

Treponoma pallidium ........

Tri-acid combinations ........

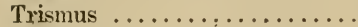

Tristezza ................

Trommer's test for sugar ...

Trypanolysin ..............

Trypannsoma americanum ....

Trypanesoma Brueei ....

Trypanosoma Calmetti ......

Trypanosoma Cazaboui . .811,

Trypanosoma congolense .....

Trypanosoma Cruzi .........

Trypanosoma dimorphon ....

.............800, s11,

Trypanosoma Elmassiani .....

Trypanosoma equinum .......

Trypanosoma equiperdum ....

Trypanosoma Evansi .......

Trypanosoma Frank ........

Trypanosoma gambiense .....

Trypanosoma gigantem .....

Trypanosoma himalayanum ...

Trypanosoma indicum ......

Trypanosoma Lewisii ........

Trypanosoma muktesari ......

Trypanosoma nanum .......

Trypanosoma Pecaudi ....S11.

Trypanosoma sudanese .......

Trypanosoma Theileri.783, 800 ,

Trypanosoma vivax ........

Trypanosomes ............

Tryoanesomes of man ......

Tsetse fly $\ldots \ldots \ldots \ldots \ldots \ldots$.

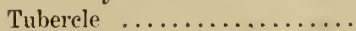

Tubercle bacillus ..........

Tubercle bacillus of birds .....

Tubercle bacillus of cattle ....

Tubercle bacillus of cold-blooded animals ...............

Tubercle bacillus, homogeneous cultures ...............

Tubercle bacillus, inoculation as evidence ..............

Tuberele bacillus of man .....

Tubercele bateillus in milk .....

Tubercle bacillus, microseopic determination ............
Tubercle bacillus, spread in the animal body.......... 533

T'ubercle bacillus staining methods ....................

Tubercle bacillus, virulence of various types ............ 509

Tuberele in birds.......611, (i36

Tubercle, fibrous .... ..... 533

Tubercle-like nodules ....... 532

Tuberele, solitary .......543, 5.57

Tubereulin ............. $57 \frac{1}{2}$

Tubereulin, reactions $\ldots \ldots \ldots$ sit

Tuberculin, test .......... .5.

Tuberculosis ........... 49.

Tuberculosis, aerogenic infection ................ 516

Tubereulosis of birds ...... 617

Tuberculosis of buffaloes ..... 544

Tuberculosis of eats ....548, 567

Tubereulosis of eattle ...... 536

Tubereulosis of cold-blonded animals .............. 630

Tuberculosis, congenital ..... .521

Tuberculosis of dogs ... T547, 56i.

Tuberculosis, eradication ..... 593

Tuberculosis of goats ....... 5til

Tuberculosis, hereditary ..... 5:0

Tuberculosis of horses ...545, 561

Tuberenlosis, latent ........ 530

Tuberculosis, local and general itt

Tubereulosis of mammals .... $49 \mathrm{~s}$

Tuberculosis of man ...... 506

Tuberculosis, miliary ....54t, 561

Tuberculosis, open and closed. 511

Tuberculosis pathogenesis . ... 523

Tubereulosis primary and secondary ................ 5tt

Tuberculosis, protective inmunization $\ldots \ldots \ldots \ldots \ldots \ldots .597$

Tuberculosis, pseudo-bacillary. 635

Tuberculosis, respiratory infection .................... 515

Tuberculosis of sheep ........ .61

Tuberculosis of swine....546, ifit

Tubulonephritis ........... 991

Tumor of the heart ........ 107t

Tumor of the kidney ........ 102s:

Trumor of the spleen. ateute ...

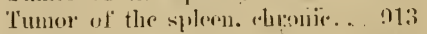

Typhoid fever .......170, 257

Typhus, bovine. contagious.... 234

Typhus of ranaries. ........ 100

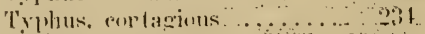

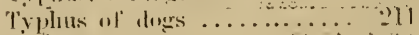

Typhus of fowls ........ 






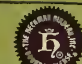

OCT 8 
